\title{
Abstracts of the 53rd ESPN Annual Meeting, Amsterdam, The Netherlands, September 2021
}

Published online: 25 August 2021

(C) IPNA 2021

\section{OP-1 EFFICACY OF IMMUNOGLOBULIN IMMUNOADSORPTION IN THE TREATMENT OF MULTIDRUG RESISTANT IDIOPATHIC NEPHROTIC SYNDROME}

Stephanie Bonneric, Theresa Kwon, Elodie Nattes,Claire Dossier, Julien Hogan

Pediatric Nephrology Department, Robert Debré Hospital

Introduction: Idiopathic nephrotic syndrome is mostly a chronic disease, which can lead to end-stage renal disease in case of cortico-resistance and multidrug resistance. The aim of this study was to analyze the evolution of pediatric patients with multidrug resistant nephrotic syndrome treated by immunoglobulin immunoadsorptions (IA) associated with intravenous immunoglobulins (IvIg).

Material and methods: In this monocentric retrospective study, 23 patients with steroid and multidrug resistant nephrotic syndrome were treated with an association of IA and IvIg, followed by B cell depletion when in remission. The primary outcome was the remission of proteinuria (uPCR $<0,05 \mathrm{~g} / \mathrm{mmol})$.

Results: A remission was obtained in 17 patients. 14 patients responded during the first cycle of treatment (10 daily sessions of IA), and 3 after a more prolonged treatment (up to 3 months). Anti-CD20 monoclonal antibodies were administered to maintain depletion in patients in remission. 10 patients relapsed after the first cycle of IA, and 6 developed a dependency to IA to remain in remission. Only 6 patients did not respond to IA therapy and 2 progressed towards end-stage renal disease. Out of 18 patients with long-term follow-up data (median 22,5 months), 10 patients were in remission ( 7 of them with no immunosuppressive therapies). Conclusions: IA therapy is effective in inducing remission in children with steroid and multidrug resistant nephrotic syndrome. However, more than $50 \%$ of the patients relapse despite B cell depletion and a significant number of these patients become dependent on IA to maintain remission. Newer treatment strategies are needed to maintain remission and allow IA discontinuation in children with steroid and multidrug resistant nephrotic syndrome.

\section{OP-2 SAFETY AND EFFICACY OF OFATUMUMAB IN REFRACTORY PEDIATRIC NEPHROTIC SYNDROME. AN STUDY COHORT}

Yolanda Calzada Baños; Elena Codina Sampera, Pedro Arango Sancho, Víctor López-Báez, Ana Vinuesa Jaca, Lina Catherine Hernández Zúñiga, Álvaro Madrid Aris

Hospital Sant Joan De Déu

Introduction: Ofatumumab (OFA) is an anti-CD-20 monoclonal antibody used in nephrotic syndrome (NS) refractory to conventional treatments and rituximab (RTX). Our objective is to evaluate the safety and efficacy of Ofatumumab in our cohort.

Material and methods: Prospective descriptive study of 2 yr follow-up (2017-2019) in children with NS refractory to first-line therapies, with anti-CD20 monoclonal antibodies indication. We divided the cohort in: steroid-dependent NS (SDNS) without response or with adverse effects related to first-line treatment (Group 1); steroid-resistant SN (SRNS) (Group 2) and SN with post-transplant recurrence (Group 3). Safety and remission rate were evaluated.

Results: 33 patients (21 SDNS, 11 SRNS, 1 recurrence) were included and administered anti-CD20. The male/female ratio was 2: 1 . Mean age at diagnosis was 5.2 years. $100 \%$ of the children (33) received RTX and $18.2 \%$ (6) OFA. RTX achieved complete remission in $87.9 \%$ (29) and $48.3 \%$ of these did not relapsed. $100 \%$ of Group 1 presented complete remission after RTX, although $52.4 \%$ (11) presented at least 1 relapse after 22.9 months (mean 2.5 relapses). In group 2, $72.72 \%$ (8) remitted and $27.2 \%$ (3) presented partial remission. $36.4 \%$ (4) relapsed after 17 months of treatment (mean 1 relapse). Of the 6 who received OFA, $83.3 \%$ presented complete remission (1 SRNS and 4 SDNS) and 1 patient (SDNS) presented relapse at 24 months (mean follow-up 1 year). The other case, a 13-year-old girl with recurrence of focal segmental glomerulosclerosis (FSGS) in kidney transplantation, presented partial remission in association with immunoadsorption sessions. Adverse reactions occurred in 6\% (2): allergic reaction with 2nd dose of RTX and cytokine release syndrome with 1st dose of OFA.

Conclusions: Ofatumumab in our series has proven to be safe and effective therapy in refractory NS, achieving complete remission in 5 patients who had not previously responded to Rituximab

\section{OP-3 EVALUATION OF THE GENETICAL FEATURES AFFECTING RENAL OUTCOME IN CHILDREN WITH STEROID RESISTANT NEPHROTIC SYNDROME}

Elif Comak ${ }^{1}$, Asli Toylu ${ }^{2}$, Ugur Bilge ${ }^{3}$, Gulsah Kaya Aksoy ${ }^{1}$, Mustafa Koyun ${ }^{1}$, Sema Akman ${ }^{1}$

${ }^{1}$ Akdeniz University Medical Faculty, Pediatric Nephrology, Antalya, Turkey, ${ }^{2}$ Akdeniz University Medical Faculty, Medical Genetics, Antalya, Turkey, ${ }^{3}$ Akdeniz University Medical Faculty, Biostatistics And Medical Informatics, Antalya, Turkey

Introduction: Although several studies in children with steroid-resistant nephrotic syndrome (SRNS) have shown that mutations in genes encoding proteins in the podocyte skeleton may be responsible for the etiology in only one-third of cases, the genetic features related with renal prognosis and responde to immunosuppressive are not fully recognized. The aim of this study was to investigate the genetic alterations associate 
with renal prognosis and resistance to immunosuppression in children with SRNS.

Material and methods: The children with SRNS were enrolled in this study. Custom genetic panel was designed for next-generation sequencing analysis of 205 SNPs in 33 target genes.

Results: A total of 25 children, 16 boys ( $64 \%)$, median age at last visit of 17.5 years (13-18 years), median age at diagnosis of 7.5 years (2$15)$, median follow-up of $9.58 \pm 4.54$ years, were included in the study. All patients was diagnosed focal segmental glomerulosclerosis on renal biopsy. 13 patients had normal renal function, while the remaning 12 had end stage renal disease (ESRD) after a median of 4.5 years (2-11 years) follow up period with a median age at ESRD diagnosis of 13.5 years (6-17 years). In study group, patients were treated five different immunosuppressive medications. All patients received steroids and cyclosporin A; 10 patients mycophenolate mofetil, 10 patients tacrolimus, 1 patient cyclophosphamide, 4 patients rituximab. The genetic analysis revealed significant differences in SNP genotype frequencies between groups of ESRD and normal renal function. Genetic alterations in several genes involved in the drug metabolism (CYP3A5, CYP2C19, GSTP1, MPDH2, SLCO1B1), immune response regulation (FCGR3A, FKBP5, CXCL12), glucocorticoid receptor gene (NR3C1) and uromodulin (UMOD) were significantly associated with the lack of response to immunosuppressive treatments and renal function $(\mathrm{p}<0.05)$.

Conclusions: Our results suggest that heterogeneous genetic alterations in children with SRNS associate with resistance to different immunosuppressive treatments and renal function.

\section{OP-4 IN VITRO ASSAYS TO DEMONSTRATE THE PRESENCE OF PLASMA-DERIVED CIRCULATING PERMEABILITY FACTORS IN THE PATHOGENESIS OF FOCAL SEGMENTAL GLOMERULOSCLEROSIS}

Susan Veissi ${ }^{1}$, Bart Smeets ${ }^{2}$, Joanna Van Wijk ${ }^{3}$, Floor Veltkamp ${ }^{5}$, Rene Classens ${ }^{1}$, Thea Van Der Velden ${ }^{1}$, Annelies Jeronimus-klaasen ${ }^{1}$, William Morello ${ }^{4}$, Giovanni Montini ${ }^{4}$, Antonia Bouts ${ }^{5}$, Lambertus P. W. J. Van Den Heuvel ${ }^{1}$, Michiel Schreuder ${ }^{1}$

${ }^{I}$ Department Of Pediatric Nephrology, Amalia Childrens Hospital, Radboud University Medical Center, Radboud Institute For Molecular Life Sciences, Nijmegen, The Netherlands, ${ }^{2}$ Department Of Pathology, Radboud University Medical Center, Radboud Institute For Molecular Life Sciences, Nijmegen, The Netherlands, ${ }^{3}$ Department Of Pediatric Nephrology, Amsterdam University Medical Center, Amsterdam, The Netherlands, ${ }^{4}$ Department Of Pediatric Nephrology, Dialysis And Transplant Unit, Foundation Irccs Cà Granda, Irccs Ospedale Maggiore Policlinico, Milan, Italy, ${ }^{5}$ Department Of Pediatric Nephrology, Amsterdam Umc, University Of Amsterdam, Amsterdam, The Netherlands

Introduction: Circulating permeability factors (CPFs) involved in the pathogenesis of idiopathic focal segmental glomerulosclerosis (FSGS) can lead to early recurrence of FSGS and kidney failure after transplantation. Identification of FSGS patients with CPFs is clinically important as it can predict treatment response and prognosis. Currently, kidney biopsy is the gold standard diagnosis. Therefore, there is an increased demand for diagnostic assays to determine the presence of CPFs in the sera of FSGS patients. Using conditionally immortalized human podocytes as a substrate, we aim to demonstrate the presence of plasma-derived CPFs using series of in vitro assays.

Material and methods: Podocytes and primary glomerular endothelial cells (GMVECs) were incubated with plasma from biopsy proven FSGS patients in relapse and remission as well as from steroidresistant nephrotic syndrome (SRNS), minimal change NS (MCNS), membranous nephropathy (MN), a non-renal control patient, and healthy controls. Cell viability, podocyte actin cytoskeleton architecture, and reactive oxygen species (ROS) formation in the presence or absence of ROS scavenger, dimethylthiourea, were investigated by CCK-8 assay, immunofluorescence staining, and CMH2DCFDA probing, respectively.

Results: We show that plasma of patients with FSGS causes a series of events in podocytes but not in endothelial cells. These events include actin cytoskeleton rearrangement, excessive formation of ROS, and eventually also cell death. These effects were solely observed in response to plasma of relapse FSGS patients, but not in response to plasma of kidney patients with SRNS, MCNS, MN and healthy controls. The co-presence of dimethylthiourea, abolished these effects.

Conclusions: Altogether, we provide a panel of assays to measure podocyte injury and predict the presence of CPFs in FSGS plasma, providing a new framework for monitoring CPF activity that can be used for diagnostics or disease monitoring purposes. Moreover, our findings suggest that the inhibition of ROS formation or facilitating rapid ROS scavenging may exert beneficial effects in FSGS patients.

\section{OP-5 KIDNEYNETWORK: USING KIDNEY-DERIVED GENE EXPRESSION DATA TO PREDICT AND PRIORITIZE NOVEL GENES INVOLVED IN KIDNEY DISEASE}

Floranne Boulogne ${ }^{1}$, Laura R Claus ${ }^{2}$, Henry Wiersma ${ }^{1}$, Roy Oelen ${ }^{1}$, Floor Schukking ${ }^{1}$, Niek De Klein ${ }^{1}$, Shuang $\mathrm{Li}^{1}$, Harm-Jan Westra ${ }^{1}$, Bert Van Der Zwaag ${ }^{2}$, Franka Van Reekum ${ }^{3}$, Dana Sierks ${ }^{4}$, Ria SchÖnauer ${ }^{4}$, Jan Halbritter ${ }^{4}$, Nine V.a.m. Knoers ${ }^{1}$, Genomics England Research Consortium ${ }^{5}$, Patrick Deelen ${ }^{2}$, Lude Franke ${ }^{1}$, Albertien M Van Eerde ${ }^{2}$

${ }^{I}$ Department Of Genetics, University Medical Center Groningen, University Of Groningen, Groningen, The Netherlands, ${ }^{2}$ Department Of Genetics, University Medical Center Utrecht, Utrecht University, Utrecht, The Netherlands ${ }^{3}$ Department Of Nephrology, University Medical Center Utrecht, Utrecht, The Netherlands, ${ }^{4}$ Medical Department Iii Endocrinology, Nephrology, Rheumatology Department Of Internal Medicine, Division Of Nephrology, University Of Leipzig Medical Center, Leipzig, Germany, ${ }^{5}$ Genomics England, London, UK

Introduction: Genetic testing in patients with suspected hereditary kidney disease does not always reveal the genetic cause for the patients disorder. Potentially pathogenic variants can reside in genes that are not known to be involved in kidney disease, which makes it difficult to prioritize and interpret the relevance of variants in these genes. To help identify candidate genes for kidney disease we have developed KidneyNetwork, in which tissue-specific expression is utilized to predict kidney-specific gene functions.

Material and methods: KidneyNetwork is a co-expression network built upon a combination of 878 kidney RNA-sequencing samples and a multi-tissue dataset of 31,499 samples. It uses expression patterns to predict which genes have a kidney-related function and which phenotypes might result from mutations in these genes. As proof of principle, we applied KidneyNetwork to prioritize rare variants in exome-sequencing data from 13 kidney disease patients.

Results: We assessed the prediction performance of KidneyNetwork by comparing it to GeneNetwork, a multi-tissue co-expression network we previously developed. In KidneyNetwork, we observe a significantly improved prediction accuracy of kidney-related HPOterms, as well as an increase in the total number of significantly predicted kidney-related HPO-terms (figure 1). Applying KidneyNetwork to exome-sequencing data of kidney disease patients allowed us to identify $A L G 6$ as promising candidate gene for kidney and liver cysts. 


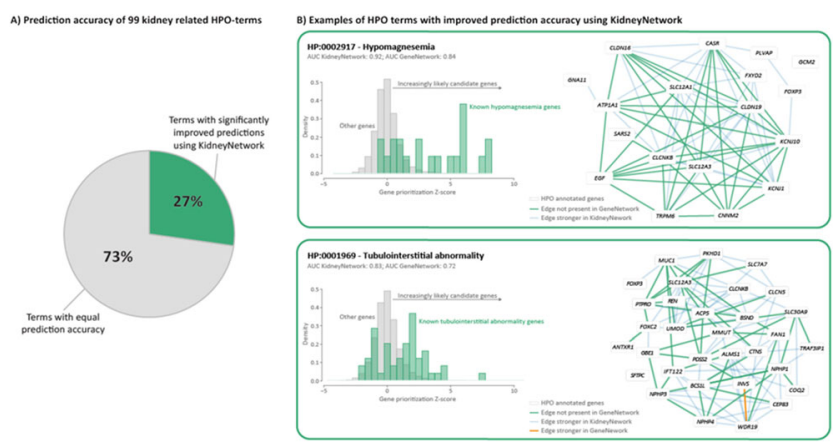

Conclusions: We present KidneyNetwork, a kidney-specific co-expression network that predicts which genes have kidney-specific functions and can result in kidney disease. Gene-phenotype associations of genes unknown for kidney-related phenotypes can be predicted by KidneyNetwork. We show the added value of KidneyNetwork by applying it to kidney disease patients without a molecular diagnosis. KidneyNetwork can be applied to clinically unsolved kidney disease cases, but it can also be used by researchers to gain insight into individual genes to better understand kidney physiology and pathophysiology.

\section{OP-6 HYPERURICEMIA IS STRONGLY DEPENDENT ON RENAL FUNCTION AND HYPERGLYCEMIA, WHICH LIMITS ITS CLINICAL UTILITY AS A PREDICTOR OF HNF1B NEPHROPATHY}

Marcin Kołbuc ${ }^{1}$, Beata Bieniaś ${ }^{2}$, Sandra Habbig ${ }^{3}$, Mateusz F. Kołek ${ }^{4}$, Maria Szczepańska ${ }^{5}$, Katarzyna Kiliś-pstrusińska ${ }^{6}$, Anna Wasilewska ${ }^{7}$, Piotr Adamczyk ${ }^{8}$, Rafał Motyka ${ }^{1}$, Marcin Tkaczyk ${ }^{9}$, Przemysław Sikora ${ }^{2}$, Bodo B. Beck ${ }^{10}$, Marcin Zaniew ${ }^{1}$

${ }^{1}$ Department Of Pediatrics, University Of Zielona Góra, Zielona Góra, Poland, ${ }^{2}$ Department Of Pediatric Nephrology, Medical University Of Lublin, Lublin, Poland, ${ }^{3}$ Department Of Pediatrics, University Hospital Of Cologne, Cologne, Germany, ${ }^{4}$ Department Of Animal Physiology, Faculty Of Biology, University Of Warsaw, Warsaw, Poland, ${ }^{5}$ Department Of Pediatrics, Faculty Of Medical Sciences In Zabrze, Medical University Of Silesia In Katowice, Zabrze, Poland, ${ }^{6}$ Department Of Paediatric Nephrology, Wroclaw Medical University, Wroclaw, Poland, ${ }^{7}$ Department Of Pediatric Nephrology, University Hospital, Bialystok, Poland, ${ }^{8}$ Department Of Pediatrics, Faculty Of Medical Sciences In Katowice, Medical University Of Silesia In Katowice, Katowice, Poland, ${ }^{9}$ Department Of Pediatrics, Immunology And Nephrology, Polish Mothers Memorial Hospital Research Institute, Lodz, Poland, ${ }^{10}$ Institute Of Human Genetics And Center For Molecular Medicine Cologne, University Of Cologne, Faculty Of Medicine And University Hospital Cologne, Cologne, Germany

Introduction: Mutations in the hepatocyte nuclear factor-1beta (HNF1B) gene are considered to be one of the most common genetic causes of congenital anomalies of the kidneys and urinary tract (CAKUT). Diagnosis of $H N F 1 B$-related disease is difficult, and individuals with mutations in $H N F 1 B$ are likely undiagnosed. One of the features, which could serve as a predictor of the disease, is hyperuricemia. However, neither a causal relationship nor its predictive value have been proven. We thus assessed this in children with CAKUT, both with (mut+) and without $H N F 1 B$ mutations (mut-).

Material and methods: We performed a retrospective analysis of clinical characteristics of pediatric patients tested for $H N F 1 B$ mutations, collected in a national registry. The most recent, age- and sex-dependent pediatric norms for sUA were used.
Results: 108 children were included in the study, comprising 43 mut+ patients and 65 mut- subjects. Mean serum uric acid (sUA) was higher in mut+ than in mut- subjects $(p=0.006)$, and hyperuricemia was more prevalent in $H N F 1 B$ carriers ( $42.5 \%$ vs. $15.4 \%, p=0.002)$. The groups were similar with respect to kidney function and fractional excretion of uric acid (FEUA). Hyperglycemia and pancreatic anomalies were more prevalent in the mut+ group. After exclusion of patients with these confounders, which are independent predictors of SUA, the difference in hyperuricemia was accounted for. Kidney function and FEUA were also independent predictors of sUA. Mutation was identified as a minor determinant of sUA. The potential of hyperuricemia to predict mutation was low, and addition of hyperuricemia to a multivariate logistic regression model did not increase its accuracy.

Conclusions: Hyperuricemia is relatively common in children with CAKUT due to HNF1B mutations, however its direct association with this molecular defect remains unproven. Its dependence on kidney function and hyperglycemia diminishes its ability to predict $H N F 1 B$ disease.

\section{OP-7 KIDNEY INJURY IN A LARGE COHORT OF CHILDREN WITH SOLITARY FUNCTIONING KIDNEY}

Sander Groen In T Woud ${ }^{1}$, Nel Roeleveld ${ }^{1}$, Wout Feitz ${ }^{2}$, Sofia Study Group ${ }^{1}$, Michiel Schreuder ${ }^{3}$, Loes Van Der Zanden ${ }^{1}$

${ }^{1}$ Radboud University Medical Center, Department For Health Evidence, Radboud Institute For Health Sciences, Nijmegen, The Netherlands, ${ }^{2}$ Radboudumc Amalia Children's Hospital, Department Of Urology, Radboud Institute For Molecular Life Sciences, Nijmegen, The Netherlands, ${ }^{3}$ Radboudumc Amalia Children's Hospital, Department Of Pediatric Nephrology, Radboud Institute For Molecular Life Sciences, Nijmegen, The Netherlands

Introduction: A solitary functioning kidney (SFK) in children is a condition resulting from a congenital anomaly of the kidney and urinary tract or acquired later in childhood. Patients with SFK are at higher risks of kidney injury, although it is unclear to which magnitude. Our objective was to investigate the risk of and risk factors for proteinuria, high blood pressure, a decreased glomerular filtration rate (GFR), or use of antihypertensive medication in children with SFK.

Material and methods: Children with congenital and acquired SFK were recruited in over 30 hospitals throughout the Netherlands. Information on risk factors for and signs of kidney injury were collected from electronic patient files. Kaplan-Meier curves were used to estimate survival without signs of kidney injury and Cox regression was used to evaluate risk factors.

Results: In total, 982 children provided informed consent and detailed clinical information was available from 898 (91\%). Median follow-up duration was 9.7 years. Proteinuria was present in $118(15 \%)$, high blood pressure in $184(22 \%)$, a GFR below $60 \mathrm{ml} / \mathrm{min} / 1.73 \mathrm{~m}^{2}$ in $23(3.2 \%)$, and antihypertensive medication was used by 90 (9.8\%). In total, 319 children (36\%) exhibited one or more signs of kidney injury and the median age at first sign of kidney injury was 4.4 year. Cumulative proportions of children with kidney injury were $20 \%$ at 5 years, $29 \%$ at 10 years, and $35 \%$ at 15 years of age. No differences in kidney injury rates were observed between boys and girls, left- or right-sided SFK, congenital or acquired SFK, and inclusion via secondary or tertiary hospital.

Conclusions: Data from this largest SFK cohort so far indicates that one third of patients with SFK has one or more signs of kidney injury at 15 years of age. Other risk factors will be investigated in our cohort to develop care strategies based on individual-patient risk profiles., 
OP-8 PHENOTYPE-GENOTYPE CORRELATION AND CLINICAL CHARACTERISTICS OF PEDIATRIC PATIENTS WITH ALPORT SYNDROME

MichaŁ Pac ${ }^{1}$, Łukasz Obrycki ${ }^{1}$, Paulina Halat-Wolska ${ }^{2}$, ElŻbieta Ciara ${ }^{2}$, Janusz Feber ${ }^{3}$, MieczysŁaw Litwin ${ }^{1}$

${ }^{1}$ Department Of Nephrology, Kidney Transplantation And Hypertension, Children`s Memorial Health Institute, Warsaw, Poland, ${ }^{2}$ Department Of Medical Genetics, The Children's Memorial Health Institute, Warsaw, Poland, ${ }^{3}$ Division Of Nephrology, Department Of Pediatrics, The Children's Hospital Of Eastern Ontario, Ottawa

Introduction: We aimed to analyse the correlation between clinical characteristics of Alport syndrome patients and their genotype.

Material and methods: Next-generation sequencing was performed in 84 patients ( 40 girls, $48 \%$; mean age $12.3 \pm 5.5$ years) referred to the Department of Nephrology, Kidney Transplantation and Hypertension in Children's Memorial Health Institute in Warsaw with suspected Alport syndrome. We determined the presence of pathogenic COL4A3, COL4A4 and COL4A5 variants and compared it with the clinical manifestations of the disease including renal function, proteinuria, hematuria, arterial hypertension, ocular abnormalities and hearing defects.

Results: Pathogenic variants were found in total of 68 patients $(81 \%)$, COL $4 A 3$ in 7 patients (8\%), COL4A4 in 13 patients $(15 \%)$, COL $4 A 5$ in 48 patients $(57 \%)$, including the most common variant p.Gly624Asp in 13 patients (15\%), 2 patients $(2 \%)$ had co-existing COL $4 A 3$ and COLAA5 common variant. Fourteen patients (17\%) had no mutation identified. Statistically significant differences in microalbuminuria $(\mathrm{p}=0.020)$ and protein excretion $(\mathrm{p}=0.048)$ between the genotypes were found. Patients with non-p.Gly624AspCOL4A5 mutation were younger at hematuria detection $(\mathrm{p}=0.034)$, had higher microalbuminuria $(\mathrm{p}=0.008)$ and protein excretion $(p=0.013)$ and more often presented ocular symptoms $(p=0.009)$. The predictors of GFR reduction for all patients were: older age of diagnosis $(p=0.005)$, hypertension $(p=0.007)$ and hypertension coexisting with proteinuria above $500 \mathrm{mg} / 24 \mathrm{~h}(\mathrm{p}=0.007)$; and for non-p.Gly624Asp-COL4A5 patients: male gender $(\mathrm{p}=0.035)$, ocular symptoms $(\mathrm{p}=0.007)$ and microalbuminuria $(\mathrm{p}=0.054)$.

Conclusions: Patients with non-p.Gly624Asp-COL4A5 mutation had the most severe clinical manifestation with male gender, ocular symptoms and microalbuminuria as predictors of lower GFR.

\section{Partially supported: CMHI-M29/18}

\section{OP-9 ECULISHU: ECULIZUMAB IN PEDIATRIC PATIENTS AFFECTED BY SHIGA-TOXIN RELATED HEMOLYTIC AND UREMIC SYNDROME}

Garnier Arnaud ${ }^{1}$, Brochard Karine ${ }^{1}$, Allain-Launay Emma ${ }^{2}$, Allard Lise ${ }^{4}$, Caillez Mathilde ${ }^{12}$, Cloarec Sylvie ${ }^{17}$, De Parscauc Loic ${ }^{7}$, Djamal-Dine Djeddi ${ }^{3}$, Fila Marc ${ }^{13}$, Fremeaux-Bacchi Veronique ${ }^{18}$, Guigonis Vincent ${ }^{11}$, Kwon Theresa ${ }^{15}$, Lahoche Annie ${ }^{9}$,

Llanas Brigitte ${ }^{6}$, Michel-Bourdat Guylène ${ }^{8}$, Nobili Francois ${ }^{5}$,

Salomon Remi ${ }^{14}$, Sellier-Leclerc Anne-Laure ${ }^{10}$, Taque Sophie ${ }^{16}$ Ulinski Tim ${ }^{1}$, Morin Christophe ${ }^{1}$, Olivier-abbal Pascal ${ }^{1}$,

Arnaud Catherine ${ }^{1}$, Brusq Clara ${ }^{1}$, Kieffer Isabelle ${ }^{1}$

${ }^{1}$ Chu Toulouse, ${ }^{2}$ Chu Nantes, ${ }^{3}$ Chu Amiens, ${ }^{4}$ Chu Angers, ${ }^{5}$ Chu Besancon, ${ }^{6}$ Chu Bordeaux, ${ }^{7}$ Chu Brest ${ }^{8}$ Chu Grenoble, ${ }^{9}$ Chu Lille, ${ }^{10}$ Chu Lyon, ${ }^{11}$ Chu Limoges, ${ }^{12}$ Chu Marseille, ${ }^{13}$ Chu Montpellier, ${ }^{14}$ Chu Necker, Paris, ${ }^{15}$ Chu Robert Debre, Paris, ${ }^{16}$ Chu Rennes, ${ }^{17}$ Chu Tours, ${ }^{18}$ Chu Hegp, Paris
Introduction: Shiga-toxin related HUS (STEC-HUS) in pediatric patients is severe with at least $50 \%$ of affected children requiring dialysis. Mortality rates can reach 5\% and long-term renal sequels occur in $30 \%$ of surviving patients. No specific treatment has proven its efficacy. Activation of the complement alternative pathway (CAP), a well known pattern of atypical HUS (aHUS) has been postulated in STEC-HUS patients. Eculizumab (EC), a monoclonal C5 antibody, which inhibits CAP activation has been proposed in STEC-HUS patients with conflicting results. Considering the lack of therapy in STEC-HUS, controlled study of EC efficiency was mandatory.

Material and methods: We conducted a multicentric, single blinded, versus placebo trial of EC in pediatric STEC-HUS patients. Patients under 18 years were randomized in a 1:1 ratio to receive either EC or placebo during 4 weeks. Follow-up lasted for 1 year after treatment. Primary end point was the occurrence of a dialysis $>48 \mathrm{~h}$. Secondary end points included renal, hematological and other organs involvement. Safety was assessed throughout the course of the trial.

Results: Of 100 patients who underwent randomization, 14 did not complete the trial. Among the 86 analyzed patients (42 in the placebo and 44 in the EC group) demographic characteristics were similar. There was no difference in the occurrence of a dialysis $>48 \mathrm{~h}$ between the 2 groups $(43 \%$ in the placebo and $57 \%$ in the EC group). No difference was found in the general course of acute renal failure or in the occurrence of mid-term renal sequels (6 and 12 months after the treatment phase). Hematological involvements as well as extrarenal manifestations of the HUS were also similar between the 2 groups.

Conclusions: In pediatric STEC-HUS patients, treatment with EC is not associated with either an improvement of the course of neither the acute phase of the disease nor a prevention of midterm renal sequels.

\section{OP-10 ECULIZUMAB THERAPY MONITORING: RESIDUAL HEMOLYSIS IN ALTERNATIVE PATHWAY TEST IS NOT CAUSED BY C5 ACTIVITY}

Bert Van Den Heuvel ${ }^{1}$, Nicole Van De Kar ${ }^{1}$, Andrei Sarlea ${ }^{1}$, Sanne Van Kraaij ${ }^{1}$, Thea Van Der Velden ${ }^{1}$, Wilhelmus Liebrand ${ }^{1}$, Caroline Duineveld ${ }^{1}$, Romy Bouwmeester ${ }^{1}$, Jack Wetzels ${ }^{1}$, Tom Eirik Mollnes ${ }^{2}$, Elena Volokhina ${ }^{1}$

${ }^{1}$ Radboud University Medical Center, Nijmegen, The Netherlands, ${ }^{2}$ Oslo University Hospital, Oslo, Norway

Introduction: Eculizumab is a C5-blocker used in the treatment of atypical hemolytic uremic syndrome (aHUS). Hemolytic assays are often used to monitor classical (CP) and alternative pathway (AP) blockade in these patients. The AP assay is known to show hemolysis in patient samples that attain target drug concentrations $>100$ $\mu \mathrm{g} / \mathrm{mL}$. This suggests incomplete complement blockade and may lead to a change of therapy (higher dose or alternative drug). Therefore, we investigated whether the residual hemolysis detected by the AP assay in the presence of eculizumab is caused by incomplete $\mathrm{C} 5$ blockade by the drug.

Material and methods: Normal human serum (NHS) spiked with eculizumab $(100,200$ and $500 \mu \mathrm{g} / \mathrm{mL})$, five aHUS samples containing $256-371 \mu \mathrm{g} / \mathrm{mL}$ of the drug and serum of $\mathrm{C} 5$ deficient donor were tested in CP and AP hemolytic assays and ELISA-based Wieslab ${ }^{\circledR}$ tests for complement activity. Eculizumab, C5 and complement activation markers were assessed by ELISAs. Mechanical fragility was assessed by exposing erythrocytes to low $\mathrm{NaCl}$ concentrations. 
Results: All eculizumab samples and C5 deficient serum had comparable high (up to $81 \%$ ) hemolysis in AP test, but no measurable complement activity in CP hemolytic assay and ELISA-based AP and CP tests. Furthermore, NHS spiked with $100 \mu \mathrm{g} / \mathrm{mL}$ of eculizumab and stimulated by $100 \mu \mathrm{g} / \mathrm{mL}$ of zymosan A produced time-dependent increase of $\mathrm{C} 3$ activation markers $\mathrm{C} 3 \mathrm{bBbP}, \mathrm{C} 3 \mathrm{bc}$ and $\mathrm{C} 3 \mathrm{a}$ but not of the $\mathrm{C} 5$ activation marker sC5b-9. Erythrocytes remaining after AP assay of eculizumab samples were fragile in osmotic test. This fragility was abolished by adding C3-inhibitor compstatin.

Conclusions: Hemolysis in AP hemolytic assay under eculizumab treatment is not caused by the residual C5 activity. Likely, it is caused by deposition of $\mathrm{C} 3$ activation products, which weaken the erythrocytes and make them more prone to hemolysis. ELISA-based methods are the better option to monitor C5 blockade during eculizumab treatment.

\section{OP-11 EXTENSIVE COMPLEMENT ANALYSIS DURING FOLLOW-UP OF A PEDIATRIC C3 GLOMERULOPATHY COHORT}

Marloes A.H.M. Michels ${ }^{1}$, Elena B. Volokhina ${ }^{1}$, Janne De Klein ${ }^{1}$, Roel A.J. Kurvers ${ }^{1}$, Kioa L. Wijnsma ${ }^{1}$, Joanna A.E. Van Wijk ${ }^{2}$, Antonia H. Bouts ${ }^{2}$, Valentina Gracchi ${ }^{3}$, Flore Horuz ${ }^{4}$,

Mandy G. Keijzer-Veen ${ }^{5}$, Eiske M. Dorresteijn ${ }^{6}$, Lambertus P.W.J. Van Den Heuvel ${ }^{1}$, Nicole C.A.J. Van De Kar ${ }^{1}$

${ }^{1}$ Department Of Pediatric Nephrology, Amalia Children's Hospital, Radboud University Medical Center, Nijmegen, The Netherlands, ${ }^{2}$ Department Of Pediatric Nephrology, Emma Children's Hospital, Amsterdam University Medical Center, Amsterdam, The Netherlands, ${ }^{3}$ Department Of Pediatric Nephrology, Beatrix Children's Hospital, University Of Groningen, Groningen, The Netherlands, ${ }^{4}$ Department Of Pediatric Nephrology, Academic Medical Center Maastricht, Maastricht, The Netherlands, ${ }^{5}$ Department Of Pediatric Nephrology, Wilhelmina Children's Hospital, University Medical Center Utrecht, Utrecht, The Netherlands, ${ }^{6}$ Department Of Pediatric Nephrology, Sophia Children's Hospital, Erasmus Medical Center, Rotterdam, The Netherlands

Introduction: $\mathrm{C} 3$ glomerulopathy $(\mathrm{C} 3 \mathrm{G})$ is a rare kidney disorder characterized by predominant glomerular depositions of complement $\mathrm{C} 3$, resulting from alternative pathway (AP) dysregulation. $\mathrm{C} 3 \mathrm{G}$ can be subdivided into dense deposit disease (DDD) and $\mathrm{C} 3$ glomerulonephritis (C3GN). In this study we retrospectively analyzed the presence of complement-directed autoantibodies and the complement biomarker profiles of 29 pediatric $\mathrm{C} 3 \mathrm{G}$ patients.

Material and methods: $\mathrm{C} 3$ levels were retrieved from the patient files. The complement activation markers $\mathrm{C} 3 \mathrm{bBbP}$ (properdin-stabilized $\mathrm{C} 3$ convertase), C3bc (C3 activation products), soluble C5b-9 (sC5b-9; terminal complement complex), complement component $\mathrm{C} 5$, positive regulator properdin, and negative complement regulators Factor $\mathrm{H}(\mathrm{FH})$ and Factor I were measured using sandwich ELISA. Convertase activity assays were used to detect the presence of $\mathrm{C} 3$ nephritic factors $(\mathrm{C} 3 \mathrm{NeFs})$ and $\mathrm{C} 4$ nephritic factors (C4NeFs). ELISA was used to screen for $\mathrm{FH}$ autoantibodies

Results: C3NeFs were found in 20/28 patients (12/18 DDD, 8/10 $\mathrm{C} 3 \mathrm{GN}$ ). One patient (DDD) additionally carried C4NeFs and two patients (1 DDD, $1 \mathrm{C} 3 \mathrm{GN}$ ) additionally carried FH autoantibodies. C3NeFs remained detectable over time despite immunosuppressive treatment. At presentation, $\mathrm{C} 3$ levels were decreased in 21/25 patients, indicating complement consumption. During follow-up, in about $50 \%$ of patients, all of them $\mathrm{C} 3 \mathrm{NeF}-$ positive, $\mathrm{C} 3$ levels remained low. Linear mixed model analysis showed that $\mathrm{C} 3 \mathrm{GN}$ patients had higher $\mathrm{SC} 5 \mathrm{~b}-9$ and lower properdin levels compared to DDD patients.

Conclusions: The majority of pediatric patients with $\mathrm{C} 3 \mathrm{G}$ show signs of complement activation in their blood. $\mathrm{C} 3 \mathrm{NeFs}$ were associated with lower $\mathrm{C} 3$ levels at last measurement. $\mathrm{C} 3 \mathrm{GN}$ patients showed higher sC5b-9 and lower properdin levels compared to DDD patients. These findings may aid in further understanding the AP dysregulation in $\mathrm{C} 3 \mathrm{G}$ and in the development and monitoring of novel complement-directed treatment strategies in the near future. Given the rarity and heterogeneity of $\mathrm{C} 3 \mathrm{G}$, prospective longitudinal studies are needed to further investigate the complement profiles over time in $\mathrm{C} 3 \mathrm{G}$.

OP-12 EVALUATION OF THE COMBINATION OF IMMUNOADSORPTIONS WITH ECULIZUMAB IN THE TREATMENT OF NEUROLOGICAL INVOLVEMENT IN PEDIATRIC SHIGATOXIN-ASSOCIATED HEMOLYTIC UREMIC SYNDROME (STEC-HUS)

Charlotte Duneton ${ }^{1}$, Florian Manca Barayre ${ }^{1}$, Olivia Gillion Boyer ${ }^{2}$, Theresa Kwon ${ }^{1}$, Julien Hogan ${ }^{1}$

${ }^{1}$ Pediatric Nephrology Department, Robert Debré Hospital, Aphp, Paris France. ${ }^{2}$ Pediatric Nephrology Department, Necker Enfants-malades Hospital, Aphp, Paris France

Introduction: Evidence to guide the treatment of STEC-HUS with neurological involvement are lacking. The use of Eculizumab, has been reported in a few pediatric series yielding conflicting results. Antibodymediated mechanisms have also been suggested and immunoadsorption of immunoglobulins (IgIA) was tested in a cohort of adult patients. We evaluated the combination of IgIA with eculizumab (IgIA-eculi) as a recue therapy in pediatric patients.

Material and methods: We retrospectively analyzed neurologically impaired HUS patients with confirmed STEC infections treated with IgIAeculi $(n=19)$ or with eculizumab $(n=24)$ in two pediatric nephrology centers in Paris between 2010 and 2021. The primary outcome was the neurological evaluation at 1 year dichotomized as normal vs. abnormal. Results: Patients' characteristics at presentation did not significantly defer between the groups, however patients in the IgIA-eculi tended to be older 3,6 vs. 2,1 years and have more severe neurological presentation with confusion in $37 \%$ (vs. $21 \%$ ), paresis in $26 \%$ (vs. $4 \%$ ), seizures in $84 \%$ (vs. $75 \%$ ) and status epilepticus in $42 \%$ (vs. $21 \%$ ). They also tend to require more often mechanical ventilation (44\% vs. $35 \%$ ) and dialysis $(95 \%$ vs. $71 \%$ ). Each patient received a mean of 7 sessions in the IgIA-eculi group and complement blockade occurred after the first injection of eculizumab in $70 \%$ of cases. No significant association between the treatment group and the neurological evaluation at 1 year of follow-up was found (OR for abnormal evaluation in the IgIA-eculi 1,4; IC95\% 0,2-8,1) after adjustment for age, sex and initial neurological presentation. The only factor independently associated with neurological status at 1 year was the presentation with status epilepticus at diagnosis (OR 12,2; IC95\% 2-76).

Conclusions: This is the first pediatric study reporting on the use of immunoadsorptions in combination with eculizumab as a rescue therapy in STEC-HUS with neurological involvement. We found no benefit to the addition of immunoadsorptions to eculizumab therapy on neurological outcome after 1 year of follow-up.

\section{OP-13 PHOSPHATE STABILIZES CARDIAC FGF23 VIA GALNT3 AND THEREBY WORSENS CARDIAC FUNCTION}

Maren Leifheit-Nestler ${ }^{1}$, Isabel Vogt ${ }^{1}$, Andrea Grund ${ }^{1}$, Beatrice Richter ${ }^{1}$, Oliver J. Müller ${ }^{2}$, Dieter Haffner ${ }^{1}$

${ }^{1}$ Department Of Paediatric Kidney, Liver And Metabolic Diseases, Hannover Medical School, Germany, ${ }^{2}$ Department Of Internal Medicine Iii, University Hospital Kiel, Germany

Introduction: FGF23 is associated with LVH in patients with and without CKD. Whether elevated FGF23 per se causes LVH or a further challenge such as high phosphate is required for FGF23 to tackle the heart is controversially discussed. 
Material and methods: By generating a mouse model with high intracardiac FGF23 synthesis using an adeno-associated virus (AAV), we investigated the effects of cardiac FGF23 on the heart per se and after feeding a high phosphate diet (HPD). In vivo data were verified in neonatal rat ventricular myocytes (NRVM) in vitro.

Results: AAV-Fgf23 mice showed increased cardiac-specific Fgf23 synthesis and enhanced circulating iFgf 23 levels. Serum of AAV-Fgf23 mice stimulated hypertrophic growth of NRVM and pro-hypertrophic gene expression. Activation of renal FGFR1/Klotho/MAPK signalling downregulating $\mathrm{NaPi} 2 \mathrm{a} / 2 \mathrm{c}$ and a concomitant reduction in tubular phosphate reabsorption were observed in AAV-Fgf23 mice, indicating bioactivity of cardiac-secreted iFgf23. However, neither cardiac dysfunction nor LVH or LV fibrosis was observed.

In contrast, HPD caused increased LV diameters, LV volumes, blood pressure and arterial elastance that were more pronounced in AAVFgf 23 than in controls. Bone $F g f 23$ was stimulated in both controls and AAV-Fgf 23 on HPD, while intra-cardiac $F g f 23$ mRNA levels were only increased in AAV-Fgf 23 groups irrespective of the diet. Interestingly, Oglycosylation of cardiac iFgf 23 was enhanced in AAV-Fgf 23 mice on HPD, indicating protein stabilization. Concomitantly, HPD increased Galnt3 mRNA expression and circulating iFgf23 levels in controls and AAV-Fgf 23 on HPD compared to their respective normal phosphate dietfed controls. Verifying in NRVM, high phosphate induced Galnt3 mRNA and protein levels, while endogenous Fgf23 synthesis was not significantly changed.

Conclusions: Chronic exposure to biologically active cardiac iFgf 23 per se does not tackle the heart, while high intra-cardiac Fgf23 synthesis in the presence of high dietary phosphate promotes the toxicity of cardiac Fgf23 that is at least partly related to stabilization of cardiac iFgf23.

\section{OP-14 HYPOCALCAEMIA AND HYPERPHOSPHATEMIA ARE ASSOCIATED WITH ALLOGRAFT DYSFUNCTION IN PAEDIATRIC KIDNEY TRANSPLANT RECIPIENTS}

Agnieszka Prytula ${ }^{1}$, Rukshana Shroff ${ }^{2}$, Justine Bacchetta ${ }^{3}$, Kai Krupka ${ }^{4}$, Ellen Deschepper ${ }^{5}$, Dieter Haffner ${ }^{6}$, Burkhard Tönshoff ${ }^{4}$

${ }^{1}$ Department Of Pediatric Nephrology And Rheumatology, Ghent University Hospital, Belgium, ${ }^{2}$ Renal Unit, Great Ormond Street Hospital London, UK, ${ }^{3}$ Hospices Civils De Lyon, France, ${ }^{4}$ University Children's Hospital Heidelberg, Germany, ${ }^{5}$ Biostatistics Unit, Ghent University, Belgium ${ }^{6}$ Department Of Pediatric Kidney, Liver And Metabolic Diseases, Hannover Medical School, Hannover, Germany ${ }^{7}$ On Behalf Of The Espn Ckd-mbd And Transplantation Wgs This Work Has Been Supported By The Espn Research Grant 05/2020

Introduction: We aimed to analyse the relationship between calcium $(\mathrm{Ca})$, phosphorus $(\mathrm{P})$ and parathyroid hormone $(\mathrm{PTH})$ levels and allograft outcomes over time in paediatric kidney transplant recipients.

Material and methods: We included patients from the European CERTAIN Registry with up to 5 years follow-up and younger than 19 years at transplantation. Laboratory measures were collected at baseline, 1, 3, 6, 9 and 12 months and every 6 months thereafter. $\mathrm{Ca}$ and $\mathrm{P}$ were adjusted for age- specific reference values, whereas PTH levels were adjusted for centre- specific reference values and expressed as upper limit of normal (ULN). We performed survival analysis where the event was death, graft loss or allograft dysfunction defined as estimated glomerular filtration rate $(\mathrm{eGFR}) \leq 30 \mathrm{ml} / \mathrm{min} / 1.73 \mathrm{~m}^{2}$. Associations between $\mathrm{Ca}, \mathrm{P}$ and allograft outcomes were investigated using a stratified Cox proportional hazards model with time-varying covariates.

Results: We included 1218 patients from 42 centres in 15 countries, $61 \%$ boys, $46 \%$ with congenital dysplasia and the mean age at transplantation $9.7( \pm 5.1)$ years. $12.3 \%$ of patients experienced allograft dysfunction, of whom $3 \%$ allograft loss and $0.6 \%$ death. There was no difference in time to event between patients with baseline PTH lower or higher than 2-fold ULN. In contrast, hypocalcaemia (HR 1.93, 95\% CI 1.29;2.89, p =
0.001), hyperphosphatemia (HR 4.05, 95\% CI 2.65;6.18, $\mathrm{p}<0.001$ ), and hyperparathyroidism (HR 4.72, 95\% CI 2.51;8.89, p $<0.001$ ) were associated with allograft dysfunction. In in a multivariable model hypocalcaemia (HR 1.89, 95\% CI 1.22;2.93, p = 0.004) and hyperphosphatemia (HR 3.0, 95\% CI 1.89;4.77, p<0.001) were independently associated with the hazard of event, adjusted for rejection, body mass index $\mathrm{Z}$ score, systolic blood pressure $\mathrm{Z}$ score and disease vintage. Conclusions: In conclusion, hypocalcaemia and hyperphosphatemia are associated with allograft dysfunction independently of known risk factors in paediatric kidney transplant recipients.

\section{OP-15 A FIXED BLOOD PRESSURE CUT-OFF APPROACH TO BLOOD PRESSURE INTERPRETATION IN CHILDREN}

\author{
Robert L. Myette, Janusz Feber \\ Childrens Hospital Of Eastern Ontario, Ottawa, Ontario, Canada
}

Introduction: Current pediatric hypertension definitions are based on a child's height, age and sex, and are complex. A more simplified method includes using fixed blood pressure cut-offs ( $\mathrm{fBPc}$ ). We sought to determine the performance of the blood pressure definition using $\mathrm{fBPc}$, when compared to the complex, percentile-based definitions.

Material and methods: We consecutively enrolled all pediatric patients, aged 5-18 years, who underwent ambulatory blood pressure monitoring (ABPM) in the last 10 years. All patients had 3 consecutive oscillometric office blood pressure readings within 90 days of their ABPM. Office normotension/hypertension was defined using: CAN1: Canadian pediatric hypertension guidelines 2020 (fBPc, age $<12,120 / 80 \mathrm{mmHg}$, age $>=12,130 / 85 \mathrm{mmHg}$ ); CAN2 (percentiles all ages), AAP (percentiles up to 13 years, $>130 / 80 \mathrm{mmHg}$ thereafter) and ESH (percentiles up to 16 years, $>140 / 90 \mathrm{mmHg}$ thereafter). We tested agreement between CAN1, CAN2, AAP, and ESH using Cohen's Kappa, and their ability to predict daytime ambulatory hypertension (daytime SBP or daytime DBP Z-score $>1.65)$ using AUC. Further, we analyzed LVMi $\left(\mathrm{g} / \mathrm{m}^{2.7}\right)$ to ascertain differences in target organ damage (TOD) between groups.

Results: Two-hundred and ninety-three children (male=157) were included in the study. Using Cohen's Kappa, we noted substantial agreement between CAN1:CAN2 $(\mathrm{k}=0.70)$, CAN1:AAP $(\mathrm{k}=0.75)$ but only moderate agreement between CAN1:ESH $(\mathrm{k}=0.46)$. Predictive power (AUC) of daytime ambulatory hypertension was: $\mathrm{CAN} 1=0.58, \mathrm{CAN} 2=0.54, \mathrm{AAP}=0.53$ and $\mathrm{ESH}=0.56$. There were no significant differences in LVMi between CAN1 and CAN2, AAP, or ESH.

Conclusions: There was substantial agreement between hypertension diagnosis using a $\mathrm{fBPc}(\mathrm{CAN} 1)$, and the more complex percentilebased definitions (CAN2, AAP); with only moderate agreement with ESH. The prediction of ambulatory daytime hypertension was limited. There were no differences in LVMi between groups, suggesting that the use of an age-dependent, fBPc definition of hypertension may be equivalent to more complex, percentile-based definitions while not leading to underdiagnosis of TOD.

\section{OP-16 RE-EVALUATING HYPERTENSION IN CHILDREN ACCORDING TO DIFFERENT GUIDELINES: A SINGLE CENTER STUDY}

Cemallye Başaran ${ }^{1}$, Belde Kasap Demİ ${ }^{2}$, Mustafa Agah Tekİndal ${ }^{3}$, Gökçen Erfidan ${ }^{1}$, Özgür Özdemİr Șimșek ${ }^{1}$, Seçil Arslansoyu Çamlar ${ }^{4}$, Demet Alaygut ${ }^{1}$, Fatma Mutlubaș ${ }^{4}$, Ferhan Elmali ${ }^{3}$

${ }^{1}$ Izmir University Of Health Sciences Tepecik Education And Research Hospital Department Of Pediatric Nephrology, Izmir, Turkey, ${ }^{2}$ Izmir Katip Celebi University Medical Faculty, Department Of Pediatrics, Division Of Nephrology And Rheumotology, Izmir, Turkey, ${ }^{3}$ Izmir Katip Çelebi University Faculty Of Medical Sciences Deprtment Of 
Biostatistics, Izmir, Turkey, ${ }^{4}$ University Of Health Sciences, Izmir Faculty Of Medicine Department Of Pediatrics, Division Of Nephrology, Izmir, Turkey

Introduction: We aimed to evaluate the agreement between the guidelines used for both office blood pressure(OBP) and ambulatory blood pressure monitoring(ABPM). Our secondary aim was to define the best threshold to assess children at risk of left ventricular hypertrophy $(\mathrm{LVH})$. Material and methods: Data of patients with ABPM between October2017 and December2020 were evaluated retrospectively. Thresholds proposed by Fourth Report(FR), European Society of Hypertension(ESH) and American Academy of Pediatrics(AAP) for OBP; Wühl et al(W), ESH and American Heart Association(AHA) for ABPM were used and Nine different BP phenotype combinations were created. The agreements between the thresholds and the sensitivity(S) of the thresholds and BP phenotype to predict $\mathrm{LVH}$ were determined for age groups and BMI levels.

Results: Of the 949 patients (M/F:502/447), 325(34.2\%) were $<12 \mathrm{y}$, $368(38.8 \%)$ were $13-15 y$, and $256(27 \%)$ were $16-18 y$; $425(35.8 \%)$ were lean, 148(12.5\%) were overweight and $376(31.6 \%)$ were obese. Echocardiographic evaluation was available in $553(58 \%)$ cases. The agreement between guidelines for OBP and ABPM were "good" and "very good" $(\mathrm{K}=0.639$; 95\%CI, 0.638-0.640, $\mathrm{K}=0.986$; 95\%CI, 0.985 0.988 ), respectively. To classify OBP and ABPM as normal, white coat, masked and sustained hypertension, we had nine different combinations that had "very good" agreement ( $\mathrm{K}=0.880$; 95\%CI, 0,879-0,880). The S of AAP for detecting $\mathrm{LVH}$ was the highest. The highest $\mathrm{S}$ was in $<12-$ year-old obese children $(\mathrm{S}=75.8,95 \% \mathrm{CI}, 56.4-89.7)$ for all. The $\mathrm{S}$ of ABPM in detecting LVH were similar among different age and BMI groups. The lean group of 13-15y ( $\mathrm{S}=88.8,95 \% \mathrm{CI}, 51.7-99.7)$ had the highest $\mathrm{S}$ for all combinations. In overweight and obese patients, the highest sensitivity was in the 13-15 age group with combinations formed according to AAP thresholds $(\mathrm{S}=75,95 \% \mathrm{CI}, 19.4-99.3, \mathrm{~S}=84.2,95 \% \mathrm{CI}$, 60.4-96.6, respectively).

Conclusions: We found that AAP guideline is more sensitive and decisive for BP phenotypes in detecting $\mathrm{LVH}$, especially in children $\leq 15$ years. This is the first study evaluating BP thresholds considering LVH with the highest patient number.

\section{OP-17 STRUCTURAL AND FUNCTIONAL CARDIOVASCULAR DISEASE IN CHILDREN AND YOUNG ADULTS WITH CKD AND ON DIALYSIS}

Alexander D. Lalayiannis ${ }^{1}$, Charles J. Ferro ${ }^{2}$, David C. Wheeler ${ }^{3}$, Neill D. Duncan ${ }^{4}$, Colette Smith ${ }^{10}$, Joyce Popoola ${ }^{5}$, Varvara Askiti ${ }^{6}$, Andomachi Mitsioni ${ }^{6}$, Amrit Kaur ${ }^{7}$, Manish D. Sinha ${ }^{8}$,

Simon P. Mcguirk ${ }^{9}$, Kristian H. Mortensen ${ }^{1}$, David V. Milford ${ }^{9}$, Rukshana Shroff ${ }^{1}$

${ }^{1}$ University College London Great Ormond Street Hospital Institute Of Child Health, London, ${ }^{2}$ University Hospitals Birmingham ${ }^{3}$ Department Of Renal Medicine, University College London, ${ }^{4}$ Imperial College Renal And Transplant Centre, Hammersmith Hospital, London, ${ }^{5}$ St. Georges University Hospital Nhs Foundation Trust, London, ${ }^{6}$ "p. \& A. Kyriakou" Childrens Hospital, Athens, ${ }^{7}$ Manchester University Nhs Foundation Trust, Manchester ${ }^{8}$ Evelina Children's Hospital, Guys \& St Thomas Nhs Foundation Trust, London, ${ }^{9}$ Birmingham Women's And Children's Nhs Foundation Trust, Birmingham, ${ }^{10}$ Institute Of Global Health, University College London

Introduction: Cardiovascular disease (CVD) is the most common cause of morbidity and mortality in young people with CKD. Calcium and phosphate deposition in the arterial medial layer causes vascular calcification. Structural changes in the vessels perhaps lead to arterial stiffness, in turn causing increased left ventricular pressure load and left ventricular hypertrophy. Our aim was to examine structural and functional CV changes in a young cohort with CKD. The skeleton continues to mineralize until the third or fourth decade of life, perhaps acting as a protective calcium buffer.

Material and methods: 79 children \& 21 young adults with CKD stages 4-5 and on dialysis aged 5-30 years underwent cardiac CT for coronary artery calcification $(\mathrm{CAC})$, ultrasound for carotid intima media thickness(cIMT), pulse wave velocity(PWV), echocardiography for left ventricular mass $\left(\mathrm{LVMI}, \mathrm{g} / \mathrm{m}^{2.7}\right)$. cIMT \& cfPWV were expressed as zscores. The vascular measures were analysed as structural changes (cIMT, LVMI and CAC) \& functional changes (carotid distensibility, and PWV). We examined carotid dilatation (lumen/wall cross sectional area) as a surrogate of the association between structural and functional changes.

Results: $69.5 \%$ of CKD and $88.3 \%$ of dialysis patients had at least one structural or functional $\mathrm{CV}$ abnormality. The odds of having any structural or functional abnormality in dialysis was 17.3 times higher compared to CKD (95\% CI 5.3 to 53.5 , p<0.0001). Carotid dilatation was lower in patients with cIMT z-scores $>2$ compared to $<1$. The presence of more than one structural abnormality increased the odds of more than one functional abnormality by 4.5 (95\% CI 1.3 to $16.6, \mathrm{p}=0.045$ ).

Conclusions: There is a high prevalence of subclinical CVD in children and young adults with CKD4-5D. Functional changes indicative of arterial stiffness were more prevalent in dialysis. Our data suggests that patients with more structural abnormalities were more likely to have functional abnormalities. Longitudinal studies are required to determine the temporal association of structural and functional $\mathrm{CV}$ changes.

\section{OP-18 SOLUBLE CD89 IS A CRITICAL FACTOR FOR MESANGIAL PROLIFERATION IN CHILDHOOD IGA NEPHROPATHY.}

Alexandra Cambier ${ }^{1}$, Patrick James Gleeson ${ }^{2}$, Lilia Abbad ${ }^{2}$, Fanny Canesi ${ }^{2}$, Jennifer Da Silva ${ }^{2}$, Julie Bex-coudrat ${ }^{2}$, Georges Deschenes ${ }^{3}$, Olivia Boyer ${ }^{4}$, Marion Rabant ${ }^{4}$, Tim Ulinski ${ }^{5}$,Michel Peuchmaur ${ }^{3}$, Laureline Berthelot ${ }^{2}$, Renato Monteiro ${ }^{2}$

${ }^{1}$ Sainte Justine Hospital, ${ }^{2}$ Centre De Recherche Sur Linflammation (cri); Inserm U1149, ${ }^{3}$ Hôpital Robert Debré ${ }^{4}$ Hôpital Necker ${ }^{5}$ Hôpital Trousseau

Introduction: Childhood $\operatorname{IgA}$ nephropathy $(\mathrm{cIgAN})$ includes a wide spectrum of clinical presentations, from isolated hematuria to acute nephritis with rapid loss of renal function. IgAN is an autoimmune disease and its pathogenesis involves galactose deficient (Gd) IgA1, IgG anti-Gd-IgA1 autoantibodies and the soluble IgA Fc receptor (sCD89). However, the implications of such factors in cIgAN pathogenesis remain unclear.

Material and methods: Here, we studied these biomarkers in a cohort of 67 cIgAN patients and 42 controls but also in vivo with human mesangial cells and in vivo with human IgA transgenic mice.

Results: While Gd-IgA1 was only moderately enhanced in patient plasma, levels of circulating IgA complexes (sCD89-IgA and IgG$\operatorname{IgA}$ ) and free sCD89 were markedly increased in cIgAN. sCD89IgA1 complexes and free sCD89 correlate with proteinuria, as well as histological markers of disease activity: mesangial, endocapillary hypercellularity and cellular crescent. Mesangial sCD89 deposits were detected in $\mathrm{cIgAN}$ biopsies. Plasma chromatography fractions 
containing sCD89-IgA1 or free sCD89 from patients induced mesangial cell proliferation in vitro. Recombinant (r) sCD89 induced mesangial cell proliferation in vitro that was inhibited by $\mathrm{rCD} 71$ or rapamycin. Injection of rsCD89 induced marked glomerular proliferation and proteinuria in human IgA1 transgenic mice.

Conclusions: In conclusion, free and IgA1-complexed SCD89 are key players in mesangial proliferation. These findings reveal a new role for sCD89 in cIgAN, making it a potentially useful biomarker and therapeutic target.

\section{OP-19 PREDICTORS OF RENAL OUTCOME IN CHILDREN WITH ANCA ASSOCIATED VASCULITIS: RESULTS OF THE ERKNET/ESPN/IPNA SURVEY}

Matko Marlais ${ }^{1}$, Tanja Wlodkowski ${ }^{2}$, Nikoleta Printza ${ }^{3}$, Dorothea Kronsteiner ${ }^{2}$, Regina Brinster ${ }^{2}$, Marina Aksenova ${ }^{4}$, Isa Ashoor ${ }^{5}$, Atif Awan ${ }^{6}$, Ramnath Balasubramanian ${ }^{7}$, Biswanath Basu ${ }^{8}$, Zivile Bekassy ${ }^{9}$, Olivia Boyer ${ }^{10}$, Eugene Chan ${ }^{11}$, Carlos Chica ${ }^{12}$, Dagmar Csaicsich ${ }^{13}$, Stephane Decramer ${ }^{14}$, Eiske Dorresteijn ${ }^{15}$, Magdalena Drozynska-Duklas ${ }^{16}$, Loai Eid ${ }^{17}$, Laura Espinosa 18, Veronica Ferraris ${ }^{19}$, Hana Flögelova ${ }^{20}$, Jessica Forero-Delgadillo ${ }^{21}$ ', Valentina Gracchi ${ }^{22}$, Mercedes López González ${ }^{23}$, Matthias Hansen 24, Motoshi Hattori ${ }^{25}$, Nakysa Hooman ${ }^{26}$, Swe Lwin Htun ${ }^{27}$, Dmytro Ivanov ${ }^{28}$, Hee Gyung Kang ${ }^{29}$, Vasiliki Karava ${ }^{30}$, Ina Kazyra ${ }^{31}$, Audrey Laurent ${ }^{32}$, Adrian Catalin Lungu ${ }^{33}$, Stephen Marks ${ }^{1}$, Andrew Maxted 34, Anna Moczulska ${ }^{35}$, Rebekka Mueller 2, Tatiana Nastausheva ${ }^{36}$, Mattia Parolin ${ }^{37}$, Carmine Pecoraro ${ }^{38}$, Iliana Principi ${ }^{39}$, Cheryl Sanchez-kazi ${ }^{40}$, Seha Saygili ${ }^{41}$, Raphael Schild ${ }^{42}$, Mohan Shenoy ${ }^{43}$, Rajiv Sinha ${ }^{44}$, Ana Paula Spizzirri ${ }^{45}$, Maria Stack ${ }^{6}$, Maria Szczepanska ${ }^{46}$, Alexey Tsygin ${ }^{47}$, Julia Tzeng ${ }^{48}$, Vaidotas Urbonas ${ }^{49}$, Tao Zhang ${ }^{50}$, Jakub Zieg ${ }^{51}$, Federica Zotta ${ }^{52}$, Franz Schaefer ${ }^{2}$, Marina Vivarelli ${ }^{52}$, Kjell Tullus ${ }^{1}$

${ }_{1}^{1}$ Great Ormond Street Hospital For Children Nhs Foundation Trust, ${ }^{2}$ Heidelberg University Hospital, ${ }^{3}$ Aristotle University Of Thessaloniki, ${ }^{4}$ Pirogov Russian National Research Medical University, ${ }^{5}$ Childrens Hospital, New Orleans, ${ }^{6}$ Children's Health Ireland At Temple Street, Dublin, ${ }^{7}$ Evelina London Hospital For Children, London, ${ }^{8}$ Nil Ratan Sircar Medical College, Kolkata, ${ }^{9}$ University Hospital Sus Lund Sweden, ${ }^{10}$ Hopital Necker-enfants Malades, Ap-hp, Paris, ${ }^{11}$ Hong Kong Childrens Hospital, ${ }^{12}$ Hospital Universitario San Jorge Pereira ${ }^{13}$ Medical University Of Vienna, ${ }^{14}$ Chu Purpan, Toulouse, ${ }^{15}$ Erasmusmc, Rotterdam, ${ }^{16}$ University Clinical Centre, Medical University Of Gdansk, ${ }^{17}$ Dubai Hospital, Dubai, ${ }^{18}$ Hospital Infantil La Paz, Madrid ${ }^{19}$ Hospital Italiano De Buenos Aires,${ }^{20}$ Faculty Hospital, Olomouc, Czech Republic, ${ }^{21}$ Fundacion Valle Del Lili, Colombia, ${ }^{22}$ Beatrix Childrens Hospital - Umcg, Groningen, ${ }^{23}$ Vall D'hebrón Hospital, Barcelona, ${ }^{24}$ Kfh-nierenzentrum FÜr Kinder Und Jugendliche Beim Clementine -kinderhospital, Frankfurt, ${ }^{25}$ Tokyo Womens Medical University, Tokyo, ${ }^{26}$ Aliasghar Clinical Research Development Center, Iran, ${ }^{27}$ Mandalay Children Hospital, Myanmar, ${ }^{28}$ Ukraine Kiev Shypuk National Medical Academy, ${ }^{29}$ Seoul National University Childrens Hospital, Seoul, ${ }^{30} 1$ st Department Of Pediatrics, Ippokratio General Hospital, Thessaloniki, ${ }^{31}$ Belarus State Medical University, Minsk 2nd Childrens Hospital, ${ }^{32}$ Hfme, Lyon, ${ }^{33}$ Fundeni Clinical Institute, Bucharest, ${ }^{34}$ Nottingham Childrens Hospital, ${ }^{35}$ Jagiellonian University Medical College, Poland, ${ }^{36}$ Voronezh State Medical University, ${ }^{37}$ Azienda Ospedaliera Di Padova, ${ }^{38}$ Santobono Childrens Hospital, ${ }^{39}$ Hospital H. Notti, Mendoza, ${ }^{40}$ Loma Linda University Childrens Hospital, California, ${ }^{41}$ Cerrahpasa Medical School, Istanbul, ${ }^{42}$ University Childrens Hospital Hamburg, ${ }^{43}$ Royal Manchester Childrens Hospital, ${ }^{44}$ Institute Of Child Health, Kolkata, ${ }^{45}$
Hospital De Niños Sor Ludovica, Argentina, ${ }^{46}$ Faculty Of Medical Sciences In Zabrze, Sum In Katowice, ${ }^{47}$ National Medical Centre For Childrens Health, Russia, ${ }^{48}$ Valley Childrens Healthcare, Madera, ${ }^{49}$ Vilnius University Clinic For Childrens Diseases, Vilnius, ${ }^{50}$ The Children's Hospital Of Fudan University, Shanghai, ${ }^{51}$ Motol University Hospital, ${ }^{52}$ Ospedale Pediatrico Bambino Gesù, Rome

Introduction: ANCA associated vasculitis (AAV) is a rare condition in children and data are limited on its treatment and renal outcomes. We aimed to determine clinical predictors of adverse renal outcome in children with AAV.

Material and methods: Retrospective international survey conducted through professional paediatric nephrology organisations from December 2019 to March 2020. Paediatric nephrologists were asked to anonymously enter demographic and clinical data through an online form on all children presenting with AAV to their centre.

Results: Adequate data were collected for 337 patients (from 41 countries, $72 \%$ female). Mean age at presentation 12.4 years, mean duration of follow-up 42 months. Mean peak serum creatinine at presentation was $377 \mathrm{umol} / \mathrm{L}(4.26 \mathrm{mg} / \mathrm{dl})$ falling to a mean of $95 \mathrm{umol} / \mathrm{L}(1.07 \mathrm{mg} / \mathrm{dl})$ at latest follow up in those not on KRT. $42 \%$ were classified as microscopic polyangiitis, $31 \%$ as granulomatosis with polyangiitis. $63 \%$ were from high income countries, $29 \%$ from upper-middle income countries (GNP 2019). There was an 5\% mortality in this cohort, with $41 \%$ of patients requiring kidney replacement therapy (KRT) at any point. In multivariate mixed regression modelling, significant predictors of adverse renal outcome (requirement for KRT) included higher peak serum creatinine at presentation $(p<0.001)$, ANCA-MPO positivity $(p=0.002)$ and neurological involvement at presentation $(p=0.02)$. Additionally, receiving plasma exchange as part of induction treatment was associated with a higher risk of having an abnormal creatinine at latest follow up in those not requiring $\mathrm{KRT}(p=0.0004)$.

Conclusions: This large international cohort of children with AAV demonstrates the significant risk of chronic kidney disease and requirement for KRT in those presenting to paediatric nephrologists. Clinical predictors of adverse renal outcome at presentation are identified but further prospective research is required to determine the impact of treatment on clinical outcomes.

\section{OP-20 KIDNEY TRANSPLANT REJECTION AND SURVIVAL IN ADOLESCENTS - THE ROTTERDAM EXPERIENCE}

Femke Vrieling-Prince ${ }^{1}$, Marian Clahsen-Van Groningen ${ }^{2}$, Huib De Jong ${ }^{1}$, Karlien Cransberg ${ }^{1}$, Joke Roodnat ${ }^{3}$

${ }^{I}$ Department Of Paediatric Nephrology And Erasmus Mc Transplant Institute, Erasmus Mc Sophia Children's Hospital, Rotterdam, ${ }^{2}$ Department Of Pathology And Erasmus Mc Transplant Institute, Erasmus Mc, Rotterdam, ${ }^{3}$ Department Of Internal Medicine, Division Of Nephrology And Erasmus Mc Transplant Institute, Erasmus Mc, Rotterdam

Introduction: Several registries report a higher risk of kidney transplant loss in adolescence compared to other age groups. The objectives of this study were to evaluate:

1. -if adolescents have a higher risk of acute rejection

2. -if adolescents have (early) transplant loss due to acute and/or chronic rejection.

Material and methods: This retrospective study was performed in pediatric and young adult kidney transplant recipients, receiving a first 
transplant between 1990-2018, at the age of 6-25 years(y), in the Erasmus MC Rotterdam. Patient files were searched for all for-cause graft biopsy reports, and cause of transplant failure and/or death. Biopsies were reevaluated according to the 2017 Banff Classification.

Results: In $65(54 \%)$ of all pediatric and $85(65 \%)$ of all adult patients at least one transplant biopsy was obtained. Sixty seven $(45 \%)$ patients had an acute rejection episode (ARE) in at least one biopsy, with the highest incidence in the $20-25 \mathrm{y}$ age group $(27 \%, \mathrm{n}=20)$ and the lowest incidence in the $6-10 y$ group $(16 \%, n=5)$.

ARE-free survival was best in the $6-10 y$ group $(n=32): 90 \%$ at $2 y$ and $86 \%$ at 8 y post-transplant, followed by the $10-15 y$ group $(n=49): 86 \%$ and $81 \%$. Older recipients showed a poorer ARE-free survival: in $15-20 \mathrm{y}$ group ( $\mathrm{n}=94$ ) of $86 \%$ at $2 \mathrm{y}$ and $68 \%$ at $8 \mathrm{y}$ post-transplant, and in the 20 $25 \mathrm{y}$ group $(\mathrm{n}=75)$ of $84 \%$ and $65 \%(\mathrm{p}=0.063)$.

Subsequently graft losses due to acute and/or chronic rejection were studied. Transplant survival was superior in the $6-10 \mathrm{y}$ group: $97 \%$ at $2 \mathrm{y}$ and $89 \%$ at $8 \mathrm{y}$ at post-transplant, followed by the $20-25 \mathrm{y}$ group: $97 \%$ and $85 \%$. Inferior transplant survival was seen in the $10-15 y$ group $(96 \%$ and $67 \%)$ and in the $15-20 y$ group (93\% and 67\%) $(\mathrm{p}=0.073)$

Conclusions: The youngest(6-10y) kidney transplant recipients had the lowest incidence of ARE, the oldest(20-25y) recipients the highest. Inferior transplant survival due to rejection was seen in the adolescent (10-20y) groups.

\section{OP-21 PHARMACOKINETICS OF TACROLIMUS IN RENAL TRANSPLANTATION RECIPIENTS DURING ADOLESCENCE}

Femke Vrieling-prince ${ }^{1}$, Romy Fleur Van Der Wouden ${ }^{1}$, Karlien Cransberg ${ }^{1}$, Antonia Bouts ${ }^{2}$, Marlies Cornelissen ${ }^{3}$, Joke Roodnat ${ }^{4}$

${ }^{1}$ Department Of Paediatric Nephrology And Erasmus Mc Transplant Institute, Erasmus Mc Sophia Children's Hospital, Rotterdam, ${ }^{2}$ Amsterdam Umc Emma Children's Hospital, Paediatric Nephrology, Amsterdam, ${ }^{3}$ Radboud Umc Amalia Children's Hospital, Paediatric Nephrology, Nijmegen ${ }^{4}$ Department Of Internal Medicine, Division Of Nephrology And Erasmus Mc Transplant Institute, Erasmus Mc, Rotterdam

Introduction: Adolescents have the highest risk of kidney transplant failure of all age groups. The objective of this study is to investigate the relation between puberty and the pharmacokinetic properties of tacrolimus after kidney transplantation.

Material and methods: This study is part of the larger multicentre cohort study Adolesce-NT. Kidney transplant recipients aged between $8-30$ years were enrolled between 2012-2021. The study contains a pretransplant and post-transplant patient group at time of inclusion. The variability of tacrolimus levels was calculated with all measurements from 6 months till 1 year after transplantation in the pre-transplant group and from inclusion till 1 year later in the post-transplant group. For each trough level we calculated the tacrolimus dose adjusted for weight.

Our primary outcome parameters are the dose-adjusted trough level and the intrapatient variability. We compared these pharmacokinetic parameters to puberty staging and gender, and corrected for confounders (including eGFR, steroid use, CYP3A4 and CYP3A5 polymorphisms).

Results: 25 patients were included in the pre-transplant and 39 patients in the post-transplant group. When corrected for confounders, we found a significant correlation between puberty staging and the dose-adjusted trough level $(p=0.017)$. There was a linearly increase of the doseadjusted trough levels by increasing age.

Dose-adjusted trough levels increased linearly with increasing age, with higher increase for females compared to males. Intrapatient variability did not correlate significantly with stages of puberty $(\mathrm{p}=0.122)$ or gender $(\mathrm{p}=0.287)$.

Conclusions: Our preliminary results indicate that the dose requirement to reach tacrolimus target levels is lower in adolescents, and in females.
We did not find a relationship between intrapatient variability as a measure of drug adherence and pubertal development. As we are in the process of including more patient data and adding data on drug compliance, future evaluations are expected to provide more information.

OP-22 RANDOMIZED, PLACEBO-CONTROLLED, PHASE 3B TRIAL OF TOLVAPTAN IN THE TREATMENT OF CHILDREN AND ADOLESCENTS WITH AUTOSOMAL DOMINANT POLYCYSTIC KIDNEY DISEASE (ADPKD): 1-YEAR DATA

Djalila Mekahli ${ }^{1}$, Lisa Guay-Woodford ${ }^{2}$, Melissa A. Cadnapaphornchai ${ }^{3}$, Larry A. Greenbaum ${ }^{4}$, Mieczyslaw Litwin ${ }^{5}$, Tomas Seeman ${ }^{6}$, Ann Dandurand ${ }^{7}$, Lily Shi ${ }^{8}$, Kimberly Sikes ${ }^{8}$, Susan E. Shoaf ${ }^{9}$, Franz Schaefer ${ }^{10}$

${ }^{1}$ Department Of Pediatric Nephrology, University Hospitals Leuven And Pkd Research Group, Department Of Development And Regeneration, Ku Leuven, Leuven, Belgium, ${ }^{2}$ Center For Translational Science, Childrens National Health System, Washington, Dc, USA, ${ }^{3}$ Rocky Mountain Pediatric Kidney Center, Rocky Mountain Hospital For Children At Presbyterian/st. Luke's Medical Center, Denver, Co, USA, ${ }^{4}$ Department Of Pediatrics, Division Of Pediatric Nephrology, Emory University School Of Medicine And Childrens Healthcare Of Atlanta, Atlanta, Ga, USA, ${ }^{5}$ Department Of Nephrology, Kidney Transplantation And Arterial Hypertension, Childrens Memorial Health Institute, Warsaw, Poland, ${ }^{6}$ Department Of Paediatrics, 2nd Faculty Of Medicine, Charles University And Motol University Hospital, Prague, Czech Republic, ${ }^{7}$ Otsuka Pharmaceutical Development \& Commercialization, Princeton, Nj, USA (previously Affiliated With Otsuka At The Time The Research Was Conducted), ${ }^{8}$ Otsuka Pharmaceutical Development \& Commercialization, Rockville, Md, USA, ${ }^{9}$ Otsuka Pharmaceutical Development \& Commercialization, Princeton, Nj, USA, ${ }^{10}$ Division Of Pediatric Nephrology, Center For Pediatrics And Adolescent Medicine, Heidelberg, Germany

Objectives: To evaluate the vasopressin V2 receptor antagonist tolvaptan for pharmacodynamic activity and preliminary efficacy and safety in children/adolescents with early manifesting ADPKD.

Methods: Phase 3b, 2-part trial (EudraCT 2016-000187-42). Phase A (reported here) was a 1-year (y), randomized, double-blind, placebo-controlled, multicenter trial; Phase B is an ongoing, 2-y, open-label extension. Eligibility criteria: ADPKD (renal cysts with family history and/or genetic diagnosis), eGFR $\geq 60 \mathrm{~mL} / \mathrm{min} / 1.73 \mathrm{~m}^{2}$, body weight $\geq 20 \mathrm{~kg}$. The target population was age $12-17 \mathrm{y}$; subjects $4-11 \mathrm{y}$ could also enter if eligible. Tolvaptan/placebo were titrated based on body weight and tolerability. Co-primary endpoints: changes from baseline in spot urine osmolality (Uosm) and specific gravity (SG) at Week 1. Additional endpoints: 12-month changes in height-adjusted total kidney volume (htTKV) and eGFR, safety/tolerability. Statistical comparisons were exploratory and post hoc.

Results: Of 91 subjects enrolled (66 age 12-17y; 25 age $<12$ y), 48 were randomized to tolvaptan and 43 to placebo. Mean reduction $( \pm \mathrm{SD})$ from baseline to Week 1 (tolvaptan vs placebo): 386 (284) vs 93 (332) $\mathrm{mOsm} / \mathrm{kg}$ for Uosm $(P<.001)$ and $0.009(0.007)$ vs $0.002(0.008)$ for urine SG $(P<.001)$. In subjects $12-17 \mathrm{y}$, mean \%htTKV increase from baseline to Month 12 was $2.3 \%$ (8.8) for tolvaptan and $6.1 \%$ (7.5) for placebo $(P=.14)$. Mean eGFR change from Day 7 to Month 12 (all subjects) was $2.7(10.7) \mathrm{mL} / \mathrm{min} / 1.73 \mathrm{~m}^{2}$ for tolvaptan, $-3.2(10.9) \mathrm{mL} / \mathrm{min} /$ $1.73 \mathrm{~m}^{2}$ for placebo $(P=.10)$. Most frequent adverse events over 12 months (tolvaptan/placebo): polyuria $(25.0 / 2.3 \%)$, elevated serum creatinine $(18.8 / 4.7 \%)$, pollakiuria $(18.8 / 0.0 \%)$, cough $(14.6 / 11.6 \%)$, and nocturia $(14.6 / 4.7 \%)$. No elevated transaminases or drug-induced liver injury. Serious adverse events in 1 tolvaptan (viral pericarditis) and 6 placebo, none treatment-related. One discontinuation due to pollakiuria (tolvaptan), 1 due to dizziness (placebo). 
Conclusions: Tolvaptan exhibited evidence of V2 receptor antagonism in pediatric ADPKD patients. Results suggestive of slowed TKV growth and eGFR decline warrant further investigation.

\section{OP-23 RITUXIMAB BIOAVAILABILITY IN PEDIATRIC PATIENTS WITH STEROID DEPENDENT IDIOPATHIC NEPHROTIC SYNDROME}

Cyrielle Parmentier ${ }^{1}$, Sabine Mignot ${ }^{2}$, Quentin Bertrand ${ }^{3}$, Tim Ulinski ${ }^{1}$, Claire Dossier ${ }^{4}$

${ }^{1}$ Trousseau Hospital, Paris France, ${ }^{2}$ Bichat Hospital, Paris, France, ${ }^{3}$ Clinique Chc, Liege, Belgique, ${ }^{4}$ Robert Debre Hospital, Paris France

Introduction: B-cell depletion is an efficient treatment option in pediatric patients with steroid dependent idiopathic nephrotic syndrome (SDNS). Despite overall satisfying results concerning tolerance and remission maintenance, some patients experience rapid relapses and/or had a short B-cell depletion with rituximab (RTX) infusions.

Material and methods: We aim to analyze the RTX bioavailability, evaluate the presence of anti-RTX antibodies (ARA) and measured RTX serum levels monthly in 43 pediatrics patients with SDNS treated with an injection of $375 \mathrm{mg} / \mathrm{m}^{2}$ RTX, of which, 24 patients received a first RTX.

Results: The median B-cell depletion was 3.8 months (IQ 2.8; 4.5). RTX serum level 1 month (M1) post RTX injection were significantly lower for the 7 patients with a short B cell depletion period ( $<3$ months), 0 $\mu \mathrm{g} / \mathrm{ml}$ (IQ $0 ; 6,2) v s .34 \mu \mathrm{g} / \mathrm{ml}$ (IQ 22,$8 ; 37$ ) for the others $(\mathrm{p}<0.0001)$. ARA were detected in 11 patients $(26 \%)$ during follow-up. Among the 5 patients with ARA after the first RTX, all achieved B-cell depletion, with a median duration of 3.0 months (IQ 2.7; 3.8). Furthermore, 4 out of 6 patients with ARA after the second or more RTX, did not achieve B-cell depletion, and developed ARA at baseline or M1 after these RTX. Seven of the 11 patients ARA+ were switched to obinutuzumab and achieved complete B-cell depletion with a median duration of 7.6 months (IQ 5.9 ; 9.01) suggesting that ARA do not have any cross-reactivity against obinutuzumab.

Conclusions: ARA should be searched for in patients with short or absent B-cell depletion following RTX treatment. The use of obinutuzumab seems to cover the specific needs in these patients. ARA and RTX serum levels are of interest in order to individualize the use of B-cell depleting agents in patients with "difficult-to treat" SDNS.

\section{OP-24 IDENTIFYING INTERPERSONAL DIFFERENCES IN GUT MICROBIOTA METABOLISM OF TRANSPLANTATION AND CHRONIC KIDNEY DISEASE DRUGS}

\author{
Maral Baghai Arassi ${ }^{1}$, Nicolai Karcher ${ }^{2}$, Burkhard Toenshoff ${ }^{1}$, \\ Georg Zeller ${ }^{2}$, Michael Zimmermann ${ }^{2}$ \\ ${ }^{1}$ Department Of Paediatrics I, University Childrens Hospital Heidelberg, \\ Heidelberg, Germany, ${ }^{2}$ Structural And Computational Biology Unit, \\ European Molecular Biology Laboratory, Heidelberg, Germany
}

Introduction: The outcome of kidney transplantation (KTx) and chronic kidney disease (CKD) depends on multiple therapeutic factors. We investigated how interpersonal differences in gut microbiome composition may impact interpersonal variability in drug metabolism and the activation of prodrugs. We specifically focused on immunosuppressants, including tacrolimus and mycophenolate mofetil, which are challenging to dose due to their narrow therapeutic index and large interpersonal variability in drug response.

Material and methods: We constructed an in vitro system mimicking the intestinal environment and tested 10 human bacterial communities for the metabolism of 27 drugs, comprising four different classes of immu- nosuppressants and relevant non-immunosuppressive drugs. To link the metabolic activity of complex microbial communities to their composition, we additionally screened our drug panel against 44 highly abundant and prevalent gut bacterial strains.

Results: Our analysis revealed donor and compound-specific differences in bacterial drug metabolism. While more than $75 \%$ of the immunosuppressants were metabolized by at least one bacterial community, less than $30 \%$ of the non-immunosuppressive drugs were metabolized. Strikingly, bacterial communities differed in compound spectrum and velocity of drug metabolism, indicating pronounced donor-specific differences. Further, we observed marked differences in metabolism between individual bacterial species and strains. Over $90 \%$ of the drug community metabolism was reproduced with single species, indicating a causal link between microbiome composition and metabolic activity.

Conclusions: We identified large interpersonal variability in gut bacterial metabolism of common immunosuppressive and non-immunosuppressive drugs used in KTx and CKD. Donor and drug-specific differences were particularly pronounced for immunosuppressants. Given the challenges associated with the dosage of immunosuppressants, we suggest that further studies should focus on how bacterial metabolism impacts immunosuppressive drug response in kidney disease patients. This may open new opportunities to establish microbial biomarkers for personalised drug selection and dosing.

\section{OP-25 EXPLORING THE EFFECTS OF CYP3A5 AND ABCB1 GENETIC VARIATIONS IN FUNCTIONAL DIFFERENCES, TACROLIMUS METABOLISM AND CTGF PRODUCTION IN HUMAN PROXIMAL TUBULE CELLS}

Yasaman Ramazani ${ }^{1}$, Noel Knops ${ }^{1}$, Jetty De Loor ${ }^{1}$, Roel Goldschmeding ${ }^{2}$, Tri Q Nguyen ${ }^{2}$, Bert P. Van Den Heuvel ${ }^{1}$, Elena Levtchenko ${ }^{3}$, Dirk Kuypers ${ }^{3}$

${ }^{1} \mathrm{Ku}$ Leuven, ${ }^{2}$ Umc Utrecht, ${ }^{3}$ Ku Leuven And Uz Leuven

Introduction: The mechanisms of calcineurin inhibitor nephrotoxicity (interstitial fibrosis and hyalinosis), is largely unknown. The interplay between CYP3A5 enzymes and the P-glycoprotein (P-gp) pump is important for tacrolimus (tac) metabolism and toxicity. There is a large genetic variation in the genes encoding CYP3A5 and P-gp. Our objectives are: 1) exploring the tacrolimus-induced profibrotic signature of the only cells in the kidney responsible for tacrolimus metabolism, the proximal tubule cells 2) exploring the underlying mechanisms for the geneticdependent tacrolimus-induced profibrotic responses.

Material and methods: Eleven clones of conditionally immortalized PTC (ciPTC) from donor kidneys with different combinations of $C Y P 3 A 5$ (rs 776746) and $A B C B 1$ (rs 1045642) genotypes were incubated with vehicle, 50 and $300 \mathrm{ng} / \mathrm{mL}$ tac (tissue range) for $72 \mathrm{~h}$. qRT-PCR and western blot were performed for $C Y P 3 A 5, A B C B 1$ (gene encoding P-gp) and connective tissue growth factor (CTGF) expression. Functional CYP3A5 expression was assessed by midazolam hydroxylation using LC-MS and P-gp activity by calcein efflux. Quantitative metabolite analysis was performed by HPLC. Immunohistochemistry of CTGF expression in protocol biopsies was performed blindly by two pathologists.

Results: 1) ciPTC, produce CTGF, a clinically relevant profibrotic protein, when exposed to increasing concentration of tacrolimus in a dosedependent manner. This increase is significantly higher in ciPTC with CYP3A5*1 ABCB1 3435 TT genotype. 2) increasing concentration of tacrolimus, decreases the function of CYP3A5 and P-gp without altering their expression. 3) P-gp activity is significantly higher in carriers of $A B C B 1$ $3435 T T$ vs $C C / C T$. 4) Proximal tubule cells carrying $C Y P 3 A 5 * 1$ allele, metabolize tacrolimus faster than the carriers of the $* 3 / * 3$ allele with a 
preferential generation of 13-O-demethyl (M1). 5) ciPTC with CYP3A5*I $A B C B 13435$ TT genotype has significantly higher production of M1 compared to other genetic combinations. 6) Staining in protocol biopsies, in a 2year follow-up period, demonstrates a higher CTGF accumulation in donor kidneys with $A B C B 13435 T T$ allele vs the $C C / C T$.

Conclusions: We show for the first time, the direct genetic-dependent profibrotic effect of tacrolimus in a novel human PTC model incorporating the genetic variation in $C Y P 3 A 5$ and $A B C B 1$. The increase of CTGF in PTC in cells with the highest M1 production and altered P-gp activity, is suggestive for a genetic-dependent interplay of tacrolimus metabolism/ efflux in fibrotic processes and long-term kidney function.

\section{OP-26 CEREBROVASCULAR COMPLICATIONS OF RENOVASCULAR DISEASE IN CHILDREN - KNOWNS AND UNKNOWNS}

Chavini Ranasinghe ${ }^{1}$, Nadeesha Mudalige ${ }^{2}$, Jelena Stojanovic ${ }^{3}$

${ }^{1}$ Department Of Undergraduate Medicine, University College London, 74 Huntley St, London Wcle 6de, ${ }^{2}$ University College London Great Ormond Street Institute Of Child Health, Nihr Great Ormond Street Hospital Biomedical Research Centre, London, United Kingdom, ${ }^{3}$ Department Of Pediatric Nephrology, Great Ormond Street Hospital For Children Nhs Foundation Trust, Great Ormond Street, London, United Kingdom

Introduction: Renovascular disease is an important cause of hypertension in childhood. Cerebrovascular complications are known to be associated with renovascular hypertension. However, the presenting features and phenotypic characteristics of this cohort with coexisting disease is undefined, therefore a literature review was conducted in order to further identify this group.

Material and methods: A systematic review of cerebrovascular complications associated with renovascular hypertension in the global paediatric $(<18$ years) population. The MEDLINE, Embase and Google Scholar databases were searched, from database inception to 26th January 2021. Primary articles were not restricted by study design and geographical location but were limited to those published in English.

Results: 303 individuals (median age: 7.6 years [range 10 days -17.9 years], M:F - 174:129) from 37 studies were included, across 13 countries. Case reports published cases of 30 children with coexisting renovascular hypertension and cerebrovascular disease. Most children had bilateral renal artery stenosis (43\%), stenosis secondary to fibromuscular dysplasia (53\%) and coexisting occlusive cerebrovascular disease $(50 \%)$. The majority $(82 \%)$ presented with neurological symptoms and cerebral complications ranged from asymptomatic cerebrovascular stenosis in one child, to acute stroke in $51 \%$ and posterior reversible encephalopathy syndrome in $10 \%$. The location or underlying etiology of the renovascular disease did not predict the location or extent of the cerebrovascular disease or the longterm prognosis of these children. The evidence from the 7 cohort studies included in the review were limited, estimating the prevalence of coexisting disease ranging from $16-79 \%$, though none specifically established a cohort of children with coexisting disease. Furthermore, the conclusions drawn were subjected to considerable bias from the treating clinicians.

Conclusions: A prospective cohort of children with renovascular hypertension and cerebrovascular complications should be established in order to better understand the long-term prognosis and impact of interventions.

\section{OP-27 HEMODIAFILTRATION IS ASSOCIATED WITH REDUCED INFLAMMATION AND INCREASED BONE TURNOVER COMPARED TO CONVENTIONAL HEMODIALYSIS IN CHILDREN - THE HDF, HEART AND HEIGHT (3H) STUDY}

Dagmar-christiane Fisher ${ }^{1}$, Colette Smith ${ }^{2}$, Francesca De Zan ${ }^{3}$, Sevcan A. Bakkaloglu ${ }^{4}$, Varvara Askiti ${ }^{5}$, Karolis Azukaitis ${ }^{6}$,
Aysun Bayazit ${ }^{7}$, Nur Canpolat ${ }^{8}$, Lukasz Obrycki ${ }^{9}$, Fabio Paglialonga ${ }^{10}$, Anja Rahn ${ }^{1}$, Bruno Ranchin ${ }^{11}$, Brankica Spasojevic ${ }^{12}$, Michel Fischbach ${ }^{13}$, Franz Schaefer ${ }^{14}$, Claus Peter Schmitt ${ }^{14}$, Rukshana Shroff ${ }^{3}$

${ }^{1}$ Department Of Pediatrics, Rostock University Medical Centre, Rostock, Germany, ${ }^{2}$ Institute Of Global Health, University College London, London, UK, ${ }^{3}$ University College London Great Ormond Street Hospital For Children And Institute Of Child Health, London, UK, ${ }^{4}$ Gazi University Hospital, Ankara, Turkey, ${ }^{5}$ A \& P Kyriakou Children's Hospital, Athens, Greece, ${ }^{6}$ Clinic Of Pediatrics, Faculty Of Medicine, Vilnius University, Vilnius, Lithuania, ${ }^{7}$ Cukurova University, Adana, Turkey, ${ }^{8}$ Cerrahpasa School Of Medicine, Istanbul, Turkey, ${ }^{9}$ Children Memorial Health Institute, Warsaw, Poland, ${ }^{10}$ Fondazione Irccs Ca' Granda Ospedale Maggiore Policlinico, Milan, Italy, ${ }^{11}$ Hôpital Femme Mère Enfant, Hospices Civils De Lyon, Université De Lyon, Bron, France, ${ }^{12}$ University Children Hospital, Belgrade, Serbia, ${ }^{13}$ Children Dialysis Center, Strasbourg, France, ${ }^{14}$ Center For Pediatrics And Adolescent Medicine, Heidelberg, Germany

Introduction: Patients on dialysis have a high burden of bone related comorbidities manifesting as bone pain, deformities, growth retardation and fractures.

Material and methods: We report a post-hoc analysis of the prospective cohort study "HDF-Heart-Height" to determine the prevalence and risk factors for CKD-related bone disease in children on hemodiafiltration (HDF) and conventional hemodialysis (HD).

Results: 144 children were included in baseline cross-sectional analysis, of which 103 (61 HD, $42 \mathrm{HDF}$ ) completed 12-month followup. Circulating biomarkers of bone formation and resorption, inflammatory markers, fibroblast growth factor-23 (FGF23) and klotho were measured. Inflammatory markers interleukin-6 [IL-6], tumor necrosis factor-alfa $[\mathrm{TNF}-\alpha]$, and high-sensitivity CRP [hsCRP] were lower in HDF compared to HD cohorts at baseline and 12 months $(\mathrm{p}<0.001)$. Concentrations of bone formation (bonespecific alkaline phosphatase, BAP) and resorption (tartrate-resistant acid phosphatase $5 \mathrm{~b}$ [TRAP5b]) markers were comparable between cohorts at baseline, but after 12-months the BAP/TRAP5b ratio increased in HDF ( $p=0.004)$ and was unchanged in HD $(p=0.44)$. On adjusted analysis the BAP/TRAP5b ratio was 2.66-fold lower $(95 \%$ CI $-3.91,-1.41 ; p<0.0001)$ in HD compared to HDF. FGF23 was comparable between groups at baseline $(\mathrm{p}=0.52)$ but increased in HD $(p<0.0001)$ and remained unchanged in HDF $(p=0.34)$ at 12 months. Klotho levels were similar between groups and unchanged during follow-up. The FGF23/klotho ratio was 3.86-fold higher $(95 \%$ CI $2.15,6.93 ; \mathrm{p}<0.0001)$ after 12 months of HD compared to HDF.

Conclusions: We conclude that children on HDF have an attenuated inflammatory profile, increased bone formation and lower FGF23/ klotho ratios compared to those on HD. Long-term studies are required to determine the effects of an improved bone biomarker profile on fracture risk and cardiovascular health.

\section{OP-28 ASSESSMENT OF ULTRAFILTRATION ACCURACY IN CHILDREN ON HOME HAEMODIALYSIS}

Iona Madden ${ }^{1}$, Jerome Harambat ${ }^{2}$, Daljit Hothi ${ }^{1}$

${ }^{I}$ Great Ormond Street Hospital, London, ${ }^{2}$ Bordeaux University Hospital, Bordeaux, France

Introduction: Paediatric home haemodialysis (HHD) is infrequently performed despite growing data in favour of intensive dialysis. One of the main concerns regarding paediatric HHD is the lack of evidence showing ultrafiltration (UF) accuracy of the dialysis machines that have been adapted to children. UF inaccuracy may cause serious complications, especially in younger children with a lower circulating volume. In this study, covering a period of 10 years' experience dialysing children at 
home using the NxStage Dialysis System OneTM, we investigated the frequency and potential determinants of UF inaccuracy.

Material and methods: This was a retrospective single centre observational study of all children under the age of 18 and undergoing HHD treatment using the NxStage System OneTM from 2011 to 2020. The primary event of interest was UF accuracy measurement. This was defined as the difference (in L) between the planned UF and the actual UF volume for each HHD session. In addition, we investigated associations between range of UF accuracy and machine-related as well as patientrelated factors.

Results: Twenty-one children undergoing HHD with a total of 2230 HHD sessions were included. The median UF accuracy was 0.1 liters (IQR 0.0-0.2) representing 6.6\% (IQR 3.1-12.3) of total blood volume. Using a mixed-effects linear regression model without covariate, the mean UF accuracy was $0.05 \mathrm{~L}$ (95\% CI -0.01-0.10). The agreement between planned and actual UF was strong (bias $0.07 \mathrm{~L}$ ). We found a statistically significant association between the patient's weight, software, dialysate flow rate, venous pressure, transmembrane pressure and UF accuracy.

Conclusions: Our study suggests good overall UF accuracy whilst dialysing with the NxStageTM with a strong agreement between the planned UF and the actual UF. These results should provide some reassurance to those considering HHD in children.

\section{OP-29 DIETARY FIBRE INTAKE IS ASSOCIATED WITH SERUM LEVELS OF URAEMIC TOXINS IN CHILDREN WITH CHRONIC KIDNEY DISEASE}

Amina El Amouri ${ }^{1}$, Evelien Snauwaert ${ }^{1}$, Aurélie Foulon ${ }^{2}$, Charlotte Vande Moortel ${ }^{2}$, Maria Van Dyck ${ }^{3}$, Koen Van Hoeck ${ }^{4}$, Nathalie Godefroid ${ }^{5}$, Griet Glorieux ${ }^{2}$, Wim Van Biesen ${ }^{2}$, Johan Vande Walle ${ }^{1}$, Ann Raes ${ }^{1}$, Sunny Eloot ${ }^{2}$

${ }^{1}$ Paediatric Nephrology, Ghent University Hospital, ${ }^{2}$ Nephrology, Ghent University Hospital, ${ }^{3}$ Paediatric Nephrology, University Hospitals Leuven, ${ }^{4}$ Paediatric Nephrology, Antwerp University Hospital, ${ }^{5}$ Paediatric Nephrology, Cliniques Universitaires St. Luc

Introduction: Unbalanced colonic microbial metabolism plays a pivotal role in generating protein-bound uraemic toxins (PBUTs), accumulating with deteriorating kidney function and contributing to the uraemic burden of children with chronic kidney disease (CKD). Dietary choices impact the gut microbiome and metabolism. Our aim was to investigate the relation between dietary fibre and gut-derived PBUTs in paediatric CKD. Material and methods: Sixty-one (44 male) CKD children 9 [5;14] years were prospectively followed at 3-month intervals for 2 years. Dietary fibre intake was evaluated by either 24 -h recalls (73\%) or 3-day food records (27\%) at the same time of blood sampling for assessment of total and free serum levels of different PBUTs (mg/dL) using liquid chromatography. Linear mixed models for (natural) log-transformed plasma concentrations were fitted with a random intercept for the patient and with fibre intake (g/day), protein intake (g/day), BSA as a proxy for age $\left(\mathrm{m}^{2}\right)$, eGFR $\left(\mathrm{mL} / \mathrm{min} / 1.73 \mathrm{~m}^{2}\right)$ to assess associations between fibre intake and PBUT levels.

Results: For every g/day increase in fibre consumption, mixed-model analysis revealed a $1.6 \%[-3.0 \% ;-0.3 \%]$ lower total IAA concentration $(\mathrm{p}=0.020)$, whereas free IAA levels were $6.6 \%[-9.3 \% ;-3.7 \%](\mathrm{p}<$ $0.001)$ lower. Total pCG levels were $3.0 \%[-5.6 \% ;-0.5 \%](\mathrm{p}=0.021)$ lower, and free serum pCG $3.3 \%[-5.8 \% ;-0.8 \%](\mathrm{p}=0.010)$ lower per $\mathrm{g} /$ day increase in daily fibre consumption. For every gram of increment in daily fibre intake, free IxS levels were $3.1 \%[-5.9 \% ;-0.3 \%](\mathrm{p}=0.035)$ lower, and free pCS were $2.5 \%[-4.7 \% ;-0.3 \%](\mathrm{p}=0.034)$ lower. In contrast, total IxS and pCS serum concentrations were not associated with daily fibre intake.
Conclusions: The observed associations between dietary fibre intake and the investigated PBUTs highlight potential benefits of fibre intake in the paediatric CKD population. The present observational findings should inform and guide adaptations of dietary prescriptions in children with CKD.

\section{OP-30 NUTRITIONAL STATUS IN CHILDREN RECEIVING HEMODIAFILTRATION VS CONVENTIONAL HEMODIALYSIS - THE HDF, HEART AND HEIGHT (3H) STUDY}

\author{
Fabio Paglialonga ${ }^{1}$, Alice Monzani ${ }^{2}$, Francesca De Zan ${ }^{3}$, \\ Claus Peter Schmitt ${ }^{4}$, Franz Schaefer ${ }^{4}$, Alberto Edefonti ${ }^{1}$, \\ Rukshana Shroff ${ }^{3}$ \\ ${ }^{I}$ Fondazione Irccs Ca Granda Ospedale Maggiore Policlinico, Milan, \\ Italy, ${ }^{2}$ Universita Del Piemonte Orientale, Novara, Italy, ${ }^{3}$ University \\ College London, Great Ormond Street Hospital For Children Nhs \\ Foundation Trust, London, UK, ${ }^{4}$ Center For Pediatrics And Adolescent \\ Medicine, Heidelberg, Germany
}

Introduction: The "HDF-Heart-Height" study showed that hemodiafiltration (HDF) is associated with better statural growth than conventional hemodialysis (HD) in children. We report a post-hoc analysis of the same study assessing the effects of dialysis modality on nutritional status.

Material and methods: We included in the baseline cross-sectional analysis (T0) 107 incident and prevalent children, 79 of whom (43 HD, 36 HDF) completed a 12-month follow-up (T12). Height (HtSDS), weight (Wt-SDS), and body mass index (BMI-SDS) standard deviations scores, waist-to-hip ratio, normalized protein catabolic rate (nPCR), insulin-like growth factor-1 (IGF-1)-SDS, des-acyl ghrelin (DAG), adiponectin, leptin, insulin, and prealbumin levels were measured.

Results: Compared to patients treated with HD, those on HDF had higher Ht-SDS both at T0 and T12 (-1.4 vs $-2.4 ; \mathrm{p}=0.01$, and $-1.6 \mathrm{vs}$ $-2.6 ; \mathrm{p}=0.009$, respectively) and higher W-SDS at T12 (-1.5 vs -2.1 ; $\mathrm{p}=0.02$ ), while no differences were observed for the other anthropometric or laboratory parameters. On univariate analysis, Ht-SDS positively correlated with IGF1-SDS and inversely with adiponectin and DAG, Wt-SDS positively correlated with IGF-1 SDS and leptin and negatively with DAG, while BMI-SDS correlated with leptin and insulin. On multivariable linear regression analyses, higher Wt-SDS at T12 was positively associated with HDF modality (beta $=0.47$ vs HD; 95\%CI 0.12-0.83; $\mathrm{p}=0.01$ ) and inversely with T0 DAG (beta=0.18 per 500 higher; $95 \%$ CI $-0.32,-0.05 ; \mathrm{p}=0.006$ ). Similarly, higher T12 Ht-SDS was associated with HDF modality (beta $=0.20$ vs HD; $95 \% \mathrm{CI}-0.05,0.44 ; \mathrm{p}=0.11$ ) and lower T0 DAG (beta $=-0.13$ per 500 higher; 95\% CI -0.22, $-0.04 ; \mathrm{p}=0.004)$.

Conclusions: Although children treated with HDF have better growth than those receiving $\mathrm{HD}$, and growth is independently associated with DAG levels, the effect of dialysis modality on growth does not appear to be related to an improvement in the measured nutritional parameters. Further studies are needed to assess the mechanisms linking HDF to improved growth.

\section{OP-31 ASSESSMENT OF ENDOGENOUS OXALATE PRODUCTION IN PRIMARY HYPEROXALURIA TYPE 1 PATIENTS BY STABLE ISOTOPE TRACER INFUSION}

Sander F. Garrelfs , Dewi Van Harskamp, Hessel Peters-Sengers, Chris H.P. Van Den Akker, Ronald J.A. Wanders, Frits A. Wijburg, Johannes B. Van Goudoever, Jaap W. Groothoff, Henk Schierbeek, Michiel J.S. Oosterveld 
Amsterdam Umc, University Of Amsterdam, Emma's Children's Hospital, Amsterdam, The Netherlands

Introduction: Primary Hyperoxaluria type 1 (PH1) is an inborn error of glyoxylate metabolism characterized by increased endogenous oxalate production. Its exact metabolic pathways have not been fully elucidated and upcoming therapies require more reliable outcome parameters than currently used plasma oxalate levels and urinary oxalate excretion rates. We therefore developed a stable isotope infusion protocol to assess endogenous oxalate synthesis rate and the contribution of glycolate to both oxalate and glycine synthesis in vivo.

Material and methods: Eight healthy volunteers and eight patients with PH1 (stratified by pyridoxine responsiveness) underwent a combined primed continuous infusion of intravenous $\left[1-{ }^{13} \mathrm{C}\right]$ glycolate, $\left[\mathrm{U}_{-}{ }^{13} \mathrm{C}_{2}\right]$ oxalate and, in a subgroup, $\left[\mathrm{D}_{5}\right]$ glycine. Isotopic enrichments of ${ }^{13} \mathrm{C}$-labelled oxalate and glycolate were measured using a newly developed gas chromatography - tandem mass spectrometry (GC-MS/MS) method. Stable isotope dilution and incorporation calculations were used to quantify glyoxylate kinetics.

Results: Total daily endogenous oxalate rates of appearance (mean (SD)) were $2.71(0.54), 1.46(0.23)$ and $0.79(0.15) \mathrm{mmol}$ per day in pyridoxine unresponsive patients, pyridoxine responsive patients and controls, respectively $(p=0.002)$. Mean (SD) contribution of glycolate to oxalate production was $47.3 \%$ (12.8) in patients and $1.3 \%(0.7)$ in controls. Using the incorporation of $\left[1-{ }^{13} \mathrm{C}\right]$ glycolate tracer in glycine revealed significant conversion of glycolate into glycine in pyridoxine responsive, but not in pyridoxine unresponsive $\mathrm{PH} 1$ patients.

Conclusions: This stable isotope infusion protocol could be applied to evaluate therapeutic efficacy of new therapies, investigate pyridoxine responsiveness and serve as a tool to further explore glyoxylate metabolism in humans.

\section{OP-32 OUTCOME OF INFANTILE OXALOSIS IN EUROPE.}

Lisa J Deesker ${ }^{1}$, Sander F Garrelfs ${ }^{1}$, Giorgia Mandrile ${ }^{5}$, Michiel J S Oosterveld ${ }^{1}$, Pierre Cochat ${ }^{3}$, Gill Rumsby ${ }^{2}$, Georges Deschenes ${ }^{4}$, Bernd Hoppe ${ }^{6}$, Sally-anne Hulton ${ }^{7}$, Rezan Topaloglu ${ }^{8}$, Jaap W Groothoff ${ }^{1}$

${ }^{1}$ Department Of Pediatric Nephrology, Emma Childrens Hospital, Amsterdam Umc, University Of Amsterdam, Amsterdam, The Netherlands, ${ }^{2}$ Department Of Clinical Biochemistry, University College London Hospitals, London, UK, ${ }^{3}$ Department Of Pediatric Nephrology, Hospices Civils De Lyon And University De Lyon, Lyon, France, ${ }^{4}$ Department Of Pediatric Nephrology, Robert DebrÉ Hospital, Paris, France, ${ }^{5}$ Departement Of Clinical And Biological Sciences, Medical Genetics Unit, S. Luigi Hospital, University Of Torino, Orbassano, Italy, ${ }^{6}$ Department Of Pediatric Nephrology, Childrens Hospital Of The University Of Bonn, Bonn, Germany, Department Of Nephrology, Birmingham Childrens Hospital Nhs Foundation Trust, Birmingham, UK, ${ }^{8}$ Department Of Pediatric Nephrology And Rheumatology, Hacettepe University Faculty Of Medicine, Ankara, Turkey

Introduction: Infantile oxalosis is the most severe form of primary hyperoxaluria $(\mathrm{PH})$, with onset of symptoms and end-stage kidney disease (ESKD) at a very young age. Our aim was to provide an overview of the course of the disease, treatment, and prognosis of this severe subgroup of $\mathrm{PH}$ patients as our current understanding is limited by a paucity of literature.

Material and methods: A retrospective cohort study was conducted using data from the European registry OxalEurope. All PH patients with infantile oxalosis, defined as development of ESKD before the age of one year, were identified.
Results: We identified 95 patients with infantile oxalosis, all diagnosed with $\mathrm{PH}$ type 1 . The median (interquartile range, IQR) age at ESKD was $0.35(0.25-0.50)$ years. Five patients were diagnosed by family screening. Eleven patients were homozygous for a B6responsive mutation and 25 were compound heterozygous. Systemic oxalosis was reported in almost all screened patients $(n=54,96 \%)$. A total of 27 patients $(28 \%)$ of this cohort died, at a median (IQR) age of $1.37(0.61-2.00)$ years. The median (IQR) age at first transplantation was $1.72(1.30-2.90)$ years. Of these patients, 43 underwent a combined liver-kidney transplantation and 24 a liver transplantation as part of a sequential procedure. Patient survival did not differ when comparing dialysis modalities (intensive versus normal dialysis) or transplantation strategies (combined versus sequential liver-kidney transplantation). However, log-rank analysis showed a significant $(\mathrm{p}=0.002)$ improved patient survival in patients born after the year 2000 .

Conclusions: The clinical outcome of patients with infantile oxalosis is severe, with ESKD at a young age, development of systemic oxalate deposits, and a poor survival. However, patient survival has improved over the years and might improve further in the light of new therapeutic modalities that are on the horizon.

\section{OP-33 CLINICAL AND METABOLIC CHARACTERISTICS OF CHILDHOOD RENAL MICROLITHIASIS IN DIFFERENT AGE GROUPS}

Onur Erișen ${ }^{1}$, Gökçen Erfidan ${ }^{2}$, Eren Soyaltin ${ }^{2}$, Özgür Özdemir Șimșek ${ }^{2}$, Seçil Arslansoyu Çamlar ${ }^{3}$, Demet Alaygut ${ }^{2}$, Belde Kasap Demir ${ }^{4}$, Fatma Mutlubaș ${ }^{3}$

${ }^{I}$ University Of Health Sciences, Tepecik Training And Research Hospital. Department Of Pediatrics, Izmir, Turkey, ${ }^{2}$ University Of Health Sciences, Tepecik Training And Research Hospital, Department Of Pediatrics, Division Of Nephrology, Izmir, Turkey, ${ }^{3}$ University Of Health Sciences, Izmir Faculty Of Medicine, Department Of Pediatrics, Division Of Nephrology, Izmir, Turkey, ${ }^{4}$ Izmir Katip Celebi University, Faculty Of Medicine, Department Of Pediatrics, Division Of Nephrology And Rheumatology, Izmir, Turkey

Introduction: Renal microlithiasis(RM) is a radiological diagnostic term used to define stones $<3 \mathrm{~mm}$ in size. It was aimed to compare the etiology, clinical and radiological findings of pediatric patients diagnosed with renal microlithiasis in infancy and later years.

Material and methods: Children under age of 18 who were followed-up with RM between 2010-2019 were analyzed retrospectively. The patients were grouped according to their age of diagnosis. The patients with $0-24$ months were included to Group-1, and $>24$ months were to Group-2.

Results: There were 72 patients in Group-1, 18 patients in Group-2. The frequency of hospitalizations was $50 \%$ higher in Group$1(p=0.046)$. Unilateral one RM was more common in Group-2, unilateral multiple RM was in Group-1( $\mathrm{p}=0.002)$. Metabolic disorders were detected in $40.3 \%$ of Group-1, and in $33.3 \%$ of Group$2(p=0.046)$. The most common metabolic disorder was hypercalciuria $(22 \%)$ in Group-1, hyperoxaluria (29\%) in Group-2. Hypocitraturia $(23.8 \%)$ and hyperuricosuria $(19 \%)$ were found more common in Group-2( $\mathrm{p}=0.043, \mathrm{p}=0.044$ respectively $)$. The incidental diagnosis $(58.3 \%)$ was higher in Group- $1(\mathrm{p}<0.001)$. In the first evaluation, the number and diameter of RM increased in $29.2 \%$ and $19.4 \%$ of patients, respectively. After two years of follow-up, the RM disappeared in $90.3 \%$ of Group- 1 and $95.2 \%$ of Group- 2 . The frequency of unilateral one RM in Group- 2 and unilateral multiple $\mathrm{RM}$ in Group-1 were statistically higher( $\mathrm{p}=0.002)$. In Group- $1, \mathrm{RM}$ was mostly located in the lower pole of the right $(47.5 \%)$ and left 
kidneys $(60.7 \%)$, while in the Group-2, it was located in the middle pole for the right $(50 \%)$ and left kidneys $(53.3 \%)$. Upper pole location was the rarest.

Conclusions: Renal microlithiasis is a common clinical condition in childhood. Since metabolic disorders mostly play a role in the etiology, metabolic tests should be performed not only in infancy but also in advanced childhood and adolescence.

\section{OP-34 LONG-TERM TRANSPLANTATION OUTCOMES IN PATIENTS WITH PRIMARY HYPEROXALURIA TYPE I: RESULTS FROM THE OXALEUROPE DATABASE}

Elisabeth Metry ${ }^{1}$, Sander Garrelfs ${ }^{1}$, Hessel Peters-sengers ${ }^{2}$, Michiel Oosterveld ${ }^{1}$, Gill Rumsby ${ }^{3}$, Graham Lipkin ${ }^{4}$, Sally-ann Hulton ${ }^{5}$, Shabbir Moochhalla ${ }^{6}$, Justine Bacchetta ${ }^{7}$, Georges Deschenes ${ }^{8}$, Cecile Acquaviva ${ }^{9}$, Bodo Beck ${ }^{10}$, Bernd Hoppe ${ }^{11}$, Markus Kemper ${ }^{12}$, Casper Franssen ${ }^{13}$, Georgia Mandrile ${ }^{14}$, Laure Collard ${ }^{15}$, Nilufar Mohebbi ${ }^{16}$, Larisa Prikhodina ${ }^{17}$, Jaap Groothoff ${ }^{1}$

${ }^{1}$ Department Of Pediatric Nephrology, Emma Childrens Hospital, Amsterdam Umc, University Of Amsterdam, Amsterdam, The Netherlands ${ }^{2}$ Center For Experimental And Molecular Medicine, Amsterdam Umc, University Of Amsterdam, Amsterdam, The Netherlands, ${ }^{3}$ Department Of Clinical Biochemistry, University College London Hospitals, London, UK, ${ }^{4}$ Department Of Nephrology, University Hospitals Birmingham Nhs Foundation Trust, Birmingham, UK, ${ }^{5}$ Department Of Nephrology, Birmingham Childrens Hospital Nhs Foundation Trust, Birmingham, UK, ${ }^{6}$ Kidney And Urology Centre, Royal Free Hospital, London, UK, ${ }^{7}$ Department Of Pediatric Nephrology, Rheumatology And Dermatology, University Childrens Hospital, Lyon, France, ${ }^{8}$ Department Of Pediatric Nephrology, Assistance Publiquehôpitaux De Paris, Hôpital Robert Debré, University Of Paris, Paris, France, ${ }^{9}$ Fédération De Génétique, Assistance Publique-hôpitaux De Paris, Hôpital Robert Debré, University Of Paris, Paris, France, ${ }^{10}$ Institute Of Human Genetics, Center For Molecular Medicine Cologne, And Center For Rare And Hereditary Kidney Disease, University Hospital Of Cologne, Cologne, Germany, ${ }^{11}$ Department Of Pediatric Nephrology, Childrens Hospital Of The University Of Bonn, Bonn, Germany, ${ }^{12}$ Division Of Pediatric Nephrology, University Childrens Hospital, Hamburg, Germany, ${ }^{13}$ Department Of Internal Medicine, University Medical Center Groningen, University Of Groningen, Groningen, The Netherlands, ${ }^{14}$ Medical Genetics Unit, Department Of Clinical And Biological Sciences, University Of Torino, Orbassano (to), Italy; Genetica E Thalassemia Unit, San Luigi University Hospital, Orbassano (to), Italy, ${ }^{15}$ Department Of Pediatric Nephrology, Chu Liège, Liège, Belgium, ${ }^{16}$ Division Of Nephrology, University Hospital Zurich, Zurich, Switzerland, ${ }^{17}$ Department Of Inherited And Acquired Kidney Diseases, Research And Clinical Institute For Pediatrics, Pirogov Russian National Research Medical University, Moscow, Russia

Introduction: Primary hyperoxaluria type $1(\mathrm{PH} 1)$ is a rare genetic disorder causing calcium-oxalate kidney stones and often kidney failure. Since this is due to overproduction of oxalate by the liver, combined liver-kidney transplantation (CLKT) is recommended. However, the advantage of a sequential procedure (SLKT) remains unclear as well as the place of isolated kidney transplant (KT) for patients in whom vitamin B6 lowers hepatic oxalate production effectively. Data on long-term transplantation outcomes are scarce.
Material and methods: We retrospectively analyzed data from the OxalEurope registry. Out of approximately $1100 \mathrm{PH}$ patients, we selected PH1 patients who underwent one or more transplantations. Survival analyses were performed using Kaplan Meier method.

Results: In total, 267 PH1 patients underwent transplantation between 1978 and 2019. Data of 244 transplantations were eligible for comparative analyses. Median (interquartile range) follow-up was 6.0 years (1.9-11.5). Event-free survival was better for CLKT compared to KT in B6 unresponsive patients $(\mathrm{p}<0.001)$, but not for CLKT compared to KT in B6 responsive patients $(\mathrm{p}=0.411)$, while death-censored kidney graft survival was superior for CLKT in both groups. Comparable outcomes were found for CLKT $(n=159)$ and SLKT $(n=37)$. Twelve patients underwent pre-emptive liver transplantations with poor outcomes.

Conclusions: Combined or sequential liver-kidney transplantation remains the preferred transplantation modality in B6 unresponsive patients with kidney failure. Our findings support the choice for isolated KT in B6 responsive patients.

\section{OP-35 EARLY CHILDHOOD HEIGHT-ADJUSTED TOTAL KIDNEY VOLUME AS A RISK MARKER OF KIDNEY SURVIVAL IN AUTOSOMAL RECESSIVE POLYCYSTIC KIDNEY DISEASE (ARPKD)}

Kathrin Burgmaier ${ }^{1}$, Samuel Kilian ${ }^{2}$, Klaus Arbeiter ${ }^{3}$, Bahriye Atmis ${ }^{4}$, Anja BÜscher ${ }^{5}$, Ute Derichs ${ }^{6}$, Ismail Dursun ${ }^{7}$, Ali Duzova ${ }^{8}$, Loai Eid ${ }^{9}$, Matthias Galiano ${ }^{10}$, Michaela Gessner ${ }^{11}$, Ibrahim Gokce ${ }^{12}$,

Karsten Haeffner ${ }^{13}$, Nakysa Hooman ${ }^{14}$, Augustina Jankauskiene ${ }^{15}$, Friederike KÖrber ${ }^{16}$, Germana Longo ${ }^{17}$, Laura Massella ${ }^{18}$,

Djalila Mekahli ${ }^{19}$, Gordana Miloševski-lomić ${ }^{20}$, Hulya Nalcacioglu ${ }^{21}$, Rina Rus ${ }^{22}$, Rukshana Shroff ${ }^{23}$, Stella Stabouli ${ }^{24}$, Lutz Weber ${ }^{1}$,

Simone Wygoda ${ }^{25}$, Alev Yilmaz ${ }^{26}$, Katarzyna Zachwieja ${ }^{27}$, Ilona Zagozdzon ${ }^{28}$, JÖrg DÖtsch ${ }^{1}$, Franz Schaefer ${ }^{29}$,

Max Christoph Liebau ${ }^{30}$

${ }^{1}$ Department Of Pediatrics, University Hospital Cologne And University Of Cologne, Faculty Of Medicine, Cologne, Germany, ${ }^{2}$ Institute Of Medical Biometry And Informatics, University OfHeidelberg, Heidelberg, Germany, ${ }^{3}$ Department Of Paediatrics And Adolescent Medicine, Medical University Vienna, Vienna, Austria, ${ }^{4}$ Department Of Pediatric Nephrology, Cukurova University Faculty Of Medicine, Adana, Turkey, ${ }^{5}$ Department Of Pediatrics Ii, University Hospital Essen, Essen, Germany, ${ }^{6}$ Pediatric Nephrology, Center For Paediatric And Adolescent Medicine, University Medical Clinic, Mainz, Germany, ${ }^{7}$ Department Of Pediatric Nephrology, Erciyes University, Faculty Of Medicine, Kayseri, Turkey, ${ }^{8}$ Department Of Pediatrics, Division Of Pediatric Nephrology, Hacettepe University Faculty Of Medicine, Ankara, Turkey, ${ }^{9}$ Department Of Pediatrics, Division Of Pediatric Nephrology, Dubai Hospital, Dubai, United Arab Emirates, ${ }_{10}^{10}$ Department Of Pediatrics And Adolescent Medicine, University Of Erlangen-nürnberg (fau), Erlangen, Germany ${ }^{11}$ Department Of General Pediatrics And Hematology/oncology, Children's University Hospital Tuebingen, Germany, ${ }^{12}$ Research And Training Hospital, Division Of Pediatric Nephrology, Marmara University, Istanbul, Turkey, ${ }^{13}$ Department Of Internal Medicine Iv, Medical Center, University Of Freiburg, Medical Faculty, Freiburg, Germany, ${ }^{14}$ Department Of Pediatric Nephrology, Ali-asghar Children Hospital, Aliasghar Clinical Research Development Center (aacrdc), Iran University Of Medical Sciences, Tehran, Iran, ${ }^{15}$ Clinic Of Children Diseases, Institute Of 
Clinical Medicine, Vilnius University, Vilnius, Lithuania, ${ }^{16}$ Pediatric Radiology, Institute Of Diagnostic And Interventional Radiology, University Hospital Of Cologne, Cologne, Germany, ${ }^{17}$ Pediatric Nephrology, Dialysis And Transplant Unit, Department Of Woman And Child Health, Azienda Ospedaliera-university Of Padova, Padova, Italy, ${ }^{18}$ Division Of Nephrology, Department Of Pediatric Subspecialties, Bambino Gesù Children's Hospital, Irccs, Rome, Italy, ${ }^{19}$ Department Of Development And Regeneration, Pkd Research Group, Ku Leuven, Leuven, Belgium; Department Of Pediatric Nephrology, University Hospitals Leuven, Leuven, Belgium, ${ }^{20}$ Department Of Nephrology, University Children's Hospital, Belgrade, Serbia, ${ }^{21}$ Ondokuz Mayis University Faculty Of Medicine, Pediatric Nephrology Department, Samsun, Turkey, ${ }^{22}$ University Medical Centre Ljubljana, University Children's Hospital, Dept Of Nephrology, Ljubljana, Slovenia, ${ }^{23}$ Ucl Great Ormond Street Hospital For Children Institute OfChild Health, Ucl, London, $U K,{ }^{24}$ First Department Of Pediatrics, School Of Medicine, Faculty Of Health Sciences, Aristotle University Of Thessaloniki, Hippokratio Hospital, Thessaloniki, Greece, ${ }^{25}$ Clinic For Children And Adolescents, Hospital St. Georg, Leipzig, Germany, ${ }^{26}$ Pediatric Nephrology Department, Istanbul Faculty Of Medicine, Istanbul University, Istanbul, Turkey, ${ }^{27}$ Department Of Pediatric Nephrology And Hypertension, Faculty Of Medicine, Jagiellonian University Medical College, Krakow, Poland, ${ }^{28}$ Department Of Nephrology And Hypertension Of Children And Adolescents, Medical University Of Gdansk, Gdansk, Poland, ${ }^{29}$ Division Of Pediatric Nephrology, Center For Pediatrics And Adolescent Medicine, University Hospital Heidelberg, Heidelberg, Germany, ${ }^{30}$ Department Of Pediatrics, University Hospital Cologne And University Of Cologne, Faculty Of Medicine, Cologne, Germany; Center For Molecular Medicine, University Hospital Cologne And University Of Cologne, Faculty Of Medicine, Cologne, Germany

Introduction: Autosomal recessive polycystic kidney disease (ARPKD) is one of the major reasons for early-onset end stage kidney disease. Fibrocystic changes result in bilateral gross kidney enlargement. Courses of kidney function differ substantially between individuals and clear associations to kidney size have not been established so far. Easily available risk markers are eagerly awaited in order to stratify patients for future clinical trials and within clinical decision-making.

Material and methods: We analyzed clinical datasets of 456 ARPKD patients from the ARegPKD registry study for their longitudinal development of sonographic kidney pole-to-pole (PTP) length and estimated total kidney volume (eTKV). In a second step, we aimed for correlations of kidney sizes with kidney function.

Results: Sonographic kidney PTP length increases over time, but height-adjusted PTP (haPTP) length decreases. While eTKV increases during childhood and adolescence, height-adjusted eTVK (haeTVK) remains rather stable. Furthermore, haeTKV inversely correlates with estimated glomerular filtration rate in a loose manner. When stratifying for quartiles of haeTKV in the first 18 months of life, pediatric kidney survival differed clearly with 10 -year kidney survival rates of $20 \%$ in the highest quartile and $94 \%$ in the lowest quartile.

Conclusions: Infancy and early childhood haeTKV may serve as an easily obtainable risk marker of kidney function in children with ARPKD and thereby qualify for the identification of patients for future clinical studies.

\section{OP-36 HETEROZYGOUS VARIANTS IN KINASE DOMAIN OF} NEK8 CAUSE AN AUTOSOMAL-DOMINANT CILIOPATHY

Laura R Claus ${ }^{1}$, Jennifer Stallworth ${ }^{2}$, Richard H Van Jaarsveld ${ }^{1}$, Ray Louie ${ }^{2}$, Josh Silver ${ }^{3}$, Jordan Lerner-ellis ${ }^{4}$, Chantal Morel ${ }^{3}$, Chloe Mighton ${ }^{4}$, Alban Ziegler ${ }^{5}$, Stefan Barakat ${ }^{6}$, Karin Dahan ${ }^{7}$, Nathalie Demoulin ${ }^{8}$, Eric Goffin ${ }^{8}$, Martin J Larsen ${ }^{9}$, Jens Michael Hertz ${ }^{9}$, Marc Lilien ${ }^{10}$, Eric Olinger ${ }^{11}$, John A Sayer ${ }^{11}$, Curtis Rogers ${ }^{2}$,

Karen J Duran ${ }^{1}$, Edith Dj Peters ${ }^{1}$, Gijs Van Haaften ${ }^{1}$, Richard Steet ${ }^{1}$, Albertien M Van Eerde ${ }^{1}$

${ }^{I}$ Department Of Genetics, University Medical Center Utrecht, Utrecht University, The Netherlands, ${ }^{2}$ Greenwood Genetic Center, Greenwood, Sc Usa ${ }^{3}$ The Fred A. Litwin Family Centre In Genetic Medicine, University Health Network And Mount Sinai Hospital, Toronto Canada, ${ }^{4}$ Pathology And Laboratory Medicine, Mount Sinai Hospital, Sinai Health, Toronto, On, Canada ${ }^{5}$ Department Of Genetics, University Hospital Of Angers, France, ${ }^{6}$ Department Of Clinical Genetics, Erasmus Mc University Medical Center, Rotterdam, Netherlands, ${ }^{7}$ Center Of Human Genetics, Institut De Pathologie Et Génétique, Gosselies, Belgium; Division Of Nephrology, Université Catholique De Louvain Medical School, Brussels, Belgium, ${ }^{8}$ Department Of Nephrology, Cliniques Universitaires Saint-luc, Université Catholique De Louvain, Belgium, ${ }^{9}$ Department Of Clinical Genetics, Odense University Hospital, Denmark, ${ }^{10}$ Department Of Pediatric Nephrology, Wilhelmina Childrens Hospital, University Medical Center Utrecht, Utrecht, The Netherlands, ${ }^{11}$ Translational And Clinical Research Institute, Newcastle University. Central Parkway, Newcastle Upon Tyne, United Kingdom

Introduction: $N E K 8 / N P H P 9$ encodes a protein that localizes to the inversin compartment of the primary cilium. Biallelic $N E K 8$ variants are known to cause multiorgan developmental defects including renal cystic dysplasia and extensive extra-renal defects, with heterozygous carrier parents being asymptomatic. This autosomal recessive inheritance is the most common inheritance mode for ciliopathies. Complementary to this, we now propose a dominant-negative effect for certain heterozygous $N E K 8$ missense variants in the kinase domain resulting in an autosomaldominant ciliopathy.

Material and methods: Genetic testing was performed in patients from several medical centers. To further explore the consequences of the identified $N E K 8$ variants we are performing cilia staining assays in both patients skin fibroblast and kidney cells, as well as in mIMCD3 kidney cells overexpressing the identified variants.

Results: We identified three distinct heterozygous $N E K 8$ variants in seven families (table 1), all leading to missense alterations in the kinase domain. Interestingly the p.Arg45Trp variant is a recurrent variant we detected in five unrelated families. All patients have a kidney phenotype that varies from mild FSGS to prenatal presentation with polycystic kidneys. Some patients have end-stage kidney disease at varying ages of onset. In all patients we thoroughly checked whether a variant could be found on the second allele. Furthermore, the large symptomatic family and the de novo occurrences are also in favor of a dominant mode of inheritance.

Our preliminary results from functional studies show normal localization of the NEK8 protein to the Golgi region, but abnormal primary cilia formation, in serum starved patient derived cells - a finding consistent with pathogenicity. 
Table 1. Clinical features of individuals with heterozygous NEK8 variants

\begin{tabular}{|c|c|c|c|c|c|c|c|c|c|c|c|c|c|c|}
\hline Family & 1 & 2 & 3 & 4 & & 5 & & 6 & \multicolumn{6}{|c|}{7} \\
\hline Inheritance & de novo & de novo & de novo & de novo & mosaic & familial & familial & de novo & & & famil & & & \\
\hline NEK8 Variant & Arg45Trp & Arg45Trp & Arg45Trp & Arg45Trp & Arg45Trp & Arg45Trp & Arg45Trp & Lys157GIn & & & Ile150 & & & \\
\hline $\begin{array}{l}\text { Age at PKD } \\
\text { diagnosis } \\
\text { (years) }\end{array}$ & 11 & Fetal & 16 & Fetal & unknown & 1.5 & 1.5 & Fetal & $>70$ & 43 & 40 & 40 & 14 & 18 \\
\hline $\begin{array}{l}\text { Suspected } \\
\text { clinical } \\
\text { diagnosis }\end{array}$ & $\begin{array}{l}\text { NPH vs. } \\
\text { ADPKD }\end{array}$ & ARPKD & $\begin{array}{l}\text { ADPKD/ } \\
\text { ARPKD }\end{array}$ & ADPKD & ADPKD & ADPKD & APKD & ARPKD & ADPKD & ADPKD & ADPKD & ADPKD & $\begin{array}{l}\text { FSGS vs. } \\
\text { ADMCKD }\end{array}$ & FSGS \\
\hline $\begin{array}{l}\text { Age ESRD } \\
\text { (years) }\end{array}$ & 11 & 4 & 19 & 5 & 28 & 14 & 14 & N/A & $70 \mathrm{~s}$ & N/A & N/A & N/A & N/A & N/A \\
\hline $\begin{array}{l}\text { Enlarged } \\
\text { kidneys }\end{array}$ & + & + & + & + & unknown & + & + & + & - & - & $+/-$ & - & - & - \\
\hline $\begin{array}{l}\text { Liver cysts } \\
\text { (age in } \\
\text { years) }\end{array}$ & - & - & - & - & unknown & - & - & - & - & $+(52)$ & - & - & - & $+(18)$ \\
\hline Hypertension & + & + & + & + & unknown & + & + & + & & - & + & - & - & - \\
\hline $\begin{array}{c}\text { Extra-renal } \\
\text { features }\end{array}$ & $\begin{array}{l}\text { short } \\
\text { stature }\end{array}$ & $\begin{array}{c}\text { short } \\
\text { stature, } \\
\text { alopecia }\end{array}$ & NA & NA & unknown & $\begin{array}{l}\text { short } \\
\text { stature }\end{array}$ & $\begin{array}{l}\text { short } \\
\text { stature }\end{array}$ & $\begin{array}{l}\text { feeding } \\
\text { difficulties }\end{array}$ & $\begin{array}{c}\text { retinal } \\
\text { dystrophy* }\end{array}$ & $\begin{array}{c}\text { retinal } \\
\text { dystrophy* }\end{array}$ & $\begin{array}{c}\text { retinal } \\
\text { dystrophy* }\end{array}$ & NA & asthma & asthma \\
\hline
\end{tabular}

$A D M C K D=$ autosomal dominant medullary cystic kidney disease; $A D P K D=$ autosomal dominant polycystic kidney disease; $A R P K D=$ autosomal recessive polycystic kidney disease; FSGS = focal segmental glomerulosclerosis; N/A = not applicable; NPH = nephronophthisis. $\mid$ *consistent with RDS variant

Conclusions: We present the first evidence for a pathogenic effect of heterozygous $N E K 8$ variants. Remarkably our patients present with a renal limited phenotype as compared to the multiorgan defects found in patients with biallelic variants. This reveals a new mode of inheritance for $N E K 8$ variants and expands genotype-phenotype correlations for this gene.

\section{OP-37 PREDICTION OF RENAL PROGNOSIS IN NEPHRONOPHTHISIS}

Jens König ${ }^{1}$, Rebeka Karsay ${ }^{1}$, Joachim Ger $3{ }^{11}$, Karl Peter Schlingmann ${ }^{1}$, Mareike Dahmer-heath ${ }^{1}$, Anna-katharina Telgmann ${ }^{1}$, Sabine Kollmann ${ }^{1}$, Gema Ariceta ${ }^{2}$, Valentine Gillion ${ }^{3}$, Detlef Bockenhauer ${ }^{4}$,

Aurelia Bertholet-thomas ${ }^{5}$, Antonio Mastrangelo ${ }^{6}$, Olivia Boyer ${ }^{7}$,

Marc Lilien ${ }^{8}$, Stephane Decramer ${ }^{9}$, Joost-peter Schanstra ${ }^{10}$,

Petra Pennekamp ${ }^{1}$, Martin Konrad ${ }^{1}$

${ }^{I}$ University Childrens Hospital, Department Of General Pediatrics, Universitätsklinikum Münster, Münster, Germany, ${ }^{2}$ Servicio De Nefrología Pediátrica, Hospital Vall Dhebron, Universitat Autónoma De Barcelona, Barcelona, Spain, ${ }^{3}$ Division Of Nephrology, Saint-luc Academic Hospital, Université Catholique Louvain, Brussels, Belgium, ${ }^{4}$ Department Renal Medicine, University College London, London, $U K,{ }^{5}$ Centre De Référence Des Maladies Rénales Rares-néphrogones-hôpital Femme Mère Enfant, Hospices Civils De Lyon-filière Orkid, Bron, France, ${ }^{6}$ Fondazione Irccs Ca Granda Ospedale Maggiore Policlinico, Milan, Italy, ${ }^{7}$ Service De Néphrologie Pédiatrique, Centre De Référence Des Maladies Rénales Héréditaires De Lenfant Et De Ladulte (marhea), Institut Imagine, Hôpital Necker-enfants Malades, Université De Paris, Paris, France, ${ }^{8}$ Department Of Paediatric Nephrology, Wilhelmina Childrens Hospital, University Medical Center Utrecht And Utrecht University, Utrecht, The Netherlands, ${ }^{9}$ Institut National De La Santé Et De La Recherche Médicale (inserm), U1048, Institut Of Cardiovascular And Metabolic Disease, Toulouse, France; Université Toulouse Iii Paulsabatier, Toulouse, France; Service De Néphrologie Pédiatrique, Hôpital Des Enfants, Chu Toulouse, Toulouse, France; Centre De Référence Des Maladies Rénales Rares Du Sud-ouest (sorare), Toulouse, France, ${ }^{10}$ Université Toulouse Iii Paul-sabatier, 31330, Toulouse, France, ${ }^{11}$ Institute Of Biostatistics And Clinical Research, University Of Münster, MÜnster, Germany
Introduction: Nephronophthisis comprises rare genetic defects accounting for $10 \%$ of end-stage kidney disease (ESKD) in children. Despite the improved molecular understanding, prediction of individual renal prognoses still poses a major challenge. To overcome this burden, we assessed differences in genotype-specific kidney survival, the impact of mutational severity and the influence of other clinical characteristics on the decline of kidney function.

Material and methods: Information was obtained from 3 independent data sources: the Network of Early Onset Cystic Kidney Diseases (NEOCYST) clinical registry $(n=105)$, an online survey sent out to the members of the European Reference Network for Rare Kidney Diseases $(n=60)$ and a complementary literature search $(n=227)$.

Results: 392 genetically characterized individuals were available for analysis: 117 NPHP1, 85 NPHP3, 87 NPHP4 and 103 NPHP11. Kidney survival curves differed between the four cohorts with a highly variable outcome for onset of age when $50 \%$ of participants reached ESKD: NPHP3 4.0 yrs, NPHP1 13.5 yrs, NPHP4 16.0 yrs and NPHP11 19.0 yrs. Above that, for NPHP3 and NPHP4 kidney survival was significantly associated with the severity of the underlying genetic defect $(p<0.01)$. Multivariate analysis based on the phenotypic details available for the NPHP1 cohort additionally revealed arterial hypertension (HR 3.298) and growth retardation (HR 2.178) as two independent factors associated with an earlier onset of ESKD. Finally, the use of ACE inhibitors initiated for the treatment of arterial hypertension $+/$ - proteinuria was significantly associated with a more rapid annual GFR decline ( 13.0 vs. $6.2 \mathrm{ml} / \mathrm{min} / 1,73 \mathrm{~m} 2$ ) in patients with underlying NPHP1 defect. Conclusions: The present study represents the largest of its kind, addressing the kidney survival of the most relevant genetic causes of nephronophthisis. The presented data will enable clinicians to further classify and estimate the renal prognosis of their patients and thereby allow for personalized counselling.

\section{OP-38 USE OF EPITHELIAL MODELS TO STUDY CYST FORMATION IN PKD}

Fatima Hassan, Thomas Pokrant, Jan Faix, Dieter Haffner, Wolfgang H Ziegler

Hannover Medical School 
Introduction: Hereditary cystic kidney disease is associated with defective epithelial morphogenesis and/or homeostasis. To address molecular aspects of genetically induced epithelial defects, canine renal tubular epithelial cells, MDCK, appear to provide a well-established model. In 3D culture, MDCK cells form monolayered epithelial spheroids with apicobasal polarity and controlled water and ion transport. These structures can be employed to study consequences of pharmacological stimulation on cell morphology and epithelial barrier. Here, we study loss of fibrocystin, the cause of ARPKD, on the behavior of epithelial spheroids to evaluate usefulness of the genetic model in two closely related MDCK cell lines.

Material and methods: Fibrocystin function was suppressed by $P k h d 1$ silencing and CRISPR/Cas9-based genetic knockout in MDCKII and plMDCK, sub-cloned principal-like cells, respectively. Cells were grown in matrigel to allow spheroid formation, within 4 days, and treated with forskolin (FSK) to stimulate cAMP-induced cystic growth, as reported for PKD conditions. Lumen and cyst size were determined based on apical and basolateral markers using ImageJ/FIJI. Cyst formation was correlated to induction of STAT3 signaling.

Results: Our data show that MDCKII and pl-MDCK respond differently to FSK. Lumen shrinkage was observed in MDCKII spheroids upon treatment, while $P k h d 1$-silencing led to mild lumen expansion with no further impact of FSK. In contrast, pl-MDCK spheroids show enhanced lumen size for $P k h d 1$-knockout lines and strong expansion upon FSK stimulation of control and knockouts, mimicking PKD behavior. As proposed for loss of fibrocystin, pSTAT3 was induced in stimulated Pkhd1knockout cells but not controls.

Conclusions: 3D-culture models require functional evaluation of epithelial behavior to adequately reproduce tissue properties in vivo. Despite formation of well-established, polarized epithelial sheet and spheroids, MDCKII appear less suitable for PKD disease modeling, while pIMDCK may constitute a useful cell model for pharmacological treatment and comparison of different disease genetics.

\section{OP-39 PREVALENCE AND RISK FACTORS FOR THE DEVELOPMENT OF KIDNEY DAMAGE IN SICKLE CELL ANEMIA CHILDREN}

Oyindamola C. Adebayo ${ }^{1}$, Dieumerci K. Betukumesu ${ }^{2}$, Agathe B. Nkoy ${ }^{3}$, Oluyomi M. Adesoji ${ }^{4}$, Pepe M. Ekulu ${ }^{2}$, Lambertus P. Van Den Heuvel ${ }^{3}$, Elena Levtchenko ${ }^{3}$, Veerle Labarque ${ }^{1}$

${ }^{1}$ Center For Molecular And Vascular Biology, Department Of Cardiovascular Sciences, Katholieke Universiteit Leuven, Leuven, Belgium, ${ }^{2}$ Division Of Nephrology, Department Of Pediatrics, University Hospital Of Kinshasa, Faculty Of Medicine, University Of Kinshasa, Democratic Republic Of Congo, ${ }^{3}$ Department Of Development And Regeneration, Katholieke Universiteit Leuven, Leuven, Belgium, ${ }^{4}$ Cologne Center For Genomics, University Of Cologne, Cologne, Germany

Introduction: Clinical and genetic factors have been reported to influence the development of sickle cell nephropathy (SCN). However, such data remain limited in the pediatric population. Our study aimed to determine the prevalence of markers of kidney damage and to examine the association between these markers and clinical and genetic factors in children with sickle cell anemia (SCA).

Material and methods: In a cross-sectional study, we enrolled 361 patients with sickle cell disease from the Democratic Republic of Congo (DRC). Participants were genotyped for beta-globin gene, Apolipoprotein-L1 (APOL1) G1 and G2 variants, and Heme oxygenase-1 (HMOX1) GT dinucleotide repeats. As main outcomes, albuminuria was defined as urinary albumin-to-creatinine ratio (ACR) $\geq$ $30 \mathrm{mg} / \mathrm{g}$, decreased estimated GFR with creatinine (eGFRcr) when $<60$ $\mathrm{ml} / \mathrm{min} / 1.73 \mathrm{~m}^{2}$ and hyperfiltration when eGFRcr $>130$ and $140 \mathrm{ml} / \mathrm{min} /$ $1.73 \mathrm{~m}^{2}$ for female and male, respectively.
Results: From participants enrolled, 326 were confirmed to be SCA patients through genetic sequencing. Albuminuria, hyperfiltration and decreased eGFRcr were presented in $65(20 \%), 93(28 \%)$ and 18 $(5.50 \%)$ patients, respectively. Regression analysis revealed more frequent blood transfusions $(\mathrm{P}=0.01)$, lower diastolic blood pressure $(\mathrm{P}=$ $0.04)$ and male gender $(\mathrm{P}=0.02)$ as clinical determinants of $\mathrm{SCN}$. APOL1 high risk genotype (HRG) $(\mathrm{G} 1 / \mathrm{G} 1, \mathrm{G} 2 / \mathrm{G} 2$, and $\mathrm{G} 1 / \mathrm{G} 2)$ was significantly associated with albuminuria (odds ratio [OR]: $3.4,95 \%$ confidence interval $[\mathrm{CI}]:-0.02-2.4 ; \mathrm{P}=0.04$ ) and hyperfiltration (OR: $28.2,95 \% \mathrm{CI}: 1.3$ $-5.4 ; \mathrm{P}=0.001) . H M O X 1$ GT dinucleotide long repeats were significantly associated with lower eGFRcr $(\mathrm{P}=0.03)$.

Conclusions: The study revealed a high burden of kidney damage among children in the DRC. Frequent blood transfusions, male gender and lower diastolic blood pressure were found as the main clinical determinants and evidence has been provided of possible role of APOL1 and HMOX1 in making individuals more susceptible to kidney complications.

\section{OP-40 HIGH PHOSPHATE INTAKE IN MICE CAUSES INFLAMMATORY KIDNEY INJURY DOMINATED BY MACROPHAGES}

Isabel Vogt ${ }^{1}$, Stefanie Walter ${ }^{1}$, Tamar Kapanadze ${ }^{2}$, Jessica Schmitz ${ }^{3}$, Jan Hinrich Braesen ${ }^{3}$, Roland Schmitt ${ }^{2}$, Florian P. Limbourg ${ }^{2}$, Dieter Haffner ${ }^{1}$, Beatrice Richter ${ }^{1}$, Maren Leifheit-nestler ${ }^{1}$

${ }^{1}$ Department Of Pediatric Kidney, Liver And Metabolic Diseases, Pediatric Research Center, Hannover Medical School, Hannover, Germany, ${ }^{2}$ Department Of Nephrology And Hypertension, Hannover Medical School, Hannover, Germany, ${ }^{3}$ Institute Of Pathology, Nephropathology Unit, Hannover Medical School, Hannover, Germany

Introduction: Dietary phosphate intake greatly exceeds the recommended daily allowance in the Western population. Epidemiologic studies link elevated phosphate levels with an increased cardiovascular and all-cause mortality, but the direct renal effects of chronic high dietary phosphate intake are still under investigation.

Material and methods: Male C57BL/6 mice were fed with a $2 \%$ high phosphate diet (HPD) or a $0.8 \%$ normal phosphate diet (NPD) for up to six months. Blood, urine and kidneys were collected to investigate phosphate metabolism and kidney function, tissue alterations and inflammation. In vitro, we distinguish whether renal toxicity was directly mediated by phosphate or its phosphaturic hormone fibroblast growth factor (FGF) 23 in murine proximal tubule cells (mPTs).

Results: Feeding a HPD increased plasma intact FGF23 levels, phosphaturia, plasma creatinine and albuminuria compared to NPD. The HPD induced proximal tubular injury characterized by loss of proximal tubular cell polarity, flattened epithelia, lack of brush border membranes, increased proliferation, mononuclear interstitial infiltration and finally fibrosis. HPD-fed mice showed renal upregulation of tubular injury marker Kim-1 and $\mathrm{Ngal}$ compared to NPD mice. Immunofluorescent staining revealed KIM-1 localization to damaged proximal tubules accompanied by enhanced $\mathrm{Ki}-67^{+}$tubular nuclei, suggesting increased proliferation. $\mathrm{F} 4 / 80^{+}$macrophages were localized around tubular lesions and dominated the inflammatory response to the HPD. Accumulation of macrophages was accompanied by increased renal expression of chemotaxis and growth factors for monocytes and macrophages Mcp-1, M-csf and Il34 in HPD mice. In mPTs, high phosphate and FGF23 enhanced proliferation and stimulated the expression of Kim-1 and Mcp-1.

Conclusions: Chronic oral phosphate loading in mice impaires kidney function and causes a robust inflammatory response dominated by macrophages that is associated with proximal tubular injury and interstitial fibrosis. Our in vitro data indicate that high phosphate and FGF23 may contribute to an inflammatory driven kidney injury in phosphate loaded mice. 
OP-41 A COMPUTATIONAL APPROACH TO INVESTIGATE MALFUNCTIONS OF CENTRAL VENOUS LINES IN CHILDREN

Claudia Bruno ${ }^{1}$, Rayan Moumneh ${ }^{1}$, Emilie Sauvage ${ }^{1}$, Alberto C.1. Redaelli $^{2}$, Ian Simcock ${ }^{3}$, Silvia Schievano ${ }^{1}$, Claudio Capelli ${ }^{1}$, Rukshana Shroff ${ }^{3}$

${ }^{1}$ University College London, Institute Of Cardiovascular Science, London, UK; ${ }^{2}$ Department Of Electronics, Information And Bioengineering, Politecnico Di Milano, Milan, Italy; ${ }^{3}$ Great Ormond Street Hospital For Children, Nhs Foundation Trust, London, UK

Introduction: Central venous lines (CVLs) for haemodialysis (HD) in children are associated with frequent complications leading to inadequate dialysis and change of devices in nearly half the patients. Computational fluid dynamics (CFD) can be used to characterize the haemodynamic of CVLs. We studied CVLs commonly used in children to investigate the correlation between fluid-dynamics parameters and clinical outcomes.

Material and methods: Four models of CVLs of varying design and sizes $(6.5 \mathrm{~F}, 8 \mathrm{~F}, 10 \mathrm{~F}$ and $14 \mathrm{~F})$ were reconstructed from microCT images. CFD analyses were set up to simulate CVL working conditions in anatomical models of superior vena cava. Haemodynamic features including velocity fields, shear stresses, and platelet lysis index (PLI) were analysed, and CFD simulations compared to clinical data on catheter dysfunction from our Centre.

Results: Analysis of velocity fields identified presence of jets through the side holes (Figure 1a) and areas of recirculation and stagnation in all the designs, especially when used in arterial configurations. Side-holes played a crucial role in fluid exchange in all the design but in the $8 \mathrm{~F}$ design. In this case area of stagnation were found in proximity of the side-holes. Highest presence of shear stresses $>10 \mathrm{~Pa}$ were also found in the $8 \mathrm{Fr}$ CVL while highest PLI in the $10 \mathrm{~F}$ model. The anatomical model showed an increased disturbance of the flow (Figure 1b) and wall shear stresses. CFD results were in accordance with the clinical data which showed a higher recurrence rate of complications for the $8 \mathrm{Fr}$ and $10 \mathrm{Fr}$ CVLs (Figure 1c).

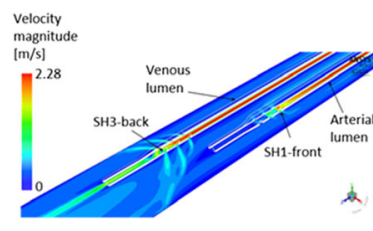

a)



b)

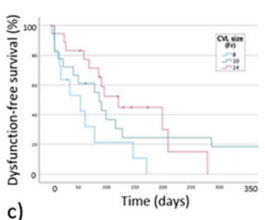

c)
Pédiatrie - Unité De Néphrologie, Chru Lille, ${ }^{6}$ Service De Pédiatrie Néphrologie, Médecine Interne Et Hypertension, Chu Toulouse, ${ }^{7}$ Département De Pédiatrie Médicale, Chu Rouen, ${ }^{8}$ Service De Pédiatrie, Chu Nancy, ${ }^{9}$ Service De Néphrologie Et Immunologie Clinique, Chu Nantes, ${ }^{10}$ Service De Néphrologie-rhumatologiedermatologie Pédiatriques, Chu Lyon, ${ }^{11}$ Service De Pédiatrie Médicale, Chu Bordeaux, ${ }^{12}$ Service De Néphrologie - Hémodialyse Pédiatrique, Chru Tours, ${ }^{13}$ Service De Pédiatrie Générale Et Spécialisée, Chu Reims

Introduction: TINU syndrome is a rare disease, defined by the association of acute tubulointerstitial nephritis and uveitis. The renal prognosis in children with this disease is usually known to be good and better than in the adulthood. Owing the small number of data available in this field, we sought to precise the long term renal prognosis in a French cohort of children with tubulointerstitial nephritis and uveitis syndrome.

Material and methods: We performed a national retrospective study including 23 french pediatric nephrology units, collecting patients with tubulointerstitial nephritis and uveitis syndrome diagnosed between January 2000 and December 2018.

Results: 46 patients were included (52\% females, median age 13.8 years). At tubulointerstitial nephritis diagnostic, the median estimated glomerular filtration rate was $30,6 \mathrm{~mL} / \mathrm{min} / 1.73 \mathrm{~m}^{2}(4,9-62,8)$. The median time between the diagnosis of uveitis and tubulointerstitial nephritis was 0.4 months $(-4.1$ to $+17.1 \mathrm{~m})$. All patients had anterior uveitis but among them $12(28.6 \%)$ had no ophthalmologic symptoms. At last follow-up (median 2.8 years), the median estimated glomerular filtration rate was $87.5 \mathrm{~mL} / \mathrm{min} / 1.73 \mathrm{~m}^{2}(60,3-152,7)$ with 20 less than $90 \mathrm{~mL} /$ $\min / 1.73 \mathrm{~m}^{2}$

Conclusions: In our study, more than half of the patients had a poor renal outcome at last follow-up. Given the possible progression to chronic renal disease, long-term monitoring of patients with tubulointerstitial nephritis and uveitis syndrome is mandatory. Worthy of note, $25 \%$ of patients had asymptomatic uveitis so an ophtalmological exam in patients with tubulointerstitial nephritis is necessary even in the absence of ocular signs.

\section{OP-43 LONG-TERM FOLLOW-UP OF PATIENTS WITH BARTTER SYNDROME TYPE III}

Leire Gondra Sangroniz ${ }^{1}$, Leire Madariaga Domínguez ${ }^{2}$, Alejandro García Castaño $^{5}$, María Herrero Goñi ${ }^{3}$, Mireia Aguirre Meñica ${ }^{3}$, Ana Vinuesa Jaca ${ }^{3}$, Nélida García Pérez ${ }^{1}$, Sara Gomez Conde ${ }^{4}$

${ }^{1}$ Cruces University Hospital. Iis Biocruces Bizkaia Health Research Institute. University Of The Basque Country; ${ }^{2}$ Cruces University Hospital. Iis Biocruces Bizkaia Health Research Institute. University Of The Basque Country. Ciberer. Ciberdem; ${ }^{3}$ Cruces University Hospital. Iis Biocruces Bizkaia Health Research Institute; ${ }^{4}$ Iis Biocruces Bizkaia Health Research Institute; ${ }^{5}$ Iis Biocruces Bizkaia Health Research Institute. University Of The Basque Country

Introduction: Bartter Syndrome Type III (BSIII) is a clinically heterogeneous hereditary salt-losing tubulopathy. Approximately $25 \%$ of patients develop chronic kidney disease (CKD) over time. The aim of this study is to describe a cohort of patients with BSIII and to identify possible factors associated with the long-term prognosis.

Material and methods: Clinical presentation, treatment, long-term follow-up (1-46, median 12.5 years) and molecular findings were evaluated in 53 patients. To study the phenotype/genotype correlation patients were classified into two groups according to the residual current in $\mathrm{ClC}-\mathrm{Kb}$ channel $(\leq 20 \%$ or $>20 \%)$.

Results: Among 53 patients, $85 \%$ were Spanish. Median age at diagnosis was $0.67(0.5-3)$ years. At diagnosis, $34 \%$ had antenatal/neonatal BS phenotype $54 \%$ classic BS, and $12 \%$ Gitelman Syndrome phenotype. Fifty percent were born prematurely (32-37 weeks). In 55\% cases homozygous p.Ala204Thr and in 
$19 \%$ heterozygous p.Ala204Thr mutation was found. All of them except two were Spanish. At last follow-up, $72 \%$ patients had normal growth, $15 \%$ had nephrocalcinosis and in $22 \%$ CKD was detected. One patient required hemodialysis. Severe hypokalemia and metabolic alkalosis at diagnosis were related with long-term growth retardation and CKD $(\mathrm{p}<0.05)$. Moreover, CKD was associated with older age at diagnosis $(\mathrm{p}=0.038)$. All patients with CKD except three had homozygous p.Ala204Thr mutation $(30 \%$ of patients with this mutation). There were no significant differences in the long-term renal prognosis depending on the clinical phenotype at diagnosis. No genotype/phenotype correlation was found in long-term prognosis.

Conclusions: Older age and severe hypokalemic alkalosis at diagnosis are related with CKD, therefore, chronic hyperaldosteronism is a relevant risk factor for renal impairment in patients with BSIII. In addition, patients with homozygous p.Ala204Thr mutation have a very variable long-term prognosis, suggesting that patients' genotype is probably not a relevant factor implicated in the long-term prognosis.

\section{OP-44 CLINICAL FEATURES AND MANAGEMENT OF DENT'S DISEASE TYPE 1 (DD1) IN EUROPE}

Carla Burballa ${ }^{1}$, Larisa Prikhodina ${ }^{2}$, Francesca Lugani ${ }^{3}$, Karlpeter Schlingmann ${ }^{4}$, Petr V Ananin ${ }^{5}$, Detlef Bockenhauer ${ }^{6}$ Leire Madariaga ${ }^{7}$, Aurelia Bertholet-thomas ${ }^{8}$, Francesca Taroni ${ }^{9}$, Dorella Delprete ${ }^{10}$, Dominique Chauveau ${ }^{11}$, Mattia Parolin ${ }^{12}$,

Marc Fila ${ }^{13}$, Andrea Pasini ${ }^{14}$, Linda Koster-kamphuis ${ }^{15}$, Isabel Castro ${ }^{16}$, Barian Mohidin ${ }^{1}$, Giacomo Colussi ${ }^{17}$, Marta Gil ${ }^{18}$, Gema Ariceta ${ }^{1}$

${ }^{1}$ Vall Dhebron Hospital Vall Dhebron Research Institute (vhir), Barcelona, Spain, ${ }^{2}$ Research \& Clinical Institute For Pediatrics, Pirogov, Russia, ${ }^{3}$ Istituto G. Gaslini, Genova, Italy, ${ }^{4}$ University Childrens Hospital, MÜnster, Germany, ${ }^{5}$ Scientific Centre Of Children Health 2, Moscow, Russia, ${ }^{6}$ Great Hormond Street Hospital, London, United Kingdom ${ }^{7}$ Hospital De Cruces, Bilbao, Spain, ${ }^{8}$ Hopital Femme Mere Enfant, Lyon, France, ${ }^{9}$ Policlinico Irccs ,milan, Italy, ${ }^{10}$ Azienda Ospedale-universita, Padova, Italy, ${ }^{11}$ Hopital Rangueil, Toulouse, France, ${ }^{12}$ Azienda Ospedaliera, Padova, Italy, ${ }^{13}$ Arnaud De Villeneuve, Montpellier, France, ${ }^{14}$ Azienda Ospedaliero-universitaria Santorsolamalpighi Bologna, Italy, ${ }^{15}$ Radboudumc Amalia Childrens Hospital, Nijmegen, The Netherlands, ${ }^{16}$ Complexo Hospitalario De Pontevedra, Pontevedra, Spain, ${ }^{17}$ Niguarda Hospital, Milan, Italy, ${ }^{18}$ Hospital Universitario Santiago De Compostela, Santiago De Compostela, Spain

Introduction: Dent's disease type 1 (DD1) is a rare X-linked nephropathy characterized by proximal tubule dysfunction, including low-molecular-weight proteinuria (LMWP), hypercalciuria, nephrolithiasis, nephrocalcinosis and often progressive renal failure (RF). A broad phenotypic variability has been reported, with no genotype-phenotype relationship and limited long-term knowledge. The prevalence of DD1 is unknown. Geographic dispersion of the few diagnosed cases makes epidemiologic and clinical studies difficult.

Material and methods: A physician-based anonymous survey supported by ERKNet, ESPN and ERA-EDTA, either distributed via post mail or e-mail, was conducted. Questions were aimed to assess the prevalence and disease traits in the target population.

Results: 73 nephrologist from 63 hospitals in 22 European countries participated in this survey. 230 patients with DD1 were reported. Demographic and clinical data were available for 152 male patients with confirmed mutations in CLCN5 gene. Median age at diagnosis was 6 years [IQR,2.5-12] and the main sign leading to diagnosis was proteinuria (see Figure 1); $11.5 \%$ had positive family history. All patients showed LMWP, $66.9 \%$ nephrocalcinosis, $44.4 \%$ hypercalciuria and $25.6 \%$ kidney stones. $60 \%$ patients had progressed to chronic kidney disease with estimated glomerular filtrataion (eGFR) $<90 \mathrm{ml} / \mathrm{min}$ at last follow-up. Eight patients presented RF at 35 years [IQR,30-43]. Patients < 18 years at last follow-up showed hypercalciuria more frequently than those older $(51.4 \%$ vs. $22.7 \%$; $<<0.001)$, less glycosuria $(8.9$ $\%$ vs. $43.2 \%$; $<<0.01)$ and better eGFR $(90 \mathrm{ml} / \mathrm{min}$ [IQR,72-120] vs. $58 \mathrm{ml} / \mathrm{min}$ [IQR,18-101]).

Conclusions: Large registries are important to increase the knowledge of this probably under-diagnosed disease. LMWP, the hallmark of DD1, should be measure in males with proteinuria, nephrocalcinosis, lithiasis or bone disease although the phenotype is very variable and evolves with age and loss eGFR. Our results confirm DD1 manifestations described in smaller cohorts and show long-term outcomes with less percentatge of renal failure.

\section{OP-45 POTASSIUM AND FIBRE: A CONTROVERSIAL COUPLE IN THE NUTRITIONAL MANAGEMENT OF CHILDREN WITH CHRONIC KIDNEY DISEASE}

Amina El Amouri ${ }^{1}$, Kato Delva ${ }^{1}$, Aurélie Foulon ${ }^{2}$, Charlotte Vande Moortel ${ }^{2}$, Koen Van Hoeck ${ }^{3}$, Griet Glorieux ${ }^{2}$, Wim Van Biesen ${ }^{2}$, Johan Vande Walle ${ }^{1}$, Evelien Snauwaert ${ }^{1}$, Sunny Eloot ${ }^{2}$, Ann Raes ${ }^{1}$

${ }^{I}$ Paediatric Nephrology, Ghent University Hospital, ${ }^{2}$ Nephrology, Ghent University Hospital, ${ }^{3}$ Paediatric Nephrology, Antwerp University Hospital

Introduction: Life-threatening hyperkalaemia is the most dreaded metabolic complication in children with chronic kidney disease (CKD). However, direct evidence in support of the widespread practice of dietary potassium restriction for the prevention and management of hyperkalaemia is lacking. Furthermore, the resultant lower fibre exposure from avoiding potassium-rich fruits and vegetables may deprive CKD patients from potential health benefits. Therefore, we examined whether potassium and fibre intake correlate and investigated associations between dietary potassium, fibre intake and serum potassium levels in paediatric CKD.

Material and methods: This study is a longitudinal analysis of a 2year, prospective, multi-institutional study, following children with CKD at 3-month intervals. 24-h recalls and 3-day food records were used to assess dietary potassium and fibre intake and coupled to serum potassium concentrations. Associations between dietary potassium intake, dietary fibre intake and serum potassium concentrations were determined using linear mixed models.

Results: 52 patients ( 7 transplant recipients, none on dialysis) aged $9[4 ; 14]$ years with an estimated glomerular filtration rate (eGFR) of $49[25 ; 68] \mathrm{mL} / \mathrm{min} / 1.73 \mathrm{~m}^{2}$, were included. For every g/day decrease in dietary potassium intake, the estimated mean daily fibre intake was $5.1 \mathrm{~g}$ (95\% confidence interval(CI), 4.3 to $5.9 \mathrm{~g} /$ day; $\mathrm{p}<0.001$ ) lower. Regarding potassium intake, the relative contribution of fruits and vegetables was $16[12 ; 33] \%$, while non-nutritious sources (fastfood, sugar...) accounted for $29[11 ; 45] \%$. Neither dietary potassium intake $(\mathrm{p}=0.403)$, nor dietary fibre intake $(\mathrm{p}=0.432)$ were associated with circulating potassium in a model adjusted for time point, eGFR, treatment with a renin-angiotensin-aldosterone system blocker, serum bicarbonate concentration and body surface area.

Conclusions: Dietary potassium and fibre intake are closely related and were not associated with circulating potassium levels in paediatric CKD. If downward adjustments of potassium intake are necessary, the focus should shift from mere potassium content to potassium sources to safeguard fibre intake. 
OP-46 RENAL ACTIVATION OF PIT-2/ERK1/2 SIGNALLING BY PHOSPHATE MEDIATES INTERNALIZATION OF NAPI2A IN THE PROXIMAL TUBULE INDEPENDENT OF FGF23

Beatrice Richter ${ }^{1}$, Stefanie Walter ${ }^{1}$, Isabel Vogt ${ }^{1}$, Roland Schmitt ${ }^{2}$, Dieter Haffner ${ }^{1}$, Maren Leifheit-nestler ${ }^{1}$

${ }^{1}$ Department Of Paediatric Kidney, Liver And Metabolic Diseases, Paediatric Research Centre, Hannover Medical School, Hannover, Germany, ${ }^{2}$ Department Of Nephrology And Hypertension, Hannover Medical School, Hannover, Germany

Introduction: In renal proximal tubule (PT) cells, bone derived fibroblast growth factor (FGF) 23 activates the FGFR1/Klotho/ERK1/2 signalling to reduce the type II sodium phosphate transporters $\mathrm{NaPi}-2 \mathrm{a}$ and $\mathrm{NaPi}-2 \mathrm{c}$ in the apical brush border membrane (BBM) causing lower serum phosphate levels. In the presence of high extracellular phosphate, the type III sodium-dependent phosphate transporters PiT-1 and PiT-2 activate ERK1/2 in bone cells in vitro. It has been shown that PiT-2 is responsible for the phosphate-mediated osseous FGF23 secretion. Yet, it is unknown if phosphate sensing plays a role in renal PT cells and thereby regulates its own excretion. Thus, we aimed to analyse renal actions of phosphate in the setting of chronic high phosphate loading independent of FGF23.

Material and methods: $\mathrm{C} 57 \mathrm{BL} / 6 \mathrm{~N}$ male mice were fed a $0.8 \%$ normal phosphate diet (NPD) or a $2 \%$ high phosphate diet (HPD) for 6 months, phosphate homeostasis was determined and kidneys were analysed by qPCR, immunoblot and histology. Cultured murine PT cells were treated with phosphate or FGF23 with or without the phosphate transporter inhibitor Foscarnet followed by analyses.

Results: Elevated plasma FGF23 levels in HPD-fed mice resulted in reduced TRP and increased FEPi with still enhanced serum phosphate levels compared to NPD. HPD diminished renal Klotho protein, while Fgfr1 was unaltered. Although ERK1/2 activation was inconsistent, $\mathrm{NaPi}-2 \mathrm{a}$ mRNA expression was significantly reduced in HPD-fed mice compared to NPD. Analysing BBM vesicles and immunofluorescence staining confirmed $\mathrm{NaPi}-2 \mathrm{a}$ internalization from the apical BBM. HPD-fed mice showed increased PiT-2 expression and enhanced accumulation on the basolateral membrane of PT. In cultured murine PT cells, Foscarnet prevented the phosphatemediated PiT-2 upregulation and ERK1/2 phosphorylation but not the FGF23-induced effects.

Conclusions: In the setting of high phosphate-mediated renal resistance of FGF23/Klotho signalling, phosphate itself may stimulate its urinary secretion via PiT-2/ERK1/2-mediated downregulation of NaPi-2a resulting in hyperphosphaturia FGF23-independent.

\section{OP-47 EPHEX: A PHASE 3 DOUBLE-BLIND, PLACEBO- CONTROLLED, RANDOMISED STUDY TO EVALUATE LONG-TERM EFFICACY AND SAFETY OF OXALOBACTER FORMIGENES IN PATIENTS WITH PRIMARY HYPEROXALURIA}

Gema Ariceta ${ }^{1}$, Gesa Schalk ${ }^{2}$, Laure Collard ${ }^{3}$, Saoussen Abroug ${ }^{4}$, Shabbir Moochhala ${ }^{5}$, Edward Gould ${ }^{6}$, Abir Boussetta ${ }^{7}$,

Mohamed Ben Hmida ${ }^{8}$, Faical Jarraya ${ }^{8}$, Sudarsana Dee ${ }^{9}$, Tracy E. Hunley ${ }^{10}$, Ana Banos ${ }^{11}$, Elisabeth Lindner ${ }^{11}$, Bastian Dehmel ${ }^{11}$

${ }^{1}$ Hospital Vall D'hebron, Barcelona, Spain, ${ }^{2}$ Kindernieren-Zentrum, Bonn, Germany, ${ }^{3}$ Chu, Liege, Belgium, ${ }^{4}$ Hôpital Universitaire Sahloul, Sousse, Tunisia, ${ }^{5}$ Royal Free Hospital, London, UK, ${ }^{6}$ Vanderbilt University Medical Center, Nashville, USA, ${ }^{7}$ Hopital Charles Nicolle, Tunis, Tunisia, ${ }^{8}$ Hôpital Hedi Chaker, Sfax, Tunisia, ${ }^{9}$ Queen's Medical Centre, Nottingham, UK, ${ }^{10}$ Vanderbilt University Medical Center, Nashville, USA, ${ }^{11}$ Oxthera Ip Ab, Stockholm, Sweden
Introduction: Primary hyperoxaluria $(\mathrm{PH})$ is a rare genetic condition characterized by recurrent kidney stones and Chronic Kidney Disease. Oxabact ${ }^{\circledR}(O$.formigenes $)$ is a commensal, gram-negative, anaerobic gut bacteria with the ability to metabolise and remove excess oxalate from the body. The main objectives of the ePHex study were to evaluate efficacy and safety of Oxabact $\AA$ in patients with $\mathrm{PH}$, an estimated glomerular filtration rate $(\mathrm{eGFR})<90 \mathrm{ml} / \mathrm{min} / 1.73 \mathrm{~m}^{2}$ and total plasma oxalate (Pox) concentration $\geq 10 \mu \mathrm{mol} / \mathrm{L}$.

Material and methods: This international 52-week study evaluated the treatment effect of Oxabact ${ }^{\circledR}$ versus placebo on Pox as the primary endpoint, and kidney function and kidney stone events as key secondary endpoints. Other endpoints included 24-h urinary oxalate (Uox) excretion, stool count for $O$. formigenes, nephrocalcinosis grade, safety labs and adverse events in patients ( $\geq 2$ years of age) with any type of $\mathrm{PH}$. Subjects receiving vitamin B6 were required to receive a stable dose prior to screening and were not allowed to change the dose during the study. Pre-study kidney function and kidney stone events were collected. Subjects were randomised in a 1:1 ratio to either twice daily Oxabact ${ }^{\circledR}$ or placebo and stratified based on PH type and 24-h Uox excretion. During the study, Pox, eGFR, kidney stone events and safety labs were assessed every 8weeks. Cardiac function was determined with echocardiography (traditional and Speckle Tracking) and kidney stones with ultrasound. Stool samples and 24-h urine were collected at regular intervals, and adverse events monitored throughout the study.

Results: Twenty-five subjects were randomized in the study. The results from the study will become available early/mid June 2021 and will be presented for the first time during the ESPN 2021 conference.

Conclusions: The ePHex study represents the longest placebo-controlled phase 3 study with the objective to investigate efficacy and safety of Oxabact ${ }^{\circledR}$ in the treatment of patients with $\mathrm{PH}$.

\section{OP-48 THE EPIDEMIOLOGY OF CHILDHOOD ACUTE KIDNEY INJURY IN ENGLAND USING AKI E-ALERTS}

Lucy Plumb ${ }^{1}$, Anna Casula ${ }^{1}$, Manish Sinha ${ }^{2}$, Carol Inward ${ }^{4}$, Stephen Marks ${ }^{3}$, James Medcalf ${ }^{1}$, Dorothea Nitsch ${ }^{1}$

${ }^{1}$ Uk Renal Registry, ${ }^{2}$ Evelina London Childrens Hospital, ${ }^{3}$ University College London Great Ormond Street Institute Of Child Health, Nihr Great Ormond Street Hospital Biomedical Research Centre, London, $U K,{ }^{4}$ University Hospitals Bristol \& Weston Nhs Foundation Trust

Introduction: Few national datasets describe the epidemiology and outcomes of childhood AKI. Since 2014, all NHS laboratories in England are required to issue electronic (e-)alerts for AKI based on changes in serum creatinine. The UK Renal Registry receive these data which are linked to electronic health record data. The aim was to describe a national cohort of children who received an AKI ealert and their clinical course.

Material and methods: AKI e-alert data from 01/01/2017- 31/12/ 2017 are described. Linkage to Hospital Episode Statistics data was undertaken to describe outcomes of hospitalised AKI including length of stay (LOS) and critical care admission (CC). Risk associations with CC (hospitalised cohort) and 30-day mortality (total cohort) are examined using age- and sex-adjusted multivariable logistic regression models.

Results: Over the study period, 7,788 children (52\% male, median age 4.4 years, IQR 0.9-11.5) experienced 8,927 AKI e-alert episodes; $8.2 \%$ occurred during birth admissions. Children aged under 5-years accounted for half of all episodes. Of 5,582 children with hospitalised AKI episodes, peak AKI stage 2 was noted in $20.8 \%$ and stage 3 in 13.2\%. CC occurred in 1,402 (25\%): children of Asian ethnicity, in the youngest $(<1)$ or oldest (16-18) age-bands (reference 1-5 years), and 
with higher peak AKI stages had higher odds of CC. The median LOS was 9 (IQR 4-25) days, with longer LOS in birth-admission episodes. There was strong evidence for higher LOS with higher peak AKI stage. There were 251 deaths within 30-days. Youngest and oldest agegroups, hospital-acquired $\mathrm{AKI}$, higher peak stage, and requiring $\mathrm{CC}$ were associated with higher odds of death.

Conclusions: Young children contributed significantly to the AKI burden. As higher peak AKI stage and hospital-acquired injury were associated with longer LOS and mortality, inpatient-based interventions may minimise morbidity and be cost-effective to healthcare providers.

\section{OP-49 DEVELOPMENT OF A SIMULATION TOOL OF THE CARE TRAJECTORIES OF CHILDREN AND YOUNG ADULTS WITH ESKD}

Julien Hogan ${ }^{1}$, Jerome Harambat ${ }^{2}$, Etienne Berard ${ }^{3}$, Cecile Couchoud ${ }^{4}$ ${ }^{I}$ Department Of Pediatric Nephrology, Robert Debré Hospital, Aphp, Paris, France, ${ }^{2}$ Pediatric Nephrology Unit, Bordeaux University Hospital, Bordeaux, France, ${ }^{3}$ Pediatric Nephrology Department, Fondation Lenval, Nice, France, ${ }^{4}$ Registre Rein, Agence De La Biomédecine, La Plaine Saint-denis, France

Introduction: Given the improved life expectancy and the limited survival of kidney allografts, children and young adults undergo sequential periods of dialysis and transplantation over their lifetime. Most studies focus on access to transplant or allograft survival but fail to study patients' complete KRT trajectories. This study aims at developing a tool able to simulate the impact of medical practices or healthcare interventions on these care trajectories.

Material and methods: Data from all ESKD patients starting KRT before 30 years old in France between 1995 and 2015 were included $(\mathrm{N}=9402)$. A multistate model was used to study patients' trajectories between 15 states and transition rates were estimated using a Poisson model.

Results: Over the 20 years following KRT initiation, patients spent on average 4.6 years on dialysis and 13.1 with a functioning transplant. The restricted mean survival was 18.5 years. The tool allowed us to assess the impact of the rate of preemptive waitlisting, the rate of living donation and the number of HLA mismatches (A/B/DR) overall and in specific age subgroups. As an example, increasing preemptive listing and transplant from living donors to $50 \%$ only resulted in a slight decrease of the time on dialysis $(3.6$ months over 20 years) in children $(<18)$ while a significant reduction of 18 months would be expected among young adults.

Conclusions: The final aim of this tool is to guide clinicians and help health policy makers by assessing the impact of changes in clinical practices or healthcare policies. Such tools may help identifying strategies to maximize the time spent with a functioning transplant and minimize the time on dialysis.

\section{OP-50 LOW-DOSE PREDNISOLONE AT THE TIME OF AN URTI DOES NOT PREVENT RELAPSES IN STEROID SENSITIVE NEPHROTIC SYNDROME BUT MAY HAVE A HEALTH ECONOMIC BENEFIT: THE PREDNOS 2 TRIAL}

Martin Christian ${ }^{1}$, Nicholas Webb ${ }^{2}$, Samir Mehta ${ }^{3}$, Rebecca Woolley ${ }^{3}$, Elizabeth Brettell ${ }^{3}$, Adam Khan ${ }^{3}$, Natalie Ives ${ }^{3}$, Nafsika Afentou ${ }^{4}$, Emma Frew ${ }^{4}$, David Milford ${ }^{5}$, Detlef Bockenhauer ${ }^{6}$, Moin Saleem ${ }^{7}$ Angela Hall ${ }^{8}$, Ania Koziell ${ }^{9}$, Heather Maxwell ${ }^{10}$, Shivaram Hegde ${ }^{11}$, Hitesh Prajapati ${ }^{12}$, Rodney Gilbert ${ }^{13}$, Caroline Jones ${ }^{14}$, Karl Mckeever ${ }^{15}$, Wendy Cook ${ }^{16}$

${ }^{1}$ Nottingham Children's Hospital, ${ }^{2}$ Royal Manchester Children's Hospital, ${ }^{3}$ Birmingham Clinical Trials Unit, University Of Birmingham, ${ }^{4}$ Health Economics Unit, University Of Birmingham, ${ }^{5}$ Birmingham Children's Hospital, ${ }^{6}$ Great Ormond Street Hospital For
Children, ${ }^{7}$ Bristol Royal Hospital For Children, ${ }^{8}$ Leicester Children's Hospital, ${ }^{9}$ Evelina Children's Hospital, London, ${ }^{10}$ Royal Hospital For Sick Children, Glasgow, ${ }^{11}$ University Hospital Of Wales, Cardiff, ${ }^{12}$ Leeds Children's Hospital, ${ }^{13}$ Southampton Children's Hospital, ${ }^{14}$ Alder Hey Children's Hospital, Liverpool, ${ }^{15}$ Department Of Paediatric Nephrology, Royal Hospital For Sick Children, Belfast, UK, ${ }^{16}$ Nephrotic Syndrome Trust (nest)

Introduction: For children with relapsing steroid sensitive nephrotic syndrome (rSSNS), previous studies have shown that giving daily lowdose prednisolone for 5-7 days during an upper respiratory tract infection (URTI) may reduce risk of relapse. It is unclear if these findings are generalisable to a large multi-ethnic population on different maintenance treatments.

Material and methods: A randomised, double-blind, placebo-controlled trial was carried out in 122 UK paediatric units. 365 children with rSSNS ( $7.6 \pm 3.5$ years) were randomised by background treatment to receive 6 days of prednisolone $15 \mathrm{mg} / \mathrm{m}^{2}$ or placebo at the start of an URTI. Primary outcome was incidence of first URTI-related relapse (URR) following any URTI. Secondary outcomes included measures of corticosteroid adverse effects and quality of life (QoL). Cost-effectiveness analysis used trial data and a decision-analytic model to estimate 1-year QualityAdjusted-Life-Years (QALYs) and costs, which were then extrapolated over 16 years.

Results: The number of patients experiencing a URR was 56 $(42.7 \%)$ and $58(44.3 \%)$ in prednisolone and placebo arms respectively (adjusted risk difference: $-0.024,95 \%$ CI: -0.14 to 0.095 ; $\mathrm{P}=0.7$ ). There was no evidence that treatment effect differed according to maintenance treatment. There were no significant differences in secondary outcomes between treatment arms. In apparent contrast, the economic evaluation found that daily prednisolone at the time of an URTI increased QALYs and decreased overall costs, a finding that was robust to sensitivity analysis.

Conclusions: PREDNOS 2 has shown no clinical benefit to 6 days of daily low-dose prednisolone at the start of an URTI in reducing relapse risk. The apparent conflicting economic result illustrates the cost implications associated with background treatment and the costs and reduced QoL associated with relapses when balanced against a cheap treatment. Literature in this area is sparse and more work is needed to evaluate the QoL and economic impact of SSNS relapses.

\section{OP-51 PHENOTYPIC VARIABILITIES IN SIBLINGS WITH ARPKD}

Ramona Ajiri ${ }^{1}$, Kathrin Burgmaier ${ }^{1}$, Nurver Akinci ${ }^{2}$, Anja BÜscher ${ }^{3}$, Ismail Dursun ${ }^{4}$, Ali Duzova ${ }^{5}$, Loai Akram Eid ${ }^{6}$, Marc Fila ${ }^{7}$, Michaela Gessner ${ }^{8}$, Ibrahim GÖkce ${ }^{9}$, Laura Massella ${ }^{10}$, Antonio Mastrangelo ${ }^{11}$, Monika Miklaszewska ${ }^{12}$, Larisa Prikhodina ${ }^{13}$, Bruno Ranchin ${ }^{14}$, Nadejda Ranguelov ${ }^{15}$, Rina Rus ${ }^{16}$, Lale Sever ${ }^{17}$, Julia Thumfahrt ${ }^{18}$, Lutz Thorsten Weber ${ }^{1}$, Elke Wuehl ${ }^{19}$, Alev Yilmaz ${ }^{20}$, Joerg Doetsch ${ }^{1}$, Franz Schaefer ${ }^{21}$, Max Christoph Liebau ${ }^{1}$

${ }^{1}$ Department Of Pediatrics, University Hospital Cologne And University Of Cologne, Faculty Of Medicine, Cologne, Germany, ${ }^{2}$ Department Of Pediatric Nephrology, Şişli Etfal Training And Research Hospital, Istanbul, Turkey, ${ }^{3}$ Department Of Pediatrics Ii, University Hospital Essen, Essen, ${ }^{4}$ Department Of Pediatric Nephrology, Erciyes University, Faculty Of Medicine, Kayseri, ${ }^{5}$ Department Of Pediatrics, Division Of Pediatric Nephrology, Hacettepe University Faculty Of Medicine, Ankara, Turkey, ${ }^{6}$ Department Of Pediatric Nephrology, Dubai Kidney Center Of Excellence, Dubai Hospital, Dubai, United Arab Emirates, ${ }^{7}$ Pediatric Nephrology Unit, Chu Arnaud De Villeneuve-université De Montpellier, Montpellier, France, ${ }^{8}$ Department Of General Pediatrics And Hematology/ Oncology, Children's University Hospital Tuebingen, ${ }^{9}$ Research And Training Hospital, Division Of Pediatric Nephrology, Marmara University, 
Istanbul, Turkey, ${ }^{10}$ Division Of Nephrology, Department Of Pediatric Subspecialties, Bambino Gesù Children's Hospital - Irccs, Rome, Italy, ${ }^{11}$ Pediatric Nephrology, Dialysis And Transplant Unit, Fondazione Irccs Cà Granda, Ospedale Maggiore Policlinico, Milan, Italy, ${ }^{12}$ Department Of Pediatric Nephrology And Hypertension, Faculty Of Medicine, Jagiellonian University Medical College, Krakow, Poland, ${ }^{13}$ Department Of Inherited And Acquired Kidney Diseases, Research Clinical Institute For Pediatrics N.a. Acad. Y. E. Veltishev, Pirogov Russian National Research Medical University, Moscow, Russia ${ }^{14}$ Pediatric Nephrology Unit, Hôpital Femme Mère Enfant, Hospices Civils De Lyon, Centre De Référence Maladies Rénales Rares, Bron, France, ${ }^{15}$ Department Of Pediatrics, Université Catholique De Louvain Medical School, Saint-luc Academic Hospital, Brussels, Belgium ${ }^{16}$ Division Of Nephrology, University Children's Hospital Ljubljana, Ljubljana, Slovenia, ${ }^{17}$ Department Of Pediatric Nephrology, Cerrahpaşa School Of Medicine, Istanbul University Cerrahpasa, Istanbul, Turkey, ${ }^{18}$ Department Of Pediatric Nephrology, Charité - Universitätsmedizin Berlin, Corporate Member Of Freie Universität Berlin, Humboldt-universität Zu Berlin, And Berlin Institute Of Health, Germany, ${ }^{19}$ Division Of Pediatric Nephrology, Center For Pediatrics And Adolescent Medicine, University Of Heidelberg, Heidelberg, Germany, ${ }^{20}$ Pediatric Nephrology Department, Istanbul University Istanbul Medical Faculty, 34662, Istanbul, Turkey, ${ }^{21}$ Division Of Pediatric Nephrology, Heidelberg University Center For Pediatrics And Adolescent Medicine, Heidelberg, Germany

Objectives: Autosomal recessive polycystic kidney disease (ARPKD) is a rare but severe pediatric disorder characterized by fibrocystic hepatorenal changes. It is mainly caused by variants in the PKHDl gene. Previous reports have documented pronounced phenotypic variability, even amongst siblings. The phenotypic spectrum is broad and the underlying causes are poorly understood. Here, we describe the courses of sibling pairs included in the ARPKD registry study ARegPKD.

Methods: We present the longitudinal clinical courses of 35 sibling pairs. Data on primary manifestation, pre- and perinatal findings, genetic testing and family history are described and data on kidney function, liver involvement and radiological findings were compared.

Results: We identified 70 siblings from 35 families with a median age of 0.73 years at initial diagnosis and a median follow-up time of 3.5 years. Only one patient deceased in the neonatal period. Genetic analysis was available for 37 patients from 21 families. Eight patients from 7 families required kidney replacement therapies. Thirty seven patients from 24 different families showed signs of portal hypertension (either thromocytopenia, splenomegaly or documentation of collateral blood flow). Nine patients from 6 different families suffered from substantial hepatic complications (either variceal bleeding or portosystemic shunt or LTx/CLKTx). Interestingly, variability in the clinical course was not very pronounced in this ARPKD cohort of neonatal survivors with many genetically confirmed cases.

Conclusions: We describe a cohort of 70 ARPKD siblings from 35 families with a high percentage of genotyped families. In patients surviving the neonatal period our longitudinal follow-up did not reveal very pronounced clinical variability.

\section{OP-52 CLINICAL DELINEATION OF THE NUP93 GLOMERULOPATHY PREVALENT IN CENTRAL AND EASTERN EUROPE.}

Jankowski Maciej ${ }^{1}$, Daca-roszak Patrycja ${ }^{2}$, Trautmann Agnes ${ }^{3}$, Milovanova Anastasiia ${ }^{5}$, Balasz-chmielewska Irena ${ }^{4}$, Grenda Ryszard ${ }^{6}$, Zieg Jakub ${ }^{7}$, Jankauskiene Augustina ${ }^{8}$, Simkova Eva ${ }^{9}$, Drożdż Dorota ${ }^{11}$,
Szymanik-grzelak Hanna ${ }^{10}$, Żurowska Aleksandra ${ }^{4}$, Tsygin Alexey ${ }^{5}$, Ziętkiewicz Ewa ${ }^{2}$, Schaefer Franz ${ }^{3}$, Lipskaziętkiewicz Beata S. ${ }^{12}$

${ }^{1}$ Department Of Biology And Medical Genetics, Faculty Of Medicine, Medical University Of Gdańsk, Gdańsk, Poland, ${ }^{2}$ Institute Of Human Genetics, Polish Academy Of Sciences, Poznań, Poland, ${ }^{3}$ Division Of Pediatric Nephrology, Center For Pediatrics And Adolescent Medicine, University Of Heidelberg, Heidelberg, Germany, ${ }^{4}$ Department Of Pediatrics, Nephrology And Hypertension, Faculty Of Medicine, Medical University Of Gdańsk, Gdańsk, Poland, Department Of Nephrology, National Medical And Research Centre For Children's Health, Moscow, Russia, ${ }^{6}$ Department Of Nephrology, Kidney Transplantation \& Hypertension, The Childrens Memorial Health Institute, Warsaw, Poland, ${ }^{7}$ Department Of Paediatrics, 2nd Faculty Of Medicine, Charles University In Prague And University Hospital Motol, Prague, Czech Republic, ${ }^{8}$ Clinic Of Pediatrics, Institute Of Clinical Medicine, Faculty Of Medicine, Vilnius University, Vilnius, Lithuania, ${ }^{9}$ Department Of Pediatric Nephrology, Dubai Hospital, Dubai, United Arab Emirates, ${ }^{10}$ Department Of Pediatrics And Nephrology, Medical University Of Warsaw, Warsaw, Poland, ${ }^{11}$ Department Of Pediatric Nephrology And Hypertension, Jagiellonian University Medical College, Cracow, Poland, ${ }^{12}$ Rare Diseases Centre And Clinical Genetics Unit, Department Of Biology And Medical Genetics, Faculty Of Medicine, Medical University Of Gdańsk, Gdańsk, Poland.

Introduction: Molecular defects in genes encoding nucleoporins (NUPs; components of nuclear pore complexes), such as NUP93, have been recently reported to cause steroid-resistant nephrotic syndrome in childhood. Here we collected clinical and genetic information on 31 patients, including 20 unpublished, to further characterize the associated phenotype.

Material and methods: The patients were recruited from the international PodoNet Registry clinical registry for patients with persistent subnephrotic proteinuria/overt steroid-resistant nephrotic syndrome and/or identified through systematic literature review. Clinical data were collected through a standardized questionnaire. The shared background haplotypes were determined and compared to general population; genetic clock methods were used to estimate the age of a mutation.

Results: Median age at onset was $4.4(0.3-16)$ yrs. Most patients presented with overt nephrotic syndrome and $5(16 \%)$ in end-stage kidney disease (ESKD), which was attained at a median age of 6 years. Extrarenal manifestations were present in a minority of cases and most commonly comprised cardiac disorders (19\%) including severe dilated cardiomyopathy in 3 pts during peritoneal dialysis, eye abnormalities and vision difficulties (15\%) and neurodevelopmental problems $(7 \%)$.

The c. $1772 \mathrm{G}>\mathrm{T}$ variant was found in 21 (including 10 homozygous) patients from Central and Eastern Europe (Poland, Czech, Germany, Hungary, Russia, Serbia) and Turkey. Patients homozygous for c. $1772 \mathrm{G}>\mathrm{T}$ had a milder disease course then other NUP93 cases with later median age at onset $(5.0$ vs $2.9, \mathrm{p}=0.05)$ and at ESKD (11.0 vs 4.9 yrs; p=0.04). Analysis of the haplotype background composed of SNPs surrounding the variant revealed a common segment of at least $360 \mathrm{~kb}$. Preliminary estimation of the nonrecombined haplotypes suggests that the mutation occurred 6001,500 years ago.

Conclusions: The study enabled us to delineate the phenotype of NUP93 glomerulopathy, including a homogeneous group of SRNS patients of Central/Eastern European ethnicity who harbor a single founder mutation associated with a milder phenotype. 
OP-53 TUBULAR DYSFUNCTION AND TREATMENTRELATED RISK FACTORS IN LONG-TERM CHILDHOOD CANCER SURVIVORS; DCCSS-LATER 2: RENA.

Esmee C.m. Kooijmans ${ }^{1}$, Helena J.h. Van Der Pal ${ }^{2}$, Saskia Pluijm ${ }^{2}$, Dorine Bresters ${ }^{2}$, Eline Van Dulmen-den Broeder ${ }^{1}$, Margriet Van Der Heiden-van Der Loo ${ }^{2}$, Marry M. Van Den Heuvel-eibrink ${ }^{2}$, Leontien C.m. Kremer ${ }^{2}$, Jacqueline Loonen ${ }^{3}$, Marloes Louwerens ${ }^{4}$, Cecile M. Ronckers ${ }^{2}$, Wim J.e. Tissing ${ }^{2}$, Andrica C.h. De Vries ${ }^{5}$, Gertjan J.l. Kaspers ${ }^{2}$, Arend Bokenkamp ${ }^{6}$, Margreet A. Veening ${ }^{2}$, On Behalf Of The Dutch Later Study Group ${ }^{2}$

${ }^{1}$ Emma Children's Hospital, Amsterdam Umc, Vrije Universiteit Amsterdam, Pediatric Oncology, Amsterdam, The Netherlands, ${ }^{2}$ Princess Maxima Center For Pediatric Oncology, Utrecht, The Netherlands, ${ }^{3}$ Department Of Hematology, Radboud University Medical Center, Nijmegen, The Netherlands ${ }^{4}$ Department Of Internal Medicine, Leiden University Medical Center, Leiden, The Netherlands, ${ }^{5}$ Department Of Pediatric Oncology, Sophia Children's Hospital/erasmus Medical Center, Rotterdam, The Netherlands, ${ }^{6}$ Emma Children's Hospital, Amsterdam Umc, Vrije Universiteit Amsterdam, Pediatric Nephrology, Amsterdam, The Netherlands

Introduction: The aim of this nationwide cross-sectional cohort study was to determine the prevalence of and risk factors for tubular dysfunction in childhood cancer survivors (CCS).

Material and methods: For the Dutch Childhood Cancer Survivor Study (DCCSS) LATER cohort (1963-2001) part 2; clinical visit \& questionnaire study; RENA project, 1,024 CCS ( $\geq 5$ years after diagnosis), aged $\geq 18$ years at time of study, treated between 1963 and 2001 with potentially nephrotoxic therapy (i.e. nephrectomy, abdominal radiotherapy, total body irradiation, cisplatin, carboplatin, ifosfamide, high-dose cyclophosphamide $(\geq 1 \mathrm{~g} / \mathrm{m} 2$ per course or $\geq 10 \mathrm{~g} / \mathrm{m} 2$ in total), or bone marrow transplantation participated. Five-hundred age- and sex matched controls were used from the Lifelines cohort study. Renal electrolyte loss was defined as low serum levels in combination with increased renal excretion, or receiving electrolyte supplementation. $\alpha 1$ microglobulin:creatinine ratio $>1.7 \mathrm{mg} / \mathrm{mmol}$ was considered as lowmolecular weight proteinuria (LMWP). Chi-Square analysis was used to compare the prevalence in both groups. Multivariable logistic regression analyses were performed to assess risk factors and expressed as odds ratio $(\mathrm{OR})$ with $95 \%$ confidence interval $(95 \% \mathrm{CI})$.

Results: Median age at diagnosis was 4.7 years (interquartile range [IQR] 2.4-9.2), at study 32.5 years (IQR 27.7-38.0), and follow-up time 25.5 years (IQR 21.4-30.3). Risk factor for magnesium loss was cisplatin (OR $10.1,95 \%$ CI 3.9-26.0), for potassium loss these were ifosfamide (OR 2.4 [1.2-4.7]) and cisplatin (OR 3.5 [1.6-7.5]) and for phosphate loss ifosfamide (OR 2.3 [1.2-4.3]). LMWP was associated with ifosfamide (OR 2.8 [2.0-4.1]). Overall prevalence of renal losses in CCS (magnesium $5.6 \%$, potassium $4.5 \%$, phosphate $5.5 \%$ ) was not higher compared to controls, while LMWP was significantly more prevalent (CCS $20.1 \%$ versus controls $0.4 \%, \mathrm{p}<0.001$ ).

Conclusions: After a median follow-up of 25.5 years CCS had an increased prevalence of LMWP compared to controls, but not of renal electrolyte losses. Ifosfamide and cisplatin were associated with tubular toxicity over time.

\section{OP-54 TRANSPLANTATION IN CHILDHOOD AND RE- TRANSPLANTATION IN ADULTHOOD: RESULTS FROM THE ERA-EDTA REGISTRY.}

Evgenia Preka ${ }^{1}$, Marjolein Bonthuis ${ }^{1}$, Jerome Harambat ${ }^{2}$, Enrico Vidal ${ }^{3}$, Lesley Rees ${ }^{4}$, Kitty J Jager ${ }^{1}$

${ }^{1}$ European Society For Pediatric Nephrology/ European Renal Association-european Dialysis And Transplant Association Registry, Amsterdam Umc, University Of Amsterdam, Department Of Medical
Informatics, Amsterdam Public Health Research Institute, Amsterdam, The Netherlands, ${ }^{2}$ Pediatric Nephrology Unit, Bordeaux University Hospital, Bordeaux, France, ${ }^{3}$ Division Of Pediatrics, Department Of Medicine, University Of Udine, Udine, Italy, ${ }^{4}$ Pediatric Nephrology, Ucl Great Ormond Street Institute Of Child Health, Ucl, London, United Kingdom

Introduction: Over $25 \%$ of pediatric kidney transplants (KTs) are lost within 7 years necessitating return to dialysis or retransplantation. Knowledge regarding the long-term outcomes after pediatric retransplantation is limited. Using ERA-EDTA Registry data, we investigated kidney retransplantation outcomes from childhood into adulthood.

Material and methods: Patients on kidney replacement therapy (KRT) between August 1979 and December 2016 were studied. Multivariable Cox regression models were applied to study access to KT.Death-censored graft survival was analyzed and retransplantation outcomes were assessed using cumulative incidence competing risk analysis.

Results: A total of 4871, 1126 and 259 children having received at least one, two and three KTs, respectively, were included. Median age at first, second and third KT was 12.6, 20.0 and 24.9 years old, respectively and median follow-up was 23.2 years. Starting KRT at younger age (0-4 years old) was associated with a lower access to first KT [adjusted HR (aHR) 0.45 ; 95\%CI 0.41-0.49]. Children receiving their first KT between 10-14 years old had lower graft survival rates compared to other age groups. Patients with recurrent diseases showed a lower access to $\mathrm{KT}$ regarding their first and second KT (aHR 0.79; 95\%CI 0.73-0.86 and aHR 0.85; 95\%CI 0.73-0.99, respectively). Regarding access to subsequent KT according to the type of previous KT, the likelihood of receiving a second KT was significantly lower when first KT was from a deceased donor (aHR, $0.79 ; 95 \%$ CI $0.68-0.92$ ). The 10 -year graft survival rate was $63.1 \%, 51.3 \%$ and $46.4 \%$ for the first, second and third KT, respectively, and graft survival was significantly better for first and second KT with living donor kidneys.

Conclusions: First KT had better overall survival compared to subsequent KTs. Survival rates are better for living donor recipients for first and second KT but comparable to DD for third KT. Having received a $\mathrm{DD}$ as first KT decreases the chances for further KTs.

\section{OP-55 VESICULAR MIRNAS AS NEW FRONTIER IN THE PREVENTION OF RENAL SUBCLINICAL REJECTION}

Andrea Carrao ${ }^{1}$, Diana Marzenta ${ }^{1}$, Emanuele Vianello ${ }^{2}$, Benedetta Bussolati ${ }^{3}$, Federica Collino ${ }^{4}$, Piera De Gaspari ${ }^{1}$, Loris Bertoldi ${ }^{5}$, Giuseppe Benvenuto ${ }^{5}$, Davide Meneghesso ${ }^{2}$, Mattia Parolin ${ }^{2}$, Germana Longo ${ }^{2}$, Luisa Murer ${ }^{1}$, Elisa Benetti ${ }^{2}$, Susanna Negrisolo ${ }^{1}$

${ }^{I}$ Laboratory Of Immunopathology And Molecular Biology Of The Kidney, Institute Of Pediatric Research Città Della Speranza, Department Of Women's And Children's Health, Padua University Hospital, Padua, Italy, ${ }^{2}$ Pediatric Nephrology Dialysis And Transplant Unit, Department Of Women's And Children's Health, Padua University Hospital, Padua, Italy, ${ }^{3}$ University Of Turin, Department Of Molecular Biotechnology And Health Sciences, Italy, ${ }^{4}$ Major General Hospital Of Milan, Ca Granda Foundation Irccs, Laboratory Of Translational Research In Pediatric Nephro-urology, Italy, ${ }^{5}$ Bmr-genomics, Padua, Italy

Introduction: The study of novel biomarkers useful to prevent subclinical rejection has grown up in the last decades. Among the various candidates emerge the microRNAs (miRNAs), short non-coding sequences of RNA ( $21-23 \mathrm{nt})$ involved in different post-transcriptional gene pathways. Their presence is confirmed in different types of tissues and 
biological fluids, such as urine. Due to their high degradability, miRNAs are often carry by extracellular vesicles (EV), lipid nano-bound particles release from all types of cells. Particularly the study of urinary extracellular vesicles (UEVs) and their miRNAs content could be helpful to reflect directly the condition of the kidney. For this reason, we try to identify a miRNAs profile from UEVs samples useful to prevent subclinical kidney rejection in pediatric transplanted patients.

Material and methods: The miRNAs were extracted from UEVs of 20 pediatric transplanted patients, with a stable condition or kidney rejection at one-year post-transplantation. The UEVs were isolated from urine samples by the ultracentrifugation method. The vesicles concentration and size were identifying by electron microscopy and scattering analysis (Nanosight 3000). The extracted miRNAs were enriched before sequencing with the Illumina sequencer.

Results: The miRNAs sequencing shows about 522 different miRNAs and 48 of them were differentially expressed between patients with subclinical rejection or stable condition. UEVs characterization show a concentration of $2,79 \times 10^{11}-9,56 \times 10^{11}$ particles with a size diameter of about $197 \pm 7 \mathrm{~nm}$. The miRNAs concentration was between $197-907$ $\mathrm{pg} / \mu \mathrm{l}$.

Conclusions: These results emphasize the relevance of vesicles miRNAs as useful biomarkers lead to the evaluation and prevention of subclinical kidney rejection in pediatric transplanted patients.

\section{OP-56 OUTCOME OF KIDNEY TRANSPLANTATION FOLLOWING DONATION AFTER CIRCULATORY DEATH IN CHILDREN}

Jessica Mcilwham ${ }^{1}$, Tom Dowsett ${ }^{2}$, Helen Stannard ${ }^{1}$, Afshin Tavakoli ${ }^{1}$, Henry Morgan ${ }^{2}$, Mohan Shenoy ${ }^{1}$

${ }^{1}$ Royal Manchester Childrens Hospital, ${ }^{2}$ Alder Hey Childrens Hospital

Introduction: Outcome of kidney transplantation (KT) from donors after circulatory death (DCD) in adults is comparable to KT from donors after brainstem death (DBD). DCD now account for nearly $30 \%$ of the total KT performed in the UK. Limited data exists for DCD KT outcomes in children. This study reports the outcome of DCD KT in children compared to age and sex matched children who received DBD KT around the same time period in the NW of England.

Material and methods: Retrospective review of case records. Results: From January 2013 to December 202010 children underwent DCD KT. All children received steroid minimisation immunosuppressive regimen with tacrolimus and MMF maintenance therapy from day 5. Mean age at KT was 11.8 years (7.0 -17.0) for DCD recipients and 10.7 years (4.9-17.3) for DBD recipients, with mean cold ischaemic times of 12.3 (8.5-18.2) and $12.3(8.4-18.3)(\mathrm{p}=0.97)$ hours, respectively. Average length of stay was similar (DCD $13.7 \mathrm{v}$ DBD 13.3 days). There was a trend towards increased delayed graft function (DGF) in DCD recipients compared with DBD recipients $(7 / 10$ v $3 / 10, p=0.07)$. eGFR was not significantly different at discharge for DCD compared to DBD (mean 50.7 vs $63.5 \mathrm{ml} / \mathrm{min} / 1.73 \mathrm{~m}^{2}, \mathrm{p}=0.36$ ), at 6 months (mean 70.7 vs $77.7 \mathrm{ml} / \mathrm{min} / 1.73 \mathrm{~m}^{2}, \mathrm{p}=0.53$ ) or at 12 months (mean 75 vs $72.8 \mathrm{ml} / \mathrm{min} / 1.73 \mathrm{~m}^{2}, \mathrm{p}=0.87$ ). There was no significant difference in incidence of hypertension - DCD 2/10 vs DBD 6/10, $(\mathrm{p}=0.07)$ at mean follow-up of 43.9 and 39.2 months respectively. Biopsy proven acute rejection episodes were similar in the 2 groups (DBD 1, DCD 2). Mean $Z$ score change for height since KT in DCD and DBD patients at latest follow-up was +0.24 and $+0.11 \quad(\mathrm{p}=0.83)$.

Conclusions: Although children who receive DCD KT have a trend towards increased risk of DGF, the long-term graft function and outcome is similar to DBD recipients.
OP-57 STEERING OF IMMUNOSUPPRESSION BY VIRUSSPECIFIC T CELLS AFTER PEDIATRIC KIDNEY TRANSPLANTATION IN THE RANDOMIZED CONTROLLED IVIST TRIAL

Thurid Ahlenstiel-grunow ${ }^{1}$, Xiaofei Liu ${ }^{2}$, Raphael Schild ${ }^{4}$, Christina Taylan ${ }^{3}$, Lutz Weber ${ }^{3}$, Jun Oh... ${ }^{4}$, Hagen Staude ${ }^{5}$, Anika Großhennig ${ }^{2}$, Lars Pape ${ }^{1}$

${ }^{1}$ University Of Essen-duisburg, ${ }^{2}$ Hannover Medical School, ${ }^{3}$ University Of Cologne, ${ }^{4}$ University Of Hamburg, ${ }^{5}$ University Of Rostock

Introduction: Pharmacokinetic monitoring alone is insufficient to estimate the intensity of immunosuppression after kidney transplantation. Levels of virus-specific CD4 T cells(CD4Tvis) have been shown to identify overimmunosuppression. The IVIST trial has demonstrated that additional steering of immunosuppressive therapy by CD4Tvis levels is safe and reduces exposure to immunosuppressants with significantly lower trough levels but without increasing the risk of acute rejections.

Material and methods: In the multicenter, randomized controlled IVIST trial, 64 pediatric kidney recipients were randomized 1:1 to a control group with trough level monitoring of immunosuppressants or to an intervention group with additional steering by CD4Tvis levels against adenovirus(ADV), cytomegalovirus(CMV) and herpes simplex virus(HSV). The immunosuppression consisted of cyclosporine A, everolimus and glucocorticoids. CD4Tvis were quantified by cytokine flow cytometry in 20 visits during the two-year study period. In the intervention group we have analyzed the CD4Tvis levels and the number of Tvisbased dose adjustments of immmunosuppressants.

Results: At time of transplantation, ADV-CD4Tvis were detectable in $30 / 31$ patients from the intervention group, CMV-CD4Tvis and HSVCD4Tvis only in $12 / 31$. No significant ADV- or HSV-DNAemia was found; only two patients showed transient CMV-DNAemia based on $\mathrm{CMV}$-reactivation. Five primary CMV-infections with seroconversion and boost of CMV-CD4Tvis were observed without significant CMVDNAemia. The mean level of ADV-CD4Tvis was 1.63(SD1.25), 2.03(SD1.8), 2.18(SD2.51) and 1.97 cells/ $\mu 1(\mathrm{SD} 1.34) 1,6,12$ and 24 months after transplantation. In case of CD4Tvis $<2$ cells/ $\mu 1125$ dose reductions of immunosuppressants (96\% based on ADV-CD4Tvis) were performed in 28/31 children with a median of 4 Tvis-based dose reductions (range $0-10$ ) per patient. $48 \%$ of the Tvis-based dose reductions were carried out in the first six months, $36.8 \%$ between month 7 and 12 and $15.2 \%$ in the second year after transplantation.

Conclusions: Under the intensified immunosuppression during the initial post-transplant period low ADV-CD4Tvis levels were observed with subsequent increase after dose reduction of the immunosuppressive therapy. ADV-CD4Tvis are most suitable for immune monitoring because of their high prevalence (even in children) and stability combined with absence of ADV-DNAemia. Routine monitoring of ADV-CD4Tvis is recommendable especially in the first post-transplant year to prematurely identify overimmunosuppression.

\section{OP-58 KIDNEY PAIRED EXCHANGE IN CHILDREN IN THE US}

Julien Hogan ${ }^{1}$, Alvin Thomas ${ }^{2}$, Jennifer Verbesey ${ }^{3}$, Dorry Segev ${ }^{4}$

${ }^{1}$ Department Of Surgery, Emory University, Atlanta, $\mathrm{Ga},{ }^{2}$ Department Of Epidemiology, University Of North Carolina, Chapel Hill, $\mathrm{Nc}{ }^{3}$ Medstar Georgetown Transplant Institute, Washington, Dc, ${ }^{4}$ Department Of Surgery, Johns Hopkins University, Baltimore, Md

Introduction: Kidney paired exchange (KPE) plays an increasing role among adult living donor transplant (LDTx) in the US. LDTx is the treatment of choice in children with ESKD. Despite the allocation of high quality transplants from deceased donors and short waiting times in 
children, improving access to LDTx is of high importance. In this study, we describe the characteristics and outcomes of pediatric transplants performed through KPE.

Material and methods: We studied 5,022 pediatric LDTx (2/2008-12/ 2019), including 128 National Kidney Registry (NKR) transplants, using the Scientific Registry of Transplant Recipients (SRTR) linked with the NKR database. We compared death-censored graft failure (DCGF) and mortality between NKR recipients and three control groups (1) all nonNKR SRTR living donor recipients, (2) all non-NKR unrelated SRTR living donor recipients, and (3) all non-NKR SRTR KPE recipients.

Results: There were 352 pediatric KPE transplants during the study period (36\% participated in NKR). Among NKR pediatric recipients, $44 \%$ were female, $23 \%$ were African American (AA), $14 \%$ were Hispanic, and the median age was 16. Compared to SRTR controls, NKR pediatric participants were more often AA ( $23 \%$ vs $9 \%, \mathrm{p}<0.001)$, less likely to receive preemptive transplant $(28 \%$ vs. $38 \% \mathrm{p}=0.02)$, more often had a previous transplant (27\% vs. 9\%), and more often had a PRA $>80(23 \%$ vs. $4 \%, \mathrm{p}<0.001)$. NKR participants experienced longer cold ischemia times (median 8 vs. 1 hour) but did not experience increased risk of delayed graft function ( $4 \%$ vs $3 \%, \mathrm{p}=0.6$ ), 5 -year DCGF ( $5 \%$ vs. $0.5 \%$, $\mathrm{p}=0.1)$, or 5 -year mortality $(1.7 \%$ vs. $1.9 \%, \mathrm{p}=0.2)$.

Conclusions: The use of KPE to improve access to LDTx in children increased over the last decade. This especially benefited children with traditionally lower access to LDTx such as AA and highly sensitized patients and demonstrated good patients and transplant outcomes similar to non-KPE LDTx.

\section{PI-1 NOVEL COMPOUND HETEROZYGOUS MUTATIONS OF CLDN16 GENE IN TWO SIBLINGS WITH NEPHROCALCINOSIS, HYPOMAGNESEMIA AND HYPERCALCIURIA}

Lovro Lamot, Ivan Jakopčić, Hana Matković, Ivanka Kos,

Kristina Vrljičak

Department Of Pediatrics, University Of Zagreb School Of Medicine, University Hospital Centre Zagreb, Zagreb, Croatia

Introduction: The pathological condition underlying nephrocalcinosis in children is not always evident and requires a detailed history and workup. Most commonly it is not associated with specific symptoms, while only a small number of children can present with recurrent urinary tract infection (rUTI). Yet, when accompanied with hypercalciuria and hypomagnesemia, it should raise a suspicion of an inherited disease, and therefore genetic testing is warranted.

Material and methods: Case report.

Results: We report a case of sister (11 years) and brother ( 7 years) born to nonconsanguineous, healthy parents. The girl initially presented rUTI at the age of 4 , while renal ultrasound revealed bilateral nephrocalcinosis. A screening renal ultrasound was performed in her brother at the age of 4 months with the same findings. Further evaluation revealed hypercalciuria, hypomagnesaemia, hypermagnesuria, hyperparathyroidism, hyperuricemia and decreased GFR in both siblings, with no other extrarenal manifestations. Finally, targeted gene sequencing (Blueprint Nephrolitiasis panel with 35 genes) identified a novel heterozygous frameshift variant c.332 333del, p.(Thr111Lysfs*15) and a heterozygous missense variant c. $358 \mathrm{~T}>\mathrm{C}$, p.(Cys120Arg) in CLDN16 gene, confirming the diagnosis of Familial hypomagnesaemia with hypercalciuria and nephrocalcinosis (FHHNC).

Conclusions: FHHNC is is a rare autosomal recessive tubular disease caused by mutations in CLDN16 and CLDN19 genes encoding for the tight-junction proteins expressed in the thick ascending limb of Henles loop, where they form an essential complex for the paracellular reabsorption of magnesium and calcium. Many patients display a marked decline in GFR at the time of diagnosis, and about one-third progress to chronic renal failure during adolescence. While most of them harbor loss-of- function mutations in both alleles, phenotypic variability has been shown even in siblings with the same homozygous mutation, emphasizing the likely contribution of epigenetic factors to the disease development. In this context, to achieve a wider health impact, reporting of novel diseasecausing mutations is of high importance in order to establish phenotypegenotype correlations, facilitate appropriate treatment and inform disease outcome.

\section{PI-2 PHARMACOLOGICAL APPROACHES IN PAEDIATRIC SIADH: TOLVAPTAN AND UREA}

Faidra Veligratli, Demitra Alexandrou, Adeola Famuboni, Sergio Camilo Lopez-Garcia, Detlef Bockenhauer Great Ormond Street Hospital For Children Nhs Foundation Trust, United Kingdom

Introduction: The first step in the management of the syndrome of inappropriate antidiuretic hormone (SIADH) is fluid restriction. This can be challenging, as patients with SIADH not only tend to be thirsty but may occasionally not tolerate restriction due to coexisting medical conditions. Data on the safety of pharmacological approaches are needed to guide clinical care in paediatric SIADH. Our objective was to review the efficacy and safety of tolvaptan and urea in paediatric patients with a clinical diagnosis of SIADH.

Material and methods: Patients were identified from pharmacy electronic records based on their tolvaptan and urea prescriptions. The prospectively collected electronic patient records during the inpatient stay were reviewed and relevant information and laboratory results were extracted.

Results: We identified 14 patients who had received tolvaptan and /or urea as part of their routine clinical care for treatment of SIADH. The majority of patients were under the care of the paediatric oncology team $(12 / 14,86 \%)$. Tolvaptan use led to plasma sodium normalisation in 10/13 (77\%) within six days (median 2.5 days, range $[1,6]$ ) with a median change of sodium concentration of $7 \mathrm{mmol} / \mathrm{L}(-1,14)$ within the first 24 hours of treatment.

Urea use led to sodium normalisation in $5 / 6(83 \%)$ patients. Median number of days to normalisation with urea was $2(1,10)$ with a median change of plasma sodium concentration of 2 $\mathrm{mmol} / \mathrm{L}(-1,6)$ within 24hours. All patients tolerated tolvaptan and urea without reported side effects. Sudden sodium concentration changes in 3 patients were not associated with clinical signs or symptoms.

Conclusions: When fluid restriction is challenging in managing paediatric SIADH, tolvaptan and urea appear to be safe and effective therapeutic options.

\section{PI-3 NEXT GENERATION SEQUENCING (NGS) DATA AMONG CHILDREN FROM EASTERN INDIA WITH SUSPECTED INHERITED TUBULAR DISORDER: EAST ZONE TUBULOPATHY GENETIC STUDY}

Rajiv Sinha ${ }^{1}$, Kausik Mandal ${ }^{2}$, Subal Pradhan ${ }^{3}$, Sushmita Banerjee ${ }^{4}$, Afsana Jahan ${ }^{6}$, Ramprasad V ${ }^{7}$, Amitava Pahari ${ }^{5}$

${ }^{1}$ Institute Of Child Health, ${ }^{2}$ Sgpg, India,${ }^{3}$ Svppgi, Cuttack, India,${ }^{4}$ Cmri, Kolkata, India, ${ }^{5}$ Apollo Hospital, Kolkata, India, ${ }^{6}$ Pratiksha Hospital, Gauhati, India, ${ }^{7}$ Medgenome, India

Introduction: Currently data are scarce on tubulopathy genotype among Indian population. We hereby present Next Generation Sequencing (NGS) generated phenotype-genotype data among children with suspected tubular disorder from Eastern India.

Material and methods: A prospective observational multi-centric study was conducted across 5 paediatric nephrology centres. After formal counselling and consent children ( $<18$ years) with clinically suspected 
tubular disorders underwent NGS. Variants were classified as per American College of Medical Genetics (2015) guidelines and pathogenic / likely pathogenic variants were considered significant.

Results: 77 index cases (median age 46, IQR 18 to 10 months; male $74 \%$ $\mathrm{n}=57$ ) underwent NGS. Phenotypic classifications were as follows: Distal Renal Tubular Acidosis i.e. RTA $(n=24)$, Bartter $(n=19)$, Isolated hypophosphatemic rickets with phosphaturia $(n=6)$, Proximal RTA $(n=12)$, Nephrogenic Diabetes Insipidus ( $\mathrm{n}=7$ ), Kidney stone / Nephrocalcinosis $(n=5)$ and Miscellaneous $(n=4)$. Among them 58 children $(75 \%)$ had 53 pathogenic or likely pathogenic variants (33 novel) detected. Percentage NGS yield was noted to be in 80 's for most of the phenotypes except distal RTA (71\%) and stone /nephrocalcinosis (20\%). NGS resulted in a new diagnosis in 9 children including non-tubulopathy disorder mimicking as tubulopathy in 7 children (Cystic fibrosis presenting with Bartter phenotype; $\mathrm{n}=4$ and one each of Cong chloride diarrhea with Bartter phenotype, Nephronopthisis with Nephrogenic Diabetes Insipidus phenotype and Hypo-parathyroidism, sensori-neural deafness and renal dysplasia phenotype mimicking miscellaneous tubulopathy). Additional change of diagnosis happened in two children (Bartter phenotype to Gitelman syndrome and Nephrogenic Diabetes Insipidus phenotype to distal RTA).

Conclusions: In our cohort of suspected tubulopathy from Eastern India NGS had a yield of $75 \%$, identified a large number of novel variants and resulted in $12 \%(n=9)$ new diagnosis. Our finding underscores the clinical utility of NGS for suspected tubulopathy and also highlights the unique mutation spectrum in Indian population and need for larger Indian studies.

\section{PI-4 TMP/GFR REFERENCE VALUES FROM CHILDHOOD TO ADULTHOOD IN THE ERA OF IDMS-STANDARDIZED CREATININE VALUES}

Laurence Dubourg, Manon Aurelle, Laurence Chardon,

Sacha Flammier, Sandrine Lemoine, Justine Bacchetta

Centre De Référence Des Maladies Rares Du Calcium Et Du Phosphore, Hospices Civils De Lyon, France

Introduction: The assessment of phosphate homeostasis in clinical practice relies on circulating phosphate levels but also on phosphate tubular reabsorption, ideally assessed using the Tubular maximum Phosphate Reabsorption per Glomerular Filtration Rate (TmP/GFR). $\mathrm{TmP} / \mathrm{GFR}$ reference values were established before the onset of IDMS-standardized creatinine assays, and thus need to be updated with these modifications in assays and laboratory standards. Our objective is to provide reference values for TmP/GFR from childhood to adulthood, using the gold-standard of GFR assessment and IDMS-standardized creatinine values.

Material and methods: We retrospectively analyzed all the inulin and iohexol clearances (mGFR) performed in children in our unit since the beginning of IDMS-creatinine assays, as well as the clearances performed in adults screened for a living-donation. TmP/GFR was calculated on a fasting sample, using the conventional formula without correction for TRP in subjects below 19 years of age.

Results: A total of 2051 subjects (1711 children and 340 adults), aged from 1.9 to 73.4 years with normal GFR, normal phosphate and normal calcium levels, was included for TmP/GFR analysis. As expected, there was a progressive decrease along puberty in both genders of plasma phosphate and TmP/GFR, the decrease occurring earlier in girls. After the age of 19, there was a stabilization of plasma phosphate and $\mathrm{TmP} /$ GFR levels until the age of 55, phosphate levels and TmP/GFR being slightly lower in men than in women.

Conclusions: We present here the largest cohort describing TmP/ GFR reference values in the era of IDMS-standardized creatinine assays. We believe that these data will help physicians to better diagnose and manage patients with abnormal phosphate metabolism in daily clinical routine.
PI-5 X-LINKED HYPOPHOSPHATEMIA, OBESITY AND CARDIOVASCULAR PARAMETERS: DATA FROM THE XLH21 STUDY

Louisa Bloudeau, Agnès Linglart, Sacha Flammier, Aurélie Portefaix, Jean-Pierre Salles, Anya Rothenbuhler, Christelle Roger,

Justine Bacchetta

Reference Center of Rare Diseases of Calcium and Phosphate, Rare Diseases French Network Oscar, France

Introduction: There is an over-incidence of obesity in X-linked hypophosphatemia (XLH), but the underlying pathophysiological mechanisms are not yet known. The objective of this study was to evaluate whether FGF21, another endocrine FGF involved in the regulation of carbohydrate-lipid metabolism, could be involved.

Material and methods: We performed a prospective multicenter crosssectional study comparing circulating FGF23, Klotho and FGF21 levels in teenagers with XLH compared to healthy controls (HC, VITADOS cohort) after a 1:1 matching on age, gender and puberty (XLH21, NCT03596554; VITADOS NCT01832623). Non-parametric tests were performed, results are presented as median (min-max).

Results: A total of 40 teenagers with XLH ( $\mathrm{N}=20$ with standard of care, SOC, $\mathrm{N}=20$ with burosumab) were included. Whilst patients receiving burosumab displayed decreased SDS for height and increased BMI as compared to patients receiving SOC, systolic blood pressure expressed as percentile progressively and significantly decreased when comparing the three groups: 77(4-99) in SOC, 47(9-98) in burosumab, and 28(0.5$94)$ in $\mathrm{HC}(\mathrm{p}=0.007)$. When compared to patients receiving SOC, patients receiving burosumab displayed significantly increased phosphate, 1-25-D and Klotho levels. No differences were found for either carbohydratelipid biomarkers or FGF21 between the three groups. In HC, FGF23 and FGF2 1 were positively associated $(\mathrm{R}=0.586, \mathrm{p}=0.002)$, but this association was lost in XLH patients. A total of 21 XLH patients (53\%) had insulin-resistance (HOMA $>2.4, \mathrm{~N}=10 \mathrm{SOC}, \mathrm{N}=11$ burosumab); there were no differences for FGF21, phosphate and Klotho levels between these two sub-groups.

Conclusions: FGF21 does not explain obesity/overweight in XLH teenagers. This study was performed in France in 2018-2019, early after the approval authorizing the treatment of patients with burosumab only in case of severe XLH despite SOC. As such, the data on systolic blood pressure highlighting a possible impact of burosumab to decrease blood pressure deserve further studies given their potential impact on long-term cardiovascular risk.

\section{PI-6 ASSESSMENT OF GLOMERULAR AND TUBULAR FUNCTION IN THE EVALUATION OF CHILDREN WITH SICKLE CELL DISEASE}

Edouard Rollot ${ }^{1}$, Iona Madden ${ }^{2}$, Justine Bacchetta ${ }^{1}$, Laurence Dubourg ${ }^{3}$ ${ }^{1}$ Centre De Référence Des Maladies Rénales Rares, Service De Néphrologie Pédiatrique, Hôpital Femme Mère Enfant, Hospices Civils De Lyon, Bron, France, ${ }^{2}$ Centre De Référence Des Maladies Rénales Rares, Unité De Néphrologie Pédiatrique, Chu De Bordeaux, Bordeaux, France, ${ }^{3}$ Département De Néphrologie, Service Dexploration Fonctionnelle Rénale, Hôpital Edouard Herriot, Hospices Civils De Lyon, Lyon, France

Objectives: Renal tubular dysfunction in children with Sickle Cell Disease (SCD) is one of the earliest manifestations of the disease, but there is a lack of data. Sickle Cell Nephropathy (SCN) is defined by hyperfiltration followed by microalbuminuria. Our aim was to compare tubular function between SCD patients with and without glomerular involvement.

Methods: This was a retrospective, single-center, cross-sectional study of all children with SCD admitted for comprehensive renal functional 
assessment at Lyon University Hospital, France, over a period of 18 years (1991-2009). Glomerular and tubular dysfunction was ascertained by collecting the following data: the measured glomerular filtration rate (mGFR) by inulin clearance, albuminuria, urine osmolality, reabsorption rates of chloride, sodium, potassium, calcium and magnesium as well as the tubular maximum re-absorption of phosphorus per unit volume of GFR (TmP/GFR).

Results: Eighty-six children were included, aged from 2 to 20 years old (mean age of 10.6 years, 53\% girls). Thirty-six children (42\%) presented SCN including $22(26 \%)$ with hyperfiltration (median mGFR $177 \mathrm{ml} /$ $\mathrm{min} / 1.73 \mathrm{~m}^{2}$ [IQR $\left.166 ; 182\right]$ and $29(34 \%)$ with albuminuria (median $5.5 \mathrm{mg} / \mathrm{mmol}$ [IQR $3.7 ; 6.7]$ ). There was not a statistically significant difference between $\mathrm{SCN}$ and non-SCN groups regarding urinary osmolarity, calcium reabsorption rate and $\mathrm{TmP} / \mathrm{GFR}$. However, the mean chloride, sodium and magnesium reabsorption rates were significantly higher in the group with SCN, with respectively 99.06 vs 98.74 (p-value $=0.01$ ), 99.51 vs 99.31 (p-value < 0.01), 97.98 vs 97.49 (p-value =0.04). The mean potassium reabsorption rate also tended to be higher in the SCN group: 91.72 vs. 89.95 (p-value $=0.05$ ).

Conclusions: Children with SCN seem to have a greater tubular reabsorption activity compared to those with normal glomerular function but exhibit similar renal concentrating ability, and urinary calcium and phosphorus excretion.

PI-7 ASSESSMENT OF QUALITY OF LIFE THROUGH A PATIENT-CENTRED APPROACH IN CHILDREN AND ADULTS WITH DRTA TREATED WITH A NEW PROLONGED-RELEASE ALKALISING DRUG (ADV7103) FOR 5 YEARS

Aurélia Bertholet-thomas ${ }^{1}$, Michael Acquadro ${ }^{2}$, Catherine Guittet ${ }^{3}$, Sophie Joukoff ${ }^{3}$, Maria A. Manso-silván ${ }^{3}$, Victor Navas ${ }^{3}$, Alexia Marrel $^{2}$

${ }^{1}$ Hospices Civils De Lyon, France, ${ }^{2}$ Icon Plc, Ireland ${ }^{3}$ Advicenne Sa

Introduction: Patients with distal renal tubular acidosis (dRTA) have negative impacts on growth, bone and kidney, sometimes with hearing loss, which may significantly affect their quality of life (QoL). This study explores their lived experience about QoL linked to dRTA and its treatment.

Material and methods: Individual 1-hour interviews $(n=19)$ were performed to gather the impressions of patients with dRTA, (and/or their parents) 5 years after enrolment in a clinical study. ADV7103 (potassium citrate and potassium bicarbonate prolonged-release granules, twice daily) was compared with the standard of care (SoC) taken before study entry. Interviewers trained in qualitative research conducted the interviews using a semi-structured interview guide and performed thematic analysis with MAXDA software.

Results: The main QoL domains impacted by dRTA and its treatment were difficulties studying, social challenges, and negative emotional and physical impacts. Difficulties associated to hearing impairment remained, but switching from previous SoC to ADV7103 was experienced as "life-changing" by patients/parents (Table 1):

- The emotional burden of disease perceived was relieved in the absence of treatment-related invasive questions from others,

- Difficulties at school due to burdensome administrative issues and need to explain disease and treatment disappeared, facilitating parents who had stopped working to return to work,

- Social/family issues improved: travel and holidays became easier to organise, patients/parents stopped thinking about managing treatment daily/nightly, reducing tension in the family/couple,
- Bad taste and gastro-intestinal adverse events improved with ADV7103. Better compliance led to milder physical impacts and less fear to be hospitalised.

Mean satisfaction score with ADV7103 vs. SoC was 9 out of 10 and ADV7103 exceeded or met the expectations of $82 \%$ patients.

Table 1. Number of patients (\%) concerned by each QoL domain after switching from $\mathrm{SoC}$ to ADV7103 $(n=19)$

\section{Reported impacts with SoC}

Emotional functioning difficulties, 13 patients $(68 \%)$

Administrative complications at school and difficulties for parents to work, 13 paediatric patients $(100 \%)$

Social functioning and family problems, 6 patients $(31 \%)$

Health and product acceptability issues, 16 patients $(84 \%)$

\section{Reported impacts with ADV7103}

Improved emotional functioning, 12 patients $(63 \%)$

No more organisation problems for families at school and work, 13 paediatric patients $(100 \%)$

Improved family functioning, 18 patients $(95 \%)$

Improved health and product acceptability, 18 patients $(95 \%)$
Conclusions: Qualitative interviews show that dRTA and its treatment have a significant impact on QoL of patients and parents and that ADV7103 helps improve daily-life and reduces treatment burden, resulting in greater overall patients' and parents' satisfaction.

\section{PI-8 AN ADOLESCENT BOY PRESENTED WITH POLYURIA:} A DIAGNOSTIC CHALLENGE

Gökçen Erfidan ${ }^{1}$, Demet Alaygut ${ }^{1}$, Eren Soyaltin ${ }^{1}$, Cemaliye Başaran ${ }^{1}$, Yaşar Kutbay $^{2}$, Seçil Arslansoyu Çamlar ${ }^{1}$, Fatma Mutlubaș ${ }^{1}$, Belde Kasap Demir ${ }^{3}$

${ }^{1}$ University of Health Sciences, Tepecik Training and Research Hospital, Department of Pediatrics, Division of Nephrology, Izmir, Turkey, ${ }^{2}$ University of Health Sciences, Tepecik Training and Research Hospital, Department of Medical Genetic Diseases Center, Izmir, Turkey, ${ }^{3}$ Izmir Katip Celebi University, Faculty of Medicine, Department of Pediatrics, Division of Nephrology and Rheumatology, Izmir, Turkey

Introduction: Polyuria in children may become a diagnostic challenge since it may be seen as presenting symptom of an underlying renal or systemic disease.

Material and methods: An adolescent boy, who did not have any known illness, was presented with polyuria. The detailed history revealed the findings of learning difficulty, speech impairment, unsteady gait and an operation of polydactyly. He had consanguineous parents and a brother who had abruptly diagnosed with the end-stage renal disease at the age of 21. These clues pointed to genetic background.

Results: On physical examination, he had pectus excavatum, atypical facial appearance, dysarthria, hypotonia, rotatory nystagmus, impaired tandem walk, hyperpigmented retinal irregularities. Laboratory examinations showed Stage-4 chronic kidney disease accompanied by tubulopathy. Ultrasonography detected cystic lesions on the corticomedullary junction. Thus, the patient had diagnosed JuvenileNephronophthisis. During further examinations, Molar Tooth Sign was detected in cranial MRI imaging. All these clinical and radiological findings indicate the spectrum of Joubert-Syndrome-Related-Disorders (JSRD). Genetic analysis of the patient and his brother revealed homozygous NPHP1 deletion. Distinctly from literature, they both had hematological involvement in the form of persistent thrombocytopenia.

Conclusions: Genetic heterogeneity and phenotypic variability of nephronophthisis are major challenges. Although NPHP1 deletions are 
mostly identified in isolated nephronophthisis, they have also been described in complex ciliopathy syndromes such as JSRD. This case is also specific due to haematological involvement additional to kidney, retina, skeleton, neurological. We think, this case may shed light on future genotype-phenotype studies.

\section{PI-9 INFANTILE HYPERCALCEMIA DUE TO HOMOZYGOUS CYP24A1 MUTATION IN A PATIENT WITH NEUROFIBROMATOSIS TYPE 1: A CASE REPORT}

Ivanka Kos, Hana Matković, Maja Ban, Maša Davidović, Lovro Lamot, Kristina Vrljičak

Department Of Pediatrics, Unviersity Of Zagreb School Of Medicine, University Hospital Centre Zagreb, Zagreb, Croatia

Abstract: Idiopathic infantile hypercalcemia (IIH) is characterized by severe symptomatic hypercalcemia. It is caused by mutations in CYP24A1 and SLC34A1 resulting in increased vitamin D sensitivity and elevated serum $1,25(\mathrm{OH}) 2 \mathrm{D} 3$ with subsequent hypercalcemia and hypercalciuria. SLC34A1 mutations additionally lead to primary renal phosphate wasting and hypophosphatemia. Contrarly, neurofibromatosis (NF) is associated with vitamin D deficiency, so supplementation of vitamin $\mathrm{D}$ is beneficial. Yet, NF is sometimes complicated by hypophosphatemic osteomalacia and hyperparathyroidism.

We report a case of a 14 years old male who initially presented at the age of 5 months with profound hypercalcemia, vomiting, dehydration, weight loss, hypotonia, and hypercalciuria. He was exclusively breastfed and received 1000 IU of vitamin D for prophylaxis. Serum $25-\mathrm{OH}$ vitamin D and $1,25(\mathrm{OH}) 2 \mathrm{D} 3$ levels were normal, while parathyroid hormone level was undetectable. Besides, he had hypophosphatemia and osteomalacia. A low calcium/vitamin D diet was initiated. Ultrasound showed increased echogenicity of the renal pyramids at the age of 3 years and kidney stones at the age of 5. Serum calcium and phosphate levels were normal and hypercalciuria was excluded. Furthermore, he fulfilled clinical criteria for neurofibromatosis type 1 and started prophylaxis with $600 \mathrm{IU}$ vitamin D. Neverthless, after two months of prophylaxis, he developed hypercalciuria with suppressed PTH and hyperphosphaturia, so vitamin D was restricted again. By the age of 7 , he suffered from recurrent renal colic. The kidney stones were fragmented with extracorporeal shock wave lithotripsy and excreted in the urine. Since then the patient has been treated with hydrochlorothiazide, which lead to normalization of urine calcium/creatinine ratio and renal ultrasound. Moreover, catch-up in growth and development was observed. Finally, genetic analysis identified homozygous missense variant CYP24A1 c.1186C >T,p. (Arg396Trp).

IIH has not yet been described in individuals with NF1. Both conditions adversely affect complex phosphocalcic metabolism. Hydrochlorothiazide is a useful treatment option. Further investigation is required to facilitate appropriate management and treatment of this challenging patients.

\section{PI-10 OUTCOME OF A COHORT OF PATIENTS WITH DENT DISEASE}

Ane Intxauspe Maritxalar ${ }^{1}$, Maria Herrero GoNii ${ }^{2}$, Leire Madariaga Dominguez $^{3}$, Leire Gondra Sangroniz ${ }^{3}$, Mireia Aguirre MeÑica ${ }^{2}$, Alejandro Garcia CastaÑo ${ }^{4}$, Ana Vinuesa Jaca ${ }^{2}$, Sara Gomez Conde ${ }^{5}$, Nelida Garcia Perez ${ }^{6}$

${ }^{1}$ Pediatric Department, Cruces University Hospital, Barakaldo, Vizcaya, Spain, ${ }^{2}$ Pediatric Nephrology, Cruces University Hospital, Iis Biocruces Bizkaia, Barakaldo, Vizcaya, Spain, ${ }^{3}$ Pediatric Neprhology, Cruces University Hospital, Iis Biocruces Bizkaia, Basque Country University, Barakaldo, Vizcaya, Spain, ${ }^{4}$ Iis Biocruces Bizkaia, Basque Country University, Vizcaya, Spain, ${ }^{5}$ Iis Biocruces Bizkaia, Vizcaya, Spain, ${ }^{6}$ Pediatric Nephrology, Basurto University Hospital, Iis Biocruces Bizkaia, Barakaldo, Vizaya, Spain
Background: Dent disease is an X-linked renal proximal tubular disorder characterized by low-molecular-weight (LMW) proteinuria, hypercalciuria and nephrocalcinosis/nephrolithiasis that frequently develops chronic kidney disease (CKD) over time. The aim of this study is to describe the long term outcome of patients with Dent disease.

Methods: This is a retrospective, observational study including affected male patients who were diagnosed during childhood. We measured urine and blood parameters related to the expected evolution of the disease and registered radiological findings, estimated glomerular filtration rate (GFR) and the treatment they received.

Results: Seven male patients were included, all of them carrying CLCN5 gene pathogenic variants. They were referred to the Pediatric Nephrology Service at the median age of 3 years (range 11months-13years) due to proteinuria (3patients), proteinuria and hypercalciuria (1), kidney failure (1) and familial study (2). The median rate follow-up time was 9 years (range 2-20.5 years). All of the patients have, at last follow-up, LMW proteinuria and 5 patients have hypercalcemia and nephrocalcinosis. None of them have nephrolithiasis or hematuria. Additional manifestations of proximal tubular dysfunction are also present with variable frequencies, such as hypokalemia (3patients) or glycosuria (1). Three patients have hypophosphatemia, low bone mineral density and take phosphate supplements from the adolescence. One patient showed growth failure and received Growth Hormone Therapy. At diagnosis, the median estimated GFR was $96 \mathrm{ml} / \mathrm{min} / 1.73 \mathrm{~m}^{2}$ (range 13235) and one patient had CKD. During follow-up period, 4 patients developed CKD (II-III), median GFR $82 \mathrm{ml} / \mathrm{min} / 1.73 \mathrm{~m}^{2}$ (range 51-157) with the median age of 18years (range 13-18).

Four patients need thiazide and amiloride combination drugs, 5 receive potassium or phosphate supplements and 3 vitamin D supplements.

Conclusion: Dent disease is a rare condition with poor long-term prognosis that progress to CKD at early adulthood and may develop hypophosphatemia with risk of osteomalacia. Genetic diagnosis is essential for the study of relatives.

\section{PI-11 POSITIVE OUTCOMES WITH EARLY STEROID TREATMENT IN A SEVERE CASE OF DRUG-INDUCED TUBULOINTERSTITIAL NEPHRITIS (DI-TIN)}

Maduri Raja ${ }^{1}$, Mushfequr R Haq ${ }^{2}$, Bhumita Vadgama ${ }^{2}$, Arvind Nagra ${ }^{2}$, Rodney D Gilbert ${ }^{2}$, Evgenia Preka ${ }^{3}$

${ }^{1}$ University College London Great Ormond Street Institute of Child Health, 30 Guilford Street, London Wcln 1eh, United Kingdom, ${ }^{2}$ Department of Paediatric Nephrology, Southampton Children's Hospital, University Hospital Southampton Nhs Foundation Trust, Tremona Road, Southampton Sol6 6yd, UK, ${ }^{3}$ European Society for Paediatric Nephrology/ European Renal Association-European Dialysis And Transplant Association Registry, Amsterdam Umc, University of Amsterdam, Department of Medical Informatics, Amsterdam Public Health Research Institute, Amsterdam, The Netherlands

Objectives: Drug-induced tubulointerstitial nephritis (DI-TIN) remains an underappreciated cause of AKI in children with delays in diagnosis and treatment contributing to further renal sequelae. Although corticosteroids are widely used in severe DI-TIN cases, knowledge of its efficacy in children is limited with no valid data from large randomised controlled trials. In order to reach generally accepted policies for these patients, all successful experiences should be reported.

Methods: Retrospective review of case notes and analysis of kidney function data (from admission up to 12 months) alongside review of current literature.

Results: A 6-year-old girl presented with oliguric acute kidney injury (AKI) [eGFR $11 \mathrm{ml} / \mathrm{min} / 1.73 \mathrm{~m}^{2}$ ] requiring continuous veno-venous haemofiltration $(\mathrm{CVVH})$. History suggested recent use of ibuprofen, paracetamol and ceftriaxone following a positive urine culture with enterococcus. She had a previous history of recurrent urinary tract infections 
associated with self-limiting AKI episodes and both times she was given regular NSAIDs and antibiotics. Kidney biopsy showed a mixed interstitial infiltrate of lymphocytes, plasma cells and eosinophils with patchy tubulitis and neutrophil casts. Ophthalmology review was normal. Despite 4 days of daily CVVH, discontinuing potential nephrotoxic drugs and subsequent improvement in urine output, her eGFR remained between $14-16 \mathrm{ml} / \mathrm{min} / 1.73 \mathrm{~m}^{2}$. A tapering steroid regime was started on day 6 and reduced over 6 weeks [initial prednisolone dose $1 \mathrm{mg} / \mathrm{kg}$ / day]. The eGFR improved to $49.7 \mathrm{ml} / \mathrm{min} / 1.73 \mathrm{~m} 2$ after the first 4 doses and normalised 1 month after commencing prednisolone. DMSA and MCUG at 6 months follow-up were normal. Her latest eGFR at 12 months follow-up is $100.6 \mathrm{ml} / \mathrm{min} / 1.73 \mathrm{~m}^{2}$.

Conclusion: Based on current literature and our case report, we suggest that early diagnosis and timely administration of corticosteroids in severe DI-TIN cases can lead to full recovery despite a critical initial presentation. Large randomised prospective studies are needed to determine bestpractice recommendations for DI-TIN treatment in children.

\section{PI-12 KIDNEY TRANSPLANTATION IN CHILDREN WEIGHING 15 KILOGRAMS OR LESS}

Ozan Özkaya ${ }^{1}$, Havva Evrengül ${ }^{1}$, Eryiğit Eren ${ }^{1}$, Mehmet Tokaç ${ }^{1}$, Ayhan Yaman 1, Taylan Șahin ${ }^{1}$, Murat Sütçü ${ }^{1}$, Belde Kasapdemir ${ }^{2}$, Ismail Dursun ${ }^{1}$, Ayhan Dinçan ${ }^{1}$

${ }^{1}$ İstinye University Liv Hospital Bahçeşehir, Turkey, ${ }^{2}$ Tepecik Ë̆itim ve Araştırma Hastanesi, Turkey, ${ }^{3}$ Erciyes Üniversitesi Tip Fakültesi, Turkey

Objective: Kidney transplantation (KTx) is the best treatment option for renal replacement therapy in pediatric patients with end-stage renal disease (ESRD). However, especially in very small children KTx can be a challenging procedure with a higher risk of peri-operative complications and poorer outcome. The aim of this study is to to analyze the outcome of KTx recipients weighting under $15 \mathrm{~kg}$, focusing on surgical and infectious complications. Methods: We reviewed our retrospective database for recipients of KTx between January 2018 and April 2021 with body weight under $15 \mathrm{~kg}$. Results: In the period between 1 January 2018 and 31 March 2021, 86 paediatric KTxs were performed at İstinye University Liv Bahçeșehir Hospital. $32(37,2 \%)$ of these 86 children had a body weight of $<15 \mathrm{~kg}$ at the time of Ktx. Twenty-seven of 32 children who had detailed followup data were included in the study. There were 13 females $(48.1 \%)$ and $14(51,9 \%)$ males. Mean weight of the patients was $11.69 \pm 2.16 \mathrm{~kg}$ and mean age was $3.60 \pm 1.59$ years. Median follow-up was 9.5 (6-17) months. The most common cause of ESRD was nephrotic syndrome (26.9\%). Six (22\%) patients were on hemodialysis whereas $9(33 \%)$ were on peritoneal dialysis treatment. In $12(44 \%)$ patients Ktx were performed preemptively. The only postoperative complication was ileus (3 patients). Five $(18.5 \%)$ patients had BK virus, 5 patients $(18.5 \%)$ had CMVand 1 $(3.75 \%)$ patient had EBV infection. Urinary tract infection was observed in $6(22,2 \%)$ patients and none of the patients lost the graft.

Conclusion: Our results suggest that KTx in children weighing under $15 \mathrm{~kg}$ is not associated with increased risk of surgical and infectious complications or early graft loss. Kidney transplantation should be considered early to allow children to reach normal physical, mental and social development.

\section{PI-13 FIRST REPORT OF LONG-TERM OUTCOME OF COMBINED LIVER AND KIDNEY TRANSPLANTATION IN SEVERE PROTEIN C DEFICIENCY}

Maduri Raja ${ }^{1}$, Maninder Bal ${ }^{1}$, David V Milford ${ }^{2}$, Mushfequr R Haq ${ }^{1}$, Rodney D Gilbert ${ }^{1}$, Patrick Mckiernan ${ }^{2}$, Arvind Nagra ${ }^{1}$

$I^{\prime}$ Southampton Children's Hospital, University Hospital Southampton Nhs Foundation Trust, Tremona Road, Southampton Sol6 6yd, United Kingdom, ${ }^{2}$ Birmingham Women's and Children's
Hospital Nhs Foundation Trust, Steelhouse Lane, Birmingham, B4 6nh, United Kingdom

Introduction: The long-term outcome of combined liver and kidney transplantation (CLKT) in a 13-year-old boy with end stage kidney disease secondary to homozygous protein $\mathrm{C}$ deficiency (PCD).

Material and methods: Retrospective review of case notes, kidney function and protein $\mathrm{C}(\mathrm{PrC})$ levels from birth to 13 years of age (10 years post-transplantation); Cost comparison pre and post CLKT.

Results: This is the first report of 10 -year post-CLKT outcome in a child with PCD born with bilateral renal venous thrombosis resulting in end stage kidney disease. He had worsening kidney function due to recurrent thrombotic episodes secondary to undetectable PrC levels. On PCD diagnosis at 7 months of age he started activated PrC infusions (levels maintained $>0.25 \mathrm{IU} / \mathrm{ml}$ ) to prevent thrombosis. He received a deceased donor CLKT at age 3 years. PrC levels were maintained above $100 \mathrm{IU} / \mathrm{ml}$ peri-operatively to prevent thrombosis and were discontinued 48 hours post-CLKT when levels were $1.03 \mathrm{IU} / \mathrm{ml}$. 10 years post CLKT they remain normal $(0.79 \mathrm{IU} / \mathrm{ml})$ with no thrombotic episodes since CLKT and he has had no further requirement for exogenous PrC. Kidney function: His creatinine improved rapidly from $350 \mu \mathrm{mol} / \mathrm{l}$ pre-CLKT to $30 \mu \mathrm{mol} / 1$ post-CLKT. Kidney function remained stable but deteriorated 6 years post-CLKT secondary to antibody mediated rejection. This responded to increased immunosuppression. Cost analysis: Pre-CLKT PrC infusions cost $£ 182,000 /$ year. The cost of the CLKT was approximately $£ 130,000$ for the first year. 10 years post-CKLT, over $£ 1,000,000$ has been saved on $\mathrm{PrC}$ infusions alone and no requirement for long-term dialysis.

Conclusions: CLKT in PCD with early renal involvement restores hepatic synthesis of $\mathrm{PrC}$ and provides definitive renal replacement therapy. CLKT has few long-term complications and is a cost-effective treatment option. This first reported case suggests CLKT should be considered as a treatment option in similar cases of PCD.

PI-14 NEUTROPHIL GELATINASE-ASSOCIATED LIPOCALIN (NGAL) AND INTERLEUKINE-18 (IL-18) IN GRAFT URINE CORRELATE WITH THE RATE OF RECOVERY OF TRANSPLANTED KIDNEYS IN CHILDREN

De Jong Huib ${ }^{1}$, Terpstra Anniek ${ }^{1}$, Meys Karlijn ${ }^{1}$, Zwiers Alexandra ${ }^{2}$, Sloots Cornelius ${ }^{2}$, Cornelissen Elisabeth ${ }^{3}$, Bouts Antonia ${ }^{4}$, De Rijke Yolanda ${ }^{5}$, Van Rosmalen Joost ${ }^{6}$, Cransberg Karlien ${ }^{1}$ ${ }^{I}$ Dept. of Pediatric Nephrology, Sophia Children's Hospital, Erasmus Universitymc, Rotterdam, The Netherlands, ${ }^{2}$ Dept. of Pediatric Surgery, Sophia Children's Hospital, Erasmus University Mc, Rotterdam, The Netherlands, ${ }^{3}$ Dept. of Pediatric Nephrology, Radboudumc Amalia Childrens Hospital, Nijmegen, The Netherlands, ${ }^{4}$ Dept. of Pediatric Nephrology, Emma Children's Hospital, Amsterdam University Medical Center, Amsterdam, The Netherlands, ${ }^{5}$ Dept. of Clinical Chemistry, Erasmus University Medical Center Rotterdam, The Netherlands, ${ }^{6}$ Dept. of Biostatistics, Erasmus University Medical Center Rotterdam, The Netherlands

Introduction: Organ transplantation includes the procurement, preservation and implantation of an organ. During this process ischemia-reperfusion injury (IRI) of the graft is inevitable. Pediatric kidney transplantation carries a high risk of IRI, because most children receive an adult donor kidney mismatched in size. Considering the tubular pathogenesis of IRI, we hypothesize that specific biomarkers of tubular damage (neutrophil gelatinaseassociated lipocalin (NGAL) and interleukin-18 (IL-18) better predict graft recovery then serum kreatinine.

Material and methods: We performed a prospective multi-center trial in pediatric kidney transplantation recipients. We measured urinary neutrophil gelatinase-associated lipocalin (uNGAL) and interleukin-18 (uIL-18) concentrations in 82 recipients, in urine separately collected from graft 
and bladder within 3 days after transplantation. Concentrations at 3,6 and 12 hours post-reperfusion were compared with the half-life of serum creatinine at reperfusion (T1/2Creat) as measure of graft recovery, and with the estimated glomerular filtration rate (eGFR) after 3 months.

Results: At all time-points post-transplantation, the median graft uNGAL concentration was lower than the bladder concentration, whereas the median graft uIL-18 concentration was structurally higher than the bladder concentration. After reperfusion, the graft UNGAL and uIL-18 concentrations peaked within 6 hours, then gradually declined. The graft uNGAL and uIL-18 concentrations at 3, 6 and 12 hours post-reperfusion correlated with the rate of graft recovery, and predicted slow graft function, with higher ROC-AUCs for UNGAL than for uIL-18. The graft function at 3 months posttransplant was only weakly predicted by the uNGAL and uIL-18 concentrations at the early time-points.

Conclusions: UNGAL and uIL-18 concentrations in urine directly drained from the graft in the hours after reperfusion are associated with graft recovery after transplantation. Thus slow graft function can be anticipated by measuring these tubular injury markers and therefore prevent us to perform more invasive diagnostic interventions in pediatric kidney recipients.

\section{PI-15 LIMITED VALUE OF THROMBOPHILIA SCREENING IN KIDNEY TRANSPLANTATION - DO WE NEED A CLINICAL RISK STRATIFICATION GUIDELINE?}

\section{Cheryl Lim , Manish Sinha, Nabil Melhem \\ Evelina London Children's Hospital, United Kingdom}

Introduction: Thrombosis is a rare but catastrophic complication in the context of paediatric kidney transplantation. Whilst thrombophilia is often implicated, there are few data to support this association. We describe the prevalence of thromboembolic events (TEs) in paediatric kidney transplant recipients and assess the clinical utility of pre-operative thrombophilia screening.

Material and methods: Single-centre, retrospective study of all paediatric kidney transplant recipients aged 1-18 over a 5 year period. Results: 84 children included with a mean age of $11.1 \pm 5.2$ years. $54(64.3 \%)$ boys, $59(70.2 \%)$ white-Caucasian, and $48(57.1 \%)$ with CAKUT. 61(72.6\%) were receiving dialysis at time of transplantation. All participants underwent thrombophilia screening pre-transplantation to identify inherited and acquired abnormalities of coagulation.

Abnormal thrombophilia screen was recorded in 37(44.0\%) with $20(23.8 \%)$ showing persistent abnormalities upon repeat testing. $11(13.1 \%)$ had anticardiolipin (AC) antibodies, 11(13.1\%) lupus anticoagulant (LA) antibodies, 2(2.4\%) elevated homocysteine, and 5(6.0\%) were heterozygous for thrombophilic gene mutations. 21(25.0\%) started prophylactic unfractionated heparin post-operatively as per haematologist advice pre-transplantation and 10(11.9\%) following intraoperative decision by transplanting surgeon.

Overall, TEs occurred in 10(11.9\%) patients. 6(7.1\%) pre-transplant of whom only two had LA antibodies. A further 4(4.8\%) TEs occurred post-transplant of whom one had abnormal screening (AC antibodies and protein S). Graft loss did not occur following TEs. There was no association between pre-operative thrombophilia screening and pre or post-transplant TEs $(\mathrm{p}=0.70)$. Dialysis and non-White ethnicity was associated with abnormal thrombophilia screening ( $\mathrm{p}=0.04$ and $\mathrm{p}<0.01$ respectively).

Conclusions: We found a high prevalence of thrombophilia in our pre-transplant population, particularly in those on dialysis and of ethnic minorities. Despite high prevalence of abnormal tests, we observed lack of significant predictive value of detailed testing. Risk stratification could improve our approach to transplant thromboprophylaxis, with focus on patients with previous TEs and on dialysis.

\section{PI-16 LEFLUNAMIDE FOR TREATMENT OF BK VIRUS} NEPHROPATHY

GÜlşah Kaya Aksoy ${ }^{1}$, Mine Erkan ${ }^{2}$, Mustafa Koyun ${ }^{1}$, Elif Çomak ${ }^{1}$, Havva Serap Toru ${ }^{3}$, Derya Mutlu ${ }^{4}$, Bahar Akkaya ${ }^{3}$, Sema Akman ${ }^{1}$

${ }^{1}$ Akdeniz University Medical Faculty, Department of Pediatric Nephrology, Antalya, Turkey, ${ }^{2}$ Akdeniz University Medical Faculty, Department of Pediatrics, Antalya, Turkey, ${ }^{3}$ Akdeniz University Medical Faculty, Department of Pathology, Antalya, Turkey, ${ }^{4}$ Akdeniz University Medical Faculty, Department of Microbiology, Antalya, Turkey

Introduction: BK virus nephropathy (BKVAN) is a clinicopathological entity that negatively affects graft function in kidney transplant recipients. In this study, we aimed to evaluate the efficacy of leflunamide treatment compared to cidofovir in patients with BKVAN.

Material and methods: The medical records of kidney transplant recipients with BK viremia between 2004 and 2019 were reviewed retrospectively, and those with a diagnosis of BKVAN were included in the study. Serum BK virus level above $10^{4}$ copies was accepted as BK viremia. BKVAN was defined as the detection of BK virus SV40 antigen in renal graft tissue by immunochemistry staining and accompanying signs of tubulointerstitial nephritis or the presence of high serum creatinine in addition to BK viremia.

Results: Of 304 kidney transplant recipients, 53 had persistent BK viremia; and 36 of these patients $(61.1 \%$ male) were included in the study with the diagnosis of BKVAN. Median age at the time transplantation was 12.23 (IQR 4.06-15.41) years. Anti-thymocyte globulin was administered in $52.8 \%$ of patients for induction therapy, and tacrolimus was used in $80.6 \%$. As a first-line treatment; MMF dose was reduced in 34 (94.4\%) patients, MMF was switched to azathioprine in $9(25.0 \%)$, calcineurin dose was reduced in 29 patients $(80.5 \%)$. Ciprofloxacin was given to all patients. 12 patients $(33.3 \%)$ received cidofovir and 14 $(38.8 \%)$ received leflunamide. Plasma BK virus load and graft functions were similar between the groups. Median viremia clearance time after treatment was lower in the leflunamide group compared to cidofovir (3.0 vs 4.0 weeks, $\mathrm{p}=0.032$ ). Graft failure occurred in 5 of 12 patients treated with cidofovir and 3 of 14 patients treated with leflunamide $(p=0.310)$. There were no advers effects associated with leflunamide and cidofovir, leading to a discontinuation of treatment.

Conclusions: Leflunamide is an effective and safe treatment option that can be used in kidney transplant recipients with BKVAN.

\section{PI-17 CIRCULATION AND HEMODYNAMICS IN LIVING DONATION OF KIDNEY TRANSPLANTATION IN CHILDREN - THE CHILD-KITC STUDY: MAGNETIC RESONANCE - ARTERIAL SPIN LABELING PERFUSION IMAGING IN PEDIATRIC KTX}

E.1. Stille, M. Voet, E.a.m. Cornelissen, P.p. Poyck, M.f.p. Van Der Jagt, A. Nusmeier, W. Klein, J. Lemson, J.i. Malagon, J.j. Fütterer Radboud University Medical Center, The Netherlands

Introduction: In pediatric kidney transplantation, adequate perfusion of adult-sized grafts demands significant hemodynamic changes in small recipients. Hypoperfusion can shorten graft survival by loss of kidney mass and function, especially in donor-recipient size mismatch. In order to adequately perfuse the transplanted adult-sized kidney, young recipients are hypothesized to develop a supraphysiological hemodynamic state. Our primary objective is to enhance knowledge and insight in hemodynamic responses after pediatric kidney transplantation with a living, adult donor. By comparing Magnetic Resonance - Arterial Spin Labeling (MR-ASL) with transabdominal and intra-operative ultrasound (US), our secondary goal is investigating the reliability of non-invasive techniques in monitoring donor kidney perfusion after transplantation. 
Material and methods: Twenty children (including donors) with a maximum age of 15 and a maximum weight of $40 \mathrm{~kg}$ will be included in this prospective clinical pilot study with 12 months of follow-up. Perioperative hemodynamics are monitored closely, including cardiac output measurements. We measured volume of the kidney graft (MR), whole organ kidney perfusion (MR-ASL) and afferent blood flow (US and MR) before, during and 6 months after transplantation. We present the first three patients with complete series of imaging in follow up.

Results: MR-ASL perfusion imaging yields good quality images and reproducible perfusion values. Kidney graft volumetrics and perfusion showed a decrease after transplant, and a subsequent compensatory increase at 6 months. Afferent flow measurements comparing US and MR are corresponding, and in line with our hypotheses.

\begin{tabular}{lllll}
\hline & $\begin{array}{c}\text { Mean graft volume } \\
(\mathbf{c m} 3)\end{array}$ & \multicolumn{2}{c}{$\begin{array}{c}\text { Mean graft perfusion } \\
(\mathbf{m l} / \mathbf{m i n})\end{array}$} \\
1. Pre-transplant & 306 & & 407 & \\
2. Post-transplant & 202 & $(\downarrow 34 \%)$ & 161 & $(\downarrow 60 \%)$ \\
3. FU 6 months & 226 & $(\uparrow 12 \%)$ & 233 & $(\uparrow 45 \%)$ \\
\hline
\end{tabular}

Conclusions: In pediatric kidney transplantation with an adult living donor, graft volume and perfusion decreased considerably after transplantation and recovered partially after 6 months. Complementing current monitoring techniques, MR-ASL proves to be a feasible non-invasive peri-operative imaging technique, yielding good quality images and reproducible perfusion values.

\section{PI-18 KIDNEY TRANSPLANTED CHILDREN NOT RESPONDING TO ANTI-REJECTION TREATMENT: HOW TO IMPROVE THE OUTCOME}

Irene Alberici ${ }^{1}$, Susanna Negrisolo ${ }^{2}$, Andrea Carraro ${ }^{2}$, Piera De Gaspari ${ }^{2}$, Diana Marzenta ${ }^{2}$, Mattia Parolin ${ }^{1}$, Germana Longo ${ }^{1}$, Davide Meneghesso ${ }^{1}$, Elisa Benetti ${ }^{1}$

${ }^{1}$ Pediatric Nephrology Dialysis and Transplant Unit, Padua University Hospital, Italy, ${ }^{2}$ Laboratory of Immunopathology And Molecular Biology of The Kidney, Institute of Pediatric Research Città Della Speranza, Department of Women's And Children's Health, Padua University Hospital, Italy

Introduction: Despite the significant improvement in immunosuppressive therapy over the years, graft rejection remains the leading cause of graft loss in kidney transplanted children. The aim of this study was to identify which are the acute rejection (AR) at major risk for nonresponding to anti-rejection treatment.

Material and methods: A retrospective analysis of all the AR diagnosed in our Hospital (Jan 2015-Aug 2020) in kidney-transplanted children, who completed the anti-rejection treatment and a follow-up biopsy after the AR treatment, was done. The immunohistochemical characterization (IC) of the cellular infiltrate with anti-CD3/CD20 antibodies was performed on all the biopsies.

Results: 73 AR (53\% subclinical) in 52 children: 33 males, median age 9 years, $19 \%$ pre-emptive, $30 \%$ living donor, $10 \% 2^{\text {nd }}$ kidney transplant. The Banff- 17 criteria classified $54 \%$ cellular, $38 \%$ antibody-mediated and $8 \%$ mixed rejections. At the IC $84 \%$ had a mixed CD3-CD20 infiltrate. Cellular AR received pulse methylprednisolone (386 mg/mq, 293-488) and ATG in 7 cases. The antibody-mediated AR: $88 \%$ pulse steroid, $94 \%$ immunoglobulin, $62 \%$ plasmapheresis and $68 \%$ Rituximab. After treatment, a follow-up biopsy proved: $32 \%$ Banffl, $42 \%$ persistent AR and $26 \%$ IFTA. At the univariate and multivariate analysis, children at risk for non-responding to AR treatment were those at the $2^{\text {nd }}$ transplant, previously dialyzed, with a poor graft function at discharge from transplant, older at the time of AR. AR, if diagnosed by a protocol biopsy, or treated with a major pulse methylprednisolone dose or switched to Tacrolimus, had a better outcome. A predominant CD3 infiltrate increased risk of nonresponding to treatment (OR 10.11, 1.05-97.00).

Conclusions: The protocol biopsy, the pulse steroid dose and the switch to Tacrolimus are the only modifiable variables that correlate with a better response to the anti-rejection therapy. The implementation of IC of the inflammatory infiltrate could be an additional tool to identify patients most likely to not respond to therapy.

\section{PI-19 RENAL GRAFT LOSS DURING ADOLESCENCE: IS THERE A RELATIONSHIP BETWEEN PUBERTAL STAGE AND ACTIVITY OF THE IMMUNE SYSTEM?}

Femke Vrieling-prince ${ }^{1}$, Sophie Van Dongen ${ }^{1}$, Marlies Cornelissen ${ }^{2}$, Antonia Bouts ${ }^{3}$, Karlien Cransberg ${ }^{1}$, Joke Roodnat ${ }^{4}$, Anton Langerak ${ }^{5}$ ${ }^{I}$ Department of Paediatric Nephrology And Erasmus Mc Transplant Institute, Erasmus Mc Sophia Children's Hospital, Rotterdam, The Netherlands, ${ }^{2}$ Department of Paediatric Nephrology, Radboud Umc Amalia Children's Hospital, Nijmegen, The Netherlands, ${ }^{3}$ Department of Paediatric Nephrology, Amsterdam Umc Emma Children's Hospital, Amsterdam, The Netherlands, ${ }^{4}$ Department of Internal Medicine, Division of Nephrology and Erasmus Mc Transplant Institute, Erasmus Mc, Rotterdam, The Netherlands, ${ }^{5}$ Department of Immunology, Laboratory of Medical Immunology, Erasmus Mc, Rotterdam, The Netherlands

Introduction: The objective of this study was to explore immunological causes of inferior graft survival in renal transplant recipients during late adolescence and young adulthood in comparison to other age categories. Material and methods: This study is part of the larger multicentre cohort study Adolesce-NT and investigates the relationship between T cell subsets and pubertal stage in patients with end-stage renal failure, renal transplant recipients and healthy controls.

Participants, with age 8 to 30 years at time of inclusion, were divided in a pre-transplantation, post-transplantation and healthy control group. Exclusion criteria: no informed consent, pubertas praecox, prior treatment with immunomodulating agents. Patients had two study visits one year apart, healthy controls had one study visit. Participants were classified as pre-pubertal, early pubertal, late pubertal or post-pubertal according to skeletal age, Tanner stadium and/or testis volume.

Using 8-color flow cytometry, mean cell counts of recent thymic emigrants (CD4+CD31+CD62Lhi and CD8+CD31+CD62Lhi) and naïve and memory T cells (CD4+ and CD8+ Temro, Temra and Tcm) were determined.

Results: In total 267 participants were included in the analysis. The increased numbers of recent thymic emigrants showed a trend towards a higher thymic $\mathrm{T}$ cell output during the late pubertal period in both patient groups compared other age groups (pre-transplantation group $\mathrm{p}=0.053$, post-transplantation group $\mathrm{p}=0.054$ ). Memory $\mathrm{T}$ cells showed a higher cell count during the post-pubertal stage, being CD8+ Temra in the pre-transplantation group $(\mathrm{p}=0.014)$ and $\mathrm{CD} 4+$ Temro in the healthy control group $(\mathrm{p}=0.027)$ compared to other age groups.

Conclusions: The current study shows a trend towards a more active immune system during the late pubertal and post-pubertal stage in transplant patients. These observations form an important starting point to further explore the possible causes of the poorer kidney transplant survival during late adolescence and early adulthood.

\section{PI-20 GROWTH AFTER PEDIATRIC KIDNEY TRANSPLANTATION: THE EFFECT OF PRETRANSPLANT RECOMBINANT GROWTH HORMONE AND POSTTRANSPLANT STEROID THERAPY}

Gilles Tourlamain ${ }^{1}$, Mandy G. Keijzer-veen ${ }^{2}$, Antonia H.m. Bouts ${ }^{3}$, Karlien Cransberg ${ }^{4}$, Huib De Jong ${ }^{4}$, Rani De Pauw ${ }^{1}$, 
Karen Vanderstraeten ${ }^{4}$, Amina El Amouri ${ }^{1}$, Ann Raes ${ }^{1}$, Noel Knops ${ }^{5}$, Agnieszka Prytula ${ }^{1}$

${ }^{1}$ Ghent University Hospital, Ghent, Belgium, ${ }^{2}$ Wilhelmina Childrens Hospital, University Medical Centre, Utrecht, The Netherlands, ${ }^{3}$ Emma Childrens Hospital, Amsterdam University Medical Centre, Amsterdam, The Netherlands, ${ }^{4}$ Sophia Childrens Hospital, Erasmus Medical Centre, Rotterdam, The Netherlands, ${ }^{5}$ Leuven University Hospitals, Leuven, Belgium

Introduction: We aimed to analyze the effect of pretransplant recombinant human growth hormone (rhGH) and corticosteroid withdrawal on longitudinal growth in the $1^{\text {st }}$ year after kidney transplantation $(\mathrm{kTx})$ and to identify factors associated with final height $(\mathrm{FH})$ in pediatric $\mathrm{kTx}$ recipients.

Material and methods: Patients who underwent kTx between 1996 and 2018 in 5 Belgian and Dutch centers before the age of 18 yrs were included in this retrospective study. We analyzed the differences between height SDS at kTx and 1 year post kTx (D height SDS) in children who were and were not treated with rhGH before $\mathrm{kTx}(\mathrm{rhGH}+$ and rhGH-) and those with and without corticosteroids at 1 year (CS+ and CS-). Univariate and multivariate linear regression analysis was performed to identify factors associated with height SDS at 1 year post kTx, D height SDS and FH SDS.

Results: 179 patients were included, with a median age at kTx 9.27 years. The median height SDS before kTx and 1 year after kTx in the CS-/ rhGH-, $\mathrm{CS}+/ \mathrm{rhGH}-, \mathrm{CS}-/ \mathrm{rhGH}+$ and $\mathrm{CS}+/ \mathrm{rhGH}+$ groups were respectively $-1.42 /-0.80,-0.90 /-0.62,-1.35 /-1.20$ and $-1.30 /-1.60(\mathrm{p}=0.001)$. Corticosteroid use at 1 year post $\mathrm{kTx}$ was the only factor associated with D height SDS on multivariate analysis $(\mathrm{p}=0.003)$. Median FH SDS, available in 124 patients, was -0.80 (range:-7.30-3.10). There was an association between $\mathrm{FH}$ and $\mathrm{D}$ height SDS at 1 year $(\mathrm{p}<0.001)$. Of 96 patients with height SDS $<-1$ at 1 year, 64 had FH $<-1$ SDS. Corticosteroid dose at 1 year was associated with $\mathrm{FH}$ on multivariate analysis $(\mathrm{p}=0.014$ ).

Conclusions: Growth in the first year post kTx was highest in the CS-/ rhGH- group and lowest in the $\mathrm{CS}+/ \mathrm{rhGH}+$ group. The use of corticosteroids at 1 year post $\mathrm{kTx}$ is the most important factor associated with growth in children in the $1^{\text {st }}$ year after kTx and their final height.

\section{PI-21 HOW LONG DOES PROTECTION BY HEPATITIS-B VACCINE LAST AFTER RENAL TRANSPLANTATION?}

Gökçen Erfidan ${ }^{1}$, Seçil Arslansoyu Çamlar ${ }^{2}$, Özgür Özdemir Șimșek ${ }^{1}$, Demet Alaygut ${ }^{1}$, Fatma Mutlubaș ${ }^{2}$, Dilek Yılmaz Çiftdoğan ${ }^{3}$, Belde Kasap Demir ${ }^{4}$

${ }^{1}$ University of Health Sciences, Tepecik Training and Research Hospital, Department of Pediatrics, Division of Nephrology, Izmir, Turkey, ${ }^{2}$ University of Health Sciences, Izmir Faculty of Medicine, Department of Pediatrics, Division of Nephrology, Izmir, Turkey, ${ }^{3}$ Izmir Katip Celebi University, Faculty of Medicine, Department of Pediatrics, Division of Infectious Diseases, Izmir, Turkey, ${ }^{4}$ Izmir Katip Celebi University, Faculty of Medicine, Department of Pediatrics, Division of Nephrology and Rheumatology, Izmir, Turkey

Introduction: Kidney transplant candidates and recipients have a high risk for infectious diseases which are preventable with vaccination. Monitoring the serology in pre- and post-transplant periods may guide during follow-up, as these patients vaccine responses have decreased due to existing renal failure and immunosuppressive treatments. The aim was to determine the course of Hepatitis-B titers in pediatric renal transplant recipients.

Material and methods: A retrospective analysis of the patients was performed in a single center. The Hepatitis-B surface antibody (Anti$\mathrm{HBs}$ ) titer $>10 \mathrm{mIU} / \mathrm{mL}$ was considered positive. The most recent pretransplant titer and the lowest post-transplant titer during follow-up were compared.
Results: A total of 30 patients were involved. Transplantation age was $9.5 \pm 4.4$ years, and current age was $15.5 \pm 5.8$ years. Male/female ratio was 2. Cadaveric transplantation was performed in $40 \%$ of them. There was a significant decrease in Anti-HBs titers after transplantation. No relationship was found between the Anti-HBs titers and primary disease/donor type. The ratio of positive serology was similar in pre- and post-transplant periods $(86.7 \%$ and $70 \%$, respectively). Pre-transplant positive patients $(\mathrm{n}=26)$ were divided into two groups as "titer $>100 \mathrm{mIU} / \mathrm{mL}$ " and "titer between 10 and $100 \mathrm{mIU} / \mathrm{mL}$ ". The second group had significantly lower titers after transplantation, while the first group had similar titers. Six $(23 \%)$ of pre-transplant positive 26 patients had became negative in a mean of $24 \pm 28$ months, and it took less than 5 years to become negative in $70 \%$ of them. Also, five patients had pre-transplant titers below $100 \mathrm{mIU} / \mathrm{mL}$. Pre-transplant Anti-HBs was negative in four patients $(13.3 \%)$. After Hepatitis-B vaccination, the Anti-HBs became positive in three of them. However, on follow-up, it became negative again in two patients.

Conclusions: After kidney transplantation, a decrease of Anti-HBs titers may be observed in recipients, especially in those with titers below 100 $\mathrm{mIU} / \mathrm{mL}$. Therefore, monitorization of the serologic responses, even after repetition of vaccine may be helpful.

\section{PI-22 RELATIONSHIP BETWEEN THE ESTIMATED GLOMERULAR FILTRATION RATE AT ONE MONTH AFTER TRANSPLANTATION AND THE CHANGE OF GRAFT FUNCTION IN PAEDIATRIC LIVING RENAL TRANSPLANT RECIPIENTS}

Junya Hashimoto ${ }^{1}$, Yuko Hamasaki ${ }^{1}$, Mai Kubota ${ }^{1}$, Yujiro Aoki ${ }^{1}$, Masaki Muramatsu ${ }^{1}$, Takeshi Kawamura ${ }^{1}$, Seiichiro Shishido ${ }^{2}$ Ken Sakai ${ }^{1}$

${ }^{I}$ Department of Nephrology, Toho University Faculty of Medicine, Japan, ${ }^{2}$ Department of Pediatric Nephrology, Toho University Faculty of Medicine, Japan

Introduction: This study was performed to evaluate the usefulness of the estimated glomerular filtration rate at one month after transplantation (eGFR-1M) in predicting the change of graft function in paediatric living renal transplant recipients.

Material and methods: The 43 paediatric recipients were classified as those with eGFR-1M $\geq 90 \mathrm{ml} / \mathrm{min} / \mathrm{m}^{2}(\mathrm{n}=19$; Group A) and those with eGFR-1M $<90(\mathrm{n}=24$; Group B). In Group A and B, changes in eGFR were evaluated for five years after renal transplantation retrospectively.

Results: The mean recipient age at transplantation in Group A / B was $6.1 \pm 3.4 / 7.8 \pm 4.0$ years $(P=0.14)$. In both groups, more than $50 \%$ of the recipients had congenital anomalies of the kidney and urinary tract (CAKUT) as their primary diseases. The mean eGFR1,12 and, $\left.60 \mathrm{M} \mathrm{(ml/min} / 1.73 \mathrm{~m}^{2}\right)$ in Group A / B were 106.8 \pm 2.99 / $78.5 \pm 1.52(P<0.0001), 79.3 \pm 3.22 / 62.7 \pm 2.38(P=0.0001)$, and 73.1 $\pm 4.16 / 59.2 \pm 2.79(P=0.006)$, respectively. During the observation period, the mean eGFR of Group A was always higher than Group B. Compared with Group A, the odds ratio for Group B to have an eGFR-60M $<60 \mathrm{ml} / \mathrm{min} / 1.73 \mathrm{~m}^{2}$ was 4.5 (CI, 1.04-19.7; $P=0.04$ ). The mean recipient body weight and body surface area ratio for recipient to donor at transplantation in Group A / B were 15.0 \pm 5.2 $/ 21.4 \pm 13.1(P=0.04)$ and $0.38 \pm 0.08 / 0.49 \pm 0.19(P=0.046)$, respectively. There were no significant differences in donor age, the number of recipients with CAKUT as primary disease, acute rejection and BK nephropathy between both groups.

Conclusions: The eGFR-1M could be useful in predicting the change of graft function in paediatric living renal transplant recipients. Our study also suggested an interesting finding that body size mismatch between donor and recipient could affect graft function up to five years after transplantation. 


\section{PI-23 PEDIATRIC ADPKD PROGRESSION STRATIFICATION MODEL BASED ON 3DUS VOLUMETRY}

Luc Breysem, Frederik De Keyzer, Stéphanie De Rechter, Chantal Van Ongeval, Djalila Mekahli

University Hospitals Leuven, Belgium

Introduction: Height-adjusted total kidney volume (htTKV) is a validated prognostic measure for adult ADPKD e.g. to categorize disease severity and progression according to the Mayo classification. Recently, our group has implemented a novel 3D ultrasound (3DUS) method to measure htTKV in pediatric ADPKD. We aim to develop a pediatric ADPKD imaging classification wherein 3DUS htTKV functions as a prognostic parameter.

Methods: Genetically-confirmed pedADPKD patients were evaluated between 2015-2020 in a tertiary hospital, and htTKV was assessed using 3DUS manual contouring. htTKV values were plotted according to patient's age. Slopes of renal growth were defined into five approximately equal-sized severity classes. The borders delineating the different slope areas were defined using $\mathrm{y}=\mathrm{A} *(1+\mathrm{B})^{\wedge} \mathrm{x}$ (applied from the MAYO classification), with "A" the starting htTKV at age 0 and "B" the yearly htTKV $\%$ increase.

Results: 67 genotyped patients ( 32 female, 35 male) underwent in total 232 3DUS assessments. Age (mean+/-stdev) at first evaluation was $10.5+/-4.8$ years (range: $0.2-19.2$ ), height $143+/-29 \mathrm{~cm}(59-198)$, and htTKV of $187+/-101 \mathrm{~mL} / \mathrm{m}$ (74-630). Extrapolating the MAYO curves $(\mathrm{A}=150 \mathrm{~mL} / \mathrm{m}, \mathrm{B}=1.5,3,4.5$ and $6 \% /$ year) below the age of 15 years strongly overestimates htTKV in children, even with correction for patient height. Therefore, we optimized renal growth cut-off lines with respective values $\mathrm{A}=70,80,90,100$ and $\mathrm{B}=6,7.5,9,10.5 \%$, splitting the 232 scans into 5 subgroups with $47,94,42,27$ and 22 patients, respectively. We demonstrated an accelerated growth after the age of 10 years comparing to the expected kidney growth in children.

Conclusion: Extrapolating htTKV cut-off growth curves of adults to pediatric patients, we developed an optimization of the previous adult model to a pediatric ADPKD population with 3DUS. This provides a tool to stratify disease severity and progression from a young age, which is a novelty in pediatric ADPKD.

\section{PI-24 DECREASED CD28 EXPRESSION IN MEMORY CD4+ T CELLS IN CHILDREN AWAITING KIDNEY TRANSPLANT IS ASSOCIATED WITH INCREASED EXPRESSION OF SENESCENCE MARKERS}

\author{
Charlotte Duneton ${ }^{2}$, Roshan George ${ }^{1}$, Mandy Ford ${ }^{1}$, \\ Pamela Winterberg ${ }^{1}$ \\ ${ }^{1}$ Emory University, Atlanta, USA, ${ }^{2}$ Robert Debré Hospital, Paris, France
}

Introduction: Despite improved patient and graft outcomes with CD28CD80/86 costimulation blockade, increased early acute rejection has hindered the widespread use of belatacept (CTLA-4Ig) for kidney transplant. Our group has previously reported lower pre-transplant frequencies of $\mathrm{CD} 28^{+} \mathrm{CD} 4^{+}$effector memory helper $\mathrm{T}$ cells $\left(\mathrm{CD} 4^{+} \mathrm{T}_{\mathrm{EM}}\right.$ : $\mathrm{CCR} 7^{-}$ $\mathrm{CD}^{2} 5 \mathrm{RA}^{-}$) with decreased functional capacity in adults that were subsequently free from early rejection on belatacept. We aimed to determine if a similar $\mathrm{T}$ cell phenotype is detectable in children awaiting kidney transplant.

Material and methods: We analyzed existing flow cytometry data of unstimulated blood cells collected from children on dialysis $(n=30)$ or healthy children $(n=18)$ and examined expression of markers of costimulation (CD28), senescence (CD57, PD1), activation (CD38) and cytotoxicity (Perforin, Granzyme B) on memory $\mathrm{CD}^{+} \mathrm{T}$ cells.

Results: None of the children had $\mathrm{CD} 28^{+} \mathrm{CD} 4^{+} \mathrm{T}_{\mathrm{EM}}$ frequencies as low as those we have previously observed in adults that were rejection-free on belatacept. However, 8 children on dialysis $(27 \%)$ had $\mathrm{CD} 28^{+} \mathrm{CD} 4^{+} \mathrm{T}_{\mathrm{EM}}$ frequencies (median 92.56\%, IQR 85.92-93,05) below the minimum value $(96 \%)$ observed in healthy children $(99.82 \%, 99.46-99.91 ; \mathrm{p}<0.0001)$. Patients with this "stable-like" $\mathrm{T}$ cell phenotype had higher frequency of $\mathrm{CD} 4^{+} \mathrm{T}_{\mathrm{EM}}$ cells bearing senescence markers $\left(\mathrm{CD} 57^{+} \mathrm{PD} 1^{+}, \mathrm{p}=0,011\right)$ and cytolytic effectors (Granzyme B, perforin, $\mathrm{p}=0,0004$ ) but decreased activation markers $\left(\mathrm{CD} 38^{+}, \mathrm{p}=0,003\right)$ compared to the rest of the dialysis patients.

Conclusions: Despite their young age and limited antigen experience, a subset of children on dialysis accumulate $\mathrm{CD}^{+} \mathrm{T}_{\mathrm{EM}}$ cells that have lost CD28 expression and bear markers suggestive of impaired function, a phenotype reminiscent of adults with decreased risk for early rejection on belatacept. The functional capacity of these cell populations in children needs further study.

\section{PI-25 EFFECT OF ELAMIPRETIDE (SS-31) ON INFLAMMASOM FORMATION IN MICE MODEL WITH OXALATE NEPHROPATHY}

Ismail Dursun ${ }^{1}$, Zuhal Hamurcu ${ }^{1}$, Serpil Taheri ${ }^{1}$, Esra Tufan ${ }^{1}$, Ecmel Mehmetbeyoglu ${ }^{1}$, Nesrin Delibas ${ }^{1}$, Zuleyha Doganyigit ${ }^{3}$, Aydin Alan ${ }^{4}$, Yurdun Kuyucu ${ }^{5}$, Sait Polat ${ }^{5}$, Gokmen Zararsız ${ }^{6}$, Hakan Poyrazoğlu ${ }^{2}$

${ }^{I}$ Betül-ziya Eren Genome And Stem Cell Center, Erciyes University, Kayseri, Turkey, ${ }^{2}$ Erciyes University Faculty of Medicine, Department of Pediatrics, Division of Nephrology, Turkey, ${ }^{3}$ Department of Histologyembryology, Faculty of Medicine, Yozgat Bozok University, Yozgat, Turkey, ${ }^{4}$ Department of Anatomy, Faculty of Veterinary Medicine, University of Erciyes, Kayseri, Turkey, ${ }^{5}$ Cukurova University Faculty of Medicine, Histology and Embryology Department, Adana, Turkey, ${ }^{6}$ Erciyes University, Department of Biostatistics, Kayseri, Turkey, ${ }^{7}$ Erciyes University, Faculty of Medicine, Department of Medical Biology, Kayseri, Turkey

Introduction: Inflammaoma complex (NOD-like receptor protein 3 (NRLP), apoptosis-associated speck-like protein (ASC), inactive butchipase-1) is a part of the innate immune system has recently been shown as the responsible mechanisms that lead to kidney damage in kidney stone disease. According to the one of the mechanisms responsible for this process, crystal structures such as oxalate enter the cell and cause mitochondrial damage. Then reactive oxygen species resulting from the damage cause the formation of the inflammasome complex. Elamipretide is a drug containing peptide in aromatic cationic structure and it has been shown that acute tubular necrosis prevents the development of chronic damage in the kidneys by reducing the formation of inflammasome. In this study, it was investigated whether the use of Elamipretide for the first time reduced the activation of the inflammatory pathway caused by oxalate crystals and the occurrence of kidney damage Material and methods: In this study, 40 C57BL6 mice at 10-12weeks of age were used ( 8 controls, 32 experimental groups). The study group was administered $200 \mathrm{mg} / \mathrm{kg}$ sodium oxalate intraperitoneally (IP) for 4 days. No application was made to the positive control group after the 5th day. Phosphate buffer saline was administered to the placebo group on days 0-9, and elamipretide at a dose of 5 and $7.5 \mathrm{mg} / \mathrm{kg} /$ day was administered via IP to the drug groups. On days of 0,5 and 10 , the mice were weighed and urinated. The experiment was terminated on the 10th day and the mice were bled and their kidneys removed. Serum kreatinine was measured. Histopathological evaluation was made in the kidney tissue. The mRNA expression levels of genes and proteins involved in the formation of the inflammasome complex were measured and protein levels were determined by western blot. This study supported by the Erciyes University Scientific Research Center (TOA-2018-7752) Results: When the mice were evaluated in terms of weight changes between the 0th and 5th days, weight gain was observed in the negative control group, while weight loss was detected in the other 
groups and the difference was statistically significant. Serum creatinine level was lower in negative control and elamipretide groups. It was observed that urine oxalate values on day 5 increased significantly in all groups compared to the negative control group $(\mathrm{p}=$ 0.037). Histologically, it was observed that kidney damage was less and mitochondrial damage was reduced in elamipretide groups. When the groups were compared in terms of IL-11, it was found that the gene expression in the placebo group was statistically significantly higher than the other groups and that caspas 1, caspas 9, caspas 11 and NLRP3 gene expression were significantly lower in the negative control group compared to the other groups. There was no significant difference in terms of caspas 1, caspas 9, caspas 11 and NLRP3 gene expression between treatment groups (plasebo $v s$ elamipretid).

Conclusions: In this study, it has been shown that elamipretidine reduces mitochondrial damage, and with increased caspas 1 and NLRP3 mRNA expressions, the inflammatory pathway is active in oxalate nephropathy, but elamipretide is not effective in suppressing this pathway

\section{PI-26 CHARACTERIZATION OF ACUTE KIDNEY INJURY IN THE SETTING OF ACUTE MYOCARDIAL INFARCTION IN MICE}

Nada J. Habeichi ${ }^{2}$, Jad Degheili ${ }^{4}$, Rana Ghali ${ }^{3}$, Sami Sanjad ${ }^{4}$, Mathias Mericskay ${ }^{1}$, Mohamed Khaled ${ }^{7}$, George W. Booz ${ }^{1}$, Fouad A. Zouein ${ }^{5}$, Bilal Aoun ${ }^{4}$

${ }^{1}$ American University of Beirut Medical Center, Lebanon, ${ }^{2}$ Inserm Department of Signaling and Cardiovascular Pathophysiology-umrs1180, University Paris-saclay, Châtenay-malabry, France, ${ }^{3}$ Institut National De La Sante Et De La Recherche Medicale (inserm), Unit 970, Paris Cardiovascular Research Center, 75015 Paris, France, ${ }^{4}$ Department of Pediatrics and Adolescent Medicine, Division of Pediatric Nephrology, American University of Beirut Faculty of Medicine, Beirut, Lebanon, ${ }^{5}$ Department of Pharmacology and Toxicology, American University of Beirut Faculty of Medicine, Beirut, Lebanon, ${ }^{6}$ Department of Pharmacology and Toxicology, School of Medicine, University of Mississippi Medical Center, Jackson, Ms, USA, ${ }^{7}$ Montfermeil Hospital, Department of Pediatrics and Adolescent Medecin, Montfermeil, France

Introduction: Cardiovascular diseases remain the leading cause of death worldwide. Epidemiological studies report that 4 out of 5 CVD related deaths are attributable to acute myocardial infarction (AMI) and ischemic diseases. Around $25 \%$ of patients with AMI progress not only to heart failure but also acute kidney injury (AKI) through the cardiorenal interrelationship, increasing mortality rate significantly.

Material and methods: In this study, we aimed at characterizing AKI as early as 4 and 7 days post-MI. 3 months old C57BLB/J male mice were enrolled in this study and divided into 2 groups: Control and MI. Mice were sacrificed 4 and 7 days post-MI. Kidney functional, structural, and molecular changes were evaluated.

Results: Cardiac hemodynamic analysis showed a significant decrease in ejection fraction (EF) and cardiac output (CO) at 4 and 7 days post-MI. EF dropped from $54.202 \pm 1.336 \%$ to $25.502 \pm 3.529 \%$ and from $56.289 \pm 1.481 \%$ to $30.774 \pm 2.371 \%$ post-MI at 4 and 7 days respectively. CO dropped from $0.914 \pm 0.044 \mathrm{ml} / \mathrm{min}$ to 0.450 $\pm 0.024 \mathrm{ml} / \mathrm{min}$ and $0.953 \pm 0.120 \mathrm{ml} / \mathrm{min}$ to $0.667 \pm 0.076 \mathrm{ml} / \mathrm{min}$ post-MI at 4 and 7 days respectively. Urine output, a marker of acute kidney injury substantially decreased from $2175 \pm 213.6 \mathrm{ml} /$ $24 \mathrm{~h}$ to $950 \pm 119.02 \mathrm{ml} / 24 \mathrm{~h}$ and from $2333 \pm 2.2 .78 \mathrm{ml} / 24 \mathrm{~h}$ to 1260 $\pm 136.38 \mathrm{ml} / 24 \mathrm{~h}$ post-MI at 4 and 7 days respectively. Kidney histological alterations characterized by proximal convoluted tubule
(PCT) dilatation and fibrosis markedly increased in MI mice. PCT dilatation increased from $699202.852 \pm 28622.352$ AU to $839509.844 \pm 44501.25 \mathrm{AU}$ and from $713231.66 \pm 24318.17 \mathrm{AU}$ to 952155.38 $\pm 38239.119 \mathrm{AU}$ post-MI at 4 and 7 days respectively. Renal fibrosis increased from $2.201 \pm 0.127 \%$ to $7.341 \pm 1.735 \%$ and from $1.621 \pm 0.119 \%$ to $4.367 \pm 0.571 \%$ post-MI at 4 and 7 days respectively. $\alpha$-smooth muscle actin ( $\alpha$-SMA), a well-known indicator of fibrosis, substantially increased in kidneys by $3.400 \pm 0.704$ and $2.852 \pm 0.836$ fold change post-MI at 4 and 7 days respectively. The pro-inflammatory cytokine interleukin- 1 significantly increased by $1.96 \pm 0.389$ fold change in the kidneys of mice at day 7 post-MI only

Conclusions: Our findings revealed enhanced AKI as early as 4 and 7 days post-MI. Further experiments are currently underway to decipher the molecular pathways involved in the observed damage.

\section{PI-27 NOVEL HUMAN PODOCYTE CELL MODEL CARRYING APOL1 G2/G2 HIGH-RISK GENOTYPE}

Oyindamola C. Adebayo ${ }^{1}$, Fanny O. Arcolino ${ }^{2}$, Pepe M. Ekulu ${ }^{3}$, Jean-paul Decuypere ${ }^{2}$, Linda Bellucci ${ }^{4}$, Mohamed A. Elmonem ${ }^{5}$, Agathe B. Nkoy ${ }^{2}$, Djalila Mekhali ${ }^{2}$, Benedetta Bussolati ${ }^{4}$, Lambertus P. Van Den Heuvel ${ }^{2}$, Elena N. Levtchenko ${ }^{2}$

${ }^{1}$ Center for Molecular and Vascular Biology, Department of Cardiovascular Sciences, Kathiolieke Universiteit Leuven, Leuven, Belgium, ${ }^{2}$ Department of Development and Regeneration, Kathiolieke Universiteit Leuven, Leuven, Belgium, ${ }^{3}$ Division of Nephrology, Department of Pediatrics, University Hospital of Kinshasa, Faculty of Medicine, University of Kinshasa, Democratic Republic Of Congo, ${ }^{4}$ Department of Molecular Biotechnology and Health Sciences, University of Turin, Turin, Italy, ${ }^{5}$ Department of Clinical and Chemical Pathology, Faculty of Medicine, Cairo University, Cairo, Egypt

Introduction: Apolipoprotein L1 (APOL1) variants, G1 and G2, increase the risk of various non-diabetic kidney diseases in the African population. To date, the precise mechanisms by which $A P O L 1$ risk variants induce injury on podocytes and other kidney cells remain unclear. Trying to unravel these mechanisms, most studies used animal or cell models created by gene editing.

Material and methods: We developed conditionally immortalized human podocyte cell lines from urine of a donor carrying APOL1 high-risk genotype (HRG), G2/G2. As control, APOL1 wild type $(\mathrm{G} 0 / \mathrm{G} 0)$ podocytes previously generated from a Caucasian donor were used. The APOL1 G2/G2 cell lines were characterized for podocyte markers at both the mRNA and the protein levels, using real-time quantitative PCR and Western blot, respectively. APOL1 expression was induced upon exposure to $50 \mu \mathrm{g} / \mathrm{mL}$ polyinosinicpolycytidylic acid (poly(I:C)). Validation of the functional features of APOL1-induced podocyte dysfunction such as cell detachment, cell viability, cell death, autophagy, cytoskeleton organization and podocyte permeability were assessed.

Results: We successfully generated for the first time APOL1 G2/G2 podocyte cell lines isolated from urine of a human donor carrying APOL1 high-risk genotype (HRG). Upon exposure to poly(I:C), G2/G2 and $\mathrm{G} 0 / \mathrm{G} 0$ podocytes upregulated APOL1 expression regardless of the APOL1 genotype. This resulted in podocytes detachment, decreased cells viability and increased apoptosis rate in a variant-independent manner. Nevertheless, G2/G2 podocyte cell lines exhibited altered features, including upregulation of synaptopodin, alteration of cytoskeleton, reduction of autophagic flux and increased permeability in an in vitro model under continuous perfusion.

Conclusions: The human APOL1 G2/G2 podocyte cell model is a useful tool for unraveling the mechanisms of APOL1-induced podocyte injury and the cellular functions of APOL1. 
PI-28 COMPARISON OF CELL BIOLOGICAL RESPONSES AFTER SHIGA TOXIN-1 EXPOSURE TO PRIMARY HUMAN GLOMERULAR MICROVASCULAR ENDOTHELIAL CELLS FROM PEDIATRIC AND ADULT ORIGIN

Wouter J.c. Feitz ${ }^{1}$, Petra A. Van Setten ${ }^{1}$, Thea J.a.m. Van Der Velden ${ }^{1}$, Christoph Licht ${ }^{2}$, Lambert P.j.w. Van Den Heuvel ${ }^{1}$, Nicole C.a.j. Van De Kar ${ }^{1}$

${ }^{1}$ Amalia Childrens Hospital, Radboudumc, Nijmegen, The Netherlands;

${ }^{2}$ The Hospital For Sick Children, Toronto, On, Canada

Introduction: The hemolytic uremic syndrome (HUS) is characterized by a triad of symptoms consisting of hemolytic anemia, thrombocytopenia and acute renal failure. The most common form of HUS is caused by an infection with Shiga toxin (Stx) producing Escherichia coli bacteria (STEC-HUS), and the kidneys are the major organs affected. The development of HUS after an infection with Stx occurs most frequently in children under the age of 5 years. However, the cause for the higher incidence of STEC-HUS in children compared to adults is still not well understood.

Material and methods: In this study, primary human glomerular microvascular endothelial cells (HGMVECs) isolated and cultured from pediatric and adult kidney tissue were investigated with respect to Stx binding and different cellular responses.

Results: Shiga toxin type 1 (Stx-1) inhibited protein synthesis in both pediatric and adult HGMVECs in a dose-dependent manner. Preincubation with TNF $\alpha$ resulted in increased Stx binding and a 20$40 \%$ increase in protein synthesis inhibition in both age groups. Decreased proliferation of cells was found when a bromodeoxyuridine (BrdU) assay was performed. A trend towards a delay in endothelial wound closure was visible when HGMVECs were incubated with Stx1. Although minor variations between pediatric HGMVECs and adult HGMVECs were found in this study, it was not significant.

Conclusions: In conclusion, in vitro primary HGMVECs isolated from pediatric and adult kidneys do not differ in their cell biological responses to Stx-1. With this in mind, we hypothesize that other extrinsic or genetic factors contribute to the sensitivity of the glomerular endothelium and the pathophysiology of STEC-HUS in young children.

\section{PI-29 UROMODULIN AND VESICO-URETERAL REFLUX. A GENETIC STUDY}

Silvio Maringhini ${ }^{1}$, Rosa Cusumano ${ }^{2}$, Ciro Corrado ${ }^{2}$, Giovanni Pavone ${ }^{2}$, Maria Michela Dalessandro ${ }^{2}$, Maria Chiara Sapia ${ }^{2}$, Serena Abbate ${ }^{1}$

${ }^{1}$ Dept Pediatrics, Ismett, Palermo, Italy, ${ }^{2}$ Ped Nephrology Unit, Childrens Hospital, Arnas Civico, Palermo, Italy

Objectives: Vesico-ureteral reflux (VUR) is associated with urinary tract infections (UTI) and renal scars. The renal damage is correlated with the grade of reflux and the number of UTI, butother factors may have a role. Uromodulin (UMOD) is a protein produced by the renal tubular cells. Aim of our study is to evaluate if some UMOD genes are associated with febrile UTI (FUTI) and/or scars in children with VUR.

Methods: Inclusion criteria: children with documented VUR, DMSA study, normal eGFR and blood pressure and no other urinary tract malformation. Age, sex, grade of VUR, DMSA report, number of FUTI, were registered. Reflux grade (5 degree) was calculated as the mean of both ureters. Genotyping of rs 4293393 was done on DNA extracted from white blood cells using PCR technique.

Results: A total of 31 patients with VUR were enrolled. Renal scars were detected in 16 children (age range 20-196, mean 89 months): 9 males and 7 females; reflux was grade 2.8 in both sexes, bilateral in 8 ; FUTI were 3.3 per patient. No scar was detected in 15 children (age range 6-170, mean 57 months): 8 males and 7 females; reflux grade was 2.3 in males and 2.2 in females, bilateral in 7; FUTI were 1.9 per patient.
Genotype rs4293393 TC (TC) was present in 10 pts, 9 (90\%) had scars; genotype rs4293393 TT (TT), the most frequent in the general population, was found in 21 patients, 7 (33\%) had scars. Among children with scars, those with TC compared to those with TT were younger (mean age 77 vs 101 months), their reflux grade was lower $(2,5$ vs 3,6), the number of FUTI was lower (2.9 vs 3.7 per patient).

Conclusions: Children with VUR carrying UMOD genotype rs4293393 TC are more prone to develop renal scars independently from FUTI.

\section{PI-30 PERSISTENT HEMATURIA DUE TO SCHISTOSOMA HAEMATOBIUM. AN IMPORTED PATHOLOGY WITH DIFFICULT TREATMENT}

Elena Codina Sampera, Pedro Arango Sancho, Yolanda Calzada Baños, Víctor López-Baez, Ana Vinuesa Jaca, Lina Catherine Hernández Zúñiga, Álvaro Madrid Aris

Hospital Sant Joan De Déu, Barcelona, Spain

Objectives: Bladder schistosomiasis is a rare cause of hematuria. We present three patients with different evolution, highlighting the importance of this pathology in the differential diagnosis of persistent hematuria.

Methods: Case 1: A 9-year-old male from Mali with macrohematuria for 4 months. The physical examination and the renal ultrasound were normal with a negative urine culture. The urine test showed hematuria, leukocyturia and proteinuria. Blood tests just showed anemia. In the microbiological urine study, Schistosoma haematobium (SH) eggs were observed. He received three doses of Praziquantel. One month later, he still had microhematuria and SH eggs in the urine, so another praziquantel cycle was repeated.

Case 2: A 10-year-old male from Guinea. He presented intermittent episodes of macrohematuria with hypogastric pain. She received antibiotic therapy for suspected acute cystitis. He had hematuria, leukocyturia, and moderate proteinuria. Renal ultrasound showed a bladder with thickened walls and inflammatory appearance, compatible with cystitis and distal ureteritis. The microbiological study showed eggs of $\mathrm{SH}$. He received three doses of Praziquantel. 3 weeks later he had a normal urine, but SH eggs persisted, so the treatment was repeated.

Case 3: A 11-year-old male from Senegal. In Spain for 3 months. He presented a 1-month of macroscopic non-painful hematuria. The urine test showed proteinuria in the nephrotic range, hematuria and leukocyturia. The renal ultrasound showed a thinned bladder without calcifications. In the urine we found SH eggs. He received three doses of praziquantel with urinary normalization in 2 weeks.

Results and Conclusions: SH has a special tropism for the perivesical plexuses. In chronically affected patients, the eggs may persist after treatment, requiring an other cycle of praziquantel at 4 weeks. If the clinic persists, we must rule out scar lesions or subsidiary granulomas of surgical resection.

\section{PI-31 THE WEIGHTED COMBINATION OF CYSTATIN C AND CREATININE EQUATIONS BROUGHT INTO PRACTICE}

Tobias Oostdam ${ }^{1}$, Emil Den Bakker ${ }^{1}$, Kajsa Åsling-monemi ${ }^{8}$, Ulla Berg ${ }^{8}$, Jonas Björk ${ }^{3}$, Karolien Goffin ${ }^{2}$, Anders Grubb ${ }^{5}$, Magnus Hansson ${ }^{7}$, Karin Littmann ${ }^{7}$, Ulf Nyman ${ }^{6}$, Arend Bökenkamp ${ }^{1}$ ${ }^{1}$ Emma Children's Hospital, Amsterdam Umc, Vrije Universiteit Amsterdam, Amsterdam, The Netherlands, ${ }^{2}$ Department of Nuclear Medicine \& Molecular Imaging, University Hospital Leuven, Katholieke Universiteit Leuven, Leuven, Belgium, ${ }^{3}$ Division of Occupational and Environmental Medicine, Lund University, Lund, Sweden ${ }^{4}$ Clinical Studies Sweden, Forum South, Skåne University Hospital, Lund University, Lund, Sweden, ${ }^{5}$ Department of Clinical Chemistry, Skåne University Hospital, Lund University, Lund, Sweden, ${ }^{6}$ 
Department of Translational Medicine, Division of Medical Radiology, Lund University, Malmö, Sweden, ${ }^{7}$ Department of Laboratory Medicine, Division of Clinical Chemistry, Karolinska Institutet, Karolinska University Hospital Huddinge, Stockholm, Sweden, ${ }^{8}$ Karolinska Institutet, Karolinska University Hospital Huddinge, Stockholm, Sweden

Background: The mean of a creatinine-based and a cystatin C-based GFR estimation (eGFR) has higher accuracy than either of the two. If the difference between the creatinine-based and the cystatin Cbased eGFR exceeds $40 \%$, however, it has been suggested that the relative contribution of the equations should be adapted based on underlying diagnosis ("Lund approach"). In this approach, $\alpha$ describes the contribution of the creatinine-based eGFR, $(1-\alpha)$ the contribution of cystatin $\mathrm{C}$.

Methods: Retrospective analysis of 1712 single plasma clearance GFR measurements from four pediatric nephrology centers. The 2 most common eGFR equation systems (i.e. Full Age Spectrum [FAS] and CKiD [bedside Schwartz-creatinine with Lyon modification in adolescent males and CKiD cystatine]) were studied. $2 / 3$ of the cohort (mean age 11.8 years, GFR $93.8 \mathrm{ml} / \mathrm{min} / 1.73 \mathrm{~m}^{2}$ ) was used to determine the $\alpha$-values yielding the highest $\mathrm{P}_{30}$ accuracy globally $\left(\mathrm{FAS}_{\alpha}\right.$ and $\left.C \mathrm{CKD}_{\alpha}\right)$ as well as in diagnosis subgroups. These $\alpha$-values were validated in the remaining $1 / 3$ of the cohort. The "Lund approach" was applied to the FAS ${ }_{\alpha}$ and $\mathrm{CKiD}_{\alpha}$ to assess clinical applicability.

Results: The optimal $\alpha$-values were determined as 0.3 for FAS and 0.4 for CKiD. $\mathrm{P}_{30}$ accuracy for $\mathrm{FAS}_{0.3}$ and $\mathrm{CKiD}_{0.4}$ in the validation cohort was $90.2 \%$ and $91.8 \%$, respectively. While $\mathrm{P}_{30}$ accuracy of $\mathrm{FAS}_{0.3}$ was significantly higher than $\mathrm{FAS}_{0.5}(90.2$ vs. $87.4 \%, \mathrm{p}<0.05)$, there was little difference between $\mathrm{CKiD}_{0.5}$ and $\mathrm{CKiD}_{0.4}$ (91.2 vs. $91.8 \%$ ). The Lund approach using disease specific $\alpha$-values improved accuracy slightly to $90.7 \%\left(\mathrm{FAS}_{0.3}\right)$ and $92.5 \%\left(\mathrm{CKiD}_{0.4}\right)$, respectively. This was only relevant in patients with neuromuscular diseases and spina bifida, where accuracy increased from 60 to $72 \%$ (FAS) and 56 to $72 \%$ (CKiD).

Conclusions: For calculation of the weighted mean, $\mathrm{FAS}_{0.3}$ and $\mathrm{CKiD}_{0.4}$ are the optimal mix but $\mathrm{CKiD}_{0.5}$ performs equally well. A disease-specific adaptation (i.e. $100 \%$ cystatin C eGFR) is clinically relevant only for patients with neuromuscular diseases and spina bifida.

\section{PI-32 MODELLING OF RENAL FUNCTION IN FORMER EXTREMELY LOW BIRTHWEIGHT INFANTS IN LATE CHILDHOOD}

Marieke Colleman ${ }^{1}$, Elena Levtchenko ${ }^{1}$, Zhenyu Zhang ${ }^{2}$, Jan A. Staessen ${ }^{3}$, Jan A. Staessen ${ }^{4}$, Karel Allegaert ${ }^{1}$, Karel Allegaert ${ }^{6}$, Karel Allegaert ${ }^{5}$, Anke Raaijmakers ${ }^{7}$

${ }^{1}$ Department Of Development And Regeneration, Katholieke Universiteit Leuven, Belgium, ${ }^{2}$ Studies Coordinating Centre, Research Unit Hypertension And Cardiovascular Epidemiology, Department Of Cardiovascular Sciences, Katholieke Universiteit Leuven, Belgium, ${ }^{3}$ Biomedical Science Group, Faculty Of Medicine, Katholieke Universiteit Leuven, Belgium, ${ }^{4}$ Research Institute Alliance For The Promotion Of Preventive Medicine, Mechelen, Belgium, ${ }^{5}$ Department Of Pharmaceutical And Pharmacological Sciences, Katholieke Universiteit Leuven, Belgium, ${ }^{6}$ Department Of Clinical Pharmacy, Erasmus Mc, Rotterdam, The Netherlands, ${ }^{7}$ Department Of Pediatrics, Campus Jan Palfijn, Zna Hospitals, Antwerp, Belgium

Objectives: Extremely low birthweight (ELBW) infants suffer from poorer outcomes in later life, including lower estimated glomerular filtration rate (eGFR). We aim to identify the perinatal factors influencing these outcomes, as previously reported for neurodevelopmental outcome, enabling targeted renal follow-up.

Methods: As part of the PREMATCH study, we evaluated and compared eGFR in 59 ELBW children and 71 controls at age 11 . Adverse renal outcome was defined as presence of chronic kidney disease (CKD) stage 2 (eGFR $\left.<90 \mathrm{ml} / 1.73 \mathrm{~m}^{2} / \mathrm{min}\right)$. We investigated perinatal factors associated with this adverse renal outcome to develop a proof-of-concept model to predict the proportional risk of CKD stage 2 in ELBW children.

Results: We confirmed that ELBW children suffer from lower average eGFR than controls $\left(94.1\right.$ vs. $106.5 \mathrm{ml} / 1.73 \mathrm{~m}^{2} / \mathrm{min}$, $p<0.001)$. Amongst ELBW children $34.5 \%$ progressed to CKD stage 2 . ELBW children with CKD stage 2 were more frequently male $(75 \%$ vs. $47.7 \%, p=0.055)$, were ventilated significantly longer post-birth (17 vs. 9 days, $p=0.006)$ and suffered more commonly from intraventricular hemorrhage (IVH) (40\% vs. $15.8 \%$, $p=0.056)$. In contrast, birthweight, gestational age, steroids/ ibuprofen use, duration of oxygen therapy, retinopathy of prematurity and bronchopulmonary dysplasia showed no significant association to renal function in adolescence. In our model, we developed a score (0-3) to determine the risk of CKD stage 2 based on the cumulative perinatal risk factors sex (male), ventilation duration ( $>10$ days), and IVH. We found this risk to increase linearly from $0 \%$ to $80 \%$, delineated as follows - 0 points: $0 \%$; 1 point: $25 \%$; 2 points: $47 \%$; and 3 points: $80 \%$.

Conclusions: ELBW children have poorer renal function and are at increased risk to develop CKD stage 2 by age 11. Renal function in ELBW children is influenced by identifiable perinatal factors, which can be scored to predict the occurrence of CKD stage 2, facilitating targeted follow-up.

\section{PI-33 SAFETY AND COMPLICATIONS OF KIDNEY BIOPSIES IN CHILDREN: A SINGLE CENTRE STUDY FROM SWEDEN}

Mia Dursun, Per Brandström, Sverker Hansson

Institute of Clinical Sciences, Sahlgrenska Academy, University of Gothenburg, Sweden

Introduction: Percutaneous kidney biopsy is considered a safe procedure in the diagnosis and management of paediatric kidney disease. We aimed to study complications to percutaneous kidney biopsies made in children in our centre and to target potential risk factors to the procedure.

Material and methods: We retrospectively studied kidney biopsies performed during a five-year period in the Swedish regional centre of Queen Silvia Children's Hospital. Our study included both native and transplanted kidneys, with exclusion of oncological biopsies. All biopsies were performed by a paediatric radiologist under general anaesthesia using ultrasound-guidance. Most of the elective biopsies followed a protocol enabling discharge after six to seven hours of observation. Complications were divided into minor, moderate and major events, where minor complications were classified as events not in need of further investigation nor intervention.

Results: 232 biopsies were performed in 198 individuals, 116 (59\%) boys and $82(41 \%)$ girls. The median age was $10.8(1.4-18.4)$ years. Nephrotic proteinuria or minimal change disease was the most common indication, followed by non-nephrotic proteinuria or haematuria and investigation of kidney transplants. One major complication with the need of blood transfusion occurred. The complication was identified as a large renal hematoma during the procedure. We found the most common complications being minor complications, involving minor reduction of haemoglobin, renal hematomas and transient macroscopic haematuria. Identification of $75 \%$ of complications was made by $<6$ hours and $95 \%$ by $<12$ hours. Increased number of needle passes and low diastolic blood pressure was significantly associated with moderate to major complications.

Conclusions: Kidney biopsy in children is a low-risk procedure. The number of needle passes and low diastolic blood pressure were associated with the risk for more serious complications in children. 


\section{PI-34 A KINETICS BASED ALGORITHM TO TREAT ACUTE} NEONATAL HYPERAMMONEMIA

Snauwaert Evelien, Derudder Jonathan, Verloo Patrick, Dhont Evelyn, Raes Ann, Van Biesen Wim, Eloot Sunny

Ghent University Hospital, Belgium

Introduction: Acute neonatal hyperammonemia is associated with poor neurological outcomes and high mortality, in which decrease in serum ammonia is crucial. We developed, based on kinetic modeling, a widely applicable algorithm to treat acute neonatal hyperammonemia.

Material and methods: 4 hyperammonemic patients $(3.24 \pm 0.40 \mathrm{~kg})$ underwent 13 hemodialyses, i.e. 5 with the 4008 and FXPaed dialyzer (Fresenius Medical Care, Germany), and 8 with the CarpeDiem (Medtronic, USA) of which 4 with the $0.15 \mathrm{~m}^{2}$ and 4 with the $0.25 \mathrm{~m}^{2}$ dialyzer. Blood flows $\mathrm{Q}_{\mathrm{B}}$ were $22-35 \mathrm{~mL} / \mathrm{min}$. Dialyzer clearance and extraction ratio were derived from the measured ammonia time-concentration curves during dialysis. Ammonia was hereby assumed being distributed in the patient in a single compartment, equal to patient's total body water. Timeconcentration profiles were simulated for different start concentrations, patient weight, dialysis machines/dialyzers and different dialysis settings. To make the model widely beneficial, an algorithm was drawn to guide clinicians.

Results: Extraction ratios were $38 \pm 5 \%$ (4008/FXPaed), $10 \pm 3 \%-13$ $\pm 3 \%$ (CarpeDiem $/ 0.15-0.25 \mathrm{~m}^{2}$ ). For a start concentration of e.g. $3000 \mu \mathrm{mol} / \mathrm{L}$ in a $3 \mathrm{~kg}$ patient, time to reach $400 \mu \mathrm{mol} / \mathrm{L}$ was $315 \mathrm{~min}$ (QB30) and 190min (QB50) with 4008/FXPaed. The CarpeDiem machine could not decrease ammonia concentrations below $400 \mu \mathrm{mol} / \mathrm{L}$ within $4 \mathrm{~h}$ for start concentrations $>800 \mu \mathrm{mol} / \mathrm{L}$. Simulations with CarpeDiem or $5 \mathrm{~kg}$ patient resulted in longer time intervals. Our institution-specific protocol therefore prescribes 4008/FXPaed for start concentrations $>400 \mu \mathrm{mol} / \mathrm{L}$. To calculate the time needed to decrease start ammonia concentration $\mathrm{C}_{\text {start }}$ to target $\mathrm{C}_{\text {target }}$, the following algorithm can be used: from patient's total body water (V) (Wells formula) and dialyzer clearance $(\mathrm{K})$ (by single measurement of ammonia at dialyzer inlet and outlet), time (T) to target $\mathrm{T}=(-\mathrm{V} / \mathrm{K}) \cdot \mathrm{LN}\left(\mathrm{C}_{\text {target }} / \mathrm{C}_{\text {start }}\right)$.

Conclusions: By implementing these formulae in a simple spreadsheet, medical staff can draw its institution-specific flowchart for patienttailored treatment of hyperammonemia. Due to some made assumptions in kinetics, our own institution-specific protocol will be applied and studied in a prospective study to validate the present results.

\section{PI-35 POSTNATAL MATURATION OF GLOMERULAR FILTRATION RATE IN TERM BORN NEONATES: AN INDIVIDUAL PARTICIPANT DATA META-ANALYSIS.}

Nori Smeets ${ }^{1}$, Joanna Inthout ${ }^{1}$, Maurice Van Der Burgh ${ }^{1}$, George Schwartz ${ }^{2}$, Michiel Schreuder ${ }^{1}$, Saskia De Wildt ${ }^{1}$

${ }^{1}$ Radboud University Medical Center, The Netherlands, ${ }^{2}$ University of Rochester School of Medicine, The Netherlands

Introduction: The evidence from individual studies to support the maturational pattern of measured glomerular filtration rate (GFR) in healthy term born neonates is inconclusive. This hampers the delineation between normal and abnormal kidney development as well as the diagnosis of acute kidney injury (AKI). Thus, we aimed to describe GFR maturation in the first month of life using an individual participant data meta-analysis (IPDMA) of measured GFR data.

Material and methods: The Pubmed and ClinicalTrials.gov databases were searched to identify studies reporting mGFR as measured by exogenous markers or creatinine clearance (CrCL) in healthy term born neonates. Articles were subsequently reviewed using Rayyan by two individual researchers. The relationship between postnatal age and clearance was investigated using penalized splines with generalized additive linear mixed models on individual data, taking into account clustering by study. Data from aggregated results were simulated to exactly reflect the reported mean/median and SD.
Results: 1521 articles were screened and 50 relevant studies reported mGFR in healthy term born neonates. In total, 1055 measured GFR values from 958 neonates were included. IPD were available for 371 neonates and 587 neonates were represented by 46 summarizing datapoints as means/medians per cohort. On average, GFR increases rapidly in the first five days after birth from a value of 20.1 (95\% CI $16.1-24.1) \mathrm{ml} / \mathrm{min} / 1.73 \mathrm{~m} 2$ at the first day to 37.6 at day 5 (95\% CI 34.2-40.9), followed by a more steady increase to 57.0 (95\% CI $41.1-72.9) \mathrm{ml} / \mathrm{min} / 1.73 \mathrm{~m} 2$ at end of the fourth week.

Conclusions: These normative values show a clear developmental pattern of GFR maturation in the first weeks of life and indicate a biphasic increase with the largest increase until day 5. Our IPDMA data can therefore serve as a useful baseline for neonatal GFR.

\section{PI-36 EFFECT OF CHIMERIC ANTIGEN RECEPTOR (CAR) T CELL THERAPY ON RENAL FUNCTION OF PEDIATRIC PATIENTS: PRELIMINARY RESULTS}

Andrea Cappoli ${ }^{1}$, Francesca Del Bufalo ${ }^{2}$, Isabella Guzzo ${ }^{1}$, Franco Locatelli ${ }^{2}$, Marina Vivarelli ${ }^{1}$

${ }^{1}$ Division of Nephrology and Dialysis, Department of Pediatric Subspecialties, Irccs Bambino Gesù Pediatric Hospital, Rome, Italy, ${ }^{2}$ Department of Pediatric Hemato-oncology and Cell and Gene Therapy, Irccs Bambino Gesù Pediatric Hospital, Rome, Italy

Introduction: Cancer immunotherapies are rapidly emerging in pediatric oncology as revolutionary approaches. However, their use may entail unique and severe toxicities that require expert management. Specifically, after chimeric antigen receptor (CAR) T-cell therapy, cytokine release syndrome (CRS) can occur in 30\%-80\% of patients and lead to acute kidney injury (AKI), owing to direct inflammatory injury, capillary leak syndrome, hypoperfusion and shock.

Material and methods: We analyzed the effect of CAR T-cell treatment on renal function in 22 children affected by neuroblastoma and treated at the Pediatric Oncology Department of our Hospital, at day $0,+3,+7,+10,+14,+21$ and +28 post-infusion. Creatinine clearance $(\mathrm{ClCr})$ was estimated using the Schwartz modified formula for children and the Cockroft-Gault formula for two patients who had just turned 18-year old. Blood pressure, fever and CRS signs and symptoms were recorded. All patients received the same lymphodepletion based on the combination of low-dose Cyclophosphamide and Fludarabine

Results: At baseline, $3 / 22$ patients had $\mathrm{ClCr}$ between $60-90 \mathrm{ml} / \mathrm{min} / 1.73 \mathrm{~m}^{2}$, while $19 / 22 \mathrm{had}>90 \mathrm{ml} / \mathrm{min} / 1.73 \mathrm{~m}^{2}$. In the 28 days following CAR T-cell treatment, we observed no change in renal function in 15/22 patients. The three children with a baseline reduction had an improvement at day +28 . In detail, two patients had $\mathrm{ClCr}>90 \mathrm{ml} / \mathrm{min} / 1.73 \mathrm{~m}^{2}$, while one child increased from 53 to $70 \mathrm{ml} / \mathrm{min} / 1.73 \mathrm{~m}^{2}$. In the remaining four, we observed a transient reduction of $\mathrm{ClCr}$. However, they all completely recovered by day 28 . CRS was observed in 16/22 patients (72\%) and severe CRS (grade 3 and 4), which required use of inotropes, in two of them (12.5\%). All patients who developed AKI presented CRS, but AKI did not correlate with CRS severity.

Conclusions: Following CAR T-cell therapy, cytokine release syndrome is frequently observed. However, with appropriate management, AKI can be avoided in the vast majority of children.

PI-37 ESCHERICHIA COLI O55:H7 ASSOCIATED HAEMOLYTIC URAEMIC SYNDROME (HUS): CASE SERIES FROM THE FIRST DOCUMENTED O55:H7 OUTBREAK IN THE UNITED KINGDOM (UK)

Maduri Raja, Mushfequr R Haq, Arvind Nagra, Rodney D Gilbert Department Of Paediatric Nephrology, Southampton Children's Hospital, University Hospital, Southampton Nhs Foundation Trust, Tremona Road, Southampton Sol6 6yd, United Kingdom 
Introduction: Shiga Toxin-producing Escherichia coli (STEC) are a well-established cause of haemolytic uraemic syndrome (HUS) causing acute kidney injury in children. In the UK, the majority of cases of STEC HUS are caused by serotype O157:H7. This series describes 5-year outcomes of ten children with HUS during England's first documented STEC O55:H7 outbreak.

Material and methods: Retrospective review of ten children with HUS following STEC O55:H7 infection diagnosed between July 2014 and September 2015. Acute clinical course, duration of renal replacement therapy (RRT) and extra-renal involvement were reviewed. 12-month and 5-year follow-up data (kidney function, proteinuria, antihypertensive requirement and neurodevelopmental outcomes) were analysed using descriptive statistics and compared to an O15:H7 HUS case cohort.

Results: Median age at presentation was 3 years; $60 \%$ were male. 9 (90\%) had bloody diarrhoea. STEC O55:H7 serology positive in 5 $(50 \%)$; Stool culture positive for STEC O55:H7 in $9(90 \%)$. Mean acute dialysis duration was 17 days with 9 children requiring more than 10 days of RRT. Neurological involvement was seen in 7 (70\%); 3 (30\%) had cardiac involvement.

At 12-month follow up, 6 (60\%) were on a single antihypertensive agent; $8(80 \%)$ had raised urine albumin/creatinine ratio; the median formal GFR of the O55:H7 cohort was significantly lower $(50-92 \mathrm{ml} / \mathrm{min} /$ $1.73 \mathrm{~m}^{2}$ ) than that of the STEC O157:H7 HUS group. At 5 years postHUS, $50 \%$ of the O55:H7 cohort had formal GFRs corresponding to CKD stage $2\left(\mathrm{G}^{3} \mathrm{a}^{1} / \mathrm{G} 2\right)$.

Conclusions: From this series it appears that HUS secondary to infection with E. coli O55 tends to be more severe than with O157. Patients spent longer on dialysis, had more severe renal sequelae and had a higher incidence of extra-renal manifestations. With E. coli strains other than O157 increasing in global incidence, it is important that microbiology laboratories are set up to find all Shiga Toxin producing organisms.

\section{PI-38 PEDIATRIC CASES DIAGNOSED WITH DRUG- RELATED ACUTE TUBULOINTERSTITIAL NEPHRITIS: A SINGLE CENTER EXPERIENCE}

Özgür Özdemir Şimșek ${ }^{1}$, Gökçen Erfidan ${ }^{1}$, Eren Soyaltin ${ }^{1}$, Seçil Arslansoyu Çamlar ${ }^{2}$, Fatma Mutlubaș ${ }^{2}$, Belde Kasap Demir ${ }^{3}$, Demet Alaygut ${ }^{1}$

${ }^{1}$ Izmir University of Health Sciences Tepecik Training and Research Hospital Department of Pediatric Nephrology, Turkey, ${ }^{2}$ University of Health Sciences Izmir Faculty of Medicine Department of Pediatrics Division of Nephrology, Turkey, ${ }^{3}$ Katip Celebi University Faculty of Medicine Department of Pediatric Nephrology and Rheumatology, Turkey

Introduction: Tubulointerstitial nephritis (TIN) is a frequent cause of acute kidney injury (AKI). TIN has multiple etiologies, including drugrelated, infectious, systemic, autoimmune, genetic, and idiopathic. In this study, drug-related TIN cases were evaluated.

Material and methods: Pediatric cases who applied to the Pediatric Nephrology Clinic of Health Sciences University Tepecik Training and Research Hospital between 2010 and 2020 and were diagnosed with drug-related acute tubulointerstitial nephritis were retrospectively evaluated. Demographic, clinical and laboratory data were obtained from the file records.

Results: Data of a total of 29 (19 girls) pediatric patients were evaluated. Mean age at diagnosis was $126.7 \pm 89.1$ (103.3-217.8) months. When the symptoms at presentation were examined, it was found that $12(41.4 \%)$ patients presented with the complaint of nausea and vomiting, one patient had arthralgia, 4 patients had high fever, and 1 patient had a rash. In the laboratory evaluation, $25(86.2 \%)$ of the cases had acute kidney injury. Proteinuria in $19(65.5 \%)$, microscopic hematuria in $18(62 \%)$ and leucocyturia in 4 patients. Proteinuria was at nephrotic level in 2 patients. 25 patients reported the names of the drugs they used before admission. 19 of them had used medication one week before the application. NSAIDs provided the largest proportion (63.1\%). In the second place, non-aminoglycoside antibiotics took place.

Uveitis was detected in one patient during follow-up. One patient showed a need for hemodialysis. Biopsy was performed in five patients because of high resistant creatinine and the diagnosis was found to be compatible with acute tubulointerstitial nephritis. Three patients required steroid therapy.

Conclusions: AIN is a common cause of AKI. Clinicians should be aware of the possibility of a drug-induced AIN particularly in patients exposed to multiple medications.

\section{PI-39 STUDY OF PREVALENCE AND CLINICAL SPECTRUM OF SEPTIC ACUTE KIDNEY INJURY (SAKI) IN STEROID RESPONSIVE NEPHROTIC SYNDROME}

Debanjan Sinha, Sanat Kumar Ghosh
Dr. B C Roy Pgips, Kolkata, India

Abstract: Systemic inflammatory response syndrome (SIRS) insult in the nephrotic background initiates interplay between inflammation and oxidative stress, leading to septic acute kidney injury (SAKI). Most paediatric studies are related to $\mathrm{AKI}$ in nephrotic syndrome while there is hardly any data on SAKI in steroid-responsive nephrotic syndrome.

Objectives: To study prevalence, clinical spectrum, contributory factors and short term outcome of SAKI in steroid responsive nephrotic syndrome.

Methods: A prospective observational study was done on inpatients paediatrics department of DR.BC RoyPGIPS including steroid responsive nephrotic syndrome up to 12 yrs of age. Children with congenital kidney anomaly and immunodeficiency were excluded. pRIFLE criteria were used for AKI while SIRS criteria was used for sepsis.

Results: Among the total 235 admitted steroid responsive nephrotic patients, 64 patients $(27.23 \%)$ developed AKI while 59 of them had features of SAKI. So, the proportion of SAKI was $92.10 \%$ (59 of 64). Severity of AKI was found to be increased as per progression of sepsis to severe sepsis to septic shock. $15.3 \%$ SAKI cases were positive for blood culture (M.C.Pneumococci) while $8.5 \%$ positive for urine culture (M.C.- E.Coli). most common presentation were Ascites $(93.2 \%)$ followed by tachycardia $(84.5 \%)$. Clinical features included peritonitis or septic shock followed by prolongation of the oliguric phase, development of hypertension, azotemia and congestive heart failure in some cases. Diuresis followed with subsidence of edema, CCF, azotemia. Among 59 SAKI cases, majority $(n=14,23.7 \%)$ was frequent relapse nephrotic syndrome (FRNS) followed by $1^{\text {st }}$ relapse $(18.7 \%), 2^{\text {nd }}$ relapse $(18.7 \%)$. Regarding contributory factors, $52.4 \%$ patients had hypovolemia and $10.17 \%$ had prior usage of nephrotoxic drugs. We found that 4 out of 55 patients $(7.27 \%$ ) had features of relapse after 6 months, $10.91 \%$ remained hypertensive.

Conclusions: Mortality and morbidity are more among SAKI patients with nephrotic syndrome than without SAKI.

\section{PI-40 A NEW CLINICAL TOOL TO SUSPECT DEHYDRATION AND ACUTE KIDNEY INJURY IN NON-FEBRILE CHILDREN}

Pierluigi Marzuillo ${ }^{1}$, Anna Di Sessa ${ }^{1}$, Felice Nunziata ${ }^{2}$, Paolo Montaldo ${ }^{1}$, Pierfrancesco Guida ${ }^{1}$, Federica Scaglione ${ }^{1}$, Rosa Melone ${ }^{1}$, Emanuele Miraglia Del Giudice ${ }^{1}$, Stefano Guarino ${ }^{1}$

${ }^{I}$ Department Of Woman, Child And Of General And Specialized Surgery, Università Degli Studi Della Campania "luigi Vanvitelli", Via Luigi De Crecchio 2, 80138, Naples, Italy, ${ }^{2}$ Department Of Pediatrics, Aorn Sant'anna E San Sebastiano, Via Ferdinando Palasciano, 81100 Caserta, Italy

Introduction: Heart rate (HR) is an easily and quickly detectable parameter and modifies on the basis of volemia. We hypothesized that the estimated HR variation in acute compared with basal conditions 
(EHR\%) could be a good clinical marker of both dehydration and acute kidney injury (AKI).

Material and methods: Two independent cohorts, one prospective comprehending children at type 1 diabetes mellitus onset (derivation) and one retrospective comprehending children with acute gastroenteritis and pneumonia (validation) were used to develop and externally validate EHR\% as predictor of dehydration and AKI. EHR\% was calculated as [(HR at admission $-50^{\text {th }}$ percentile of HR $) / \mathrm{HR}$ at admission $] * 100$. Dehydration was classified on the basis of weight loss and AKI according to the KDIGO criteria. EHR\% was evaluated as predictor of $\geq 5 \%$ dehydration and AKI by receiver-operating characteristic (ROC) curves analysis in the derivation cohort. The best cut-offs were identified and their diagnostic performance was tested both in derivation and validation cohort

Results: The prevalence of $\geq 5 \%$ dehydration and AKI were $61.1 \%$ and $43.8 \%$ in the derivation cohort and $34.4 \%$ and $24.5 \%$ in the validation cohort. The area under ROC curve (AUROC) of the EHR\% was $0.64(95 \% \mathrm{CI}, 0.56-0.71, \mathrm{p}=0.001)$ for $\geq 5 \%$ dehydration and $0.67(95 \% \mathrm{CI}, 0.59-0.73, \mathrm{p}<0.001)$ for $\mathrm{AKI}$ in the derivation cohort and $0.84(95 \% \mathrm{CI}, 0.77-0.90, \mathrm{p}<0.001)$ for $\geq 5 \%$ dehydration and $0.86(95 \% \mathrm{CI}, 0.79-0.93, \mathrm{p}<0.001)$ for AKI in the validation cohort. The best EHR\% cut-offs identified in the derivation cohort were $>23.2 \%$ for $\geq 5 \%$ dehydration and $>24.5 \%$ for AKI. In the validation cohort, EHR\% $>23.2 \%$ showed specificity $=99.1 \%(95 \%$ CI,95.2-99.9\%), positive likelihood ratio $(\mathrm{PLH})=24.6(95 \% \mathrm{CI}, 3.2-190.6)$, positive predictive value $(\mathrm{PPV})=88.9 \%(95 \% \mathrm{CI}, 50.8-98.1 \%)$, negative predictive value $(\mathrm{NPV})=79.6 \%(95 \% \mathrm{CI}, 76.7-82.2 \%)$, odds ratio $(\mathrm{OR})=20.5(2.5-167.0)$ for $\geq 5 \%$ dehydration and specificity $=99.0 \%(95 \%$ CI, $94.5-99.9 \%)$, PLR $=17.1$ (95\%CI,2.2-131.6), PPV=90.0\%(95\%CI,54.0-98.6\%), $\mathrm{NPV}=69.5 \%(95 \% \mathrm{CI}, 66.8-72.1), \mathrm{OR}=31.2(95 \% \mathrm{CI}, 3.7-259.3)$ for AKI.

Conclusions: EHR\% could predict $\geq 5 \%$ dehydration if $>23.2 \%$ and AKI if $>24.5 \%$. In case of ERH $>24.5 \%$, blood tests to detect AKI could be considered. While waiting for biochemical exams reports, a short-course of intravenous rehydration with $0.9 \% \mathrm{NaCl}$ could be warranted to counteract the progression of prerenal AKI toward acute tubular necrosis.

\section{PI-41 RAPIDLY PROGRESSIVE ACUTE RENAL FAILURE WITH FAVORABLE PROGNOSIS}

Elena Codina Sampera, Yolanda Calzada Baños, Pedro Arango Sancho, Víctor López-Báez, Lina Catherine Hérnandez Zúñiga, Ana Vinuesa Jaca, Álvaro Madrid Arias

Hospital Sant Joan De Déu, Barcelona, Spain

Objective: We present a patient with initial manifestation of rapidly progressive acute renal failure with macrohematuria. We consider the differential diagnosis between immunological diseases, post-renal obstructive pathologies and glomerulonephritis among other patologies.

Methods: A 10-year-old female patient with a one-week clinic of abdominal pain and diarrhea. Tests showed a creatinine of $0.6 \mathrm{mg} / \mathrm{dl}$, microhematuria and an abdominal ultrasound without signs of acute abdomen. Five days later, he presented a clinical worsening: macrohematuria with asthenia, anorexy, and isolation of Campylobacter in stool culture. Physical examination and blood pressure were normal. She presented: an increase in creatinine $4.5 \mathrm{mg} / \mathrm{dL}$ (GFR $12.64 \mathrm{~mL} / \mathrm{min} /$ $1.73 \mathrm{~m} 2$ ), urea: $204 \mathrm{mg} / \mathrm{dL}$, hemogram without anemia or thrombocytopenia, no ionic alterations, hematuria $>100 \mathrm{xc}$ and Protein/Creatinine in$\operatorname{dex}(\mathrm{P} / \mathrm{Cr}): 0.21 \mathrm{mg} / \mathrm{mg}$. Autoimmunity: ANA, ANCA, anti-basal membrane, Anti PLA2R, negative. C3: $1403 \mathrm{mg} / \mathrm{L}, \mathrm{C} 4: 315 \mathrm{mg} / \mathrm{L}$, IgA: $4209 \mathrm{mg} / \mathrm{L}$, ASLO $<200 \mathrm{U} / \mathrm{mL}$. Renal ultrasound showed bilateral renal hyperechogenicity without signs of lithiasis. Corticosteroid boluses at 30 $\mathrm{mg} / \mathrm{kg}$ were started every other day due to clinical suspicion of rapidly progressive glomerulonephritis, followed by oral prednisone. He presented progressive improvement, with preserved diuresis. He was discharged seven days after admission with creatinine $0.74 \mathrm{mg} / \mathrm{dL}$ (GFR $76 \mathrm{~mL} / \mathrm{min} /$
$1.73 \mathrm{~m} 2$ ), microhematuria $10-25 \mathrm{xc}$ and $\mathrm{P} / \mathrm{Cr} 0.29 \mathrm{mg} / \mathrm{mg}$. The renal biopsy revealed 7 glomeruli with slight mesangial expansion, doubtful hypercellularity, tubular atrophy and mixed inflammatory infiltrate, some dilated tubules and numerous blood casts. Immunofluorescence staining: weak mesangial positivity for $\mathrm{C} 3(+)$, irregular focal mesangial positivity for IgA (+). We diagnosed him with acute kidney failure due to IgA nephropathy. At six months of evolution, he presented normal kidney function with negative proteinuria: creatinine $0.53 \mathrm{mg} / \mathrm{dL}$ (GFR $109 \mathrm{~mL} / \mathrm{min} / 1.73 \mathrm{~m} 2$ ), cystatin $\mathrm{C}: 0.94 \mathrm{mg} / \mathrm{L}$, microhematuria $0-10 \mathrm{xc}$ and $\mathrm{P} / \mathrm{Cr}: 0.1 \mathrm{mg} / \mathrm{mg}$.

Conclusions: We want to highlight the rare presentation of a well-known pathology, where the compromise of renal function is justified by the intrarenal obstruction itself, caused by the hematic casts as well as by the tubulo-toxicity generated by hemoglobin and some of its degradation products (hematin).

\section{PI-42 AN 8-YEAR-OLD CHILD WITH MACROSCOPIC HEMATURIA AND ACUTE KIDNEY INJURY}

Hulya Nalcacioglu ${ }^{1}$, Bilge Can Meydan ${ }^{2}$, Hulya Gozde Onal ${ }^{1}$, Demet Tekcan ${ }^{1}$, Ozlem Aydog ${ }^{1}$

${ }^{1}$ Ondokuz Mayis University Faculty of Medicine, Pediatric Nephrology Department, Samsun, Turkey, ${ }^{2}$ Ondokuz, Mayis University Faculty of Medicine, Pathology Department, Samsun, Turkey

Objectives: Anti-glomerular basement membrane (GBM) disease is characterized by circulating anti-GBM antibodies with immunoglobulin deposition (Ig) G along the GBM. It manifests as rapidly progressive glomerulonephritis (RPGN) with crescentic proliferation with or without pulmonary hemorrhage. Early diagnosis and prompt treatment are essential to preserving kidney function. We present an eight -years -old boy who presented with macroscopic hematuria and was diagnosed with antiGBM.

Case presentation: An 8-year-old boy was admitted to his primary care clinic with macroscopic hematuria. He had no other complaints such as fever, dysuria, cough, dyspnea, or abdominal pain. He was initially treated outpatient with antibiotics and with symptomatic therapies. Despite that, his macroscopic hematuria persisted for ten days, and he was referred to our department when his kidney functions were high. Upon admission, vital signs and physical examination were normal. Initial lab. work was significant for anemia, elevated creatinine $(2.2 \mathrm{mg} / \mathrm{dL})$, ESR (greater than $160 \mathrm{~mm} / \mathrm{hr}$, ref $0-30 \mathrm{~mm} / \mathrm{hr})$ and CRP $(65 \mathrm{mg} / \mathrm{dl} \mathrm{ref}$ less than $0.5 \mathrm{mg} / \mathrm{dl}$ ). Urine analysis was remarkable for macroscopic hematuria - glomerular origin (numerous dysmorphic erythrocytes, erythrocytes cast). The chest graphic was normal. Renal and bladder Ultrasonography, complement analysis, was normal. He underwent a renal biopsy which showed necrotizing and crescentic glomerulonephritis with positive linear IgG along the GBM. Treatment included plasma exchange, intravenous high-dose methylprednisolone, and cyclophosphamide as a mainstay medication was stared. Anti-GBM antibody level was high of $>200 \mathrm{u} / \mathrm{ml}$ (ref less than $20 \mathrm{U} / \mathrm{ml}$ ). The treatment was effective with an immediate decrease in anti-GBM titers and creatinine levels. After two weeks of plasmapheresis, plasmapheresis was interrupted because the negative anti-GBM titer was negative. On day 7, the anti-GBM antibody came back positive. The patient was started on plasmapheresis shown to improve outcomes and survival for patients with anti-GBM nephritis.

Results: During the follow-up, he received a $15 \mathrm{mg} /$ day steroid and the second monthly dose of cyclophosphamide, and the last creatinine value was $0.7 \mathrm{mg} / \mathrm{dl}$.

Conclusions: Anti- GBM disease leads to RPGN since prompt diagnosis and early treatment are essential to improve outcomes. As seen in our case, timely diagnosis led to aggressive therapy, which ultimately translated into a favorable prognosis for our patient. 
PI-43 ACUTE KIDNEY INJURY AFTER ALLOGENEIC HAEMATOPOIETIC CELL TRANSPLANTATION IN CHILDREN - A SINGLE CENTRE OBSERVATIONAL STUDY

Erik Larsson ${ }^{1}$, Mikael Sundin ${ }^{2}$, Peter Bárány ${ }^{3}$, Milan Chromek ${ }^{3}$

${ }^{1}$ Division of Paediatrics, Department of Clinical Sciences, Intervention and Technology (clintec), Karolinska Institutet And Astrid Lindgren Children's Hospital, Karolinska University Hospital, Stockholm, Sweden, ${ }^{2}$ Division of Paediatrics, Department of Clinical Sciences, Intervention and Technology (clintec), Karolinska Institutet And Section of Haematology, Immunology and Hct, Astrid Lindgren Children's Hospital, Karolinska University Hospital, Stockholm, Sweden, ${ }^{3}$ Division of Paediatrics, Department of Clinical Sciences, Intervention and Technology (clintec), Karolinska Institutet And Paediatric Nephrology Unit, Astrid Lindgren Children's Hospital, Karolinska University Hospital, Stockholm, Sweden

Introduction: The incidence of acute kidney injury (AKI) after paediatric allogeneic haematopoietic cell transplantation (HCT) varies widely (21$53 \%$ ) and its pathogenesis remains unclear.

Material and methods: All paediatric patients who underwent HCT in 2015-2017 at our hospital were retrospectively assessed. Clinical and laboratory parameters including plasma creatinine (P-Cr) levels (pre HCT, peak value and at, or as close as possible to, 100 days) were recorded. AKI was defined as a $\geq$ twofold increase in $\mathrm{P}-\mathrm{Cr}$ (KDIGO $\geq$ II). Results: Sixty-one HCTs were done in 56 patients ( $66 \%$ boys) with a mean age of 7.5 years ( $0.1-17.4)$. Most patients ( $51 \%)$ had non-malignant indications. Reduced intensity conditioning was more frequent (52\%), as was cyclosporine as post-HCT immunosuppression (98\%). Median P-Cr was significantly higher at $41 \mu \mathrm{mol} / \mathrm{L} 100$ days post-HCT compared to $24 \mu \mathrm{mol} / \mathrm{L}$ pre-HCT $(\mathrm{p}<0.001)$, corresponding to an eGFR of 96 and 158 $\mathrm{ml} / \mathrm{min} / 1.7 \mathrm{~m}^{2}$, respectively. AKI was observed in $66 \%$ of the patients and $7 \%$ of the patients had developed eGFR $<60 \mathrm{ml} / \mathrm{min} / 1.7 \mathrm{~m}^{2}$ at the last follow-up. No patient needed renal replacement therapy. AKI incidence was significantly higher in unrelated donor (URD) HCTs compared to other donor-type HCTs ( 78 vs. $52 \%$, p<0.05). Age was negatively associated with AKI. There was no association between acute GVHD, conditioning, HCT indication, infections, sex or toxic cyclosporine levels and AKI. Occurrence of AKI did not affect eGFR 100 days post-HCT or three-year survival.

Conclusions: AKI seems to be a very common complication to HCT. However, in this study, AKI was not associated with a worse outcome as measured by eGFR at 100 days or three-year survival. The high pre-HCT eGFR in this study suggests that plasma creatinine may overestimate GFR pre-HCT, which makes diagnosing AKI challenging. The observed association between URD-HCTs and AKI is intriguing and advocates further investigation addressing immunological mechanisms in the pathogenesis of AKI after HCT.

\section{PI-44 ASSESSMENT OF THE RENAL ANGINA INDEX FOR PREDICTION OF ACUTE KIDNEY INJURY ON PATIENTS ADMITTED TO AN EUROPEAN PAEDIATRIC INTENSIVE} CARE UNIT (PICU)

Francisco Ribeiro-mourão ${ }^{1}$, Ana Carvalho Vaz ${ }^{1}$, André Azevedo ${ }^{1}$, Helena Pinto ${ }^{2}$, Marta João Silva ${ }^{3}$, Joana Jardim ${ }^{2}$, Augusto Ribeiro ${ }^{3}$

${ }^{1}$ Unidade Local De Saúde Do Alto Minho Paediatrics Department, Viana Do Castelo, Portugal, ${ }^{2}$ Centro Hospitalar E Universitário De São João, Paediatrics Department, Pediatric Nephrology Unit, Porto, Portugal, ${ }^{3}$ Centro Hospitalar E Universitário De São João, Paediatric Intensive Care Unit, Porto, Portugal

Introduction: Acute Kidney Injury (AKI) is frequent on Paediatric Intensive Care Units (PICU) patients, being associated with worse outcomes and increased morbidity and mortality. AKI is challenging and treatment's success depends on early detection and management. The Renal Angina Index (RAI) has been proposed as an early prediction tool of AKI development. Our objectives are: to evaluate RAI's performance as an AKI predictor and to compare its performance with other markers.

Material and methods: This is an observational retrospective study. All children admitted on a tertiary hospital PICU over a 3-year period were included. Electronical medical records were reviewed. Day 1 RAI was calculated as well as the presence and staging of day 3 AKI. Both were correlated with outcomes.

Results: 593 patients were included, $56 \%$ male, mean age 55 months. $17 \%$ had a positive RAI. This was associated to Day 3 AKI development and to worst outcomes, namely: greater need for renal replacement therapy, longer duration of mechanical ventilation, vasoactive support and length of stay in PICU and higher mortality. For all-stage kidney injury RAI presented sensitivity of $87.5 \%$ and specificity of $88.1 \%$. Prediction of Day 3 all-stage AKI by RAI had AUC $=0.878$; its performance increased for severe $\mathrm{AKI}$ ( $\mathrm{AUC}=0.93$ ). RAI was superior to Serum Creatinine increase and KDIGO AKI staging on day 1 on predicting AKI development.

Conclusions: RAI is a simple and inexpensive tool that can be used on PICU patients to predict AKI development and anticipate complications. Interpretation of its results should be cautious and integrated with other clinical data.

\section{PI-45 EPIDEMIOLOGICAL TRENDS OF CHRONIC KIDNEY DISEASE IN CHILDREN: A REPORT FROM SOUTH EAST OF ENGLAND 2012-2016}

Jacqueline Sit, Caroline Booth, Nabil Melhem, Manish Sinha

Evelina London Children's Hospital, Guy's And St Thomas' Nhs Foundation Trust, United Kingdom

Introduction: Chronic kidney disease (CKD) is a devastating illness with profound impact on the child and their families. To our knowledge, there are currently no national registries reporting epidemiology of pre-dialysis or non-dialysis stages of CKD in children.

The objectives of this study were (i) to describe the demographic characteristics of children $<16$ years with stages $3-5$ of CKD in South East of England; (ii) to describe the recent trends in incidence and prevalence; (iii) to describe age, gender and ethnicity-specific incidence; (iv) to compare trends with a previous 5-year period.

Material and methods: This was a retrospective study of all children $<16$ years with an estimated glomerular filtration rate of $<60 \mathrm{ml} / \mathrm{min} /$ $1.73 \mathrm{~m}^{2}$, who were followed up at Evelina London Children's Hospital between 2012 and 2016. Incidence and prevalence were calculated based on data from the 2011 Census. Results were compared with data from a previous 5-year period (2005-2009).

Results: There were 292 patients in the study. Renal dysplasia (39.3\%) and obstructive uropathy $(21.6 \%)$ remained the most common causes of $\mathrm{CKD}$. The mean incidence and prevalence of CKD amongst $<16$ years were 16.7 per million age related population (pmarp) and 110.0pmarp respectively. The incidence of CKD was higher in males (10.5pmarp) compared to females (6.1pmarp). The incidence of CKD was twice as high amongst Asian (24.7pmarp) and Black (21.2pmarp) populations when compared with the White (11.4pmarp) and Mixed (10.0pmarp) populations. The incidence of CKD had remained stable between 13.0pmarp to $20.4 \mathrm{pmarp}(\mathrm{p}=0.62)$, whilst the prevalence had increased from $105.7 \mathrm{pmarp}$ to $118.7 \mathrm{pmarp}(\mathrm{p}=0.04)$.

Conclusions: Compared with the previous period 2005-2009, the mean incidence of CKD in the recent 5 -year period was similar whilst there was a continued increase in the mean prevalence. This study describes gender, age and ethnicity-specific incidence which have not previously been reported. Understanding the epidemiology of CKD enables appropriate resource allocation and service planning. 
PI-46 SGLT2I AS RENOPROTECTION TOOL IN CHILDREN WITH CKD

Dmytro Ivanov, Mariia Ivanova

Shupyk National Healthcare University Of Ukraine, Ukraine

\begin{abstract}
SGLT2i in adults with CKD have shown impressive results in reducing total and cardiovascular mortality and reducing the rate of progression of CKD. The clinical model for imitating the intake of SGLT2i in children is renal glucosuria, which does not bring significant suffering to the kids. The aim of the study was to evaluate the safety and efficacy of SGLT2i in children with CKD.
\end{abstract}

Materials and methods: A prospective randomized, open out of label 6month study was conducted in 9 children (8-17 years old) with CKD presented by isolated urinary syndrome in the form of albuminuria 244 12.5. All children have been already receiving an ACE inhibitor or ARB to control albuminuria. The following criteria for assessing the safety of SGLT2i were selected: assessment of the childs clinical condition, blood pressure, decrease in eGFR, development of urinary tract infections. Evaluation of the effectiveness of prescribing the drug was carried out according to the dynamics of albuminuria. The drug of choice is dapagliflozin, a dose of $0.125 \mathrm{mg} / \mathrm{kg}$ in one dose.

Results and discussion: Dapagliflozin has been shown to be well tolerated. Clinical complaints in the form of transient weakness were documented in 2 children (22\%), a decrease in blood pressure by $5-10$ percentiles in $3(33 \%)$, a transient decrease in eGFR by $3 \pm 1 \mathrm{ml} /$ min in 5 children (55\%), no urinary tract infections in a single child. Efficiency in reducing albuminuria was demonstrated in 7 children $(77 \%)$. Side effects of the drug were not sufficient as well as temporally. At the same time, the addition of SGLT2i to ACE inhibitor / ARB therapy reduced albuminuria from $243 \pm 12 \mathrm{mg} / \mathrm{L}$ to $160 \pm 9 \mathrm{mg} / \mathrm{L}(\mathrm{P} \leq 0 / 01)$.

Conclusions: The preliminary results of the observations allow to consider the promising using SGLT2i in children with further determination of strict indications.

\section{PI-47 JOINING FORCES: OPTIMISING ORAL HEALTH FOR CHILDREN AND YOUNG PEOPLE WITH KIDNEY DISEASE}

\author{
Christopher Wallace ${ }^{1}$, Pallavi Prasad ${ }^{2}$, Catherine Horridge ${ }^{3}$, \\ Oliver Sumner ${ }^{3}$, Christine Pattinson ${ }^{4}$, Jayne Straker ${ }^{4}$, \\ Richard Balmer ${ }^{1}$, Virginia Hind ${ }^{3}$ \\ ${ }^{1}$ Leeds Dental Institute, United Kingdom, ${ }^{2}$ Alder Hey Childrens \\ Hospital, Liverpool, United Kingdom, ${ }^{3}$ Newcastle Dental Hospital, \\ United Kingdom, ${ }^{4}$ Great North Childrens Hospital, Newcastle, United \\ Kingdom
}

Introduction: Children and young people (CYP) with kidney diseases may face adverse impacts of chronic kidney disease (CKD) on their developing dentition, alongside increased risk of adverse side-effects from dental treatment. Those who are immunocompromised are at increased risk of severe orofacial infections which can lead to pain and poor quality of life. Our objective: To highlight the main orofacial manifestations of kidney disease and provide guidance on improving dental care and maximising oral health outcomes in CYP with kidney disease.

Material and methods: A joint dental-kidney service for CYP with CKD was piloted at our centres. Patients with CKD (IV-V) and those undergoing renal replacement therapy (RRT) are offered specialist dental reviews as part of their regular kidney out-patient clinics, or during incentre haemodialysis therapy.

Results: Enamel defects are prevalent in patients with CKD. They can result in poor aesthetics alongside pain, and breakdown of molar teeth. Drug-induced gingival overgrowth is a side-effect of ciclosporin, tacrolimus and calcium-channel blockers. It can lead to disfiguration and increased risk of periodontal disease. Other orofacial manifestations of kidney disease may include oral soft tissue changes, xerostomia, salivary stones, opportunistic infections, increased risk of malignancy and radiographic changes in those with renal osteodystrophy.

This joint service ensures CYP receive dental care by dentists with appropriate experience within paediatric dentistry to manage these more complex conditions. Furthermore, it also minimises the personal and financial burden patients and families face from attending excessive health care appointments.

Conclusions: The Kidney MDT is in a pivotal position to advocate oral health and reinforce regular dental reviews. Equally, the dental team may require support from the kidney team regarding additional precautions such as antibiotic prophylaxis, steroid cover, altered prescribing, and haemostatic measures during dental treatment. By working together, nephrology and dental teams can maximise oral health and quality of life for their patients.

\section{PI-48 CHRONIC KIDNEY DISEASE TEN YEARS AFTER PEDIATRIC ALLOGENEIC HEMATOPOIETIC STEM CELL TRANSPLANTATION}

Gertjan Lugthart ${ }^{1}$, Carlijn Jordans ${ }^{1}$, Anne De Pagter ${ }^{1}$, Dorine Bresters ${ }^{2}$, Els Jol-van Der Zijde ${ }^{1}$, Joell Bense ${ }^{1}$, Roos Van Rooij-kouwenhoven ${ }^{1}$, Ram Sukhai ${ }^{1}$, Marloes Louwerens ${ }^{1}$, Eiske Dorresteijn ${ }^{3}$, Arjan Lankester ${ }^{1}$ ${ }^{1}$ Leiden University Medical Center, The Netherlands, ${ }^{2}$ Princess Maxima Center, The Netherlands, ${ }^{3}$ Erasmus Medical Center, The Netherlands

Introduction: Chronic kidney disease (CKD) is an important sequela of hematopoietic stem cell transplantation (HSCT), but data regarding CKD after pediatric HSCT are limited.

Material and methods: In this single center cohort study, we evaluated eGFR dynamics, proteinuria and hypertension in the first decade after HSCT and assessed risk factors for chronic kidney disease in 216 pediatric long term HSCT survivors, transplanted between 2002-2012.

Results: The eGFR decreased from median 148 to $116 \mathrm{ml} / \mathrm{min} / 1.73 \mathrm{~m}^{2}$ between pre-HSCT and ten years after HSCT. CKD, defined as an eGFR $<90 \mathrm{ml} / \mathrm{min} / 1.73 \mathrm{~m}^{2}$ and/or albuminuria (KDIGO stage $\geq \mathrm{G} 2$ or $\geq \mathrm{A} 2$ ) occurred in $17 \%$ of patients. In multivariate analysis, severe prolonged stage $\geq 2$ acute kidney injury (AKI), with an eGFR $<60 \mathrm{ml} / \mathrm{min} / 1.73 \mathrm{~m}^{2}$ and duration $\geq 28$ days, was the main risk factor for CKD (HR 9.5, 95\% CI 3.4-27). Stage $\geq 2$ AKI with an eGFR $\geq 60 \mathrm{ml} / \mathrm{min} / 1.73 \mathrm{~m}^{2}$ and stage $\geq 2$ AKI with eGFR $<60 \mathrm{ml} / \mathrm{min} / 1.73 \mathrm{~m}^{2}$ but recovery within $<28$ days were not associated with CKD. Furthermore, hematological malignancy as HSCT indication (HR 3.5, 95\% CI 1.4-8.6) was an independent risk factor for CKD. One third of patients with CKD had both an eGFR $<90 \mathrm{ml} / \mathrm{min} / 1.73 \mathrm{~m}^{2}$ as well as albuminuria, one third had isolated eGFR reduction and one third only had albuminuria. Hypertension was observed in $27 \%$ of patients with CKD compared to $4.4 \%$ of patients without CKD. Tubular proteinuria was present in $7 \%$ of the subgroup of patients $(n=71)$ in which $\beta 2$-microglobulinuria was measured.

Conclusions: In conclusion, a significant proportion of pediatric HSCT recipients developed chronic kidney disease within ten years after HSCT. Our data stress the importance of structural long term monitoring of eGFR, urine and blood pressure after HSCT to identify patients with incipient CKD who can benefit from nephroprotective interventions.

\section{PI-49 PULSE WAVE VELOCITY (PWV) DOES NOT ASSOCIATE WITH CHRONIC KIDNEY DISEASE (CKD) PROGRESSION IN CHILDREN}

Karolis Azukaitis ${ }^{1}$, Marietta Kirchner ${ }^{2}$, Anke Doyon ${ }^{3}$, Mieczysław Litwin ${ }^{4}$, Aysun Bayazit ${ }^{5}$, Ali Duzova ${ }^{6}$, Nur Canpolat ${ }^{7}$, Augustina Jankauskiene ${ }^{1}$, Rukshana Shroff $^{8}$, Anette Melk ${ }^{9}$, Uwe Querfeld ${ }^{10}$, Franz Schaefer ${ }^{3}$, On Behalf Of $4 \mathrm{c}$ Study Consortium ${ }^{11}$

${ }^{1}$ Clinic Of Pediatrics, Institute Of Clinical Medicine, Faculty Of Medicine, Vilnius University, Vilnius, Lithuania, ${ }^{2}$ Institute Of Medical Biometry And Informatics, University Of Heidelberg, Heidelberg, 
Germany, ${ }^{3}$ Division Of Pediatric Nephrology, Center For Pediatrics And Adolescent Medicine, Heidelberg University Hospital, Heidelberg, Germany, ${ }^{4}$ Department Of Nephrology And Arterial Hypertension, The Childrens Memorial Health Institute, Warsaw, Poland, ${ }^{5}$ Department Of Pediatric Nephrology, Cukurova University, Adana, Turkey, ${ }^{6}$ Division Of Pediatric Nephrology, Hacettepe University Faculty Of Medicine, Ankara, Turkey, ${ }^{7}$ Department Of Pediatric Nephrology, Istanbul University Cerrahpasa Faculty Of Medicine, Istanbul, Turkey, ${ }^{8}$ Great Ormond Street Hospital For Children, London, United Kingdom, ${ }^{9}$ Department Of Kidney, Liver And Metabolic Diseases, Hannover Medical School, Hannover, Germany, ${ }^{10}$ Department Of Pediatric Nephrology, Charité University, Berlin, Germany, ${ }^{11} 4 c$ Study Consortium

Objectives: Large studies in adults with CKD have shown that arterial stiffness measured by PWV is associated with CKD progression but the association was not consistent. The association of PWV with risk of CKD progression has not been studied in children with CKD.

Methods: Post-hoc analysis of the Cardiovascular Comorbidity in Children with Chronic Kidney Disease (4C) study that prospectively followed children aged 6-17 years with CKD stages 3-5. PWV was measured with an oscillometric Vicorder device and standardized according to sex and height (PWVz). CKD progression was defined as a composite endpoint of (i) $50 \%$ decline in baseline estimated glomerular filtration rate (eGFR), (ii) eGFR < $15 \mathrm{ml} / \mathrm{min} / 1.73 \mathrm{~m}^{2}$ or (iii) start of kidney replacement therapy (KRT).

Results: 667 patients ( $12.2 \pm 3.3$ years, $65.5 \%$ boys) with a median baseline eGFR of $26.9 \mathrm{ml} / \mathrm{min} / 1.73 \mathrm{~m}^{2}$ and baseline PWVz of $0.31 \pm 1.65$ were analyzed. A total of 389 patients reached the composite endpoint of CKD progression with a median time-to-event of 2.3 years (range 0.9-4.5). Of those, $75(19.3 \%)$ patients reached eGFR $<10 \mathrm{ml} / \mathrm{min} . / 1.73 \mathrm{~m}^{2}, 158$ (40.6\%) had 50\% loss in eGFR and $156(40.1 \%)$ started KRT. CKD progression-free survival did not differ when stratifying the study cohort by PWVz tertiles (Figure; log-rank $\mathrm{p}=0.24$ ). PWVz was also not associated with the risk of CKD progression (aHR $0.964,95 \%$ CI 0.896 to 1.037) when added to a Cox proportional hazards regression model adjusted for known predictors of CKD progression (age, sex, primary kidney disease, body mass index, systolic blood pressure, eGFR and albuminuria).

Conclusions: PWV does not associate with the risk of CKD progression in children. The magnitude of PWV increase and exposure duration in childhood may be too small to cause substantial kidney damage as compared to adults with extended risk factor exposure.



PI-50 F18-CHOLINE PET/CT TO LOCATE A PARATHYROID ADENOMA IN CHILD WITH CHRONIC KIDNEY DISEASE

Sara De Lucas Volle, Ana Belén Martínez-lópez, Daniel Barraca Núñez, Paula Carrascosa García, Dany Zamudio Rodríguez, Débora Claramunt Taberner, Olalla Alvarez Blanco

Hospital General Universitario Gregorio Marañon, Madrid, Spain

Objectives: Tertiary hyperparathyroidism is an uncommon complication in children with chronic kidney disease (CKD). Once parathyroidectomy has been considered, location of parathyroid glands is essential for minimal invasive surgery and Tc99m-sestaMIBI SPECT/CT is recommended as the most sensitive imaging test. $18 \mathrm{~F}$-choline is a choline analogue that can be used for this purpose as there is choline kinase activity in PTH secretion in normal parathyroid cells. To our knowledge, there is no available data about use of this technique in children.

Methods: We report the use of F18-choline PET/CT in a paediatric patient for presurgical location of a hyperfunctioning adenoma.

Results: 4-year-old boy with end-stage CKD due to steroid-resistant nephrotic syndrome. Peritoneal dialysis was performed unsuccessfully due to intraperitoneal adherence so he started daily haemodialysis in 2017. After a year, he received a deceased donor kidney transplant but had a fast recurrence of the underlying disease forcing to graft nephrectomy. In 2019, PTH levels started to rise despite treatment optimization. The patient was in dietary phosphate restriction, vitamin D replete, he had phosphate binders, vitamin D analogue therapy and calcimimetics. Tertiary hyperparathyroidism was diagnosed and parathyroid glands ultrasound only revealed a non-specific small nodule adjacent to the inferior pole of right thyroid lobe. The Tc99m-sestaMIBI scintigraphy was negative for hyperfunctioning parathyroid tissue. As minimal invasive surgery was the preferred technique, the F18-choline PET/CT was finally performed after a multidisciplinary meeting. It revealed a right paratracheal $5 \mathrm{~mm}$ nodular image, located distal to lower pole of the right thyroid lobe, showing increased metabolic activity consistent with parathyroid adenoma. The child went on an inferior right parathyroidectomy on June 2020 without complications and he is now better controlled with calcimimetics.

Conclusions: Although there is limited experience, F18-choline PET/CT seems to be a useful and safe technique in children with suspicion of tertiary hyperparathiroidism.

PI-51 EARLY VERSUS LATE REQUIREMENT FOR RENAL REPLACEMENT THERAPY IN CHILDREN AT PRESENTATION: EARLY AND MEDIUM-TERM OUTCOMES

Jacqueline Sit, Caroline Booth, Nabil Melhem, Manish Sinha Evelina London Children's Hospital, Guy's And St Thomas' Nhs Foundation Trust, United Kingdom

Objectives: Nearly 25\% children start renal replacement therapy (RRT) within 90 days of presenting to paediatric nephrology services in the UK (early requirement for RRT). The objective of this study was to compare the clinical characteristics, the early and medium-term outcomes of children who had early requirement (ER) versus late requirement (LR) for RRT.

Methods: This was a retrospective study of all children with chronic kidney disease (CKD) who were initiated on RRT at the Evelina London Children's Hospital between 2012-2016. Demographics, primary renal disease (PRD), date of presentation, biochemical parameters, dialysis modality, complications and transplant details were recorded.

Results: There were 86 patients in the study, $32.6 \%$ had ER and $67.4 \%$ had LR for RRT. Patients with ER for RRT at presentation were likely to be female $(p=0.046)$, older $(p<0.001)$, with different PRD's (higher rates of hereditary nephropathies, miscellaneous renal disorders, and systemic diseases) and with lower eGFR ( $\mathrm{p}=0.002)$. There were no differences in ethnicity $(\mathrm{p}=0.48)$ and socioeconomic status $(\mathrm{p}=0.26)$.

Patients with ER had higher urea $(\mathrm{p}=0.02)$, higher phosphate $(\mathrm{p}<0.001)$ and were more acidotic $(\mathrm{p}=0.01)$ at initiation of RRT. They had a shorter 
time interval between presentation and transplant (1.3 vs 5.2 years, $\mathrm{p}<0.001$ ), and were more likely to change dialysis modality in the first 2 years $(\mathrm{p}<0.001)$.

There were no differences in the number transplanted $(\mathrm{p}=0.33)$, donor source $(\mathrm{p}=0.22)$, or age at transplantation $(\mathrm{p}=0.29)$. There were no differences in the need for $(p=0.7)$ and the time intervals between switching RRT modalities $(\mathrm{p}=0.6)$.

Conclusion: Patients who had early requirement for RRT had poorer outcomes and worse biochemical parameters at initiation of RRT. They were more likely to require changes in dialysis modalities in the first 2 years and have less access to preparation before initiation of dialysis or pre-emptive transplant. Research to identify opportunities for earlier detection of CKD in children is needed.

\section{PI-52 OFF-LABEL USE OF CINACALCET IN PEDIATRIC PRIMARY HYPERPARATHYROIDISM: A FRENCH MULTI-CENTRE EXPERIENCE}

Julie Bernardor, Sacha Flammier, Jean-pierre Salles, Mireille Castagnet, Laetitia Martinerie, Anne Lienhardt-roussie, Cyril Amouroux, Agnès Linglart, Justine Bacchetta From The Reference Center of Rare Diseases of Calcium and Phosphate, Rare Diseases French Network Oscar, France

Introduction: For adult patients who do not meet indications to surgery, the calcimimetic cinacalcet is approved for primary hyperparathyroidism (HPT), decreasing calcium and parathyroid hormone (PTH) levels. This drug is largely used in adults, but may be a challenge in daily practice in children, because of the risk of hypocalcemia, increased QT interval and drug interactions. A few pediatric cases have highlighted its potential interest in children with HPT; the aim here is to report the French pediatric experience in this setting.

Material and methods: We retrospectively analyzed data from 19 pediatric patients from 7 tertiary French centres who received cinacalcet for HPT. The results are presented as median (interquartile range).

Results: At a median age of 12.2(2.5-14.8) years, 19 patients received Cinacalcet for primary HPT (12 patients with inactive CaSRmutation, 1 patient with $C D 73$ mutation, 1 patient with $P H E X$ mutation, 1 patient with multiple endocrine neoplasia and 4 patients with primary HPT of unknown etiology). Cinacalcet was introduced at an estimated glomerular filtration rate (eGFR) of $123(120-131) \mathrm{mL} / \mathrm{min} / 1.73 \mathrm{~m}^{2}$, plasma calcium of $2.98(2.87-3.10) \mathrm{mmol} / \mathrm{L}$, age-standardized (z score) phosphate of $2.7(-3.2 ;-1.9)$, total ALP of 214(111-225)UI/L, 25-D of 42(22-46) $\mathrm{nmol} / \mathrm{L}$, and PTH of 59(42-80) ng/L. The starting daily dose of cinacalcet was $0.7(0.6-0.9) \mathrm{mg} / \mathrm{kg}$, with a maximum dose of $1.0(0.9-1.3) \mathrm{mg} / \mathrm{kg}$ per day. With a follow-up of 2.3(1.3-4.3) years on cinacalcet therapy, PTH remained stable at 44(35-59) ng/L ( $\mathrm{p}=\mathrm{NS})$, as did eGFR, 25-D, ALP and phosphate and urinary calcium levels. In contrast, plasma calcium levels significantly decreased to $2.73(2.58-2.90) \mathrm{mmol} / \mathrm{L}(\mathrm{p}=0.03)$. Nephrocalcinosis was not reported but one patient displayed nephrolithiasis. Cinacalcet was withdrawn in one patient (no side effect was reported).

Conclusions: These results suggest that off-label cinacalcet in pediatric HPT controls hypercalcemia without significant side effects.

\section{PI-53 WHICH FORMULAS USING TO ESTIMATE GFR IN ADOLESCENTS AND YOUNG ADULTS?}

Laurence Dubourg Derain ${ }^{1}$, Sandrine Lemoine ${ }^{1}$, Bruno Ranchin ${ }^{2}$, Aurelia Bertholet-thomas ${ }^{2}$, Anne-laure Sellier Leclerc ${ }^{2}$,

Laurence Chardon ${ }^{3}$, Justine Bacchetta ${ }^{4}$

${ }^{1}$ Nephrologie, Dialyse, Hypertension Et Exploration Fonctionnelle Renale, Groupement Hospitalier Edouard Herriot, Hospices Civils De Lyon, France Universite Claude Bernard Lyon 1, Lyon, France, ${ }^{2}$ Centre
De Reference Des Maladies Renales Et Phosphocalciques Rares, Bron, France; Universite Claude Bernard Lyon 1, Lyon, France, ${ }^{3}$ Service De Biochimie Et Biologie Moleculaire Grand Est, Hospices Civils De Lyon,lyon, France, ${ }^{4}$ Centre De Reference Des Maladies Renales Et Phosphocalciques Rares, Bron, France; Universite Claude Bernard Lyon 1, Lyon, France

Introduction: Estimating glomerular filtration rate (eGFR) in adolescents and during the pediatric-to-adult transition period is challenging since performance of specific pediatric or adult equations is poor. Some authors suggested to use the average of pediatric and adult creatininebased eGFR in young adults whereas others proposed equations designed to cover the whole life span. The aim of this study is to evaluate the performance of these various methods of GFR estimation in adolescents and during the transition period, namely between 13 and 25 years.

Material and methods: Measured GFR (mGFR) was assessed in 1861 adolescents and young adults at various CKD stages (mean age 16.0 \pm 0 .1years) by a reference method (inulin or iohexol clearance); plasma creatinine (Pcr) was determined by an IDMS-standardized assay. Mean mGFR was $87.0 \pm 0.7 \mathrm{~mL} / \mathrm{min} / 1.73 \mathrm{~m}^{2}$ [range $12-209$ ]. The performance of Schwartz2009(1), Schwartz-Lyon(2), CKDEPI(3), average(Schwartz2009CKDEPI)(4), EKFC(5), CKDEPI40(6), LMR18(7), CKiDU25ct and CKiDagedep $(8)$ was studied according to age groups (13-16 n=949; 16-18 $\mathrm{n}=685 ; 18-25$ years $\mathrm{n}=227)$. Equation performance was assessed using bias and accuracy (P30\%).

Results: Mean absolute bias in all the groups for Schwartz2009, SchwartzLyon, CKD-EPI, average(Schwartz2009-CKDEPI), EKFC, CKDEPI40, LMR18, CKiDU25ct, and CKiDagedep equation was 3.0 $\pm 0.6,-1.8 \pm 0.6$, $32.2 \pm 0.5,17.6 \pm 0.5,4.5 \pm 0.40 .8 \pm 0.4,1.2 \pm 0.4,-0.3 \pm 0.6$ and $6.6 \pm 0.7 \mathrm{ml} / \mathrm{min} /$ $1.73 \mathrm{~m}^{2}$, respectively. P30\% was $83.6,86.8,38.8,64.8,84.1,85.8,88.2,89.8$ and $84.5 \%$, respectively. For Schwartz2009, Schwartz-Lyon, EKFC, LMR18 and CKiDagedep P30\% was $>80 \%$ in all groups, except in girls 18-25 years who displayed lower P30\% (71.2, 77.9, 70.2, 76.9 , 73.1\%, respectively). CKDEPI40 and CKiDU25ct were the only equations with a comparable performance in all groups (P30\% > 82\%).

Conclusions: CKDEPI40 and CKiDU25ct have better performance all over the age groups. However the use of the whole age span CKDEPI40 could prevent the implausible increase in eGFR when switching from pediatric to adult equations.(1) Schwartz CJASN 2009 (2) Carla De Souza PLoS One 2012 (3) Levey AJKD 2010 (4) Ng Kidney Int 2018 (5) Pottel Annals of Internal Medicine 2020 (6) Björk Kidney Int 2021 (7) Björk Scand J Clin Lab Invest 2020 (8)Pierce Kidney Int 2021

\section{PI-54 AGREEMENT BETWEEN CREATININE- AND CYSTATINE C-BASED FORMULAS TO ESTIMATE GLOMERULAR FILTRATION RATE IN CONGOLESE CHILDREN}

Agathe B. Nkoy ${ }^{1}$, Therance T. Matoka ${ }^{1}$, Dieumerci K. Betukumesu ${ }^{1}$, Orly K. Kazadi ${ }^{1}$, Ernest K. Sumaili ${ }^{2}$, René M. Ngiyulu ${ }^{1}$,

Pierre Delanaye ${ }^{3}$, Etienne Cavalier ${ }^{4}$, Lambertus P. Van Den Heuvel ${ }^{5}$, Elena Levtchenko ${ }^{5}$, Pépé M. Ekulu ${ }^{1}$

${ }^{1}$ Division of Nephrology, Department of Pediatrics, University Hospital of Kinshasa, Faculty Of Medicine, University of Kinshasa, Kinshasa, Democratic Republic of Congo, ${ }^{2}$ Division of Nephrology, Department of Internal Medicine, University Hospital of Kinshasa, Faculty of Medicine, University of Kinshasa, Kinshasa, Democratic Republic of Congo, ${ }^{3}$ Department of Nephrology-dialysis-transplantation, Chu Sart Tilman, University of Liège, Liège, Belgium, ${ }^{4}$ Division of Clinical Chemistry, Chu Sart Tilman, University of Liège , Liège, Belgium, ${ }^{5}$ Department of Development and Regeneration, Ku Leuven, Leuven, Belgium

Introduction: Glomerular filtration rate (GFR) is a critical tool for the diagnosis of kidney diseases. In routine, creatinine-based formula are usually used to estimate GFR (eGFR) in children. However, 
the accuracy of these formula is highly dependent on the creatinine assay method used. Our study aimed to assess the concordance between the original and the updated Schwartz formula applied to creatinine, regardless of the measurement method used, and the cystatin $\mathrm{C}$ formula used as the reference, in healthy children living in the Democratic Republic of Congo (DRC).

Material and methods: In a cross sectional study conducted from February to December 2019, a random sample of 515 children (aged 6 to 16 years) were recruited using a multistage sampling, from 17 schools in Kinshasa, the capital of the DRC. Using kappa coefficient and BlandAltman method, we evaluated the agreement between eGFR from the original Schwartz formula applied to Jaffe-based uncalibrated creatinine, from the updated Schwartz formula applied to both Jaffe-based uncalibrated creatinine and enzymatic based-creatinine, and cystatine C-based formula.

Results: The results showed a strong agreement between eGFR from the original Schwartz formula applied to Jaffe-based uncalibrated creatinine versus cystatine $\mathrm{C}$-based formula (kappa $=0.95$ ), and the updated Schwartz formula using enzymatic based-creatinine versus cystatine Cbased formula (kappa=0.96). However, there was a significant gap between eGFR from updated Schwartz formula applied to Jaffe-based uncalibrated creatinine with eGFR from cystatine C-based formula (kappa=0.45).

Conclusions: The significant difference between the updated Schwartz formula applied to Jaffe-based uncalibrated creatinine and cystatine Cbased formula reveals that the new Schwartz formula should be used with caution, especially in low-income countries, like DRC, where Jaffe method for determining creatinine remains the most commonly used. In this setting, the use of the original Schwartz formula is more appropriate.

\section{PI-55 SCREENING FOR URINARY ABNORMALITIES AND KIDNEY FUNCTION ASSESSMENT AMONG CHILDREN LIVING IN DEMOCRATIC REPUBLIC OF CONGO}

Agathe B. Nkoy ${ }^{1}$, Therance T. Matoka ${ }^{1}$, Dieumerci K. Betukumesu ${ }^{1}$, Orly K. Kazadi ${ }^{1}$, Ernest K. Sumaili ${ }^{2}$, René M. Ngiyulu ${ }^{1}$,

Pierre Delanaye ${ }^{3}$, Etienne Cavalier ${ }^{4}$, Lambertus P. Van Den Heuvel ${ }^{5}$, Elena Levtchenko ${ }^{5}$, Pépé M. Ekulu ${ }^{1}$

${ }^{1}$ Division of Nephrology, Department of Pediatrics, University Hospital of Kinshasa, Faculty of Medicine, University of Kinshasa, Kinshasa, Democratic Republic of Congo ${ }^{2}$ Division of Nephrology, Department of Internal Medicine, University Hospital of Kinshasa, Faculty of Medicine, University of Kinshasa, Kinshasa, Democratic Republic of Congo, ${ }^{3}$ Department of Nephrology-dialysis-transplantation, Chu Sart Tilman, University of Liège, Liège, Belgium, ${ }^{4}$ Division of Clinical Chemistry, Chu Sart Tilman, University of Liège, Liège, Belgium, ${ }^{5}$ Department of Development And Regeneration, Ku Leuven, Leuven, Belgium

Introduction: Kidney function assessment and urinary screening are useful for identifying occult kidney diseases in asymptomatic children and might be an important initial step in prevention of chronic kidney disease (CKD). Our study aimed to determine the prevalence of urinary abnormalities and to assess the kidney function through the estimated glomerular filtration rate (eGFR) among school children living in the Democratic Republic of Congo (DRC).

Material and methods: In a cross sectional study conducted from February to December 2019, we randomly recruited 515 healthy children aged 6 to 16 years, using a multistage sampling, from 17 schools in Kinshasa, the capital of the DRC. Recorded parameters of interest were dipstick proteinuria, dipstick hematuria, urinary tract infection detected by dipstick, urinary albumin-to-creatinine ratio (uACR) assessed using the DCA Vantage Analyser Siemens, enzymatic serum creatinine, eGFRcr based on the updated Schwartz formula. The moderately increased albuminuria was defined as $\mathrm{UACR} \geq 30 \mathrm{mg} / \mathrm{g}$, and decreased GFR defined as eGFRcr $<60 \mathrm{ml} / \mathrm{min} / 1.73 \mathrm{~m}^{2}$.
Results: Among 515 school children (218 males and 297 females), isolated proteinuria, isolated hematuria, isolated urinary tract infection were found in $7.0 \%, 1.6 \%$ and $13.6 \%$ respectively. The prevalence of urinary tract infection combined with proteinuria was $1.4 \%$ and the prevalence of moderately increased albuminuria was $11.8 \%$ (95\% CI: $9.1-14.9 \%$ ). Decreased GFR was found in $0.97 \%$ of children (95\% CI: $0.96-0.98 \%$ ). Conclusions: Urinary abnormalities and altered kidney function are common in asymptomatic school children living in Kinshasa. This finding emphasizes the need to implement an appropriate screening program for early detection and prevention of CKD later in life.

\section{PI-56 THE EFFECTS OF POTASSIUM AND PHOSPHATE BINDERS ADDED TO FIRST AGE FORMULA: AN INTEREST IN INFANTS WITH CHRONIC KIDNEY DISEASE}

Rouba Bechara, Veronique Chambon, Corentin Naud, Bruno Ranchin, Justine Bacchetta

Hospices Civils De Lyon, Lyon, France

Background: In infants with chronic kidney disease (CKD), even more than in older children, nutrition and growth are complex entities. Management of hyperkalemia and hyperphosphatemia is challenging, and the use of potassium and phosphate binders directly in formulas may be useful, avoiding direct ingestion of resins by infants.

Materials and methods: Gallia ${ }^{1} 1^{\text {st }}$ age milk was studied. For $90 \mathrm{~mL}$ of formula, we added either carbonate sevelamer (Renvela ${ }^{2}, 200,400$ or $800 \mathrm{mg}$ ) or polystyrene sulfonate (Resikali ${ }^{\circledR}, 2000,4000$ or $8000 \mathrm{mg}$ ). We also evaluated the combined use of the resins: in $90 \mathrm{~mL}$ we added $200 \mathrm{mg} /$ $2000 \mathrm{mg}, 400 \mathrm{mg} / 4000 \mathrm{mg}$, and $200 \mathrm{mg} / 4000 \mathrm{mg}$ of Renvela ${ }^{\circledR}$ and Resikali ${ }^{\circledR}$, respectively. Milks were decanted for 10 minutes; the supernatants were then weighted and analyzed for osmolarity and $\mathrm{pH}$. After centrifugation sodium, potassium, chlore, bicarbonate, magnesium, glucose, calcium, phosphate, protein, cholesterol, triglycerides, iron, folate, vitamin B12 levels were assessed.

Results: Potassium levels decreased in milk in a dose-dependent manner with polystyrene sulfonate, by 36,52 and $68 \%$, respectively. Polystyrene sulfonate reduced magnesium levels and increased both calcium concentration (from 9.7 to $21 \mathrm{mmol} / \mathrm{L}$ ) and osmolarity (from 359 to 423 $\mathrm{mOsm} / \mathrm{kg}$ ). No effect on phosphate was observed. Sevelamer reduced both calcium and phosphate concentrations, in a dose-dependent manner: by 14,26 and $29 \%$, and by 24,36 and $40 \%$, respectively. Sevelamer increased $\mathrm{pH}$ from 6.9 to 9.1 , and also decreased folate levels by 32,66 and $81 \%$ respectively, from 465 to $88 \mathrm{mmol} / \mathrm{L}$. The combined use of sevelamer and polystyrene sulfonate decreased both potassium (by 45 , 59 and 59\%, respectively), phosphate (by 38,39 and 36\%, respectively), and folate levels, however with a slighter $\mathrm{pH}$ increase. Osmolarity was unchanged. No other relevant differences were observed.

Conclusion: Pretreating formulas by resins is reproducible and simple when specific diets for CKD are not available. However, resins may impact the global composition (osmolarity) and other nutrients (folates).

\section{PI-57 THE SURVEY OF ASSOCIATION BETWEEN PERITONEAL DIALYSIS FLUID CA125 AND PERITONEUM FUNCTION IN PET TEST IN PERITONEAL DIALYSIS PATIENTS}

Sahar Hemmati, Nakisa Hooman

Ali Asghar Hospital, Iran

Abstract: Over time, changes in the peritoneal membrane lead to changes in soluble material transfer and reduced ultrafiltration. Finding a measurable biomarker in peritoneal effluent with the ability to predict the progression of membrane damage or failure is of interest. One of these markers is CA125 antigen, which is considered as a measure of the health and mass of peritoneal lining mesothelial cells. So far, few studies have 
examined this issue in the age group of children. The aim of this study was to evaluate the relationship between dialysate CA125 concentration and peritoneal function in PET and its changes over time.

This is a cross-sectional study conducted in patients under 18 years of age with peritoneal dialysis during the years 2014-march 2019. After selection of patients according to inclusion and exclusion criteria, demographic information, PET test result, serum concentration and dialysis CA125 were collected according to the prepared checklist. To investigate the relationship between dialysis duration and dialysate CA125, patients were divided into two time groups $(\leq 12$ and $>12$ months). Patients undergoing more than two PET tests were examined for longitudinal changes in dialysis concentration of CA125 over time.

75 PET tests were performed during this period. 46 males $(62.2 \%)$ and 29 females $(38.7 \%)$ were studied. The mean and standard deviation of age was $7.81 \pm 4.12$ years. Peritoneal dialysis duration in patients was 3-91 months with mean and standard deviation $27.61 \pm 19.89$ months. None of the patients had peritonitis at the test time and 2 months prior. The dialysis concentration of CA125 protein was $0.6-40.50 \mathrm{U} / \mathrm{ml}$. CA125-AR was significantly higher in patients in the short-term group $(19.29 \pm 16.02$ compared to $11.09 \pm 10.79, \mathrm{P}=0.034$ ) but with time there was no significant change in the peritoneal transfer parameters. There was no association between CA125-AR and the occurrence of peritonitis and EPS, although it was lower in the group with a history of peritonitis or EPS (P $<0.05)$. There was no significant relationship between CA125-AR and $\mathrm{PET}$ test for creatinine and glucose $(\mathrm{P}>0.05)$. There was no significant correlation between dialysate CA125 and CA125-AR in ultrafiltration failure group campare to normal group. In the longitudinal study of patients, the trend of decrease in CA125 and CA125-AR from test 1 to 3 was significant $(\mathrm{P}=0.04$ and $\mathrm{P}=0.013$, respectively).

Conclusion: It can be assumed that in the first few years of peritoneal dialysis, the duration of dialysis has no effect on the level of dialysis CA125. In addition, age, sex, and peritoneal transport characteristics do not appear to affect the level of CA125 dialysis. Changes in peritoneal lining mesothelial cell mass cannot be assessed by cross-sectional determination of CA125. Longitudinal evaluations may be more valuable in the follow-up of patients.

PI-58 CONTINUOUS RENAL REPLACEMENT THERAPY IN LOW WEIGHT PATIENTS: A RETROSPECTIVE STUDY ON NEWBORNS AND INFANTS TREATED WITH CA.R.PE.DI.E.M (CARDIO-RENAL, PEDIATRIC DIALYSIS EMERGENCY MACHINE) IN PADUA UNIVERSITY HOSPITAL

Lisa Cusinato ${ }^{1}$, Dario Gregori ${ }^{2}$, Elisa Benetti ${ }^{1}$, Mattia Parolin ${ }^{1}$

${ }^{1}$ Pediatric Nephrology Dialysis and Transplant Unit, Padua University Hospital, Italy, ${ }^{2}$ Unit of Biostatistics, Epidemiology and Public Health, Dctvph, University of Padova, Italy

Objectives: CRRT in low weight patients is a clinical and technical challenge: Ca.R.Pe.Di.E.M. is one of the first miniaturized dialysis machine dedicated to these patients.

Aim of this study: Identify demographic, clinical and dialytic factors potentially related to mortality and length of stay in Intensive Care Unit (ICU). Methods: A retrospective analysis was performed enrolling patients treated with Ca.R.Pe.Di.E.M. between 01.01.2014 ad 30.09.2020 in University Hospital of Padua. A Random Forest model was implemented for outcomes "mortality" and "length of stay".

Results: 20 patients received 211 CRRT session (75\% CVVH, 25\% CVVHD) in 3033 hours. Their body weight was between 1610-9100g. The median age at ICU admission was 38 days [IQR 9,75-142], median weight at CRRT start 4015g [IQR 3400-4970] with median fluid overload 23\% [IQR $10-28,5]$. Most of patients (75\%) were treated using a $5 \mathrm{Fr}$ CVC (4Fr in $10 \%$, 4.5Fr in 5\% and 6.5Fr in 10\%). Circuits were primed with 5\% albumin in 16 patients; no one received blood priming. Downtime occurred in $2.3 \%$ of total time of treatment; the most common cause was clotting. Survival at CRRT discontinuation and hospital discharge were $95 \%$ and $50 \%$ respectively (4 patients were discharged in peritoneal dialysis and 6 without need of renal replacement therapy). Median ICU length of stay was 7.5 days [IQR 325,75]. A Random Forest model was implemented. Predictors best related with mortality were numbers of ICU days before CRRT, need of inotropic support, fluid overload rate, bicarbonates and haemoglobine level, anticoagulation and dialytic dose. Predictors best related with length of stay were loss of weight rate, $\mathrm{pH}$ and haemoglobin level.

Conclusions: CRRT with Ca.R.Pe.Di.E.M is safe and efficient in a heterogeneous group of low-weight patients, without major complication and significant downtime.

\section{PI-59 CARPEDIEM CONTINUOUS RENAL REPLACEMENT THERAPY FOR VERY LOW BIRTH WEIGHT NEONATES: FIRST EXPERIENCE IN EUROPE}

Julie Bernardor ${ }^{1}$, Giulia Regiroli ${ }^{2}$, Camille Faudeux ${ }^{1}$, Berengere Francois-garret ${ }^{3}$, Sebastien Damez-fontaine ${ }^{2}$, Sergio Eleni Dit Trolli ${ }^{3}$, Daniele De Luca ${ }^{2}$

${ }^{1}$ Department of Pediatric Nephrology, Chu De Nice, Hôpital Archet, Nice, France, ${ }^{2}$ Division of Paediatrics and Neonatal Critical Care, "a. Béclère" Medical Centre, Paris Saclay University Hospitals, Aphp, Paris, France, ${ }^{3}$ Department of Intensive Care and Neonatal Medecine, Chu De Nice, Hôpital Archet, Nice, France

Background: Acute Kidney Injury (AKI) is associated with higher mortality, particularly, in neonates and is a frequent complication (prevalence: $30 \%$ of hospitalized patients). Cardio-Renal Pediatric Dialysis Emergency Machine (C.A.R.PE.DI.E.M.) is a new continuous renal replacement therapy (CRRT) equipment dedicated to neonates and small infants weighting $2.0-9.9 \mathrm{~kg}$.

Methods: We aimed to retrospectively report our experience using CARPEDIEM in very low birth weight and premature neonates treated by continuous venovenous hemofiltration $(\mathrm{CVVH})$. Data are presented as median (25th-75th quartile).

Results: Four neonates (median gestational age: 31 (30-34) SA; median birth weight: 1578 (1162-1986) grams) received 145 hours of CVVH during 8 sessions and using 0.15 and $0.25 \mathrm{~m}^{2}$ hemofilters. CVVH was conducted using a $4.5 \mathrm{Fr}$ and $6.5 \mathrm{Fr}$ double-lumen central vascular access. The median prescribed $\mathrm{Qb}$ was 23 (14-35) $\mathrm{mL} / \mathrm{min}$. Circuits were primed with $\mathrm{NaCl} 0.9 \%$, using heparin anticoagulation (10 to $12 \mathrm{UI} / \mathrm{kg} / \mathrm{h})$ in all patients. Only two sessions were stopped secondary to a filter clotting. CVVH efficaciously allowed blood purification and fluid balance without hemodynamic instability in all neonates. No survival was reported secondary to basal neonate pathology.

Conclusion: CARPEDIEM is feasible, accurate and safe even in preterm neonates; it has the potential enhance the clinical management of very low birth weight neonates with multisystemic organ dysfunction.

\section{PI-60 PLASMA IOHEXOL CLEARANCE FOR RENAL FUNCTION ASSESSMENT IN PEDIATRIC PATIENTS AFTER CARDIAC SURGERY}

Evelyn Dhont ${ }^{1}$, Annick De Jaeger ${ }^{1}$, Kristof Vandekerckhove ${ }^{2}$, Siska Croubels ${ }^{3}$, Evelien Snauwaert ${ }^{4}$, Johan Vande Walle ${ }^{4}$, Peter De Paepe ${ }^{5}$, Pieter De Cock ${ }^{6}$

${ }^{1}$ Pediatric Intensive Care Department, Ghent University Hospital, Belgium, ${ }^{2}$ Pediatric Cardiology Department, Ghent University Hospital, Belgium, ${ }^{3}$ Faculty Of Veterinary Medicine, Ghent University, Belgium, ${ }^{4}$ Pediatric Nephrology Department, Ghent University Hospital, Belgium, ${ }^{5}$ Heymans Institute Of Pharmacology, Ghent University, Belgium, ${ }^{6}$ Pharmacy Department, Ghent University Hospital, Belgium 
Introduction: Identification of patients with altered renal function after cardio-pulmonary bypass is often delayed due to lack of accurate methods for evaluation of glomerular filtration rate (GFR). We compared GFR measurement by the gold standard technique plasma iohexol clearance (mGFRiohex) with estimated GFR based on the routinely used Schwartz formula (eGFRschwartz) in pediatric patients after cardiac surgery.

Material and methods: Children who underwent cardiopulmonary bypass surgery were included. After IV iohexol bolus injection, plasma iohexol clearance was calculated from the plasma concentration-time curve over a 360-minutes interval. Volumetric absorptive microsampling was used for iohexol blood sampling. Pearson's correlation coefficient for mGFRiohex and eGFRschwartz was determined and agreement between the methods was assessed visually using Bland-Altman plots. Accuracy of eGFRschwartz was determined as the percentage of GFR estimates within $\pm 30 \%$ of mGFRiohex $\left(\mathrm{P}_{30}\right)$. Acute Kidney Injury (AKI) was defined as a decrease of GFR of $\geq 25 \%$ of age-specific GFR reference values. Augmented renal clearance (ARC) was defined as a GFR exceeding normal values for age plus two standard deviations.

Results: We included 19 patients with a median age of 2 months (range 0.13-135 months) after closure of atrial septum defect $(n=1)$, ventricular septum $\operatorname{defect}(n=3)$, atrio-ventricular septum defect $(n=3)$, arterial switch procedure $(n=8)$, ROSS procedure $(n=1)$, Norwood procedure $(n=1)$, coronary surgery $(n=1)$ and truncus arteriosus repair $(n=1)$. Median mGFRiohex was $62 \mathrm{ml} / \mathrm{min} / 1.73 \mathrm{~m} 2$ (range 29-231). Median eGFRschwartz was $63 \mathrm{ml} / \mathrm{min} / 1.73 \mathrm{~m} 2$ (range $34-193$ ). $21 \%$ and $11 \%$ of patients presented with AKI according to mGFRiohex and eGFRschwartz respectively. With mGFRiohex 4 patients $(21 \%)$ were identified as having ARC versus 3 patients (16\%) with eGFRschwartz. eGFRschwartz correlated well with mGFRiohex $(r=0,83)$, however, accuracy was poor with $\mathrm{P} 30=53 \%$.

Conclusions: Widely used eGFRschwartz fails to identify a significant part of children with AKI after cardiac surgery. mGFRiohex could offer a feasible and safe alternative to accurately evaluate renal function in these vulnerable patients.

\section{PI-61 RISK FACTORS FOR ACUTE KIDNEY INJURY IN PEDIATRIC INTENSIVE CARE UNIT}

\section{Kubra Celegen ${ }^{1}$, Mehmet Celegen ${ }^{2}$ \\ ${ }^{1}$ Afyonkarahisar Health Sciences University, Pediatric Nephrology Department, Turkey, ${ }^{2}$ Afyonkarahisar Health Sciences University,} Pediatric Intensive Care Unit, Turkey

Introduction: Acute kidney injury (AKI) is one of the common risk factors for prolonged hospitalization and increased mortality in critically ill patients. The incidence of AKI was reported as $26.9 \%$. Underlying diseases, inotropic requirement, multi-organ failure, and the use of nephrotoxic treatments are associated with in-hospital AKI. In this study, we aimed to evaluate in-hospital AKI rate and risk factors for AKI

Material and methods: All pediatric patients admitted to the intensive care unit between January-2020 and March-2021 were included. Patients with primary kidney disease and hospitalization $\leq 24$ hours were excluded. Demographics, clinical features, treatments, PRISM II and PELOD scores were compared AKI and non-AKI group. Acute kidney injury (AKI) was defined based on KDIGO 2012 creatinine criteria.

Results: 142 patients were included to analyses. AKI was developed in 19 patients (13.3\%) during hospitalization median on 3.5 days. Six patients $(31.6 \%)$ had stage 1,7 patients $(36.8 \%)$ stage 2 , and 6 patients $(31.6 \%)$ stage 3 AKI. AKI was more common in patients with sepsis (p $<0.001)$ and patients with a diagnosis of malignancy $(\mathrm{p}=0.007)$. Inotrope requirement, mechanical ventilation support, and PRISM II and PELOD scores were higher in AKI group. Mortality rate was higher in patients who developed AKI $(p<0.001)$. Use of diuretics, vancomycin/aminoglycoside, blood products, and contrast agents was found to be significantly higher in AKI group. In the multivariate logistic regression analysis adjusted for PRISM II, sepsis and malignancy diagnoses, the use of diuretics (OR: 16.4; $\mathrm{p}=0.007$ ) and vancomycin/ aminoglycoside (OR: $10.5 ; \mathrm{p}=0.018$ ) were remained their significance.

Conclusions: The use of diuretic and nephrotoxic antibiotics are independent risk factors for AKI in critically ill children. Preventive strategies such as drug blood level monitoring and avoiding redundant diuretics are necessary to reduce the risk of AKI and mortality.

\section{PI-62 REGIONAL CITRATE ANTICOAGULATION IN PEDIATRIC CRRT}

Andrea Cappoli ${ }^{1}$, Raffaella Labbadia ${ }^{1}$, Emanuele Rossetti ${ }^{2}$, Gabriella Bottari $^{2}$, Isabella Guzzo ${ }^{1}$

${ }^{1}$ Division of Nephrology and Dialysis, Department of Pediatric Subspecialties, Irccs Bambino Gesù Pediatric Hospital, Rome, Italy, ${ }^{2}$ Department of Pediatric Intensive Care, Ospedale Pediatrico Bambino Gesù-ircc, Rome, Italy

Introduction: Acute kidney injury (AKI) is highly prevalent in hospitalized children, especially those in pediatric intensive care unit (PICU). Continuous renal replacement therapy (CRRT) is the treatment of choice in critically ill children with AKI but needs an anticoagulation of the extracorporeal circuit to prevent filter clotting. Regional citrate anticoagulation (RCA) is widely reported in adults, but pediatric data are still incomplete.

Material and methods: We have treated twenty-one critically ill children admitted in pediatric intensive care units with CRRT using RCA. Mean age was $56,6 \pm 71,1$ months with mean body weight of $18,42 \pm 21,6 \mathrm{~kg}$. Severe AKI, intended as stage $3 \mathrm{KDIGO}$, was present in most of the patients $(52.3 \%)$. Citrate dose adjustments were made by controlling the calcium in the circuit with a desired target, while calcium infusion was based on the ionized calcium of the patients. Filter life was recorded.

Results: Mean filter lifetime was $51.9 \pm 19.4$ hours with $62.9 \%$ of circuits lasting more than 48 hours and $38.2 \%$ of circuits lasting more than 70 hours. In our experience, metabolic acidosis was more common than metabolic alkalosis, the most common metabolic complication was hyponatremia (defined as $\mathrm{Na}<135 \mathrm{mmol} / \mathrm{L}$ ), reflecting more the clinical conditions of these patients rather than complications of RCA. We found only a few episodes of hypocalcaemia, never clinically relevant, and treated with changes in calcium compensation. Only two patients presented citrate accumulation (CA), defined as a calcium ratio $>2.5$, but neither of them presented clinical signs of CA with serum bicarbonate and lactate in order. Survival rate of our patients was $62 \%$. No patient developed treatment-related bleeding complications.

Conclusions: In our experience regional citrate anticoagulation was safe and effective in preventing circuit clotting. RCA can be used for CRRT in children, even in the ones with very low body weight.

\section{PI-63 EXAMINATION OF PATIENTS WITH ACUTE KIDNEY INJURY IN THE PEDIATRIC INTENSIVE CARE UNIT}

Burcu Gizem Teber ${ }^{1}$, Leyla Telhan ${ }^{2}$, Seçil Kezer ${ }^{3}$, Cihangir Akgün ${ }^{3}$, Önder Yavașcan ${ }^{3}$

${ }^{I}$ Istanbul Medipol University, Faculty of Medicine, Department of Pediatrics, Turkey, ${ }^{2}$ Istanbul Medipol University, Faculty of Medicine, Department of Pediatric Intensive Care, Turkey, ${ }^{3}$ Istanbul Medipol University, Faculty of Medicine, Department of Pediatric Nephrology, Turkey

Introduction: Acute kidney injury (AKI), has negatively impacting the mortality and morbidity of patients in pediaric intensive care units. We aimed to investigate AKI causes, risk factors and factors affecting survival in children while being monitored in the pediatric intensive care unit (PICU). 
Material and methods: 328 patients, who were followed up in the PICU between January 2018 and March 2021 for various reasons, were examined retrospectively. $43(13,1 \%)$ patients who diagnosed with AKI without prior chronic kidney injury were included in the study. Results of demographic data, primary diagnosis, AKI causes, risk factors and Pediatric Risk of Mortality (PRISM III) scores of surviving and deceased were compared.

Results: 15 of AKI patients (34,9\%) were girls. The mean age was $6.18 \pm$ 5.61 (median: 5) years. On the first day of hospitalization in PICU, PRISM III scores were $19.48 \pm 9.65$ (median: 19). Acute lymphoblastic leukemia was the most common primary disease with 7 patients $(16,2 \%)$. Median PICU length of stay was 10 days. We found that $30(89,7 \%)$ patients developed AKI while being followed up with a mechanical ventilator and $28(65,1 \%)$ of these patients used nephrotoxic drugs before AKI developed. It was found that $17(39,5 \%)$ cases developed oliguria and/or anuria, and $36(83,7 \%)$ patients developed multiple organ failure. Dialysis treatment was applied to $8(18,6 \%)$ patients and continuous dialysis treatment methods were preferred in all of them. Median duration of dialysis time was 3,5 days. $28(65,1 \%)$ patients died. PRISM scores, sepsis and dialysis requirement were found to be statistically significantly higher in deceased than surviving patients (respectively $\mathrm{p}=0.001$, $\mathrm{p}=0.001, \mathrm{p}=0.001$ ).

Conclusions: Despite technological advances, AKI is an important cause of mortality and morbidity in critically ill patients. PRISM scoring and the presence of sepsis can be considered as an important determinant in predicting mortality and acting early for the dialysis decision.

\section{PI-64 ACUTE KIDNEY INJURY POST CARDIAC SURGERY IN INFANTS AND CHILDREN: A SINGLE CENTER EXPERIENCE IN A DEVELOPING COUNTRY}

Bilal Aoun ${ }^{1}$, Sami Sanjad ${ }^{1}$, Ghadi Abu Daher ${ }^{2}$, Karim N. Daou ${ }^{1}$, Hani Tamim ${ }^{3}$, Issam El Rassi ${ }^{1}$, Mariam Arabi ${ }^{5}$, Jad Degheili ${ }^{1}$, Mohamed Khaled $^{7}$, Marianne Majdalani ${ }^{2}$

${ }^{I}$ Department Of Pediatrics And Adolescent Medicine, Division Of Pediatric Nephrology, American University Of Beirut Medical Center, Beirut, Lebanon, ${ }^{2}$ Department Of Pediatrics And Adolescent Medicine, Division Of Pediatric Intensive Care Unit, American University Of Beirut Medical Center, Beirut, Lebanon, ${ }^{3}$ Biostatistics Unit, Faculty Of Medicine, Clinical Research Institute, American University Of Beirut, Beirut, Lebanon, ${ }^{4}$ Division Of Cardiothoracic Surgery, Department Of Surgery, American University Of Beirut Medical Center, Beirut, Lebanon, 5 Department Of Pediatrics And Adolescent Medicine, Division Of Pediatric Cardiology, American University Of Beirut Medical Center, Beirut, Lebanon, ${ }^{6}$ Division Of Urology, Department Of Surgery, American University Of Beirut Medical Center, Beirut, Lebanon, ${ }^{7}$ Hospital Of Montfermeil,department Of Pediatrics And Adolescent Medecine, Montfermeil,ile-de-france, France

Introduction: The incidence of acute kidney injury (AKI) in pediatric patients following cardiac surgery varies between $15 \%$ and $64 \%$, with a mortality rate of $10-89 \%$ among those requiring dialysis. This variation in the incidence and mortality of AKI across studies are due to the inconsistent definitions used for AKI.

Material and methods: We evaluated the incidence of AKI using the KDIGO criteria in 150 infants and children undergoing cardiac surgeries between 2015 and 2017. Post operatively, all patients were admitted to the Pediatric Intensive Care Unit (PICU) at a tertiary care center in a developing country. This is a retrospective chart review in which data collected included: age, gender, type of heart disease, prior cardiac surgeries, RACHS-1 category, and pre- and post-operative creatinine levels. Neonates were not included in this study.

Results: Six percent of the studied patients were below one year of age, $84 \%, 1-10$ years and $10 \%, 10-18$ years. Fourteen patients $(9.3 \%)$ developed AKI. Patients with cyanotic heart disease were more prone to develop AKI (78\%) compared to those with noncyanotic heart disease (44\%). Children with AKI had a higher length of stay in PICU, $2.56 \pm$ 1.44 vs $4 \pm 2.66$ (p- 0.02). Serum lactic acid was higher in patients who developed AKI with a mean value of $6.8 \pm 6.9$ vs. $2.85 \pm 1.55$ in the nonAKI group (p- 0.03). Lower hemoglobin levels and hyperlactic acidemia were significantly more prevalent in the AKI group. There were 5 death in this series $(3.3 \%)$ and 4 of those $(80 \%)$ were in the AKI group.

Conclusions: Using KDIGO criteria, the incidence of AKI in infants and children following cardiac surgery was $9.3 \%$. This is slightly lower than previously published studies which range between $15 \%$ and $64 \%$. Children with cyanotic cardiac disease, hyperlactic acidemia and anemia were more prone to develop AKI. Identifying patients at risk might help in decreasing the risk of postoperative AKI.

\section{PI-65 URINE NGAL IN THE ASSESSMENT OF EARLY TUBULAR DAMAGE IN CHILDREN UNDERGOING HEMATOPOIETIC STEM CELL TRANSPLANTATION - A PILOT STUDY}

\author{
Kinga Musial ${ }^{1}$, Krzysztof Kalwak ${ }^{2}$, Marek Ussowicz ${ }^{2}$, \\ Danuta Zwolinska ${ }^{1}$ \\ ${ }^{1}$ Wroclaw Medical University, Department of Pediatric Nephrology, ${ }^{2}$ \\ Wroclaw Medical University, Department of Bone Marrow \\ Transplantation, Pediatric Oncology and Hematology, Poland
}

Introduction: Allogeneic hematopoietic stem cell transplantation (alloHSCT) is often complicated by acute kidney injury (AKI). Intensive hydration and forced diuresis effectively diminish the risk of AKI, yet modify the conditions of glomerular filtration evaluation. Thus, the search for early markers of tubular dysfunction, preceding changes in serum creatinine or eGFR, continues. The aim of the study was to analyze the serum and urine concentrations of cystatin C, NGAL and IL-18 as potential new markers of tubular damage in children before HSCT and in the early post-transplantation period.

Material and methods: The study was performed on 27 children undergoing alloHSCT and 20 healthy controls. eGFR values were counted based on Schwartz formula. Serum and urine cystatin C, NGAL and IL-18 concentrations were assessed by ELISA before HSCT, 24h, 2 and 4 weeks after transplantation.

Results: Serum creatinine values remained within normal range throughout the whole observation period. Most of the patients presented with hyperfiltration. Serum and urine concentrations of cysC, NGAL and IL18 were elevated vs. controls before alloHSCT. The serum values of all parameters kept increasing until the 4th week after transplantation. Urine NGAL values increased 24h after alloHSCT, remained elevated until the 2nd week, then decreased to the values lower than before alloHSCT. Urine cystatin $\mathrm{C}$ values became elevated 2 weeks after alloHSCT and decreased by the 4th week, yet remained higher than before the procedure. The raise of urine IL-18 values was also delayed by 2 weeks, although by the 4 th week it decreased below the pre-transplantation records.

Conclusions: Tubular damage is present already before alloHSCT and aggravates after the procedure. Urine markers are more specific in the assessment of tubular function than the corresponding serum concentrations. Urine NGAL seems the most promising parameter in the early detection of tubular damage, although further investigation on a larger group is required.

\section{PI-66 INVESTIGATING N-TERMINAL PRO B-TYPE NATRIURETIC PEPTIDE CONCENTRATIONS IN PAEDIATRIC PATIENTS ON HAEMODIALYSIS}

Nabil Melhem, Sarah Roy, Heidi Ramsey, Manish Sinha

Evelina London Childrens Hospital 
Introduction: N-terminal pro B-type natriuretic peptide (NTproBNP) is a biological marker of ventricular dysfunction secondary to fluid overload. Few data describe NT-proBNP concentrations and its potential utility for intravascular fluid assessments in the paediatric haemodialysis population. We describe demographic and clinical associations of NT-proBNP concentrations in children on established haemodialysis (HD).

Material and methods: Retrospective cohort study including all prevalent children on $\mathrm{HD}$, with preserved cardiac function aged $<18 \mathrm{yrs}$ between 2018 and 2019. Stepwise multiple regression analysis was performed to assess potential predictors of post-dialysis $\operatorname{logNT}$-proBNP and repeated measures ANOVA performed to analyse change in concentrations from baseline to 3 and 6 months.

Results: 24 of 31 children [14 (58\%) white-Caucasian], mean age 10.3 $\pm 5.8 \mathrm{yrs}$ of whom $16(67 \%)$ were boys; 8 (33\%) had congenital abnormalities of kidneys and urinary tract with $16(66.7 \%)$ polyuric and remainder oligo-anuric $(<150 \mathrm{ml}$ per day) at baseline.

Amongst all participants, there were 161 post-HD measurements with median (IQR) NT-proBNP 2046 (794, 5275) ng/L and following log transformation normalised data with mean $\operatorname{logNT}$-proBNP 3.34 $\pm 0.58 \mathrm{ng} / \mathrm{L} .130(80.7 \%)$ had an elevated of NT-proBNP defined as a concentration above $598 \mathrm{ng} / \mathrm{L}$ as previously described in a non-renal population. Multiple regression analysis identified post-dialysis systolic BP $\mathrm{z}$-score $(\beta=0.15, \mathrm{P}<0.01)$, haemoglobin $(\beta=0.01, \mathrm{P}<0.01)$ and non-White ethnicity $(\beta=0.10, p=0.02)$ as positive predictors of $\operatorname{logNT}$-proBNP, whilst residual urine output of $>150 \mathrm{mls}(\beta=-0.28, \mathrm{P}<0.01)$ was a negative predictor of $\operatorname{logNT}$-proBNP concentrations in this population.

On longitudinal assessment, the mean $\operatorname{logNT}$-proBNP at baseline, 3 and 6 months were $3.32 \pm 0.47 \mathrm{ng} / \mathrm{L}, 3.23 \pm 0.55 \mathrm{ng} / \mathrm{L}$ and $3.19 \pm 0.61 \mathrm{ng} / \mathrm{L}$ respectively $(\mathrm{p}=0.50)$.

Conclusions: Elevated NT-proBNP were observed in the majority of children on haemodialysis with preserved cardiac function with a dominant effect of blood pressure and residual urine output. These data demonstrate both modifiable and non-modifiable risk associations with higher NT-proBNP and highlight potential clinical relevance when fine-tuning the clinician's intravascular fluid assessments for children on chronic haemodialysis.

\section{PI-67 FGF23 CORRELATION WITH GLOMERULAR FILTRATION RATE, MEASURED WITH A GOLD- STANDARD TECHNIQUE IN CHILDREN WITH CHRONIC KIDNEY DISEASE}

\begin{abstract}
Atessa Bahadori ${ }^{1}$, Alexandra Goischke ${ }^{2}$, Carole Grasset Salomon ${ }^{3}$, Cyril Jaksic ${ }^{4}$, Christophe Combescure ${ }^{4}$, Alexandra Wilhelm-Bals ${ }^{1}$, Paloma Parvex ${ }^{1}$

${ }^{1}$ Pediatric Specialties Division, Geneva University Hospitals (hug), Geneva, Switzerland, ${ }^{2}$ Nephrology Department, University Children's Hospital (ukbb), Basel, Switzerland, ${ }^{3}$ Pediatric Clinical Research Platform, Department Of Woman-children-teenagers, Geneva University Hospitals (hug) And Faculty Of Medicine, Geneva, Switzerland, ${ }^{4}$ Division Of Clinical Epidemiology, Geneva University Hospitals (hug) And Faculty Of Medicine, Geneva, Switzerland
\end{abstract}

Objectives: Prognosis of chronic kidney disease (CKD) is closely related to early diagnosis and initiation of nephroprotective measures. Fibroblast Growth Factor 23 (FGF23) is well described as an early marker that increases proportionally to worsening CKD stages in adults. The aim of this study is to investigate the association between FGF23 and glomerular filtration rate (GFR) in children.

Methods: Children aged 5 to 20 years old requiring kidney function assessment following primary nephropathy or solid organ transplantation were recruited prospectively in a tertiary hospital of Switzerland for a gold-standard measurement of their GFR. Plasma FGF23, parathormone, proteinuria, all susceptible to worsen $\mathrm{CKD}$ prognosis, were measured simultaneously.

Results: 119 clearances in 91 children (63\% males) were collected. 51\% had primary nephropathy, $37 \%$ had solid organ transplantation. $26 \%$ of children had stage I CKD, 39\% stage II and 26\% stage III or IV. FGF23 was significantly higher in patients with stages III or IV CKD (mean 256.09 $\mathrm{UI} / \mathrm{ml} \pm 155.34$ ) versus stage I (mean $168.22 \mathrm{UI} / \mathrm{ml} \pm 180.75 ; \mathrm{p}=0.045$ ) or stage II (mean $138.82 \mathrm{UI} / \mathrm{ml} \pm 107.43 ; \mathrm{p}<0.001$ ). Protein/creatinine urinary ratio $(\mathrm{P} / \mathrm{C}$ ratio) and parathormone increased significantly with $\mathrm{CKD}$ stage (respectively $\mathrm{P}<0.001$ ). There was a moderate inverse association between FGF23 and GFR ( $\mathrm{r}=-0.373$, confidence interval (CI): -0.550 to -0.164 ). This association was stronger in patients with primary nephropathy $(\mathrm{r}=-$ 0.523 , CI: -0.729 to -0.230$)$. FGF23 was correlated with $\mathrm{P} / \mathrm{C}$ ratio $(\mathrm{r}=0.451, \mathrm{CI}: 0.242-0.621)$ and parathormone levels ( $\mathrm{r}=0.430$, CI: 0.220 $0.602)$. FGF23 discriminated CKD stages I-II from III-IV with an area under ROC curve of 0.857 (95\% CI: 0.768-0.945).

Conclusions: FGF23 is a potentially good predictor of CKD stages in children and is significantly associated with decreasing GFR. Further studies are needed to evaluate FGF23 as a marker of CKD progression

\section{PI-68 ALUMINUM, LEAD AND CADMIUM TOXICITY AND THEIR IMPACT IN HEMATOLOGICAL COMPLICATIONS IN DIALYZED PATIENTS}

Popa Doriana Maria ${ }^{1}$, Aldea Cornel Olimpiu ${ }^{2}$, Aldea Andreea Alexandra ${ }^{4}$, Moldovan Mărioara ${ }^{3}$, Prodan Doina ${ }^{3}$, Perhaiță Ioana ${ }^{3}$, Delean Dan ${ }^{2}$, Bulată Bogdan $^{2}$, Borzan Cristina ${ }^{1}$, Răchișan Andreea Liana ${ }^{2}$

${ }^{1}$ Department Of Public Health, University Of Medicine \& Phramacy "iuliu Hatieganu”, 31 Avram Iancu Street, Fl 3, 40083, Cluj-Napoca, Romania, ${ }^{2}$ Department Of Nephrology-2nd Pediatric Department, Clujnapoca, Romania, 5 Crisan Street, 400177, Cluj-Napoca, Romania, ${ }^{3}$ Department Of Polymer Composites, Babes-bolyai University, Institute Of Chemistry Raluca Ripan, 30 Fantanele Street, 400294, Cluj-Napoca, Romania, ${ }^{4}$ Neurology And Pediatric Neurology Department, University Of Medicine \& Phramacy "iuliu Hatieganu”, 46-50, Viilor Street, 40066, Cluj Napoca, Romania

Background: End Stage Renal Disease (ESRD) patients despite receiving adequate extra-renal epuration are developing significant long-term complications. Abnormalities in the plasmatic heavy metals levels may potentiate the occurrence of these complications. Hence, the present study was undertaken to evaluate the plasmatic trace elements in patients receiving extra-renal epuration. The specific aim of our work was to study the blood levels of aluminium ( $\mathrm{Al})$, lead $(\mathrm{Pb})$ and cadmium $(\mathrm{Cd})$ among ESRD patients undergoing haemodialysis (HD) and peritoneal dialysis (PD) and their impact in red blood cells, plasmatic iron and transferrin homeostasis.

Materials and methods: It was a longitudinal prospective study done in a tertiary care nephrology center. We enrolled 15 patients (female $/$ male $=9 / 6)$ with a mean age of $12.80 \pm 4.51$ years old undergoing dialysis (HD and PD) for more than six months. Patients who had history of occupational exposure to heavy metals were excluded from the study. About $5 \mathrm{ml}$ of fasting venous blood sample was obtained and analysed for trace elements. To asses the level of trace elements we performed 3 determinations for each patient in predialytic and postdialytic sessions.

Results: There was no difference in the mean values for $\mathrm{Al}, \mathrm{Pb}$ and $\mathrm{Cd}$ in pre and post dialytic blood samples. There was a positive correlation for $\mathrm{Al}$ and red blood cells ( $\mathrm{r}=0.433, \mathrm{p}$ 0.006); $\mathrm{Al}$ and transferrin $(\mathrm{r}=0.424, \mathrm{p}$ $0.011)$. A negative correlation was found concerning the $\mathrm{Pb}$ and transferrin $(r=-0.402$, p 0.03), and Cd and transferrin $(r=-0.487, \mathrm{p} 0.009)$.

Conclusions: Since high levels of trace elements are potentially harmful, the hypothesis that trace elements level can influence the iron and transferrin status is worthy of investigation. 
Keywords: trace elements, chronic kidney disease, dialysis, hematologic disturbances

\section{PI-69 ATTAINMENT OF KDIGO 2012 CLINICAL PRACTICE GUIDELINE TARGETS FOR ANAEMIA MANAGEMENT IN CHILDREN WITH PRE-DIALYSIS CKD, A SINGLE CENTRE STUDY}

Harriet Gass, Caroline Booth, Jo Clothier, Pernille Rasmussen, Nabil Melhem

Evelina London Children's Hospital, United Kingdom

Introduction: Anaemia is an independent risk factor for hospitalisation and cardiovascular disease in children with CKD. We report on the attainment of KDIGO (Kidney Disease: Improving Global Outcomes) guideline targets for the management of anaemia in the paediatric predialysis CKD population.

Material and methods: Single centre, retrospective cross-sectional study including all prevalent children aged 0-18 years with pre-dialysis advanced CKD in a dedicated clinic. Anaemia was defined as per the KDIGO 2012 clinical practice guideline for anaemia management in patients with chronic kidney disease. Modifiable and non-modifiable covariates were analysed for their associations with anaemia in this group. Results: 137 children were included with a mean age of $9.2 \pm 5.1$ years and a mean eGFR of $32.6 \pm 21.1 \mathrm{ml} / \mathrm{min} / 1.73 \mathrm{~m}^{2} .4 \%, 47 \%, 37 \%$ and $12 \%$ had stages of CKD 2, 3, 4 and 5 respectively. 90 (65.6\%) were boys, and $103(75.1 \%)$ had congenital abnormalities of kidneys and urinary tract (CAKUT). Mean length of time in the dedicated CKD clinic was $5.3 \pm 4.4$ years.

$46(33.6 \%)$ were classified as anaemic, of which $31(67.4 \%)$ were on iron supplementation and/or erythropoiesis-stimulating agent (ESA) treatment. 46 (33.6\%) maintained target haemoglobin levels with iron supplementation and/or ESA treatment and $45(32.8 \%)$ maintained target haemoglobin levels with no treatment. Non-CAKUT primary kidney disease (PKD), lower eGFR and higher parathyroid hormone (PTH) were identified as covariates significantly associated with anaemia in this population $(\mathrm{p}<0.05)$.

Conclusions: In a population of children with advanced CKD, we have shown that whilst the majority achieved target hemoglobin levels, a significant proportion were anaemic despite most receiving iron and/or ESA therapy. Non-CAKUT PKD, lower eGFR and higher PTH were associated with anaemia, and these factors should warrant further attention from clinicians. Strategies are needed to identify and overcome barriers to achieving target haemoglobin levels in children with CKD.

\section{PI-70 RENAL OUTCOMES IN A PAEDIATRIC COHORT OF AUTOSOMAL DOMINANT POLYCYSTIC KIDNEY DISEASE: WHEN SHOULD THE FOLLOW-UP START?}

Joana Simões ${ }^{1}$, Mafalda Crisóstomo ${ }^{1}$, Rute Baptista ${ }^{1}$, Diogo Rodrigues ${ }^{1}$, Catarina Borges ${ }^{1}$, Marisa Cruz ${ }^{2}$, Telma Francisco ${ }^{1}$, Raquel Santos ${ }^{1}$, Ana Paula Serrão ${ }^{1}$, Gisela Neto ${ }^{1}$, Margarida Abranches ${ }^{1}$

${ }^{1}$ Pediatric Nephrology Unit, Hospital Dona Estefânia, Centro Hospitalar Lisboa Central, Portugal, ${ }^{2}$ Genetics Department, Hospital Dona Estefânia, Centro Hospitalar Lisboa Central, Portugal

Introduction: Autosomal-dominant polycystic kidney disease (ADPKD) affects nearly 12.5 million people worldwide, with prevalence estimates between 1 in 400-2500. Most patients develop symptomatic disease in adult age, but $2-5 \%$ of all ADPKD patients present during childhood. Data on the natural history and prognostic factors for childhood ADPKD are lacking.
Methods: Longitudinal retrospective study from 2009 to 2020. Logistic regression model to evaluate predictive factors for renal injury markers. Results: Data from 59 children and adolescents with ADPKD were analysed, $34(57.6 \%)$ boys and $25(42.4 \%)$ girls. From the 37 who completed genetic testing, 32 had mutations in ADPKD-related genes: $26(70.3 \%)$ in PKD1, $2(5.4 \%)$ in PKD2, $2(5.4 \%)$ in PKD1/TSC associated with Tuberous Sclerosis Complex and 2 $(5.4 \%)$ in GANA $\beta$. Thirty-six $(61.0 \%)$ patients met at least one renal outcome: $13(22.0 \%)$ hypertension, $10(17.0 \%)$ albuminuria, $22(37.3 \%)$ reduced glomerular filtration rate (GFR) classifying to stage $2 \mathrm{CKD}$; of these, $10(16.9 \%)$ were obese or overweight. Thirteen $(22.0 \%)$ had at least one episode of hematuria and 5 $(8.5 \%)$ presented with hyperuricemia. Median ages of onset for renal outcomes were 12.0 years old for hypertension, 12.5 for albuminuria, 13.0 for hematuria, 14.5 for decreased GFR and 15.0 for hyperuricemia. Obesity/overweight was a predictive factor for hypertension $(\mathrm{p}=0.03)$, but not for decreased GFR $(\mathrm{p}=0.94)$, albuminuria $(p=0.48)$ or for the composite renal outcome $(p=0.24)$.

Conclusions: More than half of the patient cohort met the composite renal outcome at the age of 12 . Nearly one fifth of the patients were obese or overweight, which was found to be the only predictive factor for hypertension, although not predictive of the other studied renal outcomes. While screening and follow-up of adult patients' relatives during childhood remains controversial, these results support the need to identify and monitor these patients closely since childhood.

\section{PI-71 SYSTEMATIC EVALUATION OF OLFACTION IN PATIENTS WITH HEREDITARY CYSTIC KIDNEY DISEASES/RENAL CILIOPATHIES}

Mareike Dahmer-Heath ${ }^{1}$, Valentin Schriever ${ }^{2}$, Sabine Kollmann ${ }^{1}$, Carolin Schleithoff ${ }^{1}$, Andrea Titieni ${ }^{1}$, Metin Cetiner ${ }^{3}$, Ludwig Patzer ${ }^{4}$, Burkhard Tönshoff ${ }^{5}$, Matthias Hansen ${ }^{6}$, Petra Pennekamp ${ }^{1}$, Joachim Gerß $^{7}$, Martin Konrad ${ }^{1}$, Jens König ${ }^{1}$

${ }^{1}$ University Childrens Hospital, Department Of General Pediatrics, Universitätsklinikum Münster, Münster, Germany, ${ }^{2}$ Abteilung für Neuropädiatrie, Medizinische Fakultät Carl Gustav Carus, Dresden, Germany, ${ }^{3}$ Department of Pediatric Nephrology, Pediatrics Ii, University Of Duisburg-essen, Essen, Germany, ${ }^{4}$ Childrens Hospital St. Elisabeth and St. Barbara, Halle (Saale), Germany, ${ }^{5}$ Department of Pediatrics I, University Childrens Hospital Heidelberg, Heidelberg, Germany, ${ }^{6}$ Clementine Kinderhospital, Frankfurt Am Main, Germany, Institute of Biostatistics And Clinical Research, University of MÜnster, MÜnster, Germany

Introduction: One of the perceptions cilia are involved in is the sense of smell. Motile cilia are equipped with odorant receptors and play a critical role in odor transmittance and detection. As a consequence, genetic defects disrupting the functional or structural integrity of these cilia may cause olfactory dysfunction. One of the ciliopathies that has already been associated with hyposmia is Bardet-Biedl syndrome (BBS). BBS is a multivisceral syndromic disorder caused by genetic defects located at the ciliary base. However, reports of olfactory dysfunction in other hereditary kidney diseases that are also caused by mutations in genes located at the cilium (nephronophthisis, polycystic kidney diseases) are sparse. Here we provide a systematic survey on olfaction in a large cohort of children, adolescents and adults displaying genetically determined ciliopathies.

Material and methods: We performed a match- controlled systematic olfactory evaluation in a group of 75 patients with a defined genetic background using age adapted and validated odour identification tests. 
Results: Identification tests revealed a significant olfactory deficit in patients carrying TMEM67 variants $(n=4)$, while all other genetic disorders causing nephronophthisis $(n=25)$ or polycystic kidney disease $(n=18)$ were not associated with an impaired sense of smell. In patients suffering from BBS, olfactory performance was depending on the underlying molecular defect. While defects in the BBS1 gene $(n=9)$ had no impact on the sense of smell, all other BBS gene disorders $(n=19)$ were associated with significant hyposmia. Noteworthy, there was no correlation of the olfactory deficit with the level of renal impairment.

Conclusions: Hyposmia is a critical clinical feature of most but not all (except for BBS1) genetically determined BBS patients. Furthermore, $\mathrm{COACH}$ syndrome caused by mutations in the TMEM67 gene was found to display significant olfactory impairment, a feature that has not been reported as part of the clinical spectrum so far.

\section{PI-72 PROCALCITONIN SERUM LEVELS IN STAGE 5 CHRONIC KIDNEY DISEASE CHILDREN ON HEMODIALYSIS}

Antoine Mouche ${ }^{1}$, Cyrielle Parmentier ${ }^{1}$, Claire Herbez Rea ${ }^{1}$, Theresa Kwon ${ }^{2}$, Olivia Boyer ${ }^{3}$, Jean Daniel Delbet ${ }^{1}$, Tim Ulinski ${ }^{1}$

${ }^{1}$ Pediatric Nephrology Unit, Armand Trousseau Hospital, Aphp, Dmu Origyne, Sorbonne Universite, 75012, Paris, France, ${ }^{2}$ Pediatric Nephrology Unit, Robert Debre Hospital, Aphp, Paris, France, ${ }^{3}$ Pediatric Nephrology Unit, Necker Enfants Malades Hospital, Aphp, Paris, France

Introduction: Infections are responsible for morbidity and mortality in children on hemodialysis (HD). Procalcitonin (PCT) is rarely used in this population, even though it is an efficient biomarker of infection and sepsis. Our aim was to study PCT baseline level in uninfected children with stage 5 chronic kidney disease (CKD 5) on $\mathrm{HD}$, and determine how to use it in this population.

Material and methods: Prospective observational study including 40 uninfected children on classical HD or hemodiafiltration (HDF) in three pediatric HD centers in the Paris region. PCT was monitored before and after 3 consecutive sessions within 1 week.

Results: Median pre-dialysis PCT was $0.60 \mathrm{ng} / \mathrm{mL}$ [0.36-1.15], median post-dialysis PCT was $0.23 \mathrm{ng} / \mathrm{mL}$ [0.10-0.47], PCT reduction rate was $59.8 \%$ [37.5-75.8]. Seventy percent of pre-dialysis PCT were $<1 \mathrm{ng} / \mathrm{mL}$. Anuric patients had higher pre-dialysis PCT than those with residual urine output $(0.70[0.42-1.30]$ vs. $0.48[0.30-0.93] \mathrm{ng} / \mathrm{mL}, \mathrm{p}=0.01)$. HDF was more efficient than HD to clear PCT during sessions (reduction rate $75 \%$ $[67-80]$ vs. 37 [31-50], $\mathrm{p}<0.001)$.

Conclusions: PCT levels in pediatric HD patients without infection are higher than normal, but this increase is relatively moderate compared to massive increases of PCT in children with bacterial infections on HD. If PCT is measured after dialysis sessions, the specific technique-dependent reduction rates should be taken into consideration. Moderately increased PCT levels around $2 \mathrm{ng} / \mathrm{ml}$ should be interpreted with caution; however, higher PCT serum levels can be used to motivate rapid start of antibiotic treatment in pediatric HD patients.

\section{PI-73 CREATININE-BASED GFR-ESTIMATING EQUATIONS IN CHILDREN WITH OVERWEIGHT AND OBESITY}

\author{
Mark J.C.M. Van Dam ${ }^{1}$, Hans Pottel ${ }^{2}$, Anita C.E. Vreugdenhil ${ }^{1}$ \\ ${ }^{1}$ Centre for Overweight Adolescent and Children's Healthcare (Coach), \\ Department of Pediatrics, School of Nutrition and Translational \\ Research In Metabolism (Nutrim), Maastricht University Medical \\ Centre +, Maastricht, The Netherlands, ${ }^{2}$ Department of Public Health \\ And Primary Care, Ku Leuven Campus Kulak Kortrijk, Kortrijk, Belgium
}

Introduction: With the increasing prevalence of childhood obesity and related development of chronic kidney disease (CKD), there is a critical need to understand how best to assess kidney function in children with obesity. Since direct measurement of glomerular filtration rate (GFR) is still considered to be too invasive in children for routine clinical use, estimated GFR (eGFR) is preferred. With serum creatinine ( $\mathrm{SCr}$ ) being recommended as marker of first choice for GFR evaluation, we evaluated and compared creatinine-based GFR estimating equations in children with overweight and obesity.

Material and methods: In this retrospective, cross-sectional study, 600 children with overweight and obesity $(53.5 \%$ female; mean age $12.20 \pm$ 3.28 years; mean BMI z-score $3.31 \pm 0.75$ ) were included from the Centre for Overweight Adolescent and Children's Healthcare (COACH).

Results: Serum creatinine ( $\mathrm{SCr}$ ), normalized using Q-age polynomials, obtained from reference values, results in median and mean $\mathrm{SCr} / \mathrm{Q}$ value close to ' 1 ' for all age-groups, and $96.5 \%$ of the children have a $\mathrm{SCr} / \mathrm{Q}$ within the reference band [0.67-1.33], corresponding to the $2.5^{\text {th }}$ and $97.5^{\text {th }}$ percentile. There is a significant difference between boys and girls (0.97 vs. 1.01) in mean SCr/Q ( $\mathrm{p}=0.0006)$. eGFR Schwartz and Schwartz-Lyon and age were inversely correlated, whereas eGFR iCARE showed an increasing eGFR-age pattern. eGFR EKFC and modified CKD-EPI showed no age-dependency, but the distribution of eGFRvalues was not symmetrical. eGFR FAS-age, FAS-height and adjustedcreatinine revised Lund-Malmö (LMREV-18) showed a relatively symmetrical distribution and no age-dependency.

Conclusions: Serum creatinine ( $\mathrm{SCr}$ ) values of children with overweight and obesity are mostly within the normal reference range for children without CKD. Normalization of SCr using reference Q-age polynomials works very well in this cohort. After evaluation of the different equations, we suggest that FAS-age, FAS-height and LMREV-18 are the preferred creatinine-based GFR-estimating equations in children with overweight and obesity.

\section{PI-74 COMPARISON OF CREATININE AND CYSTATIN C- BASED ESTIMATED GFR WITH RESPECT TO SEVERITY OF SPINA BIFIDA}

Emil Den Bakker, Demi De Vries, Carola Mcdonald, Dewie, Eric Van Der Horst, Arend Bökenkamp

Amsterdam Umc, The Netherlands

Introduction: Renal damage is a well-recognized complication of spina bifida. Paralysis of the lower extremities results in diminished creatinine production and overestimation of GFR by creatinine-based equations. Cystatin $\mathrm{C}$ is produced by all nucleated cells and has been shown to be more accurate in spina bifida patients. As the neurological deficit in spina bifida varies with lesion height we aimed to define patients in whom cystatin $\mathrm{C}$ measurement is mandatory.

Material and methods: Cross-sectional analysis of data collected during routine outpatient visits of 73 children with spina bifida ( $\Delta$ eGFR study) and results of inulin clearance in 24 children. Comparison of the creatinine-based $F_{A S}$ age and $F S_{\text {height }}$ equations with the cystatin Cbased FAS $\mathrm{cys}_{\text {s }}$ equation. Analysis of $\Delta \mathrm{eGFR}$ age (i.e. $\mathrm{FAS}_{\mathrm{age}}-\mathrm{FAS}_{\mathrm{cys}}$ ) and $\Delta \mathrm{eGFR}_{\text {height }}$ in relation to lesion level, walking impairment and orthopaedic complications. In the clearance study, bias of $\mathrm{FAS}_{\text {age }}, \mathrm{FAS}_{\text {height }}$ and $\mathrm{FAS}_{\mathrm{cys}}$ was related to lesion level and walking impairment.

Results: $\triangle \mathrm{eGFR}$ age and $\Delta \mathrm{eGFR}$ height were directly related to lesion height and significantly higher than zero in lesions $\geq \mathrm{L} 5$, in complete wheelchair dependency, presence of scoliosis or contractures. By multivariate linear regression analysis, Both $\Delta \mathrm{eGFR}_{\mathrm{age}}$, and $\Delta \mathrm{eGFR}_{\text {height }}$ were significantly related to complete wheelchair dependency and scoliosis/ contractures, while lesion height $\geq \mathrm{L} 3$ was also related $\triangle \mathrm{eGFR}$ age. Bias

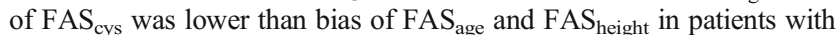
lesions $\geq \mathrm{L} 5$ and in fully wheelchair dependent patients.

Conclusions: FAS $_{\text {age }}$ and $\mathrm{FAS}_{\text {height }}$ overestimate GFR in spina bifida patients with a lesion $\geq \mathrm{L} 5$ who are completely wheelchair dependent or have scoliosis/contractures. In this subgroup, measurement of cystatin $\mathrm{C}$ is mandatory. 
PI-75 EVALUATION OF VACCINATION RATES AND ANTIBODY TITERS IN CHILDREN WITH CHRONIC KIDNEY DISEASE AND COMPARISON WITH HEALTHY CHILDREN

Merve Boyraz, Nilüfer Göknar, Cengiz Candan

Istanbul Medeniyet University, Turkey

Introduction: Children with chronic kidney disease (CKD) have an increased risk of developing bacterial and viral infections. However, vaccination rates and post-vaccination seroconversion levels of patients with CKD are lower than healthy children. In this study, it is aimed to evaluate the vaccination rates of children with CKD and the antibody titers against vaccines and to compare them with healthy children.

Material and methods: In this retrospective study, 90 pediatric patients with CKD and 36 healthy children were included.

Results: High vaccination rates in children with CKD were present in Hepatitis B (93.4\%), Measles (92.2\%), OPV (91.1\%), DaBT (90\%), BCG (87.8\%), Rubella (84.4\%), Mumps (84.4\%), HiB (77.8\%), and PCV vaccines $(67.8 \%)$. The lowest vaccination rates were seen in chickenpox (53.3\%) and hepatitis A vaccines $(54.4 \%)$. It was determined that $46(57.6 \%)$ of $84 \mathrm{CKD}$ patients who were administered hepatitis B vaccine and $22(61.1 \%)$ of 36 children from the healthy control group have positive antibody titers. There were no statistically significant difference between controls and CKD children for Hepatitis B seroconversion rates $(p=0.09)$. However, when the serum levels of vaccine antibodies were measured, significantly lower antibody titers were observed in patients in the HD-PD group compared to healthy controls and children with stage 3-4 CKD ( $p=0.034)$. In the control group, the percentage of positive antibodies to measles vaccine and antibody titer were found as significantly higher than the patients with $\operatorname{CKD}(\mathrm{p}=0.02$ and $\mathrm{p}=$ 0.0001 , respectively). Healthy children can produce a better antibody response to Rubella vaccine than patients with CKD ( $\mathrm{p}=$ 0.03). No statistically significant difference was found between the patients with CKD and the control group for mumps and chickenpox antibody titers $(\mathrm{p}>0.05)$. Positive antibody response percentages with hepatitis A vaccine were found as significantly higher in the control group than the patients with $\operatorname{CKD}(\mathrm{p}=0.03)$.

Conclusions: It has been shown that antibody response to vaccines occurs in most children with CKD, but these values were lower for some vaccines compared to healthy children and therefore these children should be followed closely. Children with CKD should receive ageappropriate vaccines and vaccination schedules must be checked in clinical visits.

\section{PI-76 KIDNEY FAILURE - AN OVERLOOKED FEATURE OF DOWN SYNDROME}

Marieke S.E. Chatelion Counet ${ }^{1}$, Hans Pottel ${ }^{2}$, Roos N. Van Der Plas ${ }^{4}$, Michel Weijerman ${ }^{3}$, Chantal J.m. Broers ${ }^{4}$, Emil Den Bakker ${ }^{1}$, Arend Bökenkamp ${ }^{1}$

${ }^{1}$ Emma Children's Hospital, Amsterdam Umc, Vrije Universiteit Amsterdam, Department Of Pediatric Nephrology, Amsterdam, The Netherlands, ${ }^{2}$ Department Of Public Health And Primary Care, Ku Leuven Campus Kulak Kortrijk, Kortrijk, Belgium, ${ }^{3}$ Alrijne Ziekenhuis, Department Of Pediatrics, Leiderdorp, The Netherland, ${ }^{4}$ Emma Children's Hospital, Amsterdam Umc, Vrije Universiteit Amsterdam, Department Of Pediatrics, Amsterdam, The Netherlands

Objectives: Life expectancy of children with Down syndrome (DS) has increased significantly in the last decades. DS is associated with a fourfold risk of urinary tract abnormalities, still data on renal dysfunction in DS patients are conflicting. The present study was set out to assess kidney function in a large pediatric tertiary DS clinic.
Methods: Retrospective analysis of data collected during routine visits at the DS outpatient clinic of VU medical center. All patients aged between 2 and 18 years in whom serum creatinine had been measured were eligible for inclusion. Exclusion criteria were glucocorticosteroid use, neuromuscular disease or primary referral to a nephrologist or urologist. Kidney function was assessed using the full-age spectrum equations, i.e. $\mathrm{FAS}_{\text {height }}(107.3 /[\mathrm{sCr}(\mathrm{mg} / \mathrm{dL}) /$ $\mathrm{Q}(\mathrm{Q}=$ height-based median reference value $)])$ and $\mathrm{FAS}_{\mathrm{cys}}=$ $(107.3 / \mathrm{sCys}(\mathrm{mg} / \mathrm{L}) / 0.82)$. In a subgroup of 74 patients, a total of 236 serial creatinine measurements with a minimum interval of 2 years were analysed by linear mixed modelling.

Results: Creatinine was available in 189 patients (63\% boys, aged $10.8 \pm$ 5.0 years), cystatin $\mathrm{C}$ in 143 (64\% boys). Mean $\mathrm{FAS}_{\text {height }}$ was $83.6 \pm 16.7$ $\mathrm{mL} / \mathrm{min} / 1.73 \mathrm{~m}^{2}$ and mean $\mathrm{FAS}_{\text {cys }} 87.3 \pm 12.0 \mathrm{~mL} / \mathrm{min} / 1.73 \mathrm{~m}^{2}$. Based on FAS $_{\text {height }} 32 \%$ of the patients had CKD stage I, $62 \%$ stage II and $6 \%$ stage III. There was no relation between kidney function and co-morbidity (i.e. celiac disease, congenital heart disease, hypothyroidism or history of leukemia). Serial measurements showed a significant decline of $\mathrm{FAS}_{\text {height }}$ (slope $-2.11 \mathrm{ml} / \mathrm{min} / 1.73 \mathrm{~m}^{2} /$ year [95\% CI -2.51 to -1.70 ], $\mathrm{p}<$ 0.0001 ).

Conclusion: Mildly to moderately impaired renal function is a common finding in children with Down syndrome. The progressive loss of GFR is troublesome and calls for regular monitoring of kidney function both in children and in adults with Down syndrome to identify potentially treatable risk factors for disease progression such as hypertension and microalbuminuria.

\section{PI-77 RETURNING TO DIALYSIS AFTER KIDNEY ALLOGRAFT FAILURE: THE EXPERIENCE OF THE ITALIAN REGISTRY OF PAEDIATRIC CHRONIC DIALYSIS}

Ester Conversano ${ }^{7}$, Edoardo La Porta ${ }^{8}$, Daniela Zugna ${ }^{1}$, Roberta Camilla ${ }^{2}$, Raffaella Labbadia ${ }^{3}$, Fabio Paglialonga ${ }^{4}$, Mattia Parolin ${ }^{5}$, Enrico Vidal ${ }^{6}$, Enrico Verrina ${ }^{8}$

${ }^{1}$ Department of Medical Sciences, Cancer Epidemiology Unit, University of Torino and Cpo-piemonte, Torino, Italy, ${ }^{2}$ Pediatric Nephrology Unit, Regina Margherita Children's Hospital, CDSS, Torino, Italy, ${ }^{3}$ Nephrology and Dialysis Unit, Department of Pediatrics, "Bambino Gesù" Childrens Hospital-IRCCS, Roma, Italy, ${ }^{4}$ Pediatric Nephrology, Dialysis and Transplant Unit, Fondazione Irccs Ca' Granda Ospedale Maggiore Policlinico, Milano, Italy, ${ }^{5}$ Pediatric Nephrology, Dialysis and Transplant Unit, Department of Woman's and Child's Health, University Hospital, Padova, Italy, ${ }^{6}$ Division of Pediatrics, Department of Medicine (Dame), University of Udine, Udine, Italy, ${ }^{7}$ Department of Pediatrics, Institute for Maternal and Child Health-IRCCS Burlo Garofolo, Trieste, Italy, ${ }^{8}$ Dialysis Unit, Department of Pediatrics, IRCCS Giannina Gaslini, Genova, Italy

Introduction: The need for dialysis after kidney allograft failure (DAGF) is among the top five reasons for initiation of dialysis, making this an important topic in clinical nephrology. However, there are few data on dialysis choice after transplantation and the clinical outcome for DAGF in children.

Material and methods: Patients receiving chronic dialysis at $<18$ year were recorded from January 1991 to January 2019 by the Italian Registry of Pediatric Chronic Dialysis (IRPCD). We investigated the factors influencing the choice of the dialysis modality, the patient outcome in terms of mortality, switching on dialysis modality, and kidney transplantation.

Results: Among 118 patients who received DAGF, 41 (35\%) were treated with peritoneal dialysis (PD), and 77 (65\%) with hemodialysis (HD). Significant predictors for being treated with PD after kidney allograft failure (KAF) were a younger age at dialysis start (OR 0.85 per year increase [95\% CI 0.72-1.00]) and PD use before kidney transplantation (OR 8.20 [95\%CI 1.82-37.01]). Patients entering DAGF in more recent 
eras (OR 0.87 per year increase [95\%CI 0.80-0.94]) and with more than one dialysis modality before kidney transplantation (OR 0.56 for being treated with PD [0.12-2.59]) were more likely to be initiated on HD. As compared to patients in $\mathrm{HD}$, those treated with $\mathrm{PD}$ exhibited an increased but non-significant mortality risk (HR 2.15 [95\%CI 0.54-8.6]; $p=0.28$ ) and a higher prevalence of dialysis-related complications during DAGF course $(\mathrm{p}=0.03)$

Conclusions: Patients entering DAGF in more recent years are more likely to be initiated on HD.

\section{PI-78 IMPACT AND SEROPREVALENCE OF SARS-COV-2 INFECTION IN CHILDREN WITH CKD OR IMMUNO- SUPPRESSION}

Federica Alessandra Vianello ${ }^{1}$, William Morello ${ }^{1}$, Antonio Pio Mastrangelo ${ }^{1}$, Enrico Widal ${ }^{2}$, Isabella Guzzo ${ }^{3}$, Luigi Annicchiarico Petruzzelli ${ }^{4}$, Andrea Pasini ${ }^{5}$, Chiara Benevenuta ${ }^{6}$, Laura Martelli ${ }^{7}$, Roberto Dallamico ${ }^{8}$, Marco Materassi ${ }^{9}$, Lisa Cusinato ${ }^{10}$, Mario Giordano ${ }^{11}$, Giuseppe Puccio ${ }^{1}$, Roberto Chimenz ${ }^{12}$, Ciro Corrado ${ }^{13}$, Elisa Benetti ${ }^{10}$, Carmine Pecoraro ${ }^{4}$, Licia Peruzzi ${ }^{14}$, Laura Massella ${ }^{3}$, Giovanni Montini ${ }^{1}$

${ }^{1}$ Pediatric Nephrology, Dialysis and Transplant Unit, Fondazione Irccs Cà Granda, Ospedale Maggio Policlinico, Milan, Italy, ${ }^{2}$ Division of Pediatrics, Department of Medicine, University of Udine, Udine, Italy, ${ }^{3}$ Nephrology and Dialysis Unit, Pediatric Subspecialties Department, Bambino Gesu'Children's Hospital, Istituto Di Ricerca E Cura A Carattere Scientifico, Rome, Italy, ${ }^{4}$ Pediatric Nephrology and Dialysis Unit, Santobono Children's Hospital, Naples, Italy, ${ }^{5}$ Nephrology and Dialysis Unit, Department of Pediatrics, Azienda Ospedaliero Universitaria, Policlinico Sant'orsola-Malpighi, Bologna, Italy, ${ }^{6}$ Pediatric Nephrology Unit, Reginamargherita Children's Hospital, Azienda Ospedaliero-Universitaria Citta`Della Salute E Della Scienza Di Torino, Turin, Italy, ${ }^{7}$ Paediatric Unit, Papa Giovanni Xxiii Hospital, Bergamo, Italy, ${ }^{8}$ Department of Pediatrics, Azienda Per Lassistenza Sanitaria N 5 Friuli Occidentale, Pordenone, Italy, ${ }^{9}$ Nephrology and Dialysis Unit, Meyer Children's Hospital, Florence, Italy, ${ }^{10}$ Pediatric Nephrology, Dialysis and Transplant Unit, Department of Womens and Childrens Health, University Hospital of Padua, Italy, ${ }^{11}$ Nephrology Unit, Giovanni XXIII Children's Hospital, Bari, Italy, ${ }^{12}$ Pediatric Nephrology And Rheumatology Unit with Dialysis, Azienda Opedaliero-Universitaria G. Martino, Messina, Italy, ${ }^{13}$ Pediatric Nephrology Unit, Children's Hospital "G. Di Cristina," Azienda Di Rilievo Nazionale Ad Alta Specializzazione. "civico," Palermo, Italy, ${ }^{14}$ Pediatric Nephrology Unit, Reginamargherita Children's Hospital, Azienda Ospedaliero-Universitaria Citta 'Della Salute E Della Scienza Di Torino, Turin, Italy

Introduction: Italy was one of the most affected Countries by COVID-19 pandemic. Children with immunosuppression and chronic kidney disease (CKD) are considered at greater risk of infections. We aimed to assess the clinical impact and the seroprevalence of SARS-CoV-2 infection in an Italian cohort of children with CKD or immunosuppression.

Material and methods: The Italian Society of Pediatric Nephrology performed a nationwide observational study to identify patients with severe COVID-19, defined as death, admission to intensive care unit, mechanical ventilation, and the need to change immunosuppressive treatment. Patients aged $<18$ years and affected by glomerular disease requiring immunosuppression, CKD stages 3-5, dialysis, or kidney transplant were enrolled. Data were collected by a phone-based questionnaire between April 13 and 24, 2020, and referred to the period February $20^{\text {th }}$ -
April $15^{\text {th }}, 2020$. To estimate the prevalence of SARS-CoV-2-IgG, 200 children were selected by random sampling from the initial population to be representative of the whole cohort and tested with COVID-19 Rapid Test (Model: GCCOV-402a) from July 15th to September 15th, 2020.

Results: A total of 1572 patients were included. No patients fulfilled the criteria for severe COVID-19. Compared with the healthy Italian pediatric population, a significantly higher percentage of patients underwent swab testing $(5 \%$ versus $1 \% ; \mathrm{p}=0.005)$ but the percentage of positive tests was not significantly different $(\mathrm{p}=0.43)$. Only $3 / 1572(0.19 \%)$ reported a previous SARS-CoV-2 infection documented by nasal swab. The serological testing for SARS-CoV-2-IgG was performed on 178/200 selected patients. A positive test was detected in $3 / 178$ patients (1.7\%). The percentage of children with kidney diseases who tested positive was not statistically different from the corresponding Italian healthy pediatric population (1.7\% vs $2.2 \%)$.

Conclusions: Children in the advanced stages of CKD or on immunosuppressive therapy have a low risk of SARS-CoV2 infection, similar to that of the healthy Italian pediatric population.

\section{PI-79 COVID-19: EXPERIENCES OF LOCKDOWN AND SUPPORT NEEDS IN CHILDREN AND YOUNG ADULTS WITH KIDNEY CONDITIONS}

Yincent Tse ${ }^{1}$, Anne-sophie E Darlington ${ }^{2}$, Kay Tyerman ${ }^{3}$,

Dean Wallace ${ }^{4}$, Tanya Pankhurst ${ }^{5}$, Sofia Chantziara ${ }^{2}$, David Culliford ${ }^{6}$, Alejandra Recio-saucedo ${ }^{7}$, Arvind Nagra ${ }^{8}$

${ }^{1}$ Great North Childrens Hospital, Newcastle Upon Tyne, United Kingdom, ${ }^{2}$ University of Southampton, School of Health Sciences, United Kingdom, ${ }^{3}$ Leeds Teaching Hospitals Nhs Trust, United Kingdom, ${ }^{4}$ Manchester Childrens Hospitals, United Kingdom, ${ }^{5}$ University Hospitals Birmingham Nhs Foundation Trust, United Kingdom, ${ }^{6}$ Nihr Applied Research Collaboration Wessex, School of Health Sciences, University of Southampton, United Kingdom, ${ }^{7}$ University of Southampton, Faculty of Medicine, United Kingdom, ${ }^{8}$ Southampton Childrens Hospital, United Kingdom

Introduction: During the initial COVID-19 pandemic, young United Kingdom (UK) kidney patients underwent lockdown and those with increased vulnerabilities socially isolated or 'shielded' at home. The experiences, information needs, decision-making and support needs of children and young adult (CYA) patients or their parents during this period is not well known.

Material and methods: UK wide online survey co-produced with patients was conducted in May 2020 among CYA aged 12-30, or parents of children aged $<18$ years with any degree of chronic kidney disease. Participants answered qualitative open text alongside quantitative closed questions. Thematic content analysis using a three-stage coding process was conducted.

Results: 118 CYA (median age 21) and 197 parents of children (median age 10) responded. Predominant concerns from CYA were heightened vigilance about viral (68\%) and kidney symptoms (77\%) and detrimental impact on education or work opportunities $(70 \%)$. Parents feared the virus more than CYA ( $71 \%$ vs $40 \%)$, that their child would catch the virus from them $(64 \%)$ and adverse impact on other children at home $(65 \%)$. CYA thematic analysis revealed: strong belief of becoming seriously ill if they contract COVID-19, lost educational opportunities, socialisation, and career development, and frustration at public for not following social distancing rules. Positive outcomes included improved family relationships and community cohesion. Only a minority (14-21\% CYA and $20-31 \%$ parents, merged questions) desired more support. Subgroup analysis identified greater negative psychological impact in the shielded group. 
Conclusions: This survey demonstrates substantial concern and need for accurate tailored advice for CYA based on individualised risks to improve shared decision making.

\section{PI-80 ACUTE KIDNEY INJURY IN PEDIATRIC COVID-19 OR MIS-C IN DUTCH HOSPITALIZED PATIENTS}

Miriam Mooij ${ }^{1}$, Anne Verbeek ${ }^{1}$, Eiske Dorresteijn ${ }^{2}$, David Slotboom ${ }^{1}$, Caroline Brackel $^{3}$, Simone Hashimoto ${ }^{3}$, Suzanne Terheggen-lagro ${ }^{3}$, Michiel Bannier ${ }^{4}$, Manouk Van Der Steen ${ }^{4}$, Rianne Oostenbrink ${ }^{2}$, Pieter Fraaij ${ }^{2}$, Naomi Ketharanathan ${ }^{2}$, Denise Rook ${ }^{5}$, Koen Van Aerde ${ }^{6}$, Ronald Petru ${ }^{6}$, Marlies Van Houten ${ }^{7}$, Sanne Hammer ${ }^{8}$,

Jantine Bolt-wieringa ${ }^{9}$, Gerdien Tramper ${ }^{10}$, Xandra Van De Tweel ${ }^{11}$, Anjali Bechan-kooter ${ }^{12}$, Jan Van Der Linden ${ }^{13}$, Arvid Kamps

${ }^{14}$, Lonneke Van Onzenoort ${ }^{15}$, Margot Ernst-kruis ${ }^{16}$, Ankie Lebon ${ }^{17}$, Stephanie De Crom ${ }^{18}$, Jolitha Bekhof ${ }^{19}$, Han Hendriks ${ }^{20}$, Wim Tissing ${ }^{21}$, Annette Vernooij ${ }^{22}$, Ingeborg Bart ${ }^{23}$, Carien Miedema ${ }^{24}$,

Annemarie Oudshoorn ${ }^{25}$, Bettina Auffarth ${ }^{26}$, Yolande Thomasse ${ }^{27}$, Maarten Rijpert ${ }^{28}$, Joyce Goris ${ }^{29}$, Monique Jacobs ${ }^{30}$, Charlie Obihara ${ }^{31}$, Erik Von Asmuth ${ }^{1}$, Emmeline Buddingh ${ }^{1}$

${ }^{1}$ Willem-Alexander Kinderziekenhuis, Lumc, The Netherlands, ${ }^{2}$ Erasmus Mc Sophia, The Netherlands, ${ }^{3}$ Emma Kinderziekenhuis, Amsterdam Umc, The Netherlands ${ }^{4}$ Maastricht University Medical Centre, The Netherlands, ${ }^{5}$ Uliana Kinderziekenhuis, Haga Ziekenhuis, The Netherlands, ${ }^{6}$ Amalia Kinderziekenhuis, Radboud Umc, The Netherlands, ${ }^{7}$ Spaarne Gasthuis, The Netherlands, ${ }^{8}$ Amphia Ziekenhuis, The Netherlands, ${ }^{9}$ Haaglanden Medisch Centrum, The Netherlands ${ }^{10}$ Franciscus Gasthuis Vlietland, The Netherlands, ${ }^{11}$ Maasstad Ziekenhuis, The Netherlands, ${ }^{12}$ Alrijne Ziekenhuis, The Netherlands, ${ }^{13}$ Bernhoven, The Netherlands, ${ }^{14}$ Martini Ziekenhuis, The Netherlands, ${ }^{15}$ Maxima Medisch Centrum, The Netherlands, ${ }^{16}$ Meander Medisch Centrum, The Netherlands, ${ }^{17}$ Albert Schweizer Ziekenhuis, The Netherlands, ${ }^{18}$ Bravis Ziekenhuis, The Netherlands, ${ }^{19}$ Isala Klinieken, The Netherlands, ${ }^{20}$ Zuyderland Medisch Centrum, The Netherlands, ${ }^{21}$ Prinses Maxima Centrum, The Netherlands, ${ }^{22}$ St Jansdal, The Netherlands, ${ }^{23}$ Canisius Wilhelmina Ziekenhuis, The Netherlands, ${ }^{24}$ Catharina Ziekenhuis, The Netherlands, ${ }^{25}$ Gelre Ziekenhuis, The Netherlands, ${ }^{26}$ Ommelander Ziekenhuis, The Netherlands, ${ }^{27}$ Dijklander Ziekenhuis, The Netherlands, ${ }^{28}$ Zaans Medisch Centrum, The Netherlands, ${ }^{29}$ Zorgsaam Ziekenhuis, The Netherlands, ${ }^{30}$ Slingeland Ziekenhuis, The Netherlands, ${ }^{31}$ Elisabeth-tweesteden Ziekenhuis, The Netherlands

Introduction: A small but significant proportion of children with COVID-19 or Multisystem Inflammatory Syndrome in Children (MISC) is severely ill, therefore we aimed to describe the incidence of Acute Kidney Injury (AKI).

Material and methods: This is a multicenter, prospective, ongoing cohort study in 53 hospitals www.covidkids.nl. Children (0-17 years) were included if they presented with COVID-19 in the hospital and had a positive real-time RT-PCR test on SARS-CoV-2 or fulfilled a clinical diagnosis for COVID-19 or MIS-C. Data was collected from April 2020 till April 2021. Patients were excluded from analysis when there were no symptoms attributed to COVID-19 (e.g. screening test positive). AKI was defined and staged according to Kidney Disease: Improving Global Outcomes criteria. Severity of COVID-19 disease was defined as non-severe, moderately severe (need of supplemental oxygen), severe (admitted to ICU), very severe (ECMO or death). Severity of MIS-C disease was defined as non-severe, moderately severe (admitted to ICU), severe (ICU admission with invasive respiratory and/or inotropic support), very severe (ECMO or death).

Results: In total 188 subjects were included in the cohort, of which 89 had at least one serum creatinine measurement. 41/89 cases had an acute
COVID-19 presentation, 48/89 had MIS-C. Five patients had a nephrological medical history (pyelonephritis [n=2], IgA nephropathy $[n=2]$, renal insufficiency $[n=1])$. One patient with acute COVID-19 required renal replacement therapy. The incidence of AKI was $29 \%$ and $40 \%$ in respectively the COVID-19 and the MIS-C group. If we assume that missing cases of creatinine had normal renal function, then the incidence of AKI is respectively 9\% (12/132) and 34\% (19/56). In our cohort, severity of disease of COVID-19/MIS-C was associated with AKI in the COVID-19 ( $\mathrm{p}=0.015)$, but not in the MIS-C group ( $\mathrm{p}=0.12$ ).

Conclusions: Children with MIS-C or severe COVID-19 had the highest incidence of AKI.

\section{PI-81 ANXIETY, DEPRESSION AND COPING OF CHILDREN WITH CHRONIC KIDNEY DISEASE AND THEIR CAREGIVERS DURING THE COVID-19 PANDEMIC}

Ozde Nisa Turkkan, Nurdan Yildiz, Neslihan Cicek, Ece Demirci Bodur, Sercin Guven, Mehtap Sak, Serim Polat Pul, Harika Alpay Marmara University Pendik Training and Research Hospital Division of Pediatric Nephrology, Turkey

Introduction: In this study, we aimed to evaluate our patients with chronic kidney disease(CKD) or kidney transplant recipients(KTx) and their parent's/caregiver's anxiety and depression levels and abilities of coping with difficulties during the COVID-19 pandemic.

Material and methods: This cross-sectional study was conducted on 80 children with predialysis $\mathrm{CKD}$, children on dialysis and KTx, 30 healthy children between 7-18 years of age and their care providers. Children were surveyed by the Strengths and Difficulties Questionnaire(SOQ-T), Screen for Child Anxiety Related Disorders(SCARED), childhood depression inventory and parents or caregivers by Coping Attitudes Assessment Scale(COPE), Beck Depression Inventory and Beck Anxiety Inventory.All questionnaires were done face-to-face or by phone and scored in accordance with the scales.

Results: Signs of anxiety (SCARED scores) was present in $26(32.5 \%)$ patients and $4(13.3 \%)$ controls, and significantly higher in children with CKD and KTx recipients $(p=0.03)$. Sixty-nine children and all controls have a depression scale of $>19$ and control group showed significantly higher depression scores than the patients (mean $\pm \mathrm{SD}, 51.6 \pm 2.8$ vs. $47.5 \pm 11.1$; respectively, $\mathrm{p}=0.009$ ). Thirteen $(16.3 \%)$ patient and $2(6.7 \%)$ controls have high SDQDysregulation Profile(SDQ-DP) scores. SDQ-DP scores of the patients were significantly higher than the controls $(9.6 \pm 5.3$ vs. 7.2 \pm 5.8 , respectively; $\mathrm{p}=0.000$ ).

There was no significant difference of mothers in coping with difficulties, anxiety and depression scores between the groups $(\mathrm{p}>0.05)$. Higher degree of anxiety of the patients was significantly associated with higher anxiety and depression symptoms of the mothers in the patients group $(r=0.5, p=0.000$ and $r=0.435, p=0.001$; respectively $)$, whereas there was no relationship between the SDQ-DP of the patients and Beck anxiety and depression scores of the mothers

Conclusions: The COVID-19 outbreak causes increased anxiety in children with CKD and KTx and increased depression in healthy children. We may speculate that higher depression scores in healthy children may be due to sudden lifestyle restrictions during the pandemic compared to CKD patients who already have certain restrictions. The association of the increased anxiety of the patients with the depression and anxiety of the mothers has shown the necessity of more attention from mental health practitioners. Identifying affected children as early as possible and providing professional psychological and behavioral interventions will ensure a better mental health for children with chronic kidney diseases and their caregivers during the pandemic. 
PI-82 HOME TESTING TO MEASURE CREATININE AND TACROLIMUS LEVELS: A COLLABORATION DURING THE COVID19 PANDEMIC

Helen Jones ${ }^{1}$, Rachel Carling ${ }^{2}$, Donna Austin ${ }^{2}$, Erin Emmett ${ }^{2}$, Louise Kipping ${ }^{1}$, Grainne Walsh ${ }^{1}$, Nick Ware ${ }^{1}$, David Marshall ${ }^{3}$, Jon Jin Kim ${ }^{4}$

${ }^{1}$ Evelina London Childrens Hospital, United Kingdom, ${ }^{2}$ St Thomas Hospital, United Kingdom, ${ }^{3}$ Manchester University Foundation Trust, United Kingdom, ${ }^{4}$ Queens Medical Centre, United Kingdom

Introduction: During the first wave of the COVID19 pandemic all paediatric renal transplant recipients (pRTR) in the UK were required to shield driving a reduction in their face to face outpatient clinics and an increase in virtual reviews in our centre. Re-configuration of the transplant service was required to enable effective monitoring of pRTRs. We describe a testing method set up responsible for reducing some hospital or primary care visits.

Material and methods: Collaboration between 2 UK paediatric nephrology centres and 2 laboratories enabled setting up of a home testing method (finger prick sample) to measure creatinine and tacrolimus level using Mitra tips which could be undertaken by pRTRs and their parent/carer. The samples are then posted to the tertiary centre.

Results: Mitra tip finger prick method measures creatinine(umol/L) and tacrolimus(ug/l). Extensive testing was carried out by our biochemistry colleagues to set up the assays, ensuring results comparable to standard laboratory testing used in our centre.

To date this home-testing has been used for 1. Monitoring between outpatient visits to reduce frequency of visits in stable pRTRs 2. Enabling monitoring of tacrolimus levels after dose adjustment 3 . Facilitating early creatinine re-check if graft function elevated from baseline at routine clinic visit 4 . Checking an accurate $12 \mathrm{~h}$ trough tacrolimus level in pRTR who is unable to do this in clinic.

Conclusions: Home testing to measure creatinine and tacrolimus levels in pRTRs using Mitra tips allows increased flexibility with respect to the monitoring of transplant function we are able to offer. Though it was established in our centre through collaboration during the pandemic, it will be used going forwards to facilitate some of the patients' monitoring enabling a reduction in missed school days for patients and less time off work for their families. We hope it can be used in other patient groups receiving tacrolimus therapy.

\section{PI-83 ACUTE KIDNEY INJURY (AKI) IN PAEDIATRIC I NFLA M M A T ORY MULTISYSTEM SYNDROME TEMPORALLY ASSOCIATED WITH SARS-COV-2 (PIMS-TS)}

\author{
Douglas Stewart ${ }^{1}$, Nadeehsa Mudalige ${ }^{2}$, Pascale Du PrÉ ${ }^{1}$, \\ Mae Johnson ${ }^{1}$, Jelena Stojanovic ${ }^{1}$ \\ ${ }^{1}$ Great Ormond Street Hospital, London, United Kingdom, ${ }^{2}$ Ucl Great \\ Ormond Street Institute Of Child Health, London, United Kingdom
}

Introduction: An observation study to evaluate the incidence of AKI in PIMS-TS, with reporting of 6-month follow-up data.

Material and methods: A retrospective chart review of 112 patients admitted to our centre between April 2020 and March 2021 with a diagnosis of PIMS-TS was performed. Classification of AKI was based on serum creatinine values above upper limit of normal (ULN) reference values as published by the British Association of Paediatric Nephrology. Results: 34 patients (30\%) met diagnostic criteria for AKI (38\% stage 1, $24 \%$ stage 2 , and $38 \%$ stage 3 ), which was most evident at time of admission. Median age of the AKI group was younger ( 8.8 years vs 10.6 years $[p=0.03])$, with higher peak triglyceride $(p=0.03)$, CRP $(p$ $=0.003)$, D-dimer $(p=0.003)$, and troponin $(p=<0.001)$ values. The AKI group were more likely to require PICU admission $(\mathrm{p}=0.016)$, require intubation and ventilation $(\mathrm{p}=0.006)$, and require inotropic support $(\mathrm{p}=<0.001)$. In univariable analysis, hypertriglyceridaemia, CRP, D-dimer, and Black African/Caribbean ethnicity were associated with AKI. Multivariable logistic regression demonstrated that hypertriglyceridaemia remained significant for the development of severe AKI (stages 2 and 3) (p 0.02). There was no increase in the proportion or severity of AKI in the first wave of the UK COVID-19 pandemic compared with the second wave (pre and post September 2020 respectively). Six-month follow-up data was available for 13 (38\%) of the AKI group. None had persistent macroalbuminuria or haematuria. All had serum creatinine values $<\mathrm{ULN}$ for age. Three $(27 \%)$ had systolic blood pressure (BP) readings $>90^{\text {th }}$ centile for age.

Conclusions: We report several risk factors for the development of AKI in PIMS-TS, which occurs in approximately one third of patients. Sixmonth follow-up data is reassuring with normalisation of serum creatinine in all. Longer term follow-up of BP is required to assess whether hypertension may be a sequelae of PIMS-TS with renal involvement.

\section{PI-84 COVID-19-ASSOCIATED KIDNEY INJURY IN CHILDREN: AN OBSERVATIONAL STUDY USING EXPANDED DIAGNOSTIC CRITERIA OF ACUTE DISEASE QUALITY INITIATIVE (ADQI)}

Seha Saygili ${ }^{1}$, Nur Canpolat ${ }^{1}$, Rümeysa Yasemin Cicek ${ }^{2}$, Ayșe Agbas ${ }^{3}$, Esra Karabag Yilmaz ${ }^{1}$, Ayse Ayzit Kilinc Sakalli ${ }^{4}$, Deniz Aygün ${ }^{5}$, Gülsen Akkoc ${ }^{6}$, Kaan Can Demirbaș ${ }^{7}$, Dildar Konukoglu ${ }^{8}$, Haluk Cokugras ${ }^{9}$, Salim Caliskan ${ }^{1}$, Lale Sever ${ }^{1}$

${ }^{1}$ Department of Pediatric Nephrology, Istanbul University-Cerrahpasa, Cerrahpasa Faculty of Medicine, Istanbul, Turkey, ${ }^{2}$ Department of Pediatric Nephrology, University of Health Sciences Kanuni Sultan Süleyman Training And Research Hospital, Istanbul, Turkey, ${ }^{3}$ Department of Pediatric Nephrology, University of Health Sciences Haseki Training and Research Hospital, Istanbul, Turkey, ${ }^{4}$ Department of Pediatric Pulmonology, Istanbul University-cerrahpasa, Cerrahpasa Faculty of Medicine, Turkey, ${ }^{5}$ Department of Pediatric Infectious Disease, University of Health Sciences Kanuni Sultan Süleyman Training and Research Hospital, Turkey, ${ }^{6}$ Department of Pediatric Infectious Disease, University of Health Sciences Haseki Training and Research Hospital, Istanbul, Turkey, ${ }^{7}$ Istanbul University-Cerrahpasa, Cerrahpasa Faculty of Medicine, Istanbul, Turkey, ${ }^{8}$ Department of Biochemistry, Istanbul University-Cerrahpasa, Cerrahpasa Faculty of Medicine, Istanbul, Turkey, ${ }^{9}$ Department of Pediatric Infectious Disease, Istanbul University-Cerrahpasa, Cerrahpasa Faculty Of Medicine, Istanbul, Turkey

Introduction: We aimed to identify COVID-19 associated kidney injury in children with mild-to-moderate disease using the expanded definition of acute kidney injury (AKI) with both functional and damage markers according to the Acute Disease Quality Initiative (ADQI) recommendations.

Material and methods: This case-control observational study included children under the age of 18 years who were hospitalized but not admitted to the ICU with a diagnosis of COVID-19 from three centers in Istanbul. Patients were classified by the recommendations on AKI biomarkers of the 23rd ADQI Consensus Report. We used a combination of functional (serum creatinine) and damage (NGAL, KIM-1, and IL-18) markers for the definition of AKI.

Results: 71 children with COVID-19 aged between $0 \cdot 1$ and $17 \cdot 9$ years and 75 healthy children were enrolled in the study. Patients had significantly higher levels of urinary biomarkers compared to the healthy controls $(\mathrm{p}<0.001) .12$ patients $(16.9 \%)$ had increased serum creatinine, and 27 patients $(38 \%)$ showed positivity for at least one urinary biomarker. Five patients had both an increase in serum creatinine and a positive biomarker. According to the ADQI staging, 34 children (47.9\%) were 
determined to have kidney injury; 22 were classified as having AKI stage $1 \mathrm{~S}(31 \%)$, seven as AKI stage 1A (10\%), four as AKI stage 1B (5.6\%), and one as AKI stage $2 \mathrm{~B}(1.4 \%)$.

Conclusions: COVID-19 associated kidney injury is common even in children with less severe disease. A significant number of children with COVID-19 develop early stages of AKI, which can be referred to as subclinical AKI.

\section{PI-85 IS COVID-19 RELATED ANXIETY HIGHER IN CHILDREN WITH CHRONIC KIDNEY DISEASE THAN HEALTHY CHILDREN?}

Gökçen Erfidan ${ }^{1}$, Gonca Özyurt ${ }^{2}$, Seçil Arslansoyu Çamlar ${ }^{3}$, Özgür Özdemir Șimșek ${ }^{1}$, Cemaliye Bașaran ${ }^{1}$, Demet Alaygut ${ }^{1}$, Fatma Mutlubas ${ }^{3}$, Belde Kasap Demir ${ }^{4}$

${ }^{1}$ University of Health Sciences, Tepecik Training and Research Hospital, Department of Pediatrics, Division of Nephrology, Izmir, Turkey, ${ }^{2}$ İmir Katip Celebi University, Faculty of Medicine, Department of Child and Adolescent Psychiatry, İzmir, Turkey, ${ }^{3}$ University of Health Sciences, Izmir Faculty of Medicine, Department of Pediatrics, Division of Nephrology, Izmir, Turkey, ${ }^{4}$ Izmir Katip Celebi University, Faculty of Medicine, Department of Pediatrics, Division of Nephrology and Rheumatology, Izmir, Turkey

Introduction: Since the emergence and worldwide spread of the new Coronavirus pandemic (COVID-19), many factors such as the high rate of spread, unpredictability and uncertainty of the prognosis, physical and social restrictions due to the measures taken, have caused people to be psychologically adversely affected in our country as well as in the world. This study aimed to assess anxiety levels during COVID-19 in children with chronic kidney disease (CKD), including nephrotic syndrome (NS) and kidney transplantation (Tx).

Material and methods: A case-control, cross-sectional study was conducted with children aged of 10 to 18 years, who had been followed with diagnosis of CKD, NS, Tx in Izmir Tepecik Training and Research Hospital, Turkey, between April and July 2020. Healthy control group was recruited age and gender-matched children. A questionnaire with printed and online version is designed in three parts: demographic characteristics, opinions about the pandemic, Turkish version of The Revised Child Anxiety and Depression Scale- Child Version (RCADS-CV).

Results: Total of 88 children completed the questionnaire. The patient and control groups were similar by gender, age, household members and history of psychiatric treatment. Both groups stated that coronavirus is a risky disease for children (63.6\%), they were afraid of contagion (69.3\%). Only half of them were receiving realistic and informative answers from family members. In RCADS-CV, $66 \%$ of them get high score from at least one subscale. Social Phobia (SP) scores of the control group was higher, although the high-scoring ratios were similar. The ratio of highscored participants was higher in CKD patients for Panic Disorder, and was lower in immunosuppressive agent group for SP.

Conclusions: The current COVID-19 pandemic is a disaster that children encounter for the first time in their lives. It does not exclusively cause anxiety among children with chronic kidney diseases, but also healthy children.

\section{PI-86 RENAL INVOLVEMENT AND HISTOLOGICAL FINDINGS IN TWO PEDIATRIC COVID-19 PATIENTS}

\author{
Antonio Mastrangelo ${ }^{1}$, Jessica Serafinelli ${ }^{1}$, William Morello ${ }^{1}$, \\ Valeria Fanny Cerioni ${ }^{2}$, Giovanni Montini ${ }^{1}$ \\ ${ }^{1}$ Fondazione Irccs Ca Granda Ospedale Maggiore Policlinico Milano, \\ Italy, ${ }^{2}$ Asst Monza E Brianza, Italy
}

Introduction: Kidney involvement and histological findings have been rarely reported in pediatric SARS-CoV-2 infection. Acute tubular necrosis is the most common histological finding in adults, but the exact mechanism of kidney involvement is unclear.

Material and methods: We describe clinical, laboratory and histological findings of two pediatric cases with an almost exclusive renal involvement by SARS-CoV2 without pre-existing diseases.

Results: A 10-years-old girl with IgA vasculitis nephritis underwent a renal biopsy, showing a diffuse and segmental mesangial-proliferative glomerulonephritis. A steroid therapy was started (methylprednisolone, prednisone). After few weeks, clinical conditions worsened (weight gain, nephritic-nephrotic syndrome) and an atypical skin rash appeared. A SARS-CoV-2 infection was diagnosed. An electron microscopy evaluation revealed cytoplasmatic blebs and virus-like particles in tubular cells. After few days, SARS-CoV-2 infections was cleared and cyclophosphamide was added to prednisone for 10 weeks. A second kidney biopsy was performed showing a crescentic glomerulonephritis with jalinosis, while virus-like particles were no more present.

The second patient was a 12-year-old girl with a 3-week history of progressive weakness and weight loss. A mild rhinitis was reported the month before. No medications were taken. Blood and urine analysis revealed rise in serum creatinine $(1.8 \mathrm{mg} / \mathrm{dL})$, hypouricemia $(1.8 \mathrm{mg} / \mathrm{dl})$, severe low molecular weight proteinuria, low urinary gravity (1008), glycosuria, and proteinuria (uPCR $0.5 \mathrm{mg} / \mathrm{mg}$ ). Patient showed a high SARS-CoV-2-IgG titre (195 AU/ml). A kidney biopsy was performed, showing features of acute tubular-interstitial nephritis. On EM, no images suggestive for virus-like particles were observed. Steroid therapy was started and resulted effective to restore a normal renal function. In both cases RT-PCR was negative for SARS-CoV-2 on renal tissue. Conclusions: Kidney involvement is uncommon in children and related histological data are lacking, being reported only in two children with SARS-CoV-2-associated AKI. The diagnosis was acute necrotizing glomerulonephritis in both cases. RT-PCR for SARS-CoV-2 tested negative while EM was not performed. We firstly completely describe renal histological and clinical finding in SARS-CoV-2 positive children. We can speculate that in both cases SARS-CoV-2 played a major role as inflammatory trigger of the renal damage. We suggest investigating the potential kidney damage by SARS-CoV-2 in children and that SARS-CoV2 can be included among infectious agents of pediatric acute tubular interstitial nephritis.

\section{PI-87 INCIDENCE AND OUTCOME OF SARS-COV-2 INFECTION IN A KIDNEY TRANSPLANT PEDIATRIC RECIPIENTS COHORT FROM A SINGLE CENTER IN NORTHERN ITALY}

Marco Cazzaniga ${ }^{1}$, Marta Brambilla ${ }^{2}$, Antonio Vergori ${ }^{2}$, Maria Viganoni ${ }^{1}$, Stefania Rotondo ${ }^{2}$, Sara Testa ${ }^{2}$, Giovanni Montini ${ }^{3}$ ${ }^{1}$ Pediatric Nephrology, Dialysis and Transplantation Unit - Fondazione Irccs Ca Granda Ospedale Maggiore Policlinico - University of Milan, Milan, Italy, ${ }^{2}$ Pediatric Nephrology, Dialysis and Transplantation Unit Fondazione Irccs Ca Granda Ospedale Maggiore Policlinico, Milan, Italy, ${ }^{3}$ Pediatric Nephrology, Dialysis and Transplantation Unit Fondazione Irccs Ca Granda Ospedale Maggiore Policlinico, Giuliana and Bernardo Caprotti, Chairs of Pediatrics, Department of Clinical Sciences and Community Health, University of Milan, Milan, Italy

Objectives: To investigate the incidence and clinical outcomes of SARSCoV-2 infection in pediatric kidney transplant (KT) recipients.

Methods: From March 2020 to April 2021, transplanted patients aged $<21$ yrs, routinely managed in our Center that underwent nasopharyngeal real time-polymerase chain reaction (RT-PCR) test to detect SARS-CoV2 infection, were studied. Tests were performed according to clinical and/ or epidemiological criteria.

Results: 100 patients were enrolled; median age was 15.7 (2.2-20.8) years; median time from KT was 2.8 (0.0-16.6) years, including 20 patients enrolled at the KT. All patients were on maintenance immunosuppressive (IS) therapy (corticosteroids, CNI and anti-proliferative agents). 
57 (35 males) pts were included according to the inclusion criteria and underwent at least one RT-PCR test, with a total of 110 swabsperformed. Indications were 43 for symptoms suggestive for SARS-CoV-2 infection, 24 for contact tracing, 40 for screening at hospitalization and 3 for positivity at serological Rapid Test. Overall, $12(10.9 \%)$ tests were positive: two $(4.6 \%)$, eight $(33,3 \%)$, one $(2,5 \%)$ and one $(33,3 \%)$ for each criteria listed above, respectively.

Among positive patients (6 males), 4 were asymptomatic (33.3\%), 7 presented fever (58.3\%), 2 upper respiratory tract symptoms (16.7\%), 1 diarrhea $(8.3 \%)$, 1 headache $(8.3 \%)$, and 3 osteo-muscular pain $(25 \%)$. None showed KT dysfunction. Mean duration of symptoms was 2.4 days. No patients required specific treatment (except for antipyretics) or IS reduction; no one was admitted to hospital. Median duration of RTPCR positivity was 17 (2-31) days.

Conclusions: Our data show that renal transplanted pediatric patients, even if immunosuppressed, are at low risk of clinically relevant COVID-19 as healthy age-related population. On the other hand, our results differed substantially from those showed by Italian elderly SOT recipients that have an high incidence and even more early and high mortality rate (27\%) [Trapani et al. - Am J Transplant. 2020; 00:1-13].

\section{PI-88 ACQUIRED RENAL TUBULAR DYSGENESIS IN A PREMATURE NEONATE.}

Martine Docx ${ }^{1}$, Vande Johan ${ }^{2}$, Dendooven Amelie ${ }^{3}$

${ }^{I}$ Department Of Chronic Paediatric Diseases And Nephrology Queen Paola Childrens Hospital Antwerp Belgium, ${ }^{2}$ Department Of Paediatric Nephrology And Rheumatology University Hospital Ghent, Belgium, ${ }^{3}$ Department Of Pathology University Hospital Antwerp, Belgium

Objectives: Renal tubular dysgenesis (RTD), genetic or acquired, is mostly a lethal disorder and characterised by the absence or poor development of proximal tubules. Clinical features include early and persistent anuria, pulmonary hypoplasia, ossification defects of the skull and refractory hypotension.

Methods: A male neonate was the first child of healthy non-consanguineous parents. He was born at 32 3/7 weeks of gestation due to fetal distress (IUGR) through caesarean section. Severe oligohydramnios was detected at 30 weeks progressing to anhydramnios by week 32 . No drug intake during pregnancy. Fetal ultrasound showed two normal sized kidneys and empty urinary bladder. Examination revealed: large anterior fontanel with broad sutures, hypoplastic nasal bridge, low set ears and bilateral clubfeet. Persistent anuria, severe metabolic acidosis, renal failure and untreatable hypotension were observed. The patient died on day 3 .

Results: Ultrasonography showed two kidneys $(3.6 \mathrm{~cm})$ with corticomedullary differentiation. Postmortem renal biopsy showed few and poorly differentiated tubular structures. The tubules expressed no CD10. Glomeruli were normal. The histopathology was representative for RTD. The accompanying placenta showed no abnormalities. Normal SNP-array and genpanel for AGT, AGTR1 and ACE.

Conclusions: (1) Consider RTD in any anuric foetuses with normal or nearly normal kidneys (size \& structure). (2) Secondary forms are often associated with IUGR. Normal fetal growth in genetic forms. (3) Questioning of the pregnant mother about her drug intake (especially RAS blockers). (4) Complete autopsy with detailed pathologic kidney examination required. (5) In genetic forms identification of the gene defect can give precise genetic counselling.

PI-89 FACTORS ASSOCIATED WITH LONGITUDINAL CHANGES OF PULSE WAVE VELOCITY (PWV) IN CHILDREN WITH CHRONIC KIDNEY DISEASE (CKD)

Karolis Azukaitis ${ }^{1}$, Marietta Kirchner ${ }^{2}$, Anke Doyon ${ }^{3}$, Mieczysław Litwin ${ }^{4}$, Aysun Bayazit ${ }^{5}$, Ali Duzova ${ }^{6}$, Nur Canpolat ${ }^{7}$,
Augustina Jankauskiene ${ }^{1}$, Rukshana Shroff ${ }^{8}$, Anette Melk ${ }^{9}$, Uwe Querfeld ${ }^{10}$, Franz Schaefer ${ }^{3}$, On Behalf Of 4c Study Consortium ${ }^{11}$ ${ }^{1}$ Clinic Of Pediatrics, Institute of Clinical Medicine, Faculty of Medicine, Vilnius University, Vilnius, Lithuania, ${ }^{2}$ Institute of Medical Biometry and Informatics, University of Heidelberg, Heidelberg, Germany, ${ }^{3}$ Division of Pediatric Nephrology, Center For Pediatrics and Adolescent Medicine, Heidelberg University Hospital, Heidelberg, Germany, ${ }^{4}$ Department of Nephrology and Arterial Hypertension, The Childrens Memorial Health Institute, Warsaw, Poland, ${ }^{5}$ Department of Pediatric Nephrology, Cukurova University, Adana, Turkey, ${ }^{6}$ Division of Pediatric Nephrology, Hacettepe University Faculty of Medicine, Ankara, Turkey, Department of Pediatric Nephrology, Istanbul University Cerrahpasa Faculty of Medicine, Istanbul, Turkey, ${ }^{8}$ Great Ormond Street Hospital for Children, London, United Kingdom, ${ }^{9}$ Department Of Kidney, Liver And Metabolic Diseases, Hannover Medical School, Hannover, Germany, ${ }^{10}$ Department Of Pediatric Nephrology, Charité University, Berlin, Germany, ${ }^{11} 4 c$ Study Consortium

Introduction: Uremia has a profound effect on arterial elasticity and increased PWV has been reported in children with CKD, but comprehensive description of arterial stiffening risk profile is still lacking. The aim of the present analysis was to evaluate risk factors associated with PWV over time in children with CKD.

Material and methods: Post-hoc analysis of the Cardiovascular Comorbidity in Children with Chronic Kidney Disease (4C) study that prospectively followed children aged 6-17 years with CKD stages 3-5. Follow-up was censored at the start of kidney replacement therapy. PWV was measured with an oscillometric device (Vicorder) and standardized to sex and height (PWVz). Multivariable linear longitudinal mixed-effects models with patient-individual random intercepts and slopes were built to determine the associations of each covariate with PWVz (censored at age 17 years due to lack of reference values thereafter) and absolute PWV (all observations).

Results: 667 patients $(12.2 \pm 3.3$ years, $65.5 \%$ boys $)$ with a median baseline eGFR of $26.9 \mathrm{ml} / \mathrm{min} / 1.73 \mathrm{~m}^{2}$ and baseline $\mathrm{PWVz}$ of $0.31 \pm 1.65$ were analyzed. Median follow-up of study participants was 1.7 years.

PWVz $(n=628)$ showed time-stable associations with younger age $(-0.055, p=0.002)$, lower height $\mathrm{z}$-score $(-0.329, \mathrm{p}<0.001)$, higher diastolic blood pressure $(\mathrm{BP}) \mathrm{z}$-score $(0.332, \mathrm{p}<0.001)$, higher serum low-density lipoprotein (LDL) cholesterol $(0.003, p=0.02)$, higher serum calcium $(0.549, \mathrm{p}=0.05)$, higher albuminuria $(0.107$, $\mathrm{p}=0.001)$ and lower ferritin $(-0.117, \mathrm{p}=0.01)$. In addition, timeinteraction analysis revealed an intensifying effect of lower height $\mathrm{z}$-score $(\mathrm{p}=0.03)$ and female gender $(0.161, \mathrm{p}=0.01$ for time-interaction) over time. In a model with absolute PWV values greater height and older age were associated with higher PWV, as expected physiologically; the effect of female gender was lost, while other associations remained.

Conclusions: Higher PWV in children with CKD associates with nonmodifiable (age, height, female gender) and potentially modifiable (BP, LDL cholesterol, albuminuria, serum calcium and ferritin) factors but not kidney function.

\section{PI-90 AORTIC PULSE WAVE VELOCITY IS PRIMARILY DETERMINED BY BLOOD PRESSURE BUT NOT OTHER CARDIOVASCULAR RISK FACTORS DURING CHILDHOOD: A CALL TO ARMS TO STANDARDISE RESULTS}

Karolis Azukaitis ${ }^{1}$, Manish Sinha ${ }^{2}$, Augustina Jankauskiene ${ }^{1}$, Bojko Bjelakovic $^{3}$, Mieczysław Litwin ${ }^{4}$, On Behalf Of Hyperchildnet Wg3 ${ }^{1}$

${ }^{1}$ Clinic of Pediatrics, Institute of Clinical Medicine, Faculty of Medicine, Vilnius University, Vilnius, Lithuania, ${ }^{2}$ Kings College London Evelina London Childrens Hospital, London, United Kingdom, ${ }^{3}$ Clinic of 
Pediatrics, Clinical Center, Nis, Serbia, Medical Faculty, University of Nis, Nis, Serbia, ${ }^{4}$ Department of Nephrology and Arterial Hypertension, The Childrens Memorial Health Institute, Warsaw, Poland, ${ }^{5}$ Cost Action Hyperchildnet

Introduction: Pulse wave velocity (PWV) is the gold-standard for non-invasive assessment of arterial stiffening in patients with hypertension. Adult studies have shown that PWV is mainly determined by blood pressure (BP) and age. We aimed to systematically analyze determinants of PWV in studies involving children with primary hypertension $(\mathrm{PH})$.

Material and methods: 196 articles retrieved from PubMed according to a predefined search strategy were screened for eligibility. 38 articles identified by title/abstract review underwent full-text screening by 2 reviewers and 12 were selected for the analysis if (i) measured carotid-femoral PWV (PWVcf); (ii) reported on risk associations using regression models; and (iii) included BMI and BP in the model.

Results: The total population included $>3700$ children with normal and elevated BP. 25\% studies used indirect methods for PWVcf estimation and $17 \%$ analyzed standardized PWVcf. Higher PWVcf in hypertensive vs non-hypertensive children was reported in $75 \%$ studies. $92 \%$ and $75 \%$ included age and sex, in regression models. In $83 \%$ studies PWV was positively associated with BP, whereas BMI associated with PWV in $25 \%$ only (positive 3 and negative association in 1). Age was positively associated in $18 \%$ and no studies reported any significant associations with sex. Association of race were reported in 2 studies only (direct effect of non-white race in 1). Models including BP among other covariates (biochemical, anthropometric and other $\mathrm{BP}$ parameters) explained $12-88 \%$ of $\mathrm{PWV}$ cf variability $\left(\mathrm{R}^{2}\right.$ not reported in $25 \%$ of studies).

Conclusions: BP is the predominate contributor to PWV, compared to the inconsistent or lesser contributions of BMI, age, sex or other CV risk factors during childhood. Additional studies are needed to determine whether increased PWV is a reflection of primary arterial disease or a secondary phenomenon. Our data also highlights the need of standardization and reporting of confounders, such as age and sex.

\section{PI-91 DIAGNOSTIC VALUE OF SERUM CARDIOVASCULAR (CV) BIOMARKERS TO DETECT SUBCLINICAL CV DISEASE (CVD) IN CHILDREN WITH CHRONIC KIDNEY DISEASE (CKD)}

Karolis Azukaitis ${ }^{1}$, Justinas Daraskevicius ${ }^{2}$, Marietta Kirchner ${ }^{3}$, Franz Schaefer ${ }^{4}$, On Behalf Of 4c Study Consortium ${ }^{5}$

${ }^{1}$ Clinic of Pediatrics, Institute of Clinical Medicine, Faculty of Medicine, Vilnius University, Vilnius, Lithuania, ${ }^{2}$ Faculty of Medicine, Vilnius University, Vilnius, Lithuania, ${ }^{3}$ Institute of Medical Biometry And Informatics, University of Heidelberg, Heidelberg, Germany, ${ }^{4}$ Division of Pediatric Nephrology, Center For Pediatrics And Adolescent Medicine, Heidelberg University Hospital, Heidelberg, Germany, ${ }^{5} 4 \mathrm{c}$ Study Consortium

Objectives: Serum levels of biomarkers associated with CV stress are increased in children with CKD and after kidney replacement therapy (KRT). We aimed to evaluate the diagnostic performance of traditional (TnT and BNP) and novel (GDF15 and ST2) CV biomarkers to detect subclinical CV disease (CVD) in children with CKD and under KRT.

Methods: Biomarker levels were measured in 120 children with CKD stage 3-5, 95 of whom started KRT ( $n=53$ dialysis and $n=42$ kidney transplantation $(\mathrm{KTx}))$. CV endpoints were defined as left ventricular hypertrophy (LVH, left ventricular mass index $>45 \mathrm{~g} / \mathrm{m}^{2.16}$ ), increased carotid intima-media thickness (cIMT; $>95^{\text {th }}$ percentile) and increased pulse wave velocity $\left(\mathrm{PWV} ;>95^{\text {th }}\right.$ percentile). Logistic regression with
ROC analysis using sum maximization was applied to assess diagnostic performance in each subgroup (non-KRT, dialysis and KTx) crosssectionally.

Results: TnT and BNP in the non-KRT group was associated with higher odds of LVH, BNP in the dialysis group was associated with increased PWV, while GDF15 associated with LVH in the KTx group. The ability of the biomarkers to discriminate patients with the presence of subclinical CVD was poor-to-fair. (Table)

\begin{tabular}{|c|c|c|c|c|c|c|}
\hline $\begin{array}{l}\text { Biomarker } \\
\quad \text { (per doubling) }\end{array}$ & $\mathrm{CV}$ endpoint & OR & AUC & Cut-off value & Sensitivity & Specificity \\
\hline \multicolumn{7}{|c|}{$\operatorname{CKD}\left(\mathrm{n}=120 ; 13.2\right.$ years, eGFR $\left.14.3 \mathrm{ml} / \mathrm{min} / 1.73 \mathrm{~m}^{2}\right)$} \\
\hline TnT & $\mathrm{LVH}$ & 2.24 & 0.66 & $0.008 \mathrm{ng} / \mathrm{mL}$ & $93 \%$ & $35 \%$ \\
\hline BNP & & 1.35 & 0.63 & $0.45 \mathrm{pg} / \mathrm{mL}$ & $37 \%$ & $79 \%$ \\
\hline \multicolumn{7}{|c|}{ Dialysis $\left(\mathrm{n}=53 ; 15.0\right.$ years, eGFR $8.8 \mathrm{ml} / \mathrm{min} / 1.73 \mathrm{~m}^{2}$ ) } \\
\hline $\mathrm{BNP}$ & PWV & 1.44 & 0.73 & $1.26 \mathrm{pg} / \mathrm{mL}$ & $77 \%$ & $72 \%$ \\
\hline \multicolumn{7}{|c|}{$\mathrm{KTx}\left(\mathrm{n}=42 ; 14.9\right.$ years, eGFR $\left.57.8 \mathrm{ml} / \mathrm{min} / 1.73 \mathrm{~m}^{2}\right)$} \\
\hline GDF15 & LVH & 3.26 & 0.72 & $1195 \mathrm{pg} / \mathrm{mL}$ & $44 \%$ & $92 \%$ \\
\hline
\end{tabular}

Conclusions: Serum biomarkers of CV stress (TnT, BNP and GDF15) are associated to a varying extent with higher odds of LVH or increased PWV in children with CKD and under KRT. Their sensitivity and specificity to detect subclinical CVD, however, is relatively poor.

\section{PI-92 ASSOCIATION BETWEEN BLOOD PRESSURE AND ANGIOTENSIN CONVERTING ENZYMES ACTIVITY IN PREPUBERTAL CHILDREN}

Gaspar Ana Rita ${ }^{1}$, Mosca Sara ${ }^{2}$, Ferreira-duarte Mariana ${ }^{3}$, Teixeira Ana ${ }^{4}$, Cosme Dina ${ }^{5}$, Albino Teixeira António ${ }^{5}$, Aparecida Ronchi Fernanda ${ }^{6}$, Leite Ana Paula ${ }^{6}$, Casarini Dulce ${ }^{6}$, Areias José Carlos ${ }^{7}$, Sousa Teresa ${ }^{5}$, Caldas Afonso Alberto ${ }^{8}$, Morato Manuela ${ }^{3}$, Correia-costa Liane ${ }^{8}$

${ }^{1}$ Instituto De Ciências Biomédicas Abel Salazar, University of Porto, Porto, Portugal, ${ }^{2}$ Instituto De Ciências Biomédicas Abel Salazar, University of Porto, Porto, Portugal; Department Of Pediatrics, Centro Materno-infantil Do Norte, Centro Hospitalar Universitário Do Porto, Porto, Portugal; Epiunit - Instituto De Saúde Pública, Universidade Do Porto, Porto, Portugal, ${ }^{3}$ Laboratory of Pharmacology, Department of Drug Sciences, Faculty of Pharmacy of University of Porto (ffup), Portugal; Laqv@requimte, Faculty of Pharmacy, University of Porto, Portugal, ${ }^{4}$ Epiunit - Instituto De Saúde Pública, Universidade Do Porto, Porto, Portugal; Division of Pediatric Nephrology, Centro Materno-infantil Do Norte, Centro Hospitalar Universitário Do Porto, Porto, Portugal, ${ }^{5}$ Department of Biomedicine - Unit Of Pharmacology and Therapeutics, Faculty of Medicine of University of Porto, Portugal; Medinup - Center For Drug Discovery and Innovative Medicines, University of Porto, Porto, Portugal, ${ }^{6}$ Department of Medicine, Nephrology Division, Escola Paulista De Medicina, Universidade Federal De São Paulo, ${ }^{7}$ Division of Pediatric Cardiology, Centro Hospitalar Universitário São João, Porto, Portugal; Faculdade De Medicina, University of Porto, Porto, Portugal, ${ }^{8}$ Instituto De Ciências Biomédicas Abel Salazar, University of Porto, Porto, Portugal; Epiunit Instituto De Saúde Pública, Universidade Do Porto, Porto, Portugal; Division of Pediatric Nephrology, Centro Materno-infantil Do Norte, Centro Hospitalar Universitário Do Porto, Porto, Portugal

Introduction: Angiotensin-converting enzyme (ACE) and angiotensin-converting enzyme 2 (ACE2) are key enzymes of the renin-angiotensin-aldosterone system (RAAS), an important regulator of blood pressure (BP). Their relationship with BP during childhood has not been clearly established. We aimed to compare ACE and ACE2 activities between body mass index (BMI) groups in a sample of prepubertal children, and to characterize the association between the activity of these enzymes and BP. 
Material and methods: Cross sectional study of 313 children aged 8 to 9 years old, included in the birth cohort Generation XXI (Portugal). Anthropometric measurements and 24-hour ambulatory BP monitoring were performed. ACE and ACE2 activities were quantified by fluorometric methods.

Results: Overweight/obese children presented significantly higher levels of ACE and ACE2 activities, when compared to their normal weight counterparts [median (P25-P75), ACE: 39.48 (30.52-48.97) vs. $42.90(35.62-47.18)$ vs. $43.38(33.49-49.89) \mathrm{mU} / \mathrm{mL}, \mathrm{p}$ for trend=0.009; ACE2: $10.41(7.58-15.47)$ vs. 21.56 (13.34-29.09) vs. $29.00(22.91-34.32) \mathrm{pM} / \mathrm{min} / \mathrm{mL}$, p for trend $<0.001$, in normal weight, overweight and obese children, respectively]. In girls, nighttime systolic BP (SBP) and diastolic BP (DBP) increased across tertiles of ACE activity. in the normal weight $(\mathrm{p}=0.010$ and $p=0.017$, respectively $)$ and overweight $(p=0.019$ and $p=0.042$, respectively) groups. ACE2 activity levels were associated with higher nighttime SBP in overweight/obese girls $(p=0.048)$ and nighttime DBP in the BMI z-score adjusted model in girls $(\mathrm{p}=0.022)$. Median ACE2 activity levels were significantly higher among non-dipper girls (16.7 vs. $11.6 \mathrm{pM} / \mathrm{min} / \mathrm{mL}, \mathrm{p}=0.009)$. No significant association was found in the daytime BP values, neither in BP values distribution across tertiles of ACE2 activity.

Conclusions: Our work shows that obesity is associated with activation of the RAAS, with significant increase of ACE or ACE2 activities already in childhood. Also, we report gender differences in the association of ACE and ACE2 activities, particularly with nighttime $\mathrm{BP}$ in prepubertal girls.

\section{PI-93 DEMOGRAPHIC CHARACTERISTICS OF CHILDHOOD HYPERTENSION IN THE UNITED KINGDOM}

Emily Haseler ${ }^{2}$, Cheentan Singh ${ }^{3}$, Joanna Newton ${ }^{1}$, Nabil Melhem ${ }^{1}$, Manish D Sinha ${ }^{1}$

${ }^{1}$ Evelina London Childrens Hospital, London, United Kingdom, ${ }^{2}$ University Hospital Lewisham, London, United Kingdom, ${ }^{3}$ North Middlesex University Hospital Nhs Trust, London, United Kingdom

Introduction: We report on demographic characteristics in Children and Young People (C\&YP) from the largest dedicated paediatric hypertension clinic in the UK.

Material and methods: Prospective data including all C\&YP over a recent 6 year period (2013-2018). Patients categorised as normotensive, white coat hypertension $(\mathrm{WCH})$, primary hypertension $(\mathrm{PH})$ and secondary hypertension $(\mathrm{SH})$ and compared across age groups $<6(n=162), 6$ to $<12(n=114)$, and 12 to $<18$ years $(n=272)$.

Results: 548 C\&YP [58.2\% girls] with median age of 11.9 (IQR $10.8)$ years. $232(42.3 \%)$ were normotensive; of those hypertensive $(\mathrm{n}=316), 46(15 \%), 83(26 \%)$ and $187(59 \%)$ were $\mathrm{WCH}, \mathrm{PH}$ and $\mathrm{SH}$ respectively. There was a bimodal age distribution for those with $\mathrm{SH}$ with peaks in $<6$ and adolescent years, whereas $\mathrm{PH}$ and WCH peaked solely in adolescence.

Children <6years significantly more likely to have normotension following evaluation using appropriate out-of-office monitoring. If hypertensive, they were significantly more likely to have $\mathrm{SH}$ and less likely to have body mass index $>95$ percentile compared with older children. In those aged <6years, prevalence of WCH and $\mathrm{PH}$ both was $<0.5 \%$ and significantly lower than older age groups.

There were lower numbers of hypertensive children of non-White Ethnicity <6years. Amongst those with hypertension, those of nonWhite Ethnicity had higher prevalence of PH but comparable rates of WCH when compared with White patients.

Children of normal weight had higher prevalence of non-White ethnicity with PH. Amongst those with excess weight $\left(>85^{\text {th }}\right.$ percentile), significantly more older children but no difference in distribution of hypertension categories by ethnicity $[\mathrm{p}=0.07]$
Conclusions: Hypertensive young children are most likely to have secondary hypertension and negligible rates of $\mathrm{WCH}$ and $\mathrm{PH}$. Primary hypertension including WCH accounts for $40 \%$ of hypertension in C\&YP in the UK with the highest prevalence in adolescence, non-White Ethnicity and excess weight.

\section{PI-94 BLOOD PRESSURE PARAMETERS AFFECTING VENTRICULAR REPOLARIZATION IN OBESE CHILDREN}

Belde Kasap Demir ${ }^{1}$, Eren Soyaltin ${ }^{2}$, Caner Alparslan ${ }^{2}$, Seçil Arslansoyu Çamlar ${ }^{2}$, Demet Alaygut ${ }^{2}$, Fatma Mutlubaș ${ }^{2}$, Elif Perihan Öncel ${ }^{2}$, Onder Yavascan ${ }^{2}$, Cem Karadeniz ${ }^{3}$

${ }^{1}$ Katip Celebi University Faculty of Medicine Department of Pediatric Nephrology and Rheumatology, Turkey, ${ }^{2}$ Izmir University of Health Sciences Tepecik Education and Research Hospital Department of Pediatric Nephrology, Turkey, ${ }^{3}$ Katip Celebi University Faculty of Medicine Department of Pediatric Cardiology, Turkey

Introduction: Ventricular repolarization (VR) increases the risk of sudden cardiac death due to ventricular arrhythmia. We aimed to evaluate the blood pessure (BP) parameters affecting VR in obese children.

Material and methods: Obese (BMI $\geq 95 \mathrm{p})$ and healthy children $\geq 120 \mathrm{~cm}$ between January 2017 and June 2019 were included. Demographic and laboratory data were recorded. Peripheral and central BPs were evaluated by a device capable of ambulatory blood pressure monitoring (ABPM) and pulse wave analysis (PWA). QT and QTc, Tp-e and Tp-e/QTc, left ventricular mass index (LVMI), cardiac geometry, aortic strain and elastic modulus were calculated.

Results: 52 obese and 41 control patients with similar age and gender were included. Uric acid, triglyceride and ALT values, systolic and diastolic office BPs, 24-hour, daytime and nighttime systolic and mean arterial BPs, daytime diastolic BP SDS levels, daytime and nighttime systolic loads, daytime diastolic load, 24-hour, daytime and nighttime central systolic and diastolic BPs and PWV values were significantly higher in obese cases $(\mathrm{p}<0.005)$. However, @ AIx75 values for 24 hours, day and night were similar between the groups $(\mathrm{p}>0.05)$. Among the VR data, QTed and Tp-ed were higher in obese patients $(p<0.005)$. While LVMI values were similar between the groups, the relative wall thickness and aortic tension were higher in obese cases $(p<0.005)$. The most important factor affecting VR in obese cases was decreasing age and increasing diastolic load at night $(\mathrm{B}=-2.83, \mathrm{p}=0.010, \mathrm{~B}=0.257, \mathrm{p}=0.007$, respectively).

Conclusions: Obese patients have a higher risk of peripheral and central BP, arterial stiffness, aortic tension and sudden cardiac death, and these develop before an increase in LVMI.

\section{PI-95 AMBULATORY BLOOD PRESSURE MONITORING AND END ORGAN DAMAGE IN CHILDHOOD HYPERTENSION}

Musa Öztürk ${ }^{1}$, Z. Birsin Özçakar ${ }^{2}$, Nilgün Çakar ${ }^{2}$, Beyza Doğanay ${ }^{3}$, Fatoș Yalçinkaya ${ }^{2}$

${ }^{1}$ Ankara University Faculty of Medicine-Departments of Pediatrics, Turkey, ${ }^{2}$ Ankara University Faculty of Medicine- Pediatric Nephrology, Turkey, ${ }^{3}$ Ankara University Faculty of Medicine- Biostatistics, Turkey

Introduction: Childhood hypertension (HT) is a chronic disease that requires careful and delicate follow-up. Many data obtained from ambulatory blood pressure monitoring (ABPM) guide us in the management of HT. The purpose of evaluating these measurements is to obtain the most accurate and earliest prediction for the severity of HT and end organ damage. Material and methods: Files of 269 children who were followed up with the diagnosis of HT were evaluated retrospectively. The 
presence of end organ damage was recorded together with the ABPM data of the patients at the time of diagnosis. Z scores were obtained from the ABPM outputs using the statistical LMS method and the relationship to end organ damage was investigated.

Results: Left ventricular hypertrophy (LVH) and hypertensive retinopathy were found in $18 \%$ and $9 \%$ of the patients who underwent cardiological and ophthalmological examination, respectively. The relationship of ABPM data $\mathrm{Z}$ scores and blood pressure (BP) loads with LVH is given in Table 1. 24-hour, day and night mean systolic BP Z scores of the patients with LVH were found to be significantly higher as compared to the patients without. In addition, systolic day and night BP loads were significantly higher in the group with LVH. It was calculated that one unit increase in the mean daytime systolic BP $\mathrm{Z}$ score increases the risk of LVH by 1.35 times and ten percent increase in systolic BP load increases the risk 1.16 times.

Table 1. Evaluation of the relationship between left ventricular involvement (LVH) and ABPM data

\begin{tabular}{|l|l|l|l|l|}
\hline \multicolumn{2}{|c|}{ Cardiological investigation (n:206) } & LVH (+) (n:37) & LVH (-) (n:169) & p \\
\hline \multirow{3}{*}{ Systolic BP } & 24 hour Z score & $2,57 \pm 2,21$ & $1,7 \pm 1,76$ & $\mathbf{0 , 0 1}$ \\
\cline { 2 - 5 } & Daytime Z score & $2,18 \pm 2,03$ & $1,32 \pm 1,64$ & $\mathbf{0 , 0 2}$ \\
\cline { 2 - 5 } & Nighttime Z score & $2,74 \pm 2,12$ & $1,68 \pm 1,48$ & $\mathbf{0 , 0 0 6}$ \\
\cline { 2 - 5 } & Daytime BP Load (\%) & $65(0-100)$ & $52(0-100)$ & $\mathbf{0 , 0 3 3}$ \\
\hline & Nighttime BP Load (\%) & $55(0-100)$ & $43(0-100)$ & $\mathbf{0 , 0 4 7}$ \\
\hline \multirow{2}{*}{ Diastolic BP } & 24 hours Z score & $0,86 \pm 1,71$ & $0,69 \pm 1,34$ & 0,56 \\
\cline { 2 - 5 } & Daytime Z score & $0,26 \pm 1,58$ & $0,11 \pm 1,21$ & 0,58 \\
\cline { 2 - 5 } & Nighttime Z score & $1,47(-1,98+7,39)$ & $0,91(-2,67+6,51)$ & $\mathbf{0 , 0 4 3}$ \\
\cline { 2 - 5 } & Daytime BP Load (\%) & $25(0-85)$ & $18(0-86)$ & 0,189 \\
\cline { 2 - 5 } & Nighttime BP Load (\%) & $33(0-100)$ & $30(0-100)$ & 0,949 \\
\hline
\end{tabular}

ABPM: Ambulatory blood pressure monitoring; BP: Blood pressure

Conclusions: These results show that evaluation of the ABPM data in childhood hypertension through the incoming studies will increase the chance of early diagnosis and treatment. At this point, the real question is what should be the ABPM cut-off points to prevent organ damage.

\section{PI-96 EVOLUTION OF CARDIOVASCULAR RISK FACTORS IN PEDIATRIC KIDNEY TRANSPLANT RECIPIENTS}

Anna Végh ${ }^{1}$, Adrienn Bárczi ${ }^{2}$, Orsolya Cseprekál ${ }^{3}$, Éva Kis ${ }^{4}$, György S. Reusz ${ }^{1}$

${ }^{1}$ First Department of Pediatrics Semmelweis University, Budapest, Hungary, ${ }^{2}$ Medical Imaging Centre Semmelweis University, Budapest, Hungary; ${ }^{3}$ Department of Surgery And Transplantation, Semmelweis University, Budapest, Hungary; ${ }^{4}$ Gottsegen György Hungarian Institute of Cardiology, Budapest, Hungary

Introduction: Cardiovascular disease is leading cause of mortality in pediatric kidney transplant recipients. Major cardiovascular events are rare in childhood however, atherosclerosis, increased arterial stiffness and left ventricular hypertrophy may be already present in early stages of kidney disease. Successful kidney transplantation may reduce the overall cardiovascular risk; however, it remains 3-5 times higher than in the general population. The aim of this study was to detect the cardiovascular risk factors in pediatric kidney transplant recipients.

Material and methods: Fifty-two children and young adults who underwent kidney transplantation before the age of 18 (median [IQR]: 10.79[8.36-23.24] years) were prospectively enrolled into the study. Anthropometric data, laboratory-, office and 24-h ambulatory blood pressure monitoring values and pulse wave velocity (PWV), a marker of arterial stiffness were evaluated at two timepoints (median [IQR]: 2.53[1.03-4.72] years and 9.3[4.0311.76] years) following transplantation.
Results: Controlled hypertension (44.2\% and 55.7\%), hypertriglyceridemia $(57.14 \%$ and $38.1 \%)$ and anemia $(19.2 \%$ and $19.6 \%)$ were the most prevalent risk factors at the two timepoints, respectively. At the second follow-up, but not at the first there was a positive correlation between PWV Z score and systolic and diastolic blood pressure $(\mathrm{R}=0.44, \mathrm{p}=0.009 ; \mathrm{R}=0.55 \mathrm{p}=0.001$ ). A significant difference in creatinine levels and GFR was also found between those who had their blood pressure controlled, and who had not (mean \pm SD: $\mathrm{GFR}_{\text {controlled }}=74.09 \pm 32.44, \mathrm{GFR}_{\text {uncontrolled }}=49.6 \pm 23.13$, $\mathrm{p}=0.025$ ).

Conclusions: Even though renal transplantation helps reduce the global cardiovascular risk, multiple cardiovascular risk factors were present in our study population at the long-term follow-ups. Our results underline the importance of adequate blood pressure control, since several years after transplantation it may improve outcome measures such as, the graft function and arterial stiffness.

Supported by NKFI-124549 grant.

\section{PI-97 THE EFFECT OF CHRONIC KIDNEY DISEASE ON ARTERIAL ELASTICITY IN PEDIATRIC POPULATION}

Şule Arici ${ }^{2}$, Ece Demirci Bodur ${ }^{1}$, Özlem Sarisoy ${ }^{3}$, Oğuzhan Tezel ${ }^{4}$, Harika Alpay ${ }^{1}$, Figen Akalin ${ }^{2}$

${ }^{1}$ Marmara University Pediatric Nephrology, Turkey, ${ }^{2}$ Marmara University Pediatric Cardiology, Turkey, ${ }^{3}$ Ümraniye Research and Training Hospital Paediatric Cardiology, Turkey, ${ }^{4}$ Marmara University Paediatrics, Turkey

Objectives: Chronic kidney disease (CKD) can cause atherosclerosis and cardiac diseases. Echocardiographic measurement of the elasticity and distensibility of the aorta is useful in detecting early vascular changes. These measurements may be affected by dialysis efficiency and fluid load. In our study, we aimed to evaluate arterial functions by 
echocardiography in patients with $\mathrm{CKD}$ and investigate its relationship with clinical findings and bioimpedance measurements which are indicators of hydration status.

Materials and Methods: Twenty six patients with CKD followed-up in our Pediatric Nephrology and Cardiology outpatient clinics were evaluated for aortic elasticity. Demographic, clinical and laboratory findings, echocardiographic measurements, 24-hour ambulatory blood pressure values, bioimpedance measurements were recorded. Aortic strain, distensibility, aortic stiffness index were compared by a correlation analysis with CKD duration, dialysis time and type, overhydration status (bioimpedance analysis), systolic and diastolic blood pressures, hemoglobin and creatinine levels. A $p$ value of $<0.05$ was considered statistically significant.

Results: Ten patients (38.5\%) were female. Mean age was $13.5 \pm 3.6$ years, CKD duration was $4.9 \pm 3.2$ years. Sixteen patients $(61.5 \%)$ were pre-dialysis patients, 10 patients $(38.5 \%)$ were on renal replacement therapy. The systolic and diastolic blood pressures of the patients were 121.13 $\pm 11.27 \mathrm{mmHg}$ and $79.07 \pm 12.57 \mathrm{mmHg}$, respectively. Mean hemoglobin levels were $11.54 \pm 2.22 \mathrm{~g} / \mathrm{dl}$, mean creatinine levels were $4.33 \pm 3.09$ $\mathrm{mg} / \mathrm{dl}$. Left ventricular functions were normal in all of the patients. Aortic valve pathology was detected in 3 patients. Ascending aorta stiffness index, strain and distensibility were $8,26 \pm 9,67,-1,62 \pm 1,15,0,47 \pm 0,57$ respectively. A positive correlation between CKD duration and stiffness of aorta was found ( $\mathrm{p}$ values were 0,039 and 0,030 for arcus aorta and abdominal aorta respectively). No correlation was found between overhydration, blood pressure, hemoglobin, creatinine levels and aortic strain, distensibility, stiffness index.

Conclusion: We found that the duration of $\mathrm{CKD}$ and the deterioration in arterial functions were parallel. CKD may accompany aortic valve pathologies. Evaluation of arterial functions in the long-term follow-up will be useful for patients with CKD.

\section{PI-98 SECONDARY HYPERTENSION WITH UNREMARKABLE RADIOLOGICAL EXAMINATIONS BUT HIGH GRADE STENOSIS IN ANGIOGRAPHY}

\author{
Mara Egerer ${ }^{1}$, Mohammed Nashawi ${ }^{1}$, Peter Müller-ABT ${ }^{2}$, \\ Frank Uhlemann ${ }^{3}$, Axel Enninger ${ }^{1}$, Martin Bald ${ }^{4}$ \\ ${ }^{1}$ Department of Paediatrics, Olgahospital, Klinikum Stuttgart, \\ Germany, ${ }^{2}$ Department of Paediatric Radiology, Olgahospital, \\ Klinikum Stuttgart, Germany, ${ }^{3}$ Department of Paediatric Cardiology, \\ Olgahospital, Klinikum Stuttgart, Germany, ${ }^{4}$ Department of Paediatric \\ Nephrology, Olgahospital, Klinikum Stuttgart, Germany
}

Objectives: About 5-25\% of arterial hypertension in children is caused by renovascular hypertension. Children suffering from this condition often show now significant clinical symptoms.

Methods: We hereby introduce a 2 years old patient with sever hypertension due to renal artery stenosis showed only in catheter angiography. Results: The patient showed no symptoms and no abnormalities in the clinical examination. The blood pressure measurements showed pressures over the $95^{\text {th }}$ percentile. Blood work, urine screening, echocardiography and kidney ultrasound showed normal results. In the MRI-Angiography no vascular abnormality could be seen, however the left kidney was smaller than the right one. Moreover, because of persisting high blood pressure despite 3 anti-hypertensive medications, a digital subtraction angiography has been done and showed a high-grade stenosis of the left renal artery with a string-of-beads appearance. A renal scintigraphy was conducted and showed a leading right kidney and a left kidney with a slightly delayed renal perfusion.

Conclusion: Secondary arterial hypertension should always be considered in children with elevated blood pressure levels. If renal and endocrinologic causes can be ruled out, stenosis of vessels close to the heart and renal vessels is the most common reason for a secondary arterial hypertension. Comprehensive diagnostic should be considered to rule out renal artery stenosis. As presented in our case, MR angiography cannot always detect arterial blood vessel abnormalities. Therefore, catheter angiography should be taken in consideration to confirm the diagnosis of renal arterial stenosis.

\section{PI-99 A BAYESIAN POPULATION APPROACH TO DETERMINE LISINOPRIL EXPOSURE IN HYPERTENSIVE PAEDIATRIC PATIENTS WITH MILD TO MODERATE RENAL IMPAIRMENT}

Louis Sandra ${ }^{2}$, Eva Degraeuwe ${ }^{1}$, Elke Gasthuys ${ }^{2}$, Paulien De Bruyne ${ }^{1}$, An Vermeulen ${ }^{2}$, Ann Raes ${ }^{1}$, Johan Vande Walle ${ }^{1}$

${ }^{1}$ Department of Internal Medicine and Paediatrics (ge35), Ghent University, Belgium ${ }^{2}$ Laboratory of Medical Biochemistry and Clinical Analysis, Faculty of Pharmaceutical Sciences, Ghent University, Belgium

Objective: Lisinopril, an angiotensin-converting enzyme (ACE) inhibitor, is the most frequently prescribed antihypertensive therapeutic in the paediatric population, despite being used off-label (1). The SAFEPEDDRUG project studied lisinopril exposure and blood pressure effects in a dose-escalation pilot study, envisioning safe and dose-optimized usage of lisinopril in children with primary or secondary hypertension including patients with mild renal impairment, corresponding to the vast majority of the day-to-day clinical paediatric population (2).

Methods: The SAFEPEDDRUG lisinopril pilot study included 13 children with primary/secondary hypertension who received oral lisinopril once daily, doses ranging from $0.05 \mathrm{mg} \mathrm{kg}^{-1}$ to $0.2 \mathrm{mg} \mathrm{kg}^{-1}$. A total of 46 peak and trough plasma samples were collected. Patients were aged between 1.9 and 17.9 years (median: 13.5 years) and had a total body weight between 9.62 and $97.2 \mathrm{~kg}$ (median: $53.2 \mathrm{~kg}$ ). eGFR was measured between 55.5 and $180 \mathrm{~mL} / \mathrm{min} / 1.73 \mathrm{~m}^{2}$ (median: $99.9 \mathrm{ml} / \mathrm{min} / 1.73 \mathrm{~m}^{2}$ ). All data were analyzed using Monolix version 2020R1 (Lixoft ${ }^{\circledR}$, France) and $\mathrm{R}$ version 3.6.2.

Results: A one-compartment model with $1^{\text {st }}$ order absorption and $1^{\text {st }}$ order elimination optimally describes the analyzed data. Significant covariate effects include total bodyweight on elimination clearance and distribution volume and eGFR on elimination clearance. The effects of total bodyweight are parametrized in terms of fixed allometric exponents $(0.75$ for clearance, 1 for volume), centered around a total body weight of $70 \mathrm{~kg}$ (3). The effects of eGFR on the elimination clearance are optimally described by a power law parametrization centered around $105 \mathrm{~mL} / \mathrm{min} /$ $1.73 \mathrm{~m}^{2}$. Estimated population elimination clearance equals 23.12 $\mathrm{L} / \mathrm{h} / 70 \mathrm{~kg}$ and distribution volume equals $32.91 \mathrm{~L} / 70 \mathrm{~kg}$ and are in line with results published by Thompson et al (4).

Conclusion: Lisinopril dose and regimen adjustments for paediatric patients should include eGFR on top of weight adjustments. Extending the model to characterize pharmacodynamic effect is required to identify the optimal dose and dosing regime.

\section{PI-100 IMPROVING ALLOGRAFT SURVIVAL BY INFORMING DONOR SELECTION IN KIDNEY TRANSPLANT RECIPIENTS UNDER 5 YEARS OLD}

Florian Manca Barayre ${ }^{1}$, Larry A Greenbaum ${ }^{3}$, Rouba Garro ${ }^{3}$, Chia-shi Wang ${ }^{3}$, Pamela D Winterberg ${ }^{3}$, Rachel Patzer ${ }^{4}$, Julien Hogan ${ }^{2}$ 
${ }^{1}$ Emory University School of Medicine, Atlanta, USA, ${ }^{2}$ Robert-debré Hospital, Aphp, Paris, France, ${ }^{3}$ Children's Healthcare of Atlanta, Atlanta, USA, ${ }^{4}$ Department of Epidemiology, Rollins School of Public Health, Emory University, Atlanta, USA

Objectives: The US kidney allocation system aims at allocating highquality kidneys to pediatric recipients but the score used to assess the quality of the donors (KDPI) may not accurately predict graft survival in pediatric recipients. We aimed at assessing KDPI accuracy to predict graft loss in pediatric recipients and at developing predictive models of graft loss for deceased and living donor transplants to inform donor selection.

Methods: We included first-time kidney transplantations in pediatric recipients from the US SRTR database of adult donors from 2005 to 2018. The primary outcome was defined as death or graft loss. We developed a Small Pediatric-KDPI (SP-KDPI) and Small Pediatric-Living KDPI (SP-LDKPI) on the same scale to compare each other by using Cox models adjusted for recipients characteristics.

Results: KDPI C-statistic was 0.52 and was not associated with graft survival in recipients under 5 years old. Six deceased donor factors were included in SP-KDPI computation: ethnicity, age, body surface area, gender, cold ischemia time, HLA-B mismatches number. Four living donor factors were included in SP-LKDPI computation: race, age, HLA-B mismatch number and donor/recipient body surface area ratio. Model accuracies were validated internally by cross-validations and Cstatistics were $0.64(95 \% \mathrm{CI}=0.57-0.70)$ and $0.65(95 \% \mathrm{CI}=0.58-$ 0.73 ) for SP-KDPI and SP-LKDPI, respectively. $16.8 \%$ of living donors allograft had a negative SP-LKDPI (meaning a better predicted survival than any deceased donor)

Conclusions: The current US kidney allocation system allocates good kidneys to pediatric recipients but provides little information to guide donor selection especially in recipients $<5$ years old. We developed an adaptation that demonstrated a higher accuracy to predict graft loss in young recipients and an extension of this score to accurately compare adult living and deceased donors offered to young recipients.

\section{PI-101 PUBLICATION RATE AND RESEARCH TOPICS OF STUDIES IN PEDIATRIC KIDNEY TRANSPLANTATION}

Patry Christian ${ }^{1}$, Cordts Stefanie ${ }^{1}$, Baumann Lukas ${ }^{2}$, Höcker Britta ${ }^{1}$, Fichtner Alexander ${ }^{1}$, Ries Markus ${ }^{1}$, Tönshoff Burkhard ${ }^{1}$

${ }^{1}$ University Childrens Hospital Heidelberg, Germany, ${ }^{2}$ Institute of Medical Biometry and Informatics, University of Heidelberg, Germany

Introduction: The quality of medical care for pediatric kidney transplant recipients also depends on evidence from clinical trials. This evidence could be negatively influenced by a publication bias resulting from selective non-publication of studies. Publication bias is currently considered a major problem in evidence-based medicine.

Material and methods: We therefore examined the publication rate, time to publication and factors associated with non- or late publication of studies in pediatric kidney transplantation registered on ClinicalTrials.gov from 1999 to 2020 . We searched for respective publications on PubMed, GoogleScholar and by directly contacting principal investigators and/or study sponsors. Late publication was defined as a publication later than 3 years after completion. Data ware compared by binary logistic regression- and Kaplan Meier analysis.

Results: We identified 138 studies on pediatric kidney transplantation with an overall enrolment of 36678 study participants. Only $55.8 \%$ of these studies were published until closure of the database (November 2, 2020). Unpublished studies included data from 12014 study patients. The median time to publication was 25 months (range, 0 - 117) with significantly earlier publications in more recent years. The most frequent research topic was immunosuppressants (68/138 [49.3\%]), followed by perioperative management $(15 / 138$ [10.9\%]) and infectiology (14/138 [10.1\%]). $41.3 \%$ of all studies were randomized controlled trials. Factors favoring publication of a study at all were a co-funding by industry $(\mathrm{P}=0.003)$ and the research topic infectiology $(\mathrm{P}=0.046)$. Factors associated with late publication were the research topic immunosuppressants $(\mathrm{P}=0.017)$, co-funding by industry $(\mathrm{P}=0.004)$ and a randomized controlled study design $(\mathrm{P}=0.019)$. The level of the respective impact factor was not associated with the time to publication.

Conclusions: Currently, nearly half of all registered and completed studies in pediatric kidney transplantation remain unpublished. This and the relative long time to publication might lead to a publication bias with a negative impact on clinical decision making.

\section{PI-102 GRAFT SURVIVAL AFTER PEDIATRIC KIDNEY TRANSPLANTATION IN THE NETHERLANDS: RESULTS OF THE LAST 16 YEARS}

Emmanuelle Gaikhorst, Antonia Bouts, Ernst Van Heurn Amsterdam Umc, The Netherlands

Introduction: This study evaluated graft survival rates of pediatric kidney transplants performed at Emma Children's Hospital Amsterdam over the last 16 years and aimed to detect factors that influence graft survival. Material and methods: A retrospective single-center study was conducted with a total of 98 pediatric ESRD patients who were transplanted between January 2004 and December 2019 at our center. Patients were split into three cohorts (transplant era's) based on their date of transplantation (2004-2009, 2009-2014 and 2014-2019). Cox regression analysis and Kaplan Meier procedure were used to analyze the data.

Results: Overall graft survival rates at one and five year post-transplant were $97 \%$ and $88 \%$, respectively. Both one- and five-year graft survival of the most recent cohort (transplants of 2014-2019) were 100\%, both for living and deceased donors. Out of ten independent variables that were analyzed, occurrence of rejection within the first year after transplantation (HR 2,86; p-value 0,020) and deceased donors were found to be the strongest negative predictors of graft survival (HR 2,79; p-value 0,029). We found an unequal proportion of deceased donors allocated to black patients. Detailed investigation of this finding was beyond the scope of this research, but should be further investigated in order to limit possible racial gaps.

Conclusions: 1- and 5-year graft survival rates improved substantially over the last 16 years in our center. Rejection within the first year after transplantation and deceased donors were significantly associated with worse graft survival in our data.

\section{PI-103 REDUCING FACE TO FACE OUTPATIENT VISITS FOR RENAL TRANSPLANT PATIENTS...VIRTUALLY IMPOSSIBLE?}

Louise Kipping, Helen Jones, Grainne Walsh, Nick Ware Guys and St Thomas Hospital, United Kingdom

Introduction: For half a century, paediatric renal transplant recipients (pRTRs) have been exclusively followed up in face to face(F2F) clinic in our tertiary centre. During the COVID-19 global pandemic adaptations were made to minimise travel/exposure for all pRTRs by re-structuring the clinic and utilising local services, for monitoring traditionally carried out by the tertiary centre. A virtual(video) transplant clinic overseen by the paediatric transplant team was developed together with postal packs to 
enable immunosuppression drug level and viral load monitoring in conjunction with laboratory colleagues.

Material and methods: Retrospective review of F2F versus virtual pRTR outpatient follow-up appointments 6 months before the COVID19 pandemic, after 6 months and $1 \mathrm{y}$ into it in a single paediatric nephrology centre.

Results: 97 pRTRs under follow up included in the study. Due to COVID-19 the transplant program was suspended for a period where no new pRTRs were added to the cohort until the end June 2020.

Median number of reviews per pRTR was $5 \mathrm{~F} 2 \mathrm{~F}$ and 0 virtual before, 3.5 F2F and 2 virtual 6 months into and $3 \mathrm{~F} 2 \mathrm{~F}$ and 2 virtual 1 year into the pandemic. To facilitate the virtual reviews pRTRs had monitoring blood tests, anthropometry, blood pressure and urinalysis at the tertiary centre $(57 \%)$, in primary care (15\%), children's community nursing team (14\%) and at their local hospital (12\%).

Conclusions: Developing a virtual transplant clinic enabled a reduction of F2F visits during the COVID-19 pandemic. We hope to further develop this service by strengthening our links with local services as well as empowering pRTRs and families to carry out some of their own monitoring (weight, blood pressure, urinalysis and finger prick blood tests) in the home environment and evaluate the outcome including patient feedback of these changes.

\section{PI-104 REVERSIBLE GLOMERULAR GRAFT DAMAGE IN DISSEMINATED INTRAVASCULAR COAGULATION}

Raffaella Labbadia ${ }^{1}$, Luca Antonucci ${ }^{4}$, Francesca Diomedi Camassei ${ }^{2}$, Laura Lucchetti ${ }^{1}$, Isabella Guzzo ${ }^{1}$, Andrea Onetti Muda ${ }^{2}$, Marco Spada ${ }^{3}$, Luca Dello Strologo ${ }^{1}$

${ }^{1}$ Nephrology, Dyalisis And Renal Transplant Unit Bambino Gesù Children's Research Hospital Irccs, Rome, Italy, ${ }^{2}$ Pathology Unit Bambino Gesù Children's Research Hospital Irccs, Rome, Italy, ${ }^{3}$ Abdominal Transplant Surgery Bambino Gesù Children's Research Hospital Irccs. Rome, Italy, ${ }^{4}$ Academic Department Of Pediatrics University of Rome "tor Vergata", Nephrology, Dyalisis and Renal Transplant Unit Bambino Gesù Children's Research Hospital Irccs, Rome, Italy

Introduction: Kidneys from a 17-year-old male donor who died from head injury in a car accident were offered. His labs at ICU showed: fibrinogen $20 \mathrm{mg} / \mathrm{dL}, \mathrm{Hb} 12 \mathrm{gr} / \mathrm{dl}$, hematocrit $36.8 \%$ and platelets $237,000 / \mathrm{mcl}$. CPK was $318 \mathrm{mg} / \mathrm{dl}$ with MB fraction of $6 \%$. Serum creatinine was $0.9 \mathrm{mg} / \mathrm{dL}$. Within 24 hours hematocrit, $\mathrm{Hb}$ and platelets dropped to $23.8 \%, 8.3 \mathrm{~g} / \mathrm{dl}$ and $32,000 / \mathrm{mcl}$, respectively. Serum creatinine rose to a maximum of $2.01 \mathrm{mg} / \mathrm{dL}$ and urinalysis showed proteinuria $(300 \mathrm{mg} / \mathrm{dL})$ at the time of organ procurement.

Material and methods: Pre-implant kidney biopsy showed acute tubular damage; glomerular capillaries were massively occluded by fibrin thrombi containing fragmented red blood cells and inflammatory cells. Arterioles and small arteries were consistently spared. A diagnosis of disseminated intravascular coagulation (DIC) was made. Thrombotic microangiopathy was also considered, but the absence of significant vascular wall damage, despite massive microthrombi formation, made it less likely.

Results: Kidneys were accepted. Delayed graft function was observed for both recipients until post-operative (pod) day 6 . Platelets dropped to a minimum value of 65,000 and $84,000 / \mathrm{mcl}$ for each recipient on pod 6 and 5and were again above $200,000 / \mathrm{mcl}$ on pod 12 and 11 . Diuresis was always present. Renal function improved quite slowly: creatinine clearance in the two children was 68 and $54 \mathrm{ml} / \mathrm{min} / 1.73 \mathrm{~m}^{2}$ on pod 30,80 and $81 \mathrm{ml} / \mathrm{min} / 1.73 \mathrm{~m}^{2}$ and 100 and $80 \mathrm{ml} / \mathrm{min} / 1.73 \mathrm{~m}^{2}$ on $3^{\text {th }}$ and $6^{\text {th }}$ months after transplant, respectively.

Conclusions: DIC occurs in $29 \%$ of potential donors and is a frequent cause for organ refusal. It consists ofpersistent coagulation activation and extensive formation of microthrombi in microvessels, especially in capillary bed. As DIC progresses, it can lead to multiorgan failure. Although renal recovery after transplantation is slow, kidneylesions are likelyreversible and do not affect long-term function of the transplanted organ. Such kidneys should therefore be considered for transplantation.

\section{PI-105 HYPERGLYCEMIA AND NODAT AFTER RENAL TRANSPLANTATION}

Muhammet Sancaktar, Gülșah Kaya Aksoy, Mustafa Koyun, Ayla Kaçar, Mehtap Adar, Elif Çomak, Sema Akman

Akdeniz University Medical Faculty Department of Pediatric Nephrology, Antalya, Turkey

Introduction: Hyperglycemia is one of the common metabolic complications in pediatric kidney transplant recipients. The aim of this study was to investigate the clinical features of pediatric kidney transplant recipients with hyperglycemia.

Material and methods: The medical records of kidney transplant recipients who developed hyperglycemia during the post-transplant period were retrospectively reviewed. Those who had diabetes mellitus (DM) before transplantion were excluded from the study. Hyperglycemia was defined as fasting blood glucose $>126 \mathrm{mg} / \mathrm{dL}$ or 2 hour post-load plasma glucose $>200$ $\mathrm{mg} / \mathrm{dL}$. In addition to these findings, patients with low $\mathrm{C}$ peptide and insulin levels were defined as new-onset diabetes after transplantation (NODAT). Patients with HOMA-IR (Homeostatic Model Assessment-Insulin Resistance) value $>2.5$ was accepted as insulin resistance.

Results: Twenty-nine $(6.1 \%)$ of 471 recipients developed hyperglycemia after transplantation. The median follow-up period was 38 months (range: $15-58)$ and $\% 58.6$ were girls. The most common (41.3\%) primary kidney disease was CAKUT. Five patients with hyperglycemia (17.2\%) had a positive family history for DM.

The detection times of hyperglycemia after transplantation were as follows; $20(68.9 \%)$ patients within the first 3 months, $2(6.8 \%)$ patients between 3-6 months, 3 (10.3\%) patients between 6-12 months and the remaining $4(13.7 \%)$ after 12 months. Insulin resistance was found in 27 $(93.1 \%)$ patients. Only $2(6.8 \%)$ patients were diagnosed with NODAT. Subcutaneous insulin therapy was started in 26 (89.6\%), 9 of whom $(31.0 \%)$ were on insulin therapy at the end of the post-transplant first year. Conclusions: Post-transplant hyperglycemia is often accompanied by insulin resistance. Short-term insulin treatments are needed in most of these patients, but it is noteworthy that this period is prolonged in up to a quarter of the patients.

\section{PI-106 FINAL HEIGHT OF CHILDREN OVER 12 YEARS INITIATED ON A STEROID MINIMISATION IMMUNOSUPPRESSIVE REGIMEN FOLLOWING KIDNEY TRANSPLANTATION}

Demetria Theodorou ${ }^{1}$, Thomas Dowsett ${ }^{2}$, Richard Holt ${ }^{2}$, Mohan Shenoy ${ }^{1}$

${ }^{1}$ Royal Manchester Children's Hospital, United Kingdom, ${ }^{2}$ Alder Hey Children's Hospital, United Kingdom

Objectives: A large European study reported that only $55 \%$ of adults who started renal replacement therapy in childhood achieved normal height. Previous studies have not demonstrated catch-up growth in children $\geq 12$ years at the time of transplantation. Our aim was to characterise final height outcome in children who underwent kidney transplantation at age $\geq 12$ years using a CSM immunosuppressive regimen.

Method: A retrospective review of electronic records was performed on patients who underwent a kidney transplant at ${ }^{3} 12$ years and were initiated on a CSM immunosuppressive protocol from 2009 to 2019. Final height (FH) was defined as height at $\geq 18$ years (or $<1 \mathrm{~cm}$ growth in the last 12 months). Normal FH was defined as SDS $\geq-1.88$. 
Results: 51 patients were included (mean follow-up 2.6 years, range 0.26-5.49). Median age at last follow-up was 18.4 years (17.4-19). A normal FH SDS was achieved in $36(71 \%)$. Median height at transplantation was SDS -1.19 and the median final height SDS was -0.86 (median FH was $152.5 \mathrm{~cm}$ in girls and $173.2 \mathrm{~cm}$ in boys). The significant factors associated with achieving normal FH were male gender $(p=0.03)$, and shorter duration of dialysis $(\mathrm{p}=0.019)$. All boys $(n=9)$ transplanted under the age of 15 years achieved normal FH (SDS -1.46 to 1.66). $73 \%$ (37) of patients had a normal BMI SDS, $12 \%$ (6) were overweight and $12 \%$ (6) were obese. $41 \%$ (21) were on antihypertensive medication at the last paediatric follow-up.

Conclusion: $71 \%$ of children ${ }^{3} 12$ initiated on a CSM immunosuppressive protocol achieved normal $\mathrm{FH}$. Boys $<15$ years were more likely to achieve a normal FH compared to girls of a similar age.

\section{PI-107 THYROID FUNCTIONS IN PEDIATRIC PATIENTS AFTER RENAL TRANSPLANTATION}

Demet Alaygut ${ }^{1}$, Gökçen Erfidan ${ }^{1}$, Eren Soyaltin ${ }^{1}$, Özgür Özdemir Şimșek ${ }^{1}$, Cemaliye Başaran ${ }^{1}$,

Seçil Arslansoyu Çamlar ${ }^{2}$, Fatma Mutlubaș ${ }^{2}$, Bumin Nuri Dündar ${ }^{3}$, Belde Kasap Demir ${ }^{4}$

${ }^{1}$ Izmir University of Health Sciences Tepecik Training and Research Hospital Department of Pediatric Nephrology, Turkey, ${ }^{2}$ University of Health Sciences Izmir Faculty of Medicine Department of Pediatrics Division of Nephrology, Turkey, ${ }^{3}$ Katip Celebi University Faculty of Medicine Department of Pediatric Endocrinology, Turkey, ${ }^{4}$ Katip Celebi University Faculty of Medicine Department of Pediatric Nephrology and Rheumatology, Turkey

Introduction: Kidney transplantation is the preferred replacement therapy for patients with end-stage renal disease (ESRD). However, long-term immunosuppression may be responsible of a variety of complications, including the development of thyroid disease. This study evaluated the incidence of thyroid disease in a pediatric population of kidney transplant recipients performed in a single center.

Material and methods: This retrospective study included 24 pediatric patients who received kidney transplantation between 2009-2019 and with regular follow-up. After transplantation, in all transplant recipients Free T3 (normal range 1.8-4.2 pg/mL) and free T4 (normal range $0.8-1.9 \mathrm{ng} / \mathrm{dL}$ ) levels were measured on a yearly basis and an ultrasonography of the neck was performed every 2 years.

Results: Data of 24 patients, 16 of whom $(66.7 \%)$ were male, were obtained. Mean transplantation ages were $11.16 \pm 4.21$ (1-17) years. There were $11(45.8 \%)$ living / $13(54.2 \%)$ cadaveric transplants. When primary diseases were examined, 12 (50.0\%) had CAKUT, 6 (25\%) had chronic glomerulonephritis, 5 (20.8\%) had chronic tubulopathy, and 1 had HUS. Preemptive transplantation was performed in three cases. $12(50.0 \%)$ patients were followed up in the peritoneal dialysis program before transplantation. When the dialysis periods were examined, the mean was $1.96 \pm 1.72(0-6)$ years. 22 cases were using the steroid + MMF + Tacrolimus regimen immunosuppressively. Induction was performed in 14 (58.3\%) cases with basiliximab. In 13 cases, post-transplantation developed acute graft dysfunction (AGD) at different times. When the mean fT3, fT4 and TSH levels are evaluated, $3.53 \pm 0.67$ (1.89-4.45), $0.93 \pm 0.22$ (0.60-1.65) and $2.07 \pm 1,25(0.52-5.59)$ were detected respectively. Anti-thyroid peroxidase antibody and anti-thyroglobulin antibody (Anti Tg) were only high in one patient. fT3 was above $4.2 \mathrm{pg} /$ $\mathrm{mL}$ in three cases and all three cases had AGD.

Conclusions: Thyroid diseases are common in transplant recipients. Thyroid disease may evolve after transplantation, probably as a consequence of immunosuppression. but we did not find abnormalities in this series.

\section{PI-108 SKIN LESIONS IN KIDNEY TRASPLANT RECIPIENT}

Ana Vinuesa Jaca ${ }^{1}$, Mireia Aguirre Meñica ${ }^{1}$, Maria Rosario Gonzalez Hermosa ${ }^{3}$, Leire Gondra Sangroniz ${ }^{4}$, Maria Herrero Goñi ${ }^{1}$, Leire Madariaga Dominguez ${ }^{4}$, Nelida Garcia Perez ${ }^{2}$

${ }^{1}$ Pediatric Nephrology, Cruces University Hospital, Iss Biocruces Bizkaia, Spain, ${ }^{2}$ Pediatric Nephrology, Basurto University Hospital, Iss Biocruces Bizkaia, Spain, ${ }^{3}$ Dermatology, Cruces University Hospital, Spain, ${ }^{4}$ Pediatric Nephrology, Cruces University Hospital, Iis Biocruces Bizkaia, Basque Country Univeristy, Spain

Introduction: Bacillary angiomatosis is an opportunistic infectious disease, caused by bacteria of the genus Bartonella that produce a vascular proliferative process. Apart from HIV infection it can affect other immunocompromised patients.

Material and methods: We describe a kidney trasplant recipient who developed bacillary angiomatosis

Results: A 12-year-old girl, adopted from Ethiopia, with chronic kidney disease diagnosed at 5 years of age of unknown origin. She received a cadaveric donor kidney transplant at the age of 11 years. After transplantation she received immunosuppressive treatment with tacrolimus, mycophenolate and prednisone with normalization of kidney function, without infectious complications. Six months after transplantation, she started with symptoms of asthenia, weight loss, nausea and joint pain in ankles and wrists. She presented skin lesions suggestive of molluscum contagiosum. Laboratory tests showed normochromic anemia, elevated CRP and ESR, renal graft dysfunction and positive BK viremia. Complement was normal and the autoimmunity study was negative. Joint ultrasound revealed a slight effusion with nodular images, suggesting schwannomas or neurofibromas as possible diagnoses. The skin lesions progressively increased in number and size to $1 \mathrm{~cm}$ nodules. She presented more than 100 generalized cutaneous lesions, including the gingival mucosa, with palmo-plantar involvement, with different morphologies: well-defined rounded pink-violaceous papules, subcutaneous nodular lesions and pedunculated warty papules. Biopsy showed bacillary angiomatosis with $16 \mathrm{~S}$ rRNA gene sequencing: Bartonella quintana and serology for Bartonella quintana $\operatorname{IgM}$ negative and high values of $\mathrm{IgG}$. She was treated with doxacycline for 3 months, with rapid progressive improvement of general symptoms, laboratory and ultrasound findings and skin lesions.

Conclusions: The main involvement of bacillary angiomatosis is cutaneous, but it can involve other organs with risk of mortality. Early diagnosis and prolonged antibiotic treatment reduces bacteremia, parenchymal involvement and mortality.

\section{PI-109 CLINICAL OUTCOME AFTER RENAL TRANS- PLANTATION IN SMALL CHILDREN (< 15 KILOS): THE DUTCH EXPERIENCE}

Mike Keizer ${ }^{1}$, Marlies Cornelissen ${ }^{2}$, Mandy Keijzer-veen ${ }^{3}$, Antonia Bouts ${ }^{1}$, Huib De Jong ${ }^{4}$

${ }^{1}$ Emma Children's Hospital, Amsterdam University Medical Center, Amsterdam, The Netherlands, ${ }^{2}$ Amalia Children's Hospital, St Radboud Hospital, Nijmegen, The Netherlands ${ }^{3}$ Wilhelmina Children's Hospital, University Medical Center Utrecht, Utrecht, The Netherlands, ${ }^{4}$ Erasmus Mc-sophia Children's Hospital, Rotterdam, The Netherlands

Introduction: In the smallest recipients $(<15 \mathrm{~kg})$ with end-stage-renaldisease (ESRD), kidney transplantation is a delicate task. We recently conducted a systematic review in which we showed that after an increase in graft survival in the early era, a stabilisation of graft survival is seen in recent years (under review). Although the better immunosuppressants have given great improvement in graft survival in the past, we hypothesize that their side effects (e.g. nephrotoxicity and infections) prevent 
further improvement. The current study investigates the clinical outcome of small children in the Netherlands, focussing on long-term post-transplantation complications (e.g infection, immunosuppression side effects and rejection) and relate these complications to the immunosuppression protocol used.

Material and methods: All children having received a renal transplantation in the Netherlands with a weight of 15 kilograms or less were included. Data regarding graft survival were obtained from the Dutch Organ Transplantation Registry. Other variables were extracted from reviewing medical charts.

Results: We included 73 transplantations performed from 1989 through 2020, of which 37 received a corticosteroid-sparing-protocol (TWIST) and 46 received a TRIPLE- immunosuppression-protocol. Overall, 5years graft survival improved from $46 \%$ in the $70-80$ 's, to $84 \%$ in the $90-00$ 's and $96 \%$ in most recent era. Our analysis will focus on factors that are potentially associated with the recent stabilisation in graft survival (infections rate, immunosuppression side effects and rejection) and will be analysed for both immunosuppression groups.

Conclusions: Our preliminary results show that clinical outcome after renal transplantation has improved over the years. However, the burden of (viral) infections and nephrotoxicity have grown, which could have a negative effect on recent graft survival data. Small children should have our special attention for two reasons. Firstly, at time of kidney transplant they are naive to most common (viral) infections. Secondly, with a lifelong immunosuppressant treatment ahead, they are more prone to side effects.

\section{PI-110 KIDNEY GRAFT WITHOUT IMMUNOSUPPRESSION IN AN ADOLESCENT WITH FANCONI APLASIA}

De Saint-Gilles David ${ }^{1}$, Berthaud Romain ${ }^{1}$, Charbit Marina ${ }^{1}$, Avramescu Marina ${ }^{1}$, Dehoux Laurene ${ }^{1}$, Boyer Olivia ${ }^{1}$,

Dorval Guillaume ${ }^{2}$

${ }^{1}$ Service De Néphrologie Pédiatrique, Hôpital Necker, Assistance Publique Des Hopitaux De Paris, Paris, France, ${ }^{2}$ Service De Génétique Moléculaire, Hôpital Necker, Assistance Publique Des Hôpitaux De Paris, Paris, France

\begin{abstract}
One of the major issues in organ transplantation is graft immune tolerance. The balance between the risk of acute/chronic rejection and infectious complications generated by immunosuppressive therapy tends to minimize their use. In the rare cases of solid organ transplantation following hematopoietic stem cell transplantation (HSCT) for immune deficiency from the same donor, a natural graft tolerance is theoretically expected.

We report herein the case of a 17-year-old girl who underwent kidney transplantation in our center. She suffered from Fanconi aplasia and received a first HSCT from an unrelated HLA-identical donor at the age of 8 years. The outcome was very poor and she received a second HSCT from her haploidentical mother two months later. This procedure was successful despite numerous infectious complications, and liver and skin graft-versus-host disease. The outcome was favorable with complete maternal chimerism (100\%) nine years after HSCT. Unfortunately, she progressed to kidney failure from multifactorial origin requiring peritoneal dialysis. The mother was a candidate for living-donor kidney donation. Since the recipient was $100 \%$ chimer with the donor's bone marrow following HSCT, we decided to perform a living-donor kidney transplant without any induction nor maintenance immunosuppression with the mother's kidney. The cross-match was negative. The renal transplantation was successful with immediate recovery of graft function and no immediate postoperative complications. Nine months after kidney transplantation, she had normal kidney function (creatinine $60 \mu \mathrm{mol} / \mathrm{L}$, eGFR: $97 \mathrm{~mL} / \mathrm{min} / 1.73 \mathrm{~m}^{2}$ ) with no medication except for phosphorus supplementation.

To our knowledge, this is the first report of a kidney transplantation after HSCT from the same donor without any immunosuppressive therapy. It
\end{abstract}

demonstrates that this strategy is conceivable when stem cells and kidney are from the same donor with a complete donor chimerism.

\section{PI-111 GENOTYPE-PHENOTYPE CORRELATIONS IN 304 PATIENTS WITH AUTOSOMAL RECESSIVE POLYCYSTIC KIDNEY DISEASE (ARPKD) AND PKHD1 VARIANTS}

Kathrin Burgmaier ${ }^{1}$, Leonie Brinker ${ }^{1}$, Florian Erger ${ }^{2}$, Bodo Beck ${ }^{2}$, Marcus Benz ${ }^{3}$, Carsten Bergmann ${ }^{4}$, Olivia Boyer ${ }^{5}$, Laure Collard ${ }^{6}$, Claudia Dafinger ${ }^{7}$, Marc Fila ${ }^{8}$, Claudia Kowalewska ${ }^{9}$, Bärbel Lange-sperandio ${ }^{10}$, Laura Massella ${ }^{11}$, Antonio Mastrangelo ${ }^{12}$ Djalila Mekahli ${ }^{13}$, Monika Miklaszewska ${ }^{14}$, Nadina Ortiz-Bruechle ${ }^{15}$, Ludwig Patzer ${ }^{16}$, Larisa Prikhodina ${ }^{17}$, Bruno Ranchin ${ }^{18}$, Nadejda Ranguelov ${ }^{19}$, Raphael Schild ${ }^{20}$, Tomas Seeman ${ }^{21}$, Lale Sever ${ }^{22}$, Przemyslaw Sikora ${ }^{23}$, Maria Szczepanska ${ }^{24}$, Ana Teixeira ${ }^{25}$, Julia Thumfart ${ }^{26}$, Barbara Uetz ${ }^{27}$, Lutz T. Weber ${ }^{1}$, Elke Wühl ${ }^{28}$, Klaus Zerres ${ }^{15}$, Jörg Dötsch ${ }^{1}$, Franz Schaefer ${ }^{28}$, Max Christoph Liebau ${ }^{7}$

${ }^{I}$ Department of Pediatrics, University Hospital Cologne and University of Cologne, Faculty of Medicine, Cologne, Germany; ${ }^{2}$ Institute of Human Genetics, University Hospital Cologne and University of Cologne, Faculty of Medicine, Cologne, Germany; Center For Molecular Medicine Cologne, University of Cologne, Faculty of Medicine, University Hospital Cologne, Germany, ${ }^{3}$ Klinik Für KinderUnd Jugendmedizin, Klinikum Dritter Orden, München, Germany, ${ }^{4}$ Medizinische Genetik Mainz, Limbach Genetics, Mainz, Germany; Renal Division, Department Of Medicine, University Freiburg Medical Center, Freiburg, Germany, ${ }^{5}$ Department of Pediatric Nephrology and Kidney Transplantation, Necker Hospital, Paris, France, ${ }^{6}$ Centre De Référence De Néphrologie Pédiatrique Sud, Clinique De Lespérance, Montegnee, Belgium, ${ }^{7}$ Department of Pediatrics, University Hospital Cologne and University of Cologne, Faculty of Medicine, Cologne, Germany; Center for Molecular Medicine Cologne, University of Cologne, Faculty of Medicine, University Hospital Cologne, Germany, ${ }^{8}$ Pediatric Nephrology Unit, Chu Arnaud De Villeneuve-université De Montpellier, Montpellier, France, ${ }^{9}$ The Childrens Memorial Health Institute, Warsaw, Poland, ${ }^{10}$ Department of Pediatrics, Dr. Von Hauner Children's Hospital, University Hospital, Lmu, Munich, Germany, ${ }^{11}$ Division of Nephrology, Department of Pediatric Subspecialties, Bambino Gesù Children's Hospital - Irccs, Rome, Italy, ${ }^{12}$ Pediatric Nephrology, Dialysis and Transplant Unit, Fondazione Irccs Cà Granda, Ospedale Maggiore Policlinico, Milan, Italy, ${ }^{13}$ Department of Development And Regeneration, Pkd Research Group, Ku Leuven, Leuven, Belgium; Department of Pediatric Nephrology, University Hospitals Leuven, Leuven, Belgium, ${ }^{14}$ Department of Pediatric Nephrology And Hypertension, Faculty Of Medicine, Jagiellonian University Medical College, Krakow, Poland, ${ }^{15}$ Institute of Human Genetics, Rwth University Hospital Aachen, Aachen, Germany, ${ }^{16}$ Childrens Hospital St. Elisabeth And St. Barbara, Halle (saale), Germany, ${ }^{17}$ Department of Inherited and Acquired Kidney Diseases, Research Clinical Institute For Pediatrics N.a. Acad. Y. E. Veltishev, Pirogov Russian National Research Medical University, Moscow, Russia, ${ }^{18}$ Pediatric Nephrology Unit, Hôpital Femme Mère Enfant, Hospices Civils De Lyon, Centre De Référence Maladies Rénales Rares, Bron, France, ${ }^{19}$ Department of Pediatrics, Université Catholique De Louvain Medical School, Saint-luc Academic Hospital, Brussels, Belgium, ${ }^{20}$ University Childrens Hospital, University Medical Center Hamburg Eppendorf, Hamburg, Germany, ${ }^{21}$ Department of Pediatrics, Dr. Von Hauner Children's Hospital, University Hospital, Lmu, Munich, Germany; Department of Pediatrics, University Hospital Motol, 2nd Faculty Of Medicine, Charles University Prague, Prague, Czech Republic, ${ }^{22}$ Department of Pediatric Nephrology, CerrahpaŞa School of Medicine, Istanbul University Cerrahpasa, Istanbul, Turkey, ${ }^{23}$ Department of Pediatric Nephrology, Medical University of Lublin, Lublin, Poland, ${ }^{24}$ Department of Pediatrics, Faculty of Medical 
Sciences In Zabrze, Sum In Katowice, Poland, ${ }^{25}$ Centro Materno-infantil Do Norte, Centro Hospitalar Do Porto, Porto, Portugal, ${ }^{26}$ Department of Pediatric Nephrology, Charité - Universitätsmedizin Berlin, Corporate Member Of Freie Universität Berlin, Humboldt-universität Zu Berlin, and Berlin Institute of Health, Germany, ${ }^{27}$ Kfh Center of Pediatric Nephrology, Childrens Hospital Munich Schwabing, Munich, Germany, ${ }^{28}$ Division of Pediatric Nephrology, Center For Pediatrics And Adolescent Medicine, University Hospital Heidelberg, Heidelberg, Germany

Objectives: Autosomal recessive polycystic kidney disease (ARPKD) is among the most severe hepatorenal diseases in childhood. It is mainly caused by variants in the PKHD 1 gene. PKHD 1 encodes the protein fibrocystin, which consists of 4074 amino acids. The basis for the pronounced phenotypic variability in ARPKD is poorly understood. Previous genotype-phenotype correlations are restricted to mainly biallelic truncating variants associating with perinatal demise or severe courses. We aimed to elucidate specific genotypephenotype correlations with respect to the functionality and localization of variants.

Methods: We analyzed datasets of 304 ARPKD patients surviving the neonatal period with $\geq$ one $P K H D 1$ variant classified as variant of unknown clinical significance, likely pathogenic or pathogenic. Genotypes were assigned to functional classes. Additionally, missense variants were categorized into four protein regions. The clinical endpoints were start of kidney replacement therapy, signs of portal hypertension and substantial hepatic complications.

Results: Patients with Null/Null variants show severe courses. Additionally, the affected region of missense variants is important: when grouping patients according to the localization of the affected amino acids, we observed better kidney survival in patients with variant combinations affecting AAs 709-1837 and better hepatic outcomes in patients with combinations affecting AAs 1838-2624. Variants affecting AAs 2625-4074 were associated with poorer hepatic outcome.

Conclusions: These results refine our understanding of genotypephenotype correlations in ARPKD during childhood and adolescence and may serve as a basis for more precise counselling and evaluation of treatment approaches.

\section{PI-112 HYPERTENSION IN CHILDHOOD CANCER SURVIVORS AFTER TREATMENT WITH POTENTIALLY NEPHROTOXIC THERAPY AND THE ADDED VALUE OF AMBULATORY BLOOD PRESSURE MONITORING; DCCSS-LATER 2: RENA.}

Esmee C.m. Kooijmans ${ }^{1}$, Helena J.h. Van Der Pal ${ }^{2}$, Saskia Pluijm ${ }^{2}$, Dorine Bresters ${ }^{2}$, Eline Van Dulmen-den Broeder ${ }^{1}$, Margriet Van Der Heiden-van Der Loo ${ }^{2}$, Marry M. Van Den Heuvel-eibrink ${ }^{2}$,

Leontien C.m. Kremer ${ }^{2}$, Jacqueline Loonen ${ }^{3}$, Marloes Louwerens ${ }^{4}$, Maxime Pilon ${ }^{1}$, Cecile M. Ronckers ${ }^{2}$, Wim J.e. Tissing ${ }^{2}$,

Andrica C.h. De Vries ${ }^{5}$, Gertjan J.1. Kaspers ${ }^{2}$, Arend Bokenkamp ${ }^{6}$, Margreet A. Veening ${ }^{2}$, On Behalf Of The Dutch Later Study Group ${ }^{2}$

${ }^{1}$ Emma Children's Hospital, Amsterdam Umc, Vrije Universiteit Amsterdam, Pediatric Oncology, Amsterdam, The Netherlands, ${ }^{2}$ Princess Maxima Center for Pediatric Oncology, Utrecht, The Netherlands, ${ }^{3}$ Department of Hematology, Radboud University Medical Center, Nijmegen, The Netherlands, ${ }^{4}$ Department of Internal Medicine, Leiden University Medical Center, Leiden, The Netherlands, ${ }^{5}$ Department of Pediatric Oncology, Sophia Children's Hospital/erasmus Medical Center, Rotterdam, The Netherlands, ${ }^{6}$ Emma Children's Hospital, Amsterdam Umc, Vrije Universiteit Amsterdam, Pediatric Nephrology, Amsterdam, The Netherlands

Introduction: We evaluated the prevalence of and risk factors for hypertension in childhood cancer survivors (CCS) compared to controls.
Furthermore, a pilot study using 24-hour ambulatory blood pressure monitoring (ABPM) was performed.

Material and methods: In the Dutch Childhood Cancer Survivor Study (DCCSS) LATER cohort (1963-2001) part2; clinical visit \& questionnaire study; RENA project, 1,024 CCS participated; $\geq 5$ years after diagnosis, aged $\geq 18$ years at study participation, treated between 1963-2001 with nephrectomy, abdominal radiotherapy (RT), total body irradiation (TBI), cisplatin, carboplatin, ifosfamide, high-dose cyclophosphamide ( $\geq 1 \mathrm{~g} / \mathrm{m} 2$ per course or $\geq 10 \mathrm{~g} / \mathrm{m} 2$ total) or bone marrow transplantation. We used 500 age- and sex matched controls from Lifelines. Hypertension was defined as blood pressure (BP) ( $\mathrm{mmHg}$ ) systolic $\geq 140$ and/or diastolic $\geq 90$ or receiving antihypertensive medication for previously diagnosed hypertension. Multivariable regression analyses were used. The ABPM-pilot study was performed in $77 \mathrm{CCS}$. Hypertension was defined as BP daytime: systolic $\geq 135$ and/or diastolic $\geq 85$, nighttime: systolic $\geq 120$ and/or diastolic $\geq 70,24$-hour: systolic $\geq 130$ and/or diastolic $\geq 80$. Outcomes were masked hypertension $(\mathrm{MH})$, white coat hypertension (WCH) and abnormal nocturnal dipping (aND).

Results: Median age at diagnosis was 4.7 years (IQR 2.4-9.2), at study 32.5 years (IQR 27.7-38.0), and follow-up time 25.5 years (IQR 21.4$30.3)$. The prevalence of hypertension was comparable in CCS (16.3\%) and controls (18.2\%). A decrease in glomerular filtration rate (GFR) per $10 \mathrm{ml} / \mathrm{min} / 1.73 \mathrm{~m} 2$ was associated with hypertension in CCS (OR 1.2, 95\%CI 1.1-13), but not in controls. Treatment risk factors were abdominal RT (OR 2.2, 95\% CI 1.3-4.0) and TBI (OR 3.0, 95\% CI 1.6-5.8). The ABPM-pilot study showed 7.8\% MH, 2.6\% WCH, and 20.8\% aND.

Conclusions: Prevalence of hypertension was comparable in CCS and controls, but an association with decreased GFR was only found in CCS. Treatment risk factors were abdominal RT and TBI. ABPM has added value by identifying masked hypertension and abnormal nocturnal dipping.

\section{PI-113 OUTCOME OF PEDIATRIC PATIENTS STARTING KIDNEY REPLACEMENT THERAPY AFTER CANCER TREATMENT- ESPN/ERA-EDTA REGISTRY DATA}

Henna Puusaari ${ }^{1}$, Henna Puusaari ${ }^{2}$, Marjolein Bonthuis ${ }^{3}$, Jerome Harambat ${ }^{4}$, Enrico Vidal ${ }^{5}$, Manish Sinha ${ }^{6}$, Kitty Jager ${ }^{3}$, Timo Jahnukainen ${ }^{2}$

${ }^{1}$ Tampere University Hospital, Department of Pediatrics, Pediatric Nephrology, Tampere, Finland, ${ }^{2}$ Department of Pediatric Nephrology And Transplantation, Children's Hospital, Helsinki University Hospital And University of Helsinki, Helsinki, Finland, ${ }^{3}$ Espn/era-edta Registry, Department of Medical Informatics, Amsterdam Umc, University Of Amsterdam, Amsterdam Public Health Research Institute, Amsterdam, The Netherlands, ${ }^{4}$ Department of Pediatrics, Bordeaux University Hospital, Bordeaux Population Health Research Center Umr 1219, University of Bordeaux, Bordeaux, France, ${ }^{5}$ Division of Pediatrics, Department of Medicine, University of Udine, Udine, Italy, ${ }^{6}$ Kings College London; Department of Paediatric Nephrology, Evelina London Childrens Hospital, London, United Kingdom

Introduction: Survival of pediatric cancer patients has improved during recent decades, leading to an increased number of children with kidney dysfunction caused by the tumor itself or by cancer treatments, like cytostatic and irradiation therapy. Only few studies reporting outcomes of pediatric cancer patients starting kidney replacement therapy (KRT) are available. Therefore, we aimed to evaluate long-term patient and graft survival in pediatric KRT patients with a malignancy history.

Material and methods: Data were collected from the ESPN/ERAEDTA Registry. All patients younger than 20 years of age starting KRT after malignancy treatment between 1980 and 2018 were included. Cancer patients were divided in two groups: (group 1) malignancy as a 
primary renal disease (PRD) and (group 2) history of malignancy but another PRD. Two non-cancer controls were selected for every patient matched on age, sex, year of KRT and country macro-economics.

Results: 130 patients in group 1 and 108 patients in group 2 were identified. Patients in group 1 were significantly younger ( $4.4 ; 1.7-8.7$ years) at start of KRT than patients in group 2 (13.0; 6.1-17.5 years). The median time to kidney transplantation (KT) was longer in the malignancy groups than in the control groups. Five-year access to KT was $72.4 \%, 76.8 \%$, $66.5 \%$ and $78.2 \%$ in group 1, their controls (group 3), group 2 and their controls (group 4), respectively. The 5-year mortality was higher in among malignancy patients: $14.7 \%$ (group 1), 10.7\% (group 2), $6.6 \%$ (group 3), and $6.8 \%$ (group 4). In group 1, 36.4\% of deaths were caused by the malignancy itself, whereas infection predominated in group 3 .

Conclusions: Five-year mortality was higher in malignancy groups, mainly due to mortality caused by malignancies. Patients with malignancies spent longer time on dialysis before receiving KT, but access to KT was much more similar at 5-year follow-up.

\section{PI-114 CHANGES IN IMMUNOSUPPRESSIVE MEDICATION AFTER KIDNEY TRANSPLANTATION}

Henna Puusaari ${ }^{1}$, Anna Bjerre ${ }^{2}$, Hannu Jalanko ${ }^{3}$, Lars Wennberg ${ }^{4}$, Søren Sørensen Schwartz ${ }^{5}$, Susanne Westphal Ladfors ${ }^{6}$,

Helle Charlotte Thiesson ${ }^{7}$, Zivile Bekassy ${ }^{8}$, Gianni Celsi ${ }^{9}$, Timo Jahnukainen ${ }^{3}$

${ }^{1}$ Tampere University Hospital, Department of Pediatrics, Pediatric Nephrology, Tampere, Finland, ${ }^{2}$ Division of Pediatric and Adolescent Medicine, Oslo University Hospital, Oslo, Norway, ${ }^{3}$ Children's Hospital and Pediatric Research Center, University of Helsinki And Helsinki University Hospital, Finland, ${ }^{4}$ Department of Transplantation Surgery, Karolinska University Hospital, Stockholm, Sweden, ${ }^{5}$ Department of Nephrology, Rigshospitalet, Copenhagen University Hospital, Copenhagen, Denmark, ${ }^{6}$ Department of Pediatrics. The Queen Silvia Children's Hospital, Sahlgrenska University Hospital, Gothenburg, Sweden, ${ }^{7}$ Department of Nephrology, Odense University Hospital, Odense, Denmark, ${ }^{8}$ Department of Pediatric Nephrology, Skane University Hospital, Lund, Sweden, ${ }^{9}$ Dept of Pediatric, Uppsala University Hospital, Uppsala, Sweden

Introduction: There are data available from both single center studies and large registries on the initial immunosuppression in pediatric kidney transplant (KT) recipients. However, the knowledge of changes in maintenance immunosuppression is scarce. In the present study, we evaluated changes in maintenance immunosuppressive medication in pediatric KT recipients in the Nordic countries.

Material and methods: Data were collected retrospectively from the Scandiatransplant pediatric registry (Nordic Pediatric Renal Transplantation Study Group, NPRTSG) including all pediatric KTs in the Nordic countries between the years 2005 and 2016. The inclusion criteria were age below 16 years at transplantation and a post-transplant follow-up time of at least two years.

Results: Of the 482 transplanted children, 345 patients met the inclusion criteria. A change in maintenance immunosuppression was recorded in 161 patients $(47.1 \%)$, occurring during the first post-transplant year in $44.7 \%$ of these patients. The most common change was conversion of cyclosporine (CsA) to tacrolimus (Tac) ( $41.6 \%$ of all changes). The age distribution was similar in patients with CsA to Tac conversion as compared to those continuing on CsA. CsA was switched to Tac in $66.7 \%$, $78.6 \%$ and $75.7 \%$ of the cases under two years old, two to five years old, and five to sixteen years old, respectively. Otherwise, initial immunosuppression was likely to remain unchanged. Concurrent rejection was present in $17.5 \%$ of all cases with immunosuppression change and in $23.9 \%$ of the cases when CsA was converted to Tac. There were 10 recorded PTLD cases, eight cases with tacrolimus as an initial calcineurin inhibitor.

Conclusions: The majority of patients with CsA as an initial immunosuppression were converted to Tac during the follow up. Rejection was the reason for drug change in less than $20 \%$ of the cases.

\section{PI-115 DEVELOPMENT OF AN OBSERVATIONAL REGISTRY FOR GENETIC HYPOPHOSPHATEMIA AND ACQUIRED RENAL PHOSPHATE WASTING IN THE NETHERLANDS: ORPHOS-NED}

A. Bosman ${ }^{1}$, N. M. Appelman-dijkstra ${ }^{2}$, J. P. Van Den Bergh ${ }^{3}$, A. BÖkenkamp ${ }^{4}$, A. M. Boot ${ }^{5}$, M. H. De Borst ${ }^{6}$, B. C. J. Van Der Eerden $^{1}$, M. C. Zillikens ${ }^{1}$

${ }^{I}$ Erasmus Mc, University Medical Center Rotterdam, Department of Internal Medicine, Erasmus Mc Bone Center, The Netherlands, ${ }^{2}$ Leiden University Medical Center, Leiden, Department of Internal Medicine, Division Endocrinology, Center For Bone Quality, The Netherlands, ${ }^{3}$ Viecuri Medical Centre, Venlo, Department of Internal Medicine, The Netherlands, ${ }^{4}$ Emma Children's Hospital, Amsterdam University Medical Center, Vrije Universiteit, Department of Pediatric Nephrology, The Netherlands, ${ }^{5}$ University Medical Center Groningen, Groningen, Department of Pediatrics, The Netherlands, ${ }^{6}$ University Medical Center Groningen, Groningen, Department of Internal Medicine, The Netherlands

Background: Phosphate is critical for skeletal development and mineral metabolism. Phosphate deficiency leads to e.g. muscle weakness and rickets or osteomalacia. Several inherited and acquired causes of renal phosphate wasting can lead to hypophosphatemic rickets (HR). X-linked hypophosphatemia (XLH) is the most common form of HR with an estimated prevalence of 1:20.000. The prevalence of chronic hypophosphatemia in the Netherlands and the clinical manifestations are currently unknown.

Purpose: ORPHOS-NED has been developed to identify and evaluate patients with XLH and other forms of chronic hypophosphatemia within a registry in the Netherlands.

Methods: ORPHOS-NED is a web-based registry that has been set up by a group of medical specialists, who are affiliated to the Dutch Federation of Nephrology (NFN) and the Bone Network of the Dutch Society of Endocrinology (NVE). Dutch endocrinologist and nephrologists are approached for eligible patients. Children and adults with chronic hypophosphatemia are considered for inclusion. After informed consent, a chart review is performed to collect data on several aspects of the disease: initial presentation; symptoms; radiological, genetic and laboratory examinations; treatment; and follow up. Furthermore, questionnaires are sent out to assess health-related quality of life including the Brief Fatigue Inventory, the Brief Pain Inventory, RAND36, the Health Assessment Questionnaire and the Pediatric Outcomes Data Collection Instrument.

Results: Currently, 107 pediatric and adult patients from 4 academic hospitals have been included in this registry. Inclusion of patients is ongoing. The data from this registry will lead to more insight in the prevalence, natural history, treatment and its effects on HR, quality of life, and into genotype-phenotype relations in the different genetic forms. Conclusion: A Dutch nationwide registry is being set up for genetic and acquired forms of chronic hypophosphatemia, which will lead to improved insight in prevalence, causes, disease manifestations and therapy. 


\section{PI-116 SOCIAL DEPRIVATION AND INCIDENCE OF PEDIATRIC END-STAGE RENAL DISEASE IN FRANCE.}

Bénédicte Driollet ${ }^{1}$, Florian Bayer ${ }^{2}$, Denis Morin ${ }^{3}$, Michel Tsimaratos ${ }^{4}$, Cécile Couchoud $^{2}$, Karen Leffondré ${ }^{6}$, Jérôme Harambat ${ }^{5}$

${ }^{1}$ University of Bordeaux, Isped, Centre Inserm U1219-bordeaux Population Health Research, Bordeaux, France, ${ }^{2}$ Agence De La Biomédecine, La Plaine-saint Denis, France, ${ }^{3}$ Pediatric Nephrology Unit, Montpellier University Hospital, Montpellier, France, ${ }^{4}$ Pediatric Nephrology Unit, La Timone Hospital, Assistance Publique-hôpitaux De Marseille, Marseille, France, ${ }^{5}$ University of Bordeaux, Isped, Centre Inserm U1219-bordeaux Population Health Research, Inserm, Clinical Investigation Center-clinical Epidemiology-cic-1401, Pediatric Nephrology Unit, Pellegrin-enfants Hospital, Bordeaux University Hospital, Bordeaux, France, ${ }^{6}$ University of Bordeaux, Isped, Centre Inserm U1219-bordeaux Population Health Research, Inserm, Clinical Investigation Center-clinical Epidemiology-cic-1401, Bordeaux, France

Introduction: The objective of this study is to describe the incidence of pediatric ESKD in metropolitan France, according to the level of social deprivation.

Methods: This study included all patients who initiated a kidney replacement therapy (KRT) from 2010 to 2015 in metropolitan France. Data were collected from the French Renal Epidemiology and Information Network (REIN registry). We used a validated ecological index of deprivation, the 2011 French version of the European Deprivation Index (EDI). Mapping has been carried out to describe the distribution of deprivation and the incidence of ESKD in France. We then estimated crude and standardized incidence as well as standardized incidence ratio (SIR) according to the quintiles of EDI.

Results: 613 patients were included (58.6\% males, $44.9 \%$ starting KRT between 11 and 17 years and 30.2\% with CAKUT). Children from the most deprived areas represented $38.8 \%$ of the sample. Although no trend could be identified geographically, a clear social gradient was found in the incidence (crude, standardized or SIR) of KRT. For example, the standardized incidence rate (per 1,000,000 children-years) increased from 5.11 (95\% CI 3.96-6.26) in the least deprived quintile to 7.81 (95\% CI 6.80-8.81) in the most deprived quintile of EDI.

Conclusion: This study is the first to show that in France, there is a relationship between the incidence of pediatric ESKD and deprivation, showing that the higher the level of deprivation the higher the incidence.

\section{PI-117 SOCIAL DEPRIVATION AT INITIATION OF KIDNEY REPLACEMENT THERAPY IN THE PEDIATRIC POPULATION}

Bénédicte Driollet ${ }^{1}$, Florian Bayer ${ }^{2}$, Theresa Kwon ${ }^{3}$, Saoussen Krid ${ }^{4}$, Bruno Ranchin ${ }^{5}$, Michel Tsimaratos ${ }^{6}$, Cyrielle Parmentier ${ }^{7}$, Robert Novo ${ }^{8}$, Gwenaëlle Roussey ${ }^{9}$, Stéphanie Tellier ${ }^{10}$, Marc Fila ${ }^{11}$, Ariane Zaloszyc ${ }^{12}$, Ludivine Launay ${ }^{13}$, Jérôme Harambat ${ }^{14}$, Karen Leffondre ${ }^{1}$

${ }^{1}$ University Of Bordeaux, Isped, Centre Inserm U1219-bordeaux Population Health Research, Bordeaux, France, ${ }^{2}$ Agence De La Biomédecine, Rein Registry, La Plaine-saint Denis, France, ${ }^{3}$ Pediatric Nephrology Unit, Robert Debré University Hospital, Assistance Publique-hôpitaux De Paris, Paris, France, ${ }^{4}$ Pediatric Nephrology Unit, Centre De Référence Des Maladies Rénales Rares, Neckerenfants Malades University Hospital, Assistance Publique-hôpitaux De Paris, Paris, France, ${ }^{5}$ Pediatric Nephrology Unit, Centre De Référence Des Maladies Rénales Rares, Femme Mère Enfants Unniversity Hospital, Hospices Civils De Lyon, Bron, France, ${ }^{6}$ Pediatric Nephrology Unit, La Timone Hospital, Assistance Publique-hôpitaux De Marseille, Marseille, France, ${ }^{7}$ Pediatric Nephrology Unit, Trousseau University Hospital, Assistance Publique-hôpitaux De Paris, Paris, France, ${ }^{8}$ Pediatric
Nephrology Unit, Lille University Hospital, Lille, France, ${ }^{9}$ Pediatric Nephrology Unit, Nantes University Hospital, Nantes, France, ${ }^{10}$ Pediatric Nephrology Unit, Centre De Référence Des Maladies Rénales Rares, Toulouse University Hospital, Toulouse, France, ${ }^{11}$ Pediatric Nephrology Unit, Montpellier University Hospital, Montpellier, France, ${ }^{12}$ Pediatric Nephrology Unit, Strasbourg University Hospital, Strasbourg, France, ${ }^{13}$ Inserm-ucn U1086 Anticipe, Centre De Lutte Contre Le Cancer FranÇois Baclesse, Caen, France, ${ }^{14}$ Pediatric Nephrology Unit, Pellegrin-enfants Hospital, Bordeaux University Hospital, Bordeaux, France

Introduction: Socioeconomic status is recognized as an important determinant of kidney health. We aimed to assess the association of social deprivation with different indicators at kidney replacement therapy (KRT) initiation in the French pediatric population.

Material and methods: All end-stage kidney disease (ESKD) patients who started KRT before 20 years old in France between 2002 and 2015 were included. We investigated different indicators at KRT initiation: KRT modality (dialysis vs. pre-emptive transplantation), dialysis modality (hemodialysis [HD] vs. peritoneal dialysis $[P D]$ ), urgent vs. planned start of dialysis, use of catheter vs. fistula for HD vascular access), and late referral to a nephrologist. An ecological index, the European Deprivation Index (EDI), was used as a proxy for social deprivation.

Results: 1115 patients were included (males 59\%, median age at dialysis 14.4 years, glomerular/vascular diseases $36.8 \%$ ). The most deprived group represented $38.7 \%$ of the patients, suggesting that pediatric ESKD patients come from a more socially deprived background. Social deprivation was significantly associated with the initial modality of KRT. Patients from the most deprived areas were more likely to initiate KRT with dialysis (adjusted OR 1.88 ; 95\% CI 1.15-3.07) than those from the least deprived areas, and more often with HD than with PD. Among HD patients, the odds of starting treatment in emergency with a catheter was two-fold higher for the most deprived compared to the least deprived children (adjusted OR 2.08 ; 95\% CI 1.07-4.04). There was a trend towards later referral in patients from the most deprived areas.

Conclusions: Children from the most deprived areas have lower access to pre-emptive kidney transplantation, lower access to PD, have more urgent initiation of HD with a catheter, and tend to be later referred to a nephrologist.

\section{PI-118 RENAL LENGTH NORMATIVE VALUES IN CHILDREN AGED 0-18 YEARS - MULTICENTER STUDY}

Lukasz Obrycki ${ }^{1}$, Jedrzej Sarnecki ${ }^{2}$, Marianna Lichosik ${ }^{1}$, Malgorzata Sopinska ${ }^{3}$, Malgorzata Placzynska ${ }^{3}$, Malgorzata Stanczyk ${ }^{4}$, Julia Mirecka ${ }^{4}$, Agnieszka Wasilewska ${ }^{5}$, Maciej Michalski ${ }^{5}$, Weronika Lewandowska ${ }^{6}$, Tadeusz Derezinski ${ }^{6}$, Michal Pac ${ }^{1}$, Natalia Szwarc ${ }^{7}$, Karol Annusewicz ${ }^{8}$, Viktoriia Rekuta ${ }^{8}$, Karolis Ažukaitis ${ }^{9}$, Augustina Jankauskiene ${ }^{9}$, Boleslaw Kalicki ${ }^{3}$, Katarzyna Jobs ${ }^{3}$, Marcin Tkaczyk ${ }^{4}$, Janusz Feber ${ }^{10}$, Mieczyslaw Litwin ${ }^{1}$ ${ }^{I}$ Department of Nephrology, Kidney Transplantation and Hypertension, Children 's Memorial Health Institute, Warsaw, Poland, ${ }^{2}$ Department of Radiology, Children's Memorial Health Institute, Warsaw, Poland, ${ }^{3}$ Department of Paediatrics, Paediatric Nephrology And Allergology, The Military Institute of Medicine, Warsaw, Poland, ${ }^{4}$ Department of Pediatrics, Immunology And Nephrology, Polish Mothers Memorial Hospital Research Institute, Lodz, Poland, ${ }^{5}$ Department of Radiology, Polish Mothers Memorial Hospital Research Institute, Lodz, Poland, ${ }^{6}$ Outpatient Clinic "esculap", Gniewkowo, Poland, ${ }^{7}$ Department of Pediatrics, Jan Bogdanowicz Children 's Hospital, Warsaw, Poland, ${ }^{8}$ 
Department of Paediatrics, Nutrition and Metabolic Diseases, Children `s Memorial Health Institute, Warsaw, Poland, ${ }^{9}$ Clinic of Pediatrics, Faculty of Medicine, Vilnius University, Vilnius, Lithuania, ${ }^{10}$ Division of Nephrology, Department of Pediatrics, The Children's Hospital of Eastern Ontario, Ottawa

Introduction: Kidney length measurement and its interpretation is an integral part of the diagnosis of pediatric nephrology diseases. The most widely used kidney length norms, published in 1984, are based on an American study including 203 non-necessarily healthy patients.

Material and methods: The aim of the study was to develop ultrasoundbased normative values of kidney length in European healthy children and adolescents aged 0-18 years.

Our study included 1769 patients aged $0-18$ years ( 877 boys; $50 \%$ ), with normal glomerular filtration rate, no history of urinary tract infections, no kidney abnormalities in abdominal ultrasound, recruited in schools and kindergartens as well as in pediatric units in Poland and Lithuania. The examination was conducted in accordance with the standards of the Polish and European ultrasound societies.

Results: Based on the ultrasound measurements, kidney length nomograms (including $2.5^{\text {th }} ; 25^{\text {th }} ; 50^{\text {th }} ; 75^{\text {th }} ; 90^{\text {th }}$ and $97.5^{\text {th }}$ percentiles) for patients aged 0-18 years were developed using quantile regression. There were no sex-related or patient position (supine or prone) differences in renal size. The median difference between the left and right kidney across all age categories was $-1.00 \mathrm{~mm}\left(5^{\text {th }}\right.$ percentile $=-6.7 \mathrm{~mm}, 95^{\text {th }}$ percentile $=+3.6 \mathrm{~mm}$ ). Out of age, weight, height, body surface area and body mass index, the height was the most significant predictor or renal length $(\mathrm{T}=9.21, \mathrm{p}<0.0001$, relative importance $=25.8 \%$ ). We also developed a formula to calculate the average kidney length $\left(50^{\text {th }}\right.$ percentile) and the cut-off values for small $\left(<2.5^{\text {th }}\right.$ percentile) and enlarged kidney $\left(>97.5^{\text {th }}\right.$ percentile) using the patients height.

Conclusions: The main determinant of kidney length was height. Our results, based on a largest pediatric cohort to date, can serve as reference values in clinical practice and research studies.

\section{PI-119 THE SPECTRUM OF DISEASE IN CHILDREN WITH ESTABLISHED KIDNEY FAILURE USING REGISTRY AND LINKED ELECTRONIC HEALTH RECORD DATA}

Lucy Plumb ${ }^{1}$, Retha Steenkamp ${ }^{1}$, Alexander Hamilton ${ }^{2}$, Heather Maxwell ${ }^{3}$, Carol Inward ${ }^{4}$, Stephen Marks ${ }^{5}$, Dorothea Nitsch ${ }^{1}$ ${ }^{1}$ UK Renal Registry, ${ }^{2}$ Population Health Sciences, University of Bristol Medical School, United Kingdom, ${ }^{3}$ Department of Paediatric Nephrology, Royal Hospital For Children, Glasgow, United Kingdom, ${ }^{4}$ University Hospitals Bristol \& Weston Nhs Foundation Trust, United Kingdom, ${ }^{5}$ University College London Great Ormond Street Institute Of Child Health, Nihr Great Ormond Street Hospital Biomedical Research Centre, London, United Kingdom

Introduction: Children with established kidney failure may have additional medical conditions that impact upon care and outcomes for their kidney disease. This data is often not collected as part of a core dataset by kidney registries, resulting in data capture of variable completeness and accuracy. The aim of this study was to audit data on coexisting disease held by the UK Renal Registry (UKRR) against national electronic health record data using Hospital Episode Statistics (HES).

Material and methods: The study population comprised English children aged $<18$ years receiving kidney replacement therapy (KRT) for $>90$ days on 31/12/2016. The proportion of children coded for co-existing disease variables as captured by the UKRR were compared to relevant ICD-10 diagnostic codes in HES.

Results: Linked HES data was available for 808 (62\% male) children at the end of 2016. The median age was 12.0 (IQR 7.8-15.3) years. Using UKRR data, $23.1 \%$ of children had no additional diagnoses. Higher proportions of disease were noted in the UKRR dataset for most disease variables (9/12); higher proportions of cerebral palsy (12 versus $4 \%$ ) and congenital anomalies ( 73 versus $47 \%$ ) were recorded by HES. HES data offered a more comprehensive picture of co-existing disease not currently captured by the UKRR dataset: a strong male preponderance was noted among gastrointestinal, genetic, respiratory, and perinatal disease. UKRR data showed a female predominance for malignancy and chromosomal anomalies while prematurity, congenital anomalies and congenital heart disease affected males more frequently.

Conclusions: This is the first study to report the spectrum of co-existing disease for UK children with established kidney failure using registry and electronic health data. Electronic health record data offers a comprehensive overview of all disease groups, which will help facilitate future audit and research of this cohort.

\section{PI-120 COMPARING DIFFERENT MORPHO-COMPOSITIONAI GROUPS OF KIDNEY STONES IN A PEDIATRIC RENAL LITHIASIS REGISTRY}

Javier Lumbreras ${ }^{1}$, Maria Dolores Rodrigo ${ }^{1}$, Rafael Maria Prieto ${ }^{2}$, Antonia Costa-bauza ${ }^{2}$, Pilar Sanchís ${ }^{2}$, Natalia Espinosa ${ }^{3}$, Iratxe Tapia ${ }^{3}$, Juan David González ${ }^{4}$, María Isabel Luis-yanes ${ }^{5}$, Inés Vergara ${ }^{6}$, Héctor Ríos ${ }^{7}$, Montserrat Antón-gamero ${ }^{8}$, Mar Espino ${ }^{9}$, Jorge Luján ${ }^{10}$, Felix Grases ${ }^{2}$

${ }^{l}$ Pediatric Nephrology Unit. University Hospital Son Espases-balearic Islands Health Research Institute (idisba). Palma De Mallorca, Spain, ${ }^{2}$ Laboratory Of Renal Lithiasis Research, Universitary Institute Of Health Sciences Research (iunics), University Of Balearic Islands-balearic Islands Health Research Institute(idisba). Palma De Mallorca, Spain, ${ }^{3}$ Pediatric Nephrology Unit, University Hospital Son Espases, Palma De Mallorca, Spain, ${ }^{4}$ Pediatric Nephrology Unit, University Hospital Santa Lucia, Cartagena, Spain, ${ }^{5}$ Pediatric Nephrology Unit, University Hospital Nuestra Señora De Candelaria. Santa Cruz. De Tenerife, Spain, ${ }^{6}$ Pediatric Nephrology Unit, Complejo Hospitalario Universitario A Coruña, La Coruña, Spain, ${ }^{7}$ Pediatric Nephrology Department, University Hospital Vall Dhebron, Barcelona, Spain, ${ }^{8}$ Pediatric Nephrology Unit, University Hospital Reina Sofia, Córdoba, Spain, ${ }^{9}$ Pediatric Nephrology Unit, University Hospital 12 De Octubre, Madrid, Spain, ${ }^{10}$ Pediatric Nephrology Unit, Hospital Sanitas La Moraleja, Madrid, Spain

Objectives: Different morpho-compositional types of kidney stones represent different etiologies. By understanding these, we can achieve a correct therapeutic approach. We expose clinical and analytical particularities among several groups of stones in children.

Material and methods: A national renal lithiasis registry is active in our country since January 2015. A specialized laboratory analyses stones. Physicians provide clinical and analytical data. For this study, similar stone types were grouped in 5 categories, according to main component: calcium oxalate monohydrate (COM, $\mathrm{n}=8)$, calcium oxalate dihydrate (COD, n=34), hydroxyapatite (HAP, $n=11$ ), magnesium ammonium phosphate (MAP, $n=16)$ and Others $(n=14)$. Clinical and analytical variables were compared.

Results: 83 stones from 69 patients were included. Male prevalence ranged from $64 \%$ in HAP to $93 \%$ in MAP. Mean age at diagnosis varied from COD (10.3 years old) to MAP and HAP (3.1). Highest 
prevalence of relatives with stones appeared in COM (100\%), lowest in MAP (36\%). Urinary tract abnormalities ranged between $40-44 \%$ in HAP and MAP respectively, and $12-14 \%$ in the rest. Past urinary infection appeared from $69 \%$ in MAP to $13 \%$ in COM. HAP had much more frequently multiple stones at diagnosis $(73 \%)$ with the lowest spontaneous passage (55\%). Lowest urine volumes were observed in COM $(0.7[0.6-1.1] \mathrm{mL} / \mathrm{kg} / \mathrm{h})$, highest urine $\mathrm{pH}$ in HAP $(7,0[7,0-8,5])$. Hypercalciuria prevalence was similar among all groups (38-50\%) except COM (17\%). Hypocitraturia was more common in COM (50\%), COD (36\%) and HAP (29\%). Calcium/citrate ratio $>0.33 \mathrm{mg} / \mathrm{mg}$ was more frequent in COD (60\%) and HAP $(57 \%)$. Conclusions: COM showed more frequently low urine volumes, hypocitraturia and stones in relatives. MAP and HAP were associated to urinary tract abnormalities, COD and HAP with high calcium/citrate ratio. HAP had higher urine $\mathrm{pH}$.

\section{PI-121 CLINICAL AND ETIOPATHOGENIC CHARACTERISATION OF HYDROXYAPATITE STONE-FORMERS IN A PEDIATRIC RENAL LITHIASIS REGISTRY}

Javier Lumbreras ${ }^{1}$, Maria Dolores Rodrigo ${ }^{1}$, Rafael Maria Prieto ${ }^{2}$, Antonia Costa-Bauza ${ }^{2}$, Pilar Sanchís ${ }^{2}$, Natalia Espinosa ${ }^{3}$, Iratxe Tapia ${ }^{3}$, Juan David González ${ }^{4}$, María Isabel Luis-Yanes ${ }^{5}$, Montserrat Antón-Gamero ${ }^{6}$, Felix Grases ${ }^{2}$

${ }^{1}$ Pediatric Nephrology Unit, University Hospital Son Espases-balearic Islands Health Research Institute (idisba). Palma De Mallorca, Spain, ${ }^{2}$ Laboratory of Renal Lithiasis Research, Universitary Institute Of Health Sciences Research (iunics), University of Balearic Islands-balearic Islands Health Research Institute(idisba). Palma De Mallorca, Spain ${ }^{3}$ Pediatric Nephrology Unit, University Hospital Son Espases, Palma De Mallorca, Spain ${ }^{4}$ Pediatric Nephrology Unit, University Hospital Santa Lucia, Cartagena, Spain, ${ }^{5}$ Pediatric Nephrology Unit, University Hospital Nuestra Señora De Candelaria, Santa Cruz De Tenerife, Spain, ${ }^{6}$ Pediatric Nephrology Unit, University Hospital Reina Sofia, Córdoba, Spain

Objectives: Hydroxyapatite stones are more frequent in pediatric patients. Hypocitraturia, hyperphosphaturia, high urine $\mathrm{pH}$, urine stasis, and distal renal tubular acidosis (dRTA) are related factors. We describe our cohort.

Material and methods: A national pediatric renal lithiasis registry is active since January 2015 , sponsored by the national pediatric nephrology association. Stones are analyzed by a specialized laboratory. Clinical and analytical data are provided by clinicians. We describe clinical variables in hydroxyapatite stone-formers and compare their metabolic urinary profiles with other types of calculi (struvite excluded).

Results: In september 2018, 83 stones from 69 patients had been included. In 11 stones (13\%) from 10 patients, main component was hydroxyapatite. Male:female ratio was 2:1. Age at diagnosis was 3,1 [1,4-13,7] vs $9,4[4,2-13,0](\mathrm{p}=0,10)$. Family history of kidney stones was present in $6(67 \%)$, past urinary tract infection (UTI) in $5(46 \%)$, preterm delivery in $2(19 \%)$. Structural or functional abnormalities of the urinary tract appeared in 4 patients (40\%): one (two stones) with ureterovesical junction obstruction, one duplicated collecting system and one neurogenic bladder. Nine stones $(73 \%)$ showed multiple localizations in image evaluation. Six stones $(55 \%)$ passed spontaneously. No urinary solute concentration (calcium, phosphate, citrate) nor solute/creatinine ratio showed significant differences compared to the rest of stones but $\mathrm{pH}$ did $(7,0[7,0$ $-8,5]$ vs $6,0[6,0-7,0], p=0,02)$. dRTA was demonstrated in 2 patients (incomplete form), discarded in 4 cases and had not been evaluated in another 4 ones.
Conclusions: Hydroxyapatite stones appeared at an early age with multiple localizations. Patients frequently had past UTI, abnormalities in urinary tract and relatives with lithiasis. The most significant urinary metabolic difference was higher $\mathrm{pH}$. dRTA was insufficiently evaluated.

PI-122 A RANDOMIZED CONTROL TRIAL COMPARING SAFETY AND EFFICACY OF SINGLE DOSE VERSUS DIVIDED DAILY DOSE PREDNISOLONE IN FIRST EPISODE OF CHILDHOOD NEPHROTIC SYNDROME

Taniya Khan, Surupa Basu, Shakil Akhtar, Subhankar Sarkar, Rajiv Sinha

Institute of Child Health, India

Objectives: Corticosteroid is the standard therapy for first episode nephrotic syndrome (FENS). Daily divided dose (DD) prednisolone is still commonly prescribed despite early morning single dose (SD) hypothetically having less hypothalamic-pituitary-adrenal axis (HPA) suppression and better medication adherence. Controlled study confirming these hypotheses are scarce. We conducted a randomized controlled trial with the primary objective to compare HPA axis suppression between SD and DD steroid at completion of 6 weeks of daily steroid before reduction. Secondary objective included comparing side effects, time to remission, time to first relapse, and frequency of relapse

Methods: Children with FENS were randomized to receive prednisolone $2 \mathrm{mg} / \mathrm{kg}$ per day, either as SD $(n=26)$ or in two DD $(n=27)$ for 6 weeks, followed by single alternative daily dose of $1.5 \mathrm{mg} / \mathrm{kg}$ for 6 weeks. After completion of $6+6$ weeks of steroid therapy follow up continued for 6 months. HPA axis was assessed by short synacthen test (SST) on completion of 6 weeks of daily steroid.

Results: In both groups all patients were steroid responsive without differences in time to remission [SD: 9 days (IQR 8-12.5) and DD: 10 days (IQR 7.5-12)], relapse rates [SD: $35 \%, \mathrm{n}=9 / \mathrm{DD}: 37 \%, \mathrm{n}=10$ ], adverse event or medication adherence. In SD group $20 \%(\mathrm{n}=5)$ passed SST vs $0 \%$ in DD group ( $\mathrm{p}=0.01)$. Time to first relapse was longer in SD (115; IQR 40.5 to 126$)$ than in DD (60; IQR 32 to 68) but did not reach statistical significance.

Conclusion: Our finding suggests that SD is equally effective as DD prednisolone in FENS with less HPA axis suppression. Further studies are required to assess role of HPA axis suppression on relapse of nephrotic syndrome.

\section{PI-123 HIGH INCIDENCE OF COL4 VARIANTS AMONG A COHORT OF CHILDHOOD STEROID RESISTANCE NEPHROTIC SYNDROME FROM EASTERN INDIA.}

Subhankar Sarkar ${ }^{1}$, Arpita Ray Choudhury ${ }^{2}$, Samartya Pulai ${ }^{2}$, Saugat Das Gupta ${ }^{2}$, Rajiv Sinha ${ }^{1}$

${ }^{1}$ Institute of Child Health, India, ${ }^{2}$ IPGMER, India

Introduction: About one third of children with steroid resistant nephrotic syndrome (SRNS) have underlying genetic origin and majority of them have poor response to immunosuppressant. Western data suggest high incidence of NPHS 2 (podocin) mutation among childhood SRNS (cSRNS) but there is scarcity of data among Indian population.

Material and methods: Retrospective multicentre cross-sectional study to evaluate genetic causal association of SRNS. cSRNS (3 months to 18 years age) who had undergone genetic analysis utilising next generation sequencing between May 2016 and December 2020 at four tertiary centers across Eastern India. Results were classified according to American 
College of Medical Genetics and Genomic criteria. Pathogenic / likely pathogenic variants were taken as significant.

Results: During the study period 70 cSRNS (33 female, 51 primary SRNS, median age of onset of nephrotic syndrome: 56 months; IQR: 10 to 66) underwent genetic analysis. 48 variants were identified out of which 19 were significant, distributed among 17 children giving a positive yield of $24 \%$ among our cohort. Just over a third $(37 \%)$ of the variants identified were in COL 4 genes $(C O L 4 A 5 \times 4, C O L 4 A 4 \mathrm{x} 1$, COL $4 A 3 \times 2$ ), followed by NPHS1 x 4, WT1 x 2, NPSH2x 1, PLCE1 x 1, COQ2 $\mathrm{x} 1$ and $P A X 2 \times 1$. Significant variants were more likely among primary SRNS $(76.5 \%)$. Only a single child with underlying significant variant (Compound heterozygous mutation in [NPHS1: Exon 14 c.1802G>C (p.Gly601Ala) \& Exon11 c.1339G>A (p.Glu447Lys)] showed response to immunosuppressant in contrast to $40 \%(21 / 53)$ among whom significant variants were not found.

Conclusions: Similar to international reports; a quarter of SRNS in our cohort were genetic in origin, majority of them were primary SRNS and had poor response to immunosuppressant. Important highlight was our high incidence of COL4 variants which is in sharp contrast to western literature.

\section{PI-124 ASSOCIATION BETWEEN DEPRIVATION AND WORSE OUTCOMES IN NEPHROTIC SYNDROME}

Abigail Townsend ${ }^{1}$, Thomas Dowsett ${ }^{2}$, Louise Oni ${ }^{2}$, Caroline Jones ${ }^{2}$ ${ }^{1}$ University of Liverpool, United Kingdom, ${ }^{2}$ Department of Paediatric Nephrology, Alder Hey Childrens Hospital, Nhs Trust, Liverpool, United Kingdom

Objectives: This study explores whether higher levels of deprivation could influence rates of relapse and treatment in childhood nephrotic syndrome (NS), and if additional support from social services could improve outcomes in these patients.

Methods: A retrospective cohort study of 27 patients with childhood NS treated at Alder Hey Children's Hospital was conducted. Inclusion criteria included a Liverpool postcode, steroid responsive NS, and age 1-18 years. Records were searched over a 24-month period and data collected on number of relapses, hospital admissions, Did Not Attend (DNA) appointments, courses of rituximab and second line immunosuppressive agents. Patients were grouped into deciles according to their postcode using the Index of Multiple Deprivation (IMD) 2019. Univariant analysis and the Mann-Whitney $U$ test were used to assess the associations between the IMD decile and outcomes.

Results: $19(70 \%)$ of 27 of patients resided in the top $20 \%$ most deprived areas. $13(93 \%)$ of 14 admissions occurred in these patients, which was statistically significant $(0.68$ vs $0.13, p=0.048) .47(81 \%)$ of 58 relapses also occurred in these patients. $9(90 \%)$ of 10 rituximab courses and 18 (95\%) of 19 DNA appointments occurred in patients living in the top $10 \%$ most deprived areas. There was no statistically significant association for number of relapses or use of second line immunosuppressants.

Conclusions: Social deprivation was associated with increased number of hospital admissions in childhood NS in a small cohort of patients residing in Liverpool, one of the most deprived regions in the United Kingdom. More research is needed to understand how deprivation may influence outcomes and therefore management of childhood NS.

\section{PI-125 CENTRAL BLOOD PRESSURE AND EARLY VASCULAR AGING IN PEDIATRIC PATIENTS WITH IDIOPATHIC NEPHROTIC SYNDROME}

Piotr Skrzypczyk ${ }^{1}$, Anna Ofiara ${ }^{1}$, Michal Szyszka ${ }^{2}$, Anna Zacharzewska ${ }^{3}$, Malgorzata Panczyk-Tomaszewska ${ }^{1}$

${ }^{1}$ Department of Pediatrics and Nephrology, Medical University of Warsaw, Poland, ${ }^{2}$ Department of Pediatrics and Nephrology, Doctoral
School, Medical University of Warsaw, Poland, ${ }^{3}$ Student Scientific Group at the Department of Pediatrics and Nephrology, Medical University of Warsaw, Poland

Introduction: Hyperlipidemia, hypercoagulability, and immunosuppressive treatment may elevate blood pressure and exert negative impact on arterial wall. Thus, the aim was to assess central blood pressure and arterial damage in pediatric patients with idiopathic nephrotic syndrome (INS).

Material and methods: In a group of 51 children with INS (10.86 \pm 4.18 years, 35 boys, 16 girls, 31 children with steroid-sensitive INS and 20 children with steroid-resistant INS) and 33 healthy children $(10.89 \pm 2.20$ years, 20 boys, 13 girls) we evaluated peripheral and central blood pressure, arterial damage: aortic pulse wave velocity $(\mathrm{aPWV})[\mathrm{m} / \mathrm{s}]$, [Z-score], augmentation index (AIx75HR [\%]) and common carotid artery intimamedia thickness (cIMT) [mm], [Z-score].

Results: Children with INS compared to healthy children had higher central systolic blood pressure (AoSBP) $(98.67 \pm 11.65$ vs. $92.09 \pm 6.31$ $[\mathrm{mm} \mathrm{Hg}], \mathrm{p}<0.001)$, aPWV (4.78 \pm 0.79 vs. $4.13 \pm 0.59[\mathrm{~m} / \mathrm{s}], \mathrm{p}=<0.001)$, and cIMT $(0.44 \pm 0.06$ vs. $0.40 \pm 0.04[\mathrm{~mm}], \mathrm{p}<0.0015)$. In children with INS, AoSBP correlated with total cholesterol $(r=0.350, p=0.027)$, LDLcholesterol $(\mathrm{r}=0.400, \mathrm{p}=0.019)$, and triglyceride $(\mathrm{r}=0.317, \mathrm{p}=0.049)$, cIMT correlated with BMI Z-score $(\mathrm{r}=0.327, \mathrm{p}=0.026)$, aPWV Zscore with systolic blood pressure $Z$-score $(r=0.327, p=0.024)$, and AIx75HR with systolic and diastolic blood pressure Z-scores ( $\mathrm{r}=0.387$, $\mathrm{p}=0.010$ and $\mathrm{r}=0.335, \mathrm{p}=0.028$ ). Children with steroid-resistant INS had significantly higher AoSBP $(103.30 \pm 11.96$ vs. $95.68 \pm 10.59$ [mm Hg], $\mathrm{p}=0.021$ ) compared to patients with steroid-sensitive INS.

Conclusions: 1 . Children with idiopathic nephrotic syndrome are characterized by elevated central blood pressure and subclinical arterial damage.

2. In children with INS, dyslipidemia and steroid-resistance are risk factors for elevated central blood pressure, and hypertension is a risk factor for early vascular aging.

\section{PI-126 COMPARATIVE STUDY ON THE THERAPEUTIC RESPONSE TO DIFFERENT DRUG GROUPS IN PATIENTS WITH CORTICODEPENDENT NEPHROTIC SYNDROME}

Pedro Arango Sancho, Elena Codina Sampera, Yolanda Calzada Baños, Víctor López-baez, Ana Vinuesa Jaca, Lina Catherine Hernández Zúñiga, Álvaro Madrid Aris

Hospital Sant Joan De Déu, Spain

Introduction: To study the effectiveness and relapses-free persistence of different treatment groups in 107 patients with corticodependent nephrotic syndrome (CDNS)

Material and methods: Retrospective study in patients with CDNS (2011-2019) who have received treatment with cyclophosphamide(CF), mycophenolate(MMF), cyclosporine(CyA), rituximab(RTX) and/or ofatumumab(OFA), establishing homogeneous groups based on the number of drugs received and the introduction order, thereby defining 7 groups. In group 1 those with only CF. Group 2a those who received $\mathrm{CF}+\mathrm{MMF}$. Group $2 \mathrm{~b}$ those who received $\mathrm{CF}+\mathrm{CyA}$. Group 3 those with 3 drugs, subdivided into $3 \mathrm{a}(\mathrm{CF}, \mathrm{MMF}$ and $\mathrm{CyA}$ ) and $3 \mathrm{~b}$ (reversing order of MMF and CyA). Finally, group 4(CF-MMF-CYA-RTX) and group 5(CF-MMF-CYA-RTX-OFA) included monoclonal antibodies. The variables analyzed were age at diagnosis, initial treatment, relapses after last treatment, time without relapses and without treatment since the last immunosuppressant.

Results: Of the total cohort, 63 patients were included (excluding those who did not the criteria of any groups). The mean age at diagnosis was 3.49 years. The distribution was: $62 \%$ (group 1), 17\%(2a), $8 \%(2 b)$, $1.5 \%(3 a), 6.3 \%(3 b), 3.1 \%(4)$ and $3.1 \%(5)$. The mean free time (years) of relapses was: 3.69 (group 1), 1.33 (group 2a), 3.39 (group 2b), 3.7 (group 3a-b), 4.22 (group 4) and 1.04 (group 5). The percentage of patients without relapses after the last treatment was $70 \%, 100 \%(2 a-b)$, 
$75 \%$ (3a-b), $100 \%, 100 \%$, respectively. Currently, within group 1, 83\% are without treatment, $10 \%$ in $2 \mathrm{a}, 80 \%$ in $2 \mathrm{~b}, 75 \%$ in $3 \mathrm{~b}, 50 \%$ in 4 and 5 and none in $3 \mathrm{a}$

Conclusions: $62 \%$ of the SNCDs were controlled only with the administration of CF. Of those who received more than one drug, the group that received $\mathrm{MMF}$ as a second immunosuppressant had a higher mean relapses-free time than the CyA group $(\mathrm{p}<0.05)$

\section{PI-127 LONG-TERM OBESITY PREVALENCE AND LINEAR GROWTH IN CHILDREN WITH IDIOPATHIC NEPHROTIC SYNDROME: IS NORMAL GROWTH AND WEIGHT CONTROL POSSIBLE WITH STEROID SPARING DRUGS AND LOW DOSE STEROIDS FOR RELAPSES?}

Nilüfer Göknar, Hazel Webb, Aoife Waters, Kjell Tullus Great Ormond Street Hospital NHS Foundation Trust London UK and UCL Institute of Child Health, United Kingdom

Introduction: Long term steroid treatment in children is known to cause obesity and negatively affect growth. The objective of this study was to determine the prevalence of obesity and overweight and analyze linear growth in children with nephrotic syndrome. Also, we aimed to investigate possible positive effects of steroid sparing drugs and low-dose steroid regimens on these parameters.

Material and methods: Our study was a retrospective analysis of patients treated at Great Ormond Street Hospital with a diagnosis of idiopathic nephrotic syndrome (NS) from 2009-2019. Height, weight and prednisolone dose was recorded at each visit. Standard deviation scores (SDS) for height, weight and BMI and rate of change between the final and initial height, weight and BMI were calculated. In a relapse without significant edema, GC treatment was introduced at low dose; $30 \mathrm{mg} / \mathrm{m}^{2} /$ day. Almost $85 \%$ of patients were treated with steroid sparing drugs.

Results: The study involved 265 children treated with glucocorticoids for nephrotic syndrome for a mean duration of 43 months (range: 6-167, IQR: 17;63.3). Mean first BMI SDS was $+1.40 \pm 1.30$ (median: 1.35 ) and final was $+0.79 \pm 1.30$ (median: 0.79 ). At the initial assessment $41.4 \%$ of the patients were obese (BMI $>95^{\text {th }}$ percentile), $19.5 \%$ were overweight (BMI $85^{\text {th }}-95^{\text {th }}$ percentile). At the last clinical visit; $24 \%$ of the patients were obese, $17 \%$ were overweight. Children with SSNS, SDNS/FRNS and SRNS had lower BMI SDS at last clinical visit compared to initial assessment. Mean first height SDS of the cohort was -0.11 \pm 1.22 and final score was $+0.078 \pm 1.14(\mathrm{p}<0.0001)$. Median height SDS had improved by 0.2 SDS at the end of the follow up.

Conclusions: These results indicate that, decreased obesity ratios and improved linear growth is possible in children with nephrotic syndrome with low dose steroid regimens and steroid sparing drugs despite frequent relapses.

\section{PI-128 CYCLOSPORINE-INDUCED NEPHROTOXICITY IN CHILDREN WITH STEROID-RESISTANT NEPHROTIC SYNDROME DUE TO FOCAL SEGMENTAL GLOMERULOS- CLEROSIS}

Larisa Prikhodina, Svetlana Papizh , Tatyana Lepaeva, Tatyana Nikishina Veltishev Research \& Clinical Institute of Pediatrics, Pirogov Russian National Research Medical University, Moscow, Russia

Introduction: Calcineurin inhibitors (CNIs) remain the first-line immunosuppressive therapy in children with steroid-resistant nephrotic syndrome (SRNS). This study aimed to identify the frequency of CNI-induced nephrotoxicity (CNIT) and its clinical and histological features in children with SRNS due to focal segmental glomerulosclerosis (FSGS).

Material and methods: We studied 35 children $(21 \mathrm{~F} / 14 \mathrm{M})$ aged at onset of SRNS due to FSGS 6.0 (IQR: $3.0 ; 9.9$ ) years. The eGFR before cyclosporine (CsA) treatment was $97.5(86.4 ; 113.8) \mathrm{ml} / \mathrm{min} / 1.73 \mathrm{~m}^{2}$. The initial CsA dosage was $4.6(4.0 ; 5.0) \mathrm{mg} / \mathrm{kg} /$ day to blood trough level of $80-150 \mathrm{ng} / \mathrm{ml}$. CNIT was defined as rise in serum creatinine $\geq 25 \%$ from baseline level. The follow-up was $54.0(35.0 ; 80.0)$ months.

Results: $24 / 35(68.6 \%)$ children with SRNS due to FSGS developed CNIT in $7.0(5.3 ; 16.5)$ months of CsA treatment. There were no significant differences in age at onset of SRNS, albumin and serum uric acid levels, proteinuria, disease duration before initiating of CsA treatment, baseline eGFR, CsA dosage and C0 blood levels in patients with CNIT compared with individuals without CNIT ( $p>0.05$ ). Histological findings at the $1^{\text {st }}$ kidney biopsy did not differ among the groups including rate of global and focal glomerulosclerosis, interstitial fibrosis and tubular atrophy ( $p>0.05$ ). After 6 months of CsA treatment children with CNIT compared with patients without CNIT had significantly higher serum uric acid level: $0.37(0.29 ; 0.41)$ vs. $0.31(0.26 ; 0.33) \mathrm{mmol} / \mathrm{l}(\mathrm{p}=0.041)$ and lower eGFR: $86.8(65.6 ; 91.2)$ vs. $85.2(82.9 ; 123.8) \mathrm{ml} / \mathrm{min} / 1.73 \mathrm{~m}^{2}$ $(\mathrm{p}=0.044)$ without any differences in C0 CsA and albumin serum levels, proteinuria and rate of response to CsA treatment ( $>0.05$ ).

Conclusions: We conclude that CNIT occurred in $68.6 \%$ of children with SRNS due to FSGS. Increased serum uric acid level and decreased eGFR in 6 months of CsA treatment were found in patients with CNIT. Identification of biomarkers of tubular dysfunction might be useful for early detection and prevention of CNIT to improve outcome of SRNS due to FSGS in children.

\section{PI-129 CLINICAL AND MOLECULAR CHARACTERISTICS IN CHILDREN WITH SCHIMKE IMMUNO-OSSEOUS DYSPLASIA}

Larisa Prikhodina, Svetlana Papizh, Olga Katysheva Veltishev Research \& Clinical Institute of Pediatrics, Moscow, Russia

Introduction: Schimke immunoosseous dysplasia (SIOD; OMIM \#242900) is an autosomal recessive multisystem disorder characterized by spondyloepiphyseal dysplasia, T-cell deficiency, and nephrotic syndrome (NS) progressing to ESKD during childhood. The aim of the study was to investigate clinical and molecular characteristics in Russian children with SIOD.

Methods: Retrospective analysis of phenotype and genotype of 10 children $(6 \mathrm{M} / 4 \mathrm{~F})$ with SIOD was conducted. Kidney biopsy performed in two subjects revealed FSGS. The median follow-up period was 14.5 (IQR: $11.8 ; 24.5)$ months. SMARCAL1 mutations were identified by direct Sanger sequencing $(n=5)$ and NGS $(n=5)$.

Results: Low birth weight (LBW) resulted from intrauterine growth restriction (IUGR) had 10/10 (100\%) children with SIOD. The median age at onset of NS was 49.0 (IQR: 38.0; 51.0) months. Among patients with SIOD NS, spondyloepiphyseal dysplasia and T-cell deficiency had 10/10 (100\%) children, cerebral ischemic events had 5/10 (50\%), hematological abnormalities and CAKUT had 4/10 (40\%), respectively. Pathogenic homozygous $(n=5)$ or compound heterozygous $(\mathrm{n}=4)$ nonsense variant $\mathrm{c} .2542 \mathrm{G}>\mathrm{T}$ (p.Glu848*) in the SMARCAL1 gene was identified in 9/10 (90\%) of individuals. One child had compound heterozygous SMARCAL1 variant c.994delA (p.R332fs); c.2070+2dupT. The median age of genetic diagnosis of SIOD was 54.0 (IQR: 44.0; 58.0) months. At the last follow-up of patients aged 5.5 (IQR: $4.5 ; 6.4$ ) years progression to $\mathrm{CKD}$ was found in all children, including stage 2 CKD in $4 / 10(40 \%)$ subjects, stage 3 CKD in $2 / 10(20 \%)$, stage 4 CKD in $1 / 10(10 \%)$ and ESKD in $3(30 \%)$ individuals.

Conclusion: We conclude that Russian children with SIOD characterized by LBW due to IUGR, NS, spondyloepiphyseal dysplasia, immunodeficiency, and common SMARCAL1 pathogenic variant c. $2542 \mathrm{G}>\mathrm{T}$ (p.Glu848*).

\section{PI-130 EFFECT OF LEVAMISOLE ON KIDNEY FUNCTION}

Lieke Hoogenboom, Hazel Webb, Kjell Tullus, Aoife Waters Great Ormond Street Hospital, United Kingdom 
Objectives: Levamisole is frequently used as a steroid sparing agent in children with nephrotic syndrome. Side effects, such as neutropenia, gastro-intestinal upset and skin rash have been reported. However, literature on the effect of renal function is lacking. Our study aims to report on effect of levamisole on eGFR.

Methods: A retrospective cohort study was conducted, including patient 0-18 years of age, treated for nephrotic syndrome with Levamisole at Great Ormond Street Hospital for Children between January 2010 and January 2020. Data was collected on clinical observations and serum creatinine values before, during and after treatment.

Results: In total, 75 children were included in the analysis. The mean duration of treatment was 19 (IQR 12-27) months. The median estimated GFR was 134 (IQR $119-160) \mathrm{ml} / \mathrm{min} / 1.73 \mathrm{~m} 2,101$ (IQR $91-113$ ) $\mathrm{ml} / \mathrm{min} / 1.73 \mathrm{~m} 2$ and 116 (IQR 106-153) $\mathrm{ml} / \mathrm{min} /$ $1.73 \mathrm{~m} 2$ respectively before, during and after treatment with Levamisole. The difference between eGFR before and after treatment compared with during treatment was statically significant $(\mathrm{P}<0.0001)$. During the treatment period the eGFR decrease was not progressive. The median Levamisole dose was 2.5 (IQR 2.32.6) $\mathrm{mg} / \mathrm{kg}$ and the dose was not correlated with the decrease in eGFR ( $\mathrm{r}=0.07,95 \%$ CI $-0.22-0.35)$.

Conclusions: Levamisole significantly decreases eGFR, however this increase is not progressive or irreversible and would not be an indication to discontinue the treatment.

\section{PI-131 IN VITRO CHARACTERIZATION OF THE DNA- BINDING AFFINITY OF THE WT1 TRANSCRIPTION FACTOR MUTANTS FOUND IN CHILDREN WITH STEROID- RESISTANT NEPHROTIC SYNDROME}

\author{
Martin BezdíČka ${ }^{1}$, Jakub Zieg ${ }^{2}$, OndŘej SouČek ${ }^{1}$ \\ ${ }^{I}$ Vera Vavrova Lab/vial, Department of Pediatrics, Second Faculty of \\ Medicine, Charles University and Motol University Hospital, Prague, \\ Czech Republic, ${ }^{2}$ Department of Pediatrics, Second Faculty of \\ Medicine, Charles University and Motol University Hospital, Prague, \\ Czech Republic
}

Introduction: Nephrotic syndrome is one of the most common glomerulopathies in children. Approximately one third of children with steroid-resistant nephrotic syndrome (SRNS) carry pathogenic variants in one of the nowadays almost 70 causative genes. WT1 gene, being one of the most frequent ones and coding the transcription factor WT1, is known to cause SRNS, which may be associated with disorder of sex development and nephroblastoma (i.e., Denys-Drash syndrome). This study aimed to characterize the DNA-binding affinity of WT1 mutants.

Material and methods: Eight distinct exonic WT1 variants were found in the national genetic database of children with SRNS. Wild type and mutant WT1 proteins were produced and the binding affinity of these proteins to the EGR1 DNA target sequence was analysed by microscale thermophoresis.

Results: Whereas three WT1 mutants (p.Arg439Pro, p.His450Arg and p.Arg463Ter) showed decreased binding affinity, other three mutants (p.Gln447Pro, p.Asp469Asn and p.His474Arg) revealed increased binding affinity and two mutants (p.Cys433Tyr and p.Arg467Trp) showed no change of binding affinity when compared to the affinity of the wild type WT1. No correlation was found between the binding affinity and the phenotype.

Conclusions: The WT1 mutants found in children with SRNS presented with variable DNA-binding affinity, with no association with the phenotype of the patients. Further investigation is needed to clarify the impact of the WT1 mutants on gene expression and changes in the numerous target signalling pathways. This might help to discover the underlying mechanisms and potentially allow for individualised treatment of the so far unfavourable disease prognosis.
PI-132 VACCINE TITERS IN CHILDREN WITH IDIOPATHIC NEPHROTIC SYNDROME ON ORAL IMMUNOSUPPRESSIVE DRUGS

Claire Herbez Rea, Claire Dossier, Cyrielle Parmentier, Olivia Boyer, Tim Ulinski, Jean-daniel Delbet

Trousseau Hospital, France

Introduction: Infections remain a major complication in children with idiopathic nephrotic syndrome (INS). This study reports post-vaccination antibody levels in patients with steroid dependent and steroid resistant INS treated with oral immunosuppressants. The primary endpoint was a positive antibody titer.

Material and methods: We carried out a retrospective study in three pediatric nephrology departments. All children with a diagnosis of INS treated with tacrolimus, mycophenolate mofetil or levamisole were included. The post-vaccine $\mathrm{Ab}$ titers and the following clinical and biological parameters were analyzed: age at diagnosis of the INS, number of relapses, duration of the INS, duration of immunosuppressive treatment, immunoglobulin levels, time between the last relapse and postvaccination serologies and time between the last vaccination and serologies.

Results: Sixty-two patients were included. The median age at INS diagnosis was 4.08 (2.78-7.11) years, the median duration of the INS 3.43 (2.06-7.09) years, the median time between the last relapse and postvaccination serologies was $1.15(0.68-2.08)$ years. 30/62 (48\%) patients received calcineurin inhibitors, $44 / 62(71 \%)$ received mycophenolate mofetil, 15/62 (24\%) received both and 11/62 (18\%) received levamisole. The percentage of positive vaccine titers for hepatitis B, measles, diphtheria / tetanus and chickenpox were $38 \%, 66 \%, 79 \%$ and $55 \%$, respectively. A higher number of relapses was associated with a negative antibody titer against hepatitis B.

Conclusions: Patients with INS treated with oral immunosuppressive therapy have inadequate post-vaccination antibody coverage, which is not correlated with any specific treatment modality. However, the number of relapses appears to be associated with the likelihood of having negative hepatitis B antibody titers.

\section{PI-133 ANTENATAL DIAGNOSIS OF TYPE 1 HYPEROXALURIA AND EARLY NEONATAL INITIATION OF LUMASIRAN DO NOT PREVENT NEPHROCALCINOSIS: A CASE REPORT}

Anne-Laure Sellier-Leclerc ${ }^{1}$, Cécile Acquaviva-bourdain ${ }^{2}$, Bruno Ranchin ${ }^{1}$, Aurelia Bertholet-Thomas ${ }^{1}$, Pierre Cochat ${ }^{3}$, Justine Bacchetta ${ }^{3}$

${ }^{1}$ Centre De Reference Des Maladies Renales Et Phosphocalciques Rares, Hcl, Bron, France, ${ }^{2}$ Laboratory of Inborn Metabolicdiseases, Centre De Biologie Est, Hcl, Lyon, France, ${ }^{3}$ Centre De Reference Des Maladies Renales Et Phosphocalciques Rares, Hcl, Bron, France; Universite Claude Bernard Lyon 1, Lyon, France

Objectives: We report on a newborn with primitive hyperoxaluria type 1 (PH1) treated with lumasiran early in life after prenatal diagnosis (familial screening).

Methods: At birth, the child displayed increased circulating oxalate levels and urinary oxalate/creatinine ratio (Uox/creat), respectively at 36 $\mu \mathrm{mol} / \mathrm{L}(\mathrm{N}<5)$ and $1263 \mathrm{mmol} / \mathrm{mmol}(\mathrm{N}<280)$. Renal function was normal.

Results: Given the familial history of end-stage renal disease at the age of 4 months, it was decided to perform the first subcutaneous injection at day 9. According to the product information template, he received monthly lumasiran (3 injections of $6 \mathrm{mg} / \mathrm{kg}$, and subsequent tapering to $3 \mathrm{mg} / \mathrm{kg}$ ). Uox $/$ creat further increased at 2850 $\mathrm{mmol} / \mathrm{mmol} 15$ days after the first injection and then started to decrease (1500 at month 1 and 1060 at month 2). However, despite 
an early initiation of lumasiran, decreased circulating oxalate levels (12 $\mu \mathrm{mol} / \mathrm{L}$ at 3 months) and persistent normal renal function, the evolution was not completely satisfying. Indeed, renal ultrasounds performed after 2 months of lumasiran therapy revealed nephrocalcinosis stage 3, with a stabilization of Uox/creat between months 2 and 3 around $1000 \mathrm{mmol} / \mathrm{mmol}$. Of note, hyperhydration and potassium citrate were associated with lumasiran from the beginning. Conversely, tolerance of lumasiran was good without any side effect: it was therefore decided to restart injections at $6 \mathrm{mg} / \mathrm{kg}$ in order to try to lower Uox/creat below the threshold of 300 $\mathrm{mmol} / \mathrm{mmol}$.

Conclusion: To our knowledge, this is the youngest child treated with lumasiran worldwide. Despite satisfying clinical and biological tolerance, the initiation of lumasiran early during the neonatal period associated with conventional management did not prevent the onset of nephrocalcinosis. The specific hepatic immaturity in this age group could justify to maintain a $6 \mathrm{mg} / \mathrm{kg}$ regimen per injection, but this hypothesis deserves to be confirmed.

\section{PI-134 COMPASSIONATE USE TREATMENT WITH RNAI MEDICATION (NEDOSIRAN) IN TWO PATIENTS WITH PRIMARY HYPEROXALURIA TYPE 1 AND MAINTENANCE HEMODIALYSIS}

\section{Cristina Martin-higueras ${ }^{1}$, Gesa Schalk ${ }^{2}$, Bernd Hoppe ${ }^{3}$ \\ ${ }^{1}$ German Hyperoxaluria Center, Germany, ${ }^{2}$ Kindernierenzentrum Bonn, Germany, ${ }^{3}$ Dicerna Pharmaceuticals, USA}

Introduction: The primary hyperoxalurias $(\mathrm{PH})$ are three ultra-rare, autosomal recessive genetic disorders characterized by oxalate overproduction in the liver. Hyperoxaluria induces recurrent kidney stones, nephrocalcinosis, progressive renal impairment, and systemic oxalosis, especially in PH1. Nedosiran is an investigational RNAi therapeutic administered monthly by subcutaneous injection. It reduces hepatic LDHA protein thereby inhibiting the final step responsible of oxalate production in all $\mathrm{PH}$.

Material and methods: We report on two PH1 patients, a 40 year old woman (a) on hemodialysis (HD) 6 × 3 hours weekly, and a 6.5 year old boy (b) receiving $5 \times 5$ hours HD, both homozygous for $A G X T$ c. $508 \mathrm{G}>\mathrm{A}$ and treated with pyridoxine. In patient (a), global longitudinal strain (GLS), an index of left ventricular contractibility, was impaired $(-13 \%$; normal $\leq-18 \%)$. Patient (b), had massive oxalate osteopathy, myocardial hypertrophy and cardiac insufficiency (GLS of - 9.98). They received Nedosiran as compassionate use medication for now 6 months. Monthly plasma oxalate (Pox in $\mu \mathrm{mol} / 1$, normal < 7.4) was measured, Speckle Echo and/or 3 Tesla MRI (left knee) were repeated.

Results: Speckle Echo improved in both (a: GLS - 23; b: GLS -14.6, but month 6 result pending). Bone MRI ameliorated in patient (b) showing a nidus of normal trabecular structure.

\begin{tabular}{llllllll}
\hline Patient & Pox & Pox & Pox & Pox & Pox & Pox & Pox \\
& Pre RNAi & Month 1 & Month 2 & Month 3 & Month 4 & Month 5 & Month 6 \\
(a) & $64.2-71.6$ & 40.9 & 60.2 & 52.8 & 37.67 & 23.2 & 32.4 \\
(b) & $73.1-101$ & 96.5 & 40.6 & 56.9 & 48.08 & 69.7 & 51.3 \\
\hline
\end{tabular}

Conclusions: Clinics improved and Pox declined over the six months of treatment. Pox was influenced in (b) by severe oxalate osteopathy and therefore possibly dissolving oxalate and in (a) when dialysis regimen was reduced to $4 \times 3$ hours at month 6 . We cautiously conclude, that liver transplantation may be avoidable in PH1 patients treated with Nedosiran.

\section{PI-135 SYSTEMIC OXALATE DEPOSITION IN PATIENTS WITH PRIMARY HYPEROXALURIA TYPE 3}

\author{
Cristina Martin-higueras ${ }^{1}$, Mark Born ${ }^{2}$, Ulrike Herberg ${ }^{2}$, \\ Johannes Birtel $^{2}$, Bernd Hoppe ${ }^{1}$ \\ ${ }^{1}$ German Hyperoxaluria Center, Germany, ${ }^{2}$ University Hospital Bonn, \\ Germany
}

Introduction: Patients with Primary Hyperoxaluria type 3 (PH3) remain, in the contrary to previous reporting, symptomatic in adulthood with recurrent kidney stones. They are also on risk to develop chronic kidney disease (CKD). We therefore speculate, that PH3 patients may develop systemic oxalate deposition.

Material and methods: We started to examine $\mathrm{PH} 3$ patients regularly seen at the German Hyperoxaluria Center (GHC) for systemic depositions by eye exam, hand $\mathrm{x}$-rays, bone MRI ( 3 Tesla of the left knee and proximal tibia) and Speckle tracking echocardiography, which measures changes in global longitudinal strain (GLS), an index of left ventricular contractibility (normal GLS is $\leq 18 \%$ ).

Results: At GHC we see 12 pediatric and 4 adult patients on a regular basis, at least twice a year. All 16 patients are in no less than CKD 2. So far the following examinations were performed:

- Eye in 8 patients, normal in all.

- X-ray left hand in 6 patients, one patient (multiple stone removal procedures, decline in GFR) with tiny sclerosing areas at caput MCP IV and the thumb.

- MRI of left knee and proximal tibia in 6 patients, textural changes in one. - Speckle tracking echocardiography in 8 patients, abnormal in one (GLS -17.3 and left ventricular hypertrophy) and borderline in another (GLS 18.6) in 2020. During 2021 GLS values returned to normal in both under increased treatment awareness ( -23 and -21 , respectively).

- Two patients had salivary stones in the parotid gland, found in a routine $\mathrm{x}$-ray of the jaw before orthodontic treatment.

Conclusions: Although this is currently only data of a small cohort, systemic oxalate deposition may also occur in $\mathrm{PH} 3$. Of course, data in more patients are needed to elucidate the true risk of systemic oxalate deposition and we therefore recommend to screen all known $\mathrm{PH} 3$ patients.

\section{PI-136 OXALATE IN URINE AND PLASMA RELATED TO KIDNEY FUNCTION, DIALYSIS OR TRANSPLANTATION IN PATIENTS WITH PRIMARY HYPEROXALURIA TYPE 1}

\section{Cristina Martin-higueras ${ }^{1}$, Julia Pick ${ }^{2}$, Bernd Hoppe ${ }^{1}$ \\ ${ }^{1}$ German Hyperoxaluria Center, Germany, ${ }^{2}$ University Hospital Bonn, Germany}

Introduction: Three different enzyme defects in primary hyperoxaluria $(\mathrm{PH})$ lead to endogenous oxalate overproduction and extremely elevated urinary oxalate excretion (UOx), which induces recurrent urolithiasis, nephrocalcinosis and chronic kidney disease, the latter especially in PH1. Uox and plasma oxalate (Pox) are used as biochemical markers for diagnosis, treatment decisions and outcome parameters. We were interested in their long term follow up related to kidney function, hemodialysis (HD) or transplantation (Tx).

Material and methods: We retrospectively analyzed biochemical data taken in genetically confirmed $\mathrm{PH} 1$ patients over the last 15 years. Both urine and plasma were analyzed for oxalate, glycolate (UGlyc/PGlyc) and creatinine (Crea). Data was examined by means of correlation/regression analysis within groups of different renal function (normal, CKD 1-5; $n=56$ patients), HD $(n=26)$, post $T x(n=32)$, and over time.

Results: Pox remained equivalent between stages of kidney function (median Pox $17 \mu \mathrm{mol} / 1$ ). Until CKD 3-4 only 17\% Pox values and 33\% in CKD 5 were $>30$. Highest Pox was found in HD (91) and HD post liver Tx $(46 \mu \mathrm{mol} / \mathrm{l})$ in sequential procedure. PGlyc was elevated in all groups of kidney function and significantly higher in HD $(211 \mu \mathrm{mol} / \mathrm{l})$. 
Correlations were found for Pox with PGlyc in CKD 3-4 ( $r=0.73$; $\mathrm{p}<0.001)$, for Pox with SCrea $(\mathrm{r}=0.78 ; \mathrm{p}<0.001)$ and negatively with eGFR in CKD $5(\mathrm{r}=-0.51 ; \mathrm{p}<0.001)$ and for Uox with eGFR in normal kidney function $(\mathrm{r}=-0.24 ; \mathrm{p}=0.04)$. Uox correlated to Pox in CKD 3-4 $(r=0.77 ; p=0.004)$. Pox and Uox slowly declined over time in combined and sequential, but also in kidney $\mathrm{Tx}$, which was only performed in adult B6-sensitive patients. PGlyc remained elevated post Tx.

Conclusions: We did not find consistent correlation of either Pox or Uox to SCrea or eGFR. Pox was surprisingly low until HD. Long term follow up post Tx shows a slow decline in Pox and Uox in all transplant procedures.

\section{PI-137 SUCCESSFUL EARLY TREATMENT WITH STIRIPENTOL IN A PATIENT WITH PRIMARY HYPEROXALURIA TYPE 1}

Priscillia Violier ${ }^{1}$, Olivia Boyer ${ }^{2}$, Romain Berthaud ${ }^{2}$, Charlotte Samaille ${ }^{1}$, Robert Novo ${ }^{1}$, Guillaume Dorval ${ }^{2}$

${ }_{1}$ Nephrologie Pédiatrique, Université De Lille, Lille, France, ${ }^{2}$ Néphrologie Pédiatrique, Marhea, Institut Imagine, Université De Paris, Hôpital Necker - Enfants Malades, Aphp, Paris, France

Abstract: Primary hyperoxaluria type 1(HP1) is a rare autosomal recessive disorder caused by biallelic pathogenic AGXT variants. The gene defect results in dysfunction of the liver-specific peroxisomal enzyme alanine amino-transferase (AGT) and consequently nephrocalcinosis, kidney stones, kidney failure and systemic oxalosis despite supportive care. Until recently, the only specific treatment was liver-kidney transplantation. Therapeutic prospects, notably with interfering RNA, would aim at reducing the substrate by upstream oxalate enzyme blockade. Stiripentol is an anti-epileptic drug registered in pediatric Dravets syndrome and well-tolerated in young children. It inhibits the lactate dehydrogenase 5 enzyme, the key enzyme of the last step in the hepatic production of oxalate. It is therefore hypothesized to reduce hepatic oxalate production and urinary excretion, thereby preventing progression to kidney failure and systemic oxalosis in HP1. We report the case of a 18 month-old girl diagnosed with HP1 with bilateral kidney stones who received stiripentol from the age of 21 months with adjunctive measures. Her urinary oxalate/creatinine ratio quickly decreased from 0.48 to $0.07 \mathrm{mmol} / \mathrm{mmol}$ and was followed by complete normalization of the kidney ultrasound after 11 months of treatment. No side effect was noted. We suggest considering a trial of stiripentol in children with HP1, alone or in combination with other therapeutic strategies such as RNA interfering drugs where available.

\section{PI-138 RNA INTERFERENCE THERAPY MAY ALLOW WITHDRAWAL OF NOCTURNAL HYPERHYDRATION IN CHILDREN WITH PRIMARY HYPEROXALURIA}

Nathalie Biebuyck, Olivia Boyer

Néphrologie Pédiatrique, Marhea, Institut Imagine, Université De Paris, Hôpital Necker - Enfants Malades, Aphp, Paris, France

\footnotetext{
Abstract: Primary hyperoxaluria type 1 (PH1) is a rare inherited disease associated with excessive liver oxalate production and consequent systemic accumulation and severe organ involvement. Treatment consists in nychthemeral hyperhydration, crystallization inhibitors, pyridoxine and eventually kidney-liver transplantation. This treatment has a high negative impact on quality-of-life, even before transplantation, especially nocturnal hyperhydration on a G-tube. Recently, ARN interference therapy (Lumasiran) was shown to significantly decrease oxaluria in PH1. There are however no recommendations about the discontinuation of the burdensome supportive measures. We present here 2 patients with $\mathrm{PH} 1$ who stopped the nocturnal hyperhydration with promising outcomes.

Patient 1, a 16 yo girl, and Patient 2, a 14 yo boy, were diagnosed with PH1 at the age of 6 months with bilateral nephrocalcinosis, elevated urinary oxalate/
}

creatinine ratio, whewellite urinary crystals and $A G X T$ variants. They were prescribed hyperhydration via a G-tube, crystallization inhibitors and pyridoxine. This allowed disappearance of crystals in the urine, reduction of urinary oxalate/creatinine ratio and regression of nephrocalcinosis. Patient 1 had adherence difficulties and psychological distress and decided to stop nocturnal hyperhydration at the age of 12 and nephrocalcinosis and bilateral lithiasis reappeared at the age of 14 . Lumasiran was begun at the age of 15 years and 13 years, respectively. We rapidly observed normal oxaluria/creatininuria and calciuria/creatininuria, negative crystalluria and regression of lithiasis (patient 1 ) or persistently normal kidney ultrasound (patient 2). We decided to withdraw nocturnal hydration after 6 months in Patient 2 due to difficult-totolerate enuresis. 3 months after withdrawal, oxaluria, calciuria and crystalluria remain normal. The control kidney ultrasound is pending.

Lumasiran seems very efficient to normalize oxaluria even in patients with no nocturnal hyperhydration because of non-adherence or in order to improve the quality-of-life. Additional data and longer follow-up are needed to guide updated treatment recommendations.

\section{PI-139 PLASMA OXALATE VALUES IN PATIENTS WITH END-STAGE KIDNEY DISEASE}

Elisabeth Metry ${ }^{1}$, Sander Garrelfs ${ }^{1}$, Joost Bijlsma ${ }^{2}$, Aegida Neradova ${ }^{2}$, Fred Vaz ${ }^{3}$, Michiel Oosterveld ${ }^{1}$, Jaap Groothoff ${ }^{1}$

${ }^{1}$ Department of Pediatric Nephrology, Emma Childrens Hospital, Amsterdam Umc, University of Amsterdam, Amsterdam, The Netherlands, ${ }^{2}$ Department of Nephrology, Amsterdam Umc, University of Amsterdam, Amsterdam, The Netherlands,${ }^{3}$ Department of Clinical Chemistry, Amsterdam Umc, University of Amsterdam, Amsterdam, The Netherlands

Introduction: Patients with end-stage kidney disease (ESKD) are known to have higher plasma concentrations of metabolic waste products than healthy individuals. Patients with Primary Hyperoxaluria $(\mathrm{PH})$, a rare congenital cause of ESKD, suffer from hepatic overproduction of the metabolic end product oxalate. Plasma oxalate (POx) levels are determined in the diagnostic and therapeutic work-up for $\mathrm{PH}$. Remarkably, correct interpretation of these values is hampered by the absence of knowledge concerning POx levels in patients with ESKD due to common causes.

Material and methods: In this observational study, we obtained POx values in patients with ESKD due to another cause than $\mathrm{PH}$, to establish reference values in this patient group. We collected blood samples from 118 patients with eGFR $<15 \mathrm{~mL} / \mathrm{min} / 1.73 \mathrm{~m} 2$ who required maintenance hemodialysis or peritoneal dialysis at the Amsterdam UMC. In addition, we collected 15 blood samples from adults with chronic kidney disease (CKD) stage 4 and 5 who received no renal replacement therapy.

Results: While there was a wide variation in patients with ESKD, the median POx level was $50 \mathrm{umol} / \mathrm{L}$ and lowest values were twice the upper reference limit that applies to healthy individuals $(6.7 \mathrm{umol} / \mathrm{L})$. Median POx level was 10.4 umol/L in patients with CKD stage 4 and 5 who received no renal replacement therapy.

Conclusions: This study shows that POx levels of $50 \mathrm{umol} / \mathrm{L}$ are not necessarily suggestive for $\mathrm{PH}$ which contradicts the current literature. This study could lead to a paradigm shift in the diagnostic and therapeutic work-up for patients with ESKD.

\section{PI-140 SPECKLE TRACKING ECHOCARDIOGRAPHY (STE) IN PATIENTS WITH PRIMARY HYPEROXULARIA TYPE 1 (PH1)}

Sander F. Garrelfs ${ }^{1}$, H.A.C.M. Bon ${ }^{1}$, Sally-Anne Hulton ${ }^{1}$, Michiel J.S. Oosterveld ${ }^{2}$, Irene M. Kuipers ${ }^{1}$, Berto J. Bouma ${ }^{1}$, Ashish Chikermane ${ }^{2}$, Jaap W. Groothoff ${ }^{1}$

I Amsterdam Umc, University of Amsterdam, Emma's Children's Hospital, Amsterdam, The Netherlands, ${ }^{2}$ Birmingham Children's Hospital Nhs Foundation Trust, Birmingham, United Kingdom 
Introduction: Primary Hyperoxaluria type 1 (PH1) is a rare, inherited metabolic disorder of glyoxylate metabolism characterized by oxalate overproduction, leading to end-stage kidney disease (ESKD) in more than half of all patients. As renal function deteriorates the ability of the kidneys to excrete the excess of oxalate is increasingly compromised resulting in elevated concentrations of oxalate in the blood and progressive systemic deposition of calcium-oxalate crystals in virtually all tissues, a situation referred to as systemic oxalosis. A wide spectrum of cardiac abnormalities are reported in $\mathrm{PH} 1$ patients, including pre-symptomatic myocardial disease early in the disease course. We aimed to further determine cardiac manifestations in a heterogenous cohort of $\mathrm{PH} 1$ patients by conventional and speckle tracking echocardiography (STE).

Material and methods: Data from 38 genetically confirmed PH1 patients were included in this two-centre study and compared to age- and sex-matched controls. Comprehensive echocardiographic analysis (including STE) was performed. PH1 patients were subdivided in three groups based on stage of chronic kidney disease (CKD) and transplantation status (CKD1-4 ( $\mathrm{n}=24), \mathrm{CKD} 5(\mathrm{n}=7)$ and post-liver transplantation $(\mathrm{n}=7))$.

Results: Left-ventricle ejection fraction (LV EF) was preserved in all subjects. PH1 patients had a decreased global longitudinal strain compared to healthy controls, with a mean difference of $-2.28(\mathrm{p}<0.001)$. In patients with CKD1-4 decreased LS values were solely found in all apical segments, with a mean difference of $-5.3(\mathrm{p}<0.001)$ as compared to healthy controls. In patients whom already progressed to end-stage kidney disease (ESKD, defined as CKD5) diastolic dysfunction is noted, with preserved systolic function.

Conclusions: Pre-symptomatic myocardial dysfunction is present in $\mathrm{PH} 1$ patients with mild renal impairment and relatively normal plasma oxalate values. This indicates that cardiac screening is warranted in all $\mathrm{PH} 1 \mathrm{pa}-$ tients, irrespective of renal function.

\section{PI-141 LIVER AND KIDNEY TRANSPLANTATION IN PRIMARY HYPEROXALURIA TYPE 1}

\author{
Maria Arena ${ }^{2}$, Raffaella Labbadia ${ }^{1}$, Isabella Guzzo ${ }^{1}$, Francesco Emma ${ }^{1}$, \\ Luca Dello Strologo ${ }^{1}$ \\ ${ }^{I}$ Nephrology, Dialysis and Renal Transplantation, Bambino Gesù \\ Children's Research Hospital, Irccs Rome, Italy, ${ }^{2}$ Università Cattolica \\ Del Sacro Cuore, Rome, Italy Nephrology, Dialysis And Renal \\ Transplantation, Bambino Gesù Children's Research Hospital, Irccs \\ Rome, Italy
}

Introduction: Primary hyperoxaluria type 1 is a rare autosomal recessive disease caused by mutation of the AGTX gene responsible for derangement of hepatic AGT enzyme. It accounts for 1 to $2 \%$ of cases of pediatric end-stage kidney disease (ESKD).

Aim of the study was to evaluate the outcomes of patients with primary hyperoxaluria who received combined or sequential liver-kidney transplantation.

Material and methods: We retrospectively evaluated 10 patients with oxalosis who received a liver-kidney transplantation, with a median age of 5.6 years (range: 2.2-17.0) at kidney transplant. Five patients received a combined transplant. In five, the kidney transplant was postponed by a median time of 8 months (range 4-20) to achieve a lower oxalate burden for the graft. One of the patient died just after intervention and was excluded from further analysis. Median follow up was 3.2 years (range 1.6-11). All patients were on hemodialysis treatment at transplantation.

Results: Median eGFR at 6 and at 12 month was 81.2 (range: 45.7-108.8) and $79.3 \mathrm{ml} / \mathrm{min} / 1.73 \mathrm{~m}^{2}$ (range $54.7-112.1$ ) in patients who underwent combined transplant, and 45.7 (range $34.5-86.7$ ) and $38.3 \mathrm{ml} / \mathrm{min} / 1.73 \mathrm{~m}^{2}$ (range: 29.9-77.5) in those with sequential transplant (p:NS).

In children with sequential transplant, serum oxalate before liver transplantation compared to pre-kidney transplant values showed a median drop of $51 \%$ (range 14-73\%).
Sixth-month protocol biopsy showed the presence of oxalate crystals in all patients except one, demonstrating the recurrence of deposition in spite of the delay between liver and kidney transplant.

Conclusions: Liver and kidney transplant is currently the only possible option in patients with oxalosis. A delay between liver and kidney transplant does not appear to improve the final prognosis.

\section{PI-142 COMPOSITION OF URINARY STONES IN CHILDREN: CLINICAL AND METABOLIC DETERMINANTS IN A FRENCH TERTIARY CARE CENTER}

Camille Rauturier, Christelle Machon, Delphine Demede, Laurence Dubourg, Justine Bacchetta, Aurelia Bertholet-Thomas Hôpital Femme Mère Enfant, France

Objectives: The aim was to describe the composition of stones of pediatric patients in a tertiary center, to illustrate current epidemiological trends in pediatric urolithiasis.

Methods: Clinical and metabolic data from all pediatric patients with at least one stone that was analyzed by Fourier Transformed Infrared Spectroscopy (FTIR) in the Hospices Civils de Lyon s Civils de Lyon between 2013 and 2017 were retrospectively collected.

Results: A total of 111 patients (sex ratio 1.4:1) were included; their median [IQR] age was 7.5 [3.1-10.5] years. The main component of stones was calcium oxalate [weddellite for $34(31 \%)$ stones, whewellite $23(21 \%)$ ], calcium phosphate [carbapatite $32(29 \%)$, brushite $6(5 \%)$, amorphous calcium phosphate $3(3 \%)$ ], struvite $5(5 \%)$, cystine $4(4 \%)$, uric acid $2(2 \%)$, and ammonium acid urate $2(2 \%)$. A total of $20(18 \%)$ stones were pure and $24(22 \%)$ were infectious. Carbapatite stones were the most frequent in patients $<2$ years and calcium oxalate stones in patients $>2$ years old. Metabolic abnormalities (most frequently hypercalciuria) were found in 50\% of tested patients and in 54\% of patients with infectious stones. Congenital anomalies of the kidney and/or urinary tract (CAKUT) or neurogenic bladder was present in $9 / 24(38 \%)$ patients with infectious stones and 12/16 (76\%) patients with bladder stones.

Conclusion: This study confirms that calcium oxalate stones are the most frequent among pediatric patients, which could reflect the nutritional habits of predisposed patients. In contrast, infectious stones are less frequent and occur mostly in association with anatomic or metabolic favoring factors.

\section{PI-143 HEALTH-RELATED QUALITY OF LIFE OF CHILDREN AND ADOLESCENTS WITH CYSTINOSIS}

\author{
Katharina Hohenfellner ${ }^{1}$, Julia Quitmann ${ }^{2}$, Stefanie Witt ${ }^{2}$, \\ Kaja Kristensen ${ }^{2}$ \\ ${ }^{1}$ Romed Klinikum Rosenheim, Germany, ${ }^{2}$ University Medical Center \\ Hamburg-Eppendorf, Germany
}

Introduction: Despite the impairments experienced by patients with cystinosis, very few studies investigate health-related quality of life ( $\mathrm{HrQoL})$ in this patient group and disease-specific $\mathrm{HrQoL}$ measures are lacking. Thus, the aim of this study is to develop a Patient-Reported Outcome measures (PROM) for children and adolescents with cystinosis. This instrument will capture the $\mathrm{HrQoL}$ from both the child/adolescent and parent perspectives. Material and methods: Based on international guidelines the PROM will be developed simultaneously in six countries (US, UK, Ireland, Germany, Spain \& France). The study will take place in four phases within 24-months. Patients and parents will be recruited by clinicians and patient organizations. A first, the state of research on cystinosis and HrQoL will be examined in a systematic literature review. In phase 2, focus interviews with patients and their parents will be conducted to create preliminary questionnaire items. In phase 3 , this preliminary questionnaire will be psychometrically tested in a pilot test. Subsequently, a cognitive debriefing will be conducted to examine the comprehensibility, 
completeness, and cultural applicability of the preliminary version from the patients and parents perspectives. In the fourth phase, the final questionnaire will be applied in a field- and re- test to evaluate its psychometric properties.

Results: The final product will be a cross-cultural, psychometrically validated, practically feasible, and conceptually suitable instrument for children and adolescents with cystinosis. It will be available in English, German, French and Spanish. With further international collaborations, this tool can be linguistically validated and cross-culturally adapted for use in a wide range of countries. And while this project refers to the development of a PROM for children, the development of a PROM for adult patients is planned as a follow-up project.

Conclusions: First results will be presented and critically discussed.

\section{PI-144 SAFETY AND EFFICACY OF LOW-DOSE OBINUTU- ZUMAB IN PEDIATRIC STEROID-DEPENDENT NEPHROTIC SYNDROME}

Claire Dossier ${ }^{1}$, Christelle Moreau ${ }^{2}$, Theresa Kwon ${ }^{1}$, Anne Couderc ${ }^{1}$, Alexandra Cambier ${ }^{1}$, Veronique Baudouin ${ }^{1}$, Anne Maisin ${ }^{1}$, Julien Hogan ${ }^{1}$

${ }^{1}$ Pediatric Nephrology, Robert-debre Hospital, Aphp, Paris - France, ${ }^{2}$ Pharmacy, Robert-debre Hospital, Aphp, Paris - France

Introduction: B-cell depletion with rituximab (RTX) induces sustained remission in children with Steroid-Dependent or Frequent Relapsing Nephrotic Syndrome (SD/FRNS). However, most patients relapse after B-cell recovery and some do not achieve B-cell depletion. We report our experience of low dose Obinutuzumab (OBI), a $2^{\text {nd }}$ generation anti-CD20 antibody in this population.

Material \& Methods: This is a retrospective study of children with FR/ SDNS and resistance or relapse after rituximab, treated at Robert-Debre hospital, with a single infusion of $300 \mathrm{mg} / 1,73 \mathrm{~m}^{2}$ of OBI, and $>6$ months follow-up. Oral immunosuppressors were discontinued within $\overline{2}$ months. Results: 32 patients were included. Median ages at INS onset, first RTX and first OBI injection were 3.9, 8.8 and 10.7 years old, respectively. Indication for OBI were: no B-cell depletion $(n=7)$ or short depletion $<3$ months $(n=2)$, Antirituximab Antibodies $(\mathrm{n}=1)$ or relapse after B-cell recovery following RTX ( $\mathrm{n}=22)$. B-cell depletion was achieved in all patients, with a longer duration compared to prior RTX. At last follow up (median 18 months), Bcell recovery had occurred in 27 patients after a median depletion of 8.3 months [IQ 6.1-12.3]. 8 patients relapsed (25\%), after a median time of 16 months. Mild infusion reactions occured in 5 patients and neutropenia grade 2-3 in 7 patients. IgM were low in 7 patients at baseline, and 21 during followup. One patient was hospitalised for infectious pneumonia and another for Acute-Lung Injury with complete recover.

Conclusion: A single low-dose of obinutuzumab is effective in children with FR/SDNS, including those resistant to rituximab. Tolerance profile is comparable to that of Rituximab but long term monitoring of Immunoglobulin levels is mandatory as well as a face to face trial with rituximab.

\section{PI-145 INCIDENCE AND RELAPSE OF IDIOPATHIC NEPHROTIC SYNDROME: META-ANALYSIS}

Floor Veltkamp, Leonie Rensma, Antonia Bouts

Amsterdam University Medical Centers, University of Amsterdam, Emma Childrens Hospital, Department of Pediatric Nephrology, Amsterdam, The Netherlands

Introduction: Idiopathic nephrotic syndrome (INS) in children is, although the most common glomerulopathy in paediatric nephrology, a rare disease in children. Corticosteroids are the cornerstone of the treatment of the first episode, however, relapses occur in the vast majority of children causing considerable morbidity. Differences between ethnicities have been suggested, yet the incidence nor the risk for relapse have been systematically reviewed. Therefore, the objective of this systematic review and meta-analysis was to estimate the overall pooled weighted incidence and risk for relapse of INS in children.

Material and methods: Using a comprehensive search strategy, MEDLINE and Embase were searched for eligible studies until December 2020. All studies reporting incidence (per 100,000 children/ year) and/or risk for relapse (proportion of total of patients who experience at least one relapse) of INS in children $(<18$ year) were eligible. After quality assessment, the following data were extracted: study (design, localisation, sample size) and patient (age, sex, steroid response, ethnicity) characteristics, incidence, and risk for relapse. The overall pooled weighted estimates were calculated using random-effects metaanalysis, as heterogeneity among studies was expected. In addition, metaregression was used to test if there was a change over time in incidence and risk for relapse. Sources of heterogeneity were explored by separate subgroup analyses. To test the robustness of the results, subsequent sensitivity analyses were performed.

Results: After screening, a total of 73 studies ( 27 incidence, 54 relapse) were included for analysis. The results will be presented during ESPN 2021.

Conclusions: The manuscript was accepted for publication by Pediatrics and its results and conclusions are still under embargo.

\section{PI-146 LOWER INCIDENCE OF IDIOPATHIC NEPHROTIC SYNDROME IN CHILDREN IN THE NETHERLANDS AND BELGIUM BETWEEN 2017-2019 AND DURING THE COVID- 19 PANDEMIC}

Floor Veltkamp ${ }^{1}$, Bas Van Lieshout ${ }^{1}$, Carlijn Mussies ${ }^{1}$, Djera Khan ${ }^{2}$, Elena Levtchenko ${ }^{3}$, Anna Bael ${ }^{4}$, Sandrine Florquin ${ }^{2}$, Antonia Bouts ${ }^{1}$ ${ }^{1}$ Amsterdam University Medical Centers, University of Amsterdam, Emma Children's Hospital, Department of Pediatric Nephrology, Amsterdam, The Netherlands, ${ }^{2}$ Amsterdam University Medical Centers, University of Amsterdam, Department of Pathology, Amsterdam, The Netherlands, ${ }^{3}$ Division of Pediatric Nephrology, University Hospitals Leuven, Leuven, Belgium; Department of Development And Regeneration, Katholieke Universiteit Leuven, Leuven, Belgium, ${ }^{4}$ Zna Queen Paola Childrens Hospital, Department of Pediatric Nephrology, Antwerp, Belgium

Background: Idiopathic nephrotic syndrome in children is a rare disease. In the Netherlands, the estimated incidence is 1.52 new cases per $100,000 /$ year (2006-2009). During the inclusion phase of an ongoing randomised controlled trial (LEARNS study), the number of new cases per year did not meet this estimate. Furthermore, during the Covid-19 pandemic, it seemed that even fewer new cases were identified, yet this needs to be confirmed. We hypothesise that the latter may be due to lower exposure to (viral) infections as a result of social distancing, school closings, and other lockdown measures.

Methods: Two separate online surveys were sent to all paediatricians in the Netherlands and Flanders, Belgium on two occasions: May 2020 and June 2021 (planned). Paediatricians were asked to report any case of new onset INS in a child (1-18 years) between January 2017 and December 2019 (survey 1) and March 2020 and March 2021 (survey 2). Census data were retrieved from the Dutch Centraal Bureau voor de Statistiek and the Belgian Statbel. Governmental websites were consulted for SARS-CoV2 (sero) prevalence, Covid-19 hospital admissions, and lockdown measures. Incidence and corresponding confidence intervals (CIs) were calculated using the exact Poisson test. Incidences were compared using a proportion $\mathrm{Z}$ test.

Results: Between 2017 and 2019, the incidence of INS was estimated to be 1.14 (95\% CI $0.94-1.37$ ) and 1.12 (95\% CI $0.82-1.52)$ per 100,000 children/year in the Netherlands and Belgium, respectively. The results of survey 2 will be available in September 2021. 
Conclusion: The results of the first survey show that the incidence of INS in children is similar in the Netherlands and Belgium, but lower (-27\%) than previously reported. A further decrease during the Covid-19 may point towards an infectious event as trigger for the onset of INS. The results will be presented during ESPN 2021.

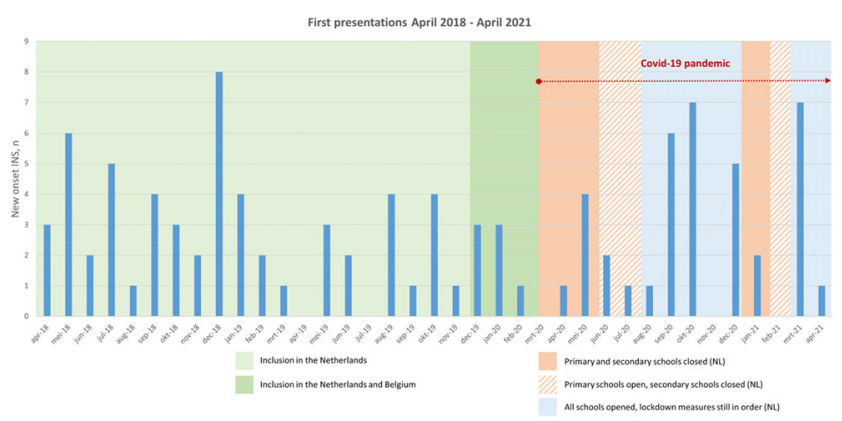

PI-147 LOWER PNEUMOCOCCAL SEROTYPE-SPECIFIC BCELLULAR RESPONSE IN CHILDREN WITH IDIOPATHIC NEPHROTIC SYNDROME (INS) UNDER CYCLOSPORIN-A (CSA)

Varvara Askiti ${ }^{1}$, Konstantina Kitsou ${ }^{2}$, Argyroula Zampetoglou ${ }^{1}$, Marianna Tzanoudaki ${ }^{3}$, Emmanouil Liatsis ${ }^{3}$, Gkikas Magiorkinis ${ }^{4}$, Andromachi Mitsioni ${ }^{1}$, Vana Spoulou ${ }^{2}$

${ }^{1}$ Pediatric Nephrology Department, "p. And A. Kyriakou" Childrens Hospital, Athens, Greece, Immunobiology and Vaccinology Research Laboratory, First Department of Peadiatrics, "Aghia Sophia" Children's Hospital, School of Medicine, National and Kapodistrian University of Athens, ${ }^{3}$ Department of Immunology \& Histocompatibility, Specific Reference Centre for Primary Immunodeficiencies-Paediatric Immunology, "Aghia Sophia" Childrens Hospital, ${ }^{4}$ Department of Hygiene, Epidemiology and Medical Statistics, Medical School, National and Kapodistrian University of Athens, Greece

Objective: Children with INS under immunosuppression often demonstrate lower vaccine-derived protection. We evaluated the effect of a booster dose of 13-valent Pneumococcal Conjugate Vaccine (PCV13) on antigen-specific B-cellular immunity against Pneumococcal Serotypes, PS1 and PS9V, in children under CsA and corticosteroids. Methods: Blood was collected from INS patients, aged 6-10 years under CsA $(n=4)$, corticosteroids ( $n=5)$ and from age-matched healthy controls $(n=6)$, prior and one-month post PCV13. Peripheral Mononuclear Cells (PBMCs) were isolated. For the identification of PS1- and PS9V-specific B-cells, PBMCs we stained using chemically-biotinylatedpolysaccharides bound to anti-biotin-coated beads and PE-conjugated anti-biotin antibodies along with CD10-APC-AlexaFluor-750, CD20ECD, CD19-PC7. PS-specific B-cell counts were calculated by multiplying the $\mathrm{CD} 19^{+} \mathrm{CD} 20^{+} \mathrm{CD} 10^{-} \mathrm{PS}^{+}$event frequencies derived from cytometry and a total B-cell count measurement. Cytometric data were analyzed using Kaluza 2.1. Mann-Whitney U-test and Wilcoxon-rank test were performed.

Results: At baseline, patients under CsA demonstrated lower antigen-specific B-cell counts than controls for both serotypes ( $p=0.019$ for PS1 and $p=0.0105$ for PS9V), while no significant differences were found between children under corticosteroids and controls. After PCV13, patients under CsA demonstrated lower antigen-specific B-cell counts compared to controls for both serotypes ( $p=0.0105$ for PS1 and $p=0.0105$ for PS9V), while no significant differences were found between children under corticosteroids and controls. Comparing pre- and post-PCV13 PS-specific Bcell counts in each group, no significant increase was found in children under CsA, while significant increases were detected in controls (2-fold increase for PS1 and 2.57-fold increase for PS9V, both $p=0.0277)$ and patients under corticosteroids (1.82-fold increase for PS1 and 2.32-fold increase for PS9V, both $p=0.043$ ).

Conclusions: These results indicate a negative effect of CsA on PSspecific B-cell responses to PCV13. This Flow-Cytometry method assisted the detection of these differences, despite the small sample. It remains to determine the potential correlation of these differences with the achievement of protective antibody titers.

\section{PI-148 EFFECTIVENESS AND KIDNEY PROGNOSIS IN THE TREATMENT OF CORTICORRESISTANT NEPHROTIC SYNDROME IN PEDIATRICS}

Elena Codina Sampera, Pedro Arango Sancho, Yolanda Calzada Baños, Víctor López-Baez, Ana Vinuesa Jaca, Lina Catherine Hernández Zúñiga, Álvaro Madrid Arias

Hospital Sant Joan De Déu, Spain

Introduction: Our aim was to report the response to different treatments and the renal prognosis in a cohort of patients with corticosteroid-resistant nephrotic syndrome (CRNS).

Material and methods: Retrospective observational study in patients with CRNS. For this, the results of the histology, the different treatment guidelines used in each case and the evolution of renal function were collected, determining the results in terms of remission and renal survival in the different groups.

Results: Of the initial cohort of 37 patients, 33 were included, excluding 4 patients with Finnish-type genetic CRNS. The mean age at diagnosis was 6.1 years. $54.5 \%$ were women. Regarding the initial biopsy, $45.5 \%$ corresponded to minimal changes (15 patients), $27.3 \%$ (9) focal and segmental glomerulosclerosis, $15.1 \%$ (5) diffuse mesangial proliferation and $12.1 \%$ (4) others. The mean follow-up was 53 months (3-115 months). 27 patients $(84.4 \%)$ received cyclosporine (CyA), $66.7 \%$ (18) of them presented complete remission and $22.2 \%$ (6) partial response. Of the patients in complete remission, $33 \%$ had at least one relapse after 17 months of treatment (7-27 months). Rituximab was administered in 12 patients (37.5\%), of which 7 had not previously responded to immunosuppressants. $100 \%$ of frequent relapsers presented complete remission after administration of Rituximab, although 3 had subsequent relapses $(60 \%)$ after 21 months of treatment (12-34 months). 57\% of the patients who did not respond to immunosuppressants did not respond to Rituximab either, with Ofatumumab allowing complete remission in one of them. When relating the results with the histology, we saw how the remission in minimal changes and diffuse mesangial proliferation was $100 \%$ and $80 \%$, respectively, although it was $33.3 \%$ in focal and segmental glomerulosclerosis. Similarly, renal failure was more frequent in patients with focal segmental glomerulosclerosis $(77.7 \%)$. Of the remissions (24; $72.7 \%), 3$ were partial $(9.1 \%)$ and $6(18.2 \%)$ did not respond to any immunosuppressive treatment, with the need for kidney transplantation in 2 of them (6\%) and with 1 deceased due to an infectious cause (3\%). Conclusions: Histology and especially focal and segmental glomerulosclerosis, plays a prognostic role in the CRNS, with a lower remission rate and a greater deterioration in renal function and the need for associated kidney transplantation.

\section{PI-149 RITUXIMAB FOR THE TREATMENT OF ANTI-FACTOR H AUTOANTIBODY-ASSOCIATED C3 GLOMERULOPATHY: IS IT EFFECTIVE?}

Federica Zotta ${ }^{1}$, Alessandra Gianviti ${ }^{1}$, Laura Lucchetti ${ }^{1}$, Elisabetta Valoti ${ }^{2}$, Elena Bresin ${ }^{2}$, Antonio Gargiulo ${ }^{1}$, Manuela Colucci ${ }^{1}$, Francesco Emma ${ }^{1}$, Marina Vivarelli ${ }^{1}$

${ }^{1}$ Irccs, Division of Nephrology and Dialysis Bambin Gesu Pediatric Hospital, Rome Italy, ${ }^{2}$ Irccs, Institute of Pharmacological Research , Mario Negri, Bergamo, Italy 
Introduction: Autoantibodies targeting factor $\mathrm{H}$ (AB-FH), which is a crucial alternative pathway (AP) of complement regulatory protein, have been described in 1-3\% of $\mathrm{C} 3$ glomerulopathy patients. It may be hypothesized that in their presence, which leads to uncontrolled AP activation, B cell depletion with rituximab may be effective

Material and methods: .

Results: CASE REPORT: A 10-yr old girl was admitted to our department for an occasional finding of proteinuria (protein/creatinine ratio 2.32 $\mathrm{mg} / \mathrm{mg}$ ), microscopic hematuria (60-150 blood cells per field) and anemia $(9,2 \mathrm{~g} / \mathrm{dl})$. She had no history of serious illness or any recent evidence of upper respiratory or gastrointestinal tract infection. Her physical examination was normal. Laboratory findings revealed normal renal function, absence of nephrotic syndrome. Low levels of C3 (3 mg/dl) and C4 (6 $\mathrm{mg} / \mathrm{dl})$ were observed with elevated sC5b9 (2270 ng/ml), ANA and antidsDNA were normal. The first kidney biopsy revealed a membranoproliferative glomerulonephritis with a full house immunofluorescence pattern. A low-salt diet, oral prednisone and mycophenolate were immediately started. A second biopsy, performed 3 months later showed a more proliferative pattern with mainly granular deposit of $\mathrm{C} 3$ detected by immunofluorescence. Treatment with 3 methylprednisolone pulses were added. Screening for complement abnormalities showed no genetic mutations in the genes coding for FH, FI, MCP, THBD, C3, C5, CFHR1-5, FB and DGKE. However, AB-FH (1069 AU/ml, upper limit of normal $>127 \mathrm{AU} / \mathrm{ml}$ ) was found to be positive and Rituximab infusion $(375 \mathrm{mg} / \mathrm{m} 2)$ was administered. Interestingly, AB-FH titer was not significantly reduced by Rituximab (739 AU/ml at 4 months) without any effect on proteinuria in the following months

Conclusions: To the best of our knowledge, this is the first report on the use of Rituximab in a patient with AB-FH-associated C3 glomerulopathy. Large collaborative studies are needed to elucidate the best therapeutic strategy for this ultra-rare disease.

\section{PI-150 A RARE CAUSE OF NON-LUPUS FULL-HOUSE NEPHROPATHY IN AN ADOLESCENT BOY: C3 GLOMERU- LOPATHY}

Gökçen Erfidan ${ }^{1}$, Özgür Özdemir Șimșek ${ }^{1}$, Cemaliye Başaran ${ }^{1}$, Seçil Arslansoyu Çamlar ${ }^{2}$, Demet Alaygut ${ }^{1}$, Fatma Mutlubaș ${ }^{2}$, Belde Kasap Demir ${ }^{3}$

${ }^{1}$ University of Health Sciences, Tepecik Training and Research Hospital, Department of Pediatrics, Division of Nephrology, Izmir, Turkey, ${ }^{2}$ University of Health Sciences, Izmir Faculty of Medicine, Department of Pediatrics, Division of Nephrology, Izmir, Turkey, ${ }^{3}$ Izmir Katip Celebi University, Faculty of Medicine, Department of Pediatrics, Division of Nephrology and Rheumatology, Izmir, Turkey

Introduction: "Full-house" pattern is a histological definition to describe glomerular immune complex deposits of IgG, IgA, IgM, C3 and C1q on Immunofluorescent (IF) microscopy. It is well known as a characteristic feature of lupus nephritis. However, full-house nephropathy in a patient without any extrarenal signs and symptoms of systemic lupus erythematosus (SLE) and/or presence of autoantibodies may become a diagnostic challange.

Material and methods: A sixteen-year-old boy, admitted with periorbital edema, which was intermittently prominent in last two months. He had no history of hematuria, hypertension, infection, drug use or family history of kidney disease. On the physical examination, he had normal development with a weight of $73 \mathrm{~kg}\left(50-75^{\text {th }}\right.$ percentile), height of $181 \mathrm{~cm}\left(75-90^{\text {th }}\right.$ percentile) and blood pressure of $110 / 75 \mathrm{mmHg}$. He had normal urine output and no edema. The laboratory examination showed microscopic hematuria, nephrotic range proteinuria $\left(95 \mathrm{mg} / \mathrm{m}^{2} / \mathrm{h}\right)$, low serum albumin $(2.1 \mathrm{~g} / \mathrm{dL})$ with elevated lipid levels. He had low $\mathrm{C} 3$ level of $0.14 \mathrm{~g} / \mathrm{L}(0.9-1.8 \mathrm{~g} / \mathrm{L})$ and normal C4 level of $0.27 \mathrm{~g} / \mathrm{L}(0.1-0.4 \mathrm{~g} / \mathrm{L})$. The Anti-Streptolysin-O, Anti-Nuclear Antibody, Anti-double stranded DNA, Anti-Neutrophil Cytoplasmic Antibody, Anti-Phospholipase A2
Receptor Antibody were all negative. Due to low C3 level and the presentation age, a renal biopsy was performed.

Results: The light microscopy showed diffuse thickening of glomerular basement membrane, mild mesangial hypercellularity, endocapillary proliferation and segmental sclerosis. Immunofluorescent microscopy revealed "full-house" pattern with $\mathrm{C} 3$ prominancy. Electron microscopic examination revealed basement membrane thickening with irregular borders, granular electron dense deposits in subepithelial, intramembranous, subendothelial areas and mesengium with matrix expansion. No cytoplasmic tubuloreticular inclusions were identified.

Conclusions: In this case, the histopathological findings were suggestive for lupus nephritis with full-house pattern on IF. Nevertheless, he had only microscopic hematuria and nephrotic proteinuria with no other manifestations of SLE. Considering light-IF-electron microscopic examination, this case is diagnosed with C3-glomerulopathy.

\section{PI-151 IgG4-RELATED DISEASE WITH ISOLATED RENAL INVOLVEMENT IN CHILDHOOD}

Özgür Özdemir Șimșek ${ }^{1}$, Gökçen Erfidan ${ }^{1}$, Neslihan Güney ${ }^{2}$, Seçil Arslansoyu Çamlar ${ }^{3}$, Demet Alaygut ${ }^{1}$, Fatma Mutlubaş ${ }^{3}$, Belde Kasap Demİr ${ }^{4}$

${ }^{I}$ University of Healthy Sciences Tepecik Training and Research Hospital Department of Pediatrics, Division of Nephrology, Izmir, Turkey, ${ }^{2}$ University of Healthy Sciences Tepecik Training and Research Hospital Department of Pathology,izmir, Turkey, ${ }^{3}$ University of Health Sciences, Izmir Faculty of Medicine Department of Pediatrics, Division of Nephrology, Izmir, Turkey, ${ }^{4}$ Izmir Katip Çelebi University Medical Faculty, Department of Pediatrics, Division of Nephrology and Rheumotology, Izmir, Turkey

Introduction: IgG4-related disease is a fibroinflammatory disorder characterized by IgG4-positive plasma cell infiltration and fibrosis. We present a case of isolated IgG4-related kidney disease (RKD) with tubulointerstitial nephritis (TIN) and several glomerular lesions.

Material and methods: A 6-year-old boy was admitted to the emergency room with nausea, vomiting and fatigue that started 5 days ago. He had a body weight of $21.4 \mathrm{~kg}(25-50 \mathrm{p})$, a height of $118.5 \mathrm{~cm}(25-50 \mathrm{p})$, blood pressure of $158 / 100 \mathrm{mmHg}(>95 \mathrm{p}+12)$, and $(+1)$ pretibial pitting edema. Laboratory tests revealed urea $198 \mathrm{mg} / \mathrm{dL}(\mathrm{N}: 10-38)$; serum creatinine $6.9 \mathrm{mg} / \mathrm{dL}(\mathrm{N}: 0.5-1.2)$; uric acid $6.4 \mathrm{mg} / \mathrm{dL}(\mathrm{N}: 2-5.5)$; albumin $2.3 \mathrm{~g} /$ $\mathrm{dL}(\mathrm{N}: 3.5-5.5)$; sodium $130 \mathrm{mmol} / \mathrm{L}(\mathrm{N}: 134-150)$, potassium $4.91 \mathrm{mmol} / \mathrm{L}(\mathrm{N}: 3.5-5.5)$, calcium $7.7 \mathrm{mg} / \mathrm{dL}(\mathrm{N}: 8.8-10.8)$, phosphorus $6.6 \mathrm{mg} / \mathrm{dL}(\mathrm{N}: 4-7)$, erythrocyte sedimentation rate(ESR) $108 \mathrm{~mm} / \mathrm{h}(\mathrm{N}: 0$ 20); parathormone $248 \mathrm{ng} / \mathrm{L}(\mathrm{N}: 18.5-88)$; total $\operatorname{IgE} 24600 \mathrm{IU} / \mathrm{mL}(\mathrm{N}: 0-$ 90), total $\operatorname{IgG}: 8.44 \mathrm{~g} / \mathrm{dL}(\mathrm{N}: 7-16), \operatorname{IgG} 4: 2.91 \mathrm{~g} / \mathrm{dL}(\mathrm{N}: 0.012-1.699), \mathrm{pH}$ 7.13, $\mathrm{HCO} 311.6 \mathrm{mmol} / \mathrm{L}$, and $\mathrm{BE}-16.7 \mathrm{mmol} / \mathrm{L}$. His urine output was $0.3 \mathrm{cc} / \mathrm{kg} / \mathrm{h}$. Urinalysis revealed specific gravity 1018 ; glucose +2 ; protein+3; erythrocyte+2. Spot urine protein/creatinine was 24.4. Serum C3$\mathrm{C} 4$ were normal; ANA and anti-dsDNA were negative. The kidney biopsy was consistent with chronic TIN. There was global sclerosis in 3, cellular/fibrocellular crescents in 4 , ischemic collapse in 10-11 of the 43 glomeruli. $\operatorname{IgG}(+2)$ and $\operatorname{IgM}(+1)$ staining were observed in immunofluorescence microscopy. There were $\geq 10 \mathrm{IgG} 4$ stained cells/hpf. The patient was diagnosed with IgG4-RKD and USG, CT, MRI and PET-CT were performed to detect other organ involvements. There were no signs of malignancy or extrarenal symptoms. Hemodialysis was initiated. Results: He received 6 doses of pulse methylprednisolone followed by 2 doses of intravenous cyclophosphamide 15 days apart and Rituximab. Serum creatinine decreased to $1.7 \mathrm{mg} / \mathrm{dL}$ and he had no need for dialysis anymore. The treatment was supposed to be personalized due to laboratory tests in the follow-up.

Conclusions: IgG4-RKD is should be kept in mind in patients with high IgE levels and chronic TIN even in childhood. Our case is the smallest patient with IgG4-RKD and the first child with isolated IgG4-RKD. 
PI-152 EVALUATION OF EPICARDIAL ADIPOSE TISSUE IN PEDIATRIC PATIENTS WITH NEPHROTIC SYNDROME DIAGNOSIS

Özgür Özdemir Șimșek ${ }^{1}$, Tülay Demİrcan ${ }^{2}$, Gökçen Erfİdan ${ }^{1}$, Cemallye Başaran ${ }^{1}$, Caner Alparslan ${ }^{1}$, Seçill Arslansoyu Çamlar ${ }^{3}$, Fatma Mutlubaş ${ }^{3}$, Belde Kasap Demİr ${ }^{4}$, Demet Alaygut ${ }^{1}$

${ }^{1}$ Izmir University of Health Sciences Tepecik Education and Research Hospital Department of Pediatric Nephrology, Izmir, Turkey, ${ }^{2}$ Izmir University of Health Sciences Tepecik Education and Research Hospital Department of Pediatric Cardiology, Izmir, Turkey, ${ }^{3}$ University of Health Sciences, Izmir Faculty of Medicine Department of Pediatrics, Division of Nephrology, ${ }^{4}$ Izmir Katip Çelebi University Medical Faculty, Department of Pediatrics, Division of Nephrology and Rheumotology, Izmir, Turkey

Introduction: Nephrotic syndrome (NS) is a syndrome characterized by nephrotic proteinuria, hypoalbuminemia, edema and hyperlipidemia. These patients have been shown to have a higher risk of cardiovascular disease (CVD), obesity, and dyslipidemia. The aim of this study is to evaluate the epicardial adipose tissue that plays an active role in the development of CVD and atherosclerosis by secreting inflammatory substances in patients with nephrotic syndrome. The study was conducted with the inclusion of pediatric patients aged 0-18 years with NS who were followed up in the Pediatric Nephrology Outpatient Clinic of SBU Tepecik Training and Research Hospital. Retrospectively, from the file data of these patients, age of diagnosis, current disease status (remission, attack), treatments used, findings of biopsy, weight, height, BMI, mean blood pressure, presence of edema, laboratory parameters and ECHO findings were recorded (Group 1). Healthy, screening ECHO cases were taken as the control group (Group 2).

Material and methods: The study and control groups consisted of 32 patients (20 males) and 15 patients ( 7 males), respectively. There was no difference between the groups in terms of gender, age, BMI, systolic and diastolic blood pressure. In Group 1, there were steroid dependent NS patients with $37.5 \%$ (12). The mean age at diagnosis was $80.0 \pm 57.8$ (17.0-207) months. 22 patients were clinically in remission. Biopsy diagnosis was present in 23 patients and 10 of them (43.5\%) were compatible with MLH. There was no patient with ascites. Six patients were clinically edematous.

Results: The demographic and clinical characteristics of Group 1 are shown in Table 1. When both groups were evaluated in terms of ECHO findings, a significant difference was found between the groups in terms of IVSD, LVWD, epicardial steatosis (viewed from the right ventricle and parasternal short screen in the parasternal long axis) (Table 2). In addition, there were significant differences between the patient and control groups in terms of parameters indicating repolarization in ECG evaluations in both groups (Table 3 ).

Conclusions: Cardiovascular morbidity and mortality are high globally in renal diseases and early diagnosis of cardiovascular diseases is important in this group of patients. Measurement and tracking of EYD can give us information in this sense as a non-invasive parameter.

\section{PI-153 FREQUENTLY RELAPSING NEPHROTIC SYNDROME IN A -11-YEAR OLD BOY WITH HETEROZYGOSITY FOR TWO VARIANTS IN NPHS1 GENE.}

\author{
Martine Docx ${ }^{1}$, Segers Nathalie ${ }^{1}$, Vande Johan ${ }^{2}$ \\ ${ }^{1}$ Department of Chronic Paediatric Diseases and Nephrology Queen Paola \\ Childrens Hospital Antwerp, Belgium, ${ }^{2}$ Department of Paediatric \\ Nephrology and Rheumatology University Hospital Ghent, Belgium
}

Objectives: An international research collaboration recently found that NPHS1 is also known as disease-susceptibility gene for steroid-sensitive nephrotic syndrome. Mutations in NPHS1 gene could occur in both SRNS and SSNS patients.
Methods: We report on a 11-year old Surinamese-Creole male patient who presented since the age of $211 / 12$ years with a steroid sensitive frequently relapsing nephrotic syndrome (SSFRNS). Kidney biopsy showed minimal change disease (MCD). He was treated first with the classical schedule steroids with tapering. After a steroid-free period of 6 months he developed 4 flares, for which cyclosporine was added. $\mathrm{He}$ remained without relapses for 2 years. Then frequent relapses were seen on tapering off his medication. Rituximab was started. Patient remained in remission for 14 months and then 2 flare-ups occurred each time after a viral infection. Steroids and cyclosporine were restarted. A second rituximab treatment was given at the age of 10 with subsequent tapering of steroids and retention of cyclosporine. Currently: (GFR: $101.9 \mathrm{ml} / \mathrm{min} /$ $1.73 \mathrm{~m}^{2}$ ), no hematuria, normal $\mathrm{C} 3$ and $\mathrm{C} 4$.

Results: Because of the SSFRN a genetic study was carried out. A heterozygosity was found for 2 variants in NPHS 1: c.2734 $\mathrm{G}>\mathrm{A}(\mathrm{p} .($ Ala912Thr)) and c.1747 G>A(p.(Gluc583Lys)).

Conclusions: The association of these variants has been previously reported as novel and potentially pathogenic variants by Bierzynska A. et al in 2017. Variations in heterozygous variants are not adequate to make structural damage and so are manifested with a milder phenotype. To conclude it is advisable to perform a genetic analysis, especially in SRNS, in familial cases of NS, but also in SSFRNS like in our case.

\section{PI-154 CONGENITAL NEPHROTIC SYNDROME: A CASE REPORT OF TWO SISTERS WITH THE SAME MUTATIONS BUT DIFFERENT PHENOTYPE}

Fiorenza Alfier ${ }^{1}$, Susanna Negrisolo ${ }^{2}$, Germana Longo ${ }^{3}$, Andrea Carraro ${ }^{2}$, Davide Meneghesso ${ }^{3}$, Mattia Parolin ${ }^{3}$, Leonardo Salviati ${ }^{4}$, Luisa Murer ${ }^{2}$, Elisa Benetti ${ }^{3}$

${ }^{1}$ Department of Woman and Childs Health, University of Padova, Padova, Italy, ${ }^{2}$ Laboratory of Immunopathology and Molecular Biology of The Kidney, Institute of Pediatric Research Città Della Speranza, Department of Women's and Children's Health, Padua University Hospital, Padua, Italy, ${ }^{3}$ Pediatric Nephrology Dialysis and Transplant Unit, Department of Women's and Children's Health, Padua University Hospital, Padua, Italy, ${ }^{4}$ Clinical Genetics Unit, Department of Woman and Child Health, University of Padova, And Irp Città Della Speranza, Padova, Italy

Introduction: Congenital nephrotic syndrome (CNS) is a heterogeneous kidney disorder characterized by early onset of symptoms caused by genetic defects in specific podocyte proteins disrupting the integrity of the filtration barrier. The most common genetic defects involve NPHS1 gene, which encodes nephrin and causes the Finnish type CNS.

Material and Methods: Herein we report the case of two Chinese sisters with the same mutations but different clinical severity. The younger sister was referred at three months because she developed diffuse oedema associated with massive proteinuria $(\max 11,6$ $\mathrm{g} / \mathrm{L}$ ), hypoalbuminemia (min $14 \mathrm{~g} / \mathrm{L}$, range $38-54 \mathrm{~g} / \mathrm{L}$ ), hypothyroidism and hypertension. Renal biopsy showed mesangial hypercellularity and diffuse microcystic dilation of the proximal tubules and ultrastructural analysis showed complete foot process effacement and villous transformation of podocytes with endothelial swelling. On the suspect of CNS, NGS analysis was performed. The girl was treated with albumin, antithrombin III and immunoglobulin supplements, ACE-inhibitor (ACE-i) and antihypertensive drugs. In her family history, parents were healthy and not consanguineous, but her older sister was diagnosed with proteinuria at the age of 2 and was treated with ACE-i in China. At 9 years of age, she had mild proteinuria (1 g/day) and her renal biopsy showed mild mesangial hypercellularity, little microcystic dilation of the proximal tubules and foot process effacement. The genetic test was extended to her. 
Results: NGS analysis highlighted the Fin-minor mutation c.3325C $>\mathrm{T}$ (p.Arg1109Ter) and the missense mutation c.928G $>$ A (p.Asp310Asn) in compound heterozygous state in both sisters.

Conclusions: In CNS is emerging evidence of the possibility of a variable phenotype despite the same genetic variants. Our case highlights profound differences both in clinical presentation and evolution and in histological features between sisters with similar environmental exposure and the same compound heterozygous mutations in NPHS1.

\section{PI-155 RARE COLLAGENOUS HETEROZYGOTE VARIANTS IN CHILDREN WITH IGA NEPHROPATHY}

Alexandra Cambier ${ }^{1}$, Thomas Robert ${ }^{2}$, Julien Hogan ${ }^{3}$, Marion Rabant ${ }^{4}$, Michel Peuchmaur ${ }^{3}$, Olivia Boyer ${ }^{4}$, Tim Ulinski ${ }^{5}$, Renato Monteiro ${ }^{6}$, Laurent Mesnard $^{7}$

${ }^{1}$ Sainte Justine Hospital, Montreal, Canada, ${ }^{2}$ Centre De Néphrologie Et Transplantation Rénale, Aphm,Marseille, France, ${ }^{3}$ Hôpital Robert Debré, Paris, France, ${ }^{4}$ Hôpital Necker, Paris, France,${ }^{5}$ Hôpital Trousseau, Paris, France, ${ }^{6}$ Centre De Recherche Sur Linflammation, Paris (cri); Inserm U1149, ${ }^{7}$ Service Des Urgences Néphrologiques Et Transplantation Rénales, Aphp, Hôpital Tenon, Paris, France

Introduction: Childhood $\operatorname{IgAN}(\operatorname{cIg} A N)$ is one of the primary glomerulonephritis clinically characterized by microscopic hematuria and proteinuria, the presence of which might potentially overlap with Alport syndrome. Interestingly, previous works suggest that familial IgAN could be linked to the chromosome $2 \mathrm{q} 36$ region, also the coding region for COL4A3/A4.

Material and methods: To investigate a possible relationship or phenocopy between Alport syndrome and cIgAN, COL4A3, COL4A4 as COL4A5 exons were sequenced in 36 cIgAN patients. Clinical data and treatment were retrospectively collected. COL4A3/A4/A5 variants were classified according to American College of Medical Genetics and the Association for Molecular Pathology (ACMG/AMP) guidelines.

Results: Four of $36 \mathrm{cIgAN}$ patients were affected by ACMG class $4 / 5$ COL4A3 heterozygous variants (COL4A3-cIgAN). We found no COL4A4 or COL4A5 variant. Despite having rare and deleterious COL4A3 variants, 3 of 4 COL4A3-cIgAN children developed the clinical and biological features of active IgAN rather than Alport syndrome. Response to intensive immunosuppressive treatment was favorable, leading to the reduction of endocapillary and extracapillary proliferation lesions. High levels of immune $\mathrm{IgG} / \mathrm{IgA}$ complexes, reduction of proteinuria and gradual eGFR stabilization argued against Alport syndrome. Nevertheless, COL4A3-cIgAN patients seem predisposed to more serious IgAN presentation compared to the non-COL4A3-cIgAN group, with more glomerulosclerosis and a lower eGFR over time. One of the four patients was kidney transplanted with subsequent IgAN recurrence.

Conclusions: Predisposition factors for developing serious cIgAN flairs should be considered for cIgAN with COL4A3 pathological heterozygous variants. COL4A3 variants, usually responsible for Alport syndrome in adults, should not automatically exclude an immunosuppressive regimen in cIgAN. Moreover, evidence of an ACMG class 4/5 COL4A3 variant in early stage cIgAN could be a helpful tool for stratifying severity of cIgAN beyond the Oxford classification.

PI-156 NO CLEAR INDICATION FOR DIGENIC INHERITANCE IN 60 INDIVIDUALS WITH DISEASE-CAUSING VARIANTS IN COL4A3, COL4A4 OR COL4A5 AND IN A MENDELIAN GENOMICS EXOME DATABASE OF MORE THAN 22,000 INDIVIDUALS

Jasmina Ćomić ${ }^{1}$, Korbinian M. Riedhammer ${ }^{1}$, Roman GÜnthner ${ }^{2}$, Patrick Richthammer ${ }^{1}$, Hannes Simmendinger ${ }^{1}$, Donald Kieffer ${ }^{1}$,
Lutz Renders $^{2}$, Uwe Heemann ${ }^{2}$, Matthias C. Braunisch ${ }^{2}$, Ute Moog ${ }^{3}$, Thomas Meitinger ${ }^{1}$, Julia Hoefele ${ }^{1}$

${ }^{1}$ Institute of Human Genetics, Klinikum Rechts Der Isar, Technical University of Munich, School of Medicine, Munich, Germany, ${ }^{2}$ Department of Nephrology, Klinikum Rechts Der Isar, Technical University of Munich, School of Medicine, Munich, Germany, ${ }^{3}$ Institute of Human Genetics, Heidelberg University, Heidelberg, Germany

Introduction: Disease-causing variants in COL4A3-5 are known to be associated with type-IV-collagen-related nephropathy, comprising Alport syndrome (AS) and thin basement membrane nephropathy (TBMN). Initial symptoms of individuals with AS are microscopic hematuria $\mathrm{MH})$ followed by proteinuria leading to end-stage renal disease (ESRD). Extrarenal manifestations like hearing impairment and eye abnormalities can additionally be observed in AS. In contrast, individuals with TBMN mostly present with $\mathrm{MH}$, only some of these individuals develop ESRD. Digenic inheritance has been proposed as causative for type-IV-collagen-related nephropathy and discussed as a possible aggravating factor of the phenotype in type-IV-collagen-related nephropathy. However, up to now only little and inconclusive information concerning this topic can be found in the literature.

Material and methods: 60 index cases, in whom exome sequencing had been performed and with (likely) pathogenic variant(s) in COL4A3-5 (summarized to "disease-causing variant"), were reanalyzed for additional (likely) pathogenic variants in COL4A3-5. Moreover, a Mendelian genomics database (Munich Exome Server) comprising 22,000 exomes was scrutinized for individuals with a combination of disease-causing variants in at least two of the three collagen genes COL4A3, COL4A4, or COL4A5.

Results: Of 60 reanalyzed individuals, $83 \%$ had AS phenotypically, $12 \%$ TBMN and 5\% had focal segmental glomerulosclerosis. No additional disease-causing variants were identified in one of the alternate COL4A35 genes in these individuals. In the Munich Exome Server, only one individual with a non-renal phenotype and a combination of a heterozygous disease-causing variant in COL4A4 and COL4A5, respectively, was identified.

Conclusions: In this study, no clear indication for digenic inheritance could be observed implicating that this phenomenon might be very rare and not that common than postulated in the literature. Future research on digenic inheritance in type-IV-collagen-related nephropathy should focus on statistic evidence from case-control studies and experimental proof of functional impact of digenic variants in COL4A3-5.

\section{PI-157 VALVULAR ABNORMALITIES IN CHILDREN WITH AUTOSOMAL DOMINANT POLYCYSTIC KIDNEY DISEASE}

Alexandra Savis, John Simpson, Manish Sinha

Evelina London Childrens Hospital, United Kingdom

Introduction: The incidence of Mitral valve prolapse (MVP) in children with ADPKD has previously been reported as $12 \%$ and in adult populations between 26-33\%. Our objective in this study was to establish the prevalence of valvular abnormalities in Children \& Young People (C\&YP) with Autosomal Dominant Polycystic Kidney Disease (ADPKD)

Material and methods: Retrospective, single centre cross-sectional analysis of all children seen in a dedicated paediatric ADPKD clinic. Mitral valve prolapse (MVP) was diagnosed if one or both MV leaflets breaks the plane of the MV annulus in a non-symmetric manner and/or may demonstrate myxomatous degeneration. Mitral regurgitation, aortic valve abnormalities and variation of normal valvular anatomy, if any, were additionally evaluated.

Results: The echocardiograms of 102 C\&YP with ADPKD (range 0.25$18 \mathrm{yrs})$ were analysed. Only $1(0.98 \%), 12$ year old boy had MVP. There was no associated mitral regurgitation. Five (4.9\%) patients, 3 boys, aged 
7-14 years had trivial/mild aortic regurgitation. Evaluating variations in normal valvular anatomy, $9(8.82 \%)$ patients, aged 3 to 15.9 years, had minor buckling +/- visual elongation of either the anterior or posterior leaflet of the mitral valve, none of which fall within the criteria of true MVP, but may be early signs of developing MVP. Of the 9 patients with minor bowing +/- leaflet elongation, only 4 had a trace of MR. None of the patients had any valvular abnormality that was of any clinical significance.

Conclusions: We conclude that following careful evaluation in this contemporary cohort of C\&YP with ADPKD, the incidence of mitral valve prolapse is significantly lower than previously reported in both children and in adults with ADPKD. Those with variants of normal anatomy may represent early signs of developing MVP in this population and longitudinal studies are needed.

\section{PI-158 LOW PREVALENCE OF HYPERTENSION IN CHILDREN WITH RENAL CYSTS AND DIABETES SYNDROME IS THE HALLMARK OF THE DISEASE}

Tomas Seeman ${ }^{1}$, Friederike Weigel ${ }^{2}$, Kveta Blahova $^{1}$, Filip Fencl ${ }^{1}$, Stepanka Pruhova ${ }^{1}$, Katharina Hermes ${ }^{3}$, Richard Klaus ${ }^{3}$, Baerbel Lange-sperandio ${ }^{3}$, Veit Grote ${ }^{3}$, Ulrike John-kroegel ${ }^{2}$

${ }^{1}$ Charles University Prague, 2nd School of Medicine, Czech Republic, ${ }^{2}$ Friedrich-Schiller University Jena, Germany, ${ }^{3}$ Ludwig-maximillian Universität Munich, Germany

Objectives: Cystic kidney diseases such as autosomal recessive or dominant polycystic kidney disease (ARPKD and ADPKD) are associated with high prevalence of arterial hypertension. On the contrary, studies on hypertension in children with renal cysts and diabetes (RCAD) syndrome caused by abnormalities in the $H N F 1 B$ gene are rare. Therefore, the aim of our study was to investigate the prevalence of hypertension in children with RCAD syndrome due to HNF1B gene abnormalities and to search for possible risk factors for development of hypertension.

Methods: Data on all children with genetically proven RCAD syndrome from three pediatric nephrology tertiary centers were retrospectively reviewed (office blood pressure (BP), ambulatory blood pressure monitoring (ABPM), creatinine clearance, renal ultrasound, echocardiography, albuminuria/proteinuria). Hypertension was defined using the current ESH 2016 criteria and/or by the use of antihypertensive drugs.

Results: Thirty-two children with RCAD syndrome were investigated. Three children received ACE-inhibitors for hypertension and/or proteinuria. Hypertension was diagnosed using office BP in $22 \%$ of the children $(\mathrm{n}=7)$ In the 7 performed ABPM (one in a child with office hypertension and five with normal office BP) only one child (14\%) was diagnosed with hypertension by ABPM. Creatinine clearance, proteinuria, albuminuria, body mass index, enlargement or hypodysplasia of the kidneys and prevalence of $H N F 1 B$-gene deletion or mutation were not significantly different between hypertensive and normotensive children.

Conclusions: Hypertension is rare in children with RCAD syndrome. The low prevalence of hypertension seems to be the hallmark of the disease.

PI-159 THERAPEUTIC MANAGEMENT OF PAEDIATRIC SCHIMKE IMMUNO-OSSEOUS DYSPLASIA PATIENTS WITH KIDNEY TRANSPLANTATION: A 20-YEAR EXPERIENCE OF A FRENCH CENTRE

Laurene Dehoux ${ }^{1}$, Benedicte Neven ${ }^{1}$, Nathalie Gouge-biebuyck ${ }^{1}$, Despina Moshous ${ }^{1}$, Sophie Taque ${ }^{2}$, Philippe Eckart ${ }^{3}$,

Jerome Harambat ${ }^{4}$, Gwenaelle Roussey ${ }^{5}$, Marina Avramescu ${ }^{1}$, Mathilde Grapin ${ }^{1}$, Marina Charbit ${ }^{1}$, Olivia Gillion-boyer ${ }^{1}$

${ }^{1}$ Hopital Necker Enfants Malades, Universite Paris Descartes, Aphp, Paris, France, ${ }^{2}$ Chu Pontchaillou, Rennes, France, ${ }^{3}$ Chu Caen
Normandie, Caen, France, ${ }^{4}$ Chu Pellegrin, Bordeaux, France, ${ }^{5}$ Chu Nantes-hotel Dieu, Nantes, France

Objectives: Schimke immuno-osseous dysplasia (SIOD) is a rare autosomal recessive disease (SMARCAL1 variants) characterized by T-cell immunodeficiency and steroid-resistant nephrotic syndrome progressing to kidney failure with early mortality mainly due to infections and stroke. Indication and modalities of kidney transplantation (KTx) and bone marrow transplant (BMT) are not standardised. We described the management proposed by our unit.

Methods: Retrospective, observational, single-centre study including paediatric SIOD KTx recipients followed between January 2002 and January 2021.

Results: Eight patients of 7 year median age (3-10) at deceased donor KTx time were included. Seven patients required chronic dialysis of 6 month median duration (3-6) before KTx. Induction treatment was basiliximab and steroids (6 patients), combined with rituximab (1 patient), rituximab and steroids (1 patient). Maintenance therapy associated calcineurin inhibitors, antiproliferative agents and/or steroids. Three patients followed before 2015 received only KTx. Two brothers died of acute cardiac failure at 10 years old, one of them on dialysis due to chronic rejection. The second had prior CMV and pneumocystis carinii severe infections 1 year before. The third one had a stable eGFR $(110 \mathrm{ml} / \mathrm{min} /$ $1.73 \mathrm{~m}^{2}$ ) with no extra-renal complication 9 years after KTx. Among the 5 other patients, 3 developed severe graft versus host disease (GVHD) 19 days, 71 days and 134 days after KTx. GVHD was successfully treated by increased immunosuppression and renal donor-targeted serotherapy. One received emergency BMT. Nevertheless, 2 died of aspergillosis. The third had BM colonisation by kidney donor hematopoietic precursor with $100 \%$ chimerism and stable eDFG after 3 years. The 2 remaining patients received successful BMT 1 and 4.7 years after KTx (1 year follow-up).

Conclusion: SIOD patient management evolved over time and is challenging. Therapy management by KTx followed by BMT may be a reasonable alternative if GVHD risk is prevented or rapidly treated by specific donor-targeted serotherapy.

\section{PI-160 NPHS2 VARIANTS AND DISEASE RECURRENCE AFTER TRANSPLANTATION: EXPERIENCE FROM A LARGE SINGLE CENTER COHORT}

Dorval Guillaume ${ }^{1}$, Gribouval Olivier ${ }^{3}$, Laurence Heidet ${ }^{2}$, Berthaud Romain ${ }^{2}$, Antignac Corinne ${ }^{1}$, Boyer Olivia ${ }^{\mathrm{z}}$

${ }^{1}$ Service De Génétique Moléculaire, Hôpital Necker Enfants Malades, Aphp, Paris, France, ${ }^{2}$ Service De Néphrologie Pédiatrique, Marhea Et Sni, Hôpital Necker Enfants Malades, Aphp, Paris, France, ${ }^{3}$ Laboratoire Des Maladies Renales Hereditaires - Institut Imagine - Paris, France

Abstract: Unlike idiopathic nephrotic syndrome, which is thought to be of immune origin, hereditary nephrotic syndrome is not expected to recur after kidney transplantation. However, recurrence of proteinuria or nephrotic syndrome on the kidney graft has been occasionally reported in patients with the p.Arg138Gln NPHS2 variant (podocin gene) in the homozygous or compound heterozygous state. These data raise the question of kidney transplantation from living donors. The objective of this study was to refine the risk of recurrence after kidney transplantation in patients with $N P H S 2$ variants.

Since January $2010,61 / 175$ patients with causative bi-allelic NPHS2 variants have been transplanted at our center. Of these, 11 carried the p.Arg $138 \mathrm{Gln}$ variant in the homozygous state and 9 in the compound heterozygous state. The additional 41 patients carried other NPHS2 variants in the homozygous [mostly p.Val260Glu $(n=6 / 20)$ ] or compound heterozygous state [mostly p.Arg229Gln $(n=11)$; associated to the p.Ala284Val $(n=6 / 11)]$. During the same 
period, 48 children with steroid-resistant nephrotic syndrome and a negative genetic testing regarding a panel a genes involved in nephrotic syndromes. No patient with a NPHS2 variant recurred after kidney transplantation (mean follow-up time $15.6+/-8$ years). Conversely, $7 / 48(14.5 \%)$ patients without any identified causative variant(s) recurred within a maximum of 7 days after renal transplantation (mean follow-up 15.6+/-10.1 years).

Our data show that the risk of disease recurrence after kidney transplantation is extremely low in case of identified NPHS2 causal variants. These data are consistent with the pathophysiology of the disease due to structural defects of the glomerular filtration barrier in the native kidney and with recent data highlighting that the rare reported cases of post-transplant proteinuria in patients with $N P H S 2$ variants are probably multifactorial. All together, these data reinforce the possibility of kidney donation from living donors in individuals carrying the NPHS2 p.R138Q variant in the heterozygous state.

\section{PI-161 EFFECTS OF RAAS INHIBITION AND IMMUNO- SUPPRESSIVE THERAPY IN PEDIATRIC PATIENTS WITH X-LINKED ALPORT SYNDROME}

Gulsah Ozdemir ${ }^{1}$, Bora Gulhan ${ }^{1}$, Eda Didem Kurt Sukur ${ }^{1}$, Emine Atayar ${ }^{2}$, Ismail Dursun ${ }^{3}$, Zeynep Birsin Ozcakar ${ }^{4}$, Seha Saygili ${ }^{5}$, Alper Soylu ${ }^{6}$, Oguz Soylemezoglu ${ }^{7}$, Alev Yilmaz ${ }^{8}$, Aysun Karabay Bayazit ${ }^{9}$, Fehime Kara Eroglu ${ }^{10}$, Belde Kasap Demir ${ }^{11}$, Selcuk Yuksel ${ }^{12}$, Yilmaz Tabel ${ }^{13}$, Ayse Agbas ${ }^{14}$, Ali Duzova ${ }^{1}$, Mutlu Hayran ${ }^{15}$, Fatih Ozaltin ${ }^{1}$, Rezan Topaloglu ${ }^{1}$

${ }^{1}$ Division of Pediatric Nephrology, Hacettepe University Faculty of Medicine, Ankara, Turkey, ${ }^{2}$ Division of Pediatric Nephrology, Nephrogenetics Laboratory, Hacettepe University Faculty of Medicine, Ankara, Turkey, ${ }^{3}$ Division of Pediatric Nephrology, Erciyes University Faculty of Medicine, Kayseri, Turkey, ${ }^{4}$ Division of Pediatric Nephrology, Ankara University Faculty of Medicine, Ankara, Turkey, ${ }^{5}$ Division of Pediatric Nephrology, Istanbul University CerrahpaŞa Faculty of Medicine, Istanbul, Turkey, ${ }^{6}$ Division of Pediatric Nephrology, Dokuz, Eylül University Faculty of Medicine, Izmir, Turkey, ${ }^{7}$ Division of Pediatric Nephrology, Gazi University Faculty of Medicine, Ankara, Turkey, ${ }^{8}$ Division of Pediatric Nephrology, Istanbul University Capa Faculty of Medicine, Istanbul, Turkey, ${ }^{9}$ Division of Pediatric Nephrology, Cukurova University Faculty of Medicine, Adana, Turkey, ${ }^{10}$ Division of Pediatric Nephrology, Dr. Sami Ulus Maternity and Childrens Health Hospital, Ankara, Turkey, ${ }^{11}$ Division of Pediatric Nephrology, Izmir Katip Celebi University, Tepecik Research And Training Hospital, Izmir, Turkey, ${ }^{12}$ Division of Pediatric Nephrology, Pamukkale University Faculty of Medicine, Denizli, Turkey, ${ }^{13}$ Division of Pediatric Nephrology, İnönü University Faculty of Medicine, Malatya, Turkey, ${ }^{14}$ Division of Pediatric Nephrology, Haseki Training and Research Hospital, Istanbul, Turkey, ${ }^{15}$ Department of Preventive Oncology, Hacettepe University Faculty of Medicine, Ankara, Turkey

Introduction: Alport syndrome (AS) is a hereditary nephritis characterized by progressive renal failure. There is increasing evidence that renin-angiotensin-aldosterone system (RAAS) inhibition slows progression to chronic kidney disease (CKD) in AS patients. On the other hand, the effectiveness of immunosuppressive (IS) therapy and the number of studies is limited. This study aimed to analyze the outcomes of RAAS inhibitors and IS therapy in pediatric patients with X-linked AS (XLAS).

Material and methods: A total of 74 pediatric XLAS patients were included in this multicenter study. Data from medical records were collected retrospectively, other centers were requested to fill out a questionnaire which includes data regarding patient demographic features, clinical and laboratory characteristics, treatment, and follow-up results.
Results: A total of 74 pediatric patients with XLAS (41 male, 33 female) were studied. The median age at first presentation was 6.0 years (interquartile range (IQR) $3.4-9.9$ ). The median follow-up duration was 4.0 years (IQR 1.7-7.3). Fifty-two (70.2\%) patients received only RAAS inhibitors, $11(14.9 \%)$ patients received RAAS inhibitors and IS, and $11(14.9 \%)$ patients were followed up without treatment. During follow up, glomerular filtration rate (GFR) decreased below $60 \mathrm{ml} / \mathrm{min} / 1.73 \mathrm{~m}^{2}$ in $7(9.5 \%)$ of 74 patients $(1$ female, 6 male) at the median 16.2 years (IQR 15.2 - 16.6). The patients who were followed up without treatment did not progress to CKD, however, the follow-up period was significantly shorter than other groups $(p=0.02)$. The median age at onset of RAAS inhibitor therapy were significantly higher in patients who progressed to CKD than those who did not (12.3 vs 8.7 years, $p=0.007)$. There was no difference in time to progression to CKD and GFR loss between patients in RAAS and RAAS+IS group ( $\mathrm{p}=0.40$ and $\mathrm{p}=0.87$ ).

Conclusions: Our study showed that early initiation of RAAS inhibitors in patients with XLAS may delay progression to CKD. IS treatment did not improve renal survival in XLAS patients.

\section{PI-162 FIRST DESCRIPTION OF A CASE OF HYPO- SULFATEMIC HYPERSULFATURIA CAUSED BY A HOMOZYGOUS VARIANT IN THE SLC13A1 GENE}

\author{
Arend Bokenkamp ${ }^{1}$, Martijn Finken ${ }^{1}$, Jiddeke Van Der Kamp ${ }^{2}$ \\ ${ }^{1}$ Emma Childrens Hospital, Amsterdam UMC, The Netherlands, ${ }^{2}$ \\ Department of Clinical Genetics, Amsterdam UMC, The Netherlands
}

Background: Sulfate is an important mineral for cartilage metabolism and hepatic detoxification. Like other ions, sulfate is absorbed via dedicated transporters in the gut and in the proximal tubule. As sulfate is not commonly measured in clinical medicine, sulfate deficiency is not recognized and little is known on the phenotype of sulfate deficiency. This is the first report describing hyposulfatemic hypersulfaturia caused by a homozygous nonsense variant in the SLC13A1 gene, encoding the sodium-sulfate cotransporter, which is responsible for intestinal and renal sulfate (re)absorption.

Case description: The boy presented at 4 months with enlargement of the knees. X-ray revealed spiky widening of all metaphyses, ovoidshaped vertebral bodies, slightly delayed epiphyseal ossification, irregular tarsal bones and flared ribs, which improved over the years. At the age of 11 years, he has broad knees and elbows, winged scapulae, a relatively shorter trunk with reduced movement of the lumbar spine and knees. He has a normal cognitive development, and no kidney or liver dysfunction.

Trio-based Whole Exome Sequencing showed a homozygous g.122839967G $>$ A (Chr7(GRCh37)), c.34C $>$ T, p. $($ Arg12*) variant in SLC13A1.

Plasma sulfate concentrations were consistently diminished (27-70 $\mu \mathrm{mol} / 1$; N 166-383), fractional excretion of sulfate was elevated to $89 \%$ (N 13-34). The heterozygous parents had an intermediate phenotype (sulfate concentrations 58 and $93 \mu \mathrm{mol} / 1$, fractional excretions 28 and 49\%, respectively). As sulfate is critical for paracetamol elimination, we performed an 8-hour iv sulfate loading test with 225 $\mathrm{mg} / \mathrm{kg}$ acetylcysteine in analogy to the treatment of paracetamol intoxication. This led to a transient normalization of sulfate (max 349 $\mu \mathrm{mol} / \mathrm{l})$, which returned to baseline after 24 hours.

Conclusion: Hyposulfatemic hypersulfaturia due to a homozygous variant in the SLC13A1 gene manifests with a distinct skeletal phenotype probably due to abnormal cartilage metabolism that improves with age. Drugs metabolized by sulfation should be avoided, in case of intoxication plasma sulfate can be normalized transiently with high-dose acetylcysteine. 
PI-163 LONG-TERM OUTCOME OF A SERIES OF PATIENTS WITH PRIMARY DISTAL RENAL TUBULAR ACIDOSIS

Leire Madariaga ${ }^{4}$, Sara Gomez-conde ${ }^{1}$, Alejandro Garcia-castano ${ }^{1}$, Mireia Aguirre ${ }^{3}$, Maria Herrero ${ }^{3}$, Leire Gondra ${ }^{4}$, Ana Vinuesa ${ }^{3}$, Nelida Garcia-perez ${ }^{2}$, Gema Ariceta ${ }^{5}$, Luis Castaño ${ }^{4}$

${ }^{1}$ Iis Biocruces Bizkaia, Spain, ${ }^{2}$ Pediatric Nephrology Department, Basurto University Hospital, Iis Biocruces Bizkaia, University of The Basque Country, Spain, ${ }^{3}$ Pediatric Nephrology Department, Cruces University Hospital, Iis Biocruces Bizkaia, Spain, ${ }^{4}$ Pediatric Nephrology Department, Cruces University Hospital, Iis Biocruces Bizkaia, University of The Basque Country, Spain, ${ }^{5}$ Pediatric Nephrology Department, Vall De Hebron University Hospital, Universidad Autonoma De Barcelona, Spain

Introduction: Primary Distal renal tubular acidosis (dRTA) is a rare genetic disorder caused by mutations in ATP6V0A4, ATP6V1B1 and $S L C 4 A 1$ genes, which encode transporters that regulate acid-base balance in the collecting duct.

Material and methods: This is a retrospective study on the molecular and clinical data from diagnosis and long-term follow-up of 16 patients with primary dRTA.

Results: Molecular analyses revealed that 9 patients had mutations in ATP6VOA4, 5 in ATP6V1B1 and 2 in SLC4A1. We found a novel intragenic deletion in the ATP6V0A4. We reported a common ATP6VOA4 gene variant (c.1691+2dupT) in two thirds of these patients, suggesting a founder effect. The mean age at last follow-up was 26.6 (SD 15) years. Median SDS height was -1.17 (IQR $-1.71 ;-0.3$ ), and it did not change significantly from diagnosis $(-1.02$, IQR $-1.79 ;-0.14, P=0.28)$. Final SDS height was significantly correlated with the age at diagnosis $(P=0.03)$. Patients with an adequate metabolic control (plasma bicarbonate $>22$ $\mathrm{mEq} / \mathrm{L}$ and urinary calcium $<0.2 \mathrm{mg} / \mathrm{mg}$ of creatinine) had a significantly better SDS height at last follow-up $(P=0.02)$. Six out of 16 patients $(37.5 \%)$ had a chronic kidney disease (CKD) at last follow-up, with a mean GFR of 48.9 (SD 29.1) $\mathrm{ml} / \mathrm{min} / 1.73 \mathrm{~m} 2$. The mean age of patients with CKD was 37.5 (SD 9.3) years. The development of CKD was not significantly associated with the age at diagnosis or with an adequate metabolic control at last follow-up. The incidence of sensorineural hearing loss (SNHL) was very high in ATP6V1B1 patients, though not universal. Patients harboring ATP6VOA4 mutations also developed SNHL at a high rate $(80 \%)$ over time. All except one patient developed nephrocalcinosis or lithiases over time.

Conclusions: Patients with dRTA may develop growth failure over time. An early diagnosis can ameliorate the prognosis of height. The development of CKD is not rare (37.5\% of patients) despite adequate metabolic control.

\section{PI-164 BLOOD PRESSURE AND GLOMERULAR FILTRATION RATE IN YOUTH WITH TUBEROUS SCLEROSIS COMPLEX}

Efthymia Vargiami ${ }^{1}$, Stella Stabouli ${ }^{1}$, Christina Sidira ${ }^{1}$, Maria Kyriazi ${ }^{1}$, Athanasia Anastasiou ${ }^{2}$, Athanasios Notopoulos ${ }^{3}$, Dimitrios Zafeiriou ${ }^{1}$

${ }^{1}$ First Department of Pediatrics, School of Medicine, Faculty of Health Sciences, Aristotle University of Thessaloniki, Hippokratio Hospital, Thessaloniki, Greece, ${ }^{2}$ Department of Radiology, Hippokratio Hospital, Thessaloniki, Greece, ${ }^{3}$ Department of Nuclear Medicine, Hippokratio Hospital, Thessaloniki, Greece

Objectives: To assess kidney function and clinical features of renal involvement in tuberous sclerosis complex (TSC), including glomerular filtration rate (GFR), albuminuria, and blood pressure (BP) levels in children, adolescents and young adults.

Methods: Patients with a confirmed TSC diagnosis followed up at the Paediatric Neurology department of a tertiary hospital were included in the current cross-sectional study. All participants had kidney imaging within six months of ambulatory blood pressure and GFR assessment.
Data on demographics, history, genotype, kidney function at diagnosis and last imaging were also collected.

Results: The median age of the patients was 15 years (IQR range 9 to 18 ). $23.5 \%$ of the participants had ambulatory BP hypertension. Significant

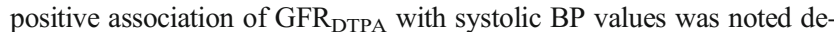
spite the absence of hyperfiltration. Greater increase in GFR from initial TSC diagnosis to assessment date resulted in higher GFR levels in childhood and adolescence in those that developed hypertension and possibly in those with angiomyolipoma or cysts. All patients with ambulatory BP hypertension had angiomyolipomas or cysts on renal imaging studies.

Conclusions: Hypertension may be prevalent in young patients with TSC-related kidney disease. Routine ambulatory BP measurement could be part of the annual clinical assessment of the TSC population.

\section{PI-165 RENAL AND EXTRA RENAL MANIFESTATIONS IN ADULT ZEBRAFISH MODEL OF CYSTINOSIS}

Sante Princiero Berlingerio ${ }^{1}$, Junling $\mathrm{He}^{2}$, Lies De Groef ${ }^{1}$, Pieter Baatsen ${ }^{3}$, Sara Cairoli ${ }^{4}$, Bianca Maria Goffredo ${ }^{4}$, Francesco Emma ${ }^{4}$, Lieve Moons ${ }^{1}$, Bert Van Den Heuvel ${ }^{1}$, Hans Baelde ${ }^{2}$, Elena Levtchenko ${ }^{1}$

${ }^{1}$ Ku Leuven, Belgium, ${ }^{2}$ Leiden University Medical Center (LUMC), The Netherlands, ${ }^{3}$ VIB-kuleuven, Belgium, ${ }^{4}$ Bambino Gesù Children's Hospital, Italy

Introduction: Cystinosis is a rare, incurable autosomal recessive disease caused by mutations in the CTNS gene, which encodes for the cystine transporter cystinosin leading to lysosomal cystine accumulation in all cells, with kidneys being the first affected organs. Treatment with cysteamine decreases the cystine accumulation, but does not reverse the renal Fanconi syndrome, glomerular injury or loss of renal function. We have developed a zebrafish larvae model having truncating mutation in ctns, which recapitulates kidney phenotype of cystinosis. In this study, we characterized the adult cystinosis zebrafish model to evaluate the long term effects of cystinosis on kidney and extra renal organs.

Material and methods: Cystinosis $\left(c^{-t n s^{-/}}\right)$zebrafish of 18 months and control (WT) zebrafish were studied. Histological studies of kidneys and extrarenal organs were performed. Cleaved caspase- 3 staining was used to evaluate apoptosis in the kidney. Cystine accumulation was evaluated via liquid chromatography-mass spectrometry and toluidine blue staining. For the fertility studies, the number of total eggs and fertile eggs produced by breeding female and male ctns $^{-1}$ zebrafish compared to WT zebrafish was evaluated.

Results: $c_{t n s^{--}}$zebrafish show cystine accumulation, glomerular hypertrophy and proximal tubular accumulation of hyaline-like eosinophilic droplets and vacuolated cytoplasm. Moreover, the cystinotic zebrafish show increased apoptosis in proximal tubules. In addition, instead of the typical striped pattern, $\mathrm{ctns}^{-/}$zebrafish presents an altered melanin skin pigmentation resulting in spotted skin. Lastly, male $\mathrm{ctns}^{-/-}$zebrafish presents spermatogenic cysts enriched in spermatozoa in the testis, while female presented increased percentage of unfertilized eggs.

Conclusions: The $c$ tns $^{-/}$zebrafish model reproduces several human phenotypes of cystinosis such as cystine accumulation, altered kidney morphology, impaired skin pigmentation and decreased fertility. This model may be useful for studying long-term effects of cystinosis and for testing novel drugs for correcting cystinosis phenotype.

PI-166 WHAT ARE THE PSYCHOSOCIAL FACTORS INFLUENCING ACCESS TO KIDNEY TRANSPLANTATION AND TRANSPLANT OUTCOMES FOR CHILDREN? A SYSTEMATIC LITERATURE REVIEW

Ji Soo Kim, Jo Wray, Stephen D Marks

Great Ormond Street Hospital For Children Nhs Foundation Trust, London, United Kingdom 
Introduction: Kidney transplantation is often seen as the gold standard treatment for children and young people (CYP) with End Stage Kidney Disease (ESKD). However, psychosocial factors have been cited as a barrier to accessing a kidney transplant, although it is unclear what these are.

Through a systematic literature review, this study explores the range of psychological and social factors that influence how soon a CYP with ESKD accesses a kidney transplant. This includes factors that influence kidney transplantation outcomes and factors deemed important to patients and their families in terms of their QoL.

Material and methods: We included quantitative, qualitative and mixed-method studies that were peer-reviewed and included primary data. Medline, PsycInfo, CINAHL and Web of Science were searched for papers published in English between January 1964 and September 2020.

Results: After removing duplicates, a total of 6235 studies were retrieved through database searches, hand-searching references and consulting experts in the field. Fifty-seven studies remained after full-text screening against inclusion criteria. There were 46 quantitative, 8 qualitative and 3 mixed-method studies. Most study designs were retrospective longitudinal registry studies. Factors influencing access to transplantation included maternal education, social support network and therapy non-adherence. Race, socioeconomic status and geographic remoteness were often cited as contributory factors. Although factors such as anxiety, depression and avoidant coping strategies were described in the literature in relation to patient family experience and wellbeing, evidence linking these with accessibility to, or outcomes of, paediatric kidney transplantation was limited.

Conclusions: Longitudinal and prospective studies are needed to fully assess the relationship between psychological factors and the relationship with social factors and a CYP's subsequent access to, or outcomes after, kidney transplantation.

\section{PI-167 PERSPECTIVES ON LIFE PARTICIPATION BY YOUNG ADULTS WITH CHRONIC KIDNEY DISEASE: AN INTERVIEW STUDY}

Jasmijn Kerklaan ${ }^{2}$, Elyssa Hannan ${ }^{1}$, Camilla Hanson ${ }^{1}$, Chandana Guha ${ }^{1}$, Yeoungjee Cho ${ }^{3}$, Martin Christian ${ }^{4}$, Lorraine Hamiwka ${ }^{5}$, Jessica Ryan ${ }^{6}$, Aditi Sinha ${ }^{7}$, Germaine Wong ${ }^{1}$, Jonathan Craig ${ }^{8}$, Jaap Groothoff ${ }^{2}$, Allison Tong ${ }^{1}$

${ }^{1}$ Centre for Kidney Research, The Children's Hospital at Westmead, Westmead, Nsw, Australia, ${ }^{2}$ Department of Pediatric Nephrology, Emma Children's Hospital, Academic Medical Center, Amsterdam, The Netherlands ${ }^{3}$ Department of Neprohology, Princess Alexandra Hospital, Brisbane, Australia, ${ }^{4}$ Department of Paediatric Nephrology, Nottingham Children's Hospital, Nottingham, United Kingdom, ${ }^{5}$ Division of Pediatric Nephrology, Alberta Children's Hospital, University of Calgary, Calgary, Alberta, Canada, ${ }^{6}$ Department of Nephrology, Monash Medical Centre, Melbourne, Australia, ${ }^{7}$ Division of Nephrology, Department of Pediatrics, All India Institute of Medical Sciences, India, ${ }^{8}$ College of Medicine and Public Health, Flinders University, Adelaide, South Australia, Australia

Objective: To describe the perspectives on life participation by young adults with childhood-onset chronic kidney disease (CKD).

Methods: We conducted semi-structured interviews with 30 young adults aged 18-35 years diagnosed with CKD during childhood and across six countries (Australia, Canada, India, United Kingdom, United States of America and New-Zealand). Interview transcripts were analyzed thematically.

Results: We identified six themes: struggling with daily restrictions (debilitating symptoms and side effects, giving up valued activities, impossible to attend school and work, trapped in a medicalized life, overprotected by adults, and cautious to avoid health risks); lagging and falling behind (delayed independence, failing to keep up with peers, and socially inept); defeated and hopeless (incapacitated by worry, an uncertain and bleak future, unworthy of relationships, and low self-esteem and shame); reorienting plans and goals (focusing on the day to day, planning parenthood, and forward and flexible planning); immersing oneself in normal activities (refusing to miss out, finding enjoyment, determined to do what peers do, and being present at social events); and striving to reach potential and seizing opportunities (encouragement from others, motivated by the illness, establishing new career goals, and grateful for opportunities).

Conclusions: Young adults encounter lifestyle limitations and missed school and social opportunities as a consequence of developing CKD during childhood and as a consequence lack confidence and social skills, are uncertain of the future, and feel vulnerable. Some re-adjust their goals and become more determined to participate in "normal" activities to avoid missing out. Strategies are needed to improve life participation in young adult 'graduates' of childhood CKD and thereby strengthen their mental and social wellbeing and enhance their overall health.

\section{PI-168 VIRTUAL ART THERAPY: MEETING THE PSYCHOLOGICAL NEEDS OF CHILDREN AND YOUNG PEOPLE WITH CHRONIC KIDNEY DISEASE DURING THE COVID-19 PANDEMIC}

Eleanor Stubbs ${ }^{1}$, Sarah Shameti ${ }^{1}$, Mushfequr R Haq ${ }^{1}$, Rodney Gilbert ${ }^{1}$, Caroline Anderson ${ }^{1}$, Anne-sophie Darlington ${ }^{2}$, Alejandra Recio-saucedo ${ }^{2}$, Kirsty Edwards ${ }^{3}$, Arvind Nagra ${ }^{1}$

${ }^{I}$ Southampton Childrens Hospital, United Kingdom, ${ }^{2}$ University of Southampton, United Kingdom, ${ }^{3}$ Teapot Trust Charity, United Kingdom

Introduction: Children and young people (CYP) with chronic kidney disease (CKD) are known to have increased psychosocial needs. During the covid-19 pandemic psychological needs increased. A Children's Hospital, in partnership with the Teapot Trust Charity offered a virtual art therapy intervention to CYP with CKD to support these psychological needs. Objectives: To assess the effectiveness of virtual art therapy in CYP with CKD

Material and methods: The hospital MDT identified and shared referrals with the Teapot Trust art therapist. CYP completed a WarwickEdinburgh Mental Wellbeing Scale (WEMWBS) survey at the start and end of the intervention to assess impact on psychological wellbeing 31.8.20 - 21.12.2020: 8 CYP (aged 6-14 years) received virtual art therapy support. Sessions were delivered 1:1 $(\mathrm{N}=4)$ or group session $(\mathrm{N}=4)$. CYPs needs were identified at their initial session and a support plan put in place. Progress was reviewed after 8 weeks and sessions extended as needed. An enjoyment form was completed at the end of the intervention. Parent feedback about their child's well-being and enjoyment was collected at the end of the intervention.

Results: CYP: $\mathrm{N}=8$.

CYP received a mean of 8 (range $6-18$ ) sessions.

Mean WEMWBS score increased from 82.7 at baseline to 91.3 post intervention.

$100 \%$ showed improved self-esteem, improved mood, increased energy, motivation and increased relaxation.

Parents $(\mathrm{N}=5)$ completed the feedback forms. $100 \%$ responses were positive.

CYP said "it helped me understand all those feelings that were hidden for such a long time."

Parent feedback "the difference in R's mental health from when she started the art therapy and finished was like night and day"..... "is being very proactive in her own self-care, seemed happier ..........extremely pleased with the result".

Conclusions: Psychological needs of CYP with CKD can be supported by virtual art therapy. 


\section{PI-169 ADAPTING AND EVOLVING YOUTH WORK SUPPORT THROUGHOUT THE COVID-19 PANDEMIC}

Sarah Shameti ${ }^{1}$, Eleanor Stubbs ${ }^{1}$, Mushfequr R Haq ${ }^{1}$, Rodney Gilbert ${ }^{1}$, Caroline Anderson ${ }^{1}$, Anne-Sophie Darlington ${ }^{2}$, Alejandra Recio-Saucedo ${ }^{2}$, Arvind Nagra ${ }^{1}$

${ }^{1}$ Southampton Childrens Hospital, United Kingdom, ${ }^{2}$ University of Southampton, United Kingdom

Introduction: Children and Young people (CYP) with chronic kidney disease (CKD) are known to have increased psychosocial needs. Youth workers (YW) have a valuable place in providing a holistic approach to meet the psychosocial and needs of CYP (aged 11-18 years). Youth work delivered as part of the PEEER (building Patient, Empowerment, selfEsteem, Employability \& Resilience) project offers this support. Pre covid-19 YW support was delivered face to face (F2F) in a 1:1 setting or group sessions in the hospital, youth spaces or educational settings.

Due to the Covid-19 pandemic YW support was delivered virtually to meet the psychosocial needs of CYP with CKD

Objectives: To assess the effectiveness of PEEER project youth work delivered virtually compared to F2F youth work.

Material and methods: Group 1: Pre-Covid-19 lockdown: (1.1.2020 22.3.2020) CYP received YW support F2F

Group 2: During Covid-19 lockdown:(23.3.2020 - 31.7.2020) CYP received YW support virtually.

CYP in both groups received 1:1 sessions, fun group activities and workshops.

Feedback on effectiveness of sessions was collected $(1=$ strongly disagree $-6=$ strongly agree) - mean scores were collated

Results: Group $1 \mathrm{~N}=105$. Group $2 \mathrm{~N}=236$. The demand for YW support increased by $69 \%$ per month during the covid-19 pandemic. In Group 2 there was a 56\% increase in CYP accessing PEEER events.

CYP in Group 1 and Group 2: 100\% said they enjoyed the sessions (5.6 vs 5.3), would like to attend future sessions (5.5 vs 5.5), felt part of a friendship group 5.2 vs 4.3 ), felt more confident (5 vs 4.17), felt more independent (4.68 vs 5.0), felt good about themselves (4.88 vs 4.0$)$ and learnt a new skill (4.5 vs 4.0)

Conclusions: Psychosocial needs of CYP with CKD can be effectively met through the virtual delivery of YW support.

\section{PI-170 ILLNESS-RELATED PARENTAL STRESS, QUALITY OF LIFE AND PROBLEM BEHAVIOR IN CHILDREN WITH CHRONIC KIDNEY DISEASE}

Lore Willem ${ }^{1}$, Noël Knops ${ }^{1}$, Djalila Mekahli ${ }^{1}$, Elke De Bruyne ${ }^{2}$, Johan Vande Walle ${ }^{2}$, Ann Raes ${ }^{2}$, Sarah Reynaert ${ }^{3}$, Dominique Trouet ${ }^{3}$, Koen Van Hoeck ${ }^{3}$, Sylvie Vankerckhove ${ }^{4}$, Joy Assenmaker ${ }^{4}$, Brigitte Adams ${ }^{4}$, Stephanie Leroi ${ }^{5}$, Laure Collard ${ }^{5}$, Aline Michaux ${ }^{6}$, Nathalie Godefroid ${ }^{6}$, Elena Levtchenko ${ }^{1}$

${ }^{1}$ University Hospital Leuven, ${ }^{2}$ University Hospital Ghent, ${ }^{3}$ University Hospital Antwerpen, ${ }^{4}$ Huderf Brussels; ${ }^{5}$ Groupe Sante Chc Liege, Universite Catholique De Louvain

Introduction: Tertiary centers of pediatric nephrology in Belgium provide multidisciplinary care for children with chronic kidney disease (CKD). This care is essential as studies have shown that CKD has an impact on the psychosocial development of these children and their families. Many studies have focused on QoL. However, less is known about the illness-related stress of parents and its relation with child psychosocial functioning. Therefore the aim of the study was to investigate (1) illnessrelated stress in parents of children with CKD with and without kidney transplantation (Tx) and (2) explore associations between illness-related parental stress, QoL and problem behavior in the child.

Material and methods: QoL was assessed by means of PedsQL Core 4.0, child problem behavior by means of the Child Behavior Checklist
(CBCL) and Illness-related parental stress by means of Pediatric Inventory for Parents (PIP).

Results: In total 49 children (30 boys; $M$ age $=10.06$, range $0-18$ ) and their parents were included. Parents of children with Tx reported higher illnessrelated stress $(p<.05)$, compared to parents of children without Tx. Results show negative correlations between PIP and PedsQL $(p<0.01)$ and positive correlations between PIP and CBCL $(p<.01)$.

Conclusions: Our study show that CKD also affects the parents, and especially parents of children with a Tx. Parental stress, child QoL and problem behavior are also interrelated. These results show the importance of a multidisciplinary team that focus on the entire family of children with CKD.

\section{PI-171 PSYCHOSOCIAL CHALLENGES FOR PARENTS OF CHILDREN WITH CHRONIC KIDNEY DISEASE}

Elke De Bruyne ${ }^{1}$, Sunny Eloot ${ }^{2}$, Johan Vande Walle ${ }^{3}$, Ann Raes ${ }^{3}$, Wim Van Biesen ${ }^{2}$, Liesbeth Goubert ${ }^{4}$, Eline Van Hoecke ${ }^{1}$, Evelien Snauwaert $^{3}$

${ }^{1}$ Pediatric Psychology, Department Of Pediatrics, Ghent University Hospital, Belgium, ${ }^{2}$ Department Of Nephrology, Ghent University Hospital, Belgium, ${ }^{3}$ Department Of Pediatric Nephrology \& Rheumatology, Ghent University Hospital, Belgium, ${ }^{4}$ Department Of Experimental-clinical And Health Psychology, Ghent University, Belgium

Introduction: Little is known about the impact of pediatric chronic kidney disease (CKD) on the parents. This study aims to explore the psychosocial functioning in this parent group.

Material and methods: Fourthy-four parents (32 mothers) of children with CKD completed our psychosocial questionnaires. Parental stress was evaluated by the Parenting Stress Index - Short Form (PSI-SF), anxiety and depression were measured using the Hospital Anxiety and Depression Scale (HADS). The Child Vulnerability Scale (CVS) and Parent Overprotection Measure (POM) were used to explore parents' perception of child's vulnerability and protective behavior, respectively. A matched control group of parents of healthy children was used for comparison.

Results: Since the CKD diagnosis, $47 \%$ of the parents perceived a deterioration of their own health, and in $38 \%$ of the families at least one of the parents reduced work activities. Averagely parents took 17 days of work leave in the last 6 months because of the child's treatment. Compared to our control group, parents of children with CKD reported higher stress levels (59.6 versus 43.5 ; $p<0.001$; normal range $43-61$ ) and more symptoms of anxiety ( 7.8 versus $5.1 ; p<0.05$; normal range $0-7)$ and depression (5.3 versus $3.7 ; \mathrm{p}<0.05$; normal range $0-7$ ). Significantly higher scores on child vulnerability perception $(8.6$ versus $2.5 ; \mathrm{p}<$ $0.001)$ and protective behaviour $(41.7$ versus $36.8 ; p<0.05)$ could be measured in parents of children with CKD.

Conclusions: Parents of CKD patients report more health problems and a significant professional impact. Compared to parents of healthy children, a higher presence of stress, anxiety and depression symptoms can be seen. Also, these parents perceive their child as more vulnerable and use more protective behavior towards their child. As the impact of CKD goes beyond the child and affects the entire family, a multidisciplinary family-based therapy should be recommended.

\section{PI-172 DIAGNOSTIC PERFORMANCE OF URINE DIAGNOSTICS IN CHILDREN WITH A SUSPECTED URINARY TRACT INFECTION}

Stephanie Middelkoop ${ }^{1}$, Janneke Ludwig-Roukema ${ }^{2}$, Valentina Gracchi ${ }^{2}$, Elizabeth Legger ${ }^{3}$, Nataliya Hilt ${ }^{4}$, Joost Van Pelt ${ }^{5}$, Coen Stegeman ${ }^{1}$, Martine Besouw ${ }^{2}$

${ }^{1}$ University Of Groningen, University Medical Center Groningen, Department Of Internal Medicine, Division Of Nephrology, Groningen, 
The Netherlands, ${ }^{2}$ University Of Groningen, University Medical Center Groningen, Department Of Pediatric Nephrology, Groningen, The Netherlands, ${ }^{3}$ University Of Groningen, University Medical Center Groningen, Department Of Pediatric Infectious Diseases, Rheumatology And Immunology, Groningen, The Netherlands, ${ }^{4}$ University Of Groningen, University Medical Center Groningen, Department Of Medical Microbiology And Infection Prevention, Groningen, The Netherlands, ${ }^{5}$ University Of Groningen, University Medical Center Groningen, Department Of Laboratory Medicine, Groningen, The Netherlands

Introduction: The diagnosis of urinary tract infection (UTI) can be challenging in children. Many studies are based on laboratory results alone and exclude patients with underlying urological abnormalities, hence they do not represent general practice. We investigated the performance of UTI screening methods in a broad pediatric population.

Material and methods: A 3 year period cohort study in children with suspected UTI, in whom urine dipstick, automated urinalysis (UF-1000i) and urine culture was performed. Diagnostic performance was assessed for the total population and separately for children $<3$ months (group 1), $\geq 3$ months without clean intermittent self-catheterisation (CIC) (group 2), $\geq 3$ months with CIC (group 3). Diagnosis of UTI was based on urine culture, symptomatology and the conclusion of the treating physician.

Results: 292 periods were included, UTI was diagnosed in $74(25.3 \%)$, including 6/50 (12.0\%) in group 1, 56/211 (26.5\%) in group 2 and 12/31 $(38.7 \%)$ in group 3. Dipstick: sensitivity and specificity for leucocyte esterase $3+$ was $64.9 \%$ and $83.0 \%$ for all children, $66.7 \%$ and $88.6 \%$ in group 1, $60.7 \%$ and $85.2 \%$ in group 2, and $83.3 \%$ and $52.6 \%$ in group 3 . For nitrite it was $33.8 \%$ and $91.7 \%$ for all children, $16.7 \%$ and $100 \%$ in group $1,39.3 \%$ and $91.6 \%$ in group 2, and $16.7 \%$ and $73.7 \%$ in group 3. Automated urinalysis: at $90 \%$ sensitivity, the leucocyte cut-off was $13 / \mu \mathrm{L}$ for all children (specificity $22.9 \%$ ), $15 / \mu \mathrm{L}$ in group 2 (specificity $27.1 \%$ ), and $70 / \mu \mathrm{L}$ in group 3 (specificity $36.8 \%$ ). Bacterial count cut-off was $63 / \mu \mathrm{L}$ for all children (specificity $62.4 \%$ ), $98 / \mu \mathrm{L}$ in group 2 (specificity $65.2 \%$ ), and $54 / \mu \mathrm{L}$ in group 3 (specificity $36.8 \%$ ). Cut-off values could not be determined for group 1 .

Conclusions: Diagnostic performance of urinary screening parameters in children is moderate, especially $<3$ months, emphasizing the need to incorporate clinical presentation when diagnosing UTI. Specific cut-off values for children with CIC could be useful.

\section{PI-173 EXOME SEQUENCING IMPLICATES HETEROZYGOUS VARIANTS IN DSTYK IN FUNCTIONAL URINARY BLADDER DISTURBANCE}

Clara Vidic ${ }^{1}$, Marcin Zaniew ${ }^{2}$, Holger Thiele ${ }^{3}$, Janine AltmÜller ${ }^{3}$, Heiko Reutter ${ }^{4}$, Alina Hilger ${ }^{4}$

${ }^{1}$ Institute of Human Genetics, University of Bonn, Germany, ${ }^{2}$ Department of Pediatrics, University of Zielona Góra, Poland, ${ }^{3}$ Cologne Center For Genomics, University of Cologne, Germany, ${ }^{4}$ Department of Pediatrics, Children's Hospital, University of Bonn, Germany

Introduction: $D S T Y K$ encodes dual serine/threonine and tyrosine protein kinase. DSTYK has been associated with autosomal dominant congenital anomalies of the kidney and urinary tract and with autosomal-recessive hereditary spastic paraplegia. Here we report a father and his two dizygotic twin sons with a novel, heterozygous variant in DSTYK, all presenting with voiding dysfunction due to functional urinary bladder disturbance.

Materials and Methods: We performed whole exome sequencing of the family. All three presenting clinically with hesitancy, abnormal voiding pattern and night incontinence till adolescence. In the sons cystoscopy excluded urethral valves but showed hypertrophy of the bladder neck and trabeculated bladder. Additionally, both sons were diagnosed with epilepsy. Based on the pedigree, filtering of exome data focused on rare $(\mathrm{MAF}<0.01 \%$ ), autosomal-dominant variants, predicted to be deleterious, residing in highly conserved regions of the exome. Validation of prioritized variants was performed using Sanger sequencing.

Results: We identified a novel, heterozygous c.271C $>$ A (p.Leu91Met) variant in DSTYK segregating with the disease. The amino acid is highly conserved. Rated deleterious by 3 different prediction programs (SIFT, PolyPhen, MutationTaster) and with a CADD score of 24.5, this variant was prioritized as likely disease causing.

Conclusion: To the best of our knowledge, we describe the first familial case with autosomal dominant inherited variants in DSTYK and specific functional bladder outlet obstruction and epilepsy. PI-174 THE ROLE OF PRO-ADRENOMEDULLIN IN THE
DIAGNOSIS OF URINARY TRACT INFECTION IN
CHILDREN AND COMPARISON WITH C-REACTIVE PROTEIN, PROCALCITONIN, BLOOD LEUKOCYTE COUNT

Șeyma Köksal Atiș , Asuman Kiral, Nilüfer Göknar

Istanbul Medeniyet University Göztepe Prof. Dr. Süleyman Yalçin City Hospital

Introduction: Urinary system infections (UTI) are one of the most common bacterial infections in childhood. UTI should be diagnosed quickly and treatment should be initiated as soon as possible due to its significant morbidity and mortality. In this study, we aimed to investigate the diagnostic value of Pro-adrenomedullin in children with UTI and compare with Procalcitonin, C-Reactive Protein and blood leukocyte count.

Material and methods: In this prospective study we enrolled 106 children (62 girls) with a diagnosis of UTI and 31 (21 girls) controls. Urinalysis, complete blood count, Procalcitonin, C-Reactive Protein, BUN, creatinine and, urine culture was done. Venous blood samples for the analysis of Pro-adremonedullin were obtained from all patients before starting antibiotic therapy and one week later.

Results: The patients with UTI had higher Pro-Adrenomedullin levels compared to control group $(\mathrm{p}=0.001)$. The diagnostic accuracy for predicting the UTI, reflected by the area-under-the-curve of receiver operating characteristics (ROC) were: Pro-adrenomedullin 0,91 (95\% CI $0,861-0,971)$ and, procalcitonin 0,94 (95\% CI 0,904-0,989). The ROC analysis showed a cut-off value of $11679 \mathrm{pmol} / \mathrm{l}$ for Pro-adrenomedullin. We showed that the risk of UTI was 33,8 times higher in children with Pro-adrenomedullin level above $11679 \mathrm{pmol} / \mathrm{l}$. Based on the cut-off values obtained for Proadrenomedullin and Procalcitonin, we demonstrated that the probability of predicting the diagnosis of UTI was 189.8 times higher when both biomarkers were used. Also, Pro-adrenomedullin levels at initial diagnosis were well correlated with Procalcitonin $(r=0,470$, $\mathrm{p}=0.001), \mathrm{CRP}(\mathrm{r}=0.395, \mathrm{p}=0,001)$ and leukocyte count $(\mathrm{r}=0,387$, $\mathrm{p}=0.001)$. Although Procalcitonin, CRP and leukocyte count decreased one week after treatment $(\mathrm{p}=0,001)$, there was no statistically significant difference in terms of Pro-adrenomedullin levels $(\mathrm{p}>0.05)$.

Conclusions: Our results demonstrated that Pro-adrenomedullin is a powerful biomarker in the diagnosis of UTI and increases the probability of predicting the diagnosis when used with other biomarkers but not useful for follow-up.

PI-175 IS A DIFFERENT FOLLOW-UP PROCEDURE NECESSARY FOR INFANTS WITH FIRST FEBRILE URINARY TRACT INFECTION CAUSED BY NON-E.COLI AND/OR EXTENDED-SPECTRUM B-LACTAMASE-PRODUCING BACTERIA?

Mustafa Kavruk ${ }^{1}$, Eren Soyaltin ${ }^{2}$, Gökçen Erfidan ${ }^{2}$, Seçil Arslansoyu Çamlar ${ }^{3}$, Demet Alaygut ${ }^{2}$, Fatma Mutlubaş ${ }^{3}$, Nisel Yilmaz ${ }^{4}$,

Belde Kasap Demir ${ }^{5}$

${ }^{1}$ University Of Health Sciences, Tepecik Training And Research Hospital, Department Of Pediatrics, Izmir, Turkey, ${ }^{2}$ University Of 
Health Sciences, Tepecik Training And Research Hospital, Department Of Pediatrics, Division Of Nephrology, Izmir, Turkey, ${ }^{3}$ University of Health Sciences, Izmir Faculty Of Medicine, Department Of Pediatrics, Division Of Nephrology, Izmir, Turkey, ${ }^{4}$ University Of Health Sciences, Tepecik Training And Research Hospital, Department Of Clinical Microbiology, Izmir, Turkey, ${ }^{5}$ Izmir Katip Celebi University, Faculty Of Medicine, Department Of Pediatrics, Division Of Nephrology And Rheumatology, Izmir, Turkey

Introduction: We aimed to evaluate the effects of non-E.coli or extended-spectrum beta-lactamase-producing bacterial (ESBL-PB) growth in the first febrile UTI of infants

Material and methods: The data of patients aged between 2-24 months and were followed up for at least 6 were retrospectively analyzed. Ultrasonography was performed in all cases at the time of the infection and dimercaptosuccinic acid (DMSA) at least 4 months after the infection. Voiding cystourethrography (VCUG) was performed only if ultrasonography findings were suggestive of vesicoureteral reflux (VUR), the up-take deformity was detected in DMSA scan or the patients experienced recurrent UTIs. The patients were grouped concerning E.coli or non-E.coli and ESBL$\mathrm{PB}$ or non-ESBL-BP growth in urine cultures.

Results: There were 277 (M/F:153/124) infants followed up for $28.55 \pm 15.24$ (6-86) months. The causative microorganisms were non-E.coli in 73 (26.4\%); and ESBL-PB in 58 (20.9\%) cases. CRP values, pyuria, and leukocyte esterase positivity were significantly higher in UTIs caused by E.coli compared to non-E.coli bacteria $(\mathrm{p}=0.027, \mathrm{p}<0.001$ and $\mathrm{p}<0.001$, respectively). All clinical and laboratory findings were similar between the ESBL-PB and non-ESBL groups, but abnormal ultrasonography findings were more common in the non-E.coli group $(\mathrm{p}=0.029)$. The white blood cell count was significantly higher in patients with VUR compared to those without VUR $(\mathrm{p}=0.012)$. Multivariate analysis revealed that leukocyte esterase positivity $(\mathrm{p}=0.024)$ and high-grade VUR $(\mathrm{p}=0.004)$ were independent risk factors for renal damage in DMSA scan.

Conclusions: Prioritizing the date of further radiological examinations would not be mandatory, just because the patients are infected with nonE.coli or ESBL-PB, as long as all they undergo urinary USG after the first febrile UTI.

\section{PI-176 HOW DOES FEBRILE NEUTROPENIA AFFECT THE PRESENCE AND CLINIC OF URINARY TRACT INFECTION IN CHILDREN?}

Gökçen Erfidan ${ }^{1}$, Deniz Kızmazoğlu ${ }^{2}$, Özgür Özdemir Șimșek ${ }^{1}$, Cemaliye Başaran ${ }^{1}$, Seçil Arslansoyu Çamlar ${ }^{3}$, Fatma Mutlubaș ${ }^{3}$, Belde Kasap Demir ${ }^{4}$, Demet Alaygut ${ }^{1}$

${ }^{1}$ University Of Health Sciences, Tepecik Training And Research Hospital, Department Of Pediatrics, Division Of Nephrology, Izmir, Turkey, ${ }^{2}$ University Of Health Sciences, Tepecik Training And Research Hospital, Department Of Pediatrics, Division Of Hematology And Oncology, Izmir, Turkey, ${ }^{3}$ University Of Health Sciences, Izmir Faculty Of Medicine, Department Of Pediatrics, Division Of Nephrology, Izmir, Turkey, ${ }^{4}$ Izmir Katip Celebi University, Faculty Of Medicine, Department Of Pediatrics, Division Of Nephrology And Rheumatology, Izmir, Turkey

Introduction: Febrile neutropenia (FN) is a common life-threatening infectious complication of cancer chemotherapy. In approximately $8 \%$ of pediatric FN patients, urinary tract infection (UTI) has been reported. Clinical findings of UTI may seen obscure due to low infection response, and leukocyturia may not present in FN. This study aims to analyze the urine culture (UCx) results of children who were hospitalized with FN.
Material and methods: The episodes of FN in children hospitalized in Pediatric Hematology-Oncology Clinic during one year of period were analyzed retrospectively. Demographic, clinical and laboratory results were noted. Patients without UCx on admission were excluded. Urine samples were collected via midstream urination or urinary bag. UCx considered positive and diagnosed UTI, if $\geq 10^{5} \mathrm{cfu} / \mathrm{ml}$ of a single urine pathogen was found.

Results: Thirty-four patients with $53 \mathrm{FNs}$ (79.1\%) were included. In $28 \%$ of the FN episodes, a microbial growth on urine culture was detected. The most common isolation was Klebsiella spp $(66.7 \%)$. The mean age in UTI group was smaller. In $80 \%$ of the episodes with UTI, the patients were younger than 4 years old. None of the patients with abdominal/flank pain had diagnosed UTI. Only one in 2 patients with dysuria had UTI. Also, in $21 \%$ of the episodes with UTI, the patients had some symptoms suggestive of other sites of infection. Leukocyte esterase and nitrite reactions were negative in all study group. There was a significant negative correlation with the frequency of UTI and neutropenia level. C-reactive protein level was found lower in the UTI group.

Conclusions: In this study, the frequency of UTI in FN episodes was three times higher compare to literature. The most commonly isolated microorganism was Klebsiella spp. During FN episodes, most patients with UTIs didn't have specific symptom, and pyuria may not present. Therefore, if $\mathrm{UCx}$ is not taken routinely, the diagnosis can easily be missed.

\section{PI-177 EVALUATION OF PATIENTS WITH A DIAGNOSIS OF ECTOPIC KIDNEY}

Özgür Özdemir Șimșek ${ }^{1}$, Gökçen Erfídan ${ }^{1}$, Cemallye BaȘaran ${ }^{1}$, Seçill Arslansoyu Çamlar ${ }^{2}$, Demet Alaygut ${ }^{1}$, Fatma Mutlubaș ${ }^{2}$, Belde Kasap Demİ ${ }^{3}$

${ }^{1}$ University Of Healthy Sciences Tepecik Training And Research Hospital Department Of Pediatrics, Division Of Nephrology, Izmir, Turkey, ${ }^{2}$ University Of Health Sciences, Izmir Faculty Of Medicine Department Of Pediatrics, Division Of Nephrology, Izmir, Turkey, ${ }^{3}$ Izmir Katip Çelebi University Medical Faculty, Department Of Pediatrics, Division Of Nephrology And Rheumotology, Izmir, Turkey

Introduction: We aimed to evaluate the accompanying urological and urinay anomalies including vesicoureteral reflux(VUR), hypoplasia, urinary tract infection(UTI), hematuria and proteinuria and long-term results of patients who were followed up with a diagnosis of ectopic kidney (EK).

Material and methods: The files of the patients with EK followed up between 2008-2020 in the outpatient clinics were retrospectively reviewed. Gender, age at presentation, complaints, urinary findings (hematuria, proteinuria), and radiological imaging of the patients were evaluated.

Results: The mean age at presentation of 78 cases $(\mathrm{F} / \mathrm{M}=41 / 37)$ was 53.42 $\pm 58.5(0-274.03)$ months. $45 \%(n=35)$ of the patients were diagnosed incidentally, while $22 \%(n=17)$ presented with abdominal pain, $15 \%(n=12)$ had antenatal history, $12 \%(\mathrm{n}=9)$ had UTI and $\% 6.5(\mathrm{n}=5)$ of them applied for voiding disorder. $69 \%(\mathrm{n}=54)$ of the cases had pelvic, $11 \%(\mathrm{n}=9)$ had cross $\mathrm{EK}$, and $19 \%(\mathrm{n}=15)$ had horseshoe kidney. $24 \%(\mathrm{n}=19)$ of the patients had recurrent UTI. VUR was detected in 9 of these cases. In $35 \%$ of the patients $(n=25)$, EKs were hypoplastic. The frequency of hematuria was statistically significantly higher in patients without pelvic EKs $(p=0.039)$. No significant difference was found in terms of age at presentation, follow-up time, current age, size difference between two kidneys on USG between patients with and without hematuria and between those with and without proteinuria. The fractional functions of the kidneys in DMSA were similar between patients with and without hematuria, while having $>10 \%$ difference between the two kidneys in DMSA was significantly higher in patients with proteinuria $(\mathrm{p}=0.023)$.

Conclusions: Recurrent UTI and VUR can be seen in patients with renal ectopia. Hematuria in non-pelvic EKs and kidneys with high differential difference in DMSA are more common in patients with proteinuria. 
PI-178 DIAGNOSTIC YIELD AND BENEFITS OF WHOLEEXOME SEQUENCING IN PATIENTS WITH CONGENITAL ANOMALIES OF THE KIDNEY AND URINARY TRACT (CAKUT) DIAGNOSED IN THE FIRST THOUSAND DAYS OF LIFE

Lina Werfel ${ }^{2}$, Helge Martens ${ }^{1}$, Imke Hennies ${ }^{2}$, Anne Christians ${ }^{1}$, Ann Christin Gjerstad ${ }^{3}$, Kerstin Fröde ${ }^{2}$, Barbara Magda Ludwikowski ${ }^{4}$, Robert Geffers ${ }^{5}$, Heiko Billing ${ }^{6}$, Martin Kirschstein ${ }^{7}$,

Alejandro D. Hofmann ${ }^{8}$, Anna Bjerre ${ }^{3}$, Dieter Haffner ${ }^{2}$,

Ruthild G. Weber ${ }^{1}$

${ }^{1}$ Department Of Human Genetics, Hannover Medical School, Hannover, Germany, ${ }^{2}$ Department Of Pediatric Kidney, Liver And Metabolic Diseases, Hannover Medical School, Hannover, Germany, ${ }^{3}$ Division Of Paediatric And Adolescent Medicine, Oslo University Hospital, Oslo, Norway, ${ }^{4}$ Department Of Pediatric Surgery And Urology, Auf Der Bult Kinder- Und Jugendkrankenhaus, Hannover, Germany, ${ }_{5}^{5}$ Genome Analytics Research Group, Helmholtz Centre For Infection Research, Braunschweig, Germany, ${ }^{6}$ Department Of Paediatrics I, University Children's Hospital, Tübingen, Germany, ${ }^{7}$ Department Of Pediatrics, General Hospital, Celle, Germany, ${ }^{8}$ Department Of Pediatric Surgery, Hannover Medical School, Hannover, Germany

Introduction: Congenital anomalies of the kidney and urinary tract (CAKUT) are the predominant cause of chronic kidney disease (CKD) in children and adolescents. CAKUT can occur in an isolated form or with extrarenal comorbidities. Although over 50 genes are known to cause CAKUT if mutated, the diagnostic yield of whole-exome sequencing (WES) studies is typically lower than $15 \%$. Here, we asked for the diagnostic yield in young CAKUT patients and whether an early genetic diagnosis may impact patient management.

Material and methods: In 100 patients diagnosed with CAKUT in the first 1,000 days of life, WES was performed and variants in 58 established CAKUT-associated genes were extracted and classified according to the ACMG guidelines. The translational value of the genetic findings was assessed.

Results: In $26 \%$ of patients diagnosed with CAKUT early in life, we identified a likely pathogenic (LP) or pathogenic $(\mathrm{P})$ rare variant in one or two of 17 CAKUT-associated genes, including HNF1B, LIFR, SALL1 and $U M O D$. Of the 24 different variants detected, 12 were loss-offunction and five de novo variants. Thirteen of the affected genes were potentially associated with extrarenal morbidities, e.g. diabetes mellitus or hyperuricemia. Patients with $\mathrm{LP} / \mathrm{P}$ variants were significantly more likely to have end-stage CKD under three years of age and extrarenal manifestations than those without.

Conclusions: Our data demonstrate a relatively high diagnostic yield of WES in children diagnosed with CAKUT early in life, particularly with end-stage CKD and extrarenal manifestations, and suggest a benefit for managing comorbidities (Else Kröner-Fresenius-Stiftung, grant no. 2018_Kolleg.12).

\section{PI-179 A NATIONAL SURVEY OF MANAGEMENT OF UNILATERAL MULTICYSTIC DYSPLASTIC KIDNEY (MCDK) IN THE UNITED KINGDOM}

\author{
Douglas Stewart ${ }^{1}$, Matthew Harmer ${ }^{2}$, Pallavi Prasad ${ }^{3}$, \\ Faidra Veligratli ${ }^{1}$, Charles Pickles ${ }^{6}$, Ji Soo Kim ${ }^{4}$, Maduri Raja ${ }^{5}$ \\ ${ }^{1}$ Evelina London Childrens Hospital, ${ }^{2}$ Bristol Royal Hospital For \\ Children, ${ }^{3}$ Alder Hey Childrens Hospital, ${ }^{4}$ Great Ormond Street \\ Childrens Hospital, ${ }^{5}$ Southampton Childrens Hospital, ${ }^{6}$ Great North \\ Childrens Hospital
}

Objectives: To explore variation in management of unilateral MCDK amongst paediatricians.
Methods: A nationwide online survey, with multiple choice and free text questions, was open to tertiary paediatric nephrology and general paediatric consultants between August and December 2020.

Results: Of 60 respondents, two-thirds were paediatric nephrologists. $62 \%$ routinely perform a DMSA scan to confirm diagnosis (paediatric nephrologists [58\%]; specialist interest paediatricians [67\%]; nonspecialist interest paediatricians [80\%]). $8 \%$ routinely perform a cystogram to investigate contralateral vesicoureteric reflux. $62 \%$ would undertake routine renal function assessment with frequency ranging from once only $(62 \%)$ to "every two years". Nephrectomy thresholds quoted were: "still present at $3 / 4$ years", "still present at 5 years", " $3 \mathrm{~cm}$ at 5 years", and " $6 \mathrm{~cm}$ at 6 years". $25 \%$ recalled MCDK nephrectomy within the previous five years. Respondents voiced concerns that national guidance may result in an overcautious approach but could (1) balance between consensus and safe variation, and (2) offer families choice and reassurance.

Estimated costs were calculated from the respondents' answers. Mean estimated cost from birth to 18 years was $£ 1,962$ (range: $£ 258$ - $£ 3,854$ ). Although the average costs were greater for general paediatricians compared to paediatric nephrologists, this was not statistically significant ( $11,950 \mathrm{SD} \pm £ 871$ versus $£ 1,485 \mathrm{SD} \pm £ 829$ ).

Conclusions: Management of unilateral MCDK varies amongst UK paediatricians. Despite consensus for avoiding invasive testing, the risks of hypertension and progression to chronic kidney disease remains. This highlights the need for a clear pathway to decrease unwanted variability to ensure early recognition of those at high-risk of renal sequelae whilst avoiding undue investigatory burden on the patient.

\section{PI-180 EARLY RENAL ULTRASOUND IN CONGENITAL SOLITARY KIDNEY MAY HELP TO SELECT PATIENTS AT LOWER RISK OF ASSOCIATED VESICO-URETERAL REFLUX}

Pierluigi Marzuillo, Anna Di Sessa, Rossella Francesca De Simone, Davide Ursi, Michele Sabatino, Maria Cecilia Russo, Emanuele Miraglia Del Giudice, Stefano Guarino Department Of Woman, Child And Of General And Specialized Surgery, Università Degli Studi Della Campania "luigi Vanvitelli", Via Luigi De Crecchio 2, 80138, Naples, Italy

Introduction: The identification of vesico-ureteral reflux (VUR) in children with congenital solitary functioning kidney (CSFK) identifies patients at risk of kidney injury but exposes to radiations and pain. VUR may be associated with renal dysplasia and reduced renal length (RL). Our hypothesis was that RL $>2$ standard deviation score (SDS) in the first months of life (renal hyperplasia) could identify patients with CSFK with lower probability of presenting VUR. We also hypothesized that the utility of RL in selecting patients with lower risk of VUR is limited to the first months of life because -later- the compensatory renal growth of CSFK could not allow to distinguish patients with renal hyperplasia from those with renal hypertrophy. We aimed testing our hypotheses.

Material and methods: We retrospectively selected 207 CSFK patients with prenatal diagnosis of CSFK and having undergone renal ultrasound (RUS) both at 0-3 and 10-13 months of life, renal scintigraphy and cystourethrography/cystoscintigraphy. We compared the cumulative proportion of RL $>2$ SDS by Kaplan-Meier analysis and evaluated the odds to present VUR of patients with RL $>2$ SDS both at first and second RUS. Results: RL $>2$ SDS at first RUS was shown by $3.3 \%$ of patients with VUR and $22.0 \%$ of patients without VUR $(\mathrm{p}=0.02)$. At the second RUS, RL $>2$ SDS was presented by $53.3 \%$ of patients with VUR and $52.5 \%$ of patients without VUR ( $\mathrm{p}=0.93$ ). The cumulative proportion of RL $>2$ SDS at 3months of life was higher in patients without than in those with VUR ( $\mathrm{p}=0.02)$. This difference however, disappeared at 11 and 13 months of age ( $\mathrm{p}=0.17$ and $\mathrm{p}=0.54$, respectively). A RL $>2$ SDS within 3 months predicted absence of VUR $(\mathrm{OR}=0.12 ; 95 \% \mathrm{CI}, 0.02-0.92 ; \mathrm{p}=0.005)$ 
while a RL $>2$ SDS at 12 months of life presented an OR for VUR of 0.96 (95\% CI, $0.45-2.1 ; \mathrm{p}=0.93)$.

Conclusions: A RUS made early in life (within 3months) identifies a population of CSFK patients at lower risk of presenting an associated VUR.

\section{PI-181 THE PREDICTIVE VALUE OF THE RENAL LENGTH TOWARD KIDNEY INJURY IN PATIENTS WITH CONGENITAL SOLITARY KIDNEY IS TIME-DEPENDENT}

Pierluigi Marzuillo, Stefano Guarino, Simona Riccio, Anna Di Sessa, Rosa Melone, Emilia Pirozzi, Ivan Maddaluno, Emanuele Miraglia Del Giudice, Cesare Polito

Department Of Woman, Child And Of General And Specialized Surgery, Università Degli Studi Della Campania "luigi Vanvitelli", Via Luigi De Crecchio 2, 80138, Naples, Italy.

Introduction: Since a high percentage of congenital solitary functioning kidney (CSFK) in adulthood present a RL $>2$ SDS, we hypothesized that the utility of renal ultrasound (RUS) to obtain prognostic information is time-dependent, with the highest predictivity at birth and later progressive reduction. In fact, more precocious RUS is, higher could be the probability to identify patients born with renal hyperplasia (higher nephron number). To test our hypothesis and to evaluate the prognostic value toward kidney injury (KI) of RUS during childhood we made a post-hoc analysis of one cohort of CSFK followed-up from birth to adulthood.

Material and methods: CSFK patients followed-up between 19932020 , aged 1-3months at first observation and having reached adulthood at last observation were enrolled. The patients attended to our observation yearly until 5years of age and then every 2years. If presenting KI, the returned yearly after KI detection. Data about renal length(RL) at each follow-up visit were collected. The RL standard deviation score(SDS) were calculated. KI was defined by hypertension and/or proteinuria and/or reduced estimated glomerular filtration rate. The prognostic value of RL $>2$ SDS at each follow-up visit was evaluated by logistic regression.

Results: We evaluated 56patients(20females) with a mean age at last follow-up of 21.1years(3.4SDS)(range:18-33years). KI was found in $15 / 56$ patients $(26.8 \%)$ with a mean age at KI onset of 20.9 years $(3.4$ SDS)(range:18-26years). Increasing the age, the prevalence of RL $>2$ SDS constantly increased reaching $85.7 \%$ in adulthood. A RL $>2$ SDS was predictive of absence of KI both at 1-3months (none of the patients with RL>2SDS at this age presented $\mathrm{KI}$ in adulthood) and 1year of age $(\mathrm{OR}=0.13 ; 95 \% \mathrm{CI}: 0.03-0.66 ; \mathrm{p}=0.01)$. At the following RUS, RL $>2$ SDS was not useful to predict absence of kidney injury in adulthood.

Conclusions: The predictive value of RUS is time-dependent. A RL $>2$ SDS at 1-3months and 1year of life predicts lower risk of KI. The predictive value of RUS reduces later in life.

\section{PI-182 THE SPECTRUM OF KIDNEY FUNCTION ALTERATIONS IN ADOLESCENT WITH A SOLITARY FUNCTIONING KIDNEY.}

Mathilde Grapin ${ }^{1}$, Nathalie Biebuyck ${ }^{1}$, FranÇois Gaillard ${ }^{2}$, Melissa Ould-rabah ${ }^{4}$, Carole Hennequin ${ }^{1}$, Romain Berthaud ${ }^{3}$, Guillaume Dorval $^{3}$, Thomas Blanc ${ }^{3}$, Nicolas Garcelon ${ }^{3}$,

Frank Bienaimé ${ }^{1}$, Olivia Boyer ${ }^{1}$

${ }^{1}$ Service De Néphrologie Pédiatrique, Centre De Référence Marhea, Hôpital Necker-enfants Malades, Assistance Publique-hôpitaux De Paris, Paris France, ${ }^{2}$ Université De Paris, Institut Necker-enfants Malades Inserm U1151, Paris, France, ${ }^{3}$ Institut Imagine, Inserm U1163, Université De Paris, Paris, France, ${ }^{4}$ Service De Physiologie , Hôpital Necker-enfants Malades, Assistance Publique-hôpitaux De Paris, Paris, France
Introduction: A precise assessment of glomerular filtration rate is key to delineate the care of children with a solitary functioning kidney (SFK). Data regarding measured GFR (mGFR) in this population is restricted to a single study of 77 individuals, which suggested that a GFR estimation (eGFR) method based on creatinine and cystatin C (eGFR-CKiD2) performed better than Schwartz's equation (eGFR-Schwartz).

Material and methods: We measured GFR in 210 consecutive adolescents ( 7 to 22 years old) with a SFK referred to our institution between 2014 and 2019 and in 43 young candidates to kidney donation (18 to 25 year old). We compared the distribution of MGFR in both groups and determined the factors associated with reduced $\mathrm{mGFR}$ in adolescent with a SFK. We further compared different eGFR formulas with mGFR and assessed the association of mGFR and eGFRs with PTH and FGF23, two early indicators of GFR reduction.

Results: While adolescent with a SFK had a similar median mGFR than healthy controls $\left(103 \pm 24 \mathrm{ml}\right.$ vs $\left.107 \pm 12 \mathrm{ml} / \mathrm{min} / \mathrm{min} / 1.73 \mathrm{~m}^{2}\right)$, the fraction of individuals with a mGFR below $90 \mathrm{ml} / \mathrm{min} / 1.73 \mathrm{~m}^{2}$ was higher in patients with SFK ( $23 \%$ vs $5 \%$ in controls; $\mathrm{P}=0.005)$. Multiple linear regression identified older age, ipsilateral abnormalities of the urinary tract, lack of compensatory hypertrophy and treated hypertension as independent factors associated with reduced mGFR. eGFR-Schwartz showed a smaller bias [ $95 \%$ confidence interval $(95 \% \mathrm{CI}): 3$ to 7] than other eGFR. mGFR showed a stronger correlation with PTH $(\mathrm{r}=0.1$ vs $\mathrm{r}=0.04)$ and FGF23 ( $r=0.05$ vs $\mathrm{r}=0.03)$ than eGFR-Schwartz.

Conclusions: SFK is not a benign condition, since $20 \%$ of the patients display altered renal function. Our results raise caution regarding the use of the cystatin-based equation. mGFR shows a better ability than eGFRSchwartz to discriminate patients showing early homeostatic adaptation to GFR reduction.

\section{PI-183 RENAL OUTCOME OF CHILDREN WITH SOLITARY FUNCTIONING KIDNEY}

Rabia Gundogan ${ }^{1}$, Elif Comak ${ }^{2}$, Mustafa Koyun ${ }^{2}$, Aygül Kernak Elmali ${ }^{3}$, Gulsah Kaya Aksoy ${ }^{2}$, Sema Akman ${ }^{2}$

${ }^{1}$ Akdeniz University Medical Faculty, Pediatrics, Antalya, Turkey, ${ }^{2}$ Akdeniz University Medical Faculty, Pediatric Nephrology, Antalya, Turkey, ${ }^{3}$ Akdeniz University Medical Faculty, Radiology, Antalya, Turkey

Introduction: Children with a Solitary Functioning Kidney (SFK) have an increased risk of developing hypertension, albuminuria and chronic kidney disease. The aim of this study was to evaluate the clinical characteristics and renal outcome in children with SFK

Material and methods: The medical records of children with SFK who had followed at our clinic were reviewed retrospectively. Renal injury was defined as hypertension, proteinuria, an impaired glomerular filtration rate, and/or the use of renoprotective medication.

Results: A total of 206 children, 125 boys (60.7\%), median age at last visit of $9.36 \pm 5.27$ years, median follow-up of $56.14 \pm 46.52$ months were included in the study. Among 206 children with SFK, $121(58.73 \%)$ presented with unilateral renal agenesis, 53 (25.72\%) multicystic dysplastic kidney and 32 (15.53\%) children had underwent nephrectomy secondary to congenital anomalies of the kidney and urinary tract (CAKUT). 81 patients had renal injury (44.5\%), 25 patients GFR $<60 \mathrm{ml} / \mathrm{min} / 1.73 \mathrm{~m} 2(14.4 \%), 32(15.5 \%)$ had hypertension, 22 had $(11.5 \%)$ hyperfiltration, $22(10.7 \%)$ had proteinuria, $49(23.8 \%)$ had use of renoprotective drugs, and 143 patients $(69.8 \%)$ had compensatory hypertrophy; anemia was detected in $32.5 \%$. In univariate analysis, male gender, presence of additional urological anomaly, premature birth, presence of urinary tract infection history, presence of hyperuricemia, birth weight $<2500 \mathrm{~g}$ were found to be associated with renal injury. However, in multivariate analysis, only hyperuricemia and prematurity were associated with renal injury [(OR:4.83, CI:1.75-13.34) and (OR:3.026, CI:1.103-8.303) repectively]. In study group, a statistically 
significant decrease was observed between creatinine and eGFR values of 6 th month, 1 st year and 5 th year $(\mathrm{p}<0.001)$.

Conclusions: Our findings demonstrate that renal injury is present in children with SFK at early age. Kidney functions of these patients start to deteriorate over years starting from young age. Kidney protective approaches should be taken at follow-up of these children.

\section{PI-184 CONSERVATIVE MANAGEMENT OF NON- REFLUXING NON-OBSTRUCTIVE MEGAURETER: A LONGITUDINAL OBSERVATIONAL STUDY}

\author{
Seçil Kezer, Cihangir Akgün, Önder Yavașcan \\ İstanbul Medipol University, Faculty Of Medicine, Department Of \\ Pediatric Nephrology
}

Introduction: There is a lack of prospective studies that include a selected population of patients with non-refluxing non-obstructive megaureter (MU). We aimed to examine the 2-year follow-up results of non-refluxive non-obstructive MU patients who dont require surgery.

Material and methods: $17[(22$ kidney units (KU))MU cases who were followed up between 2018 and 2021 at Medipol University, Faculty of Medicine, Division of Pediatric Nephrology were included in the study. Group 1 was defined as improved or regressed $\mathrm{KU}$ according to hydronephrosis degrees and diameters. Group 2 was defined as worsened or remained stable KU. Cases were analyzed retrospectively ultrasonographic (US) findings, in terms of urinary tract infection (UTI) frequency, stone and growth (height and weight SDS scores) parameters.

Results: 13 (76.4\%) of the 17 cases were diagnosed antenatally. 13 of cases were boys. 3 of the 17 cases were found on the right side, 5 were bilateral and 9 were found on the left side. We determined no statistically significant difference in terms of hydronephrosis severity, UTI frequency or initial and final growth scores between Group 1 and Group 2 (Table 1). Table 1. Comparison of groups

\begin{tabular}{lll}
\hline & $\begin{array}{l}\text { Group 1 } \\
\text { (regressed, } \\
\text { improved) }\end{array}$ & $\begin{array}{l}\text { Group 2 } \\
\text { (worsened, } \\
\text { stable) }\end{array}$ \\
patient(n) & 10 & 7 \\
$\begin{array}{l}\text { Follow-up time(mean } \pm \text { SD, } \\
\text { month) }\end{array}$ & $22 \pm 13$ & $16,3 \pm 7$ \\
US(initial-final), median,mm & & \\
AP diameter & $10,6-6,6$ & $14,9-18$ \\
Proximal ureter & $7,5-4$ & $8,4-8$ \\
Distal ureter & $9,2-6$ & $9,1-11$ \\
Parenchymal thickness & $4,7-9,3$ & $4,5-7,8$ \\
Kidney Zscore & $2,8-2$ & $3,2-2,2$ \\
Stone n & 0 & 3 \\
Grade 1-2 echogenity n & 2 & 1 \\
UTI n & 3 & 2 \\
Growth initial-finalSDS & & \\
(median) & & $0,15 / 0,15$ \\
Weight & $0,37 / 0,80$ & $0,30 / 0,15$ \\
Height & $0,35 / 0,28$ & \\
\hline
\end{tabular}

SD:Standard deviation, AP:Anteriorposterior , SDS: Standard deviation score.

Conclusions: Although US findings cause concern for the doctor and parents in MU cases without reflux and obstruction, we could be interpreted to these cases having a low risk of causing problems in terms of UTI, parenchymal damage and growth.

\section{PI-185 PREVALENCE OF REFLUX NEPHROPATHY IN IRANIAN CHILDREN WITH SOLITARY KIDNEY: RESULTS OF A MULTI-CENTER STUDY}

Maryam Esteghamati ${ }^{1}$, Hadi Sorkhi ${ }^{2}$, Hamid Mohammadjafari ${ }^{3}$, Ali Drakhshan ${ }^{4}$, Simin Sadeghi-bojd ${ }^{5}$, Hossein Emad Momtaz ${ }^{6}$, Masoumeh Mohkam ${ }^{7}$, Baranak Safaeian ${ }^{8}$, Nakysa Hooman ${ }^{9}$, Afshin Safaeiasl ${ }^{10}$, Mohsen Akhavan Sepahi ${ }^{11}$, Khadijeh Ghasemi ${ }^{12}$, Zahra Bazargani ${ }^{13}$, Elham Emami ${ }^{14}$

${ }^{I}$ Department Of Pediatric Nephrology, Clinical Research Development Center Of Childrens Hospital, Hormozgan University Of Medical Sciences, Bandar Abbas, Iran, ${ }^{2}$ Non-communicable Pediatric Diseases Research Center, Health Research Institute, Babol University Of Medical Sciences, Babol, Iran, ${ }^{3}$ Department Of Pediatric Nephrology, Mazandaran University Of Medical Sciences, Sari, Iran, ${ }^{4}$ Shiraz Nephro-urology Research Center, Shiraz University Of Medical Sciences, Shiraz, Iran, ${ }^{5}$ Genetics Of Non-communicable Disease Research Center, Zahedan University Of Medical Sciences, Zahedan Iran, ${ }^{6}$ Division Of Pediatric Nephrology, Besat Hospital, School Of Medicine, Hamadan University Of Medical Sciences, Hamadan, Iran, ${ }^{7}$ Pediatric Nephrology Research Center, Research Institute For Children's Health, Shahid Beheshti University Of Medical Sciences, Tehran, Iran, ${ }^{8}$ Taleghani Pediatric Hospital, Golestan University Of Medical Sciences, Gorgan, Iran, ${ }^{9}$ Clinical Research Development Center, Iran University Of Medical Sciences, Tehran, Iran, ${ }^{10}$ Department Of Pediatrics, Guilan University Of Medical Sciences, Guilan, Iran, ${ }^{11}$ Department Of Pediatrics, School Of Medicine, Qom University Of Medical Sciences, Qom, Iran, ${ }^{12}$ Department Of Pediatric Nephrology, Bushehr University Of Medical Sciences, Bushehr, Iran, ${ }^{13}$ Department Of Pediatrics, School Of Medicine, Fasa University Of Medical Sciences, Fasa, Iran, ${ }^{14}$ Department Of Pediatrics, Hajar Shaherkord University Of Medical Sciences, Shahrekord, Iran

Introduction: Given the importance of the function of the remnant kidney in children with unilateral renal agenesis and the significance of timely diagnosis and treatment of reflux nephropathy to prevent further damage to the remaining kidney, we aimed to determine the prevalence of reflux nephropathy in this subgroup of pediatric patients.

Material and methods: In general, 274 children referred to pediatric nephrologists in different parts of Iran were evaluated, from which 199 had solitary kidney and were included in this cross-sectional study. The reason for referral included urinary tract infection (UTI), abnormal renal ultrasonography, being symptomatic, and incidental screening. Demographic characteristics consisting of age and gender were recorded. History of UTI and presence of vesicoureteral reflux (VUR) were evaluated.

Results: From the 274 children evaluated in this study with the mean age (SD) of 4.71 (4.24) years, 199 (72.6\%) had solitary kidney. Among these $118(59.3 \%)$ were male and $81(60.7 \%)$ were female, $21.1 \%$ had a history of UTI, and VUR was present in $23.1 \%$. The most common cause of referral was abnormal renal ultrasonography $(40.2 \%)$, followed by incidental screening $(21.1 \%)$, being symptomatic $(14.1 \%)$, and UTI $(5.5 \%)$. In 116 children $(58.3 \%)$, the right kidneys and in $83(41.7 \%)$ the left kidneys were absent. Besides, $14.6 \%$ of the participants had consanguineous parents and $3 \%$ had a family history of solitary kidney. Upon DMSA scan, the single kidney was scarred in $13.1 \%$. In addition, proteinuria and hematuria were observed in $6.5 \%$ and $1.5 \%$ of children, respectively.

Conclusions: The prevalence of reflux nephropathy was $23.1 \%$ in children with solitary kidney with a slight female predominance. Given the relatively high prevalence of reflux nephropathy in these 
children, screening for VUR in the remnant kidney appears to be essential in this population.

\section{PI-186 PRESENTATION, MANAGEMENT, AND OUTCOME OF POSTERIOR URETHRAL VALVES IN A TERTIARY HOSPITAL}

\author{
Mehmet Yan ${ }^{1}$, Demet Alaygut ${ }^{2}$, GökÇen Erfidan ${ }^{1}$, Eren Soyaltin ${ }^{1}$, \\ Tunç Özdemir ${ }^{3}$, Seçil Arslansoyu Çamlar ${ }^{2}$, Fatma Mutlubaș ${ }^{1}$, \\ Belde Kasap Demir ${ }^{1}$ \\ ${ }^{1}$ Izmir University Of Health Sciences Tepecik Education And Research \\ Hospital Department Of Pediatrics, ${ }^{2}$ Izmir University Of Health Sciences \\ Tepecik Education And Research Hospital Department Of Pediatric \\ Nephrology, ${ }^{3}$ Izmir University Of Health Sciences Tepecik Education \\ And Research Hospital Department Of Pediatric Surgery
}

Introduction: The aim of this study was to evaluate the presentation, management, challanges and outcome of the disease in a tertiary hospital in Izmir inTurkey.

Material and methods: Retrospectively, medical records of male children with a diagnosis of PUVs over a 10 year period (2009-2019) were retrieved. All data in relation to the study objectives were recorded and analyzed.

Results: A total of 43 cases, 38 Turkish and 5 Syrian, were included in the study. 21 had been diagnosed under 1 month. The mean age of diagnosis of the remaining 22 was $32,45 \pm 45.43$ (1-132) months. Average gestational weeks were $37.4 \pm 2.59$ median 38 weeks, 17 (39.5\%) cases were diagnosed antenatally. Seven cases had antenatal hydronephrosis and one patient had intrauterine vesicoamniotic shunt. The most common complaint was urinary tract infection and $55 \%$ of these cases were diagnosed with urosepsis. In ultrasonographic examinations at the time of diagnosis, $33(76.7 \%)$ cases had hydronephrosis. Nine cases $(20.9 \%)$ had an increase in parenchymal echogenicity, and eight (18.6\%) had atrophy. Systemic anomaly was accompanied by $13.9 \%$ of the cases and urinary system anomaly was accompanied by $74.4 \%$. Among these anomalies, vesicoureteral reflux was the first with $62.7 \%$. Postnatal surgery was performed in 38 cases. 22 (51.2\%) were PUV resection only, 9 $(20 \%, 9)$ PUV resection and vesicostomy operation. The urodynamics report of $25(58.1 \%)$ cases was available. Nine cases had flask bladder.14 $(32.6 \%)$ cases were performing clean intermittent catheterization. Seven $(16.3 \%)$ of the cases received renal replacement therapy. Six patients had undergone renal transplantation. In total, $12(27.9 \%)$ cases received hypertension treatment and $10(23.3 \%)$ cases had proteinuria.

Conclusions: Valve ablation was very helpful in establishing satisfactory urine flow in affected patients. Renal transplantation can be applied in selected cases. PUV is a disease with long-term morbidity.

\section{PI-187 A CASE REPORT OF DUPLEX KIDNEY: MASSIVE DILATATION OF THE UPPER MOIETY WITHOUT VESICOURETERAL REFLUX}

Miriam Acunzo ${ }^{1}$, Alessandra Mari ${ }^{1}$, Patrizia Carlucci ${ }^{1}$, Valentina Fabiano ${ }^{1}$, Giorgio Selvaggio ${ }^{2}$, Gian Vincenzo Zuccotti ${ }^{1}$ ${ }^{1}$ Pediatric Department, V. Buzzi Childrens Hospital, University Of Milan, Via Castelvetro 32, 20154 Milan, Italy, ${ }^{2}$ Pediatric Surgery Department, V. Buzzi Childrens Hospital, University Of Milan, Via Castel-vetro 32, 20154 Milan, Italy

Introduction: Duplicated renal collecting system is one of the congenital anomalies of the kidney and of the urinary tract (CAKUT), which are the most common cause of chronic renal disease in children.

Material and methods: S. is a 7-month-old male infant, born at term with an antenatal diagnosis of bilateral, complete duplex system. Renal ultrasound at birth confirmed the diagnosis and antibiotic prophylaxis with amoxicillin-clavulanate was started in the first week of life. A month later, voiding cystourethrography was instead normal, with no evidence of passive or active vesicoureteral reflux (VUR). Abdominal MRI showed an ectopic outlet into prostatic urethra of both the upper ureters, leading to an obstruction of urine flow and to a massive dilatation of these systems, associated with normal bilateral lower systems. A Technetium99m-MAG3 Scan detected a good function of upper bilateral mojety.

Results: Corrective elective surgery was performed at 6 months of age by carrying out a right termino-lateral ureteroureterostomy with stent placement. Due to a multiresistant P. aeruginosa urinary tract infection though, S. was admitted to hospital to remove the ureteral stent and to start IV antibiotic therapy with meropenem. Renal function and BP were within normal range. Contralateral hydropyonephrosis required an invasive approach with percutaneous nephrostomy, left in place until left ureteroureterostomy was carried out. Clinical conditions improved and no other intercurrent episode of UTI occurred.

Conclusions: The most common complications of duplex kidney are obstruction of the upper moiety and VUR from the lower one. S. did not present the latter, but the severity of the first one required surgical intervention. Upper ureteroureterostomy is a safe and conservative alternative to upper pole heminephrectomy. Prenatal diagnosis is of crucial importance as it allows to plan a multidisciplinary intervention with diagnostic workup, and, if needed, elective corrective surgery. In our case, an early start of antibiotic prophylaxis allowed us to have a safer surgical timing.

\section{PI-188 AN ATYPICAL CASE OF TYPICAL HUS}

Marina Avramescu ${ }^{1}$, Laura Soyer ${ }^{1}$, Romain Berthaud ${ }^{1}$, Marion Rabant ${ }^{1}$, Aline Broch ${ }^{1}$, Patricia Mariani ${ }^{2}$, Veronique Fremeaux-bacchi ${ }^{3}$, Olivia Boyer $^{1}$

${ }^{I}$ Ap-hp Hopital Necker, ${ }^{2}$ Ap-hp Hopital Robert Debre, ${ }^{3}$ Ap-hp Hegp

Abstract: Shiga toxin-producing Escherichia coli (STEC) is the leading cause of hemolytic-uremic syndrome (HUS) in children. Patients with STEC HUS are generally ill-appearing at presentation and have the biological triad of microangiopathic hemolytic anemia, thrombocytopenia and acute renal failure. A previously healthy 7-month-old girl presented to the emergency room with periumbilical inflammation. An abdominal ultrasound revealed the presence of a urachal cyst. Blood workup showed: hemoglobin $8.7 \mathrm{~g} / \mathrm{dl}$, white blood cell count $6300 /$ $\mathrm{mm} 3$, platelet count $287000 / \mathrm{mm} 3$, C-reactive protein $3 \mathrm{mg} / \mathrm{l}$, serum creatinine $101 \mu \mathrm{mol} / \mathrm{l}$; peripheral blood smear found no schizocytes. The infant had no history of recent infection or toxic exposure. She had a normal urine output, no edema and normal blood pressure. Renal ultrasound was normal. Urinalysis revealed leukocyturia, mixed proteinuria and hematuria. Further laboratory tests were conducted and found normal values of serum albumin, LDH, haptoglobin, liver and pancreatic enzymes. Immunologic workup found normal complement levels and negative antinuclear antibodies. Creatinine levels did not decrease after IV hydration. A percutaneous kidney biopsy was performed and was in favour of a thrombotic microangiopathy. The urachal abscess was drained and culture was positive for Escherichia coli. Polymerase chain reaction identified the strain as producing shiga toxin-2. Stool samples were negative for STEC. No genetic mutation of atypical HUS was found. Evolution was favourable: at 6-month followup, the patient had a normal clinical examination and an improved renal function with a serum creatinine of $50 \mu \mathrm{mol} / \mathrm{l}$. The abdominal ultrasound found no sign of urachus remnants. The patient is closely monitored every three months and receives an anti-proteinuric treatment with ACEi. This very atypical case raises awareness of the possibility of STEC HUS following E. Coli abcess. Our patient had no prodromal diarrhea, no signs of hemolysis, a normal platelet count, a fortuitous discovery of renal failure and STEC was identified in a urachal abscess and not in stool. 
PI-189 THE DEVELOPMENT AND PRELIMINARY VALIDATION OF A SCORING TOOL FOR MONITORING DISEASE ACTIVITY IN PATIENTS WITH IGA VASCULITIS (HSP).

Chloe Williams ${ }^{3}$, Jared Murphy ${ }^{2}$, Tom Dowsett ${ }^{1}$, Louise Oni ${ }^{1}$

${ }^{1}$ Department Of Paediatric Nephrology, Alder Hey Children's Nhs Foundation Trust Hospital, Liverpool, United Kingdom, ${ }^{2}$ Royal Liverpool And Broadgreen University Hospitals, Liverpool, United Kingdom, ${ }^{3}$ Department Of Women's And Children's Health, Institute Of Life Course And Medical Sciences, University Of Liverpool, Liverpool, United Kingdom

Introduction: IgA vasculitis (IgAV, HSP) is the most common form of childhood vasculitis. The Paediatric Vasculitis Activity Score (PVAS) is used for scoring vasculitis, however it may not be specific enough for IgAV. The aim is to develop and perform preliminarily validation of a vasculitis activity scoring tool for IgAV (IgA-VAS).

Material and methods: The IgA-VAS was derived from existing tools and underwent face validity prior to this study. It consists of 40 manifestations, each scored 0-10, within 5 domains: cutaneous, gastrointestinal, musculoskeletal, renal and other. A retrospective cohort of paediatric patients were scored by two clinicians using the IgA-VAS and PVAS. Test validity, concurrent validity and inter-rater agreement were assessed. A subgroup were scored using a visual analogue scale.

Results: For preliminary validation, retrospective scoring was performed (February 2021) from a single tertiary centre over a 5-year period. 196 children were identified; 153 met inclusion criteria. $54 \%$ were male, median age of 5.7 years (range 0.6-16.7). Median total IgA-VAS scores were 7/125 (range 2-31) and 5/125 (range 2-29) and median PVAS scores were $6 / 63$ (range 2-25) and 5/63 (range 2-20) for rater 1 and 2 respectively. Inter-rater reliability was low for both tools $(0.131,0.225, p<0.001)$. For the renal domains, median IgA-VAS scores were $2 / 52$ (range $0-24$ ) and 0/52 (range 0-24), and renal PVAS domain score was 0/12 (range 0-12) for both raters. Inter-rater agreement for the renal domains of both tools was similar $(0.237$ and $0.304, p<0.001)$. Correlation between the renal domain scores of the tools was strong (rater $1=0.747$, rater $2=0.825$, $p<0.001)$. The total IgA-VAS score moderately correlated with the visual analogue scale for both raters $(0.482$ and $0.362, p<0.05)$, the PVAS strongly correlated with rater $1(0.504, p=0.004)$ and moderately correlated with rater $2(0.372, p=0.043)$.

Conclusions: The IgA-VAS continues to evolve following preliminary validation however refinement is needed before prospective validation.

\section{PI-190 A SYSTEMATIC REVIEW OF URINE BIOMARKERS IN CHILDREN WITH IGA VASCULITIS NEPHRITIS.}

Chloe Williams ${ }^{1}$, Aileen Toner ${ }^{1}$, Rachael D Wright ${ }^{2}$, Louise Oni ${ }^{3}$ ${ }^{1}$ School Of Medicine, University Of Liverpool, Liverpool, United Kingdom, ${ }^{2}$ Department Of Women's And Children's Health, Institute Of Life Course And Medical Sciences, University Of Liverpool, Liverpool, United Kingdom, ${ }^{3}$ Department Of Paediatric Nephrology, Alder Hey Children's Nhs Foundation Trust Hospital, Liverpool, United Kingdom

Introduction: Nephritis is a recognised complication of IgA vasculitis (IgAV, Henoch-Schoenlein purpura, HSP) and contributes to 1-2\% of all chronic kidney disease (CKD) stage 5. Improved detection of kidney inflammation may allow earlier intervention and reduce irreversible kidney damage in IgA vasculitis nephritis (IgAV-N). The primary aim of this study was to perform a comprehensive systematic review to evaluate the current literature to identify promising urine biomarkers that can detect and assess the severity of kidney disease in children with IgAV.

Material and methods: A systematic literature review was performed using 4 search engines and a search term strategy with predefined inclusion and exclusion criteria. Promising biomarkers were divided in terms of clinical or pre-clinical and described using statistical significance and area under the curve (AUC) values.

Results: 21 studies were identified; 13 were eligible. A total of 2,446 paediatric patients were included: healthy controls $(n=761)$, children with $\operatorname{IgAV}-\mathrm{N}(\mathrm{n}=1,236)$ and children with IgAV without nephritis (IgAVnoN, $n=449$ ). $51 \%$ were male, median age 7.9 years. The clinical markers, 24-hour protein quantity and urine protein:creatinine ratio were deemed acceptable for assessing severity of nephritis (AUC <0.8). Urinary albumin concentration (Malb) performed well (AUC 0.81$0.98)$. The most promising pre-clinical urinary biomarkers in predicting presence of nephritis were kidney injury molecule-1 (KIM-1) (AUC 0.93 ), monocyte chemotactic protein-1 (MCP-1) (AUC 0.83), N-acetylb-glucosaminidase (NAG) (0.76-0.96), and angiotensinogen (AGT) (AUC not available). Urinary KIM-1, MCP-1, and NAG appeared to correlate with disease severity.

Conclusions: Further longitudinal studies are needed to assess whether these biomarkers enhance standard of care in the management of IgAV-N.

\section{PI-191 EVALUATION OF RISK FACTORS FOR PROGRESSION OF PRIMARY IGA-NEPHROPATHY IN CHILDREN}

\author{
Mariia Proskura ${ }^{1}$, Edita Petrosyan ${ }^{1}$, Dmitry Korostin ${ }^{1}$, \\ Berta Kushnir ${ }^{1}$, Patrisia Povilaitite ${ }^{2}$ \\ ${ }^{1}$ Pirogov Russian National Research Medical University, ${ }^{2}$ State \\ Institution Of Health, Rostov Region, Pataloanatomical Bureau
}

Introduction: The course and outcomes of primary $\operatorname{IgA}$ nephropathy (IgAN) in children are variable. Unlike adults, no accurate progression factors for children have been identified yet. If timely detected it may help stratify patients, improve treatment for high-risk individuals and delay end-stage renal failure.

Material and methods: 124 children with primary IgAN were enrolled in a retrospective study. 75 children were followed up more than 2 years (the median follow-up was 28 months). The median age of onset was 11.0 years (IQR is 7.0-13.5). Patients were divided into 2 groups: group \#1 idiopathic IgAN ( $\mathrm{n}=92)$, group \#2 - Shenlein-Henoch purpura $(\mathrm{n}=32)$. The age of onset and primary admission, the level of proteinuria and glomerular filtration rate (GFR) at the onset, after 12, 24 months, at the end of follow up, mean blood pressure, medications before nephrobiopsy were analyzed. Nephrobiopsy data were classified according to the Oxford Scale. 70 children, followed for at least 2 years, showed disease progression (assessed by the GFR decline curve and the presence of persistent proteinuria), underwent next generation sequencing (NGS). Single nucleotide polymorphisms (SNP) were assessed according to the IgAN panel (panelapp.genomicsengland.co.uk/panels/156/). Progression was estimated by a decrease in GFR less than $60 \mathrm{ml} / \mathrm{min} / 1.73 \mathrm{~m} 2$. Outcomes were classifyed into absence or presence of remission. A search for factors influencing GFR after 24 months of observation was carried out. Comparison and analysis of data was performed using Students t-test, Mann-Whitney, $\chi 2$, Fisher, linear regression model, Cox regression model.

Results: Unlike adults, the predictive value of the Oxford scale in children has not been proven and is not associated with a decrease in GFR $<60 \mathrm{ml} / \mathrm{min} / 1.73 \mathrm{~m} 2$. GFR at the end of follow-up was lower in the first group than in the second. The level of GFR at the onset, mean blood pressure and the age of primary admission turned out to be independent factors helping detect children with an expected decrease in GFR less than $90 \mathrm{ml} / \mathrm{min} / 1.73 \mathrm{~m} 2$ within 2 years. Analysis of SNP, their associations did not have a statistically significant effect on the clinical, morphological data of patients, outcomes. Perhaps the NGS results may not be representative due to the limited statistical power of the sample.

Conclusions: The influence of morphological and genetic factors on the outcome and course of primary IgA nephropathy has not been proven. The use of multiple linear regression predicts GFR on average after 24 months of observation. 
PI-192 THE EVALUATION OF CLINICAL, LABORATORY AND PATHOLOGIC FINDINGS OF CHILDREN WITH HENOCH-SCHÖNLEIN NEPHRITIS AND INVESTIGATING THE EFFECT ON PROGNOSIS

Caner Aslan, Cengiz Candan, Nilüfer Göknar Istanbul Medeniyet University

Background: Henoch-Schönlein Purpura (HSP) is the most common vasculitis seen in children. The most important prognostic factor for HSP vasculitis is the renal involvement. Our study is aiming to investigate the relation of clinical, laboratory and pathologic findings with long term prognosis.

Material and Methods: Children with HSP nephritis between January 2010 and December 2019 were included in this retrospective study. All children were classified with Meadow Classification according to initial and final clinical findings and grouped into mild and severe cases.

Results: Ninty children (59 male) with a mean age of $8.8 \pm 3.2$ years were included. Nephritic syndrome, nephrotic syndrome and nephritic/ nephrotic syndrome was present in $4 \%, 10 \%$ and $4 \%$ of the cases respectively. Seventy-two children $(80 \%)$ were in mild group, eighteen were in severe group. Thirty-three children had undergone kidney biopsy; fifteen $(15 / 72)$ were in mild group and eighteen (18/18) were in severe group. The indications were persistent proteinuria, nephrotic syndrome, nephritic/nephrotic syndrome, and decreased eGFR. We compared mild and severe group according to histopathologic findings and ISKDC stage $3 \mathrm{~B}$ and more lesions were more frequently seen in children severe group $(\mathrm{p}=0.22)$. Immunosuppressive treatment were used in $44.4 \%$ in mild cases and $100 \%$ in severe cases( $\mathrm{p}<0.01)$. On follow up only four children (Two in mild group) had persistent proteinuria.

Conclusion: All children with severe clinical findings had undergone kidney biopsy and received immunosuppressive treatment. Clinical findings in initial assessment can help to predict histopathologic findings and treatment protocols in HSP nephritis. Persistent proteinuria on long term follow up can be seen even if children with mild clinical presentations. Long term follow up is required in all children with HSP nephritis.

\section{PI-193 OUTCOME OF CHILDREN WITH IGA VASCULITIS WITH NEPHRITIS TREATED WITH STEROIDS: A MATCH CONTROL STUDY.}

Jean-daniel Delbet ${ }^{1}$, Stephanie Clave ${ }^{5}$, Julien Hogan ${ }^{2}$, Olivia Boyer ${ }^{3}$, Jerome Harambat ${ }^{4}$, Cyrielle Parmentier ${ }^{1}$, Tim Ulinski ${ }^{1}$

${ }^{1}$ Pediatric Nephrology, Armand Trousseau Hospital, Aphp, Paris, France, ${ }^{2}$ Pediatric Nephrology, Robert Debré Hospital, Aphp, Paris, France, ${ }^{3}$ Pediatric Nephrology, Necker Enfants Malades, Aphp, Paris, France, ${ }^{4}$ Pediatric Nephrology, Chu De Bordeaux, France, ${ }^{5}$ Pediatric Nephrology, Chu De Marseille, France

Introduction: $\operatorname{IgA}$ vasculitis (IgAV) is the most common vasculitis in children. IgAV long-term prognosis depends on kidney involvement or IgA vasculitis with nephritis (IgAVN). Up to now, steroid treatment (oral steroids or methylprednisolone pulses) have not proven to be formally efficient. The objective of our study is to assess steroid impact on IgAVN prognosis.

Material and methods: mAll children with IgAVN diagnosed between 2000 and 2019 in French paediatric nephrology units were retrospectively included. The outcomes of patients treated with steroids were compared with those of a control group of untreated patients matched for age, sex, proteinuria, eGFR and histological features. The primary endpoint was IgAVN remission defined as a proteinuria $<200 \mathrm{mg} / \mathrm{L}$ without renal failure.

Results: 359 patients with IgAVN were included with a median follow-up time of 249 days (43-809). 108 (30\%) patients received oral steroids alone and $207(51 \%)$ patients received three methylprednisolone pulses followed by oral corticosteroids, 44 patients $(12.5 \%)$ did not receive steroids. Thirty-four children treated with oral steroids alone were compared with 34 matched control patients who did not receive steroid. IgAVN remission proportion was not different between these two groups: $76 \%$ versus $77 \%$ respectively. Ninety children treated with oral steroids alone were compared with 180 matched patients treated with three methylprednisolone pulses followed by oral corticosteroids. IgAVN remission proportion was not different between these two groups: $76 \%$ versus $73 \%$ respectively.

Conclusions: The benefit of oral steroids alone and methylprednisolone pulses could not be established based on our findings. Randomized controlled trials are thus required to determine the efficacy of stroids in IgAV with nephritis.

PI-194 EFFICACY OF COMBINED THERAPY OF AZATHIOPRINE, PREDNISONE AND ENALAPRIL IN IGAN AND IGAVN CHILDREN INCLUDING CONTROL KIDNEY BIOPSY

Malgorzata Mizerska-wasiak ${ }^{1}$, Jadwiga Maldyk ${ }^{2}$, Milosz Starczynski ${ }^{3}$, Wojciech Wasiak ${ }^{3}$, Malgorzata Panczyk-tomaszewska ${ }^{1}$

${ }^{1}$ Department Of Pediatrics And Nephrology, Medical University Of Warsaw, Poland ${ }^{2}$ Department Of Pathology, Medical University Of Warsaw, Poland, ${ }^{3}$ Student's Scientific Group At The Department Of Pediatrics And Nephrology, Medical University Of Warsaw, Poland

Abstract: IgA nephropathy (IgAN) and IgA vasculitis nephritis (IgAVN) are glomerulonephritis in which immunosuppressive therapy may be used.

The aim of this study was to evaluate the efficacy of 1-year treatment with Azathioprine with Prednisone and Enalapril including control renal biopsy in children with IgAN and IgAVN.

Material and Methods: This retrospective study consist of 36 children :15 IgAN, $21 \operatorname{IgAVN}$, diagnosed in kidney biopsy. All children were treated with Azathioprine 12 months, Prednisone- 15 months (gradually diminished according to the protocol) and Enalapril. Before the biopsy and at the end of treatment we analyzed: proteinuria, hematuria, creatinine, GFR. After the treatment control kidney biopsy was performed in all patients ; results of biopsies were classified ac. to the Oxford classification.

Results: At the onset of the disease proteinuria and GFR were higher in IgAVN children than IgAN (median 64 (5.79-190) $\mathrm{mg} / \mathrm{kg} / \mathrm{d}$ vs 15 (7-177) $\mathrm{mg} / \mathrm{kg} / \mathrm{d}, \mathrm{p}<0.01 ; 127.15 \pm 35.11$ vs $102.41 \pm 30.1, \mathrm{p}<0.05)$; hematuria (NS). Kidney biopsy was performed significantly later in children with IgAN than IgAVN $(1.01 \pm 1.03$ vs $0.11 \pm 0.11$ years, $p<0.0002$. In first biopsy MEST-C score was not significantly different between IgAN and IgAVN.

Et the end of combined therapy we found significant reduction of proteinuria (proteinuria $=0$ in 35 patients, and hematuria. GFR and creatinine were normal in all patients of both groups. In Oxford classification we noted significant improvement in M,E between first and second biopsy in both groups $(\mathrm{M} p<0.01$ in $\operatorname{IgAN}$ and IgAVN , $\mathrm{E} p<0.01$ in $\operatorname{IgAN}, \mathrm{p}<0.05$ in IgAVN) and additionally T only in $\operatorname{IgAVN}(\mathrm{p}<0.001)$.

We observed significantly higher percent of patients with S0,T0 and C0 after treatment in IgAVN than IgAN.

Conclusions: 1- year combined therapy of Azathioprine, Prednisone and Enalapril leads to clinical and histological improvement in children with IgAN and IgAVN.

\section{PI-195 IGA NEPHROPATHY - CHARACTERIZATION AND EVOLUTION: A SINGLE CENTER EXPERIENCE}

Cátia Granja, Filipa Neves, Carolina Cordinhã, Marta Machado, Carmen Carmo, Clara Gomes

Unidade De Nefrologia Pediátrica - Hospital Pediátrico, Centro Hospitalar E Universitário De Coimbra 
Objectives: Describe forms of presentation, therapy performed and evolution of patients diagnosed with IgA nephropathy (IgAN) in a Pediatric Nephrology Unit of a level III hospital.

Material and methods: Retrospective, descriptive analysis of demographic, clinical, analytical and histological data of children with kidney biopsy proven IgAN from January 2001 to December 2020.

Results: Thirteen children included, $54 \%$ girls. Initial findings were one or more episodes of macroscopic hematuria in five (four linked to respiratory tract infection, two with microscopic hematuria in-between); one had persistent microscopic hematuria and nephrotic proteinuria one year before admission. Seven children had no previous complaints.

The average age at the time of biopsy was $11 \pm 4.4$ years. In the six children with previous symptoms, biopsy was performed on average 25 (5-60) months. At that time, seven patients had nephritic syndrome, associated to nephrotic proteinuria (three), significative nonnephrotic proteinuria (two) and nephrotic syndrome (one); two had nephrotic syndrome with microscopic hematuria; one had macroscopic hematuria and nephrotic proteinuria; two had microscopic hematuria and nephrotic proteinuria; one was diagnosed with stage 4 chronic kidney disease.

Histopathologically, ten children had glomerular crescents and/or interstitial fibrosis. Of these, nine received corticoids and angiotensinconverting enzyme inhibitor (ACEI), five with cyclophosphamide association (persistent nephrotic proteinuria or relapse of nephritic syndrome). One child had only ACEI (spontaneous resolution).

Mean follow-up was 3.94 (0.6-11.7) years. At last evaluation, seven children (54\%) had normal blood pressure, unchanged sediment, no proteinuria and normal renal function. The child with stage 4 chronic kidney disease did not worsen and five children maintained hematuria and/or proteinuria (nephrotic-three).

Conclusions: IgA nephropathy is the most common glomerulonephritis with a high clinical and histological variability and an important cause of end-stage renal disease.

Our results show that outcome is good, however some patients need immunosuppressive therapies and careful long-term observation is required.

\section{PI-196 DEFINING RENAL REMISSION IN AN INTERNATIONAL COHORT OF CHILDREN WITH LUPUS NEPHRITIS}

\author{
Chiara De Mutiis ${ }^{1}$, Kjell Tullus ${ }^{2}$ \\ ${ }^{1}$ Pediatric Department, Maggiore Hospital, Bologna, Italy, ${ }^{2}$ Renal Unit, \\ Great Ormond Street Hospital For Children Nhs Ft, London, UK
}

Objectives: Lupus Nephritis (LN) occurs in up to $80 \%$ of children with SLE and it affects the long term outcome. Achieving and maintaining renal remission is the goal of treatments. However definition of remission in children is not clearly defined. We compared outcomes using different published definitions of complete and partial remission.

Methods: 248 children with biopsy proven LN class $\geq$ III (ISN/RPS), diagnosed in the last 10 years in 23 international centers were studied up to 24 months of follow-up. Children were treated according to local protocols. Seven definitions of remission from published studies and international guidelines were compared.

Results: The number of children in complete and partial remission varied significantly between the different definitions. At 24 month between $50 \%$ and $78.8 \%$ of the children were in complete remission according to the different criteria. A relatively small percentage of children, between $2.3 \%$ to $25 \%$, achieved partial remission during the follow-up.

Using 3 of the 7 definitions, we found no difference in terms of gender and age of children entering remission, while patients with East Asian ethnicity achieved remission more often than other ethnic groups $(\mathrm{p}<0.05)$. Also children treated in high income countries showed a higher percentage of complete remission $(\mathrm{p}<0.05)$.
Conclusion: Rate of remission varied hugely applying the different definitions. Ethnicity and income of the country where the patients were treated influenced outcomes.

Our findings open the discussion on what to consider remission also in the perspective of new possible treatments in children.

\section{PI-197 INDUCTION THERAPY FOR PEDIATRIC ONSET LUPUS NEPHRITIS : MYCOPHENOLATE MOFETIL VERSUS CYCLOPHOSPHAMIDE}

Laurye-anne Eveillard ${ }^{1}$, Marwa Chbihi ${ }^{2}$, Quentin Riller ${ }^{3}$, Marion Rabant ${ }^{4}$, Laurene Dehoux ${ }^{1}$, Marina Charbit ${ }^{1}$,

Nathalie Biebuyck ${ }^{1}$, Marina Avramescu ${ }^{1}$, Brigitte Bader-meunier ${ }^{2}$, Olivia Boyer ${ }^{1}$

${ }^{1}$ Service De Néphrologie Pédiatrique, Hôpital Necker Enfants Malades, Marhea, Institut Imagine, Université De Paris, Paris, France, ${ }^{2}$ Unité D'immuno-hémato-rhumatologie Pédiatrique, Hôpital Necker Enfants Malades, Marhea, Institut Imagine, Université De Paris, Paris, France, ${ }^{3}$ Inserm Umr1163, Immunogenetics Of Pediatric Autoimmune Diseases, Imagine - Institut Des Maladies Génétiques, Paris, France, ${ }^{4}$ Service De Pathologie, Hôpital Necker Enfants Malades, Inem, Université De Paris, Paris, France

Introduction: Class IV lupus nephritis (LN) is one of the most severe involvements in systemic lupus erythematosus and is particularly frequent in case of pediatric onset. The gold standard induction treatment consists of intravenous (IV) pulses of Cyclophosphamide (CYC) in association with corticosteroids. It has considerably improved the renal prognosis but has potential short and long-term toxic effects. Recent studies in adults have shown similar efficacy of oral Mycophenolate Mofetil (MMF) as induction therapy with a lower toxicity. However, the pediatric literature is scarce and current treatment guidelines are extrapolated from the adult population.

The aim of the study was to compare the efficacy and tolerance of CYC and MMF as induction treatment of a first episode of class IV LN in children. Material and methods: We conducted a monocentric retrospective study including all consecutive children ( $<18$ years) with at least 4 American College of Rhumatology criteria for lupus, and biopsy-proven class IV LN according to the IRS/RPS classification, and who had not received any prior immunosuppressive treatment.

Results: Among the 33 patients, 17 had been treated with oral MMF (51\%) and 16 with IV CYC. The basic characteristics were similar in both groups except for more neurological involvement in the CYC group (6/17 vs. $0 / 16$ ). There was a non-significant trend for more severity in the CYC group with higher grade proteinuria, lower albuminuria, and more frequent acute kidney injury. At one year, 53\% of the patients from the MMF group and $77 \%$ from the CYC group had achieved remission ( $\mathrm{p}=0.25$ ). $59 \%$ of the patients from the MMF group had relapsed, versus $50 \%$ of patients from the CYC group ( $\mathrm{p}=0.87$ ), respectively at 3.4 years and 4.7 years after beginning of treatment $(\mathrm{p}=0.41)$. The severe and mild complication rates were not significantly different between the two groups.

Conclusions: In conclusion, we found no difference in the kidney outcome and side effects in children receiving either MMF or CYC as induction therapy of class IV LN. However, in this retrospective study, no patient from the MMF group had neurological involvement, and there was a trend for more severity in the CYC group. Further studies are needed to confirm these results with stratification of children by disease severity.

\section{PI-198 EVALUATION OF HYDROXYCHLOROQUINE BLOOD CONCENTRATIONS AND EFFECTS IN CHILDHOOD-ONSET SYSTEMIC LUPUS ERYTHEMATOSUS}

Margaux Boistault ${ }^{1}$, Saik Urien ${ }^{1}$, Christian Funck-brentano ${ }^{1}$, Hélène Vantomme ${ }^{1}$, Nicolas Garcelon ${ }^{1}$, Isabelle Melki ${ }^{1}$, Brigitte Bader-meunier ${ }^{1}$, Noel $\mathrm{Zahr}^{2}$, Olivia Boyer ${ }^{1}$ 
${ }^{1}$ Ap-hp. Université De Paris, Necker Hospital, Department Of Pediatric Nephrology, Reference Center For Idiopathic Nephrotic Syndrome In Children And Adults, Imagine Institute, Laboratory Of Hereditary Kidney Diseases, Inserm U1163, Paris, France, ${ }^{2}$ Ap-hp. Sorbonne Université, Pitié-salpêtrière Hospital, Department Of Pharmacology And Clinical Investigation Center; Inserm, Cic-1901 And Umr Ican 1166, Sorbonne Université, Faculty Of Medicine; Sorbonne Université, Faculty Of Medicine, F-75013 Paris, France

Introduction: Hydroxychloroquine (HCQ) is an antimalarial agent given to patients with systemic lupus erythematosus (SLE) as first-line therapy. It alleviates childhood-onset systemic lupus erythematosus cSLE skin and musculoskeletal disease, decreasing disease activity and flares. HCQ concentration-effect relationships in children remains unknown. Therefore, this study aimed to investigate the pharmacokinetics of HCQ and possible concentration-effect relationships.

Methods: HCQ blood concentrations and effects were obtained on a daily basis during clinical routine at different occasions during the treatment. cSLE flares were defined using the SLE Disease Activity Index (SLEDAI); flare was denoted by a SLEDAI score $>6$. Blood concentration was measured using high-performance liquid chromatography with fluorometric detection. Statistical analysis was performed using a non linear mixed-effect approach via Monolix.

Results: A total of 168 blood samples were obtained from 55 pediatric patients. HCQ apparent blood clearance (CL/F) was dependent on patients' body weight and platelet count. Patients with active cSLE had a lower mean blood HCQ concentration compared with inactive cSLE patients $\left(536 \pm 294\right.$ vs $\left.758 \pm 490 \mathrm{ng} / \mathrm{mL}, p=5 \times 10^{-6}\right)$. Among patients with HCQ blood concentration $\geq 750 \mathrm{ng} / \mathrm{mL}, 87.6 \%$ had inactive cSLE. Moreover, HCQ blood concentration was a significant predictor of disease status.

Conclusion: We developed the first HCQ blood concentration-effect relationship for cSLE in terms of active or non-active disease status. To confirm these results, a prospective analysis is necessary.

\section{EP-1 PREDICTIVE VALUE OF RENAL OXYGENATION IN THE FIRST DAY OF LIFE IN PREMATURE INFANTS WITH HEMODYNAMICALLY SIGNIFICANT PATENT DUCTUS ARTERIOSUS IN EARLY DIAGNOSIS OF ACUTE KIDNEY INJURY}

Svitlana Samsonenko ${ }^{1}$, Tamara Borysova ${ }^{1}$, Olha Obolonska ${ }^{1}$, Denis Surkov ${ }^{2}$

${ }^{1}$ Dnipro State Medical University, ${ }^{2}$ Mi "dnepropetrovsk Regional Childrens Clinical Hospital" Drc"

Introduction: To assess the prognostic value of renal oxygenation in the first day of life in premature infants with hemodynamically significant patent ductus arteriosus (HSPDA) in the early diagnosis of acute kidney injury (AKI).

Material and methods: We examined 74 premature infants (gestational age 29-36 weeks). The patients were divided into three groups: I - 40 children with HSPDA, II - 17 children with patent ductus arteriosus (PDA) without hemodynamic disorders, III - 17 children with closed ductus arteriosus (CDA). In each of the groups were identified subgroups with the presence of AKI. Doppler echocardiography was performed at 511 hours of life. Regional renal oxygenation $\left(\mathrm{RrSO}_{2}\right)$ was monitored using Near-Infrared Spectroscopy (NIRS).

Results: AKI on the third day of life was diagnosed in $52.5 \%$ of children with HSPDA, which is 2.2 times more often than in children with PDA without hemodynamic significance $(\mathrm{p}<0.05)$ and 4.4 times more often than with CDA $(\mathrm{p}<0.007)$. Renal oxygenation $\left(\mathrm{RrSO}_{2}\right)$ in the first day of life was below $68 \%$, mainly in children with HSPDA+AKI. Extremely low levels of $\mathrm{RrSO}_{2}$ on the first day, associated with the development of AKI on the third day, in the group of HSPDA+AKI were registered in 49-52\%. In children with HSPDA who developed AKI by the third day of life, the $\mathrm{RrSO}_{2}$ index in the first day of life was $56.1 \pm 4.5 \%$ versus $63.9 \pm 9.72 \%$ in children without AKI $(p<0.01)$. An inverse correlation was established between the $\mathrm{RrSO}_{2}$ indicator on the first day of life and the blood creatinine concentration on the third day of life $(\rho=-0.434, p<0.02)$.

Conclusions: Low renal oxygenation in the first day of life is associated with the development of AKI on the third day of life in premature infants with HSPDA.

\section{EP-2 RENAL VEIN THROMBOSIS IN NEONATES : A FRENCH EXPERIENCE FROM 2005 TO 2020}

Bellaure Ndoudi Likoho ${ }^{1}$, Theresa Kwon ${ }^{1}$, Romain Berthaud ${ }^{2}$, Claire Dossier ${ }^{1}$, Jean-daniel Delbet ${ }^{3}$, Olivia Boyer ${ }^{2}$, Véronique Baudouin ${ }^{1}$, Julien Hogan ${ }^{1}$, Anne Couderc ${ }^{2}$

${ }^{1}$ Department Of Pediatric Nephrology, Robert-debré University Hospital, Assistance Publique-hôpitaux De Paris, And University Of Paris, Paris, France, ${ }^{2}$ Department Of Pediatric Nephrology, Neckerenfants-malades University Hospital, Assistance Publique-hôpitaux De Paris, And University Of Paris, Paris, France, ${ }^{3}$ Department Of Pediatric Nephrology, Trousseau University Hospital, Assistance Publiquehôpitaux De Paris, And Sorbonne University, Paris, France

Introduction: Neonatal renal vein thrombosis (NRVT) is a wellrecognized but rare condition, with only a few retrospective studies published to date.

Material and methods: We retrospectively analyzed newborns (age from birth to 28 days old) diagnosed with NRVT admitted to 3 pediatric nephrology units in Paris from 2005 to 2020. Results were expressed as median and range.

Results: Twenty-nine patients were analyzed (male $=59 \%)$. The median age at diagnosis was 3 days $[1 ; 7]$ and 3 patients $(10 \%)$ were diagnosed antenatal. Thirteen patients $(45 \%)$ were premature. In 26 patients, diagnosis was suspected based on at least one of the three cardinal signs of RVT: palpable mass in the flank (62\%), hematuria $(62 \%)$ and thrombocytopenia (69\%). Only 9 patients $(31 \%)$ presented with the full triad at diagnosis. In all patients, diagnosis was confirmed by ultrasound. All patients had at least one known obstetrical or neonatal risk factor: birth by cesarean section $(69 \%)$, perinatal anoxia $(41 \%)$ or maternal diabetes $(21 \%)$. NRVT was unilateral in 21 infants (72\%), involving the left renal vein in $52 \%$. NRVT was associated with inferior vena cava thrombosis in 21 cases $(72 \%)$ and with adrenal glands hemorrhage in 11 patients $(38 \%)$. Prothrombotic abnormalities were found in 12 patients $(41 \%), 11$ patients $(92 \%)$ had factor V Leiden mutation. Among 27 treated patients, 20 received low molecular weight heparin, 2 unfractionated heparin and 4 fibrinolysis. The median length of treatment was 8 weeks [4; 12]. Most patients achieved partial repermabilization $(59 \%)$. After a median follow-up of 4 years and 5 months [1; 7 years], 16 patients $(55 \%)$ had lost function of their kidney, $1(3 \%)$ had chronic kidney disease (CKD) and 2 (7\%) had high blood pressure (HBP).

Conclusions: NRVT remains a challenging condition, which need to be early diagnosed to avoid CKD and HBP.

\section{EP-3 THE ROLE OF EXTRACELLULAR DNA IN ACUTE} KIDNEY INJURY

Alexandra Gaál Kovalčíková ${ }^{1}$, Lubomíra Tóthová ${ }^{2}$, Peter Celec ${ }^{2}$, Ludmila Podracká ${ }^{1}$

${ }^{I}$ Department Of Pediatrics, National Institute Of Children's Diseases And Faculty Of Medicine, Comenius University, Bratislava, Slovakia, ${ }^{2}$ Institute Of Molecular Biomedicine, Faculty Of Medicine, Comenius University, Bratislava, Slovakia 
Introduction: Acute kidney injury (AKI) is a global clinical problem, affecting almost one third of critically ill children. The conventional diagnostic criteria of AKI are based on serum creatinine and urinary output. However, changes in serum creatinine lag behind changes in glomerular filtration rate (GFR), which might take several days. It does not allow early intervention. Therefore, extensive effort is put into searching for more reliable biomarkers allowing early detection of kidney impairment before rapid decrease of kidney functions. A potential non-specific marker of tissue damage is extracellular DNA (ecDNA). ecDNA is present in various body fluids, where enters from disintegrated cells in processes of apoptosis and necrosis, or during inflammatory damage - NETosis. The aim of our study was to assess concentrations of ecDNA in animal model of AKI and cohort of pediatric patients.

Material and methods: In the first part of the study, 93 Wistar rats were used. To induce AKI, glycerol-induced rhabdomyolysis was performed $(n=58)$. Healthy controls received saline $(n=35)$. Blood and urine were collected 1, 3 and 24 hours after AKI induction. In the clinical part of the study, 20 children $(1-18$ years) diagnosed with AKI were included. Thirty age-matched healthy children were recruited as controls.

Results: Plasma concentrations of creatinine and urea in rats with AKI significantly increase 24 hours after AKI induction. Total ecDNA and nuclear DNA was significantly increased already after 1 hour, and this rise continued during the observed period. Plasma mitochondrial DNA in AKI group raised after 3 hours. Urinary ecDNA significantly increased 24 hours after AKI induction. Total DNA and nuclear DNA were significantly higher in plasma of pediatric patients compared to healthy controls ( 70 vs. $190 \mathrm{ng} / \mathrm{ml}, \mathrm{p}<0.01 ; 1983$ vs. $3823 \mathrm{GE} / \mathrm{ml}, \mathrm{p}<0.05$ ). Moreover, their negative relation with eGFR was found $(r=-0.43, p<0.05 ; \mathrm{r}=-0.47$, $\mathrm{p}<0.05$ ).

Conclusions: The rise of plasma ecDNA precedes an increase of conventional markers - creatinine and urea. Our results indicate that ecDNA has potential to serve as an early marker of AKI. Further studies should elucidate the relation between plasma and urinary ecDNA i.e. whether urinary ecDNA is trans-renal origin or comes only from damaged kidneys. Moreover, the dynamics between 3 and 24 hours should be better studied.

The work was support by the Grant Agency of Ministry of Education, Science, Research and Sport of the Slovak Republic VEGA 1/0234/18 and APVV-18-0287.

\section{EP-4 COULD ROBO2 GENE VARIANT BE CAUSATIVE OF RENAL HYPODYSPLASIA?}

\author{
Elena Cavallaro ${ }^{1}$, Susanna Negrisolo ${ }^{2}$, Andrea Carraro ${ }^{2}$, \\ Germana Longo ${ }^{3}$, Davide Meneghesso ${ }^{3}$, Mattia Parolin ${ }^{3}$, \\ Elisa Benetti ${ }^{3}$ \\ ${ }^{1}$ Department Of Woman And Childs Health, University Of Padova, \\ Padua, Italy, ${ }^{2}$ Laboratory Of Immunopathology And Molecular Biology \\ Of The Kidney, Institute Of Pediatric Research Città Della Speranza, \\ Department Of Women's And Children's Health, Padua University \\ Hospital, Padua, Italy, ${ }^{3}$ Pediatric Nephrology Dialysis And Transplant \\ Unit, Department Of Women's And Children's Health, Padua University \\ Hospital, Padua, Italy
}

Introduction: Renal hypodysplasia is part of the broad spectrum of congenital anomalies of the kidney and urinary tract (CAKUT). The presence of familial clustering supports a genetic basis for these diseases. More than 40 genes involved in kidney development are known to be associated with CAKUT and these genes mutations have often different expression even within the same family.

Material and methods: An 18-month-old baby was evaluated at our Pediatric Nephrology Department after E.Coli urinary tract infection (UTI). The renal US showed a smaller right kidney with parenchymal thinning, whereas the left one had compensatory hypertrophy. Imaging analysis (e.i. voiding cystourethrography and DMSA scintigraphy) revealed a mild right vesicoureteral reflux (VUR) and the presence of scar tissue. Monolateral renal hypodysplasia and VUR were diagnosed. Clinical family history pointed out that the mother had right kidney ptosis. To rule out which specific variants were involved, we performed the NGS analysis considering a panel of 74 CAKUT genes.

Results: The genetic analysis highlighted a $\mathrm{ROBO} 2$ missense variant with an uncertain significance (NM 002942.4: c.3788C $>$ T p.Pro1263Leu). This variation, never reported in literature, localized in a conserved protein position and in silico analysis with PolyPhen2 predicted that the variation might be "probably damaging" with a 0.994 score. ROBO2 encode for the SLIT2 receptor which act as mediator of GDNF signaling in renal branching morphogenesis. $\mathrm{ROBO} 2$ variants have been already reported in isolated autosomal dominant CAKUT and in VUR. Therefore, we are currently performing segregation analysis. Conclusions: The ROBO2 heterozygous missense variant $p$.Pro1263Leu could be related to CAKUT, as already observed for other variant of the same gene. Due to the family history, segregation analysis will finally elucidate the pathogenic role of this new variant.

\section{EP-5 A RARE CAUSE OF ACUTE KIDNEY INJURY: RETROPERITONEAL FIBROSIS ASSOCIATED WITH SLC29A3 GENE MUTATION}

Sibel Yel ${ }^{1}$, Neslihan GÜnay ${ }^{1}$, Aynur Gencer Balaban ${ }^{1}$, Aylin Inal ${ }^{1}$, Ebru Yilmaz ${ }^{2}$, Ibrahim Boga ${ }^{3}$, Atil Bisgin ${ }^{3}$, Deniz Demirci ${ }^{4}$, Ismail Dursun ${ }^{1}$, Muammer Hakan PoyrazoĞlu ${ }^{1}$

${ }^{1}$ Erciyes University Department Of Pediatric Nephrology Kayseri, Turkey, ${ }^{2}$ Erciyes University Department Of Pediatric Hematology\&oncology Kayseri, Turkey, ${ }^{3}$ Cukurova University Agentem (adana Genetic Diseases Diagnosis And Treatment Center) \& Medical Genetics Department Of Medical Faculty Adana,Turkey, ${ }^{4}$ Erciyes University Department Of Urology Kayseri, Turkey

Introduction: The mutations in the SLC29A3 gene cause a wide range of different clinical manifestations, including cardiac anomalies, growth retardation, insulin-dependent diabetes and, chronic inflammatory syndrome. Here, we report a case with early-onset diabetes and hydroureteronephrosis secondary to retroperitoneal fibrosis associated with chronic inflammation of the peritoneal membrane.

Material and methods: A 10-year-old boy with diabetes mellitus presented with fever and right flank pain was admitted. Ultrasound showed bilateral hydronephrosis without any stone. DTPA was remarkable for bilateral ureteropelvic junction obstruction. A ureteral stricture on the left side and retroperitoneal fibrosis (RPF) was detected during the surgical procedure. Bilateral double $\mathrm{J}$ stent was placed for urine drainage. On follow-up, iliac venous thrombosis was detected. Since he had increased acute phase reactants and creatinine levels with normal immunoglobulin levels, he was given Coumadin, steroid, and azathioprine for idiopathic retroperitoneal fibrosis. During follow-up, he underwent recurrent double J stent procedures. Sometimes nephrostomy was needed for acute kidney injury due to drainage problems or infectious complications of double $J$ catheter. Because of the early onset autoantibody negative insulin-dependent diabetes mellitus and idiopathic RPF, he underwent a genetic study that revealed a novel homozygous frameshift mutation (p. F71fs*24) on the SLC29A3 gene. Therefore, our case was diagnosed as a rare phenotype of histiocytosis plus lymphadenopathy. $\mathrm{He}$ is stable with MMF treatment.

Results: Histiocytosis plus lymphadenopathy associated with SLC29A3 mutations are very rare in childhood and can present with retroperitoneal fibrosis.

Conclusions: An early diagnosis is warranted in order to start systemic therapy and to treat obstructive complications such as ureteral obstruction that may lead to chronic kidney disease. 


\section{EP-6 ACUTE KIDNEY INJURY IN PAEDIATRIC INTENSIVE} CARE AFTER CARDIAC SURGERY

Joanne Hemingway, Joseph Mcallister, Eve Blanshard, Pallavi Yadav Leeds Teaching Hospitals Trust

Introduction: Acute Kidney Injury (AKI) is a common complication after cardiac surgery associated with increased morbidity and mortality. Chronic Kidney Disease and hypertension burden is high after paediatric cardiac surgery. This audit aimed to characterise the epidemiology and risk factors associated with $\mathrm{AKI}$ and need for dialysis.

Material and methods: A single centre retrospective study of patients admitted to the Paediatric Intensive Care Unit (PICU) following cardiac surgery between January 2019 and December 2020 who developed an AKI. Patients were identified from electronic AKI warnings. Electronic medical notes and blood tests were reviewed.

Results: There were 681 admissions to PICU following cardiac surgery. 9\% (61) developed AKI - the majority (93\%) underwent cardiopulmonary bypass (CPB) procedures. Three patients required Extracorporeal Membrane Oxygenation. $48 \%$ of patients with AKI had a peritoneal dialysis (PD) catheter inserted at the time of surgery.

Eighteen patients with AKI (30\%) required renal replacement therapy. 89\% were neonates $(<1$ month, $<5 \mathrm{~kg}) .81 \%$ had a CPB time of over 180 mins. $50 \%$ of the procedures had a RACHS-1 score of 6 . Other risk factors included inotrope requirements (75\%), fluid overload (56\%) and low cardiac output syndrome $(34 \%)$. No pre-existing renal abnormalities were identified. The reasons for initiating dialysis were fluid overload $(61 \%)$, hyperkalaemia $(22 \%)$, uraemia $(11 \%)$ and acidosis $(6 \%)$. Mortality within this group was $17 \%$. At discharge from PICU 93\% had recovered from AKI.

There was minimal nephrology input into AKI $-5 \%$ of patients were discussed. Conclusions: Our cohort had similar incidence of AKI to published reports. We identified the same risk factors for AKI post cardiac surgery including younger age, lower weight, and longer bypass time. AKI resolved in the majority of patients but there was no renal follow up, despite national AKI guidance. We need a drive to institute follow up for these high-risk patients to improve quality of care.

\section{EP-7 EARLY URINARY SYSTEM COMPLICATIONS IN CHILDREN WITH HEMATOPOIETIC STEM CELL TRANSPLANTATION}

Anar Gurbanov ${ }^{1}$, Bora Gülhan ${ }^{2}$, Barıș Kușkonmaz ${ }^{3}$, Fatma Visal Okur ${ }^{3}$, Duygu Uçkan Çetinkaya ${ }^{3}$, Gülșah Özdemir ${ }^{2}$, Kübra Çeleğen ${ }^{2}$,

Eda Didem Kurt Șükür ${ }^{2}$, Fatih Ozaltin ${ }^{2}$, Ali Düzova ${ }^{2}$, Rezan Topaloglu ${ }^{2}$

${ }^{1}$ Hacettepe University School Of Medicine, Department Of Pediatrics, Ankara, Turkey, ${ }^{2}$ Hacettepe University School Of Medicine, Department Of Pediatrics, Division Of Pediatric Nephrology, Ankara, Turkey, ${ }^{3}$ Hacettepe University School Of Medicine, Department Of Bone Marrow Transplantation, Ankara, Turkey

Introduction: Urinary system complications after hematopoietic stem cell transplantation (HSCT) cause severe morbidity and mortality. The aim of the study is to investigate the incidence and risk factors of urinary system complications in patients who had HSCT during their childhood. Material and methods: Patients who had HSCT between January 20102019 with a minimum follow-up period of 6 months were included. Data were collected from the medical records of the patients. pRIFLE and KDIGO classification systems were used for the definition of acute kidney injury (AKI).

Results: 167 patients (108 males and 59 females) with allogeneic $(n=165)$ and autologous $(n=2)$ HSCT were investigated. In cohort, HSCT was performed in 41 patients (\%24.6) secondary to malignant diseases and in 126 patients secondary to non-malignant diseases. Hemorrhagic cystitis (HC) developed in $16.8 \%$ patients. Among patients with $\mathrm{HC}, 17$ had concomitant viral infection. Presence of viral infection, gender, disease group, history conditioning regime, total body irradiation, acute graft-versus-host disease and veno-occlusive disease (VOD) did not have any effect on the development of $\mathrm{HC}$ in logistic regression analysis. In cohort, 126 patients $(75.4 \%)$ developed AKI according to KDIGO (stage 1; 55 patients, stage 2; 36 patients, stage $3 ; 35$ patients). The mean period of development of AKI after HSCT was $34 \pm 22$ days. The risk of AKI (according to KDIGO) was higher in patients who had HSCT secondary to malignant diseases and/or who developed viral infections after HSCT $(\mathrm{p}=0.034$ and $\mathrm{p}=0.013$, respectively). Among patients, 71 patients (\%42.5) developed AKI according to pRIFLE. The risk of AKI (according to pRIFLE) was higher in patients who had HSCT secondary to malignant diseases, who developed viral infections and/or who developed VOD ( $\mathrm{p}=0.043, \mathrm{p}=0.001$ and $\mathrm{p}=0.006$, respectively). Conclusions: Patients who had HSCT secondary to malignant disease, history of viral infections and/or VOD had higher risk of AKI and should be closely monitored.

\section{EP-8 EVALUATION OF SODIUM STATUS IN PATIENTS WITH CYSTIC FIBROSIS}

Lieselot Peremans ${ }^{1}$, Stephanie Van Biervliet ${ }^{2}$, Dimitri Declercq ${ }^{3}$, Ann Raes ${ }^{4}$, Evelien Snauwaert ${ }^{4}$, Johan Vande Walle ${ }^{4}$

${ }^{1}$ Ghent University, Faculty Of Medicine And Health Sciences, C. Heymanslaan 10, Ghent, Belgium, ${ }^{2}$ Department Of Paediatric Gastroenterology, Ghent University Hospital, C. Heymanslaan 10, Ghent, Belgium, ${ }^{3}$ Cystic Fibrosis Reference Center, Ghent University Hospital, C. Heymanslaan 10, Ghent, Belgium, ${ }^{4}$ Department Of Paediatric Nephrology, Ghent University Hospital, C. Heymanslaan 10, Ghent, Belgium

Introduction: Cystic fibrosis (CF) is characterized by increased sodium and chloride losses related to defects in the CF transmembrane conductance regulator gene. The circulating volume, electrolyte as well as acid-base homeostasis in CF are constantly challenged in basal conditions, activating compensatory mechanisms in different renal functions. Current guidelines on monitoring sodium status in CF patients are based on fractional sodium excretion (FENa), which is unpractical since it requires paired blood and urine samples. The aim of this study was to identify urinary parameters as a surrogate for sodium-status in different age-groups.

Material and methods: Blood and urine samples for electrolyte status determination were collected and analyzed retrospectively from 214 patients followed at the Ghent University Hospital CF-center.

Results: The urinary sodium/creatinine ratio ( $\mathrm{UNa} / \mathrm{Creat})$ showed a strong correlation with FENa $(r=0,93)$. The target FENa of $0.5-1.5 \%$ corresponded to a UNa/Creat of $13,125-38,575 \mathrm{mmol} / \mathrm{mmol}$. However, the correlation between $\mathrm{FENa}$ and $\mathrm{UNa} / \mathrm{Creat}$ did differ significantly between different age-groups. There was no association found between a decreased FENa $(<0,5 \%)$ and several clinical characteristics.

Conclusions: The strong correlation between $\mathrm{FENa}$ and $\mathrm{UNa} / \mathrm{Creat}$ in a population of $214 \mathrm{CF}$ patients implies that $\mathrm{UNa} / \mathrm{Creat}$ would be a more practical and less invasive marker to follow the sodium status in this patient population compared to the current gold standard FENa. In addition, we showed that there were clear age differences which highlights the need for different reference values according to age.

\section{EP-9 OXIDATIVE STRESS BIOMARKERS IN AN EXPERIMENTAL MODEL OF MILD RENAL FUNCTION IMPAIRMENT}

Tomsa Anamaria Magdalena ${ }^{1}$, Rachisan Andreea Liana ${ }^{1}$, Pandrea Stanca Lucia ${ }^{2}$, Benea Andreea ${ }^{2}$, Junie Lia Monica ${ }^{1}$

1 "iuliu Hatieganu" University Of Medicine And Pharmacy, Clujnapoca, Romania, " "prof. Dr. Octavian Fodor" Regional Institute Of Gastroenterology And Hepatology, Cluj-napoca, Romania

Introduction: Kidney disease represents a burden for the health system worldwide. As the prevalence continues to rise, discovering new 
biomarkers of early kidney damage becomes acute. Oxidative stress (OS) represents one of the main factors involved in the early stages of many syndromes leading to kidney damage, therefore it must be studied in detail. So far, many studies focused on OS in advanced stages of acute kidney injury, with great success. The purpose of our study was to determine if even mild renal function impairment can be linked to specific systemic markers of OS and systemic antioxidants in order to pinpoint certain biomarkers for early kidney damage.

Material and methods: We used male rats (Rattus norvegicus) in which we induced kidney damage by injecting gentamicin for 7 days. Blood was collected 24 hours after the last dose of gentamicin. Urea, creatinine, 3nitrotyrosine (3NT), nitric oxide (NO), malondialdehyde (MDA), thiols (TS), total oxidative stress (TOS), interferon- $\gamma($ IFN- $\gamma$ ) were measured. Also, for the antioxidant status we measured total antioxidant capacity (TAC) and interleukin-10 (IL-10).

Results: Our results show that our rats had mild renal impairment consistent with a pre-AKI stage due to the nephrotoxic effect of gentamicin. However, TOS, MDA and NO were significantly higher in the gentamicin group compared to the control group. Also, TAC was higher in the control group.

Conclusions: Oxidative stress markers reach higher levels even in the pre-AKI stage and might potentially be used as markers of kidney damage when mild renal function impairment occurs.

\section{EP-10 OVERLAP GLOMERULONEPHRITIS IN AN ADOLESCENT WITH AUTOIMMUNE LYMPHOPROLIFERATIVE SYNDROME}

Emre Leventoğlu ${ }^{1}$, Bahar Büyükkaragöz ${ }^{1}$, Zühre Kaya ${ }^{2}$, Kibriya Fidan ${ }^{1}$, Oğuz Söylemezoğlu ${ }^{1}$, Sevcan A. Bakkaloğlu ${ }^{1}$

${ }^{I}$ Gazi University, Faculty Of Medicine, Department Of Pediatric Nephrology, ${ }^{2}$ Gazi University, Faculty Of Medicine, Department Of Pediatric Hematology

Introduction: Autoimmune lymphoproliferative syndrome (ALPS) is an immune dysregulation syndrome characterized by chronic, nonmalignant lymphoproliferation and autoimmunity due to defective lymphocyte apoptosis. Clinical features include lymphadenopathy, splenomegaly, and cytopenia; glomerulonephritis occurred in 3\%.

Material and methods: We present a patient with ALPS phenotype who developed an overlap syndrome of ANCA-associated crescentic glomerulonephritis with immune complex-mediated glomerulopathy as a case report. Results: 14-year-old male with a 5-year history of hepatosplenomegaly, presented to emergency department with tea colored urine. There was no history of infection or dysuria and rash. Laboratory analysis showed pancytopenia and acute kidney injury; creatinine $1.55 \mathrm{mg} / \mathrm{dl}$. Urinalysis showed dysmorphic erythrocytes and proteinuria $(4728 \mathrm{mg} /$ day). Ultrasound showed hepatosplenomegaly and echogenic kidneys. Haptoglobulin was low $(2 \mathrm{mg} / \mathrm{dl})$, direct coombs was $2+\mathrm{C} 3$ and $\mathrm{C} 4$ were at $49 \mathrm{mg} / \mathrm{dl}(79-152)$ and $12 \mathrm{mg} / \mathrm{dl}(16-38)$, respectively. IgG was elevated $(2860 \mathrm{mg} / \mathrm{dl})$. ANA, anti-dsDNA, anti-GBM were negative; c-ANCA, pANCA were positive. Double negative T cells were high as 3.8\%. Viral markers were negative except for Ebstein-Barr virus (EBV) PCR (750 copies $/ \mathrm{ml}$ ) which was increased to 11500 copies $/ \mathrm{ml}$ one week later. He was diagnosed as probable ALPS. Renal biopsy showed diffuse proliferative glomerulonephritis with $\mathrm{IgG}$ deposition. Also, the presence of glomerular basement membrane fracture and fibrinoid necrosis was interpreted in favor of a second glomerular disease in the form of a necrotizing glomerulonephritis when evaluated together with the patients positive ANCA serology.

Induction treatment consisted of intravenous pulse methylprednisolone for five days and rituximab; which was preferred considering the positive EBV titers. In addition, five sessions of plasma exchange were performed. During the maintenance phase, oral steroids and mycophenolatemofetil were given. After these treatments, serum creatinine has normalized $(0.53 \mathrm{mg} / \mathrm{dl})$; his proteinuria and hematuria were significantly reduced and pancytopenia improved.

Conclusions: ALPS should be considered in the differential diagnosis of a patient presenting with hematuria, proteinuria, acute kidney injury and pancytopenia and hepatosplenomegaly.

\section{EP-11 ANALYSIS OF LATE RENAL COMPLICATIONS AND RISK FACTORS IN CHILDREN WITH HEMATOPOIETIC STEM CELL TRANSPLANTATION}

Anar Gurbanov ${ }^{1}$, Bora Gulhan ${ }^{2}$, Barıș Kușkonmaz ${ }^{3}$, Fatma Visal Okur ${ }^{3}$, Duygu Uçkan Çetinkaya ${ }^{3}$, Gülșah Özdemir ${ }^{2}$, Nesrin Taș ${ }^{2}$,

Eda Didem Kurt Șükür ${ }^{2}$, Fatih Ozaltin ${ }^{2}$, Ali Düzova ${ }^{2}$, Rezan Topaloglu ${ }^{2}$ ${ }^{1}$ Hacettepe University School Of Medicine, Department Of Pediatrics, Ankara, Turkey, ${ }^{2}$ Hacettepe University School Of Medicine, Department Of Pediatrics, Division Of Pediatric Nephrology, Ankara, Turkey, ${ }^{3}$ Hacettepe University School Of Medicine, Department Of Bone Marrow Transplantation, Ankara, Turkey

Introduction: The aim of the study is to investigate the incidence and risk factors of hypertension (HT) and chronic kidney disease (CKD) in patients who had hematopoietic stem cell transplantation (HSCT) during their childhood.

Material and methods: Patients who had HSCT between January 20102019 were included in the study. Guidelines of European Society of Hypertension (ESH) and American Academy of Pediatrics (APA) were used for the evaluation of HT. Ambulatory HT is diagnosed when systolic and/or diastolic blood pressure (BP) load is higher than $25 \%$. Ambulatory prehypertension is diagnosed when mean systolic and/or diastolic BP is less than $95^{\text {th }}$ percentile with systolic and/or diastolic BP load higher than $25 \%$.

Results: A total of 72 patients (41 males and 31 females) were included. The mean age of the patients at last visit was $10.8 \pm 4$ years. ABPM revealed ambulatory HT in 6 patients $(8.8 \%)$ and ambulatory prehypertension in 12 patients (17.6\%). Office BP revealed HT in 3 patients $(4.2 \%)$ and increased BP in four patients $(5.6 \%)$ according to APA guideline. In cohort, 12 patients with normal office BP (according to APA guideline) had ambulatory prehypertension or hypertension with ABPM. Office BP revealed HT in 1 patient $(1.4 \%)$ and high-normal BP in 3 patients $(4.2 \%)$ according to ESH guideline. In cohort, 15 patients with normal office BP (according to ESH guideline) had ambulatory prehypertension or HT with ABPM. After a mean follow-up period of $4.4 \pm 2.5$ years, CKD developed in 8 patients $(11.1 \%)$. Patients with chronic graft-versus-host disease, with HLA-mismatched HSCT and/or transplantation of peripheric or cord blood hematopoietic stem cells had increased risk of CKD ( $\mathrm{p}=0.041, \mathrm{p}=0.033$ and $\mathrm{p}=0.002$, respectively).

Conclusions: Patients with HSCT should be regularly followed for the development of HT and ABPM should be used on regular basis. Patients with risk factors should be closely monitored for the development of CKD.

\section{EP-12 RHABDOMYOLYSIS IN CHILDREN - A 10-YEAR EXPERIENCE}

Veronica Nijloveanu, Matthew Harmer Bristol Royal Hospital For Children

Introduction: Aims to identify the aetiologies of paediatric rhabdomyolysis and explore the medium to long term renal consequences. We describe a single centre's experience of paediatric rhabdomyolysis over 10years. This is the largest dataset reported on in children with the condition.

Material and methods: Retrospective, single-centre review of children presenting to a tertiary children's hospital with rhabdomyolysis as defined 
by a laboratory measured creatinine kinase (CK) of greater than $1000 \mathrm{IU} /$ litre. Exclusion criteria applied (children post cardiac surgery, cardiomyopathy, HIE).

Results: 232 children met inclusion criteria for the analysis. Age at presentation was 8.4years. Median follow-up was 6.3 (interquartile range $+/-43.1)$ months. Infection $(28 \%)$ and trauma(18\%) were the most common causes of rhabdomyolysis. There was no association between aetiology and severity of the condition. Acute kidney injury (AKI) was present in $32 \%$ of cases. Children with AKI tended to be younger with higher peak $\mathrm{CK}$ and active urinary sediment on urinalysis at presentation ( $\mathrm{p}=0.001$ to $<0.0005)$. The $38 \%$ of cases defined biochemically as having 'severe' disease (CK >5000 IU/ litre) were no more likely to require admission to the Paediatric Intensive Care Unit (PICU) than the rest of the cohort. AKI and need for renal replacement therapy were associated with a prolonged hospital stay $(\mathrm{p}<0.0005)$. Over the period of the study, $9 \%$ of children died and $2 \%$ met criteria for a diagnosis of chronic kidney disease (CKD).

Conclusions: Although limited by its retrospective single-centre design, this large retrospective analysis of paediatric rhabdomyolysis provides new and unique insights into the condition. Results highlight the common aetiologies and provide evidence of good renal recovery overall, even in the most severely affected cases. Abnormalities of urinalysis appear to be important in predicting the development of AKI.

\section{EP-13 DIAGNOSTICS AND PREDICTION OF GLOMERU- LOPATHIES IN CHILDREN BY DETECTION NANO- MOLECULAR BIOMARKERS IN BLOOD}

Tatsiana Makhouskaya ${ }^{1}$, Ina Kazyra ${ }^{1}$, Andrei Astashonok ${ }^{2}$, Aliaxander Sukalo ${ }^{1}$, Nikolai Poleshchuk ${ }^{2}$, Hanna Bialkevich ${ }^{1}$

${ }^{1}$ Belarusian State Medical University, ${ }^{2}$ The Republican Research And Practical Center For Epidemiology And Microbiology, Minsk, Belarus

Introduction: We analyzed use of nanomolecular biomarkers of apoptosis (sAPO-1/FAS, annexin V) and endothelial dysfunction (sVCAM-1, sICAM-1) in blood serum in the diagnostics and prognosis of glomerulopathies (GP) in children.

Material and methods: The study included 52 children ( 27 boys and 25 girls aged 3-17 years, median 15). Main group of patients consisted of 38 children with GP, comparison group - of 14 patients with congenital anomalies urinary tract (CAKUT). The main study group was divided into two subgroups: I nonimmune $(n=13)$ and II immune $(n=25)$ kidney disorders.

Results: In subgroup I the concentration of the sAPO-1/FAS factor was from 0,046 to $0,24 \mathrm{ng} / \mathrm{ml}$ (normal value of $0,023 \pm 0,002 \mathrm{ng} / \mathrm{ml}$ ) in serum of 12 children $(92,3 \%)$. The AnV level in this subgroup was normal. In subgroup II the concentration of sAPO-1/FAS was significantly higher (from 0,2 to $0,24 \mathrm{ng} / \mathrm{ml}$ ) than normal in all specimens. In 7 patients (28\%) from this subgroup, values of AnV were increased too - 1.1-1.2 times (7,5-8,49 ng/ml) in 6 cases and 3 times $(21,06 \mathrm{ng} / \mathrm{ml})$ in one patient with $\mathrm{IgA}$-vasculitis. In CAKUT group the concentration of both biomarkers was in the reference range. Expression of sVCAM-1had variations from 0 to $1.2 \mathrm{ng} / \mathrm{ml}$ (normal $0,7-0,9 \mathrm{ng} / \mathrm{ml}$ ) in 10 children $(40 \%)$ of subgroup II. Quantity of sVCAM-1 in subgroup I and CAKUT remained normal. The level of sICAM-1 in all groups was in the normal range from 50 to $67 \mathrm{ng} / \mathrm{ml}$.

Conclusions: Increased levels of sAPO-1/FAS are present in the majority of patients with GP and aren't detected in the CAKUT group. The AnV is elevated only in some children with immune GP. Increased expression of VCAM-1 in the blood of some patients with immune nephropathies was revealed.
EP-14 ASSESSMENT OF THE REPEATABILITY OF 24-HOUR BLOOD PRESSURE MONITORING IN CHILDREN REFERRED FOR THE FIRST DIAGNOSIS OF HYPERTENSION PRELIMINARY RESULTS

Malgorzata Stanczyk ${ }^{1}$, Julia Mirecka ${ }^{1}$, Jill Dybiec ${ }^{2}$, Karolina Drążek ${ }^{2}$, Mateusz Kaliszczuk ${ }^{2}$, Marcin Tkaczyk ${ }^{1}$

${ }^{I}$ Department Of Paediatrics, Immunology And Nephrology, Polish Mothers Memorial Hospital Of Lodz, ${ }^{2}$ Department Od Paediatrics, Preventive Cardiology And Immunology Of Developmental Age, Medical Univeristy Of Lodz.

Introduction: Studies in adults have shown that the results of $24 \mathrm{~h}$ blood pressure monitoring conducted for the first time were higher than the results of subsequent measurements, especially among women. The aim of the study was to assess the repeatability of 24-hour blood pressure monitoring result in children.

Material and methods: 46 children ( $58 \%$ boys), aged 8 to 17 (Me 14) admitted for the first time for the diagnosis of hypertension were examined. No participant had prior 24-hour blood pressure monitoring. Median BMI was $25.15 \mathrm{~kg} / \mathrm{m} 2$ (93 pc). 24-hour blood pressure monitoring was performed twice - on the day of admission to the hospital and after 2 days of hospitalization.

Results: Hypertension was confirmed in $26 \%$ of patients and high normal pressure in $28 \%$ of patients. In $43 \%$ of patients there was a discrepancy between the records - in $85 \%$ of them (37\% of total) blood pressure in the second measurement was significantly lower.

In the whole group, the second measurement showed significantly lower systolic blood pressure during the day ( $128 \mathrm{vs} 125 \mathrm{mmHg}, \mathrm{p}=0.03$ ) and at night (115 vs $111 \mathrm{mmHg}, \mathrm{p}=0.04$ ) and a slower heart rate at night (69 vs 66, $p=0.005$ ). The diastolic, mean and central blood pressure did not differ. The drop in blood pressure in the second measurement was significant mainly in the overweight group, especially in boys. The mean blood pressure during the day in the group of overweight boys was 94 and $85 \mathrm{mmHg}(\mathrm{p}=0.009)$ in the first and second measurement, and 85 and $75 \mathrm{mmHg}$ at night $(\mathrm{p}=0.007)$, respectively. A significant change was observed in all blood pressure values Conclusions: The results of the study indicate that the first result of blood pressure monitoring may be confusing - in more than $1 / 3$ of patients it gives an overestimated result. This is significant especially for overweight boys. The study shows that the 24-hour blood pressure monitoring results should be verified with the second measurement, especially in overweight boys.

\section{EP-15 VITAMIN D IN CHILDREN WITH HYPERTENSION}

Živa Radulović ${ }^{1}$, Zarja Polak Zupan ${ }^{1}$, Aljoša Tomazini ${ }^{1}$, Nataša Marčun Varda ${ }^{2}$

${ }^{1}$ Medical Faculty, University Of Maribor, Maribor, Slovenia, ${ }^{2}$ University Medical Centre Maribor, Department Of Paediatrics, Unit For Paediatric Nephrology And Hypertension, Maribor, Slovenia

Introduction: Vitamin D is very important element in human organism and plays a key role in many physiological as well as pathological processes. The purpose of this study was to find potential correlations in serum levels of vitamin $\mathrm{D}(25(\mathrm{OH}) \mathrm{D})$ in overweight children with hypertension (group A ) and normal-weight children with hypertension (group B) compared to healthy control group (group C).

Material and methods: The parameters we determined in all groups of children were serum $25(\mathrm{OH}) \mathrm{D}$ levels, anthropometric measurements (weight, height, body mass index (BMI)), age, sex and blood pressure (BP). We have analyzed the results with the help of SPSS statistic tool (independent t-test and Pearson correlation test).

Results: The study included 120 children between 4 and 18 years of age. The results obtained showed distinction in values of serum $25(\mathrm{OH}) \mathrm{D}$ in group A compared to group $\mathrm{B}$, although the difference was not statistically significant 
$(\mathrm{p}=0.084)$. There was statistically significant difference in values of serum $25(\mathrm{OH}) \mathrm{D}$ between group $\mathrm{A}$ and group $\mathrm{C}(\mathrm{p}=0.001)$. Results also showed differences in 25(OH)D levels between group B and group C, but it was not significant $(\mathrm{p}=0.117)$. We have also managed to prove the correlation between serum vitamin $\mathrm{D}$ levels and systolic $\mathrm{BP}$ values $(\mathrm{r}=-0.288 ; \mathrm{p}=0.01)$ as well as between serum vitamin $\mathrm{D}$ levels and body weight $(\mathrm{r}=-0.155$; $\mathrm{p}=0.045$ ). The correlation between vitamin $\mathrm{D}$ levels and systolic $\mathrm{BP}$ was most apparent in group $\mathrm{B}(\mathrm{r}=-0.338 ; \mathrm{p}=0.033)$.

Conclusions: The pilot study shows that there are differences in serum vitamin D levels between investigated groups, most significant in the group A compared to group C. The biggest correlation was observed between vitamin D levels and systolic blood pressure values. We conclude that the obtained results show that vitamin D supplementation is needed in pediatric patients with hypertension and obesity.

\section{EP-16 SORBENT HEMADSORPTION ON EXTRACORPOREAL CONTINUOUS MEMBRANE OXYGENATION AND CONTINUOUS RENAL REPLACEMENT TREATMENT IS A USEFUL TOOL FOR SEVERE HEMOPHAGOCYTIC LYMPHOHISTIOCYTOSIS}

Pietro Lonardi ${ }^{1}$, Emilia Parodi ${ }^{2}$, Sergio Grassitelli ${ }^{3}$, Roberta Camilla ${ }^{5}$, Davide Montin ${ }^{4}$, Licia Peruzzi ${ }^{5}$

${ }^{1}$ Department Of Paediatric And Public Health Sciences, Postgraduate School Of Paediatrics, University Of Torino, Turin, Italy, ${ }^{2}$ Pediatric Hematology, Department Of Pediatric And Public Health Sciences, University Of Torino, Turin, Italy, ${ }^{3}$ Pediatric Intensive Care Unit, Regina Margherita Childrens Hospital, Turin, Italy, ${ }^{4}$ Division Of Pediatric Immunology And Rheumatology, Department Of Public Health And Pediatrics, Regina Margherita Children Hospital, University Of Turin, Turin, Italy, ${ }^{5}$ Pediatric Nephrology Unit, Intensive Care Unit, Regina Margherita Dept, Cdss University Hospital Turin Italy

Introduction: Hemophagocytic Lymphohistiocytosis (HLH) is a lifethreatening disease. Therapy relies on protocols based on high dose immunosuppressors, with high risk of infection and toxicity.

Extracorporeal sorbent hemadsorption (HA) techniques are a promising land for patients with cytokine storm. Among HA devices, Cytosorb ${ }^{\circledR}$ is among the most used in clinical practice, though evidence to support its use is still lacking.

Mantaining a sufficient blood flow rate and managing the priming volume can be challenging in the setting of hemodynamic instability or in pediatric patients. Extracorporeal membrane oxygenation (ECMO) offers an ideal setting for HA.

So far only few experiences of Cytosorb®/HA techniques use in HLH are reported.

Here we report our experience in a multidisciplinary handling of a case. Material and methods: A 17-year-old boy with new onset systemic juvenile idiopathic arthritis rapidly evolved in secondary $\mathrm{HLH}$, requiring intensive care unit admission for vasopressor support, high dose corticosteroids, Etoposide, Cyclosporin and Anakinra. After initial improvement, the patient's condition worsened with critical reduction of cardiac output, increased ferritin, multiorgan failure. The patient was started on ECMO.

Considering the cytokine storm and ferritin levels despite aggressive immunosuppression and secondary shock requiring high doses of vasopressors, Cytosorb® was applied to ECMO and CRRT circuit.

The patient improved dramatically. After five days he was weaned from ECMO, vasopressor support and HA.

Sadly, after a week, he developed bone marrow failure and died of pulmonary aspergillosis.

Results:

Conclusions: In severe HLH prompt ECMO with CRRT and HA techniques can be considered to obtain a rapid hemodynamic support and efficient cytokine removal. The cytokine storm with high levels of IL6 typical of HLH can be an ideal setting for Cytosorb ${ }^{\circledR}$ cartridge use.
EP-18 ACUTE TUBULOINTERSTITIAL NEPHRITIS IN CHILDREN: A SINGLE CENTER EXPERIENCE

Mehtap Adar ${ }^{1}$, Havva Serap Toru ${ }^{2}$, Bahar Akkaya ${ }^{2}$, Elif Çomak ${ }^{1}$, Mustafa Koyun ${ }^{1}$, Ayla Kaçar ${ }^{1}$, Muhammet Sancaktar ${ }^{1}$, Gülșah Kaya Aksoy ${ }^{1}$, Sema Akman ${ }^{1}$

${ }^{I}$ Akdeniz University Medical Faculty, Department Of Pediatric Nephrology, Antalya, ${ }^{2}$ Akdeniz University Medical Faculty, Department Of Pathology, Antalya

Introduction: Acute tubulointerstitial nephritis (ATIN), a rare cause of acute kidney injury in children, is characterized by inflammation of the tubulointerstitial region in the kidneys. In this study, it was aimed to evaluate the clinical features, etiology, treatment and prognosis of pediatric patients with ATIN who were diagnosed by biopsy and followed up at our center.

Material and methods: The patients who underwent kidney biopsy between January 1th 2008 and December 31th 2020 at our hospital were reviewed and patients diagnosed with ATIN were included in the study. The demographic and clinical characteristics of the patients were obtained from records.

Results: Twenty-one patients who were diagnosed with ATIN out of 325 patients $(6.5 \%)$ having biopsy were included in the study. 11 were boys $(52.4 \%)$, the mean age was 13.6 years (2-17 years) and median follow-up period was 27 months. All patients had acute kidney injury at presentation; mean creatinine was $4.36 \mathrm{mg} / \mathrm{dl}$. Nephrotoxic drug usage was observed in $11(52.4 \%)$ patients: NSAID in four, paracetamol in two, and pseudoephedrine, benzoyl peroxide and gentamicin-containing cream, gingko biloba, heroin, and chemotherapeutic drugs in one patient each. Etiology was not detected in the remaining 10 patients, who were accepted as idiopathic ATIN. Renal functions were improved in 6 patients (28.6\%) spontaneously within a few days. 9 patients $(42.9 \%)$ were treated with corticosteroids and 4 patients with both corticosteroids and azathioprine. At follow-up, 6 patients had eGFR lower than age-appropriate values, three of whom progressed to end-stage renal failure, all of whom were in idiopathic group. Chronic renal failure did not develop in any patient with ATIN due to nephrotoxic medications.

Conclusions: ATIN develop mostly due to nephrotoxic drug usage. Early diagnosis and treatment is important, because it may lead to chronic renal failure.

\section{EP-19 RISK FACTORS FOR LOWER URINARY TRACT DYSFUNCTION}

Mehmet Çagri Özkara, Mustafa Koyun, Elif Çomak, Gulsah Kaya Aksoy, Sema Akman

Akdeniz University Medical Faculty, Department Of Pediatrics

Introduction: Lower urinary tract dysfunction (LUTD) is a common problem in children. The risk factors is not well defined. In the present study, we aimed to investigate the risk factors such as voiding position (standing, sitting and squatting in boys, sitting and squatting in girls) and age and duration of toilet training on the development of LUTD.

Material and methods: 150 children who had a diagnosis of LUTD and 300 healthy children above 5 years of age were included in the study. The data was collected by questionnaire in the control group whereas the data of the patient group were evaluated retrospectively. The patients with anatomical or neurological problems were excluded.

Results: The mean ages of the patient and control group were $8.1 \pm 2.5$ and 9.2 \pm 3.1 years, respectively ( $p>0.05$ ). Girls were more in patient than control group ( $64 \%$ vs $51 \%, \mathrm{p}=0.009$ ). Age at toilet training were similar between the groups, whereas having $>1$ month of duration of toilet training was more common in patient than control group ( $86.7 \%$ vs $51.0 \%, \mathrm{p}<0.001)$. The ratio of boys who void on standing was more in patient than control group ( $64.8 \%$ vs $40.8 \%$, $\mathrm{p}=0.006$ ). Also, the ratio of girls who void on squatting position was more in patient than control group (40.6\% vs $17.6 \%, \mathrm{p}<0.001)$. On logistic regression 
analysis, boys who void on standing had 4.9 fold and girls who void on squatting position had 3.2 fold increased risk for LUTD (95\% CI: 1.9-12.5, $\mathrm{p}=0.001$ and 95\% CI: 1.8-5.7, $\mathrm{p}<0.001)$; also, having $>1$ month of duration of toilet training had 6.7 fold increased risk for LUTD (95\% CI: 4.0-11.4, p <0.001).

Conclusions: In this study, we found that duration, rather than age, at toilet training affects the development of LUTD. Also, standing voiding in boys and squatting position in girls increased the risk of LUTD.

\section{EP-20 THROMBOTIC MICROANGIOPATHY IN A PATIENT WITH DIFFUSE CUTANEOUS MASTOCYTOSIS}

Carlos Echecopar ${ }^{1}$, Alejandro Zarauza ${ }^{1}$, Marta Feito ${ }^{2}$, Iván Álvareztwose ${ }^{3}$, Carlota Fernández ${ }^{1}$, Marta Melgosa ${ }^{1}$

${ }^{1}$ Pediatric Nephrology. Hospital Universitario La Paz. Madrid, ${ }^{2}$ Dermatology. Hospital Universitario La Paz. Madrid, ${ }^{3}$ Haematology. Instituto De Estudios De Mastocitosis De Castilla La Mancha. Hospital Virgen Del Valle, Toledo, Spain

Introduction: We present a case of recurrent episodes of thrombotic microangiopathy (TMA) in a patient with diffuse cutaneous mastocytosis. Material and methods: Case report

Results: Female infant with neonatal diagnosis of diffuse cutaneous mastocytosis, biopsy-proven. Chronic treatment wih cromoglycate, antihistamine (ketotifen, ranitidine, dexchlorpheniramine), prednisolone.

At 8 month-old addmited to emergency department for pallor and hypoactivity. Concurrent worsening of cutaneous mastocytosis. No fever, nor diarrhea. Blood analysis revealed hemolytic non-immune anemia $(5.9 \mathrm{~g} / \mathrm{dL})$ and thrombocitopenia $\left(74.000 / \mathrm{mm}^{3}\right)$. Further evaluation showed schistocytes (11\%) and elevated LDH (2100 $\mathrm{UI} / \mathrm{L})$. Serum creatinine was mildly elevated $(0.56 \mathrm{mg} / \mathrm{dL})$ and urinalysis showed proteinuria and microsopic hematuria. Normal blood pressure. She was addmited to hospital for complete evaluation. Etiologic study of Hemolytic Uremic Syndrome (HUS) was negative: stool culture, ADAMTs-13 activity, serum complement levels and complement genetic study, autoantibodies, serum homocystein. Drugs were investigated and dexchlorpheniramine was discontinued due to potential association with HUS.

She received 5 blood transfusions. Quick recovery of glomerular filtration rate (72h) and total disappearance of proteinuria and hematuria in 4 weeks.

After this first episode, she has had two more relapses of selflimited HUS, at 9 and 12 months of age. Both episodes had slightly elevated creatinine, transient proteinuria and hematuria and completely recovery. Anemia required 2 transfusions per episode. Each HUS relapse was coincident with a worsening of his cutaneous disease. At 12 months she started PUVA-therapy for mastocytosis with good dermatologic results. She has had no new episodes of HUS since then (6 months follow-up).

Kidney biopsy was offered repeatedly but parents refused due to high anesthetic risk.

Conclusions: We present a case of an association not described to date in the literature. We think that thrombogenic and vasoactive effect of mast cell mediators may play a role in recurrent TMA episodes.

\section{EP-21 RITUXIMAB THERAPY IN CHILDREN WITH STEROID-DEPENDENT NEPHROTIC SYNDROME}

Varvara Obukhova, Marina Aksenova, Larisa Prikhodina, Vladimir Dlin Veltischev Research Clinical Institute For Pediatrics Of The Pirogov Russian National Research Medical University

Introduction: Rituximab therapy (RTX) is recommended for children with SDNS relapsing on therapy with 2 or more immunosuppressive drugs, with cyclosporine toxicity/dependence.
Material and methods: 11 patients $(7 \mathrm{M}, 4 \mathrm{~F})$ with SDNS were treated with RTX in our clinic. Median age was 4.8 years $(1.8 ; 5.9)$ at disease onset and 12.9 years $(10.5 ; 14.2)$ at the beginning of RTX treatment. The median disease duration before RTX treatment was 8.17 years $(4.9 ; 9.5)$. A renal biopsy was done in 6/11 children after CNI treatment: FSGS were found in 5 patients, MCNS in 1. Only one patient had biopsy-proven CyA nephrotoxicity. All patient were on MMF therapy during RTX treatment. Results: All patients had B-cells depletion after the first RTX infusion with median duration for 5 months $(3.5 ; 6.0)$. NS relapses were seen in two patients ( $q=0.18$ ): in first - from 8 months after RTX in dose $375 \mathrm{mg} /$ $\mathrm{m}^{2}\left(\mathrm{CD} 1947.8 \%, 1374 / \mathrm{mm}^{3}\right)$ and in second one - from 3 months after MMF discontinuation (RTX in dose $1125 \mathrm{mg} / \mathrm{m}^{2}$; CD19 9.5\%, 190/ $\mathrm{mm}^{3}$ ). Immediate adverse effects (fever, headache, nausea) were seen in 2 patients $(\mathrm{q}=0.18)$ and did not require to stop the infusion. The incidence of hypogammaglobulinemia depended on cumulative RTX dose $(0.71$ vs 0.61 vs 0.3 in high $\left(1125-1500 \mathrm{mg} / \mathrm{m}^{2}\right)$, medium $\left(750 \mathrm{mg} / \mathrm{m}^{2}\right)$ and low $\left(375 \mathrm{mg} / \mathrm{m}^{2}\right)$ received doses, respectively, $\mathrm{p}>0.05$ ) and age of treatment starting ( 1.0 vs 0.67 in pts $<10$ and $\geq 10$ years, respectively, $p>0.05$ ).

Conclusions: Medium and high doses of RTX prevent $82 \%$ of NS recurrence. Infusion-dependent side effects of RTX are infrequent and controlled. The risk of hypogammaglobulinemia is higher in young children and in patients received medium/high RTX doses

\section{EP-22 BIOELECTRICAL IMPEDANCE ANALYSIS OF BODY COMPOSITION IN SOME GROUPS OF CHILDREN AND ADOLESCENTS WITH CARDIOVASCULAR RISK}

Nataša Marčun Varda, Sonja Golob Jančič, Mirjam Močnik, Marjetka Švigelj

Department Of Paediatrics, University Medical Centre Maribor, Slovenia

Introduction: Chronic kidney disease (CKD), obesity and hypertension are important health problems in both adults and children. Moreover, obesity is still increasing in some countries, influencing the prevalence of CKD and hypertension. Nutrition and evaluation of body composition are important part of management of all three groups of patients. The aim of the study was to perform body composition measurement of patients with $\mathrm{CKD}$, obesity-related hypertension and normal-weight hypertension, using bioelectrical impedance.

Material and methods: 114 children and adolescents were included in the study and divided into 4 groups, 25 patients with CKD, 31 patients with obesity-related hypertension, 28 patients with normalweight hypertension and 30 healthy controls. Body composition was measured on an empty stomach and bladder in the same body position using Nutricomb device. Anthropometric measurements (height, weight, waist and hip circumference) were also performed. Bioelectrical impedance gave the results about fat free mass (FFM), total body water (TBW), extracellular water (ECW), body cell mass (BCM), fat mass (FM) and phase angle (PA).

Results: Our results showed that some bioimpedance parameters differ significantly between our control group and normal-weight hypertensive patients ( $p<0.0001$ for FFM, $p<0.0001$ for TBW, $p=0.002$ for $E C W, p<0.0001$ for $\mathrm{BCM}, \mathrm{p}=0.020$ for $\mathrm{FM}, \mathrm{p}<0.0001$ for PA) as well as between the controls and patients with obesity-related hypertension ( $\mathrm{p}<0.0001$ for FFM, $\mathrm{p}<0.0001$ for TBW, $\mathrm{p}<0.0001$ for ECW, $\mathrm{p}<0.0001$ for BCM, $\mathrm{p}<0.0001$ for FM, $\mathrm{p}<0.0001$ for PA, respectively). No statistically significant differences for CKD were found, however, all investigated parameters were in lower range compared to the control group.

Conclusions: Our pilot study confirmed that bioimpedance parameters of paediatric patients with both obesity-related and normal-weight hypertension differ significantly compared to the healthy controls. The results give insight into the importance of monitoring the nutritional status of patients with presumed risk. 


\section{EP-23 CONGENITAL FINNISH NEPHROTIC SYNDROME:} A PATIENT WITH SPONTANEOUS REMISSION

Laura García Espinosa, Marta Melgosa Hijosa, Alejandro Zarauza Santoveña, Julian Nevado Blanco, Juan Bravo Feito, Laura Espinosa Roman Hospital Universitario La Paz

Introduction: We report a patient with genetically confirmed Finnish congenital nephrotic syndrome (CNS) and spontaneous remission in the first 2 years of life. Anecdotical cases of spontaneous remission in CNS have been reported in the literature, only two cases, we have found with a genetic confirmation.

Material and methods: 5-year retrospective evolution of a clinical case Results: An 11-day-old male neonate with no previous history was admitted in hospital with a generalized seizure secondary to cerebral venous sinus thrombosis. Physical examination showed palpebral and lower limb edema. Blood pressure was normal. Blood tests showed albumin $0.5 \mathrm{~g} / \mathrm{dl}$, total protein $3.2 \mathrm{~g} / \mathrm{dl}$, creatinine $<0.15 \mathrm{mg} / \mathrm{dl}$, hypocalcemia $6.7 \mathrm{mg} / \mathrm{dl}$, total cholesterol $230 \mathrm{mg} / \mathrm{dl}$ with protein/creatinine index in urine $36.1 \mathrm{mg} / \mathrm{mg}$. Etiological study ruled out connatal infection and the genetic study showed a compound heterozygosis for two different mutations in NPHS1 gene (exon 9 and 27) inherited from both parents. Hypothyroidism, vitamin D deficiency and antithrombin III deficiency were diagnosed and treated. Antiproteinuric treatment was started with captopril, hydrochlorothiazide and aldactone and he could be discharged at 86 days of life. Edema was controlled and no albumin infusions were required, although the biochemical pattern of nephrotic syndrome was maintained. After 6 months, a progressive rise in serum albumin was observed, with a parallel decrease in proteinuria until normalization at 18 months (figure). Antiproteinuric treatment could be withdrawn at 2 years of age. At present he is 5 years old and remains asymptomatic, without proteinuria and minimal albuminuria $(72.6 \mathrm{mg} / \mathrm{g}$ creatinine).

Conclusions: FNS is a rare entity, genetically determined, progressing unavoidably to end-stage renal disease in the first years of age. Multiple complications are expected; spontaneous remission is exceptional. Clinical presentation and genetical study confirm FNS diagnosis but the evolution has been surprisingly good. The development of genetics will lead to the discovery of new variants, which will identify the prognosis of the disease.

\section{EP-24 SEVERE INFANTIL HYPERCALCEMIA ASSOCIATED WITH WILLIAMS BEUREN SYNDROME AFTER A SINGLE INTRAVENOUSLY ADMINISTERED PAMIDRONATE}

\section{Rumeysa Yasemin Çiçek ${ }^{1}$, Feride Tahmiscioglu ${ }^{1}$ \\ ${ }^{1}$ Başakşehir Çam And Sakura City Hospital, ${ }^{2}$ Bagcllar Education, Research Hospital}

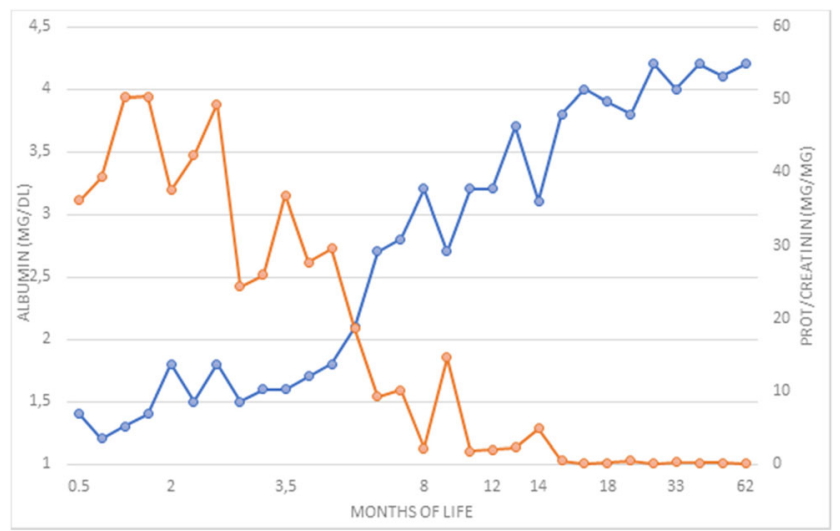

Introduction: Williams syndrome is an autosomal dominant disorder caused by a hemizygous submicroscopis deletion of 7q11.23 detectable by fluorescence in situ hybridization (FISH). Children with WS usually are recognized during infancy or childhood with dysmorphic facies (\%100), cardiovasculer disease (most commonly supravalvular aortic stenosis, \%80), mental retardation (\%75), and a characteristic cognitive profile (\%90). Infantile hypercalcemia occurs in $\% 15$ of children with Williams syndrome (WS) and is typically not clinically severe and usually occurs in the first years of life. The cause of hypercalcemia is unknown and hypercalcemia resolves in most cases by 4 years of age. However, some patients with WS can have severe hypercalcemia and do not respond well to traditional therapies. Recently, pamidronate has been used in the treatment of childhood hypercalcemia.

Material and methods: A 15 -month-boy was admitted to our clinic for the investigation of medullary nephrocalcinosis. He had constipation, polyuria, and polydipsia since he was 9 months. We learned that he had been diagnosed as having mental and growth retardation and being a syndromic baby due to having an abnormal face appearance and nephrocalcinosis. He was neither walking nor speaking at 15 months. He was born via section, her birth weight was 2580 gr. At the age of 2 months he got a operation to his hearth disease. He had healthy parents who did not have consanguinity. On physical examination, the following were measured: fewer, 36,8 ; heart rate $100 / \mathrm{min}$, blood pressure, $100 / 60 \mathrm{~mm} \mathrm{hg}$; and respiratory rate, $30 / \mathrm{min}$. Weight was $6900 \mathrm{~g}$ deviation height $69 \mathrm{~cm}$ and head circumference $40 \mathrm{~cm}$. He had the characteristic facial appearance of WBS, i.e., elongated philtrum, low nasal bridge, broad nasal tip, eyes set apart, periorbital fullness, wide mouth, widely spaced teeth, the so- called elfin face. Systolic ejection murmur was detected at the cardiovascular system examination. On laboratory examination, hemoglobin, $14 \mathrm{~g} / \mathrm{dL}$, total white blood cell count, $11000 / \mathrm{mm} 3$, platelet count, $213000 / \mathrm{mm} 3$, serum calcium $(\mathrm{Ca})$, phosphorus, and alkaline phoshatese were found to be $18,7 \mathrm{mg} / \mathrm{dL}, 3,8$ $\mathrm{mg} / \mathrm{dL}, 105 \mathrm{U} / \mathrm{L}$, respectively. Parathormone : $4 \mathrm{pq} / \mathrm{mL}$ and 25 hydroxyvitamin D levels were $20,8 \mathrm{ng} / \mathrm{mL}$ (normal ranges: $20-80$ ). Spot urine $\mathrm{Ca}$ /creatinin: $1,1 \mathrm{mg} / \mathrm{mg}$ (normal: $<0,2$ )

Bun: 20,56mg/dl, üre:44mg/dl, creatinin 0,84mg/dL,üric acid: 7,5mg/dL, $\mathrm{Na}: 133 \mathrm{mmol} / \mathrm{L}, \mathrm{K}: 5,5 \mathrm{mmol} / \mathrm{L}, \mathrm{Cl}: 94 \mathrm{mmol} / \mathrm{L}$. Urinalysis revealed a $\mathrm{ph}$ of 7,5, density:1004. Abdominal ultrasonography (USG) of the patient showed grade 3 nephrocalcinosis. Echocardiography was reported as tricuspid atrezia, ventrikuler septal defect, nonrestriktif asd, pulmoner stenosis. There were no abnormalities except for nephrocalcinosis on renal ultrasonography.

Results: Intravenous fluid, furosemide administration $2 \mathrm{mg} / \mathrm{kg} /$ day, prednisolone $2 \mathrm{mg} / \mathrm{kg} /$ day, and dietary Ca restriction( $400 \mathrm{mg} /$ day) were started to treat the hypercalcemia. However, the patient's $\mathrm{Ca}, \mathrm{BUN}$, and $\mathrm{Crl}$ levels did not reduce to the normal range after 24 hour of standart treatment. Then the patient received 1-day intravenously administered pamidronate $1 \mathrm{mg} / \mathrm{kg}$ over 4 hour although the level of Ca reduced from $18 \mathrm{mg} / \mathrm{dL}$ to $15 \mathrm{mg} / \mathrm{dL}$, the follow up Ca level was detected as $12,5 \mathrm{mg} / \mathrm{dL}$ on the next day. Ca levels were found as,10.6, 10.7 and $9.1 \mathrm{mg} / \mathrm{dL}$, respectively on subsequent days. Renal function also gradually improved after serum calcium level was restored through pamidronate treatment. On the 7th day of admission the laboratory tests results were: $\mathrm{Ca}, 10.5$ $\mathrm{mg} / \mathrm{dL}$, üre, $13 \mathrm{mg} / \mathrm{dL}$, creatinin $0.46 \mathrm{mg} / \mathrm{dL}$, spot urine $\mathrm{Ca} /$ creatinine ratio was found $0.7 \mathrm{mg} / \mathrm{mg}$. The infusion of pamidronate was well tolerated, and no side effects were observed. The patient was discharged from hospital with a low $\mathrm{Ca}$ diet. At the last follow- up visit, the patient was 20month of age and serum $\mathrm{Ca}, \mathrm{BUN}$ and $\mathrm{Cr}$ levels were found to be in the normal range.

Conclusions: There are clearly no defined criteria of bisphosphonate therapy in pediatric practice. However, intravenous pamidronate therapy seems to be a reasonable way of treating life threatening hypercalcemia in WS, despite the potential long effects which are yet not well characterized. 
EP-25 SEVERE NEUROLOGICAL INVOLVEMENT IN TWO CASES WITH SHIGA TOXIN-RELATED HEMOLYTIC UREMIC SYNDROME

Hulya Nalcacioglu ${ }^{1}$, Ozlem Aydog ${ }^{1}$, Demet Tekcan ${ }^{1}$, Hulya Gozde Onal ${ }^{1}$, Gokcen Oz Tuncer ${ }^{2}$, Ayse Aksoy ${ }^{2}$

${ }^{1}$ Ondokuz Mayis University Faculty Of Medicine, Pediatric Nephrology Department, Samsun, Turkey, ${ }^{2}$ Ondokuz Mayis University Faculty Of Medicine, Pediatric Neurology Department, Samsun, Turkey

Introduction: Neurologic involvement is the most threatening complication of the diarrhea-associated hemolytic uremic syndrome (D+HUS). We presented two cases with severe initial neurologic involvement that occurred in the course of D+HUS.

Material and methods: Case I. A 3.5-year-old female patient was followed up for five days with bloody diarrhea and referred to our clinic for high creatinine levels and oliguria. Midazolam was administered because of a generalized seizure during the referral. On physical examination, she exhibited fluctuating levels of alertness. Initial laboratory evaluation showed hyponatremia and the triad of HUS. Supportive treatment and peritoneal dialysis initiated for hyponatremia and renal failure. In admission, she had severe neurological symptoms, with convulsions and decreased consciousness; cranial MRI showed signal increases in the basal ganglia. One dose of Eculizumab was administered. Plasmapheresis was started for diffuse encephalopathy findings and ongoing focal seizures in EEG. After five sessions of plasmapheresis and two doses of Eculizumab, urine output begun on the 25th day of hospitalization. Enterohemorrhagic and EPEC PCR were positive in stool examination. On the 40th day of her admission, she was discharged. One week later, in the outpatient clinic control, creatinine value was $0.67 \mathrm{mg} /$ $\mathrm{dl}$, and hematological parameters were normal.

Case II: A 6-year-old boy presented with bloody diarrhea, fever, and diffuse abdominal pain. Physical examination revealed a fever of $38.6 \mathrm{C}$, tenderness, and defense in the abdomen. No pathological findings were found in abdominal ultrasonography and non-contrast abdominal MRI. HUS was diagnosed, and hemodialysis was started. On the third day of hospitalization, we checked the acute phase levels due to ongoing fever and abdominal pain. High levels of ferritin, triglyceride, $\mathrm{LDH}$, and decreased fibrinogen, sedimentation values with hemophagocytic cells in the bone marrow suggested macrophage activation. IVIG and steroid were initiated. Two times COVID (PCR) test was negative. Hypertension $(180 / 110 \mathrm{~mm} \mathrm{hg})$ and seizure were observed at the third dose of pulse steroid. Diffusion Cranial MRI was normal. Hypertension was controlled with the Ca channel blocker. Two days later, he had a newonset headache and convulsion with a fever. Intracranial hemorrhage was detected and drained. Ferritin values increased to $36.000 \mathrm{ng} / \mathrm{ml}$ under plasmapheresis and Eculizumab. Enterohemorrhagic, EPEC, and EAgEC PCR tests were positive in stool examination. Twenty days after the initial presentation, the patient died due to cardiac failure combined with neurological impairment.

Results: Neurological involvement in STEC-HUS; may develop due to uremia, hypertension, electrolyte disturbances, and thrombotic microangiopathy in cerebral microcirculation.

Conclusions: These two cases aimed to emphasize the neurological involvement of STEC-HUS with high mortality and morbidity.

\section{EP-26 DAMAGE OF GASTROINTESTINAL TRACT IN CHILDREN WITH HEMOLYTIC UREMIC SYNDROME}

Elena Gunkova, Igor Zorin, Albina Vyalkova

Orenburg Medical University

Introduction: HUS is actual problem of pediatrics and nephrology. There is not enough data of damage of gastrointestinal tract (GIT) in children with HUS. The aim of the study is to determine the state of GIT in children with HUS.
Material and methods: We examined 20 children with HUS in age from 9 months till 6 years old. There were 2 groups: 1 - children who underwent RRT $(\mathrm{n}=10), 2$ - children whose AKI was stopped without $\operatorname{RRT}(\mathrm{n}=10)$.

Results: Patients under 3 years old are prevailed (70\%). Thrombocytopenia, hemolytic anemia, AKI were found in $100 \%$ of children with HUS. Typical form of HUS had damage of GIT with diarrheal syndrome in $100 \%$, haemocolitis in $45 \%(\mathrm{n}=9)$, vomiting syndrome in $75 \%(\mathrm{n}=15)$, liver damage with increasing of transaminases in blood - 80\% $(\mathrm{n}=16)$, and hepatomegaly - $55 \%$, abdominal syndrome - $25 \%(\mathrm{n}=5)$. Damage of GIT in 1 group had more often hemocolitis syndrome $(60 \%, \mathrm{p}<0.01)$, liver damage (hepatomegaly 60\%, increased transaminases in blood, 100\%). Damage of GIT in 2 group had more often vomiting syndrome, abdominal pain syndrome $(40 \%)(\mathrm{p}<0.01)$.

Conclusions: Damage of GIT in children with HUS is more hard in patients who need RRT.

\section{EP-27 KIDNEY FUNCTION IN CHILDREN WITH ACUTE LEUKEMIA}

Mariya Skrylnikova, Olga Zhdanova, Elena Kulakova , Inna Kondratyeva, Timofey Chubarov

Voronezh State Medical University Named After N.n. Burdenko

Introduction: Changes of glomerular filtration rate (GFR) in children with acute lymphoblastic leukemia (ALL) were published in few articles and have controversial results due to different methods of estimated GFR (eGFR). The aim of our study was to determine GFR in children with ALL before, during and after chemotherapy (ALL-MB 2015 protocol). Material and methods: In 49 patients (average age $5.34 \pm 0.51$ years) with ALL, hospitalized at Voronezh Regional Children's Hospital, the GFR was estimated using Bedside Schwartz formula.

Results: The average value of eGFR before the start of the intensive therapy (IT) was $107.53 \pm 4.20 \mathrm{ml} / \mathrm{min} / 1.73 \mathrm{~m}^{2}$. During the IT there was a significant increasing of eGFR up to $154.61 \pm 5.90 \mathrm{ml} / \mathrm{min} / 1.73 \mathrm{~m}^{2}$ ( $\left.\mathrm{p}<0.001\right)$. After the IT eGFR began to decrease $-120.26 \pm 4.77 \mathrm{ml} / \mathrm{min} / 1.73 \mathrm{~m}^{2}(\mathrm{p}=0.04)$ and after of maintenance therapy it reached $102.77 \pm 3.25 \mathrm{ml} / \mathrm{min} / 1.73 \mathrm{~m}^{2}$ ( $p=0.38$ compared to eGFR before the therapy and $p<0.001$ compared to eGFR during the IT). Serum creatinine increased by 1.5-2 times and a GFR decreased by $25-50 \%$ in 23 children $(46.9 \%)$ during IT.

Conclusions: GFR increases during the IT and returns gradually to the original values by the end of the maintenance therapy. Acute kidney injury (AKI) occurred in $46.9 \%$ of the patients during the IT period. It is necessary to determine further dynamics of GFR in children with ALL, who have AKI, as a group of risk for chronic kidney disease.

\section{EP-28 ACUTE KIDNEY INJURY IN SEVERE BACTERIAL COMMUNITY-ACQUIRED PNEUMONIA REQUIRING PAEDIATRIC INTENSIVE CARE UNIT}

Chon In Kuok, Mei Lam Natalie Hsu, Kin Nam Karen Wong, Hui Fung Stephanie Lai, Winnie Kwai Yu Chan

Department Of Paediatrics, Queen Elizabeth Hospital, Hong Kong Sar

Introduction: Our study aimed to identify the prevalence and outcome of acute kidney injury (AKI) in severe bacterial community-acquired pneumonia in children.

Material and methods: We conducted a retrospective review on paediatric patients younger than 18 years old who were admitted to the paediatric intensive care unit (PICU), Queen Elizabeth Hospital, Hong Kong due to bacterial community-acquired pneumonia in 2019. AKI staging was defined by KDIGO serum creatinine criteria.

Results: A total of 14 patients were included in the study. Eight patients had Streptococcal pneumoniae infection, whilst Mycoplasma 
pneumoniae was identified in 4 patients. Four of them (28.6\%) developed AKI (Stage I AKI in 2 patients, Stage III AKI in 2 patients). There was no significant difference in the causative organisms in the AKI and non-AKI group ( $\mathrm{p}=0.307$ ). Children with AKI required longer hospitalisation as compared to non-AKI group (Median 39.6 vs 20.9 days, $p=0.024$ ), but the two groups had similar length of stay in PICU (11.5 vs 10.2 days, $\mathrm{p}=1.000$ ). All patients survived to discharge.

Streptococcus pneumoniae infection was the causative organism in both patients with stage III AKI. Both of them required mechanical ventilation and inotropic support. One of the patients developed fluid overload due to pneumococcal-associated haemolytic uraemic syndrome, and received CVVHDF for 3 days. His urine output improved with diuretics, and his serum creatinine normalised 2 weeks later. In both patients, the renal ultrasound 1 month after the initial insult were unremarkable. Subsequent renal function tests were normal. Further follow-up is needed to monitor the kidney growth and their blood pressure.

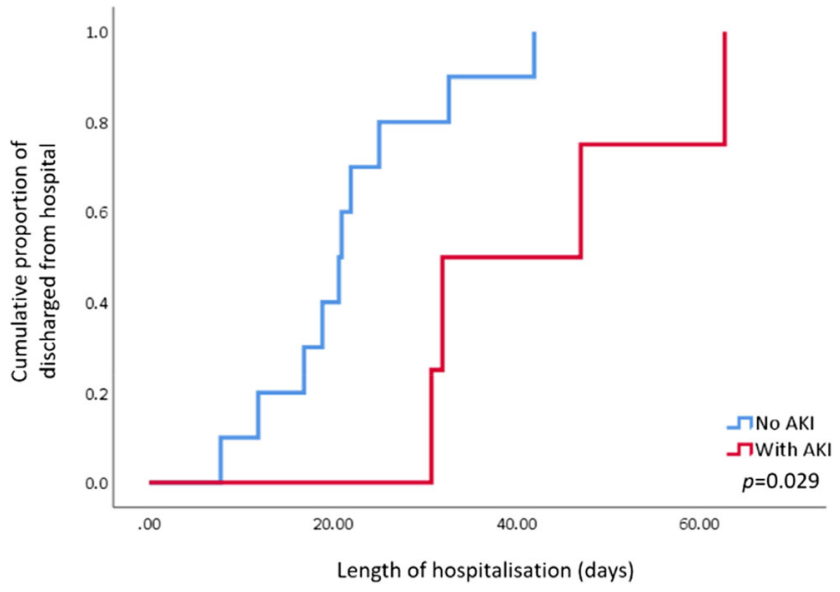

Conclusions: Acute kidney injury occurred in $28.6 \%$ of children who had severe bacterial community-acquired pneumonia. Patients with AKI required longer hospitalisation. Paediatricians should be aware of potential severe complications of pneumococcal infection including haemolytic uraemic syndrome.

\section{EP-29 MULTISYSTEM INFLAMMATORY SYNDROME IN CHILDREN AND SHOCK: A CASE PRESENTATION}

Asli Çelebi Tayfur ${ }^{2}$, Esra Bildik ${ }^{1}$, PaŞa Balci ${ }^{1}$, Meyrí Arzu YoldaȘ ${ }^{1}$, AyȘegÜl Daniș ${ }^{3}$

${ }^{1}$ Bolu Abant İzzet Baysal University, Department Of Pediatrics, ${ }^{2}$ Bolu Abant İzet Baysal University, Department Of Pediatric Nephrology, ${ }^{3}$ Bolu Abant İzet Baysal University, Department Of Pediatric Neurology

Introduction: Multisystem inflammatory syndrome in children (MIS-C) is a life-threatening post-infectious complication of SARS-CoV2 infection.

Material and methods: We describe a case of MIS-C in a child who had distributive shock, multi-organ dysfunction and systemic inflammation associated with COVID-19.

Results: A 17 years old boy admitted to Pediatric Emergency Departmet of Bolu Abant İzzet Baysal University with symptoms of fever, nausea, diarrhea, neck ache and dyspnea. He was conscious and had fever, tachypnea, tachycardia, sweating and hypotension on admission. Physical examination revealed mild pharyngeal erythema, non-blanching papular rash on back of both hands and fingers. Fluid resuscitation failed to normalize blood pressure and heart rate. Inotropic support, antibiotics and nazal $\mathrm{O} 2$ support were started. On follow up, acute dystonia developed and stoppped with biperiden. The parents denied any contact with a patient with COVID-19 firstly but when they queried repeatedly they indicated that his brother had had COVID-19, 1 month ago. The laboratory studies revealed leucocytosis, lymphopenia, hyperferritinemia, significant increase in acute phase reactant levels, D-dimer level and cardiac enzyme levels and disturbed renal and liver function tests. The reverse transcription polymerase chain reactions for detection of SARS-CoV-2 from 2 nasopharengeal swabs were negative. The echocardiography was normal, the ejection fraction was $50 \%$. The patient was diagnosed as MIS-C and treated by IVIG infusion, prednisolone, tocilizumab, enoxaparine, aspirin at antiinflammatory dose. The patient was transferred to a center that had a pediatric intensive care unit with a higher level of care. The patient recovered and discharged soon after hospitalization.

Conclusions: We should consider MIS-C in the differential diagnosis if the patient had multisystem organ involvement and a contact history to a patient with COVID-19. We should query the patient and parents for contact tracing, repeatedly if we have any doubt about the veracity of the statements

\section{EP-30 DELAYED DIAGNOSIS OF BK NEPHROPATHY AFTER KIDNEY TRANSPLANT DUE TO COVID19 PANDEMIC}

Laura Fraile García ${ }^{1}$, Olalla Álvarez Blanco ${ }^{2}$, Francisco Díaz-crespo ${ }^{2}$, Daniel Barraca Núñez ${ }^{2}$, Begoña Santiago García ${ }^{2}$,

Belén Fernández Monteagudo ${ }^{2}$, Paula Carrascosa García ${ }^{2}$, Ana Belén Martínez-lópez ${ }^{2}$

${ }^{1}$ Hospital Clínico Universitario De Valladolid, ${ }^{2}$ Hospital General Universitario Gregorio Marañón

Introduction: Present the case of a delayed diagnosis and treatment of severe BK virus-associated nephropathy (BKVAN) in a pediatric kidney transplant recipient due to the COVID-19 pandemic.

Material and methods: Patient's medical history review.

Results: Eight-year-old male from Pakistan. He debuted at four years old with nephrotic syndrome in Pakistan, presenting three relapses in the first months after diagnosis, requiring corticosteroid therapy and tacrolimus.

Upon arrival to Spain we performed a biopsy consistent with minimal change disease. He suffered additional relapses and finally triggered a corticosteroid resistance. Despite adding mycophenolate, plasmapheresis and rituximab, he developed end-stage chronic kidney disease. Bilateral nephrectomy was performed with histological finding of focal segmental glomerulosclerosis. He required hemodialysis until deceased donor kidney transplant in December 2019.

He suffered an acute mixed rejection (humoral and cellular), intensifying immunosuppressive therapy. Control biopsy in February 2020 was normal. BK virus was monitored monthly after transplant. In the context of the COVID-19 pandemic, BK-DNAemia samples were not processed for four months and July's result was delayed until September. In September 65-million copies were observed, confirming the presence of class 3 BKVAN in renal biopsy according to the Banff-2019 classification with signs of acute tubular necrosis. Immunosuppression was decreased as first measure with a gradual rise up to 130-million copies. Tacrolimus and mycophenolate were replaced with everolimus and leflunomide. Subsequent initiation antiviral therapy with cidofovir and intravenous immunoglobulin was effective, presenting in March 2021 4.8-million BK copies and stable creatinine around $2 \mathrm{mg} / \mathrm{dl}$.

Conclusions: Consumption of resources due to COVID19 pandemic has caused a delay in diagnosis and treatment of other diseases. BK monitoring during the first year after kidney transplantation is essential. The current guidelines suggest that antiviral therapy may be considered in patients with sustained high-level plasma BK load despite reduction of immunosuppression. 


\section{EP-31 RISK FACTORS FOR THE OUTCOME IN CHILDREN} WITH STEC-HUS/ D+ HUS: A SINGLE-CENTER EXPERIENCE

Hulya Nalcacioglu, Demet Tekcan, Hulya Gozde Onal, Ozlem Aydog Ondokuz Mayis University Faculty Of Medicine, Pediatric Nephrology Department, Samsun, Turkey

Introduction: Hemolytic uremic syndrome (HUS) is one of the common causes of acute kidney injury in childhood. We aimed to evaluate the epidemiological, clinical, laboratory findings and identify risk factors for short-term and long-term outcomes in Shiga toxin-producing Escherichia coli (STEC)-HUS and other diarrhea positives (D+) HUS

Material and methods: A retrospective chart review was obtained of 16 pediatric patients treated for STEC-HUS and other D+ HUS between January 2018 and January 2021.

Results: The mean \pm SD age of our patients was $3.1 \pm 2.7$ (0.6-11.6 )years. Gender distribution was $56.3 \%$ girls and $43.8 \%$ boys. Eight patients were $<2$ years old. 11 of the 16 patients had a history of bloody diarrhea. Stool examination was done on 13 patients. Shiga toxin was identified in one case, and the remaining five patients had shown growth of $\mathrm{E}$ Coli to establish the diagnosis of STEC-HUS/D+ HUS. Nine patients $(56.3 \%)$ required dialysis. Dialysis was more likely initiated if a patient was oliguric (urine output $<0.5$ $\mathrm{mL} / \mathrm{kg} / \mathrm{h}, \mathrm{P}<.0001$ ), hyponatremia(Na) level $<130 \mathrm{meq} / \mathrm{L}$ ( $\mathrm{p}=0.003$ ), increased LDH levels and CRP levels ( $\mathrm{p}=0.016, \mathrm{p}=0.031$ respectively) at admission. Complement $\mathrm{C} 3$ was measured in 15 children and was low in six patients. Neurological complications developed only among three dialyzed patients. Five patients received Eculizumab, and two patients received both Eculizumab and plasmapheresis. Two patients developed CKD with a mean GFR level of $63.5 \pm 14.8 \mathrm{ml} / \mathrm{min}$; one patient died due to neurological complications (intracranial hemorrhage).

Conclusions: Children with STEC-HUS/D+ HUS under dialysis treatment are identified by decreased urine output, lower sodium level, and higher LDH -CRP levels at admission.

\section{EP-32 FRASER SYNROME (ORPHA: 2052) IN CHILD: CASE REPORT}

Zhanna Leviashvili, Nadezhda Savenkova, Vladimir Brzheskiy, Pavel Pavlov, Oksana Gorkina

Saint-petersburg State Pediatric Medical University, Russian Federation

Introduction: Fraser syndrome is an autosomal recessive disease caused by mutations of tree genes: FRAS1, FREM2 and GRIP. The incidence of Fraser syndrome is 1 in 230,000 live born infants. Fraser syndrome type 1 is caused by mutation in the FRAS1 gene. The diagnosis of Fraser syndrome according to the major and minor criteria.

Material and methods: We present a clinical case of a boy 1 years 2 months from a closely related marriage.

Results: The child from the 4th pregnancy, proceeding with the threat of interruption and fetal hypoxia, was born at 39 weeks, weighing $4000 \mathrm{~g}$, length $57 \mathrm{~cm}$. In child were diagnosed 5 major and 1 minor clinical criteria for the Fraser syndrome: microphthalmos, left-sided cryptophthalmos; narrowing of the auditory canals in the bony sections, atresia of the external auditory canals on both sides, bilateral conductive hearing loss 3rd, congenital intervocal membrane. The only right kidney due to agenesis contralateral, kidney function is preserved GFR $59.7 \mathrm{ml} / \mathrm{min}$. Syndactyly of 3-4 fingers of both hands; congenital malformations of the nose and ears - stenosis of the nasolacrimal canal, shortening of the frenulum of the tongue, hypoplasia of the auricles. A multistage surgical treatment of cryptophthalmos on the left was performed: the formation of the palpebral fissure, plastic eyelids, enucleation of the left eyeball and early prosthetics of the orbital cavity. Genetic research revealed a mutation in the FRAS1 gene. Fraser syndrome type 1 was diagnosed.

Conclusions: We report a case of a boy 1years 2 months with Fraser syndrome type 1 (ORPH: 2052) by mutation FRAS1 gene. Fraser syndrome was characterized the 5 major and 1 minor diagnostic criteria.

\section{EP-33 EPIDEMIOLOGY OF ACUTE KIDNEY INJURY (AKI)} IN TUNISIAN CHILDREN : A SINGLE CENTER STUDY

Selsabil Nouir, Sameh Mabrouk, Houda Ajmi, Fadwa Majdoub, Tfifha Meniar, Chamli Jaleleddine, Noura Zouari, Abroug Saoussen Pediatric Department, Sahloul Hospital, Tunisia

Introduction: The incidence of AKI in children appears to be increasing, and the etiology of AKI over the past decades has shifted from primary renal disease to multifactorial causes, particularly in hospitalized children. It's at the origin of a high morbidity and mortality rate in hospitalized patients. Early positive and etiological diagnosis are needed for the development of successful therapeutic strategies for the treatment of AKI. The goal of our study is to determine epidemiological data, clinical manifestations and evolutionary modalities of AKI in hospitalized children. Material and methods: Descriptive and retrospective study, conducted in our pediatric department in the period between February 2020 and February 2021. All children 01 month to 15 years aged and presented with ARI defined according KDIGO diagnostic criteria were included in the study.

Results: 56 cases of AKI were included, predominantly girls (sex ratio $=0.46$ ), a mean age of $64.8 \pm 55.8$ months was found in our patients. On examination, $61 \%$ of patients presented with dehydration, $40 \%$ with dyspnea, proteinuria was found in $25 \%$ of children. Etiological investigations concluded to hypovolemia (61\%), septic shock (29\%) and multiple organ failure (19.6\%). A severe form with a stage 3 ARI was observed in $32 \%$ of cases, intrinsic renal disease was found in $51 \%$ of children. Only 2 patients were first treated with peritoneal dialysis, $66.8 \%$ of children had evolved well.

Many factors were correlated to Evolution, medical history of congenital heart disease $(p=0.002)$, multi organ failure $(p=0.000)$, septic shock $(\mathrm{p}=0.012)$, heart failure $(\mathrm{p}=0.05)$ and type of ARI $(\mathrm{p}=0.02)$ had a positive significant influence on mortality rate. Neither stage of ARI nor eGFR influenced mortality $(\mathrm{p}=0.34)$

Conclusions: Acute kidney injury is common and is associated with poor outcomes in children, including increased mortality among critically ill children. For it early and adequate management can improve outcomes.

\section{EP-34 BOTRYROID WILMS' TUMOR PRESENTING WITH ACUTE KIDNEY INJURY IN AN INFANT}

Mehtap Adar ${ }^{1}$, Ayşe Keven ${ }^{2}$, Aygül Elmali ${ }^{2}$, Elif Güler ${ }^{3}$, Gülşah Kaya Aksoy ${ }^{1}$, Mustafa Koyun ${ }^{1}$, Muhammet Sancaktar ${ }^{1}$, Ayla Kaçar ${ }^{1}$, Elif Çomak ${ }^{1}$, Sema Akman ${ }^{1}$

${ }^{1}$ Akdeniz University Medical Faculty, Department Of Pediatric Nephrology, Antalya, ${ }^{2}$ Akdeniz University Medical Faculty, Department Of Radiology, Antalya, ${ }^{3}$ Akdeniz. University Medical Faculty, Department Of Pediatric Oncology, Antalya

Introduction: Botryroid Wilms' tumor is located in the intrapelvic region of the kidney, often presenting with hematuria and rarely with anuric renal failure. We present an infant who presented with anuric renal failure and was diagnosed with Botryroid Wilms tumor.

Material and methods: A 13-month-old boy was admitted with anuria lasting for 24 hours and periorbital swelling. He had no fever or any other systemic complaint. His anthropometric measurements were normal (height: 50 percentile, weight: $25-50$ percentile) and blood pressure was $100 / 60 \mathrm{mmHg}$ ( 95 th percentile $102 / 54 \mathrm{mmHg}$ ). On physical examination, a generalized diffuse edema and abdominal distention was seen. Laboratory tests were as follows: Blood ure nitrogen $60 \mathrm{mg} / \mathrm{dL}$, serum creatinine $3.77 \mathrm{mg} / \mathrm{dL}$, sodium $137 \mathrm{mEq} / \mathrm{L}$, potassium $7.7 \mathrm{mEq} / \mathrm{L}$, serum phosphorus $6.9 \mathrm{mg} / \mathrm{dL}$ and serum uric acid $8.5 \mathrm{mg} / \mathrm{dL}$.

Results: An ultrasonography yielded enlarged kidneys with renal parenchymal thinning and increased echogenicity; renal calyces were dilated and deformed; multiple masses originating from the lower pole of both kidneys extending to renal pelvis, the largest being $\sim 6 \mathrm{~cm}$. On tomography, 
polypoid mass lesions, with a size of 40x40x45 and 47x38x32 mm at right and left kidneys, filling renal pelvis extending to the whole calyceal system and to the proximal part of the ureters were observed. As hyperkalaemia and anuria persisted, dialysis was started. The patient was diagnosed with Botryoid Wilms' tumor based on imaging findings, and neoadjuvant chemotherapy (doxorubicin, actinomycin and vincristine) was given. Urine output began 48 hours after chemotherapy, and serum creatinine decreased to $0.4 \mathrm{mg} / \mathrm{dL}$ on the $5^{\text {th }}$ day after chemotherapy.

Conclusions: Botryroid Wilms' tumor should be considered in the differential diagnosis of a child with renal failure and a mass in the kidney.

\section{EP-35 URINARY MARKERS OF KIDNEY INJURI IN CHILDREN WITH OBESITY}

Tatiana Nastausheva, Andrey Savchenko, Timofey Chubarov, Olga Zhdanova, Anna Khan

Voronezh State Medical University Named After N.n. Burdenko

Introduction: It is well - known that obesity is one of the factors of kidney injury. It is difficult to diagnose this injury in the early stages. Purpose of our study was to find diagnostic significance of urinary markers: KIM-1, NGAL, $\beta 2-m$, IL-18 for kidney injury in children with obesity.

Material and methods: We investigated 34 patients with exogenously constitutional obesity (21 boys and 13 girls) aged 11 years [6-15]. All patients had BMI $>2$ SDS (Z score). Obesity of the $1^{\text {st }}$ stage had 12 children $(35.3 \%)$, the $2^{\text {nd }}$ stage was in 13 patients (38.2), the $3 \mathrm{~d}$ stage had 8 children $(23.5 \%)$ and the $4^{\text {th }}$ stage was diagnosed in 1 boy $(2.9 \%)$. Control group included 50 children ( 25 boys and 25 girls) aged 10 years [10-14] without obesity and any kidney diseases. All children had normal GFR $\left(>90 \mathrm{ml} / \mathrm{min} / 1.73 \mathrm{~m}^{2}\right)$. Urinary markers measured by ELISA methods. The results were presented as median and interquartile range $\mathrm{Me}$ [IQR]. Comparison between groups was performed using Kolmogorov-Smirnov test.

Results: In children with obesity urinary KIM -1/UCr was $1442.0 \mathrm{pg} / \mathrm{mg}$ [856.5- 2433.3]; NGAl /UCr - 1.27 ng/mg [0.46- 9.57]; IL-18 /UCr $0.07 \mathrm{pg} / \mathrm{mg}$ [0.04- 0.1]. These results were significantly higher than in control group of children: KIM -1/UCr was $135.9 \mathrm{pg} / \mathrm{mg}$ [60.0- 248.7], $\mathrm{p}$ $<0.001 ; \mathrm{NGAl} / \mathrm{UCr}-0.17 \mathrm{ng} / \mathrm{mg}$ [0.03- 0.8], $\mathrm{p}<0.01$; IL-18/UCr -0.04 $\mathrm{pg} / \mathrm{mg}$ [0.03- 0.05], $\mathrm{p}<0.005$. Urinary $\beta 2-\mathrm{m} / \mathrm{UCr}$ was equal in patients and healthy children: $4.1 \mathrm{mkg} / \mathrm{mg}$ [1.54- 9.68] and $4.6 \mathrm{mkg} / \mathrm{mg}$ [1.759.73], $\mathrm{p}>0.05$ correspondently.

Conclusions: Urinary KIM-1, NGAL, IL-18 may be early markers of kidney injury in children with exogenously constitutional obesity.

\section{EP-36 EXCESS OF SODIUM CHLORIDE INDUCES INFLAMMATION AND PROFIBROTIC RESPONSE IN PERITONEAL CELLS}

Domonkos Pap ${ }^{2}$, Csenge Pajtók ${ }^{1}$, Apor Veres-székely ${ }^{2}$, Beáta Szebeni ${ }^{2}$, Eszter Lévai ${ }^{1}$, Attila J. Szabó ${ }^{1}$, Ádám Vannay ${ }^{2}$, Tivadar Tulassay ${ }^{1}$ ${ }^{1} 1$ st Department Of Paediatrics, Semmelweis University, Budapest, Hungary, ${ }^{2}$ Elkh-se Pediatrics And Nephrology Research Group, Budapest, Hungary

Introduction: Peritoneal scarring leads to decreased peritoneal ultrafiltration capacity which is the main cause of technical failure in peritoneal dialysis patients. High dietary salt $(\mathrm{NaCl})$ intake was reported to induce peritoneal fibrosis via osmotic stimuli due to accumulation of sodium in the peritoneal membrane, however, the underlying mechanisms are not clear. Therefore, our aim was to investigate the effect of high salt environment on various peritoneal cell types with focus on fibrosis related gene expression changes.

Material and methods: The effect of high $\mathrm{NaCl}$ concentrations and mannitol as osmotic control was investigated in vitro on the changes of inflammation and fibrosis related gene expression in human primary mesothelial cells (HPMC) and human primary peritoneal fibroblasts (HPF), endothelial cells (HUVEC) and immune cells (PBMC), respectively.

Results: High salt loading induced epithelial-mesenchymal transition in HPMCs evidenced by decreased epithelial marker E-cadherin and increased mesenchymal marker $\alpha$-SMA and SNAI1 expressions. Increased salt environment also resulted in elevated profibrotic growth factor TGF- $\beta$, PDGF-B or CTGF expression in HPMCs, HUVECs and PBMCs. In addition, high salt induced the MCP-1 and IL-1 expression of HUVECs and/or PBMCs. Increased salt concentration induced the collagen production of HPFs. Furthermore, our results also demonstrated the role of increased osmolarity on the detected gene expression changes.

Conclusions: Our results showed that elevated peritoneal sodium concentration and osmolarity induced a comprehensive inflammatory and profibrotic response in cells responsible for the impaired integrity and decreased ultrafiltration capacity of peritoneum during peritoneal dialysis.

\section{EP-37 VEGF GENE RS699947 AND RS2010963 POLY- MORPHISMS AND VEGF SERUM CONCENTRATION IN CHILDREN WITH LUPUS NEPHRITIS}

Ina Kazyra ${ }^{1}$, Aleksandr Sukalo ${ }^{1}$, Anna Bialkevich ${ }^{1}$, Anna Krylova-alefirenko ${ }^{1}$, Natalia Nikitchenko ${ }^{2}$, Roza Goncharova $^{2}$

${ }^{1}$ Belarusian State Medical University, ${ }^{2}$ Institute Of Genetics And Cytology Of The National Academy Of Sciences Of Belarus

Introduction: It was reported that VEGF, one of key factors in vascular formation, is associated with kidney involvement in SLE. We investigated prevalence of rs699947 and rs2010963 VEGF gene polymorphisms and their association with serum VEGF concentration in children with Lupus nephritis (LN).

Material and methods: 22 children with LN aged 10 to 17 years were enrolled ( $m: f 2: 20$ ). Serum concentration of VEGF was measured twice in each child - during the exacerbation of nephritis and in remission. VEGF gene polymorphysms were analyzed by polymerase chain reaction.

Results: Serum concentration of VEGF in active LN was significantly higher $(\mathrm{p}<0.001)$ in patients with AA genotype of polymorphic locus of rs699947 (1506 ng/ml) compared to CC and CA genotypes (413 and 560 $\mathrm{ng} / \mathrm{ml}$ respectively). When analyzing serum concentration of VEGF according to genotypes of rs2010963 we found its significant elevation $(\mathrm{p}=0,036)$ in patients with at least one minor allele C $(\mathrm{GC}, 893 \mathrm{ng} / \mathrm{ml}$ and $\mathrm{CC}, 1339 \mathrm{ng} / \mathrm{ml})$ compared to GG carriers $(485 \mathrm{ng} / \mathrm{ml})$ during exacerbation period. In remisssion concentration of VEGF did not differ in groups of patients with rs6999947 or rs2010963 genotypes.

Conclusions: VEGF gene polymorphisms correlate with gene expression in patients with active LN. AA genotype of rs699947 and GC+CC genotype of rs2010963 are associated with higher serum concentration of VEGF in children with the exacerbation of LN.

\section{EP-38 EPIDEMIOLOGY OF HYPERTENSION IN CHILDREN POPULATION IN CENTRAL POLAND IN 2017- 2019}

Julia Mirecka ${ }^{1}$, Malgorzata Stanczyk ${ }^{1}$, Aleksandra Olejniczak ${ }^{2}$, Justyna Zamojska $^{3}$, Marta Gruca ${ }^{3}$, Karolina Kowara- Dzik ${ }^{4}$, Agnieszka Wosiak $^{5}$, Agnieszka Szadkowska ${ }^{2}$, Elzbieta Smolewska ${ }^{3}$, Marcin Tkaczyk ${ }^{1}$

${ }^{I}$ Department Of Pediatrics, Immunology And Nephrology, Polish Mother's Memorial Hospital, Research Institute Lodz, ${ }^{2}$ Department Of Pediatrics, Diabetology Endocrinology And Nephrology, Medical University Of Lodz, ${ }^{3}$ Department Of Paediatric Cardiology And Rheumatology, Medical University Of Lodz, ${ }^{4}$ Department Of Pedietrics, Hospital In Skierniewice, ${ }^{5}$ Institute Of Information Technology, Lodz University Of Technology 
Introduction: The prevalence of hypertension in children population is estimated at about $4 \%$. It is different depending on the region of the world because of various definitions of hypertension and methodology of measuring. The available epidemiological data shows that essential hypertension concerns the group of teenagers and adolescents. However, observations in recent years have shown, that this type of hypertension is more common among increasingly young children.

The aim of the study was to evaluate the current epidemiology of hypertension in children from central Poland. These patients were either suspected of hypertension or have already been diagnosed with hypertension.

Material and methods: The study was retrospective. The data of 471 patients aged 5 to 18 , who were hospitalized in 4 pediatric departaments dealing with hypertension in central Poland in the period 2017-2019 were analyzed.

Patients' medical records were analyzed in the study. The primary criterium for qualifying the patient to the study group was the suspicion of hypertension in outpatient conditions or during hospitalization. The analysis covered: age (patients were divided into 2 age groups - 5-10 years of age and 11-18 years of age), gender, residence, anthropometric data (absolute and percentile), clinical symptoms of hypertension, data obtained from an Ambulatory Blood Pressure Measurement, family history, presence of complications of hypertension and treatment.

Results: The study group consisted of 175 girls (37\%) and 296 boys $(63 \%)(\mathrm{p}<0.001)$.

Hypertension was confirmed in $42.5 \%$ of patients $(61 \%$ of boys, $p=$ 0.62 ), high normal blood pressure was diagnosed in $36.5 \%$ and normal blood pressure in $21 \%$.

$61 \%$ children were overweight or obese, as far as the group with confirmed hypertension or high normal blood pressure is concerned, $63 \%$ children were overweight or obese $(62 \%$ boys, $\mathrm{p}=0.61)$.

More than half of the patients $(55.5 \%)$ did not present clinical symptoms of hypertension. The most common symptom reported by patients in the whole group was headache (132 patients, 63\%). Among patients who reported headaches, the most common diagnosis was normal blood pressure (43.5\%). In both age groups secondary hypertension was the rarest diagnosis- $6 \%$ in younger and $1,5 \%$ in older children. High normal blood pressure was diagnosed in $53 \%$ of younger children and $34 \%$ of older children and primary hypertension in $29 \%$ and $42 \%$, respectively.

Conclusions: Hypertension was confirmed in less than half of the patients. We found no specific clinical symptoms indicative for hypertension in this group. Essential hypertension is more often confirmed than secondary hypertension in both age groups.

\section{EP-39 BESIDES THE PROTECTIVE EFFECT OF CILASTATIN ON DRUG-INDUCED NEPHROTOXICITY, DOES IT HAVE AN EFFECT ON THE RECOVERY OF DRUG-INDUCED NEPHROTOXICITY ? AN IN VITRO STUDY}

\section{Tülay Becerir, Onur Tokgün, İlknur Girișgen, SelÇuk Yüksel Pamukkale University}

Introduction: By creating nephrotoxicity models with cisplatin, vancomycin and gentamicin in HK-2 (human renal proximal tubule cell) and HEK293T (human embryonic kidney epithelial cells) cell lines, we aimed to evaluate the effect of cilastatin on recovery of cell damage after toxicity occurred.

Material and methods: In the first phase of the study, the doses of cisplatin, vancomycin and gentamicin $(50 \%$ inhibitive concentration; $\mathrm{IC}_{50}$ ) were determined. In the second phase, the effective dose of cilastatin against these drugs was determined, and $\mathrm{IC}_{50}$ doses of nephrotoxic agents were administered simultaneously. In the third phase of our study, to evaluate the possible therapeutic effect of cilastatin after toxicity occurs; Analyzes of cell viability, apoptosis, oxidative stress and expression of kidney injury molecule-1 (KIM-1), neutrophil gelatinase associated lipocalin (NGAL) were performed.
Results: In the second phase of the study, it was observed that cilastatin increased cell viability when treated simultaneously with a nephrotoxic agent. In the third phase, cilastatin provided a significant increase in cell viability. We determined that after treatment with each agent for 24 hours, adding cilastatin to the medium had an effect on the recovery of cell damage by increasing cell viability, reducing apoptosis, and oxidative stress. The expression of KIM-1 and NGAL increased when nephrotoxicity occurred and decreased with the addition of cilastatin to the medium. Conclusions: The findings of the study suggest that cilastatin may have a healing effect after development of nephrotoxicity.

\section{EP-40 LEVAMISOLE, A POTENTIAL ADJUVANT DRUG FOR IDIOPATHIC NEPHROTIC SYNDROME, ACTS AS A SUPPRESSOR OF T-CELL ACTIVATION}

Gerarda H. Khan ${ }^{1}$, Floor Veltkamp ${ }^{2}$, Antonia H.m. Bouts ${ }^{2}$, Sandrine Florquin ${ }^{1}$, Jeroen E.j. Guikema ${ }^{1}$

${ }^{1}$ Pathology, Amsterdam Umc, University Of Amsterdam, Amsterdam, The Netherlands, ${ }^{2}$ Paediatric Nephrology, Amsterdam Umc, University Of Amsterdam, Amsterdam, The Netherlands

Introduction: Levamisole (LMS) is a small molecule used in the treatment of idiopathic nephrotic syndrome (INS). The pathogenesis of INS remains unknown, but evidence points towards an immunological basis of disease. Recently, LMS was shown to be effective in combination with corticosteroids in children with frequently relapsing INS ${ }^{1}$. However, the mechanism of action of LMS in INS remains unknown. To provide insight into the mechanism of LMS, we studied its immunomodulatory activity on in vitro cultured human T-cells.

Material and methods: Untouched human T-cells were isolated from three healthy donors. The effects of LMS on CD3/CD28-induced T-cell proliferation were assessed by the CFSE dilution assay in a three-day culture experiment. T-cell activation was determined by flow cytometry staining for the early activation marker CD69 and cytokine production by real-time PCR.

Results: Treatment with LMS decreased expression of CD69 in all donors after two and three days of activation. LMS treatment showed an average 5.0-fold decrease of CD69 expression at day 2 and 3.0-fold decrease at day 3. No differences were seen in CD4 and CD8 frequencies between treatment groups. LMS-treated CD4+ T-cells showed an average 5.4-fold decrease of CD69 expression at day 2 and 3.0-fold decrease at day 3. LMS-treated CD8+ T-cells showed an average 3.6-fold and 2.2fold decrease at day 2 and day 3 , respectively. In agreement with the dampening effect on T-cell activation, we found that proliferation was decreased in LMS-treated T-cells with an average 9.9-fold decrease in division index (DI) at day 3. An average 10.9-fold decrease in DI was calculated for LMS-treated CD4+ T-cells, compared to 7.7 for CD8+. Finally, LMS-treated T-cells showed a decrease in IL-2 transcription. Conclusions: Our preliminary findings indicate that LMS acts as an immunomodulatory drug that directly affects the activation and proliferation of T-cells. Further experiments are necessary to identify the exact mechanism.

\section{EP-41 HAEMATURIA IN CHILDREN - A REGIONAL GUIDELINE}

John Tolliday ${ }^{1}$, Shuman Haq ${ }^{1}$, Ramnath Balasubramanian ${ }^{2}$, Evgenia Preka ${ }^{1}$

${ }^{1}$ University Hospitals Southampton; ${ }^{2}$ Guys And St Thomas Nhs Foundation Trust

Introduction: Haematuria represents a presenting feature in a wide range of conditions in children. In many cases the cause is benign or transient. However, haematuria may be the sole presenting feature in serious 
pathology. This guideline looks to aid clinicians in the investigation of haematuria in addition to providing guidance regarding referral to tertiary services.

Material and methods: During the guideline creation process we reviewed latest evidence and practices regarding the investigation and management of haematuria in children in order to formulate pathways based on clinical presentation. Following review by the paediatric nephrology team this was sent to paediatric nephrourology leads in the hospitals within the region for further input. Further guidance was added particularly regarding management of microscopic haematuria ahead of publication on the Paediatric Innovation, Education and Research (PIER) network website, providing guidance for the Wessex region.

Results: We formulated an investigation and referral pathway based on four different clinical scenarios. These were macroscopic haematuria with altered or with fresh appearance of blood and microscopic haematuria which was transient or persistent. We also included further guidance where a specific diagnosis was suspected and where a referral to a tertiary centre was recommended.

Conclusions: We hope that the collaborative approach to guideline creation will provide pragmatic support to clinicians in the region to investigate and refer cases of haematuria in children appropriately and therefore improve the patient journey. Our aim is to standardise practice in a safe manner and utilise investigations effectively. Our guidance will be updated based on feedback from hospitals in the region in parallel with updated evidence.

\section{EP-42 SARA IN THE KIDNEY: REGULATION OF CELL PHENOTYPE AS A POTENTIAL THERAPEUTIC TARGET IN RENAL FIBROSIS}

Vidhi Dalal ${ }^{1}$, Xiaoyan Liang ${ }^{2}$, William Schnaper ${ }^{1}$, Tomoko Hayashida ${ }^{2}$ ${ }^{1}$ Lurie Childrens Hospital; ${ }^{2}$ Northwestern University

Introduction: Epithelial cells play an important role in renal fibrosis. After injury, podocytes and renal tubular epithelial cells (TECs) dedifferentiate. When dedifferentiated, podocytes detach from the glomerular basement membrane, while TECs stimulate surrounding cells to transdifferentiate into myofibroblasts, thus resulting in glomerulosclerosis and tubulointerstitial fibrosis respectively. Our laboratory has identified a protein called Smad Anchor for Receptor Activation (SARA) as a key factor for maintaining cellular phenotype in the face of fibrogenesis. Here, we aim to determine if SARA overexpression in podocytes and TECs can prevent their dedifferentiation and reduce fibrosis in mouse models of glomerular and tubulointerstitial disease.

Material and methods: SARA ${ }^{\text {podo }}$ and SARA ${ }^{\mathrm{TEC}}$ mice that overexpress SARA selectively in podocytes or TECs respectively and SARA negative littermates $\left(\mathrm{Ctrl}{ }^{\text {podo }}\right.$ and $\mathrm{Ctrl}{ }^{\mathrm{TEC}}$ mice) were used. SARA/Ctrl ${ }^{\text {podo }}$ mice were treated with Adriamycin to induce glomerulosclerosis, and SARA/Ctrl ${ }^{\text {TEC }}$ mice with aristolochic acid to induce tubulointerstitial fibrosis. Urine, blood, and kidneys were harvested for histological and molecular analysis.

Results: SARA ${ }^{\text {podo }}$ mice showed less glomerulosclerosis histologically and less proteinuria than $\mathrm{Ctr}^{\text {podo }}$ mice after Adriamycin treatment. In SARA ${ }^{\mathrm{TEC}}$ mice, upregulation of markers of fibrosis and tubular injury (measured by qPCR) was less apparent than in $\mathrm{Ctrl}^{\mathrm{TEC}}$ mice after AA treatment. Expression levels of markers specific for podocytes and TECs will be measured to confirm that SARA maintained cellular phenotype. Gene expression profiles of podocytes isolated from SARA/Ctr ${ }^{\text {podo }}$ mice by flow cytometry are being analyzed by RNA sequencing. Kidneys from SARA/Ctrl ${ }^{\mathrm{TEC}}$ mice were digested into single cell suspensions for single cell RNA sequencing. These high throughput analyses will provide insight into the mechanisms by which SARA maintains cellular phenotype and protects against renal fibrosis.

Conclusions: SARA overexpression in podocytes and TECs protects against glomerulosclerosis and tubulointerstitial fibrosis respectively.
Elucidating the mechanisms by which SARA functions will help unearth new molecular targets for therapies directed at renal fibrosis.

\section{EP-43 DOSE OPTIMISATION OF COLECALCIFEROL IN PAEDIATRIC PATIENTS WITH CHRONIC KIDNEY DISEASE}

Wan Mandy ${ }^{1}$, Bruce Green ${ }^{2}$, Arpana Iyengar ${ }^{3}$, Nivedita Kamath ${ }^{3}$, Hamsa Reddy ${ }^{3}$, Jyoti Sharma ${ }^{4}$, Jyoti Singhal ${ }^{4}$, Susan Uthup ${ }^{5}$, Sudha Ekambaram $^{6}$, Sumithra Selvam ${ }^{3}$, Greta Rait ${ }^{7}$, Rukshana Shroff ${ }^{8}$, Jignesh Patel ${ }^{1}$

${ }^{I}$ Kings College London; ${ }^{2}$ Model Answers R\&D; ${ }^{3}$ St John's Medical College Hospital; ${ }^{4}$ King Edward Memorial Hospital; ${ }^{5}$ Government Medical College; ${ }^{6}$ Mehta Multispecialty Hospital; ${ }^{7}$ University Of College London; ${ }^{8}$ Great Ormond Street Hospital Institute Of Child Health

Introduction: The prevalence of vitamin D deficiency is high in children with chronic kidney disease (CKD). This study aimed to develop a population pharmacokinetic $(\mathrm{PK})$ model of colecalciferol that can be used to optimise colecalciferol dosing in this population.

Material and methods: Data from 83 children with CKD were used to develop a population PK model using a nonlinear mixed effects modelling approach. Serum creatinine and glomerular disease were investigated as covariates, and optimal dosing was determined based on achieving and maintaining 25-hydroxyvitamin D (25(OH)D) concentrations of $30-48$ $\mathrm{ng} / \mathrm{mL}(75-120 \mathrm{nmol} / \mathrm{L})$.

Results: The time course of $25(\mathrm{OH}) \mathrm{D}$ concentrations was best described by a one compartment model with the addition of a basal concentration parameter to reflect endogenous $25(\mathrm{OH}) \mathrm{D}$ production from diet and sun exposure. Colecalciferol showed wide between-subject variability in its PK, with total body weight scaled allometrically the only covariate included in the model. Simulations from the model demonstrated that current dosing recommendation for colecalciferol can be optimised using a weight-based dosing strategy.

Conclusions: This is the first study to describe the population PK of colecalciferol in children with CKD. PK model-informed dosing is expected to improve the probability of achieving target $25(\mathrm{OH}) \mathrm{D}$ concentrations while minimising the risk of overdosing.

\section{EP-44 ENDOTHELIAL DYSFUNCTION IS COMMON IN CHILDREN WITH CKD STAGE III-V}

\author{
Martin Kreuzer ${ }^{1}$, Lavin Mohamad ${ }^{2}$, Maren Leifheit-nestler ${ }^{2}$, \\ Dieter Haffner ${ }^{2}$ \\ ${ }^{I}$ Essen University Hospital; ${ }^{2}$ Hannover Medical School
}

Introduction: In children with chronic kidney disease (CKD), damage to the cardiovascular system occurs frequently. Earliest manifestation is an endothelial dysfunction. With cutaneous laser Doppler fluximetry (LDF) a non-invasive method is available to detect early changes in microcirculation in children.

Material and methods: In this pilot study we examined 52 children and adolescents aged 6 to 20 years with a glomerular filtration rate (GFR) $<60 \mathrm{ml} / \mathrm{min} / 1.73 \mathrm{~m}^{2}($ CKD stage III $(\mathrm{n}=25)$, IV $(\mathrm{n}=14), \mathrm{V}(\mathrm{n}=13))$. In the patients, the change in cutaneous LDF during thermal stimulation, and blood pressure were measured during a regular outpatient visit. In addition to regular blood samples, we determined angiopoietin 1 and 2, fetuin A, c-term FGF-23, intact FGF-23 and sKlotho. The data were compared to those of healthy controls. All parameters of the linear regression analyzes with a $\mathrm{p}<0.1$ were included in a multiple regression analysis.

Results: 52 patients aged $13.3 \pm 4.6$ years were compared with 77 healthy controls. In the demographics, the groups only differed in body length (0.91 vs. $0.35 \mathrm{SDS}, \mathrm{p}<0.0001)$. LDF AUC was significantly reduced in patients $\left(157 \pm 17\right.$ vs. $\left.528 \pm 21 \mathrm{rPU}^{*} \min , \mathrm{p}=0.0007\right)$. As a marker of 
impaired endothelial function, the angiopoietin2/angiopoeietin1 ratio differed significantly between the groups ( 0.63 vs. $0.12, \mathrm{p}<0.0001)$. Although systolic blood pressure differed significantly from normal mean $(0.64 \pm 1.4$ SDS vs. $0, \mathrm{p}=0.002$ ), all patients had blood pressure within physiologic range. 38/52 patients received antihypertensives. In the multiple regression analysis, only GFR remained as an independent predictor for LDF AUC. Conclusions: Endothelial dysfunction can often be detected in children and adolescents with CKD, regardless of stage (III-V). This pilot study reveals only GFR as independent risk factor for impaired endothelial function. Notably, in this population FGF-23 was not associated with endothelial dysfunction, though, none of the patients presented with Klotho deficiency.

\section{EP-45 LONG TERM KIDNEY OUTCOME AFTER ALLOGENEIC HEMATOPOIETIC STEM CELL TRANSPLANTATION IN PEDIATRIC PATIENTS - A SINGLE-CENTER STUDY}

Chiara Benevenuta ${ }^{1}$, Francesca Matarozzo ${ }^{1}$, Francesca Carraro ${ }^{2}$, Bruno Gianoglio ${ }^{1}$, Elena Ernestina Vassallo ${ }^{2}$, Franca Fagioli ${ }^{2}$, Licia Peruzzi ${ }^{1}$

${ }^{1}$ Pediatric Nephrology Unit, Regina Margherita Children's Hospital, Department Of Pediatrics, University Of Turin, Italy, ${ }^{2}$ Oncology And Stem Cell Transplantation Unit, Regina Margherita Children's Hospital, Department Of Pediatrics, University Of Turin, Italy

Introduction: Chronic kidney disease (CKD) is a major cause of morbidity among hematopoietic stem cell transplantation (HSCT) survivors, but conclusive evidence on its prevalence, risk factors and physiopathology is still lacking, especially at pediatric age.

Material and methods: We performed a retrospective longitudinal study to characterize CKD incidence and risk factors in patients who underwent allogeneic HSCT for either malignant or non-malignant indication between December 2016 and December 2019 at Regina Margherita Childrens Hospital - Torino, Italy and survived to day +100 .

Results: Seventy-three patients were included in the study. Median age was $7.47(0.4-22.8)$ years old. Median GFR at baseline was $153 \mathrm{ml} / \mathrm{min} /$ $1.73 \mathrm{mq}$ (range $74-298 \mathrm{ml} / \mathrm{min} / 1.73 \mathrm{mq}$ ). The three-year cumulative incidence of CKD was $10 \%$. Older age at transplantation, previous grade 2 or 3 AKI events, and the clinical evidence of a leak syndrome were significantly associated with $\mathrm{CKD}$ development on univariate analysis $(p<0.01, p<0.05$ and $p=0.05$ respectively). Age at time of transplantation persisted as an independent risk factor for $\mathrm{CKD}$ on multivariate analysis. Immunosuppressive drugs showed no impact on kidney function in the majority of patients, except for minor transient AKI events. With regard to the potential role of under-immunosuppression and consequent GvHD mechanism in CKD development, we report the case of a histologically confirmed renal GvHD.

Conclusions: Late kidney dysfunction is a significant complication of HSCT, even in a pediatric setting. CKD patients require adequate diagnosis and specific follow up, and so do patients affected by mild persistent alteration of kidney function who do not meet the full CKD criteria yet, but represent a vulnerable population that may progress to CKD in adulthood. Very long term, prospective follow up studies are needed to better characterize renal outcome in adults who survived HSCT in childhood.

\section{EP-46 EVALUATION OF PERFORMANCE USING MOXO-CPT IN CHILDREN WITH ADVANGED STAGES OF CHRONIC KIDNEY DISEASE, DIALYSIS AND RENAL TRANSPLANTATION}

Bahar Büyükkaragöz ${ }^{1}$, Șebnem Soysal Acar ${ }^{2}$, Mesiha Ekim ${ }^{3}$, Umut Selda Bayrakçi ${ }^{4}$, Mehmet Bülbül ${ }^{5}$, Aysun Çaltik Yilmaz ${ }^{6}$, Sevcan A. Bakkaloğlu ${ }^{1}$

${ }^{1}$ Gazi University, Department Of Pediatric Nephrology, Ankara, Turkey, ${ }^{2}$ Gazi University, Department Of Pediatrics, Ankara, Turkey, ${ }^{3}$ Ankara University, Department Of Pediatric Nephrology, Ankara, Turkey, ${ }^{4}$ Ankara City Hospital, Department Of Pediatric Nephrology,
Ankara, Turkey, ${ }^{5}$ Dr. Sami Ulus Maternity And Childrens Hospital, Department Of Pediatric Nephrology, Ankara, Turkey, ${ }^{6}$ BaŞkent University, Department Of Pediatric Nephrology, Ankara, Turkey

Introduction: Cognitive and psychiatric disorders are relatively higher in children with chronic kidney disease (CKD) compared to their healthy peers. Although renal transplantation (RTx) corrects many of the metabolic abnormalities belonging to the pre-RTx period and improves the quality of life, behavioral or cognitive disorders may persist, especially in adolescents with RTx. Attention-deficit/hyperactivity disorder (ADHD) is the most common neurobehavioral disorder of the childhood. On the other hand, studies focusing on ADHD in children with CKD are scarce. Herein, we aimed to evaluate the ADHD symptoms in children with $\mathrm{CKD}$, dialysis and RTx, and investigate differences between groups. Material and methods: MOXO-continuous performance test (CPT), which provides the analysis of four specific domains of ADHD including attention, timeliness, hyperactivity and impulsivity was applied to 72 patients ( 23 children with RTx, 23 children undergoing dialysis and 26 children with stages 3-5 CKD).

Results: Mean age of the patients was $13.35 \pm 3.39$ years. Majority were boys $(61.1 \%)$. Although serum creatinine was lowest in RTx group, stage 2-4 CKD-T was detected in 69.6\% $(n=16)$. Mean pre-RTx CKD duration was $71.13 \pm 46.44$ years, with similar overall CKD duration in all groups. There were no significant differences between the groups in respect to the MOXO-CPT total and z scores. Attention and timeliness z scores were significantly higher in girls $(\mathrm{p}=0.004$ and $\mathrm{p}=0.008$, respectively). Attention and timeliness total scores were also significantly higher ( $p=0.006$ and $p=0.047$ ) and hyperactivity total scores were significantly lower in older patients (12-18 years) compared to the younger ones (6-12 years, $\mathrm{p}=0.017$ )

Conclusions: Similar MOXO-CPT scores in all groups was attributed to presence of long CKD duration in all groups as well as presence of allograft dysfunction in the majority of RTx group. Regardless of the groups, older age and female sex were associated with better performance in respect to attention and timeliness, besides hyperactivity was found to be decreased as time passes.

\section{EP-47 51CR-EDTA PLASMA CLEARANCE IN CHILDREN: ONE, TWO OR MULTIPLE SAMPLES?}

Pottel Hans ${ }^{1}$, Goffin Karolien ${ }^{1}$, De Waele Liesbeth ${ }^{1}$, Levtchenko Elena 1 , Delanaye Pierre ${ }^{2}$

${ }^{1}$ Ku Leuven; ${ }^{2}$ University Of Liège

Introduction: Plasma disappearance curves using multiple blood samples are a recognized reference method for measuring Glomerular Filtration Rate (GFR). However, there is no consensus on the protocol for this type of measurement. A two-compartment model is generally considered acceptable for the mathematical description of the concentration-time decay curve. The impact of the fitting procedure on the reported GFR has not been questioned so far.

Material and methods: We defined eight different fitting procedures to calculate the area under the curve (AUC), and from this AUC, the GFR. We applied the eight fitting methods (all considering a full concentrationtime curve) on the multiple sample data ( 8 samples) of 20 children diagnosed with Duchenne Muscular Dystrophy. We evaluated the effect (variability) on the reported GFR from the different fitting methods and compared these results with GFR-values calculated from late samples only (samples after 120 minutes) and from one-sample methods.

Results: In 6 out of 20 cases, the fitting methods on the full concentration-time curve resulted in very different reported GFR-values, mainly because some methods were not able to fit the data, or methods resulted in GFR-values ranging from zero to $120 \mathrm{~mL} / \mathrm{min}$. The reported GFR-result therefore strongly depends on the fitting method making the full concentration-time method less robust than expected. Compared to a 
consensus reference GFR, the late sample models did not show fitting issues and may therefore be considered as more robust. Also the onesample methods showed acceptable accuracy.

Conclusions: The late sample methods (using 3 time-points) provide robust and reliable methods to determine GFR.

\section{EP-48 BETA TRACE PROTEIN APPEARS TO BE AN IDEAL MARKER SHOWING DETERIORATION IN GLOMERULAR FILTRATION RATE IN CHILDREN WITH CHRONIC KIDNEY DISEASE}

\author{
Betül Șenyürek ${ }^{2}$, İbrahim GökÇe ${ }^{1}$, Alİ Yaman ${ }^{3}$, Haríka Alpay ${ }^{1}$ \\ ${ }^{1}$ Marmara University Paediatric Nephrology, ${ }^{2}$ Marmara University \\ Paediatrics, ${ }^{3}$ Marmara University Biochemistry
}

Introduction: The glomerular filtration rate (GFR) used in the diagnosis, follow-up and evaluation of renal diseases is considered to be the best indicator of renal function. Beta-trace protein (BTP) is a low molecular weight protein produced in the cerebrospinal fluid. Our aim in this study is to investigate the serum and urine levels of BTP in children with chronic kidney disease (CKD) and to examine its relationship with impaired GFR and development of end-stage renal disease.

Material and methods: 136 children with CKD stage 1-4 were included in the study. The control group consisted of 43 healthy children. In addition to serum creatinine, BUN, cystatin-C, spot urine creatinine, microalbumin, 24-hour-urine creatinine, protein and microalbumin were measured. Both serum and urine samples for BTP and beta-2 microglobulin (B2M) measurements were also taken at the same visit. Serum-BTP (S-BTP), urine-BTP (U-BTP), $\mathrm{BTP} /$ creatinine (U-BTP/creatinine) and fractional clearance of BTP (FEBTP) were calculated.

Results: S-BTP level was significantly higher in the patient group compared to the control group $(0.36 \pm 0.14$ vs $0.29 \pm 0.13$, p: $<0.001)$. U-BTP/ creatinine and FEBTP demonstrating U-BTP excretion were significantly higher in the patient group compared to the control group $(87.69 \pm 107.9$ vs $21.47 \pm 30.41, \mathrm{p}:<0.001$ and $0.44 \pm 0.71$ vs $0.04 \pm 0.06$, p: $<0.001$, respectively). S-BTP, U-BTP/creatinine and FEBTP were detected to be elevated as the stage increased in children with CKD. Especially, U-BTP/creatinine and FEBTP were detected to have strong negative correlation with GFR (p:0.000, r:-0,690 and p:0.000, r:-0.784, respectively), and positive correlation with cystatin- $\mathrm{C}$ and urine protein, microalbumin and $\mathrm{B} 2 \mathrm{M}$ excretions. In multiple regression analysis, it was found that the U$\mathrm{BTP} /$ creatinine was mostly affected by the microalbumin excretion in the urine $(\mathrm{p}:<0.001, \mathrm{t}: 5.670)$. GFR and S-BTP were other factors affecting U-BTP/creatinine levels.

Conclusions: Serum and urine BTP levels are increased in children with $\mathrm{CKD}$ and it is thought that it may be an ideal marker demonstrating deterioration in GFR.

\section{EP-49 CROSSTALK BETWEEN SECONDARY HYPERPARA- THYROIDISM AND BODY COMPOSITION IN PEDIATRIC PATIENTS WITH CHRONIC KIDNEY DISEASE}

Vasiliki Karava ${ }^{1}$, Antonia Kondou ${ }^{1}$, John Dotis ${ }^{1}$, Athanasios Christoforidis ${ }^{2}$, Vassilios Liakopoulos ${ }^{3}$, Dimitrios Zafeiriou ${ }^{1}$, Nikoleta Printza ${ }^{1}$

${ }^{1}$ Pediatric Nephrology Unit, 1st Department Of Pediatrics, Hippokratio General Hospital,aristotle University Of Thessaloniki, Greece; ${ }^{2}$ Pediatric Endocrinology Unit, 1st Department Of Pediatrics, Hippokratio General Hospital,aristotle University of Thessaloniki, Greece; ${ }^{3} 1$ st Department Of Internal Medicine, Division Of Nephrology And Hypertension, Aristotle University Of Thessaloniki, Greece
Introduction: This cross-sectional study explores the association between secondary hyperparathyroidism and body composition in pediatric patients with moderate (stage 3 ) and severe (stage 4-5) chronic kidney disease (CKD).

Material and methods: 61 patients (median age: 13.4 years) were included. Body composition data, using multi-frequency bio-impedance spectroscopy, including lean tissue index (LTI) and fat tissue index (FTI) were recorded. Muscle wasting was defined as LTI height-age (HA) z-score <-1.65 SD and increased adiposity as FTI z-score $>1.65$ SD. Serum parathormone (PTH), calcium, phosphorus, 25hydroxyvitamin D and leptin were collected. 6-month mean serum values of mineral laboratory parameters were recorded in severe CKD patients. Alfacalcidol index, defined as alfacalcidol dose (mcg/week) per $\mathrm{pg} / \mathrm{ml}$ of PTH x 1000, was calculated in severe CKD patients.

Results: In moderate CKD (31 patients), high PTH ( $>90 \mathrm{ng} / \mathrm{ml}, 10 \mathrm{pa}-$ tients) was associated with higher FTI $z$-score $(\mathrm{p}=0.022)$. Serum PTH was correlated to LTI HA $z$-score ( $r s=-0.486, p=0.006$ ) and to serum leptin levels ( $\mathrm{rs}=0.369, \mathrm{p}=0.041$ ), while the correlation between FTI $\mathrm{z}$-score and PTH ( $r s=0.393, p=0.039$ ) lost significance after adjustment for serum leptin. PTH was associated with increased adiposity (12 patients) after adjustment for the other mineral metabolism parameters (OR 1.023, 95\% CI 1.002-1.045, $\mathrm{p}=0.028$ ). In severe CKD (30 patients), no correlation was observed between PTH and body composition indices. Eleven patients with muscle wasting presented lower alfacalcidol index $(\mathrm{p}=0.017)$. Alfacalcidol index $<25$ was associated with muscle wasting, after adjustment for CKD stage and other mineral metabolism parameters (OR $17.143,95 \%$ CI $1.794-163.806, \mathrm{p}=0.014$ ).

Conclusions: Secondary hyperparathyroidism is associated with increased adiposity in moderate but not severe CKD, probably mediated by serum leptin. In severe CKD, low alfacalcidol index maybe a marker of muscle wasting.

\section{EP-50 THE EGFR VARIABILITY ASSESSMENT IN CHILDREN AFTER HEMATOPOIETIC STEM CELL TRANSPLANTATION IN 1 YEAR OBSERVATION}

Katarzyna Zachwieja ${ }^{1}$, Katarzyna Mikrut ${ }^{1}$, Aleksandra Krasowska-kwiecień ${ }^{2}$, Monika Miklaszewska ${ }^{1}$, Jolanta Goździk ${ }^{1}$, Przemysław Korohoda ${ }^{3}$, Dorota Drożdż ${ }^{1}$

${ }^{1}$ Departement Of Pediatric Nephrology And Hypertension, Jagiellonian University Medical College, ${ }^{2}$ Departement Of Clinical Immunology Nad Transplatantation Jagiellonian University Medical College, ${ }^{3}$ Agh University Of Science And Technology, faculty Of Electronics, Krakow

Introduction: Hematopoietic stem cell transplantation (HSCT) is often performed in various oncologic and other diseases. The renal function assessment is obligatory for safe treatment.

Material and methods: eGFR variability was assesed in 40 children (av. 8,6 yrs) treated with HSCT (11 autologous, 29 allogenic) in one year period. The eGFR was estimated before, one week, 1, 6 and 12 months after HSCT based on: cystatin C-eGFR(cysC); Schwartz bed-side - eGFR(BS) and Schwartz trimarker - eGFR $(3 \mathrm{M})$ with our correction and with creatinine clearance - eGFR(U24h). The Iohexol method eGFR(IOH)- reference method was performed before, 6 and 12 months after HSCT.

Results: eGFR values - in $\mathrm{ml} / \mathrm{min} / 1,73 \mathrm{~m} 2$. The mean value of eGFR(IOH) was in normal range before: $(131,8 \pm 35,7)$ and in 6 months $(104,2 \pm 45,6, \mathrm{p}=0,024)$ and 12 months after HSCT $(109,6 \pm 33,0$; $\mathrm{p}=0,052$ ). One year after HSCT eGFR were lower statistically according to all but one equation (eGFR-U24h) with mean difference from 14,2 to 31,2. One week after HSCT increase of eGFR was observed with mean difference $(15,9$ to 27,9$)$. 1 month later eGFR decreased of mean difference $(6,4$ to 31,8$)$; then in 6 months from 16,4 to 27,9 . One year later eGFR was higher compared to 6 months eGFR (mean difference 6,4 to $31,2)$. The highest variability of GFR was seen during first 1 month after procedure. In $25 \%$ if children eGFR was $<90$ one year after HSCT. The 
eGFR(3M) showed the best concordance with Iohexol method, the lowest agreement was noted for eGFR (U24h)

Conclusions: The renal function in 12 months after HSCT is well preserved with non clinically significant decrease in eGFR. The temporary deterioration of eGFR was confirmed in 1 up to 6 months after with improvement tendency in 1 year. We suggest to use trimarker formula for eGFR estimation.

\section{EP-51 GROWTH PATTERN AND RISK FACTORS FOR SHORT STATURE IN NON-DIALYSIS CKD CHILDREN}

Matheus Dantas Soeiro ${ }^{1}$, Hendersson Edward Firmino Cavalcanti ${ }^{1}$, Lucas Cavalcanti Tenorio De Albuquerque ${ }^{1}$, Virgina Barbosa De Melo ${ }^{2}$, Danielle Barbosa Da Silva ${ }^{2}$, Marcela Correa De Araujo Pandolfi ${ }^{2}$, Emilia Maria Dantas Soeiro ${ }^{2}$

${ }^{1}$ Faculdade Pernambucana De Saude, ${ }^{2}$ Instituto De Medicina Integral Professor Fernando Figueira

Introduction: Growth failure in the context of chronic kidney disease in children is related to low quality of life, low self-esteem and worse school performance. Growth retardation in this case is multifactorial. Knowing the modifiable factors and establishing strategies that can improve care for these children is of utmost importance. Here we described growth pattern in children with chronic kidney disease and associated risk factors for short stature.

Material and methods: We retrospectively analyzed anthropometric, epidemiological, birth weight, prematurity and laboratory data (bicarbonate, hemoglobin, serum bone biomarkers), from medical record of 43 children and adolescents with non-dialysis chronic kidney disease.

Results: Short stature was present in $63 \%$ of children at the beginning of the follow-up, which remained $51 \%$ at the end of the study period $(\mathrm{p}=$ 0.096). There was an improvement in serum bicarbonate levels $(18.2 \pm$ 4.5 and $20.7 \pm 4.0 \mathrm{mEq} / \mathrm{L}, \mathrm{p}=0.005)$, and hemoglobin $(10.2 \pm 1.68$ and $11.5 \pm 1,97 \mathrm{mg} / \mathrm{dl}, \mathrm{p}=0.001$ ) throughout the follow-up period. Despite the improvement in laboratory data, $60 \%$ of children maintained metabolic acidosis (bicarbonate $<22 \mathrm{mEq} / \mathrm{L}$ ) and $34.9 \%$ still had anemia (hemoglobin $<11 \mathrm{mg} / \mathrm{dL}$ ). When comparing children with adequate stature and short stature, we observed lower serum levels of bicarbonate in the latter group, respectively, $22.0 \pm 3.1$ and $19.4 \pm 4.5(\mathrm{p}=0.03)$. There was an increase in PTH levels over the follow-up period ( $p=0.029)$, but when compared PTH levels $>2 \mathrm{xUNL}$ in both groups (adequate and short stature), there was no difference $(\mathrm{p}=0.9)$. Hemoglobin, bone disease markers, birth weight and gestational age did not show any association with short stature in our population.

Conclusions: We observed a high prevalence of short stature which was associated with low serum bicarbonate levels. Our results point out the need for better control of metabolic acidosis.

\section{EP-52 A CASE OF HUPRA SYNDROME PRESENTING WITH EXTRA-RENAL MANIFESTATIONS}

\section{Nilüfer Göknar , Emre Keleșoğlu , Cengiz Candan , Diana Üçkardeș Istanbul Medeniyet University}

Introduction: HUPRA syndrome is a rare mitochondrial disease characterized by hyperuricemia, pulmonary hypertension, metabolic alkalosis and, renal failure in infancy. This syndrome was previously described in five patients with a homozygous mutation in SARS2, encoding the mitochondrial seryl-tRNA synthetase. The principal affected tissue in HUPRA syndrome is the kidney.

Material and methods: We report here a girl, who presented with hyperuricemia, increased urea and creatinine values for her age and diagnosed HUPRA syndrome.
Results: A two years old girl was admitted to our emergency department with nausea, vomiting and fever. She had previous history of recurrent admissions to emergency department with similar manifestations. Family history was significant for a similar presentation of a cousin who died at seven years old age. Blood tests showed renal failure, anemia, mild acidosis, hypomagnesemia and, hyperuricemia. Echocardiography revealed pulmonary hypertension. Genetic analysis showed homozygous mutation in SARS gene (c.515A $>\mathrm{G}$ (p.N172S) resulting in p.Asn172Ser). She was investigated for other system involvements and hyperthyroidism and immunodeficiency were diagnosed. She had cranial MRI imaging which demonstrated Arnold-Chiari Type 1 malformation. On last visit, our patient was 4 years and 4 months old age and she had stage $3 \mathrm{~b}$ renal failure. She was under the treatments of allopurinol for hyperuricemia, sodium bicarbonate for acidosis, vitamin D for low vitamin D levels, alpha lipoic acid, Coenzyme Q10, and L-carnitine for their antioxidant effect and to improve mitochondrial enzymes, thyromazol and propranolol for hyperthyroidism and darbepoetin for anemia of chronic kidney disease.

Conclusions: In conclusion, our patient had important renal failure, severe pulmonary hypertension, hyperuricemia and other extrarenal malformations which were not reported yet. HUPRA syndrome is a multisystem fatal mitochondrial cytopathy and children with this syndrome must be checked for other system involvements.

\section{EP-53 RESISTANCE TO ERYTROPOESIS STIMULATING AGENTS IN CHILDREN WITH END STAGE RENAL DISEASE}

\author{
Mehtap Sak, Neslihan Cicek, Ibrahim Gokce, Harika Alpay \\ Marmara University Medical School
}

Introduction: Anemia is one of the most important cause of morbidity in chronic kidney disease (CKD). Erytropoesis stimulating agents (ESAs) are the mainstay of the therapy. In this paper we aimed to analyse the risk factors associated with ESA hyporesponsiveness in children receiving renal replacement therapy (RRT).

Material and methods: We evaluated 33 clinically stable ESRD patients (14 males, 19 females) on hemodialysis or peritoneal dialysis. EPO hyporesponsiveness evaluated at the last visit by eritropoesis resistance index (ERI). We also evaluated serum hemoglobin, iron, ferritin, total iron binding capacity, transferrin saturation index, folic acid, vitamin B12, platelet, WBC, parathyroid hormone $(\mathrm{PTH})$, calcium, phosphorus, magnesium, alkaline phosphatase, $25-\mathrm{OH}$ vitamin-D, albumin, blood pressure and body fluid volume (overhydration) measured by bioimpedance spectroscopy. Then, we analysed the correlation between ERI and all of the parameters related to CKD above. We also divided patients into two groups by the median value of the ERI as high ERI group $(>16)$ and low ERI group $(<16)$ and compared the parameters between two groups.

Results: The mean follow-up time was $4.83 \pm 4.37$ (range: $0.3-14.1$ ) years. ERI was positively correlated with serum phosphorus level $(\mathrm{p}=0.016)$ and although not statistically significant, presence of hypertension was also found to be correlated with ERI ( $p=0.058)$. When two groups were compared mean serum phosphorus levels were found to be significantly higher in high ERI group than low ERI group $(6.61 \pm 1.67$ vs $5.14 \pm 1.14 \mathrm{mg} / \mathrm{dl}$ respectively, $\mathrm{p}=0.007)$. Mean PTH levels were also higher in high ERI group than low ERI group but it was not statistically significant $(599 \pm 351$ vs $392 \pm 320$ $\mathrm{pg} / \mathrm{ml}$ respectively, $\mathrm{p}=0.088$ ). Presence of hypertension was significantly higher in high ERI group than low ERI group $(p=0.041)$ in the presence of comparable body fluid volumes of two groups.

Conclusions: ERI seems to be associated with secondary hyperparathyroidism and hypertension in children with ESRD receiving RRT. 
EP-54 WHAT TO DO WITH POLYURIA AFFECTING ACADEMIC PERFORMANCE AND MOOD-RELATED COMPLAINTS IN IDIOPATHIC FANCONI SYNDROME?

Rishika Geda ${ }^{1}$, Guido Filler ${ }^{2}$, Fabio Salerno ${ }^{2}$, Andy Zhang ${ }^{2}$, Maria E. Díaz-gonzález De Ferris ${ }^{3}$, Christopher William Mcintyre ${ }^{2}$

${ }^{1}$ University Of Western Ontario, ${ }^{2}$ Schulich School Of Medicine And Dentistry, ${ }^{3}$ The University Of North Carolina At Chapel Hill

Introduction: Polyuria is a common problem in patients with tubular diseases, especially for those with and Fanconi syndrome and chronic kidney disease (CKD). There are currently no guidelines on how to treat debilitating polyuria. Vasopressin is usually not effective.

Material and methods: A 13-year-old female with idiopathic Fanconi syndrome and CKD stage II had an estimated glomerular filtration rate (eGFR) of $69 \mathrm{~mL} / \mathrm{min} / 1.73 \mathrm{~m}^{2}$ and was severely affected by polyuria of 5 L per day (voiding at least 11 times during the day and up to 8 times at night), impacting her mood (RCADS-child) and academic performance at school. In the absence of guidelines and with literature discouraging the use of indomethacin in this condition, we nonetheless attempted indomethacin treatment because of its effect on reduction of sodium wasting. RCADS scores were obtained before and during treatment. Sodium-23 $\left({ }^{23} \mathrm{Na}+\right) \mathrm{MRI}$ of her thigh was performed at 18 months on treatment.

Results: Indomethacin was started at a dose of $1 \mathrm{mg} / \mathrm{kg}$ every 12 hours. The patient's urine output dropped to $2.5 \mathrm{~L}$ and this was accompanied by a substantial decrease of her sodium wasting from 24.6 to $7.7 \mathrm{mmol} / \mathrm{kg} /$ day. Over the course of 18 months, her eGFR dropped temporarily to 60 $\mathrm{mL} / \mathrm{min} / 1.73 \mathrm{~m}^{2}$ and recovered to $68 \mathrm{~mL} / \mathrm{min} / 1.73 \mathrm{~m}^{2}$ at last follow up. However, MRI revealed ongoing moderate sodium decrease in her skin substantial $\mathrm{Na}+$ decrease in her muscle (z-score -2.5 when compared to age-matched peers with normal renal function). The self-reported RCADS-Child survey and academic performance improved significantly. Conclusions: We report the successful use of indomethacin in an adolescent female with idiopathic Fanconi syndrome, which was safe and substantially improved the urinary frequency. We also demonstrated a substantial reduction of the $\mathrm{Na}+$ wasting with indomethacin while the patient's muscle $\mathrm{Na}+$ concentration remained substantially depleted, suggesting no further role with $\mathrm{Na}+$ intake reduction.

\section{EP-55 ASSESSMENT OF AWARENESS AND OPINIONS ABOUT INFLUENZA VACCINATION OF PARENTS OF CHILDREN DIAGNOSED WITH CHRONIC KIDNEY DISEASE}

Eren Soyaltin ${ }^{1}$, Eda Karadağ Öncel ${ }^{2}$, Seçil Arslansoyu Çamlar ${ }^{3}$, Gökçen Erfidan ${ }^{1}$, Özgür Özdemir ${ }^{1}$, Cemaliye Bașaran ${ }^{1}$, Demet Alaygut ${ }^{1}$, Fatma Mutlubaș ${ }^{3}$, Dilek Yilmaz Çiftdoğan ${ }^{4}$, Belde Kasap Demir ${ }^{5}$

${ }^{1}$ University Of Health Sciences, İmir Tepecik Training And Research Hospital, pediatric Nephrology Clinic, İzmir, Turkey; ${ }^{2}$ University Of Health Sciences, İzmir Tepecik Training And Research Hospital, pediatric Infectious Diseases Clinic, İzmir, Turkey; ${ }^{3}$ University Of Health Sciences, İzmir Faculty Of Medicine, Pediatric Nephrology Clinic, Izmir Turkey; ${ }^{4}$ Izmir Katip Çelebi University, Department Of Pediatric Infectious Diseases, Izmir, Turkey; ${ }^{5}$ Izmir Katip Çelebi University, Department Of Pediatric Nephrology And Rheumatology, İmir, Turkey

Introduction: Children diagnosed with chronic kidney disease(CKD); have more risk for the incidence and severity of infections due to immune deficiency, repeated dialysis sessions, and frequent contact with medical care facilities. With its seasonal epidemic, influenza creates a high risk of mortality and morbidity for children with CKD. We aimed to evaluate the knowledge and opinions of the parents of children who were followed with CKD. Material and methods: A seasonal influenza vaccine questionnaire was administered to a voluntary parent of patients with CKD who admitted to outpatient Pediatric Nephrology Clinic between November 2019 and
February 2020, and those who completed the questionnaire were included in the study.

Results: Parents of 64 patients diagnosed with CKD completed the questionnaire. 44(68.8\%) participants were informed about the vaccine. 54 participants who had not had their child vaccinated were asked about the reasons for not getting vaccine. The most frequent reason was reported as insufficient information about vaccine( $33.3 \%$ ) requirement and the second reason was the fear of possible side effects of the vaccine (14.8\%). There was no significant difference about the rate of vaccination between the two groups of patients who use and did not use immunosuppressive drugs (71.4\% and $40 \%$ respectively) The knowledge about influenza vaccine of parents of children with kidney transplantation was significantly higher than other CKD (p:0,006). All participants were informed about infections caused by influenza and flu vaccines. Athough information 18(28.1\%) participants did not want to vaccinate, $17(26.6 \%)$ participants stated that they still have no idea.

Conclusions: In our study, the most common cause of having no vaccination was lack of knowledge about vaccination. Therefore, influenza vaccination rates can be can be increased by informing parents about influenza infection and vaccination in pediatric nephrology clinics.

\section{EP-56 TOCILIZUMAB AS A SUCCESSFUL TREATMENT OF AA-TYPE AMYLOIDOSIS IN A CHILD WITH FAMILIAL MEDITERRANEAN FEVER}

Houda Nassih, Rabiy El Qadiry, Aicha Bourrahouat, Imane Ait Sab B Pediatric Ward, Department Of Pediatrics, Child And Mother Hospital, Mohammed Vi University Hospital Center, Marrakesh, Morocco

Introduction: Familial Mediterranean fever (FMF) is an autoinflammatory disease. One of its dreadful complications is systemic AA-type amyloidosis.

Material and methods: We report the case of a child with end stage kidney disease and nephrotic syndrome complicating AA-type amyloidosis secondary to FMF.

Results: A first degree consanguineous six-year-old boy presented for progressive onset of generalized oedema and poor urine output. The family reported a three years history of recurrent fever and abdominal pain treated symptomatically. First assessment found hypertension, oliguria, and pallor. His height was less than -2 standard deviations. He has microscopic hematuria on dipstick. Laboratory assessment found kidney failure, microcytic anemia, acidosis, hyperkalemia, hyperphosphatemia, hyperparathyroidism, hypoalbuminemia, and nephronic proteinuria. Renal ultrasound found large dedifferentiated kidneys. Furosemide, nicardipine and bicarbonate were started. After four hours, the child was still oliguric and his blood pressure raised. Hemodialysis was soon started, and prednisone was prescribed. Renal biopsy showed AA-type amyloidosis. Genetic testing found mutation of the MEFV gene in favor of FMF. Colchicine was soon started. Evolution was marked by persistent nephrotic syndrome and inflammation despite a course of four weeks high-dose steroids. Anti-interleukin 6 (tocilizumab) was then started at $8 \mathrm{mg} / \mathrm{kg} / \mathrm{month}$ by IV route. After three months, the child had no more abdominal pain, arthritis. Also, laboratory tests showed no inflammation, normal albumin, and less proteinuria. At the same time, his blood pressure normalized, his GFR increased by $75 \%$, and he was dialysis free for two years. Conclusions: Tocilizumab should be used as a first line therapeutic option in renal AA-type amyloidosis complicating FMF.

\section{EP-57 CHRONIC RENAL FAILURE IN A CHILD WITH CORNELIA DE LANGE'S SYNDROME}

Zehra Aydin ${ }^{1}$, Umut Selda Bayrakci ${ }^{2}$, Fatma Semsa Cayci ${ }^{1}$

${ }^{I}$ The University Of Health Sciences, Ankara City Hospital, Department Of Pediatric Nephrology, Ankara, Turkey, ${ }^{2}$ The University Of Yildirim Beyazit, Ankara City Hospital, Department Of Pediatric Nephrology, Ankara, Turkey 
Introduction: Cornelia de Langes syndrome (CdLS) is a rare disease that affects multiple organs. It is characterized by atypical facial features, growth retardation, hirsutism, intellectual disability, malformations of the skeleton, and genitourinary system. Up to $40 \%$ of patients with CdLS have structural kidney and/or urinary tract anomalies.

Material and methods: Here we present a 6-year-old girl diagnosed with CdLS coexisting with chronic renal failure (CRF), horseshoe kidney, and bilateral VUR.

Results: A-six-month-girl was admitted to the hospital because of a recurrent urinary tract infection. The facial features of the child seemed dysmorphic, microcephaly, facial hirsutism, hypertelorism, microphthalmia, nystagmus, thick bushy eyebrows, phocomelia. Laboratory examination revealed normal whole blood count, serum biochemistry. Urinary ultrasound demonstrated a horseshoe kidney. CdLS was considered in the patient who was investigated for dysmorphic findings. A prophylactic antibiotic was administered because of a recurrent urinary tract infection. The voiding cystourethrography revealed bilateral grade 3 reflux. DMSA renal scintigraphy determined horseshoe kidney with bilateral renal scars. Despite the antibiotic prophylaxis, the patient who underwent recurrent urinary tract infection was operated for VUR. During the follow-up period, it was observed that the patient had an increase in creatinine with a decrease in the eGFR. At the last visit, the seven-year-old patient has been followed up with the diagnosis of CRF with a $36 \mathrm{ml} / \mathrm{dk} / 1.73 \mathrm{~m} 2$ eGFR.

Conclusions: Various urinary tract pathologies such as pelvic dilation, horseshoe kidney, VUR, renal dysplasia have been reported in CdLS. The urine and biochemistry analysis, urinary ultrasound should be regularly performed in CdLS.

\section{EP-58 SOLITARY KIDNEY WITH GLOMERULOPATHY}

Katarzyna Mikrut, Iwona Ogarek, Dorota Drożdż

Jagiellonian University Medical College, Department Of Pediatric Nephrology And Hypertension

Introduction: Prenatal screening rules out majority of renal agenesis, however up to $38 \%$ cases are diagnosed postnatally. Glomerulopathy occurring in solitary kidney may result in rapid kidney function loss.

Material and methods: 16-year-old male with solitary kidney was hospitalized due to accidentally discovered erytrocyturia and proteinuria. Ultrasound exam revealed absence of left kidney. Renal scintigraphy confirmed its null function. Laboratory tests presented with eleveated IgA level and serum creatinine. Prednisone and antihypertensive therapy with enalapril were started. A few weeks later patient presented with nephrotic-range proteinuria and erythrocytes found in urine sediment. His GFR corresponded to the II stage of CKD. Follow-up ultrasound shown hyperechogenity of renal parenchyma and indistict border between renal medulla and cortex of the right kidney. Antineuthrophil cytoplasmic antibodies were found. Granulomatosis with polyangitis was suspected, therefore the patient underwent a CT scan of sinuses - resulting in polyps and mucosal thickening. To finally verify the diagnosis, biopsy of patient's solitary kidney was performed. The histopathological report shown features of proliferative glomerulopathy with IgA deposits. Methylprednisolone and cyclophosphamide were the implemented in therapeutical protocol. Enalapil was converted to ramipril, beta-blocker was added.

Results: Kidney function, serum cANCA levels normalized, the patient no longer presented with signs of nephrotic syndrome, renal sonogram returned to normal, blood pressure remained within limits. Low-range proteinuria occured, cyclophosphamide was discontinued and cyclosporine was administered. Since then patient has presented with no abnormalities in urinalysis and urine sediment and his filtration rate is stable.

Conclusions: Glomerulopathy in solitary kidney carries significant risk in progression towards CKD. Detailed diagnostic test are required. Proper therapy results in stable kidney function and improves patients' quality of life.

\section{EP-59 URINARY MARKERS OF KIDNEY INJURI IN CHILDREN} WITH CHRONIC KIDNEY DISEASE AND OBESITY

Anna Khan, Tatiana Nastausheva, Elena Kulakova, Ekaterina Chichuga, Elena Stenshinskaya, Lilia Stahurlova

Voronezh State Medical University Named After N.n. Burdenko

Introduction: Purpose of our study was to compare urinary markers of kidney injury: KIM-1, NGAL, $\beta 2-\mathrm{m}$, IL-18 in children with chronic kidney disease (CKD) with and without obesity.

Material and methods: We studied 2 group of children. First group - 38 patients with CKD 1-2 stages and obesity (16 boys and 22 girls) aged 11.8 years [4.4 - 13]. All patients had BMI $>2$ SDS ( $Z$ score). The reasons of CKD in this group were: UTI or/and CAKUT in 31 patients $(81.6 \%)$, NS in 7 (18.4\%). Second group included 40 children (18 boys and 22 girls) aged 11.4 years [4.2-11] with CKD 1-2 stages without obesity. The reasons of CKD in this group were: UTI or/and CAKUT had 34 patients (85.0\%), NS had $6(15.0 \%)$. Urinary markers measured by ELISA methods. The results were presented as median and interquartile range - Me [IQR]. Comparison between groups was performed using Kolmogorov-Smirnov test.

Results: Urinary KIM -1 in children of $1^{\text {st }}$ group was $2209.8 \mathrm{pg} / \mathrm{ml}$ [1252.9- 2846.6] and in patients $2^{\text {nd }}$ group $-1462.0 \mathrm{pg} / \mathrm{ml}[1098.0-$ 1917.5], $\mathrm{p}<0.001$. The level of NGAL in urine did not differ in patients of 2 groups: $3.9 \mathrm{ng} / \mathrm{ml}$ [1.5- 7.9] and $3.4 \mathrm{ng} / \mathrm{ml}$ [1.1-7.8] in patients of $1^{\text {st }}$ and $2^{\text {nd }}$ group, $p>0.05$. Also urinary $\beta 2-\mathrm{m}$ and IL-18 were equal in 2 groups: $\beta 2-\mathrm{m}-9.8 \mathrm{mkg} / \mathrm{ml}[2.5-18.7]$ in $1^{\text {st }}$ group and $12.3 \mathrm{mkg} / \mathrm{ml}[4.1-$ $20.2]$ in $2^{\text {nd }}$ group, $p>0.05 ;$ IL- $18-1.1 \mathrm{pg} / \mathrm{ml}[0.9-1.4]$ in children of $1^{\text {st }}$ group and $1.1 \mathrm{pg} / \mathrm{ml}[0.9-1.3]$ in $2^{\text {nd }}$ group, $\mathrm{p}>0.05$.

Conclusions: Urinary KIM-1 reflects a negative influence of obesity for kidney in children with early stages of CKD.

\section{EP-60 26-YEAR EXPERIENCE WITH GROWTH HORMONE THERAPY IN CHILDREN WITH CHRONIC KIDNEY DISEASE IN STAGE II-V IN POLAND - RESULTS OF NATIONAL MULTICENTER STUDY}

Beata Leszczynska ${ }^{1}$, Maria Daniel ${ }^{1}$, Anna Deja ${ }^{1}$, Anna Majcher ${ }^{2}$, Maria Szczepanska ${ }^{3}$, Ilona Zagozdzon ${ }^{4}$, Aleksandra Zurowska ${ }^{1}$, Maecin Tkaczyk ${ }^{5}$, Anna Jander ${ }^{5}$, Przemyslaw Sikora ${ }^{6}$, Anna Wasilewska ${ }^{7}$, Irena Makulska ${ }^{8}$, Danuta Zwolińska ${ }^{8}$, Katarzyna Zachwieja ${ }^{9}$, Dorota Drozdz ${ }^{9}$, Jacek Rubik ${ }^{10}$, Wioletta Jarmuzek ${ }^{10}$, Jacek Zachwieja ${ }^{11}$, Roman Stankiewicz ${ }^{12}$, Andrzej Brodkiewicz ${ }^{13}$, Malgorzata Panczyk-tomaszewska ${ }^{1}$

${ }^{1}$ Department Of Pediatrics And Nephrology, Medical University Of Warsaw, Warsaw, Poland, ${ }^{2}$ Department Of Pediatrics And Endocrinology, Medical University Of Warsaw, Warsaw, Poland, ${ }^{3}$ Dialysis Division For Children, Department Of Pediatrics, School Of Medicine With The Division Of Dentistry In Zabrze, Medical University Of Silesia In Katowice, Zabrze, Poland, ${ }^{4}$ Department Pediatrics, Nephrology \& Hypertension, Medical University Of Gdańsk, Poland, ${ }^{5}$ Department Of Pediatrics, Immunology And Nephrology, Polish Mothers Memorial Hospital Research Institute, Łódź, Poland, ${ }^{6}$ Department Of Pediatric Nephrology, Medical University Of Lublin, Lublin, Poland, ${ }^{7}$ Department Of Pediatrics And Nephrology, Medical University Of Biatystok, Biatystok, Poland, ${ }^{8}$ Department Of Paediatric Nephrology, Wrocław Medical University, Poland, ${ }^{9}$ Department Of Pediatric Nephrology And Hypertension, Jagiellonian University Medical College, Kraków, Poland, ${ }^{10}$ Department Of Nephrology, Kidney Transplantation And Hypertension, The Childrens Memorial Health Institute, Warsaw, Poland, ${ }^{11}$ Department Of Pediatric Cardiology And Nephrology, Poznań University Of Medical Sciences, Poznań, Poland, ${ }^{12}$ Department Of Pediatric Nephrology, Specialist Municipal Hospital, Toruń, Poland, ${ }^{13}$ Department Of Pediatrics, Child Nephrology, Dialysotherapy And Management Of Acute Poisoning, Pomeranian Medical University, Szczecin, Poland 
Introduction: Recombinant human growth hormone (rhGH) has been available in Poland for children with chronic kidney disease (CKD) since 1994. The aim of the study was to assess growth velocity (GV) and factors affecting it in Polish children with CKD in stage II-V (excluding dialysis patients) treated with rhGH.

Material and methods: We analyzed 156 children (age of start of treatment rhGH median 10.8 years; 118 ) among 345 qualified patients who were treated for at least 6 months. We evaluated: age of CKD diagnosis, cause of CKD, growth and skeletal age at baseline and after year of the treatment and mean annual values of selected biochemical parameters.

Results: The cause of CKD was CAKUT $66.7 \%$, genetic diseases $18.6 \%$, glomerulonephritis $6.4 \%$ and other $8.3 \%$. The time from diagnosis of $\mathrm{CKD}$ to initiation of rhGH treatment was median 6.68 years, rhGH duration was 28 months (IQR 18-38).

Skeletal age was 8 median years (IQR 4.5-11) at the start of treatment and after $12 \mathrm{mths}$ and $24 \mathrm{mths}$ were $-2.1,-1,5,-1,3$ respecitively.

The median rate of growth was $8 \mathrm{~cm} /$ year (IQR 4.5-11). During first-year rhGH treatment GV was significantly faster compared to the second year (median $9.5 \mathrm{~cm} /$ year and $7.6 \mathrm{~cm} /$ year, $\mathrm{p}<0.001$, respectively). There were no differences according to the stage of CKD.

We found no significant relation between GV and sex, cause of CKD, hemoglobin, total protein, albumin, urea, creatinine, calcium, phosphorus, Ca x P, PTH and alkaline phosphatase.

The median eGFR at onset of the treatment group was $25 \mathrm{ml} / \mathrm{min} / 1.73 \mathrm{~m}^{2}$, after 1 year of treatment 22.1 and after 2 years of treatment $23.3 \mathrm{ml} / \mathrm{min} /$ $1.73 \mathrm{~m}^{2}$, respecitively.

Conclusions: 1 . Treatment with rhGH is effective in children with every stage of CKD

2. Early initiation of rhGH treatment in patients with $\mathrm{CKD}$ allows for long-term treatment and enables the children to reach the prognostic growth percentile.

\section{EP-61 POLYCYTHEMIA IN A PEDIATRIC PATIENT WITH CHRONIC KIDNEY DISEASE：OVERUSE OF ERYTHROPOERIN DURING COVID-19 ISOLATION}

Yasar Kandur ${ }^{1}$, Aysegul Alpcan ${ }^{2}$, Mehmet Yozgat ${ }^{2}$, Serkan Tursun ${ }^{1}$

${ }^{1}$ Department Of Pediatric Nephrology, School Of Medicine, Kirikkale University, Kirikkale, Turkey, ${ }^{2}$ Department Of Pediatrics, School Of Medicine, Kirikkale University, Kirikkale, Turkey

Introduction: Anemia in chronic kidney disease (CKD) is correlated to a decrease in erythropoietin (EPO) production due to kidney dysfunction . Erythropoiesis-Stimulating Agents (ESAs) are very capable drugs, targeting a significant hemoglobin $(\mathrm{Hb})$ level. Polycythemia refers to an increased hemoglobin concentration in peripheral blood. Drug-induced polycythemia can occur with excess use of ESAs. On 12 January 2020, the World Health Organization (WHO) confirmed that a novel coronavirus was the cause of a pandemic respiratory illness. This pandemic has affected many countries including our country. Patients under risk were suggested to be isolated. In the present report, we describe a case of a CKD patient who developed polycythemia due to ESA overuse during COVID-19 isolation. Material and methods: A 12-year-old male was admitted to the hospital with a high hemoglobin level that was detected at routine outpatient evaluation. He had not been able to attend routine controls since he had been in isolation for 4 months after the COVID-19 outbreak. He had continued to take ESA at the dose of $100 \mathrm{U} / \mathrm{kg} /$ week that had been started upon the the diagnosis of anemia at a level of $\mathrm{Hb} 8.7 \mathrm{gr} / \mathrm{dl}, 6$ months ago. On physical examination, his blood pressure was 140/80 $\mathrm{mm} \mathrm{Hg}$ (he had been on an ACE inhibitor and a calcium channel blocker). Additionally, he had slight facial edema. Laboratory investigation revealed a $\mathrm{Hb}$ level of $20.8 \mathrm{~g} / \mathrm{dl}$, hematocrit level of $66 \%$, creatinine level of $6.5 \mathrm{mgr} / \mathrm{dl}$, urea level of $135 \mathrm{mg} / \mathrm{dl}$, potassium level of $5.3 \mathrm{meq} / \mathrm{L}$, and a parathormone level of $132 \mathrm{pg} / \mathrm{ml}$. Venous blood gas analysis revealed ph:7.39, bicarbonate $25.2 \mathrm{mEq} / \mathrm{L}$. His previous $\mathrm{Hb}$ results were as follows: December
2019: $8.7 \mathrm{gr} / \mathrm{dl}$, February 2020: $11.2 \mathrm{gr} / \mathrm{dl}$. He was admitted to the hospital and underwent daily phlebotomy sessions $(10 \mathrm{cc} / \mathrm{kg} / \mathrm{session})$. During this period aspirin was also started $(5 \mathrm{mg} / \mathrm{kg})$. After 5 sessions, his $\mathrm{Hb}$ level decreased to $14 \mathrm{gr} / \mathrm{dl}$ and hematocrit to $40 \%$. He was discharged to return to the outpatient clinic for a check later. ESA was stopped until the time when anemia will re-appear.

Results:

Conclusions: During this pandemic,patients with CKD should be closely monitored by their family physicians at home by telephone, or by visiting them in person. In addition, as pediatric nephrologists, we should inform other pediatricians and family physicians for risks such as the one experienced by our patient.

\section{EP-62 THE ASSESSMENT OF THE USEFULNESS OF SELECTED MARKERS IN THE PROGNOSIS OF CHRONIC KIDNEY DISEASE IN CHILDREN}

Agata Będzichowska, Katarzyna Jobs

Department Of Pediatrics, Pediatric Nephrology And Allergology, Military Institute Of Medicine

Introduction: The kidney deterioration, which starts in childhood often leads to end-stage renal failure in the future. Therefore, searching for an early, sensitive and specific biomarkers became a paramount for chronic kidney disease diagnosis. The aim of the study was the appraisal of the usefulness of new markers: KIM-1, FGF-23, NAG, NGAL, uromodulin in predicting the development of chronic kidney disease in children.

Material and methods: 97 children (27 boys, 70 girls from 6 months to 17 years old) with kidney disorders, which had clinical indications for renoscintigraphy, were included in the study. The exclusion criteria were: chronic diseases and infectious diseases, acute kidney injury, previously diagnosed chronic kidney disease and prematurity. Firstly, the patients were divided depending on the result of renoscintigraphy (impaired uptake of the tracer corresponding to renal hypodysplasia or postinflammatory scars in renal parenchyma vs normal kidney picture). Secondly, depending on the level of estimated glomerular filtration rate (glomerular hyperfiltration vs normal filtration rate). Serum and urine concentration of KIM-1, FGF-23, NAG, NGAL and uromodulin were measured in all patients and compared between the groups.

Results: There were no significant differences in the concentration of tested markers in blood and urine between the groups divided based on the result of renoscintigraphy.

Statistically, significantly higher serum NAG and NGAL levels were found in children with glomerular hyperfiltration compared to children with normal glomerular filtration rate.

Conclusions: The results of the study do not allow to indicate new markers, which could be useful in the diagnosis of the early stage and prognosis of kidney damage visible in the scintigraphic examination. The study confirmed the possible usefulness of NAG and NGAL in detecting early stage of kidney disease associated with glomerular hyperfiltration in children.

\section{EP-63 CHARACTERISTICS OF NEPHROPATHIES IN} CHILDREN WITH DIFFUSE CONNECTIVE TISSUE DISEASES

Guzel Karymova, Igor Zorin, Albina Vyalkova Orenburg Medical University

Introduction: The aim of study was to determine frequency and structure of renal damage in children with DCTD.

Material and methods: We examined retrospective 199 case histories of patients with Schönlein-Genoch vasculitis (SGV) and 326 case histories of patients with rheumatoid arthritis (RA) hospitalized in paediatric clinic of Orenburg medical university in period 2010-2020. 
Results: We detected renal damage in $31.5 \%$ patients with SGV $(\mathrm{n}=$ 60 ). Renal damaged was combined with skin, articular, abdominal syndromes. Renal damaged had microhematuria in combination with proteinuria $(41.6 \%, \mathrm{n}=25)$, isolated microhematuria $(35 \%, \mathrm{n}$ $=21)$, isolated proteinuria $(11.6 \%, \mathrm{n}=7)$. Nephrotic syndrome was in 1 child $(1.6 \%)$. We diagnosed articular form of RA in $89.7 \%$, systemic form in $10.3 \%$ of patients. We detected renal damage in $20.8 \%$ of patients $(n=68)$ with RA. Renal damage had isolated microhematuria $(20.5 \%, \mathrm{n}=14)$, isolated proteinuria $(19.1 \%, \mathrm{n}=$ $13)$, microhematuria in combination with proteinuria $(4.4 \% \mathrm{n}=3)$. Nephrotic syndrome was in 1 child $(1.5 \%)$. Renal damage was more often in boys $(63.3 \%, \mathrm{n}=38)$ than in girls $(36.6 \%, \mathrm{n}=22, \mathrm{p}<0.05)$ in SGV, in girls $(70.6 \% \mathrm{n}=48)$ than in boys $(29.4 \% \mathrm{n}=20)$ in RA. Renal damage was more often in children 4-6 years old $(40 \%)$ in $\mathrm{SGV}$, in children over 10 years old $(41.1 \%)$ in RA.

Conclusions: Renal damage in DCTD is formed in children with a severe forms of the disease and had isolated urinary syndrome.

\section{EP-64 MARKERS OF EARLY DIAGNOSIS AND PROGRESSION OF CKD IN CHILDREN}

Svetlana Tchesnokova, Albina Vyalkova, Svetlana Plotnikova, Igor Zorin

Orenburg Medical University

Introduction: Diagnosis of formation and progression of CKD in children is actual problem of pediatrics and nephrology. The aim of the study was to determine markers of early diagnosis and progression of CKD.

Material and methods: We examined 90 children with different stages of CKD (I -n=30, II-n=34, III-n=16) and 30 children with chronic renal diseases (CRD) without $\mathrm{CKD}$ at age from 1 to 17 years. All children underwent serum level of endothelin-1 (ET-1), asymmetric dimethylarginine (ADMA), plasminogen activation inhibitor-1 (PAI-1) by ELISA.

Results: We established level of endothelial dysfunction markers: in CKD I - ET-198 01 \pm 2.73 pg/ 1, ADMA $31 \pm 0.06$ ng/ml, PAI-1 $39.5 \pm 0.52 \mathrm{ng} / 1$. We revealed increasing of markers of endothelial dysfunction during progression of CKD: ET-1 (CKD II 107.09 $\pm 1.54 \mathrm{pg} / \mathrm{ml}$, CKD III 111.4 \pm 1.3 $\mathrm{pg} / \mathrm{ml}$ ), ADMA (CKD II $38.86 \pm 1.86 \mathrm{ng} / \mathrm{ml}$, CKD III $44.25 \pm 1.9 \mathrm{ng} / \mathrm{ml}$ ), PAI-1 (CKD II $41.2 \pm 0.4 \mathrm{ng} / 1$, CKD III $73.4 \pm 4.96 \mathrm{ng} / \mathrm{ml}$. In patients with CRD without CKD: ET $82.4 \pm 2.62 \mathrm{pg} / \mathrm{ml}$, ADMA $30.68 \pm 0.12 \mathrm{ng} / \mathrm{ml}$, PAI$138.4 \pm 0.12 \mathrm{ng} / \mathrm{ml}$. So we established significance difference between levels of ET, ADMA, PAI-1 in comparing groups $(\mathrm{p}<0,05)$.

Conclusions: Levels of ET, ADMA, PAI-1 can be used for early diagnosis and progression of CKD in children

\section{EP-65 HOMOCYSTEINE IS EARLY BIOMARKER OF RENAL INJURY AND PROGRESSION IN CHILDREN WITH CKD}

Svetlana Tchesnokova, Albina Vyalkova, Igor Zorin Orenburg Medical University

Introduction: Homocysteine $(\mathrm{HC})$ is one of sensitive markers of vascular endothelial dysfunction. The aim of study is to evaluate diagnostic role of homocysteine in children as a biomarker of early diagnose and progression of renal damage in children with CKD.

Material and methods: We examined of 90 children with different stages of CKD (I $-n=30$, II-n=34, III- $n=16$ ) and 30 children with chronic renal diseases without $\mathrm{CKD}$ at age from 1 to 17 years. All children underwent serum level of HC by ELISA.

Results: $60 \%$ of patients with CKD had increase of HC: CKD I $2963.8 \pm$ $330 \mathrm{pg} / \mathrm{ml}$, CKD II $4083.5 \pm 282.5 \mathrm{pg} / \mathrm{l}$, with CKD III-IV 7640.2 $\pm 30 \mathrm{pg} /$ $\mathrm{ml}$. Children with chronic renal diseases without $\mathrm{CKD}$ had $\mathrm{HC}=756,2$ $\pm 54,2 \mathrm{pg} / \mathrm{ml}$. So we established significance difference between levels of $\mathrm{HC}$ in comparing groups (with $\mathrm{p}<0,05$ ).
Conclusions: Level of $\mathrm{HC}$ can be used for early diagnosis and progression of CKD in children

\section{EP-66 ORAL FUNGAL INFECTION IN CKD POPULATION}

Dr. Nandita Shenoy, Mohankumar Nedunchezhiyan, Afza Anjum Manipal University

Introduction: Chronic Kidney Disease is an increasing health alarm worldwide with systemic signs like hematologic changes, bone metabolic error, and compromised immune status presenting to a dental practitioner with oral complications. Hence a study was done to assess the oral health condition, cytological changes, and presence or absence of candida in patients with chronic renal disease undergoing dialysis aged between 30 to 65 years and controls in South India.

Material and methods: A cross sectional study was conducted among 80 adults with CKD and controls, attending KMC and MCODS, Mangalore. Oral manifestations and oral hygiene wereassessed; Cytological smears were taken for morphometric analysis of buccal mucosal cells from the subjects. Oral smears were cultured to check for the growth of fungal species in subjects and controls. Statistical analysis was done using SPSS Version 13. Chi square test was used to compare categorical variables between the groups.

Results: There was a significant difference in oral hygiene status and cytological changes in patients with CKD. Oral Fungal Infection was found in $32 \%$ of our patients on dialysis and oral lesions, defined as clinical signs associated with OFI such as erythematous oral stomatitis, membranous candidiasis, or angular cheilitis, were found in $37 \%$ of the patients with OFI, while $5 \%$ of the patients without findings of fungal infection presented oral lesions associated with OFI $(\mathrm{p}=0.0002)$. Furthermore, patients with self-reported mouth dryness were three times more likely $(\mathrm{p}=0.02)$ to be diagnosed with OFI.

Conclusions: Hemodialysis patients are found to have significantly more OFI than controls. Oral dryness and dental plaque formation also seem to be at risk of developing OFI. Detection of oral lesions associated with OFI should be combined with a histopathological diagnosis before antifungal treatment. The prevalence of oral candida in these cases isalarming and can be a predictor of a poor prognostic index. As medicine advances, oral health care professionals should have a holistic approach to the management of patients with complex medical problems especially to diseases of the renal system as it is pivotal in maintaining a stable internal environment and homeostasis.

EP-67 PHASE 3, RANDOMIZED, MULTICENTER STUDY TO EVALUATE THE EFFICACY AND SAFETY OF PEGCETACOPLAN IN TREATMENT OF C3G OR IC-MPGN

Bradley P. Dixon ${ }^{1}$, Fadi Fakhouri ${ }^{2}$, Matthew C. Pickering ${ }^{3}$, Terence Cook ${ }^{3}$, David Kavanagh ${ }^{4}$, Giuseppe Remuzzi ${ }^{5}$, Patrick Walker ${ }^{6}$, Christoph Licht ${ }^{7}$, Gerald Appel ${ }^{8}$, Marina Vivarelli ${ }^{9}$, Zhiqun Zhang ${ }^{10}$, Hetal Kocinsky ${ }^{10}$

${ }^{1}$ Department Of Pediatrics, University Of Colorado School Of Medicine, ${ }^{2}$ Lausanne University Hospital And University Of Lausanne, ${ }^{3}$ Imperial College, ${ }^{4}$ National Renal Complement Therapeutics Centre, ${ }^{5}$ Mario Negri Institute For Pharmacological Research, ${ }^{6}$ Arkana Laboratories, ${ }^{7}$ The Hospital For Sick Children, ${ }^{8}$ Columbia University, ${ }^{9}$ Division Of Nephrology And Dialysis, Bambino Gesù Pediatric Hospital, ${ }^{10}$ Apellis Pharmaceuticals

Introduction: Complement 3 glomerulopathy $(\mathrm{C} 3 \mathrm{G})$ and immune complex membranoproliferative glomerulonephritis (IC-MPGN) are rare diseases characterized by excessive deposition of $\mathrm{C} 3$ breakdown products in renal glomeruli leading to proteinuria and 
progressive renal disease. Pegcetacoplan is a targeted C3 investigational therapy for diseases related to complement overactivation. This is a phase 3, randomized, placebo-controlled, double-blind, multicenter study of the efficacy and safety of pegcetacoplan in individuals with $\mathrm{C} 3 \mathrm{G}$ or IC-MPGN.

Material and methods:Approximately 90 patients (age, $\geq 12$ years; weight, $20-100 \mathrm{~kg}$ ) diagnosed with $\mathrm{C} 3 \mathrm{G}$ or IC-MPGN, either as primary disease or posttransplant disease recurrence, will be recruited. Inclusion criteria include $2+$ staining for $\mathrm{C} 3 \mathrm{c}$, global glomerulosclerosis $<50 \%$, urine protein-to-creatinine ratio (uPCR) $\geq 1000 \mathrm{mg} / \mathrm{g}$, and estimated glomerular filtration rate $(\mathrm{eGFR})>30$ $\mathrm{mL} / \mathrm{min} / 1.73 \mathrm{~m}^{2}$. Exclusion criteria include previous pegcetacoplan exposure, $\mathrm{C} 3 \mathrm{G} / \mathrm{IC}-\mathrm{MPGN}$ secondary to other conditions, and significant infection/malignancy. Patients will be randomized $1: 1$ to receive subcutaneous infusions of pegcetacoplan $(1080 \mathrm{mg} / 20 \mathrm{~mL}$, equivalent to minimum dose of $15.9 \mathrm{mg} / \mathrm{kg}$ ) or matching volume of placebo twice weekly for 26 weeks (in addition to standard care). Thereafter, in the open-label period, all participants will receive pegcetacoplan twice weekly for 26 weeks. Assessments include first-morning uPCR every 4 weeks and renal biopsies at baseline/ screening and weeks 26 and 52. Primary endpoints include proportion of participants with reduction in $\mathrm{UPCR} \geq 50 \%$ relative to baseline and stable/improved eGFR (defined as no decrease $>20 \%$ from baseline) at week 52 . Secondary endpoints include proportion of participants with reduction in $\mathrm{UPCR} \geq 50 \%$ relative to baseline at week 52, stable/improved eGFR relative to baseline at week 52, proportion of participants with decreased $\mathrm{C} 3 \mathrm{c}$ staining from baseline at week 26, and statistical decrease in $\mathrm{C} 3 \mathrm{G}$ histologic index activity score from baseline at week 26 . Safety outcomes will also be monitored throughout the study. Participants may enter a subsequent 8week follow-up period or long-term extension study.

Results: NA

Conclusions: NA

\section{EP-68 COMMUTE-P: A PHASE III TRIAL EVALUATING THE EFFICACY AND SAFETY OF CROVALIMAB IN PAEDIATRIC PATIENTS WITH ATYPICAL HAEMOLYTIC URAEMIC SYNDROME}

Larry A. Greenbaum ${ }^{1}$, Fadi Fakhouri ${ }^{2}$, Chantal Loirat ${ }^{3}$, Ito Shuichi ${ }^{4}$, Minghui Zhao ${ }^{5}$, Khaled Benkali ${ }^{6}$, Federico Mattiello ${ }^{7}$, Mona Shah ${ }^{8}$, Camelia S. Sima $^{8}$, Alexandre Sostelly ${ }^{7}$, Sasha Sreckovic ${ }^{7}$, Neil Sheerin ${ }^{9}$

${ }^{1}$ Emory University And Childrens Healthcare Of Atlanta, Atlanta, Ga, Usa, ${ }^{2}$ Vaudois University Hospital Center (chuv), Lausanne, Switzerland, ${ }^{3}$ University Hospital Robert DebrÉ, Paris, France, ${ }^{4}$ Department Of Pediatrics, Graduate School Of Medicine, Yokohama City University, Kanagawa, Japan, ${ }^{5}$ Renal Division, Department Of Medicine, Peking University First Hospital, Beijing, China, ${ }^{6}$ Certara, Inc., Paris, France, ${ }^{7}$ F. Hoffmann-la Roche Ltd, Basel, Switzerland, ${ }^{8}$ Genentech, Inc., South San Francisco, Ca, Usa, ${ }^{9}$ Translational And Clinical Research Institute, Newcastle University, Newcastle Upon Tyne, United Kingdom

Introduction: Atypical haemolytic uraemic syndrome (aHUS) is a life-threatening disease, characterised by thrombotic microangiopathy (TMA), due to complement dysregulation. While C5 inhibition is effective, intravenous infusions impose a significant treatment burden. Crovalimab is a novel anti-C5 monoclonal antibody, with a 30-day half-life, allowing for small volume subcutaneous (SC) self-injection every 2 or 4 weeks (weight-based tiered dosing). Crovalimab has been studied in patients with paroxysmal nocturnal haemoglobinuria, another complement-mediated disorder, in the COMPOSER trial (Röth et al, Blood 2020), where crovalimab showed rapid complement inhibition and promising efficacy and safety. COMMUTE-p is a global Phase III single- arm trial evaluating the efficacy and safety of crovalimab in paediatric patients with aHUS.

Material and methods: COMMUTE-p will enrol three cohorts of paediatric (aged $\geq 28$ days to $<18$ years) patients with aHUS: 1 ) Naive: patients treatment-naive to complement inhibitors and with active TMA; 2) Switch: patients with controlled TMA switching from eculizumab or ravulizumab; and 3) Pretreated: patients who discontinued previous eculizumab or ravulizumab treatment. All cohorts will receive weight-based crovalimab loading dose(s), followed by self-injected SC maintenance dosing. The primary efficacy objective is to evaluate the effect of crovalimab in treatment-naive patients, measured by the proportion of patients with complete TMA response anytime from baseline to Week 25 . Secondary efficacy endpoints for the Naive and Switch cohorts include change in dialysis status, changes in estimated glomerular filtration rate and haematological parameters, changes in chronic kidney disease staging and changes in fatigue, measured using age-appropriate Pediatric Quality of Life Inventory questionnaires. Safety will be evaluated, along with pharmacokinetics (including dose confirmation for patients $<40 \mathrm{~kg}$ ), immunogenicity, biomarker and treatment preference objectives.

Results: Enrolment will initiate October 2021.

Conclusions: COMMUTE-p will evaluate the efficacy, safety, pharmacokinetics and pharmacodynamics of crovalimab in paediatric patients with aHUS, a therapy which may reduce the treatment burden for these patients.

\section{EP-69 OUTCOMES IN PATIENTS DIAGNOSED WITH ATYPICAL HAEMOLYTIC URAEMIC SYNDROME}

\section{Emma Y X Walker, Stephen D Marks, Aoife Waters} Great Ormond Street Hospital

Introduction: Atypical haemolytic uraemic syndrome (aHUS) is a rare disease, the morbidity and mortality of which, has improved since the introduction of Eculizumab.

Material and methods: Retrospective study of disease manifestation and outcome in a single-centre cohort.

Results: 15 patients ( 8 female, 7 male) were diagnosed with aHUS from October 2002 to March 2021. Median [IQR] follow up of 8.3 [2.6-11.7] years; $93 \%$ patient and $80 \%$ renal survival.

Age at presentation was 4.2 [1.6-5.6] years, C3 was 0.73 [0.59$0.74] \mathrm{g} / \mathrm{l}$, peak creatinine was 215 [159-348] umol/L and eGFR was $15[10-21] \mathrm{mls} / \mathrm{min} / 1.73 \mathrm{~m}^{2}$. 6 required dialysis, 4 plasma exchange.

Genetic mutations were identified in $9 / 15$ patients in genes including: CD46, CFHR1/3, CFI, C3 and DGKE, five patients had complement factor $\mathrm{H}$ antibodies (six unknown, four negative).

Seven patients initially presented prior to routine availability of intravenous eculizumab. Of these, $71 \%$ relapsed, two of whom then received eculizumab.

10/15 patients received eculizumab, To date, none have relapsed following eculizumab with four patients on maintenance treatment. 6 patients have ceased treatment: duration 1.2 [0.8-2.8] years; 2 had CD46 mutation, 1 CFHR1/3 deletion. All are followed up, except two (one transitioned to adults, one died).

At last review, eGFR was 93 [78-103] $\mathrm{mls} / \mathrm{min} / 1.73 \mathrm{~m}^{2}$. One patient has $\mathrm{CKD}$, one died (no genetic mutations identified for either). Two further patients progressed to renal transplants (one has $\mathrm{C} 3$ mutation, the other CFHR1/3 deletion); aHUS recurred in one of these post-transplant, they continue on eculizumab for the past 7.4 years.

Conclusions: aHUS is rare and patients can present very unwell. Introducing eculizumab resulted in noticeable improvement. There is still some way to go to further understand the genetic influence on outcomes. This study hopes to add to the growing knowledge of this rare disease. 
EP-70 PATTERNS OF PRESENTATIONS AND OUTCOMES IN CHILDREN WITH C3 GLOMERULOPATHY: ASPECTS FROM A DEDICATED CLINIC AT GREAT ORMOND STREET HOSPITAL

Mariana Capela, Emma Walker, Aoife Waters

Great Ormond Street Hospital

Introduction: $\mathrm{C} 3$ glomerulopathy in children is a rare disease with an incidence of 1-2 per 1,000,000 population and a has a high risk of progressing to end stage renal disease (ESRD). Cluster hierarchical analysis has recently shown that patients tend to fall into four major subgroups. Each subgroup has their own different pathogenetic patterns and therefore may respond to anti-complement therapies differently. Current strategies involve treatment with RAAS blockade, immunosuppression and complement inhibition, albeit with a lack of randomised controlled trials for any of the above. The objective of establishing this clinic, designed to assess and manage children with $\mathrm{C} 3$ glomerulopathy, is to evaluate the efficacy of a harmonised treatment approach. Thereafter, attempt to define management therapies and review who may benefit from enrolment in clinical trials with complement inhibitors.

Material and methods: Sixteen children with the diagnosis of C3 glomerulopathy were followed in the last two years at GOSH. Clinical, histological characteristics and biochemical analysis were assessed before and after treatment.

Results: At onset, median [IQR] age was 9.5 [8.0-11.5] years, with an eGFR of 108 [88-129] mL/min/1.73 m2, serum albumin of 25 [22-31] $\mathrm{g} / \mathrm{L}$ and urine albumin:creatinine ratio of 616 [262-825] $\mathrm{mg} / \mathrm{mmol}$. All patients had microhaematuria and five presented with macrohaematuria. Oedema was present in $69 \%$. C3/C4 levels were low in $93 \% / 37 \%$ of the patients and $\mathrm{C} 3$ nephritic factor was positive in four cases (25\%). The majority (15/16) of patients demonstrated immune complex glomerulonephritis with $\mathrm{C} 3$ predominance associated with $\mathrm{IgG}, \mathrm{Clq}$ and IgM positivity. Electron microscopy revealed that only 3 cases had intramembranous lesions.

Twelve were treated with oral prednisolone, five of whom also received mycophenolate mofetil (MMF) and one with cyclophosphamide.

At twelve months of follow-up, all patients were on ACE inhibitors (ACEI) with improvement of albuminuria in all, except one. Six patients continue with prednisolone, eight with MMF and two commenced Tacrolimus. At present, three patients are in remission, two who were treated with immunosuppressive medication, the third was only treated with ACEI and had spontaneous recovery. One patient is in stage $3 \mathrm{CKD}$ and two patients are in ESRD. These latter two were treated with Rituximab without success.

Conclusions: Establishing this clinic, learning to identify which of these clusters the patients fall into and collating further data will help predict treatment response and therefore create more personalised management plans and guide prognosis.

\section{EP-71 NONGROUPABLE NEISSERIA MENINGITIDIS DISEASE} IN VACCINATED CHILDREN RECEIVING ECULIZUMAB

Nilüfer Göknar, Diana Üçkardeş, Cengiz, Candan, Emre Keleşoğlu

Istanbul Medeniyet University

Introduction: Atypical hemolytic uremic syndrome (aHUS) is a rare and severe disease characterized by hemolytic anemia, thrombocytopenia and acute renal failure. The dysregulation of the alternative complement pathway is caused by the mutations in the complement gene or acquired autoantibodies against complement regulatory proteins. Eculizumab, which is the first-line therapy in aHUS, blocks the formation of $\mathrm{C} 5$ convertase and inhibits the formation of terminal membrane attack complex. It is known that the treatment with eculizumab increases the risk of meningococcal disease 1000-2000 times.

Material and methods: In this case report, we presented a case who developed meningococcemia under Eculizumab treatment despite vaccinations against meningococcus.
Results: A 1-year-7-months-old girl admitted with fever and low appetite but no rash, or neck stiffness. She was under the treatment of eculizumab with the diagnosis of atypical hemolytic uremic syndrome in the past 11 months of period. She received meningococcal $\mathrm{A}, \mathrm{C}, \mathrm{W}, \mathrm{Y}$ and $\mathrm{B}$ serotype vaccination previous to eculizumab. Homozygous mutation was present in the Complement Factor I gene. Laboratory evaluation revealed leukocytosis (WBC: 20.200/ $\mathrm{mm} 3)$, neutrophilia $(17.640 / \mathrm{mm} 3)$ and increased $\mathrm{CRP}(2.9 \mathrm{mg} / \mathrm{dl})$ and procalcitonin $(15.9 \mathrm{ng} / \mathrm{ml})$ levels. Treatment with ceftriaxone 75 $\mathrm{mg} / \mathrm{kg}$ was started. In the blood culture of the patient Neisseria meningitidis was grown and serotyping resulted with nontypeable $N$. meningitidis. On follow up, she had a rapid recovery of the disease. Eculizumab treatment was discontinued and biweekly fresh-frozen plasma infusions were given.

Conclusions: In conclusion, despite vaccination against other serotypes, meningococcemia with non-typeable N.Meningitidis occurred in our patient. The differential diagnosis of meningococcemia should be decided in children receiving eculizumab.

\section{EP-72 TREATMENT OF TWO CHILDREN WITH COMPLEMENT H RELATED ATYPICAL HEMOLYTIC} UREMIC SYNDROME WITH REGULAR PLASMA INFUSION Nilüfer Göknar, Tuğçe Arman, Diana Üçkardeş, Emre Keleşoğlu, Cengiz Candan

Istanbul Medeniyet University

Introduction: Atypical hemolytic uremic syndrome (HUS) is a chronic disease related to dysregulation and/or excessive activation of the complement system. Plasmapheresis/plasma infusion forms the basis of managing aHUS in absence of access to Eculizumab which is the first line treatment of aHUS.

Material and methods: In this case report, we present two brothers who were diagnosed complement factor $\mathrm{H}$ related aHUS and successfully treated with two weekly plasma infusions.

Results: Case1: An eight years old Syrian refugee boy had first aHUS attack when he was three years old age. He was treated with hemodialysis and plasmapheresis. His brother admitted on same day with similar symptoms. Genetic analysis was heterozygous positive for factor $\mathrm{H}$ mutation. Two years later he had second attack with neurologic involvement. Because of insurance problems eculizumab couldn't be administered and plasma infusion treatments were started. Two weekly plasma infusions were given without any adverse effect.

Case 2: Ten years old boy had first TMA attack when he was five years old age. Genetic analysis was homozygous positive for factor $\mathrm{H}$ mutation. He had second TMA attack two years later and treated with plasma infusions. One year later he had experienced a third TMA attact after a one-week delay of plasma infusion.

On follow up two weekly plasma infusions were continued and both patients did not experience a new event for two years and had hematological and renal remission. Both children were under enalapril treatment for persistent proteinuria.

Conclusions: The outcome of aHUS has improved after eculizumab treatment. If there is lack of availability of eculizumab, plasma exchange/plasma infusions are the preferred treatment. Both patients were successfully treated with plasmapheresis during aHUS attack and treatments were continued with regular plasma infusions without adverse effect and control of the disease.

EP-73 OUTCOMES OF PEDIATRIC ATYPICAL HEMOLYTICUREMIC SYNDROME DUE TO ANTI-FACTOR H ANTIBODIES IN CHILDREN: A SINGLE CENTER STUDY

Marion Ferri, Claire Dossier, Theresa Kwon , Julien Hogan

Pediatric Nephrology Department, Robert Debre Hospital, Aphp, Paris 
Introduction: Previous guidelines suggest combining plasma exchange (PE) and immunosuppressive drugs to treat children with aHUS due to anti-CFH antibodies but the place of other treatments remains controversial.

Material and methods: Since 2011, our treatment protocol combines eculizumab with corticosteroids and mycophenolate mofetil (MMF). Rituximab was added in patients >1year old and immunoadsorption (IA) in patients requiring dialysis. We report the clinical course and outcomes of 5 patients treated with this protocol.

Results: Patients were aged 5 months to 12 years and anti-CFH antibodies titers varied from 14000 to 129500 IU/L. Median duration of Eculizumab treatment was 14 [5;23] months. All but one patient presented platelets count normalization within one week. Kidney function improved rapidly and only one patient did not recover a normal function at 1 -year $\left(\mathrm{eGFR}=68.9 \mathrm{~mL} / \mathrm{min} / 1.73 \mathrm{~m}^{2}\right)$. Anti-CFH antibody titers decreased rapidly over the first 3 months to reach titers below $3000 \mathrm{IU} / \mathrm{L}$ in 1 to 12 months in 4 patients. Only one patient reached titers below $1000 \mathrm{UI} / \mathrm{L}$ after 2 years of follow-up. No increase in Ab titers was observed at B-cell reconstitution (median time to reconstitution 4 months). Immunosuppressant treatments were stopped in one patient (titers 2470 $\mathrm{IU} / \mathrm{L}$ ) and no relapse was observed 36 months after discontinuation. The youngest patient was only treated with ECZ for 6 months and IvIg infusions. Anti-CFH antibody titers remained low and no relapse was observed 40 months after ECZ discontinuation. No infectious complication was reported in this cohort.

Conclusions: These data suggest that ECZ can be safely used to induce rapid remission and that maintenance treatment with steroids and MMF. The benefit of B-cell depletion on relapse prevention and antibody titers remains to be demonstrated. Finally, treatment discontinuation seems possible despite antibody titers $>1000 \mathrm{UI} / \mathrm{L}$ and larger studies are needed to assess predictors of relapse in these patients.

\section{EP-74 CATASTROPHIC ANTIPHOSPHOLIPIDE SYNDROME ACCOMPANIED WITH DYSREGULATION OF THE COMPLEMENT SYSTEM \\ Serim Pul ${ }^{1}$, İbrahim Gökçe ${ }^{1}$, Serçin Güven ${ }^{1}$, Cemile Pehlivanoğlu ${ }^{2}$, Neslihan Çiçek ${ }^{1}$, Ece Demirci Bodur ${ }^{1}$, Betül Sözeri ${ }^{2}$, Harika Alpay ${ }^{1}$ \\ ${ }^{I}$ Marmara University Medícal School, Istanbul \\ 2 Ümranlye TraİIng And Research HospItal, İstanbul}

Introduction: In this case report, a case of Catastrophic Antiphospholipid Syndrome (CAPS) with Thrombotic Microangiopathy (TMA) that is accompanied by a genetic disorder in the complement system is presented. Material and methods: ...

Results: A 13-year-old patient presented with menstrual bleeding and thrombocytopenia. On physical examination pretibial edema was present. Laboratory findings showed abnormal kidney function tests (creatinine: $3.97 \mathrm{mg} / \mathrm{dL}$ ), anemia ( $\mathrm{Hb}: 9.7 \mathrm{~g} / \mathrm{dL})$, thrombocytopenia $(35.000 / \mathrm{mm} 3)$, reticulocytosis (4\%), low haptoglobin $(<8 \mathrm{mg} / \mathrm{dl})$, elevated LDH $(1080$ $\mathrm{U} / \mathrm{L}$ ) and low C3 levels. Anti-nuclear antibody (ANA) and direct coombs were positive. There were signs of hemolysis in peripheral blood smear. The patient was considered to have "TMA". Cortical necrosis was detected in the kidney biopsy that were consistent with clinical diagnosis of TMA. Lupus nephritis was not detected in the kidney biopsy. Three doses of pulse-steroid and intravenous immunoglobulin treatments were given and continued with oral high dose prednisolone. Plasmapheresis was initiated for antibody and complement-related TMA causes. Three doses of eculizumab and rituximab were also administered to the patient. In autoimmune antibody screening; anticardiolipin antibody (ACA) was negative, anti-beta-2-glycoprotein-I antibody ( $\beta 2 \mathrm{GPI}$ ) IgM and Lupus anticoagulant (LA) were positive. The patient was evaluated as "primary $A P S^{\prime \prime}$ with histopathology and antibody positivity. After a while, the patient presented with severe abdominal pain. Abdominal CT angiography images were consistent with mesentery, spleen and liver ischemia. In addition, deterioration was observed in kidney functions. With these findings, the patient was evaluated as "CAPS". Homozygous deletion in the Complement Factor H-Related 1 (CFHR1) gene was detected. She is still being followed up with anticoagulant and immunosuppressive treatments. Despite therapy, she eventually progressed to end stage kidney disease.

Conclusions: Antiphospholipid Syndrome should be kept in mind and investigated in patients presenting with TMA. Genetic mutations related to the complement system in CAPS patients may be responsible for the severe clinical course.

EP-75 FAMILIAL FORM OF ATYPICAL HEMOLYTIC UREMIC SYNDROME: THE HISTORY OF ONE FAMILY

Tamara Makarova ${ }^{1}$, Yulia Melnikova ${ }^{1}$, Lyudmila Poladova ${ }^{2}$, Lilia Davlieva ${ }^{1}$

${ }^{1}$ Kazan State Medical University

${ }^{2}$ Childrens Republican Clinical Hospital Of The Ministry Of Health Of The Republic Of Tatarstan

Introduction: Atypical hemolytic uremic syndrome (aHUS) is a rare complement-mediated life-threatening disease belonging to the group of thrombotic microangiopathies (TMA).

Material and methods: anamnestic, clinical, laboratory

Results: The father has a debut of the disease at the age of 12 , the children were diagnosed in their infancy (6 months for son, 7 months for daughter). The peculiarity of the course of the disease in all family members was the development of the classical symptoms of aHUS without extrarenal symptoms. The severity of clinical manifestations differed among family members: the father had 3 episodes of activity of complement-mediated TMA, the daughter - 1, and the son - 5. For father and daughter, the severity of hematological manifestations of aHUS prevailed over nephrological ones. For the son, this was observed only in the first 2 episodes. The severity of the disease was due to the severity of hemolysis over the remaining symptoms of TMA, and in subsequent relapses the severity was caused by the development of dialysis-dependent renal failure. All family members were plasma sensitive. Complement-blocking therapy (eculizumab) in children made it possible to achieve aHUS remission with complete restoration of renal function. The clinical diagnosis was supported by the results of a genetic study of the complement system that identified the $\mathrm{C} 3$ complement component gene mutation (p.Ile1157Thr) in a son and daughter.

Conclusions: The presented clinical observation demonstrates the development of aHUS in three family members with indications of an autosomal dominant type of disease inheritance. Given that patients with aHUS have constant uncontrolled activation of complement, the risk of developing sudden complications persists throughout their lives, therefore, long-term therapy with eculizumab is recommended for such patients.

\section{EP-76 HEMOLYTIC UREMIC SYNDROME IN CHILDREN: EXPERIENCE FROM A TURKISH TERTIARY REFERRAL CENTER}

Serra Surmeli Doven, Esra Danaci Vatansever, Ali Delibaş

Mersin University Faculty Of Medicine, Department Of Pediatric Nephrology, Mersin, Turkey

Introduction: Hemolytic Uremic Syndrome (HUS) is characterized as microangiopathic hemolytic anemia, trombocytopenia and acute kidney injury. This study aimed to evaluate etiological factors, follow-up and treatment results of the patients with HUS who were followed-up at Pediatric Nephrology Department of Mersin University Faculty of Medicine.

Material and methods: Nineteen patients who were admitted to Department of Pediatric Emergency and Pediatric Nephrology Units between December 2010, December 2020 with HUS diagnosis were included in the study. Demographic and clinical features, treatments and extra- 
renal involvement of the patients were garnered from the records, retrospectively.

Results: Mean age of the patients was 28,8 $\pm 33,6$ months. Atypical and STEC-HUS were diagnosed in $10(52.6 \%)$ and nine (47.4\%) of the patients, respectively. Sixteen $(84.2 \%)$ patients developed hypertension. Eighteen patients $(94.7 \%)$ received fresh frozen plasma infusions. Eight patients with atypical HUS received Eculizumab (8/10, 80\%). Plasma exchange was applied to three patients $(15.8 \%)$. Renal replecament therapy was required to three of the patients with STEC-HUS (3/9, 33.3\%) and to all of the patients with atypical HUS. Extra-renal involvement was observed in one patient with STEC-HUS and in all patients with atypical HUS. Two patients with atypical HUS (20\%) died due to gastrointestinal bleeding and heart failure, respectively. Three patients (15.8\%) with atypical HUS developed chronic kidney disease.

Conclusions: Hemolytic Uremic Syndrome is an important etiology for acute kidney injury in children. Hemolytic Uremic Syndrome is usually accompanied by hypertension. The frequency of extra-renal involvement, requirement of renal replacement therapy and mortality rate were higher in patients with atypical HUS compared to STEC-HUS.

EP-77 SUCCESSFUL ECULIZUMAB THERAPY FOR A STREPTOCOCCCUS PNEUMONIAE - ASSOCIATED HAEMOLYTIC UREMIC SYNDROME CONCOMITANT TO A COVID-19 INFECTION

Julie Tenenbaum ${ }^{l}$, Agnès Chevalier ${ }^{1}$, Marc Fila ${ }^{1}$, Julien Baleine ${ }^{2}$, Christophe Milesi ${ }^{2}$, Veronique Fremeaux-bacchi ${ }^{3}$, Denis Morin ${ }^{1}$

${ }^{1}$ Department Of Pediatric Nephrology, Centre Hospitalier Universitaire De Montpellier, Montpellier, France

${ }^{2}$ Pediatric Critical Care Department, Centre Hospitalier Universitaire De Montpellier, Montpellier, France

${ }^{3}$ Laboratory Of Immunology, Hôpital Européen Georges Pompidou, Paris, France

Introduction: Haemolytic uremic syndrome (HUS) is a rare but known complication of infection by Streptococcus pneumoniae (SP-HUS), especially in children.

Material and methods: We report the case of a sixteen-months boy presenting COVID-19 infection with positive nasopharyngeal RT-PCR test and SP-HUS with neurological impairment.

Streptococcus pneumoniae serotype 10A was found in blood culture and lombar puncture, realised after generalized tonic-clonic seizure.

SP-HUS associated microangiopathic haemolytic anemia, thrombocytopenia and oligoanuric renal failure leading to continuous hemofiltration. After diagnosis of SP infection, antibiotherapy was also beginned.

One single dose of eculizumab (ECZ) was administrated six days after admission regarding persistant complexe seizures with extended ischemic lesions at MRI.

Results: Hemofiltration was stopped two days after eculizumab injection, renal function fully recovered within a few days with a normal serum creatinine and neurological condition gradually improved.

Investigations into the cause of thrombotic microangiopathy found a normal ADAMTS13 activity.

Analysis of plasma levels of complement factors showed an initial decrease of C3 antigen ( $483 \mathrm{mg} / \mathrm{L})$ and a high level of plasmatic SC5b-9 $(1222 \mathrm{ng} / \mathrm{mL})$, involving an activation of the alternate complement pathway.

Moreover, CH50 activity was low (20\%). Other plasma levels of complement factors ( $\mathrm{C} 4$ antigen, $\mathrm{C} 1 \mathrm{q}$ antigen, factor $\mathrm{H}$ and factor $\mathrm{I}$ ) were normal. Screening for anti-CFH, anti-CFB and anti-C3b was negative.

Control of $\mathrm{C} 3$ complement factor was normal six months after HUS episode.

Conclusions: To date, only very few cases of SP-HUS treated by ECZ have been reported.

In this case, we report a co-infection with COVID-19 and Streptococcus pneumoniae complicated with HUS and severe neurological impairment.
Activation of complement alternate and classical pathways was found and ECZ was successfully use with recovery of renal condition.

ECZ could provide a new therapeutic weapon for the most serious forms of SP-HUS with further studies required to document the benefit of complement blockade therapy in severe forms of SP-HUS.

\section{EP-78 DGKE MUTATION IN A CHILD TREATED WITH ECULIZUMAB}

Demet Tekcan ${ }^{1}$, Hülya Nalçacıoğlu ${ }^{1}$, Sefika İlknur Kökcü Karadağ ${ }^{2}$, Hülya Gözde Önal ${ }^{1}$, Fatih Özaltın ${ }^{3}$, Özlem Aydoğ ${ }^{1}$

${ }^{1}$ Ondokuz Mayis University Faculty Of Medicine, Pediatric Nephrology, Samsun, Turkey

${ }^{2}$ Ondokuz Mayis University Faculty Of Medicine, Pediatric Allergy And Immunology, Samsun, Turkey

${ }^{3}$ Hacettepe University Faculty Of Medicine, Pediatric Nephrology, Nephrogenetics Laboratory, Ankara, Turkey

Introduction: Atypical hemolytic uremic syndrome (HUS) is an alternative complement pathway disease. One of the rare underlying genetic causes is the Diacylglycerol Kinase E (DGKE) gene mutation, and the optimal treatment strategy is still unclear.

Material and methods: A three-year-old boy was referred to our clinic because of vomiting and high creatinine level after using amoxicillin and paracetamol for fever and sore throat. Physical examination revealed no pathological finding other than edema. Laboratory evaluation showed the triad of HUS; anemia, thrombocytopenia, increased creatinine and hemolysis in peripheral blood smear. He was anuric. Supportive treatments, hemodialysis, and plasmapheresis were initiated. Adamts13 activity, complement three, four, $\mathrm{H}$ levels were in normal range. Enterohemorrhagic E. coli (verotoxin) PCR test were positive in stool. Treatment continued with fresh frozen plasma under premedication because of anaphylaxis after the third plasmapheresis. On the seventh day the first dose of Eculizumab was given. Urine output began on the 21 st day. He was discharged with a $0,67 \mathrm{mg} / \mathrm{dl}$ creatinine value on the 36 th day. He was followed up with enalapril for his proteinuria. There was a history of death due to DGKE mutation in the grandson of his fathers uncle. We also detected compound heterozygous mutation in the patient DGKE gene, exon 5: C. 1009C $>$ T (p. R337X), heterozygous; exon 1: C.427C $>$ T (p. Q143*), heterozygous. Creatinine decreased to $0.4 \mathrm{mg} / \mathrm{dl}$. After four-dose, Eculizumab treatment was interrupted. 6 months after discharge, he presented with a second attack triggered by fever. He didn't need dialysis, achieved remission after eculizumab treatment. One week after the second eculizumab dose, he presented a widespread rash on the skin and mucous membranes. Skin biopsy was compatible with StevensJohnson Syndrome. His rashes regressed with intravenous immunoglobulin, steroid, and antihistamines. We discontinued Eculizumab due to its side effect.

Results: In the follow-up, there was no new attack, creatinine was 0.4 $\mathrm{mg} / \mathrm{dl}$ (GFR $103 \mathrm{ml} / \mathrm{min} / 1.73 \mathrm{~m} 2$ ), and hematological parameters were normal, urine protein was normal with enalapril.

Conclusions: The optimal treatment strategy for patients with DGKE mutations is not clear. The attacks of our case were controlled with plasmapheresis and eculizumab treatments.

\section{EP-79 PREDICTIVE FACTORS OF PROLIFERATIVE LUPUS NEPHRITIS IN TUNISIAN CHILDREN}

Abir Boussetta $^{1}$, Dalia Louati ${ }^{2}$, Manel Jellouli ${ }^{1}$, Ouns Naija ${ }^{1}$,

Hanene Gaidi ${ }^{3}$, Tahar Gargah ${ }^{1}$

1 Pediatric Nephrology Department, Charles Nicolle Hospital, Tunis, Tunisia

${ }^{2}$ Faculty Of Medicine Of Sfax, Sfax, Tunisia

${ }^{3}$ Nephrology Department, Mongi Slim Hospital, Tunisia 
Introduction: To identify predictive factors for the occurrence of proliferative lupus nephritis (LN) in pediatric Tunisian cohort

Material and methods: This was a multi-center retrospective study conducted in 5 centers in Tunisia. Children included were those under 18 years of age with LN confirmed by renal biopsy. Proliferative LN, characterized by endocapillary and/or extracapillary glomerulonephritis, encompasses classes III and IV of the International Society of Nephrology/ Renal Pathology Society LN classification scheme, or a combination of classes III $+\mathrm{V}$ or IV+V. Statistical significance was considered with a Pvalue $<0.05$

Results: Among our 40 patients, $\mathrm{LN}$ was proliferative in 35 patients (87.5\%). These were 29 women and 6 men. Class IV was the most frequent $(40 \%)$, followed by the association of class IV+V (20\%), and class III $(15 \%)$. The age at diagnosis of NL was between 10 and 14 years in $54.2 \%$ of cases. Female gender $(29 / 4, \mathrm{p}=0.875)$, age of $\mathrm{LN}$ diagnosis $(19 / 2, p=0.55)$, presence of inaugural LN $(26 / 5, p=0.198)$, hypertension $(16 / 3, p=0.547)$, hematuria $(23 / 4, p=0.582)$, nephrotic syndrome $(25 / 4$, $\mathrm{p}=0.688)$, initial function kidney impairment $(19 / 3, \mathrm{p}=0.81)$, and immunologic abnormalities were more common in the group with proliferative lesions but without statistically significant correlations.

Conclusions: Because proliferative LN is an aggressive disease that may lead to ESRD, it is important to identify to identify the predictive factors of its occurrence in order to establish a diagnosis as soon as possible and institute the appropriate treatment.

\section{EP-80 ANTI-FACTOR H AUTO-ANTIBODY-ASSOCIATED} AHUS: A SINGLE CENTRE EUROPEAN EXPERIENCE

Laura Lucchetti ${ }^{1}$, Federica Zotta ${ }^{1}$, Elena Bresin ${ }^{2}$, Elisabetta Valoti ${ }^{2}$, Isabella Guzzo ${ }^{1}$, Francesco Emma ${ }^{1}$, Marina Vivarelli ${ }^{1}$

${ }^{1}$ Ircss, Division Of Nephrology And Dialysis , Bambin Gesu Pediatric Hospital, Rome Italy

${ }^{2}$ Irccs, Institute Of Pharmacological Research, Mario Negri, Bergamo, Italy

Introduction: Anti-factor $\mathrm{H}(\mathrm{FH})$ antibodies cause atypical hemolytic uremic syndrome (aHUS). Plasma exchange, immunosuppression and Eculizumab represent the main treatment strategies.

Material and methods: ..

Results: Patient 1 (male) presented with aHUS at 3 years- 8 months of age, with abdominal pain without diarrhea, moderate kidney failure, proteinuria, macrohematuria, petechiae, anemia, and low $\mathrm{C} 3$. He did not have neurological involvement and STEC serology was negative. We administered Eculizumab with rapid normalization of kidney function, hemoglobin and platelets. After 3 months, results of positive high titer $(>2000$ $\mathrm{AU} / \mathrm{ml}$ ) anti-FH antibodies with homozygous deletion of CFHR1CFHR3 were found. Immunosuppression was not started due to concomitant COVID-19 pandemic and parent refusal. Anti-FH antibody titers at 6 months were $1506 \mathrm{AU} / \mathrm{ml}$, at 12 months $509 \mathrm{AU} / \mathrm{ml}$ with normalization of $\mathrm{C} 3$ and FH levels. Therapy with Eculizumab was discontinued after 14 months without concomitant immunosuppression.

Patient 2 is a 6-year-old male with a history of HUS diagnosed in Albania with terminal renal failure requiring hemodialysis and neurological involvement associated with non-bloody diarrhea, positive for E.coli O:157. No treatment was given and after 8 weeks the family left Albania to seek treatment in our center. We performed a kidney biopsy, which showed thrombotic microangiopathy lesions. Kidney function improved spontaneously, and hemodialysis was stopped after 1 month. Complement analysis revealed a low positive titer of anti-FH antibodies (386 AU/ml) with homozygous deletion of CFHR1-CFHR3. Six months later antibody titers spontaneously reduced to $143 \mathrm{AU} / \mathrm{ml}$. At last followup (+ 18 months), proteinuria was absent and kidney function only mildly reduced with no neurological sequelae.

Conclusions: Complement FH is the main regulatory protein of the complement alternative pathway. Mutations in genes encoding for $\mathrm{FH}-$ related proteins are associated with autoantibodies against $\mathrm{FH}$, which can cause aHUS. We describe two cases of anti-FH antibodyaHUS with a good outcome and, in both cases, spontaneous decrease in anti-FH antibody titer.

\section{EP-81 HEMOLYTIC UREMIC SYNDROME WITH AN UNUSUAL KIDNEY BIOPSY TREATED WITH ECCULIZUMAB}

Nasrin Esfandiar

Pediatric Nephrology Research Center, Research Institude For Children Health, Shahid Beheshti University Of Medical Sciences, Tehran, Iran

Introduction: Hemolytic Uremic Syndrome (HUS) is diagnosed by microangiopathic hemolytic anemia, thrombocytopenia and kidney involvement. Thrombotic microangiopathy(TMA) is a well-known pathological renal involvement in HUS. In this study, a case of HUS is introduced with crescent formation in about $10 \%$ of glomeruli in renal pathology with minimal TMA that was treated with Eculizumab.

Case presentation: Patient was a fourteen-month old boy presented with recurrent vomiting after breastfeeding and icterus and was suffering from anorexia and had discoloration of urine. In past medical history there was no sign of diarrhea or fever in last days. He was born full term and weighted 2.5 kilograms. His parents are second-degree relatives. He was pale and icteric in the physical exam. Laboratory data showed hemolytic anemia and thrombocytopenia with anisocytosis, schistocyte and burr cell which are the indicators of microangiopathy. Decreased level of C3 component of complement was detected. Urine analysis showed protein $(1+)$, blood $(3+)$ and many red blood cells. Ultrasound imaging showed mild increased size of kidney,increased corticomedullary echo pattern and differentiation for both kidneys. He was treated with methylprednisolone and intravenous immunoglobulin (IVIG). Because of unavailability of Eculizumab, plasma infusion was started. His hemolysis was improved but within 2 weeks his creatinine increased gradually from 0.5 to $0.9 \mathrm{mg} / \mathrm{dl}$. His blood pressure increased severely and he started to have gross hematuria. Consequently, he underwent renal biopsy which showed focal proliferative, necrotizing glomerulitis and cellular crescents in 7 out of 75 glomeruli. Features of possible acute tubular damage was detected. Finally, Eculizumab was provided with difficulty. The patient received $300 \mathrm{mg}$ Eculizumab in week zero, one and then every 3 weeks with penicillin prophylaxis. After 4 doses, he had only microscopic hematuria without hypertension and his creatinine dropped to $0.5 \mathrm{mg} / \mathrm{dl}$.

Conclusion: An unusual kidney biopsy in HUS was successfully treated with Eculizumab.

EP-82 A HOMOZYGOUS HOXA11 VARIATION AS A POTENTIAL NOVEL CAUSE OF AUTOSOMAL RECESSIVE CONGENITAL ANOMALIES OF THE KIDNEY AND URINARY TRACT

Seha Saygili ${ }^{1}$, Emine Atayar ${ }^{2}$, Nur Canpolat ${ }^{1}$, Mehmet Elicevik ${ }^{3}$, Sebuh Kurugoglu ${ }^{4}$, Lale Sever ${ }^{1}$, Salim Caliskan ${ }^{1}$, Fatih Ozaltin ${ }^{5}$

${ }^{1}$ Department Of Pediatric Nephrology, Istanbul University-cerrahpasa, Cerrahpasa Faculty Of Medicine, Istanbul, Turkey

${ }^{2}$ Nephrogenetics Laboratory, Department Of Pediatric Nephrology, Hacettepe University Faculty Of Medicine, Ankara, Turkey

${ }^{3}$ Department Of Pediatric Surgery, Istanbul University-cerrahpasa, Cerrahpasa Faculty Of Medicine, Istanbul, Turkey

${ }^{4}$ Department Of Pediatric Radiology, Istanbul University-cerrahpasa, Cerrahpasa Faculty Of Medicine, Istanbul, Turkey

${ }^{5}$ Department Of Pediatric Nephrology, Hacettepe University Faculty Of Medicine, Ankara, Turkey

Introduction: Congenital anomalies of the kidney and urinary tract (CAKUT) is the leading cause of end-stage kidney disease in children. Until now, more than 50 monogenic causes for CAKUT have been 
described, all of which only explain $10 \%$ to $20 \%$ of all patients with CAKUT, suggesting the presence of additional genes that cause CAKUT when mutated.

Material and methods: Herein, we report two siblings of a consanguineous family with CAKUT, both of which rapidly progressed to chronic kidney disease in early childhood. Whole-exome sequencing (WES) was performed in the two affected boys, one unaffected boy and their parents. A homozygosity mapping was performed using WES data via HomSI software.

Results: WES identified 49.737 variants with the average $147 \mathrm{x}$ depth of coverage in affected individuals. Homozygosity mapping identified two variants and thereby two candidate genes; RNA binding motif protein (RBM19) and Homeobox A11 (HOXA11). The former has been detected in healthy individuals and has knock-out mice models without any urinary abnormality. On the other hand, knocking-out of Hoxal1 in mouse has been associated with CAKUT.

Conclusions: We therefore showed for the first time an association between a homozygous HOXA11 variation with CAKUT in humans, expanding the genetic spectrum of the disease.

\section{EP-83 THE YIELD OF FETAL CHROMOSOMAL MICROARRAY ANALYSIS DURING PREGNANCIES WITH AN ISOLATED RENAL MALFORMATION \\ Hadas Shasha-lavsky ${ }^{1}$, Chen Nozik ${ }^{2}$, Irith Weissman ${ }^{1}$, Ziv Paz $^{1}$, Ariel Berl ${ }^{2}$, Pierre Awadia ${ }^{1}$, Lama Majadla ${ }^{2}$, Shir Sivan ${ }^{1}$, Orly Yakir ${ }^{1}$, Tzipora Falik-zaccai ${ }^{1}$ \\ ${ }^{1}$ Galilee Medical Center \\ ${ }^{2}$ Bar Ilan University}

Introduction: Chromosomal microarray analysis (CMA) is part of the prenatal evaluation of congenital anomalies. The prevalence of pathogenic CMA test results in cases of prenatal isolated renal malformations is unknown.

Objective: To determine the risk of pathogenic CMA results in fetuses with an isolated renal malformation compared to normal pregnancies and to fetuses with combined renal and extra-renal malformations.

Material and methods: A single center, retrospective observational study based on medical records of pregnant women who were referred for a prenatal nephro-genetic consultation at the Galilee Medical Center, Israel, between the years 1997-2018. The rate of pathogenic CMA findings was compared between fetuses with isolated renal malformation and fetuses with combined renal and extra-renal malformation. Each group was then compared to the general population based on a national registry which includes 5541 CMA results of fetuses with a normal prenatal ultrasound.

Results: A total of 70 fetuses were included in this study. Of those, 38 fetuses had an isolated renal malformation and 32 had renal and extra-renal malformations. Pathogenic CMA test results were found in 8 cases $(11.4 \%)$ with no statistically significant difference between the subgroups within our cohort [5 cases $(13.2 \%)$ in the isolated renal malformation and 3 cases $(9.4 \%)$ in the renal and extra renal malformations]. The risk for pathogenic CMA test result in isolated renal malformations was higher compared to the control group which represent the general population) $13.2 \%$ vs $1.4 \%$, $\mathrm{P}<0.001)$ with a relative risk of 10.148 (95\% CI 1.63-63.13).

Conclusions: The risk for a pathogenic CMA test result among fetuses with an isolated renal malformation is higher compared to fetuses with normal prenatal ultrasound. CMA test should be considered in cases of prenatal renal malformation.

EP-84 MULTICYSTIC DYSPLASTIC KIDNEY: WHOLE GENOME SEQUENCING DATA ANALYSIS IN 30 CASEPARENT TRIOS

Maud Kerstholt ${ }^{1}$, Sander Groen In T Woud ${ }^{2}$, Laura R. Claus ${ }^{1}$,

Flip Mulder ${ }^{3}$, Isaac J. Nijman ${ }^{3}$, Lude H. Franke ${ }^{4}$, Michiel F. Schreuder ${ }^{5}$,
Wout F.j. Feitz ${ }^{6}$, Gijs Van Haaften ${ }^{1}$, Loes F.m. Van Der Zanden ${ }^{2}$, Albertien M. Van Eerde ${ }^{1}$, Marc R. Lilien ${ }^{7}$, Kirsten Y. Renkema ${ }^{1}$

${ }^{1}$ Department Of Genetics, University Medical Center Utrecht, Utrecht, The Netherlands

${ }^{2}$ Radboud Institute For Health Sciences, Department For Health Evidence, Radboud University Medical Center, Nijmegen, The Netherlands

${ }^{3}$ Utrecht Bioinformatics Expertise Core, Center For Molecular Medicine, University Medical Center Utrecht, Utrecht, The Netherlands

${ }^{4}$ Department Of Genetics, University Medical Center Groningen, Groningen, The Netherlands

${ }^{5}$ Department Of Pediatric Nephrology, Radboud University Medical Center, Nijmegen, The Netherlands

${ }^{6}$ Department Of Urology, Radboud University Medical Center, Nijmegen, The Netherlands

${ }^{7}$ Department Of Pediatric Nephrology, University Medical Center Utrecht, Utrecht, The Netherlands

Introduction: Congenital anomalies of the kidney and urinary tract (CAKUT) comprise a spectrum of structural malformations that form the major cause of end-stage renal disease in children. Despite the occurrence of familial cases that clearly suggest a genetic background, the exact patterns and mechanisms of inheritance have not been conclusive. Although $10-20 \%$ of CAKUT cases seem to have a monogenic background, disease etiology is probably more complex in most cases. We currently investigate a cohort of 30 cases with multicystic dysplastic kidney and their parents, using a whole genome sequencing-based approach to identify underlying molecular mechanisms.

Material and methods: Patients and their parents were included in the AGORA data- and biobank. The selected cohort was phenotypically homogeneous in the sense that all patients presented with unilateral multicystic dysplastic kidney. All participants gave informed consent for whole genome sequencing (WGS). WGS was performed in 30 caseparent trios. First, WGS data was analyzed for single nucleotide and structural variants in known CAKUT genes. Next, potentially deleterious variants were listed and prioritized for further investigation using KidneyNetwork (https://kidney.genenetwork.nl/) based on phenotyperelated and tissue-specific expression data.

Results: A de novo variant in the GREBIL gene was identified in a patient with unilateral multicystic dysplastic kidney and absence of the vas deferens. The variant was classified as pathogenic. The patient was referred for genetic counseling at the nephrogenetics outpatient clinic for children.

The upcoming integration of WGS data with KidneyNetwork will lead to the prioritization of high impact variants in new CAKUT-related genes for follow-up studies.

Conclusions: Case-parent trio WGS revealed one de novo diseasecausing variant in a known disease gene for CAKUT. This finding extended the phenotypic spectrum related to GREB1L gene variants. The integration of trio WGS, expression and phenotypic data will bring more insight into the genetic background of multicystic dysplastic kidney.

EP-85 POSTNATAL MANAGEMENT OF ASYMPTOMATIC INFANTS WITH ANTENATAL HYDRONEPHROSIS DUE TO URETEROPELVIC JUNCTION OBSTRUCTION

Çă̆la Serpil Doğan ${ }^{1}$, Gülşah Kaya Aksoy ${ }^{1}$, Sibel Saraçoğlu ${ }^{2}$, Duygu Kuşlu ${ }^{3}$, Hüseyin Emre Çakar ${ }^{4}$, Ahmet Șükrü Alparslan ${ }^{2}$

${ }^{1}$ Antalya Training And Research Hospital, Department Of Pediatrics, Division Of Pediatric Nephrology

${ }^{2}$ Antalya Training And Research Hospital, Department Of Radiology, Division Of Pediatric Radiology

${ }^{3}$ Antalya Training And Research Hospital, Department Of Nuclear Medicine

${ }^{4}$ Antalya Training And Research Hospital, Department Of Pediatrics 
Introduction: The management of asymptomatic infants with antenatal hydronephrosis $(\mathrm{AHN})$ due to high-grade ureteropelvic junction obstruction (UPJO) remains controversial. In this retrospective study, we evaluated the infants with isolated unilateral UPJO, with special emphasis on the timing of surgical intervention.

Material and methods: Pyeloplasty was performed at first presentation if the initial differential renal function (DRF) was $\leq 40 \%$ or if there was severe hydronephrosis on ultrasound along with poor drainage on diuretic renography. Surgical indicaitons at follow-up were worsening hydronephrosis on subsequent ultrasounds, persistence of obstructive curve on second renogram, and the presence of any symptom.

Results: A total of 45 infants (38 boys) were icluded. Of 45 infants, $28(62.2 \%)$ were followed only conservatively for median 23 months (12-60). Hydronephrosis resolved spontaneously in 19, improved in 8 and remained stable in 1 . Surgical correction required in a total of $17(37.8 \%)$ infants with a median age of 5.8 months (1-12), 4 infants at first presentation and 13 while on conservative protocol. In these 13 infants, pyeloplasty was performed at a median age of 6.7 months (112) before a decrease in renal function on second renogram or parenchimal thinning on subsequent ultrasounds occured. For all 17 patients, worsening hydronephrosis was the main indication for surgery. Conclusions: Pyeloplasty was performed after a short follow-up period in our center, based on many studies suggesting that early surgical aproach prevents renal impairment and even improves renal function in infants with antenatal UPJO. We think that this approach is effective and reliable in young infants.

\section{EP-86 PROGNOSTIC FACTORS OF CHRONIC KIDNEY DISEASE IN CHILDREN WITH POSTERIOR URETHRAL} VALVE

Cagla Cagli, Bahriye Atmis, Derya Cevizli, Emel Saribas, Aysun K. Bayazit

Cukurova University, Faculty Of Medicine, Department Of Pediatric Nephrology, Adana, Turkey

Introduction: Posterior urethral valve (PUV) is the main cause of bladder outflow obstruction in males. PUV is also the most common obstructive uropathy leading to chronic kidney failure. We aimed to identify the clinical course, kidney outcomes and prognostic factors in children with PUV.

Material and methods: We retrospectively analyzed of 83 children with PUV who were follow-up in our tertiary center. We reviewed medical records of children with PUV and recorded demographic features, growth parameters, clinical course, laboratory and radiological data of the patients.

Results: The median age at diagnosis was 2.5 months (range 1 day-99 months). The median surgical intervention age was 3 months. The median follow-up period was 2.5 years and the longest was 20 years. The most presenting symptom was urine dribbling (42.1\%). Fourteen (16.9\%) patients had chronic kidney disease (CKD) stage 5 and required kidney replacement therapy (KRT) on their follow-up. The mean age of patients with end stage kidney disease was $8.82 \pm 5.65$ years old. KRT was kidney transplantation at four $(4.8 \%)$ patients, peritoneal dialysis at $6(7.2 \%)$ patients and hemodialysis at $5(6 \%)$ patients. Vesicoureteral reflux (VUR) was present in 47 patients $(56.6 \%) ; 28$ (33.8\%) unilateral and $19(22.9 \%)$ bilateral. Hypertension was developed in $16(20.8 \%)$ patients. The development of kidney failure was significantly higher $(p=0.024)$ in the group of patients with VUR compared to those without. The mean estimated glomerular filtration rate (eGFR) at the time of diagnosis (18.41 $\pm 10.39 \mathrm{ml} / \mathrm{min} / 1.73 \mathrm{~m}^{2}$ ) was lower in patients with kidney failure at last visit compared to those without $\left(49.87 \pm 40.96 \mathrm{ml} / \mathrm{min} / 1.73 \mathrm{~m}^{2}\right)(p=$ $0.024)$. The mean eGFR at diagnosis $\left(24.14 \pm 18.24 \mathrm{ml} / \mathrm{min} / 1.73 \mathrm{~m}^{2}\right)$ was lower in patients with hypertension compared to those without (47.83 $\pm 40.45 \mathrm{ml} / \mathrm{min} / 1.73 \mathrm{~m}^{2}$ ), but no statistically significant difference was found $(p=0.208)$. There was no significant difference between patients with kidney failure and those without in terms of prematurity, age at diagnosis, kidney scar at presentation and kidney scarring during follow-up.

Conclusions: VUR is common in children with PUV. Coexistence of PUV and VUR, and low eGFR at diagnosis are affecting factors in the development of kidney failure during follow-up.

\section{EP-87 URINARY UROMODULIN AND VESICO-URETERAL REFLUX \\ Rosa Cusumano ${ }^{1}$, Ciro Corrado ${ }^{1}$, Giovanni Pavone ${ }^{1}$, \\ Maria Michela Dalessandro ${ }^{1}$, Maria Chiara Sapia ${ }^{1}$, \\ Serena Abbate ${ }^{2}$, Silvio Maringhini ${ }^{2}$ \\ ${ }^{1}$ Ped Nephrology Unit, Childrens Hospital, Arnas Civico, Palermo, Italy ${ }^{2}$ Dept Pediatrics, Ismett, Palermo, Italy}

Introduction: Vesico-ureteral reflux (VUR) is associated with febrile urinary tract infections (UTI) and renal scars. Uromodulin (UMOD) may protect from UTI. Aim of our study is to evaluate if low concentration of UMOD in the urine is associated with UTI and/or scars in children with VUR.

Material and methods: We studied 42 patients with VUR, normal eGFR, and no other urinary tract malformation; 18 were males (age range $1-15$, mean 7.7 years) and 24 females (age range $2-15$, mean 7,4 years). A group of 17 controls (no VUR, no UTI) included 9 males (age range 3-15, mean 8.0 years) and 8 females (age range $4-13$, mean 8.8 years). UMOD concentration $(\mathrm{U})$ was measured $(\mu \mathrm{g} / \mathrm{ml})$ in first morning urine using an ELISA method.

Results: U was lower in patients with VUR (Mean \pm SD: $24.9 \pm 12.4$ ) and in 7 of these with $>3$ UTI $(12.1 \pm 5.4)$ compared to controls $(40.5 \pm 21.5)$. Renal scars were detected in 22 children (Group A): 11 males (age range $1-14$, mean 7.3 years) and 11 females (age range 3-15, mean 9.6 years). No scar was detected in 20 patients (Group B): 7 males (age range 1-15, mean 8.4 years) and 13 females (age range $2-11$, mean 5.6 years). $U$ was lower in Group A $(21.7 \pm 12,2)$ compared to Group B $(28.4 \pm 11.9)$. In patients with $>3$ UTI U was low in both Group A (11.2 \pm 5.9$)$ and B (13.3 \pm 5.7). Among patients with VUR of 3-to-5-degree $\mathrm{U}$ was lower in 11 Group A (22.2 \pm 11.8$)$ than in 5 Group B $(26.7 \pm 13.5)$.

Conclusions: U was lower in children with VUR and recurrent UTI, especially in those with scars. Renal scars may be related to low $U$ levels independently from UTI. Genetic studies of UMOD in VUR are needed.

\section{EP-88 COMPARISON OF THE COMPENSATORY RENAL HYPERTROPHY IN CONGENITAL AND ACQUIRED SOLITARY KIDNEYS}

Gizem Yildiz $^{1}$, Meral Torun Bayram ${ }^{1}$, Bahadir Senol $^{2}$, Salih Kavukcu $^{l}$, Alper Soylu ${ }^{1}$

${ }_{1}^{1}$ Dokuz Eylul University Medical Faculty, Department Of Pediatric Nephrology, İzmir, Turkey

${ }^{2}$ Dokuz Eylul University Medical Faculty, Department Of Pediatrics, Izmir, Turkey

Introduction: Solitary functional kidney can develop secondary to congenital and acquired causes. Absence of one kidney results in adaptive compensatory hypertrophy of the contralateral healthy kidney in all age groups. We aimed to evaluate the rate of compensatory renal hypertrophy and the development of adverse events as hypertension, proteinuria and decrease in estimated glomerular filtration rate (eGFR) in children with congenital or acquired solitary kidneys.

Material and methods: Children with solitary functioning kidneys were evaluated retrospectively for the following parameters: Cause of solitary kidney, age, kidney length, eGFR, proteinuria and blood pressure at diagnosis and during follow-up. Children with congenital solitary kidneys (Group 1) were compared with those having acquired solitary kidneys due to nephrectomy for various causes (Group 2). 
Results: The total number of subjects was 63 (male/female: 38/25). Groups 1 and 2 included 44 and 19 cases, respectively. Patients in Group 2 were older, their kidneys were longer and they tended to have higher eGFR at onset. Follow-up period (median 7 years) were similar in both groups. At last visit kidney length and eGFR were not different between the groups. Percent change in GFR [(last GFR - first GFR)/first GFR] was also not different, but percent change in kidney length per year was significantly higher in Group 1 . Increase in renal length was correlated with increase in eGFR. Groups 1 and 2 were not different with respect to proteinuria $(3 / 39$ vs $0 / 19)$, however hypertension rate was higher in Group $2(0 / 42$ vs $2 / 16, p=0.028)$.

Conclusions: Compensatory hypertrophy develops in both congenital and acquired solitary kidneys, but growth rate of congenital solitary kidneys is higher. Children with acquired solitary kidneys may be more prone to develop hypertension.

\section{EP-89 FEBRILE URINARY TRACT INFECTIONS IN CHILDREN WITH PRIMARY NON-REFLUXING MEGAURETER: A SYSTEMATIC REVIEW AND METAANALYSIS \\ Katharina Rohner ${ }^{2}$, Kathrin Buder ${ }^{2}$, Sara Mazzi ${ }^{2}$, Marcus Weitz ${ }^{1}$ \\ ${ }^{1}$ Paediatric Nephrology, University Childrens Hospital Tübingen, Germany \\ ${ }^{2}$ Paediatric Nephrology, University Childrens Hospital Zürich, Switzerland}

Introduction: Knowledge of the baseline risk of febrile urinary tract infections (fUTI) in patients with primary non-refluxing megaureter can help clinicians to make informed decisions for offering continuous antibiotic prophylaxis (CAP). The objective of this systematic review was to determine the pooled prevalence of fUTI in order to assess the value of CAP.

Material and methods: MEDLINE, EMBASE, and Cochrane Controlled Trials Register electronic databases were searched for eligible studies without language and time restriction. The systematic review was carried out following the recommendations of the Cochrane Collaboration and the Preferred Reporting Items for Systematic Reviews and Meta-Analyses.

Results: Of 25871 records, 16 studies ( $n=749$ patients) were eligible for inclusion. The overall pooled prevalence of fUTI in patients with primary non-refluxing megaureter was $14.35 \%$ (95\% confidence interval: 8.8 22.6), and increases with grade of upper urinary tract obstruction and ureteral dilatation. The calculated number needed to treat for patients on CAP to prevent one single fUTI over the course of 1-2 years was 4.3.

Conclusions: Based on the current available evidence the use of continuous antibiotic prophylaxis for children with primary nonrefluxing megaureter selected for primary non-surgical treatment should be taken into consideration, at least in patients with urinary outflow impairment, higher grade of ureteral dilatation, and for children in the first months of life. However, there is an urgent need for high quality prospective studies.

\section{EP-91 A SUCCESSFUL RESOLUTION OF NUTCRACKER SYNDROME WITH 3D PRINTED PEEK EXTRAVASCULAR STENT IN AN ADOLESCENT BOY}

Da-li He ${ }^{3}$, Heng-en Wang ${ }^{3}$, Yong Jiao ${ }^{3}$, Bin $\mathrm{Wu}^{3}$, Dong Cui ${ }^{3}$,

Yan-yan $\mathrm{Li}^{4}$, Yong Yang ${ }^{5}$, Tie-sheng Cao ${ }^{6}$, Vinko Vidjak ${ }^{7}$,

Danko Milosevic ${ }^{1}$, Bo Zhang ${ }^{3}$

${ }^{1}$ University Of Zagreb School Of Medicine, Zagreb, Croatia

${ }^{2}$ General Hospital Zabok And Croatian Veterans Hospital, Zabok, Croatia

${ }^{3}$ Department Of Urology, Tangdu Hospital, Fourth Military Medical University, Xian, Shaanxi 710038, China

${ }^{4}$ Department Of Radiology, Tangdu Hospital, Fourth Military Medical

University, Xian, Shaanxi 710038, China
${ }^{5}$ Department Of Ultrasonic Diagnosis, Tangdu Hospital, Fourth Military Medical University, Xian, Shaanxi 710038, China

${ }^{6} 3 d$ Printing Research Center, Fourth Military Medical University, Xian, Shaanxi 710038, China

${ }^{7}$ Department Of Diagnostic And Interventional Radiology, University Hospital Merkur, Zajceva Ul.19, 10000 Zagreb, Croatia

Introduction: A 14-year-old boy with nutracker syndrome and a history of constant and intense periumbilical pain (pain scale, 8-9) for the last 24 months was overviewed for surgical treatment. All clinical examinations and possible psychogenic upgrades were insufficient to explain the pains cause, including autoimmune thyroiditis as comorbidity.

Material and methods: Digital subtraction angiography revealed a segment of the left renal vein with reduced contrast filling immediately before its inflow into inferior vena cava and high-pressure gradient. The three-dimensional reconstruction model demonstrated that the LRV and the duodenum were contracted at the aortomesenteric angle, resulting in left renal vein compression from the abnormal high-level duodenal compartment. When duodenum coarses between the abdominal aorta and superior mesenteric artery (duodenal interposition), the left renal vein entrapment occurs even at $<90$ aortomesenteric degrees.

Results: Three-dimensional PEEK printed extravascular stent was chosen to elevate the superior mesenteric artery and lower the duodenum position, thus relieving left renal vein compression. This extravascular application has significant advantages over open surgery, endovascular stenting and artificial vessel procedures with expanded polytetrafluoroethylene. It provides better cellular vitality by ensuring soft tissue proliferation. Such a stent has a distinctive personalized design, good stiffness, and durability that allows blood vessel growth, preventing stent migration and thrombosis. Therefore, it is suitable for both adult and pediatric patients. According to the abdominal ultrasound and MSCT scan, the post-operative follow-up results are satisfactory one year after surgery. The patient feels well, the blood flow in the left renal vein is not obstructed, and the blood flow velocity was average. The external stent is in place.

Conclusions: By reducing external acceleration and centrifugal force, a three-dimensional printed PEEK extravascular stent reduces adverse side effects. Surgical treatment of NCS should be considered for children whose conservative therapy has remained inefficient for more than 24 months or in children with severe pain, debilitating their everyday life. We believe that treating patients with nutcracker syndrome by the laparoscopic placement of a 3D printed PEEK extravascular stent around the compressed LRV is safe has advanteges over endovascular stenting. 3DPPEEK extravascular stent is an effective method of treating nutcracker syndrome in children as it allows them to grow/expand their blood vessels. To our knowledge, this is th first european patient treated with such stent.

\section{EP-92 RETROSPECTIVE EVALUATION OF CHILDREN WITH ECTOPIC KIDNEYS}

Zehra Aydin ${ }^{1}$, Umut Selda Bayrakci ${ }^{2}$, Fatma Semsa Cayci ${ }^{1}$

${ }^{1}$ The University Of Health Sciences, Ankara City Hospital, Ankara, Turkey

${ }^{2}$ The University Of Yildirim Beyazit, Ankara, Turkey

Introduction: Renal ectopia is an embryological renal anomaly characterized by abnormal anatomical location of one or both of the kidneys. It can occur in various forms such as simple renal ectopia or crossed renal ectopia. In most cases it is asymptomatic however in others its presentation could vary from UTI to renal impairment. We assessed the clinical and laboratory profile of renal ectopia in children.

Material and methods: We retrospectively analyzed pediatric cases of renal ectopia diagnosed at our hospital between 2010-2020.

Results: A total of 127 patients, 73 girls (57.4\%) and 54 boys ( $42.5 \%)$ with a mean age of $8.09 \pm 4.5$ years and a mean follow-up time of $4.29 \pm 2.1$ years were included in. Renal ectopia was incidentally found in 101 
patients (79.5\%). 76 patients had right renal ectopia. Cross renal ectopia was seen in 21 patients of all. Hydronephrosis(n=23), VUR(n=10), nephrolithiasis $(n=5)$, hypospadias $(n=4)$, ureteropelvic junction obstruction( $(n=2)$, and ureterovesical junction obstruction $(n=1)$ were identified as additional anomalies. 20 patients $(15.7 \%)$ had additional systemic abnormalities. VCU was performed in 59 patients $(46.4 \%)$. While 6 patients had contralateral VUR, 4 patients had bilateral VUR. 24 patients $(18.8 \%)$ had renal scarring on DMSA scintigraphy. None of the patients had renal failure, proteinuria, hypertension, or hematuria.

Conclusions: Patients with ectopic kidneys may require further attention for additional anomalies.

\section{EP-93 USEFULNESS OF BASIC RENAL FUNCTION TESTS IN DILATED URINARY TRACT WITH A RENAL PELVIS DIAMETER LESS THAN 2 CM}

Maria Isabel Luis Yanes Sandra Teresa Moraleda Mesa Cristina Batista Gonzalez, ${ }^{1}$, Jorge De Luis Yanes ${ }^{2}$, Andrea Gonzalez Palau ${ }^{1}$, Victor Manuel Garcia Nieto ${ }^{1}$, Patricia Tejera Carreño ${ }^{1}$, Eva Virginia Mejias Gonzalez ${ }^{1}$, Antonio Luis Lopez Figueroa ${ }^{1}$

${ }^{1}$ Hospital Universitario Nuestra Señora E Candelaria

${ }^{2}$ Hospital General Universitario Gregorio Marañon

Introduction: The recent multidisciplinary consensus on the classification of prenatal and postnatal urinary tract dilatation (UTD classification system) proposes that six ultrasound morphological parameters be assessed (J Pediatr Urol, 2014). It is considered that anteroposterior pelvis diameters (APD) smaller than $10 \mathrm{~mm}$ should not be studied. Likewise, the study of renal function is also neglected. The objective of this work was to study renal function in children with mild-moderate dilatation of the urinary tract and to check whether the results are useful in deciding to order other imaging tests.

Material and methods: A retrospective cross-sectional study included 102 infants $(75 \mathrm{~V}, 27 \mathrm{M})$ with a APD between $5 \mathrm{~mm}$ and less than $20 \mathrm{~mm}$ at the first ultrasound; 88 were diagnosed in utero (86.3\%).All had performed, in addition to ultrasonography before the age of one year, cystography and at least maximum urinary osmolality (UOsm) determined with desmopressin. When possible, the albumin/creatinine ratio value was collected. A renogram was performed in 33 cases.

Results: Three patients were diagnosed with ureteropelvic junction obstruction (UPJ)(APD between 12 and $18.2 \mathrm{~mm}$ ). Vesicoureteral reflux (VUR) was observed in 17 infants (16.8\%) (1 grade I, 4 grade II, 10 grade III, 2 grade IV; APD between 5.6 and $19 \mathrm{~mm}$ ). When distributed according to APD $(5$ to $<10 \mathrm{~mm}, 10$ to $<15 \mathrm{~mm}, 15$ to $<20 \mathrm{~mm})$ and excluding patients with VUR and PUS, significant differences in UOsm were observed (lower in the last of these subgroups, $\mathrm{p}=0.03$ ). UOsm was reduced in $64.7 \%$ of VUR cases and in $34.1 \%$ of children without VUR or UPJ. Albuminuria was increased in $16.9 \%$ of infants without VUR or PUS. The sensitivity of UOsm in cases of PUS and VUR III-IV was $66.7 \%$ and the negative predictive value (NPV) was $91.5 \%$ (Odds ratio: 3.86; 95\%CI: $1.20-12.38 ; \mathrm{p}=0.02)$. In the same cases, the NPV of the albumin/creatinine ratio was $85.5 \%$. In cases with APD between 5 and $<10 \mathrm{~mm}, 27.3 \%$ had abnormal UOsm and there were seven cases of VUR (three grade II and four grade III).

Conclusions: In cases without VUR or UPJ, UOsm may be altered and albumin clearance increased. When these two tests are normal, ordering further imaging tests is not indicated initially (high negative predictive value).

EP-94 CASE REPORT: NEONATAL RENAL FAILURE: PECULIAR PRESENTATION OF THE BRANCHIO-OTORENAL SPECTRUM DISORDER

Somyyah Kobeisy ${ }^{2}$, Afaf Bafageeh ${ }^{1}$

${ }^{1}$ King Fahad Specialist Hospital

${ }^{2}$ Soliman Faqeeh Hospital
Introduction: Branchio-oto-renal syndrome (BOR) is a spectrum of disorders with variable clinical presentations. Limited articles have been published in literature regarding this syndrome. This condition is quite rare, and it is estimated that 1 in 40,000 individuals are affected by the BOR spectrum [i]. It has been found that around $2-3 \%$ of the pediatric population who are affected by BOR syndrome have profound deafness[ii] and $5-29 \%$ of those affected develop renal failure[iii].

A literature review conducted on PubMed yielded 156 case reports related to branchio-oto-renal syndrome without any restrictions on language. $57 \%$ of the cases reported have been published in genetic, otorhinolaryngology and surgical journals. The rest of the cases have been published in European and Asian journals, in journals related to dermatology, anesthesia and radiology. Only 23 articles have been published since 1978 in either pediatric and or nephrology journals with most subjects in the cases presenting with ENT manifestations or novel genetic mutations. Only one article in the literature review presented with right hypoplastic kidney. Therefore, it is quite exceptional to have a patient present initially with renal symptoms and then to have a suspicion of branchio-oto-renal spectrum disorder made.

In this article, three cases, which presented with renal manifestations in the first few days of life and were found to have features of BOR postnatally, will be discussed along with a review of the literature to acquaint clinicians with this syndrome for early detection and prevention of its long-term adverse renal effects.

[i] "Branchiootorenal/Branchiootic Syndrome - Genetics Home Reference - NIH." U.S. National Library of Medicine, National Institutes of Health, ghr.nlm.nih.gov/condition/branchiootorenalbranchiootic-syndrome\#resources.

[ii] "Branchiootorenal Spectrum Disorders." NORD (National Organization for Rare Disorders), rarediseases.org/rare-diseases/ branchio-oto-renal-syndrome/.

[iii] "Branchiootorenal Syndrome." Genetic and Rare Diseases Information Center, U.S. Department of Health and Human Services, rarediseases.info.nih.gov/diseases/10147/branchiootorenal-syndrome.

Material and methods:

\begin{tabular}{|c|c|c|c|}
\hline & Case 1 & Case 2 & Case 3 \\
\hline \multicolumn{4}{|l|}{ History: } \\
\hline $\begin{array}{l}\text { Age at } \\
\quad \text { presentatio- } \\
n\end{array}$ & First day of life & 11 days old & 11 days old \\
\hline $\begin{array}{r}\text { Antenatal } \\
\text { history: }\end{array}$ & $\begin{array}{l}\text { 34-week } \\
\text { gestation } \\
\text { Oligohydramnios } \\
\text { right kidney not } \\
\text { visualized }\end{array}$ & $\begin{array}{l}\text { 35-week } \\
\text { gestation }\end{array}$ & 35-week gestation \\
\hline Consanguinity & Negative & Negative & Negative \\
\hline Ethnicity & Saudi & Saudi & Saudi \\
\hline $\begin{array}{l}\text { Year of } \\
\quad \text { presentatio- } \\
n\end{array}$ & 2018 & 2018 & 2018 \\
\hline \multicolumn{4}{|l|}{ Examination: } \\
\hline $\begin{array}{l}\text { Blood } \\
\quad \text { Pressure }\end{array}$ & Normal & Normal & Normal \\
\hline $\begin{array}{l}\text { Auricular } \\
\quad \text { manifestati- } \\
\quad \text { on }\end{array}$ & $\begin{array}{l}\text { Bilateral } \\
\text { preauricular } \\
\text { pits } \\
\text { Right pinna } \\
\text { malformation } \\
\text { (Figure 1) }\end{array}$ & $\begin{array}{l}\text { Bilateral } \\
\text { preauricular } \\
\text { pits } \\
\text { Pinnae } \\
\text { malforma- } \\
\text { tion } \\
\text { (Figure 2) }\end{array}$ & $\begin{array}{l}\text { Right preauricular } \\
\text { pit } \\
\text { Pinnae } \\
\text { malformation } \\
\text { (Figure 3) }\end{array}$ \\
\hline
\end{tabular}




\begin{tabular}{|c|c|c|c|}
\hline $\begin{array}{l}\text { Branchial } \\
\quad \text { malformati- } \\
\quad \text { on }\end{array}$ & $\begin{array}{l}\text { Bilateral } \\
\text { branchial } \\
\text { fistulae } \\
\text { High arched } \\
\text { palate } \\
\text { (Figure 1) }\end{array}$ & $\begin{array}{l}\text { Right } \\
\text { branchial } \\
\text { fistulae } \\
\text { (Figure 2) }\end{array}$ & $\begin{array}{l}\text { Right branchial } \\
\text { fistula } \\
\text { (Figure 3) }\end{array}$ \\
\hline \multicolumn{4}{|l|}{ Laboratory: } \\
\hline $\begin{array}{l}\text { Serum } \\
\quad \text { Creatinine } \\
\text { (mg/dl) }\end{array}$ & 1.6 & 4.6 & 2.1 \\
\hline $\begin{array}{l}\text { Blood urea } \\
\text { nitrogen } \\
(m g / d l)\end{array}$ & 17.7 & 19 & 25.2 \\
\hline $\begin{array}{l}\text { Serum } \mathrm{Na} \\
\quad(\mathrm{mmol} / \mathrm{L})\end{array}$ & 130 & 142 & 135 \\
\hline $\begin{array}{l}\text { Serum } K \\
\quad(\mathrm{mmol} / \mathrm{L})\end{array}$ & 6 & 6.3 & 5.6 \\
\hline $\begin{array}{l}\text { Serum } \mathrm{Cl} \\
\quad(\mathrm{mmol} / \mathrm{L})\end{array}$ & 102 & 107 & 102 \\
\hline $\begin{array}{l}\text { Serum } \\
\quad \text { bicarbonate } \\
\quad(\mathrm{mmol} / \mathrm{L}) \\
\text { Imaging }\end{array}$ & 16 & 16 & 19 \\
\hline & $\begin{array}{l}\text { MRI: Bilateral } \\
\text { hypoplastic } \\
\text { kidneys } \\
\text { MCUG: } \\
\text { posterior } \\
\text { urethral valve }\end{array}$ & $\begin{array}{l}\text { US: Bilateral } \\
\text { hypoplastic } \\
\text { kidneys }\end{array}$ & $\begin{array}{l}\text { US: Bilateral } \\
\text { hypoplastic } \\
\text { kidneys (left } \\
\text { kidney smaller } \\
\text { than the right) }\end{array}$ \\
\hline \multicolumn{4}{|c|}{ Renal Management } \\
\hline & $\begin{array}{l}\text { Special formula: } \\
\text { high calorie } \\
\text { and low } \\
\text { potassium } \\
\text { Sodium } \\
\text { bicarbonate } \\
\text { Calcium } \\
\text { resonium } \\
\text { Vitamin D } \\
\text { Erythropoietin }\end{array}$ & $\begin{array}{l}\text { Special } \\
\text { formula: } \\
\text { high calorie } \\
\text { and low } \\
\text { potassium } \\
\text { Sodium } \\
\text { bicarbonate } \\
\text { Calcium } \\
\text { resonium } \\
\text { Vitamin D }\end{array}$ & $\begin{array}{l}\text { Special formula: } \\
\text { high calorie and } \\
\text { low potassium } \\
\text { Sodium bicarbonate } \\
\text { Calcium resonium } \\
\text { Vitamin D }\end{array}$ \\
\hline
\end{tabular}

*consent was taken from the legal guardian of each patient

* Case 2 and Case 3 are twin brothers

Results: Branchio-oto- renal syndrome, which is also known as banchiootorenal dysplasia, Melnick-Fraser syndrome, branchiootorenal spectrum disorders (BOS)[i] are all synonymous for a condition with a wide variability of clinical presentations that has head and neck, auditory and genitourinary variances.

Head and neck variabilities range from hemifacial hypoplasia, cleft palate, branchial cysts, to lacrimal duct stenosis. Auditory abnormalities range anywhere from preauricular skin tags, hearing impairment either conductive or sensorineural, malformations of the external ear to incomplete partition of the cochlea. The scope of genitourinary involvement differs from each affected individual and can include renal steatosis, malrotation, collecting system abnormalities, vesicoureteral reflux, polycystic kidney dysplasia, renal agenesis or dysplasia[ii] and in some patients can even progress to end stage renal disease[iii].

The three cases discussed in this review presented due to renal malformations and were in end stage renal failure and were subsequently found to have preauricular pits and signs consistent with the BOR syndrome in contrast to those in review of the literature who presented initially with symptoms of auditory infections, preauricular pits, progressive hearing loss and branchial fistulae[iv] [v] [vi].
Although approximately $90 \%$ of BOR cases are inherited in an autosomal dominant fashion, there can be vast differences in manifestations of symptoms and signs among those affected, even amongst family members[vii]. The breadth of the observed defects can be explained by variable expressivity of different genetic loci including, EYA1 , SIXI and SIX5[viii]. Around $40 \%$ of the with BOR have a mutation in the EYA 1 gene[ix]. A $S I X 1$ mutation affects approximately $4 \%$ of people with BOR spectrum and a SIX5 mutation is present in about $5 \%$ of those affected[x]. The gene in approximately $50 \%$ of patients is unknown[xi].

The remaining $10 \%$ of affected individuals are due to the effect of de novo genetic mutations. The genomic defects can cause anomalous development of the genitourinary system along with abnormal development of the second branchial arch in the developing embryo which can cause the head and neck and ear abnormalities in the maturing fetus[xii].

In some individuals with BOR syndrome, no genetic mutation was identified in the EYA 1, SIXI and SIX 5 genes[xiii], therefore there are probably other gene mutations that have not been identified that can cause this syndrome[xiv].

The differential diagnosis can include but is not limited to: branchio-otorenal syndrome, branchio-oculo-facial facial syndrome $[\mathrm{xv}] /$ Goldenhar syndrome[xvi], oculo-auriculo-vertebral spectrum[xvii], CHARGE[xviii], Treacher Colllins, Alport Syndrome[xix] and Towns Brocks syndrome[xx].

Table 2. branchio-Oto-Renal Spectrum Disorder Diagnostic Criteria[xxi] [xxii] [xxiii]

\section{Major Criteria}

Pinnae malformation

Hearing impairment

Preauricular pits

Renal anomalies

Second branchial arch anomalies

External auditory canal stenosis
* Only two major criteria are needed if the proband hus hroid goiter affected family members/ family history of brachial, otologic or renal anomalies.

*Three major criteria or two major criteria and two minor criteria required if negative family history.

Those with a suspected or confirmed diagnosis of BOR require a multidisciplinary team approach that is tailored to each patient's needs. The team should comprise of a "geneticist, otolaryngologist, speech therapist, occupational therapist, radiologist and renal specialist, with the pediatrician coordinating the care" [xxiv]. An audiological evaluation is also required including computerized tomography of the ear and laboratory and radiological investigations for renal defects $[\mathrm{xxv}]$.

The treatment of those with BOR syndrome, depends on the degree of affection. Hearing aids and rehabilitation may be required for those with auditory deficit. Antimicrobial agents can be used to treat otitis media or inflamed branchial sinuses. Surgery may be beneficial for those with structural deformities of the pinna or those with branchial cleft deformities that can cause cysts or fistulae. Due to the incidence of renal involvement, it is recommended to involve a nephrologist to assess the patient for any renal impairment. The patient may require surgery, dialysis or renal transplant[xxvi].

[i] "Branchiootorenal Syndrome." Genetic and Rare Diseases Information Center, U.S. Department of Health and Human Services, rarediseases.info.nih.gov/diseases/10147/branchiootorenal-syndrome.

[ii] Human Phenotype Ontology, hpo.jax.org/app/browse/disease/ OMIM:113650.

[iii] "Branchiootorenal/Branchiootic Syndrome - Genetics Home Reference - NIH." U.S. National Library of Medicine, National 
Institutes of Health, ghr.nlm.nih.gov/condition/branchiootorenalbranchiootic-syndrome\#resources.

[iv] Gangaraj, Smithas, and Jagannath Bisanna. "Branchio-Oto-Renal Syndrome: A Case Report and Review of Literature." Indian Journal of Otology, vol. 17, no. 4, 2011, p. 176., doi:10.4103/0971-7749.94500.

[v] Prasanth, Kaninghat, et al. "Bilateral Preauricular Pits." NeoReviews, vol. 12 , no. 10, 2011, doi:10.1542/neo.12-10-e606.

[vi] Jalil, Jawad, et al. "Branchio-Oto-Renal Syndrome." Journal of the College of Physicians and Surgeons Pakistan, vol. 24, no. 5, 2014, pp. 367-3

[vii] "Branchiootorenal/Branchiootic Syndrome - Genetics Home Reference - NIH." U.S. National Library of Medicine, National Institutes of Health, ghr.nlm.nih.gov/condition/branchiootorenalbranchiootic-syndrome\#resources.

[viii] Human Phenotype Ontology, hpo.jax.org/app/browse/disease/ OMIM:113650.

[ix] "Branchiootorenal/Branchiootic Syndrome - Genetics Home Reference - NIH." U.S. National Library of Medicine, National Institutes of Health, ghr.nlm.nih.gov/condition/branchiootorenalbranchiootic-syndrome\#resources.

[x] "Branchiootorenal Spectrum Disorders." NORD (National Organization for Rare Disorders), rarediseases.org/rare-diseases/ branchio-oto-renal-syndrome/.

[xi] Smith, Richard JH. "Branchiootorenal Spectrum Disorder." Current Neurology and Neuroscience Reports., U.S. National Library of Medicine, 6 Sept. 2018, www.ncbi.nlm.nih.gov/books/NBK1380/.

[xii] "Branchiootorenal/Branchiootic Syndrome - Genetics Home Reference - NIH." U.S. National Library of Medicine, National Institutes of Health, ghr.nlm.nih.gov/condition/branchiootorenalbranchiootic-syndrome\#resources.

[xiii] "Branchiootorenal/Branchiootic Syndrome - Genetics Home Reference - NIH." U.S. National Library of Medicine, National Institutes of Health, ghr.nlm.nih.gov/condition/branchiootorenalbranchiootic-syndrome\#resources.

[xiv] "Branchiootorenal Syndrome." Genetic and Rare Diseases Information Center, U.S. Department of Health and Human Services, rarediseases.info.nih.gov/diseases/10147/branchiootorenal-syndrome. [xv] "Branchiootorenal Spectrum Disorders." NORD (National Organization for Rare Disorders), rarediseases.org/rare-diseases/ branchio-oto-renal-syndrome/.

[xvi] Human Phenotype Ontology, hpo.jax.org/app/browse/disease/ OMIM:113650.

[xvii] "Branchiootorenal Spectrum Disorders." NORD (National Organization for Rare Disorders), rarediseases.org/rare-diseases/ branchio-oto-renal-syndrome/.

[xviii] Human Phenotype Ontology, hpo.jax.org/app/browse/disease/ OMIM:113650.

[xix] Alkis M. Pierides, Yiannis Athanasiou, Kyproula Demetriou, Michael Koptides, C. Constantinou Deltas; A family with the branchiooto-renal syndrome: clinical and genetic correlations, Nephrology Dialysis Transplantation, Volume 17, Issue 6, 1 June 2002, Pages 1014-1018, https://doi.org/10.1093/ndt/17.6.1014

[xx] "Branchiootorenal Spectrum Disorders." NORD (National Organization for Rare Disorders), rarediseases.org/rare-diseases/ branchio-oto-renal-syndrome/.

[xxi] "Branchiootorenal Spectrum Disorders." NORD (National Organization for Rare Disorders), rarediseases.org/rare-diseases/ branchio-oto-renal-syndrome/.

[xxii] "Iowa Head and Neck Protocols." Required Courses (and Encouraged Electives) | Department of Anatomy and Cell Biology, medicine.uiowa.edu/iowaprotocols/branchio-oto-renal-syndromemelnick-fraser-syndrome.

[xxiii] Smith, Richard JH. "Branchiootorenal Spectrum Disorder." Current Neurology and Neuroscience Reports., U.S. National Library of Medicine, 6 Sept. 2018, www.ncbi.nlm.nih.gov/books/NBK1380/. [xxiv] Prasanth, Kaninghat, et al. "Bilateral Preauricular Pits." NeoReviews, vol. 12, no. 10, 2011, doi:10.1542/neo.12-10-e606.

[xxv] "Branchiootorenal Spectrum Disorders." NORD (National Organization for Rare Disorders), rarediseases.org/rare-diseases/ branchio-oto-renal-syndrome/.

[xxvi] "Branchiootorenal Spectrum Disorders." NORD (National Organization for Rare Disorders), rarediseases.org/rare-diseases/ branchio-oto-renal-syndrome/.

Conclusions: For those that present with features of BOR, swift recognition and supplementary investigations might prevent the potential severe long-term effects of hearing impairment and end stage renal disease[1].

Due to the $90 \%$ chance of BOR being inherited in an autosomal dominant manner, genetic counseling and screening is recommended for affected individuals and their relatives to inhibit disease progression. The patient and their siblings should have hearing and renal function examination. The patient should then be reevaluated every 6 months[2] to monitor for disease advancement. Prenatal ultrasound done at 16 to 17 weeks of gestation can be offered to pregnant women with a family history of BOR syndrome looking for renal anomalies in the fetus. Despite the availability of genetic testing, gene analysis may be postponed if the patient has features and family history suggestive of BOR spectrum disorder[3].

Patients with BOR syndrome who receive sufficient treatment and follow up can lead natural and healthy lives[4]. Therefore, it is imperative to provide early diagnosis and multidisciplinary team approach and to include the patient and their families in the medical management and planning.

Further studies are needed to shed light on genetic pathways and transcriptional regulators that may be involved in the development of the varying clinical picture of the BOR spectrum and whether or not any other factors may contribute or have an association to the de novo mutations of BOR syndrome.

[1] Gangaraj, Smithas, and Jagannath Bisanna. "Branchio-Oto-Renal Syndrome: A Case Report and Review of Literature." Indian Journal of Otology, vol. 17, no. 4, 2011, p. 176., doi:10.4103/0971-7749.94500.

[2] "Branchiootorenal Spectrum Disorders." NORD (National Organization for Rare Disorders), rarediseases.org/rare-diseases/ branchio-oto-renal-syndrome/.

[3] Prasanth, Kaninghat, et al. "Bilateral Preauricular Pits." NeoReviews, vol. 12 , no. 10, 2011, doi:10.1542/neo.12-10-e606.

[4] Morisada, Naoya, et al. "Branchio-Oto-Renal Syndrome: Comprehensive Review Based on Nationwide Surveillance in Japan." Pediatrics International, vol. 56, no. 3, 2014, pp. 309-314., doi:10.1111/ped.12357.

\section{References:}

1. Acierno, Stephanie, and John H.T. Waldhausen. "Branchio-Oto-Renal Syndrome." NeuroImage, Academic Press, www.sciencedirect.com/ topics/medicine-and-dentistry/branchio-oto-renal-syndrome.

2. Alkis M. Pierides, Yiannis Athanasiou, Kyproula Demetriou, Michael Koptides, C. Constantinou Deltas; A family with the branchio-oto-renal syndrome: clinical and genetic correlations, Nephrology Dialysis Transplantation, Volume 17, Issue 6, 1 June 2002, Pages 1014-1018, https://doi.org/10.1093/ndt/17.6.1014

3."Branchiootorenal/Branchiootic Syndrome - Genetics Home Reference - NIH." U.S. National Library of Medicine, National Institutes of Health, ghr.nlm.nih.gov/condition/branchiootorenal-branchiooticsyndrome\#resources.

4. "Branchiootorenal Spectrum Disorders." NORD (National Organization for Rare Disorders), rarediseases.org/rare-diseases/ branchio-oto-renal-syndrome/.

5. "Branchiootorenal Syndrome." Genetic and Rare Diseases Information Center, U.S. Department of Health and Human Services, rarediseases.info.nih.gov/diseases/10147/branchiootorenal-syndrome. 
6. "Branchiootic Syndrome 3 - Conditions - GTR - NCBI." Current Neurology and Neuroscience Reports., U.S. National Library of Medicine, www.ncbi.nlm.nih.gov/gtr/conditions/C1842124/.

7. Gangaraj, Smithas, and Jagannath Bisanna. "Branchio-Oto-Renal Syndrome: A Case Report and Review of Literature." Indian Journal of Otology, vol. 17, no. 4, 2011, p. 176., doi:10.4103/0971-7749.94500.

8. Human Phenotype Ontology, hpo.jax.org/app/browse/disease/ OMIM:113650.

9. "Iowa Head and Neck Protocols." Required Courses (and Encouraged Electives) | Department of Anatomy and Cell Biology, medicine.uiowa.edu/iowaprotocols/branchio-oto-renal-syndromemelnick-fraser-syndrome.

10. Jalil, Jawad, et al. "Branchio-Oto-Renal Syndrome." Journal of the College of Physicians and Surgeons Pakistan, vol. 24, no. 5, 2014, pp. 367-368.

11. Morisada, Naoya, et al. "Branchio-Oto-Renal Syndrome: Comprehensive Review Based on Nationwide Surveillance in Japan." Pediatrics International, vol. 56, no. 3, 2014, pp. 309-314., doi:10.1111/ped.12357.

12. Prasanth, Kaninghat, et al. "Bilateral Preauricular Pits." NeoReviews, vol. 12 , no. 10, 2011, doi:10.1542/neo.12-10-e606.

13. Smith, Richard JH. "Branchiootorenal Spectrum Disorder." Current Neurology and Neuroscience Reports., U.S. National Library of Medicine, 6 Sept. 2018, www.ncbi.nlm.nih.gov/books/NBK1380/.

\section{EP-95 NEXT GENERATION SEQENCING RESULTS IN PATIENTS WITH BILATERAL MEGAURETER \\ Zukhra Sabirzyanova, Vladimir Solodkiy, Galina Snigireva, Andrey Pavlov \\ Russuan Scientific Center Of Roentgenradiology}

Introduction: Patients with bilateral megaureter (BM) constitute the most severe group among congenital anomalies of the kidney and urinary tract (CAKUT-syndrome). Because its play a causative role of cases of end-stage renal disease, it is important to diagnose these anomalies and initiate therapy to minimize renal damage, prevent or delay the onset of ESRD, and provide supportive care to avoid complications of ESRD. Moreover, it's known that several genes and epigenetic factors have been implicated in the pathogenesis of renal malformations and CAKUTsyndrome is considered as monogenic disease.The aim of this study was to identify mutations in genes in patients with severe forms of bilateral megaureter with beginning-stage renal disease for possibility prognosis its development.

Material and methods: 25 boys in the age of 3-16 years old with BM, complicated acute renal disease in infancy and preserved renal function at the moment of investigation. Next generation sequencing (NGS) includes the analysis of encoding sequences of 4812 genes and bioinformation analysis of founded mutations with the help of genetic data bases OMIM, ClinVar and MedGen.

Results: All patients were operated step by step during the life. Among the treatment options the posterior urethral valve incision were done in 11 , temporary urinary diversion (ureterostomy, nephrostomy, internal stenting) in 22 , ureter reimplantation in 13 boys. Mandatory urological evaluation includes US, DMSA scanning, MAG3 dynamic scintigraphy, VCUG, and bladder urodynamic investigation. Abnormal mutations were found in 15 boys. Among them there were mutations which are known as pathological in different kidney or smooth muscle diseases in 18 genes: AGT, COL4A4, PKHD1, ACE, PKD1, CFH, CFHR2, COL9A3, COMP, NID1, EYA1, MYH9, FRAS1, TP63, ITGB4, MYLK, HNF1B, HSPG2. Each patient has from 1 to 3 mutations in these genes. Three of these genes: AGT, PKHD1 and ITGB4 were abnormal in not one of them. Moreover, the mutations, related to another diseases, were found in 4 from these 15 patients. There were mutations in well-known BRCA1, MAN2B1, MEFV and NSD1 genes. In 10 patients without any kidney or smooth muscles related mutations any others mutations also weren't founded.

Conclusions: In $40 \%$ patients with clinical picture of CAKUT-syndrome on the background of bilateral megaureter genetic base of disease not confirmed and they must be assessed as malformation without dysplasia. We may suppose that nongenetic patients have better prognosis for renal function failure. Further investigations will help to understand the pathogenesis of malformation better.

EP-96 HYPERTENSION AND SALT LOSS: AN UNUSUAL ASSOCIATION OF SYMPTOMS WITH A UNIQUE EXPLANATION

Giulia Ranucci ${ }^{1}$, Giovanni Russo ${ }^{1}$, Francesca Caprio ${ }^{2}$, Claudia Elefante ${ }^{3}$, Gabriele Malgieri ${ }^{4}$, Luigi Annicchiarico Petruzzelli ${ }^{4}$, Francesco Esposito ${ }^{5}$, Pierluigi Marzuillo ${ }^{6}$, Gianpaolo Santini ${ }^{7}$, Carmine Pecoraro ${ }^{4}$

${ }^{1}$ Department Of Translational Medical Science, Section Of Paediatrics, University Of Naples Federico Ii, Naples

${ }^{2}$ Department Of Medical Translational Sciences, University Of Campania Luigi Vanvitelli, Naples, Italy

${ }^{3}$ Nephrology Unit, Advanced Surgical And Medical Sciences Department Of University Of Campania Luigi Vanvitelli, Naples, Italy

${ }^{4}$ Nephrology And Dialysis Unit, Santobono-pausilipon Childrens Hospital, Naples, Italy

${ }^{5}$ Radiology Department, Santobono-pausilipon Children's Hospital, Naples, Italy

${ }^{6}$ Department Of Woman, Child, And General And Specialized Surgery, Seconda Univesità Degli Studi Di Napoli, Napoli, Italy

${ }^{7}$ Department Of Radiology, Ospedale Del Mare, Naples, Italy

Introduction: We describe a case of a child with hyponatriemic hypertensive syndrome (HHS) to emphasize the importance of early diagnosis and treatment of this condition

Material and methods: A previously healthy 16 months old child was referred for vomiting, clouding of consciousness, polyuria. Blood pressure measured 150/110 $\mathrm{mmHg}$. Laboratory tests revealed hyponatriemia, hypokalemia, and metabolic alcalosis. Urinalysis revealed proteinuria in nephrotic range, hypercalciuria, hyperuricuria, natriuresis (FENa 8.2\%) and glycosuria. He was hydrated and his electrolytes were corrected by intravenous sodium and potassium replacement. Renal sonography showed small right kidney (3.14 SD below the mean) with normal left kidney. Angio-TC and arteriography both demonstrated bilateral duplication of renal arteries with stenosis of right ones so percutaneous transmural angioplasty was performed.

Results: Symptoms and laboratory findings were completely reversed within 10 days after angioplasty and dual renin-angiotensin-aldosterone (RAA) blockade: blood pressure gradually normalized with resolution of polyuria and electrolyte imbalance $(\mathrm{FENa}<1 \%$, resolution of glycosuria and proteinuria). Postoperatively, Doppler sonography showed increase in the size of the right kidney ( $0.5 \mathrm{SD}$ below the mean $)$ with improvement in renal parenchyma vascularization and normalization of renal arterial resistive index.

Conclusions: HHS is characterized by unilateral renal artery stenosis, secondary systemic hypertension and activation of the RAA with glomerular and tubular dysfunction due to contralateral hyperfiltration. High levels of angiotensin II and subsequent "aldosterone escape" phenomenon are central to the pathogenesis of the syndrome. In our case the development of HHS allowed early detection and treatment of an underlying silent condition, so that irreversible renal damage was prevented. Although HHS has been reported often in adults, it is uncommon in children. This condition should be suspected in patients with severe hypertension associated with hyponatremia in the absence of other parameters of glomerular disease or tubulopathy. 
EP-97 CLINICAL CASE OF RENAL COLOBOMA SYNDROME IN A CHILD WITH PAX2 GENE MUTATION

I.v. Sheuchuk ${ }^{1}$, O.c. Mazur ${ }^{2}$, Sergey Baiko ${ }^{3}$, A.p. Mikhalenka ${ }^{2}$, A.v. Sukalo ${ }^{3}$, A.v. Kilchevsky ${ }^{2}$, Nicholas Yafremau ${ }^{2}$

${ }^{1}$ 2nd City Children's Clinical Hospital, Minsk, Belarus

${ }^{2}$ Institute Of Genetics And Cytology Of The National Academy Of Sciences Of Belarus

${ }^{3}$ Belarusian State Medical University

Introduction: Congenital anomalies of the kidney and urinary tract (CAKUT) are the leading cause of end stage renal disease in children. One of the variants of syndromic CAKUT is coloboma renal syndrome caused by $P A X 2$ gene mutations.

Material and methods: We describe a clinical case of renal coloboma syndrome identified by Next Generation Sequencing (NGS).

Results: A 3-year-old boy was referred to our hospital with a history of chronic kidney disease. He was the second child of healthy and nonrelated parents. He was born full-termed, bilateral renal hypoplasia was detected by ultrasound in the third trimester. In the 1 month of life, ophthalmological examination revealed a coloboma of the optic disc, which was later confirmed by MRI. Laboratory findings included mild anemia, hypocalcemia, hypomagnesemia, hyperkalemia, increased creatinine values $(80 \mu \mathrm{mol} / \mathrm{l})$ with the estimated glomerular filtration rate (eGFR) $25 \mathrm{ml} / \mathrm{min} / 1,73 \mathrm{~m}^{2}$. By the age of 3 , eGFR had improved to 52 $\mathrm{ml} / \mathrm{min} / 1,73 \mathrm{~m}^{2}$, the electrolyte composition and blood hemoglobin were within the normal range, subcompensated metabolic acidosis, growth retardation (z: -1,2) and arterial hypertension (to 110/75) were noted. By performing NGS on a patient's blood sample we identified a previously undescribed heterozygous deletion in $P A X 2$ gene c.226delG (p.G76Afs*6), which represents stopcodone mutation. This mutation is probably pathogenic, as it leads to the termination of protein synthesis. Conclusions: All children with renal hypodysplasia require an ophthalmologic examination, as approximately $10 \%$ have a point mutations in the $P A X 2$ gene with accompanying eye abnormalities.

EP-98 CLINICAL PRESENTATION OF SLOVENIAN COHORT OF CHILDREN WITH CONGENITAL ANOMALIES OF KIDNEY AND URINARY TRACT (CAKUT)

Jakob Zapušek ${ }^{1}$, Boris Zagradišnik ${ }^{2}$, Nataša Marčun Varda ${ }^{3}$

${ }^{1}$ Department Of Paediatrics, General Hospital Slovenj Gradec, Gosposvetska Cesta 1, 2380 Slovenj Gradec

2 2laboratory Of Medical Genetics, University Medical Centre Maribor, Ljubljanska 5, 2000, Maribor, Slovenia

3 3department Of Paediatrics, Nephrology Unit, University Medical Centre Maribor, Ljubljanska 5, 2000, Maribor, Slovenia

Introduction: CAKUT are common pathology in paediatric nephrology and important cause of chronic kidney disease in children. The aim of our study was to find out the clinical presentation of our children with CAKUT, their treatment and complications

Material and methods: 324 CAKUT patients with all the relevant clinical data available, were included in the study and clinically screened. In addition, cohort included patients, in whom, after parent consent, a blood sample for DNA isolation was taken, allowing further genetic studies to be compared to phenotype findings. Their clinical characteristics, diagnostic methods, treatment and complications were collected.

Results: Commonest CAKUT was vesicoureteral reflux (VUR), diagnosed in 273 children $(84.2 \%$ ), followed by hydronephrosis due to pyeloureteral junction stenosis in 76 children $(23.4 \%)$, dilation of pyelon in $43(13.3 \%)$, megaureter in $27(8.3 \%)$, duplex collecting system in 19 $(5.6 \%)$ and hypoplastic kidney in 12 included children (3.7\%). All other CAKUTs together were present less frequently, diagnosed in 32 cases (9.9\%).
Regarding diagnostics, ultrasound was done in $100 \%$ of patients, voiding cystourethrogram in $316(97.5 \%)$ and dynamic renal scintigraphy in 22 $(6.8 \%)$ of them. Urologist was consulted in $137(42.3 \%)$ cases.

Regarding treatment, observation only was sufficient in 48 (14.8\%) of patients, just antibiotic prophylaxis in $138(42.6 \%)$, endoscopic treatment of VUR with injection of synthetic or natural materials in $109(33.5 \%)$, classic surgical procedure in $29(9 \%)$ patients. Complications were few, mainly before the year 2010. Reflux nephropathy was diagnosed in 19 patients $(5.8 \%)$, hypertension in 17 patients $(5.2 \%)$ and chronic renal disease in 11 patients $(3,3 \%)$.

Conclusions: Commonest CAKUT diagnosed, was VUR. Diagnostic and treatment management was in accordance with newest global standards. Complications were rare, especially in the last decade.

EP-99 AETIOLOGY OF RENAL AND URINARY TRACT ANOMALIES DEFINES DIAGNOSTIC EFFICACY AND CLINICAL OUTCOME - STUDY PROTOCOL OF THE ARTDECO STUDY

Albertien Van Eerde ${ }^{1}$, Jaap Mulder ${ }^{2}$, Kirsten Renkema ${ }^{1}$,

Michiel Schreuder ${ }^{3}$, Loes Van Der Zanden ${ }^{4}$, Rik Westland ${ }^{5}$

${ }^{1}$ Department Of Genetics, University Medical Center Utrecht, Utrecht University, Utrecht, The Netherlands

${ }^{2}$ Department Of Pediatric Nephrology, Erasmus Mc-sophia Children's Hospital, Rotterdam, The Netherlands

${ }^{3}$ Department Of Pediatric Nephrology, Radboudumc Amalia Children's Hospital, Nijmegen, The Netherlands

${ }^{4}$ Department For Health Evidence, Radboudumc, Nijmegen, The Netherlands

${ }^{5}$ Department Of Pediatric Nephrology, Amsterdam Umc-emma Children's Hospital, Amsterdam, The Netherlands

Introduction: Congenital anomalies of the kidney and urinary tract (CAKUT) are the major cause of kidney failure in childhood and adolescence. Nevertheless, a substantially larger subset of individuals have a better prognosis of kidney function. This strong clinical variability highlights the need for accurate decision-making in the management of patients. Unfortunately, the largely unknown aetiology of CAKUT and the limited early predictability of kidney function currently hamper this. We have set up a nation-wide research framework - the ArtDECO Consortium - to define the genetic and environmental aetiology of CAKUT with the ultimate goal to improve the clinical management of patients.

Material and methods: The ArtDECO consortium will establish a nation-wide data- and biobank for CAKUT based on the existing protocols, infrastructure, and licenses of the AGORA data- and biobank. ArtDECO will include 3,750 CAKUT cases with comprehensive data on clinical parameters and foetal environmental exposures as well as DNA collections. We aim to increase the diagnostic yield of genetic testing in CAKUT patients by identification of new monogenic causes by whole-exome sequencing in a subset of patients. Next, we will perform multi-angle analyses using environmental information from questionnaires as well as genome-wide microarray data to elucidate the complex multifactorial background of study participants without a monogenic cause. By associating potential genetic factors with identified environmental risk factors, we also aim to detect genetic variants and environmental exposures that are associated with the development of kidney injury among CAKUT patients. Finally, genetic and environmental factors will be functionally characterized by combining innovative modelling using patient-specific induced-pluripotent stem cells and human kidney organoids with tailor-made animal-based modelling.

Results: N/A

Conclusions: The ArtDECO study is a large-scale national multicentre study that integrates genetics, environmental exposures and clinical data for the identification of molecular causes of kidney and urinary tract malformations and potential prognostic factors for kidney injury. 
EP-100 UNILATERAL PERIRENAL MASS IN A NEWBORN Özgür Özdemİr Şimşek ${ }^{I}$, SeçIl Arslansoyu Çamlar ${ }^{2}$, Gökçen Erfidan ${ }^{1}$, Yeli̇z Pekçevik ${ }^{3}$, Cemallye Bașaran ${ }^{1}$, Demet Alaygut ${ }^{1}$, Fatma Mutlubaș ${ }^{2}$, Mehmet Yekta Öncel ${ }^{4}$, Belde Kasap Demir ${ }^{5}$

${ }^{1}$ University Of Healthy Sciences Tepecik Training And Research Hospital Department Of Pediatrics, Division Of Nephrology, Izmir, Turkey; ${ }^{2}$ University Of Health Sciences, Izmir Faculty Of Medicine Department Of Pediatrics, Division Of Nephrology; ${ }^{3}$ University Of Health Sciences, Tepecik Training And Research Hospital, Department Of Radiology; ${ }^{4}$ Izmir Katip Çelebi University Medical Faculty, Department Of Pediatrics, Division Of Neonatology, Izmir, Turkey; ${ }^{5}$ Izmir Katip Çelebi University Medical Faculty, Department Of Pediatrics, Division Of Nephrology And Rheumatology, Izmir, Turkey

Introduction: Perirenal fluid collection in children may be due to urinoma or lymphangiectasis. Renal lymphangiectasis is a rare disease that gives the appearance of a cystic mass characterized by malformation in the lymphatics. Urinoma is usually traumatic or iatrogenic; it is rare in newborns. A male patient with posterior urethral valve(PUV) who developed anuric acute kidney injury(AKI) due to perirenal cystic fluid collection is presented.

Material and methods: A male newborn born in the neonatal intensive care unit at the $36^{\text {th }}$ week was admitted to the neonatal intensive care unit due to diffuse free intra-abdominal fluid and bilateral increased renal echogenicity in antenatal ultrasonography. The physical examination revealed birth weight of $2650 \mathrm{~g}$ (50-90p), height of $50 \mathrm{~cm}(90-99 \mathrm{p})$, and head circumference of $34 \mathrm{~cm}$ ( 90 percentile), and blood pressure of $62 / 39 \mathrm{mmHg}(50 / 50-75 \mathrm{p})$. He had $3 \mathrm{cc} / \mathrm{kg} / \mathrm{h}$ urine output. Renal ultrasonography revealed right perirenal anechoic cystic mass that compressed the kidney and was compatible with renal lymphangectasis. The renal parenchyma was normal with mild dilatation of the pelvis and calices. The right ureter could not be visualized. The left kidney had dilatation of the pelvis and calices. The left ureter was dilated and tortuous throughout its course. On postnatal day $3^{\text {rd }}$, the patient suddenly had abdominal distention and anuria. Serum creatinine increased to $1.5 \mathrm{mg} / \mathrm{dL}$.

Results: The magnetic resonance urography (MRU) showed perirenal cystic mass with thin septations and bilateral hydroureteronephrosis (Fig1). There was also intra-abdominal free fluid and mild trabeculation and thickening of the bladder wall. Chemical analysis drained fluid was revealed as; cholesterol $1 \mathrm{mg} / \mathrm{dL}$, triglyceride $3 \mathrm{mg} / \mathrm{dL}$, total protein $0.6 \mathrm{~g} /$ $\mathrm{dL}$, albumin $0.1 \mathrm{~g} / \mathrm{dL}$, glucose $14 \mathrm{mg} / \mathrm{dL}$, sodium $54 \mathrm{mmol} / \mathrm{L}$ and potassium $10.1 \mathrm{mmol} / \mathrm{L}$. The $\mathrm{pH}$ was 8 , the density was 1019 . It was urinoma. Urine output restarted after drainage and creatinine regressed to $0.9 \mathrm{mg} /$ dL. PUV was considered, since the patient with postrenal AKI had hydroureteronephrosis, increased bladder wall thickness was observed and the fluid sample in the perirenal area was compatible with urinoma. PUV was diagnosed by cystoscopy and resected. Urinoma did not recur after resection.

Conclusions: Fluid sampling is important in differentiating urinoma and lymphagectasis. PUV, which requires early diagnosis and intervention, should not be forgotten in the differential diagnosis of male newborns with urinoma.

\section{EP-101 EXPERIENCE IN THE USE OF MINIMALLY INVASIVE EQUIPMENT IN THE SURGICAL TREATMENT OF CONGENITAL MALFORMATIONS OF THE URINARY TRACT IN CHILDREN IN A TERTIARY REGIONAL HOSPITAL}

Dmytro Shevchuk

Zhytomyr Regional Childs Clinical Hospital

Introduction: Minimally invasive surgeries (MIS) are becoming more common due to the minimization of traumatic urinary tract injuries. Such interventions become especially relevant in childhood.
Recently, endoscopic methods of treatment of diseases of the upper urinary tract are being discussed more often. The issue of endoscopic correction of vesicoureteral reflux in children is widely covered in the modern medical literature. Successful use of minimally invasive endourological interventions in the valves of the posterior urethra is noted by many authors.

Material and methods: MIS interventions for congenital pediatric urological diseases have been introduced on the basis of surgical departments of the Zhytomyr Regional Childrens Clinical Hospital since 2000. Pediatric models of endoscopic equipment are used for it.

The range of surgical interventions is quite wide: laparoscopic pyeloplasty \& nephrectomy, laparoscopic and retroperitoneal excision of renal cysts, correction of vesicoureteral reflux, introduction of Botulinum toxin A into the detrusor, removal / installation of ureteral stents, dissection / puncture of ureterocele, dissection of valves / strictures of the urethra, etc.

Results: A total of 346 surgical interventions were performed. Age of children from 1 month of life. The duration of interventions decreased with the growth of surgical practice, the advent of endoscopic equipment. The use of MIS has made it possible to reduce trauma to the urinary tract, and minimize postoperative pain and blood loss, as well as faster recovery of the urinary tract. Such interventions are especially important in the elimination of diseases that lead to impaired evacuation function of the bladder. During the introduction of MIS (laparoscopic pyeloplasty) there were 1 complication (dysfunction of the pyeloureteral anastomosis, which required only short-term drainage of the abdominal cavity). There were no fatal complications.

Conclusions: Thus, MIS are a good alternative to open surgery in congenital pathology of the childs urinary system, including in violation of the reservoir and / or evacuation function of the bladder.

Often, with the help of modern minimally invasive equipment, it is possible to perform such surgical interventions that cannot be performed by open methods of surgical intervention.

\section{EP-102 RARE UNOPERATED SEVERE MYELOMENINGOCELLE WITH KIDNEY AGENESIS}

Valbona Stavileci, Rinor Bejiqi, Fitore Abazi

Pediatric Clinic, University Clinical Centre Ofkosovo

Introduction: A boy of 16teen with Myelomeningocele never operated for, paraplegic with severe spine deformity which represents the natural evolution of spina bifida anomaly. Ever treated for his neurogenic bladder and bowel.

Material and methods: Boys weight was $30 \mathrm{~kg}, \mathrm{HC}: 56.5 \mathrm{~cm}$, length hands finger-finger: $163 \mathrm{~cm}$, head-waist: $53 \mathrm{~cm}$, waist-foot $60 \mathrm{~cm}$. He was frightened for his health and life, never intended school but able to read and write. He had some blurred vision and loss of perception for a few seconds throw the day. And episodes of vomiting were frequent. He had severe bilateral inguinal hernia. Increased inflammatory parameters.

Results: Scintigram: left kidney afunctional type. Right kidney increased size, decreased accumulation and prolonged parenchymal transport time and half elimination time. GFR: $51.4 \mathrm{ml} / \mathrm{min}$

MR detected severe L/S angular kyphosis, positive "Baltalimani" on segments above and under the angulation, Cobb angle is $>90^{\circ}$. Intraspinal structures (the thecal sac) through angulation are herniated, with tethered cord at L4-L5. Thecal-sac was intraabdominal. Severe sinistro-convex scoliosis. Siringomielia Th3-Th5. Modulla oblongata and cerebellum Herniation, $30 \mathrm{~mm}$ though foramen magnum with obstructive hydrocephalus-Chiari II. A skin fistulation not clearly determined, was natural way to decompress the ICL.

ENG: no amplitude of nerve conduction. EMG: fibrillation, fasciculation's and positive denervated waves.

Abdominal MR: left kidney agenesis. Right kidney was enlarged with gr $\mathrm{V}$ hydronephrosis with severe megaureter. Bladder enlarged with diverticulosis and thick and hypervasculated bladder wall. 
Cystometry and found noncompliance bladder with high pressures at $30 \mathrm{ml}$ of filling. Pressures at beginning of testing were $30 \mathrm{cmH} 2 \mathrm{O}$ and at the end $57 \mathrm{cmH} 2 \mathrm{O}$. Low bladder capacity $90 \mathrm{ml}$, incontinence at $75 \mathrm{ml}$ and residual $100 \mathrm{ml}$, no filling sensation, no voiding reflex. Bladder Cooling Test negative.

Conclusions: Parents left this boy un-operated, as were told he will pass way soon after birth. Raising awareness that MMC anomalies could live a normal life if they get medical support early in life is important.

\section{EP-103 COVID-19 INFECTION IN CHILDREN WITH CHRONIC KIDNEY DISEASE}

Mine Erkan ${ }^{1}$, Gülşah Kaya Aksoy ${ }^{2}$, Bedirhan Satici ${ }^{1}$, Mehtap Adar ${ }^{2}$, Ayla Kaçar ${ }^{2}$, Muhammet Sancaktar ${ }^{2}$, Mustafa Koyun ${ }^{2}$, Elif Çomak ${ }^{2}$, Sema Akman ${ }^{2}$

${ }^{1}$ Akdeniz University Medical Faculty, Department Of Pediatrics, Antalya ${ }^{2}$ Akdeniz University Medical Faculty, Department Of Pediatric Nephrology, Antalya

Introduction: Data on COVID-19 infection in children with chronic kidney disease (CKD) is limited. We aimed to share the frequency and the clinical status of COVID-19 infection in patients with CKD at our center.

Material and methods: Pediatric patients who were on the waiting list for kidney transplantation in February 2021 were included in the study. A questionnaire was applied to the patients or their parents who agreed to participate. The questionnaire sought information about demographic characteristics, primary kidney disease, transplantation status, renal replacement therapy, comorbid diseases, medications used, in the last 6 months COVID-19 contact and COVID-19 disease.

Results: 44 children were included in the study; 28 (63.6\%) were male, the median age was 11.2 years (IQR 5.16-14.97). The most common cause of chronic kidney disease was CAKUT ( $\mathrm{n}=15$ 34,1\%). Seventeen $(38.6 \%)$ of the patients were on hemodialysis, $21(47.7 \%)$ peritoneal dialysis, and $6(13.6 \%)$ were on CKD stage 4 . Five $(11,4 \%)$ had a history of previous graft loss. None of the patients were receiving immunosuppressive therapy. Hypertension was present in $26(59.1 \%)$ of the patients. In the last 6 months, 19 (43.2\%) of patients had symptoms that could be associated with COVID-19, the most common being fever ( $\mathrm{n}=1578,9 \%$ ) and cough $(\mathrm{n}=746,7 \%)$. PCR test was applied to a total of $25(56,8 \%)$ patients. PCR test was performed for $15(34.1 \%)$ of the patients due to suspicion of COVID-19 and for screening in 18 (40.9\%). Two patients $(4,5 \%)$ had COVID PCR positive. None of them needed hospitalization and additional treatment was not required.

Conclusions: The frequency of COVID-19 in children with chronic kidney disease was relatively low and the clinical symptoms were mild in those with COVID-19.

\section{EP-104 THROMBOTIC MICROANGIOPATHY IN A SEVERE PAEDIATRIC CASE OF COVID-19}

Tahir Dalkiran ${ }^{1}$, Yasar Kandur ${ }^{2}$, Besra Dagoglu ${ }^{3}$, Sevgin Taner ${ }^{4}$, Dogan $\mathrm{Oncu}^{5}$

${ }^{1}$ Department Of Pediatric Intensive Care, Necip Fazil City Hospital, Kahramanmaras, Turkey

${ }^{2}$ Department Of Pediatrics, Faculty Of Medicine, Kirikkale University, Kirikkale, Turkey

${ }^{3}$ Department Of Radiology, Necip Fazil City Hospital, Kahramanmaras, Turkey

${ }^{4}$ Department Of Pediatric Nephrology, Adana City Hospital, Adana, Turkey

${ }^{5}$ Department Of Pediatrics, Necip Fazil City Hospital, Kahramanmaras, Turkey

Introduction: Coronavirus disease 2019 (COVID-19) has become a public health threat to people all over the world during the last year. Acute respiratory distress syndrome (ARDS), septic shock, and coagulation dysfunction are severe complications of this infection, but such severity is rare in children. COVID-19 is also associated with a thrombotic state with increased risk of thrombosis and disseminated intravascular coagulation (DIC). However, there has not yet been definitive evidence linking COVID-19 with thrombotic microangiopathies(TMA) such as hemolytic uremic syndrome (HUS). In this case report, we aimed to report a pediatric patient with COVID-19 and HUS. This is a rare COVID-19 presentation of its kind.

Material and methods: A three-year-old girl with fever and respiratory distress was admitted to the hospital. The patients COVID PCR test resulted positive; thus, hydroxychloroquine and favipiravir were added to the treatment. As her respiratory distress increased and blood gas indicated deep respiratory acidosis (pH:6.96, pCO2:91 mmHg, $\mathrm{HCO} 3: 22$ $\mathrm{mEq} / \mathrm{L}$ ) on the 3 rd day of the pediatric intensive care unit follow-up, the patient was intubated and ventilated. Thorax computerized tomography (CT) showed bilateral effusion and atelectasis. During her follow up, the pleural effusion resolved but there were marked consolidation areas and ground glass appearances compatible with COVID-19 on the followup CT. On the 10th day, she became anuric and developed progressive thrombocytopenia and persistent anemia, which were suggestive of HUS given a high creatinine level $(1.9 \mathrm{mg} / \mathrm{dl})$, an undetectable haptoglobuline level, reticulositosis (\%8), and a LDH level of $2540 \mathrm{U} / \mathrm{L}$. Direct Coombs test returned negative. Examination of a peripheral blood smear revealed schistocytes. In the light of the available findings, we considered the patient to have HUS possibly triggered by COVID-19. Hence, plasmapheresis was performed with fresh frozen plasma (FFP). LDH values started to fall and blood pressure was reduced. Her platelet count also started to rise. After 4 weeks, she became completely asymptomatic, and her hemodynamic parameters normalized.

Results:

Conclusions: COVID-19 patients are at potential risk of HUS. To confirm this association, a renal biopsy can provide valuable information about the pathogenesis HUS in COVID-19 infection. Further research should be directed at assessing the role of complement in COVID-19 and its association with hyperinflammatory and prothrombotic states.

EP-105 COVID-19 IN CHILDREN WITH CHRONIC DIALYSIS AND KIDNEY TRANSPLANTATION: A MULTICENTER STUDY FROM ISTANBUL

Nur Canpolat ${ }^{1}$, Zeynep Yürük Yildirim ${ }^{2}$, Nurdan Yildiz ${ }^{3}$,

Mehmet Taşdemir ${ }^{4}$, Nilüfer Göknar ${ }^{5}$, Havva Evrengül ${ }^{6}$,

Rüveyda Gülmez ${ }^{1}$, Bağdagül Aksu ${ }^{2}$, Hasan Dursun ${ }^{7}$, Gül Özçelik ${ }^{8}$,

Önder Yavașcan ${ }^{9}$, Rümeysa Yasemin Ciçek ${ }^{10}$, Sebahat Tülpar ${ }^{11}$,

Duygu Övünç Hacihamdio Ğlu ${ }^{12}$, Ahmet Nayir ${ }^{2}$, Harika Alpay ${ }^{3}$

${ }^{1}$ Department Of Pediatric Nephrology, Istanbul University-cerrahpaŞa, CerrahpaŞa Faculty Of Medicine, Istanbul, Turkey

${ }^{2}$ Department Of Pediatric Nephrology, Istanbul University Faculty Of Medicine, Istanbul, Turkey

${ }^{3}$ Department Of Pediatric Nephrology, Marmara University Faculty Of Medicine, Istanbul, Turkey

${ }^{4}$ Department Of Pediatric Nephrology, Koç University Hospital, Istanbul, Turkey

${ }^{5}$ Department Of Pediatric Nephrology, Istanbul Medeniyet University, Faculty Of Medicine, Istanbul, Turkey

${ }^{6}$ Department Of Pediatric Nephrology, Istinye University Liv Hospital Bahçeşehir, Istanbul, Turkey

${ }^{7}$ Department Of Pediatric Nephrology, Prof. Dr. Cemil Taşçioğlu City Hospital, Istanbul, Turkey

${ }^{8}$ Department Of Pediatric Nephrology, Istanbul ŞiŞli Hamidiye Etfal Education And Research Hospital, Istanbul, Turkey

${ }^{9}$ Department Of Pediatric Nephrology, Medipol University Hospital, Istanbul, Turkey

${ }^{10}$ Department Of Pediatric Nephrology, BaȘakȘehir Çam And Sakura City Hospital, Istanbul, Turkey 
11 Department Of Pediatric Nephrology, Bakirkoy Dr. Sadi Konuk Training And Research Hospital, Istanbul, Turkey

${ }^{12}$ Department Of Pediatric Nephrology, Acibadem University Hospital, Istanbul, Turkey

Introduction: We aimed to present incidence of COVID-19 in children with renal replacement therapy (RRT) and compare the severity and outcomes of the disease between the groups undergoing dialysis and kidney transplantation (KTx).

Material and methods: This multicenter observational study was based on data collected from children receiving RRT, either chronic dialysis or KTx, diagnosed with COVID-19. All members of the Istanbul branch of the Turkish Pediatric Nephrology Association were requested to submit all their confirmed COVID-19 cases between April 1 and December 31, 2020. They were also asked to report the number of their prevalent patients on RRT under the age of 20.

Results: A total of 46 RRT patients diagnosed with COVID-19 were reported from 12 centers. Of these, 29 were KTx recipients, and 17 were on dialysis ( 10 hemodialysis and 7 peritoneal dialysis). COVID19 cases represented $9.3 \%$ of all prevalent dialysis patients and $9.2 \%$ of all prevalent KTx recipients. Twelve KTx recipients $(41.4 \%)$ and three dialysis patients $(17.6 \%)$ were asymptomatic $(\mathrm{p}=0.12)$. The hospitalization rate was higher in dialysis patients than KTx recipients $(82.4 \%$ vs. $27.6 \%, \mathrm{p}=0.001)$. Two patients, one in each group, experienced a severe disease course, and only one hemodialysis patient had a critical illness that required mechanical ventilation. Acute kidney injury developed in $36 \%$ of KTx recipients, but none of them required dialysis or lost their graft. In the entire cohort, one patient on hemodialysis with multiple comorbidities died.

Conclusions: Although most of cases were asymptomatic or had a mild disease, children on RRT seem to have an increased risk for COVID-19.

\section{EP-106 IMPACT OF CORONAVIRUS DISEASE-2019 ON PEDIATRIC NEPHROLOGY PRACTICE AND EDUCATION: AN ESPN SURVEY \\ Burcu Yazicioğlu, Sevcan Bakkaloğlu \\ Gazi University Department Of Pediatric Nephrology}

Introduction: Coronavirus disease 2019 (COVID-19) is challenging for patients and medical staff. Radical changes were implemented for preventing disruptions in patient care as well as medical education.

Material and methods: A web-based survey was sent to European Society for Pediatric Nephrology (ESPN) members via the ESPN mailing list to evaluate the effects of the COVID-19 pandemic on delivery of pediatric nephrology $(\mathrm{PN})$ care and educational activities. There were ten questions and their subheadings.

Results: Seventy-six centers from 24 countries were enrolled. Significant decreases in number of patients' admissions in the nephrology wards and outpatient clinics (2.2 and 4.5 times) were noted. Tele-medicine tools including telephone/visual calls, whatsapp/e-mail correspondences, electronic prescriptions and online applications for off-label drugs were used in $40-60 \%$ of the centers. Despite staff training and protective measures, $44 \%$ of centers reported COVID-19 infected staff, whereas $24 \%$ centers reported infected patients. Difficulties in receiving pharmaceuticals and health related products were reported in $19 \%$ of centers. Half of the centers suspended living related kidney transplantation and one third cadaveric transplantation. Hands-on education have been suspended in $84 \%$ of medical schools and face to face teaching almost completely replaced by online system (89\%). Multidisciplinary training in PN was also largely affected.

Conclusions: This survey showed a sharp decline in patient admissions and a significant decrease in kidney transplantation. Tele-medicine became an important tool in outpatient clinics. Almost solely online medical education and limitations in multidisciplinary meetings in PN training is an area of debate. The prolonged and fluctuating course of the pandemic may pose additional challenges deserving close attention. While awaiting safe and effective vaccines or treatment options, staff infections are alarming and requires further precautions.

\section{EP-107 COVID-19 ASSOCIATED THROMBOTIC MICROANGIOPATHY}

Esra Karabă̆ Yilmaz ${ }^{1}$, Iremnaz Karahan ${ }^{2}$, Memnune Nur Cebi ${ }^{2}$, Seda Aksoy ${ }^{2}$, Cansu Durak ${ }^{3}$, Fatih Aygün ${ }^{3}$, Salim Çalişkan ${ }^{1}$

${ }^{I}$ Department Of Pediatric Nephrology, Istanbul University-cerrahpasa, Cerrahpasa Faculty Of Medicine, Istanbul, Turkey

${ }^{2}$ Department Of Pediatrics, Istanbul University-cerrahpasa, Cerrahpasa Faculty Of Medicine, Istanbul, Turkey

${ }^{3}$ Department Of Pediatric Intensive Care Unit, Istanbul Universitycerrahpasa, Cerrahpasa Faculty Of Medicine, Istanbul, Turkey

Introduction: Coagulopathy and microangiopathic disease occur due to coronavirus disease-2019 (COVID-19) caused by severe acute respiratory syndrome coronavirus 2 (SARS-CoV-2). So far, there have been a few reported cases of thrombotic microangiopathy (TMA) associated with COVID-19.

Material and methods: We present two children with COVID-19 related TMA.

Results: The first case was a 23-month-old girl, who admitted to the hospital with watery diarrhea and vomiting. Her physical examination revealed fever, edema, and severe hypertension. She was anuric and required hemodialysis. The PCR test and IgG for SARS-CoV-2 were negative, but IgM was positive. The second case was an 8-month-old boy, who admitted with vomiting and focal seizure. He had a history of close contact with a family member with confirmed COVID-19. On admission, his physical examination was unremarkable, except a low-grade fever. The PCR test for SARS-CoV-2 was positive. On the third day of hospitalization, oliguria and anemia developed.

Laboratory findings of both cases revealed non-immune hemolytic anemia, thrombocytopenia, and acute kidney injury. Their coagulation tests (prothrombin time and partial thromboplastin time), ADAMTS13 activity, and serum complement levels were within normal ranges. Stool tests for shiga-toxin producing Escherichia Coli were negative.

Eculizumab was administered to the first case who had no response to plasmapheresis. Hemolytic episode stopped three days after eculizumab, kidney functions improved, and hemodialysis was discontinued on the 20th day of hospitalization. The second case was successfully treated with plasmapheresis. He did not need dialysis. At the last follow-up, both children had normal kidney functions without proteinuria or hypertension.

Conclusions: As a result, COVID-19 infection can be a trigger for TMA like influenza and streptococcus pneumoniae.

\section{EP-108 A CASE OF GRANULOMATOSIS OF POLYANGIITIS PRESENTING WITH COVID-19 INFECTION: FALSE- POSITIVITY OR CO-EXISTENCE?}

Ibrahim Gokce, Mehtap Sak, Sercin Guven, Serim Pul, Harika Alpay

Marmara University Medical School, Istanbul

Introduction: Coronavirus disease 2019 is declared as a pandemic and public health emergency all around the world. COVID-19 has nonspecific symptoms of upper airway and pulmonary system which can overlap with other diseases having respiratory system involvement. Granulomatosis with polyangiitis (GPA), is an uncommon ANCA associated vasculitis presenting with at least three of the following six criteria; granulomatous inflammation, upper airway, pulmonary, renal system involvement, laryngo-tracheo-bronchial obstruction and positive cytoplasmic ANCA. Both COVID-19 and GPA have a high morbidity and mortality rate, that indicates the importance of rapid diagnosis and treatment. Evaluation through real-time reverse transcriptase polymerase chain reaction (RT-PCR) is currently recommended method for the diagnosis of 
COVID-19. In addition to RT-PCR, antibody tests help to diagnose both previous and current infection.

Material and methods: We present a a thirteen year old girl who was previously healty, presented with upper respiratory tract symptoms and pulmonary involvement and progressed to acute kidney failure. Computed tomography (CT) of the lungs depicted multiple nodules, cavities and ground glass opacities (GGOs) We collected upper respiratory nasopharyngeal swab on two alternate days and RT-PCR tests were negative for SARS-CoV-2. Because of progression of respiratory findings and subfebrile status, we searched immunglobuline levels for SARS-CoV-2 twice and found that IgM levels were positive $(1+)$ in both consecutive tests and $\mathrm{IgG}$ level was negative in the first test and returned to weak positive in the second test whereas RT-PCR test repeated for the third time was negative again. According to positive c-ANCA, upper airway, pulmonary and renal involvement, she was diagnosed as GPA.

Results:

Conclusions: It is important to make a rapid differential diagnosis of COVID-19 infection with chronic diseases especially those presenting with respiratory symptoms like GPA. This report highlights that COVID-19 antibody tests can be false positive in patients with autoimmune disease including Granulomatosis with polyangiitis.

\section{EP-109 SODIUM IMBALANCE IN CHILDREN WITH COVID-19 INFECTION}

Sofia Karagiannidou ${ }^{1}$, Vasiliki Karava ${ }^{1}$, Antonia Kondou ${ }^{1}$, John Dotis ${ }^{1}$, Eleni Papadimitriou ${ }^{2}$, Athina Papadopoulou ${ }^{I}$, Nikoleta Printza ${ }^{1}$

${ }^{1}$ Pediatric Nephrology Unit, 1st Department Of Pediatrics, Hippokratio General Hospital, Aristotle University Of Thessaloniki, Greece

${ }^{2}$ Pediatric Infectious Disease Unit, 1st Department Of Pediatrics, Hippokratio General Hospital, Aristotle University Of Thessaloniki, Greece

Introduction: SARS-CoV-2 infection in children is less severe compared to adult population, expect from cases of Multisystem Inflammatory Syndrome (MIS-C). Sodium homeostasis imbalance is commonly described in COVID-19 patients, however, the possible correlation with MIS-C remains unclear in pediatric population.

Material and methods: We conducted an observational single-center study. Children with confirmed COVID-19 were eligible. After hospital admission full blood count, serum biochemical profile and urine biochemical profile on urine sample was performed. Fractional excretions, blood/urine osmolarity and proteinuria were calculated. Length of hospital stay (LOS) was recorded.

Results: A total of 30 children (20 males, median age 6 years), were studied, 23 were symptomatic $(23.3 \%)$ and 5 presented MIS-C (16.7\%). Moreover, 5 children were hyponatriemic (16.7\%) while no other pathological blood or urine biochemical values were observed. All patients with hyponatremia were euvolemic with urinary osmolarity $>100$ mosm $/ \mathrm{kg}$ (median level $285 \mathrm{mosm} / \mathrm{kg}$ ) and urinary sodium $>20 \mathrm{mEq} / \mathrm{L}$ (median level $112 \mathrm{mEq} / \mathrm{L}$ ), indicative of syndrome of inappropriate antidiuretic hormone. Serum sodium was negatively correlated to maximum fever $(\mathrm{r}=-0.61, \mathrm{p}=0.006)$ and $\operatorname{LOS}(\mathrm{r}=-0.342, \mathrm{p}=0.0750)$ and was negatively associated with subsequent diagnosis of MIS-C (OR 0.334 , $95 \%$ CI $0.115-0.969, \mathrm{p}=0.044)$. Hyponatriemia was strongly associated with the presence of MIS-C $(\mathrm{p}=0.001)$. Multiple regression analysis, showed that blood serum sodium values $(\mathrm{p}<0.001)$ and sex (female) $(p=0.03)$ were independently associated with presence of MIS-C.

Conclusions: Children with COVID-19 infection may present hyponatriemia, probably due to syndrome of inappropriate antidiuretic hormone, especially in case of MIS-C. Further research around the explanatory pathophysiological paths of this sodium balance disorder is indicated.
EP-110 SARS-COV-2 INFECTION IN PEDIATRIC KIDNEY TRANSPLANT RECIPIENTS

Gülşah Kaya Aksoy ${ }^{1}$, Mehtap Adar ${ }^{1}$, Ayla Kaçar ${ }^{1}$, Muhammet Sancaktar ${ }^{1}$, Tuğçe Tural Kara ${ }^{2}$, Elif Çomak ${ }^{1}$, Mustafa Koyun ${ }^{1}$, Derya Mutlu ${ }^{3}$, Bülent Aydinli ${ }^{4}$, Sema Akman ${ }^{1}$

${ }^{1}$ Akdeniz University, Medical Faculty, Department Of Pediatric Nephrology, Antalya

${ }^{2}$ Akdeniz University Faculty Of Medicine, Department Of Pediatric Infectious Diseases, Antalya

${ }^{3}$ Akdeniz University Faculty Of Medicine, Department Of Medical Microbiology, Antalya

${ }^{4}$ Akdeniz University Faculty Of Medicine, Department Of General Surgery, Tuncer Karpuzoglu Organ Transplantation Center

Introduction: The SARS-CoV-2 virus has caused a new type of coronavirus disease (COVID-19), which has affected all ages. We aimed to share the clinical characteristics of kidney transplant recipients diagnosed with COVID-19 at our center.

Material and methods: Among pediatric kidney transplant recipients on follow-up, the patients with a diagnosis of COVID-19 confirmed by PCR in the nasopharyngeal sample were included in the study. The most common immunosuppressive modification was to increase the prednisolone dose with MMF dose reduction/withdrawn.

Results: COVID-19 PCR was positive in 13 of 158 (8.2\%) kidney transplant recipients who were followed up during the pandemic period. Nine $(69.2 \%)$ of 13 patients were girls; mean age was $13.4 \pm 4.6$ years. Three patients $(23.0 \%)$ had a deceased donor; nine patients received induction therapy with basiliximab $(n=3)$ or anti-thymocyte globulin $(A T G)(n=6)$. 8 of 13 patients had mild symptoms; the most common symptom was fever $(76.9 \%)$. Two patients had severe symptoms; one of them was 6year-old boy, who had at post-transplant 32 days at the diagnosis of COVID with living donor recipient; he had acute respiratory distress syndrome and multisystem inflammatory syndrome. MMF and tacrolimus treatment was interrupted, steroid dose was increased; however, as no response was observed, plasma exchange and intravenous immunoglobulin were administered. The other was a 17 -year-old girl; COVID PCR positivity was detected on the 5th day after pulse methyl prednisolone and ATG treatment with a diagnosis of acute cellular rejection. High flow oxygen support was required to the patient with severe pneumomia. No graft or patient loss was observed; there was no difference between the eGFR before and at the time of COVID-19 diagnosis $(83.54 \mathrm{ml} / \mathrm{min} /$ $1.73 \mathrm{~m}^{2}$ vs $76.12 \mathrm{ml} / \mathrm{min} / 1.73 \mathrm{~m}^{2}, \mathrm{p}=0.168$ ).

Conclusions: COVID19 infection was present with mild symptoms in most of the pediatric kidney transplant recipients. Clinical findings may be severe in early post-transplant period. Early diagnosis and modification of immunosuppressive therapy may be important.

\section{EP-111 KIDNEY IMPAIRMENT IN PEDIATRIC COVID-19: ABOUT A SERIES OF 13 CHILD}

Houda Nassih, Rabiy El Qadiry, Aicha Bourrahouat, Imane Ait Sab

$B$ Pediatric Ward, Department Of Pediatrics, Child And Mother Hospital, Mohammed Vi University Hospital Center, Marrakesh, Morocco

Introduction: We describe the clinical phenotype and evolution of kidney impairment $(\mathrm{KI})$ related to COVID-19 in children

Material and methods: A single center retrospective study of 13 children with KI related to COVID-19

Results: The mean age was of 9-year-old. Medical history found a Churg-Strauss syndrome, auto-immune hepatitis, CAKUT with stage 4 CKD, ARPKD with ESRD on hemodialysis, recurrent pancreatitis and nephrosis in one case each. Renal involvement was an anuria in $7.7 \%$, oliguria in $23 \%$, hemoglobinuria in $7.7 \%$, proteinuria in $38.5 \%$, hematuria in $46 \%$ (microscopic in $30.8 \%$ and gross in $15.4 \%$ ), leukocyturia in $38.5 \%$, and glycosuria in $7.7 \%$. One child presented a relapse of his 
idiopathic nephrotic syndrome, and two had a nephritic syndrome. KI was caused by an acute urine retention in one case (CAKUT), dehydration in two cases, acute glomerulonephritis in one case, rapidly progressive glomerulonephritis in one case, acute tubulointerstitial nephritis in 6 cases, and thrombotic microangiopathy in one case. KI was classified according to the pediatric-RIFLE into kidney injury in 3 cases and kidney failure in 7 cases. Two cases had hypovolemia with isolated high uremia and one case of CKD had a decline of eGFR. Type 1 and type 2 renal tubular acidosis were present in one case each. Renal biopsy found features of a nonspecific pyelonephritis, hemolytic and uremic syndrome, acute glomerulonephritis with endocapillary deposits of IgG and C3, and chronic tubulointerstitial nephritis in one case each. Steroids were given IV in 7 cases and/or oral in 8 cases. Tocilizumab and immunoglobulins were given in one case each. Hemodialysis was started in three cases during the acute phase. One child had a remaining stage 2 CKD.

Conclusions: Pediatric COVID-19 can cause very serious KI with good prognosis in the long term

\section{EP-112 WHAT MATTERS AT THE END? PAEDIATRIC COVID- 19 MORTALITY}

Joseph Mcallister, Amanda Newnham, Hitesh Prajapati, Kay Tyerman, Eric Finlay, Sergio Camilo Lopez Garcia, Pallavi Yadav

Leeds Childrens Hospital

Introduction: We have little experience of managing COVID-19 mortality in our cohort; therefore we must share our cases and learn from each other.

Material and methods: We detail a case of COVID-19 mortality in a tertiary paediatric hospital, highlighting specific challenges.

Results: Our patient was a 17-year-old male with Lowe's syndrome and severe learning difficulties, from a Punjabi speaking family. He presented with four days of cough. He was hypoxic and had bilateral pneumonia on $\mathrm{x}$-ray and was positive for covid- 19 . He deteriorated, was admitted to intensive care and ventilated on maximal support. Unfortunately his parent also tested positive, his remaining family were symptomatic and prevented from visiting.

Over the next four days he received dexamethasone, proning, inotropes and blood products. He deteriorated further, developing AKI 3, and coagulopathy. The multidisciplinary team did not believe renal replacement therapy was in his best interests and recognised that he was dying. The team communicated this to his family on a three-way telephone call with an interpreter. There was no advanced care plan. The palliative team and hospice were consulted and a care plan was made, to give him a "good" death, with family present virtually. A memory box was made including photographs, hand casts and footprints. He was compassionately extubated and passed away peacefully.

Conclusions: COVID-19 has resulted in children presenting late, as may have been the case here. Although COVID-19 rarely causes critical illness in children, this patient could be considered high risk (male, ethnic minority, low socioeconomic background, learning disabilities). Paediatricians should discuss advanced care planning with families of patients at high risk of COVID-19 mortality. COVID-19 has brought a new challenge and pressure to caring for dying patients. We cannot be perfect under these extraordinary circumstances but technology can allow families to be involved at the end of life.

\section{EP-113 COVID-19 IN PEDIATRIC NEPHROLOGY CENTERS IN TURKEY}

Sevcan A. Bakkaloğlu ${ }^{1}$, Yeşim Özdemir Atikel ${ }^{2}$, Emre LeventoĞlu ${ }^{l}$, Hülya Nalçacioğlu ${ }^{3}$, Ismaill Dursun ${ }^{4}$, Hakan PoyrazoĞlu ${ }^{4}$, Hasan Dursun ${ }^{5}$, Zeynep Yürük Yildirim ${ }^{6}$, Nurdan Yildiz, ${ }^{7}$, Gülşah Kaya Aksoy ${ }^{8}$, Sema Akman ${ }^{8}$, Mehmet Taşdemir ${ }^{9}$, Ilmay Bilge ${ }^{9}$, Mehtap Çelakil ${ }^{10}$, Beltinge Demircioğlu Kiliç ${ }^{11}$, Şenay Zirhli Selçuk ${ }^{12}$, Nur Canpolat ${ }^{13}$, Evrim Kargin Çakici ${ }^{14}$, Sare Gülfem Özlü ${ }^{15}$,
Sebahat Tülpar ${ }^{16}$, Selçuk Yüksel ${ }^{17}$, Bahriye Atmiş ${ }^{18}$, Serra Sürmeli Döven ${ }^{19}$, Sevgin Taner ${ }^{20}$, Pelin Ertan ' ${ }^{21}$, Asli Kavaz ${ }^{22}$, Meral Torun Bayram ${ }^{23}$, Mukaddes Kalyoncu ${ }^{24}$, Kaan GÜllero Ğlu ${ }^{25}$, Caner Kabasakal ${ }^{26}$, Belde Kasap Demir ${ }^{27}$, Rümeysa Yasemin Çiçek ${ }^{28}$, Osman Dönmez ${ }^{29}$, Aslihan Kara ${ }^{30}$, Önder Yavaşcan ${ }^{31}$, Gül Özçelik ${ }^{32}$, Deniz Gezgin Yildirim ${ }^{33}$, Muhammet Akif Güler ${ }^{34}$, Ferah Sönmez ${ }^{35}$, Rezan Topaloğlu ${ }^{36}$, Harika Alpay ${ }^{7}$

${ }^{1}$ Gazi University Faculty Of Medicine, Ankara, Turkey

${ }^{2}$ Eskişehir City Training And Research Hospital, EskiŞehir, Turkey

${ }^{3}$ Ondokuz Mayis University Faculty Of Medicine, Samsun, Turkey

${ }^{4}$ Erciyes University Faculty Of Medicine, Kayseri, Turkey

${ }^{5}$ University Of Health Sciences, Prof. Dr. Cemil Taşçioğlu City Hospital, Istanbul, Turkey

${ }^{6}$ Istanbul University, Istanbul Faculty Of Medicine, Istanbul, Turkey

${ }^{7}$ Marmara University, Istanbul Pendik Education And Research

Hospital, Istanbul, Turkey

${ }^{8}$ Akdeniz University Faculty Of Medicine, Antalya, Turkey

${ }^{9}$ KoC University Faculty Of Medicine, Istanbul, Turkey

${ }^{10}$ Hatay State Hospital, Hatay, Turkey

${ }^{11}$ Gaziantep University Faculty Of Medicine, Gaziantep, Turkey

${ }^{12}$ Inonu University, Turgut Ozal Medical Center, Malatya, Turkey

${ }^{13}$ Istanbul University, Cerrahpasa Faculty Of Medicine, Istanbul, Turkey

${ }^{14}$ University Of Health Sciences, Dr. Sami Ulus Maternity And Child Health And Diseases Training And Research Hospital, Ankara, Turkey

${ }_{15}$ Ankara City Training And Research Hospital, Ankara, Turkey

${ }^{16}$ University Of Health Sciences, Istanbul Bakirkoy Dr. Sadi Konuk Research And Training Hospital, İstanbul, Turkey

${ }_{17}^{17}$ Pamukkale University Faculty Of Medicine, Denizli, Turkey

${ }^{18}$ Cukurova University Faculty Of Medicine, Adana, Turkey

${ }_{19}^{19}$ Mersin University Faculty Of Medicine, Mersin, Turkey

${ }^{20}$ University Of Health Sciences, Adana City Training And Research Hospital, Ankara, Turkey

${ }^{21}$ Celal Bayar University, Manisa, Turkey

${ }_{22}$ Osmangazi University Faculty Of Medicine, Eskisehir, Turkey

${ }^{23}$ Dokuz Eylul University Faculty Of Medicine, Izmir, Turkey

${ }^{24}$ Karadeniz Technical University, Faculty Of Medicine, Trabzon, Turkey

${ }^{25}$ Baskent University Faculty Of Medicine, Ankara, Turkey

${ }^{26}$ Ege University Faculty Of Medicine, Izmir, Turkey

${ }^{27}$ Izmir Katip Çelebi University Medical Faculty, Izmir, Turkey

${ }^{28}$ Başakşehir Çam And Sakura City Hospital, Istanbul, Turkey

${ }^{29}$ Uludag University Faculty Of Medicine, Bursa, Turkey

${ }^{30}$ Firat University Faculty Of Medicine, Elaziğ, Turkey

${ }^{31}$ Medipol University Faculty Of Medicine, Istanbul, Turkey

${ }^{32}$ University Of Health Sciences, Sisli Hamidiye Etfal Training And Research Hospital, Istanbul, Turkey

${ }^{33}$ Diyarbakir Childrens Health And Diseases Hospital, Diyarbakir, Turkey

${ }^{34}$ Atatürk University Faculty Of Medicine, Erzurum, Turkey

${ }_{35}$ Adnan Menderes University Faculty Of Medicine, Aydin, Turkey

${ }^{36}$ Hacettepe University Faculty Of Medicine, Ankara, Turkey

Introduction: Contrary to our initial expectations, the prolonged and fluctuating course of COVID-19 pandemic has a significant impact on our medical practice. Despite significantly lower disease transmission among children, cases have been increasing steeply. We aimed to evaluate demographic findings and outcomes of pediatric nephrology patients with COVID-19 in Turkey.

Material and methods: An online survey was sent to all centers to collect the data.

Results: Diagnosis of COVID-19 was made in 203 children with 114 infected family members. While the disease was most commonly seen in the age group of $10-15$ years (37\%), it was less common in children under 2 years $(5,4 \%)$ and those between $2-5$ years of age $(8,9 \%)$. Renal transplantation $(27,6 \%)$, hemodialysis $(16,3 \%)$, and glomerulopathies $(15,3 \%)$ were the most common diagnostic categories. At least one comorbid 
condition was observed in half of the patients, while $15 \%$ had multiple comorbidities.

Only $42,4 \%$ of patients were admitted to the hospital due to complaints and $43,8 \%$ were diagnosed with screening tests because of infected family members or, routine PCR test before hospital admission. The remaining $13.8 \%$ of the patients were diagnosed at another center. The primary method for diagnosis was SARS CoV-2 PCR test and it was positive in $84 \%$ of the patients. Other methods for diagnosis were SARS CoV-2 serology $(5,9 \%)$, thorax CT $(5,4 \%)$ and high clinical suspicion $(4,9 \%)$. $60 \%$ of patients were asymptomatic or had mild disease, while among 82 hospitalized ones, 23 (28\% of hospitalized patients, $11,3 \%$ of all patients) required ICU care. Treatment regimens were highly heterogenous. Half of the patients $(55 \%)$ were not treated, while remaining patients were mostly given favipiravir $(20,7 \%)$, steroid $(16,3 \%)$ and hydroxychloroquine $(11,3 \%)$. Some of the patients were treated with additional combination regimens of intravenous immunoglobulin, low molecular weight heparin or biologic anti-cytokine therapies.

The patients were most frequently hospitalized for $>3-7$ days $(35,4 \%)$ and $>7-14$ days $(31,7 \%)$. Only seven patients required $>30$ days of hospitalization. Acute kidney injury developed in $20 \%$ of the hospitalized patients during the course of the disease. Five patients had the diagnosis of MIS-C and three of them died. Another two patients died due to COVID pneumonia, one post-COVID cardiac arrest at home after discharge and one COVID-unrelated reasons. Fortunately, $83 \%$ of the patients were discharged without any apparent sequelae. On the other hand, 108 health care staff including 42 physician ( 25 of them pediatric nephrologist), 15 secretary, 15 cleaning staff, 14 pediatric's nurse and 13 hemodialysis (HD) nurse were infected during the study period.

Conclusions: COVID-19 was most commonly seen in patients with renal transplantation, undergoing HD and having glomerulopathy. Combined immunosuppressive therapy and exposure in-center HD may increase these patients susceptibility. A mortality rate of $3.4 \%$ and staff infections deserve more attention and further precautions.

\section{EP-114 COVID-19 INFECTION IN PEDIATRIC KIDNEY TRANSPLANT RECIPIENTS}

Ana Dias Curado, Ana Zagalo, Filipa Durão, Patrícia Costa-reis, Ana Rita Sandes, José Eduardo Esteves Da Silva, Rosário Stone Pediatric Nephrology And Kidney Transplantation Unit, Department Of Pediatrics, Hospital De Santa Maria, Lisbon, Portugal

Objectives: To describe the clinical outcomes of pediatric kidney transplant (KT) recipients with COVID-19, followed in a pediatric KT center, and assess immunity to SARS-CoV-2.

Methods: We performed a prospective study for 1 year (March/2020 to March/2021), with descriptive analysis of demographic, baseline and transplant characteristics; immunosuppression; comorbidities; clinical and laboratory data at diagnosis; intervention and follow-up, including SARS-CoV-2 serologic tests.

Results: We report 5 cases of COVID-19 infection from a cohort of 55 KT recipients (median age at diagnosis: 13 years [7-16 years]; four females; median follow-up of 2 months [2-9 months]). Regarding the cause of chronic kidney disease: 1 was unknown, 2 had congenital anomalies of the kidney and 2 had steroid-resistant nephrotic syndrome. Cardiovascular disease was the most common comorbidity (5/5): all had hypertension, 1 left ventricular hypertrophy, 1 mild aortic regurgitation and 1 history of patent arterial duct surgically treated. Hyperuricemia was detected in 2 . None had lung disease, diabetes, dyslipidemia or obesity. All were on immunosuppression with glucocorticoids, mycophenolate mofetil and tacrolimus. Median time after KT at COVID-19 diagnosis was 38 months [ 1 month-11 years]. The most common symptoms were fever (2/5), cough (2/5), rhinorrhea (2/5), myalgia (2/5) and headache $(2 / 5)$. None had signs of respiratory distress. One was asymptomatic. Laboratory findings at diagnosis were lymphopenia (2/5), thrombocytopenia (1/5) and slight increased creatinine (3/5), which returned to baseline after infection. C-reactive protein was negative in all who were tested (3/5). None needed hospital admission. The treatment was only supportive, with no need to reduce immunosuppression. There were no episodes of allograft loss. All had serological immunity [102-461 $\mathrm{U} / \mathrm{mL}]$ at 3-6 months after COVID-19 infection.

Conclusions: Unlike reports of COVID-19 infection in KT adults, in our series children were asymptomatic or had mild disease, being managed as outpatients. Despite the immunosuppression, short-term outcome was excellent.

\section{EP-115 COVID-19 DISEASE IN PEDIATRIC NEPHROLOGY PATIENTS- A SINGLE CENTER EXPERIENCE}

Ece Demirci Bodur, Nurdan Yildiz, Serim Pul, Serçin Güven, Neslihan Çiçek, Özde Nisa Türkkan, İbrahim Gökçe, Harika Alpay Marmara University Pediatric Nephrology

Objectives: In this study,we aimed to evaluate the clinical course and outcomes of COVID-19 in children with kidney disease .

Materials and Methods: Twenty five patients(14 male,11 female) with kidney disease followed-up in Pediatric Nephrology outpatient clinic and diagnosed with COVID-19 confirmed by nasopharyngeal PCR or COVID-19 IgM tests were evaluated retrospectively.

Results: The mean age on admission was $12.5 \pm 5.8$ years. Four $(16 \%)$ patients were kidney transplantation (KTx) recipients, $8(32 \%)$ had end stage kidney disease(ESKD-4 hemodialysis, 4 peritoneal dialysis),4(16\%) had chronic kidney disease(CKD) and 2(8\%) had steroid sensitive nephrotic syndrome. Thirteen $(52 \%)$ patients presented primarily through contact exposure with positive family members. Seven patients $28 \%$ ) were asymptomatic, $16(64 \%)$ had mild disease and $2(8 \%)$ had severe disease. The most common presenting symptoms were fe$\operatorname{ver}(44 \%)$ and myalgia(36\%) followed by fatigue/weakness(20\%) and headache $(\% 20)$. Other symptoms included nausea, cough, chest pain and sore throat. Thirteen (52\%) patients were hospitalized. Median of the hospitalization was $17(4$ to 37 ) days. Acute kidney injury developed in $3 \mathrm{KTx}$ recipients and $1 \mathrm{CKD}$ patient, and completely recovered without dialysis. Chest tomography findings in 6 of 13 patients were consistent with COVID 19 pneumonia. One patient needed mechanical ventilation and one high flow oxygene support. Nine patients(36\%) were using immunsupressive therapy. Mycofenolate mofetil was discontinued in one KTx patient, and the dose was reduced in one KTx patient. Commonly used therapies are antimicrobials in 10(40\%), favipiravir in $5(20 \%)$, hydroxychloroquine in $5(20 \%)$, acetylsalicylic acid in $2(8 \%)$, steroids in $2(8 \%)$ and enoxaparin in $4(16 \%)$ patients. Patient with granulomatosis with polyangiitis was lost due to multiorgan failure and fungal sepsis in spite of aggressive treatment including ECMO.

Conclusion: KTx recipients and ESKD patients receiving dialysis appear to develop mild COVID-19 disease. However, clinical course may be severe in those with pulmoner involvement. We suggest to perform diagnostic tests in the presence of contact exposure or suspicious clinical findings and to monitor closely to prevent complications in these patients.

EP-116 A YEAR OF THE COVID19 PANDEMIC: REFLECTION ON THE TRANSPLANT ACTIVITY IN OUR CENTRE

Helen Jones, Grainne Walsh, Louise Kipping, Joanna Clothier, Kate Mythen, Nick Ware

Evelina London Childrens Hospital

Introduction: To review the transplant activity in our tertiary paediatric nephrology centre during the COVID19 pandemic.

Material and methods: Retrospective review of paediatric renal transplant recipients (pRTRs) under follow up from initial national lockdown 23/3/2020 until 23/3/2021. 
Results: 94 pRTRs under follow up at the start of the first national lockdown in our centre with 86 under follow up a year later.

18 patients transferred to 4 adult nephrology centres facilitated using video appointments enabling joint working between paediatric and adult teams which was previously conducted in person.

3 patients developed de Novo donor specific antibodies during this period ( 2 teenage with admitted non-adherence) all with evidence of rejection on allograft biopsy though only $1 / 3$ had acute antibody medicated rejection (ABMR). 1 patient required plasma exchanged and immunoglobulin treatment for ABMR and all received pulsed steroids. 2 other patients (both teenagers) had borderline $\mathrm{T}$ cell mediated rejection during the course of the year.

No graft loss recorded in those remaining under follow up in the paediatric setting and 1 graft loss secondary to significant non-adherence and haemodialysis commenced in adult nephrology centre. 1 patient was diagnosed with PTLD refractory to treatment in this period and sadly died. The transplant programme was suspended in March 2020 but resumed in June 2020 enabling 14 children to receive transplants in the period described (11 living donor including 1 via the UK kidney sharing scheme, 3 deceased donor). 10/14 followed up in our centre and no episodes of rejection to date. All new recipients shielded for 3 months post-transplant. 4 pRTRs tested positive for SARS-CoV-2 PCR DNA test (all male) and 1 requiring an inpatient stay but displayed no respiratory symptoms. None of the patients required a reduction in their background immunosuppression.

Conclusions: We describe an overview of the pRTR cohort managed during the COVID19 pandemic. Despite the challenges our multidisciplinary team adapted and has strived to maintain seamless follow up for this patient group.

EP-117 ARE WE LOSING OUR AWARENESS OF OTHER INFECTIONS DURING THE COVID 19 OUTBREAK?

Gökçen Erfidan ${ }^{1}$, Özgür ÖZdemir Șimşek ${ }^{1}$, Ahu Kara Aksay ${ }^{2}$, Gülnihan Üstündag ${ }^{1}$, Seçil Arslansoyu Çamlar ${ }^{1}$, Fatma Mutlubaş ${ }^{I}$, Dilek Yilmaz Ciftdoğan ${ }^{3}$, Belde Kasap Demir ${ }^{4}$, Demet Alaygut ${ }^{1}$

${ }^{1}$ Izmir University Of Health Sciences Tepecik Education And Research Hospital Department Of Pediatric Nephrology

${ }^{2}$ Izmir University Of Health Sciences Tepecik Education And Research Hospital Department Of Pediatric Infectious Diseases

${ }^{3}$ Katip Celebi University Faculty Of Medicine Department Of Pediatric Infectious Disease

${ }^{4}$ Katip Celebi University Faculty Of Medicine Department Of Pediatric Nephrology And Rheumatology

Introduction: After the identification of novel Coronavirus disease 2019 (COVID-19) in Wuhan, the dramatic outbreak of COVID-19 has spread globally in months. Although it usually affects children mildly, its most severe form, "Multisystem inflammatory syndrome in children" (MISC), may be seen in some rare cases. Fever is one of the indespensible symptom for the diagnosis of MISC. However, during the pandemic, other infections should also considered in the differential diagnosis of fever, besides MISC.

Material and methods: An eight-year-old girl (Case 1) and an sixteenyear-old girl (Case 2) admitted to hospital with fever for three days. Both had headache, flank pain, vomiting and lethargy. They had no known COVID-19 contact. The COVID-PCR tests were negative for both cases. But Rapid COVID-19 antigen test was positive IgG and negative IgM in first case. Due to leukocytosis, high inflammatory markers and high Ddimer levels, they had diagnosed MISC. In first case, urine analysis showed microscopic hematuria without piyuria and urine culture showed no bacterial growth.

Results: The second case had pyuria and Escherichia coli growth. However, on the physical examination, they both had persisted costovertebral angle tenderness. On the abdomen computed tomography with contrast, they had wedge-shaped hypodense multipl lesions. These lesions were present in both kidneys in Case 1 and in right kidney in Case 2. They diagnosed acute focal bacterial nephritis (AFBN).

Conclusions:Acute focal bacterial nephritis is rare form of urinary tract infection which is considered between APN and renal abscess. It is characterized by localized renal inflammatory mass-like lesion. The common presenting symptoms are persistent high fever, flank and/or abdominal pain. It may considered in differential diagnosis.

\section{EP-118 PSYCHOLOGICAL CONCERNS OF CHILDREN UNDERGOING KIDNEY TRANSPLANTATION AND THEIR FAMILIES DURING THE COVID-19 PANDEMIC \\ Alicia Paessler ${ }^{1}$, Sheila Boyle ${ }^{1}$, Stephen D. Marks ${ }^{1}$, Nicos Kessaris ${ }^{2}$, Jelena Stojanovic ${ }^{1}$ \\ ${ }^{1}$ Great Ormond Street Hospital For Children Nhs Foundation Trust \\ ${ }^{2}$ Guys And St Thomas Nhs Foundation Trust}

Introduction: The current COVID-19 pandemic has been an anxious time for children and young people (CYP) with end stage kidney disease and their families, particularly as they were identified as a vulnerable group. Many transplant programmes closed and reopening brought new concerns for patients and professionals. We report experience on being transplanted during the pandemic.

Objectives: To obtain a better understanding of the concerns and experiences of CYP and families have about receiving a kidney transplant during the pandemic.

Material and methods: A questionnaire was sent to patients and families of 10 paediatric patients transplanted in the first six months of reopening our transplant programme. One patient refused transplant and one lacked social support to proceed with transplant.

Results: All participants felt that their questions were answered before transplantation and $75 \%$ felt well informed about the SARS-CoV-2 effects on transplantation. $62.5 \%$ reported feeling nervous, $37.5 \%$ were anxious, $25 \%$ scared and $12.5 \%$ relaxed about transplantation during the pandemic. The majority of participants reported surgical complications being their biggest fear; two participants were worried about catching SARS-CoV-2. 87.5\% felt that care was delivered safely in inpatient and outpatient setting. $75 \%$ of participants found shielding easy. Overall, $87.5 \%$ of patients were glad to have received a kidney transplant during the pandemic with one patient struggling with feeling isolated.

Conclusions: Receiving a kidney transplant can be a stressful experience, particularly during a pandemic. Our results show that a significant number of patients felt scared, that detailed counselling of CYP and families about risks and addressing their concerns related to SARS-CoV-2 contributed to a good patient and family experience on transplantation during the pandemic. Further studies are needed to look into the long-term effects of the pandemic on this vulnerable group of patients and strategies to mitigate them.

EP-119 HEMATURIA AS AN EARLY SIGN OF MULTISYSTEM INFLAMMATORY SYNDROME IN CHILDREN (MIS-C): A CASE REPORT OF A BOY WITH MULTIPLE COMORBIDITIES Ana Generalić, Maša Davidović, Ivanka Kos, Kristina Vrljičak, Lovro Lamot

Department Of Pediatrics, Unviersity Of Zagreb School Of Medicine, University Hospital Centre Zagreb, Zagreb, Croatia

Introduction: While clinical course of SARS-CoV-2 infection seems to be milder or asymptomatic within pediatric population, growing attention has been laid to the rare complication elicited by virus, multisystem inflammatory syndrome in children temporarily associated with COVID-19 (MIS-C). Published definition and criteria of MIS-C include persistent fever, multisystem involvement and elevated markers of inflammation, without obvious microbial inflammation or other plausible diagnosis. The aim of this case report is to emphasize diversity of symptoms of MIS-C. 
Case description: We present a case of a ten-year-old boy with multiple comorbidities who developed MIS-C after asymptomatic SARS-CoV-2 infection. The patient initially presented hematuria, persistent fever and elevated markers of inflammation, with no other sign of renal affection. Besides, he had a discrete erythematous maculopapular rash on the right lower leg. Within the next two days, his condition worsen despite the broad-spectrum antibiotic therapy. He started to vomit and developed abdominal pain, conjunctivitis, arrhythmia and mild left ventricular systolic dysfunction with hypotension and pleural effusion. High level of clinical suspicion for MIS-C was supported by laboratory findings (elevated ESR, CRP, proBNP, D-dimers and fibrinogen) along with positive IgG SARS-CoV-2 antibodies and negative microbiological cultures. The patient was given intravenous immunoglobulin (IVIG) at a dose of $2 \mathrm{~g} / \mathrm{kg}$ and began to show instantaneous clinical improvement, including downtrend of fever and inflammatory markers.

\section{Conclusion:}

Despite the growing reports of the MIS-C in the literature, there is still paucity of studies describing the various clinical manifestation and laboratory finding in this serious condition which can be easily mistaken for many others inflammatory diseases. Therefore, pediatric professionals must be aware of (many) unusual presentations of COVID-19 associated disease in order to early recognize and treat such challenging patients.

\section{EP-120 HYPERTENSIVE CRISIS AS A PRESENTATION OF COVID-19 IN CHILDREN}

Nakysa Hooman, Golnaz Gharehbaghi, Masoud Hashemzadeh esfahani, Ali Manafi-anari, Behzad Haghighi-aski, Maryam Sakhaei Aliasghar Clinical Research Development Center, Aliasghar Children Hospital, Iran University Of Medical Sciences, Tehran

Background \& Aim: Hypertension consider as a risk factor for the severity of Covid-19 but there is no report of hypertension crisis as an earlier presentation of Covid-19 in pediatrics that is the aim of this study.

Methods: This is a case series collected from March to February 2021. Confirmed SARS-Covid 2 defined by positive RT-PCR of nasopharynx swab, or antibody against it (IgG or IgM), or imaging pattern in favor of it on chest CT-scan . suspicious cases described by clinical sign (fever, diarrhea, cough) and laboratory tests (leucopenia, lymphopeia, rise of CRP, d-dimer, LDH, CPK-MBA, Ferritin, or ALT, with negative cultures for bacteria) and close contact with an infected person. Hypertensive crisis elucidated by acute rise of blood pressure more than stage 2 cut off with potential risk of end organ damage. Results: Four confirmed and two suspicious SARS-COvid-19 cases ( 4 males, 2 females) were reported. The mean age of patients was 4.2 (range, 1-12) years. Four cases with newly diagnosis of nephrotic syndrome who were on $2 \mathrm{mg} / \mathrm{kg}$ /day prednisolon developed rapid rises of blood pressure. The other two cases were suffered from Chronic kidney disease stage 5 being on regular dialysis with no edema or sign of fluid overload (HD and CAPD). The lowest systolic blood pressure at the time of admission was $160 \mathrm{mmHg}$ and the highest was $200 \mathrm{mmHg}$. The diastolic blood pressures were between $100-155 \mathrm{mmHg}$. All the patients had normal blood pressure prior to the admission. Three patients (two CKD) received anticovid 19 medications. All patients treated with labetalol infusion titrated to maximum dosage and continued for at least one week and because of poor control of oral antihypertensive medications were added. On follow up, one patient on CAPD died, patient on HD complicated with pericardial effusion, pancreatitis and chronic diarrhea. All nephrotic syndrome patients were resistant to steroid, went on renal biopsy, and calcineurin inhibitor started for massive proteinuria despite being on ARBS /ACEI. All children had resistant hypertention and their blood pressure controlled with four or five antihypertensive medications.

Conclusion: Whether the virus itself can cause exacerbation of hypertension is not yet known but it seems that there is a correlation between
COVID-19 infection and hypertension crisis in these cases. Further clinical research with a larger population would be required to determine the clinical significance of these findings.

\section{EP-121 PHENOCOPIES OF CLINICALLY DIAGNOSED ARPKD REVEALED IN CHILDREN}

Abdul Halawi ${ }^{1}$, Kathrin Burgmaier ${ }^{1}$, Anja Buescher ${ }^{2}$, Ismail Dursun ${ }^{3}$, Matthias Galiano ${ }^{4}$, Michaela Gessner ${ }^{5}$, Ibrahim Gökce ${ }^{6}$,

Claudia Kowalewska ${ }^{7}$, Djalila Mekahli ${ }^{8}$, Sevgi Mir ${ }^{9}$, Rukshana Shroff ${ }^{10}$, Stella Stabouli ${ }^{11}$, Maria Szczepanska ${ }^{12}$, Ana Teixeira ${ }^{13}$, Lutz Weber ${ }^{1}$, Elke WÜhl ${ }^{14}$, Simone Wygoda ${ }^{15}$, Katarzyna Zachwieja ${ }^{16}$, Jörg Dötsch ${ }^{1}$, Franz Schaefer ${ }^{14}$, Max Liebau ${ }^{1}$

${ }^{I}$ Department Of Pediatrics, Faculty Of Medicine, University Hospital Cologne And University Of Cologne, Cologne, Germany

${ }^{2}$ Department Of Pediatrics Ii, University Hospital Essen, Essen, Germany

${ }^{3}$ Department Of Pediatric Nephrology, Erciyes University, Faculty Of Medicine, Kayseri, Turkey

${ }^{4}$ Department Of Pediatrics And Adolescent Medicine, University Of Erlangen-nÜrnberg, Erlangen, Germany

${ }^{5}$ Department Of General Pediatrics And Hematology/ Oncology, Children's University Hospital Tuebingen, Germany

${ }^{6}$ Research And Training Hospital, Division Of Pediatric Nephrology, Marmara University, Istanbul, Turkey

${ }^{7}$ The Children's Memorial Health Institute, Warsaw, Poland

${ }^{8}$ Department Of Pediatric Nephrology, University Hospitals Leuven, Leuven, Belgium

${ }^{9}$ Department Of Pediatric Nephrology, Ege University Medical Faculty, Izmir, Turkey

${ }^{10}$ Great Ormond Street Hospital For Children Nhs Foundation Trust, London, United Kingdom

${ }^{11}$ First Department Of Pediatrics, Hippokration Hospital, Aristotle University Of Thessaloniki, Thessaloniki, Greece

${ }^{12}$ Department Of Pediatrics, Faculty Of Medical Sciences In Zabrze, Sum In Katowice, Katowice, Poland

${ }^{13}$ Pediatric Nephrology, Centro Hospitalar São João, Porto, Portugal

${ }^{14}$ Division Of Pediatric Nephrology, Center For Pediatrics And Adolescent Medicine, University Of Heidelberg, Heidelberg, Germany

${ }^{15}$ Clinic For Children And Adolescents, Hospital St. Georg, Leipzig, Germany

${ }^{16}$ Department Of Pediatric Nephrology And Hypertension, Faculty Of Medicine, Jagiellonian University Medical College, Krakow, Poland

Objectives: Autosomal recessive polycystic kidney disease (ARPKD) is a rare genetic disorder ciliopathy with an incidence estimated to 1:20,000. ARPKD is mainly caused by variants in the PKHD1 gene and rarely in DZIPIL. The disease presents with a phenotypical spectrum with high variation in severity that could lead to an early impact on child health. While there are defined criteria for the clinical diagnosis, the clinical variability can make it challenging to differentiate ARPKD from other genetic causes of cystic kidney diseases. In some of these cases of phenocopies genetic testing can lead to a change in the initial clinical diagnosis.

Methods: Patients secondarily excluded from ARegPKD due to genetic diagnosis of another cystic kidney disease were analyzed. Variants found in phenocopy genes were evaluated using in silico pathogenicity prediction tools and classified using ACMG criteria. Initial and follow-up clinical presentation data in this cohort was then compared to ARPKD cases with detection of relevant $P K H D 1$ variants (at least one variant ACMG class $\geq 3$ ).

Results: At the time of analysis 665 patients were included in the ARegPKD registry. 284 patients had relevant PKHD1 variants. We identified 32 individuals with variants in other cystic kidney disease-causing genes (18 PKD1, 5 TMEM67, 5 HNF1B, 2 NPHP3, 1 PKD2, 1 LRP5) but without detection of PKHD1 or 
DZIP1L variants. All variants identified in these genes showed in silico predicted disease-causing pathogenicity and compatible results in variant frequencies in the genome aggregation database. The majority of these patients presented prenatally or postnatally in the first year of life with $34 \%$ and $41 \%$, respectively. Extrarenal manifestations were seen in 9 patients. 8 patients started kidney replacement therapy with a median age at onset of 4.4 years.

Conclusions: Utilizing our international cohort, we extend the data on ARPKD phenocopies and present their genetic and clinical data.

\section{EP-122 AUTOSOMAL DOMINANT POLYCYSTIC KIDNEY DISEASE IN TUNISIAN CHILDREN: A SINGLE CENTRE EXPERIENCE}

Ben Guedria Mouna, Selsabil Nouir, Mabrouk Sameh, Mansour Kais, Majdoub Fadwa, Ajmi Houda, Tfifha Meniar, Chemli Jaleleddine, Zouari Noura, Abroug Saoussen

Pediatric Department, Sahloul Hospital, Tunisia

Introduction: Autosomal dominant polycystic kidney disease (ADPKD) is a systemic disease involving cysts in the kidneys and abdominal organs as well as abnormalities in the heart and vasculature. Although it typically presents in adults, ADPKD has been diagnosed in foetuses, infants, children, and adolescents. The aim of this study was to describe the clinical features and outcomes of ADPKD among Tunisian children.

Material and methods: Cross sectional observational study of the paediatric cases of ADPKD managed in the department of paediatrics in the University hospital (Sousse - Tunisia)

Results: 21 patients, predominantly girls (57\%) were included. Family history of cystic disease was positive in $80 \%$ of cases. Mean age of diagnosis was 11 years, however one patient was diagnosed at neonatal period. Half of our patient were symptomatic when the disease was diagnosed. Main symptoms were abdominal pain, fever and gross hematuria. Hypertensionwas found in $16 \%$ of patients. Renal function was normal at the diagnosis in 20 cases. One patient had End stage renal disease at presentation. Renal ultrasound showed renal enlargement with multiple cysts. For the extra renal manifestations, liver cysts were found in 3 patients and pancreatic cysts in one patient. The main complication was Hypertension in $20 \%$ of patients, and Urinary tract infection occurred in 3 cases. Management was based on ACE inhibitors. $14 \% 3$ patients progressed to end-stage renal disease requiring haemodialysis in 2 cases followed by renal transplantation, and one was on peritoneal dialysis.

Conclusions: ADPKD in children is relatively rare in children, often asymptomatic, however symptoms may be observed during childhood, mainly hypertension and hematuria. Besides End stage renal disease can occur during childhood

\section{EP-123 KIDNEY DIMENSION IS THE MOST IMPORTANT PARAMETER ASSOCIATED WITH DETERIORATION IN KIDNEY FUNCTION IN CHILDREN WITH AUTOSOMAL RECESSIVE POLYCYSTIC KIDNEY DISEASE}

Neslihan Cicek ${ }^{1}$, Ibrahim Gokce ${ }^{1}$, Ceren Alavanda ${ }^{2}$, Sercin Guven ${ }^{1}$, Mehtap Sak ${ }^{1}$, Ozde Nisa Turkkan ${ }^{1}$, Ece Demirci Bodur ${ }^{1}$, Serim Polat ${ }^{1}$, Pinar Ata ${ }^{2}$, Nurdan Yildiz ${ }^{\text {, Harika Alpay }}{ }^{1}$

${ }^{1}$ Marmara University School Of Medicine, Department Of Pediatric Nephrology

${ }^{2}$ Marmara University School Of Medicine, Department Of Genetics Introduction: In this study, we described clinical, biochemical, imaging and genetic findings and investigated parameters that may influence renal prognosis in pediatric patients with autosomal recessive polycystic kidney disease(ARPKD).
Material and methods: Patients who were diagnosed before birth or during the first month were classified as perinatal presenters( 9 patients), diagnosed later classified as non-perinatal presenters (10 patients). We also divided patients according to estimated glomerular filtration rates(eGFR) at last visit as patients with eGFR $\geq 30$ and $e G F R<30 \mathrm{ml} /$ $\min / 1.73 \mathrm{~m}^{2}$. We analyzed clinical, demoghraphic findings, kidney dimensions adjusted to height, urinary protein excretions, liver functions, office blood pressures at diagnosis and at last visit, genetic analysis and genetic types. The correlation between time of diagnosis (perinatally or non-perinatally, eGFR at last visit(eGFR $\geq 30$ or eGFR $<30 \mathrm{ml} / \mathrm{min} /$ $1.73 \mathrm{~m}^{2}$ ) and other parameters were evaluated.

Results: Seven patients(36.84\%) were diagnosed antenatally, the mean follow up time was $7.7 \pm 5.21$ years. Among six(31.6\%) patients who reached stage-5-CKD, two had a renal transplantation. Eleven patients $(57.9 \%)$ had hypertension and five $(26.3 \%)$ had proteinuria. Liver disease was diagnosed in 13 patients $(68.4 \%)$. Genetic tests of 15 patients were evaluated. Eleven patients had homozygous mutation in PKHD1 gene, 1 truncating and 10 non-truncating. Four patients had compound heterozygous mutation in PKHD1 gene. When two groups were compared, kidney dimension at diagnosis was higher in perinatal presenters $(\mathrm{p}=0.01)$ and although not statistically significant, eGFR at diagnosis was lower in perinatal presenters $(\mathrm{p}=0.065)$. Liver and spleen involvements were higher in nonperinatal presenters $(\mathrm{p}=0.04, \mathrm{p}=0.033)$. When patients are compared according to eGFR at last visit, kidney dimension at diagnosis was higher in patients with $\mathrm{eGFR}<30 \mathrm{ml} / \mathrm{min} / 1.73 \mathrm{~m}^{2}(\mathrm{p}=0.045)$.

Conclusions: In patient with perinatal presenters kidney dimension at diagnosis is higher. Kidney dimension is the parameter most associated with low eGFR at last visit. Non-perinatal presenters, on contrary, had more liver and spleen involvements.

EP-124 CLINICAL AND MUTATIONAL SPECTRUM OF CHILDREN WITH AUTOSOMAL RECESSIVE AND AUTOSOMAL DOMINANT POLYCYSTIC KIDNEY DISEASE Ozum Tutal ${ }^{1}$, Bora Gulhan ${ }^{2}$, Emine Atayar ${ }^{3}$, Selçuk Yüksel ${ }^{4}$,

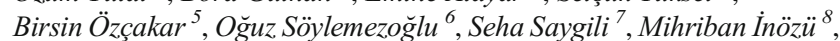
Esra Baskin ${ }^{9}$, Ali Düzova ${ }^{2}$, Mutlu Hayran ${ }^{10}$, Rezan Topaloglu ${ }^{2}$, Fatih Ozaltin $^{2}$

${ }^{I}$ Hacettepe University School Of Medicine, Department Of Pediatrics, Ankara, Turkey

${ }^{2}$ Hacettepe University School Of Medicine, Department Of Pediatrics, Division Of Pediatric Nephrology, Ankara, Turkey

${ }^{3}$ Hacettepe University School Of Medicine, Nephrogenetics Laboratory, Ankara, Turkey

${ }^{4}$ Pamukkale University School Of Medicine, Department Of Pediatrics, Division Of Pediatric Nephrology, Denizli, Turkey

${ }^{5}$ Ankara University School Of Medicine, Department Of Pediatrics, Division Of Pediatric Nephrology, Ankara, Turkey

${ }^{6}$ Gazi University School Of Medicine, Department Of Pediatrics, Division Of Pediatric Nephrology, Ankara, Turkey

${ }^{7}$ Istanbul University CerrahpaȘa School Of Medicine, Department Of Pediatrics, Division Of Pediatric Nephrology, Istanbul, Turkey

${ }^{8}$ Ankara City Hospital, Department Of Pediatric Nephrology, Ankara, Turkey

${ }^{9}$ Baskent University School Of Medicine, Department Of Pediatrics, Division Of Pediatric Nephrology, Ankara, Turkey

${ }^{10}$ Hacettepe University School Of Medicine, Department Of Preventive Oncology, Ankara, Turkey

Introduction: In this study, we aimed to investigate clinical and genetic features of autosomal recessive polycystic kidney disease (ARPKD) and autosomal dominant polycystic kidney disease (ADPKD) in a group of Turkish patients.

Material and methods: A total of 69 children with genetically confirmed ARPKD (10 females, 11 males) or ADPKD ( 28 females, 20 males) from 
seven pediatric nephrology centers were included in this study. Demographic features, family history, clinical findings at presentation and during 12 months intervals were collected.

Results: For ARPKD patients, the median age at diagnosis was 10.5 (IQR; 0.75-58.5) months. At the time of diagnosis, 14 (66.7\%) patients had eGFR $<90 \mathrm{ml} / \mathrm{min} / 1.73 \mathrm{~m}^{2}$. Mean duration of follow-up was $4.1 \pm 3.7$ years. At the last visit, median eGFR was 74 (IQR; 43-126) $\mathrm{ml} / \mathrm{min} /$ $1.73 \mathrm{~m}^{2}$. Preparations for renal transplantation are made for the other patient. For ADPKD patients, the mean age at diagnosis was $5.5 \pm 4.6$ years. At the time of diagnosis 11 (22.9\%) patients had eGFR $<90 \mathrm{ml} / \mathrm{min} / 1.73 \mathrm{~m}^{2}$. Mean duration of follow-up was $2.6 \pm 2.1$ years. At the last visit, median eGFR was 114 (IQR; $98-135) \mathrm{ml} / \mathrm{min} / 1.73 \mathrm{~m}^{2}$. A total of 42 patients $(87.5 \%)$ had a heterozygous PKD1 mutation while $6(12.5 \%)$ had a heterozygous $P K D 2$ mutation. The rate of growth retardation, hypertension at diagnosis and progression to chronic kidney disease (CKD) were higher in patients with bi-allelic PKHD1 mutation than the patients with heterozygous $P K D 1$ or PKD2 mutation ( $\mathrm{p}<0.001, \mathrm{p}<0.001$ and $\mathrm{p}=0.001$, respectively). In renal survival analysis, mutation type, growth retardation and malnutrition at presentation, increased renal echogenity in ultrasonography were found as independent risk factors for progression to CKD.

Conclusions: Differentiation of polycystic kidney diseases and establishing the predictors for CKD development are important to provide appropriate management including choosing appropriate donor in renal transplantation.

\section{EP-125 CLINICAL-GENETIC CHARACTERISTICS AND PREDICTORS OF DISEASE PROGRESSION IN PATIENTS WITH AUTOSOMAL DOMINANT POLYCYSTIC KIDNEY DISEASE}

Harika Alpay ${ }^{\prime}$, Neslihan Cicek ${ }^{1}$, Ceren Alavanda ${ }^{2}$, Sercin Guven ${ }^{1}$, Mehtap Sak ${ }^{1}$, Ozde Nisa Turkkan ${ }^{1}$, Ece Demirci Bodur ${ }^{1}$, Serim Polat ${ }^{1}$, Pinar Ata ${ }^{2}$, Nurdan Yildiz ${ }^{1}$, Ibrahim Gokce ${ }^{1}$

${ }^{1}$ Marmara University School Of Medicine, Department Of Pediatric Nephrology; ${ }^{2}$ Marmara University School Of Medicine, Department Of Genetics

Introduction: We evaluated pediatric patients with autosomal dominant polycystic kidney disease (ADPKD) and investigated the parameters that may influence renal prognosis.

Material and methods: Thirty one patients (19 males, 12 females) with ADPKD are included in the study. We evaluated kidney outcome with estimated glomerular filtration rates (eGFR) at diagnosis, eGFR change (eGFRc) and urinary protein excretion (UPE). We analyzed clinical and demographic findings, kidney dimensions adjusted to height, office blood pressures at diagnosis and at last visit. The correlation between eGFR at diagnosis, eGFRc, UPE and other parameters were evaluated. We also evaluated patients' datas according to genetic types (PKD1 or PKD2, truncating or non-truncating) and subtypes (nonsense, misense, inframe, frameshift, splice-site).

Results: The mean age at diagnosis and the mean follow-up time were $9.07 \pm 4.9(0-16.6)$ and $6.51 \pm 4.7(0.8-19.3)$ years, respectively. Antenatal diagnosis was present in two patients $(6.45 \%)$. The mean eGFR at diagnosis and annual eGFR change were $175.5 \pm 47.4$ and $1.11 \pm 17.64 \mathrm{ml} / \mathrm{min} /$ $1.73 \mathrm{~m}^{2}$ respectively. Only three patients $(9.7 \%)$ had hypertension and five $(16.1 \%)$ had proteinuria. Genetic tests of 28 patients were evaluated. Twenty-two patients had mutations in PKD1, 15 truncating, 7 nontruncating mutations. Six patients had mutations in PKD2, all of them were truncating. Eleven of the 28 mutations were novel. eGFR change was negatively correlated with age at diagnosis $(p=0.039)$ and UPE was positively correlated with kidney dimension at last visit $(\mathrm{p}=0.015)$. eGFR at diagnosis was negatively correlated with kidney dimension at diagnosis, hypertension at diagnosis and antenatal diagnosis $(p=0.002, p=0.015$, $\mathrm{p}=0.015$ ). When we compared the patients with PKD1 and PKD2 mutation, there was no significant difference in terms of all parameters.
Truncating type of mutation was negatively correlated with age at diagnosis $(\mathrm{p}=0.042)$.

Conclusions: The type of genetic mutation usually does not have clinical effects in childhood, since phenotypic features and prognostic predictors usually occur later in life.

\section{EP-126 GANAB MUTATION IDENTIFICATION IN A PAEDIATRIC AUTOSOMAL DOMINANT POLYCYSCTIC KIDNEY DISEASE COHORT}

Filipa Marujo, Mafalda Casinhas Santos, Telma Francisco , Ana Paula Serrão, Inês Carvalho, Rute Baeta Baptista, Margarida Abranches Hospital Dona Estefânia, Chulc

Introduction: Autosomal Dominant Polycystic Kidney Disease (ADPKD) is one of the most common inherited disorders, caused by PKD1 and PKD2 gene mutations, resulting in non-functioning polycistin-1(PC1) and polycistin-2(PC2), respectively. Mutations in GANAB, which encodes glucosidase II subunit $\alpha(\mathrm{GII} \alpha)$, have recently been described in a small percentage of cases of ADPKD and usually presents with mild kidney disease.

Material and methods: ...

Results: We describe two 17-year-old patients with GANAB mutations, corresponding to $3.4 \%$ of our ADPKD paediatric cohort. Patient 1 presented with macroscopic haematuria since he was one-year-old. His family history was positive for nephrolithiasis, and he was initially diagnosed with nephrolithiasis. Patient 2 presented with flank pain and intermittent micro and macroscopic haematuria after renal trauma. He also had congenital partial deficit of factor XI and a positive family history for nephrolithiasis. Further investigation revealed bilateral renal cortical cysts and hypocitraturia in both patients. No hepatic involvement or hypertension were reported and the estimated glomerular filtration rate was normal. Next-Generation Sequencing revealed GANAB heterozygous mutations in both patients: c. $1452+1 \mathrm{G}>\mathrm{A}$ in patient 1 and p.Arg834Ter (c. $2500 \mathrm{C}>\mathrm{T}$ ) in patient 2.

Conclusions: Our patients presented with intermittent haematuria and hypocitraturia, not typically described in the literature. Further workup showed bilateral renal cysts with preserved renal function and no other organ involvement. Although most ADPKD paediatric patients are asymptomatic, a small proportion presents with early-onset disease during childhood. GANAB mutations have been recently implicated in $0.3 \%$ of ADPKD cases, typically with milder kidney involvement. The knowledge of the genetic spectrum of cystic kidney disease may prompt an earlier diagnosis.

\section{EP-127 ENDOCAN LEVELS IN CHILDREN WITH POLYCYSTIC KIDNEY DISEASE}

Zehra Aydin ${ }^{1}$, İsmail Selcuk Aygar ${ }^{2}$, Beg ̈̈m Avci ${ }^{1}$, Mihriban İnozu ${ }^{1}$, Umut Selda Bayrakci ${ }^{3}$, Fatma Semsa Cayci ${ }^{1}$

${ }^{1}$ The University Of Health Sciences, Ankara City Hospital, Department Of Pediatric Nephrology, Ankara, Turkey

${ }^{2}$ The University Of Health Sciences, GÜlhane Education And Research Hospital, Medical Microbiology Laboratory, Ankara, Turkey

${ }^{3}$ The University Of Yildirim Beyazit, Ankara City Hospital, Department Of Pediatric Nephrology, Ankara, Turkey

Introduction: Autosomal dominant polycystic kidney disease (ADPKD) is a genetic disorder that causes many fluid-filled cysts in the kidneys. The increasing number of renal cysts results in regional renal ischemia, vasoconstriction, and vascular endothelial dysfunction. Many biomarkers have been investigated for understanding these molecular alterations in ADPKD progress such as endocan. Endocan is a new soluble biomarker that synthesis from various organs as well as kidneys. It plays a key role in cell proliferation, migration, inflammation, neovascularization, and 
endothelial function. It is involved in endothelial dysfunction suggests that endocan may be a potential early marker in ADPKD patients. Also, an adult study showed high serum endocan levels is ADPKD patients. For this reason, we aimed to evaluate the serum endocan levels in normotensive children with ADPKD and normal renal function.

Material and methods: Twenty normotensive children with ADPKD and normal renal function were accepted as PKD group, and 20 healthy children were accepted as the control group. Serum endocan levels were determined by enzyme-linked immunosorbent assay techniques and compared between the two groups.

Results: The mean age of patients was $9.9 \pm 4.12$ years, and the mean age of the control group was $10.2 \pm 3.83$ years. There was no significant difference between the two groups in terms of gender, age, and BMI $(\mathrm{p}=0.751, \mathrm{p}=0.813, \mathrm{p}=0.781$, respectively). The mean serum endocan level in the PKD group was $345.8 \pm 169.5 \mathrm{pg} / \mathrm{ml}$, and the control group was $448.61 \pm 258.2 \mathrm{pg} / \mathrm{ml}$. Serum endocan levels did not change between groups $(\mathrm{p}=0.159)$.

Conclusions: Serum endocan level was normal in children with ADPKD without hypertension and renal failure. It could be explained by the absence of hypertension, renal failure, and mainly tubular expression of endocan.

\section{EP-128 RABSON-MENDENHALL SYNDROME}

\section{AND MEDULLARY SPONGE KIDNEY: A CASE REPORT}

Gizem Yildiz ${ }^{l}$, Meral Torun Bayram ${ }^{\text {}}$, Ozge Besci ${ }^{2}$,

Handan Guleryuz Ucar ${ }^{3}$, Korcan Demir ${ }^{2}$, Salih Kavukcu ${ }^{1}$, Alper Soylu ${ }^{1}$

${ }^{I}$ Dokuz Eylül UnIversity Faculty Of MedİIIne, Department Of Pediatric Nephrology, Izmir, Turkey

${ }^{2}$ Dokuz Eylül Unİversity Faculty Of MedícIne, Department Of Pedíatric Endocrinology, İzmir, Turkey

${ }^{3}$ Dokuz Eylül UnIversity Faculty Of MedícIne, Department Of Radíology, Dívisílon Of PedIIatríc Radíology, Izmİr, Turkey

Introduction: Rabson-Mendenhall syndrome (RMS) is an autosomal recessive disease due to mutations in the insulin receptor gene (INRS). Clinical findings of RMS include severe insulin resistant diabetes, hyperinsulinemia, postprandial hyperglycemia, growth retardation and dysmorphisms. The syndrome may have renal manifestations such as nephromegaly, nephrocalcinosis, hydronephrosis and medullary sponge kidney (MSK). Here, we report a 10-years-old girl with a homozygous mutation in the INSR gene and MSK.

Material and methods: A 10-years-old girl who have RMS was admitted for proteinuria in urine analysis. On physical examination, there were the classic morphologic features of RMS, including severe acanthosis nigricans of the neck, axillae external genitalia and antecubital regions, mildly decreased subcutaneous fat, polydactyly in her left hand, mental retardation and hearing loss. Blood pressure was normal and there were no signs of edema. In laboratory tests, complete blood count, serum creatinine, electrolytes, $\mathrm{Ca}, \mathrm{P}, \mathrm{ALP}, \mathrm{Mg}$, albumin, $\mathrm{HCO} 3$ and uric acid values were normal. Urine density was 1015 , protein $(+1)$, erythrocyte (-) in urine analysis, 2-3 erythrocytes in each area in microscopy. $\mathrm{Na}, \mathrm{P}, \mathrm{Mg}$, uric acid excretions were normal, protein excretion was $5.5 \mathrm{mg} / \mathrm{m}^{2} /$ hour $(\mathrm{N}: 0-4)$, microalbuminuria was $29 \mathrm{mg} /$ day $(\mathrm{N}<30)$, calcium excretion was $6.9 \mathrm{mg} / \mathrm{kg} /$ day in the urine. A renal ultrasound showed nephromegaly and increased medullary echogenicity. In postcontrast series of computer tomography, papillary enhancement was observed in a brush-like pattern in the proximal part of the calyxial structures of the slightly dilated collecting system in both kidneys. The findings were consistent with the MSK

Results: Mutations in INSR can lead to abnormalities in kidney function, most prominently to hypercalciuria and nephrocalcinosis, suggesting an important role for INSR in renal calcium handling.
Conclusions: Our patient is one of the few cases with RMS and MSC coexistence in the literature. We recommend evaluation for nephrocalcinosis, MSK, and hypercalciuria in all children diagnosed with RMS.

\section{EP-129 ADPKD: A NOVEL PKD1 VARIANT}

Claudio Ancona ${ }^{1}$, Susanna Negrisolo ${ }^{2}$, Andrea Carraro ${ }^{2}$, Piera De Gaspari ${ }^{2}$, Germana Longo ${ }^{3}$, Mattia Parolin ${ }^{3}$, Davide Meneghesso ${ }^{3}$, Davide Colavito ${ }^{4}$, Elisa Benetti ${ }^{3}$

${ }^{1}$ Department Of Woman And Childs Health, University Of Padova, Padua, Italy

${ }^{2}$ Laboratory Of Immunopathology And Molecular Biology Of The Kidney, Institute Of Pediatric Research Città Della Speranza, Department Of Women's And Children's Health, Padua University Hospital, Padua, Italy

${ }^{3}$ Pediatric Nephrology Dialysis And Transplant Unit, Department Of Women's And Children's Health, Padua University Hospital, Padua, Italy

${ }^{4}$ Ier, Padua, Italy

Introduction: Autosomal dominant polycystic kidney disease (ADPKD) is a genetic dominant disorder mostly caused by PKD1 or PKD2 gene mutations. Here we describe a family (father and two children) with ADPKD due to a new heterozygous PKD1 gene mutation.

Material and methods: A five-year-old boy was admitted to ER with haematuria due to a mild abdominal trauma. Physical examination was negative, renal function was normal, whereas the laboratory analysis confirmed haematuria and pointed out proteinuria. Clinical family history disclosed that his father had polycystic disease and underwent kidney transplant two years earlier. Screening analysis was conduct in the older sister too. She had micro-haematuria and a mild proteinuria. The ultrasound confirmed the presence of bilateral renal cysts also in her case. PKD1sequencing test for was performed to confirm the hypothesis of ADPKD.

Results: The PKD1 sequencing highlighted a novel splicing variation c. $215+2 \quad 215+3 \mathrm{del}(\mathrm{NM}$ 001009944.3). The one base deletion is located in the first intron of PKD1, in a canonical splice site and it is not present in the population databases. Bioinformatics and computational analysis predict that the associated protein had an elevated pathogenicity rank. The genetic test performed on the two children confirmed the presence of the same deletion.

Conclusions: Following the American College of Medical Genomics recommendations, this variant should be considered potentially pathogenic and was submitted to the PKD1 mutation databases. This case report highlights the importance of applying genetic testing in paediatric patients with suspected ADPKD to achieve an early diagnosis, to make specific follow-ups and prevent or delay long-term complications.

\section{EP-130 A LATE DIAGNOSING OF ARPKD IN A CHILD: A CASE REPORT \\ Ganna Zvenigorodska ${ }^{\text {, }}$ Lyudmyla Starynets ${ }^{2}$, Galina Guminska ${ }^{2}$, Oleksandr Moskaliuk ${ }^{1}$, Tetyana Stepankevich ${ }^{2}$ \\ ${ }^{1}$ National Pirogov Memorial Medical University \\ ${ }^{2}$ Vinnytsya Regional Clinical Childrens Hospital}

Introduction: Autosomal recessive polycystic kidney disease (ARPKD) belongs to a group of congenital hepatorenal cystic syndromes. The majority of cases are caused by mutations in the PKHD1 gene, which encodes for fibrocystin/polycystin. There is significant variability in age and presenting clinical symptoms related degree of renal and biliary abnormalities.

Material and methods: A retrospective case review of child with ARPKD at a single center diagnosed on the basis of clinical features, 
radiological and endoscopic evidence of portal hypertension, and genetic findings.

Results: A 18-month-old male patient presented to the emergency room with a five-day history of enlarged distended abdomen. Examination showed hepatosplenomegaly. His prenatal, birth history and neonatal period were unremarkable. Family history was uneventful. His abdominal ultrasound showed bilaterally enlarged echogenic kidneys with loss of cortico-medullary differentiation and multiple cysts. His kidney function tests were normal. Hepatic findings included nonobstructed dilatation of the intrahepatic bile ducts (Caroli syndrome) and dilatation of the common bile duct. Gastroscopy visualized a low-grade esophageal varices located in the lower third secondary to portal hypertension. To confirm diagnosis genetic testing was performed. A pathogenic variant, c. $107 \mathrm{C}>\mathrm{T}$ (p.Thr36Met), was identified in PKHD1. It is estimated that approximately $13 \%$ of ARPKD patients of European origin have this variant, making it the most common mutation in that population.

Conclusions: The majority of ARPKD patients are identified late in pregnancy or at birth. ARPKD has multisystem effects, and often requires a multidisciplinary team for follow-up and is a cause of significant renal and liver-related morbidity and mortality in children.

\section{EP-131 RENAL CYSTS AND DIABETES SYNDROME (RCAD) - CASE REPORT \\ Malgorzata Placzynska, Katarzyna Jobs \\ Department Of Pediatrics, Nephrology And Allergology, Military Institute Of Medicine}

Introduction: Renal cysts and diabetes syndrome (RCAD) is an autosomal dominant multisystemic disorder caused by heterozygous mutation in the HNF1B gene. It is characterized by renal disease resulting from abnormal renal development and maturityonset diabetes of the young (MODY). The renal disease is highly variable and it may include renal cysts, glomerular tufts, irregular collecting systems. Some patients have hyperuricemic nephropathy with tubulointerstitial changes on biopsy.

Material and methods: Presented male patient was antenatally diagnosed with renal pelvic dilatation. Postnatal sonographic evaluation revealed both kidneys with increased renal cortical echogenicity, poor visibility of the renal pyramids and numerous small cysts. Parents didnt have any history of policystic kidney disease. Ultrasound examination also showed several small echogenic foci without acoustic shadowing but with present twinkle artifact on color Doppler. Thus the computed tomography was performed but it did not showed any kidney stones. The markers of renal function were normal. Metabolic evaluation of potential causes of urolithiasis showed only hyperuricemia. Renal scintigraphy performed in the age of 12 months showed no abnormalities. Results: In the age of 8 months the boy was genetically tested for the causes of policystic kidney disease. Comparative genomic hybridisation showed a deletion of the long arm of chromosome 17 in the $17 \mathrm{q} 12$ region. The aberration covers a region of the RCAD deletion syndrome (OMIM: 137920), described in patients with renal cystic disease and MODY diabetes.

The patient remains under constant nephrological care. At the present time at the age of 3.5 years the kidney function remains normal and the ultrasound image of the kidneys has not changed significantly. Diabetes has not yet been established.

Conclusions: HNF1B - associated polycystic kidney disease should be included in the differential diagnosis of all children presenting with renal cysts. Genetic testing is necessary to confirm a diagnosis.
EP-132 COMPARISON OF FRESENIUS 5008® VERSUS 6008® GENERATORS IN CHILDREN BELOW 20 KG

Melodie Mosca, Sarra Rachedi, Rouba Bechara, Justine Bacchetta, Bruno Ranchin

Centre De Référence Des Maladies Rénales Rares, Hôpital Femme Mère Enfant, Hospices Civils De Lyon, Bron, France

Introduction: In 2018, 119 French children (22 below 5 years) received maintenance hemodialysis. Most of new-generation dialysis machines do not propose pediatric lines anymore but Fresenius had validated pediatric sets above $10 \mathrm{~kg}$.

Material and methods: We performed a retrospective single-center evaluation of daily practice with novel Fresenius $6008 \AA$ dialysis machines, using either low volume pediatric sets $(83 \mathrm{~mL})$ or standard adult lines $(122 \mathrm{~mL})$, as compared to $5008 \AA$ machines with pediatric $(108 \mathrm{~mL})$ or adult lines $(136 \mathrm{~mL})$. Each child underwent indifferently online hemodialysis with both Fresenius $5008 \AA$ and $6008 \AA$ generators. All dialysis sessions were performed on a central venous catheter $8 \mathrm{Fr}-18 \mathrm{~cm}$ Medcomp Hemo-cath ${ }^{\circ}$ T $(\mathrm{N}=3)$ and $15 \mathrm{Fr}-28 \mathrm{~cm}$ Metronic Permcath ${ }^{\circledR}$ $(\mathrm{N}=1)$. Results are presented (mean $\pm \mathrm{SD})$.

Results: A total of 115 sessions were performed over 6 weeks in 4 children (body weight $13.1 \pm 4.1 \mathrm{~kg}$, age $3.7 \pm 1.6$ years). For the three smallest children $(9.1,11.1$ and $13.0 \mathrm{~kg})$, treated with pediatric lines, blood flow was higher with $5008 \AA$ vs $6008 \AA(86 \pm 4$ versus $80 \pm 8 \mathrm{~mL} / \mathrm{min}, 87 \pm 4$ versus $84 \pm 3 \mathrm{~mL} / \mathrm{min}$ and $95 \pm 5$ versus $86 \pm 6 \mathrm{~mL} / \mathrm{min}$, all $\mathrm{p}<0.05)$ and so was the blood volume treated per session, due to higher arterial aspiration and venous pressures requiring reduced blood flow rate. In contrast, we observed lower mean arterial aspiration $(96 \pm 18$ versus $130 \pm 19 \mathrm{mmHg}, \mathrm{p}=0.003$ ) and venous pressures $(82 \pm 8$ versus $96 \pm 16 \mathrm{mmHg}, \mathrm{p}=0.018)$ in the $4^{\text {th }}$ child $(18.8 \mathrm{~kg})$, when dialyzed on Fresenius 6008 @ with adult lines as compared to sessions performed with adult lines on Fresenius $5008 \AA$, without significant changes in blood flow, volume of blood treated per session and duration of sessions.

Conclusions: These results suggest that children between $9-13 \mathrm{~kg}$ be treated with pediatric lines on 5008® if possible to avoid higher arterial aspiration and venous pressures, and that children above $13 \mathrm{~kg}$ be treated with adult lines on $6008 \AA$. The interest of pediatric lines with $6008 \AA$ is likely below $10 \mathrm{~kg}$ and deserves further studies.

\section{EP-133 PERITONEAL DIALYSIS EFFLUENT DERIVED EXTRACELLULAR VESICLES REDUCE RISK OF DEVELOPMENT OF PERITONEAL DIALYSIS ASSOCIATED PERITONEAL FIBROSIS}

Beáta Szebeni ${ }^{1}$, Zoltán Varga ${ }^{3}$, István Márton Takács ${ }^{2}$, Domonkos Pap ${ }^{1}$, Apor Veres-székely ${ }^{1}$, Attila J. Szabó ${ }^{2}$, GyÖrgy S. Reusz, ${ }^{2}$, Ádám Vannay ${ }^{1}$

${ }^{I}$ Elkh-se Pediatrics And Nephrology Research Group

${ }^{2}$ Semmelweis University, 1st Department Of Pediatrics

${ }^{3}$ Elkh-ttk Biological Nanochemistry Research Group

Introduction: Extracellular vesicles (EVs) have significant therapeutic potential. They can protect against myocardial infarction, acute kidney injury or liver fibrosis, as well. However, the impact of EVs derived from dialysis effluents (PDE) of PD patients on the development of peritoneal fibrosis during PD remained unclear. Our aim was to determine, whether PDE-EVs can alter progression of peritoneal fibrosis.

Material and methods: PDEs were collected from children receiving continuous ambulatory peritoneal dialysis treatment in the $\mathrm{I}^{\mathrm{st}}$ Department of Paediatrics, Semmelweis University. PDE derived EVs (EVs) were isolated by size exclusion chromatography following ultrafiltration and were characterized according to the recommendations of the International Society for Extracellular Vesicles. Their impact on the proliferation and collagen production of primary peritoneal fibroblasts 
(pPFs) was tested by MTT or Sirius Red assay, respectively. The effect of EVs was also studied in a chlorhexidine gluconate (CG) induced mice model of peritoneal fibrosis in vivo. Submesothelial thickness was analysed after Periodic Acid Schiff- and Masson's Trichrome staining.

Results: EVs showed spherical morphology in the $100 \mathrm{~nm}$ size range. The infrared spectrum of EVs after buffer subtraction presented typical spectral features of EVs. Flow cytometry certified their CD9, CD63 and annexin positivity and lack of calnexin. PDE derived EVs entered into the cytoplasm of pPFs and significantly reduced PDE induced proliferation and collagen production of them. Finally, EVs penetrated into the peritoneal membrane of C57/BL6 mice after their i.p. injection and reduced the peritoneal thickness of CG treated mice almost to the control level.

Conclusions: PDE derived EVs have strong effect on PDE induced activation of pPFs in vitro and peritoneal thickness in vivo. This Project was supported by the FIKP (61830-69271) and STIA-KFI-2020 (61830-69319).

\section{EP-134 IDENTIFICATION OF EARLY BIOMARKERS OF PERITONEAL MEMBRANE DYSFUNCTION IN CHILDREN ON PERITONEAL DIALYSIS USING METABOLOMICS ANALYSIS -PRELIMINARY RESULTS OF AN ONGOING PROSPECTIVE STUDY \\ Antonia Kondou ${ }^{1}$, Vasiliki Karava ${ }^{1}$, John Dotis ${ }^{1}$, Olga Begou ${ }^{2}$, Helen Gika ${ }^{2}$, Georgios Theodoridis ${ }^{2}$, Dimitrios Zafeiriou ${ }^{3}$, Nikoleta Printza ${ }^{1}$ \\ ${ }^{1}$ Pediatric Nephrology Unit, 1st Department Of Pediatrics, Aristotle University Of Thessaloniki \\ ${ }^{2}$ Laboratory Of Analytical Chemistry, Department Of Chemistry, Aristotle University Of Thessaloniki Laboratory Of Forensic Medicine And Toxicology, School Of Medicine, Aristotle University Of Thessaloniki \\ ${ }^{3} 1$ st Department Of Pediatrics Aristotle University Of Thessaloniki}

Introduction: Peritoneal dialysis (PD) is the main renal replacement treatment for children with end stage renal disease (ESRD). Peritoneal fibrosis is a major complication in long-term PD patients. Aim of the study was to record the metabolic "fingertip" of children on PD and to investigate its correlation with PD vintage and dialysis adequacy as well as the emergence of potential biomarkers that could early detect or predict peritoneal dysfunction.

Material and methods: Samples of serum, urine and peritoneal effluent collected from 15 ESRD children on PD. At zero time and 6 months later PET-test was performed as well as assessment of KT/V and creatinine clearance. Samples were subjected to targeted metabolomic analysis of amino-acids and derivatives by mass spectrometry (HLIC- MS/MS) method and high flow (LC-qTOF analysis- HPLC/MS) analysis. Univariate and multivariate non -parametric statistical analysis tests were performed while level of significance was set at 0.01 .

Results: We found out that PD duration, presence or absence of diuresis and PD creatinine clerance values are associated with significant differences in the levels of several metabolites, including glycine, creatinine, proline and 4-hydroxyproline, leucine, valine, glutamine and glutamic acid. Using LC-qTOF analysis-HPLC/MS, approximately 200 metabolites were analyzed and many of them showed significant differnce accordingly to PD duration, creatinine clerance and presence or absence of diuresis. Concenring serum analysis, more than 25 metabolites were statistically different between the examined subgroups. Concerning urine analysis, uridine and itaconic acid showed statistical difference based on $\mathrm{PD}$ vintage.

Conclusions: Metabolomics may be a tool in the evaluation of patients with ESRD on PD as it appeas to reflect the clinical phenotype of the patient and the functional phenotype of the peritoneal membrane. These results are the preliminary results of an ongoing prospective study. There are also preliminary evidences that possibly may predict the decline in clearance adequacy.
EP-135 EARLY RECOGNITION OF ULTRAFILTRATION PROBLEMS BY REMOTE PATIENT MONITORING IN PEDIATRIC PERITONEAL DIALYSIS PATIENTS

Kyriaki Apostolidou ${ }^{I}$, Vasiliki Karava ${ }^{1}$, John Dotis ${ }^{1}$, Antonia Kondou ${ }^{I}$, Kyriaki Charpantidou ', Anestis Apostolidis ${ }^{2}$, Dimitrios Zafeiriou ', Nikoleta Printza ${ }^{1}$

${ }^{1}$ Pediatric Nephrology Unit, 1st Department Of Pediatrics, Hippokratio General Hospital,aristotle University Of Thessaloniki, Greece

${ }^{2}$ Department Of Electrical And Computer Engineering, Faculty Of Engineering, Aristotle University Of Thessaloniki, Greece

Introduction: Remote patient monitoring (RPM) technologies are used in peritoneal dialysis (PD) patients in order to improve patient life quality and healthcare efficacy. This is a six-moth prospective observational single-center pediatric study aiming to determine the impact of lost PD treatment recorded by RPM on peritoneal ultrafiltration volume (UF).

Material and methods: Nine pediatric patients, 5 males and 4 females, with a median age of 15 years old, on automated PD were enrolled. 5 patients were oligoanuric and 4 presented residual renal function (RRF). All patients' cyclers were connected to a web-based software (ClariaSharesource Baxter) and PD session data were obtained from this database. Weekly UF, lost PD time, as well as number of alarms were recorded for each patient. In total, $1617 \mathrm{PD}$ prescribed sessions data were analyzed. Results: Lost PD sessions were observed in $2.5 \%$ of total prescribed PD sessions, while weekly mean lost PD time was $188.34 \mathrm{~min}$. At least 1 lost PD session per week was significantly associated with lower weekly nocturnal UF $(p<0.001)$. Number of alarms per week did not negatively affect nocturnal UF, except for patients with RRF $(r=-0.613, p<0.001)$. Weekly lost PD time percentage was negatively correlated to nocturnal UF (r:-0.323, p<0.001). In detail, weekly lost PD time of $1 \%$ in oligoanuric patients $(\mathrm{p}<0.001)$ and of $10 \%$ in patients with RRF $(\mathrm{p}<0.001)$ was significantly associated with lower nocturnal UF.

Conclusions: Access to PD sessions data by RPM is vital for optimal pediatric PD monitoring because it facilitates pediatric nephrologist awareness of treatment adherence and PD efficacy, which ultimately affects UF.

\section{EP-136 MICRODIALYSIS TO STUDY TISSUE PENETRATION OF DRUGS IN THE JUVENILE PIG: AN OPPORTUNITY FOR PEDIATRIC DRUG RESEARCH}

Eline Hermans ${ }^{1}$, Pieter De Cock ${ }^{1}$, Johan Vande Walle ${ }^{2}$, Peter De Paepe ${ }^{2}$, Mathias Devreese ${ }^{I}$

${ }^{1}$ Ghent University

${ }^{2}$ Ghent University Hospital

Introduction: Juvenile pig models have proven to provide an accurate and reliable prediction of pediatric pharmacokinetic behavior. Microdialysis is currently the gold standard for studying drug concentrations in tissues. In microdialysis a small probe, consisting of a semipermeable hollow fiber membrane, is implanted into the tissue of interest. Mainly because of the invasiveness of microdialysis, data on tissue distribution of drugs remains largely understudied in children. The aim of this pilot study was to investigate the feasibility of multiple day microdialysis in awake and free moving piglets.

Material and methods: Six piglets of four weeks old were individually housed in pens. During short sedation a microdialysis probe was implanted in the paraspinal musculature under ultrasound guidance. Thereafter, the microdialysis pump was connected and fixated on the body by means of an elastic band encircling the thorax. Over the course of four days intravenous antibiotics were administered and blood and microdialysis samples were taken while the piglets were awake and free moving in their pen.

Results: In five of the six piglets the microdialysis catheter remained functional until the end of the fourth sampling day. In one piglet, the probe membrane broke on day 2 after intense struggling of the piglet 
during blood sampling. No adverse events (bleeding, infection, pain or other discomfort) were observed during the placement of the microdialysis catheter and whole study period.

Conclusions: This study shows that it is feasible to use microdialysis to study tissue drug disposition in free moving piglets over multiple days, adding to the potential of the juvenile pig in pediatric drug research.

\section{EP-137 SEVERE REFRACTORY BULLOUS PEMPHIGOID IN A PEDIATRIC PATIENT SUCCESSFULLY TREATED WITH IMMUNOADSORPTION}

Perez-beltran Victor ${ }^{1}$, Pujol-manresa Anna ${ }^{2}$, Muñoz Marina ${ }^{1}$, Seidler-nussenbaum Laura ${ }^{2}$, Lopez-corbeto Mireia ${ }^{3}$,

Garcia-patos VicenÇ ${ }^{4}$, Ariceta Gema ${ }^{1}$

${ }^{1}$ Pediatric Nephrology Department, Hospital Universitari Vall

D’hebron, Barcelona, Spain

${ }^{2}$ Pediatric Critical Care Department, Hospital Universitari Vall D'hebron, Barcelona, Spain

${ }^{3}$ Rheumatology Department, Hospital Universitari Vall D’hebron, Barcelona, Spain

${ }^{4}$ Dermatology Department, Hospital Universitari Vall D'hebron, Barcelona, Spain

Introduction: Pathogenic autoantibodies removal by immunoadsorption (IA) has been described as an effective adjuvant treatment in some bullous diseases, especially in pemphigus, but the experience is limited. Bullous pemphigoid is not listed as disease treatable by IA at the American Apheresis Society (ASFA) guidelines. Further, bullous pemphigoid occurs very rarely in children and usually exhibits good outcome and response to corticosteroids.

Material and methods: We describe a very aggressive case of bullous pemphigoid refractory to conventional treatment that was treated effectively with IA associated to high-dose corticosteroids, mycophenolate, rituximab and IV immunoglobulin (IVIg).

Results: A previously healthy 8 -year-old boy with pruritic papular rash that progressed to papular erythematous rash, associated with multiple bullae, initially more pronounced in hands and feet but later generalized, is described. Skin biopsy and positive serum autoantibodies against BP180 confirmed the diagnosis of bullous pemphigoid. Despite treatment with systemic corticosteroids and sulphones, patient's lesions worsen to superimposed bullae and erosions with concomitant oral mucosa involvement.

The patient was admitted to the pediatric critical care department and topical silver sulfadiazine and high protein diet were initiated. Further, 5 IA sessions were performed (days 1, 2, 3, 5 and 8) and treatment was completed with IVIg $(2 \mathrm{gr} / \mathrm{kg})$ and 4 Rituximab doses $(375 \mathrm{mg} / \mathrm{m} 2$ weekly). Oral mycophenolate was initiated in order to spare corticosteroid treatment. After the first immunoadsorption session, no new skin lesions appeared, and complete remission was achieved 3 days after the last session. BP180 auto-antibodies turned weakly positive at the end of treatment. At 6-months follow up, the patient remains stable without new lesions and weakly positive BP180 antibodies.

Conclusions: Even with spare evidence in uncommon diseases, immunoadsorption has to be considered in refractory and life-threating cases of demonstrated antibody mediated diseases. This case is another example of IA therapy's safety and feasibility in pediatric patients.

\section{EP-138 HEMATOPOIETIC STEM CELL TRANSPLANTATION IN CHRONIC KIDNEY DISEASE \\ Katarzyna Mikrut, Katarzyna Zachwieja, Dorota Drożdż \\ Jagiellonian University Medical College, Department Of Pediatric Nephrology And Hypertension}

Introduction: Performing hematopoietic stem cell transplantation (HSCT) in children with advanced chronic kidney disease (CKD) carries significant risk of complications.
Material and methods: 16-year-old male with stage IV CKD due to obstructive nephropathy/posterior urethral valvae and recently diagnosed with Fanconi anemia was prepared to an allogenic HSCT procedure. Adequate dosage of cyclophosphamide (Cy) used in conditioning regimen appeared challenging because of patients lowered GFR $(30 \mathrm{ml} / \mathrm{min} / 1,73 \mathrm{~m} 2)$ but also due impossible monitoring of $\mathrm{Cy}$ concentration in serum and tracking its pharmacokinetics.

The patient received full dose of Cy provided in the protocol and he underwent CVVHDF for 90 hours (from 20 hours after first dose of Cy till 20 hours following the last dose of Cy). CRRT parameters were continuously customized according to patient's clinical and metabolic condition. No complications of CRRT were seen. As a result correct myeloablative function of $\mathrm{Cy}$ was obtained and the patient succeed undergoing HSCT. After the procedure he presented laboratory features of transient acute kidney injury with signs of increased catabolism managed with conservative treatment only and hemorrhagic cystitis. Typical Graft-versusHost disease prophylaxis was administered.

Results: The patient has been living over 2 years since the HSCT so far, lymphoproliferative disease and central line -associated infection have been observed as complications in post-transplant period.

Conclusions: The patient remains in good clinical condition with CKD treatment with no need of dialysis, he undergoes regular check-ups by nephrologists, his current GFR $=42 \mathrm{ml} / \mathrm{min} / 1,73 \mathrm{~m} 2$ - actually higher than before HSCT.

\section{EP-139 A RARE CAUSE OF CAPD-RELATED PERITONITIS} Aynur Gencer Balaban Esra Aykutlu ${ }^{2}$, Usame Takas ${ }^{2}$, Aylin Inal ${ }^{1}$, Sibel Yel ${ }^{1}$, Neslihan Gunay ${ }^{1}$, Ismail Dursun ${ }^{1}$, Muammer Hakan Poyrazoglu ${ }^{1}$

${ }^{1}$ Erciyes University Department Of Pediatric Nephrology Kayseri, Turkey

${ }^{2}$ Erciyes University Department Of Pediatrics Kayseri, Turkey

Introduction: Kocuria species are bacteria commonly found in the environment and human skin and mucosa. There are limited reported cases of Kocuria kristinae related peritonitis in the literature. Here, we would like to report a case of Kocuria kristinae infection related with continuous ambulatory peritoneal dialysis (CAPD) peritonitis leading to catheter loss.

Material and methods: A 4-year-old boy was admitted to the pediatric nephrology department because of turbid dialysis effluent for 3days. $\mathrm{He}$ had end-stage renal disease as a result of posterior urethral valve and had been undergoing continuous ambulatory peritoneal dialysis (CAPD) for 45 days. Upon physical examination, he was afebrile, with a normalappearing catheter exit site. However,the peritoneal dialysate fluid was fibrinous and cloudy, with a total leukocyte count of $2655 /$ microliter, PMN:1648/microliter, monocyte: 1007/microliter. Intraperitoneal administration of vancomicin and ceftazidime was started for empirical treatment of CAPD peritonitis. No microorganisms were seen on a Gram stain. Culture of the dialysate yielded a pure culture of gram positive cocci in pairs or clusters and resulted in contamination. Due to our high suspicion of bacterial peritonitis, different techniques were used for microorganism identification and Kocuria kristinae was determined. By day twenty one of treatment, the peritoneal fluid became clear, with dialysate white cell count was 22 leucocytes $/ \mu$ l. The patient's complaints including backpain, fatigue and loss of appetite resolved completely. At the first control visit, the peritoneal dialysate was turbid anew, and cell count revealed the presence of 422 leucocytes $/ \mu 1$.

Results: In spite of the administration of intraperitoneal vancomycin, the response remained unsatisfactory. The catheter of peritoneal dialysis was removed and the patient improved with antibiotic therapy for 7 days after catheter removal and was discharged. 
Conclusions: Kocuria kristinae is a rare agent in PD peritonitis. In cases of CAPD-related peritonitis, we should consider K. Kristinae especially if the culture results in contamination.

\section{EP-140 EVALUATION OF CLARIA SHARESOURCE SYSTEM FROM THE PERSPECTIVES OF PATIENT/CAREGIVER, PHYSICIAN, AND NURSE IN CHILDREN UNDERGOING AUTOMATED PERITONEAL DIALYSIS}

Bahriye Uzun Kenan ${ }^{1}$, Beltinge Demircioglu Kilic ${ }^{2}$,

Mehtap Akbalik Kara ${ }^{3}$, Aysel Taktak ${ }^{4}$, Aysun Karabay Bayazit ${ }^{5}$, Zeynep Nagehan Yuruk Yildirim ${ }^{6}$, Ali Delibas ${ }^{7}$, Mehmet Baha Aytac ${ }^{8}$, Secil Conkar ${ }^{9}$, Gulsah Kaya Aksoy ${ }^{10}$, Osman Donmez ${ }^{11}$, Sibel Yel ${ }^{12}$, Seha Saygili ${ }^{13}$, Okan Akaci ${ }^{14}$, Bahar Buyukkaragoz ${ }^{1}$, Harika Alpay ${ }^{15}$, Sevcan A. Bakkaloglu ${ }^{l}$

${ }^{1}$ Gazi University School Of Medicine ,Ankara, Turkey

${ }^{2}$ Gaziantep University School Of Medicine, Gaziantep, Turkey

${ }^{3}$ Diyarbakir Children's Hospital, Diyarbakir, Turkey

${ }^{4}$ Diyarbakir Gazi Yaşargil Training And Research Hospital, Diyarbakir, Turkey

${ }^{5}$ Cukurova University School Of Medicine, Adana, Turkey

${ }^{6}$ Istanbul University School Of Medicine, İstanbul, Turkey

${ }^{7}$ Mersin University School Of Medicine, Mersin, Turkey

${ }^{8}$ Kocaeli University School Of Medicine, Kocaeli, Turkey

${ }^{9}$ Ege University School Of Medicine, Izmir, Turkey

${ }^{10}$ Akdeniz University School Of Medicine, Antalya, Turkey

${ }^{11}$ Uludag University School Of Medicine, Bursa, Turkey

${ }^{12}$ Erciyes University School Of Medicine, Kayseri, Turkey

${ }^{13}$ Istanbul University-cerrahpasa Medical School, Istanbul, Turkey

${ }^{14}$ Bursa Yuksek Ihtisas Training And Research Hospital, Bursa, Turkey

${ }^{15}$ Marmara University School Of Medicine, İstanbul, Turkey

Introduction: Peritoneal dialysis (PD) remains the most commonly used dialysis modality in children, and automated PD (APD) has been increasingly preferred worldwide. By application of a software (Homechoice Claria with sharesource system) that can be used with a mod-M added to the APD device, the details of the home dialysis treatment become visible for PD nurse and physician to allow for close supervision.

Objective: This study was planned to evaluate patient/caregiver, PD nurse, and physician's perception and knowledge about advantages and disadvantages of the Claria resource sharing system regarding its effect on patient-oriented clinical recovery parameters.

Method: A web-based questionnaire consisting of yes-no questions and multiple-choice questions was sent to 15 pediatric nephrology centers, where remote patient monitoring (RM-APD) applied with more than a year of experience with Claria. It was answered by 30 patients/caregivers, 15 PD nurses, and 22 pediatric nephrologists.

Results: $86 \%$ of the nurses reported that the Claria system increased medical control over the patients. $75 \%$ of physicians thought that the Claria system increased medical supervision over the patients and therefore created a safe zone and time saving for both patients and healthcare providers, while no impact on financial burden of the family and healthcare system. Half of the physicians believe that Claria can reduce the need for frequent visits and $2 / 3$ had a belief that it increased patients compliance with PD treatment and other medical treatments. While $60 \%$ of the patients/ caregivers stated that they had concerns about the quality-of-care delivery at the beginning, they were convinced about the system when they saw that the problems were solved through online based on an objective and reliable data.

Conclusion: RM-APD application can be successfully applied to children for increasing compliance with the dialysis prescription and can be considered an important tool for early recognition of problems and their proactive interventions/solutions by the physicians and nurses.

Key words: Peritoneal dialysis, remote monitoring, children, quality of life
EP-141 FIRST VASCULAR ACCESS FOR PEDIATRIC HEMODIALYSIS PATIENTS - IMPACT ON HOSPITALIZATION AT ONE YEAR AFTER STARTING DIALYSIS

Ana Domingos ${ }^{1}$, Mariana Bastos Gomes ${ }^{2}$, Liane Costa ${ }^{3}$, Ana Teixeira ${ }^{3}$, Liliana Rocha ${ }^{3}$, Paula Matos ${ }^{3}$, Teresa Costa ${ }^{3}$, Maria Sameiro Faria ${ }^{3}$, Conceição Mota ${ }^{3}$, Alberto Caldas Afonso ${ }^{3}$

${ }^{1}$ Centro Hospitalar E Universitário Do Algarve - Hospital De Faro

${ }^{2}$ Unidade Local Saúde Alto Minho - Hospital De Santa Luzia

${ }^{3}$ Centro Hospitalar E Universitário Do Porto - Centro Materno-infantil Do Norte

Introduction: Central venous catheter (CVC) is by far the most used vascular access (VA) in incident pediatric patients starting on Hemodialysis (HD), with considerable rates of morbimortality. We present our experience, regarding several outcomes at one year follow-up, namely hospital admissions and according to their VA type at baseline.

Material and methods: A retrospective descriptive study included patients admitted to our pediatric HD Unit between 2005-2020. Patients were followed from their first HD session until one-year follow-up, or concurrent event.

Results: 42 patients were enrolled, mean age at admission $10.8 \pm 5.3$ years. Most patients initiated HD with a CVC $(n=32,76.2 \%)$. At the end of follow-up, $50 \%$ of patients ended up with an arteriovenous fistula (AVF), comparing to $23.8 \%$ at the beginning. Twenty-four patients (57\%) had, at least, one hospitalization (total of 46 hospitalizations and 204 cumulative days). "CVC at baseline" group represents $36.9 \%$ of all admissions. Seven of 10 patients (70\%) with "AVF at baseline", and 17 of 32 patients $(53 \%)$ starting with CVC experienced hospitalization. There was not a statistically significant difference between total number of hospitalizations and length of stay (LOS) on group "CVC at baseline" versus "AVF at baseline". VA-related hospitalizations accounted for $36.9 \%$ of total admissions. We did not find a statistically significant difference between the type of VA and the cause of hospitalization. Admissions due to VA mechanical complications were responsible for the longest mean LOS, with $7.1 \pm 9.3$ days.

Conclusions: Although there has been a trend towards "Fistula First", over-all hospitalizations were significant in both VA groups. More than $75 \%$ of our patients had a CVC at baseline, but this is mainly justified by the anatomical limitations of younger and smaller patients, and is similar to several international reports. We highlight the increase in AVF placement at the end of the follow-up.

\section{EP-142 PERITONEAL DIALYSIS (PD) COMPLICATIONS IN CHILDREN WITH ESRD: A LOW-INCOME COUNTRY SINGLE CENTER EXPERIENCE}

Houda Nassih, Rabiy El Qadiry, Aicha Bourrahouat, Imane Ait Sab B Pediatric Ward, Department Of Pediatrics, Child And Mother Hospital, Mohammed Vi University Hospital Center, Marrakesh, Morocco

Introduction: We report the complications of PD in children from a lowincome country

Material and methods: A single center retrospective study of 11 children undergoing PD for ESRD

Results: The mean age was 12-year-old. We had 5 boys and 6 girls. The mean time under PD was of 2.5 years. Etiology of ESRD was severe tubulointerstitial nephritis in one case, rapidly progressive glomerulonephritis in 4 cases, nephroblastoma and contralateral hypoplastic kidney in one case, focal segmental glomerulosclerosis in one case, Alport syndrome in one case, and CAKUT with recurrent pyelonephritis in 2 cases. PD was initiated as first line renal replacement therapy in 10 cases, while one child was switched from hemodialysis to PD after a period of two years. PD type was APD/CCPD in 10 cases, and tidal PD in one case. The mean overnight treatment time was 9 hours and the mean number of cycles was 5 . The mean total therapeutic volume was of $5944 \mathrm{ml}$. 
Dialysis fluids used in our patients were $1.36 \%$ dextrose in 11 cases, $2.25 \%$ dextrose in 2 cases. Icodextrine was prescribed as a unique daytime dwell in 3 cases with a mean duration of 14.6 hours. Complications were noted in 10 patients. Peritonitis was present in 6 patients with a mean of 3 episodes for each patient. Retention was seen in 6 patients. Catheter dysfunction was noted in 5 cases, from whom 3 had migration and 2 had obstruction of the PD catheter. One child had Sclerosing peritonitis and was switched to hemodialysis after 3 years under PD. Unfortunately, two of our patients died, one after massive alveolar hemorrhage and the second one after septic choc.

Conclusions: The training on PD catheter placement technics as well as the practice of aseptic techniques by the family are the keys to limit PD complications in our population.

\section{EP-143 CHARACTERISTICS OF PATIENTS WITH DISTAL RENAL TUBULAR ACIDOSIS AND ITS ASSOCIATION WITH HYPERAMMONEMIA: A SINGLE-CENTER EXPERIENCE}

Ece Demirci Bodur ${ }^{\text {}, ~ S e r c ̧ i n ~ G u ̈ v e n ~}{ }^{1}$, Burcu Hışmi Öztürk ${ }^{2}$, Neslihan Çiçek ${ }^{1}$, Mehtap Sak ${ }^{l}$, Özde Nisa Türkkan ${ }^{1}$, Serim Pul ${ }^{1}$, Nurdan Yuldız ${ }^{1}$, Ibrahim Gökçe ${ }^{1}$, Harika Alpay ${ }^{1}$

${ }^{1}$ Marmara University Pediatric Nephrology

${ }^{2}$ Marmara University Pediatric Metabolism

Introduction: Distal renal tubular acidosis (dRTA) is a tubular disorder characterized by metabolic acidosis with a normal anion gap and alkaline urine. Mutations in ATP6V1B1, ATP6V0A4, SLC4A1, FOXI1 and WDR72 can cause dRTA. Since chronic metabolic acidosis and hypokalemia increase renal ammoniagenesis and ammonia reabsorption, it leads to hyperammonemia in dRTA. Treatment of acidosis and hypokalemia usually correct hyperammonemia. In this study, we described the presenting features and long-term outcomes and investigated the relationship with hyperammonemia of seven patients with dRTA.

Material and methods: Biochemical and clinical results were collected at the presentation and last clinic visit. Genetic studies were performed. The estimated glomerular filtration rate (eGFR) was calculated using the original Schwartz formula. Hyperammonemia was defined as serum ammonia level $>60 \mu \mathrm{mol} / \mathrm{L}$.

Results: Three patients $(42,8 \%)$ were female. The median age at diagnosis was six months (min-max:1-114 months). The median follow-up time was 90 months (min-max:34-150 months). Median plasma concentrations of electrolytes at presentation were nonremarkable. All patients had nephrocalcinosis at presentation. Median eGFR $(\mathrm{ml} / \mathrm{dk} / 1.73 \mathrm{~m} 2)$ at presentation was 123 (min-max:24-240) and 126 (min-max:105-192) at last visit. The most common presenting symptom was vomiting $(57,2 \%)$ followed by fever $(28,5 \%)$ and polyuria-polydipsia $(14,2 \%)$. At the last visit, six of seven patients $(85,7 \%)$ improved their weight percentiles and five of seven patients $(71 \%)$ improved their height percentiles. Homozygous mutation was found in the ATP6V0A4 gene in 5 of 7 patients and in the SLC4A1 gene in one patient. No mutation was found in one patient. In our study, four patients (\%57) had hyperammonemia and it resolved in all except one patient with correction of acidosis and hypokalemia.

Conclusions: Early recognition of dRTA and correction of metabolic acidosis and dehydration are important. Since hyperammonemia may accompany dRTA, hyperammonemia should be kept in mind especially in patients with neurocognitive problems or persistant vomiting.

\section{EP-144 AUDIOLOGICAL FINDINGS IN DISTAL RENAL TUBULAR ACIDOSIS \\ Ezgİ Ay ${ }^{1}$, Emre GÜrses ${ }^{2}$, Filiz Arslan ${ }^{2}$, Bora GÜlhan ${ }^{3}$, Asuman AlniaÇik ${ }^{1}$, Ali DÜzova ${ }^{3}$, MÜnir Demir Bajin ${ }^{4}$, Levent SennaroĞlu ${ }^{4}$, Gülsüm Aydan GenÇ ${ }^{2}$, Fatih Ozaltin ${ }^{3}$, Rezan Topaloglu ${ }^{3}$}

${ }^{I}$ Baskent University Faculty Of Health Sciences, Department Of Audiology, Ankara, Turkey

${ }^{2}$ Hacettepe University Faculty Of Health Sciences, Department Of Audiology, Ankara, Turkey

${ }^{3}$ Hacettepe University School Of Medicine, Department Of Pediatrics, Division Of Pediatric Nephrology, Ankara, Turkey

${ }^{4}$ Hacettepe University School Of Medicine, Department Of Ear Nose And Throat, Ankara, Turkey

Introduction: Different degrees of hearing loss may accompany different mutations in distal renal tubular acidosis (dRTA). The aim of this study is to examine the characteristics of hearing loss in dRTA patients.

Material and methods: A group of 21 patients with confirmed dRTA were included in the study. Clinical and laboratory evaluations of the patients were collected. Otological examination and holistic audiological evaluation of all patients were performed.

Results: The population consisted of 13 females and 8 males (range; 8 months-33 years) with dRTA. A genetic analysis could be performed in 17 patients(four participants did not give consent). Among 17 patients, nine had ATP $6 V 1 B 1$, six had ATP6VOA4, one had SLC4A2 and one had WDR72 mutation. In the group, hearing loss configurations tended to increase towards higher frequencies. Based on pure tone average (PTA), all patients with ATP6V1B1 mutation had various degrees of hearing loss from mild to profound. We also found large vestibular aqueduct syndrome (LVAS) in $83 \%$ of the patients with ATP6V1B1 mutation. Patients with bi-allelic ATP6V0A4 mutation showed that one patient had severe sensorineural hearing loss (SNHL) in one ear and moderately-severe SNHL in the other. The rest of the patients had normal PTAs. One patient with SLC4A2 mutation had bilateral mild mixed hearing loss based on her PTA's. We found that the pure tone hearing thresholds of the patients with ATP6V0A4 mutations were statistically significantly higher than those obtained in patients with ATPVIB1 mutations for both air and bone conduction on the right and the left ears $(\mathrm{p}<0.05)$.

Conclusions: In our dRTA patients, hearing loss in high frequencies is commonly observed in all mutations. Besides, the type of the mutation seems to have an effect on the hearing thresholds. Our study is the first to demonstrate the association of SLC4A2 mutation and hearing loss.

EP-145 CLINICAL COURSE AND PROGNOSIS OF
TUBULOPATHIES CHARACTERIZED BY METABOLIC ALKALOSIS IN CHILDREN

Bahruz Huseynli ${ }^{1}$, Bahriye Atmis ${ }^{1}$, Derya Cevizli ${ }^{1}$, Atil Bisgin ${ }^{2}$, Aysun K. Bayazit ${ }^{I}$

${ }^{1}$ Cukurova University, Faculty Of Medicine, Department Of Pediatric Nephrology, Adana, Turkey

${ }^{2}$ Cukurova University, Faculty Of Medicine, Department Of Medical Genetics, Adana, Turkey

Introduction: Bartter and Gitelman syndromes are rare inherited tubulopathies characterized by hypokalemic, hypochloremic metabolic alkalosis. Three phenotypes exist in Bartter syndrome (BS); antenatal (aBS), classical (cBS) and mixed type. Also, BS is classified genotypically into five types as SLC12A1 (BS I), KCNJ1 (BS II), CLCNKB (BS III), BSND (BS IV) and CASR (BS V). It is aimed to clarify the frequency of the phenotypic and genotypic subgroups, clinical features, long-term management and prognosis of children diagnosed with BS and GS in this study.

Material and methods: Twenty seven patients with BS and six patients with GS, who were followed-up between 2004 and 2020 in Department of Pediatric Nephrology, Çukurova University Faculty of Medicine, were included in our study.

Results: Fifteen (55.5\%) of the patients with BS were male and two $(33.3 \%)$ of the GS patients were male. The mean age of diagnosis was 14.9 months 
(0.5- 84) in patients with BS and 158 months (84-192) in patients with GS. Fourty eight percent of BS patients had a history of polyhydramnios. Ten patients with BS had $C L C N K B, 3$ patients had SLC12A1, 1 patient had $B S N D, 1$ patient had $C L C N K A$, and 6 patients with GS had SLC12A3 genetic mutations. Growth retardation was found in $81.8 \%$ of patients with aBS, $68.7 \%$ of patients with cBS and mixed type, and $50 \%$ of patients with GS. Chronic kidney disease (CKD) stage 3 was detected in $14 \%$ of patients with $\mathrm{BS}$ at the time of diagnosis, while the rate of patients with CKD stage 3 and above at the last follow-up was $25.9 \%$. Estimated glomerular filtration rates (eGFR) of patients with GS were normal at the time of diagnosis and at the last follow-up. eGFR values were found to be statistically significantly lower in patients with aBS than in patients with $\mathrm{cBS}$ and GS in the last follow-up. Conclusions: Patients with BS and GS may have a different clinical course due to the underlying genetic mutation. Since BS and GS require life-long treatment, treatment compliance is important to prevent advanced stage $C K D$.

\section{EP-146 A CASE SERIES OF FAMILIAL HYPOMAGNESEMIA WITH HYPERCALCIURIA AND NEPHROCALCINOSIS}

Zehra Aydin ${ }^{1}$, Fatma Semsa Cayci ${ }^{1}$, Ibrahim Sahin ${ }^{2}$, Mihriban Inozu ${ }^{1}$, Begum Avci ${ }^{1}$, Umut Selda Bayrakci ${ }^{l}$

${ }^{1}$ Ankara City Hospital, Department Of Pediatric Nephrology, Ankara, Turkey

${ }^{2}$ Diskapi Yildirim Beyazit Training And Research Hospital, Department Of Medical Genetics, Ankara, Turkey

Introduction: Familial hypomagnesemia with hypercalciuria and nephrocalcinosis (FHHNC) is a rare autosomal-recessive renal tubular disorder. It is characterized by renal wasting of magnesium $(\mathrm{Mg})$ and calcium $(\mathrm{Ca})$, subsequently leads to bilateral nephrocalcinosis, renal stones, and chronic renal failure. It is also caused by variants in the CLDN16 or CLDN19 genes. Treatment is supportive. Here, we report seven patients with FHHNC.

Material and methods: We retrospectively analyzed pediatric cases of FHHNC diagnosed at our hospital between 2010-2020.

Results: A total of 7 patients, 5 girls $(71.4 \%)$ and 2 boys $(28.5 \%)$ with a median age of 4 years (min:4 months, max:13 years) and a mean followup time of $4.4 \pm 3.5$ years were included in the study. All patients had normal body and height percentiles. Three of them had low urine citrate levels. All of the patients had nephrocalcinosis, normal urine oxalate levels, and normal blood gas analysis. One patient had recurrent urinary tract infection and urolithiasis. Five patients had CLDN 16 mutation, and two patients had CLDN19 mutation. None of them had ocular findings. All patients had high parathormone levels $(216,7 \pm 169,5(\mathrm{pg} / \mathrm{mL})$. At the end of the follow-up period, two patients had chronic renal failure.

Conclusions: FHHNC frequently leads to renal failure during childhood or adolescence. FHHNC must be kept in mind when severe hypomagnesemia or hypercalciuria is accompanied by nephrocalcinosis.

\section{EP-147 MEDULLARY NEPHROCALCINOSIS IN A BOY WITH ATP6V0A4 AND PKD1 MUTATION}

Zehra Aydin ${ }^{1}$, Fatma Semsa Cayci ${ }^{1}$, Ibrahim Sahin ${ }^{2}$, Umut Selda Bayrakci ${ }^{1}$

${ }^{1}$ Ankara City Hospital, Department Of Pediatric Nephrology, Ankara, Turkey

${ }^{2}$ Diskapi Yildirim Beyazit Training And Research Hospital, Department Of Medical Genetics, Ankara, Turkey

Introduction: Nephrocalcinosis (NC) is a rare condition defined by the accumulation of calcium phosphate or calcium oxalate deposits in the renal parenchyma, particularly in tubular epithelial cells and interstitium. The most common form is the medullary NC form and it may be a sign of some metabolic disorders as distal renal tubular acidosis. In addition, it may be rarely associated with other anomalies.
Material and methods: We present a case of a 1-month old boy who was referred for metabolic evaluation of bilateral NC.

Results: The patient had hypokalemia, hyperchloremia, and metabolic acidosis with a normal anion gap. Urine density was 1008 and urine ph was 8 with no evidence of glycosuria, proteinuria, or phosphaturia. Further investigations showed high urine anion gap and high urine potassium-to-creatinine ratio. He was diagnosed with dRTA. The patient was subsequently treated with potassium citrate, a low sodium diet accompanied by high fluid intake. After a 12-year therapy bilateral multiple parapelvic renal cysts with $\mathrm{NC}$ were observed by renal ultrasonography. To provide a specific diagnosis, we performed genetic analysis, and homozygous ATP6VOA4 and heterozygous PKD1 mutations have been detected in our patient.

Conclusions: We conclude that dRTA should always be considered as a cause of medullary NC. Since simple cortical renal cysts are considered as a complication of the hypokalemia in dRTA, the concomitant multiple renal cysts should also aware of polycystic kidney disease and the final diagnosis requires specific genetic analysis.

\section{EP-148 ANTENATAL BARTTER SYNROME (ABS) TYPE 1 WITH LOW URINARY CHLORIDE EXCRETION: AN UNUSUAL PRESENTATION}

Varvara Askiti ${ }^{1}$, Anastasia Garoufi ${ }^{2}$, Eleni-maria Papatesta ${ }^{2}$, Mato Nagel ${ }^{3}$, Andromachi Mitsioni ${ }^{1}$

${ }^{1}$ Pediatric Nephrology Department Athens Childrens Hospital "p\&a Kyriakou"

${ }^{2}$ 2nd Department Of Pediatrics National And Kapodistrian University Of Athens, Childrens Hospital "p\&a Kyriakou"

${ }^{3}$ Center For Nephrology And Metabolic Disorders, Laboratory For Molecular Diagnostics, Weisswasser, Germany

Introduction: It is known the utility of urinary chloride measurement in the differential diagnosis of Bartter syndrome. We report a case of ABS with low urinary chloride excretion.

Material and methods: Premature newborn of 31 weeks of gestation with history of polyhydramnios, birth weight of $1650 \mathrm{gr}$. He remained to the neonatal intensive care unit for 3 months due to respiratory distress, acute kidney injury (Ur:230, Cre:1.13) which gradually resolved. At the age of 4 months he was severely growth retarded (height and weight far below the $3^{\text {rd }}$ percentile), normotensive, with polyuria, metabolic alkalosis, hypernatremia (144$154 \mathrm{meq} / \mathrm{l})$, serum chloride normal, serum potassium $(3,6-4,4 \mathrm{mEq} /$ L), bilateral nephrocalcinosis grade II-III. Plasma renin $>31 \mathrm{ng} / \mathrm{mL}$ ( 0.32-1,84 ng/mL), serum aldosterone $1700 \mathrm{pg} / \mathrm{mL}(20-1100)$. In serial spot urine, calcium to creatinine ratio $2-2,88$, potassium $60-69$ $\mathrm{mEq} / \mathrm{L}$, sodium $11-16 \mathrm{mEq} / \mathrm{L}, \mathrm{FE} \mathrm{Na} 0.10-0.15 \%$, chloride $11-20$ $\mathrm{mEq} / \mathrm{L}$, chloride/creatinine ratio 5.5-6.8, serial values $\mathrm{FE} \mathrm{Cl}<0.5$. Although there was suspicion of BS, the consistently low urinary chloride excretion were debated the diagnosis. Whole exome sequencing analysis was performed.

Results: Two heterozygous mutations likely pathogenic were found in SLC12A1 gene (c.2168_2169delTG, c.3164+2T>G), that are not yet published. A mutation in the CLCNKB was found (c700T>C) and another variant (c.936_937insAATA) of unknown significance. The father has one of the two SLC12A1 mutations and both the CLCNKB variants, while the other SLC12A1 was found in the mother. As by parental screening have been confirmed that the SLC12A1 mutations are bi-allelic, this is consistent with ABS type 1. Therapy with indomethacin and potassium citrate was started. At the age of 4 yrs old, his height and weight have improved $\left(10^{\text {th }}\right.$ percentile $)$.

Conclusions: We have described a child with atypical clinical presentation of ABS type 1 confirmed with genetic testing. This demonstrates the utility of whole exome analysis in improving the diagnostic accuracy of atypical ABS. 
EP-149 CIRCADIAN RHYTHM OF URINARY WATER AND SODIUM EXCRETION IN CHILDREN WITH MNE AND NMNE ENURESIS

Sevasti Karamaria ${ }^{3}$, Vincent Delens ${ }^{2}$, Lien Dossche ${ }^{3}$, Ann Raes ${ }^{3}$, Evelien Snauwaert ${ }^{1}$, Johan Vande Walle ${ }^{3}$

${ }^{1}$ Ghent University Hospital

${ }^{2}$ Ghent University

${ }^{3}$ Ghent University Hospital, Ghent University

Introduction: Nocturnal enuresis (NE) is caused by a mismatch of nocturnal urine production and functional bladder capacity. Nocturnal polyuria (NP) is defined as nocturnal diuresis (ND) exceeding the Expected Bladder Capacity (EBC) by $130 \%$ and is well documented as pathogenetic mechanism. NP is attributed to abnormal circadian rhythm of vasopressin (AVP). However, 40-60\% of patients treated with desmopressin, an AVP agonist, show insufficient response, suggesting other factors may be involved.

Material and methods: We aimed to study the kidney circadian rhythm on water and solute excretion in fractionated urine samples over $24 \mathrm{~h}$ (4 day and 4 night samples); we analyzed retrospectively data from 402 enuretic. The children were divided into 3 subgroups: a) low-normal ND $(<90 \%$ EBC, $n=113)$ b) high-normal ND (90-130\% EBC, $n=103)$ c) NP $(>130 \%$ EBC, $n=91)$.

Results: Increased diuresis rate and free water clearance overnight is present in the NP and the high-normal ND group. Both groups demonstrate an abnormal circadian rhythm of diuresis $(\mathrm{p}=0,001)$ and osmolary excretion $(\mathrm{p}<0,001)$, predominantly in the two first nighttime samples. Children with NP produced more urine $(\mathrm{p}<0,003)$ and excreted more osmols $(\mathrm{p}<0,001)$ overnight but also during the daytime and in a 24h-period.

Conclusions: Where the clinical indication for desmopressin is often restricted to children with $\mathrm{NP}(>130 \% \mathrm{EBC})$, our data suggest that:

- NP is better defined as ND>100\% EBC; this population might be equally desmopressin responsive

- pathogenesis is more complex than an abnormal AVP circadian rhythm; in $60 \%$ of patients abnormalities of other renal circadian rhythms and/or increased nutritional intake might be involved

- NP is the highest and osmolality the lowest in the early night collections; treatment should target fast rather than long-term action

\section{EP-150 FEATURES OF THE PROTEOMIC SPECTRUM OF URINE IN CHILDREN WITH MICROHEMATURIA \\ Ekaterina P. Krivonosova, Gadgy M. Letifov \\ Federal State Budgetary Educational Institution Of Higher Education "rostov State Medical University" Of The Ministry Of Health Of The Russian Federation}

Introduction: The high incidence of microhematuria and predominantly latent course of nephropathies with microhematuria leads to seek for new criteria for early diagnosis and prognosis of the disease. The aim of the study was to examine the proteomic spectrum of urine in various diseases of the urinary system in children in order to predict the nature of the course of kidney pathology.

Material and methods: Proteomic urine analysis was performed using proteomics methods (MALDI-TOF-MS / MS, Ultraflex II, Bruker, USA). Molecular interaction data were obtained using the STRING 10.0 database. The study included 30 children aged 1 to 18 years with various kidney pathologies, the leading laboratory symptom in which was microhematuria.

Results: Isolated 39 different proteins. The most frequently detected antigen of tubulointerstitial nephritis (100\%), antiepithelial membrane antigen $(50 \%)$, aminopeptidase $\mathrm{N}(42 \%)$, alpha subunit of smooth muscle fiber actin (33\%), sinfilin $1 \mathrm{~A}(33 \%)$, hepcidin $(33 \%)$. With the addition of proteinuria, the frequency of detection of a molecule of renal tissue damage increased $(75 \%)$. In 3 patients with polycystic kidney disease in the proteomic spectrum of urine in $100 \%$ of cases, the collagen chain alpha-1 (VI), afamin, alpha-subunit of smooth muscle fiber actin, profilin-1 were determined. In a patient with gouty nephritis (KellySigmiller syndrome), the proteomic spectrum of urine was dominated by a factor stimulating prostacyclin, interleukin 16 , a molecule of renal tissue damage, matrix metalloproteinase, tolloid-like protein 2 , aquaporin-1, apoptosis-inducing factor.

Conclusions: Thus, the study of the protein spectrum of urine opens up wide opportunities for the search for new non-invasive diagnostic markers for assessing the level of damage to the renal structures, the effectiveness of nephroprotective therapy, as well as determining the prognosis for various pathologies of the urinary system.

\section{EP-151 PHENOTYPIC AND GENOTYPIC CHARACTERISTICS} OF CHILDREN WITH BARTTER SYNDROME

Serçin Güven ${ }^{1}$, Ibrahim Gokce ${ }^{1}$, Ceren Alavanda ${ }^{2}$, Ece Demirci Bodur ${ }^{1}$, Neslihan Cicek ${ }^{l}$, Mehtap Sak ${ }^{1}$, Serim Pul ${ }^{1}$, Ozde Nisa Turkkan ${ }^{l}$, Pinar Ata ${ }^{2}$, Nurdan Yildiz, ${ }^{1}$, Harika Alpay ${ }^{I}$

${ }^{1}$ Marmara University, Department Of Pediatric Nephrology

${ }^{2}$ Marmara University, department Of Medical Genetics

Introduction: Bartter syndrome is classified into five genetic subtypes. SLC12A1, KCNJ1, CLCNKB, BSND and CASR encode proteins in the thick ascending limb of loop Henle and distal convulated tubule in BS type I, II, III, IV and V, respectively. Bartter syndrome can also be classified by phenotype (antenatal, classic). Patients with mutations in the same gene can present different phenotypes. In the present study, target gene sequencing was performed to evaluate the genotype-phenotype relationship

Material and methods: Biochemical, clinical, imaging and genetic results were collected at the presentation and the last clinic visit. The findings at presentation of patients with classical BS (cBS) and antenatal BS (aBS) were compared

Results: Our study included 21 patients (12 female, \%57.1) from 20 families with BS. The median age at diagnosis was 8 months (minmax: 1-139 months) and the median follow-up period was 39 months (min-max: 3-236). The most frequent complaint was growth failure (10/ 21). Height persentiles were $<3 p$ in 13 (\%61) patients at presentation, in 9 (\%42) patients at the last follow-up. We have found 18 variants in 4 genes, including 8 CLCNKB gene, 7 in SLCA12A1 gene, 2 in KCNJ1 gene and 1 in BSND gene, 11 of them were novel. When we compare patients with aBS (11/21) and cBS (10/21), there was no difference between the two groups in terms of age of diagnosis and follow-up time. Prematurity was significantly higher in the group with aBS than the group with cBS with a rate of $90 \%$. Median plasma potassium and chloride concentrations were significantly lower in cBS group $(2.2 \mathrm{vs} 3.0 \mathrm{mEq} /$ L; 85 vs. $94 \mathrm{mg} / \mathrm{L}$, respectively).

Conclusions: This investigation revealed the mutation characteristics and phenotype-genotype relationship of our patients and provided valuable data for genetic counseling.

EP-152 TRANSIENT PSEUDOHYPOALDOSTERONISM SECONDARY TO URINARY TRACT INFECTION IN A MALE INFANT WITH UNILATERAL HYDRONEPHROSIS DUE TO PRIMARY OBSTRUCTIVE MEGAURETER: CASE REPORT

Matea Crnković Ćuk, Ana Kovačević, Orjena Žaja, Marija Požgaj Šepec, Goran Roić, Bernardica Valent Morić, Ivana Trutin

Department Of Pediatrics Sestre Milosrdnice University Hospital Center, Zagreb, Croatia

Introduction: We present a case of transient form of type 1 pseudohypoaldosteronism (S-PHA) in a 1,5-month-old male infant who presented with lethargy, failure to thrive, severe hyponatremia $(\mathrm{Na}=118$ $\mathrm{mmol} / \mathrm{L})$, hypochloremia $(\mathrm{Cl}=93 \mathrm{mmol} / \mathrm{L})$ and fever due to urinary tract infection. 
Material and methods: Potassium levels were normal. Markedly elevated serum aldosterone level and elevated serum renin confirmed the diagnosis of pseudohypoaldosteronism. Renal ultrasound showed grade III hydronephrosis on the left kidney while contrast-enhanced voiding urosonography excluded the existence of vesicoureteral reflux which raised suspicion of opstructive uropathy on the level of vesicoureteral junction.

Results: Serum sodium normalized after several days of intravenous fluids and antibiotic therapy after which oral supplementation of sodium was introduced. Levels of 17-hydroxyprogesterone, ACTH, cortisole and TSH were normal. Functional MR urography conducted at the age of 3 months confirmed the diagnosis of primary congenital obstructive megaureter and the infant was referred to a pediatric surgeon.

Conclusions: Although a rare occurence, S-PHA can be a potentially life threatening condition in infants if not recognized and treated adequately. Therefore, serum concentrations of electrolytes should be obtained in every child with diagnosed obstructive anomaly of the urinary tract and/ or acute cystopyelonephritis. On the other hand, every child with diagnosed S-PHA should be evaluated for obstructive anomaly of the urinary tract.

\section{EP-153 AN ORPHAN DISEASE MIMICKING BARTTER SYNDROME: CONGENITAL DISORDERS OF GLYCO- SYLATION TYPE IIK}

Bahriye Atmis ${ }^{1}$, Fatma Derya Bulut ${ }^{2}$, Deniz Kor $^{2}{ }^{2}$ Derya Cevizli ${ }^{1}$, Emel Saribas ${ }^{1}$, Cagla Cagli ${ }^{1}$, Aysun K. Bayazit ${ }^{1}$, Neslihan Onenli Mungan ${ }^{2}$

${ }^{1}$ Cukurova University, Faculty Of Medicine, Department Of Pediatric Nephrology, Adana, Turkey

${ }^{2}$ Cukurova University, Faculty Of Medicine, Department Of Pediatric Metabolism And Nutrition, Adana, Turkey

Introduction: Congenital disorders of glycosylation (CDG) are rare and severe inherited diseases causing glycosylation defects lead to severe growth and psychomotor retardations in patients.

Material and methods: Here, we reported an infant who presented with hypokalemic metabolic alkalosis and diagnosed CDG type IIk.

Results: A 7-month-old girl presented to the pediatric nephrology department due to an incidental finding of hypokalemia and metabolic alkalosis in hospitalization for pneumonia. She was born from second-degree consanguineous parent. On physical examination, she had a weight of $5300 \mathrm{~g}$ $(-3.21 \mathrm{SDS})$, the height of $56 \mathrm{~cm}$ ( -4.69 SDS $)$, head circumference of $39 \mathrm{~cm}(-3.45 \mathrm{SDS})$ and blood pressure $85 / 50 \mathrm{mmHg}$. Examination revealed generalized hypotonia, inverted nipples, pectus carinatum, large and flap ears, and long eyelashes. In past medical history, she had seizures and was on phenobarbital treatment. Her family history was remarkable for her elder brother had mental retardation and developmental delay. Blood biochemistry revealed blood urea nitrogen $11 \mathrm{mg} / \mathrm{dl}$, creatinine $0.21 \mathrm{mg} / \mathrm{dL}$, sodium $129 \mathrm{mmol} / \mathrm{L}$, potassium $2.1 \mathrm{mmol} / \mathrm{L}$, chloride 81 $\mathrm{mmol} / \mathrm{L}$, calcium $9.6 \mathrm{mg} / \mathrm{dl}$, phosphorus $3.7 \mathrm{mg} / \mathrm{dl}$, magnesium $0.63 \mathrm{mg} /$ $\mathrm{dl}$, albumin $34 \mathrm{~g} / \mathrm{L}$, alanine aminotransferase $23 \mathrm{U} / \mathrm{L}$, aspartate aminotransferase $115 \mathrm{U} / \mathrm{L}$, lactate dehydrogenase $318 \mathrm{U} / \mathrm{L}$ and creatine phosphokinase $77 \mathrm{U} / \mathrm{L}$. Venous blood gas analysis was $\mathrm{pH} 7.58$, pCO2 32 $\mathrm{mmHg}$, bicarbonate $45.4 \mathrm{mmol} / \mathrm{L}$, and base excess $21.6 \mathrm{mmol} / \mathrm{L}$. Plasma renin activity was elevated and serum aldosterone was normal. Her urine analysis results were: $\mathrm{pH} 8$, density 1010 , protein (+) positive, leukocyte esterase negative, nitrite negative and leukocytes in urinary sediment were 2-3 every high-power field. Urine calcium creatinine ratio was $0.04 \mathrm{mg} / \mathrm{mg}$. Urine chloride level was $82 \mathrm{mmol} / \mathrm{l}$. Fractional excretion of sodium was $>4 \%$. The sweat test revealed sweat chloride concentration of $26 \mathrm{mmol} / \mathrm{L}$. Renal ultrasonography was normal. Treatment regimen consisting of sodium chloride, potassium citrate, magnesium oxide, and indomethacin was started. She had no mutations in CLCNKB and SLC12A3 genes. Ophthalmic examination, hearing test, and echocardiography were normal. She was hospitalized in the pediatric intensive care unit due to sepsis and pneumonia at the age of 19 months, and at that time, hypokalemic metabolic alkalosis became marked and rhabdomyolysis occurred. Hemodiafiltration and plasmapheresis were done. The involvement of multiple organs comprising hypotonia, seizure, liver (elevated transaminase levels), elevated creatine phosphokinase and growth retardation with remarkable family history led us to examine an inherited metabolic disease in this case. Basic metabolic tests were all in normal limits. Transferrin isoelectric focusing test showed CDG type II. Whole exome sequencing was performed and identified a pathogenic homozygous TMEM165:c.376C $>\mathrm{T}$ in exon 2 of the TMEM165 gene.

Conclusions: In conclusion, several studies reported that many inherited and acquired diseases could mimic Bartter syndrome. Besides these, our case showed that inherited metabolic diseases should be taken into consideration among the diseases mimicking Bartter syndrome.

\section{EP-154 ANTENATAL BARTTER SYNDROME MIMICKING PSEUDOHYPOALDESTERONISM}

Muhammet Sancaktar, Mustafa Koyun, GÜlȘah Kaya Aksoy,

Mehtap Adar, Ayla KaÇar, Elif Çomak, Sema Akman

Akdeniz University Medical Faculty Department Of Pediatric

Nephrology, Antalya

Abstract: Antenatal Bartter syndrome is a rare disease characterized by unexplained polyhydramnios and a history of premature birth, polyuria, salt wasting, and severe dehydration. We present an infant with antenatal Bartter syndrome without metabolic alkalosis and hypopotasemia.

An 2.5-month-old boy was admitted to our hospital due to polyuria, polydipsia and decreased nutrition. He was born 1440 grams at 30 weeks of gestation from a 24-year-old mother due to polyhydramnios. The diagnosis of pseudohypoaldosteronism was suspected due to hyponatremia $(124 \mathrm{mEq} / \mathrm{L})$ and hyperkalemia $(10 \mathrm{mEq} / \mathrm{L})$ at neonatal intensive care unit and therefore hydrocortisone and fludrocortisone treatments were administered, which was stopped at postnatal 29th day. His mother and father were first degree cousins. Physical examination was unremarkable. On laboratory examination, serum creatinine was $0.32 \mathrm{mg} / \mathrm{dL}$, serum sodium $132 \mathrm{mEq} / \mathrm{L}$, potassium $4.6 \mathrm{mEq} / \mathrm{L}$, calcium $10.9 \mathrm{mg} / \mathrm{dL}$, phosphorus was $5.8 \mathrm{mg} / \mathrm{dL}$, bicarbonate was $12 \mathrm{mmol} / \mathrm{L}$. Urinary analysis revealed that; $\mathrm{pH} 6.5$, dansity 1004 , urinary $\mathrm{Ca} / \mathrm{Cr}$ ratio was $0.76 \mathrm{mg}$ / $\mathrm{mg}$. Serum aldosterone was $177 \mathrm{ng} / \mathrm{dl}(\mathrm{N}<86)$, renin $34 \mathrm{ng} / \mathrm{ml} / \mathrm{h}(\mathrm{N}<37)$. On ultrasonography, increased medullary echogenicity was seen. Treatments with sodium chloride, potassium citrate and ibuprofen were started. Genetic analysis yielded a homozygous p.Trp99Gly (c.295T> G) mutation on KCNJ1 gene, compatible with type 2 Bartter syndrome.

Antenatal Bartter syndrome should be considered in patients with polyuria with a history of severe polyhydramnios and prematurity in the antenatal period; metabolic alkalosis may not be present.

\section{EP-155 NEONATAL DEHYDRATION: APPEARANCES DECEIVE} Pedro Arango Sancho, Yolanda Calzada, Elena Codina Sampera, Víctor López-baez, Lina Catherine Hernández Zúñiga, Ana Vinuesa Jaca, Álvaro Madrid Aris

Hospital Sant Joan De Déu

Introduction: In salt-wasting syndromes, adrenal pathology and tubulopathy should be the main differential diagnoses. Tubular maturation in the neonate can make it difficult to initially develop the classic biochemical pattern. We propose the differential diagnosis between two entities that in the first weeks may be indistinguishable: primary pseudohypoaldosteronism(PHA) and type II Bartter syndrome(BS)

Clinical observations: A 15-month-old patient(non-consanguineous parents), second pregnancy of a 35 -year-old mother with a previous cesarean section with intrauterine death of one of the fetuses. Severe polyhydramnios.In amniotic fluid biochemical study, high levels of chlorine and extremely high levels of aldosterone are found, orienting itself from the beginning as a salt-wasting syndrome. Urgent cesarean section 
at 28 weeks after sustained bradycardia in context of placental abruptio. During first 2 weeks, presented severe dehydration with sustained polyuria( $(18 \mathrm{ml} / \mathrm{Kg} / \mathrm{h}$ ), hyponatremia(minimum of $128 \mathrm{mmol} / 1$ ), hyperkalemia(up to $7.3 \mathrm{mmol} / \mathrm{l}$ ), metabolic acidosis(treatment with bicarbonate and frequent corrections needed). A response test with hydrocortisone is performed, changed to fludrocortisone on 4th day of life without response, oriented to tubular origin. Serial analyzes show an increase in renal losses of chlorine and calcium(calciuria up to $\mathrm{Ca} / \mathrm{Cr} 3.02 \mathrm{mg} / \mathrm{mg}$ ), hyperaldosteonic hyperreninema and a finding of moderate nephrocalcinosis(grade 2), considering at this point the differential diagnosis between primary type 1 PHA and BS. From the 2 nd week, the acidbase balance changes with a progressive tendency to hypokalemia and metabolic alkalosis, requiring treatment with increasing amounts of sodium(up to $17 \mathrm{mEq} / \mathrm{kg} / \mathrm{d}$ ) and potassium(up to $3.5 \mathrm{mEq} / \mathrm{Kg} / \mathrm{d}$ ), being discharged at 21 days pending the genetic study that will result in a type II Bartter Syndrome(ROMK mutation), initiating indomethacin with good subsequent metabolic control

Comments: Type II Bartter syndrome can simulate a pseudohypoaldosteronism picture during the first weeks of life, which can make it difficult to manage and diagnose

\section{EP-156 PRIMARY HYPOMAGNESEMIA? A CASE OF DIFFICULT INTERPRETATION}

Gabriele Malgieri ${ }^{1}$, Giulia Ranucci ${ }^{2}$, Giovanni Russo ${ }^{2}$, Rossella Colantuono ${ }^{3}$, Ilaria Luongo ${ }^{1}$, Daniela Molino ', Luigi Annicchiarico Petruzzelli ${ }^{1}$, Ilaria Battagliere ${ }^{1}$

${ }_{1}$ Pediatric Nephrology And Dialysis Unit, Santobono Childrens Hospital, Naples, Italy

${ }^{2}$ Department Of Translational Medical Science, Section Of Paediatrics, University Of Naples Federico Ii, Naples

${ }^{3}$ Pediatrics Section, Department Of Medicine, Surgery And Dentistry "scuola Medica Salernitana", University Of Salerno, Baronissi, Italy

Introduction: to describe a case of a child suffering from hypomagnesemia with secondary hypocalcemia, presenting with recurrent generalized seizures. The cause of hypomagnesemia in this patient is still unknown and she continues to require oral magnesium supplements to maintain normocalcemia.

Material and methods: A 2-month-term healthy girl was brought to the emergency department because of generalized seizures. On admissionshe was found to have hypocalcemia (total blood calcium: $6.3 \mathrm{mg} / \mathrm{dL}$ ). Further investigations revealed normal level of creatinine, inorganic-phosphorus, alkaline phosphatase, vitamin D3, parathyroid hormone, but low serum magnesium $(0.6 \mathrm{mg} / \mathrm{dL})$. Urinary electrolytes prior to magnesium repletion found an inappropriately elevated fractional excretion of magnesium at $4.6 \%$ with age-appropriate excretions of potassium, sodium, and phosphate. There was no hypercalciuria (calcium to creatinine ratio 0.56). Renal ultrasound was normal. After initial treatment with parenteral calcium and magnesium, followed by oral magnesium at $3.28 \mathrm{mmol} / \mathrm{kg}$ / day seizure disappeared completely over the next few days.

Results: After 6 months of follow up, there has been no seizure recurrence. The patient had normal height and weight for age and neurologic examination and electroencephalogram were normal. Her serum and urinary calcium and magnesium levels were in range. However she required oral supplementation to maintain normocalcemia. Other causes of primary hypomagnesemia were excluded. A next-generation sequencing panel for known mutations associated with hypomagnesemia including 22 genes was negative.

Conclusions: Magnesium levels should always be measured when trying to determine the etiology of seizures. Although rare, genetic causes of hypomagnesemia should be considered, once common causes of hypomagnesemia are ruled out. Despite the identification of several genes with rare Mendelian disorders our understanding of renal $\mathrm{Mg} 2+$ homeostasis remains still incomplete.
EP-157 INFANTILE HYPERCALCEMIA DUE TO HOMOZYGOUS CYP24A1 MUTATION IN A PATIENT WITH NEUROFIBROMATOSIS TYPE 1: A CASE REPORT

Ivanka Kos, Hana Matković, Maja Ban, Maša Davidović, Lovro Lamot, Kristina Vrljičak

Department Of Pediatrics, Unversity Of Zagreb School Of Medicine, University Hospital Centre Zagreb, Zagreb, Croatia

Introduction: Idiopathic infantile hypercalcemia (IIH) is characterized by severe symptomatic hypercalcemia. It is caused by mutations in CYP24A1 and SLC34A1 resulting in increased vitamin D sensitivity and elevated serum 1,25(OH)2D3 with subsequent hypercalcemia and hypercalciuria. SLC34A1 mutations additionally lead to primary renal phosphate wasting and hypophosphatemia. Contrarly, neurofibromatosis (NF) is associated with vitamin D deficiency, so supplementation of vitamin D is beneficial. Yet, NF is sometimes complicated by hypophosphatemic osteomalacia and hyperparathyroidism.

Material and methods: We report a case of a 14 years old male who initially presented at the age of 5 months with profound hypercalcemia, vomiting, dehydration, weight loss, hypotonia, and hypercalciuria. $\mathrm{He}$ was born at term after an uncomplicated pregnancy as the first and only child of healthy parents without known consanguinity. He was exclusively breastfed and received 1000 IU of vitamin D for prophylaxis. At admission his serum 25-OH vitamin $\mathrm{D}$ and 1,25(OH)2D3 levels were normal, while parathyroid hormone level was undetectably low. Besides, he had hypophosphatemia. A low calcium/vitamin D diet was initiated. Ultrasound showed increased echogenicity of the renal pyramids at the age of 3 years and kidney stones at the age of 5. Serum calcium and phosphate levels and the urine calcium/creatinine ratio were normal. Furthermore, he fulfilled clinical criteria for neurofibromatosis type 1 and started prophylaxis with $600 \mathrm{IU}$ vitamin D. After two months of prophylaxis, he developed hypercalciuria with suppressed PTH and hyperphosphaturia, so vitamin D was restricted again. By the age of 7 , he suffered from recurrent renal colic. The kidney stones were fragmented with extracorporeal shock wave lithotripsy and excreted in the urine. Since then the patient has been treated with hydrochlorothiazide. His urine calcium/creatinine ratio and renal ultrasound are normal. Moreover, catch-up in growth and development was observed.

Results: Finally, genetic analysis has recently been done. Homozygous missense variant CYP24A1 c.1186C>T,p. (Arg396Trp) was identified. Conclusions: IIH has not yet been described in individuals with NF1. Both conditions adversely affect complex phosphocalcic metabolism. Hydrochlorothiazide is a useful treatment option. Further investigation is required to facilitate appropriate management and treatment of these challenging patients.

EP-158 COMPARISON OF THE 2017 AAP AND 4TH REPORT/ ESH GUIDELINES FOR THE DIAGNOSIS OF HYPERTENSION AND DETECTION OF LEFT VENTRICULAR HYPERTROPHY IN CHILDREN AND ADOLESCENTS: A SYSTEMATIC REVIEW AND META-ANALYSIS

Ioannis Goulas ${ }^{1}$, Ioannis Doundoulakis ${ }^{3}$, Ioannis Farmakis ${ }^{2}$, Stella Stabouli ${ }^{1}$

${ }^{1}$ 1st Department Of Pediatrics, Hippokratio General Hospital, Aristotle University Of Thessaloniki, Thessaloniki, Greece

${ }^{2} 1$ st Department Of Cardiology, Ahepa University Hospital, Aristotle University Of Thessaloniki, Thessaloniki, Greece

${ }^{3}$ Department Of Cardiology, 424 General Military Hospital, Greece.

Introduction: In 2017, the American Academy of Pediatrics presented new practice guidelines for the diagnosis and management of Hypertension in children and adolescents. We aimed to assess the impact of the new guidelines, compared with, the already existing guidelines ("Fourth Report on the Diagnosis, Evaluation, and Treatment of High 
Blood Pressure in Children and Adolescents", "2016 European Society of Hypertension guidelines"), on the prevalence of hypertension and associations with Left ventricular hypertrophy in children and adolescents.

Material and methods: We searched bibliographic databases (MEDLINE, Web Of Science, CENTRAL) for studies evaluating the impact of the 2017 AAP guidelines on the prevalence of hypertension and left ventricular hypertrophy compared to the $4^{\text {th }}$ Report or the 2016 ESH guidelines. Meta-analysis was performed to compare the overall risk of LVH between the two guidelines. We used a random-effects model to synthesize quantitative data.

Results: We included 18 observational studies in our systematic review with an overall moderate to high risk of bias. The AAP guidelines resulted in higher prevalence of HTN than the ESH guidelines. In the metaanalysis of three observational studies, the two guidelines revealed similar associations with $\mathrm{LVH}(\mathrm{OR}=3.89,95 \%[\mathrm{CI}]=1.68-8.99$ for AAP - OR $=3.19,95 \%[\mathrm{CI}]=1.14-8.88$ for $4^{\text {th }}$ Report $/$ ESH). Likewise, a qualitative analysis of two observational studies revealed similar predictive capacity of the two guidelines for $\mathrm{LVH}$ in adult life.

Conclusions: The incorporation of the new AAP guidelines increased the prevalence of Hypertension in children and adolescents. However, the new nomograms did not yield any advantage in identifying children and adolescents with LVH.

\section{EP-159 ALBUMINURIA IN CHILDREN: ASSOCIATION WITH CARDIOVASCULAR RISK MARKERS AND PERSISTENCE THROUGHOUT CHILDHOOD}

Valentina Gracchi ${ }^{1}$, Hiddo Jl Heerspink ${ }^{2}$, Sophie M Van Den Belt ${ }^{2}$, Eva Corpeleijn ${ }^{3}$, Dick De Zeeuw ${ }^{2}$, Henkjan Verkade ${ }^{1}$

${ }^{I}$ Dept Pediatrics, University Medical Center Groningen, Groningen, The Netherlands

${ }^{2}$ Dept Clinical Pharmacy And Pharmacology, University Medical Center Groningen, Groningen, The Netherlands

${ }^{3}$ Dept Epidemiology, University Medical Center Groningen, Groningen, The Netherlands

Introduction: In adults, albuminuria is a recognized marker for cardiovascular risk, and it is associated with obesity and hypertension. In children, however, the association between albuminuria and cardiovascular risk has not been demonstrated, nor is it clear whether albuminuria persists throughout childhood. We aimed to determine whether albuminuria at 12 years is associated with concurrent cardiovascular risk factors and whether albuminuria at 2 years sustained until the age of 12 .

Material and methods: In the population-based GECKO (Groningen Expert Center for Kids with Obesity) cohort, urine was collected from 1352 children at the age of 2 years and 1311 children at the age of 12 years (random urine and first morning void urine, respectively). In a cross-sectional analysis, we determined the association of urinary albumin concentration (UAC) and urinary albumin-creatinine ratio (UACR) at 12 years with blood pressure (BP), Body Mass Index (BMI) and Waist Circumference (WC). In a longitudinal analysis we evaluated in 835 individuals whether albuminuria at 2 years persisted until 12 years.

Results: Both UAC and UACR at 12 years were negatively associated with BMI and WC (UACR: $\beta-0.45$ and $\beta-1.06$, respectively; both $\mathrm{p}<0.001)$. UAC was positively associated with systolic BP $(B 0.69$, $\mathrm{p}=0.046)$, UACR with diastolic BP $(\beta 0.58, \mathrm{p}=0.047)$. UACR $\geq 3 \mathrm{mg} /$ mmol was present in $23.4 \%$ of children at 2 years, but only in $3.3 \%$ at 12 years. Only $10.7 \%$ of the children with $U A C \geq 20 \mathrm{mg} / \mathrm{L}$ at 2 years had $\mathrm{UAC} \geq 20 \mathrm{mg} / \mathrm{L}$ at 12 years $(\mathrm{p}=0.43$ ).

Conclusions: In contrast to the hypothesized positive association, albuminuria at 12 years is negatively associated with BMI and WC, and the positive association with BP is weak and barely statistically significant. Albuminuria at age 2 does largely not persist at the age of 12 years, indicating that many children at 2 years of age have transient albuminuria.
EP-160 COMPARISON OF ESHG2016 AND AAP2017 HYPERTENSION GUIDELINES IN ADOLESCENTS BETWEEN THE AGES OF 13 AND 16; EFFECT OF BODY MASS INDEX ON GUIDELINES

Gülşah Kaya Aksoy ${ }^{1}$, Dilek Yapar ${ }^{2}$, Nevin Semerci Koyun ${ }^{1}$, Çağla Serpil Doğan ${ }^{1}$

${ }_{I}$ Antalya Training And Research Hospital, Department Of Pediatrics, Division Of Pediatric Nephrology

${ }^{2}$ Gazi University School Of Medicine, Department Of Public Health

Introduction: The diagnosis of hypertension in adolescents aged $\geq 13$ and $<16$ years is based on the percentile according to age, gender, and height in the European Society of Hypertension guidelines (ESHG2016) guideline; whereas the American Academy of Pediatrics guideline (AAP2017) uses blood pressure above $130 / 80 \mathrm{mmHg}$ as a single criterion. Therefore, this study aimed to evaluate the compatibility of these two guidelines in adolescents aged $\geq 13$ and $<16$ years.

Material and methods: This study was designed by retrospectively screening the records of 395 adolescents with both office and 24-hour ambulatory blood pressure measurements. Each blood pressure measurement was classified according to both the ESGH2016 and AAP2017 guidelines. Patients were divided into 3 subgroups according to body mass index. Cohens kappa analysis was used to evaluate the agreement of the two guidelines.

Results: The majority of adolescents were normotensive according to both guidelines, $55.9 \%$ by ESHG2016 and $43.1 \%$ by AAP2017. For the whole group, the frequency of hypertension was $32.4 \%$ with ESHG2016 and $34.4 \%$ with AAP2017; while, in obese patients, hypertension frequencies were $38.8 \%$ and $43.3 \%$, respectively. The diagnosis of hypertension demonstrated with the two guidelines, and there was significant agreement at a substantial level, both for the obese subgroup and the whole study group (kappa value $=0.738$ and 0.785 , respectively). The frequency of white-coat hypertension was higher with the AAP2017 guideline $(29.1 \%$ vs $19.4 \%, \mathrm{p}<0.001)$

Conclusions: With our experience in this single-center study, it seems that both the AAP2017 and the ESHG2016 guidelines can be used in the diagnosis of hypertension in adolescents.

\section{EP-161 METABOLIC SYNDROME - AN IMPORTANT LVH} RISK FACTOR IN CHILDREN WITH CKD

Monika Drozdz ${ }^{l}$, Anna Moczulska ${ }^{1}$, Andrzej Rudzinski ${ }^{2}$, Dorota Drozdz $^{1}$ ${ }^{1}$ Jagiellonian University Medical College, Department Of Pediatric Nephrology And Hypertension, Krakow, Poland

${ }^{2}$ Jagiellonian University Medical College, Department Of Pediatric Cardiology, Krakow, Poland

Introduction: The metabolic syndrome (MetS) is a cluster of risk factors including insulin resistance, dyslipidemia and hypertension, which are often found in chronic kidney disease (CKD).

Left ventricular (LV) hypertrophy (LVH) is a major target organ damage in hypertension and an important cardiovascular risk factor.

The study aimed to identify risk factors that contribute to LVH in children with (CKD).

Material and methods: The study was conducted in a group of 71 children ( 28 girls and 43 boys) with CKD stage 1 to 5 . The patients' mean age was 12,8 years and mean GFR was $64,8 \pm 38 \mathrm{ml} / \mathrm{min} / 1.73 \mathrm{~m}^{2}$. Serum creatinine and lipids levels were measured. MetS was diagnosed according to De Ferranti (DF) criteria. Ambulatory blood pressure measurements (ABPM) were performed. Echocardiographic examinations were carried out with a HP 5500 device. The $95^{\text {th }}$ percentile of LV mass index relative to height age was used to define $\mathrm{LVH}$.

Results: LVH was detected in $21(29,6 \%)$ children. MetS (DF) was diagnosed in $20 \mathrm{pts}(28,2 \%)$. Glucose $\geq 110 \mathrm{mg} / \mathrm{dL}$ was present in 3 pts $(4,2 \%)$; waist circumference $\geq 75^{\text {th }}$ pc in 16 pts $(22,5 \%)$; triglycerides $\geq 100 \mathrm{mg} / \mathrm{dL}$ in $35 \mathrm{pts}(49,3 \%)$; high-density lipoprotein $<50$ 
$\mathrm{mg} / \mathrm{dL}$ in $31 \mathrm{pts}(43,7 \%)$ and blood pressure $\geq 90^{\text {th }} \mathrm{pc}$ in $29 \mathrm{pts}$ $(40,8 \%)$, respectively. In multivariate analysis with logistic regression model (logit) the most important risk factors for LVH in CKD children were: diagnosis of MetS based on DF criteria $(\mathrm{OR}=24.11$, Chi2 $=8.38, \mathrm{p}=0.0038)$; low HDL according to DF criteria $(\mathrm{OR}=$ 0.066 , Chi2 $=6.38, \mathrm{p}=0.0116$ ); high mean arterial pressure standard deviation score $(\mathrm{SDS})(\mathrm{OR}=2.812, \mathrm{Chi}=5.91, \mathrm{p}=0.015)$ and low height SDS (OR=0.078, Chi2 $=25.01, \mathrm{p}<0.001)$.

Conclusions: In children with chronic kidney disease LVH is associated with the individual components of the metabolic syndrome, and a cluster of these factors significantly increases the risk.

\section{EP-162 COULD ARTERIAL STIFFNESS BE EARLY REVERSIBLE TARGET ORGAN DAMAGE TEST IN CHILDHOOD HYPERTENSION?}

Duygu Övünç Hacthamdioğlu ${ }^{1}$, Özben Ceylan ${ }^{2}$, Aytül Hande Yardımcl ${ }^{2}$

${ }^{1}$ Bahçeşehir University Medicine Faculty

${ }^{2}$ Ministry Of Health, İstanbul Research And Training Hospital

Introduction: This study is to present the changes of target organ damage (TOD) and arterial stiffness parameters after treatment in children with primary hypertension (HTN) who were managed in accordance with the European Society of Hypertension Guidelines 2016.

Material and methods: The patients included in the study were newly diagnosed and untreated ones and they had a follow-up period of minimum 6 months. All patients underwent the following assessments: Anthropometrical measurements, 24-hour ambulatory blood pressure monitoring (ABPM), left ventricular mass index (LVMI), plasma creatinine, urea, electrolytes, uric acid, fasting plasma glucose, blood lipids, urinalysis, urine culture, first morning urine albumin: creatinine ratio. The ABPM device was able to perform measurements like central blood pressure (cBP) and pulse wave velocity (PWV). At the end of the study, patients were divided into normotensive and hypertensive. Pre- and post-treatment parameters of the patient groups were compared.

Results: Thirty-two of 104 patients were enrolled. Seventeen of the patients were male and the frequency of obesity was $53 \%$. Compared with pretreatment, creatinine, urea, systolic blood pressure (SBP), diastolic blood pressure (DBP), central SBP (cSBP), cSBP z-score, cDBP, and PWV z-score decreased, whereas LVMI z-score and BMI z score were not changed. When the normotensive and hypertensive were compared, while there was cSBP and PWV z score regression in the normotensive group, both parameters increased in the hypertensive group. However there was no change in LVMI z score in hypertensive patients (Table 1).

Table 1. Comparison of normotensive and hypertensive patients

\begin{tabular}{llll}
\hline Parameters & $\begin{array}{c}\text { Normotensive } \\
(\mathrm{n}=25)\end{array}$ & $\begin{array}{c}\text { Hypertensive } \\
(\mathrm{n}=7)\end{array}$ & $\mathrm{p}$ \\
Age (years) & $12.65 \pm 3.84$ & $13 \pm 3.45$ & 0.000 \\
Gender & $44 \%(\mathrm{n}=11) \mathrm{M}$ & $85,7 \%(\mathrm{n}=6) \mathrm{M}$ & 0.000 \\
BMI z score pre-T & $0,69[(-0,45)-1.43]$ & $1,99(1,73-2,05)$ & 0.000 \\
LVM z score preT & $0,5[(-0,82)-1,47]$ & $0,76(0,21-1,05)$ & 0.000 \\
LVMI z score preT & $0.84(0,25-2,32)$ & $1.43(0,95-1,75)$ & 0.005 \\
cSBP z score preT & $0,87(0,18-1,15)$ & $1,15(0,59-2,17)$ & 0,000 \\
PWV z score preT & $0,41(0,1-0,87)$ & $1,15(0,59-1,34)$ & 0,000 \\
EF preT $(\%)$ & $70.4 \pm 4$ & $68.1 \pm 1$ & 0.000 \\
FS preT $(\%)$ & $39 \pm 3$ & $37 \pm 3$ & 0.000 \\
Creatinine preT & $0.55 \pm 0.21$ & $0.83 \pm 0.22$ & 0,000 \\
$\quad(m g / d l)$ & & $7.85 \pm 0.18$ & 0,000 \\
Uric acid preT (mg/dl) & $3.73 \pm 1.43$ & $53 \pm 8$ & 0,000 \\
Pulse Pressure preT & $49 \pm 11$ & $71,4 \%$ & 0,000 \\
RWT post $>0.42$ & $56 \%$ & &
\end{tabular}

\begin{tabular}{llll} 
LVH ESHG16 post & $4 \%(\mathrm{n}=1)$ & $71,4 \%(\mathrm{n}=5)$ & 0,000 \\
Obesity $(\%)$ & $\% 40(\mathrm{n}=10)$ & $\% 100(\mathrm{n}=7)$ & 0,000 \\
$\Delta$ LVM z score & $-0,62[(0,92)-0,56]$ & $-0,21$ & 0.000 \\
& & {$[(0,21)-0,65]$} & \\
$\Delta$ LVMI z score & $-0,04[(0,14)-0,07]$ & $-0,01$ & 0,000 \\
$\Delta$ cSBP z score & $-0,17[(-0,94)-0,62]$ & $0,12([(0,06)-0,16]$ & \\
$\Delta$ PWV z score & $-0,2[(-0,67)-0.0]$ & $0,04(0.0-0,23)$ & 0,000 \\
$\Delta$ EF & $0,5[(-1,5)-1]$ & $0,0[(-1)-1]$ & 0,000 \\
$\Delta$ KF & $1[(-0,5)-1,5]$ & $0,5(0-1,5)$ & 0,537 \\
\hline
\end{tabular}

F: Female, BMI: Body Mass Index, preT:pretreatment, LVM: left ventricular mass, LVMI: left ventricular mass index, EF:Ejection Fraction, FS: Fractional shortening, RWT: Relative wall thickness, BP: Blood Pressure, DBP:diastolic blood pressure, SBP: systolic blood pressure, AASI: ambulatory arterial stiffness index. Variable were compare with Mann Whitney $U$ test and expressed as median and interquartile ranges.

Conclusions: The finding suggest that vascular function tests may detect an earlier response of BP regression in the cardiovascular system. For hypertensive group, it seems that both obesity and HTN presence have a greater impact on arterial stiffness than does cardiac remodeling. Finally, in a short-term follow-up, in children with low CVD risk as BP normalizes, regression of cSBP and PWV may occur without LVMI reversal.

\section{EP-163 LONG TERM FOLLOW UP IN RENAL SCARRING OF URINARY TRACT INFECTION: ALBUMINURIA, DIASTOLIC BLOOD PRESSURE}

Demet Baltu ${ }^{1}$, Bilge Volkan Salanci ${ }^{2}$, Bora Gulhan ${ }^{1}$,

Tugba Tastemel Ozturk ${ }^{1}$, Eda Didem Kurt Sukur ${ }^{1}$, Fatih Ozaltin ${ }^{l}$, Ali Duzova ${ }^{1}$, Rezan Topaloglu ${ }^{1}$

${ }^{I}$ Department Of Pediatric Nephrology, Faculty Of Medicine, Hacettepe University

${ }^{2}$ Department Of Nuclear Medicine, Faculty Of Medicine, Hacettepe University

Introduction: The aim of the study was to detect complications and associated risk factors in patients with renal scarring (RS) secondary to recurrent urinary tract infections (UTI).

Material and methods: Fifty patients with RS with a mean follow-up time of 9.69 years were compared with 25 patients without RS by means of, serum creatinine, 24-hour urinary creatinine clearance, and 24-hour urinary albumin levels. Office blood pressure (BP) examination and ambulatory BP monitoring (ABPM) were also performed.

Results: Vesicoureteral reflux was detected in 50 patients and its grade was correlated with the severity of RS $(r=0.45, p=0.01)$. Glomerular filtration rate $(\mathrm{GFR})<90 \mathrm{~mL} / \mathrm{min} / 1.73 \mathrm{~m}^{2}$ was observed in 5 patients with RS but in no patient without RS. Urinary albumin excretion was significantly higher in patients with bilateral RS and severe RS. Patients with albuminuria had a significantly lower GFR than those without albuminuria. All patients with ambulatory hypertension (HT) were in the RS group, and $60 \%$ of those had isolated nocturnal HT. Compared to those without RS, patients with RS had significantly higher standard deviation score (SDS) values for all BP readings, 24-hour and nighttime systolic and diastolic BP loads with significantly lower systolic dipping. GFR was negatively correlated with diastolic BP SDS and diastolic BP load in patients with RS. Daytime diastolic BP load was significantly higher in those with severe RS than in those with mild RS.

Conclusions: Isolated nocturnal HT could be an early indicator of complications in RS of UTI. Albuminuria is related to increased BP and impaired renal function. Therefore, ABPM and assessing albuminuria should be a routine part of the follow-up. Diastolic BP elevations could be associated with worse outcomes in these patients. 
EP-164 ASSOCIATION BETWEEN HYPERTENSION AND COGNITIVE FUNCTION IN CHILDREN AND ADOLESCENTS

Katerina Chrysaidou, Athanasia Chainoglou, Filimon Tzovaras, Eleni Liazou, Efthimia Vargiami, Dimitrios Zafeiriou, Stella Stabouli Aristotle University of Thessaloniki

Introduction: In the recent years, the effect of blood pressure (BP) on cognitive functions of the brain has gained increasing interest. The aim of the present study was to study if hypertensive children and adolescents present lower cognitive function scores.

Material and methods: Children and adolescents aged 5-18 years with diagnosis of primary arterial hypertension underwent 24-hour ambulatory BP monitoring and assessment of cognitive functions with WISC-III test.

Results: Hypertensive children showed statistically significantly lower performance on the scales of similarities, cube patterns, as well as practical intelligence and general intelligence scales, compared to children with normal BP on 24-hour ambulatory BP monitoring. Night systolic $\mathrm{BP} \mathrm{z}$ score showed a negative correlation with the verbal intelligence quotidient $(\mathrm{r}=-0.439, \mathrm{p}<0.05)$, and the general intelligence quotidient $(\mathrm{r}=-0.429, \mathrm{p}<0.05)$, while night diastolic $\mathrm{BP} \mathrm{z}$ score showed a negative correlation with the practical intelligence quotidient $(r=-0.356, p<0.05)$ and the general intelligence quotidient $(r=-0.394, p<0.05)$. For each standard deviation (SD) increase of systolic and diastolic night BP intelligence quotidient was reduced by 0.462 and 0.421 respectively (image $1,2)$


Conclusions: Night BP is closely correlated with cognitive function in children and adolescents as shown by the associations with intelligence quotidients in WISC-III test. 24-hour ambulatory BP monitoring in children and adolescents with hypertension is important for identifying children at increased risk for lower cognitive performance.

\section{EP-165 FACTORS ASSOCIATED WITH CHANGES OF SERUM CARDIOVASCULAR (CV) BIOMARKER LEVELS FOLLOWING THE START OF KIDNEY REPLACEMENT THERAPY (KRT) IN CHILDREN \\ Justinas Daraskevicius ${ }^{1}$, Karolis Azukaitis ${ }^{2}$, Marietta Kirchner ${ }^{3}$, Franz, Schaefer ${ }^{4}$, On Behalf Of 4c Study Consortium ${ }^{5}$ \\ ${ }^{1}$ Faculty Of Medicine, Vilnius University, Vilnius, Lithuania \\ ${ }^{2}$ Clinic Of Pediatrics, Institute Of Clinical Medicine, Faculty Of \\ Medicine, Vilnius University, Vilnius, Lithuania \\ ${ }^{3}$ Institute Of Medical Biometry And Informatics, University Of Heidelberg, Heidelberg, Germany \\ ${ }^{4}$ Division Of Pediatric Nephrology, Center For Pediatrics And Adolescent Medicine, Heidelberg University Hospital, Heidelberg, Germany \\ ${ }^{5} 4 c$ Study Consortium}

Introduction:Serum levels of traditional (TnT and BNP) and novel (GDF15 and ST2) CV biomarkers increase after start of dialysis and decrease after kidney transplantation (KTx). The aim of this analysis was to analyse covariates associated with these changes.
Material and methods: CV biomarker levels were measured in children before and after dialysis $(n=53)$ or pre-emptive KTx $(n=42)$. Association between the changes $(\Delta)$ of biomarkers and various covariates (including left ventricular mass index (LVMI), pulse wave velocity $\mathrm{z}$-score $(\mathrm{PWVz})$ and carotid intima-media thickness $\mathrm{z}$-score (cIMTz)) was analysed by linear regression adjusted for time between biomarker measurements and baseline biomarker level.

Results: Median age and estimated glomerular filtration rate (eGFR) was 13.6 years and $13.5 \mathrm{ml} / \mathrm{min} / 1.73 \mathrm{~m}^{2}$ before the start of dialysis, and 12.9 years and $13.0 \mathrm{ml} / \mathrm{min} / 1.73 \mathrm{~m}^{2}$ before KTx, respectively. GDF15, TnT and BNP but not ST2 increased after the start of dialysis (median change $24 \%, 50 \%, 201 \%$ and $3 \%$, respectively) and decreased after KTx (-58\%, $27 \%,-73 \%$ and $-3 \%$, respectively).

The biomarker changes after initiation of dialysis associated inversely with $\triangle \mathrm{eGFR}(\Delta \mathrm{TnT}$ and $\Delta \mathrm{BNP})$ and $\Delta$ high-density lipoprotein cholesterol $(\Delta \operatorname{TnT}$ and $\Delta$ GDF15), and directly with $\Delta$ serum phosphate $(\Delta \operatorname{TnT}$ and $\Delta$ GDF15), $\Delta$ ferritin $(\Delta \mathrm{TnT}, \Delta \mathrm{BNP}$ and $\Delta \mathrm{GDF} 15)$ and $\Delta \mathrm{LVMI}(\Delta \mathrm{TnT}$ and $\triangle \mathrm{BNP}) . \Delta \mathrm{ST} 2$ was inversely associated with $\Delta$ serum albumin and $\Delta$ hemoglobin.

In children who underwent pre-emptive KTx, $\triangle \mathrm{BNP}, \triangle \mathrm{GDF} 15$ and $\triangle \mathrm{ST} 2$ associated inversely with $\Delta$ hemoglobin. In addition, $\triangle \mathrm{BNP}$ was directly associated with $\Delta$ ferritin and $\Delta$ LVMI, while $\Delta$ GDF15 was inversely associated with $\Delta \mathrm{eGFR}$ and $\Delta \mathrm{ST} 2$ directly with $\Delta$ total cholesterol. $\Delta \operatorname{TnT}$ was inversely related to $\Delta$ serum calcium and directly to $\triangle \mathrm{PWVz}$.

Conclusions: Changes of serum CV biomarker levels following the start of KRT are only partially explained by changes in kidney function and additionally associate with markers reflecting uremic and $\mathrm{CV}$ risk burden. Change of $\mathrm{CV}$ endpoints associated only with traditional $\mathrm{CV}$ biomarkers (TnT and BNP).

\section{EP-166 A RARE CAUSE OF SECONDARY HYPERTENSION IN CHILDHOOD}

Rossella Colantuono ${ }^{1}$, Francesca Caprio ${ }^{2}$, Claudia Elefante ${ }^{3}$, Giovanni Russo ${ }^{4}$, Raffaella Niola ${ }^{5}$, Francesco Esposito ${ }^{6}$, Pasquale Esposito ${ }^{7}$, Michela Grieco ${ }^{8}$, Vittorio Serio ${ }^{9}$, Gabriele Malgieri ${ }^{9}$, Carmine Pecoraro ${ }^{9}$

${ }^{1}$ Pediatrics Section, Department Of Medicine, Surgery And Dentistry "scuola Medica Salernitana", University Of Salerno, Baronissi, Italy

${ }^{2}$ Department Of Medical Translational Sciences, University Of Campania Luigi Vanvitelli, Naples, Italy

${ }^{3}$ Nephrology Unit, Advanced Surgical And Medical Sciences Department Of University Of Campania "luigi Vanvitelli", Naples, Italy ${ }^{4}$ Department Of Translational Medical Science, Section Of Paediatrics, University Of Naples Federico Ii, Naples

${ }^{5}$ Interventional Radiology Department, Aorn "a. Cardarelli", Naples, Italy

${ }^{6}$ Radiology Department, Santobono-pausilipon Children's Hospital, Naples, Italy

${ }^{7}$ Department Of Emergency, Nicu-aorn Santobono-pausilipon, Napoli, Italy ${ }^{8}$ Cardiology Unit, Santobono-pausilipon Childrens Hospital, Naples, Italy ${ }^{9}$ Nephrology And Dialysis Unit, Santobono-pausilipon Childrens Hospital, Naples, Italy

Introduction: Mid Aortic Syndrome (MAS) is a rare vascular malformation characterized by segmental narrowing of the abdominal aorta and stenosis of its principal branches. Patients affected by MAS typically present malignant renovascular hypertension, with variable clinical symptoms like claudication, abdominal angina, headache.

Material and methods: A newborn was admitted to our hospital with systemic shock. At the clinical resolution patient showed hypertension with systolic blood pressures ranging from 110-150 $\mathrm{mmHg}$ (greater than $99 \%$ percentile) and absence of femoral pulses. An echocardiogram showed mild left ventricle (LV) hypertrophy (Z score 2.7 ) with increased LV mass index $(130 \mathrm{~g} / \mathrm{m} 2$, vn $49-115 \mathrm{~g} / \mathrm{m} 2)$ and aortic coarctation was 
excluded. Diagnostic imaging of the abdominal vessels showed narrowing of abdominal aorta to the proximal tract of superior mesenteric artery, right filiform renal artery and left stenotic renal artery with size prevalence of right kidney confirming the diagnosis of MAS.

Results: Our patient was successfully treated with right percutaneous transcatheter renal angioplasty (PTRA) and left nephrectomy.

Progressively blood pressure values improved with beta-blockers, calcium channel blockers and angiotensin-converting enzyme inhibitors.

Furthermore, in our case cerebral MRI angiography showed a tortuous course of the A2 tracts of the internal cerebral arteries raising the suspicion of Moyamoya Disease.

Genetic testing is still ongoing.

Conclusions: Patients can develop hypertensive encephalopathy, vascular brain accidents and systemic shock as occurred in our patient. MAS may be acquired, caused by Takayasus or temporal arteritis, neurofibromatosis, Williams syndrome and Moyamoya disease, or congenital, ascribed to a developmental anomaly in the fusion and maturation of the paired embryonic dorsal aortas. Therefore, in patients with multidrug resistant hypertension, vascular causes such as MAS, isolated or syndromic, should be suspected. Renovascular hypertension often requires a surgical approach to treat clinical symptoms and prevent or avoid organ damage.

\section{EP-167 PULSE WAVE VELOCITY AND SITE OF MEASUREMENT IN CHILDREN AND ADOLESCENTS \\ Mirjam Močnik, Nataša Marčun Varda \\ Department Of Paediatrics, University Medical Centre Maribor}

Introduction: Pulse wave velocity is a method of functional vascular evaluation. The gold standard is the carotid-femoral pulse wave velocity measurement. However, the software allows measurement among radial, carotid and femoral artery. The goal of our prospective pilot study was to compare pulse wave velocity measurement among different arteries, not only carotid-femoral, in children and adolescents to find out, if the measurement can be performed in a simplified version with comparable results which would be especially important in younger children.

Material and methods: 58 children were analyzed using SphygmoCor Cardiovascular Management Suite ${ }^{\circledR}$ and PWV was measured in three different ways: carotid-femoral pulse wave velocity (cfPWV), carotidradial pulse wave velocity (crPWV) in lying position and crPWV in sitting position PWV results and pulse wave analysis with central hemodynamic data were collected.

Results: There was statistically significant difference between: cfPWV and crPWV in lying position and cfPWV and crPWV in sitting position $(\mathrm{p}<0.0001$ for both). There was no significant difference between crPWV in lying or sitting position ( $\mathrm{p}=0.158)$. Analysis of central hemodynamic data showed statistically significant differences between subendocardial viability ratio $(p=0.001)$, end systolic pressure $(p=0.001)$ and central diastolic pressure $(p=0.001)$ when central hemodynamic data of carotid-radial measurements lying and sitting were compared, but there were no differences when the same parameters were compared between hemodynamic data of both lying positions, except for subendocardial viability ratio.

Conclusions: The site of measurement significantly affects pulse wave velocity and can not be interchangeable. On the contrary, the position of the child does not affect pulse wave velocity, but could be important in hemodynamic data analysis.

EP-168 THE EFFECT OF CORTISOL AND IMPAIRED GLUCOSE TOLERANCE ON HYPERTENSION IN ADOLESCENTS WITH OBESITY

Emel DÜzg̈̈n, Asli Çelebİ Tayfur, AyȘe Derya BuluŞ

University OfHealth Sciences, Ankara KeÇiÖren Tarining And Research Hospital
Introduction: The hypothalamic-pituitary-adrenal (HPA) axis abnormalities and insulin resistance are involved in the pathogenesis of obesityassociated hypertension in adolescents. The aim of this study was to assess the correlation between serum cotisol levels, presence of insulin resistance and presence of hypertension in adolescents with obesity.

Material and methods: Height, body weight, waist circumference, office blood pressure measurements of the patients were recorded. Ambulatory blood pressure monitoring (ABPM) was performed in each patient. Fasting glucose, insulin, total cholesterol, high density lipoprotein (HDL) cholesterol, triglyceride, troid stimulating hormone (TSH), free $\mathrm{T} 4$, cortisol and adrenocorticotropic hormone levels and 24 hour urine cortisol and creatinine levels were studied. The patients were grouped according to the presence of hypertension and/or insulin resistance.

Results: Seventy five adolescent patients with obesity (37 girls, 38 boys) were enrolled in the study. Twenty-one (28\%-group 1) patients had hypertension and insulin resistance, 24 (32\%-group 2) patients had only hypertension; 14 (18.7\%-group 3) patients had only insulin resistance and 16 (21.3\%, group 4) patients had neither hypertension nor insulin resistance. There were statistically signicant differences in body weight sds, body mass index sds, systolic blood pressure, waist circumference, insulin, ACTH and triglyceride levels between patient groups. The patients in group 1 had higher values of these parameters than patients in other groups. Also the patients without hypertension had lower values of insulin, ACTH, cortisol and 24 hour urine cortisol than patients with hypertension.

Conclusions: Primary hypertension is often associated with obesity and insuline resistance. The disturbance in HPA axis may relate to hypertension in children with obesity. There is a need for further studies with higher number of patients and controls.

\section{EP-169 ARTERIAL HYPERTENSION IS PREDICTOR OF CKD IN CHILDREN WITH HUS}

Elena Gunkova, Igor Zorin, Albina Vyalkova

Orenburg Medical University

Introduction: CKD detected in $20-50 \%$ of children who had HUS Arterial hypertension (AH) is one of the most frequent complications of HUS. AH is not only complication of HUS but also factor of formation and progression of CKD. The aim of the study was to determine frequency and role of $\mathrm{AH}$ in formation of CKD in children with HUS.

Material and methods: Materials and methods: we had retrospective analyze of 75 case reports of children with HUS and had prospective study of these patients. All children were underwent 24h BPM.

Results: 3 years children $(81 \%$, p0,05) prevailed in patients with HUS. AH was diagnosed in $35 \%(\mathrm{n}=26)$ of patients with HUS, in $100 \%$ of children with atypical HUS. Children with AH underwent RRT in $92.3 \%$ $(n=24)$ in acute period of HUS. AH was stopped together with acute renal injury in 25 patients. AH was diagnosed in $8(11 \%)$ patients after HUS in their prospective study. 1 patient had AH from acute period of HUS till present time. 2 children who had stopped $\mathrm{AH}$ in acute period of HUS have AH in present time. 5 children had AH after stopped HUS. All children treated by antihypertensive drugs. $58 \%(\mathrm{n}=15)$ of children with $\mathrm{AH}$ in acute period of HUS have CKD in present time.

Conclusions: $\mathrm{AH}$ is predictor of formation and progression of CKD in children with HUS.

\author{
EP-170 A PATIENT WITH BEHCETS DISEASE PRESENTING \\ WITH HYPERTENSION \\ Mehtap Adar ${ }^{1}$, Elif Çomak ${ }^{1}$, Mustafa Koyun ${ }^{1}$, Firat Kardelen ${ }^{2}$, \\ Gülşah Kaya Aksoy ${ }^{\text {, Sema Akman }}{ }^{I}$ \\ ${ }^{I}$ Akdeniz University Medical Faculty, Department Of Pediatric \\ Nephrology, Antalya \\ ${ }_{2}$ Akdeniz University Medical Faculty, Department Of Pediatric \\ Cardiology, Antalya
}


Introduction: Behcets disease is a chronic recurrent systemic inflammatory disease characterized by inflammation in the vascular wall. We aimed to present an adolescent with Behçets Disease whose first presentation was with hypertension.

Material and methods: A fifteen-year-old girl was admitted with complaints of dyspnea, palpitations, right arm numbness and cough. Rash, fever, oral aphthae or myalgia were not described. Her family history was unremarkable. On physical examination, her blood pressure was $219 / 126 \mathrm{mmHg}\left(99^{\text {th }} \mathrm{p}\right.$ : $\left.124 / 80 \mathrm{mmhg}\right)$ in the right arm, lower-extremity blood pressure was normal $(110 / 70$ $\mathrm{mmHg}$ ). Laboratory results were as follows: hemoglobin $11.3 \mathrm{~g} / \mathrm{dL}$, leukocyte $9780 / \mathrm{mm}^{3}$, erythrocyte sedimentation rate $24 \mathrm{~mm} / \mathrm{hour}$, C-reactive protein $2.07 \mathrm{mg} / \mathrm{dL}$, serum creatinine $0.64 \mathrm{mg} / \mathrm{dL}$, serum sodium $138 \mathrm{mEq} / \mathrm{L}$, serum potassium $4.1 \mathrm{mEq} / \mathrm{L}$. Anti-nuclear antibody and antineutrophil cytoplasmic antibodies were negative. No proteinuria or hematuria was observed on urinalysis. A renal Doppler ultrasonography (US) showed a stenosis of $90 \%$ of the abdominal aorta. Fluorodeoxyglucose (FDG)-positron emission tomography was applied for large vessel vasculitis, showing diffuse wall thickening and accompanying significant pathological activity in the proximal abdominal aorta (L1 level) was observed. Propranolol and captopril were administered for antihypertensive treatment.

Results: At follow-up, doppler US was performed due to pain and hyperemia at right arm, revealing a thrombus in the vena basilica. Low molecular weight heparin and pulse methyl prednisolone were given to the patient with the diagnosis of vasculitis. Genetic analysis yielded a heterozygous "p.Thr68Met (c.203C> T) mutation on PSTPIP1 gene, which was previously reported to be associated with Behcets Disease.

Conclusions: Although Behçets disease usually presents with arteriovenous thrombus, it should be kept in mind that it may present with hypertension.

\section{EP-171 CORRELATIONS BETWEEN CARDIOVASCULAR RISK FACTORS AND BODY COMPOSITION PARAMETERS FROM BIOIMPENDANCE MEASUREMENT \\ Mirjam Močnik ${ }^{1}$, Sonja Golob Jančič ${ }^{1}$, Marjetka Švigelj ${ }^{2}$, Nataša Marčun Varda ${ }^{1}$ \\ ${ }^{1}$ Department Of Paediatrics, University Medical Centre Maribor \\ ${ }^{2}$ Medical Faculty, University Of Maribor}

Introduction: The aim of our study was to evaluate correlations between body composition and several clinical parameters.

Material and methods: 206 children and adolescents (120 male, 86 female) have been prospectively included in this study. Body impedance measurement has been performed in all participants. During the hospital work-up several clinical parameters were obtained and correlated to body composition measurement.

Results: Body composition parameters were significantly associated with anthropometric measurements, systolic blood pressure, insulin levels, serum creatinine, urate, liver function tests, triglycerides, cholesterols and apolipoproteins, homocysteine, vitamin D and proteins in 24-hour urine. Body composition differed between boys and girls, between participants with hepatic steatosis and normal liver parenchyma as well as between left ventricular hypertrophy and normal left ventricular wall. Interestingly, body composition did not correlate with diastolic blood pressure, pulse wave velocity and intima media thickness.

Conclusions: Several clinical parameters are associated with body composition and fat mass in children. Obesity and body composition play an important role in development of other cardiovascular risk factors, not dependent on fat mass alone, and the latter might be used for cardiovascular risk determination.
EP-172 THE RELATIONSHIP BETWEEN BODY COMPOSITION AND BLOOD PRESSURE IN CHILDREN

Gulcin Kaymakoglu ${ }^{1}$, Meral Torun Bayram ${ }^{2}$, Gizem Yildiz ${ }^{2}$, Salih Kavukcu ${ }^{2}$, Alper Soylu $^{2}$

${ }^{1}$ Dokuz Eylül Unİversity Faculty Of Medİİne, Department Of Pedíatrícs, İzmir, Turkey

${ }^{2}$ Dokuz Eylul University Medical Faculty, Department Of Pediatric Nephrology, İzmir, Turkey

Introduction: Blood pressure is influenced by adipose tissue in obesity. We aimed to investigate the relationship between blood pressure and body composition in normotensive and hypertensive children.

Material and methods: Children who underwent ambulatory blood pressure monitoring were grouped as hypertensive (+) (Group 1) and normotensive (Group 2). Bioelectric impedance analysis (Bodystat Qudscan 4000, Bodystat Limited, British Isles) were performed to all patients and the following parameters were recorded: body mass index (BMI), body fat mass index (BFMI), lean mass index (LMI), fat free mass index (FFMI), total body water (TBW\%), extracellular fluid (ECF\%), intracellular fluid (ICF\%). Groups were compared for these parameters and age, gender, waist and hip circumference, waist-hip ratio, uric acid and lipid levels. In addition, the correlation of these parameters with mean blood pressure (BP) (systolic, diastolic and total) and BP loads (day and night) was evaluated.

Results: There were 32 children in Group 1, 16 in Group 2. The age and gender were similar in both groups. The waist and hip circumference, BMI, BFMI, LMI, FFMI and uric acid level were higher in Group 1 than Group 2, while ECF\% was lower in Group 1. There was a positive correlation between uric acid level and systolic or total mean BP during the day and night. In addition, there were a positive correlation between BFMI, LMI, waist circumference with systolic or total mean BP during the day or night $(\mathrm{p}<0.05)$. There was a negative correlation between ECF\% and BMI $(r=-0.58, \mathrm{p}<0.05)$.

Conclusions: The waist circumference, uric acid, fat and fat free mass may have an impact on BP.

\section{EP-173 AN UNUSUAL CASE OF RENOVASCULAR HYPERTENSION IN A PATIENT WITH CONGENITAL ADRENAL HYPERPLASIA \\ Ganna Zvenigorodska ${ }^{2}$, Galina Guminska ${ }^{1}$, Lyudmyla Starynets ${ }^{1}$, Oleksandr Moskaliuk ${ }^{2}$ \\ ${ }^{1}$ Vinnytsya Regional Clinical Childrens Hospital \\ ${ }^{2}$ National Pirogov Memorial Medical University}

Introduction: Renovascular hypertension is not very common in the pediatric population. Because high blood pressure in children is rare and most of these patients are asymptomatic, many are overlooked until they present with a hypertensive crisis. The aim of this study was to review a case of a child with malignant hypertension caused by renal artery stenosis (RAS).

Material and methods: We have a follow up of the patient with congenital adrenal hyperplasia $(\mathrm{CAH})$ and uncontrolled hypertension caused by bilateral renal artery stenosis, determined clinically in a Vinnytsya Regional Children's Hospital. His clinical course was retrospectively reviewed and results analyzed.

Results: A 3 years old child presented with vomiting, headache. Blood pressure (BP) was discovered 260/180 $\mathrm{mmHg}$. Opthalmological examination revealed retinal exudates. Drugs couldn't control his BP. From the history it was known that the child had $\mathrm{CAH}$, deficiency of 21-hydroxylase. The diagnosis was confirmed by neonatal screening test and replacement therapy with fludrocortisone and glucocorticoid started. Hypertension was thought to be the adverse effect of drugs. Changing of doses of these medications gave no effect. Medical treatment with nifedipine, amlodipine, moxonidin failed to control his BP. Biochemical analysis showed impaired kidney function. GFR decreased 
progressively. An ultrasound scan showed two normal-sized kidneys. Echocardiography showed hypertrophy of left ventricle. Because of resistant high grade hypertension we suspect renovascular origion. CT angiography revealed right renal artery high grade stenosis, which was the cause of near total occlusion, as well as left artery stenosis. Bilateral RAS with renal insufficiency was diagnosed. The child was sent for surgical revascularization.

Conclusions: Systemic hypertension in children is usually secondary to a specific pathology, and RAS is the most common cause in children. Surgical revascularization has been an effective therapy. Hypertension may persist despite endovascular or surgical management of childhood RAS highlighting the importance of close monitoring and ongoing medical management.

\section{EP-174 A BILATERAL RENAL STENOSIS}

Marta Giambrone ${ }^{1}$, Ciro Corrado ${ }^{1}$, Rosa Cusumano ${ }^{1}$, Giovanni Pavone ${ }^{1}$, Maria Chiara Sapia ${ }^{1}$, Ilenia Chillura ${ }^{\text {, }}$ Melania Guardino ${ }^{1}$, Maria Michela Dalessandro ${ }^{1}$, Maria Cristina Maggio ${ }^{2}$

${ }^{1}$ Pediatric Nephrology. G. Di Cristina Hospital. Palermo, Italy

${ }^{2}$ University Hospital, Palermo, Italy

Introduction: A 9-year-old male was referred to the emergency department of our hospital for headache and vomiting, his blood pressure was 203/130 mmHg. He was admitted to the pediatric nephrology unit for investigation.

Material and methods: The family history was negative for hypertension but positive for renal stenosis (maternal uncle). His weight and height were normal for his age. Physical examination was unremarkable with no cardiac or abdominal bruits and normal peripheral pulses. Routine laboratory and urine examination were normal. An abdominal ultrasound showed the left kidney to be smaller than the right one (right 9,2 and left 7,9 cm) with regular corticomedullary differentiation. Fundoscopic examination and electrocardiogram were normal, where the echocardiogram showed no left ventricular hypertrophy. A bilateral renal stenosis was detected by a renal doppler. Further analysis revealed a significant increase of renin activity and aldosterone levels. Clonidine, amlodipine and furosemide were administrated with clinical benefit. At 48 hours of admission the patient was asymptomatic and his blood pressure reduced to $150 / 100 \mathrm{mmHg}$. An ambulatory blood pressure monitoring (ABPM) was performed showing an average $24 \mathrm{~h}$ value of $147 / 104 \mathrm{mmHg}$, being daytime and nighttime values 149/105 and $141 / 100 \mathrm{mmHg}$ respectively.

Results: An abdominal magnetic resonance documented ostial bilateral renal artery stenosis with post-stenotic dilatation, ectasia of abdominal aorta $(24 \mathrm{~mm})$ associated with increased intima and media thickness, ectasia of celiac trunk, and narrowing of superior mesenteric artery. We performed a percutaneous transluminal angioplasty of both renal arteries, with ultrasoft SV Boston-Scientific $4 \mathrm{x}$ 20 with no complications. Two days later ABPM was performed. At discharge, ABPM showed average $24 \mathrm{~h}$ value of $117 / 75 \mathrm{mmHg}$, daytime and nighttime blood pressure values were $117 / 75$ and $115 / 75 \mathrm{mmHg}$ respectively.

Conclusions: We suspected a middle aortic syndrome (MAS) related to Takayasu arteritis. One month later his blood pressure values were 110/ 60 , under treatment with amlodipine.

EP-175 SHOULD AN ACCESSORY RENAL ARTERY BE CONSIDERED AS A CAUSE OF HYPERTENSION IN CHILDREN: A CASE REPORT

Bernardica Valent Moric, Tomislav Krpan, Josipa Josipovic

Sestre Milosrdnice University Hospital Center

Introduction: The most common pattern of kidney vascularisation is a single renal artery originating from the abdominal aorta. However, in 20-
$30 \%$ of general population an accessory renal artery can be found being more frequently present (up to $80 \%$ ) in patients with essential hypertension.

A possible pathomechanism of hypertension is the impaired renal perfusion since the diametar of a single renal artery is usually larger than when multiple arteries are present. Whether an accessory renal artery could be a cause of hypertension is still controversial.

Material and methods: We describe a case of a 16-year old girl who presented with hypertension crisis. She was previously healthy with no record of abnormal blood pressure (BP).

At admission she reported nausea and severe headache while her BP was 220/120 mmHg. Her body mass index was normal and physical examination unremarkable.

Results: Initial workup showed normal renal function with normal serum electolytes and plasma glucose. Urin dipstick and urin toxicology screen were also normal. She had hypercholesterolemia and mild proteinuria but no signs of other target organ damage (electrocardiogram, echocardiography, fundoscopy and computed tomography of the brain were normal). Ambulatory blood pressure monitoring confirmed severe ambulatory hypertension.

Further evaluation was aimed at determining the possible cause of secondary hypertension. Urine metanephrines, urinary free cortisol, plasma cortisol, ACTH and thyroid function tests were within reference ranges. High normal plasma renin with elevated plasma aldosteron led to a suspicion of renovascular hypertension.

Magnetic resonance imaging revealed no pathology of the adrenal glands. Although renal ultrasonography with Doppler was normal, magnetic resonance angiography and later CT angiography showed two nonstenotic right renal arteries.

A satisfactory blood pressure control was eventually achieved with combination therapy including ACE inhibitor.

Conclusions: Although nonstenotic, an accessory renal artery should be considered as a possible cause of renovascular hypertension in children and adolescents.

\section{EP-176 IDIOPATHIC NEPHROTIC SYNDROME AFTER 10 YEARS-OLD: IS SYSTEMATIC RENAL BIOPSY APPROPRIATE? \\ Anna Smith ${ }^{1}$, Eric Xu ${ }^{2}$, Olivia Boyer ${ }^{3}$, Jean Daniel Delbet ${ }^{4}$, Michel Peuchmaur ${ }^{5}$, Julien Hogan ${ }^{1}$, Claire Dossier ${ }^{1}$ \\ ${ }^{1}$ Pediatric Nephrology, Robert-debre Hospital, Aphp, Paris - France \\ ${ }^{2}$ Augustin Morvan Regional University Hospital, Brest, France \\ ${ }^{3}$ Pediatric Nephrology, Necker Hospital, Aphp, Paris - France \\ ${ }^{4}$ Pediatric Nephrology, Armand-trousseau Hospital, Aphp, Paris - France \\ ${ }^{5}$ Anatomopathology, Robert-debre Hospital, Aphp, Paris - France}

Introduction: Renal biopsy (RB) is recommended at diagnosis for children presenting with isolated nephrotic syndrome (NS) after the age of $10-12$ years old, unlike in younger children, because of a higher frequency of other diagnosis than MCD or FSGS. Recent reports, local practices and new serological biomarkers for membranous nephropathy now question this approach. Therefore, the objective of our study was to evaluate causes of $\mathrm{NS}$ and indications for RB in a population-based cohort of adolescents with NS.

Material and methods: We conducted a retrospective multicenter study, including children aged 10 to 18 years diagnosed with Idiopathic NS between December 2007 and June 2020 in 35 Pediatric and 3 Pediatric Nephrology (PN) departments of the Paris area (NEPHROVIR network) and patients with Idiopathic-looking NS biopsied in the 3 PN departments.

Results: Hundred and three children were included (54 males). Mean age at diagnosis was $13,4 \pm 2$ years. A renal biopsy was performed in 76 patients: at presentation because of atypical symptoms (such as hypertension, macroscopic hematuria, organic 
renal failure) in 15 or for no other reason than age in 39 , and after 4 weeks for steroid resistance +/- atypies in 22 patients. Histological findings were MCD $(n=49)$, FSGS $(n=13)$, mesengial proliferation $(n=3)$, Membranous Nephropathy $(n=6)$ and $\operatorname{IgAN}$ $(\mathrm{n}=5)$.

Conclusions: NS was secondary in only $11 \%$ of adolescents presenting with Idiopathic-like NS, and first line treatment with oral prednisone was appropriate in all cases. In addition, more than $40 \%$ of RB could have been avoided in Steroid Sensitive NS patients. Finally, we propose that RB should only be performed in case of atypical clinical or biological history or steroid resistance after 4 weeks, as in the younger population.

\section{EP-177 PRIMARY MEMBRANOUS NEPHROPATHY IN CHILDREN: A MONOCENTRIC RETROSPECTIVE STUDY}

Claire Dossier ', Yekkeang Leng ', Anne Couderc ', Anne Maisin ', Veronique Baudouin ${ }^{1}$, Michel Peuchmaur ${ }^{2}$, Julien Hogan ${ }^{1}$

${ }^{1}$ Pediatric Nephrology, Robert-debre Hospital, Aphp, Paris - France

${ }^{2}$ Anatomopathology, Robert-debre Hospital, Aphp, Paris - France

Introduction: In the last decade, the identification of PLA2R antigenes has modified both diagnosis and treatment of adult Membranous Nephropathy (MN). In children, $\mathrm{MN}$ is a rare cause of Nephrotic Syndrome (NS) and treatment approaches are heterogeneous. We describe here the presentation, treatment and outcomes of children diagnosed with Primary MN at Robert-Debre Hospital.

Material and methods: This a monocentric retrospecive study of all patients, aged $<18$ years, diagnosed with MN, between 2005 and 2020, based on the local kidney biopsy charts.

Results: Eight patients were diagnosed with Primary MN, while 21 with secondary MN. Median age at diagnosis of primary MN was 11.4 years (range 7.9-17) with a 1:1 sex ratio. All patients presented with oedema and nephrotic syndrome +/- macroscopic $(n=2)$ or microscopic $(n=1)$ hematuria, and normal eGFR. Four patients had a positive PLA2R serology. Initial treatment included steroids and tacrolimus, in addition to ACE-inhibitors. B-cell depletion was added in 5 patients at onset and after relapse in the 3 others. Complete remission was obtained in all patients after a median time of 3.7 months (range 2-6). Five patients experienced $>1$ relapse, following tacrolimus discontinuation or B-cell recovery. At last follow-up (median 60 months), all were in complete remission, receiving ACE-inhibitors +/- B-cell depletion. One patient with negative PLA2R-Ab was diagnosed 5 years after with systemic lupus with skin involvement and arthritis.

Conclusions: In this small series, all patients went into complete remission within 6 months with a combined induction therapy with steroids and tacrolimus +/- B-cell depletion, but $60 \%$ relapsed. There are no guidelines for the treatment of pediatric primary $\mathrm{MN}$, and there is a real need for larger data. Therefore, a European survey and registry initiative is currently in process.

\section{EP-178 SARCOIDOSIS-RELATED INTERSTITIAL NEPHRITIS IN A PEDIATRIC POPULATION: A MONOCENTRIC RETROSPECTIVE STUDY \\ Oumayma Taroua ${ }^{1}$, Theresa Kwon ${ }^{\text {, }}$, Veronique Baudouin ${ }^{\text {, }}$ Anne Maisin ${ }^{1}$, Alexandra Cambier ${ }^{1}$, Anne Couderc ${ }^{1}$, Michel Peuchmaur ${ }^{2}$, Julien Hogan ${ }^{1}$, Claire Dossier ${ }^{i}$ \\ ${ }^{1}$ Pediatric Nephrology, Robert-debre Hospital, Aphp, Paris - France \\ ${ }^{2}$ Anatomopathology, Robert-debre Hospital, Aphp, Paris-France}

Introduction: Sarcoidosis is a systemic disease histologically characterized by the presence of non-caseating granuloma in multiple organs. Renal involvement is infrequent $<25 \%$ in adults with most frequently tubulo-interstitial nephritis with or without granuloma. Pediatric sarcoidosis is extremely rare, and there are no trials nor treatment guidelines concerning renal granulomatous nephritis.

Material and methods: This is a retrospective study at Robert-Debre Hospital, Paris, including patients aged $<18$ yrs, diagnosed with Acute Interstitial Nephritis, associated to non necrotizing, epithelioid and giant cell granulomas either on renal or salivary gland biopsies, between 2006 and 2019.

Results: Nineteen patients, 15 females and 4 males, were included. Mean age at diagnosis of interstitial nephritis was 14 years old. Ten patients presented with fever, 12 with digestive symptoms and 13 weight loss. Only 1/19 had a prior diagnosis of sarcoidosis. At presentation, mean serum creatinine level was $242 \mu \mathrm{mol} / \mathrm{L}$ and proteinuria $0.83 \mathrm{~g} / \mathrm{L}$. Granulomas were detected on renal biopsy in 6 cases, on salivary gland biopsy in 9 and both tissues in 4 patients. Extrarenal involvement included uveitis in 6/19 and lung in 6 patients with only asymptomatic test dysfunction in 5 . All patients received steroids, after methylprednisolone pulses in 15/ 19. Mean duration of oral prednisone was 16 months and 15 received Mycophenolate mofetil as maintenance therapy. At 2 years follow-up, GFR was $88 \mathrm{~mL} / \mathrm{min} / 1.73 \mathrm{~m}^{2}$.

Conclusions: The prevalence of renal involvment in pediatric sarcoidosis is unknown but this series highlights that renal granulomatous sarcoidosis is mostly inaugural, associated to general signs and/or uveitis rather than pulmonary or multiorgan disease. All patients had a favorable renal outcome with this intensive treatment strategy. Longer follow-up and larger series are required to better describe the disease's course and evaluate treatment options.

\section{EP-179 ACUTE TUBULOINTERSITITAL NEPHRITIS IN PEDIATRIC PRIMARY SJöGREN SYNDROME}

Ivana Trutin, Ingrid Sitas, Gabrijela Peric Markovic, Mandica Vidovic Clinical Hosiptal Center Sestre Milosrdnice

Introduction: Sjögren syndrome (SS) is a chronic autoimmune disease primarily affecting lachrymal and salivary glands with varying degrees of systemic involvement. Clinical manifestations differ in children and do not meet currently available adult criteria (American European consensus group - AERCG). Recurrent parotid swelling is often observed in children unlike oral and ocular involvement (sicca syndrome) which are specific for adults. Extraglandular involvement ia rare, only $9.9 \%$ patients having renal involvement. Pathologic and laboratory findings include lymphocytic infiltration of the exocrine glands, presence of rheumatoid factor (RF) and specific antinuclear antibody (ANA). We present a case of primary SS with renal involvement.

Material and methods: A 12-year-old girl with history of recurrent left-sided parotid enlargement episodes and sicca syndrome was admitted to our Department for evaluation. Her mother was diagnosed with systemic lupus in adolescence.

Results: Laboratory screening showed highly positive ANA (1:1280), anti-SS-A $122 \mathrm{U} / \mathrm{mL}$, anti-SS-B $176 \mathrm{U} / \mathrm{mL}$ and $\mathrm{RF}$ (762 U/mL). Schimmer's test verified moderate hypolacrimation. Salivary gland biopsy confirmed pSS diagnosis. Since there was persistent proteinuria in a 24 -hour urine $(247 \mathrm{mg} / 24 \mathrm{~h})$ renal biopsy was performed and acute tubulointerstitial nephritis was confirmed by pathohystological evaluation. The patient received pulse corticosteroid therapy. On her last follow there was no proteinuria with low dose prednisone and hydroxichlorochine therapy.

Conclusions: pSS is rare and often unrecognized condition in children. The lack of pediatric diagnostic criteria and underdiagnosed extraglandular involvement can affect course of disease. Early renal involvement recognition and adequate therapy can prevent progressive disease. Additional studies in children are needed in establishing clasification criteria and therapeutic algoritams to enable further clinical and translational research. 
EP-180 CONCOMITANT MEMBRANOUS NEPHROPATHY AND ANCA VASCULITIS IN A 4 YEAR-OLD CHILD

Vasiliki Karava ${ }^{1}$, Vasiliki Sgouropoulou ${ }^{2}$, John Dotis ${ }^{1}$, Antonia Kondou ${ }^{1}$, George Liapis ${ }^{3}$, Maria Trachana ${ }^{2}$, Nikoleta Printza $^{1}$

${ }^{1}$ Pediatric Nephrology Unit, 1st Department Of Pediatrics, Hippokratio General Hospital,aristotle University Of Thessaloniki, Greece

${ }^{2}$ Pediatric Rheumatology Unit, 1st Department Of Pediatrics, Hippokratio General Hospital,aristotle University Of Thessaloniki, Greece

${ }^{3} 1$ st Department Of Pathology, School Of Medicine, National And Kapodistrian University Of Athens, Greece

Introduction: Concurrent membranous glomerulonephritis (MGN) and ANCA vasculitis, commonly associated with necrotizing/crescentic glomerulonephritis, is rarely described.

Material and methods: We report a case of MGN and ANCA vasculitis in a pediatric patient.

Results: A 4 year-old boy was admitted to a local public hospital for acute febrile infection of lower respiratory tract, with bilateral interstitial lung infiltrates at chest X-ray. On $3^{\text {rd }}$ day, he presented nephrotic syndrome, necessitating prednisolone therapy initiation. C3, $\mathrm{C} 4$ titers and antistreptolysin levels were normal, while antinuclear and anti-DNA antibodies were negative. He was discharged on $20^{\text {th }}$ day, but eight days later, he presented acute respiratory distress and was transferred to our hospital. Pulmonary CT scan revealed scattered ground-glass opacities throughout the pulmonary parenchyma and disperse alveolar infiltrates indicative of alveolar hemorrhage. Kidney function and serum albumin were normal, while moderate proteinuria $\left(527 \mathrm{mg} / \mathrm{m}^{2} / 24 \mathrm{~h}\right)$ without hematuria was present. Positive serum cANCA antibodies indicated granulomatosis with polyangiitis (GPA) vasculitis. Nevertheless, kidney biopsy revealed MGN with $\operatorname{IgG}, \operatorname{IgM}$ and $\operatorname{IgA}, \mathrm{C} 3$ and $\mathrm{C} 1 \mathrm{q}$ subepithelial deposits, moderate mesangial proliferation and minimal endocapillary hypercellarity, without glomerular necrotizing lesions or crescent formation. Induction therapy included methylprednisolone pulses ( 3 doses) switched to prednisolone therapy for 6 months and cyclosphophamide infusions (6 doses), while rituximab therapy (4 doses) was added due to respiratory function deterioration 4 days after admission. One week after therapy onset, cANCA levels turned negative, proteinuria resolved, and respiratory function was improved. 10 months later, the patient remains in remission under maintenance therapy with mycophenolate mofetil.

Conclusions: We report a paediatric case of GPA and concurrent MGN. In case of nephrotic syndrome with ANCA vasculitis, the existence of other types of GN, especially MGN, may be considered.

\section{EP-181 PULMONARY HYPERTENSION IN CHILDREN AND YOUNG PEOPLE WITH SYSTEMIC LUPUS ERYTHEMATOSUS Catherine Longthorpe ${ }^{1}$, Matthew Harmer ${ }^{1}$, Caroline Platt ${ }^{l}$, Jacqueline Sit ${ }^{2}$, Stephen Marks ${ }^{2}$, Michael Beresford ${ }^{3}$, Athimalaipet Ramanan ${ }^{4}$ \\ ${ }^{1}$ Bristol Royal Hospital For Children \\ ${ }^{2}$ Great Ormond Street Hospital For Children \\ ${ }^{3}$ University Of Liverpool On Behalf Of The Uk Jsle Cohort Study Group \\ ${ }^{4}$ University Of Bristol}

Introduction: Systemic Lupus Erythematosus (SLE) is a rare multisystem autoimmune disorder with a variable clinical phenotype. Pulmonary hypertension (PHTx) is a recognised complication and in adults with SLE, associated with a poor prognosis (3 year survival $89 \%$ ). A proportion of cases will be asymptomatic (18\% from prospective studies). PHTx in children with SLE is relatively unexplored. Estimates of the prevalence of sub-clinical PHTx in children with minor SLE manifestations have been reported at $2 \%$ from a Brazilian cohort.
Material and methods: Retrospective case series of children in the UK with Juvenile-onset SLE (JSLE) complicated by pulmonary hypertension. All cases were identified through the UK JSLE Cohort Study (data collected from 2006 onwards). Clinical data was extracted through use of a standardised proforma.

Results: Of 665 children with JSLE in the UK cohort study to date, 4 were identified as having PHTx. 1 patient was excluded (PHTx diagnosis $>18$ years). Of the remaining 3 cases, all were female, 2 white Caucasian, 1 black African ethnicity. Age of JSLE onset was between 4 and 9 years. Case 1 presented with haematological involvement, case 2 with neuropsychiatric symptoms and class III lupus nephritis, case 3 with haematological and musculoskeletal disease.

PHTx was identified at an interval of between 2 months to 8 years from JSLE diagnosis. 2 cases presented symptomatically, the remaining case was identified through a routine screening echocardiogram. All received treatment with intravenous Methylprednisolone, Rituximab, Mycophenolate mofetil and Hydroxychloroquine. 2 children also received Cyclophosphamide (1 with additional plasma exchange) and Sildenafil. There were no shared immunological features other than the anti-nuclear antibody (ANA) positivity. Conclusions: This small UK-based descriptive series highlights PHTx as an entity worthy of early consideration in JSLE, particularly noting that it can present asymptomatically. This disease complication may be even more relevant in those with significant SLE manifestations.

\section{EP-182 RENAL INVOLVEMENT IN HYPOCOMPLEMENTEMIC URTICARIAL VASCULITIS SYNDROME WITH DNASE1L3 MUTATION}

Laura Lucchetti ${ }^{1}$, Federica Zotta ${ }^{1}$, Alessandra Gianviti ${ }^{1}$, Antonio Gargiulo ${ }^{1}$, Isabella Guzzo ${ }^{1}$, Claudia Bracaglia ${ }^{2}$, Fabrizio De Benedetti ${ }^{2}$, Francesco Emma ${ }^{1}$, Marina Vivarelli ${ }^{1}$ ${ }^{1}$ Division Of Nephrology, Irccs Bambino Gesù Children Hospital ${ }^{2}$ Division Of Rheumatology, Irccs Bambino Gesù Children Hospital

Introduction: Hypocomplementemic urticarial vasculitis syndrome (HUVS) is a rare condition characterized by recurrent urticaria associated with multisystemic features (ocular, pulmonary, gastrointestinal, musculoskeletal and renal involvement). HUVS evolves in systemic lupus eritematosus (SLE) in $>50 \%$ of patients. We report 3 patients with HUVS and renal involvement. In all patients, we found the same mutation in the DNASE1L3 gene

Material and methods: A genetic analysis was performed using a panel including genes associated to Lupus-Like Interferonopathies (DNASE1, DNASE2, DNASE1L3, TREX1) on the Illumina NextSeq platform

Results: Patient 1 is 9-year-old male who presented with joint, abdominal, pulmonary and cutaneous involvement. Subsequently he developed kidney involvement with mesangial proliferative glomerulonephritis with "full-house" immunofluorescence and normal kidney function. He did not develop ANA, anti-dsDNA and ANCA antibodies.

Patient 2 and 3 are female (14 and 13 year-old) and presented anemia, urticarial lesions and renal involvement with pauci-immune glomerulonephritis. Patient 2 had only transient mild kidney failure. Patient 3 developed severe kidney failure requiring peritoneal dialysis.

At onset, they had positive ANCA and negative ANA and anti-ds DNA antibodies. All three patients had elevated anti-C1q antibody titers and low circulating $\mathrm{C} 3$ levels. All patients were treated with steroids, cyclophosphamide, mycophenolate mofetil and dapsone. None of them have developed SLE up to now. In all patients genetic analysis reveals a homozygous DNASE1L3 variant (c.290_291 del CA)

Conclusions: HUVS is a rare disease that is observed in $7 \%$ of SLE patients and can evolve into SLE in $>50 \%$ of cases. DNASE1L3 variants have been described in other patients with HUVS and early-onset SLE. These mutations seem to be associated with a more severe phenotype which can resemble either lupus nephritis or pauci-immune glomerulonephritis, as observed in our patients. This clinical picture requires aggressive, life -long immunosuppression and a stringent follow-up, considering the high risk of SLE. 
EP-183 TAKAYASU ARTERITIS WITH RENAL ARTERY INVOLVEMENT IN CHILDREN: 12 YEARS EXPERIENCE OF A TERTIARY CENTER

Tugba Tastemel Ozturk ${ }^{1}$, Demet Baltu ${ }^{1}$, Eda Didem Kurt-sukur ${ }^{1}$, Bora Gulhan ${ }^{1}$, Seza Ozen ${ }^{2}$, Ali Duzova ${ }^{1}$, Fatih Ozaltin ${ }^{1}$,

Rezan Topaloglu ${ }^{l}$

${ }^{1}$ Division Of Pediatric Nephrology, Hacettepe University Faculty Of Medicine, Ankara, Turkey

${ }^{2}$ Division Of Pediatric Rheumatology, Hacettepe University Faculty Of Medicine, Ankara, Turkey

Introduction: The aim of this study was to determine characteristics, management, and outcome of Takayasu arteritis (TA) with renal artery involvement in children.

Material and methods: Medical records of patients diagnosed with TA at İhsan Doğramacı Childrens Hospital, Hacettepe University, between 2009 and 2020 were evaluated, and those with renal artery involvement were included in the study. Characteristics of the patients in the initial and final visits and the treatments they received were presented.

Results: Six of 16 patients $(37.5 \%$; 1 male, 5 female) with TA had renal artery involvement. The median age of TA onset was 14.4 years (11.316.2 ) in the patients with renal artery involvement. All of the patients presented with hypertension, 3 of 6 patients had bilateral renal artery involvement. During initial evaluation, functional renal asymmetry was revealed in 4 patients with MAG3, DMSA and DTPA scintigraphy. The median follow-up period was 26 months (4-73). During follow-up, two patients underwent surgery (both thoracic aorta to abdominal aorta bypass and aortorenal bypass). Radiological findings of 2 patients with single renal artery wall irregularities improved on follow-up. eGFRs were $>60$ $\mathrm{ml} / \mathrm{min} / 1.73 \mathrm{~m}^{2}$ during the follow-up in all patients. At last visit, need for antihypertensive persisted in all of the patients.

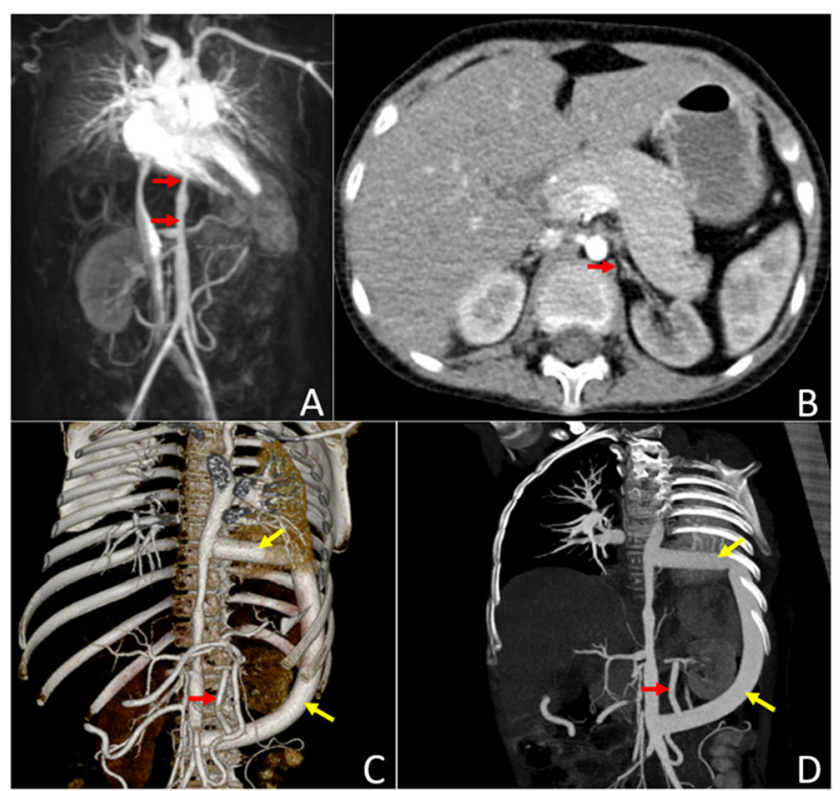

Figure: MRI and CT images of a patient with thoracoabdominal aorta and left renal artery stenosis before (A, B) and after surgery (C, D).

(A) MRI image reveals stenosis in the thoracic and abdominal aorta (red arrows). (B) CT image shows the stenosis in left renal artery (red arrow). $(\mathrm{C}, \mathrm{D}) \mathrm{CT}$ images show the graft placed between the descending thoracic aorta and the terminal part of the abdominal aorta (yellow arrows) and the graft placed between this graft and the left renal artery (red arrows).

Conclusions: In TA renal arteries are mostly affected, and patients frequently manifests with hypertension. In addition to immunosuppressive drugs used for primary disease, surgical procedures and multiple antihypertensive drugs may be required in the management of hypertension. Imaging of kidney differential functions at the time of diagnosis may be a guide for the surgical plan.

\section{EP-184 POST-INFECTIOUS GLOMERULONEPHRITIS IN THE} COVID-19 ERA: A CASE REPORT

Georgia Malakasioti ${ }^{1}$, Ariadne Neophytou ${ }^{2}$, Eirini Sakkou ${ }^{2}$, Maria Mila $^{1}$, Andromachi Mitsioni ${ }^{1}$

${ }^{1}$ Renal Unit, P. \& A. Kyriakou Children's Hospital, Athens, Greece

2 Second Department Of Pediatrics, National And Kapodistrian University Of Athens School Of Medicine And P. \& A. Kyriakou Children's Hospital, Athens, Greece

Introduction: Several mechanisms have been suggested for the COVID19 associated kidney involvement. Direct viral invasion of podocytes, renal endothelial and tubular cells together with a cytokine releasemediated immune dysregulation and a hypercoagulable state have all been implicated in the pathogenesis of glomerular diseases elicited by SARS-CoV-2. We describe the case of a toddler with post-infectious glomerulonephritis (PIGN) following COVID-19.

Material and methods: A 32-month old boy was admitted with gross hematuria two weeks after a brief febrile illness with coryza and cough. Results: Physical examination was unremarkable including normal blood pressure and absence of peripheral edema. Leukocytosis (WBC 20000/ $\mathrm{LL}$ ), raised ESR $(90 \mathrm{~mm} / \mathrm{hr}), \mathrm{CRP}(41 \mathrm{mg} / \mathrm{L})$ and d-dimers $(1.8 \mathrm{mg} / \mathrm{L})$, together with numerous dysmorphic $\mathrm{RBC}$ on urinalysis and urine protein/creatinine of $2.4 \mathrm{mg} / \mathrm{mg}$ were the cardinal laboratory abnormalities. Serum creatinine, albumin and electrolytes remained within normal range. Kidneys were enlarged and echobright on ultrasound. Immunological and infectious work-ups (including serum immunoglobulins, ASOT, anti-DNAse B, ANA, dsDNA Abs, hepatitis serology, urine culture and pharyngeal swab) were negative apart from low $\mathrm{C} 3$ and $\mathrm{C} 4$ (86 and $11 \mathrm{mg} / \mathrm{dL}$ ) and positive SARS-CoV-2 IgM, IgA and IgG antibodies. Macrohematuria and proteinuria resolved within 5 days with normal diuresis, blood pressure and kidney function; serum complement levels normalized within 4 weeks.

Conclusions: The history of a previous upper respiratory tract infection with raised inflammatory markers and positive SARS-CoV-2 antibodies are suggestive of recent COVID19 illness in our patient with the hypocomplementemic PIGN likely being a sequela of the viral driven cytokine storm. Pediatric nephrologists need to be aware of this novel cause of PIGN while more data emerge regarding its distinct pathophysiology, epidemiology and natural history.

\section{EP-185 LUPUS NEPHRITIS IN CHILDREN - CLINICAL AND PATHOMORPHOLOGICAL FEATURES}

Hanna Szymanik-grzelak ${ }^{1}$, Małgorzata Barabasz, ${ }^{2}$,

Irena Wikiera-magott ${ }^{3}$, Beata Banaszak ${ }^{4}$, Anna Wieczorkiewicz-płaza ${ }^{5}$, Beata Bienias ${ }^{5}$, Magdalena Drożynska-duklas ${ }^{6}$, Marcin Tkaczyk ${ }^{7}$, Małgorzata Pańczyk-tomaszewska ${ }^{1}$

${ }^{1}$ Department Of Pediatrics And Nephrology, Medical University Of Warsaw, Warsaw, Poland

${ }^{2}$ Department Of Pediatric Cardiology And Nephrology, Poznan University Of Medical Sciences, Poznan, Poland

${ }^{3}$ Department And Clinic Of Paediatric Nephrology, Wroclaw Medical University, Wroclaw, Poland

${ }^{4}$ Department Of Paediatrics, Faculty Of Medical Sciences In Zabrze, Medical University Of Silesia, Katowice, Poland

${ }^{5}$ Department Of Pediatric Nephrology, Medical University Of Lublin, Lublin, Poland

${ }^{6}$ Department Pediatric \& Adolescent Nephrology \& Hypertension, Medical University Of Gdansk, Gdansk, Poland

${ }^{7}$ Department Of Pediatrics, Immunology And Nephrology, Polish Mother's Memorial Hospital Research Institute, Lodz, Poland 
Introduction: The aim of the study was to evaluate the clinical course and pathomorphological features in children with lupus nephritis (LN). Methods: We retrospectively analyzed the medical records of 39 children with LN, hospitalized in 7 pediatric nephrology units in Poland between 2010-2019. Demographic data, clinical symptoms at the onset of LN and laboratory parameters were reviewed. Systemic Lupus Erythematosus Disease Activity Index (SLEDAI), histological LN findings with the activity (IA) and chronicity index (IC), were analyzed

Results: 39 children (32 girls; 7 boys), median age at LN onset 14.75 (IQR 13.0-16.0) years, SLEDAI of 22.0 (IQR 18.0-27.0) points; LN histological class: IV (59.4\%), III (18.9\%), III/V (10.8\%), IV/V (8.1\%), VI (2.7\%); IA 8.0 (IQR 6.0-11.0) points, IC 1.05 (IQR 0-2.0) points, were examed. ANA were positive in all patients, anti-dsDNA in $85 \%$.The median eGFR was 68.6 (IQR 40.5-106.0) $\mathrm{mL} / \mathrm{min} / 1.73 \mathrm{~m}^{2}$, median serum albumin level 3.25 (IQR 2.4-3.6) g/dL, median proteinuria 2.84 (IQR 1.08-6.30) g/per day. Children with nephrotic $(\mathrm{n}=22)$ and nonnephrotic $(\mathrm{n}=17)$ proteinuria differed in $\mathrm{Hb}$ level $(\mathrm{P}<0.05)$, serum albumin level $(\mathrm{P}<0.001)$, daily proteinuria $(\mathrm{P}<0.0001)$, eGFR $(\mathrm{P}<0.01)$ and occurrence of hypertension $(\mathrm{P}<0.01)$. We did not observe statistically significant differences in the age at the onset of $\mathrm{LN}, \mathrm{WBC}$ count, platelet count, C3 level, C4 level and SLEDAI between the nephrotic group and non-nephrotic group.

Conclusions: Proliferative forms of $\mathrm{LN}$ in children may have a varying clinical presentation. Children with $\mathrm{LN}$ with nephrotic range proteinuria have lower $\mathrm{Hb}$ level, lower eGFR and higher occurrence of hypertension.

\section{EP-186 CLINICAL PRESENTATION AND OUTCOME OF PEDIATRIC ANCA ASSOCIATEDVASCULITIS GLOMERULONEPHRITIS}

Luigi Annicchiarico Petruzzelli, Gabriele Malgieri, Maria Concetta Lonardo, Angela De Luca, Vittorio Serio, Carmine Pecoraro

A.o.r.n. Santobono-pausilipon - Ospedale Santobono, Nephrology And Dialysis, Napoli, Italy

Introduction: Associated vasculitis-ANCA (AAV) are rare in pediatric age (incidence 10.6 cases / 100,000 / year). Renal involvement is common in up to $90 \%$ of cases.

Material and methods: In this study, the cases of AAV diagnosed in the last 15 years at the Pediatric Nephrology of the AORN Santobono Pausilipon in Naples were retrospectively analyzed, describing the clinical characteristics, renal involvement, treatment and outcome of the disease.

Results: 9 patients were identified. Five (55\%) were affected by microscopic polyangiitis (MPA) and $4(44 \%)$ by granulomatosis with polyangiitis (GPA) according to the EULAR / PReS classification criteria.All patients received steroid boluses as induction therapy and in addition cyclophosphamide was used in 6 patients while rituximab in 2 others.

Plasma exchange was performed in two patients for a total of 10 sessions in combination with steroid boluses and rituximab.

The median follow up of the entire population is 88 months.

Maintenance therapy involved the use of prednisone with gradual decalage and mycophenolate.

Conclusions: The analysis of therapeutic efficacy showed that patients treated with rituximab and plasmapheresis did not relapse after remission, although one of the two patients had chronic kidney disease stage V D KDOQI residual.

EP-187 RECURRENT NEUTROPENIA IN A PATIENT WITH PAUCI-IMMUNE CRESCENTIC GLOMERULONEPHRITIS Aynur Gencer Balaban ${ }^{1}$, Sibel Yel ${ }^{1}$, Aylin Inal ${ }^{1}$, Hulya Akgun ${ }^{2}$, Ebru Yilmaz ${ }^{3}$, Neslihan Gunay ${ }^{1}$, Ismail Dursun ${ }^{1}$, Muammer Hakan Poyrazoglu?

${ }^{1}$ Erciyes University Department Of Pediatric Nephrology Kayseri, Turkey
${ }^{2}$ Erciyes University Department Of Pathology Kayseri, Turkey

${ }^{3}$ Erciyes University Department Of Pediatric Hematology\&oncology Kayseri, Turkey

Introduction: Various glomerular diseases are associated with many hematologic and solid malignancies. Here, we present a case of a 13year-old boy who presented with glomerulonephritis, which was not encountered in the literature and was diagnosed with T-cell acute lymphoblastic leukemia (T-ALL) in four months.

Material and methods: A 13-year-old boy was admitted to our hospital with complaints of left plank pain. He had tachypnea, weakness, hepatosplenomegaly, and bilateral pretibial +1 edema. No breath sounds were heard on the basal of the left hemithorax. Pleural fluids characteristic was exudative, and Mycobacterial, fungal and bacterial cultures were negative. The result of the cytology was reported as class II (mesothelial cells covered with proteinous material). Laboratory investigations revealed increased levels of blood urea nitrogen and creatinine, acute phase reactants and $\mathrm{LDH}$, anemia, pyuria, proteinuria, hematuria. Kidney biopsy was performed, and with ANCA-negative pauci-immune glomerulonephritis was detected. He had recurrent neutropenia attack with anemia and pancytopenia developed and persisted during the last follow-up. We excluded infection, immunosuppressive drugs, macrophage activation syndrome.

Results: Bone marrow biopsy with flow cytometric study was performed and demonstrated T-ALL. In conclusion, it has been speculated that ANCA-negative pauci-immune glomerulonephritis is associated with TALL.The patient's kidney biopsy specimen was reevaluated for neoplastic lymphoid infiltrate retrospectively. No malign cells were detected.

Conclusions: We should consider evaluating patients with glomerulonephritis for malignancy if hematological abnormalities persist and perform further examinations. Also, kidney biopsy specimens should be carefully assessed for inflammatory and malign cell infiltrates.

\section{EP-188 GLOMERULAR INVOLVEMENT AS IGA NEPHROPATHY IN A CHILD WITH H SYNDROME} $\ddot{O}_{\text {zge Besci }}{ }^{1}$, Kashyap A. Patel ${ }^{2}$, Gizem Yildiz ${ }^{3}$, Ozlem Tufekci ${ }^{4}$, Kübra Yüksek Acinikli ${ }^{1}$, Ibrahim Mert Erbaș ${ }^{1}$, Ayhan Abaci ${ }^{1}$, Ece Bober ${ }^{1}$, Meral Torun Bayram ${ }^{3}$, Sebnem Yilmaz ${ }^{4}$, Korcan Demir ${ }^{1}$

${ }^{1}$ Dokuz Eylül UnÍversity Faculty Of MedİcIne, Department Of Pedİatríc Endocrinology, İzmir, Turkey

2 2institute Of Biomedical And Clinical Science, University Of Exeter, England

${ }^{3}$ Dokuz Eylul University Medical Faculty, Department Of Pediatric Nephrology, İzmir, Turkey

${ }^{4}$ Department Of Pediatric Hematology, Dokuz Eylul University Medical Faculty, Izmir

Introduction: $\mathrm{H}$ syndrome is a novel form of histiocytosis characterized by multisystem inflammatory disease due to mutations in SLC29A3 which encodes the human equilibrative nucleoside transporter 3 (hENT3). Clinical manifestations include hyperpigmentation, hypertrichosis, hepatosplenomegaly, hearing loss, heart anomalies, hypogonadism, low height, skeletal deformities like hallux valgus, and hyperglycemia (diabetes mellitus). Renal manifestations of $\mathrm{H}$ syndrome have included congenital anomalies of the kidney and urinary tract in $6 \%$ of patients. Glomerular involvement as part of the autoinflammatory process has been reported recently. We report a child with $\mathrm{H}$ syndrome having $\mathrm{IgA}$ nephropathy.

Material and methods: The 6-year-old boy was diagnosed with insulin dependent type 1 diabetes mellitus (IDDM). After 1.5 years, he presented with Henoch-Schonlein purpura nephritis. Renal findings improved with 6 months of prednisolone treatment, however pure red cell anemia developed necessitating regular red blood cell transfusions. Genetic analysis for SLC29A3 showed compound heterozygous pathogenic variations 
consistent with diagnosis of $\mathrm{H}$ syndrome. The patient had a second attack of HSP nephritis after 2.5 years and improved with prednisolone. After that, he had recurrent inflammation characterized by fever and elevated acute phase response that responded to tocilizumab.

Results: Symmetrical cutaneous hyperpigmentation involving inner thighs accompanied by hypertrichosis and sclerodermatous induration are the most common features observed and considered hallmark of the disease. Our patient did not have these characteristic findings. However, pure red cell aplasia has been described in H syndrome, and IDDM may be the sole presentation in a few patients. Thus, genetic mutational analysis aids in confirmation of the diagnosis when characteristic cutaneous changes are absent.

Conclusions: Glomerular pathology is rare in $\mathrm{H}$ syndrome and our patient is the first case implying that HSP nephritis may be part of the spectrum of inflammatory process of $\mathrm{H}$ syndrome.

\section{EP-189 POSTSTREPTOCOCCAL ACUTE GLOMERULO- NEPHRITIS IN CHILDREN: LONG-TERM COURSE AND FACTORS AFFECTING SEVERITY}

Gizem Yildiz, Meral Torun Bayram, Alper Soylu, Salih Kavukcu

Dokuz Eylül University Medical Faculty, Department Of Pediatric Nephrology, Izmir, Turkey

Introduction: Post-streptococcal acute glomerulonephritis (PSAGN) is the most common cause of acute glomerulonephritis in children in developing countries. We aimed to describe the clinical characteristics and outcomes of pediatric PSAGN and look for predictive factors of severity in Western Turkey.

Material and methods: This was a retrospective-prospective study of patients with PSAGN admitted to Dokuz Eylül University Childrens Hospital during 1992-2019. Diagnostic criteria for PSAGN were microscopic or macroscopic hematuria, decreased $\mathrm{C} 3$ with spontaneous recovery and evidence of recent streptococcal infection. We evaluated the clinical and laboratory data of the patients at disease onset and at the time of study. Primary outcome measures were time to resolution of macro and microhematuria, proteinuria, and low $\mathrm{C} 3$ level. In addition, chronic renal disease at the time of study was assessed by the presence of hypertension, microhematuria, proteinuria and/or decreased GFR.

Results: The total number of subjects was 50 (male/female: $35 / 15$ ) with a mean age of $7.9 \pm 3.0$ (range 2.5-15.0) years. Clinical features included edema (80\%), macroscopic hematuria (62\%) and hypertension $(64 \%)$. Laboratory tests revealed microscopic hematuria (100\%), decreased GFR $(72 \%)$, proteinuria ( $96 \%$; nephrotic range in $38 \%$ ), hypoalbuminemia $(21 / 46=45.7 \%)$, low C3 $(96 \%)$, low C4 $(9 / 40=22.5 \%)$. Patients with decreased $\mathrm{C} 4$ had lower rate of macroscopic hematuria $(33.3 \%)$, but higher rate of hypertension (100\%), impaired GFR (88.9\%), and nephrotic proteinuria $(66.7 \%)$. Resolution of proteinuria $(3.8 \pm 4.7$ vs $1.6 \pm 1.1$ months) and microscopic hematuria ( $6.9 \pm 3.6$ vs $4.3 \pm 3.6$ months $)$ took longer in these patients, and one of them had GFR $<90 \mathrm{ml} / \mathrm{min} / 1.73 \mathrm{~m}^{2}$ after 3 years of follow up. In addition, at disease onset patients with decreased GFR had lower C4 levels compared to those with normal GFR ( $18.3 \pm 9.9$ vs $29.0 \pm 13.4, \mathrm{p}=0.01)$. Mean follow-up period was $10.6 \pm 9.1$ years (range 1-31). Hypertension, decreased GFR, microhematuria and proteinuria were present in $2 \%, 4 \%, 8 \%$ and $4 \%$ at last visit, respectively.

Conclusions: Although the presentation of PSAGN may be severe, longterm prognosis in children is good. Decreased C4 level is associated with severe presentation and late recovery of hematuria and proteinuria.

\section{EP-190 A CASE OF SYSTEMIC LUPUS ERYTHEMATOSUS PRESENTING LIKE HENOCH SCHÖNLEIN PURPURA}

Ece Demirci Bodur, Ibrahim GÖkÇe, Neslihan ÇiÇek, SerÇin GÜven, Nurdan Yildiz, Harika Alpay

Marmara University Pediatric Nephrology
Introduction: Henoch-Schönlein purpura (HSP) is the most common vasculitis of childhood period affecting small vessels. Purpuric skin rash on the extensor surfaces is characteristic. Systemic lupus erythematosus (SLE) is an autoimmune, chronic inflammatory disease that can affect many systems. In this report, a 5.5 year-old girl who presented with HSP findings and was diagnosed as SLE is presented to draw attention to SLE in the differential diagnosis of HSP.

Material and methods:

Results: CASE REPORT: A 5.5-year-old girl presented with purpuric rashes on the legs and dorsal surfaces of the hands that did not fade with pressure. Petechial rashes under the left gluteal area and perianal region and edema on the back of the hands and feet were seen. She had a history of weakness, cough, diarrhea, and abdominal pain that started 2 months ago. Body weight was $21 \mathrm{~kg}(50-75 \mathrm{p})$, height $120 \mathrm{~cm}(90-97 \mathrm{p})$ and blood pressure $130 / 70 \mathrm{mmHg}$ ( $>95 \mathrm{p}$ ). Laboratory findings showed creatinine level of $0.41 \mathrm{mg} / \mathrm{dL}$, total leukocyte of $6200 / \mathrm{mm}^{3}, \mathrm{Hb}$ of $10.2 \mathrm{~g} / \mathrm{dL}$, thrombocyte of $157.000 / \mathrm{mm} 3$, reticulocyte of $1 \%$ and a positive direct coombs test. Urinalysis showed $1+$ protein in dipstick, and 71 erythrocytes in microscopic examination. Protein excretion in 24-hour urine was $22 \mathrm{mg} / \mathrm{m}^{2} /$ hour. With the present clinical and laboratory findings, renal involvement due to HSP vasculitis was considered in the initial diagnosis. Since the rash was petechial and not typical, other small and medium vessel vasculitis were investigated for the differential diagnosis. Skin biopsy could not be performed because there was no fresh skin lesion. Microscopic hematuria and hypertension persisted. ANA, antidsDNA, anti-histon-antibody and p-ANCA tests were resulted as positive, complement levels of C3 and C4 were low. Kidney biopsy showed class-3 focal lupus nephritis.

Conclusions: SLE should be considered in the differential diagnosis of patients diagnosed with HSP especially in which skin findings are not typical.

\section{EP-191 CLINICAL AND HISTOPATHOLOGICAL PROFILE OF CHILDREN WITH LUPUS NEPHRITIS AND ASSESSMENT OF RENAL FUNCTION}

Vanessa Tenório Rodrigues ${ }^{1}$, Bruna Malta Castro ${ }^{1}$, Maria Luísa De Oliveira Maximino Pessoa ${ }^{1}$, Zelina Barbosa De Mesquita ${ }^{1}$, Emilia Maria Dantas Soeiro ${ }^{1}$

${ }^{1}$ Faculdade Pernambucana De Saude, Recife, Brazil

${ }^{2}$ Instituto De Medicina Integral Professor Fernando Figueira, Recife, Brazil

Introduction: Lupus nephritis is a frequent and serious clinical manifestation in systemic lupus erythematosus in children. Here we analyzed clinical, laboratory, histopathological, and assess renal function in children with lupus nephritis.

Material and methods: We retrospectively evaluated clinical, laboratory, renal biopsy, and renal function data from 45 children with systemic lupus erythematosus diagnosed according to Systemic Lupus International Collaborating Clinics classification criteria in a referral hospital in Recife, Brazil

Results: The median age at onset of symptoms was 11 (8-13.5) years, 36 $(80 \%)$ were female, and the follow-up period was 61 (23-98) months. Thirty-five children (78\%) had lupus nephritis as an initial condition, and during follow-up, two more patients. Fifteen patients (40.5\%) had asymptomatic urinary abnormalities, $14(37.8 \%)$ nephritic syndrome, six (16.2\%) nephrotic syndrome, and two (5.4\%) acute renal failure. The serum complement was low in $59.4 \%(\mathrm{n}=22)$ and the anti-DNA reagent in $54 \%$ of the patients $(n=20)$. Eighteen patients underwent renal biopsy, with classes IV $(n=7)$, III $(n=5)$, and V $(n=2)$ being the most frequent. Sixteen biopsies showed a full house pattern for immunofluorescence. Three patients underwent a second biopsy. All patients used prednisone $(\mathrm{n}=37), 59.4 \%(\mathrm{n}=22)$ mycophenolate mofetil, $37.8 \%(\mathrm{n}=14)$ azathioprine, $51.3 \%(19)$ hydroxychloroquine, $8.1 \%(\mathrm{n}=3)$ cyclophosphamide, $5.6 \%(\mathrm{n}=2)$ rituximab and $2.8 \%(\mathrm{n}=1)$ tacrolimus. The median initial 
serum creatinine was $0.58(0.5-0.7)$. At the end of the period, one patient with Class V renal biopsy is in chronic kidney disease and, two Class IV and VI, respectively, underwent kidney transplantation.

Conclusions: Our study showed a high prevalence of lupus nephritis at the diagnosis of SLE. Despite treatment, there was an evolution to chronic kidney disease in $8 \%$ of cases during the study period.

\section{EP-192 CELIAC DISEASE ASSOCIATED WITH AN UNUSUAL PRESENTATION OF PRIMARY GOUGEROT-SJöGREN SYNDROME IN A CHILD}

Houda Nassih, Rabiy El Qadiry, Aicha Bourrahouat, Imane Ait Sab B Pediatric Ward, Department Of Pediatrics, Child And Mother Hospital, Mohammed Vi University Hospital Center, Marrakesh Medical And Pharmacy Faculty, Caddy Ayad University, Marrakesh, Morocco

Introduction: We describe an unusual presentation of Gougerot-Sjögren syndrome (GSS) associated with celiac disease (CD) in a child Material and methods: A 14-year-old girl with CD presented with nephrogenic insipidus diabetes, distal tubular acidosis and peripheral neuropathy

Results: A non-consanguineous 14-year-old girl with a medical history of several family cases of $\mathrm{CD}$ and a type 1 diabetic father; was diagnosed at tenyear-old with celiac disease. At that time, the girl had an alternation of diarrhea and constipation, evolving for two years, associated with abdominal bloating, and stunting. Her anti-transglutaminase antibodies were positive. Her jejunal biopsy showed total villous atrophy. A gluten-free diet was started, but unfortunately, the child was not compliant. A year later, she presented to the hospital for polydipsic-polyuria syndrome with ataxia evolving for three months. Physical examination noted a failure to thrive, and pubertal delay. Laboratory workup revealed an Iron deficiency anemia, hypocholesterolemia, associated with features of hypogonadotropichypogonadism. Pelvic sonography showed no ovaries and a small bladder strip corresponding probably to the uterus. The girl had polyuria. Her water restriction test showed a nephrogenic insipidus diabetes pattern. Serum electrolytes showed metabolic acidosis, with hyperchloremia, hypokalemia, and normal plasma anion-gap. Her urinary $\mathrm{PH}$ was alkaline, and her urinary anion gap was positive. Neurological examination showed a peripheral neurogenic syndrome. The electromyogram showed a motor polyneuropathy. Also, the girl reported a dry syndrome of the mouth and eyes. Biopsy of salivary glands showed a chronic sialadenitis. Keratoconjunctivitis was found on ophthalmological examination, and the Schirmer test was positive. The presence of distal tubular acidosis, dry syndrome, and polyneuropathy was very suggestive of Gougerot-Sjögren syndrome. Serological tests were done, and the rheumatoid factor came positive along with anti-nuclear antibodies. Therapeutic management consisted of a strict gluten-free diet, physical rehabilitation, artificial tears, oral steroids and amiloride-hydrochlorothiazide association. Evolution was marked by normalization of stool and gait, with remarkable improvement of polydipsic-polyuria syndrome within three months Conclusions: In the course of pediatric CD, GSS should be considered when atypical clinical manifestations occur

\section{EP-193 LUPUS NEPHRITIS IN CHILDREN - A SINGLE-CENTRE ANALYSIS \\ Marcin Tkaczyk ${ }^{l}$, Ewa Malinowska ${ }^{l}$, Monika Pawlak-bratkowska ${ }^{l}$, Anna Jander ${ }^{1}$, Chrul Stawomir ${ }^{1}$, Elżbieta Smolewska ${ }^{2}$, Krzysztof Zeman ${ }^{1}$ \\ ${ }^{1}$ Polish Mother;s Memorial Hospital Research Institute \\ ${ }^{2}$ Medical University Of Lodz}

Introduction: Lupus nephritis $(\mathrm{LN})$ in children has a more severe clinical course than in adults, is a factor that worsens the prognosis, and its course is difficult to predict. The aim of the study was to assess the course of childhood-onset lupus nephritis in pediatric nephrology centre.
Material and methods: The study included 10 patients $(9$ females and 1 male) hospitalised in the tertiary pediatric nephrology centre between 20062020 , with systemic lupus nephritis diagnosed at the average age of $14 \pm 4$ years. Nephropathy was confirmed by renal biopsy in all patients.

Results: In five patients (50\%), lupus nephritis was the first symptom of cSLE. At the time of cSLE diagnosis, one of the patients was in the 20th week of pregnancy In the renal biopsy class IV LN was diagnosed in $60 \%$ of the patients. Treatment comprised glucocorticoids (GCS) (100\%), mycophenolate mofetil (MMF) (50\%), cyclophosphamide (33.3\%) and azathioprine $(0.16 \%)$. Patients diagnosed with class III $(20 \%)$ were treated with GCS (100\%) and MMF (100\%). Two patients (one with class II lupus nephritis $-10 \%$ and one with class I lupus nephritis $-10 \%$ ) were treated only with GCS. The course of the disease in one of the patients was associated with the need for renal replacement therapy. In $90 \%$ of the patients a remission was achieved. One patient died as a result of disseminated infection with Aspergillus spp. The treatment with glucocorticoids was associated with the occurrence of adverse reactions in half of the patients. In one of the patients, the use of GCS intensified psychiatric symptoms - paranoid personality disorder was diagnosed.

Conclusions: Clinical course of LN in chidren varies between cases. In most subjects, early diagnosis and treatment of cSLE leads to the remission, although the disease and its treatment may have serious complications.

\section{EP-194 ANCA VASCULITIS IN CHILDREN}

Paula Carrascosa García, Ana Belén Martínez-lópez, Daniel Barraca Núñez, María Escobar Castellanos,

Francisco Díaz-crespo, Jacqueline Apaza Chávez, Paloma Sánchez-mateos Rubio, Jose Ignacio Camuña Correa, Olalla Álvarez Blanco

Hospital General Universitario Gregorio Marañon

Objectives: Anti-neutrophil cytoplasmic antibody (ANCA) associated vasculitis is a rare but severe condition in childhood. Clinical presentation is often heterogeneous and treatment is not well defined.

Methods: We describe three cases of ANCA vasculitis in children with renal involvement.

Results: First case is a 16-month-old girl with a history of preterm birth, large interatrial communication and resistant hypertension since the first month of age. Glomerular filtration rate (GFR), blood biochemistry and renal ultrasound were normal. Positive A-ANCAs were found, her mother's serology was negative. After five plasmapheresis sessions and four weekly doses of rituximab, she was clinically better but A-ANCA persisted. Currently, hypertension is well controlled with captopril. Next case is a 2-year-old boy with end stage renal disease (ESRD) because of rapidly progressive glomerulonephritis. He was diagnosed when he was 3 months old during a hospital admission because of a suspected urinary tract infection. He started with oliguria, hematuria, proteinuria, elevated serum creatinine and refractory hypertension with positive AANCA. Eventually, he required continuous hemodiafiltration. He had a bilateral nephrectomy and kidney biopsy showed pauci-immune extracapillary proliferative glomerulonephritis. Despite treatment with intravenous bolus of methylprednisolone, oral prednisone and rituximab he is on peritoneal dialysis and is awaiting a kidney transplant. Last case is a previously healthy 5 -year-old patient who presented with nephritic syndrome of torpid evolution and low $\mathrm{C} 3$ levels. Renal function worsened progressively and he needed extrarenal depuration techniques. P-ANCA was positive and kidney biopsy showed glomerular crescents in above $70 \%$ glomeruli. He received five doses of rituximab (last one in April 2020), ten plasmapheresis sessions and intravenous and oral corticoids tapering regimen until July 2020 with clinical improvement. Currently, he has normal GFR and is not taking medication.

Conclusions: Treatment of pediatric ANCA-vasculitis is based on adult guidelines. More studies in children are needed to establish better therapies. 
EP-195 ANGIOEDEMA ASSOCIATED WITH INTRAVENOUS CALCIUM-CHANNEL BLOCKER USE IN A CHILD WITH ATYPICAL HUS

Claire Herbez, Rea, Cyrielle Parmentier, Antoine Mouche, Jean-daniel Delbet, Tim Ulinski

Trousseau Hospital

Introduction: Intravenous nicardipine is an efficient drug for severe arterial hypertension and belongs to the standard treatments in pediatric nephrology and intensive care units. We report the case of a boy with atypical HUS who developed severe macroglossia and angioedema while treated with intravenous nicardipine.

Material and methods: A 1-year-old boy presented with generalized oedema, asthenia, pallor and poor dietary intake at the clinical examination. Blood sampling revealed mechanical hemolytic anemia (hemoglobin $4.4 \mathrm{~g} / \mathrm{dL})$, low platelets $(12,000 / \mathrm{mm} 3)$, low serum albumin with nephrotic proteinuria and acute renal failure (serum creatine $91 \mathrm{umol} / \mathrm{L}$, urea $21 \mathrm{mmol} / \mathrm{L}$ ).

The search for shigatoxin in a rectal swab and stool were negative twice, ADAMTS 13 was normal, vitamins B9/B12 normal, ANF, ANCA, anti dsDNA were all negative. C5b9 activity was increased $(638 \mathrm{ng} / \mathrm{mL}$; normal value 420) and $C 3$ was decreased, allowing the diagnosis of atypical HUS. Serum levels of Factor H, I, B and CD46 were normal. Mutation analysis of respective genes is still ongoing. Eculizumab injections were performed and resulted in a rapid clinical and biological improvement. $\mathrm{He}$ received 5 blood transfusions, hemodialysis and loop diuretics to manage fluid overload and severe arterial hypertension was managed using intravenous nicardipine between 2 and $4.5 \mathrm{mc} / \mathrm{kg} / \mathrm{min}$.

Results: Thirteen days after initiation of nicardipine therapy, the child developed facial swelling and significant macroglossia. Nineteen days after nicardipine initiation, he was transferred to the intensive care unit for respiratory distress secondary to obstructive macroglossia. At that time, the antihypertensive treatment was changed, nicardipine was interrupted and intravenous urapidil (alpha-1 antagonist) was introduced with good blood pressure control and without recurrence of angioedema. Within one day after the withdrawal of nicardipine, the boy's macroglossia/angioedema had completely resolved and never relapsed. Conclusions: Severe angioedema may be related to the treatment with intravenous calcium antagonists. As soon as this association is detected, nicardipine should be immediately withdrawn and replaced by other intravenous drugs such as alpha-1-antagonists. Awareness about this rare association may help to switch the treatment quickly and avoid potentially life threatening situations.

\section{EP-196 WHAT ARE EDEMAS DUE TO?}

Yolanda Calzada Baños, Elena Codina Sampera, Pedro Arango Sancho, Víctor López-báez, Lina Catherine Hernández Zúñiga, Ana Vinuesa Jaca, Álvaro Madrid Aris

Hospital Sant Joan De Déu

Background: Only 20\% of Idiopathic Nephrotic Syndrome is associated to microscopic hematuria, and post-infectious glomerulonephritis (GN) with biochemical nephrotic syndrome only represents $2.4 \%$ of cases. Post-infectious GN with activation of classical pathway of complement cascade is also an infrequent presentation $(<10 \%)$. The aim is to discuss differential diagnosis in patient with fluid overload, nephrotic proteinuria and hematuria.

Clinical observation: 5 -year-old patient admitted with facial edema and increased abdominal perimeter. Intermittent fever was referred for the last 2 weeks. He presented hypertension, tachypnea and respiratory distress, anasarca and bad general condition. Radiological signs of pulmonary edema were observed. Blood test showed anemia (hemoglobin $8.7 \mathrm{~g} / \mathrm{dl}$ ), hypocomplementemia C3 $(<110 \mathrm{mg} / \mathrm{l})$ and C4 $(94 \mathrm{mg} / \mathrm{l})$, hypoalbuminemia $(24 \mathrm{mg} / \mathrm{l})$, hypocalcemia $(8.3 \mathrm{mg} / \mathrm{dl})$ with normal kidney function. The urinary test showed microscopic hematuria (25-50 erythrocytes/field) and nephrotic proteinuria (protein/creatinine index $5.4 \mathrm{mg} / \mathrm{mg}$ ). He received intravenous albumin and furosemide; hypertension was corrected once the fluid overload was controlled. Autoimmunity test was normal but ANA 1/180. Kidney biopsy was performed: all glomeruli (17) had endocapillary proliferation, none with extracapillary proliferation or sclerotic lesions. Positive staining for $\mathrm{C} 3(+++), \operatorname{IgA}(+)$ and $\operatorname{IgG}(+++)$ was informed with granular mesangiocapillary pattern. He was diagnosed of post-infectious GN and symptomatic treatment was decided. During follow-up patient improved all biomarkers (anemia, biochemical nephrotic syndrome and complement) and remained asymptomatic. Due to activation of classical pathway of complement (C3 and C4 decreased), therefor we performed a genetic and functional complement test with no findings.

Conclusion: The approach to patients who consult for edema and hematuria can be difficult because of atypical presentations of frequent entities. Renal biopsy is essential to reach the diagnosis and to adopt the appropriate therapeutic measures.

EP-197 PRIMARY COQ10 DEFICIENCY: CLINICAL SPECTRUM AND GENOTYPE-PHENOTYPE CORRELATIONS Stefania Drovandi ${ }^{1}$, Beata S. Lipska-ziętkiewicz ${ }^{3}$, Fatih Ozaltin ${ }^{4}$, Francesco Emma ${ }^{5}$, Bora Gulhan ${ }^{4}$, Olivia Boyer ${ }^{6}$, Agnes Trautmann ${ }^{2}$, Qian Shen ${ }^{7}$, Jia Rao ${ }^{7}$, Korbinian M. Riedhammer ${ }^{8}$, Julia Hoefele ${ }^{9}$, Sarah L. Stenton ${ }^{10}$, Alexej Tsygin ${ }^{11}$, Kar-hui $\mathrm{Ng}^{12}{ }^{12}$, Svitlana Fomina ${ }^{13}$, Elisa Benetti ${ }^{14}$, Aurelle Manon ${ }^{15}$, Larisa Prikhodina ${ }^{16}$, Anne M. Schijvens ${ }^{17}$, Monsoureh Tabatabaeifar ${ }^{2}$, Maciej Jankowski ${ }^{18}$, Sergey Baiko ${ }^{19}$, Jianhua Mao ${ }^{20}$, Chunyue Feng ${ }^{20}$, Cuihua Liu ${ }^{21}$, Shuzhen Sun ${ }^{22}$, Fang Deng ${ }^{23}$, Xiaowen Wang ${ }^{24}$, Caroline Rousset-rouviere ${ }^{25}$,

Matgorzata Stańczyk ${ }^{26}$, Irena Bałasz-chmielewska ${ }^{27}$, Marc Fila ${ }^{28}$, Anne M. Durkan ${ }^{29}$, Tanja Kersnik Levart ${ }^{30}$, Ismail Dursun ${ }^{31}$, Nasrin Esfandiar ${ }^{32}$, Dorothea Haas ${ }^{33}$, Anna Bjerre ${ }^{34}$, Ali Anarat ${ }^{35}$, Marcus Benz ${ }^{36}$, Saeed Talebi ${ }^{37}$, Hong Xu, ${ }^{7}$, Nakysa Hooman ${ }^{37}$, Gema Ariceta $^{38}$, Franz Schaefer ${ }^{2}$

${ }^{1}$ Division Of Nephrology, Dialysis And Transplantation, Department Of Internal Medicine, Ospedale Policlinico San Martino And University Of Genoa, Genoa, Italy

${ }^{2}$ Division Of Pediatric Nephrology, Center For Pediatrics And Adolescent Medicine, University Of Heidelberg, Heidelberg, Germany

${ }^{3}$ Rare Diseases Centre And Department Of Biology And Medical Genetics, Medical University Of Gdansk, Gdansk, Poland

${ }^{4}$ Department Of Pediatric Nephrology, Hacettepe University Faculty Of Medicine, 06100, Ankara, Turkey

${ }^{5}$ Department Of Pediatric Subspecialties, Division Of Nephrology And Dialysis, bambino Gesù Childrens Hospital, Irccs, Rome, Italy

${ }^{6}$ Assistance Publique-hôpitaux De Paris, Hôpital Necker-enfants Malades, service Néphrologie Pédiatrique, Centre De Référence Marhea Et Sni, Institut Imagine, Université De Paris, Paris, France

${ }^{7}$ Childrens Hospital Of Fudan University, National Children's Medical Center, Shanghai, China

${ }^{8}$ Institute Of Human Genetics And Department Of Nephrology, Klinikum Rechts Der Isar, School Of Medicine, Technical University Of Munich,

Munich, Germany

${ }^{9}$ Institute Of Human Genetics, Klinikum Rechts Der Isar, School Of Medicine, Technical University Of Munich, Munich, Germany

${ }^{10}$ Institute Of Human Genetics, technical University Of Munich, And Institute Of Neurogenomics, Helmholtz, Zentrum MÜnchen, Munich, Germany

${ }^{11}$ Department Of Nephrology,national Medical And Research Centre For Children's Health, Moscow, Russia

${ }^{12}$ Department Of Paediatrics, Yong Loo Lin School Of Medicine, National University Of Singapore, Singapore

${ }^{13}$ Department Of Pediatric Nephrology, Institute Of Nephrology Of Nams Of Ukraine, Kiev, Ukraine

${ }^{14}$ Pediatric Nephrology, Dialysis And Transplant Unit, Department Of Women's And Children's Health, Padua University Hospital, Padua, Italy 
15 Department Of Pediatric Nephrology, The Mother And Childrens Hospital, bron, France

${ }^{16}$ Research \& Clinical Institute For Pediatrics, Pirogov Russian National Research Medical University, Moscow, Russia

${ }^{17}$ Radboud University Medical Center, Radboud Institute For Molecular Life Sciences, Amalia Children's Hospital, Department Of Pediatric Nephrology, Nijmegen, The Netherlands

18 Department Of Biology And Medical Genetics, Clinical Genetics Unit, Medical University Of Gdansk, Gdansk, Poland

${ }_{19}$ Belarusian State Medical University, Minsk, Belarus

${ }^{20}$ Department Of Nephrology, The Children Hospital Of Zhejiang, university School Of Medicine, hangzhou, China

${ }^{21}$ Henan Childrens Hospital, Zhengzhou, China

${ }^{22}$ Shandong Provincial Hospital, China

${ }^{23}$ Anhui Provincial Childrens Hospital, China

${ }^{24}$ Wuhan Childrens Hospital Of Tongji Medical College, Huazhong University Of Science And Technology, China

${ }^{25}$ Department Of Pediatric Nephrology, Timone Hospital, Marseille, France

${ }^{26}$ Department Of Pediatrics, Immunology And Nephrology, Polish Mother's Memorial Hospital Research Insitute, Rzgowska, Lodz, Poland

${ }^{27}$ Department Of Pediatrics, Nephrology And Hypertension, Medical University Of Gdansk, Gdansk, Poland

${ }^{28}$ Pediatric Nephrology Department, Arnaud De Villeneuve Hospital,montpellier University,montpellier, France

${ }^{29}$ Paediatric Nephrologist,the Children's Hospital At Westmead Locked Bag 4001, Westmead,sydney,Australia

${ }^{30}$ Department Of Nephrology, division Of Paediatrics, university Medical Centre,bohoričeva ,ljubljana,Slovenia

${ }^{31}$ Erciyes University, Faculty of Medicine,department Of Pediatrics, division Of Nephrology, Kayseri,turkey

${ }^{32}$ Pediatric Nephrology Research Center,shahid Beheshti Medical University, Tehran, Iran

${ }^{33}$ Division Of Pediatric Neurology And Metabolic Medicine, Center For Pediatric And Adolescent Medicine, University Hospital Heidelberg, Heidelberg, Germany

${ }^{34}$ Division Of Pediatric And Adolescent Medecin And Institute Of Clinical Medicine, oslo University Hospital,oslo ,Norway

${ }^{35}$ Department Of Pediatric Nephrology, Çukurova University, Adana,turkey

${ }^{36}$ Pediatric Nephrology, Dachau, Germany

${ }^{37}$ Department Of Medical Genetics And Molecular Biology, School Of Medicine, Iran University Of Medical Sciences, Tehran, Iran

${ }^{38}$ Department Of Pediatric Nephrology, University Hospital Vall Dhebron, Barcelona, Spain

Introduction: Primary CoQ10 deficiency, a mitochondrial disorder caused by recessive mutations in components of the CoQ10 biosynthesis pathway, leads to a wide spectrum of organ involvement, including steroid-resistant nephrotic syndrome (SRNS). Accounting for $10 \%$ of genetic SRNS cases, the disease is potentially treatable by CoQ10 supplementation.

Material and methods: We examined the clinical characteristics and genotype-phenotype associations of individuals with defects in $C O Q 2$, COQ6 and COQ8B, the three genes mainly associated with glomerular involvement. Through systematic literature review, interrogation of registries (PodoNet, MitoNET, CCGKDD) and an online survey among the ERKNet, PodoNet and ESCAPE consortia, ESPN and AsPN we identified 251 patients (63 COQ2, 48 COQ6, 140 COQ8b), comprising 173 published (47 updated) and 78 new cases from 71 families with 23 known and 34 newly described mutations.

Results: Median (interquartile range) age at kidney disease onset was 1 (0.5-2), 2 (0.9-4.5) and 9.9 (5.3-14.4) years in COQ2/COQ6/COQ8b patients, respectively. Isolated kidney disease was present at time of diagnosis in $21 \%, 25 \%$ and $81.4 \%$ of COQ2, COQ6 and COQ8B patients respectively. ESRD was reached at median age $2.5 \mathrm{yrs}$ in COQ2, $3.4 \mathrm{yrs}$ in COQ6 and and 13 yrs in COQ8b disease. Multiorgan disease developed in $22 \%$ of COQ2 and sensorineural deafness in $73.9 \%$ of COQ6 patients. $69.9 \%$ of COQ2, $72.6 \%$ of COQ6 and $95.6 \%$ of COQ $8 \mathrm{~b}$ patients survived to adult age. Age at kidney disease onset varied by $35 \%$ within families sharing the same mutation, by $40 \%$ within families across different mutations, by $64 \%$ between families sharing the same mutation and by $106 \%$ across different families and mutations, suggesting that the phenotype is determined both by the genotype and other genetic or environmental factors.

Conclusions: Our study expands the knowledge about the genetic and clinical spectrum of Primary CoQ10 Deficiency and yields important new insights into genotype-phenotype associations in this rare disease.

EP-198 ADPEDKD: A GLOBAL ONLINE PLATFORM TO EXPLORE THE CHILDHOOD PHENOTYPE OF AUTOSOMAL DOMINANT POLYCYSTIC KIDNEY DISEASE

Angelique Dachy ${ }^{1}$, Stephanie De Rechter ${ }^{1}$, Lisa M Guay-woodford ${ }^{2}$, Andrew J Mallett ${ }^{3}$, Tess Harris ${ }^{4}$, Detlef Bockenhauer ${ }^{5}$, Franz Schaefer ${ }^{6}$, Max C Liebau ${ }^{7}$, Djalila Mekahli ${ }^{1}$

${ }^{I} \mathrm{Ku}$ Leuven, Department Of Development And Regeneration, Pkd Research Group, Laboratory Of Pediatric Nephrology, Ku Leuven, Leuven, Belgium

${ }^{2}$ Center For Translational Science, Children's National Health System, Washington, District Of Columbia, Usa

${ }^{3}$ Faculty Of Medicine And Institute For Molecular Bioscience, The University Of Queensland, Brisbane, Australia

${ }^{4}$ Pkd International, Geneva, Switzerland

${ }^{5}$ Great Ormond Street Hospital Nhs Foundation Trust, London, United Kingdom

${ }^{6}$ Division Of Pediatric Nephrology, Centre For Pediatrics And Adolescent Medicine, Heidelberg University Medical Centre, Heidelberg, Germany

${ }^{7}$ Department Of Pediatrics And Center For Molecular Medicine, University Hospital Of Cologne, Cologne, Germany

Introduction: Autosomal Dominant Polycystic Kidney Disease (ADPKD) is the most common inherited kidney disorder. As it has been historically considered an adult-onset disease, there is a lack of longitudinal data from large pediatric cohorts. However, evidence is growing that first manifestations of ADPKD already begins in childhood. To better define the pediatric spectrum of the disease, a global multicenter observational study on childhood-diagnosed ADPKD was launched in 2017.

Material and methods: The ADPedKD registry is a worldwide webbased database, including both retrospective and prospective longitudinal data from young ADPKD patients (19 years). Australia, North-America and the United Kingdom joined the initiative with their source databases, namely the KidGen Collaborative (KidGen), NIH-funded Hepato-Renal Fibrocystic Disease (HRFD) and National Registry of Rare Kidney Diseases (RaDaR). Under informed consent, de-identified patient data, including genetics, radiological and laboratory findings, treatments and follow-up were enrolled in the database accessible via https:// www.ADPedKd.org/.

Results: 1019 ADPKD children (from 89 centers and 33 countries) are enrolled in the registry of which 167 patients from RaDaR, 17 from KidGen, 11 from HRFD and 824 from ADPedKD (401 male/ 423 female) with a mean (SD) age at diagnosis of $6.3 \pm 5.2$ years. 81 children $(9.8 \%)$ were diagnosed prenatally at a mean gestational age of 26.87 .8 weeks. Reasons for initial visit were: family screening in $325(39.4 \%)$, postnatal incidental finding in 223 (27.0\%), presenting features (such as hematuria, hypertension, urinary tract infections and flank or back pain) in $150(18.2 \%)$ or unknown/not available in 126 $(15.3 \%)$. Genetic testing was performed in $42.8 \%$ of the population, with the following results: PKD1 mutation (85.4\%), PKD2 mutation $(11.7 \%)$ and others $(6.0 \%)$. 
Conclusions: The ADPedKD registry is a unique source of clinical observational data that will provide deep phenotyping of children with ADPKD and will allow to define unified diagnostic and follow-up recommendations.

EP-199 OSTEOCLASTS OBTAINED FROM PATIENTS WITH NEPHROPATHIC CYSTINOSIS AND RESPONSE TO CYSTEAMINE : A GENOTYPE/PHENOTYPE CORRELATION Thomas Quinaux ${ }^{1}$, Aurélia Bertholet-thomas ${ }^{1}$, Julien Hogan ${ }^{2}$, Olivia Boyer ${ }^{3}$, Aris Pongas ${ }^{4}$, Sandrine Lemoine ${ }^{5}$, Aude Servais ${ }^{6}$, Ségolène Gaillard ${ }^{7}$, Diane Platel ${ }^{8}$, Cécile Acquaviva-bourdain ${ }^{9}$, Olivier Peyruchaud ${ }^{8}$, Irma Machuca-gayet ${ }^{8}$, Justine Bacchetta ${ }^{\prime}$

${ }^{1}$ Centre De Référence Des Maladies Rénales Rares, Centre De Référence Des Maladies Rares Du Calcium Et Du Phosphore, Filières De Santé Maladies Rares Oscar, Orkid Et Erk-net, Hôpital Femme Mère Enfant, Bron, France

${ }^{2}$ Service De Néphrologie Pédiatrique, Aphp, Hôpital Robert Debré, Paris, France

${ }^{3}$ Service De Néphrologie Pédiatrique, Centre De Référence Des Maladies Rénales Héréditaires De L'enfant Et De L'adulte, Hôpital Necker-enfants Malades, Ap- $h p$, Paris, France

${ }^{4}$ Service De Néphrologie Dialyse Et Transplantation Pédiatriques, Hôpital D'enfants, Chru De Nancy, France

${ }^{5}$ Service De Néphrologie, Dialyse Et Hypertension Artérielle, Hôpital Edouard Herriot, Lyon, France

${ }^{6}$ Service De Néphrologie, Hôpital Necker, Paris, France

${ }^{7}$ Epicime-cic 1407, Département D'epidémiologie Clinique, Groupement Hospitalier Est, Hospices Civils De Lyon, Bron, France

${ }^{8}$ Inserm 1033 Research Unit, Lyon, France

9 Service De Biochimie Et Biologie Moléculaire, Unité Maladies Héréditaires Du Métabolisme, Groupement Hospitalier Est, Hospices Civils De Lyon, Bron, France

Introduction: Bone complications of nephropathic cystinosis (NC) have been recently described. The main objectives of this study were to determine in vitro the impact of CTNS mutations and cysteamine therapy on $\mathrm{NC}$ human osteoclasts and to carry out a genotype-phenotype analysis related to osteoclastic differentiation and response to cysteamine therapy. Material and methods: Seventeen patients were included, at a median age of 14 (7-18) years. Most patients (71\%) were under conservative kidney management. NC human osteoclasts were differentiated from peripheral blood mononuclear cells (PBMCs) treated with increasing doses of cysteamine $(0,50,200 \mu \mathrm{M})$. TRAP-positive multinucleated cells were then counted to assess for osteoclastic differentiation. Results are presented as mean osteoclast number per well with SEM (standard error of the mean). Three functional patient groups were distinguished according to CTNS mutations : 1 / cystinosin variant with residual cystin efflux activity $(n=5), 2$ / inactive cystinosin variant $(n=4), 3$ / absent protein $(n=8)$.

Results: PBMCs from patient group 1 generated significantly less osteoclasts than those obtained from group 2 and $3: 135 \pm 8$ versus $175 \pm 12$ versus $168 \pm 7$ per well, respectively $(\mathrm{p}<0.01)$. In all groups, cysteamine exerted an inhibitory effect on osteoclastic differentiation at high doses. Conclusions: Bone metabolism in cystinosis depends on numerous factors. This study highlights a link between genotype and osteoclastic differentiation, as well as a significant impact of cysteamine therapy on osteoclast formation in humans.

EP-200 POTENTIAL OF URINE-DERIVED KIDNEY PROGENITOR CELLS FOR DISEASE MODELING IN CYSTINOSIS AND AS TARGET FOR GENE THERAPY

Veys Koenraad, Dries David, Bondue Tjessa, Sante Princiero Berlingerio, Ahmed Reda, Joris Vriens, Lambertus Van Den Heuvel, Rik Gijsbers, Fanny Oliveira Arcolino, Elena Levtchenko

Ku Leuven
Objective: Nephropathic cystinosis is a rare lysosomal storage disorder, caused by mutations in lysosomal cystinosin (CTNS). The kidney is the first and most severely affected organ. In cystinosis patients, there is an excessive loss of proximal tubular cells (PTEC's) and podocytes in urine. Therefore, we hypothesized that a maladaptive regenerative response is present in cystinosis, reflected by the presence of kidney progenitor cells in the urine.

Methods: Kidney progenitor cells were isolated and cultured from urine of cystinosis patients (Cys-uKPC's), quantified in urine, and characterized, including via RNA sequencing. Cys-uKPC clones were differentiated towards a functional PTEC or podocytes as demonstrated at the gene expression level and via functional assays. Complementation of CTNS was performed via lentiviral vector technology, while the intracellular cystine levels and distribution of the LAMP1 lysosomal compartment were studied to assess restoration of the healthy phenotype.

Results: We demonstrated the exclusive presence of kidney progenitor cells in urine of cystinosis patients compared to controls. Potent CysuKPC clones showed expression of nephron progenitor markers, while some Cys-uKPCs showed the potential to exclusively differentiate towards a functional PTEC or podocyte. Cys-uKPCs demonstrated distinct signatures at the transcriptomic level according to their cell fate. Complementation of CTNS was successful as demonstrated by a significant reduction in cystine levels and redistribution of the LAMP1 lysosomal compartment.

Conclusion: Kidney progenitor cells are present in the urine of cystinosis patients that can be differentiated in vitro into a functional PTEC or podocyte and which show distinct signatures at the transcriptomic level according to their cell fate. Complementation of CTNS ex vivo is feasible. We conclude that these Cys-uKPC's might serve as a source for disease modelling, and a base for a potential autologous cell-based ex vivo gene therapeutic approach for the kidney disease in cystinosis.

\section{EP-201 RENAL MANIFESTATIONS OF TUBEROUS SCLEROSIS IN CHILDREN - SINGLE LITHUANIAN CENTRE EXPERIENCE}

Agata Kunigel, Austeja Stankute-kolosova, Ruta Praninskiene, Agne Cerkauskaite, Viktoras Sutkus, Augustina Jankauskiene,

Rimante Cerkauskiene

Vilnius University Faculty Of Medicine

Introduction: Tuberous sclerosis complex (TSC) is a rare genetic disease characterized by development of hamartomas in multiple organs, including brain, kidney, heart, liver, and lungs. Approximately $50 \%$ of pediatric TSC patients have renal angiomyolipomas (AML) and the incidence increases with age. This study aims to determine renal manifestations of TSC in children.

Material and methods: This study represents a retrospective analysis of children diagnosed with TSC and followed in Vilnius university Hospital Santaros klinikos between 2015- 2020.

Results: Data of 38 patients aged $\leq 18$ years were analyzed. The mean age at the time of TSC diagnosis was $3.44 \pm 4.23$ years. Diagnosis was confirmed for $20(52.6 \%)$ individuals based only on clinical criteria. Eighteen $(47.4 \%)$ patients met clinical and genetic criteria: six $(33.3 \%)$ with TSC1 mutation and $12(66.7 \%)$ with TSC2 mutation. Eleven (28.9\%) patients had renal involvement. There were four cases $(10.5 \%)$ of AML, four cases $(10.5 \%)$ of multiple renal cysts, three patients $(7.9 \%)$ had both renal lesions. The mean age of patients $(n=7)$ diagnosed with multiple renal cysts was $1.14 \pm 1.12$ years whereas patients with AML $(n=7)$ were aged $4.29 \pm 4.77$ years. The mean GFR of all patients was $113.97 \pm 41.54\left(\mathrm{~mL} / \mathrm{min} / 1.73 \mathrm{~m}^{\wedge} 2\right)$.

Conclusions: Genetic cause of TSC was only identified in $47.4 \%$ of patients. TSC2 mutation was more common. One-third of the individuals had renal lesions. Renal function remains normal for most children with TSC. All children with TSC should be screened for renal function and monitored regularly. 
EP-202 A CASE OF PRIMARY HYPEROXALURIA TYPE 1 AS A RESULT OF PATERNAL UNIPARENTAL DISOMY

Thomas Dowsett, Richard Holt

Alder Hey Childrens Hospital

Introduction: Primary Hyperoxaluria type 1 (PH1) is an autosomal recessive (AR) condition that results in the overproduction of oxalate. Alanineglyoxalate aminotransferase is an enzyme that catalyses glyoxalate to glycine in the liver. A deficiency of this enzyme leads to a build-up of oxalate levels that crystallise in the kidney. PH1 can present in early childhood with chronic or end stage kidney disease, faltering growth and urolithiasis. As $\mathrm{PH} 1$ is an AR condition, it usually requires both parents to be carriers of a mutated allele for a patient to inherit the condition.

Material and methods: A 3-month-old boy presented to hospital with a 7-day history of vomiting and diarrhoea. Blood tests identified significant renal impairment and electrolyte disturbance requiring acute dialysis. Further assessment confirmed Chronic Kidney Disease stage 5 associated with high urinary oxalate levels leading to a diagnosis of PH1. The patient's father had a history of urolithiasis secondary to PH1.

Results: Polymerase Chain Reaction (PCR) of genomic DNA and Sangar sequencing of exons and intron-exon boundaries identified a homozygous mutation for a known pathogenic variant in the AGXT gene, g.9287del, c. $577 \mathrm{del}$, p.(Leu193Phefs*19). Analysis of the parents confirmed his father had a compound heterozygous mutation which included the same genetic allele as his son, alongside another known pathogenic allele c.454T >A variant. His mother's analysis did not identify any known abnormal alleles for PH1. No genetic deletions were identified and Single Nucleotide Polymorphism (SNP) array confirmed that the patient had paternal Uni-parental Disomy (UPD) of chromosome 2 that accounted for his inheritance of PH1.

Conclusions: This is the first reported case of $\mathrm{PH} 1$ occurring as a result of paternal UPD of chromosome 2. The identification of an alternative inheritance pattern such as UPD has a profound impact on genetic counselling and risk to future offspring.

\section{EP-203 ALPORT SYNDROME IN CHILDREN: A 15-YEAR SINGLE CENTRE EXPERIENCE}

Jacqueline Sit, Detlef Bockenhauer , Aoife Waters

Great Ormond Street Hospital

Introduction: Alport syndrome (AS) accounts for $0.2-0.6 \%$ adult and 2$3 \%$ paediatric patients with end stage renal disease (ESRD). Clinical trials of newer therapies e.g. bardoxolone methyl, sparsentan and antimicroRNA-21 are underway. The objective of this report is to review the characteristics of our cohort of AS patients as according to their genetic-based classifications.

Material and methods: A retrospective review was undertaken on all patients with genetic confirmation of pathogenic COL4A3, COL4A4 and COL4A5 variants between 2005 and 2020 at Great Ormond Street Hospital. Patients were classified as males with X-linked AS (XLAS), autosomal recessive AS (ARAS), autosomal dominant AS (ADAS) and females with XLAS.

Results: Twenty-nine children received genetic confirmation of AS during the period. The most common presenting symptom was isolated microscopic haematuria (38\%). One patient with male XLAS progressed to ESRD at the age of 14.

Table 1. Comparison between the different groups. Data presented as median (interquartile range).

\begin{tabular}{llllll}
\hline & $\begin{array}{c}\text { Males with } \\
\text { XLAS } \\
\mathrm{n}=9\end{array}$ & $\begin{array}{l}\text { ARAS } \\
\mathrm{n}=7\end{array}$ & $\begin{array}{l}\text { ADAS } \\
\mathrm{n}=5\end{array}$ & $\begin{array}{l}\text { Females with } \\
\text { XLAS } \\
\mathrm{n}=8\end{array}$ & p-value \\
$\begin{array}{c}\text { Positive family } \\
\text { history }\end{array}$ & $8(88.9 \%)$ & $4(57.1 \%)$ & $4(80.0 \%)$ & $8(100.0 \%)$ & 0.16 \\
$\begin{array}{c}\text { Age of presentation } \\
\text { (years) }\end{array}$ & $6.6(5.0,11.7)$ & $4.7(3.4,9.2)$ & $7.5(4.9,12.8)$ & $5.4(4.1,11.8)$ & 0.70 \\
& & & & &
\end{tabular}

\begin{tabular}{|c|c|c|c|c|c|}
\hline $\begin{array}{l}\text { Length of follow-up } \\
\text { (years) }\end{array}$ & $3.5(2.0,8.2)$ & $1.4(0.5,4.9)$ & $2.6(1.4,3.0)$ & $4.8(1.6,8.5)$ & 0.30 \\
\hline $\begin{array}{l}\text { Age at last } \\
\text { follow-up } \\
\text { (years) }\end{array}$ & $13.8(11.8,14.7)$ & $10.2(5.3,11.9)$ & $10.5(6.7,15.1)$ & $14.2(7.7,16.1)$ & 0.28 \\
\hline $\begin{array}{l}\text { Albumin-creatinine } \\
\text { ratio at latest } \\
\text { follow up } \\
(\mathrm{mg} / \mathrm{mmol})\end{array}$ & $\begin{array}{l}45.0 \\
\quad(18.4,126.6)\end{array}$ & $\begin{array}{l}135.8 \\
\quad(65.5,421 .- \\
0)\end{array}$ & $1.2(0.8,21.7)$ & $9.5(6.0,301.0)$ & 0.03 \\
\hline $\begin{array}{l}\text { Serum albumin } \\
\text { at last follow-up } \\
(\mathrm{g} / \mathrm{L})\end{array}$ & $\begin{array}{c}40.5(38.08, \\
44.0)\end{array}$ & $\begin{array}{c}38.0(31.0 \\
43.0)\end{array}$ & $\begin{array}{c}45.0(43.3, \\
46.8)\end{array}$ & $41.5(37.8,46.0)$ & 0.07 \\
\hline $\begin{array}{l}\text { eGFR at last } \\
\text { follow-up } \\
\left(\mathrm{ml} / \mathrm{min} / 1.73 \mathrm{~m}^{2}\right)\end{array}$ & $\begin{array}{l}131.0 \\
\quad(104.3,137 .- \\
2)\end{array}$ & $\begin{array}{l}104.8 \\
\quad(97.4,135 .- \\
1)\end{array}$ & $\begin{array}{l}142.6 \\
\quad(97.6,168 .- \\
4)\end{array}$ & $\begin{array}{l}106.0 \\
\quad(100.5,132 .- \\
7)\end{array}$ & 0.60 \\
\hline
\end{tabular}

Conclusions: Microalbuminuria was the earliest event observed in the progression of AS, most significant in male X-linked and autosomalrecessive AS patients. Majority of children had normal serum albumin and eGFR during the study period. Eight patients have been identified for consideration of clinical trials.

EP-204 THE FIRST CASE OF AMYLOIDOSIS DUE TO HOMOZYGOUS P.V377I MUTATION IN A PATIENT WITH HYPERIMMUNOGLOBULIN D SYNDROME

Ece Demirci Bodur ${ }^{1}$, İbrahim Gökçe ${ }^{1}$, Betül Sözeri ${ }^{2}$, Ceren Alavanda ${ }^{3}$, Orkhan Farmanli ${ }^{4}$, Pinar Ata ${ }^{3}$, Handan Kaya ${ }^{4}$, Harika Alpay ${ }^{1}$

${ }^{1}$ Marmara University Pediatric Nephrology

${ }^{2}$ Ümraniye Research And Training Hospital Pediatric Romatology

${ }^{3}$ Marmara University Medical Genetics

${ }^{4}$ Marmara University Pathology

Introduction: Hyperimmunoglobuline D syndrome (HIDS) is an autoinflammatory hereditary disorder caused by mutations in mevalonate kinase (MVK) gene. HIDS is characterized by recurrent attacks of fever lasting 4-7 days and accompanied by lymphadenopathy, abdominal pain, arthralgia, diarrhea, vomiting, skin lesions, aphthous ulcers and splenomegaly. Amyloidosis is a rare but serious long-term complication of HIDS and can cause end stage kidney disease (ESKD). In this report 10 year old patient who was admitted with steroid resistant nephrotic syndrome (SRNS) and had amyloidosis at the time of HIDS diagnosis is presented.

Material and methods:

Results: CASE REPORT: A 10-year old girl admitted to hospital with fever, cough, wheezing and edema. Laboratory evaluation revealed hypoalbuminemia, massive proteinuria and normal creatinine level. Complement levels of C3 and C4 were normal, anti-nuclear antibody, anti-dsDNA, anti-neutrophil cytoplasmic antibodies were negative. In her medical history recurrent lower respiratory tract infections were noteworthy since infancy period. Fever was not a prominent symptom. On physical examination, her weight and height percentiles were normal. She had clubbing, hepatosplenomegaly, periorbital and pretibial edema. First, a diagnosis of nephrotic syndrome (NS) was made and $2 \mathrm{mg} / \mathrm{kg} /$ day methylprednisolone treatment was started orally. No response to steroid therapy was seen. Genetic analysis for SRNS showed no mutation. Kidney biopsy was performed and showed AA amyloidosis. Steroid therapy was stopped. Genetic investigation for hereditary periodic fever syndromes were performed. Analysis of MVK gene revealed a homozygote mutation in exon 11 , c.1129G $>$ A (p.Val377Ile), diagnosing the HIDS. Canacinumab, an interleukin-1 antagonist, was started. Despite therapy, she eventually progressed to ESKD.

Conclusions: Although allele frequancy for V377I mutation in patients with MKD is much more common than other mutations, AA amyloidosis has not been reported previously secondary to the p.V377I homozygous mutation. To our knowledge, this patient is the first case of amyloidosis due to homozygous p.V377I mutation. 
EP-205 JUST A SIMPLE HEADACHE OR IT CAN BE SOMETHING ELSE?

Carmen Luna Arana, Olalla Álvarez Blanco, Francisco Díaz-crespo, Julia Suárez-gonzález, Daniel Barraca Núñez, Paula Carrascosa García, Fernando Almeida Ruiz, Ismael Buño, Ana Belén Martínez-lópez

Hospital General Universitario Gregorio Marañón

Introduction: Present the case of a 5-year-old patient with severe hypertension that required kidney transplant and led to diagnosis of a rare inherited glomerular and cystic kidney disorder.

Material and methods: Patient's medical history review.

Results: Five-year-old healthy boy who went to his primary healthcare centre because of a week of severe headache and asthenia. Physical examination showed paleness, enlarged liver and hypertension (blood pressure 194/134mmHg). Blood test showed hemolytic anemia, mild increased liver enzymes and impaired kidney function (creatinine $1.56 \mathrm{mg} / \mathrm{dL}$, urea $124 \mathrm{mg} / \mathrm{dL}$ ). He was referred to an intensive care unit with the diagnosis of hypertensive emergency. On our centre we started hypertension treatment, requiring six antihypertensive medications, and began etiological study. We requested ocular fundus, echocardiogram, immunological study (without abnormalities), abdominal ultrasound (unspecified hepatomegaly with normal kidneys) and complement study (normal C3 and C4). Renal biopsy showed tubulointerstitial fibrosis, atrophic tubules, global glomerular sclerosis and vascular changes that suggest thrombotic microangiopathy, vascular predominance.

With the diagnostic suspicion of atypical hemolytic uremic syndrome, patient started treatment with eculizumab and we requested complement system genetic test, but only risk polymorphisms in $\mathrm{CFH}$ y MCP were detected. Therefore, the genetic study was extended to the analysis of a gene panel associated with glomerular and cystic kidney diseases: two pathogenic variants in TTC21B gene in compound heterocygosis [c.626C\&gt; T, p.(Pro209Leu); c.1276C\&gt;G, p.(His426Asp)] were detected, which explained impaired kidney function and increased liver enzymes. Despite treatment he developed end-stage chronic kidney disease, requiring peritoneal dialysis until deceased donor kidney transplant in January 2021 . He also maintained refractory hypertension, requiring bilateral native nephrectomy.

Conclusions: Increasing availability of next generation sequencing techniques has revolutionized nephrogenetics, allowing to diagnose a wide variety of inherited kidney disorders. Alterations in TTC21B gene are associated with glomerular and cystic kidney diseases and it can imply liver involvement.

\section{EP-206 LATE-ONSET BARTTER SYNDROME TYPE 2 PRESENTING WITH ISOLATED NEPHROCALCINOSIS WITHOUT HYPOKALEMIC ALKALOSIS MIMICKING PRIMARY HYPERPARATHYROIDISM}

Gizem Yildiz ${ }^{1}$, Meral Torun Bayram ${ }^{1}$, Tayfun Cinleti ${ }^{2}$, Altug Koc ${ }^{3}$, Alper Soylu ${ }^{1}$, Salih Kavukcu ${ }^{1}$

${ }^{1}$ Dokuz Eylul University Medical Faculty, Department Of Pediatric Nephrology

${ }^{2}$ Dokuz Eylul University Medical Faculty, Department Of Pediatric Genetic

${ }^{3}$ Dokuz Eylul University Medical Faculty, Department Of Medical Genetics

Introduction: Bartter syndrome (BS) is an inherited tubulopathy characterized by hypokalemic metabolic alkalosis. BS type 2 is caused by ROMK disorder due to loss of function mutations in KCNJ1 gene. These patients typically present during early infancy with polyhydramnios, prematurity, polyuria, dehydration and failure to thrive. Hypercalciuria and nephrocalcinosis are also common, but marked parathyroid hormone (PTH) abnormalities have not been described. Although rare patients with BS type 2 present with late-onset isolated nephrocalcinosis, they also have typical hypokalemic metabolic alkalosis. We describe a child with BS type 2 presenting with nephrocalcinosis and normal serum electrolytes mimicking primary hyperparathyroidism.

Material and methods: A 7-year-old girl presented with bilateral nephrocalcinosis. She had a history of obstructive calcium-oxalate stones at 1.5 years of age. Past and family history was normal otherwise. She was normotensive with borderline growth retardation. Serum creatinine, electrolytes and $\mathrm{HCO}_{3}$ were normal; phosphate and $25(\mathrm{OH}) \mathrm{D}_{3}$ were decreased; calcium, $1,25(\mathrm{OH}) \mathrm{D}_{3}$ and PTH were elevated. Urine tests revealed hypercalciuria and hyperphosphaturia. Wrist x-ray, parathyroid ultrasonography and parathyroid scintigraphy were normal ruling out primary hyperparathyroidism. Tubular diseases panel showed homozygous likely pathogenic variation (c.658C> T; Chr11: 128709538, L220F, rs200320892) in exon 2 of $K C N J 1$. This variant was reported to cause functional changes in ROMK in vitro and described in adults with BS type 2 previously.

Results: Absence of hypokalemia after newborn period is atypical in patients with ROMK dysfunction. Since the positive transepithelial potential in lumen created by ROMK enables paracellular calcium reabsorption, hypercalciuria is observed in type 2 BS patients. Similarly, high PTH levels can be seen in type 2 BS due to persistent hypercalciuria. High level of 1,25(OH)D3 has been attributed to increased prostaglandin E2 mediated 1-a-hydroxylase activation.

Conclusions: The present case suggests that the KCNJ1 gene should be included in genetic analysis even in older children and adults with isolated nephrocalcinosis.

\section{EP-207 A RARE PHENOTYPE OF X-LINKED ALPORT SYNDROME. TIME TO RETHINK?}

Cristina Julia Blazquez Gomez, Lara Sanchez-trujillo, Joao Pedro Vieira Dos Santos, Juan De Dios García

Hospital Universitario Príncipe De Asturias

Introduction: Alport syndrome(AS) is caused by a mutation in one of the type IV collagen chains. It is characterized by glomerular basement membrane disorder, along with hearing loss and eye abnormalities.

Material and methods: Two-year-old girl, with no prior medical history, undergoing follow-up consultation due to gross haematuria coinciding with respiratory infections, with a presumed diagnosis of IgA Mesangial glomerulonephritis. Two years later, the episodes of haematuria present farther apart; however, proteinuria in the non-nephrotic range was detected. Family history reveals that her mother was suddenly diagnosed with end-stage chronic kidney disease(ESRD) in the second decade of life and a left ventricular haemangioma stands out as the only extrarenal presentation.

Results: Given the mother's medical history and the persistent prognosis risk factor detected in our patient, the performance of a renal biopsy and genetic study was recommended, as well as initiating treatment with enalapril. Ocular and audition tests showed no defects. The histological study of the kidney showed a a slight expansion of the mesangial matrix without an increase in mesangial cellularity, direct immunofluorescence were negative and electronic microscopy could not be performed. The patient's proteinuria progressed; therefore losartan was added. The molecular genetic study revealed the mother was a carrier of the heterozygous c. $3257 \mathrm{G}>\mathrm{A}$ mutation in the COL4A5 gene, transmitting it to her daughter.

Conclusions: This is an atypical presentation of X-linked AS, due to late diagnosis and severe renal impairment in the absence of extrarenal disorders in female patients. The etiological search for ESRD in early life can determine long-term prognosis and is decisive for preconception genetic counselling. 
EP-208 FSGS IN ALPORT SYNDROME: IS IT SO RARE?

Marina Aksenova ${ }^{1}$, Ekaterina Stolyarevich ${ }^{2}$, Patrisia Povilaitite ${ }^{3}$

${ }^{1}$ Y.veltischev Research And Clinical Institute For Pediatrics At N.pirogov Russian National Research Medical University, Moscow, Russia

${ }^{2}$ City Clinical Hospital №52, Moscow, Russia

${ }^{3}$ Rostov Region Pathoanatomical Bureau, Rostov-on-don, Russia

Introduction: FSGS is an inavitable stage of progressive glomerulopathies. The aim of the study was to determine the frequency of FSGS in children with Alport syndrome (AS).

Material and methods: The study included 60 children (age Me $11(8,5 ; 13)$ yrs; $40 \mathrm{M})$ with AS; X-linked AS had 43 pts. The inclusion criteria were: availability of light and immunofluorescence renal tissue microscopy, genetic/morphological verification of AS; the exclusion criteria were: the presence of concomitant kidney disease/podocytic genes' mutation and $<10$ glomeruli for analysis. The clinical (gender, age, deafness, blood pressure (BP)), laboratory (eGFR, proteinuria) data at the moment of biopsy, and renal tissue morphology (\% of FSGS) were analyzed. The criteria for proteinuria was urine protein excretion ( $\mathrm{Pr}$ ) $>100 \mathrm{mg} / \mathrm{m}^{2} /$ day; the high-level proteinuria and nephrotic proteinuria were defined as $\operatorname{Pr} \geq 500 \mathrm{mg} / \mathrm{m}^{2} /$ day and $>1000 \mathrm{mg} / \mathrm{m}^{2} /$ day, respectively. Blood hypertension (BH) was defined as $\mathrm{BP} \geq 95 \%$ o for sex, age, height. Results: FSGS was reveled in 35 ( $\mathrm{q}=0.58$ ) children. The pts were divided into 3 groups: gr1 (FSGS $\leq 10 \%, n=28$ ), gr2 (FSGS 11-19\%, $n=13$ ), gr3 (FSGS $\geq 20 \%, \mathrm{n}=19)$. The groups did not differ in age $(11[8.5 ; 13]$ vs $11[8 ; 13]$ vs $13[10 ; 14]$ yrs, $p>0.05)$. In groups 2 and 3 there were more boys $\left(\mathrm{q}_{1}=0.44\right.$ vs $\left.\mathrm{q}_{2}=0.76 \mathrm{vs} \mathrm{q}_{3}=0.9, \mathrm{p}_{1,3}=0.048\right)$ including the boys with $X$-linked AS $\left(\mathrm{q}_{1}=0.52\right.$ vs q $\mathrm{q}_{2}=0.66$ vs $\left.\mathrm{q}_{3}=1, \mathrm{p}_{1,3}=0.049\right)$, children with $\mathrm{BH}$ $\left(\mathrm{q}_{1}=0.14\right.$ vs $\mathrm{q}_{2}=0.38$ vs $\left.\mathrm{q}_{3}=0.73, \mathrm{p}_{1,3}=0.036\right)$, proteinuria $\left(\mathrm{q}_{1}=0.47\right.$ vs $\mathrm{q}_{2}=0.79$ vs $\left.\mathrm{q}_{3}=0.81, \mathrm{p}_{1,2}=0.043, \mathrm{p}_{1,3}=0.034\right)$ including high-level $\mathrm{Pr}$ $\left(\mathrm{q}_{1}=0.05\right.$ vs $\mathrm{q}_{2}=0.46$ vs $\left.\mathrm{q}_{3}=0.72, \mathrm{p}_{1,2}=0.02, \mathrm{p}_{1,3}=0.001\right)$, pts with decreased eGFR $\left(\mathrm{q}_{1}=0.06\right.$ vs $\mathrm{q}_{2}=0.23$ vs $\left.\mathrm{q}_{3}=0.55, \mathrm{p}_{1,3}=0.038\right)$. Pr $(\mathrm{r}=0.3207, \mathrm{p}=0.004)$, eGFR $(\mathrm{r}=-0.2650, \mathrm{p}=0.018)$ depended on FSGS degree $(\%)$. The risk of FSGS was higher in boys $\left(\chi^{2}=9.1, p=0.004\right)$, children with proteinuria $\left(\chi^{2}=9.14, p=0.002\right)$ and $\mathrm{BH}\left(\chi^{2}=14.6\right.$, $\mathrm{p}=0.03)$. The proteinuria $\left(\chi^{2}=11.31, \mathrm{p}=0.01\right)$ independently associated with FSGS.

Conclusions: FSGS is not uncommon in children with AS and expected in pts with proteinuria.

\section{EP-209 THE ISOLATED AND SYNDROMAL HEREDITARY NEPHROTIC SYNDROME IN CHILDREN \\ Nadezhda Savenkova, Elena Snezhkova, Olga Lubimova, \\ Vera Barsukova, Karina Papayan, Olga Grigoreva \\ Saint-petersburg State Pediatric Medical University, Russian Federation}

Introduction: It is proved that mutations of genes encoding the main components of the glomerular basement membrane, slit diaphragm, structural and functional proteins of podocyte are responsible for the development of hereditary NS in children. Describe the clinical phenotype and genotype of isolated and syndromic forms of NS in children.

Material and methods: We analyzed phenotype and genotype of genetic testing in 15 children with NS. Isolated and syndromal NS were identified in patients.

Results: Congenital NS isolated forms were diagnosed in 5 children by mutations of genes: NPHS1 (4) and WT1 (1) and syndromic forms in 2 children by mutation of SGPL1 gene with phenotype genital abnormalities, deafness and by mutation $L A M B 2$ gene in cause Pierson syndrome with ocular abnormalities (microcoria, cataracts) and retrocerebellar cyst. Infantile NS were diagnosed in 4 children: isolated (1) by mutation WT gene and syndromic forms (3) by AD mutation $C D 151$ gene in 2 sibs with pretibial epidermolisis bullosa, onychodistrophy, deafness, preserved kidney function and in boy by
AR mutation PLCE1gene (NPHS3) associated with disease Willibrand type 3 by mutation $V W F$ gene. Isolated childhood-onset NS was diagnosed in one boy by AR mutation PDCN gene (NPHS2) and in one girl by mutation AR COQ6 gene. Fechtner syndrome with macrothrombocytopenia, inclusions in neutrophils similar to Deles body, deafness, cataracts, steroid resistant NS, hematuria with FSGS and Epstein syndrome with macrothrombocytopenia, deafness, NS, hematuria were diagnosed in 2 patients due to the AD mutation MYH9 gene.

Conclusions: NS in patients is associated with extra-renal manifestations due to mutations SGPL1, LAMB2, CD151, PLCE1, MYH9 genes. Results of molecular-genetic test allow personalize treatment strategy in children with hereditary NS.

\section{EP-210 RENAL IMPAIRMENT AND OTHER CLINICAL CHARACTERISTICS IN OUR COHORT OF CHILDREN WITH TUBEROUS SCLEROSIS COMPLEX}

Sonja Golob Jančič, Martina Filipič, Andreja Osterc Koprivšek, Peter Gradišnik, Nataša Marčun Varda

Department Of Paediatrics, University Medical Centre Maribor, Slovenia

Introduction: Tuberous sclerosis complex presents with variety of symptoms than begin in childhood and increase with age. Renal manifestations are the leading cause of mortality in patients with tuberous sclerosis. Renal impairment includes mostly cystic renal disease and angiomyolipomas. The aim of our study was to determine the extent of renal impairment and other clinical characteristics as well as treatment of children with tuberous sclerosis complex, followed-up in our department.

Material and methods: Ten patients with tuberous sclerosis (median age of 13.5 years) were in our cohort. Their history, clinical characteristics, including renal manifestations and treatment regime were highlighted.

Results: Renal manifestations, mostly angiomyolipomas, were present in six of our patients. In 2 patients renal impairment was found to be substantial. However, at the moment decreased renal function is present in only one. From extra renal characteristics: two thirds of patients had skin pathology, both cardiac rhabdomyomas and retinal hamartomas were present in 3 and brain lesions in 7 patients. In 7 patients' epileptic seizures and in 3 cognitive disorders were diagnosed, with 4 and 2 of them necessitating antiepileptic or psychiatric therapy, respectively. In 2 patients systemic everolimus was used in one because of angiomyolipoma size and other because of astrocytoma. In one patient embolization due to renal haemorrhage in angiomyolipoma was performed. In 2 patients topical everolimus for skin lesions was also prescribed. Until now, genetic analysis was performed in 7 patients and was positive in 5 and negative in 2 of them.

Conclusions: Our results confirmed common renal involvement in patients with tuberous sclerosis complex. However, in most of the paediatric patients' renal function is stable and the treatment in rarely needed. Extra renal characteristics were present according to literature.

\section{EP-211 MYSTERIOUS DIAGNOSIS IN A CHILD WITH FAILURE TO THRIVE}

Sevasti Karamaria ${ }^{1}$, Lisa Gheuens ${ }^{2}$, Annelies Matthys ${ }^{2}$, Lien Dossche ${ }^{1}$, Evelien Snauwaert ${ }^{l}$, Johan Vande Walle ${ }^{l}$, Agnieszka Prytula ${ }^{l}$, Ann Raes ${ }^{1}$

${ }^{1}$ Department Of Pediatric Nephrology

${ }^{2}$ Department Of Pediatrics

Introduction: Mutations of several genes encoding the transporters involved in salt reabsorption in the thick ascending limb cause different types of Bartter syndrome (BS), with variable phenotypic expression 
and severity. Type I and II are the most severe presenting with polyhydramnios, prematurity and characteristically hypokalemia, metabolic alkalosis, polyuria and hypercalciuria.

Material and methods: We report the case of a 9 month old girl referred because of fever, vomiting, dehydration and electrolyte abnormalities despite fluid administration. Medical history revealed unexplained maternal polyhydramnios, prematurity (34weeks) and dysmaturity (birth weight $1,75 \mathrm{~kg}$ ). She was admitted in a neonatal unit and after a smooth course, was discharged after 36 days (weight $2,2 \mathrm{~kg}$ ). At 4 months she presented with feeding difficulties and failure to thrive with no biochemical abnormalities or polyuria were .At admission, laboratory examination revealed plasma potassium $(\mathrm{K})$ level $<3.0 \mathrm{mmol} / \mathrm{L}$, combined with inappropriately high excretion (44\%), metabolic alkalosis and hypernatremia (154 mmol/l). Despite IV fluids the biochemical abnormalities persisted but polyuria became prominent Blood pressure (BP) was $115 / 64 \mathrm{mmHg}$ with normal vital parameters. She had pronounced frontal bossing, small hands and a wide nose bridge. Neurological examination was normal. Additional findings of hyperreninemia hyperaldosteronism, hypercalciuria and nephrocalcinosis were suggestive of a tubulopathy, namely BS. However hypernatremia and high BP are no typical features of BS. The introduction of indomethacin treatment, in addition to $\mathrm{K}$ supplementation, compensation of fluid losses and hypercaloric nutrition lead to a stable condition with gradual weight gain. Mutational screening revealed a homozygous variant of SLC12A1 gene, (c.1216G >T(p.Asp406Tyr)), and a heterozygous mutation of SCNN1B gene, (c.1904G>A(p.Ser635Asn)). Further genetic testing, including of the parents, is pending.

Results:..

Conclusions: This is the first report of a girl with a phenotypic overlap between Bartter and Liddle syndrome. Although genetic analysis revealed homozygosity for a variant of unknown clinical significance of SLC12A1, a gene involved in BS type I, the clinical picture is suggestive for BS. The heterozygosity for SCNN1B, a gene involved in Liddle syndrome, leads us to hypothesize that this variant may balance the renal salt wasting caused by BS.

EP-212 VALUE AND DIAGNOSTIC YIELD OF TARGETED NEXT GENERATION SEQUENCING IN CHILDREN WITH SUSPECTED GENETIC KIDNEY DISEASE: LITHUANIAN TERTIARY CENTER EXPERIENCE

Karolis Azukaitis ${ }^{1}$, Laima Ambrozaityte ${ }^{2}$, Birute Burnyte ${ }^{2}$ Kristina Grigalioniene ${ }^{3}$, Gabija Mazur ${ }^{3}$, Dovile Ruzgiene ${ }^{1}$, Rimante Cerkauskiene ${ }^{1}$, Augustina Jankauskiene ${ }^{1}$

${ }^{1}$ Clinic Of Pediatrics, Institute Of Clinical Medicine, Faculty Of Medicine, Vilnius University, Vilnius, Lithuania

${ }^{2}$ Institute Of Biomedical Sciences, Faculty Of Medicine, Vilnius University, Vilnius, Lithuania

${ }^{3}$ Center For Medical Genetics, Vilnius University Hospital Santaros Klinikos, Vilnius, Lithuania

Introduction: Genetic conditions account for a relatively large proportion of pediatric kidney disease. Technologies such as next generation sequencing (NGS) have significantly advanced diagnostic precision and understanding of the molecular basis of these disorders. Our aim was to evaluate the diagnostic utility of NGS using targeted gene-panel in children with suspected genetic kidney disease.

Material and methods: We retrospectively reviewed all pediatric patients referred to Center of Medical Genetics at Vilnius University Hospital for genetic testing due to suspected genetic kidney disease during 2019-2020. Each patient completed an extensive clinical, laboratory and instrumental evaluation to exclude potential acquired causes. Then, NGS (Illumina or Ion Torrent technologies) was applied for all patients with variant filtering using a targeted kidney gene panel (260 - 464 genes).

Results: The analysis included a total of 38 patients. Molecular diagnosis was established in 14/38 (36.8\%) patients: $3 / 8$ referred for urolithiasis/ nephrocalcinosis, 7/17 for cystic kidney diseases/ciliopathies, 2/3 for tubulopathies and 2/8 for glomerulopathies. 4 novel pathogenic variants were identified in 3 patients: 2 in SLC5A2 gene and 2 in PKHD1 gene. Establishment of genetic diagnosis changed clinical diagnosis in 4 patients (3 patients with cystic kidney diseases and 1 with urolithiasis) and led to change in the diagnosis of parents with previously unspecified disease (2 families: $H N F 1 B$ and $P A X 2$ ).

Conclusions: Our experience shows a relatively high diagnostic yield with the application of targeted NGS in carefully selected patients with suspected genetic kidney disease. The considerably high potential of reverse phenotyping, particularly in cases of cystic kidney diseases, may have important management implications for the patient and/or family members.

\section{EP-213 A CASE OF INHERITED RENAL TUBULAR DYSGENESIS: RARE CAUSE OF OLIGOHYDRAMNIOS \\ Shital Mehta \\ Hamad Medical Corporation}

Introduction: Renal tubular dysgenesis (RTD) is a lethal, developmental anomaly of the fetal kidney characterized by a defect in differentiation of the proximal and distal convoluted tubules. Inherited RTD is genetically heterogeneous and linked to mutations in the genes encoding the renin-angiotensin system (RAS). We describe a neonate with typical clinical features of RTD whose definitive diagnosis was confirmed by genetic analysis.

Material and methods: Clinical presentation, investigations, management and outcome of the baby were followed from the health records.

Results: Our patient was a male born to first degree consanguineous parents. Oligohydramnios appeared after 26 weeks gestation, leading to anhydramnios and lung hypoplasia. The baby was delivered via emergency $\mathrm{C}$ section at 32 weeks gestation with severe lung hypoplasia, potters facies and skull ossification defects. He had severe systemic hypotension unresponsive to triple inotropes. He was anuric since birth with gradually increasing edema and deranged renal functions. Ultrasound kidneys showed normal kidney size and echogenecity with normal bladder. The baby expired on day 6 of life. Family refused autopsy but whole exome sequencing showed mutation in AGT gene shomozygous $\mathrm{G}$ to A substitution at nucleotide position 1124 (c.1124, G>A) in exon 3 \} confirming the diagnosis of inherited RTD.

Conclusions: The diagnosis of RTD should be considered in a neonate who presents with late onset oligohydramnios, normal or near normal kidneys on ultrasound examination, skull ossification defects and refractory hypotension. Diagnosis of RTD is important for its severe clinical course and the risk of $25 \%$ recurrence in case of the inherited form. Identification of the genetic defect allows for precise genetic counselling and early prenatal diagnosis.

EP-214 PHENOTYPE AND GENOTYPE COMPARATIVE ANALYSIS IN CHILDREN WITH BIALLELIC AND MONOALLELIC SLC34A1 MUTATIONS

Svetlana Papizh ${ }^{l}$, Larisa Prikhodina ${ }^{1}$, Mikhail Skoblov ${ }^{2}$

${ }^{1}$ Research And Clinical Institute Of Pediatrics, Russian National Research Medical University

${ }^{2}$ Research Centre For Medical Genetics

Introduction: Mutations in $S L C 34 A 1$, encoding the proximal tubular sodium-phosphate transporter NaPi-IIa, may cause few clinical phenotypes infantile hypercalcemia type 2 (MIM 616963) with AR inheritance and hypophosphatemic nephrolithiasis with osteoporosis, type 1 (MIM 612286) with $\mathrm{AD}$ trait. The aim of the study was to compare clinical features at the first year of life and mutational spectrum in Russian children with biallelic and monoallelic SLC $34 A 1$ mutations.

Material and methods: 10 children $(5 \mathrm{M} / 5 \mathrm{~F})$ with biallelic $(n=5)$ and monoallelic (n=5) SLC 34A1 mutations were examined. The median age at the first evaluation was $3.0(2.0 ; 6.0)$ months. Molecular genetic analysis was performed in all children using $N G S$. 
Results: All 5/5 (100\%) patients with biallelic SLC34A1 mutations had hypercalcemia, hypercalciuria and medullary nephrocalcinosis (NC) Failure to thrive and decreased serum PTH had 4/5 (80\%) children, hypophosphatemia $2 / 4(50 \%)$ individuals with biallelic SLC34A1 mutations. All 5/5 (100\%) patients with monoallelic SLC34A1 mutations had hypercalcemia and decreased serum PTH, NC had 4/5 (80\%), hypercalciuria had 2/3 (66.7\%), failure to thrive and urolithiasis had 2/5 (40\%) of cases, none of them had hypophosphatemia. Previously reported SLC34A1 nonsense pathogenic variant c.1614G $>$ A (p.Trp538Ter) identified in 3/10 (30\%) children. In patients with biallelic SLC34A1 mutations 3 novel mutations: c.1438_1440delTTC, c.1016T $>C$ and c. $1449 \mathrm{G}>\mathrm{A}$, and one intronic mutation $(20 \%)$ were detected. Children with monoallelic SLC34A1 mutations had 2 novel mutations: c. $388+$ $2 \mathrm{~T}>\mathrm{C}$ and c. $1291+2 \mathrm{~T}>\mathrm{C}$, intronic mutations had $3 / 5(60 \%)$ of cases.

Conclusions: The most prevalent features in children with mono-and biallelic SLC34A1 mutations were hypercalcemia, hypercalciuria, medullary $\mathrm{NC}$ and suppressed serum PTH. Intronic heterozygous mutation was common in patients with monoallelic SLC34Al mutations. Patients with monoallelic SLC34A1 mutations require more sensitive genetic testing (Sanger sequencing, MLPA) to identify potential the $2^{\text {nd }}$ heterozygous SLC 34A1 mutation.

\section{EP-215 SCHIMKE IMMUNO-OSSEOUS DYSPLASIA: NOT AN OPTIMISTIC SCENARIO}

Marina Aksenova ${ }^{1}$, Oksana Piruzeeva ${ }^{1}$, Olga Katysheva ${ }^{l}$,

Svetlana Papizh ${ }^{I}$, Tatiana Lepaeva ${ }^{I}$, Aleksey Tsygin ${ }^{2}$, Diana Khalikova ${ }^{3}$, Yulia Rodina ${ }^{4}$, Anna Khoreva ${ }^{4}$, Nelli Kan ${ }^{4}$, Oksana Shvets ${ }^{4}$,

Anna Shcherbina ${ }^{4}$

${ }^{I}$ Y.veltischev Research And Clinical Institute For Pediatrics At N.pirogov Russian National Research Medical University, Moscow, Russia

${ }^{2}$ National Scientific Center Of Children Health, Moscow, Russia

${ }^{3}$ Shumakov Federal Research Center Of Transplantology And Artificical Organs, Moscow, Russia

${ }^{4}$ Dmitry Rogachev National Research Center Of Pediatric Hematology, Oncology And Immunology, Moscow, Russia

Introduction: Schimke immuno-osseous dysplasia (SIOD) is a multisystem disorder characterized by a poor prognosis due to immunodeficiency and end-stage kidney disease.

Material and methods: We summarized the data of 10 children ( 9 families) with SIOD syndrome observed in several Russian federal medical centers.

Results: Renal disease was diagnosed at Me 3 [2-5] yrs; 6 pts were treated with prednisone/calcineurin inhibitors $(\mathrm{q}=0.4) ; 5$ pts progressed to end-stage kidney disease at Me 7[5;8] yrs. In all cases SIOD was diagnosed by nephrologists at Me $4[3 ; 5]$ yrs. All pts had a history of intrauterine growth retardation and extrarenal features/complications: prominent growth retardation ( $\mathrm{q}=1)$, multiple pigmented macules $(\mathrm{q}=1)$, photophobia $(\mathrm{q}=0.8)$, laterally displaced capital femoral epiphysis $(\mathrm{q}=0.4)$, cerebral ischemic events $(\mathrm{q}=0.3$; Me $6[4 ; 8] \mathrm{yrs})$, severe infections ( $\mathrm{q}=0.5$ ) with ex.let. in 4 children at Me 8[7;9] yrs. The homozygous $(\mathrm{q}=0.38)$ and heterozygous $(\mathrm{q}=0.38)$ variant of SMARCAL1 c. $2542 \mathrm{G}>\mathrm{T}$ (p.Glu848Ter) was observed in most our pts.

Conclusions: Despite the specific phenotype the SIOD diagnosed late. The patients have a poor prognosis due to early ESKD or potentially fatal infections within the first decade of life. The c.2542G $>\mathrm{T}$ (p.Glu848Ter) is the most common variant in our cohort.

\section{EP-216 ATYPICAL RETROAORTIC LEFT RENAL VEIN AS A CAUSE OF HEAMATURIA AND PROTEINURIA IN CHILDREN}

Anna Moczulska, Piotr Moczulski, Joanna Bieniek, Katarzyna Zachwieja, Monika Miklaszewska, Dorota DroŻḋ̇

Jagiellonian University Medical College, Dpt. Of Pediatric Nephrology

And Hypertension, Krakow, Poland
Introduction: The aim of the study was to assess the atypical retroaortic left renal vein as a cause of hematuria and proteinuria in children.

Material and methods: The study group consisted of 13 children (6 girls, 7 boys), aged 12,5 (7-16 ys), with haematuria or proteinura, which were diagnosed having the atypical retroaortic location of left renal vein. This group was a part $(6,2 \%)$ of bigger study in 209 children presenting with haematuria and/or proteinuria of unclear origin. Every patient underwent abdominal ultrasound with Colour and Spectral Doppler of renal vessels, both in supine and standing position. Aloka Prosound alpha 6 with convex transducer $2-6 \mathrm{MHz}$ was used. Ultrasound results were correlated with clinical and laboratory data.

Results: Patients were evaluated 1-72 months (mean 24 months) after first diagnosis of haematuria or proteinuria. Proteinuria measured in a spot urine reached 2,4 g/l, mean 0,74 g/l; in 24h-urine up to $15 \mathrm{mg} / \mathrm{kg} / \mathrm{d}$. In 3 children positive orthostatic test was observed. 2 children presented with left flank pain. In 2 children kidney biopsy was performed due to proteinuria $>1,5 \mathrm{~g} / \mathrm{l}$ with coexisting heamaturia. In one of them unspecific slight mesangial proliferation was found suggesting possible glomerulopathy. In ultrasound the diameter of left renal vein was similar in both positions, $6-11 \mathrm{~mm}$, mean $8,38 \mathrm{~mm}$ vs. $6-10 \mathrm{~mm}$ mean $7,88 \mathrm{~mm}$; with similar flow velocity $12-30$ mean $21,1 \mathrm{~cm} / \mathrm{s}$ vs. $5-28$ mean $14 \mathrm{~cm} / \mathrm{s}$ respectively. Flow spectrum analysis showed severe obstruction of left renal vein in 6 cases (46\%). Retroaortal velocity increased significantly both in supine $(50-150$ mean $97 \mathrm{~cm} / \mathrm{s})$ and standing position ( $40-240$ mean $110 \mathrm{~cm} / \mathrm{s})$ in all cases. In 2 children retroperitoneal anastomoses led to scrotal or periuteral varices

Conclusions: Atypical anatomical variant of retroaortal left renal vein may lead to vein compression independent from body position, which may be the cause of heamaturia and/or proteinuria in childhood. Doppler ultrasound evaluation of renal and retroperitoneal vessels in supine and standing position is crutial to set this diagnosis. In children with nephrotic range proteinuria and hematuria kidney biopsy should be considered to rule out coexisting glomerulopathy.

\section{EP-217 NEPHROPATHIC CYSTINOSIS PRESENTING AS BARTTER'S SYNDROME \\ Shital Mehta \\ Hamad Medical Corporation}

Introduction: Nephropathic cystinosis usually presents in infancy with failure to thrive and acidosis with features of Fanconi's syndrome. Our case at presentation had features of Bartter's syndrome along with generalised proximal tubular dysfunction.

Material and methods: Clinical presentation, investigations, and management of the patient were followed from the health records.

Results: Our case is a 3 year old male child who presented at 2 years of age with vomiting, failure to thrive and dyselectrolytemia. He had triangular facies, polyuria and had normal blood pressure. At presentation, he had normal renal functions with hyponatremia, hypokalemia, hypochloremia, hypophosphatemia, hypomagnesemia mild metabolic alkalosis, and hypercalciuria. He had high urine chloride with elevated renin and aldosterone levels suggestive of Bartter's syndrome. He also had features of generalised proximal tubular dysfunction with proteinuria, glycosuria, aminoaciduria, and phosphaturia. His initial ophthalmologic evaluation was normal. Ultrasound KUB showed normal size echogenic kidneys. He required large doses of electrolyte supplements to correct his dyselectrolytemia. At 21/2 years of age, he developed photophobia, ophthalmologic evaluation revealed cystine crystals in the cornea suggesting a diagnosis of cystinosis. At 3 years of age, he now has developed mild metabolic acidosis with slight rise in creatinine and he is being considered for cysteamine therapy.

Conclusions: Till date, 10 cases with nephropathic cystinosis have been reported that initially presented as Bartter's syndrome. Patients who present with hypokalemic metabolic alkalosis along with other features of Fanconis sydnrome should be re-evaluated and followed up regularly to diagnose cystinosis early. Early diagnosis and specific treatment of cystinosis is effective in slowing down or preventing complications. 
EP-218 MULTIPLE FACES OF A RARE DISEASE - 3 CASES OF INFANTILE NEPHROPATHIC CYSTINOSIS

Sören Bäumner ${ }^{l}$, Julian Schäuble ${ }^{l}$, Kai-dietrich Nüsken ${ }^{l}$, Felix Lechner ${ }^{2}$, Sandra Habbig ${ }^{1}$, Lutz Thorsten Weber ${ }^{1}$

${ }^{1}$ University Of Cologne, Faculty Of Medicine And University Hospital Cologne, Department Of Pediatrics, Pediatric Nephrology, Immunology And Hypertensiology, Cologne, Germany

${ }^{2}$ Hospital Of Memmingen, Department Of Pediatric And Adolescent Medicine, Memmingen, Germany

Introduction: Nephropathic Cystinosis is a rare autosomal-recessive inherited lysosomal storage disorder. Mutations in the CTNS-gene lead to defective clearance of cystine from lysosomes resulting in accumulation of cystine crystals with consecutive cell death over time. Furthermore, other key cellular functions like autophagy, lysosomal dynamics or regulation of oxidative state might be impaired by defective cystinosin transporter. Even if all patients develop renal Fanconi syndrome early in infantile nephropathic cystinosis, the apparent clinical symptoms may vary considerably.

Material and methods: We present three cases of children suffering from nephropathic cystinosis with different leading symptoms at initial manifestation. The diagnostic process revealed renal Fanconi syndrome that in the end led to the diagnosis of nephropathic cystinosis.

Results: Patient 1, 7 months, suffered from vomiting, dehydration and metabolic acidosis. First cystine level in leukocytes was $0.7 \mathrm{nmol}$ hemicystine/mg protein (norm $<0.2$ ). Genetic diagnostic identified a homozygous point mutation in the CTNS-gene (c. $225+3 \mathrm{~A}>\mathrm{T})$.

Patient 2, $19 / 12$ years, presented with motoric regression refusing to walk or to stand. X-ray showed significant rickets. First cystine level was $3.88 \mathrm{nmol}$ hemicystine/mg protein. Genetic testing revealed a $57-\mathrm{kb}$ deletion, which is the most common mutation in northern Europe.

Patient 3 failed to thrive since birth (length $78 \mathrm{~cm}$, weight $9.0 \mathrm{~kg}$ at $32 / 12$ years, $<<3^{\text {rd }}$ percentile), but did not have any other symptoms. Renal function was significantly impaired at diagnosis. First measured cystine level was 10.46 hemicystine/mg protein. Genetic testing has not been done yet.

Conclusions: Diagnosis of a rare disease like nephropathic cystinosis is difficult and may be delayed, since clinical symptoms at presentation may differ. Proximal tubulopathy was the key symptom in all patients and opened the door to correct diagnosis. Early diagnosis is very important in cystinosis, because depleting therapy with cysteamine delays disease progression effectively, but requires conscientious therapy adherence.

EP-219 ORPHA HEREDIRARY HYPOPHOSPHATEMIC RICKET WITH HYPERCALCIURIA (HHRH), NEPHROCALCINOSIS BY MUTATIONS SLC34A3 GENE: CASE STADY

Zhanna Leviashvili, Nazi Levi, Margarita Amiryan, Nadezhda Savenkova Saint-petersburg State Pediatric Medical University, Russian Federation

Introduction: HHRH (ORPHA:157215) AR is caused by mutations in the SLC34A3 gene encoding NaPiIIc, clinical manifested by hyperphosphaturia, hypercalciuria, hypophosphatemia, high $1,25(\mathrm{OH})_{2} \mathrm{D}_{3}$, low parathyroid hormone $(\mathrm{PTH})$, osteomalacia, growth retardation.

Material and methods: We present a clinical case of a boy 7years 2months.

Results: Examination of an attack of renal colic in a 5year 11 month boy revealed a stone in the bladder; epicystolithotomy was performed. At 6years 3 months - stones in the right ureter, installed a urethral catheter. Renal colic attack at 6years 4 months, ureterolithotomy, epicystolithotomy were performed. There is no family history of rickets or other metabolic bone diseases. At 6years 5 months, height $114 \mathrm{~cm}$, body weight $18 \mathrm{~kg}$, without rickets, hypophosphatemia, low PTH 3.5-6.6pg/ml and $25(\mathrm{OH}) \mathrm{D}_{3} 12-29 \mathrm{ng} / \mathrm{ml}$, high $1.25(\mathrm{OH})_{2} \mathrm{D}_{3} 0.025 \mu \mathrm{g} / \mathrm{ml}$. Ca, alkaline phosphatase, $\mathrm{K}, \mathrm{Na}, \mathrm{Cl}, \mathrm{Mg}$ are normal. GFR $111 \mathrm{ml} / \mathrm{min} * 1.73 \mathrm{~m}^{2}$. Maximum phosphate reabsorption (TmP) 1.08 and TmP/GFR 0.99 $\mathrm{mmol} / \mathrm{l}$ are low. Increased urinary $\mathrm{Ca}$ excretion $7.3-10.1 \mathrm{mg} / \mathrm{kg} /$ day, $\mathrm{P}$ $15.3-24.6 \mathrm{mg} / \mathrm{kg} /$ day. U Ca/Cr 1.5-2.1 mmol $/ \mathrm{mmol}, \mathrm{P} / \mathrm{Cr} 2.41-5.0$ $\mathrm{mmol} / \mathrm{mmol}$. On ultrasound - the kidneys are enlarged in volume on the right V $50.63 \mathrm{~cm}^{3}$; left V $53.53 \mathrm{~cm}^{3}$, bilateral medullary nephrocalcinosis. The stone to be removed consists of calcium oxalate crystals and an association of carbonatapatitphosphate. Moleculargenetic sequencing revealed a heterozygous mutation c.1382G $>$ A (p.Trp461Ter) in the 13th exon and c.1094-3C> T in the 10th intron of the SLC34A gene, which made it possible to confirm the diagnosis.

Conclusions: We described patient with phenotype and genotype of HHRH, nephrocalcinosis, nephrourolithiasis by mutations in the $S L C 34 A 3$ gene. The administration of therapy with the exclusion of active metabolites of vitamin D prevents the progression of the disease.

\section{EP-220 PHENOTYPIC HETEROGENEITY OF PRIMARY HYPEROXALURIA TYPE 1: THE FIRST SUCCESSFUL EXPERIENCE COMBINED LIVER AND KIDNEY TRANS- PLANTATION IN CHILD \\ Sergey Baiko ${ }^{1}$, Sergey Korotkov ${ }^{2}$, Ivan Shturich ${ }^{2}$, Aleksey Shcherba ${ }^{2}$, Oleg Kalachik ${ }^{2}$, Oleg Rummo ${ }^{2}$ \\ ${ }^{1}$ Belarusian State Medical University \\ ${ }^{2}$ Minsk Scientific-practical Center Of Surgery, Transplantation And Hematology}

Introduction: Primary hyperoxaluria type $1(\mathrm{PH} 1)$ is a rare autosomal recessive disorder which leads to increased production of oxalates with development of nephrocalcinosis / urolithiasis and end stage renal disease (ESRD). Combined liver-kidney transplantation (CLKT) allows saving lives of children with PH1 and ESRD.

Material and methods: We present two clinical cases of children from the same family with identical homozygous mutation in AGXT gene, but different phenotypic manifestations: in an older child - severe nephrocalcinosis with onset of hemodialysis at 9,5 y.o., in a younger child - recurrent urolithiasis.

Results: The first child at 11,5 y.o. underwent CLKT from a deceased donor. There was a delayed renal graft function, which required hemodialysis. Despite several surgical complications (intraabdominal bleeding, stenosis in hepatic artery anastomosis), which were successfully solved, the function of kidney graft recovered on the $14^{\text {th }}$ day, and on the $30^{\text {th }}$ day the child was discharged from the hospital. For the last 3,5 postoperative years the child has a satisfactory function of the grafts. In the second child, since the diagnosis of PH1 at the age of 7,7 y.o. and the beginning of conservative therapy, there is a regular discharge of calculi with episodes of renal colic and obstruction of the urinary tract. Despite this, stable kidney function has been maintained over the past 5 years.

Conclusions: These clinical cases showed different phenotypes with the same mutation of $A G X T$ gene. The clinical case confirms the possibility of a successful simultaneous liver and kidney transplantation in a child with ESRD.

\section{EP-221 A RARE CASE OF METABOLIC ACIDOSIS WITH NEPHROCALCINOSIS}

Gabriele Malgieri ${ }^{1}$, Giovanni Russo ${ }^{2}$, Giulia Ranucci ${ }^{2}$, Claudia Elefante ${ }^{3}$, Luigi Annicchiarico Petruzzelli ${ }^{1}$, Vittorio Serio ${ }^{1}$, Angela De Luca ${ }^{1}$, Carmine Pecoraro ${ }^{1}$

${ }^{1}$ Pediatric Nephrology And Dialysis Unit, Santobono Childrens Hospital, Naples, Italy

${ }^{2}$ Department Of Translational Medical Science, Section Of Paediatrics, University Of Naples Federico Ii, Naples

${ }^{3}$ Nephrology Unit, Advanced Surgical And Medical Sciences Department Of University Of Campania "luigi Vanvitelli", Naples, Italy 
Introduction: Neonatal nephrocalcinosis isn't a disease itself, but the sign of underlying pathological condition. When nephrocalcinosis is associated with metabolic acidosis and electrolyte imbalance, distal renal tubular acidosis (dRTA) must be investigated. dRTA occurs with hypokalemia, hypercalciuria, nephrocalcinosis, with alkaline urinary $\mathrm{pH}$ and sometimes deafness. We describe a case of dRTA with neonatal onset.

Material and methods: genetic investigation, blood gas analysis, urine tests, kidney ultrasound

Results: G.F is a one- month- old infant, hospitalized for severe hyperchloremic metabolic acidosis with normal anion gap and electrolyte imbalance. He appeared consciousness, dehydrated, inappetent and showed poor weight gain. After the lack of response to hydration, he was referred to intensive care unit. On admission he had blood $\mathrm{pH}=7.1$ and $\mathrm{HCO} 3=3.34 \mathrm{mmol} / \mathrm{L}$, $\mathrm{K} 2.2 \mathrm{mEq} / \mathrm{L}$, normal anion gap, urinary $\mathrm{pH}$ 8. Renal ultrasound showed bilateral nephrocalcinosis. Intravenous therapy with Sodium bicarbonate and Chloride Potassium was started, and clinical condition promptly improved. Therapy was switched to oral route within 72 hours. Later the genetic analysis revealed a genomic variant c2089C $>\mathrm{T}$ in heterozygosity of the ATP6V0A4 gene responsible of dRTA. At subsequent post-discharge checks, the patient has regular weight gain, blood $\mathrm{pH}$ and electrolytes within the norm.

Conclusions: metabolic acidosis associated with nephrocalcinosis can be a sign of dRTA. It's important to diagnose it in order to set the most appropriate therapy. The early diagnosis and treatment of dRTA can prevent renal and growth failure.

\section{EP-222 REPORT OF SIX SCHIMKE IMMUNO-OSSEOUS DYSPLASIA PATIENTS WITH A NOVEL MUTATION}

Mehtap Akbalik Kara, Beltinge Demircioğlu Kiliç, Mithat Büyükçelik, Ayşe Balat

Gaziantep University, Faculty Of Medicine, Department Of Pediatric Nephrology, Gaziantep, Turkey

Introduction: To report the clinical, laboratory and genetic evaluations and follow-up of six children with Schimke Immuno-osseous Dysplasia (SIOD).

Material and methods: The files of patients suspected of SIOD due to clinical and laboratory findings and genetically confirmed were retrospectively analyzed.

Results: In this study, all of the patients except one had SIOD mutations which were already defined with Sanger sequencing method. This patient had a novel homozygous mutation in the SMARCAL1 gene (c.1177C $>$ T). Since her father lived in Syria, genetic analysis could not be obtained from the parents. All patients had nephrotic syndrome. The patients did not respond to steroid therapy or other immunsupressive agents. But in one patient we had been continued to give CyclosporinA (Cy-A) because she had been suffering from severe hypoalbuminemia and edema when $\mathrm{Cy}-\mathrm{A}$ was withdrawn. Three patients had cerebral ischemia. Three patients died at follow-up while the survived ones were in end-stage renal disease. Two of our cases had an auto-immune disease; one had coombs positive hemolytic anemia and the other had bone marrow failure. Although the patient with coombs positive hemolytic anemia responded to steroid therapy, she died from bacteriemia and acute respiratory distress syndrome in follow-up. Similarly the other patient with bone marrow failure also died because of septicemia.

Conclusions: SIOD is a rare, multisystemic disorder and it may be diagnosed late or underdiagnosed. As in our cases, SIOD should be considered in all children of growth failure with unclassifiable bone dysplasia. A urine test for proteinuria should be performed in all cases with growth failure to help the correct diagnosis. All patients diagnosed with SIOD should be evaluated for cerebrovascular complications, prompt management and renal transplantation should be considered in the earlier period.
EP-223 FAMILIAL KIDNEY DISEASE: GENETICS TO THE RESCUE

Yolanda Calzada Baños, Elena Codina Sampera, Pedro Arango Sancho, Víctor Lóepz-báez, Ana Vinuesa Jaca, Lina Catherine Hernández Zúñiga, Álvaro Madrid Aris

Hospital Sant Joan De Déu

Background: The aim of the study is to present a familial nephropathy and how genetic studies and the recording of the findings make it possible to reach diagnosis

Clinical observations. Case_1. 12-year-old girl with no medical history, his father underwent to kidney transplantation at age of 19 years (unknown kidney disease). Biochemical nephrotic syndrome is detected in urine and blood tests during the evaluation of chronic epigastric pain: hypoalbuminemia $(26 \mathrm{~g} / \mathrm{l})$, dislipemia, hypocalcemia $(8.3 \mathrm{mg} / \mathrm{dl})$, nephrotic proteinuria (protein/creatinine index $10 \mathrm{mg} / \mathrm{mg}$ ). There was no kidney impairment either hematuria. Autoimmunity was negative (complement $\mathrm{C} 3$ and $\mathrm{C} 4$, ANA, ANCA, anti-MBG, anti-PLA2R). The kidney biopsy showed diffuse glomerulosclerosis with negative immunofluorescence staining. The proband received prednisone and cyclosporine without response with progression to end stage chronic kidney disease. Genetic test initially informed a variant of unknown significance in INF2 gene (p.Glu220Lys / c.658G > A), related to focal and segmental glomerulosclerosis. She underwent to kidney transplantation after 6 months of the onset. During segregation study, nephrotic proteinuria was detected in her sister. Case 2. Proband's sister, 15-year-old girl with urinary test showing nephrotic proteinuria (protein/creatinine index $4.6 \mathrm{mg} / \mathrm{mg}$ ). In the blood test only hypoalbuminemia stands out $(26 \mathrm{mg} / \mathrm{l})$. She was asymptomatic at diagnosis. The genetic test showed the same heterozygous mutation of INF2 gene also present in proband's father. Being present in all tree family members, it could be considered as pathogenic. No immunosuppressive treatment was indicated and she underwent to kidney transplantation 15 months after diagnosis.

Conclusions. Genetic test and family screening with multicenter registries are needed for diagnosis familial nephropathies.

\section{EP-224 THE CHALLENGE DIAGNOSTIC AND THERAPEUTIC OF PRIMARY HYPEROXALURIA TYPE 1 (PH1): A CASE REPORT}

Gabriele Malgieri ${ }^{1}$, Caprio Francesca ${ }^{2}$, Colantuono Rossella ${ }^{3}$, Minale Bruno ${ }^{1}$, Enrico Zulli ${ }^{4}$, Rosamunda Darcangelo ${ }^{1}$, Carmine Pecoraro ${ }^{1}$

${ }^{1}$ Pediatric Nephrology And Dialysis Unit, Santobono Childrens Hospital, Naples, Italy

${ }^{2}$ Nephrology Unit, Advanced Surgical And Medical Sciences Department Of University Of Campania "luigi Vanvitelli", Naples, Italy

${ }^{3}$ Pediatrics Section, Department Of Medicine, Surgery And Dentistry "scuola Medica Salernitana", University Of Salerno, Baronissi, Italy

${ }^{4}$ Department Of Public Health, University Of Naples Federico Ii, Naples, Italy

Abstract: A 13 years old adolescent with history of kidney stones and urinary infections from the age of 3 months was admitted for fever, asthenia and weight loss. Unrelated parents, family history of kidney stones. No drug therapy on admission. Laboratory tests identified Acute Kidney Injury (GFR sec. Schwartz $<10 \mathrm{ml} / \mathrm{min} / 1.73 \mathrm{~m}^{2} \mathrm{sc}$ ). A renal ultrasound and urinary tract CT scan without contrast were promptly performed detecting a large stone in the medium tract of right ureter and a staghorn stone on the left kidney. Lithotrissia and stent placement were performed with gradual improvement of kidney function (GFR sec. Schwartz $22 \mathrm{ml} / \mathrm{min} / 1.73 \mathrm{~m}^{2} \mathrm{sc}$ ). Diagnostic evaluation for nephrolithiasis showed normal blood $\mathrm{pH}$ and elevated Parathormone. Considering uOx 165 (v.n. 70-82 $\mu \mathrm{mol} / \mathrm{mmol} \mathrm{Cr}$ ), genetic research was started for primary hyperoxalurias (PHs) with a finding of PH1 (mutation c.603C $>$ A of gene AGXT inhomozygous). Extrarenal involvement was excluded. 
Nephrolithiasis treatment was started without any further improvement of renal function. Therefore Lumasiran is going to be started.

DISCUSSION AND CONCLUSIONS

$\mathrm{PH} 1$, the most common and severe form of PHs, is due to a deficiency of glyoxylate aminotransferase in the liver. Diagnosis is often delayed due to atypical imaging: our patient presented obstructive urolithiasis. Current treatments for PH1 focus on preserving renal function by decreasing oxalate production through increased fluid intake, oxalate crystal inhibitors and pyridoxine. Inadequate response can be followed by dialysis or combined liver/kidney transplant. Recently, Lumasiran received its first global approval in the EU for the treatment of PH1 in all age groups. It's a small interfering RNA that inhibits the synthesis of oxalate, silencing the gene glycolate oxidase. It induces a rapid and prolonged reduction in24hour uOx levels until their normalization at 6 months.

\section{EP-225 AGTR1-RELATED RENAL TUBULAR DYSGENESES MAY NOT BE FATAL}

Ebru Burcu Demirgan ${ }^{1}$, Seha Saygili ${ }^{1}$, Nur Canpolat ${ }^{1}$, Lale Sever ${ }^{l}$, Isin Kilicaslan ${ }^{2}$, Doruk Taylan ${ }^{3}$, Salim Caliskan ${ }^{1}$, Fatih Ozaltin ${ }^{4}$

${ }^{I}$ Department Of Pediatric Nephrology, Istanbul University-cerrahpasa, Cerrahpasa Faculty Of Medicine, Istanbul, Turkey

${ }^{2}$ Department Of Pathology, Istanbul Faculty Of Medicine, Istanbul University, Istanbul, Turkey

${ }^{3}$ Nephrogenetics Laboratory, Department Of Pediatric Nephrology, Hacettepe University Faculty Of Medicine, Ankara, Turkey

${ }^{4}$ Department Of Pediatric Nephrology, Hacettepe University Faculty Of Medicine, Ankara, Turkey

Introduction: Renal tubular dysgenesis (RTD) is an ultra-rare disease that is associated with pathogenic variations in the genes of the reninangiotensin system (RAS), including AGT, REN, ACE and AGTR1 genes. The condition is associated with a very high mortality during the pregnancy and in the early postnatal period. There are only a few individuals surviving beyond the perinatal period. Here, we present two siblings with a homozygous AGTR1 pathogenic variation related RTD, who have survived for longer period than the previously reported individuals with AGTR1 mutation.

Material and methods: We had two siblings of a consanguineous parents with a similar clinical picture recognized in the early postnatal period that is characterized by oligohydramnios, multiple joint contractures, central hypotonia, wide cranial sutures, severe arterial hypotension, impaired kidney functions, hyperkalemia, and metabolic acidosis. We performed whole exome sequencing to both siblings and the parents to uncover the underlying genetic abnormality.

Results: We identified homozygous pathogenic variation in AGTR1 [c.376C > T (p.Arg126*)], thereby diagnosed as RTD in the course. In addition, both patients also had a homozygous variation in platelet derivated growth factor $\mathrm{D}(P D G F D)$ [c.737A $>\mathrm{G}$ (p.His246Arg)] variation, which has previously been reported to be associated with kidney fibrosis in humans and mice.

Conclusions: Although rare, RTD should be considered in a pregnant woman who has severe oligohydramnios without any apparent fetal urinary tract defect. Such patients should be evaluated for the mutations in the genes encoding the RAS. Additional genetic/epigenetic factors might also affect the prognosis. In our cases, we thought that variation in $P D G F D$ may modulate the clinical course and explain our patients' prolonged survival.

EP-226 GROWTH RETARDATION AND NEPHROTIC SYNDROME REVEALING SCHIMKE SYNDROME: A CASE REPORT STUDY

Rihab Sghir, Selsabil Nouir, Sameh Mabrouk, Houda Ajmi, Fadwa Majdoub, Meniar Tfifha, Chamli Jaleleddine, Noura Zouari, Saoussen Abroug
Pediatric Department, Sahloul Hospital, Tunisia

Introduction: Schimke immune-bone dysplasia is a rare multisystemic disease, with a large spectrum of manifestations, its aetiology and physiopathology remain unknown. Different forms can be found from where diagnosis difficulties.

Material and methods: We report a case of a girl of Algerian origin presented with a severe spectrum form of schimke syndrome complicated with prenatal growth retardation and End Stage renal failure (ESRF)

Results: It's about a 5 years old girl, was referred to our pediatric department for a cortico-resistant nephrotic syndrome. She's a second child of a healthy consanguineous couple, her personal history was marqued by unexplained intrauterine growth retardation with a short stature followed since the age of 2 years.

On first examination, she had short stature and a distinctive physical feature : fine hair, thin upper lip, low nasal bridge and excessive inward curvature of the lumbar spine

Initial biological check-up revealed nephrotic syndrome (high proteinuria $=175 \mathrm{mg} / \mathrm{kg} / 24 \mathrm{H}$ ), renal failure with a rapid worsening of the renal function, Cell Blood count showed severe leucopenia. Peripheral blood lymphocytes showed a significant decreased number of total CD3 T cells.

Skeletal X rays were normal, Renal biopsy showed segmental and focal hyalinosis of the "collapsing" type. Genetic study confirmed Schimke syndrome with homozygous mutation in exon 12 of the SMARCAL1 gene.

In view of the rapid evolution towards end-stage renal failure, Renal replacement therapy was required 3 months later, renal transplantation was indicated but our patient was lost of follow up.

Conclusions: Although it is likely a primary immune disease, schimke disease may affect kidneys with rapid progression to ESRF, it is more difficult to accept that it may interfere with bone growth starting before birth as happens to our patient.

\section{EP-227 ATYPICAL SEVERE EARLY-ONSET NEPHROTIC} SYNDROME

Berthaud Romain ${ }^{1}$, Heidet Laurence ${ }^{1}$, Oualha Mehdi ${ }^{2}$, Brat Roselyne ${ }^{4}$, Talmud Déborah ${ }^{5}$, Garaix Florentine ${ }^{6}$, Rabant Marion ${ }^{3}$, Frémeaux-bacchi Véronique ${ }^{7}$, Antignac Corinne ${ }^{8}$, Boyer Olivia ${ }^{1}$, Dorval Guillaume ${ }^{8}$

${ }^{1}$ Department Of Pediatric Nephrology, Hôpital Necker-enfants Malades, Assistance Publique-hôpitaux De Paris, Paris, France

${ }^{2}$ Pediatric Intensive Care Unit, Hôpital Necker-enfants Malades, Assistance Publique-hôpitaux De Paris, Paris, France

${ }^{3}$ Department Of Pathology, Hôpital Necker-enfants Malades, Assistance Publique-hôpitaux De Paris, Paris, France

${ }^{4}$ Department Of Neonatology, Hôpital De La Source, Orléans, France

${ }^{5}$ Department Of Pediatric Nephrology, Hôpital De La Source, Orléans, France

${ }^{6}$ Department Of Pediatric Nephrology, Hôpital De La Timone Enfants, Assistance Publique-hôpitaux De Marseille, Marseille, France

${ }^{7}$ Department Of Biological Immunology, Hôpital Européen Georges Pompidou, Assistance Publique-hôpitaux De Paris, Paris, France

${ }^{8}$ Department Of Genetics, Hôpital Necker-enfants Malades, Assistance Publique-hôpitaux De Paris, Paris, France

Introduction: Congenital nephrotic syndrome (CNS) is mostly caused by podocyte gene defects. Among them, Wilms tumor 1 (WT1) variants, may be responsible for Denys-Drash syndrome or isolated CNS with diffuse mesangial sclerosis. Atypical hemolytic uremic syndrome (HUS) may be due to a genetic mutation in the alternative complement pathway or in $D G K E$. The association of HUS and $W T 1$ variants has been described in 4 children aged 8 to 26 months, but the pathophysiology remains unclear. 
Material and methods: We report here a fifth case of CNS with biopsyproven thrombotic microangiopathy (TMA) and a mutation in the WT1 gene.

Results: A 14 day-old girl from unrelated healthy parents with past medical history of intrauterine growth restriction and anamnios was admitted for grunting and anorexia.

She had no dysmorphic features. Lab investigations showed kidney injury (creatinine $108 \mu \mathrm{mol} / \mathrm{L}$ ) with nephrotic syndrome (hypoalbuminemia $11 \mathrm{~g} / \mathrm{L}$, proteinuria $>30 \mathrm{~g} / \mathrm{L}$ ) and uncountable erythrocytes in the urine. Kidney ultrasonography showed no thrombosis. Her kidney function only slightly improved despite optimized hemodynamic conditions and she remained oliguric on high dose diuretics. She quickly developed thrombocytopenia and mechanical hemolytic anemia (schistocytes 1-2\%) needing recurrent erythrocyte transfusions. She had no severe hypertension. The kidney biopsy revealed signs of TMA without diffuse mesangial sclerosis. The search for shigatoxin and anti-factor $\mathrm{H}$ autoantibodies was negative. ADAMTS13 activity, homocysteinemia and analysis of a comprehensive panel of genes involved in atypical HUS were normal. Eculizumab and plasma infusions had no effect. Screening for genetic causes of CNS revealed a de novo monoallelic pathogenic variant in the WT1 gene. The evolution was pejorative with sepsis and worsening of anasarca leading to death.

Conclusions: As for DGKE gene, WT1 mutations may be a cause to consider in front of the association of CNS and HUS. Although HUS may be a confounding factor in the diagnosis of WT1-associated CNS, it should not challenge the diagnosis.

EP-228 RISK OF CELLULAR AND/OR ANTIBODY-MEDIATED TRANSPLANT REJECTION IN PAEDIATRIC KIDNEY TRANSPLANT RECIPIENTS WITH BK POLYOMAVIRUS REPLICATION - A MULTICENTRE CERTAIN ANALYSIS Britta Höcker ${ }^{1}$, Jeremy Schmidt ${ }^{1}$, Caner Süsal ${ }^{2}$, Luisa Murer ${ }^{2}$, Matthias Zirngibl ${ }^{2}$, Sabine König ${ }^{2}$, Luca Dello Strologo ${ }^{2}$, Lutz T. Weber ${ }^{1}$, Raphael Schild ${ }^{1}$, Atif Awan ${ }^{1}$, Laura Gauché ${ }^{1}$, Seha Saygili ${ }^{2}$, Kai Krupka ${ }^{1}$, Tom Bruckner ${ }^{2}$, Paul Schnitzler ${ }^{2}$, Hans H Hirsch ${ }^{2}$, Alexander Fichtner ${ }^{1}$, Burkhard Tönshoff ${ }^{1}$

${ }^{1}$ University Childrens Hospital Of Heidelberg, Germany

${ }^{2}$ Certain Research Community

Introduction: To determine the risk of alloimmune responses ( $\mathrm{T}$ cellmediated rejection (TCMR) including borderline changes and de novo HLA donor-specific antibodies (dnDSA) and/or antibody-mediated rejection $(\mathrm{ABMR})$ ) as a consequence of reduced immunosuppression for management of $\mathrm{BK}$ polyomavirus $(\mathrm{BKPyV})$ replication in paediatric kidney transplant recipients

Material and methods: In the framework of the Cooperative European Paediatric Renal Transplant Initiative (CERTAIN), we studied 195 paediatric kidney transplant recipients $(10.5 \pm 5.5$ years $)$ in whom plasma $\mathrm{BKPyV}$ viral load and dnDSA were measured regularly over a period of up to 5 years post-transplant. Risk factors for the development of dnDSA and transplant rejection were analysed using univariate and multivariable Cox regression.

Results: BKPyV replication was observed in 65 (33.3\%), and biopsyproven BKPyV associated nephropathy in $13(6.7 \%)$ patients. Ninety (46.2\%) patients developed TCMR/borderline rejection, and $56(28.7 \%)$ recipients developed dnDSA/ABMR during the 5-year period. The overall TCMR/borderline rate was comparable in patients with $(20(37.0 \%))$ or without BKPyV replication $(70(49.3 \%), p=0.150)$ but recipients with $\mathrm{BKPyV}$ replication developed TCMR/borderline rejection significantly $(\mathrm{p}=0.040)$ later than those without, presumably due to reduced immunosuppression for BKPyV management. Independent risk factors for TCMR/borderline rejection were cold ischemia time $>24 \mathrm{hrs}$ (OR 3.0, $\mathrm{p}=0.024)$, delayed graft function $(\mathrm{OR} 3.1, \mathrm{p}=0.011)$ and desensitisation at transplant (OR 2.5, $\mathrm{p}=0.031$ ). The overall $\mathrm{dnDSA} / \mathrm{ABMR}$ rate was also similar in patients with $(16(28.1 \%))$ or without BKPyV replication (40
(29.0\%), $\mathrm{p}=0.898)$. Independent risk factors for dnDSA/ABMR development were re-transplantation (OR 5.8, $\mathrm{p}=0.000)$, HLA-DR mismatch (OR $1.8, \mathrm{p}=0.005$ ) and preformed DSA (OR 3.5, $\mathrm{p}=0.002$ ). In patients with $\mathrm{BKPyV}$ replication, independent risk factors for $\mathrm{dnDSA} / \mathrm{ABMR}$ were retransplantation (OR 8.1, $\mathrm{p}=0.011)$ and an immunosuppressive regimen consisting of CSA/EVR vs. TAC/MMF (OR 3.9, $\mathrm{p}=0.034$ ).

Conclusions: Reduced immunosuppression as BKPyV management is not significantly associated with an increased risk of TCMR/borderline rejection and/or dnDSA/ABMR in paediatric kidney transplant recipients.

EP-229 THE EFFECTS OF PARENTAL ATTITUDE AND QUALITY OF LIFE ON MEDICATION ADHERENCE IN PEDIATRIC RENAL TRANSPLANT RECIPIENTS

Ayla Kaçar ${ }^{1}$, Özge Gizli Çoban ${ }^{2}$, Mehtap Adar ${ }^{1}$, Muhammet Dutucu ${ }^{1}$, Gülşah Kaya Aksoy ${ }^{1}$, Elif Comak ${ }^{1}$, Mustafa Koyun ${ }^{1}$, Sema Akman ${ }^{1}$

${ }^{1}$ Akdeniz University Faculty Of Medicine, Department Of Pediatric Nephrology, Antalya, Turkey

${ }^{2}$ Akdeniz University Faculty Of Medicine, Department Of Child And Adolescent Psychiatry, Antalya, Turkey

Introduction: The adherence to immunosuppressive therapy in pediatric kidney transplant recipients is associated with multiple variables relevant to the patient and his/her parents. In this study, we aimed to evaluate the effect of the quality of life of the patients and parenting attitude on medication adherence.

Material and methods: Renal transplant recipients aged 7-20 years and having at least post-transplant 3 months of follow-up were included in the study. The study was carried out between March and July 2019. The "Immunosuppressive Therapy Compliance Scale" was used to assess immunosuppressive therapy compliance and score $<12$ was defined as non-adherence. "Pediatric Quality of Life Inventory (PedsQL)" was administered to all patients. Evaluation of parental attitude was based on "Parental Attitude Research Instrument" and 5 main parent characteristics were discussed in this scale (factor 1: over-parenting, factor 2: democratic approach, factor 3: hostile and rejective approach, factor 4: marital discordance, factor 5: authoritarian approach).

Results: A total of 45 patients ( 26 boys) with a mean age of $14.1 \pm 3.0$ years were included in the study. Non-adherence to medication was detected in $19(42.2 \%)$ patients. There was no difference between the PedsQL scores of the patients with and without medication adherence (70.0 \pm 18.2 vs $66.3 \pm 20.5$, respectively, $\mathrm{p}=0.557$ ). It was found that the over-parenting score was weakly and inversely correlated with the PedsQL score $(r=-0.410, p=0.018)$. Democratic parenting was found to be an effective factor on treatment adherence (OR=1.290, 95\% CI: $1.004-$ 1.657; $\mathrm{p}=0.047$ ). The graft functions of patients with and without medication adherence were similar (eGFR: $64.92 \pm 21.11$ vs $59.95 \pm 18.82$, respectively, $\mathrm{p}=0.420$ ).

Conclusions: Parental attitudes may effect medication adherence in pediatric renal transplant recipients.

\section{EP-230 LONGITUDINAL FOLLOW-UP ON CARDIOPULMONARY EXERCISE CAPACITY AND CARDIOVASCULAR HEALTH IN CHILDREN WITH RENAL TRANSPLANTS}

Susanne Westphal Ladfors ' ${ }^{1}$, Per Brandström ${ }^{1}$, Sverker Hansson ${ }^{1}$, Ebba Bergdahl ${ }^{2}$, Frida Dangardt ${ }^{2}$

${ }^{1}$ Institute Of Clinical Sciences, Sahlgrenska Academy, University Of Gothenburg, Sweden

2 Institute Of Medicine, Sahlgrenska Academy, University Of Gothenburg, Sweden

Introduction: Chronic kidney disease and kidney transplantation (KT) are associated with an increased risk of cardiovascular disease. The aim of this study was to examine the cardiovascular health after KT compared to 
matched controls, to relate the results to physical activity, blood pressure and biochemical findings and to follow the cardiovascular health over time.

Material and methods: Patients with KT ( $\mathrm{n}=38$, age 7.7-18 years), with a mean time from transplantation of 3.7 years $(0.9-13.0)$ were examined at inclusion and annually for up to four years. Matched healthy controls $(\mathrm{n}=17$, age 7.3-18.6) were examined once. All subjects underwent a cardiopulmonary exercise test, ultra-high frequency ultrasound (UHFUS), pulse wave velocity (PWV) assessment, resting blood pressure measurement, anthropometry and activity assessment. Patients also underwent echocardiography, Dual-energy X-ray absorptiometry (DXA), 24-h ambulatory BP measurements (ABPM), assessment of glomerular filtration rate (GFR) and blood sampling annually.

Results: As compared to healthy controls, KT patients showed significantly decreased exercise capacity measured as $\mathrm{VO}_{2 \text { peak }}$ ( 34.5 vs $43.9 \mathrm{ml} / \mathrm{kg} / \mathrm{min}$ ), also when comparing z-scores. The KT patients were shorter and had higher BMI z-score than controls, as well as increased resting SBP and DBP z-scores. There was a significant difference in activity score (1.7 vs 2.5$)$ and a tendency towards higher aortic PWV (5.9 vs $5.4 \mathrm{~m} / \mathrm{s}$ ), but no significant differences in arterial wall thickness measured by UHFUS, compared to matched healthy controls. Within the KT group, low exercise capacity was associated with high fat mass index (FMI), low activity score, low GFR and high blood lipids.

Conclusions: Patients with KT showed decreased exercise capacity and increased BP as compared to matched healthy controls, but morphological vascular changes are minimal. Low exercise capacity was associated with low activity score, high FMI, low GFR and high blood lipids. There were no changes in exercise capacity or vascular measurements in patients over time.

\section{EP-231 CHANGES AND AFFECTING FACTORS OF HEMOGLOBIN LEVELS IN RENAL TRANSPLANT RECIPIENTS}

Onur Ozer ${ }^{1}$, Ismail Dursun ${ }^{2}$, Neslihan Gunay ${ }^{2}$, Aynur Balaban ${ }^{2}$, Sibel Yel ${ }^{1}$, Ruhan Dusunsel ${ }^{2}$, Hakan Poyrazo $\breve{G l}{ }^{2}$

${ }^{1}$ Erciyes University Faculty Of Medicine, Department Of Pediatrics, Kayseri, Turkey

${ }^{2}$ Erciyes University Faculty Of Medicine, Department Of Pediatrics, Division Of Nephrology, Kayseri, Turkey

Introduction: we aimed to determine the prevalence of anemia in pediatric kidney graft recipients and patients with CKD predialysis group, investigate the results of these patients and the factors that may play a role in the pathogenesis of anemia, to evaluate the effect of inflammation on the development of anemia, to determine the anemia-related markers in pediatric renal transplant patients and to investigate the relationship between graft function and anemia.

Material and methods: This prospective study was done with $29 \mathrm{CKD}$ patients with GFR value $<30 \mathrm{ml} / \mathrm{min} / 1,73 \mathrm{~m} 2$ (group 1) and 31 kidney transplant patients (group 2) and 20 healthy controls (group 3). Anemia and inflammation paramethers including hepcidin were measured and compared between groups, then, the affecting factors of $\mathrm{Hb}$ levels in kidney transplant recipients were determined.

Results: The frequency of anemia was statistically significantly higher in group 1 compared to group 2. Average serum phosphorus, ALP, PTH, BUN, creatinine, triglyceride levels were significantly lower in group 2. whereas $\mathrm{Hb}, \mathrm{Htc}$, iron binding capacity, total protein, albumin, HDL and GFR values were significantly higher in group 2 . Blood hepcidin levels were higher in group 1 than group 2 and 3 . It was determined that both the group 1 and the group 2 patients had high inflammation indicators in the pre-transplant period and the inflammation improved after transplantation. The blood hepcidin levels in patients with anemia and those without anemia were similar in both groups during the study. It was determined that the blood hepcidin level of transplant patients was related with ferritin and PTH value. In contrast, blood hepcidin levels of predialysis patients were found to be moderately negatively associated with $\mathrm{Hb}$, Htc, iron binding, Ret $\mathrm{Hb}$, delta $\mathrm{Hb}$, HDL and GFR, positively associated with ferritin level, hCRP and erythrocyte sedimentation rate.

Conclusions: In patients with CKD, anemia frequency and hepcidin level decreases in post-transplant period compared to predialysis period. While the blood hepcidin level was positively correlated with ferritin in both predialysis and kidney transplant patients, it is negatively correlated with $\mathrm{Hb}$, Htc and GFR, positively correlated with hCRP and erythrocyte sedimentation rate.

\section{EP-232 PEDIATRIC RENAL TRANSPLANT GRAFT NEPHRECTOMY: IMPACT ON OUTCOME,} COMPLICATIONS AND IMMUNOLOGICAL BURDEN

Gander Romy ${ }^{1}$, Perez-beltran Victor ${ }^{2}$, Muñoz Marina ${ }^{2}$, Lopez-gonzalez Mercedes ${ }^{2}$, Asensio Marino ${ }^{1}$, Ariceta Gema ${ }^{2}$

1 Pediatric Urology Unit, Pediatric Surgery Department, Hospital Universitari Vall D'hebron, Barcelona, Spain

2 Pediatric Nephrology Department, Hospital Universitari Vall D'hebron, Barcelona, Spain

Introduction: Literature regarding transplant graft nephrectomy (TGN) in children is sparse, with only two published papers focused on outcome. Indications for TGN are not well defined, being complication rate and allosensitization risk unknown in pediatric kidney transplant (KT) recipients. The aim of this study was to determine patient outcomes, complications and immunological burden associated with TGN.

Material and methods: Retrospective study of every pediatric KT patient who underwent TGN from 2000 to 2020 in our center. Collected data included primary renal disease, cause of graft failure (GF), indications for TGN, surgical technique, intra and postoperative complications and immunological impact on allosensitization analyzed by Complement-Dependent Cytotoxicity (CDC) in all patients and by Single Antigen Bead Assay (SAB) in $46.4 \%$.

Results: Out of 229 pediatric KT, 28 TGN (12.2\%) were performed in 22 patients. Median time to graft failure was 17.5 months (IQ(25-75): 0-37.5). Median age at intervention was 5.5 years (IQ(25-75): $2.25-11.25)$. Main indications for TGN were refractory hypertension (10), graft intolerance syndrome (8) and unsalvageable acute graft thrombosis (5). Neoadjuvant renal artery embolization (RAE) was performed in 1 . One patient (3.6\%) suffered intraoperative hemorrhage without hemodynamic instability. Incidence of postoperative complications was $21 \%$ (6): bleeding (3), hematoma (2) and seroma (1). Symptoms disappeared in all but one patient who remained hypertensive. No mortality related with TGN was seen after median followup of 124.5 months (IQ(25-75): 81.5-158). We observed an increase in Panel Reactive Antibody (PRA) value after the procedure in $50 \%$ of patients studied by CDC. Although, in all patients studied by SAB appeared Donor Specific Antibodies (DSA) or suffered a rise in MFI values after the surgery.

Conclusions: Surgical morbidity of TGN in our series was low and clinical symptoms improved overall. However, our data suggested that allosensitization often occurs, a complication better identified due improving diagnostic immunologic tools.

EP-233 PRIMARY CENTRAL NERVOUS SYSTEM EPSTEIN BARR POST TRANSPLANT LYMPHOPROLIFERATIVE DISORDER (EBV- PCNSPTLD) IN A CHILD WITH KIDNEY TRANSPLANT AND NEGATIVE BLOOD EBV VIRAL LOAD

Varvara Askiti ${ }^{1}$, Georgia Malakasioti ${ }^{1}$, Argyroula Zampetoglou ${ }^{1}$, Evgenios Gousetis ${ }^{2}$, Kalliopi Stefanaki ${ }^{3}$, Maria Mila ${ }^{1}$, Andromachi Mitsioni ${ }^{l}$

${ }^{1}$ Pediatric Nephrology Department Athens Childrens Hospital "p\&a Kyriakou"

${ }^{2}$ Stem Cell Transplant Unit Agia Sophia Childrens Hospital

${ }^{3}$ Histopathology Department Agia Sophia Childrens Hospital 
Introduction: EBV- PCNSPTLD is a rare complication of kidney transplantation, usually within the first months, during peak immunosuppression, that often bodes poor prognosis. Most children have significant elevations in serum EBV PCR before or at the time of diagnosis. We describe an EBV-PCNSPTLD, 8 years after kidney transplantation with negative blood EBV PCR.

Material and methods: A boy (primary diagnosis hypoplastic-dysplastic kidneys) underwent living-related renal transplantation at six years of age. Immunosuppression included prednisone, tacrolimus, MMF. There was EBV mismatching (recipient negative, donor positive). Serial blood EBV PCR were negative. Eight years after transplantation, with baseline creatinine at $3.5 \mathrm{mg} / \mathrm{dl}$, he presented with status epilepticus. After the episode, he was asymptomatic with unremarkable neurologic examination. Brain MRI showed multiple lesions in white matter and hippocampus enhanced with gadolinium. CSF cytology was normal. CSF EBV PCR was positive $\left(2,25 \times 10^{3} \mathrm{IU} / \mathrm{ml}\right)$, while blood EBV PCR was negative. Brain biopsy showed lymphoproliferative lesion CD20 positive, with EBER $1 / 2$ (in situ hybridization) in nucleus of large and medium lymphocytes, consistent with polymorphic EBV-PCNSPTLD. MMF was discontinued and tacrolimus was decreased. He received four cycles of rituximab intravenously $\left(375 \mathrm{mg} / \mathrm{m}^{2} /\right.$ dose) despite of concern over limited penetration of rituximab across the blood-brain barrier. Follow-up MRIs showed a gradual decrease in size and number of lesions. After three months, he received two additional doses of rituximab, due to lack of further improvement on imaging. Eight months after diagnosis, he had almost complete resolution of his brain lesions in MRI. His graft function further deteriorated.

Results: We report the occurrence of polymorphic EBV-PCNSPTLD with negative blood PCR EBV before or at diagnosis and its successful treatment with reduction of immunosuppression and rituximab intravenously.

Conclusions: The negative blood EBV PCR does not exclude EBVPCNSPTLD. Once the diagnosis is confirmed, immunosuppression should be reduced and rituximab should be used as first-line treatment.

\section{EP-234 CALCINEURIN-INHIBITOR INDUCED PAIN SYNDROME IN A CHILD AFTER KIDNEY TRANS- PLANTATION}

Eva Flachsova ${ }^{1}$, Patrik Konopasek ${ }^{2}$, Jan Burkert ${ }^{3}$, Jakub Zieg ${ }^{2}$

${ }^{1}$ Department Of Pediatrics, Second Faculty Of Medicine, Charles University In Prague And Motol University Hospital, Prague, Czech Republic. Department Of Pediatrics, First Faculty Of Medicine, General University Hospital, Prague, Czech Republic

${ }^{2}$ Department Of Pediatrics, Second Faculty Of Medicine, Charles University In Prague And Motol University Hospital, Prague, Czech Republic

${ }^{3}$ Department Of Cardiovascular Surgery, Second Faculty Of Medicine, Charles University In Prague, And Motol University Hospital, Prague, Czech Republic

Introduction: Calcineurin inhibitors (CNI) are the mainstay of current posttransplant immunosuppression. They provide excellent allograft and patient outcomes. However, CNI exhibit a number of adverse effects. Calcineurin-inhibitor induced pain syndrome (CIPS) has been recently described as a rare complication of CNI therapy after organ transplantations. CIPS is clinically characterized by severe disabling bone pain, the diagnosis is made on the basis of clinical symptoms and imaging techniques. While the bone X-ray is normal, magnetic resonance imaging (MRI) shows edema of bone marrow and the skeletal scintigraphy exhibits increased radiotracer uptake.

Material and methods: We report a 10-year-old boy with renal cysts and diabetes syndrome, who developed CIPS after kidney transplantation. After 5 months on hemodialysis he received a kidney allograft from a deceased donor. Induction regimen with basiliximab was used along with maintenance therapy consisting of mycophenolate mofetil, tacrolimus and corticosteroids with early withdrawal. The perioperative period was uneventful, but on day 4 posttransplant severe pain of his right leg occured. He presented with complaints of hyperesthesia with maximum pain localized to the right thumb, limitation in movements and pain irradiation to the thumb while walking.

Results: Neurologic examination did not explain the cause of the pain. The plain X-ray of the leg and the spine MRI were normal. Finally, MRI of the right thumb showed localized bone marrow edema. As the pain sustained for 2 weeks despite the adequate tacrolimus dosing, we switched tacrolimus to sirolimus with successful prompt pain relief. Six months later, sirolimus was switched back to tacrolimus because of the suspicious drug induced liver enzymes elevation with no recurrence of CIPS and subsequent normalization of transaminases.

Conclusions: Clinicians should be aware of CIPS as a very rare adverse effect of CNI therapy. CNI discontinuation results in prompt pain relief. Tacrolimus may be restarted later without recurrence of CIPS.

EP-235 ORALLY ADMINISTERED IMMUNOGLOBULIN IN THE TREATMENT OF CHRONIC NOROVIRUS GASTROENTERITIS IN A KIDNEY TRANSPLANT RECIPIENT

Ruveyda Gulmez ${ }^{1}$, Yusuf Gunay ${ }^{2}$, Seha Saygili ${ }^{1}$, Pinar Onal ${ }^{3}$, Omer Faruk Beser ${ }^{4}$, Ebru Burcu Demirgan ${ }^{1}$, Nil Urganci ${ }^{5}$, Mert Ahmet Kuskucu ${ }^{6}$, Nur Canpolat ${ }^{1}$

${ }^{1}$ Division Of Pediatric Nephrology, Cerrahpasa Faculty Of Medicine, Istanbul University-cerrahpasa, Istanbul, Turkey

${ }^{2}$ Department Of Pediatrics, Cerrahpasa Faculty Of Medicine, Istanbul University-cerrahpasa, Istanbul, Turkey

${ }^{3}$ Division Of Pediatric Infectious Diseases, Cerrahpasa Faculty Of Medicine, Istanbul University-cerrahpasa, Istanbul, Turkey

${ }^{4}$ Division Of Pediatric Gastroenterology, Hepatology, And Nutrition, Cerrahpasa Faculty Of Medicine, Istanbul University-cerrahpasa, Istanbul, Turkey

${ }^{5}$ Department Of Pathology, Cerrahpasa Faculty Of Medicine, Istanbul University-cerrahpasa, Istanbul, Turkey

${ }^{6}$ Department Of Medical Microbiology, Cerrahpasa Faculty Of Medicine, Istanbul University-cerrahpasa, Istanbul, Turkey

Introduction: There are many challenges in the treatment of viral infections in pediatric solid organ transplantation recipients. Norovirus (NoV) is a self-limiting disease in the general population, but it may cause chronic infection with an increased risk of prolonged diarrhea complicated by malabsorption, weight loss, organ failure, and even death in immunocompromised patients.

Material and methods: We present a kidney transplant patient with prolonged and persistent symptoms due to NoV infection.

Results: An 18-year-old male kidney transplant recipient was admitted to the hospital with the complaints of recurrent diarrhea attacks, abdominal pain, vomiting, loss of appetite, and weight loss $(5 \mathrm{~kg}$ in 6 months) for two years. Physical examination revealed short stature, cachexia, and dehydration. Stool tests by PCR revealed positive results for Adenovirus, NoV Genotype II (GII), and C. jejuni. Symptoms did not improve after the use of azithromycin for $\mathrm{C}$. jejuni and the modification of immunosuppressive therapy. Diarrhea and intermittent vomiting persisted; dehydration attacks and acute kidney injury occurred. NoV GII was detected by a PCR assay in the gastroscopic biopsy materials. A significant decrease in stool frequency and volume was observed after oral intravenous immunoglobulin therapy. No side effects were observed during or after the treatment. The patient had a good appetite and appropriate hydration, diarrhea resolved entirely, and he gained weight.

Conclusions: Norovirus gastroenteritis should be kept in mind in kidney transplant children with persistent and intractable diarrhea. Oral administration of intravenous immunoglobulin seems to be an easy and effective treatment option. 
EP-236 POST-TRANSPLANT LYMPHOPROLIFERATIVE DISORDER IN A PEDIATRIC KIDNEY TRANSPLANT POPULATION

Mercedes Lopez Gonzalez, Marina Muñoz Lopez, Victor Perez Beltran, Alejandro Cruz Gual, Hector Rios Duro, Gema Ariceta Iraola

Vall D'hebron Hospital

Introduction: Post-transplant lymphoproliferative disorder (PTLD) after transplantation remains a significant problem with high morbidity, with an incidence around $1.2-4 \%$ in pediatric kidney recipients.

Principal risk factors described are recipient EBV seronegativity and viremia, young age and degree of immunosuppression.

Material and methods: Description of PTLD after kidney transplantation (KT) in our center between 2010-2020.

The following patient data were collected: age, sex, immunosuppressive treatment, clinical presentation, EBV recipient serological status prior to $\mathrm{KT}$ and viremia at diagnosis, histopathological classification, treatment and graft loss.

Results: Out of 142 KT performed between 2010-2020, 9 (6.3\%) patients (7 boys) suffered PTLD. Their age at KT was 3.3 years (IQR: 2-4.6). PTLD diagnosis occurred 52.6 months (IQR: 9.9-97.7) after KT. 3 cases during the first year. Induction regimen included basiliximab in 7 patients (77.8\%) and antithymocyte globulin (ATG) in 2. At diagnosis, all patients were receiving tacrolimus, associated with mycophenolate mofetil $(n=3)$, azathioprine $(\mathrm{n}=2)$ or mTOR inhibitors $(\mathrm{n}=4)$.

The most common presentation was gastrointestinal symptoms $(n=4)$. Others: lymphadenopathy and mediastinal mass $(n=1)$, parotid tumor $(n=1)$, renal dysfunction $(n=1)$, severe aphthous stomatitis $(n=1)$ and hypergammaglobulinemia $(\mathrm{n}=1)$.

$77.8 \%$ of cases were EBV related. $6(66.7 \%)$ polymorphic and 3 monomorphic PTLD (2 diffuse large B-cell lymphoma and 1 Burkitt lymphoma).

Immunosuppression was reduced in all patients; even in 2 of them tacrolimus was temporarily discontinued.

8 patients received Rituximab. Due to aggressive presentation, 2 cases were also treated with R-COP (Rituximab, Cyclophosphamide, Vincristine and Prednisone).

In a median follow-up time of 4 years (IQR: 3-6.1) all patients survived but 3 of them progressed to graft failure, at 1.2 years (SDS \pm 1.2 ) after PTLD.

Conclusions: A high incidence of PTLD was observed in children who received first KT in early childhood, most of them related to EBV. Individualization of immunosuppressive treatment and early diagnosis is mandatory to improve patient and graft survival.

\section{EP-237 THE EVALUATION OF BONE HEALTH PARAMETERS IN CHILDREN WITH RENAL TRANSPLANTATION}

Sevda YeȘim Yilmaz ${ }^{1}$, Seda Pinarbasi ${ }^{2}$, Ummuhan Abdurrezzak ${ }^{3}$, Neslihan Gunay ${ }^{2}$, Sibel Yel ${ }^{2}$, Ismail Dursun ${ }^{2}$, Hakan Poyrazo Ğlu ${ }^{2}$, Ruhan Dusunsel ${ }^{2}$

${ }^{1}$ Erciyes University Faculty Of Medicine, Department Of Pediatrics

${ }^{2}$ Erciyes University Faculty Of Medicine, Department Of Pediatrics, Division Of Nephrology

${ }^{3}$ Erciyes University Faculty Of Medicine, Department Of Nuclear Medicine

Introduction: Although renal transplantation is the best treatment option in advanced CKD patients, many problems such as poor bone health and growth retardation may be seen at follow-up. The aim of this study was to evaluate and determine the factors affecting the bone health parameters of patients who had undergone renal transplantation

Material and methods: 30 kidney transplant patients and healthy controls were included into study. This study had both retrospective and prospective properties. Anthropometric measurements and serum biochemical parameters of the pre-transplant period were obtained retrospectively from the hospital records. Retrospective date including anthropometric measurements and serum biochemical These variables were compared to post- transplantation period of the patients. The data obtained from the patients after transplant were compared with healthy controls. Patients were grouped according to serum levels of iPTH, vitamin D and also status of steroid treatment. DEXA parameters of patients were compared between these groups. Finally, correlation analysis between all clinical and laboratory parameters of patients and DEXA parameters were studied in order to determine bone health related factors in kidney transplanted children.

Results: Nineteen (63.4\%) patients were male. There was no difference for the age of transplantation between the genders. The most common cause of CKD was CAKUT $(46.7 \%)$ in patient group. In the pretransplant period, approximately $50-60 \%$ of the patient's height and body weight percentiles were less than three percentiles. Height SDS, weight SDS and BMI of patients after transplantation period were found to be higher than those of before transplantation. in $40 \%$ of the patients, the bone age was found to be lower than the chronologic age. The anthropometric parameters of patient group were significantly lower than the control group. When the serum parameters were compared between the study groups, it was found that $\mathrm{PTH}, \mathrm{Cr}$, hCRP values were significantly higher, eGFR, $\mathrm{Mg}$, albumin and $\mathrm{HCO} 3$ values were significantly lower in patient group than the control group. There was no difference between two groups in terms of bone specific ALP (bALP). According to height age-matched lumbar vertebra $\mathrm{Z}$ score (LVZS), 3,6\% of the patients were osteoporotic and $21,4 \%$ of the patients had osteopenia. When the DEXA parameters were compared between groups according to the cumulative steroid dose, the lumbar vertebra (LV) BMD was found to be higher in the low cumulative steroid dose group. There was a negative correlation between height age-matched LVZS and duration of steroid treatment and cumulative dose. There was a positive correlation between height age-matched femur neck Z score (FBZS) and duration of tacrolimus and MMF treatment, a negative correlation between height age-matched FBZS and serum iPTH, duration of steroid and calcium treatment. There was a negative correlation between LV, FB and forearm BMD and the duration and dose of steroid treatment in the post-transplant period.

Conclusions: Although height SDS, weight SDS and BMI values of the patients after transplantation were better than the pre-transplant values, there was still significant growth retardation compared to healthy children. Serum bALP levels were not predictive for renal osteodystrophy. DEXA parameters of patients showed improvement after transplantation. In this study, corticosteroid treatment was found to be the most important factor of bone health and growth in renal transplant patients. In addition, we need more research for the use of forearm DEXA application routinely in this population.

\section{EP-238 URINAORWERY CONCENTRATIONS OF PHTHALATE METABOLITES, BISPHENOLS AND PARABENS IN PEDIATRIC RENAL TRANSPLANT RECIPIENTS}

Hjordis Thorsteinsdottir ${ }^{1}$, Jan Ludvig Lyche ${ }^{2}$, Ahmad Tsjokajev ${ }^{3}$, Amrit Kaur Sakhi ${ }^{3}$, Anna Bjerre ${ }^{1}$

${ }^{1}$ Oslo University Hospital

${ }^{2}$ Norwegian University Of Life Sciences

${ }^{3}$ Norwegian Institute Of Public Health

Introduction: Phthalates, parabens and bisphenols are added to a wide variety of consumer products including medical equipment, pharmaceuticals and personal care products. These contaminants are classified as chemicals of emerging concern because of the ubiquitous occurrence and their endocrine disrupting properties. Pediatric renal transplant recipients (RTR) might be at increased risk of exposure of these potentially harmful chemicals due to frequent health care and their lifelong medication. The aim was to measure urinary concentration of phthalate metabolites, parabens and bisphenols in pediatric RTR. 
Material and methods: Morning urine samples were collected from 30 RTR (aged $4.5-18$ years, 17 males) and phthalates metabolites, parabens and bisphenols were measured and adjusted for urinary creatinine. Associations with age, time since transplantation, anthropometrics, glomerular filtration rate (GFR) and previous dialysis were examined.

Results: The preservatives, methylparaben and ethylparaben, the sunscreen oxybenzone and all the phthalate metabolites were detected in $100 \%$ of the urine samples, whereas bisphenol A (BPA), propylparaben and triclosan were found in more than $90 \%$ of the samples. The BPA analogs (substitutes), bisphenol $\mathrm{S}$ and bisphenol $\mathrm{F}$ were detected in the $70 \%$ and $20 \%$ of the urine samples, respectively. The concentrations of phthalate metabolites and bisphenols measured in this study are comparable with the levels detected in the general Norwegian population. However, the levels of methylparaben, propylparaben and ethylparaben were substantially higher than the general population in $25 \%, 15 \%$ and $10 \%$ of the study participants, respectively. In univariate analysis were 11 of 26 compounds negatively associated with age and monoethyl phthalate was positively associated with GFR $(r=0.506, p=0.005)$. Other significant associations were not found.

Conclusions: The source for the higher levels of parabens in $25 \%$ of the patients compared to the general Norwegian population is unknown, but personal care products, drugs and medical equipment may be suspected. There is widespread exposure to bisphenol $\mathrm{S}$ which is a BPA substitute.

\section{EP-239 RENAL INVOLVEMENT IN SCHIMKE IMMUNO OSSEOUS DYSPLASIA (SIOD): CLINICAL COURSE AND OUTCOME AFTER RENAL TRANSPLANT \\ Abdaldafae Mohammed ${ }^{I}$, Khalid Alhasan ${ }^{1}$, Adi Alherbish ${ }^{l}$, Amir Eltayeb $^{2}$, Dieter Broering ${ }^{2}$, Hamad Almojalli ${ }^{2}$ \\ ${ }^{1}$ Paediatric Department, College Of Medicine, King Saud University, Riyadh, Saudi Arabia \\ ${ }^{2}$ King Faisal Specialist Hospital And Research Centre, Organ Transplant Centre, Riyadh, Saudi Arabia}

Introduction: Schimke immuno osseous dysplasia (SIOD) is a rare autosomal recessive disease caused by mutations of SMARCAL1, which is an encoding gene for a chromatin remodelling protein. This multi system disorder is characterized by disproportionate short stature, $\mathrm{T}$ cell immune deficiency, progressive CKD due to FSGS, and recurrent CVA/TIA. Classical renal involvement starts with nephrotic range proteinuria around the age of 5 years and progresses to ESRD in 1 to 11 years, at median age of 8.7 years. Renal transplant remains the key therapeutic option for ESRD, with excellent allograft survival. However, optimal immunosuppressive is challenging with high risk of death from severe infections.

Material and methods: We retrospectively studied 3 patients diagnosed with SIOD referred to our transplant centre with ESRD in the period between 2015 and 2017. Genetic diagnosis was established in all patients. We studied the length of the period of progression to the ESRD, infection rate post transplant, rejection episode and eGFR at one month, 6 months, 18 months and the current eGFR.

Results: All patients achieved stable renal function without any episode of rejection during the follow up. Infection episode required hospital admission was report in one patient for which mycophenolate mofetil was discontinued in view of severe neutropenia and lymphopenia. Patient 2 was admitted to the intensive care 6 months post-transplant with hypertensive crisis and TIA, but retained normal neurological function with good blood pressure control.

Conclusions: SIOD patients with significant T cell immunodeficiency, selecting appropriate immunosuppression remains a challenge as there is high risk of infection such as BKVAN, severe disseminated cutaneous papilloma, EBV, and CMV. In this case series, all patients were given Basiliximab as induction along with maintenance of Tacrolimus, mycophenolate mofetil $300 \mathrm{mg} / \mathrm{m} 2 /$ dose twice daily, and a 6-month course of prednisolone. All 3 patients achieved excellent graft survival with low infection rate.
EP-240 CHARACTERISTICS AND OUTCOME OF BK VIRUS INFECTION IN PEDIATRIC RENAL TRANSPLANT RECIPIENT

Eda Didem Kurt Sukur, Gulsah Ozdemir, Tugba Tastemel, Demet Baltu, Bora Gulhan, Fatih Ozaltin, Ali Duzova, Rezan Topaloglu

Hacettepe University School Of Medicine, Department Of Pediatrics, Division Of Pediatric Nephrology

Introduction: The aim of this study was to describe clinical characteristics, treatments and outcomes of renal transplant recipients with BK virus (BKV) in our center.

Material and methods: This retrospective study included 10 pediatric renal transplant recipients diagnosed with BK viremia and/or nephropathy between January 2011-January 2021. BKV screening was performed routinely at $1^{\text {st }}, 3^{\text {rd }}, 6^{\text {th }}, 12^{\text {th }}$ and $24^{\text {th }}$ months after transplantation, when renal functions were impaired and after any rejection treatment. Viremia was diagnosed by polymerase chain reaction when viral load was $>10^{4}$ copies $/ \mathrm{ml}$ in the blood. BKV nephropathy was shown by intranuclear viral inclusions with SV40 staining in the biopsy.

Results: Median age at transplantation was 13 (7-17) years, and male/female ratio 7:3. In four patients congenital anomalies of the kidney and urinary tract, in two glomerular, three vascular/systemic diseases, in 1 patient nephronophthisis were the underlying diseases. Four patients had immunosuppression history targeting the primary disease. All recipients had their first renal transplants. Nine patients presented with impaired renal functions and one patient was asymptomatic. All patients were seronegative for Ebstein-Barr virus and all patients but one were negative for cytomegalovirus at the time of BK detection. Renal biopsy was performed in all patients and BK nephropathy was shown in nine. Except for one patient with acute cellular rejection, immunosuppression was reduced and tailored to patient's clinical condition. In eight patients mycophenolate mofetil was discontinued. In seven patients tacrolimus was changed to sirolimus, in two patients both agents were used with lowered doses. Ciprofloxacin was used in nine, intravenous immunoglobulin eight, cidofovir seven and leflunomide in six patients. Median follow-up was 38,5 months (17-118). Median estimated glomerular filtration rate increased from 56 to $84 \mathrm{ml} / \mathrm{min} / 1.73 \mathrm{~m}^{2}$ at last visit $(\mathrm{p}=0.114)$.

Conclusions: BKV infection is a risk factor for graft loss. With early detection and prompt intervention preventing renal deterioration is possible.

\section{EP-241 SECONDARY IMMUNOSUPPRESSION IN PEDIATRIC KIDNEY TRANSPLANT RECIPIENTS}

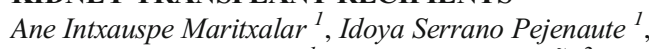

Elene Lejarzegi Anakabe ${ }^{1}$, Maria Herrero Go $\tilde{N i}^{2}$,

Leire Madariaga Dominguez, ${ }^{3}$, Marta Alonso Varela ${ }^{4}$,

Laura Santos-dÍez Vazquez ${ }^{5}$, Mireia Aguirre MeÑica ${ }^{2}$,

Leire Gondra Sangroniz ${ }^{3}$, Ana Vinuesa Jaca ${ }^{2}$

${ }^{1}$ Pediatrics Department. Cruces University Hospital. Barakaldo. Vizcaya. Spain

${ }^{2}$ Pediatric Nephrology. Cruces University Hospital. Iis Biocruces Bizkaia. Barakaldo. Vizcaya. Spain

${ }^{3}$ Pediatric Nephrology. Cruces University Hospital. Iis Biocruces Bizkaia. University Of The Basque Country. Barakaldo. Vizcaya. Spain

${ }^{4}$ Department Of Immunology. Cruces University Hospital. Barakaldo. Vizcaya. Spain

${ }^{5}$ Department Of Pediatric Immunoallergology. Cruces University Hospital. Barakaldo. Vizcaya. Spain

Introduction: Current immunosuppressive treatments for kidney transplant recipients (KTR) have improved the viability of the graft at the expense of impaired immune surveillance. The tools for monitoring immune status in pediatric KTR have not been widely investigated. Periodic testing could help recognising over-immunosuppression and setting up individualized preventive strategies.

Material and methods: This is a retrospective and observational study. We included 28 pediatric KTR managed in a tertiary hospital. We 
measured peripheral blood lymphocyte sub-populations (PBLS), immunoglobulins, immunosuppressive drug levels and viral loads. Reference analytical values for different age ranges were used to determine immune status. We registered overall hospitalizations due to opportunistic infections $(\mathrm{OI})$ and positive viral loads since the transplant.

Results: We found hippogammaglobulinemia and lymphopenia in 19\% and $41 \%$ of the patients, respectively. PBLS were below normal limits for the age in one third of the sample. These parameters were not related to the current number of immunosuppressive drugs or drug plasma levels. During follow-up, CMV, Epstein-Barr and BK viremia was detected in $60.7 \%$ of the patients. Admissions due to OI happened in $64.3 \%$, mainly related to severe viral disease (34\%) or gastrointestinal OI (26\%). Most of them occurred in patients transplanted younger and during the first 2 years post-transplant (74\%). We found no significant relation between PBLS and admissions for OI or positive viral loads during follow-up.

Conclusions: Recurrent hospitalizations for OI and analytical disorders in the immune system suggest that secondary immunosuppression in KTR is frequent. Immunosuppression was not directly related to plasma drug levels or the number of immunosuppressive drugs.

EP-242 INTEREST OF IMMUNOGLOBULINS IN BK VIRUS NEPHROPATHY IN PAEDIATRIC KIDNEY TRANSPLANTATION: STUDY OF A MONOCENTRIC COHORT FROM 2010 TO 2019

Mélodie Mosca ${ }^{1}$, Justine Bacchetta ${ }^{1}$, Valérie Chamouard ${ }^{2}$,

Valérie Dubois ${ }^{3}$, Anne-laure Sellier-leclerc ${ }^{l}$

${ }^{1}$ Centre De Référence Des Maladies Rénales Rares, Hôpital Femme Mère Enfant, Hospices Civils De Lyon, Bron, France

${ }^{2}$ Unité Dhémostase Clinique, Hôpital Louis Pradel, Université Claude Bernard, Lyon, France

${ }^{3}$ Efs Auvergne Rhône Alpes, Laboratoire Hla, Décines, France

Introduction: BK virus-associated nephropathy (BKPyVAN) induces renal allograft dysfunction. Even though decreasing immunosuppression is considered the reference standard for managing $\mathrm{BK}$ virus (BKPyV) infection, this strategy is not always effective. The use of polyvalent immunoglobulins (IVIg) may be interesting in this setting.

Material and methods: We performed a retrospective single-center evaluation on the management of BKPyV in pediatric kidney transplantation. Among the 171 patients who were transplanted between January 2010 and December 2019, 54 patients were excluded (combined transplant $\mathrm{N}=15$, follow-up in another center $\mathrm{N}=35$, early post-operative graft loss $\mathrm{N}=4$ ). Thus, 117 patients (120 transplants) were included. Results are presented as median (IQR).

Results: After transplantation, 34 (20\%) patients displayed BKPyV urinary replication, and $15(9 \%)$ patients displayed positive viremia, including 3 with biopsy-confirmed BKPyVAN. The median time to appearance of BKPyV in blood after urinary detection was 0.8 (0.4-1.8) months, 2.7 (1.6-7.7) months after transplantation. As compared to patients without $\mathrm{BKPyV}$, patients with circulating BKPyV displayed a higher prevalence of CAKUT and HLA antibodies before transplant, with lower tacrolimus through levels between 3 and 6 months post-transplant. After the detection of BKPyV replication and/or BKPyVAN, the immunosuppressive regimen was modified in 13 patients $(87 \%)$ : either by decreasing or changing the calcineurin inhibitor $(\mathrm{N}=13)$, and/or switching mycophenolate mofetil for mTor inhibitors $(\mathrm{N}=10)$, and/or adding IVIg $(\mathrm{N}=7)$. The IVIg group had significantly higher viremia $\left(3.5 \log _{10}\right.$ versus $5.4 \log _{10}$, $\mathrm{p}<0.005)$. With a follow-up of $2.0(1.2-2.6)$ years, among the 15 viremic patients, eGFR is $73(61-85) \mathrm{mL} / \mathrm{min} / 1.73 \mathrm{~m}^{2}, 10$ patients display a viral load less than $3 \log _{10}(67 \%)$, including 2 after the addition of IVIg. Three patients displayed humoral rejection (20\%), and one lost his graft as a result of BKPyVAN.

Conclusions: As long as specific antivirals are not available in pediatrics, polyvalent IVIg seem interesting for the management of severe BKPyV viremia, in combination with decreased immunosuppression.
EP-243 RITUXIMAB AS INDUCTION THERAPY IN PEDIATRIC KIDNEY TRANSPLANTATION: A SINGLE-CENTRE EXPERIENCE

Josselin ,Bernard, Anne-laure Sellier-leclerc, Delphine Demede, Valerie Chamouard, Bruno Ranchin, Justine Bacchetta

Reference Center For Rare Renal Diseases, Orkid And Erknet Networks, Hospices Civils De Lyon, Bron, France

Introduction: In pediatric nephrology, the anti-CD20 rituximab (RTX) is considered in several situations, including steroid-resistant nephrotic syndrome (SRNS) and EBV-induced post-transplant lymphoproliferative disorder (PTLD).

Material and methods: We retrospectively analyzed the five children (aged 6.1-14.7 years) who received RTX as induction therapy for kidney transplantation (KTx) since 2016 in our tertiary unit, out of a total of 81 KTx performed between $1 / 1 / 16$ and $31 / 12 / 20$.

Results: RTX was chosen because of a positive EBV viral load before transplantation in 4 of them. Between M3 and M6 post-transplantation, all patients displayed a negative EBV viral load, but the viral load became positive again in three patients after B cell repletion, occurring after 6 months post-transplantation, and remaining between 3.3 and $4.3 \log$ at last follow-up. In the last patient, RTX induction was performed to prevent a relapse of SRNS after 2 previous early failures of KTx. Despite RTX induction, SRNS relapsed but remission was obtained in one month by using two other anti-CD20 antibodies (ofatumumab and obinutuzumab) and immunoadsorptions ( $\mathrm{N}=23$ sessions). All patients displayed neutropenia, two of them even requiring granulocyte colony stimulating factor. After a median follow-up of 2.1 years, no PTLD was observed. None of the 5 patients presented rejection or de novo donor specific antibodies; the glomerular filtration rate remained above $70 \mathrm{~mL} /$ $\mathrm{min} / 1.73 \mathrm{~m}^{2}$ in all.

Conclusions: RTX may be an option as induction therapy with good results at 2 years post-transplantation, in term of graft survival, immunological biomarkers and EBV control (in case of positive viral load prior to transplantation). It could also be a strategy in patients with SRNS, in order to help managing an eventual relapse of the disease after transplantation. The risk of neutropenia appears significant, as an adverse effect of multiple concomitant therapies, and neutrophils should be assessed regularly.

\section{EP-244 QUICK RECOVERY OF BK VIRUS NEPHROPATHY} AFTER TREATMENT WITH LEFLUNOMIDE

Varvara Askiti ${ }^{1}$, Evangelia Gole ${ }^{1}$, Georgia Malakasioti ${ }^{1}$, Maria Darema ${ }^{2}$, Andromachi Mitsioni ${ }^{1}$

${ }^{1}$ Pediatric Nephrology Department, Athens Childrens Hospital "p\&a Kyriakou"

${ }^{2}$ Department Of Nephrology And Transplantation, National And Kapodistrian University Of Athens Medical School

Introduction: BK viremia is a significant complication post renal transplantation (RTx) and can progress to BK virus nephropathy (BKVN) and graft dysfunction. There is no consensus on the management of BKVN in pediatric RTx recipients. The most common therapeutic option is reduction of immunosuppression, however can increase rejection risk. The role of leflunomide, an agent with antiviral and immunosuppressive effects, as treatment for BKVN remains unclear. We describe the effectiveness of treatment with leflunomide in 3 children with BKVN.

Material and methods: All patients (age 8,12,14 years) developed BKVN in a mean period of 3.5 months post RTx. Significant viremia was evident in blood PCR with highest viral loads being in patient $1: 73521 \mathrm{IU} / \mathrm{ml}$, in patient 2: $532210 \mathrm{IU} / \mathrm{ml}$ and in patient 3: 15998551 $\mathrm{IU} / \mathrm{ml}$. Urine was positive for decoy cells. One patient that developed hemorrhagic cystitis had also histologic detection of BK on bladder mucosa biopsy. Graft dysfunction was evident in all patients with $50 \%$ $100 \%$ elevation of creatinine from baseline. Our therapeutic strategy 
included lower Tacrolimus levels, discontinuation of MMF and active treatment with leflunomide. Two of the patients received initially IVIG. Treatment with leflunomide was continued until BK was undetectable in at least 2 blood PCR samples 2 weeks apart.

Results: Within a mean period of 3.3 months all patients had undetectable levels of BK in plasma PCR in at least 2 different samples and also normalization of the renal function back to the baseline. None of our patients had evidence of hepatotoxicity or anaemia on regular monitoring none any other adverse events. Renal function remained stable in the follow up period up to date with no re-occurrence of BK viremia.

Conclusions: Our cases show that treatment with leflunomide resulted in quick BK clearance and preservation of renal function with no adverse effects and prevention of graft rejection.

\section{EP-245 EFFECT OF RENAL TRANSPLANTATION ON ATTAINMENT OF NORMAL FINAL HEIGHT IN PATIENTS WITH ESRF}

Evangelia Gole ${ }^{1}$, Dominic Wilson ${ }^{2}$, Mordi Muorah ${ }^{1}$, Renuka Dias ${ }^{3}$

${ }_{1}$ Department Of Paediatric Nephrology, Birmingham Childrens Hospital

${ }^{2}$ Department Of Paediatric Endocrinology, Birmingham Childrens Hospital

${ }^{3}$ Department Of Paediatric Endocrinology, Birmingham Childrens Hospital, University Of Birmingham

Introduction: Impaired linear growth is a common complication of end stage renal failure (ESRF), with recombinant human growth hormone (rhGH) being the suggested treatment until renal transplantation (RTx). Growth improvement post RTx has been reported, however data on the degree of catch up growth post RTx and final height $(\mathrm{FH})$ is limited. We aimed to evaluate the effect of RTx on FH standard deviation score (SDS) and the contribution of age of transplantation on height improvement.

Material and methods: We performed a retrospective review of medical records of RTx recipients from a single UK centre. We recorded demographic data, height SDS before RTx and FH SDS, treatment with rhGH and eGFR until FH. We analyzed change in height SDS prior to RTx and at $\mathrm{FH}$ and possible predictive factors.

Results: 33 RTx patients (17 males/16 females) with normal graft function were included. Mean age of RTx was 9.5 years. RhGH was administered before RTx in 12 patients. Height SDS increased significantly from pre RTx (mean Ht SDS -2.0, median -2.0, range -4.33 to 1.88) to FH (mean Ht SDS -0.9, median -1.13, range -3.72 to 1.37), p<0.001. 29 patients $(88 \%)$ had a normal height $>-2.0 \mathrm{SD}$. The mean change on height SD from before RTx to FH was +1.1 . There was a significant correlation between age of RTx and increase of height SD from before RTx to FH, R: $-0.373, p=0.032$. Multiple regression analysis (including age of RTx, rhGH treatment before RTx and gender) showed that age of RTx was an independent predictive factor of the height SD increase.

Conclusions: In our group, we observed a sustained increase in height post RTx resulting in normal FH. RTx especially in younger recipients seems to result in adequate catch up growth that can compensate for the deficit occurring during ESRF.

\section{EP-246 DE NOVO THROMBOTIC MICROANGIOPATHY AFTER KIDNEY TRANSPLANTATION IN A PEDIATRIC PATIENT}

Maria Herrero GoNi ${ }^{1}$, Leire Gondra Sangroniz ${ }^{2}$,

Mireia Aguirre MeÑica ${ }^{1}$, Ana Vinuesa Jaca ${ }^{1}$,

Leire Madariaga Dominguez, ${ }^{2}$, Aitor Fernandez De Larrinoa ${ }^{4}$,

Nelida Garcia Perez, ${ }^{3}$

${ }^{1}$ Pediatric Nephrology, Cruces University Hospital. Iis Biocruces Bizkaia

${ }^{2}$ Pediatric Nephrology. Cruces University Hospital. Iis Biocruces Bizkaia. Pais Vasco University
${ }^{3}$ Pediatric Nephrology. Basurto University Hospital. Iis Biocruces Bizkaia

${ }^{4}$ Pathological Anatomy. Cruces University Hospital

Introduction: Thrombotic microangiopathy (TMA) is a rare and serious complication of kidney transplantation which is associated with poor graft prognosis. De novo TMA is a heterogeneous entity and it is related to inmunosupressive treatment or antibody mediated rejection. Recently genetic mutations in complement genes have been described as a cause of de novo TMA.

Material and methods: Our aim is to report the case of a 12-year-old adolescent with X-linked Alport syndrome who developed de novo TMA one year after transplantation.

Results: The patient had been admitted several times for non-infectious diarrhea. He reconsulted for severe diarrhea and graft dysfunction (creatinine $3.85 \mathrm{mg} / \mathrm{dl}$, glomerular filtration rate $30 \mathrm{ml} / \mathrm{min} / 1.73 \mathrm{~m} 2$ ). Intravenous serum therapy was prescribed, tacrolimus was adjusted and mycophenolic acid was replaced by rapamycin. Stool cultures and determination of viral loads were negative. Doppler ultrasound of the graft was normal and the autoimmunity study was negative. He had non-hemolytic anemia with positive fecal occult blood test. A panendoscopy was performed with normal result. The normal platelet count and the absence of hemolysis data did not raise suspicion of TMA. Although donor-specific anti-HLA antibodies were negative, a kidney biopsy was performed to rule out rejection. Biopsy showed data of TMA. As potential causal agents of TMA tacrolimus and rapamycin were stopped. Belatacept was started and corticosteroids and mycophenolate mofetil were restarted with normalization of creatinine up to $0.9 \mathrm{mg} / \mathrm{dl}$. Currently, 6 months after diagnosis, the patient is asymptomatic, with normal kidney function. The complement genetic study results are pending.

Conclusions: De novo TMA is a diagnostic challenge in the assessment of graft dysfunction in pediatric patients. As in our case, it usually requires a biopsy for diagnosis because systemic signs of TMA are frequently absent. The use of new therapies such as belatacept to avoid other immunosuppressants associated with TMA opens a door for the treatment of such complicated cases.

\section{EP-247 HEPATIC PELIOSIS ASSOCIATED WITH AZATHIOPRINE USE IN A PEDIATRIC KIDNEY TRANSPLANT RECIPIENT}

Antoine Mouche, Cyrielle Parmentier, Claire Herbez Rea, Jean Daniel Delbet, Tim Ulinski

Pediatric Nephrology Unit, Armand Trousseau Hospital, Aphp, Dmu Origyne, Sorbonne Universite, 75012, Paris, France

Introduction: Azathioprine, an antimetabolite drug interfering with purines synthetisis, is frequently used in solid organ transplant or dysimmune disease. In pediatric kidney transplant, immunosuppressive (IS) maintenance therapy usually combines 2 or 3 IS drugs: a calcineurine inhibitor (e.g. tacrolimus, ciclosporin), an antimetabolite (e.g. mycophenolic acid, azathioprine) and potentially oral steroids.

Material and methods: We report the case of a 6 year-old-girl who developed hepatic peliosis due to azathioprine use 9 months after kidney transplant.

Results: A girl with stage 5 chronic kidney disease due to Denys-Drash syndrome received a kidney transplant at the age of 6 years. IS maintenance therapy initially consisted in the association of tacrolimus and mycophenolate mofetil (MMF). Seven months post transplant, MMF was switched to azathioprine, because of persistant diarrhea attributed to this treatment.Two months later, hepatomegaly with micronodular liver infiltration and moderate portal hypertension with splenomegaly was fortuitously discovered on abdominal sonography. Liver fonction was normal, aspartate transaminase (AST) and gamma-glutamyltransferase (GGT) were moderately increased (respectively $53 \mathrm{UI} / \mathrm{L}$ and $105 \mathrm{UI} / \mathrm{L}$ ). Complete infectious investigations were negative. A hepatic biopsy was 
performed. Histopathology study found nodular regenerative hyperplasia with severe peliosis, i.e. multiple, randomly distributed, blood-filled cavities, without central venous occlusion. The biopsy lead to hemoperitoneum which required blood transfusion.Hepatic peliosis was attributed to azathioprine, which was interrupted and switched to mycophenolic acid.One year later, control sonography found a persistent non dysmorphic micronodular hepatomegaly. Splenomegaly and portal hypertension had resolved. Biological liver tests were unchanged.

Conclusions: Hepatic peliosis is a rare but known complication of azathioprine treatment. Its long-term outcome and the reversibility remain uncertain, even after interruption of the causal treatment.

\section{EP-248 USING SOCIAL MEDIA/ MAINSTREAM MEDIA TO PROMPT ORGAN DONATION/ TRANSPLANTATION EDUCATION \\ Shahid Muhammad \\ The University Of The West Of England (uwe)}

Introduction: The use of social media (SM) today provides unparalleled opportunities to provide and receive education, access to communication and engagement. SM/ mainstream media (MM) such as television, newspapers, magazines, and radio stations are also being used to prompt education surrounding specific Long-Term Conditions (LTCs). Certainly, use of SM is not limited by constraints of time and geography.

Material and methods: Clarity of terminology to help increase HL on topics relating to organ donation/ transplantation is now especially required owing to more time being spent online. Three questions being proposed here: 1) Is there an Islamic stance on using SM/ MM to prompt organ donation/ transplantation education? 2) Can Muslims donate their organs? and 3) Does the Healthcare Scientist have a role providing the public education surrounding organ donation/ transplantation? Chronic Kidney Disease (CKD) will be used to provide a LTC example.

Results: Bridging gaps in health literacy (HL) is also important. This is where healthcare scientists have an important role, to provide health science transparency where other health professionals are challenged. There is a need for multi-channel approaches so that the issue is a more widely known social norm in primary care, where healthcare scientists will know underlying pathologies. If a Muslim decides to donate an organ must do so out of free will without being morally or socially forced and without economic pressures. If the deceased Muslim indicated during life (in a will) that they do not want to donate organs, then no one is authorized to do this on the deceased person's behalf.

Conclusions: 1) The Islamic stance on use of social media to prompt organ donation/ education has not been investigated. 2) Muslims can donate their organs, and 3) there is a role for healthcare scientists to provide education, however more research is required to shed light on what is the most effective approach for the healthcare scientist to become proactive.

\section{EP-249 PRIMARY HYPEROXALURIA TYPE 1 PROGRESSING TO END-STAGE RENAL FAILURE AT INFANCY}

Ayla KaCar ${ }^{1}$, Mehtap Adar ${ }^{1}$, Muhammet Sancaktar ${ }^{1}$, GÜlŞah Kaya Aksoy ${ }^{1}$, Elif Çomak ${ }^{l}$, Mustafa Koyun ${ }^{l}$, Afig Berdeli ${ }^{2}$, Sema Akman ${ }^{1}$

${ }^{1}$ Akdeniz University Faculty Of Medicine, Department Of Pediatric Nephrology, Antalya, Turkey

2 Department Of Pediatrics, Molecular Medicine Laboratory, Ege University, Izmir, Turkey

Introduction: Primary hyperoxaluria type-1 ( $\mathrm{PH} 1)$ is a rare autosomal recessive metabolic disorder characterized by the accumulation of oxalate in the kidney. We aimed to present a patient with PH1 who developed end-stage renal failure (ESRD) at infancy and underwent combined liver and kidney transplantation.
Material and methods: A four-month-old girl was admitted with having nephrocalcinosis on ultrasonography performed for lower urinary tract infection. There was consanguinity between his parents. On examination; body weight was $7.4 \mathrm{~kg}(50 \mathrm{p})$, height was $62.7 \mathrm{~cm}$ (25p), blood pressure was $90 / 50 \mathrm{mmHg}(95 \mathrm{p}$ : $94 / 54 \mathrm{mmHg}$ ) and daily urine output was $1200 \mathrm{ml} / \mathrm{m}^{2} /$ day. Her laboratory result were as follows: serum creatinine $0.76 \mathrm{mg} / \mathrm{dL}$, eGFR $34 \mathrm{ml} / \mathrm{min} / 1.73 \mathrm{~m} 2$, serum electrolytes were within the normal range. Urinary calcium, citrate, magnesium, uric acid and oxalate excretions (oxalate/creatinine ratio was $0.08 \mathrm{mg} / \mathrm{mg}$ ) were within normal ranges. The patient was followed up with hydration and urinary alkalinization; but 2 months later, she presented with a decrease in urine volume following vomiting and diarrhea. Serum creatinine was $7.2 \mathrm{mg} / \mathrm{dL}$. Hemodialysis treatment was initiated because oliguria continued despite furosemide treatment and accompanying hyperpotasemia. On biopsy, extensive crystal accumulation in the tubules and bowman space were detected. Eye examination revealed crystal deposits on the retina and fovea. On genetic examination, two nucleotide insertions on exon 8 of AGXT gene (c.824_825insAG) were found to be homozygous. In addition, there was a p.His402Tyr (c.1204C> T) (Homozygous) mutation in the $\mathrm{CFH}$ gene and this mutation was associated with aHUS.

Results: When the patient was 1 year old and reached $8 \mathrm{~kg}$ body weight, she underwent combined liver and kidney transplantation. Kidney donor was father and liver donor was non-related living donor. After 33 months of post-transplant follow-up, graft functions were preserved.

Conclusions: Primary hyperoxaluria type- 1 should be suspected in infants with both nephrocalcinosis and ESRD, even with normal urinary oxalate excretion.

\section{EP-250 THE FIRST EXPERIENCE OF USING THE BIOANALOGUE OF ORIGINAL ECULIZUMAB FOR THE PREVENTION OF ATYPICAL HEMOLYTIC-UREMIC SYNDROME POST-TRANSPLANT RECURRENCE}

Sergey Baiko

Belarusian State Medical University, Minsk, Belarus

Introduction: Atypical hemolytic uremic syndrome (aHUS) is a rare form of a systemic thrombotic microangiopathy (TMA) developing as a result of uncontrolled activation of the complement system. The risk of aHUS post-transplant recurrence is higher in patients with genes mutations encoding plasma complement regulatory proteins (factors $\mathrm{H}, \mathrm{I}, \mathrm{B}$, etc.).

Material and methods: The goal of the report is to present a patient with aHUS and heterozygous mutations of two genes: $C F I$ (p. $772 \mathrm{G}>\mathrm{A}$ (p.Ala258Thr)) and $M C P$ (p.1058C $>$ T (p.Ala353Val)) which were identified by NGS.

Results: A 10-year-old boy was admitted to the hospital with complaints of fever, vomiting, bloody diarrhea, severe abdominal pain. After diagnostic laparoscopy, surgical pathology was excluded. Typical HUS and anuria were identified after surgery. After 3 sessions of hemodialysis (HD) the child had hallucinations during 2 days. In a month of HD kidney biopsy was performed and TMA was confirmed. After 4 months of HD there was a stabilization of creatinine levels of 300-330 $\mu \mathrm{mol} / 1$ and the next 9 months he didnt need the HD. That time NGS was performed with changing diagnosis to aHUS. The child had average risk of aHUS posttransplant recurrence considering the $C F I$ gene mutation. In two years after aHUS onset the child received a kidney graft from a deceased donor. The Russian biosimilar eculizumab was administered an hour before surgery, on days 1, 8, 22 and then every 2 weeks. There were no episodes of TMA after surgery. And now after 7 months the levels of hemoglobin, platelets, $\mathrm{LDH}$ are normal, blood creatinine is $58 \mu \mathrm{mol} / 1$.

Conclusions: In this clinical case it is shown that diarrhea can be a trigger for aHUS and the use of biosimilar eculizumab can prevent the aHUS post-transplant recurrence. 
EP-251 STUDY OF TUBULAR DAMAGE AFTER KIDNEY TRANSPLANTATION IN A COHORT OF PEDIATRIC PATIENTS

Pedro Arango Sancho, Yolanda Calzada Baños, Elena Codina Sampera, VÍctor LÓpez-baez, Ana Vinuesa Jaca, Lina Catherine HernÁndez. ZúNĩiga, Álvaro Madrid Aris

Hospital Sant Joan De Déu

Introduction: To analyze the tubular function the first 3 months after kidney transplantation (KT) in a group of pediatric patients, taking into account all the possible causes that could determine these alterations.

Material and methods: Retrospective observational study in which we included a cohort of 17 kidney transplant patients,median age of 13 years $(3-18 y)$ to whom we evaluated tubular function at one week,one month and three months post-transplant, excluding those patients with GFR IDMS $<60 \mathrm{ml} / \mathrm{min} / 1.73 \mathrm{~m}^{2}$ one month after transplantation, avoiding the confounding factor of chronic kidney disease.The excretion of different parameters in the first-morning urine was evaluated, such as urinary volume per $100 \mathrm{ml}$ of GFR (V/GFR) or levels of B2-microglobulin (B2M) as a marker of tubular damage.

Results: The most frequent transplant was cadaveric (76.5\%) and preemptive (52.9\%). In the first 3 months, up to $35.3 \%$ of the patients $(6 / 17)$ had episodes of acute tubular necrosis (ATN), 2 of them (11.8\%) in context of BK virus infection (BKV). Corticosteroids and anticalcineurinics doses, as well as their levels, were stable. The most frequently tubular alterations observed were the V/GFR increase $(100 \%, 94.12 \%$ and $88.24 \%)$, followed by the increase in B2M $(88.2 \%$, $35.3 \%$ and $41.4 \%)$, FENa $(94.1 \%, 55.6 \%$ and $71.4 \%)$ and calciuria $(82.4 \%, 29.4 \%$ and $23.5 \%)$. The most frequent blood disorder was hypomagnesemia (12\%) followed by hypouricemia $(8.5 \%)$, without any alteration of acid-base balance at 3 months.

Conclusions: There are multiple factors that can cause kidney graft damage (ATN, infections, drugs, rejection). Previous studies determine the frequency of post-transplant tubular damage in children and adults, determining "Dent-like" alterations in adults. The same pattern seems to be observed in our work, which could be explained by defects in endocytosis at the proximal tubular cell level after exposure to multiple damage factors, such as the anti-calcineurin drugs.

\section{EP-252 THE 15 YEARS (2004-2019) EXPERIENCE OF PEDIATRIC KIDNEY TRANSPLANTATION IN THE HOSPITAL OF LITHUANIAN UNIVERSITY OF HEALTH SCIENCES KAUNO KLINIKOS}

Gintare Iveta Semenaite ${ }^{1}$, Jurate Masalskiene ${ }^{2}$, Diana Dobiliene ${ }^{2}$, Sarunas Rudaitis ${ }^{2}$

${ }^{1}$ The Lithuanian University Of Health Sciences, Faculty Of Medicine, Kaunas, Lithuania

${ }^{2}$ The Lithuanian University Of Health Sciences, Department Of Pediatrics, Kaunas, Lithuania

Objectives:To analyze and present the results and outcomes of pediatric kidney transplantation in the Hospital of Lithuanian University of Health Sciences Kauno Klinikos (HLUHSKK).

Methods: The research was carried out with the permission of the HLUHSKK Centre of Bioethics. Data was collected from depersonalized medical histories of all 17 patients who underwent a kidney transplantation in the year of $2004-2019$ at the HLUHSKK. IBM SPSS Statistics 13 package was used for statistical data analysis.

Results: The study involved $52,9 \%$ boys and $47,1 \%$ girls. The average age was $13,35 \pm 2,89$ years. Congenital kidney disease acounted $29,4 \%$, glomerular causes accounted $41,2 \%$, tubulointerstitial nephritis caused $29,4 \%$ patients of pediatric chronic kidney disease cases. Before renal transplantation $88,2 \%$ patients had treatment with haemodialysis, $11,8 \%$ - peritoneal dailysis, on average 14,9 months. Patients avarage time for waiting kidney transplantation was 9.35 \pm 8.91 months. $23,5 \%$ childrens have waited for the kidney transplantation longer than a year, 11,8\% longer than two years. Induction immunosuppressive therapy consisting of mycophenolate mofetil (MMF), basiliximab and corticosteroids was given for $58.8 \%$ patients, while MMF, daclizumab and corticosteroids was intended for $41,2 \%$ children. Maintenance immunosuppressive therapy consisting of cyclosporine, MMF and corticosteroids was given for $52,9 \%$ recipients, while tacrolimus, MMF, corticosteroids was intended for $47,1 \%$ patients. The frequency of the early onsetcomplications after a kidney transplantation is $64,9 \%$ : homeostatic imbalance - for $23,6 \%$, infection - for $23,6 \%$, acute rejection - for $11,8 \%$ patients. The 1 - and 5 -year graft survival rates among our recipients were $100 \%, 85,7 \%$. By the end of 2020 years, $69,2 \%$ graft have survival. One patient died during the follow-up period.

Conclusions: The most common cause of CKD in children who undergo transplantation is glomerular diseses. Homeostatic imbalance and infection are the most common early complications after renal transplantation. The most commonly chosen drugs for immunotherapy was cyclosporine, MMF and corticosteroids.

EP-253 UROLOGICAL MANAGEMENT OF KIDNEY TRANSPLANT RECIPIENTS WITH DEFUNCTIONALIZED BLADDER: A SINGLE CENTER EXPERIENCE Georgia Malakasioti $^{1}$, Varvara Askiti ${ }^{1}$, Eleni Kapsia ${ }^{2}$, Ioannis Bokos ${ }^{3}$, Andromachi Mitsioni ${ }^{I}$

${ }^{1}$ Renal Unit, P. \& A. Kyriakou Children's Hospital, Athens, Greece

${ }^{2}$ Department Of Nephrology And Kidney Transplantation, Laiko General Hospital And National And Kapodistrian University Of Athens School Of Medicine, Athens, Greece

${ }^{3}$ Kidney Transplantation Unit, Laiko General Hospital, Athens, Greece

Objectives: The optimal urological management of pediatric kidney transplant recipients (pKTR) with longstanding anuria resulting in defunctionalization of a previously normal bladder remains controversial. Current practices vary from pretransplant bladder augmentation, to suprapubic catheter placement at the time of surgery. We aimed to evaluate postoperative urological complications and graft function following the implementation of a more conservative urological approach in pKTR with normal lower urinary tracts prior to their anuric ESKD.

Methods: Five children with ESKD of non-urological etiology and protracted anuria underwent LRD transplantation; donor ureter was anastomosed onto native bladder with subsequent $\mathrm{JJ}$ stent and urethral catheter (UC) placement. No bladder reconstructive surgery, preoperative bladder cycling or suprapubic drainage were implemented.

Results: Median age at transplant was 12.9 years with bladders defunctionalized for a median of 9 years (range 6 to 11) representing $73.6 \%$ of the patients total lifetime. JJ stents and UC were removed at 28 and 7 days post transplant respectively as per protocol in all but one patient with bladder capacity (BC) of $20 \mathrm{ml}$ and vesicoureteral reflux in the pretransplant MCUG, in whom the UC was maintained for 25 days while bladder cycling was undertaken until a BC of $180 \mathrm{ml}$ was reached. During a median follow-up of 15.6 weeks no hydroureteronephrosis or urinary leaks were detected; only one patient developed recurrent UTIs despite an improving $\mathrm{BC}$ z-score of -0.67 and satisfactory bladder emptying likely related to underlying diabetes and persistent urothelial colonization. At last follow-up, median $\mathrm{BC} \mathrm{z}$-score and eGFR decline were 0 (range -1.65 to 0.3 ) and $0 \mathrm{ml} / \mathrm{min} / 1.73 \mathrm{~m}^{2}$ (range -10 to 0 ) respectively.

Conclusions: We suggest that invasive urological procedures can be avoided in pKTR with anuric ESKD of non-urological background with successful natural postoperative rehabilitation of a priorly defunctionalized bladder. 
EP-254 COPING WITH CHRONIC ILLNESS - A QUESTION OF FAITH

Shahid Muhammad ${ }^{1}$, Maggi Banning ${ }^{2}$, Mohammed Sadiq ${ }^{3}$

${ }^{1}$ The University Of The West Of England (uwe)

${ }^{2}$ Brunel University

${ }^{3}$ Shifa Psychological Services

Introduction: With exception to individuals with severe learning difficulties and neurological illnesses (which of course are devastating in their own right), there has never existed a human being who has not been aware not only of his body but also of his individuality, both physical and spiritual. Muslims and non-Muslims can have a number of chronic illnesses affecting their health and ability to cope. This article seeks to provide understanding of Islam and definition of knowledge, an understanding of what Muslims believe and health and coping from an Islamic context. Coping is explored providing Chronic Kidney Disease (CKD) perspective.

Material and methods: This article also looks at Kidney Transplantation and Organ Donation in an Islamic context. More evidence-based research is required on Islam and coping, and Allah (God) informs that No Muslim is afflicted with harm because of sickness or some other inconvenience, but that Allah will remove his sins for him as a tree sheds its leaves (Bukhari). There is also a requirement for health professionals to acknowledge the Islamic faith when attending to specific healthcare needs. Results: A holistic treatment plan that takes into consideration all aspects (i.e., Tawheed - oneness of God) and employs appropriate health care strategies to deal with each aspect of the illness would likely produce the best, fastest and enduring results. Through its benevolent verses, The Holy Qur'an highlights that suffering in this life is short-term.

Conclusions: Allah informs that for every disease, there is a cure. Those who do not believe in mercy or practice it cannot expect to be saved by it. Reciting and reflecting on verses of the Holy Qur'an can be comforting and promote positive coping. InAllah Ma'Sabireen (Indeed Allah is with those who are patient).

\section{EP-255 INFECTION VS REJECTION, THE TRANSPLANT CONUNDRUM \\ Karen Norman, Andrew Maxted \\ Renal And Urology Unit, Nottingham Childrens Hospital, Nottingham}

Introduction: Infections are an important cause of morbidity following kidney transplantation, with those transplanted at a younger age at particular risk. We present a patient transplanted at 2 years 8 months of age, who developed chronic EBV viraemia, aplastic anaemia secondary to Parvovirus B19, persistent symptoms of lower respiratory tract infection and episodes of acute cellular rejection.

Material and methods: A 32 month old boy with a background of genetic negative congenital nephrotic syndrome received a kidney transplant from a live, unrelated donor. EBV serology was D+, R EBNA negative. Maintenance immunosuppression comprised tacrolimus, azathioprine and prednisolone.

Results: EBV DNA was detected at 4 months post-transplant, and persisted at levels of 600-227000 IU/ml, despite weaning and cessation of anti-proliferative immunosuppression. No signs or symptoms of PTLD have been identified.

The patient developed a wet cough at 20 months post-transplant which persists at time of reporting, despite multiple courses of antibiotics. Rhino/enterovirus was identified on one occasion, in association with left-sided consolidation on chest radiograph.

Anaemia refractory to erythropoietin therapy was noted at 21 months post-transplant associated with $>1$ billion $\mathrm{IU} / \mathrm{ml}$ Parvovirus B19 DNA. Haemoglobin reached a nadir of $51 \mathrm{~g} / \mathrm{L}$, with mild leukopaenia and thrombocytopaenia. A 5 day course of IVIG (total dose $2 \mathrm{~g} / \mathrm{kg}$ ) reduced serum parvovirus DNA IU/ml, maintained post-transfusion $\mathrm{Hb}$ above $100 \mathrm{~g} / \mathrm{L}$ and improved leucocyte and platelet counts. Parvovirus DNA persists in serum 2 months posttreatment, however.

1 month post-IVIG a rise in creatinine was noted with subsequent biopsy showing acute cellular rejection. This was treated with 3 days of high dose oral prednisolone.

Conclusions: Viral infection is common in the renal transplant population due to effective post-transplant immunosuppression regimes. Parvovirus B19 infection should be considered in the post-transplant patient with anaemia resistant to erythropoietin. Management of infection in the immunosuppressed transplant patient is challenging with the balance against rejection a difficult line to tread.

\section{EP-256 POST-TRANSPLANT FOCAL SEGMENTAL GLOMERULOSCLEROSIS RECURRENCES ASSOCIATED WITH BOWEL OBSTRUCTION \\ Sarah Faggetter, Ebru Surgun, Elise Hennaut, Nathalie Tram, Benedetta Chiodini, Xenia Lolin, Khalid Ismaili, Brigitte Adams Hôpital Universitaire Des Enfants Reine Fabiola, Université Libre De Bruxelles (ulb), Belgium}

Abstract: One of the principal histopathological entities encountered in paediatric steroid resistant nephrotic syndrome (SRNS) is focal and segmental glomerulosclerosis (FSGS), which often leads to end-stage renal disease (ESRD). The rates of post-transplant FSGS recurrence vary from 6 to $59 \%$ and can lead to graft loss. Treating the relapse in transplanted patients is challenging but the outcomes are quite good with the current management. Therapeutic plasma exchange and rituximab are two of the standards treatments.

We present a case of a paediatric female patient, known for a renal transplantation secondary to SRNS with FSGS that led to terminal renal failure, who presented two episodes of recurrence both associated to a bowel obstruction. Therapeutic plasma exchange and Rituximab on the first episode, and only plasma exchange in the second, led to the complete remission of the proteinuria.

Although FSGS recurrences after renal transplant are well reported, the association with bowel obstruction has not been yet described to our knowledge.

\section{EP-257 EVOLUTION OF CALCIURIA AND CITRATURIA IN PATIENTS WITH IDIOPATHIC HYPERCALCIURIA.} A 20-YEAR LONGITUDINAL STUDY

Sandra Teresa Moraleda Mesa ${ }^{\text {, }}$, María Isabel Luis Yanes ${ }^{1}$, German Perez Suarez, ${ }^{2}$, Margarita Monge Zamorano ${ }^{1}$, Nayade Izquierdo Reyes ', Ismael Jose Dorta Luis ${ }^{1}$, Victor Manuel Garcia Nieto ${ }^{1}$

${ }^{1}$ Hospital Universitario Nuestra Señora De Candelaria

${ }^{2}$ Hospital Universitario Insular De Gran Canaria A

Introduction: Normalisation of calciuria in children with idiopathic hypercalciuria $(\mathrm{IH})$ was published by Aladjem et al. in 1996. Two Brazilian groups have described the association of IH with hypocitraturia. We have frequently observed that, in some families of children with IH, one of their parents with urolithiasis had hypocitraturia with normal calcitruria.

Material and methods: We studied 34 patients (12M, 22F) diagnosed with $\mathrm{IH}$ at the age of $7.9 \pm 3$ years (range: 1-14). Hypercalciuria was defined as calcium excretion of more than $4 \mathrm{mg} / \mathrm{kg} /$ day in two successive samples. Calciuria and citraturia were collected in the first urine of the day at the time of bone densitometry.

The first (D1) was performed at age $10.5 \pm 2.7$ years (range: $6.5-16.8$ ), the second (D2) at age $14.5 \pm 2.7$ years (range: 9.9-19.5) and the third (D3) at age $28.2 \pm 2.8$ years (range: $24.1-35.9$ ).

Results: A gradual decrease in calcium/creatinine $(\mathrm{p}=0.005)$ and citrate/ creatinine $(\mathrm{p}<0.001)$ ratios and an increase in calcium/citrate ratio $(\mathrm{p}=$ 
0.008) were observed. The percentage of patients with a calcium/ creatinine ratio higher than $0.20 \mathrm{mg} / \mathrm{mg}$ decreased from $100 \%$ at diagnosis to $52.9 \%(18 / 34)$ on D1 and to $26.5 \%(9 / 34)$ on both D2 and in adulthood (D3). The behaviour of the calcium/citrate ratio was uneven, with a decrease in the percentage of patients at lithogenetic risk from diagnosis $(15 / 28 ; 53.6 \%)$ to D1 $(13 / 32 ; 40.6 \%)$ and D2 $(8 / 28 ; 28.6 \%)$ and a marked increase in adulthood $(21 / 30 ; 70 \%)$.

Conclusions: It is difficult to explain why some of our patients with $\mathrm{IH}$ reduced, usually in adolescence and early adulthood, both calcium and citrate excretion, which has an impact on lithogenetic risk. The interpretation of these results is complex. Bone is the largest reservoir of alkaline salts in the body. We hypothesise that the late hypocitraturia observed in patients with $\mathrm{IH}$ would be an indirect sign of high functional osteoblastic activity. Increased proximal citrate reabsorption would denote an increased body production of bicarbonate which is necessary as osteoblastic activity increases, thus requiring increased inputs of alkali, calcium (normalised calciuria) and phosphate. Another option would be an acidification defect.

\section{EP-258 PEDIATRIC URINARY STONE DISEASE}

Ferhan Taş, Z.birsin Özçakar, Fatoş Yalçinkaya, Nilgün Çakar

Ankara Üniversity Faculty Of Medicine Departments Of Pediatrics

\section{Introduction:}

Environmental, metabolic, anatomic, infectious, nutritional and genetic factors play a role in the formation of urinary system stones. We aimed to evaluate the demographic and clinical features, etiologies and treatment approaches of pediatric urolithiasis.

Material and methods: Medical records of children with urolithiasis were evaluated retrospectively. Demographic data and medical history of the patients, serum and urine biochemical and metabolic analysis, blood gas analysis, urine stone analysis, imaging findings, medical and surgical treatments were recorded..

Results: The study included 177 female and 187 male patients (F/M:0,94). The mean age at diagnosis and follow-up duration were $4.97 \pm 5.00$ years and $40.4 \pm 38.3(6-194)$ months, respectively. The most common complaints were urinary tract infection (UTI) (23\%), discoloration of urine (12\%) and abdominal pain (11\%). Fifty-five percent of the patients had a history of UTI. At least one metabolic risk factor was found in $120(88 \%)$ of 137 patients who had all metabolic investigations for stone etiology. Of these 120 patients, $51 \%$ had hypercalciuria, $45 \%$ hypocitraturia, $42 \%$ hyperoxaluria, $25 \%$ hyperuricosuria and $10 \%$ cystinuria. Surgical interventions were performed in 86 patients (extracorporeal shock wave lithotripsy in 70 patients, ureteroscopy in 46, percutaneous nephrolithotomy in 16 and open surgery in 6 ). $71 \%$ of 226 patients with stone size $<5 \mathrm{~mm}$ and $54 \%$ of 125 patients with $\geq 5 \mathrm{~mm}$ were stone free at the last visit. A significant inverse correlation was found between stone size and stone-free rates $(p=0.002)$. During the follow-up, unilateral nephrectomy was performed in 3 patients ( 1 cystinuria, 1 xanthinuria, 1 hypercalciuria) due to non-functioning kidney, and 2 patients with primary hyperoxaluria developed chronic renal failure.

Conclusions: Metabolic causes are common in children with urinary stone disease. Therefore, understanding the etiology is important for the treatment of stone and prevention of new stone formation.

\section{EP-259 CALCULATED URINARY CALCIUM-OXALATE SATURATION (BCAOX) IS NOT SPECIFICALLY ELEVATED IN PATIENTS WITH PRIMARY HYPEROXALURIA}

Cristina Martin-higueras ${ }^{1}$, Wolfgang BÖhm ${ }^{2}$, Bernd Hoppe ${ }^{1}$

${ }^{1}$ German Hyperoxaluria Center

${ }^{2}$ Centre Hospitalier De Luxembourg

Introduction: Three different enzyme defects in primary hyperoxaluria $(\mathrm{PH})$ lead to endogenous oxalate overproduction and extremely elevated urinary oxalate excretion (UOx). Thus, urine should be supersaturated for calcium-oxalate $(\mathrm{BCaOx})$. But are computed calculation programs useful to express this supersaturation?

Material and methods: We calculated urinary $\mathrm{BCaOx}$ using the computed equilibrium program EQUIL2 in $\mathrm{PH}$ patients according to type, as well as in non-PH patients with urolithiasis (UL) or nephrocalcinosis (NC). Data from multiple $24 \mathrm{~h}$ urine collections per patient at different ages were available (table). All patients were in stable kidney function (eGFR $>45 \mathrm{ml} / \mathrm{min}$ ).

Results: Uox was higher in $\mathrm{PH}$ as compared to non-PH patients $(\mathrm{p}<$ 0.05 ), but not statistically different between PH types. Urinary calcium excretion was significantly lower in $\mathrm{PH} 1 . \mathrm{BCaOx}$ in $\mathrm{PH} 2$ patients (small number of urines available) was significantly higher.

\begin{tabular}{|c|c|c|c|c|c|c|}
\hline & PH 1 & PH 2 & PH 3 & $\mathrm{NC}$ & UL & Normal \\
\hline $\begin{array}{l}\mathbf{N}=\text { patients } / \\
\mathbf{N}=\text { urines }\end{array}$ & $57 / 239$ & $7 / 14$ & $17 / 30$ & $70 / 131$ & $146 / 252$ & \\
\hline $\mathbf{P}=$ percentile & $\begin{array}{l}\text { Median } \\
(25 / 75 \text { P) }\end{array}$ & $\begin{array}{l}\text { Median } \\
\text { (25/75 P) }\end{array}$ & $\begin{array}{l}\text { Median } \\
(25 / 75 \text { P) }\end{array}$ & $\begin{array}{l}\text { Median } \\
(25 / 75 \text { P) }\end{array}$ & $\begin{array}{l}\text { Median } \\
\text { (25/75 P) }\end{array}$ & \\
\hline Age & $\begin{array}{l}10.41 \\
(6.6-14.2)\end{array}$ & $\begin{array}{l}5.66 \\
(4.86-13.4)\end{array}$ & $\begin{array}{l}7.25 \\
(4.4-9.8)\end{array}$ & $\begin{array}{l}6.41 \\
(3.33-10.7)\end{array}$ & $\begin{array}{l}8.66 \\
(4.9-13.7)\end{array}$ & \\
\hline $\begin{array}{l}\text { Oxalate } \\
\mathrm{mmol} / 1.73 \mathrm{~m}^{2} / \mathrm{d}\end{array}$ & $\begin{array}{l}1.34 \\
(0.88-2.18)\end{array}$ & $\begin{array}{l}1.40 \\
(0.98-1.98)\end{array}$ & $\begin{array}{l}1.26 \\
(0.89-1.57)\end{array}$ & $\begin{array}{l}0.47 \\
(0.37-0.59)\end{array}$ & $\begin{array}{l}0.48 \\
(0.36-0.66)\end{array}$ & $<0.5$ \\
\hline $\begin{array}{l}\text { Calcium } \\
\mathrm{mg} / \mathrm{kg} / \mathrm{d}\end{array}$ & $\begin{array}{l}1.39 \\
(0.88-2.13)\end{array}$ & $\begin{array}{l}3.21 \\
(1.78-4.81)\end{array}$ & $\begin{array}{l}2.67 \\
(1.68-3.74)\end{array}$ & $\begin{array}{l}2.52 \\
(1.68-4.94)\end{array}$ & $\begin{array}{l}2.87 \\
(1.67-5.11)\end{array}$ & $<4$ \\
\hline $\begin{array}{l}\text { Citrate } \\
\mathrm{mmol} / 1.73 \mathrm{~m}^{2} / \mathrm{d}\end{array}$ & $\begin{array}{l}2.92 \\
(1.66-4.21)\end{array}$ & $\begin{array}{l}4.7 \\
(4.22-6.63)\end{array}$ & $\begin{array}{l}3.9 \\
(2.46-4.93)\end{array}$ & $\begin{array}{l}2.43 \\
(1.3-3.5)\end{array}$ & $\begin{array}{l}2.44 \\
(1.3-3.72)\end{array}$ & $\begin{array}{l}>1.6(\mathrm{f}) \\
>1.9(\mathrm{~m})\end{array}$ \\
\hline $\begin{array}{l}\mathrm{BCaOx} \\
\text { rel-units }\end{array}$ & $\begin{array}{l}4.8 \\
(2.56-6.66)\end{array}$ & $\begin{array}{l}8.7 \\
(3.88-11.48)\end{array}$ & $\begin{array}{l}5.63 \\
(3.95-6.48)\end{array}$ & $\begin{array}{l}4.35 \\
(2.6-6.65)\end{array}$ & $\begin{array}{l}4.31 \\
(2.61-7.15)\end{array}$ & $\begin{array}{l}<5.5(\mathrm{f}) \\
<8.4(\mathrm{~m})\end{array}$ \\
\hline
\end{tabular}

Conclusions: With the exception of the small $\mathrm{PH} 2$ group, $\mathrm{BCaOx}$ is similar in $\mathrm{PH}$ and non-PH patients. In PH1 this miscalculation is related to a rather lowish urinary calcium excretion. Therefore, calculation of $\mathrm{BCaOx}$ using computed programs is not a reliable parameter to define the definitively extreme $\mathrm{CaOx}$ supersaturation of urines from $\mathrm{PH}$ patients.

\section{EP-260 OPHTALMOLOGICAL INVOLVEMENT DURING PRIMARY HYPEROXALURIA TYPE}

Abir Boussetta ${ }^{1}$, Rym Maamouri ${ }^{2}$, Manel Jellouli ${ }^{1}$, Rihab Fatnassi ${ }^{3}$, Ouns Naija ${ }^{I}$, Tahar Gargah ${ }^{1}$

${ }^{I}$ Pediatric Nephrology Department, Charles Nicolle Hospital, Tunis, Tunisia; ${ }^{2}$ Ophtalmology Department, Habib Thameur Hospital, Tunisia; ${ }^{3}$ Faculty Of Medicine Of Tunis, Tunis, Tunisia

Introduction: To describe the characteristics of ophthalmologic involvement in children with primary hyperoxaluria type 1 ( $\mathrm{PH} 1)$.

Material and methods: This was a retrospective study over a period of 20 years. The patients included were those followed up for primary hyperoxaluria confirmed by biochemical assays or by molecular study in whom ophthalmological involvement was demonstrated. Ophthalmic examination included best corrected visual acuity (BCVA) testing and multimodal retinal imaging, including fundus photography and optical coherence tomography (OCT). In selected cases, fundus autofluorescence imaging was performed.

Results: A total of 11 patients from 70 children with primary hyperoxaluria were included, among them 8 girls and 3 boys. Infantile PH1 was diagnosed in 5 patients, and non-infantile PH1 in 5 children. The mean age at diagnosis of $\mathrm{PH}$ was $3.43 \pm 3.22$. The most frequent mutation was $\mathrm{I} 244 \mathrm{~T}$ in 6 cases, followed by G190R and the c.406 410 in one case respectively. A mutation of the AGXT gene could not be identified in 3 cases. The mean creatinine clearance was $29.8 \mathrm{ml} / \mathrm{min} / 1.73 \mathrm{~m}^{2} \mathrm{BA}$. All eyes $(\mathrm{n}=22)$ of PH1 patients revealed severe retinal alterations and oxalate deposits, including macular crystals and hyperpigmentations. Deposits appeared as focal hyperreflective subretinal lesions on OCT imaging and were hyperautofluorescent on autofluorescence images.

Conclusions: Severe ocular alterations may occur in patients with PH1, the natural history of (sub)retinal oxalate deposits, the pathogenesis of 
subretinal fibrosis, and exact factors influencing the overall severity of ocular disease manifestation remain to be determined.

\section{EP-261 PREVALENCE OF KIDNEY CALCULI IN IRANIAN} CHILDREN

Amin Sadat Sharif ${ }^{1}$, Mohsen Akhavan Sepahi ${ }^{2}$, Nakysa Hooman ${ }^{4}$ Anoush Azarfar ${ }^{17}$, Hamid Mohammadjafari ${ }^{7}$, Khadijeh Ghasemi ${ }^{3}$, Maryam Esteghamati ${ }^{5}$, Zahra Pournasiri ${ }^{6}$, Reza Dalirani ${ }^{6}$,

Nasrin Esfandiar ${ }^{6}$, Nasrin Esfandiar ${ }^{6}$, Ali Derakhshan ${ }^{8}$, Hadi Sorkhi ${ }^{9}$, Elham Emami ${ }^{10}$, Farzaneh Ghazanfaripour ${ }^{11}$, Banafsheh Arad ${ }^{12}$,

Simin Sadeghi-bojd ${ }^{13}$, Rama Naghshizadian ${ }^{14}$, Banafshe Dormanesh ${ }^{15}$, Kambiz Ghasemi ${ }^{5}$, Mojgan Mazaheri ${ }^{16}$, Sahar Sadr ${ }^{9}$,

Fatemeh Ghane Sharbaf ${ }^{17}$, Mohammad Reza Razavi ${ }^{2}$,

Fatemeh Emamghorashi ${ }^{18}$, Masoumeh Mohkam ${ }^{6}$, Alireza Eskandarifar ${ }^{14}$, Mahmood Maleknejad ${ }^{17}$

${ }^{1}$ Pediatric Department, Kashan University Of Medical Sciences, Kashan, Iran

${ }^{2}$ Department Of Pediatric Nephrology, School Of Medicine, Qom University Of Medical Sciences And Health Services, Qom, Iran

${ }^{3}$ Department Of Pediatrics Persian Gulf Shohada Hospital, Bushehr University Of Medical Sciences, Bushehr, Iran

${ }^{4}$ Aliasghar Clinical Research Development Center, Iran University Of Medical Sciences, Tehran, Iran

${ }^{5}$ Department Of Pediatric Nephrology, Clinical Research Development Center Of Children's Hospital, Hormozgan University Of Medical Sciences, Bandar Abbas, Iran

${ }^{6}$ Pediatric Nephrology Research Center, Research Institute Center For Children's, Shahid Beheshti University Of Medical Sciences, Tehran, Iran

${ }^{7}$ Pediatric Infectious Diseases Research Center, Communicable Diseases Institute, Mazandaran University Of Medical Sciences, Sari, Iran

${ }^{8}$ Shiraz, Nephro-urology Research Center, Shiraz University Of Medical Sciences, Shiraz Iran

${ }_{9}$ Non-communicable Pediatric Diseases Research Center, Health Research Institute, Babol University Of Medical Sciences, Babol, Iran

${ }^{10}$ Department Of Pediatric Nephrology, Clinical Research Development Center Of Children's Hospital, Shahrekord University Of Medical Sciences, Shahrekord, Iran

${ }^{11}$ Pediatric Department, Afzalipour Hospital, Kerman University Of Medical Sciences, Kerman, Iran

${ }^{12}$ Children Growth Research Center, Research Institute For Prevention Of Non-communicable Disease, Qazvin University Of Medical Science, Qazvin, Iran

${ }^{13}$ Genetics Of Non-communicable Disease Research Center, Zahedan University Of Medical Science, Zahedan, Iran

${ }^{14}$ Department Of Pediatric Nephrology, Clinical Research Development Center Of Children's Hospital, Kurdestan University Of Medical Sciences, Sanandaj, Iran

${ }^{15}$ Pediatric Department, Aja University Of Medical Sciences, Tehran, Iran

${ }^{16}$ Pediatric Department, Amiralmomenin Hospital, Semnan University Of Medical Sciences, Semnan, Iran

${ }^{17}$ Mashhad University Of Medical Sciences, Mashhad, Iran

${ }^{18}$ Ghadir Mother And Child Hospital, Shiraz University Of Medical Sciences, Shiraz, Iran

Introduction: The studies on the prevalence of kidney stones in Iranian children are very limited and only two studies have examined this issue and reported its prevalence of about $1 \%$. Children with nephrolithiasis have a high chance of developing recurrent stones, so they should be thoroughly evaluated. Due to little information in Iranian children, the aim of this study was to investigate the prevalence of kidney stones in this population.

Material and methods: This cross-sectional study investigates the prevalence of kidney stones in different university centers among outpatients and inpatients from March-December 2019 in Iran. Twenty-six pediatric nephrologists from 13 centers were participated in this study and completed questionnaire containing information on the total number of inpatients and outpatients and the number of kidney calculi in them. The diagnosis of the stone was based on radiological findings.

Results: A total of 97912 patients were examined, of which 20327 were hospitalized and the rest were outpatients. The prevalence of kidney stones was $1.5 \%$ among hospitalized patients and $3.2 \%$ among outpatients.

Conclusions: In this study, we examined a large population of children and assessed the prevalence of kidney stones in them. Also, we showed that the prevalence of kidney stones in Iranian children has an increasing trend compared to the previous two studies and most of them are outpatients. The total prevalence of stone in Iranian children was reported\%2.86. According to the few studies that have been done in this field and also to justify this upward trend it is recommended that more extensive studies should be performed in collaboration with more centers and demographic characteristics such as age, sex, stone composition, family history and diet.

\section{EP-262 THE INFLUENCE OF BODY MASS ON METABOLIC DISORDERS THAT CONTRIBUTE TO THE DEVELOPMENT OF UROLITHIASIS IN CHILDREN}

Sopińska Matgorzata

Military Institute Of Medicine

Introduction: Urolithiasis is a medical condition marked by presence of calculi in the kidney or urinary tract. These are composed of chemical substances that are normally or pathologically found in urine. About $2 \%$ of children are affected by the kidney stone disease. Another serious health issue is the prevalence of obesity that reached epidemic proportions among children and adults. The increasing incidence of urolithiasis in developed countries may be partially due to greater overweight and obese population. American studies revealed a relationship between obesity and incidence of urolithiasis in adults, especially among men. Metabolic disturbances predisposing to urolithiasis linked to excessive body weight include: low urine $\mathrm{pH}$, hypercalciuria, hyperoxaluria, hyperuricosuria and hypocytraturia.

Material and methods: The study consisted of 134 children (69 girls, 65 boys) aged 9-18 years with diagnosed urolithiasis. Patients with urolithiasis associated with urinary tract infection, urinary tract malformations, significant urinary retention and rare genetic determinants were excluded. Two study groups were distinguished: group I (95 children :45 girls, 50 boys) consisted of patients with BMI $<85$ centile, group II( 39 children: 24 girls, 15 boys) included children with BMI $\geq 85$ centile. Urolithiasis was diagnosed using abdominal ultrasound. All children underwent anthropometric measurements and laboratory tests. Excretion of following crystalloids was evaluated in 24hour urine collection and from a fasting second-void morning urine. All patients underwent height, body weight.

Results: Following results were obtained using the collected data:

- group with excessive body weight had statistically significant higher serum uric acid concentration compared to children with normal body weight ( $\mathrm{p}=0.000009)$. Uric acid concentration correlated statistically positively with the body weight of the subjects ( $\mathrm{p}=0.0001$ ),

- children with excessive body weight had statistically significant lower serum concentration of liver-produced 25-hydroxyvitamin D compared to children with normal body weight $(\mathrm{p}=0.007)$,

- children with normal body weight had higher daily urinary calcium excretion (mean $3.42 \mathrm{mg} / \mathrm{kg} /$ day) than children with excessive body weight (mean $2.75 \mathrm{mg} / \mathrm{kg} /$ day). This may be considered a trend $(p=0.059)$. No statistically significant differences were noted in the $\mathrm{Ca} /$ $\mathrm{Cr}$ ratio between the examined groups,

- children with excessive body weight had significantly higher urinary uric acid concentration in the second morning urine compared to children with normal body weight ( 0.26 vs. 0.23$)(\mathrm{p}=0.04)$. No statistically 
significant differences were noted in the daily urinary excretion of uric acid between the examined groups,

- there was a negative correlation between body weight and daily urinary uric acid excretion $(\mathrm{p}=0.002)$,

- children with normal body weight had significantly higher daily urinary oxalate excretion compared to children with excessive body weight $(0.39$ vs. 0.31$)(\mathrm{p}=0.049)$,

- daily urinary citrate excretion was significantly lower among children with excessive body weight compared to children with normal body weight (201.89 vs 277.72) ( $\mathrm{p}=0.040)$,

- there was a negative correlation between body weight and $\mathrm{Ca} / \mathrm{Cr}$ ratio in the second void urine $(\mathrm{p}=0.003)$. No statistically significant differences were noted in the daily urinary calcium excretion between the examined groups, - there was a negative correlation between body weight and daily urinary magnesium excretion $(\mathrm{p}=0.001)$ as well as $\mathrm{Mg} / \mathrm{Cr}$ ratio in the second morning urine $(\mathrm{p}=0.0001)$,

- there was no statistically significant correlation between the concentration of liver-produced 25-hydroxyvitamin D and daily urinary calcium excretion,

- furthermore, there was no statistically significant correlation between the concentration of liver-produced 25-hydroxyvitamin D and the daily urinary calcium excretion and $\mathrm{Ca} / \mathrm{Cr}$ ratio once patients were divided into groups with normal and excessive body weight.

Conclusions: 1. In the examined material, urolithiasis was more often observed in children with normal body weight, which may indicate that excessive body weight is not a risk factor for the disease.

2. Excessive body weight promoted the occurrence of hyperuricemia and reduced hepatic metabolite of vitamin $\mathrm{D}$. Low excretion of citrates, which are crystallization inhibitors, was more frequently observed in this group. 3. Hypercalciuria and hyperoxaluria were found more frequently in children with normal body weight, and higher magnesium excretion than in overweight children, which did not protect against deposits.

4. Vitamin D concentration did not affect urinary calcium excretion, regardless of body weight.

\section{EP-263 URINARY STONE DISEASE IN CHILDREN UNDER 2 YEARS OF AGE \\ Aysun Çaltik Yilmaz ${ }^{1}$, Necla Unal ${ }^{2}$, Asli Çelebi Tayfur ${ }^{2}$, Bahar Büyükkaragöz. ${ }^{3}$ \\ ${ }^{1}$ Başkent University, Department Of Pediatric Nephrology \\ ${ }^{2}$ University Of Health Sciences, Keçiören Training And Research Hospital Department Of Pediatrics \\ ${ }^{3}$ GazI University, Department Of Pediatric Nephrology}

Introduction: The purpose of this study is to investigate the demographic, etiological, clinical characteristics and prognosis of UL in children under 2 years of age. The mainly etiological factors in pediatric patients with UL can be categorized as metabolic, urinary system infection related, anatomical anomalies causing urinary stasis and idiopathic.

Material and methods: Sixty children were diagnosed with UL under the age of 2 years were recruited to study. All patients have been completely evaluated for etiological factors. Patients have been classified as age at diagnosis, sex, presenting symptoms, family history, physical examination findings, localization and size of urinary stones, laboratory and radiological findings, urinary biochemical examinations, medical and surgical treatments and prognosis.

Results: The average age at diagnosis is $8.5 \pm 4.5$ months and the male to female ratio was 1.6 . Forty one of the cases $(68.3 \%)$ were diagnosed less than 1 year of age. The family history of stone disease was found in 41 $(68.3 \%)$ cases. Restlessness was noted as the main symptom. Hypercalciuria was found to be the most common metabolic factor. Eight $(15 \%)$ of patients receiving surgical treatment. Sixteen of the patients $(26.7 \%)$ received medical treatment and eight patients received both surgical and medical treatments. The recurrence rate was found $15 \%$.
Conclusions: The most important thing is to identify the high-risk populations and take measures if necessary. In all patients with infantile stone disorder, anatomical and metabolic tests must be done.

EP-264 AGE-SPECIFIC EXCRETION OF CALCIUM, OXALATE, CITRATE, AND GLYCOSAMINOGLYCANS AND THEIR RATIOS IN HEALTHY CHILDREN AND CHILDREN WITH UROLITHIASIS

Daniel Turudic ${ }^{1}$, Anja Tea Golubic ${ }^{2}$, Marko Bilic ${ }^{3}$, Danko Milosevic ${ }^{4}$

${ }^{I}$ University Hospital Centre Zagreb, Department Of Pediatrics, Zagreb, Croatia

${ }^{2}$ University Hospital Centre Zagreb, Department Or Nucelar Medicine And Radiation Protection, Zagreb, Croatia

${ }^{3}$ University Hospital "sveti Duh", Department Of Urology, Zagreb, Croatia

${ }^{4}$ University Of Zagreb, School Of Medicine, Zagreb, Croatia

Introduction: We analyzed children with urolithiasis with age- and gender-matched healthy children as a retrospective study to ascertain any differences in urinary stone formation.

Material and methods: We used 24-h urine of $\mathrm{Ca} \mathrm{mmol} / \mathrm{mmol}$ creatinine), Ox ( $\mathrm{mmol} / \mathrm{mol} \mathrm{cr})$, Cit ( $\mathrm{mmol} / \mathrm{mol} \mathrm{cr})$, GAGs $(\mathrm{mg} / \mathrm{mmol} \mathrm{cr}), \mathrm{Ca} /$ Cit $(\mathrm{mol} / \mathrm{mmol}), \mathrm{Ox} / \mathrm{GAGs}(\mathrm{mmol} / \mathrm{g}), \mathrm{Ox} / \mathrm{Cit}(\mathrm{mmol} / \mathrm{mmol}), \mathrm{Ox} /$ $(\mathrm{Cit} \times \mathrm{GAGs})(\mathrm{mol} \mathrm{Ox} \times \mathrm{mol} \mathrm{cr}) /(\mathrm{mol} \mathrm{Cit} \times \mathrm{g} \mathrm{GAGs})$, and $\mathrm{Cit} / \mathrm{GAGs}$ $(\mathrm{mmol} / \mathrm{g})$ were analyzed. Data analysis was performed by using Statistica for Windows version 8 and GraphPad Prism version 5. Additionally, J48 classifier was used to construct classification model for discrimination between subgroups.

Results: Calcium ( $\mathrm{mmol} / \mathrm{mmol}$ creatinine) and the calcium/citrate ratio $(\mathrm{mol} / \mathrm{mmol})$ are the only variables that differentiate children before puberty from healthy children (ROC analysis confirmed only calcium/ citrate as a significant variable with cut-off value $>0.84$ ). Pubertal /postpubertal children are distinguished from age- and gender-matched healthy children by the following variables: citrate ( $\mathrm{mmol} / \mathrm{mol}$ creatinine), calcium / citrate ( $\mathrm{mol} / \mathrm{mmol})$, oxalate / glycosaminoglycans ( $\mathrm{mmol} / \mathrm{g}$ ), oxalate / citrate ratios $(\mathrm{mmol} / \mathrm{mmol}$ ) and oxalate / (citrate $\times$ glycosaminoglycans) (mol oxalate $\times$ mol creatinine $) /($ mol citrate $\times \mathrm{g}$ glycosaminoglycans), all were confirmed by ROC analysis with cut-off values $\leq 327.87,>1.02,>11.24,>0.12,>0.03$, respectively.

Conclusions: These results indicate a different risk of urinary stones development before puberty vs. pubertal/postpubertal children and increasing importance (deficiency) of citrate and glycosaminoglycans in such children. J48 classifier confirmed the importance of the oxalate/ (citrate $\times$ glycosaminoglycans) and the calcium/citrate ratios with the practically applicable classification tree for distinguishing between pubertal/postpubertal children with urolithiasis with age- and gendermatched healthy children.

\section{EP-265 DIGESTIVE MANIFESTATIONS OF PRIMARY HYPEROXALURIA TYPE1(PH1) IN CHILDREN}

Souhir Gazzeh, Selsabil Nouir, Sameh Mabrouk, Meniar Tfifha,

Houda Ajmi, Fadwa Majdoub, Chemli Jaleleddine, Noura Zouari,

Abroug Saoussen

Pediatric Department, Sahloul Hospital, Tunisia

Introduction: PH1 is a group of rare inherited diseases, linked to autosomal recessive transmission. It's characterized by excessive production of oxalate leads to exessive urinary oxalate excretion. The clinical presentation ranges from asymptomatic through to isolated or recurrent renal stones, nephrocalcinosis and renal impairment.

Insoluble oxalate is progressively stored by all organs, gastrointestinal involvement remains rare,this work has to illustrate the characteristics of digestive impairment during HP1 
Material and methods: Cross sectional observational study of the paediatric cases of PH1 managed in the department of paediatrics in the University hospital (Sousse - Tunisia)

Results: 60 cases of PH1 were followed up during study period, 5 patients $(15.1 \%)$ had gastro-intestinal involvment, mean age of digestive signs was 16.1 years with an average time of $35+/-27$ months compared to diagnosis.

the desease was more frequent in girls ( 3 girls Vs 2 boys), clinical manifestations were recurrent vague abdominal pain in 3 cases, abdominal bloating in one case and chronic diarrhea in the last case.

Physical examination found no abnormalities outside of abdominal bloat in one case. Oesophago-gastro-duodenoscopy was performed in all patients show no abnormalities. The abdominal ultrasound and abdominal scan locate calcium deposits in the gastric wall, colic or ileal loops. All children were symptomatically treated with an evolution towards death Conclusions: Systemic impairment in HPI appears at an advanced stage of the disease, Digestive involvelent of HP Is a serious impact during the évolution of the disease, it schoud be researched in all systemic forms of the disease.

\section{EP-266 NUTRITIONAL FACTORS AND INFANTILE UROLITHIASIS \\ Aysun Çaltik Yilmaz ${ }^{1}$, Necla Unal ${ }^{2}$ \\ ${ }^{1}$ BaŞkent University, Department Of Pediatric Nephrology \\ ${ }^{2}$ University Of Health Sciences, KeÇiÖren Training And Research Hospital Department Of Pediatrics}

Introduction: The main etiological factors in pediatric patients with UL can be categorized as metabolic, urinary tract infection, anatomical anomalies causing urinary stasis, and idiopathic for which the cause cannot be demonstrated. The aim of this study is to investigate the effect of nutritional factors on those diagnosed with idiopathic infantile urolithiasis.

Material and methods: The study group included 44 infants without a predictable etiologic factor for urolithiasis. The control group consisted of 60 ages and gender matched full healthy infants. Each infant in the patient and control groups was carefully questioned by the same researcher for their nutritional characteristics.

Results: Forty-four patients included in the study had a total of 65 stones were detected in urinary system. None of the patients in study group had bladder stone. In 20 of the patients, there were stones in both kidneys. The duration of intake of breast milk in children with urolithiasis was found to be longer than those without urolithiasis. No statistical differences were observed between the study and control groups during the duration of breast milk consumption. In the study groups, the duration of use of the formula was longer than in control groups $(\mathrm{p}=0.003)$. The daily intake of cows milk and dairy products was much higher in the group of infants with urolithiasis $(\mathrm{p}=0.033$ and $\mathrm{p}=0.001)$.

Conclusions: We concluded that dietary conditions could also be a risk factor for idiopathic urolithiasis at the conclusion of our research. We believe that nutritional factors for infantile urolithiasis should be better described, in addition to genetic, anatomical, and metabolic factors.

\section{EP-267 BUROSUMAB IN TWO PATIENTS WITH X-LINKED HYPOPHOSPHATAEMIA}

Silvia Acuñas-soto, Ana Castellano-martinez, Virginia Roldan-cano, Moises Rodriguez-gonzalez

Puerta Del Mar University Hospital

Introduction: X-linked hypophosphataemia (XLH) is a rare genetic disorder with $\mathrm{x}$-linked dominant inheritance caused by mutation on the PHEX gen, which increases fibroblast growth factor 23 causing loss of phosphorus at the proximal tubule. Low height and bone deformities, especially at the lower limbs, are typical. Conventional treatment consists of oral supplements with phosphorus and calcitriol. Since 2018, burosumab is approved as a novel therapeutic option with promising results. Our objective is to describe two cases of XLH treated with Burosumab.

Material and methods: Data collection from medical records of two paediatric patients with XLH under burosumab therapy in a tertiary hospital.

Results: The first case is a boy diagnosed with XLH at 22 month-old during the study of low height and bowing limbs. He had hypophosphataemia with hyperphosphaturia and a mutation in hemizygosis on the PHEX gene. Conventional treatment was initiated, with poor biochemical and radiographic control, requiring hemiepiphysiodesis at 4 years-old due to the significant deformity in the lower limbs. At 6 years-old, burosumab therapy was started. After three years of treatment, serum phosphorus values have increased to near normal values with radiological improvement and better quality of life. The second case is a girl diagnosed with XLH at 24 month-old during genu varus study. She had hypophosphataemia with hyperphosphaturia and a mutation in heterozygosis on the PHEX gene. She received conventional treatment, with persisting hypophosphataemia and worsening deformity of the lower limbs, requiring hemiepiphysiodesis at 5.5 yearsold, which was effective, but hypophosphataemia persisted with poor tolerance to oral treatment. Therefore, burosumab treatment was started at 9 year-old. After 1.5 years, hypophosphataemia has improved with radiological progress.

Conclusions: Our two patients have very different phenotypic expression. It is important to know the disease in order to suspect it and start early treatment, with special interest in new therapies. In our short-term experience, burosumab is an excellent therapeutic option for XLH in children, as it improves their quality of life and allows rapid biochemical stabilization without significant side effects.

\section{EP-268 PATIENT DRIVEN RESEARCH IN NEPHROPATHIC CYSTINOSIS IN SPAIN: A TOOL FOR BETTER CARE}

Gema Ariceta, Grupo Cistinosis -cystinosis Patient Advocacy GroupPediatric Nephrology. Hospital Universitari Vall D Hebron. Barcelona

Introduction: Patient driven research is a key tool to improve knowledge in the field of rare diseases which facilitates research focus on patient and caregivers' needs.

Objectives: to better understand current characteristics of patients with cystinosis in Spain, quality of care, changes on diagnosis and multidisciplinary approach over time.

Material and methods: Anonymized telephone surveys among patients with nephropathic cystinosis and/or caregivers (mothers in 94\%) were performed by the cystinosis patient advocacy group (PAG), after informed consent/assent to participate.

Results: Out of 40 patients and families represented at the cystinosis PAG, 34 (85\%) -18 male \& 16 female- agreed to participate but 6 adult patients refused. Patient characteristics:

Current age median(range) 18(3-50) years. Age of diagnosis: cystinosis was identified at 6.7 months (mean) and $<2$ years in $82.3 \%$, with 2 patients identified beyond the 3 rd decade. Decreasing mean time diagnosis delay from birth (months) was observed over decades: 129.4 (19671976), 118.5 (1977-1986), 43.2 (1987-1996), 23.1 (1997-2006) and 9.8 (2007-2016) respectively. Kidney transplantation prevalence by patient age (years) was $0 \%<10 ; 25 \%$ between $11-20 ; 83 \% 21-30$ and $100 \%>30$. Multidisciplinary care: $100 \%$ of patients were followed by nephrologist and ophthalmologist. $12.5 \%$ of adults remained under pediatric care. Frequency of visits are higher in pediatrics. $72.2 \%$ of pediatrics and 62 . $5 \%$ of adult patients see additional specialists (endocrinologist, gastroenterologist, orthopedic surgeon and neurologist).

Patient education: all children were students. Adult distribution: primary education $25 \%$, secondary $31.25 \%$, university $43.75 \%$. $86.1 \%$ of pediatric patients' caregivers worked as professionals and $66.7 \%$ of adult ones. 
Perceived QoL in pediatric caregivers was reduced due school absenteeism and learning difficulties, whereas in adult patients absence to work, professional limitations and social issues predominated.

Conclusions: This patient driven research project describes the contemporary clinical picture of patients with cystinosis in Spain, characterized by earlier diagnosis, later progression to renal failure and better multidisciplinary care over time, both in children and adults.

EP-269 STUDY DESIGN AND METHODOLOGY OF BONAPH1DE, A PROSPECTIVE OBSERVATIONAL STUDY OF PATIENTS WITH PRIMARY HYPEROXALURIA TYPE 1 Felix Knauf $^{1}$, Jaap Groothoff ${ }^{2}$, Christian Conradt ${ }^{3}$, David Danese ${ }^{3}$, Thomas Brown ${ }^{3}$, John Lieske ${ }^{4}$

${ }^{1}$ Department Of Nephrology And Medical Intensive Care, Charite Universitatsmedizin Berlin, Berlin, Germany

${ }^{2}$ Department Of Pediatric Nephrology, Emma Childrens Hospital, Amsterdam Umc, University Of Amsterdam, Amsterdam, The Netherlands

${ }^{3}$ Alnylam Pharmaceuticals, Cambridge, Ma, Usa

${ }^{4}$ Division Of Nephrology And Hypertension, Mayo Clinic, Rochester, Mn, Usa

Introduction: Primary hyperoxaluria type 1 (PH1) is a rare, progressive, serious, and life-threatening autosomal recessive disease, resulting in increased production of oxalate, the key toxic metabolite in PH1. Excretion of excess oxalate by the kidneys leads to recurrent kidney stones, nephrocalcinosis, progressive kidney disease, and multiorgan damage from systemic oxalosis. Lumasiran, a subcutaneously administered RNAi, is the first therapy to be approved by the EC and FDA for the treatment of PH1. Here, we present the study design of BONAPH1DE ${ }^{\mathrm{TM}}$, an observational study to characterize the long-term real-world safety and effectiveness of lumasiran in patients of all ages with PH1.

Material and methods: BONAPH1DE is a global, multicenter, prospective, observational, longitudinal study designed to characterize the longterm, real-world safety of lumasiran in patients with PH1. This study will also evaluate the natural history and progression of patients with $\mathrm{PH} 1$, and characterize the long-term, real-world effectiveness of lumasiran. Patients at participating sites are eligible to enroll in this study with a documented diagnosis of PH1, regardless of treatment status, provided they are not enrolled in a clinical trial for any investigational agent. Patients will be managed and treated per routine clinical practice; the protocol does not recommend the use of any specific treatments, visits or procedures. No medication is provided as a part of study participation. Data collected will include patient characteristics (general and PH1-related), laboratory assessments (including urinary oxalate excretion and plasma oxalate concentration), clinical outcomes, and safety outcomes of interest.

Results: BONAPH1DE is expected to begin enrollment in Q3 2021.

Conclusions: BONAPH1DE will collect real-world data in patients of all ages to further evaluate the long-term safety and effectiveness of lumasiran for the treatment of PH1. In addition, these data are expected to complement existing disease registries that describe the natural history of PH1 and characterize factors that affect the course of disease.

\section{EP-270 PRESENTING FEATURES OF NEPHROCALCINOSIS AND UROLITHIASIS IN LITHUANIAN CHILDREN - A SINGLE CENTER EXPERIENCE 2016 - 2020 \\ Austeja Ivaskeviciene, Violeta Sevcenko, Birute Burnyte, Karolis Azukaitis, Augustina Jankauskiene, Rimante Cerkauskiene Clinic Of Pediatrics, Institute OfClinical Medicine, Faculty Of Medicine, Vilnius University, Lithuania}

Introduction: Urolithiasis (UL) and nephrocalcinosis (NC) are rare in children, but their incidence and prevalence continue to rise. For the majority of individuals NC / UL has a multifactorial etiology involving genetic factors. This study aims to describe the clinical presentation of UL / NC in children.

Material and methods: A retrospective study of all children ( $0-18$ years) referred to Vilnius University Hospital Santaros Klinikos for NC / UL between 2016-2020 was performed. Patients were stratified into 3 age categories: <1, 1-7 and 8-18 years. The following data was analyzed additionally: (i) symptoms; (ii) urinary tract infections (UTI); (iii) potentially predisposing conditions; (iv) family history; and (v) results of genetic testing for NC / UL (if performed).

Results: Analysis included 279 children ( $52 \%$ boys). Mean age of the patients was $3 \pm 4.98$ years: 153 were $<1$ year, 64 were $1-7$ years and 62 were $8-18$ years old. Presenting symptoms encompassed abdominal pain in $56(20 \%)$, hematuria in $62(22 \%)$, UTI in $27(9.6 \%)$, non-specific symptoms in $10(3.5 \%)$ and asymptomatic / incidental finding in 124 (44\%) patients. Abdominal pain and hematuria were more frequent in older children while infants were mostly asymptomatic (Table). The most common potentially predisposing condition was hypervitaminosis D $(\mathrm{n}=60,21 \%)$. Family history of NC / UL was reported in $28(10 \%)$ cases and genetic mutations were detected in $13(6 \%)$ patients.

\begin{tabular}{llll}
\hline Age group (years) & Abdominal pain & UTI & Hematuria \\
$<1$ & 0 & $12(7.8 \%)$ & $18(11.8 \%)$ \\
$1-7$ & $19(29.7 \%)(4$ non- & $7(10.9 \%)$ & $19(29.7 \%)$ \\
& $\quad$ specific and 15 colic) & & \\
$>8$ & $37(59.7 \%)$ colic & $8(12.9 \%)$ & $25(40.3 \%)$ \\
\hline
\end{tabular}

Conclusions: Older children are more likely to have symptomatic presentation of NC / UL. Genetic testing may have important role in selected cases of children with NC / UL.

\section{EP-271 MINI URETERORENOSCOPY TREATMENT OF} UROLITHIASIS IN CHILDREN IN AN ENDEMIC REGION Ahmet Gokhan Guler ${ }^{1}$, Ali Erdal Karakaya ${ }^{1}$, Ahmet Burak DoĞan ${ }^{1}$, Yasar Kandur ${ }^{2}$

${ }^{1}$ Faculty Of Medicine,sutcu Imam University, Kahramanmaras, Turkey

${ }^{2}$ Faculty Of Medicine,kirikkale University, Kirikkale, Turkey

Introduction: Urolithiasis is an important disease that can lead to permanent kidney dysfunction and severe clinical symptoms in pediatric patients. We aimed to present our findings of rigid ureteroscopy combined with Ho:YAG laser lithotripsy in pediatric patients .

Material and methods: We retrospectively reviewed the ultrasonography (US), and medical records of pediatric patients with a urolithiasis of pediatric patients.

Results: Sixty-one pediatric patients $(\mathrm{M} / \mathrm{F}=38 / 23)$ with urolithiasis were enrolled in this study. The mean age was $6.7 \pm 4.9$ years (range 3 month- 17 years). Thirteen $(34.2 \%)$ male patients need an urgent operation while the corresponding number was $4(17.4 \%)$ in female patients. The difference between two sexes was statistically non-significant $(\mathrm{p}=0.156)$. Thirty-two male patients $(84.2 \%)$ and $12(52.2 \%)$ females patients had a successful operation. The difference between the two sexes was statistically significant $(\mathrm{p}=0.019)$. The ratio of female patients with right-sided stone was greater than the corresponding rates of male patients $(\mathrm{p}=<0.001)$.

Conclusions: Considering that more than one operation can be performed in infants and children with mini ureteroscopy and holmium laser, safe and effective stone treatment can be performed. Flexible or PNL may be preferred in kidney calyces.

EP-272 MINI URETERORENOSCOPY TREATMENT OF UROLITHIASIS IN CHILDREN IN AN ENDEMIC REGION

Ahmet Gokhan Guler ', Ahmet Burak Do Ğan ', Ali Erdal Karakaya ${ }^{\text {, }}$ YaŞar Kandur ${ }^{2}$ 
${ }^{1}$ Department Of Pediatric Surgery, Faculty Of Medicine, Sutcu Imam University, Kahramanmaras, Turkey

${ }^{2}$ Department Of Pediatric Nephrology, Faculty Of Medicine, Kirikkale University, Kirikkale, Turkey

Introduction: Urolithiasis is an important disease that can lead to permanent kidney dysfunction and severe clinical symptoms in pediatric patients. We aimed to present our findings of rigid ureteroscopy combined with Ho:YAG laser lithotripsy in pediatric patients .

Material and methods We retrospectively reviewed the ultrasonography (US), and medical records of pediatric patients with a urolithiasis of pediatric patients.

Results: Sixty-one pediatric patients $(\mathrm{M} / \mathrm{F}=38 / 23)$ with urolithiasis were enrolled in this study. The mean age was $6.7 \pm 4.9$ years (range 3 month- 17 years). Thirteen $(34.2 \%)$ male patients need an urgent operation while the corresponding number was $4(17.4 \%)$ in female patients. The difference between two sexes was statistically non-significant ( $\mathrm{p}=0.156$ ). Thirty-two male patients $(84.2 \%)$ and $12(52.2 \%)$ females patients had a successful operation. The difference between the two sexes was statistically significant $(\mathrm{p}=0.019)$. The ratio of female patients with right-sided stone was greater than the corresponding rates of male patients $(\mathrm{p}=<0.001)$. There was no difference between infants $(<3$ years of age) and toddlers $(>3$ years of age) with respect to gender, side of stone, procedural success, and need for urgent surgery ( $>0.05$ ) (Table 2). Regression analysis showed that female gender was a risk factor for a failed attempt $(\mathrm{OR}=-$ $0.313,95 \%$ CI: $0.052-0.528 ; \mathrm{p}=0.0018$ ).

Conclusions: Considering that more than one operation can be performed in infants and children with mini ureteroscopy and holmium laser, safe and effective stone treatment can be performed. Flexible or PNL may be preferred in kidney calyces.

EP-273 A REPORT OF A SEVERE CASE OF X-LINKED HYPOPHOSPHATEMIA DUE TO A NOVEL PHEX MUTATION Madalena Almeida Borges ${ }^{1}$, Rute Baeta Baptista ${ }^{1}$, Ana Laura Fitas ${ }^{2}$, Telma Francisco ${ }^{\prime}$, Margarida Abranches ${ }^{I}$

${ }^{1}$ Unidade De Nefrologia, Área Da Pediatria, Chulc

${ }^{2}$ Unidade De Endocrinologia, Área Da Pediatria, Chulc

Introduction: X-linked hypophosphatemia (XLH), a disorder caused by mutations in PHEX, impairs the inactivation of FGF-23 resulting in renal wasting of phosphorus, hypophosphatemia and rickets. Early diagnosis and treatment are important to prevent subsequent sequelae. We report a severe case of XLH due to a novel PHEX mutation. Our aim is to raise awareness about this disease and the potential factors contributing to its severity.

Material and methods: Clinical case description.

Results: A five-year-old girl from Guinea-Bissau was referred to our tertiary hospital for evaluation of progressive limb deformities since the age of two. Physical examination showed enamel defects, absent superior incisive teeth, genu valgum, severe tibial curvature in the sagittal plane, widening of metaphyseal ends and height-for-age index below -3 z-score. Laboratory workup revealed elevated alkaline phosphatase $(1251 \mathrm{U} / \mathrm{L})$, hypophosphatemia $(2.8 \mathrm{mg} / \mathrm{dL})$, borderline low serum calcium levels (total calcium $9.1 \mathrm{mg} / \mathrm{dL}$; Ca++ $1.08 \mathrm{mmol} / \mathrm{L})$; low 25-hydroxyvitamin-D (18 ng/mL), high 1,25dihydroxycholecalciferol (124.18 pg/mL) and PTH $(231.7 \mathrm{pg} / \mathrm{mL})$; tubular reabsorption of phosphate $(57 \%)$ and TmP/GFR $(2.22 \mathrm{mg} /$ $\mathrm{dL}$ ) were low. The $\mathrm{x}$-rays showed metaphyseal widening, diaphyseal trabeculation, rarefaction and pseudofractures. The patient was started on phosphate and calcium supplementation, alfacalcidol, and cholecalciferol. Additionally, she underwent orthopedic surgery twice resulting in partial improvement of the genu valgum. At the age of eight, a novel heterozygous PHEX mutation (c.156_174del) was identified. She started receiving treatment with burosumab, showing clinical improvement.
Conclusions: The patient was referred to us only three years after the identification of bone deformities, which led to a significant delay in the genetic diagnosis and appropriate treatment initiation. The coexisting vitamin D deficiency may have also contributed to the severity of the disease presentation. Additionally, we question whether the truncate mutation may have played a role in this case severity. Considerable controversy exists regarding the correlation between truncating mutations and a more severe phenotype in XLH.

\section{EP-274 HEMATURIA IN A BOY AFTER BOTOX TREATMENT FOR LOWER LIMB SPASTICITY}

Gamze Akca Dinc, Fatma Semsa Cayci, Husniye Nese Yarali, Melek Isik, Hazal Cavusoglu

The University Of Health Sciences, Ankara City Hospital, Ankara, Turkey

Introduction: Hematuria is a rare condition defined by the side effect of botulinum toxin injection when it is used for overactive bladder. However, there are few reports of hematuria when it is used for limb spasticity.

Material and methods: Here we report a rare case of hematuria following a botulinum toxin injection for lower limb spasticity in a 6-year-old male patient with a history of cerebral palsy.

Results: He had a history of recurrent nose bleeding. Of note, he had no hematuria in the past. The patient had normal hemogram, biochemical analysis, C3 and urinary ultrasonography. ANA and dsDNA were negative. Macroscopic hematuria started three days after botulinum toxin injection ( 35 units for right Adductor muscles, 15 units for right Gracilis muscle, 35 units for left Adductor muscles, 15 units for left Gracilis muscle) and resolved without any additional treatment. However, microscopic hematuria continued for 3 weeks. Further hematological investigations showed high levels of collagen ADP and collagen epinephrine. Although studies on the effect of botulinum toxin treatment on coagulation are needed, there are studies showing that it may play a role in the coagulation cascade by preventing the formation of fibrin.

Conclusions: We want to emphasize the importance of the evaluation of children for the underlying hematological disorders in patients who develop hematuria after Botulinum toxin injection for limb spasticity.

\section{EP-275 IMPROVING DIAGNOSTIC ACCURACY AND GUIDELINE ADHERENCE BY REGISTRY-BASED PROSPECTIVE MONITORING: THE CASE OF MULTIDRUG RESISTANT NEPHROTIC SYNDROME}

Stefanie Drovandi ${ }^{1}$, Victor Perez-beltran ${ }^{2}$, Gema Ariceta ${ }^{2}$, Imeraj Amantia ${ }^{3}$, Atif Awan ${ }^{4}$, Irena Bałasz-chmielewska ${ }^{5}$, Francesca Lugani ${ }^{6}$, Elena Levtchenko ${ }^{7}$, Marjorie Viallon ${ }^{8}$, Miriam Zacchia ${ }^{9}$, Luisa Murer ${ }^{10}$, Germana Longo ${ }^{10}$, Lorna Rouhaud ${ }^{11}$, Marina Vivarelli ${ }^{12}$, Roberta Fenoglio ${ }^{13}$, Agnes Trautmann ${ }^{14}$, Franz Schaefer ${ }^{15}$

${ }^{1}$ Division Of Nephrology, Dialysis And Transplantation, Department Of Internal Medicine, Ospedale Policlinico San Martino And University Of Genoa, Genoa, Italy

${ }^{2}$ Department Of Pediatric Nephrology, University Hospital Vall Dhebron, Barcelona, Spain

${ }^{3}$ Department Of Nephrology, Asst Papa Giovanni Xxiii, Bergamo, Italy

${ }^{4}$ National Paediatric Haemodialysis Centre And Renal Transplant Unit, Temple Street Childrens University Hospital, Dublin, Ireland

${ }^{5}$ Department Of Pediatrics, Nephrology And Hypertension, Medical University Of Gdansk, Gdansk, Poland

${ }^{6}$ Division Of Nephrology, Dialysis, Transplantation, Irccs Giannina Gaslini, Genoa, Italy

${ }^{7}$ Department Of Pediatric Nephrology And Development And Regeneration, University Hospitals Leuven, University Of Leuven, Leuven, Belgium 
${ }^{8}$ Department Of Pediatric Nephrology, Rheumatology And Dermatology, University Childrens Hospital, Lyon, France

9 Department Of Translational Medical Sciences, University Of Campania "luigi Vanvitelli", Naples, Italy

${ }^{10}$ Pediatric Nephrology, Dialysis And Transplant Unit, Department Of Womens And Childs Health, Hospital Of Padua, Padua, Italy

${ }^{11}$ Néphrologie Pédiatrique, Institut Imagine, Université De Paris, Cetres De Référence Sni Et Marhea, Hôpital Necker, Aphp, Paris, France

12 Division Of Nephrology And Dialysis, Bambino Gesù Childrens Hospital, Irccs, Rome, Italy

${ }^{13}$ Nephrology And Dialysis Unit, San Giovanni Hospital And University Of Turin, Turin, Italy

14 Division Of Pediatric Nephrology, Center For Pediatrics And Adolescent Medicine, University Of Heidelberg, Heidelberg, Germany

${ }^{15}$ The Erknet Workgroups For Inherited And Acquired Glomerulopathies

Introduction: The diagnosis "idiopathic multidrug resistant nephrotic syndrome" (MDRNS, Orphacode 567550) was recently introduced to describe patients in whom proteinuria neither responds to steroid nor to other immunosuppressive therapies. The recent IPNA SRNS Guideline recommends performing comprehensive genetic screening in these cases to rule out a genetic disease cause.

Material and methods: Adherence to this recommendation is monitored in the European Rare Kidney Disease Registry (ERKReg) as one of 55 key performance indicators (KPIs) at 47 pediatric and adult nephrology centers. At a first assessment in 12/2020, NGS panel or exome screening had been performed in a surprisingly low fraction of MDRNS patients (1/18 adult and 2/46 pediatric) reported at 14 expert units. We subsequently inquired the centers about the causes and circumstances of this apparent diagnostic performance issue.

Results: Updates were received on 59 of the 61 cases. In 38 of these, genetic screening had not or incorrectly been reported or performed after the initial data entry. A genetic diagnosis had been established in 7 patients. In 24 patients MDRNS was confirmed by negative genetic screening. 13 patients showed late responsiveness to 2nd-line immunosuppressive therapy and 3 were re-classified as secondary nephrotic syndrome. One patient was deceased. The documentation of genetic testing and/or reclassification of diagnosis increased KPI compliance from $4.3 \%$ to $82.1 \%$ in the pediatric and from $5.5 \%$ to $30.7 \%$ in the adult MDRNS cohort. In 8 of the 11 remaining patients genetic testing was scheduled for the next patient visit, which will improve KPI compliance even further.

Conclusions: This proof-of-concept study demonstrates how guideline adherence can be prospectively monitored and optimized by case documentation in an international registry. It illustrates the potential of online KPI monitoring for quality improvement, but also the substantial effort required to monitor data quality and alert treating physicians in a clinical network setting.

\section{EP-276 URINARY CD80 LEVEL IN IDIOPATHIC NEPHROTIC SYNDROME OF CHILDHOOD; IS IT A RELIABLE DIAGNOSTIC AND PROGNOSTIC BIOMARKER? \\ Parisa Honarpisheh, Nakysa Hooman , Hasan Otukesh \\ Department Of Pediatric Nephrology lali Asghar Children Hospital}

Introduction: The most common type of nephrotic syndrome in children is minimal change disease (MCD). one of the suggested mechanisms in its pathogenesis is the presence of circulating factors that cause damage to podocytes. One of these circulating factors is the CD80 (B7-1) molecule which is said to be expressed in kidney tissue in MCD patients. The aim of this study was to assess whether urinary level of CD80 is different in histopathological types of INS and also between responsive and nonresponsive types and by this way specify whether CD80 can be used as a diagnostic and prognostic biomarker.

Material and methods: This case-control study included 35 healthy children and 47 patients with INS (biopsy-proven $\mathrm{MCD}=21$, non-
$\mathrm{MCD}=8 ; \mathrm{FSGS}=6$ and MPGN=2). patients with age $<1 \mathrm{yr}$ and $\mathrm{GFR}<60 \mathrm{cc} /$ $\mathrm{min} / 1.73 \mathrm{~m} 2$ were excluded from the study. Morning urine samples were centrifuged at 2000rpm for 20 minutes and supernatant was then removed and urinary creatinine and CD80 levels were measured using a special kit (ELISA Genie) and with enzyme-linked immunosorbent assay.

Results: A significant increase in urinary CD80, normalized to urinary creatinine, was found in patients with MCD in relapse $(215.44 \mathrm{ng} / \mathrm{gr} \mathrm{cr})$ compared to those in remission $(3.23 \mathrm{ng} / \mathrm{gr} \mathrm{cr}, \mathrm{p}=0.002)$ or those with non-MCD in relapse ( $4.45 \mathrm{ng} / \mathrm{gr} \mathrm{cr}, \mathrm{p}=0.002)$. No significant differences were seen between urinary CD80 in patients with MCD in remission compared to those with non-MCD. Using ROC (receiver operating characteristic) curve a cutoff value of $83.5 \mathrm{ng} / \mathrm{g}$ creatinine was obtained, with a sensitivity of $87 \%$ and specificity of $100 \%$ to differentiate between MCD-relapse and non-MCD-relapse and AUC (the area under the ROC curve) was 0.98 . Using ROC curve, a CD80 cutoff level of $108.7 \mathrm{ng} / \mathrm{gr} \mathrm{Cr}$ with a sensitivity of $71 \%$ and specificity of $100 \%$ was obtained to differentiate between MCD-relapse and MCD-remission and AUC was 0.85. the mean urine $\mathrm{CD} 80$ level between the steroid-responsive patient in relapse $(511.78 \mathrm{ng} / \mathrm{gr} \mathrm{cr}$ ) compared to non-responsive patients in relapse (161.83) was statistically significant $(\mathrm{p}=0.00)$. No significant differences were seen between urinary CD80 in responsive (15.25) and nonresponsive groups (6.31) in remission phase $(\mathrm{p}=0.219)$

Conclusions: This study confirmed that urinary CD80 level was significantly higher in MCD and steroid-responsive children than in the nonMCD and non-responsive patients respectively. Therefor high level of CD80 is correlated with good prognosis and steroid responsiveness in children with nephrotic syndrome and can be used as a non-invasive diagnostic and prognostic biomarker in idiopathic nephrotic syndrome.

\section{EP-277 LONG-TERM OUTCOME AFTER CYCLOPHOS- PHAMIDE TREATMENT IN CHILDREN WITH NEPHROTIC SYNDROME}

Åsa Funkquist Md, Sverker Hansson Phd, Per Brandström Phd

Institute Of Clinical Sciences, University Of Gothenburg

Introduction: Minimal change nephrotic syndrome (MCNS) is the most common form of nephrotic syndrome among children. Corticosteroids is the first treatment of choice but many children relapse and need repeated or prolonged courses of steroids and are defined as frequently relapsing (FRNS) or steroid dependent (SDNS) nephrotic syndrome. To reduce the risk of side effects of steroids alternative treatment with cyclophosphamide (CYP), cyclosporin, tacrolimus, mycophenolate mofetil and rituximab has been used. The objectives of this study were to determine the long-term outcome after CYP and factors associated with remission.

Material and methods: Retrospective note review of children with biopsy-proven MCNS at the Queen Silvia Children's Hospital, Sahlgrenska University Hospital between 2004 and 2016. We correlated time to first relapse to gender, steroid-dependence, age at presentation, age at start of treatment and cumulative dose of CYP.

Results: 16 boys and 13 girls were treated with CYP during the period. Median age at presentation of nephrotic syndrome was 4.1 years and CYP was introduced at a median age of 6.6 years. The median follow-up period after CYP was 6.5 years. 13 children (45 $\%)$ remained in remission during the follow-up period. $16(55 \%)$ children had by the end of follow-up at least one relapse. Median time to relapse was 11 months. The last noted relapse occurred after 3.5 years. There was no difference in median age at presentation or cumulative dose of CYP between the group with or without relapse. Children with FRNS had a higher remission rate than patients with SDNS although the difference was not significant $(\mathrm{p}=0.07)$.

Conclusions: Almost half of the children were in long-term remission at the end of follow-up after treatment with CYP and therefore in no need of more steroids. When relapse occurred it was within the first years after treatment. Treatment with CYP seemed to be more beneficial within the group FRNS. 
EP-278 NEUROBLASTOMA PRESENTING WITH NEPHROTIC SYNDROME AND LIVER FAILURE IN AN INFANT: A CASE REPORT.

Houda Nassih, Rabiy El Qadiry, Aicha Bourrahouat, Imane Ait Sab B Pediatric Ward, Department Of Pediatrics, Child And Mother Hospital, Mohammed Vi University Hospital Center, Marrakesh, Morocco

Introduction: We present a case of nephrotic syndrome revealing a neuroblastoma in an infant

Material and methods: A case report of an 18-month-old nonconsanguineous boy presenting with generalized edema evolving for 7 days

Results: The infant was well-appearing. Medical history was unremarkable. The child wasn't under any medication. Clinical examination found a massive hepatomegaly of $11 \mathrm{~cm}$. Meanwhile, there were no signs of heart failure, nor chronic diarrhea or undernutrition. Dipstick found massive proteinuria and microscopic hematuria. Laboratory tests found hypoalbuminemia of $11 \mathrm{~g} / \mathrm{L}$, proteinuria of $3 \mathrm{~g} / 24 \mathrm{~h}$, and low PTT of $37 \%$. The child had subclinical cholestasis (GGT of $156 \mathrm{ui} / \mathrm{L}$ ) and mild hepatic cytolysis (transaminases of three times the upper normal limit). Renal function was normal with urea of $0.24 \mathrm{~g} / \mathrm{L}$ and creatinine of $3 \mathrm{mg} / \mathrm{L}$. Abdominal ultrasound found a mass enveloping the neighboring vessels. Abdominal scan showed a retroperitoneal calcified tumor resulting in the encasement of the inferior vena cava and a partial extrinsic obstruction of the vessel. Puncture biopsy of the mass followed by pathological study confirmed the diagnosis of neuroblastoma. The child was managed by symptomatic measures (IV albumin with diuretics), and neoadjuvant chemotherapy followed by surgery and maintenance chemotherapy. Evolution was marked by complete resolution of the generalized edema, negative proteinuria, and normal PTT by the second month of treatment. Conclusions: Neuroblastomas can present with features of inferior vena cava syndrome. That is why abdominal ultrasound is mandatory in every infant with nephrotic syndrome. Treatment is based on chemotherapy followed by surgery. Complete remission of the nephrotic syndrome is usual.

\section{EP-279 NEPHROTIC SYNDROME: DISEASE IMPACT BEYOND CHILDHOOD}

Laura Bucchino $^{1}$, Enrico Bodrero ${ }^{2}$, Roberta Camilla ${ }^{3}$, Silvia Pieretti ${ }^{3}$, Francesca Mattozzi ${ }^{3}$, Bruno Gianoglio ${ }^{3}$, Licia Peruzzi ${ }^{3}$

${ }^{1}$ Pediatric School, University Of Eastern Piedmont, Novara, Italy

${ }^{2}$ Pediatric School, University Of Turin, Turin, Italy

${ }^{3}$ Pediatric Nephrology Unit, Regina Margherita Childrens Department

Introduction: Long term outcome of childhood nephrotic syndrome (NS) beyond pediatric age is difficult to evaluate due to patient dispersion after transition to adult centers. Aim of this study was to retrieve long term follow up information on disease behavior and complications.

Material and methods: Data from a retrospective cohort of childhood onset steroid-sensitive (SS), steroid-dependent (SD) and steroid-resistant (SR) NS over 18 at January 2021 were collected.

Results: 46 subjects were identified: 36 SS (78\%), 29 SD (63\%), 14 SR (30\%; 9 primary resistant and 5 secondary resistant). Among SR 7/14 (50\%) were multi drugs resistant. The median time of follow up was 19.5 years (IQR 9.0).

18 patients (39\%) [3 SS (6.5\% of total patients), 11 SD (24\%), 3 SR $(6.5 \%)]$ achieved remission defined as absence of relapses during the previous 5 years while being off-therapy.

9 patients reached CKD stage 5 and underwent renal transplant ( $8 \mathrm{SR}$ and $1 \mathrm{SS}), 3$ of them had disease recurrence on the graft.

Moreover, we analyzed the relapse frequency during adult age: 15 SS patients presented at least 1 relapse (33\% of total patients), 15 remained SD $(33 \%)$ and 5 SR (11\%) under other maintenance therapy (CNI, RTX or MMF).
22 patients (48\%) suffered from at least one complication among arterial hypertension, obesity, adult height impairment, sterility, the most frequent being height impairment (18 patients; 39\%).

Conclusions: NS pediatric onset has a strong impact in adult life, due to disease relapses, prolonged therapy or permanent long term complications: it is remarkable that in our cohort relapses continued over 18 in SS and SD.

Long-term follow up multicenter studies are needed to offer better treatment planning and counseling during childhood.

\section{EP-280 SCLEROSTIN LEVELS IN CHILDREN WITH IDIOPATHIC NEPHROTIC SYNDROME}

Argyroula Zampetoglou ${ }^{~}$, Varvara Askiti ${ }^{1}$, Kyriaki Karavanaki ${ }^{l}$, Charalampos Tsentidis ${ }^{2}$, Konstantinos Stefanidis ${ }^{3}$, Andromachi Mitsioni $^{1}$

${ }^{I}$ P. And A. Kyriakou Children Hospital

${ }^{2}$ Geniko Kratiko Nikaias

${ }^{3}$ Mitera Children Hospital

Introduction: Children with idiopathic nephrotic syndrome (INS) are usually managed with long-term administration of glucocorticoids and cysclosporin, which have a negative skeletal effect. Serum sclerostin is a glycoprotein, secreted by osteocytes which acts as a negative regulator of bone formation. Limited data exist regarding sclerostin in glucocorticoid-treated patients and its relation with bone mineral density (BMD). Our aim was to study sclerostin in INS children and adolescents and its relation with various metabolic bone markers and BMD.

Material and methods: In this cross-sectional study various bone metabolism markers of 65 pediatric INS patients [mean \pm SD age: $9.0 \pm 3.9$ years, median INS (IQR) duration: 3.8 years $(2.7-5.7)]$ were measured and compared with those of a similar number of controls (age:8.7 \pm 3.7 years). About half of the patients $(53.8 \%)$ were on glucocorticoids on examination day. Lumbar and total body BMD were measured with a dual energy x-ray absorptiometry (DEXA).

Results: Patients with INS had significantly lower sclerostin $(44.3 \pm 12.2$ vs $50.6 \pm 17 \mathrm{pmol} / \mathrm{L}, \mathrm{p}=0.027)$ and $25(\mathrm{OH}) \mathrm{D} 3$ levels $(21.3 \pm 9.2$ vs 25.4 $\pm 8.7 \mathrm{ng} / \mathrm{ml}, \mathrm{p}=0.019)$, and lower Z-score of BMD in lumbar spine $(-0.2$ \pm 1.0 vs $0.3 \pm 0.95, \mathrm{p}=0.02)$ and total body $(-0.02 \pm 0.96$ vs $0.63 \pm 0.77$, $\mathrm{p}=0.001$ ), compared to controls. There was a positive correlation of sclerostin with alkaline phosphatase $(r=0.33, p=0.008)$ and PTH $(\mathrm{r}=0.26, \mathrm{p}=0.038)$ in INS patients. Sclerostin was correlated negatively with time since diagnosis $(r=-0.36, p=0.004)$ and positively with age at diagnosis $(r=0.42, p=0.001)$. Moreover, sclerostin was lower in INS patients treated with cyclosporine A compared to those without (40.0 \pm 13.0 vs $47.7 \pm 10.5 \mathrm{pmol} / \mathrm{L}, \mathrm{p}=0.011$ ). A negative correlation was also noted between sclerostin levels and months of cyclosporine exposure $(\mathrm{r}=-0.42$, $\mathrm{p}=0.001$ ).

Conclusions: Sclerostin and BMD were lower in pediatric INS patients compared to controls. Sclerostin was associated with cyclosporine, but not with glucocorticoid treatment. Sclerostin levels were affected by disease duration, age at diagnosis and cyclosporine A in INS patients.

\section{EP-281 SEVERE ACUTE KIDNEY INJURY IN A CHILD WITH NEPHROTIC SYNDROME: WHAT WAS THE TRIGGER?}

Bahar Büyükkaragöz, Burcu Yazicioğlu, Sevcan A. Bakkaloğlu, Oğuz Soylemezoğlu, Necla Buyan

Gazi University, Department Of Pediatric Nephrology, Ankara, Turkey

Introduction: Nephrotic syndrome (NS), a common nephrologic disorder in childhood, may manifest with important complications like infection, thromboembolism, acute kidney injury (AKI) and hypovolemia. However, severe AKI presenting itself with anuria in the course of NS is quite unusual and rarely reported. 
Material and methods: Herein, we present a pediatric patient who developed severe acute kidney injury soon after the diagnosis of NS, as a case report.

Results: Nine year-old previously healthy girl was admitted with the clinical and laboratory findings of NS (serum albumin: 1,7 g/dl, spot urine protein/creatinine: 27). She had recent history antibiotic and ibuprofen use for acute pharyngitis. Despite albumin and furosemide treatment, as she became anuric on the third day of hospitalization, hemodialysis was immediately started and pulse methylprednizolone was given. Renal biopsy revealed minimal mesangial proliferation with widespread acute tubular injury findings. She remained completely anuric for seven days. Urine output restarted beginning from the $11^{\text {th }}$ day of hospitalization. Overall 11 sessions of hemodialysis were performed. She was discharged after 15 days of follow-up with normal daily urine volume. After six weeks of oral prednisolone, complete remission in NS was achieved. Steroids were discontinued at the end of fifth month. On the $10^{\text {th }}$ month of follow-up, she was in good clinical condition with normal serum creatinine, albumin and urine protein levels.

Conclusions: Acute tubular necrosis was the histopathologic diagnosis underlying anuric AKI in the setting of NS. We believe that heavy proteinuria leading to tubular obstruction, presence of decreased effective intravascular volume due to severe hypoalbuminemia, and use of nonsteroid anti-inflammatory drug (ibuprofen) may have caused acute tubular necrosis. This case emphasizes the importance of awareness for possibility of severe AKI in NS due to multifactorial causes.

\section{EP-282 NIVOLUMAB-ASSOCIATED IGA NEPHROPATHY IN A CHILD WITH MALIGNANT MELANOMA \\ Gizem Yildiz, Meral Torun Bayram, Salih Kavukcu, Alper Soylu \\ Dokuz Eylul University Medical Faculty, Department Of Pediatric Nephrology}

Introduction: Nivolumab is increasingly used in the treatment of many cancers. The kidney damage related to nivolumab is usually caused by acute interstitial nephritis. However, glomerulonephritis (GN) associated nivolumab has been encountered with increasing frequency. Here, we report a patient who developed IgA nephropathy (IgAN) after using nivolumab.

Material and methods: A seven-year-old male patient was admitted with proteinuria. In his history, it was learned that two years ago malignant melanoma developed on the scalp, tumor excision was performed, various chemotherapy drugs were used, finally nivolumab treatment was initiated and proteinuria was determined after four cycles. On physical examination, blood pressure was normal and there were no edema. In laboratory tests, complete blood count, serum creatinine, electrolytes, $\mathrm{HCO} 3$ values were normal, albumin was $3.4 \mathrm{~g} / \mathrm{dL}$. Urine density was 1020 , protein (+3), erythrocyte (-) in urine analysis, 5-6 erythrocytes in microscopy. $\mathrm{Na}, \mathrm{P}, \mathrm{Ca}, \mathrm{Mg}$, uric acid excretions were normal, protein excretion was $130 \mathrm{mg} / \mathrm{m}^{2} /$ hour in the urine. Tests (C3/4, ANA, ANCA) performed for proteinuria were normal. Nivolumab-associated proteinuria was considered and the treatment was discontinued. Renal biopsy was performed because of the persistence proteinuria and development of microcopic hematuria. In the light microscopy; enlargement of mesangial matrix, increase in mesangial cellularity, endocapillary proliferation, mesangial $\operatorname{IgA}(+3), \operatorname{IgG}(-), \operatorname{IgM}($ minimal), C1q (-), Kappa (-), Lambda $(+3)$ was detected. The patient was diagnosed with IgAN with these findings and prednisolone was started. Proteinuria decreased and hematuria regressed in the first month. Complete recovery occurred in the sixth month.

Results: The mechanisms of IgAN due to nivolumab remain unclear. For treatment, while only drug discontinuation is sufficient in some cases, others may recovery with immunosuppressives.

Conclusions: Close monitoring of kidney function, proteinuria and hematuria, as well as early biopsy and intervention in renal involvement are important during the use of nivolumab.
EP-283 AGE AT ONSET OF STEROID SENSITIVE NEPHROTIC SYNDROME IN CHILDREN AND TIME TO FIRST RELAPSE

Polina Nyrkova, Nadezda Savenkova

Saint-petersburg State Pediatric Medical University, Russian Federation

Introduction: The aim of the study was to assess age at onset and time to first relapse of steroid sensitive nephrotic syndrome (NS) in children who developed and didn't develop steroid dependency.

Material and methods: The retrospective and prospective study was carried out to assess age at onset and time to first relapse in 118 children with infrequent and frequent relapsing steroid sensitive at onset NS.

Results: Median age at onset of steroid sensitive NS in 118 children was 3,5 years $[2,3-5,2]$, male $75(64 \%)$ and female $43(36 \%)$. Out of 118 children with steroid sensitive at onset NS $60(51 \%)$ and $58(49 \%)$ had infrequent and frequent relapsing course of NS, respectively. Out of 118 children with steroid sensitive at onset NS 75 (64\%) had steroid dependency. Statistically significant differences were established: median time to first relapse from onset in 75 children with NS who developed steroid dependency was 4 months [4-5], while median time to first relapse from onset in 43 children with NS who didn't develop steroid dependency was 11 months [7-17], $(p<0,0001)$; median age at onset in children with NS who developed and didn't developed steroid dependency was 3,4 years $[2,2-4,8]$ and 4,2 years $[3,0-5,8]$, retrospectively $(p<0,05)$, median age at first relapse in children with NS who developed and didn't develop steroid dependency was 3,8 years $[2,6-5,1]$ and 5,7 years $[4,0-7,4]$, respectively $(\mathrm{p}<0,001)$.

Conclusions: Age at onset and first relapse in children with NS who developed steroid dependency was younger than in those who didn't develop steroid dependency. Children with NS, who relapsed within first half a year after disease onset, more often developed steroid dependency.

\section{EP-284 OUTCOMES OF RENAL TRANSPLANTATION IN CHILDREN WITH NEPHROTIC SYNDROME}

Jacqueline Sit, Stephanie Dufek, Becky Foxler, Anthony Kershaw, Stephen D Marks, Aoife Waters

Great Ormond Street Hospital For Children Nhs Foundation Trust

Introduction: Disease recurrence post-transplant occurs in $17-34 \%$ of congenital nephrotic syndrome (CNS), and in up to $80 \%$ of steroid resistant nephrotic syndrome (SRNS). The objectives of this study are to describe and compare the transplantation outcomes in CNS, Mendelian or syndromic SRNS and non-Mendelian SRNS.

Material and methods: A retrospective review was undertaken on all patients with NS who were transplanted between 2000 and 2020 at Great Ormond Street Hospital for Children NHS Foundation Trust. Data on histology, genetics, clinical phenotype, transplant and latest estimated glomerular filtration rate (eGFR) were collected.

Results: A total of 35 patients with NS were transplanted, with $100 \%$ and $88.6 \%$ patient and renal allograft survival respectively at median followup of 7.6 years. The overall recurrence rate was $31.4 \%$, with median time to remission of 21.5 days. Patients who experienced recurrence had significantly worse eGFR at last follow-up $(\mathrm{p}=0.004)$.

Table 1. Data presented as median (interquartile range) or number (\%).

\begin{tabular}{|c|c|c|c|c|}
\hline & CNS $(n=19)$ & $\begin{array}{c}\text { Mendelian or } \\
\text { Syndromic } \\
\text { SRNS }(\mathrm{n}=6)\end{array}$ & $\begin{array}{c}\text { Non-Mendelian } \\
\text { SRNS }(\mathrm{n}=10)\end{array}$ & p-value \\
\hline Genetic mutations & $\begin{array}{l}\text { NPHS1 (89.5\%) } \\
\text { WT1 (5.3\%) } \\
\text { Negative (5.3\%) }\end{array}$ & $\begin{array}{l}\text { NPHS2 } \\
(33.3 \%) \\
\text { MYO1E } \\
(16.7 \%) \\
\text { Negative (50\%) }\end{array}$ & All negative & - \\
\hline \multirow{2}{*}{$\begin{array}{l}\text { Age at transplant } \\
\quad \text { (years) }\end{array}$} & $3.1(2.2,5.1)$ & $9.2(5.2,12.6)$ & $9.0(5.9,12.2)$ & 0.002 \\
\hline & $9(47.4 \%)$ & $3(50.0 \%)$ & $7(70.0 \%)$ & 0.50 \\
\hline
\end{tabular}




\begin{tabular}{|c|c|c|c|c|}
\hline \\
\hline \multicolumn{5}{|l|}{$\begin{array}{c}\text { transplant } \\
\text { Post-transplant: }\end{array}$} \\
\hline \multicolumn{5}{|l|}{$\begin{array}{l}\text { Post-transplant: } \\
\text { Duration of } \\
\text { follow-up } \\
\text { (vears) }\end{array}$} \\
\hline Recurrence & $2(10.5 \%)$ & $0(0 \%)$ & $9(90 \%)$ & $<0.001$ \\
\hline Remission & $2 / 2(100 \%)$ & - & 6/9 (66.7\%) & - \\
\hline Thromboembolism & $2(10.5 \%)$ & $2(40.0 \%)$ & $2(22.2 \%)$ & 0.29 \\
\hline $\begin{array}{l}\text { eGFR at last } \\
\text { follow-up } \\
\left(\mathrm{ml} / \mathrm{min} / 1.73 \mathrm{~m}^{2}\right)\end{array}$ & $58.0(40.3,68.0)$ & $\begin{array}{l}65.0 \\
\quad(52.6,69.4)\end{array}$ & $32.0(10.0,45.5)$ & 0.004 \\
\hline
\end{tabular}

Conclusions: Patients with non-Mendelian SRNS had significantly worse transplantation outcomes, with $90 \%$ recurrence and worse renal function. Recurrence has also been observed in CNS with NPHS1 mutations, all of which showed favourable responses. This highlights the importance of pre-transplant genetic testing in SRNS, as it can help prognosticate outcomes and enable a personalised approach to patient management.

\section{EP-285 NEPHROTIC SYNDROME (INS) AND TYPE 1 DIABETES MELLITUS (DMI): TEMPORAL COINCIDENCE OR A COMMON BACKGROUND? \\ Cristina Julia Blazquez Gomez, ${ }^{1}$, Mar Espino Hernandez ${ }^{2}$, Irene Fuentes Muñoz ${ }^{1}$, Joao Pedro Vieira Dos Santos ${ }^{1}$, Julia Vara MartÍn ${ }^{2}$, Joaquin Ramirez, Fernandez ${ }^{1}$ \\ ${ }^{1}$ Hospital Universitario Príncipe De Asturias \\ ${ }^{2}$ Hospital Universitario 12 De Octubre}

Introduction: Idiopathic Nephrotic Syndrome (INS) and Type1 Diabetes Mellitus (DM1) are common in paediatrics, but their association is rare. The incidence of INS is $1-7 / 100,000$ and that of DM1 $17.7 / 100,000$ children-year. In both diseases there is evidence of a genetic and immunological basis.

Material and methods: We report two children with INS and DMI, clinical presentation, laboratory, immunological and genetic test.

Results: Case 1: 15-year-old male with medical history of allergic diseases. At 4 years of age steroid-dependent INS was diagnosed and treated with levamisole and cyclophosphamide. At 14 years of age, after five years in drug-free remission, he presented a new relapse, and while receiving steroids $\left(40 \mathrm{mg} / \mathrm{m}^{2} / 48 \mathrm{~h}\right)$ glycosuria with hyperglycaemia was detected without symptoms of DM1. Laboratory studies: $\mathrm{HbA1C} 6.6 \%$, peptide C $0.9 \mathrm{ng} / \mathrm{mL}$, anti-GAD 98.2 U/mL and HLA-DQ2. Due to poor glycaemic control and partial remission of SNI, he received mycophenolate mofetil (MMF), allowing steroid withdrawal. He is currently in remission of INS with MMF and adequate metabolic DM1 control with insulin therapy. Case 2: 15-year-old-girl without prior medical history. At 3 years of age was diagnosed with steroid-dependent INS, treated with MMF for three years without subsequent relapses. At the age of 10, after 3 years of remission without treatment INS, she debuted with cardinal symptoms of DM1. Laboratory results: anti-GAD $73.9 / \mathrm{mL}$, anti-IA2 $3054 \mathrm{U} / \mathrm{mL}$, anti-insulin $2.17 \mathrm{U} / \mathrm{mL}$, anti-ZnT $324 \mathrm{U} / \mathrm{mL}$ and HLA-DQ2-DQ4. No genetic mutation was identified. After starting with subcutaneous insulin therapy with an insulin therapy pump, the patient now has good glycaemic control and is currently in remission for his SNI.

Conclusions: Association of INS and DM1 in paediatrics is extremely rare, although it may be a temporal coincidence. In patients with long-follow-up of DM1, kidney damage is well documented. In our cases, SNI preceded DM1 diagnosis. A common genetic and immunological background that predisposes patients to both disorders may be possible.
EP-286 RITUXIMAB TREATMENT OF STEROID, CYCLOSPORINE AND MYCOPFENOLATE- DEPENDENT NEPHROTIC SYNDROME - ONE CENTER EXPERIENCE

Iwona Ogarek, Elżbieta Szczesny-choruz, Ewa Wierzchowska-slowiaczek, Joanna Kwinta-rybicka, Anna Moczulska, Dorota Drożdż

Jagiellonian University Medical College, Department Of Pediatric

Nephrology And Hypertension, Krakow, Poland

Introduction: Children with nephrotic syndrome predominantly respond well to corticosteroid therapy, nonetheless $30 \%$ of those patients have frequent relapses and a steroid-dependent course of disease. Immunosuppressive drugs are being used in treatment of steroid-dependency, including rituximab in the last years- a chimeric monoclonal antibody, which selectively binds CD20 antigen on lymphocytes B, causing apoptosis of those cells.

The aim of thy study was asssessment of efficacy and safety of rituximab in patients with steroid-dependent nephrotic syndrome, in whom previous, long-standing, multidrug immunosuppressive therapy (prednisone, CsA, MMF) gave no grounds for drug dose reduction and/or resulted in significant side effects.

Material and methods: 12 patients, who met the criteria of steroid-dependent, cyclosporin-dependent, MMF-dependent nephrotic syndrome were enrolled in the study. Rituximab was given in single, recommended dose of $375 \mathrm{mg} / \mathrm{m}^{2}$. Evaluation included treatment effect at 6 months, as well as indications for subsequent doses.

Results: The mean time of remission after first rituximab infusion was $9,21 \pm 3,89$ months. 11 patients remained with no relapse for at least 6 months. In 8 patients within a month after RTX infusion CsA and prednisone treatment was discontinued completely, in others the doses were significantly reduced. In all patients the dose of MMF was reduced. Moreover a relevant reduction of serum creatinine, cystatin $\mathrm{C}$ concentration and BMI has been achieved. None of the patients had significant adverse effects of the given treatment.

Conclusions: Rituximab appears to be an effective and safe drug for patients with steroid-, cyclosporin- and MMF-dependent nephrotic syndrome in the aspect of long-term remission, and withdrawal of steroid therapy and calcineurin inhibitors.

\section{EP-287 INFANTILE NEPHROTIC SYNDROME WITH PLCE1 MUTATION; TREATMENT OF CYCLOSPORINE - 3-YEAR FOLLOW-UP}

Demet Tekcan ${ }^{1}$, Hülya Nalçacioğlu ${ }^{1}$, Hülya Gözde Önal ${ }^{1}$, Fatih Özaltin ${ }^{2}$, Özlem Aydog ${ }^{1}$

${ }^{1}$ Ondokuz Mayis University Faculty Of Medicine, Pediatric Nephrology, Samsun, Turkey

${ }^{2}$ Hacettepe University Faculty Of Medicine, Pediatric Nephrology, Nephrogenetics Laboratory, Ankara, Turkey

Introduction: Congenital and infantile nephrotic syndrome is a rare disease with edema, proteinuria, hypoalbuminemia, and may cause end-stage renal failure. They may occur due to perinatal infections and mutation of genes involving structural and functional proteins in the glomerular filtration barrier. Although PLCE1 is the major gene for diffuse mesangial sclerosis, its mutation has also been reported in patients with steroid-resistant nephrotic syndrome with focal segmental glomerulosclerosis. This report presents a case with infantile nephrotic syndrome with PLCE1 gene mutation treated with Cyclosporine.

Material and methods: A four-month-old female was referred to our clinic after detected incidental proteinuria. Her parents were first-degree cousins, and her mother's grandmother had a history of dialysis due to kidney failure of unknown origin. Physical examination was normal at admission. Initial laboratory evaluation; creatinine $0,19 \mathrm{mg} / \mathrm{dl}$, albumin $1.9 \mathrm{~g} / \mathrm{dl}, 24$-hour urine protein $147 \mathrm{mg} / \mathrm{m}^{2} / \mathrm{h}$, complement three and com- 
plement four levels were normal, viral tests were negative. Biopsy was detected as focal segmental glomerulosclerosis. A genetic test was sent, and steroid therapy was initiated. There was no response to full-dose steroid treatment for eight weeks, and the steroid treatment was tapered and stopped. We started enalapril. A homozygous mutation (Exon 23: C.5276C > T (p. S1759L), Homozygous) was detected in the Phospholipase C epsilon 1 (PLCE1) gene. During the follow-up, cyclosporine was added to her treatment. In the first year of cyclosporine treatment, the spot urine protein/creatinine ratio decreased to $1.1 \mathrm{mg} / \mathrm{mg}$ creatinine, and albumin increased to $3.7 \mathrm{~g} / \mathrm{dl}$. Our patient was considered to have a partial response to cyclosporine.

Results: At the last control, she was three years old, using enalapril and cyclosporine, creatinine $0,5 \mathrm{mg} / \mathrm{dl}$ (GFR $75,1 \mathrm{ml} / \mathrm{min} / 1,73 \mathrm{~m} 2$ ), albumin $3.1 \mathrm{~g} / \mathrm{dl}, 24$ hour urine protein was $67 \mathrm{mg} / \mathrm{m}^{2} / \mathrm{h}$.

Conclusions: Most PLCE1 mutation cases are not responsive to treatment with steroid and cyclosporine. In our case we had a partial response with cyclosporine.

\section{EP-288 NEPHROTIC SYNDROME WITH MUTATION IN SPHINGOSINE-1-PHOSPHATE LYASE: 6 CASES \\ Tugba Tastemel Ozturk ${ }^{1}$, Nur Canpolat ${ }^{2}$, Seha Saygili ${ }^{2}$, Umut Selda Bayrakci ${ }^{3}$, Oguz Soylemezoglu ${ }^{4}$, Fatih Ozaltin ${ }^{\prime}$, Rezan Topaloglu ${ }^{I}$ \\ ${ }^{1}$ Department Of Pediatric Nephrology, Hacettepe University Faculty Of Medicine, Ankara, Turkey \\ ${ }^{2}$ Department Of Pediatric Nephrology, Cerrahpasa Faculty Of Medicine, Istanbul University-cerrahpasa, Istanbul, Turkey \\ ${ }^{3}$ Department Of Pediatric Nephrology, Ankara City Hospital, Ankara Yildirim Beyazit University Faculty Of Medicine, Ankara, Turkey \\ ${ }^{4}$ Department Of Pediatric Nephrology, Gazi University Faculty Of Medicine, Ankara, Turkey}

Introduction: This study aimed to determine the characteristics and outcomes of nephrotic syndrome patients with sphingosine-1-phosphate lyase (SGPL1) mutation.

Material and methods: Nephrotic syndrome patients with SGPL1 mutation diagnosed in Hacettepe University, Department of Pediatric Nephrology, Nephrogenetics Laboratory were included in the study. The patients medical records were retrospectively evaluated, and characteristics of the patients and outcomes were presented.

Results: There were 6 nephrotic syndrome patients ( 3 female, 3 male) with SGPL1 mutation. Four of the patients (\%67) were diagnosed with nephrotic syndrome before the age of one. The median age of nephrotic syndrome diagnosis was 5 months (0.5-63). Renal biopsy was performed on 4 patients, 2 had focal segmental glomerulosclerosis, one had diffuse mesangial sclerosis, and one had mesangial cell increase and hyaline degeneration in arterioles. Three patients who were given steroid were steroid-resistant. All 6 patients progressed to renal failure and renal replacement therapy (RRT) was initiated in all but one (median age of RRT onset was 6 months, 1-41). Deceased kidney transplantation was performed in a patient at the age of 3 years. All 6 patients also had adrenal insufficiency. Five patients were diagnosed with adrenal insufficiency before 6 months of age; one was diagnosed at 29 months old. Besides, 3 of the patients had hypothyroidism, 2 had ichthyosis, 4 had immunodeficiency, 4 had various neurological findings, and 2 had genitourinary system anomalies. Four patients died at a median age of 30.5 months (1-103); 2 patients are still being followed up with RRT (peritoneal dialysis and kidney transplantation).

Conclusions: The prognosis of nephrotic syndrome seen in SGPL1 mutations is poor, and many extrarenal organ/system involvement, especially adrenal insufficiency, can be seen in patients with this mutation. SGPL1 mutation should be considered in patients with nephrotic syndrome accompanied by adrenal insufficiency.
EP-289 A RARE COMPLICATION OF NEPHROTIC SYNDROME: SAGITTAL SINUS THROMBOSIS

Ece Demirci Bodur, Ibrahim Gökçe, Neslihan Ciçek, Mehtap Sak, Serçin Güven, Nurdan Yildiz, Harika Alpay

Marmara University Pediatric Nephrology

Introduction: Thrombosis is one of the most important complications of nephrotic syndrome (NS). Renal vein, pulmonary artery, deep veins of lower extremities, inferior vena cava and femoral artery are affected most commonly while cerebral venous thrombosis is extremely rare. A patient with NS and superior sagittal sinus thrombosis is presented to draw attention to this rare complication.

Material and methods:

Results: CASE REPORT:An 18 year-old girl who was diagnosed with steroid-sensitive-NS one year ago, admitted to hospital with severe headache. She was using steroid treatment for 25 days for her second attack of NS. On physical examination she had periorbital and pretibial pitting edema. Neurological examination was normal. Laboratory findings showed albumin level of $1.8 \mathrm{~g} / \mathrm{dl}$ and $3+$ proteinuria in urine dipstick. Fundus examination of the eye was normal. Cranial MRI showed hyperintense pathological signal changes of superior sagittal sinus and left sigmoid sinus. In MR venography, defects and contour irregularities were detected in the posterior part of the superior sagittal sinus, left transverse sinus and left sigmoid sinus suggesting of thrombosis. Subcutaneous low molecular weight heparin was started for venous thrombosis and acetazolamide was started for headache. Lumbar puncture was performed to reduce the intracranial pressure. She responded to steroid treatment with remission of the NS and her headache was also resolved. One month later, control imaging of MRI and MR venography showed a complete resolution of the thrombosis. She continued to use anti-thrombotic treatment for the next 6 months and has been followed for 2 years without any problem. Conclusions: Cerebral sinus thrombosis should be considered in patients with NS with symptoms such as headache, vomiting, and behavioral disorders especially in acute attack period. Imaging methods such as cranial MRI and MR venography should be used for diagnosis. Early diagnosis and treatment prevents the development of neurological deficits in most patients. Cerebral sinus thrombosis should be considered in patients with NS with symptoms such as headache, vomiting, and behavioral disorders especially in acute attack period. Imaging methods such as cranial MRI and MR venography should be used for diagnosis. Early diagnosis and treatment prevents the development of neurological deficits in most patients.

\section{EP-290 SEVERE THROMBOTIC COMPLICATIONS IN A CHILD WITH NEPHROTIC SYNDROME}

Joanna Kwinta-rybicka ${ }^{1}$, Karina Madej-Światkowska ${ }^{2}$, Iwona Ogarek ${ }^{1}$, Krzysztof Kobylarz ${ }^{3}$, Dorota Drożd $\dot{z}^{1}$

1 Jagiellonian University Medical College; Dept Of Paediatric Nephrology And Hypertentionic

${ }^{2}$ University Childrens Hospital; Dept Of Paediatric Nephrology And Hypertentionic

${ }^{3}$ University Childrens Hospital; Dept Of Paediatric Anesthesiology

Introduction: A 10 year-old female with steroid-sensitive nephrotic syndrome was admitted to the Paediatric Nephrology Department with severe relapse and thromboembolic complications. The exact time of relapse could not be established due to poor parental care.

Material and methods: On admission, the patient presented massive generalized oedema, movement-impairing pedal oedema, pleural cavity transudate and ascites.

Intravenous steroids, 20\% albumin infusion, diuretics and Fraxiparine were administered.

Imaging yielded massive thrombosis in deep leg veins, common iliac veins and inferior vena cava. Continuous infusion of alteplase was added peripherally. 
Despite the treatment, thrombotic changes progressed with the occurrence of left pulmonary embolism. A central catheter was urgently inserted into the left pulmonary artery and thrombolytic treatment with alteplase was continued in an ICU simultaneously with continuous unfractionated heparin infusion. Treatment was monitored in terms of coagulation system parameters, echocardiographic assessment of right ventricle function and pulmonary arterial flow. For a few days, the patient was kept in pharmacological coma, ventilated. Occasional episodes of hypertension required additional treatment. Bleeding complications necessitated RBC transfusion.

Results: Nephrotic syndrome relapse treatment was first continued with methylprednisolone; later, CsA was introduced. When thrombolytic therapy was completed and the patient's general condition stabilized, she was returned to the Paediatric Nephrology Department. Regression of thrombotic changes (femoral and iliac veins, VCI), improvement of left lung aeration and remission of nephrotic syndrome were achieved.

The patient was discharged in good general condition with no symptoms of post-thrombotic syndrome; LMWH treatment was continued for 12 months after the thrombotic episode, followed by antithrombotic prophylaxis with acenocoumarol.

Steroids and CsA were discontinued and replaced with mycophenolate mofetil. Genetic tests yielded the presence of MTHFR gene 1298A>C variant and F5 gene R506Q variant, both heterozygous.

Conclusions: In children with nephrotic syndrome, the presence of "thrombophilic" genetic variants even of low clinical significance may trigger thrombotic episodes.

\section{EP-291 SCHIMKE IMMUNO-OSSEOUS DYSPLASIA: A CASE} REPORT OF TOO LATE DIAGNOSTICS

Sergey Baiko ${ }^{1}$, O.v. Raikevich-liachovskaya ${ }^{2}$, O.c. Mazur ${ }^{3}$,

E.p. Michalenko ${ }^{3}$, Nicholas Yafremau ${ }^{3}$

${ }^{1}$ Belarusian State Medical University

${ }^{2}$ 2nd City Children's Clinical Hospital, Minsk, Belarus

${ }^{3}$ Institute Of Genetics And Cytology Of The National Academy Of Sciences Of Belarus

Introduction: Schimke immuno-osseous dysplasia (SIOD) is a rare autosomal recessive multisystem disorder caused by biallelic mutation in SMARCAL1 gene.

Material and methods: We describe a clinical case of SIOD identified by Next Generation Sequencing (NGS).

Results: A boy is the first child of unrelated parents with negative family history of bone and kidney disease. He was born full-termed with intrauterine growth retardation, $44 \mathrm{sm}$ of birth height and $1980 \mathrm{~g}$ weight. Patient's characteristic features were a broad low nasal bridge on the triangular face and disproportionate short stature (short neck and trunk) with protruding abdomen and long arms. He also had pigmented macules and microdontia. At the age of 3, he was diagnosed with nephrotic syndrome (morphologically: focal segmental glomerulosclerosis) resistant to steroids and cyclosporine A (treatment 6 months) which led to the end-stage renal disease and required peritoneal dialysis by the age of 5 . Among endocrine disorders hypothyroidism and partial deficiency of growth hormone were detected. Hypothyroidism was corrected with L-thyroxine, whereas body length was not improved by growth hormone supplementation for 4 months. At the age of 6 he received kidney graft from a deceased donor. Seizures and neutropenia, frequent herpes infections appeared after transplantation. At the age of 11, SIOD was suspected, which was confirmed by NGS. Compound heterozygous mutations in 15 and 17 exons in SMARCAL1 gene: c.C2290G (p.R764G) and c.G2542T (p.E848X) were identified.

Conclusions: Schimke immuno-osseous dysplasia is an extremely rare disease - this is the first patient described in Belarus. The existence of steroid-resistance nephrotic syndrome and short stature should make doctors suspect Schimke diagnosis.
EP-292 SCHIMKE IMMUNO-OSSEUS DYSPLASIA IN TWO SISTERS WITH NEPHROTIC SYNDROME

Silvia Acuñas-soto, Ana Castellano-martinez, Virginia Roldan-cano, Moises Rodriguez-gonzalez.

Puerta Del Mar University Hospital

Introduction: Schimke immuno-osseous dysplasia (SIOD) is a rare autosomal recessive genetic disease caused by mutations in SMARCAL1 gene. It is charaterized by spondyloepiphyseal dysplasia, T-cell immunodeficiency, hypercromic nevi, hypercholesterolemia and steroid-resistant nephrotic syndrome caused by a focal segmental glomerulosclerosis with a progressive renal failure to end-stage kidney disease. Our objective is to describe two cases of SIOD in two sisters.

Material and methods: Data collection from the medical records of two paediatric patients admitted to a tertiary hospital for debut of nephrotic syndrome. Informed consent were obtained from parents.

Results: We report two cases of SIOD in two sisters, diagnosed after the debut of steroid-resistant nephrotic syndrome. Both had personal history of short stature, acetabular hip dysplasia and hypercholesterolemia. Laboratory data were compatible with nephrotic syndrome, immunophenotypes were normal, skeletal radiography showed spondyloepiphyseal dysplasia and physical exam revealed characteristic hypercromic nevi. The first case, a 6-year-old girl, presented peripheral refractory edema, severe arterial hypertension and progressive decrease of the glomerular filtration rate. Steroid-resistance of nephrotic syndrome was confirmed, so tacrolimus was started without response. Renal function progressively worsened over the following 4 months, so haemodialysis was started while awaiting renal transplantation. Her sister, a 5-year-old girl, had a steroid-resistant nephrotic syndrome currently with good edema control, normal blood pressure and normal renal function under enalapril treatment. In view of the suspicion of SIOD, the genetic studies were carried out which revealed the same mutation in homozygosis in SMARCAL1 gene.

Conclusions: SIOD has a variable expression with multi-systemic involvement and short life expectancy. It is important the early diagnosis, which can encourage early start of treatment and anticipation of possible complications that may be life-threatening.

EP-293 A NOVEL MUTATION IN DIACYLGLYCEROL KINASE EPSILON GENE CAUSING STEROID RESISTANT NEPHROTIC SYNDROME

Neslihan GÜnay ${ }^{1}$, Ayşe Seda Pinarbaşi ${ }^{~}$, Muhammed Ensar Doğan ${ }^{2}$, Sibel Yel ${ }^{1}$, Aynur Gencer Balaban ${ }^{1}$, Ismail Dursun ${ }^{1}$, Ahmet Eken ${ }^{3}$, HÜlya AkgÜn ${ }^{4}$, Munis Dündar ${ }^{2}$, Muammer Hakan PoyrazoĞlu ${ }^{l}$

${ }^{1}$ Erciyes University Medical Faculty, Department Of Pediatric Nephrology, Kayseri, Turkey

${ }^{2}$ Erciyes University Medical Faculty, Department Of Medical Genetics, Kayseri, Turkey

${ }^{3}$ Erciyes University Medical Faculty, Department Of Medical Biology, Kayseri, Turkey

${ }^{4}$ Erciyes University Medical Faculty, Department Of Pathology, Kayseri, Turkey

Introduction: It has been recently identified that mutations in the gene encoding diacylglycerol kinase epsilon (DGKE) lead to hemolytic uremic syndrome (HUS) and/or membranoproliferative glomerulonephritis (MPGN). There are few cases in the literature with DGKE mutations with MPGN. Here, two sibling cases who presented with the clinic of nephrotic syndrome in which a novel mutation was identified in the DGKE gene were reported.

Material and methods: Case Report: A 4-year-old girl presented with complaints of swelling around the eyes. In her physical exam four positive pitting pretibial edema. Her past medical history was unremarkable. Her parents were first-degree cousins, and her parents cousins suffered from chronic kidney failure. When her six-year-old brother was screened 
for familial nephrotic syndrome, hypoalbuminemia and non-nephrotic range proteinuria was found. They underwent kidney biopsy which demonstrated mild mesangial and endocapillary cell proliferation and basement membrane thickening without any immunofluorescence staining. The genetic testing for nephrin, podocin, and WT1 and genes was unremarkable. She was given multiple immunosuppressive medications including cyclosporine, tacrolimus, MMF and rituximab but reached at CKD 5 at the $5^{\text {th }}$ year of follow-up. Her brother was followed up with steroid and cyclosporine treatment.

Results: Clinical exon sequencing was performed. A c.1383-1384 ins28p.Met462 nonsense homozygous mutation was detected in the DGKE gene. The DGKE mRNA expression and gel electrophoresis supported the pathogenicity of variant (Figure 1). At the $6^{\text {th }}$ year of the follow-up, the immunosuppressive treatments were stopped and peritoneal dialysis was initiated to her. Her brothers non-nephrotic proteinuria continued, and he is being followed up with supportive therapy.

Conclusions: Clinicians should keep in mind that patients with nephrotic syndrome who do not respond to immunosuppressive therapy may rarely have DGKE nephropathy. Even in patients in whom common genetic mutations are not detected, DGKE mutations should be investigated as a part of genetic screening of SRNS.

EP-294 RITUXIMAB TREATMENT OF STEROID, CYCLOSPORINE AND MYCOPFENOLATE- DEPENDENT NEPHROTIC SYN-DROME - ONE CENTER EXPERIENCE

Iwona Ogarek, Elżbieta Szczesny-choruz, Ewa Wierzchowska-słowiaczek, Joanna Kwinta-rybicka, Anna Moczulska, Dorota Drożdż

Jagiellonian University Medical College, Department Of Pediatric Nephrology And Hypertension, Krakow, Poland

Introduction: Children with nephrotic syndrome predominantly respond well to corticosteroid therapy, nonetheless $30 \%$ of those patients have frequent relapses and a steroid-dependent course of disease. Immunosuppressive drugs are being used in treatment of steroid-dependency, including rituximab in the last years.

The aim of thy study was asssessment of efficacy and safety of rituximab in patients with steroid-dependent nephrotic syndrome, in whom previous, long-standing, multidrug immunosuppressive therapy (prednisone, CsA, MMF) gave no grounds for drug dose reduction and/or resulted in significant side effects.

Material and methods: 12 patients, who met the criteria of steroid-dependent, cyclosporin-dependent, MMF-dependent nephrotic syndrome were enrolled in the study. Rituximab was given in single, recommended dose of $375 \mathrm{mg} / \mathrm{m}^{2}$. Evaluation included treatment effect at 6 months, as well as indications for subsequent doses.

Results: The mean time of remission after first rituximab infusion was 9,21 $\pm 3,89$ months. 11 patients remained with no relapse for at least 6 months. In 8 patients within a month after RTX infusion CsA and prednisone treatment was discontinued completely, in others the doses were significantly reduced. In all patients the dose of MMF was reduced. Moreover a relevant reduction of serum creatinine, cystatin $\mathrm{C}$ concentration and BMI has been achieved. None of the patients had significant adverse effects of the given treatment.

Conclusions: Rituximab appears to be an effective and safe drug for patients with steroid-, cyclosporin- and MMF-dependent nephrotic syndrome in the aspect of long-term remission, and withdrawal of steroid therapy and calcineurin inhibitors.

\section{EP-295 EVALUATION OF CLINICAL AND FOLLOW-UP RESULTS OF PATIENTS WITH CONGENITAL NEPHROTIC SYNDROME}

Hulya Nalcacioglu, Demet Tekcan, Hulya Gozde Onal, Ozlem Aydog Ondokuz Mayis University Faculty Of Medicine, Pediatric Nephrology Department, Samsun, Turkey
Introduction: Congenital nephrotic syndrome (CNS) is characterized by severe proteinuria, hypoalbuminemia, and edema seen within the first three months after birth. CNS can occur due to perinatal infections or mutation of genes encoding structural or regulatory proteins of the glomerular filtration barrier. Treatment includes albumin infusions, nephrectomy, dialysis, and transplantation. In this study, we aimed to evaluate the demographic, clinical, and follow-up results of patients with CNS who were followed up in our center between 2010 and 2020.

Material and methods: Demographic, clinical, laboratory values of 8 patients diagnosed with CNS between 2010 and 2020, kidney biopsy results, genetic examinations, and follow-up results were retrospectively evaluated.

Results: A total of 8 patients ( 4 girls) were included in the study. The median age at diagnosis was 36 days (3 days- 8 months) and the follow-up period was 34 months (7-114 months). There was a history of prematurity and consanguinity in 5 patients in the history. Edema (expected in 5 patients) was detected at the admission of all patients. Albumin infusion and captopril therapy were started from the diagnosis. No pathology was seen in the tests and ultrasonographic examinations sent for perinatal infection. In the genetic analysis, NPHS1 (Nephrin) homozygous mutation was detected in six patients, and coenzyme Q2 mutation was detected in one patient. Peritoneal dialysis treatment was performed in four patients during the follow-up, and unilateral nephrectomy was completed in one patient. During the follow-up, four of our patients (three due to sepsis while on dialysis, one on the postoperative first day after transplantation) died. Three of our patients are followed up with kidney transplantation and one with supportive treatment.

Conclusions: According to our results, most CNS cases are due to genetic reasons, and nephrin mutation is the most common cause. Management of complications in CNS is crucial for patient survival.

\section{EP-297 A RARE CAUSE OF INFANTILE NEPHROTIC SYNDROME: DENYS-DRASH SYNDROME}

Güldane Aylin Inal ${ }^{1}$, Neslihan Günay ${ }^{~}$, Aynur Gencer Balaban ${ }^{l}$, Muhammed DoĞan ${ }^{2}$, Sibel Yel ${ }^{1}$, IsmaIl Dursun ${ }^{1}$,

Muammer Hakan Poyrazoğlu ${ }^{1}$

${ }^{1}$ Erciyes University Medical Faculty, Department Of Pediatric Nephrology, Kayseri, Turkey

${ }^{2}$ Erciyes University Medical Faculty, Department Of Medical Genetics, Kayseri, Turkey

Introduction: Denys-Drash syndrome (DDS) is a rare disease caused by mutations in exons 8 and 9 of the Wilms tumor suppressor gene (WT1). Since the WT1 gene is a transcription protein that plays a role in the development of kidneys and gonads, patients can present with steroidresistant nephrotic syndrome (NS), Wilms' tumor, and sexual differentiation disorders. In this article, a patient with DDS presented with infantile NS is reported

Material and methods: Case Report:A previously healthy 16-monthold female patient was referred to our clinic because of proteinuria while being examined with fatigue, fever, and rash. Clinical findings were compatible with the sixth disease without any edema. Laboratory investigations revealed massive proteinuria, hypoalbuminemia $(1.7 \mathrm{~g} / \mathrm{dl})$. Renal functioning test and electrolytes were within normal range. On pelvic ultrasonography, uterus didelphys was observed, and bilateral ovaries were absent. The karyotype analysis was detected as $46 \mathrm{XY}$. WT1 gene mutation was studied in terms of differential diagnosis with Frasier syndrome. Mutation in exon 8 of the WT1 gene was detected. All findings were consistent with DDS. Enalapril and L-thyroxine treatments were initiated to reduce proteinuria and for hypothyroidism, respectively. During follow-up, proteinuria decreased, and albumin levels were increased. No wilms tumor image was found in the abdominal laparoscopy of the patient. Biopsy taken from streak gonads and reported compatible with gonadoblastoma. 
Results: By this case, we emphasize the necessity to consider DDS, which is a rare condition, in the differential diagnosis of patients with steroid-resistant infantile nephrotic syndrome.

Conclusions: DDS should be considered in the differential diagnosis, especially in children presenting with steroid-resistant nephrotic syndrome in the first years of life. Ultrasound can be used as an early diagnostic tool in the evaluation of the pelvic area. Early diagnosis of this syndrome is essential to avoid unnecessary immunosuppressive treatment, and periodic assessment is required about the risk of gonadal tumor.

\section{EP-298 EFFECT OF CYCLOSPORINE A TREATMENT IN CHILDREN WITH STEROID RESISTANT NEPHROTIC SYNDROME - A SINGLE CENTER EXPERIENCE}

Galia Zlatanova, Dimitar Roussinov, Maria Gaydarova, Stefka Yankova, Polina Miteva, Kremena Dimitrova, Greta Mihneva

University Children Hospital "prof. Ivan Mitev", Medical UniversitySofia, Bulgaria

Introduction: The purpose of this retrospective study is to determine the efficacy of cyclosporine A (CyA) in children with steroid-resistant nephrotic syndrome (SRNS). Patients with genetic mutations were excluded.

Material and methods: We reviewed the data of 41 nephrotic children with primary SRNS who received CyA between 2010 and 2020. Ten patients were treated with cyclophosphamide prior to CyA therapy without clinical effect. CyA starting dose was $5 \mathrm{mg} / \mathrm{kg} /$ day and was readjusted to maintain a $\mathrm{C} 2$ blood level of $600-800 \mathrm{ng} / \mathrm{ml}$. The drug was received for 6-165 months (mean: 39.16 months). The observational period was 5 168 months (mean: 41.04 months).

Results: Complete long lasting remission (proteinuria $<4 \mathrm{mg} / \mathrm{h} / \mathrm{m}^{2}$ for more than 12 months), complete remission with relapses of the disease, partial remission (proteinuria $4.0-40 \mathrm{mg} / \mathrm{h} / \mathrm{m}^{2}$ ) and resistance to CyA (proteinuria $>40 \mathrm{mg} / \mathrm{h} / \mathrm{m}^{2}$ ) were observed in $36.5 \%, 19.1 \%$, $19.1 \%$ and $24.4 \%$ of patients, respectively. A combination with MMF was usually the next-line immunosuppressive treatment. Only two out of ten patients (20\%) achieved remission additionally. During the observational period hypertrichosis, elevated uric acid and anemia occurred in $36.6 \%, 43.9 \%$ and $29.3 \%$ of patients, respectively. Gingival hyperplasia was not observed. Arterial hypertension was found in 16 children (39\%). Renal function impairment $(7.3 \%)$ was a part of the disease progression toward CKD and not related to CyA treatment. CyA was discontinued in $72.5 \%$ of patients. Among them long lasting remission was observed in $60 \%$, while $40 \%$ showed CyA dependency.

Conclusions: CyA was able to induce complete remission in only half (55.6\%) of the children with SRNS. The rest of the patients needed prolonged steroid treatment or a combination of two or more immunosuppressants still. Hypertension, elevated uric acid and anemia required additional therapy.

EP-299 EVALUATION OF CHILDREN WITH STEROID SENSITIVE IDIOPATHIC NEPHROTIC SYNDROME: A SHORT-TERM OUTCOME STUDY

Mahir Cevizoğlu ${ }^{1}$, Gülşah Kaya Aksoy ${ }^{2}$, Çağla Serpil Doğan ${ }^{2}$

${ }^{1}$ Antalya Training And Research Hospital, Department Of Pediatrics

${ }^{2}$ Antalya Training And Research Hospital, Department Of Pediatrics, Division Of Pediatric Nephrology

Introduction: We evaluated demographic and clinical characteristics of children with steroid sensitive idiopathic nephrotic syndrome (SSINS) who experienced one or more relapses.
Material and methods: 26 patients with SSINS who were followed-up for $\geq 12$ months were included. The patients were divided into two groups: group 1; the patients with infrequent relapse (IFR) and group 2; those who had frequent relapse (FR) and steroid dependence (SD).

Results: A total of 26 patients, 17 boys (65.4\%), with a mean age of 7.4 \pm 2.8 years (range; $3-12.6$ years) and mean follow-up period of $3.3 \pm 1.8$ years were enrolled. All patients had normal renal function and received prednisolone ( 12 weeks) for the treatment of the first episode. The mean follow-up period was $3.3 \pm 1.2$ vs $2.9 \pm 2.8$ years for group $1(\mathrm{n}=19,73 \%)$ and group $2(\mathrm{n}=7,27 \%)$. Time to first relaps was $10.4 \pm 8.8$ and $5.1 \pm 2.6$ weeks for group 1 and 2, respectively.

There was no statistically significant difference between two groups with respect to sex, follow-up period, age at the onset of disease, time to diagnosis, time to first remission, time to first relapse and laboratory findings (serum albumin, creatinine, triglyceride, cholesterol, platelet, complement 3, IgA levels, eGFR, urine protein/creatine ratio $(\mathrm{pr} / \mathrm{cr})$, the presence of microscopic hematuria) (all $\mathrm{p}>0.05$ ). The time to first remission was significantly higher in those with urine $\mathrm{pr} / \mathrm{cr}>10 \mathrm{mg} / \mathrm{mg}$ compare to those who had urine $\mathrm{pr} / \mathrm{cr}$ between $5-10 \mathrm{mg} / \mathrm{mg}(\mathrm{p}=0.015)$.

Conclusions: Demographic, clinical and laboratory findings were not statistically significant between two groups. The time to first remission correlated with the severity of proteinuria.

\section{EP-300 MANAGEMENT OF CONGENITAL AND INFANTILE NEPHROTIC SYNDROME: ABOUT 9 CASES}

Kaoutar Danaoui, Radia Laanait, Rabiy Elqadiry, Houda Nassih, Aicha Bourrahouat, Imane Ait Sab

B Pediatric Ward, Mohammed Vi University Hospital Center, Marrakesh, Morocco

Objectives: to analyze the etiologies, clinical features and evolution of the patients with congenital and infantile nephrotic syndrome (NS)

Methods: A retrospective single center study during a period of 5 years including 7 cases of congenital and infantile NS

Results: we had 5 boys and 4 girls. The mean age at presentation was of 20-day-old, with extremes of age at diagnosis of 1 month and 9 months. Four patients were from an inbred marriage, and 1 patient had a sibling with neonatal NS who died from anasarca. Generalized oedema was present at diagnosis in all our cases. All our patients had hyperlipemia and hypocalcemia. Meanwhile, 7 patients had hypothyroidism. Growth delay was found in 5 patients and infection in 2 patients. Five patients had congenital NS and four had infantile NS. The NS was related to the congenital Finnish-type NS and the diffuse mesangial sclerosis disease in 6 patients, congenital CMV infection in one case, congenital rubella infection in one case, and congenital syphilis infection in one case.

All patients received symptomatic treatment, with regular IV albumin infusions, high protein diet, and calcium and iron supplements. Oral Levothyroxine was started in 7 patients. ACE inhibitors (captopril starting at $1 \mathrm{~m} / \mathrm{kg} /$ day) and anticoagulant therapy (aspirin at $3 \mathrm{mg} / \mathrm{kg} /$ day) was given to all our patients. Penicillin G was prescribed in the case of syphilis infection and ganciclovir was given for a total period of six weeks in the case of CMV infection. Two of our children died, one of massive anasarca, and the other one of septic shock. Four of our cases are lost to follow-up and one patient had evolved to ESRD.

Conclusions: Genetic forms of congenital and infantile NS are the most frequent. Symptomatic measures awaiting for a renal transplantation are the key of treatment. 
EP-301 GENETIC STUDY, KIDNEY BIOPSY AND RESPONSE TO TREATMENT IN STEROID-RESISTANT NEPHROTIC SYNDROME

Mar Espino Fernandez ${ }^{1}$, Cristina Julia Blazquez Gomez ${ }^{2}$,

Pablo Bello Gutierrez ${ }^{3}$, Julia Vara Martin ${ }^{1}$, Carmen Gallego Herrero ${ }^{1}$, Juan Francisco Quesada-espinosa ${ }^{1}$

${ }^{1}$ Hospital Universitario 12 De Octubre

${ }^{2}$ Hospital Universitario Príncipe De Asturias

${ }^{3}$ Hospital Universitario Rey Juan Carlos

Introduction: Steroid-resistant nephrotic syndrome (SRNS) in children, due to its aggressive nature, requires follow-up renal biopsy for histological study. However, limited resources for genetic testing, as well as low test availability invites us to start calcineurin inhibitors (CNI) before attempting kidney biopsy. Genetic testing now allows screening for mutations which could help determine treatment response expectation without a kidney biopsy. Our objective is to know if detected mutations can influence SRNS patient care, and substitute kidney biopsy.

Material and methods: A retrospective study was done on children diagnosed with SRNS within the last five years. Clinical, histological, genetic and therapeutic data were collected from debut up to the last follow-up.

Results: 12 patients ( 7 female) with a mean age at debut between 9 months -13 years old were included. Patients were treated either with standard oral prednisone therapy for 4 weeks or 6-8 weeks. In both groups, 9 were additionally treated with methylprednisolone pulses. Kidney biopsies showed: 5 focal segmental glomerulosclerosis (FSGS), 4 minimal changes disease (MCD), 2 IgM mesangial glomerulonephritis (MGN). Genetic tests revealed mutations in APOL1 in one patient, INF2, NPHS2 and cubillin gene in another each. At the end of follow-up: 7 cases were in complete remission, 4 patients in partial remission, and only 1 without answer. Regarding patients with detected mutations: 3 cases are in partial remission (1 FSGS, $1 \mathrm{MCD}$ and $1 \mathrm{MGN}$ ) and 1 patient with a genetic mutation associated with FSGS evolved to end-stage renal disease.

Conclusions: The presence of a genetic mutation confers a poorer prognosis in our patients regardless of histological diagnosis. Our study suggests CNI response may allow, according to some current recommendations, to delay or avoid kidney biopsy in patients with SRNS.

\section{EP-302 BENEFICIAL EFFECT OF TACROLIMUS ON NEPHROTIC SYNDROME RELATED TO SCHIMKE GLOMERULOPATHY}

Steven Tran, Hazel Webb, Aoife Waters

Great Ormond Street Hospital

Introduction: Schimke immuno-osseous dysplasia (SIOD) is associated with steroid resistant nephrotic syndrome with focal segmental glomerulosclerosis and usually progresses to end-stage renal disease. We describe a case where Tacrolimus had an early beneficial effect in achieving partial remission with normalisation of albumin and reduction of proteinuria in a child with SIOD.

Material and methods: A 5-year-old female was referred with steroid resistant nephrotic syndrome for assessment and management of oedema. Extra-renal features included short stature $\left(<<0.4^{\text {th }}\right.$ centile for height), truncal lentigines, platyspondyly with ovoid vertebrae and lymphopaenia associated with combined immunodeficiency syndrome. Despite having reduced T-cell population, she had no history of recurrent infection and had adequate vaccine responses.

Results: Genetic testing revealed a homozygous deletion in exon 3 \& 4 in SMARCAL1 mutation confirming our clinical diagnosis of Schimke immune-osseous dysplasia. Renal histopathology showed focal segmental glomerulosclerosis and $15 \%$ chronic change. Urine albumin to creatinine ratio peaked at $4172 \mathrm{mg} / \mathrm{mmol}$, an albumin of $20 \mathrm{~g} / \mathrm{l}$ and a creatinine of $34 \mathrm{umol} / \mathrm{L}$. Estimated GFR was $90 \mathrm{ml} /$ $\mathrm{min} / 1.73 \mathrm{~m}^{2}$. A trial of Tacrolimus was initiated at $0.1 \mathrm{mg} / \mathrm{kg} / \mathrm{dose}$ twice a day.



Following the introduction of Tacrolimus, the patient's albumin normalised with a reduction in albuminuria to $429 \mathrm{mg} / \mathrm{mmol}$ by month 6 from a peak of $4172 \mathrm{mg} / \mathrm{mmol}$.

Relapse occurred at month 8 with hypoalbuminaemia and oedema, following which, renal function declined with current eGFR at $15 \mathrm{ml} / \mathrm{min} /$ $1.73 \mathrm{~m}^{2}$.

Conclusions: Tacrolimus was associated with partial remission of steroid-resistant nephrotic syndrome associated with SIOD was achieved for 7 months.

\section{EP-303 ANALYSIS OF PSYCHO-SOCIAL IMPACT AND QUALITY OF LIFE (QOL) IN CAREGIVERS OF PEDIATRIC PATIENTS WITH NEPHROPATHIC CYSTINOSIS}

Karina Gonzalez ${ }^{1}$, Teresa Eixarch ${ }^{1}$, Laura Nuñez, ${ }^{1}$, Gema Ariceta ${ }^{2}$

${ }^{1}$ Pediatric Nephrology. Hospital Universitari Vall D Hebron. Barcelona

${ }^{2}$ Pediatric Nephrology. Universitat Autonoma Barcelona. Hospital Universitari Vall D Hebron. Barcelona

Introduction: Strict and complex lifelong treatment and monitoring requirements in pediatric patients with cystinosis often lead to caregiver burn-out and exhaustion. Prevalence of anxiety-depression (AD) and care overload manifestations in caregivers of pediatric patients with cystinosis are thought to negatively impact QoL perception, and to increase healthcare budget.

Aims:

1) to evaluate and compare QoL perception and $\mathrm{AD}$ symptoms in caregivers of pediatric patients with cystinosis

2 ) to compare QoL and overload perception between asymptomatic caregivers and those with $\mathrm{AD}$

Material and methods: Hypothesis: caregivers with $\mathrm{AD}$ have lower $\mathrm{QoL}$; presence of $\mathrm{AD}$ in caregivers is associated with larger care overload perception and reduced QoL

Population: 9 patients (6 boys) of $12.6( \pm 4.2)$ years with nephropathic cystinosis. Their chronic kidney disease (CKD) stage was 1 (4 patients), 2 (1 patient), 3 (4 patients). 4 had a kidney transplant (KT). All studied caregivers were patients' mothers.

Methods: validated scales for caregiver overload (HADS, ZARIT) and QoL (SF-36) were used after inform consent and ethical committee approval. Statistics tests were applied as corresponded.

Results: Only 3 out of 9 caregivers did not suffer AD; 4 caregivers manifested care overload (which was intense) whereas 5 did not. Health perception was reduced in all cases. Decreased physical activity, increased pain, reduced social life, mental health issues, negative emotional impact and decreased vitality were observed in nearly half mothers $(5,3,4,6,5,4$ out of 9 , each one respectively). QoL perception was significantly better among caregivers without $\mathrm{AD}(p=0.02)$.

Conclusions: Pediatric cystinosis patient caregivers mental health issues, were associated with larger care overload perception and reduced QoL. Those results remark the need of detection and treatment of caregivers' 
psycho-social issues to mitigate family stress and facilitate patient empowerment, adherence, transition, and emotional health.

\section{EP-304 HEALTHCARE TRANSITION READINESS ASSESSMENT TOOL FOR ADOLESCENTS WITH CHRONIC CONDITIONS: TRANSLATION, CULTURAL ADAPTATION, AND LINGUISTIC VALIDATION TO RUSSIAN \\ Elena Kulakova ${ }^{1}$, Tatjana Nastausheva ${ }^{1}$, Oleh Akchurin ${ }^{2}$, Maria Koltakova ${ }^{1}$, Tatjana Zvyagina ${ }^{1}$, Anna Stebletsova ${ }^{1}$, Anastasija Ledovskih ${ }^{I}$, Maria Diaz-gonzalez De Ferris ${ }^{3}$ \\ ${ }^{1}$ N.n. Burdenko Voronezh State Medical University \\ ${ }^{2}$ Weill Cornell Medical College \\ ${ }^{3}$ Unc School Of Medicine}

Introduction: The healthcare transition (HCT) is a vulnerable time in the lives of adolescents with chronic conditions. HCT requires readiness measurement, however, there was no instrument to evaluate HCT readiness in Russia. We aimed to develop the Russian version of the UNC $\mathrm{TR}_{\mathrm{x}}$ ANSITION Index ${ }^{\mathrm{TM}}$, a tool that assesses HCT readiness among adolescents with chronic kidney disease (CKD) and type 1 diabetes mellitus (DM). Material and methods: The UNC $\mathrm{TR}_{\mathrm{x}} \mathrm{ANSITION}$ Index ${ }^{\mathrm{TM}}$ is a provider-administered/verified 32-question HCT measure with ten domains and a maximum score of 10. In accordance with international guidelines, the following steps of translation, cross-cultural adaptation, and linguistic validation were conducted: preparation, two forward translations, reconciliation, back-translation, revision, cognitive interviewing, pilot testing, final review, and proofreading. This process was carried out by an international multidisciplinary team. Cognitive interviewing was conducted with 5 adolescents and pilot testing was conducted with 15 adolescents with CKD and 15 adolescents with DM.

Results: Through the iterative process of translation and cultural adaptation, a Russian test version of the UNC $\mathrm{TR}_{\mathrm{x}}$ ANSITION Index ${ }^{\mathrm{TM}}$ was developed. The cognitive interviewing of adolescents did not lead to any significant revisions but helped obtain qualitative data about patients' perspectives on HCT readiness. The test version was then administered through semi-structured interviews during pilot testing. The most difficult questions for Russian adolescents were about reproduction, health insurance, and new adult healthcare providers. The median transition score was 5.86 (IQR 4.81-6.16). Based on the pilot testing we developed an algorithm for automated score calculations. After the final review and proofreading, the Russian version was finalized.

Conclusions: In this study, we developed a Russian HCT readiness assessment tool, based on the UNC TRxANSITION Index ${ }^{\mathrm{TM}}$. The median scores in our adolescents with CKD and DM were low, indicating the need for HCT preparation.

\section{EP-305 CLINICIAN PERSPECTIVES ON COMMUNICATION AND DECISION-MAKING IN PEDIATRIC CHRONIC KIDNEY DISEASE: AN INTERVIEW STUDY}

Jasmijn Kerklaan ${ }^{2}$, Camilla Hanson ${ }^{1}$, Talia Gutman ${ }^{1}$, Simon Carter ${ }^{1}$, Noa Amir ${ }^{1}$, Allison Tong ${ }^{1}$, Aditi Sinha ${ }^{3}$, Allison Eddy ${ }^{4}$

Chandana Guha ${ }^{1}$, Debbie Gipson ${ }^{5}$, Detlef Bockenhauer ${ }^{6}$,

Elyssa Hannan ${ }^{1}$, Hui-kim Yap ${ }^{7}$, Jaap Groothoff ${ }^{2}$, Michael Zappitelli ${ }^{8}$ Nicholas Webb ${ }^{9}$, Stephen Alexander ${ }^{1}$, Stuart Goldstein ${ }^{10}$, Susan Furth ${ }^{11}$, Susan Samuel ${ }^{12}$, Jonathan Craig ${ }^{13}$

${ }^{1}$ Centre For Kidney Research, The Children's Hospital At Westmead, Westmead, Nsw, Australia

${ }^{2}$ Department Of Pediatric Nephrology, Emma Children's Hospital, Academic Medical Center, Amsterdam, The Netherlands

${ }^{3}$ Division Of Nephrology, Department Of Pediatrics, All India Institute Of Medical Sciences, India

${ }^{4}$ Department Of Pediatrics, University Of British Columbia, Vancouver, British Columbia, Canada
${ }^{5}$ Division Of Nephrology, Department Of Pediatrics, University Of Michigan, Ann Arbor, Michigan, Usa

${ }^{6}$ University College London Department Of Renal Medicine, Great Ormond Street Hospital For Children, National Health Service Foundation Trust, London, United Kingdom

${ }^{7}$ Department Of Pediatrics, Yong Loo Lin School Of Medicine, National University Of Singapore, Singapore, Singapore

${ }^{8}$ Department Of Nephrology, Sickkids, Toronto, Ontario, Canada

${ }^{9}$ Department Of Paediatric Nephrology And National Institute For Health Research/welcome Trust Clinical Research Facility University Of Manchester, Manchester Academic Health Science Centre, Royal Manchester Children's Hospital, Manchester, United Kingdom

${ }^{10}$ Division Of Nephrology And Hypertension, Cincinnati Children's Hospital Medical Center, Cincinnati, Oh, Usa

${ }^{11}$ Department Of Pediatrics, Perelman School Of Medicine At The University Of Pennsylvania, Children's Hospital Of Philadelphia, Philadelphia, Pennsylvania, Usa

${ }^{12}$ Department Of Pediatrics, Section Of Nephrology, University Of Calgary, Calgary, Alberta, Canada

${ }^{13}$ College OfMedicine And Public Health, Flinders University, Adelaide, South Australia, Australia

Introduction: To describe clinicians' perspectives on communication and decision-making in pediatric chronic kidney disease (CKD) and investigate to what extend their intensions on care and management interfere with the interests of children with CKD and their parents. This to identify opportunities to improve shared decision-making, care and outcomes for children with CKD and their families.

Material and methods: Forty-three semi-structured interviews with clinicians, including pediatric nephrologists, nurses, social workers, surgeons, dietitians, and psychologists, with at least one year of experience providing care to children with CKD, from multiple centers (18 hospitals and 4 University research departments) across eleven (United States of America, Canada, Australia, China, United Kingdom, Germany, France, Italy, Lithuania, New Zealand and Singapore) countries were analyzed thematically.

Results: We identified four themes: striving to blend priorities (minimizing treatment burden, emphasizing clinical and long-term risks, achieving common goals), focusing on medical responsibilities (carrying decisional burden and pressure of expectations, working within system constraints, ensuring safety is foremost concern), collaborating to achieve better long-term outcomes (individualizing care, creating partnerships, encouraging ownership and participation in decision-making, sensitive to parental distress) and forming cumulative knowledge (balancing reassurance and realistic expectations, building understanding around treatment, harnessing motivation for long-term goals).

Conclusions: Clinicians strive to blend priorities, minimize treatment burden and collaborate with their patients to support decision-making, manage expectations, and achieve the best outcomes and common goals despite many varied challenges

(the conclusion is not finalized yet... this is not the finale version!)

EP-306 A CASE REPORT OF THE RARE CONDITION OF COLISTIMETHATE SODIUM INTOXICATION IN A CHILD

Özgür Özdemir Şimşek ${ }^{l}$, GökÇen Erfidan ${ }^{l}$, Ayşe Berna Anil ${ }^{2}$, Belde Kasap DemIr ${ }^{3}$, Demet Alaygut ${ }^{1}$, SeçIl Arslansoyu Çamlar ${ }^{4}$, Doğa Lüleyap ${ }^{5}$, Fatma MutlubșS ${ }^{4}$

${ }^{1}$ University Of Health Sciences, Tepecik Training And Research Hospital, Department Of Pediatrics, Division Of Nephrology, Izmir, Turkey

${ }^{2}$ Izmir Katip Çelebi University Medical Faculty, Department Of Pediatrics, Division Of Pediatric Intensive Care Unit, Izmir, Turkey

${ }^{3}$ Izmir Katip Çelebi University Medical Faculty, Department Of Pediatrics, Division Of Nephrology And Rheumatology, Izmir, Turkey 
${ }^{4}$ University Of Health Sciences, Izmir Faculty Of Medicine Department Of Pediatric Division On Nephrology, Izmir, Turkey

${ }^{5}$ University Of Health Sciences, Tepecik Training And Research Hospital, Department Of Pediatrics, Division Of Pediatric Intensive Care Unit, Izmir, Turkey

Introduction: Colistimethate sodium(CMS) is an antibiotic with neurological and nephrological side effects. They have a narrow antibacterial spectrum limited to some gram-negative bacilli, they have an important role in the treatment of pan-resistant infections. We present a case of CMS toxicity developing iatrogenically for pan-resistant urinary tract infection. Material and methods: A 5-month-old male patient, who was followed up with a diagnosis of operated posterior urethral valve (PUV), was hospitalized for positive urine culture obtained before control cystoscopy. Physical examinations were normal. Laboratory investigations revealed as serum urea $34 \mathrm{mg} / \mathrm{dL}(\mathrm{N}: 10-38)$, creatinine $0.5 \mathrm{mg} / \mathrm{dL}(\mathrm{N}: 0.4-0.7)$, uric acid $3.1 \mathrm{mg} / \mathrm{dL}(\mathrm{N}: 2-5.5)$, sodium $135 \mathrm{mmol} / \mathrm{L}(\mathrm{N}: 134-150)$, potassium $4.95 \mathrm{mmol} / \mathrm{L}(\mathrm{N}: 3.5-5.5)$, calcium $8.7 \mathrm{mg} / \mathrm{dL}(\mathrm{N}: 7.6-10.4)$, phosphorus $4.78 \mathrm{mg} / \mathrm{dL}(\mathrm{N}: 4-7)$, CRP $1.2 \mathrm{mg} / \mathrm{L}(\mathrm{N}: 0-5)$. Blood gas analysis revealed as $\mathrm{pH} 7.35, \mathrm{HCO} 321.2 \mathrm{mmol} / \mathrm{L}$. Urine analysis was nitrite positive with Klebsiella pneumonia(100000KOU $/ \mathrm{mL})$ growth. Bacteria was resistant to carbapenems, so CMS was initiated $(5 \mathrm{mg} / \mathrm{kg} /$ day) intravenously. On the $8^{\text {th }}$ day of CMS treatment, the patient developed sudden restlessness, sleepiness, vomiting, intolerance to feeding, anuria, hypertension and tachycardia.

Results: It was observed that CMS treatment, was applied as $50 \mathrm{mg} / \mathrm{kg} /$ day. Laboratory findings displayed, abnormal liver function tests, acidosis, acute kidney injury and the patient progressed to anuric, hypertensive, tachycardiac, neurological findings ended with pediatric intensive care unit(PICU). Hemodiofiltration(HDF) was applied to the patient for 10 hours to reduce nephrotoxic side effects and to remove the drug from the circulation. Polyuria developed in the $24^{\text {th }}$ hours. Ibuprofen was added to the treatment because the diuresis that started as $18 \mathrm{cc} / \mathrm{kg} / \mathrm{h}$ at the $24^{\text {th }}$ hour was still polyuric on the $9^{\text {th }}$ day. Ibuprofen treatment was discontinued after 14 days and polyuria didn't recur in the patient.

Conclusions: CMS is still needed as a potent drug in PICU and pediatric practices where nosocomial infections are common. Literature information on toxicity in childhood is limited. Early HDF may play an essential role for side-effects reversity.

\section{EP-307 FIBROSIS IN VISCERAL TISSUES DUE TO NITROFURANTOIN IN RAT PUPS \\ Ilke Beyitler ${ }^{1}$, Bilgen Basgut ${ }^{2}$, Abdikarim Abdi ${ }^{2}$, Hanife Ozkayalar ${ }^{3}$, Alper Soylu ${ }^{4}$, Sarah Khamis ${ }^{2}$, Nerin Bahceciler ${ }^{5}$, Salih Kavukcu ${ }^{4}$ \\ ${ }^{1}$ Near East University, Faculty Of Medicine, Department Of Pediatrics, Division Of Nephrology, Nicosia, North Cyprus \\ ${ }^{2}$ Near East University, Faculty Of Pharmacy, Department Of Clinical Pharmacy, Nicosia, North Cyprus \\ ${ }^{3}$ Near East University, Faculty Of Medicine, Department Of Pathology, Nicosia, North Cyprus \\ ${ }^{4}$ Dokuz Eylul University, Faculty Of Medicine, Department Of Pediatrics, Division Of Nephrology, Izmir, Turkey \\ ${ }^{5}$ Near East University, Faculty Of Medicine, Department Of Pediatrics, Division Of Allergy And Immunology, Nicosia, North Cyprus}

Introduction: Nitrofurantoin used for prophylaxis of urinary infections may cause visceral fibrosis in adults but this effect may not be clinically observed in children. We investigated histopathological alterations due to nitrofurantoin in rat pups.

Material and methods: 64 rat pups were grouped and given 1: drinking water, $2: 60 \mathrm{mg} / \mathrm{kg}$ nitrofurantoin and 3: $120 \mathrm{mg} / \mathrm{kg}$ nitrofurantoin for 60 , 90 and 120 days. Fibrosis was investigated histopathologically in lung, liver and kidney with $0-4$ scoring (0:None, 1:Rare, 2:Moderate, 3:Frequent, 4:Abundant).
Results: There were no fibrosis in group 1.

In group 2, lung fibrosis on 120 . day (mean $=2,3$ ) was higher than 90 . and 60 . days $(p<0,0001)$. In group 3 , lung fibrosis in 90 . day $($ mean $=1,5)$ was higher than 60. day $($ mean $=1)(\mathrm{p}=0,04)$. Fibrosis on 120 . $($ mean=3,3) was higher than 90 . and 60 . days $(\mathrm{p}<0,0001)$.

In group 2 , liver fibrosis on 90 . day (mean $=0,75$ ) was higher than 60 . day (mean=0,25) $(\mathrm{p}=0,01)$. Regarding group 3 , liver fibrosis on 120 . day (mean=1) was higher than 60 . day $(\mathrm{p}=0,002)$.

In group 2, renal fibrosis on 90 . (mean=1,125) was higher than 60 . day (mean $=0,5)(\mathrm{p}=0,007)$. Renal fibrosis on 120 . $($ mean $=2,5)$ was more than 60. and 90. days $(\mathrm{p}<0,0001)$. In group 3 , renal fibrosis on 120 . $(\mathrm{mean}=3,33)$ was higher than 60 . and 90 . days $(\mathrm{p}<0,0001)$.

Conclusions: Fibrosis in lung, liver and kidney tissues increased parallel to increased dose and duration of nitrofurantoin. As in adults, fibrosis due to nitrofurantoin may be risk in children too.

\section{EP-308 WHAT MAY HAVE CAUSED THE BLACKENING OF THE TONGUE AND TEETH?}

Özgür Özdemír Șimşek ${ }^{l}$, Candan Semra Paksoy ${ }^{2}$, Gökçen Erfídan ${ }^{l}$, Seçil Arslansoyu Çamlar ${ }^{3}$, Demet Alaygut ${ }^{1}$, Fatma Mutlubaş ${ }^{3}$, Belde Kasap Demir ${ }^{1}$

${ }^{1}$ University Of Healthy Sciences Tepecik Training And Research Hospital Department Of Pediatrics, Division Of Nephrology, Izmir, Turkey

${ }^{2}$ Ankara University Faculty Of Dentistry, Division Of Oral Diagnosis And Radiology, Ankara, Turkey

${ }^{3}$ University Of Health Sciences, Izmir Faculty Of Medicine Department Of Pediatrics, Division Of Nephrology, Izmir, Turkey

${ }^{4}$ Izmir Katip Çelebi University Medical Faculty, Department Of Pediatrics, Division Of Nephrology And Rheumotology, Izmir, Turkey

Introduction: Discoloration of theeth(DT) and black hairy tongue(BHT) are frequent in our society because of the poor oral hygiene, physical agents, environmental chemicals, mouth rinses, some dental procedures, general systemic conditions and drugs. In case of suddenly developing DT and BHT, the etiology should be carefully evaluated.

Material and methods: A six-year-old boy was admitted to the emergency room with acute renal injury(AKI) due to nausea, vomiting and diarrhea. The patient, who was anuric and hypertensive, had acidosis, uremia, and hyperpotasemia. Renal replacement therapy(RRT) was planned for the patient and a catheter was placed. The patient received hemodialysis(HD) and renal biopsy was performed for etiology. Immediately after biopsy, intravenous pulse methylprednisolone(PMP) treatment was initiated for the patient who has a rapidly progressive course and had a serum creatinine level of $0.6 \mathrm{mg} / \mathrm{dL} 6$-months ago. While the patient was receiving daily $\mathrm{HD}$, he had fever on the $4^{\text {th }}$ day of prednisolone treatment and became septic. Laboratory tests of predialysis revealed serum urea $98 \mathrm{mg} / \mathrm{dL}(\mathrm{N}: 10-38)$; creatinine $3.4 \mathrm{mg} /$ $\mathrm{dL}(\mathrm{N}: 0.5-1.2)$; uric acid $4.6 \mathrm{mg} / \mathrm{dL}(\mathrm{N}: 2-5.5)$; albumin $2.4 \mathrm{~g} /$ $\mathrm{dL}(\mathrm{N}: 3.5-5.5)$; sodium $131 \mathrm{mmol} / \mathrm{L}(\mathrm{N}: 134-150)$, potassium $4.8 \mathrm{mmol} / \mathrm{L}(\mathrm{N}: 3.5-5.5)$, calcium $8.3 \mathrm{mg} / \mathrm{dL}(\mathrm{N}: 8.8-10.8)$, phosphorus $4.9 \mathrm{mg} / \mathrm{dL}(\mathrm{N}: 4-7)$; $\mathrm{CRP} 44 \mathrm{mg} / \mathrm{L}(\mathrm{N}: 0-5)$; procalcitonin $185 \mu \mathrm{g} / \mathrm{L}(\mathrm{N}: 0.04-0.1)$.

Results: Meropenem and linezolid treatment was initiated. Methicillin sensitive staphylococcus aureus grew in blood culture. Therefore, meropenem treatment was discontinued on the $5^{\text {th }}$ day. On the $8^{\text {th }}$ day of antibiotic treatment DT and BHT developed. Treatments were reviewed. It has been observed in the literature that linezolid and prednisolone can cause reversible DT and BHT. First, we stopped linezolid treatment. 72 hours after the cessation of treatment, all findings regressed with the effect of regular tooth brushing.

Conclusions: Antibiotic side effects are important in patients using medication. It should be kept in mind that sudden situations can be iatrogenic. 
EP-309 MOLECULAR-BASED SURVEILLANCE OF PEDIATRIC HEMOLYTIC UREMIC SYNDROME (HUS) CAUSED BY SHIGA TOXIN-PRODUCING ESCHERICHIA COLI (STEC) IN ITALY, 2016-2020

Eleonora Ventola ${ }^{1}$, Valeria Michelacci ${ }^{1}$, Alessandra Gianviti ${ }^{2}$, Carmine Pecoraro ${ }^{3}$, Mattia Parolin ${ }^{4}$, Mario Giordano ${ }^{5}$,

Rosangela Tozzoli ${ }^{1}$, Paola Chiani ${ }^{1}$, Fabio Minelli ${ }^{1}$, Federica Gigliucci ${ }^{1}$, Arnold Knijn ${ }^{1}$, Stefano Morabito ${ }^{1}$, Gaia Scavia ${ }^{1}$

${ }^{1}$ Istituto Superiore Di Sanità

${ }^{2}$ Ospedale Bambino Gesù, Irccs, Rome, Italy

${ }^{3}$ Ospedale Santobono, Naples, Italy

${ }^{4}$ Ospedale Di Padova, Padua, Italy

${ }^{5}$ Ospedale "giovanni Xxiii", Bari, Italy

Introduction: Hemolytic Uremic Syndrome (HUS) is the leading cause of acute renal failure in children and is mainly caused by infection with Shiga toxin-producing Escherichia coli (STEC). We report the surveillance results of the Italian HUS Registry, established by the Italian Pediatric Nephrology Society and the National Reference Laboratory for $E$. coli (NRL-ISS), for the years 2016-2020.

Material and methods: Stool and blood samples from HUS patients were submitted from all over Italy nephrology units to NRL-ISS for STEC testing, through Real-Time PCR targeting stx genes, isolation and serology. 125 isolates were subjected to whole genome sequencing (WGS) and analyzed through IRIDA-ARIES bioinformatic platform (https://irida.iss.it/irida-aries), engaging an IRIDA instance and Galaxy cluster ARIES, respectively working as web-based interface for data collection and workflow engine for automatic analysis, including serotyping, virulotyping and core genome Multi Locus Sequence Typing. IRIDAARIES is connected with the Italian HUS Registry, automatically collecting clinical information and returning typing results.

Results: 322 HUS cases were identified, corresponding to 0.72 mean annual cases per 100,000 (0-15 years). The top-5 STEC serogroups O26 $(\mathrm{N}=107), \mathrm{O} 111(\mathrm{~N}=39), \mathrm{O} 157(\mathrm{~N}=29), \mathrm{O} 145(\mathrm{~N}=27)$ and $\mathrm{O} 103$ $(\mathrm{N}=12)$ were identified in 214 cases and prevailed among the 247 STEC positive HUS cases. WGS allowed to identify other serogroups, in particular STEC O80 ( 10 cases between 2016 and 2020 vs 1 case in the six previus years). All but 2 isolates were $s t x 2$-positive. The subtype $s t x 2 a$ was present in $86,4 \%$ of these. Furthermore, 14 clusters $(<15$ allelic difference) were identified, nine including strains from patients without clear epidemiological links.

Conclusions: HUS increased of $22 \%$ compared with the six previous years. The developed integrated system for surveillance allows fast communication of STEC-HUS diagnosis and the STEC detailed typing through genomics. The identification of isolates with high genetic similarity in different years suggests circulation of certain clones leading to persistent contamination of the sources.

EP-310 END STAGE KIDNEY DISEASE IN ISRAELI CHILDREN BETWEEN 1990 AND 2020 - EPIDEMIOLOGICAL AND CLINICAL CHARACTERISTICS

Lilach Camilla Regev ${ }^{1}$, Michal Stern-zimmer ${ }^{1}$, Pazit Bekerman ${ }^{2}$, Lital Keinan-boker ${ }^{3}$, Ronit Calderon-margalit ${ }^{4}$, Asaf Vivante ${ }^{1}$

${ }^{1}$ Department Of Pediatrics B, Edmond And Lily Safra Childrens Hospital, Sheba Medical Center, Tel-hashomer, Israel

${ }^{2}$ Department Of Nephrology And Hypertension, Sheba Medical Center, Tel Hashomer, Israel

${ }^{3}$ Israel Center For Disease Control, Ramat Gan, Israel

${ }^{4}$ School Of Public Health, Hadassah-hebrew University, Jerusalem, Israel

Introduction: Childhood end stage kidney disease (ESKD) is a rare disease, with clinical characteristics and underlying etiologies of patients that may vary between countries and populations. We aimed to study secular trends in incidence of childhood ESKD and to study the etiologies, treatments, and prognosis by population group in Israel, over a period of 30 years.

Material and methods: In this nationwide, population-based, historical cohort study we collected and analyzed medical and demographic data of all children treated with renal replacement therapy and reported to the Israeli ESKD Registry from 1990 to 2020. Statistical analysis was performed with incidence rate corrected for age, ethnicity and calendar year, using as denominator the appropriate population as reported by the Israeli Central Bureau of Statistics.

Results: During 30 years of follow-up, childhood ESKD 5-year average incidence decreased from 12.1 to 10.6 cases per million age related population (pmarp). Arab and Druze children exhibited higher incidence rates of ESKD compered to Jewish children (23.4 and 19.4 vs. 7.0 cases pmarp, respectively). The most common etiologies for ESKD among Jews and Arabs were congenital anomalies of the kidney and urinary tract (27.2\% and $26.6 \%$, respectively), followed by glomerulonephritis among Jews (16.1\%) and renal cystic diseases among Arabs (11.3\%). The most common etiology among Druze children was Hyperoxaluria $(33.3 \%$ of ESKD). Arabs had a lower rate of living donor transplantations compared to Jews and thus longer waiting time for transplantation. Patients survival has improved during the study period, however, Arab and Druzes patient survival rates were lower than Jews survival rates (5-year survival of $86.7 \%$ versus $93.3 \%$, respectively).

Conclusions: In Israel, minority children have a higher incidence of ESKD compared to Jewish children. There are disparities between children with ESKD from different ethnicities in terms of etiology, treatment modalities and outcomes.

\section{EP-311 REGULAR EDUCATION IS THE KEY FOR A SUCCESSFUL TRANSITION OF CHRONIC PEDIATRIC NEPHROLOGICAL PATIENTS TO ADULT CARE}

Mirjam Močnik, Sonja Golob Jančič, Nataša Marčun Varda

Department Of Paediatrics, University Medical Centre Maribor

Introduction: Many children with chronic diseases survive into adulthood, meaning that there is an increasing need for an appropriate transition from a pediatrician to adult care. The goal of the transition is an integrated, developmentally appropriate and coordinated transition from pediatric to further treatment, which requires a lot of patient education. The aim of our study was to investigate the readiness of our patients of age for transition.

Material and methods: A questionnaire about patient readiness for the transition was sent to all patients aged 18 years and older. The questionnaire included different questions about their readiness for the transition, such as knowing the disease they are being followed and treated for, the medications they are taking, etc. SPSS Statistics (IBM, version 22) was used for basic statistical analyses.

Results: 100 questionnaires were returned. The average age of participants was 19.1 years. They were mostly high school (55\%) or college students (39\%), living with their parents (94\%). 15\% do not know, what disease they have, and $14 \%$ are not aware of its long-term consequences. About one third regularly takes medications and mostly they know what they are taking on their own, but $15 \%$ of them do not know the side effects of the medications. $42 \%$ visit doctors with their parents and $18 \%$ will do the same after transition. They are mostly aware of alcohol and drug effects on their health, but one third does not know how the disease is affecting their profession choice and reproductive health. $46 \%$ of the asked do not know who will continue with their care and $44 \%$ still have a primary pediatrician.

Conclusions: Regular education about the transition is the key for successful transfer of pediatric patients to adult care. 
EP-312 EPIDEMIOLOGICAL REPORT ON PEDIATRIC RENAL DISEASES IN FLANDERS: FCGG RENAL BIOPSY NETWORK Sevasti Karamaria ${ }^{1}$, Johan De Meester ${ }^{5}$, Amelie Dendooven ${ }^{1}$, Elena Levtchenko ${ }^{2}$, Noel Knops ${ }^{2}$, Koen Van Koek ${ }^{3}$, Dominique Trouet ${ }^{3}$, Reiner Mauel ${ }^{4}$, Ben Sprangers ${ }^{2}$, Wim Laurens ${ }^{5}$, Johan Vande Walle ${ }^{1}$

${ }^{1}$ Ghent University Hospital

${ }^{2}$ Leuven University Hospital

${ }^{3}$ Antwerp University Hospital

${ }^{4}$ Brussels University Hospital

${ }^{5}$ Nbvn, Az Nikolaas, Sint-niklaas

Introduction: The Flemish Collaborative Glomerulonephritis Group, FCGG, was founded in 2016 as a collaboration to collect all native kidney biopsies in Flanders. Uniform renal biopsy request and report forms, and a new comprehensive list of renal pathology diagnoses for coding were introduced. The 2017-2019 epidemiological data of the pediatric patients are presented

Material and methods: Following informed consent and complying to GDPR, basic patient and categorical renal data, semi-structured medical information of renal histopathology and clinical renal disease were collected.

Results: In 2017-2019, 127 renal biopsies in children were reported in Flanders $[3.3 / 100,000$ children per year $]-6 \%$ of all biopsies; boys $\mathrm{N}=73$, girls $\mathrm{N}=54$. Three "clinical" patterns were equally represented: proteinuria $>1 \mathrm{~g}$ /day; hematuria; and combination of both. Acute or chronic renal failure were present in $25 \%$. In each age/gender group glomerulopathy was most common. In the youngest age group ( 0 -5years; $\mathrm{N}=33)$ minimal change disease/nephrotic syndrome predominated $(50 \%)$, followed by Henoch-Schönlein nephritis and Alport's disease. The middle age group (6-11years; $\mathrm{N}=47$ ) mainly presented with diseases with hematuria $(47 \%)$ : IgA nephropathy, Henoch-Schönlein nephritis, Alport's disease; followed by minimal change disease (28\%). Greater impact of gender was noted in teenagers (12-18years; $\mathrm{N}=47)$ : IgA- and GBM mediated nephritis was present in $50 \%$ of the boys, whereas a more diverse palette of kidney diseases was present in girls. Children of Western-European descent presented with hematuric renal diseases $(50 \%)$ and nephrotic diseases $(25 \%)$, while the reverse was noted in the group without a Western-European descent.

Conclusions: FCGG network provides a structured format for cross-talk between renal pathologists and nephrologists. Reliable estimates of pediatric renal diseases based on histology are now available in our region. Due to the diverse renal spectrum of the teenager group, a renal biopsy may have an added value.

\section{EP-313 A RETROSPECTIVE MULTICENTRE ONE-YEAR ANALYSIS OF PREVALENCE AND RESISTANCE PATTERNS OF UROPATHOGENS IN CHILDREN IN FLANDERS (BELGIUM)}

Greet Pauwels ${ }^{1}$, Lien Dossche ${ }^{2}$, Noël Knops ${ }^{3}$, Joke Van Vlaenderen ${ }^{4}$, Mieke Bouvry ${ }^{5}$, Elien Baert ${ }^{6}$, Valerie Van Bogaert ${ }^{7}$, Anke Raaijmakers ${ }^{8}$, Ellen Putseys ${ }^{9}$, Loes Lambrecht ${ }^{10}$, Johan Vande Walle ${ }^{2}$

${ }^{1}$ Az Sint-jan Brugge-oostende, Department Of Pediatrics And Pediatric Nephrology, Bruges, Belgium

${ }^{2}$ Ghent University Hospital, Department Of Pediatric Nephrology, Ghent, Belgium

${ }^{3}$ University Hospitals Leuven, Department Of Pediatric Nephrology, Leuven, Belgium

${ }^{4}$ Az Jan Palfijn Ghent, Department Of Pediatrics And Pediatric Nephrology, Ghent, Belgium

${ }^{5}$ Az Groeninge - Kortrijk, Department Of Pediatrics And Pediatric Nephrology, Kortrijk, Belgium

${ }^{6}$ Az Sint Lucas Ghent, Department Of Pediatrics And Pediatric Nephrology, Ghent, Belgium

${ }^{7}$ Az Nikolaas, Department Of Pediatrics And Pediatric Nephrology, Sintniklaas, Belgium
${ }^{8}$ Zna Hospitals Antwerp, Department Of Pediatrics And Pediatric Nephrology, Antwerp, Belgium

${ }^{9}$ Az Diest, Department Of Pediatrics And Pediatric Nephrology, Diest, Belgium

${ }^{10}$ Az. Herentals, Department Of Pediatrics And Pediatric Nephrology, Herentals, Belgium

Introduction: International guidelines regarding urinary tract infections (UTIs) in children state that the choice of the initial empirical antibiotic should be based on local resistance data. To guide this clinical decision in Flanders (Belgium), a multicentre data-analysis was performed.

Material and methods: Prevalence and resistance patterns of all urinary isolates of children $<16$ years from 9 centres in Flanders during a one-year (2020) period, were retrospectively analysed regarding age, sex and one vs multiple urine collections (samples) per patient.

Results: 3512 isolates from 3156 samples and 2636 unique patients (male/female ratio 1/2, 57\% <2 years, $18 \%$ with multiple samples) were analysed. E. coli was the most prevalent uropathogen (58\%; girls $65.5 \%$ vs boys $43.5 \%$; boys $<2$ years $48.5 \%$ vs boys $>2$ years $29 \%$ ), comparable with a smaller 2015-multicentre study in Flanders (overall prevalence $61 \%$ ). In patients with multiple samples vs one sample, prevalence of Klebsiella and Pseudomonas species was higher $(9.73 \%$ vs $6.79 \%$ and $7.15 \%$ vs $3.35 \%$ respectively). E. coli showed a resistance-rate to coamoxiclav of $46 \%$, with an increasing resistance for 2 centres that were included in the 2015 - and 2020 -analysis (29\% to $52 \%$ and $32 \%$ to $39 \%$ ). For patients with multiple samples vs one sample, resistance to ampicillin, co-amoxiclav and co-trimoxazol was higher ( $64 \%$ vs $54 \%, 52 \%$ vs $42 \%$ and $35 \%$ vs $28 \%$ respectively).

Conclusions: E. coli remains the most prevalent uropathogen in children, but with an increasing co-amoxiclav resistance-rate. In patients with multiple samples, the prevalence of Klebsiella and Pseudomonas species and the antibiotic resistance-rate for E. coli is higher, probably reflecting a patient group with more uropathies and more frequent antibiotic use. This needs to be taken into account when selecting the empirical antibiotic to treat UTIs in children in Flanders. A regular multicentre data-review in the future will further demonstrate the local trends in time.

\section{EP-314 RISK FACTORS FOR VOIDING DYSFUNCTION IN CHILDREN}

Öznur Ege ${ }^{l}$, Zeynep Birsin Özçakar ${ }^{2}$, Nilgün Çakar $^{2}$, Fatih Günay ${ }^{l}$, Eda Nisa Çullas İlarslan ${ }^{1}$, Fatoş Yalçinkaya ${ }^{2}$

${ }^{1}$ Ankara University Faculty Of Medicine Department Of Pediatrics

${ }^{2}$ Ankara University Faculty Of Medicine Department Of Pediatrics, Division Of Pediatric Nephrology

Introduction: In this study, we aimed to investigate the frequency of voiding dysfunction (VD) in the pediatric outpatient clinics and to determine the risk factors that were related with this disorder.

Material and methods: The study included children aged 5-18 years who were admitted to the pediatric outpatient clinic of Ankara University Children's Hospital with any complaints. Patients with known anatomical and neurological defects of the lower urinary system and those who had chronic diseases were excluded. A questionnaire with 18 items including demographical information and possible risk factors for VD and Dysfunctional Voiding and Incontinence Scoring System were applied to the patients.

Results: The study included 1000 patients (54,1\% girls; mean age 9,52 $\pm 3,57$ years). Voiding dysfunction was detected in 115 (11.5\%) patients (53 boys, 62 girls). Voiding dysfunction was most frequently detected in the age group of $7(18,6 \%)$ and its frequency decreased with increasing age. The most common symptoms were urgency $(75 \%)$, holding maneuvers $(71 \%)$, night-time incontinence $(69 \%)$ and urge incontinence $(60 \%)$. Quality of life was affected in $84 \%$ of the patients with VD. Living in a crowded family, having $\geq 3$ children in the family, having a housewife mother, presence of VD in the other members of family, history of urinary 
tract infection (UTI) and constipation were found to be risk factors related with VD $(p<0,05)$. Although statistically insignificant, the prevalence of VD was found to be higher in obese children and in children of mothers who gave birth under the age of 20 .

Conclusions: Voiding dysfunction was detected in one out of every ten children who applied to the pediatric outpatient clinic and the quality of life was affected in majority of these children. Some familial risk factors, history of UTI and constipation were found to be associated with the increased prevalence of VD.

\section{EP-315 LOW BACTERIAL COUNTS IN INFANT UTI COUNT Magnus Lindén, Per Brandström}

Institute Of Medical Sciences, Sahlgrenska Academy, University Of Gothenburg, Sweden

Introduction: Several paediatric guidelines on UTI suggest a cut-off for bacterial colony count $\geq 50000$ or $100000 / \mathrm{mL}$ in urine culture required for the diagnosis. The objective of this study was to investigate if clinical parameters and imaging results differ in infant UTI with low versus high bacterial counts.

Material and methods: In a prospective nationwide study at paediatric hospital departments in Sweden, 1165 children $<1$ year of age treated for their first UTI were included. No predetermined diagnostic criteria were required and instructions to the managing paediatrician were to treat and investigate according to the Swedish national guidelines and local practice. Clinical parameters, urine collection method, bacterial data and results from imaging investigations were reported. Three groups of infants with bacterial colony counts $<10000,10000-100000$ and $>100000 \mathrm{CFU} / \mathrm{mL}$ respectively were compared regarding age, gender, CRP, non-E. Coli, dilatation on renal sonography, VUR and kidney involvement on DMSA scan.

Results: Reports on urine cultures with growth of a single urinary pathogen were available in 1122 cases ( 522 boys and 600 girls). Clean catch was used as urine collection method in $93 \%$ of cases. The frequency of high, medium and low bacterial numbers were 932, 156 and 34 respectively. Girls had lower bacterial numbers than boys. No significant difference in age, diagnostic rate of pelvic dilatation on ultrasound, abnormality on DMSA-scintigraphy or dilating VUR was seen between the groups. Non-E.Coli infection was significantly more prevalent in lower bacterial numbers $(\mathrm{p}<0.001)$ whereas $\mathrm{CRP}$ was higher in high bacterial numbers $(\mathrm{p}=0.039)$.

Conclusions: Significant renal abnormalities are present in infants with UTI in similar rate regardless of bacterial count in urine culture. These may be undetected, and adequate treatment delayed, if a high threshold of bacterial numbers is required for diagnosis and subsequent action.

\section{EP-316 METHOD OF TREATMENT OF VULVOVAGINITIS IN PATIENS WITH RECURRENT URINARY TRACT INFECTION OF PRESCHOOLAGE \\ Gadgy M. Letifov ${ }^{1}$, Yulia Yu Chebotareva ${ }^{1}$, Zareta A. Kostoeva ${ }^{2}$ \\ ${ }^{1}$ Federal State Budgetary Educational Institution Of Higher Education "rostov State Medical University" Of The Ministry Of Health Of The Russian Federation \\ ${ }^{2}$ State Budgetary Institution "center For Maternal And Child Health"}

Introduction: The aim of the study was to investigate the effectiveness of combination therapy for vulvovaginis (VV) in preschoolers with urinary tract infections (UTI).

Material and methods: We examined 52 girls aged 3-6 years with VV and UTI. The patients were divided into groups: $1 \quad(n=22)-$ standard treatment and $2(n=30)$ - combined treatment. Inclusion criteria: established diagnosis of VV, UTI, preschool age, absence of other extragenital diseases, enterobiosis, informed parental consent. Standard treatment: capsules, containing neomycin+polymyxin $\mathrm{B}+$ nystatin, intravaginally once a day for 6 days. Combination therapy: candles Viferon 150000 IU rectally twice a day for 5 days, then once a day - 5 days; gel with bacteriophages Phagogin 2-3 $\mathrm{ml}$ per area of the vestibule of the vagina twice a day for 7 days, then once a day -7 days; Kanefron $\mathrm{H} 15$ drops 3 times daily, 14 days. Monitoring of the state of the vaginal microflora was carried out after treatment, the frequency of relapses was evaluated during 12 months of follow-up. The program "SPSS Statistics 17.0 for Windows" is used for statistical processing.

Results: The high efficiency of combined therapy of VV was revealed. After treatment, the patients of group 2 in the vagina decreased microbial colonization of E. coli from $6.0 \pm 0.05 * 10^{6}$ to $1.7 \pm 0.03 * 10^{2} \mathrm{GE} / \mathrm{S}$, $(p<0.05)$, the presence of a variety of obligate anaerobic microflora, with no growth (less than $10^{2} \mathrm{GE} / \mathrm{S}$ ) and pathogenic properties, while in group 1 , a significant decrease in microbial colonization was not detected. After 1 - year follow-up, relapses were observed in $46 \%$ of patients in group 1 and $4.8 \%$ in group $2(\mathrm{p}<0.05)$.

Conclusions: Combination therapy of VV in girls with recurrent UTIs, including recombinant interferon alpha $2 \mathrm{~b}$, a complex of bacteriophages and a drug of phytoniring nature, reduces relapses, the need for antibacterial therapy, preserves the vaginal microbiome, improving reproductive health.

\section{EP-317 EFFECT OF NEPHROPROTECTIVE THERAPY ON URINARY B-2 MICROIGLOBILIN LEVELS IN CHILDREN WITH VESICOURETERAL REFLUX AND REFLUX NEPHROPATHY \\ Natalia Zaikova Vladimir Dlin Anatolii Korsunsckyi \\ ${ }^{I}$ Pirogov Russian National Research Medical University \\ ${ }^{2}$ Sechenov University}

Introduction: To determine the diagnostic value of $\beta 2-\mathrm{MG}$ ( $\beta 2$ microglobulin) in urine to determine the severity of tubulointerstitial fibrosis (TIF) in children with vesicoureteral reflux (VUR), depending on nephroprotective therapy.

Material and methods: We examined 117 patients aged 3 to 16 years (mean age $10.2 \pm 4.5,70.1 \%$ girls) with VUR. The control group consisted of 20 healthy children. All children underwent a complete nephrological examination. B2-MG levels were determined in morning urine by ELISA and calculated as urine creatinine. DMSA static scintigraphy was performed to determine the degree of damage to the renal parenchyma. Depending on the therapeutic approach, the children were divided into 2 groups: 1 gr. - 74 p. $(63.2 \%)$ who received enalapril $(0.1 \mathrm{mg} / \mathrm{kg})$ and 2 gr. -43 p. $(36.8 \%)$ did not receive enalapril. Each group was divided into subgroups according to the degree of TIF according to DMSA: A subgr. - no signs of sclerosis (15.4\%), B prep. - 1-2 lesions $(44.74 \%)$ and $\mathrm{C}$ subgr $->3-4$ lesions of sclerosis (40.1\%).

Results: After six months of treatment and follow-up, data were collected and analyzed in both groups. Patients of both groups with sclerosis showed a high urinary excretion of $\beta 2-\mathrm{MG}$ compared to the control group and the group of children without sclerosis $(p<0.05)$. Patients gr. 1 (subgr. B) had a statistically significantly lower level of $\beta 2-\mathrm{MG}$ excretion compared to patients from subgr. $\mathrm{C}(\mathrm{p}<0.05)$. In gr. 2 , in children with sclerosis (B and $\mathrm{C}$ subgr.) without enap therapy, a significantly high level of $\beta 2-\mathrm{MG}$ in urine was revealed when compared with children on enalapril therapy.

Conclusions: A significant dependence of $\beta 2-\mathrm{MG}$ excretion in urine on the severity of TIF was established according to DMSA scintigraphy in children with VUR. A high level of $\beta 2-\mathrm{MG}$ was revealed in children with sclerosis in the absence of enalapril therapy. Therefore, the study of urinary $\beta 2-\mathrm{MG}$ excretion as non-invasive markers can be used as a criterion for the effectiveness of nephroprotective therapy and for determining the degree of tubulointestinal fibrosis. 
EP-318 THE USE OF QUESTIONNAIRES AND VOIDING DIARIES AT INTAKE-VISIT FOR ENURESIS. FIRST RESULTS FROM A NATIONAL BELGIAN STUDY

Sevasti Karamaria ${ }^{1}$, Nadejda Ranguelov ${ }^{2}$, Pernille Hansen ${ }^{3}$, Veerle De Boe ${ }^{4}$, Pieter Verleyen ${ }^{5}$, Johan Vande Walle ${ }^{1}$, An Bael ${ }^{6}$

${ }^{1}$ Ghent University Hospital

${ }^{2}$ Université Catholique De Louvain

${ }^{3}$ Chu Tivoli

${ }^{4}$ Brussels University Hospital

${ }_{6}^{5} \mathrm{Az}$ Groeninge

${ }^{6}$ Zna Koningin Paola Kinderziekenhuis, , University Of Antwerp

${ }^{7}$ Ghent University Hospital, Ghent University

Introduction: International guidelines agree that stratifying enuresis into non-monosymptomatic (NMNE) and monosymptomatic (MNE) based on the presence or absence of Lower Urinary Tract Symptoms (LUTS) respectively, is mandatory at intake to optimize therapy. To identify LUTS, a checklist (Clinical Management Tool (CMT)) and/or voiding diaries can be used. However, home recordings are time-consuming for the family. Moreover, the added value to CMT is rather expert-opinion than evidence-based.

Material and methods: We conducted a study in 7 Belgian Hospitals to document the prevalence of MNE and the added value /correlation of CMT and/or diary in differentiating enuresis in treatment-naïve patients $>5$ years. At visit $1 \mathrm{CMT}$ following thorough medical history was used to set a diagnosis. Afterwards, a 2day voiding diary was registered at home. During a second visit, this diary was evaluated and used to differentiate enuresis.

Results: 109 children included, 19 lost to follow-up. Mean age 7,7years $( \pm 2) ; 62$ boys $(68,9 \%)$ and 27 girls $(30 \%)$. Based on the CMT 13 children had MNE (16,7\%) and 75 NMNE $(83,3 \%)$. Based on the diary 16 children had MNE $(17,8 \%)$ and 74 NMNE $(82,2 \%) .25(27,8 \%)$ had the same diagnosis with both methods. Regarding the presence of LUTS we observed significant inconsistencies between CMT and diary. There was fair agreement between the two modalities for urge $(\kappa=0,219)$, moderate for daytime incontinence $(K=0,432)$ and none for abnormal voiding frequency $(\mathrm{K}=-0,057)$.

Conclusions: NMNE is frequenter than MNE in treatment-naïve patients. CMT alone versus CMT+diary had a different sensitivity and specificity of identifying LUTS: in absence of validation of the importance by a therapeutic trial-outcome, we state that MNE can only be considered when both methods do not demonstrate LUTS.

\section{EP-319 RISK FACTOR FOR BACTEREMIA IN VERY YOUNG CHILDREN WITH URINARY TRACT INFECTION}

Hyesun Hyun ${ }^{1}$, Yeon Hee Lee ${ }^{2}$, Jin-Soon Suh ${ }^{3}$

${ }^{1}$ St. Vincents Hospital, The Catholic University Of Korea College Of Medicine, Suwon, Korea

${ }^{2}$ Seoul St. Marys Hospital, The Catholic University Of Korea College Of Medicine, Seoul, Korea

${ }^{3}$ Bucheon St. Marys Hospital, The Catholic University Of Korea College Of Medicine, Bucheon, Korea

Introduction: Urinary tract infections (UTI) is one of the most common bacterial infections in children. When UTI is accompanied by bactermia, it can lead to more serious complications. Therefore, prompt decisionmaking on the initial management of young infants suspected of having bacteremia is important but not feasible because UTI without bacteremia and bacteremic UTI are clinically indistinguishable. This study was conducted to evaluate the initial clinical and laboratory risk factors of concomitant bacteremia in very young children with febrile UTI.

Material and methods: We performed a multicenter retrospective study of very young children aged 0 to 24 months who admitted with febrile UTI from January 2000 to December 2019.

Results: We analyzed 2178 patients with febrile UTI, of whom $40(1.8 \%)$ patients were classified into bacteremic UTI. No significant differences in gender and pasts medical histories including previous UTI, hospitalization, or antibiotic therapy were observed between children with bacteremic UTI and non-bactermic UTI. Although the rates of vesicoureteral reflux were not significantly different between two groups, higher rates of abnormal findings of ultrasonography such as hydronephrosis or pyelonephritis were significantly associated with increased risk of bacteremic UTI. Bacteremic UTI group had a significantly younger age $(\leq 3 \mathrm{month})$ and low levels of lymphocyte, hemoglobin or albumin on admission. The C-reactive protein (CRP) levels were significantly higher in bacteremic UTI group $(P<0.001)$. Multivariate analysis showed that younger age (odds ratio [OR]: 1.35, 95\% CI: 1.03-1.77, $P=0.029$ ); lower serum albumin (OR 0.178, 95\% CI:0.057-0.560, $P=0.003$ ); higher CRP level (OR: $1.025,95 \%$ CI: $1.006-1.045, P=0.009)$ were independent risk factors of bacteremic UTI.

Conclusions: The present study found that $1.8 \%$ of very young children with febrile UTI had a concomitant bacteremic UTI. Age $\leq 3$ month, lower serum albumin and higher CRP are risk factors for bacteremic UTI in children under 24 months of age.

\section{EP-320 THE EFFECTIVENESS OF ANTIBACTERIAL AND RENOPROTECTIVE THERAPY IN THE PROGRESSION OF RENAL SCARRING IN EXPERIMENTAL RATS}

Natalia Zaikova Lilia Sinitcina Verdjil Petrovici Vladimir Dlin Anatolii Korsunsckyi

${ }^{I}$ Pirogov Russian National Research Medical University

${ }^{2}$ Institute Of Mother And Cild Care

Introduction: The goal is to establish the development of tubulointerstitial fibrosis (TIF) in rats and the level of collagen formation (free (FO) and peptide-bound (PBO) oxyproline) in experimental nephrosclerosis (NS) and to determine the effects of antibiotic therapy and ACE inhibitors to prevent scar development.

Material and methods: Experimental modeling of NS was induced in 24 Wistar rats by urethral scarification with the development of intravesical obstruction and inoculating E.coli in the cavity of the bladder and 2 groups were formed: gr.1 - 9 rats (antiobiotic - cefepim - $40 \mathrm{mg} / \mathrm{kg}$ ), gr. $2-15$ rats (antiobiotic+ enalapril $-0.1 \mathrm{mg} / \mathrm{kg}$ ). Antibacterial therapy was used 2 days after the development of acute pyelonephritis (for 5 days), enalapril a month later (for 6 months). The level of FO and PBO in the urine (method ELISA) was determined at 1,3,6 months of the experiment. The level of TIF was determined histologically after decapitation of animals in 1, 3, 6 months of the experiment.

Results: At 1 month urinary FO and PBO levels were not significant difference between the groups. The urinary level of FO was significantly higher in gr. 1 at $3(\mathrm{p}<0.05)$ and 6 months $(\mathrm{p}<0.001)$, the level of PBO in urine was statistically lowest in gr. 2 at 6 month $(\mathrm{p}<0.05)$.

the development of TIF for 1 month of the experiment did not differ in both groups. Significant low development of TIF was detected in individuals of gr. 2 at 3 and 6 months of the experiment.

Conclusions: Antibiotic does not prevent the development of TIF. The combined use of an antibiotic and enalapril reduces the activity of the urinary level of FO, which is involved in the active synthesis and breakdown of collagen and also prevents further sclerosis of the renal tissue.

\section{EP-321 ANTIBIOTIC RESISTANCE IN CHILDREN WITH URINARY TRACT INFECTION}

Betul Unverdi Elden ${ }^{\text {, }}$ Zehra Aydin ${ }^{\text {}, ~ U m u t ~ S e l d a ~ B a y r a k c i ~}{ }^{2}$,

Fatma Semsa Cayci ${ }^{1}$

${ }^{1}$ The University Of Health Sciences, Ankara City Hospital, Department Of Pediatric Nephrology, Ankara, Turkey

${ }^{2}$ The University Of Yildirim Beyazit, Ankara City Hospital, Department Of Pediatric Nephrology, Ankara, Turkey 
Introduction: Urinary tract infections are one of the most common diseases in childhood and appropriate antibiotic treatment should be started as early as possible. However, the use of antibiotics results in increasing antibiotic resistance. In this study, we aimed to investigate the relationship between antibiotic resistance and prophylactic antibiotics in children with recurrent urinary tract infections.

Material and methods: Patients with urinary tract infections ages between 1-24 months were retrospectively analyzed.

Results: 219 patients had been diagnosed with urinary tract infections, $113(51.6 \%)$ were girls and $106(48.4 \%)$ were boys. The mean age of all patients was $9.68 \pm 5.74$ months. The mean age of girls was significantly higher than the boys $(\mathrm{p}<0.001) .133$ of all patients were using the antibiotic prophylaxis (female/male: 63/70), 86 of all patients were not using antibiotic prophylaxis (female/male:50/36). The mean age of patients with prophylaxis was significantly higher than the patients without prophylaxis. $(10.33 \pm 5.88$ months, and $8.66 \pm 5.38$ months, respectively $\mathrm{p}=0.036$ ). The most frequently isolated microorganisms in urine culture were; Escherichia coli (65.8\%), Klebsiella spp. (21.9\%) and Enterobacter spp. (4.6\%). Klebsiella was significantly higher in patients with prophylaxis $(\mathrm{p}=0,001)$. The highest antibiotic resistance was observed in; Ampicillin (74\%), TMP-SXT (66\%), and ceftazidime (64\%), and the lowest was fosfomycin and imipenem (7\%), and amikacin $(6 \%)$. Although the resistance of ampicillin and amikacin is higher in patients with prophylaxis, there was no significant difference between patients with and without prophylaxis according to total antibiotic resistance $(\mathrm{p}>0.05)$.

Conclusions: We would recommend that antibiotic resistance should be investigated for each institution and treatment plans should be revised according to these reports.

EP-322 EPIDEMIOLOGY AND RISK FACTORS OF UTI IN CHILDREN - ONE CENTER 3 YEAR-OBSERVATION

Maria Daniel ${ }^{1}$, Hanna Szymanik-grzelak ${ }^{1}$, Edyta Podsiadly ${ }^{2}$,

Janusz Sierdzinski ${ }^{3}$, Magdalena Kowalewska-mlot ${ }^{1}$,

Malgorzata Panczyk-tomaszewska ${ }^{l}$

${ }^{1}$ Department Of Pediatrics And Nephrology, Medical University Of Warsaw, Warsaw, Poland

${ }^{2}$ Department Of Pharmaceutical Microbiology, Centre For Preclinical Research, Faculty Of Pharmacy, Medical University Of Warsaw, Warsaw, Poland

${ }^{3}$ Department Of Medical Informatics And Telemedicine, Medical University Of Warsaw, Warsaw, Poland

Introduction: Urinary tract infection (UTI) is one of the most common bacterial infection in the pediatric population. The main etiological factor for UTI is Escherichia coli, regardless of sex and age and presence of congenital anomaly of kidney and urinary tract (CAKUT).

The aim of the study was to analyse epidemiology and risk factors of UTI in children admitted to the tertiary pediatric hospital between 2015-2017. Material and methods: A total of 1,195 positive urine cultures were analysed. In 1,143 patients with positive urine cultures UTI was diagnosed, in the remaining 52 patients vulvovaginitis, vulvitis or sample contamination were diagnosed.

The demographic data, epidemiology and risk factors in patients with UTI were further analysed.

Results: 540 patients (325 $q, 215 \hat{\partial}$, mean age 2.17 years) were diagnosed with acute pyelonephritis, 401 patients $(204$ \&, 197 Oे, mean age 4.51 years) with cystitis and 202 patients $(101$ +, 101 , , mean age 4.14 years) with asymptomatic bacteriuria.

The most frequently isolated bacterial strains were Escherichia coli, Klebsiella pneumoniae, Pseudomonas aeruginosa, Proteus mirabilis both in patients without risk factors $(84.76 \% ; 6.69 \% ; 2.97 \% ; 0.93 \%$, respectively) and in patients with risk factors $(59.75 \% ; 11.5 \% ; 7 \%$; $8.5 \%$, respectively).
Minimum one risk factor of UTI was presented in $43 \%$ patients with pyelonephritis or cystitis. The main risk factor of UTI were: CAKUT $(26.1 \%$; 246), bladder dysfunction (neurogenic bladder 5.7\%; 54; non-neurogenic bladder $4.8 \%$; 45), constipation $(5.6 \% ; 53)$, immunosuppressive drugs $(1.5 \% ; 14)$, urolithiasis $(1.1 \%, 11)$, diabetes mellitus $(1.06 \% ; 10)$.

Conclusions: Acute pyelonephritis was more common in hospitalized girls and cystitis in boys.

Escherichia coli was the most common etiologic agent of UTI in children with/without risk factors, while Klebsiella pneumoniae, Pseudomonas aeruginosa and Proteus mirabilis were more frequently in children with risk factors. CAKUT and neurogenic/non-neurogenic bladder dysfunction were significant risk factors of UTI in children.

\section{EP-323 RECURRENT FOCAL BACTERIAL NEPHRITIS - A CASE REPORT AND REVIEW OF THE LITERATURE} Nina Vaezipour, Hanna Schmid, Katrina Evers, Nicole Ritz, Alexandra Goischke

University Childrens Hospital, University Of Basel, Switzerland

Introduction: Acute focal bacterial nephritis (AFBN) is a rare infection in children. AFBN shows an inflammatory intrarenal lesion without evidence of an abscess wall and is therefore considered as an intermediate condition between pyelonephritis and intrarenal abscess. We describe an illustrative case of a recurrent AFBN and a literature review on AFBN.

Material and methods: We performed a literature review using pubmed, EMBASE and google scholar. Data extraction was done for the following variables: patient demographics, antimicrobial duration, underlying conditions and details on relapses or recurrences.

Results: Illustrative case: An 11-year-old Swiss girl presented with multiple episodes pyelonephritis followed by AFBN. After initial oral antimicrobial courses for first five episodes of pyelonephritis, evidence of a pronounced bilateral nephritis on magnetic resonance imaging (MRI) during her 6th episode prompted treatment with intravenous antimicrobial treatment, followed by prophylactic oral therapy and physiotherapy for a coexisting micturition disorder. Despite this management and a normal uroflow examination, she presented with new focal lesions on a repeated MRI 4 weeks after treatment cessation, prompting us to resume a prolonged intravenous antibiotic therapy over 4 weeks.

The literature search identified 24 studies. No single study reported about recurrent AFBN with event-free intervals of several weeks. One prospective study reported about 7 out of 41 individuals with non-further specified treatment failure following a 2-week antimicrobial protocol, whereas no one out of 39 patients treated with a 3-week protocol experienced therapeutic failure. The overall data of the studies showed no clear association between risk of AFBN or relapse and treatment duration or vesico-urethral reflux.

Conclusions: This case highlights a rare presentation of a patient with recurrent $\mathrm{AFBN}$, several weeks following treatment cessation. Despite heterogeneity of the studies, a minimum treatment duration of 3 weeks, investigation for underlying conditions and repeated imaging might be safe approaches in the management of AFBN.

\section{EP-324 DO THE RESISTANCE PATTERNS OF BACTERIAL UROPATHOGENS CHANGE OVER THE YEARS IN CHILDREN?}

İkbal Nur Şafak ${ }^{1}$, Asli Kavaz Tufan ${ }^{2}$, Nuran Çetİn ${ }^{2}$, Ömer Kiliç ${ }^{3}$, Gül Durmaz, ${ }^{4}$

${ }^{I}$ Eskisehir Osmangazi University, Faculty Of Medicine, Department Of Pediatrics

${ }^{2}$ Eskisehir Osmangazi University, Faculty Of Medicine, Department Of Pediatric Nephrology

${ }^{3}$ Eskisehir Osmangazi University, Faculty Of Medicine,department Of Pediatric Infectious Disease

${ }^{4}$ Eskisehir Osmangazi University, Faculty Of Medicine, Department Of Microbiology 
Introduction: Urinary tract infections are among the most common infections of childhood and the development of resistance to antibiotics causes treatment failure. Therefore, the aim of this study is to evaluate the resistance patterns of uropathogens by years and to find risk factors affecting the development of antibiotic resistance.

Material and methods: This study retrospectively assessed 1852 patients (1308 females) between 1 month and 18 years of age with a diagnosis of urinary tract infection and 2340 urine cultures of these patients with uropathogen growth above $10^{5} \mathrm{CFU} / \mathrm{mL}$ from January 2010 to December 2019.

Results: The mean age was $73 \pm 61$ months (1-215 months) and the number of urinary tract infection episodes was $2.5 \pm 3.8$ (1-39). Urinary tract anomalies were found in $15.7 \%$ of the patients, among which hydronephrosis $(8.8 \%)$ was the most common. The most common uropathogens in urine culture were respectively E.coli $(59.6 \%)$ Klebsiella spp. $(11.5 \%)$ and Enterococcus spp. (9.5\%). There was a statistically significant increase in the growth rate of E.coli, Klebsiella pneumoniae and Acinetobacter spp. by years $(\mathrm{p}<0.001)$. The frequency of ESBL positive uropathogens was $16.2 \%$ at ten-year follow-up. ESBL positivity was higher in infants, patients with recurrent UTIs, urinary anomalies, and those who had used prophylactic antibiotics $(\mathrm{p}<0.05)$. Antibiotics with the highest resistance were penicillin (60.8\%), ampicillin $(55.7 \%)$ and trimethoprim-sulfamethoxazole $(40.8 \%)$. While there was no increase in resistance to carbapenems in ESBL positive E.coli, it was found that imipenem and meropenem resistance increased in Klebsiella strains $(\mathrm{p}<0.05)$.

Conclusions: In the treatment of UTI, especially in infants younger than 12 months, and those with urinary anomalies and a history of recurrent UTIs, appropriate antibiotic should be chosen in accordance with local studies.

\section{EP-325 BLADDER BOWEL DYSFUNCTION AND QUALITY OF LIFE IN OBESE CHILDREN AND ADOLESCENTS \\ Seçil Arslansoyu Camlar ${ }^{1}$, Abbasqulu Baghirov ${ }^{2}$, Kayi Eliaçik ${ }^{2}$, Özlem $\ddot{U ̈ z u ̈ m}^{2}{ }^{2}$, Gonca Özyurt ${ }^{3}$, Eren Soyaltin ${ }^{4}$, Gönül Çatli ${ }^{5}$, Demet Alaygut ${ }^{4}$, Fatma Mutlubaș ${ }^{1}$, Bumin Nuri Dündar ${ }^{5}$, Belde Kasap Demir ${ }^{6}$ \\ ${ }^{1}$ University Of Health Sciences, izmir Faculty Of Medicine, Pediatric Nephrology Clinic, izmir Turkey \\ ${ }^{2}$ University Of Health Sciences, izmir Tepecik Training And Research Hospital,pediatric Clinic,izmir,turkey \\ ${ }^{3}$ Izmir Katip Çelebi University,department Of Child And Adolescent Psychiatry,izmir,turkey \\ ${ }^{4}$ University Of Health Sciences, Izmir Tepecik Training And Research Hospital,pediatric Nephrology Clinic,izmir,turkey \\ ${ }^{5}$ Izmir Katip Çelebi University,department Of Pediatric Endocrinology,izmir,turkey \\ ${ }^{6}$ Izmir Katip Çelebi University,department Of Pediatric Nephrology And Rheumatology, Izmir,turkey}

Introduction: Obesity has become a serious health problem with increasing frequency in children and adolescents. Besides metabolic problems, it can also cause bladder-bowel dysfunction (BBD). It is known that both obesity and BBD have negative effects on the quality of life. In this study, we aimed to investigate the effect of BBD on the quality of life in obese children and adolescents.

Material and methods: Obese children and adolescents aged 5 to 17 years who were admitted to pediatric outpatient clinics between April 2019 and December 2019 were included in the study. The cases with normal weight, similar age and sex to the other groups, who came for respiratory tract infection and presented to the Pediatric Nephrology outpatient clinic with signs of bladder-bowel dysfunction were included. The cases who scored 11 or above according to the Bladder Bowel Symptom Score (BBSS) were considered as BBD.

Results: A total of 289 patients, 148 obese and 141 normal weight, with a mean age of $11.7 \pm 3.1$ years were included in the study. In the obese group, the quality of life subscale and total scores were significantly lower than the control group with normal weight. In obese cases, the quality of life subscale and total scores were significantly lower in the group with BBSS $\geq 11$ ( $p<0.001)$. There was a statistically significant difference in the physical health total score and the total scale score in the obese patients with $\mathrm{BBSS} \geq 11$.

Conclusions: In this study, while the negative effects of both obesity and $\mathrm{BBD}$ on the quality of life were revealed, it was seen that BBD affects the quality of life even more negatively, and both of them are comorbid situations with each other. In addition, it was concluded that the quality of life is most affected when both MBD and obesity coexist.

\section{EP-326 GROSS HEMATURIA IN PATIENTS OF AFRICAN ORIGIN, WHAT SHOULD WE THINK ABOUT?}

Nélida García Pérez ${ }^{1}$, Maite Goicoechea Irigaray ${ }^{2}$,

Leire Gondra Sangroniz ${ }^{3}$, María Herrero Goñi ${ }^{4}$,

Leire Madariaga Domínguez, ${ }^{5}$, Mireia Aguirre Meñica ${ }^{4}$,

Ana Vinuesa Jaca ${ }^{4}$

${ }^{1}$ Basurto University Hospital. Iis Biocruces Bizkaia Health Research

Institute. University Of The Basque Country

${ }^{2}$ Basurto University Hospital

${ }^{3}$ Cruces University Hospital. Iis Biocruces Bizkaia Health Research Institute. University Of The Basque Country

${ }^{4}$ Cruces University Hospital. Iis Biocruces Bizkaia Health Research Institute

${ }^{5}$ Cruces University Hospital. Iis Biocruces Bizkaia. University Of The Basque Country. Ciberer. Ciberdem

Introduction: The esquistosomiasis (bilharziasis) is a parasitic disease produced by the Schistosoma. It is endemic of sub-Saharan Africa, Latin America and Asia, affecting more than 200 million people/year. Moreover, the immigration and the international trips have contributed to the growing prevalence in European countries. Schistosoma haematobium predominantly leads to genitourinary complications.

Material and methods: We present 3 cases of African children diagnosed with schistosomiasis who presented with hematuria.

Results: We present three children aged 8,11 and 13 years from Mali. They consulted for recurrent macrohematuria during the last months. Physical examination was normal. All presented significant eosinophilia $(35 \%, 28 \%, 17 \%)$. All presented ultrasound images of segmental thickening of the bladder wall. Clinical and complementary tests were suggestive of bladder squistosomiasis, which was confirmed by isolation of Schistosoma haematobium in urine. All had positive Schistosoma serology. They received treatment with praziquantel with good response. Hematuria disappeared with absence of squistosomes in urine, and subsequently, in the bladder ultrasound a normalization of lesions was observed in the first year of follow-up.

Conclusions: Although schistosomiasis is a typically tropical disease, the large number of migratory movements and the international tourism make necessary to take it into account in the differential diagnosis of hematuria, and in the comprehensive assessment of the reception of an immigrant child. At present, there is no effective preventive drug. Preventive measures are recommended, avoiding bathing in endemic areas. A serological screening should be done in travelers or immigrants who are in contact with contaminated water, and in positive cases, a determination of parasites in stool and urine should be done. Patients with parasitism should be treated even if they are asymptomatic.

\section{EP-327 IS BLADDER DYSFUNCTION UNDERDIAGNOSE IN FUNCTIONAL CONSTIPATION}

Eren Soyaltın ${ }^{1}$, Seçil Arslansoyu Çamlar ${ }^{2}$, Yeliz Çă̆an Appak ${ }^{3}$, Maşallah Baran ${ }^{3}$, Demet Alaygut ${ }^{1}$, Betül Aksoy ${ }^{4}$, Fatma Mutlubaş ${ }^{2}$, Belde Kasap Demir ${ }^{5}$ 
${ }^{1}$ University Of Health Sciences,izmir Tepecik Training And Research Hospital,pediatric Nephrology Clinic,izmir,turkey

${ }^{2}$ University Of Health Sciences, Izmir Faculty Of Medicine;pediatric Nephrology Clinic, izmir, turkey

${ }^{3}$ Izmir Katip Celebi University, Department Of Pediatric Gastroenterology,izmir,turkey

${ }^{4}$ University Of Health Sciences, Izmir Tepecik Training And Research Hospital, Pediatric Gastroenterology Clinic,izmir,turkey

${ }^{5}$ Izmir Katip Çelebi University, Department Of Pediatric Nephrology And Rheumatology, Izmir,turkey

Introduction: Bladder-bowel dysfunction (BBD) is a common problem in childhood. Due to the existing anatomical and physiological conditions and chronic functional constipation (FC) can accompany to voiding disorders. Constipation should be questioned and treated frequently in children presenting with voiding dysfunction, because it is known that BBD does not improve before constipation improves. However, in patients presenting with the complaint of constipation, BBD is generally not considered and can be overlooked. We aimed to determine the frequency of $\mathrm{BBD}$ in pediatric cases presenting with $\mathrm{FC}$ and evaluate it with the Bladder-Bowel Symptom Scoring questionnaire.

Material and methods: Children ages of 5-18 diagnosed with functional constipation completed the Bladder Bowel Symptom Score (BBSS). Children with BBSS above 11 were considered bladder-bowel dysfunction. Rome IV criteria were used for diagnosis of FC.

Results: Eighty-four patients with FC included into the study. Mean age of participants was 9.03 (5.0-16.0) years. Forty-eight $(57.1 \%)$ of the participants were girls, and 36(42.9\%) were boys. The mean duration of constipation of the patients was between 2 - 168 months. Presence of painful and hard defecation (79.8\%) and abdominal pain(75.0\%) were the most common constipation symptoms. In 39 (46.4\%) patients, BBSS was found above 11 points and they were diagnosed with bladder-bowel dysfunction. The most common urinary tract symptoms were urgency $(63.1 \%)$ and frequency $(50.0 \%)$. A significant positive correlation was detected between the BBSS and the duration of constipation (p:0.042). Significant negative correlation was also found between constipation age and BBSS score (p:0.008). Patients with a younger age of constipation had higher BBSS.

Conclusions: Bladder dysfunction often accompanies children with functional constipation. It is important to evaluate children with $\mathrm{FC}$ in terms of accompanying BBSS findings.

\section{EP-328 AN EVALUATION OF THE BOWEL AND BLADDER DYSFUNCTION SYMPTOMS OF THE PATIENTS WITH PSYCHIATRIC PROBLEMS IN TERTIARY PEDIATRIC PSYCHIATRY CENTER}

Tugba Samur ${ }^{1}$, Bahadir Samur ${ }^{1}$, Sevgi Ozmen ${ }^{3}$, Neslihan Gunay ${ }^{2}$, Esra Demirci ${ }^{3}$, Aynur Balaban ${ }^{2}$, Sibel Yel ${ }^{2}$, Hakan Poyrazo Ğlu ${ }^{2}$, Ismail Dursun ${ }^{2}$

${ }^{1}$ Erciyes University, Faculty Of Medicine, Kayseri

${ }^{2}$ Erciyes University Faculty Of Medicine, Department Of Pediatrics, Division Of Nephrology

${ }^{3}$ Erciyes University Faculty Of Medicine, Department Of Pediatric Physiatry, Kayseri, Turkey

Abstract: In this study, our aim was to evaluate psychosocial risk factors, functional bladder and bowel problems, bladder bowel dysfunction (BBD) and other related parameters in children who admitted to child psychiatry outpatient clinic based on the underlying psychiatric disorders. This study was performed in patients who applied to Erciyes University Medical Faculty Child Psychiatry Outpatient Clinic. Patients with organic problems and autistic spectrum disorders were excluded. Bladder intestinal dysfunction questionnaire, depression and anxiety scales, prepared on the basis of international standardized scales, were administered to children aged 6-14. In our study, 440 patients between the ages of 6 and 14 who applied to Child Psychiatry outpatient clinic between March 2019 and December 2019 were evaluated. 227 (51.6\%) of the patients were attention deficit and hyperactivity disorder (ADHD), 23 (5.2\%) of the patients were anxiety disorder, $23(5.2 \%)$ of the patients were adjustment disorder, $89(20.2 \%)$ of the patients were grouped as other psychopathologies besides $72(16.4 \%)$ were considered as no psychopathology. A statistically significant relationship was found with ADHD in all three urinary incontinence patterns including daytime incontinence, nighttime incontinence and diurnal incontinence. A significant relation was found between anxiety disorders and nighttime urinary incontinence. Other psychopathologies were not being associated with incontinence. A statistically significant relationship was found between cigarette exposure and all three urinary incontinence patterns. There was a significant relationship between having a new sibling and daytime urinary incontinence and between lower urinary tract symptoms and BBD. To the best of our knowledge, this is the first study evaluating children with broad spectrum of psychiatric disorders in terms of BBD. We found a strong relationship between $\mathrm{ADHD}$, anxiety, social stressors, and BBD and urinary incontinence. In some patients, this situation should be considered in diagnosis and treatment since functional bladder and intestinal disorders and psychiatric problems may be intertwined.

\section{EP-329 UNEXPECTED CAUSE AND SUCCESSFUL MANAGEMENT OF TYPICAL URINARY TRACT INFECTION SYMPTOMS}

Demet Alaygut ${ }^{1}$, Özgür Özdemir Şimşek ${ }^{1}$, Fatma Ceren Sarioglu ${ }^{2}$, SeÇil Arslansoyu Çamlar ${ }^{1}$, Fatma Mutlubaş ${ }^{1}$, Belde Kasap Demir ${ }^{3}$

${ }^{I}$ Izmir University Of Health Sciences Tepecik Education And Research Hospital Department Of Pediatric Nephrology

${ }^{2}$ Izmir University Of Health Sciences Tepecik Education And Research Hospital Department Of Radiology Divison Pediatric Radiology

${ }^{3}$ Katip Celebi University Faculty Of Medicine Department Of Pediatric Nephrology And Rheumatology

Introduction: Emphysematous pyelonephritis (EPN) is a necrotizing infection with gas formation, affecting the renal parenchyma and, in some cases, perirenal tissue. Here, a patient presenting with a typical upper urinary system clinic but diagnosed with emphysematous pyelonephritis is presented. Here, we present a case that presents with a typical upper urinary system clinic but diagnosed with emphysematous pyelonephritis is presented.

Material and methods: Case: A 6-year-old girl was brought with fever, vomiting and burning pain while urinating 3 days ago. It was learned that amoxicillin-clavulanate treatment was initiated with the initial diagnosis of urinary tract infection, but persistent fever $\left(39^{\circ} \mathrm{C}\right)$, weakness, nausea, vomiting, deficient oral intake, flank pain and widespread abdominal pain continued despite the treatment. On physical examination she had tenderness on the left costovertebral angle and in the left lower abdominal quadrant on palpation. Laboratory test showed that leucocytosis, high acute phase reactants high D-dimer and fibrinogen levels. Kidney and liver function test results and electrolytes were within normal limits. In urinalysis, there was protein +1 , leukocyte +3 , erythrocyte +3 , abundant leukocytes and bacteria. Renal ultrasonography were normal. The COVID-19 PCR test result was negative. In contrast-enhanced abdomen MRI T1-weighted coronal sections showed in the upper pole of the left kidney and millimetric hypointense air signals.

Results: The presence of gas supported the diagnosis of EPN due to gas formation. Empirical treatment with meropenem and amikacin was initiated. At the end of the treatment, control MRI imaging revealed that the lesion had completely healed. Dimercaptosuccinic acid (DMSA) examination performed after 4 months revealed normal. No scar or signs of infection were seen 
Conclusions: EPN is a rare urinary system infection characterized by gas formation in the urinary system, especially in children. It is difficult to differentiate it from classical pyelonephritis cases with clinical and laboratory findings.

\section{EP-330 BEYOND RECURRENT URINARY TRACT INFECTIONS IN ADOLESCENTS: CASE REPORT OF A RARE DISEASE \\ Diana Mota Almeida, Catarina Neves, Teresa Almeida Lopes, João Filipe Nico, Hugo Antunes, Nádia Brito \\ Hospital Distrital Figueira Da Foz}

Introduction: Urinary tract infections (UTI) are very common in pediatric age. Cases of recurrent UTI with other risk factors should lead to the suspicion of an underlying disease. The objective of this work is to raise awareness of an unusual cause of recurrent UTI.

Material and methods: Information gathering on a patient with recurrent UTI that lead to the diagnosis of a rare disease.

Results: A 16-year-old male was admitted to the emergency department with fever, dysuria, pollakiuria and macroscopic hematuria that started the day before. He was a nonsmoker, with no previous history of kidney/ urologic diseases. Urine culture revealed an Escherichia Coli, that was treated with oral Amoxicillin-Clavulanate. Within the next 2 months, he was admitted several times with similar complaints, always with the diagnosis of UTI (Escherichia Coli for two times and Enterobacter cloacae with multiple antibiotic resistances in the last time).

The bladder ultrasound revealed an $18 \mathrm{~mm}$ exophytic and branched mass adjacent to the right ureteric meatus, that was later confirmed and removed with cystoscopy. The anatomopathological analysis revealed a well differentiated papillary urothelial carcinoma of low malignant potential. After cystoscopy, no more UTI were reported, and the patient remains asymptomatic.

Conclusions: The authors present this case to warn that recurrent UTI in male adolescents should lead to further investigation.

Although bladder cancer is a common disease, it is very rare in young, nonsmoking individuals. The most common presentation is gross and painless hematuria, like in this case. However, hematuria has a wide range of differential diagnosis, with the most likely being UTI, which can delay the diagnosis. Moreover, a relationship between recurrent UTI and bladder cancer has been described.

We intend to draw attention to this rare but potentially fatal disease to avoid a late diagnosis and poorer prognosis.

EP-331 MILD ENCEPHALOPATHY AND REVERSIBL SPLENIAL LESION (MERS) WITH ACUTE FOCAL BACTERIAL NEPHRITIS: A RARE CASE

Pelin Elibol ${ }^{1}$, Gökçen Erfidan ${ }^{2}$, Alper Ciçek ${ }^{l}$, Emel Berksoy ${ }^{l}$, Seçil Arslansoyu Çamlar ${ }^{2}$, Belde Kasap ${ }^{3}$, Demet Alaygut ${ }^{2}$

${ }^{1}$ Izmir University Of Health Sciences Tepecik Education And Research Hospital Department Of Pediatric Emergency

${ }^{2}$ Izmir University Of Health Sciences Tepecik Education And Research Hospital Department Of Pediatric Nephrology

${ }^{3}$ Izmir Katip Celebi University Faculty Of Medicine, Department Of Pediatric Nephrology And Rheumatology

Introduction: Acute focal bacterial nephritis (AFBN), formerly known as lobar nephronia, is a localized, bacterial and inflammatory involvement of the renal lobules without liquefaction and is a process located between pyelonephritis and renal abscess. Here, a case is presented with MERS and AFBN.

Material and methods: A nine-year-old male patient was brought to the emergency room with complaints of convulsion for 5 minutes. On physical examination, her body temperature was $38.4^{\circ} \mathrm{C}$ and the other were in norrmal area. Hyperemic and hypertrophic tonsils were present on oropharyngeal examination, and there was significant tenderness on the left side of the abdomen. Blood biochemistry tests were normal except for hyponatremia and high acute phase reactants. Urinalysis was negative for protein, occult blood, and leukocytes. Lumbar puncture was performed and CSF was found normal. Abdominal ultrasound (US) and abdominal tomography $(\mathrm{CT})$ were performed because of the patients high C-reactive protein (CRP) level, high fever and septic appearance. Abdominal CT showed hypodense patches in the upper and lower poles of the left kidney consistent with AFBN (Figure 1) Cefotaxime treatment was started in the patient.

Results: Brain diffusion-weighted magnetic resonance imaging (DW-MRI) was obtained to exclude other pathologies affecting the central nervous system. Hyperintensity and diffusion restriction were noted in the corpus callosum splenium (Figure 2). The pathological signal change in the splenium region of the corpus callosum disappeared in the brain DW-MRI repeated on the 14th day of treatment (Figure 3). The patient was discharged after 21 days of antibiotic therapy.

Conclusions: AFBN should be kept in mind in cases with toxic appearance, resistant fever, and high acute phase reactant findings. It could be together with MERS.

EP-332 AN ALTERNATIVE URINARY SYSTEM IRRIGATION METHOD IN FUNGAL URINARY TRACT INFECTIONS

Gökçen Erfidan ${ }^{1}$, Eren Soyaltin ${ }^{1}$, Lale Dadashova ${ }^{2}$,

Özgür Özdemir Şimşek ${ }^{l}$, Cemaliye Başaran ${ }^{1}$, Seçil Arslansoyu Çamlar ${ }^{3}$, Fatma Mutlubaș ${ }^{3}$, Belde Kasap Demir ${ }^{4}$, Demet Alaygut ${ }^{1}$

${ }^{I}$ University Of Health Sciences, Tepecik Training And Research Hospital, Department Of Pediatrics, Division Of Nephrology, Izmir, Turkey

${ }^{2}$ University Of Health Sciences, Tepecik Training And Research Hospital. Department Of Pediatrics, İzmir, Turkey

${ }^{3}$ University Of Health Sciences, Izmir Faculty Of Medicine, Department Of Pediatrics, Division Of Nephrology, Izmir, Turkey

${ }^{4}$ Izmir Katip Celebi University, Faculty Of Medicine, Department Of Pediatrics, Division Of Nephrology And Rheumatology, Izmir, Turkey

Introduction: Fungal urinary tract infections (UTI) are increasingly encountered in every age. However, the difficulty in distinguishing colonization and infection complicates diagnosis and treatment. Also, there are questions about who, for how long and which agent to treat. In some cases, local irrigations may also come up, apart from systemic treatment. Material and methods: One child with vesicostomy tube (VT) and two children with nephrostomy tubes (NT) due to obstructive hydronephrosis, and were diagnosed fungal UTI. Due to resistant candiduria despite systemic treatment, Amphotericin-B irrigation was started in all three cases. Results: NT/VT, drainage bag and serum infusion set were connected with a three-way stopcock. Amphotericin-B $(50 \mathrm{mg} / \mathrm{L})$ was sent into renal pelvis through the stopcock and left for 60 minutes, then drained out. The procedure was performed continuously for 24-hours/5-day. With this technique, we didn't need a second NT, and we gave enough time for anti-fungal agent to reach bladder.

Conclusions: In conclusion, fungal UTIs remains an important problem and irrigation should be started quickly in cases with resistant fungal growth before fungus ball development.

\footnotetext{
EP-333 NEONATAL HYPERTENSION: WHEN IS ONLY TRANSITORY

Beatriz Parreira De Andrade ${ }^{1}$, Catarina Menezes ${ }^{2}$, Helena Silva ${ }^{l}$, Ana Sofia Rodrigues ${ }^{1}$, Aida Azevedo ${ }^{1}$, Filipa Almeida ${ }^{1}$, Marco Pereira ${ }^{3}$, Álvaro Sousa ${ }^{l}$

${ }^{1}$ Centro Hospitalar Do Médio Ave

${ }^{2}$ Centro Materno Infantil Do Norte

${ }^{3}$ Unidade Local De Saúde Matosinhos
} 
Introduction: Neonatal hypertension is an uncommon clinical problem. The main cause is renovascular followed by other vascular causes, congenital disorders, pulmonary, iatrogenic and endocrine causes. The authors present a rare cause of transitory hyperaldosteronism secondary to renal infection.

Material and methods: Case report and systematic review.

Results: A 22 day- old boy, term newborn, without any prenatal or postnatal complications presented to paediatric emergency due to poor weight gain. After blood and urine workup he was diagnosed with urinary tract infection (UTI) and mild hyponatraemic dehydration associated with hyperkalaemia and immediately initiated ampicillin and cefotaxime and fluid therapy. During hospitalization, persistent high blood pressure (BP) levels (>P97) were observed. Promptly renal ultrasound and eco-Doppler showed no alteration as well as normal hormonal study and renal analytical function so congenital adrenal hyperplasia was excluded. At D9 of hospitalization, he presented normalization of BP values. Further laboratory tests revealed aldosterone levels $335.4 \mathrm{ng} / \mathrm{dl}$ (N 2-70 ng/dl) and renin $1684 \mathrm{mUI} / \mathrm{L}(\mathrm{N}<46)$, renal function and electrolytes were normal. No medication was introduced. 4 weeks later he presented a UTI relapse (multisensible Escherichia coli) with normal BP and renal function. Follow-up paediatric outpatient revealed a decreasing level of aldosterone and renin, normal BP and renal function. No further UTI, no imaging evidence of renal sequalae and good weight evolution.

Conclusions: Transitory hyperaldosteronism is more frequent in premature neonates due to renovascular system immaturity; some studies reveal that aldosterone levels gradually reach normal levels around 12 months of age. Transitory hyperaldosteronism is rarely seen in post term neonates and secondary to neonatal pyelonephritis. This case enhances the importance of a accurate information of normal range plasma aldosterone and renin based on gestational age and day of life as well as a proper approach to this pathology.

\section{EP-334 THE FREQUENCY OF OVERACTIVE BLADDER IN} CHILDREN IN THE PERIOD OF COVID-19 PANDEMIC

Irina Balalaeva ${ }^{1}$, Anastasiya Egorkina ${ }^{2}$

${ }^{1}$ Voronezh Children Polyclinic № 11

${ }^{2}$ Moscow Children Polyclinic № 30

Introduction: We determined the frequency of the overactive bladder $(\mathrm{OAB})$ in children during the COVID-19 isolation quarantine measures. Material and methods: The study was conducted in 2282 patients of 317 years old in April-December of 2020 and 2019 years.

Results: The frequency of OAB has been twice more in April-June of 2020 than 2019 year. It was the biggest in May and June of 2020. Girls and children after 7 years old were dominated.

Conclusions: Increased amount of children with $\mathrm{OAB}$ was noticed after COVID-19 lockdown in 2020 under the influence of stressful factors and anxiety.

Publisher's note Springer Nature remains neutral with regard to jurisdictional claims in published maps and institutional affiliations. 